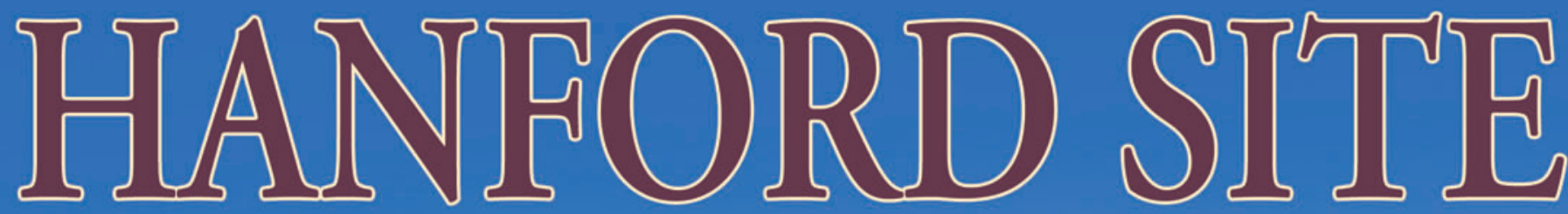

\title{
ENVIRONMENTAL SURVEILLANCE DATA REPORT
}

\section{for Calendar Year 2008}

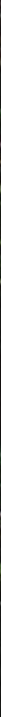




\title{
DISCLAIMER
}

This report was prepared as an account of work sponsored by an agency of the United States Government. Reference herein to any specific commercial product, process, or service by trade name, trademark, manufacturer, or otherwise does not necessarily constitute or imply its endorsement, recommendation, or favoring by the United States Government or any agency thereof, or Battelle Memorial Institute. This report is a summary of major or significant activities occurring at the Hanford Site only, and is not a full disclosure of all details associated with Hanford-related activities, nor a substitute for legally required information subject to reporting requirements regarding releases, violations, etc.

\author{
PACIFIC NORTHWEST NATIONAL LABORATORY \\ operated by \\ BATTELLE \\ for the \\ UNITED STATES DEPARTMENT OF ENERGY \\ under Contract DE-AC05-76RL01830
}

Printed in the United States of America
May be available to DOE and DOE contractors from the
Office of Scientific and Technical Information,
P.O. Box 62, Oak Ridge, TN 37831-0062;
ph: (865) $576-8401$
fax: (865) 576-5728
email: reports@adonis.osti.gov

Available to the public from the National Technical Information Service, U.S. Department of Commerce, 5285 Port Royal Rd., Springfield, VA 22161

ph: (800) 553-6847

fax: (703) 605-6900

email: orders@ntis.fedworld.gov

online ordering: http://www.ntis.gov/ordering.htm

The cover photo shows swards of exotic purple mustard (Chorispora tenella) and the remains of last year's Russian thistle (Salsola tragus) that dominate swales in the Cold Creek Valley burned by past wildfires. At higher elevations on Rattlesnake Ridge, native perennial bunchgrasses persist after being burned and present green slopes during the spring and early summer. Photo is courtesy of S Butner, Pacific Northwest National Laboratory, Richland, Washington. The cover design is by SB Colson, Pacific Northwest National Laboratory, Richland, Washington. 


\title{
Hanford Site Near-Facility Environmental Monitoring Data Report For Calendar Year 2008
}

\author{
C. J. Perkins \\ M. C. Dorsey \\ S. M. McKinney \\ J.W. Wilde \\ EnergySolutions \\ Richland, Washington 99354
}

September 2009

Prepared for

the U.S. Department of Energy

under Contract DE-AC06-76RL01830

Submitted by

Pacific Northwest National Laboratory

Richland, Washington 99354 


\section{LIST OF TERMS}

$\mathrm{Bq}$

CERCLA

CFR

CSB

CVDF

D\&D

DCG

DCRT

DOE

dpm

EDE

EDP (code)

ERDF

$\mathrm{FH}$

FSWO

GEA

IDF

LERF

LLBG

mrem/yr

NFM

PFP

PHMC

PNNL

PUREX

QA

RCC

RCRA

RMA

RPP

TEDF

TLD

WAC

WDOH

WSCF
Becquerel

Comprehensive Environmental Response, Compensation, and Liability Act of 1980

Code of Federal Regulations

Canister Storage Building

Cold Vacuum Drying Facility

decontamination and decommissioning

derived concentration guides

double-contained receiver tank

U.S. Department of Energy

disintegrations per minute

effective dose equivalent

environmental data point (identification number indicating sample

location)

Environmental Restoration Disposal Facility

Fluor Hanford, Inc.

EnergySolutions Federal Services, Inc., Western Operations

gamma energy analysis

Integrated Disposal Facility

Liquid Effluent Retention Facility

low-level burial ground

millirems/year

Near-facility monitoring

Plutonium Finishing Plant

Project Hanford Management Contract

Pacific Northwest National Laboratory

Plutonium-Uranium Extraction

quality assurance

River Corridor Closure

Resource Conservation and Recovery Act of 1976

radioactive material area

River Protection Project

Treated Effluent Disposal Facility

thermoluminescent dosimeter

Washington Administrative Code

Washington State Department of Health

Waste Sampling and Characterization Facility 


\section{CONTENTS}

1.0 NEAR-FACILITY ENVIRONMENTAL MONITORING AT HANFORD ......1-1

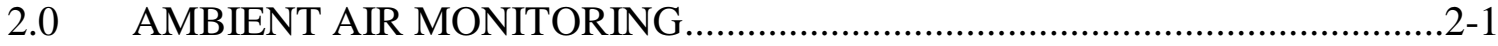

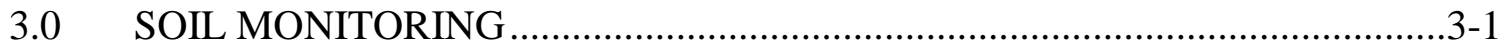

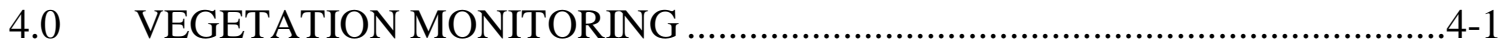

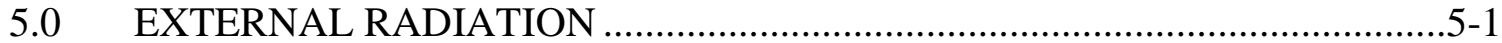

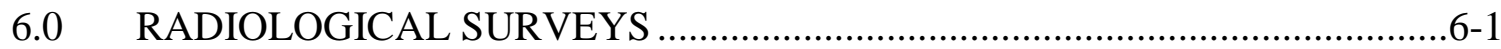

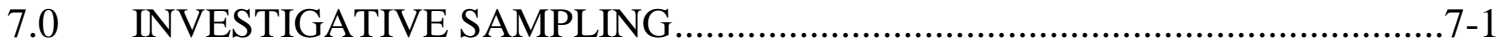

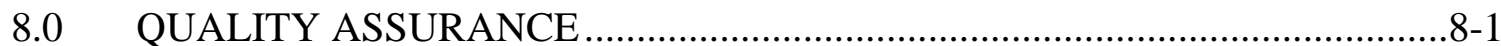

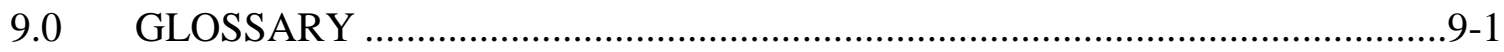

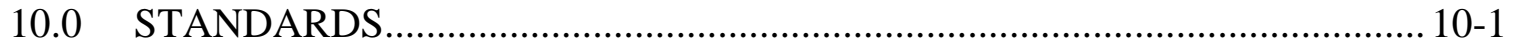

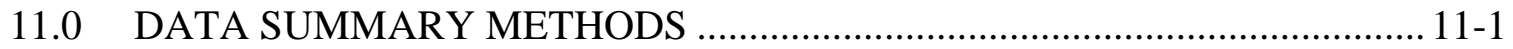

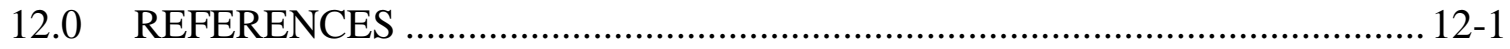




\subsection{NEAR-FACILITY ENVIRONMENTAL MONITORING AT HANFORD}

Near-facility environmental monitoring is defined as monitoring near facilities that have the potential to discharge or have discharged, stored, or disposed of radioactive or hazardous materials. Monitoring locations are associated with nuclear facilities such as the Plutonium Finishing Plant (PFP) and the Canister Storage Building (CSB); inactive nuclear facilities such as N Reactor and the Plutonium-Uranium Extraction (PUREX) Facility; and waste storage or disposal facilities such as burial grounds, cribs, ditches, ponds, tank farms, and trenches.

Much of the monitoring consists of collecting and analyzing environmental samples and methodically surveying areas near facilities. The program is also designed to evaluate acquired analytical data, determine the effectiveness of facility effluent monitoring and controls, assess the adequacy of containment at waste disposal units, and detect and monitor unusual conditions. The program implements applicable portions of U.S. Department of Energy (DOE) Orders 435.1 (DOE 2001), 450.1A (DOE 2008), and 5400.5 (DOE 1993); DOE Manual 231.1-1A (DOE 2007), Environment, Safety, and Health Reporting Manual; Washington Administrative Code (WAC) 246-247; Title 40, Code of Federal Regulations (CFR) Part 61 (40 CFR 61), Subpart H; and 10 CFR 835.

Several types of environmental media are sampled near facilities to monitor waste management and environmental restoration activities, and to evaluate the effectiveness of effluent treatment and control practices. Routine sampling and monitoring includes ambient air, soil, vegetation, and external radiation. The parameters typically monitored are radionuclide concentrations and radiation fields. Sampling methods are discussed in detail in the EnergySolutions Federal Services, Inc., Western Operations (FSWO) Manual FSWO-OEM-001, Operational Environmental Monitoring (FSWO 2008) and Fluor Hanford, Inc. (FH) EP-DI-611, Environmental Protection Desk Instruction Near-Facility Environmental Monitoring, (FH 2008).

Samples are collected from known or expected effluent pathways. These pathways are generally downwind of potential or actual airborne releases and down gradient of past liquid discharges. Table 1-1 shows the type and location of routine near-facility monitoring (NFM) samples collected in 2008.

Table 1-1. Near-Facility Routine Environmental Monitoring Samples and Locations, 2008.

\begin{tabular}{cccccccccccccc}
\multicolumn{10}{c}{$\begin{array}{c}\text { Number of } \\
\text { Sample }\end{array}$} & \multicolumn{110}{c}{ Operational Area } \\
\cline { 3 - 13 } Sample Type & Locations & $100-\mathrm{B} / \mathrm{C}$ & $100-\mathrm{D}$ & $100-\mathrm{F}$ & $100-\mathrm{H}$ & $100-\mathrm{K}$ & $100-\mathrm{N}$ & East & West & 400 & 600 & ERDF $^{(\text {a) }}$ \\
\hline Air & 92 & 3 & 4 & 5 & 4 & 10 & 3 & 21 & 24 & 7 & 8 & 3 \\
Soil & 85 & 3 & 4 & 5 & 4 & 0 & 0 & 14 & 25 & 14 & 15 & 1 \\
Vegetation & 66 & 0 & 0 & 0 & 0 & 0 & 3 & 12 & 24 & 13 & 14 & 0 \\
External Radiation & 124 & 4 & 0 & 0 & 0 & 18 & 6 & 43 & 25 & 25 & 0 & 3
\end{tabular}

(a) Environmental Restoration Disposal Facility in the 200 West area. 
This Appendix contains brief discussions, specific sampling location information, and complete analytical data results for the various near-facility environmental monitoring efforts for 2008. Detailed discussions and summarized analytical results are provided in PNNL-18427, Hanford Site Environmental Report for Calendar Year 2008 (PNNL 2009a)

\subsection{AIR MONITORING}

Near-facility air sampling monitors the effectiveness of waste management and environmental remediation controls and effluent treatment systems in reducing effluents and emissions. These air samplers also monitor diffuse source emissions.

Ambient air monitoring is conducted to determine baseline concentrations of radionuclides in the operations areas, assess the impact of operations on the local environment, and monitor diffuse and fugitive emissions from sources located within the operations area. These measurements also provide an indication of the Project Hanford Management Contract (PHMC), River Protection Project (RPP), and River Corridor Closure (RCC) Project managed facilities' performance and are used to demonstrate compliance with environmental protection criteria.

In 2008, air radioactivity was sampled by a network of continuously operating samplers at 92 locations. Location-specific maps and monitoring results are provided in Section 2.0, "Ambient Air Monitoring."

\subsection{SOIL SAMPLING}

Soil samples were collected on or adjacent to waste disposal units and from locations downwind and near or within the boundaries of the operating facilities. Soil samples were collected to detect potential migration and deposition of facility effluents. Migration of radionuclides can occur as the result of resuspension from radioactively contaminated surface areas or intrusion by animals.

Radiological analyses of soil samples included strontium-90, plutonium-239/240, isotopic uranium, and gamma-emitting radionuclides. Location-specific maps and the analytical results are presented in Section 3.0, "Soil Monitoring."

\subsection{VEGETATION SAMPLING}

Vegetation samples were collected on or adjacent to waste disposal units and from locations downwind and near or within the boundaries of the operating facilities. Vegetation samples were collected to detect potential migration of facility effluents. Migration of radionuclides into vegetation can occur primarily as the result of absorption by the roots growing on or near underground and surface water disposal units. 
Radiological analyses of vegetation samples included strontium-90, plutonium-239/240, isotopic uranium, and gamma-emitting radionuclides. Location-specific maps and the analytical results are presented in Section 4.0, "Vegetation Monitoring."

\subsection{EXTERNAL RADIATION}

External radiation levels were monitored near facilities and waste handling, storage, and disposal sites to measure, assess, and control the impacts of operations. Thermoluminescent dosimeters (TLD) are used at numerous fixed locations to gather dose rate information over extended periods of time. TLD results can be used individually or averaged to determine dose rates in a given area for a particular sampling period.

Environmental dosimeters measure dose rates from all types of external radiation sources, including cosmic radiation, naturally occurring radioactivity in air and soil, and fallout from nuclear weapons testing, as well as any contribution from Hanford Site activities. During any year, changes in soil moisture and snow cover can cause external radiation levels to vary from $15 \%$ to $25 \%$ at any given location. The results are reported in units of millirems per year (mrem/yr). Individual TLD results and their locations are provided in Section 5.0, "External Radiation.”

\subsection{RADIOLOGICAL SURVEYS}

Waste disposal sites and the surrounding terrain are surveyed to detect and characterize radioactive surface contamination. Routine radiological surveys are conducted across the surfaces of underground radioactive material areas and along the perimeters of contamination areas. Locations include cribs, trenches, retention basins, ponds, ditches, solid waste disposal sites, unplanned release sites, tank farm perimeters, stabilized waste disposal sites, roads, and firebreaks in and around the Site operational areas. A discussion and survey location maps are provided in Section 6.0, "Radiological Surveys."

In 2008, the Hanford Site had approximately 3,583 ha (8,853 acres) of posted outdoor surface contamination, and 584 ha (1,443 acres) of posted underground radioactive material, not including the production facilities (e.g., PUREX, T-Plant, etc.). The total area of surface contamination was approximately six times larger than the area of underground radioactive material.

\subsection{INVESTIGATIVE SAMPLING}

Investigative sampling was conducted in the operations areas to confirm the absence or presence of radioactive and/or hazardous contaminants. Investigative sampling took place near facilities, such as storage and disposal sites, for at least one of the following reasons:

- To follow up radiological surface surveys that had indicated radioactive contamination was present. 
- To conduct preoperational surveys to characterize the radiological/hazardous conditions at a site prior to facility construction, operation, or ultimate remediation.

- To determine if biotic intrusion (e.g., animal burrows or deep-rooted vegetation) has created a potential for contaminants to spread.

- To determine the integrity of waste containment systems.

Generally, the predominant radionuclides detected during these efforts were activation and fission products in the 100 Areas, fission products in the 200 Areas, and uranium in the 300 Area. Hazardous chemicals generally have not been identified above background levels in preoperational environmental monitoring samples. Complete results and general discussion of special characterization samples analyzed in 2008, are provided in Section 7.0, "Investigative Sampling.” 


\subsection{AMBIENT AIR MONITORING}

Air samplers are located primarily at or near (within approximately $500 \mathrm{~m}[1,600 \mathrm{ft}]$ ) sites and/or facilities having the potential for, or history of, environmental releases, with emphasis on potential source terms as well as prevailing wind direction. Meteorological conditions are monitored continuously by the Pacific Northwest National Laboratory (PNNL) meteorology stations, which are strategically positioned in and around the Hanford Site.

A network of continuously operating samplers at 92 NFM locations sampled radioactivity in air during 2008. Some air sampling stations provided monitoring for more than one project (Table 2-1). Data from several PNNL ambient air monitoring stations were utilized in 2008 to provide additional air monitoring information for several RCC remediation projects. The RCC projects and the associated PNNL stations are listed in Table 2-2. The 2008 PNNL air monitoring results can be found in PNNL-18427, Appendix 1.

Near-facility air monitoring location maps are provided in Figures 2-1 through 2-12. Historical air sampling results for selected radionuclides for the 100-K, 100-N, 200, and 300 Areas are represented in graph form in Figures 2-13 through 2-24.

A summary of near-facility ambient air sampling results collected during 2008 is presented in Table 2-3. The 2008 composited, sampler-specific monitoring results are provided in Table 2-4. Additional discussion of the 2008 air sampling results can be found in Section 10.2 of PNNL-18427.

The Hanford Site Air Operating Permit (Federal Facility License FF-01) requires regulatory notification for composite (isotopic) air sample results that exceed $10 \%$ of the U.S. Environmental Protection Agency's Table 2 (40 CFR 61, Appendix E, Table 2) values. During 2008, the following notifications were submitted to the Washington State Department of Health (WDOH):

- NFM sampling station N165 (200 West Area)

- Plutonium-239/240 and Americium-241, first-half of 2008

- Plutonium-239/240, second-half of 2008

One suspected source of the elevated plutonium results is the nearby, retired 216-ZP-1C crib/trench. This facility received liquid waste from the PFP until 1995. In November 2002, the building that had provided electrical service to N165 was demolished and the station was relocated to the present location. Historical plutonium-239/240 results observed at monitoring station N165 are included in Figure 2-22.

- NFM sampling station N977 (200 East Area, southeast of PUREX)

- Plutonium-239/240, first-half of 2008 
No facility/project activity was determined to be a probable cause of this elevated result. Review of the biweekly air sample results obtained during the composite period revealed that there were no statistically elevated total alpha/beta results during the period.

- NFM sampling station N570 (600 Area, BC Controlled Area remediation project)

- Cesium-137, second-half of 2008

Field remediation activity at this known, contaminated site was the suspected cause of this elevated result.

- NFM sampling stations at 100-K East

- N401, Plutonium-239/240 and Americium-241, second-half of 2008

- N402, Plutonium-239/240 and Americium-241, second-half of 2008

- N403, Cesium-137, Plutonium-239/240 and Americium-241, second-half of 2008

- N404, Plutonium-239/240 and Americium-241, second-half of 2008

A radiological contamination event that occurred in July during decontamination and decommissioning (D\&D) activities was the probable cause of these elevated results.

Near-facility environmental air samplers operate at a flow rate of $0.057 \mathrm{~m}^{3} / \mathrm{min}\left(2 \mathrm{ft}^{3} / \mathrm{min}\right)$, drawing a sample through a $47 \mathrm{~mm}$ (2-inch), open-faced filter about $2 \mathrm{~m}$ (6 feet) aboveground. All sample filters are exchanged biweekly, held one week (to allow for decay of short-lived natural radioactivity), and then sent to the analytical laboratory for initial analysis of total alpha and total beta activity. These initial analyses serve as an indicator of potential environmental problems.

Depending on project/facility requirements, the filters were stored until the end of either a three- or six-month sample period, then segregated and composited by sample location for specific radionuclide analysis as shown in Table 2-1. Segregating and compositing air filters by site provides a larger sample size and, thus, a more sensitive and accurate measurement of the concentration of airborne radionuclides.

All air sampling results are compared to DOE derived concentration guides (DCG) and/or U.S. Environmental Protection Agency concentration levels and are also statistically evaluated. To help assess the impact of Site operations, monitoring results are compared to the results obtained from the distant communities of Yakima and Sunnyside as reported by the PNNL Site Environmental Surveillance Program, and to data acquired from collocated sampling locations managed by Near-Facility Monitoring, PNNL, and WDOH. Collocated sampling results are used for comparability and precision of data. 
Table 2-1. Near-Facility Air Sampling Locations and Analyses, 2008.

\begin{tabular}{|c|c|c|c|c|}
\hline \multirow[b]{2}{*}{ Site } & \multirow{2}{*}{$\begin{array}{r}\text { Number of } \\
\text { Samplers }\end{array}$} & \multirow[b]{2}{*}{ EDP Code $^{(a)}$} & \multicolumn{2}{|r|}{ Analyses } \\
\hline & & & Bi-weekly & Composite $^{(\mathbf{b})}$ \\
\hline 100-B/C Area Field Remediation project ${ }^{c}$ & 3 & N466, N496, N497 & Gross $\alpha, \beta$ & GEA, Sr-90, Pu-iso, U-iso \\
\hline 100-D Area Field Remediation project ${ }^{c}$ & 4 & N467, N468, N514, N515 & Gross $\alpha, \beta$ & GEA, Sr-90, Pu-iso, U-iso, Am-241 \\
\hline 100-F Area Field Remediation project ${ }^{\mathrm{c}}$ & 5 & N519, N520, N521, N552, N553 & Gross $\alpha, \beta$ & GEA, Sr-90, Pu-iso, U-iso \\
\hline 100-H Area Field Remediation project ${ }^{\mathrm{c}}$ & 4 & N508, N509, N510, N574 & Gross $\alpha, \beta$ & GEA, Sr-90, Pu-iso, U-iso \\
\hline 100-K Spent Nuclear Fuels & 8 & $\begin{array}{l}\text { N401, N402, N403 }{ }^{\mathrm{d}} \text {, N404, } \\
\text { N476, N477, N478, N479 }\end{array}$ & Gross $\alpha, \beta$ & $\begin{array}{l}\text { GEA, Sr-90, Pu-iso, U-iso } \\
\text { Pu-241, Am-241 }\end{array}$ \\
\hline 118-K-1 Field Remediation project ${ }^{\mathrm{C}}$ & 3 & N403, N534, N535 & Gross $\alpha, \beta$ & GEA, Sr-90, Pu-iso, U-iso \\
\hline $100-\mathrm{N}$ Area D4 project & 3 & N102, N103, N106 & Gross $\alpha, \beta$ & GEA, Sr-90, Pu-iso, U-iso, Am-241 \\
\hline 100-IU2/6 Field Remediation ${ }^{c}$ & 2 & N565, N566 & Gross $\alpha, \beta$ & GEA, Sr-90, Pu-iso, U-iso, Am-241 \\
\hline 200 East Area & 17 & $\begin{array}{l}\text { N019, N158, N498, N499, N957, } \\
\text { N967, N968, N969, N970, N972, } \\
\text { N973, N976, N977, N978, N984, } \\
\text { N985, N999 }\end{array}$ & Gross $\alpha, \beta$ & GEA, Sr-90, Pu-iso, U-iso \\
\hline BC Controlled Area $^{c}$ & 7 & $\begin{array}{l}\text { N957, N569, N570, N571, N572, } \\
\text { N573, N978 }\end{array}$ & Gross $\alpha, \beta$ & $\begin{array}{l}\text { GEA, Sr-90, Pu-iso, U-iso } \\
\text { Pu-241, Am-241 }\end{array}$ \\
\hline Canister Storage Building (200 East Area) & 2 & N480, N481 & Gross $\alpha, \beta$ & $\begin{array}{l}\text { GEA, Sr-90, Pu-iso, U-iso } \\
\text { Pu-241, Am-241 }\end{array}$ \\
\hline Integrated Disposal Facility (200 East Area) & 2 & N532, N559 & Gross $\alpha, \beta$ & GEA, Sr-90, Pu-iso, U-iso \\
\hline 200 West Area & 23 & $\begin{array}{l}\text { N155, N161, N165, N168, N200 } \\
\text { N304, N433, N441, N442, N449, } \\
\text { N456, N457, N554, N555, N956, } \\
\text { N963, N964, N965, N966, N974, } \\
\text { N975, N987, N994 }\end{array}$ & Gross $\alpha, \beta$ & GEA, Sr-90, Pu-iso, U-iso \\
\hline $\begin{array}{l}\text { 200-UW-1 Decontamination \& Demolition project } \\
\text { (200 West Area) }\end{array}$ & 4 & N168, N550, N956, N963 & Gross $\alpha, \beta$ & GEA, Sr-90, Pu-iso, U-iso \\
\hline 300 Area Decontamination \& Demolition project ${ }^{c}$ & 1 & N557 & Gross $\alpha, \beta$ & GEA, Sr-90, Pu-iso, U-iso \\
\hline 300-FF-2 Field Remediation project (300 Area) ${ }^{c}$ & 6 & $\begin{array}{l}\text { N130, N527, N537, N538, N539, } \\
\text { N540 }\end{array}$ & Gross $\alpha, \beta$ & GEA, Sr-90, Pu-iso, U-iso \\
\hline Environmental Restoration Disposal Facility ${ }^{c}$ & 4 & N482 ${ }^{\mathrm{d}}, \mathrm{N} 517, \mathrm{~N} 518, \mathrm{~N} 963$ & Gross $\alpha, \beta$ & GEA, Sr-90, Pu-iso, U-iso \\
\hline 600 Area (WYE Barricade) & 1 & N981 ${ }^{\mathrm{e}}$ & Gross $\alpha, \beta$ & GEA, Sr-90, Pu-iso, U-iso \\
\hline
\end{tabular}

(a) EDP Code = Sampler location code. See PNNL-18427, APP 2.

(b) GEA = Gamma energy analysis; Pu-iso = isotopic plutonium-238 and plutonium-239/240; U-iso = isotopic uranium-234, uranium-235, and uranium-238.

(c) PNNL air sampling station(s) provide supplemental air monitoring data. See Table 2-2 for a listing of locations.

(d) Collocated sampling location with Washington State Department of Health.

(e) Collocated sampling location with Washington State Department of Health and PNNL. 
Table 2-2. PNNL Supplemental Air Sampling Locations ${ }^{\mathrm{a}}$, 2008.

Site

100-B/C Field Remediation project

100-D Area Field Remediation project

100-F Field Remediation project

100-H Field Remediation project

118-K-1 Field Remediation project

100-IU2/6 Field Remediation

BC Controlled Area

300 Area Decontamination \& Demolition project

300-FF-2 Field Remediation project

Environmental Restoration Disposal Facility

\section{Sampling Location}

100 B, 100 B SE, Yakima Barricade

Yakima Barricade

WYE Barricade, Yakima Barricade

Yakima Barricade

E $100 \mathrm{~K}$

100-F Met Tower, Hanford Townsite

200 ESE

300 NE, 300 South Gate, 300 Trench, 300 Water Intake, 300 South West

300 NE, 300 Trench, 300 Water Intake

200 W SE

${ }^{\mathrm{a}}$ Maps showing specific locations are available in PNNL-18427. 
Figure 2-1. 100-B/C Area Air Sampler Locations.

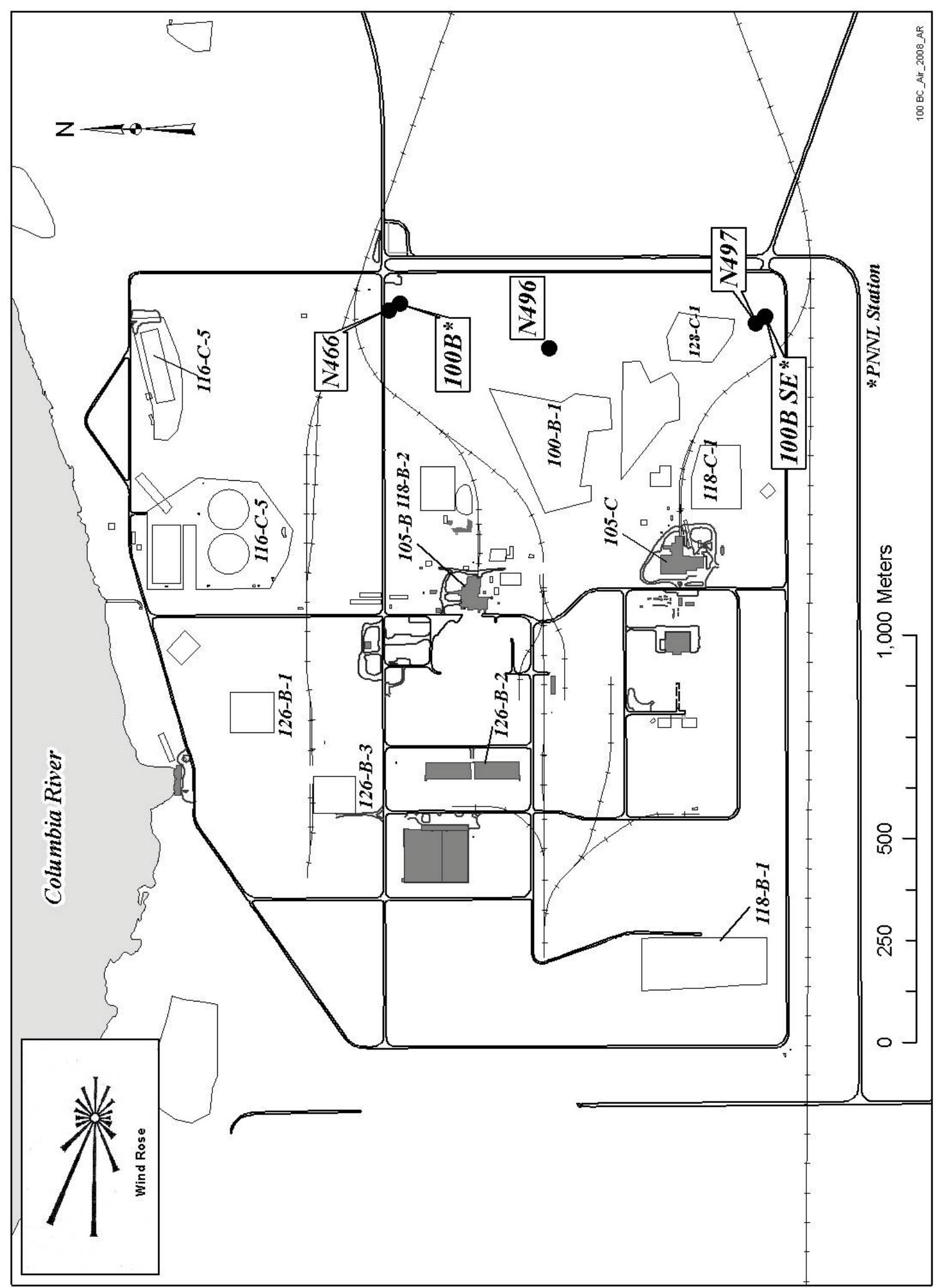


Figure 2-2. 100-D Area Air Sampler Locations.

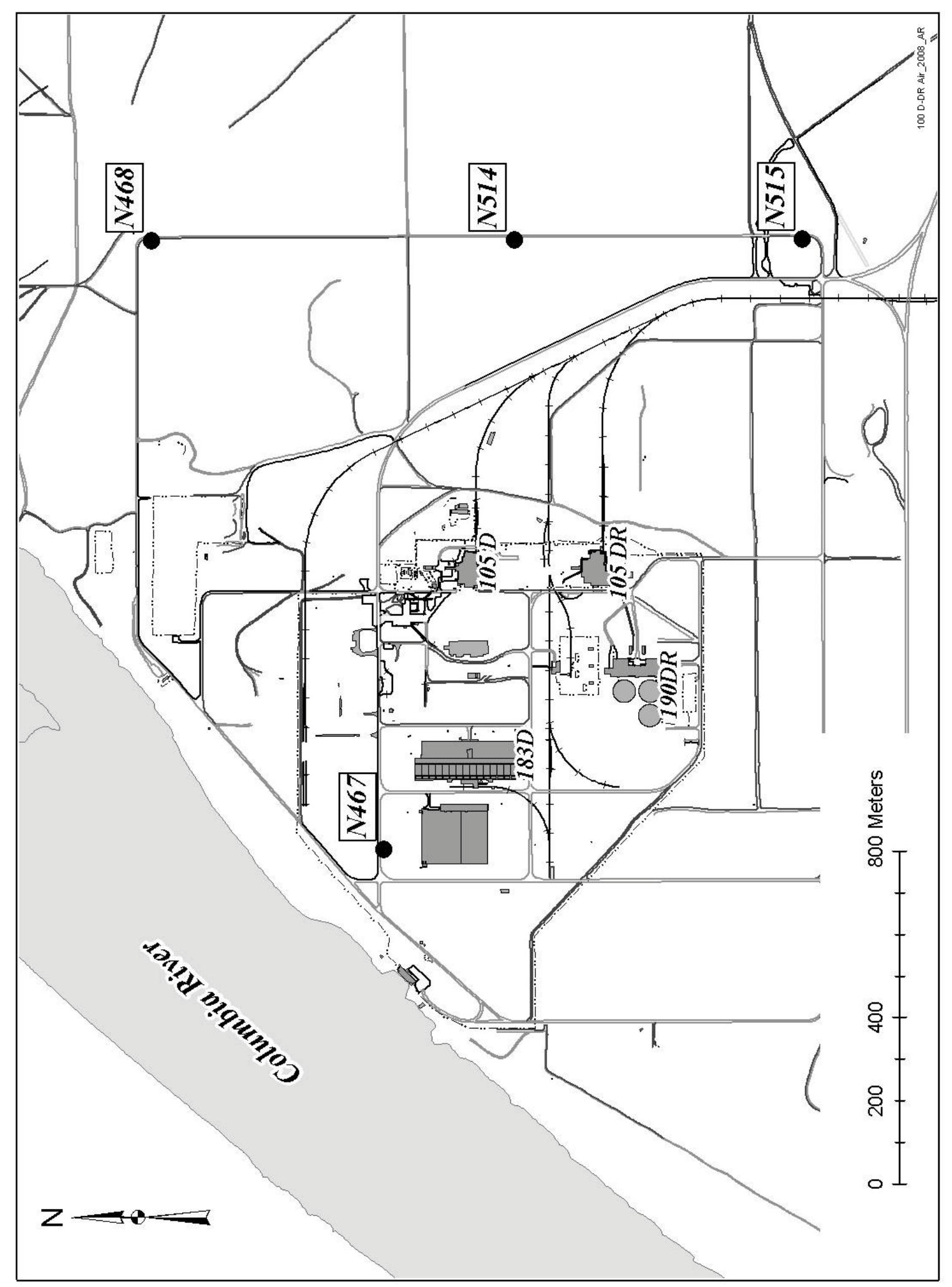


Figure 2-3. 100-F Area Air Sampler Locations.

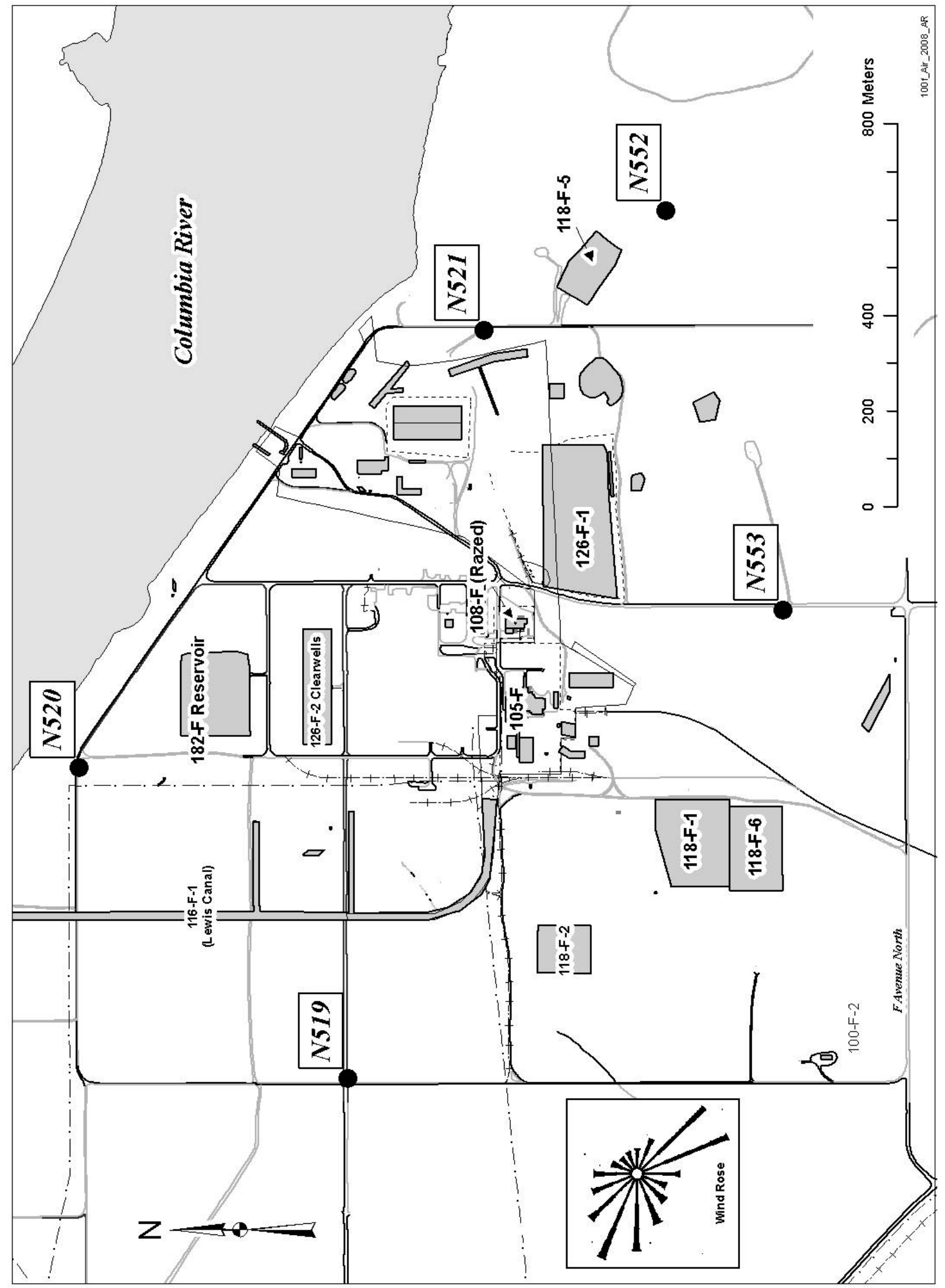


Figure 2-4. 100-H Area Air Sampler Locations.

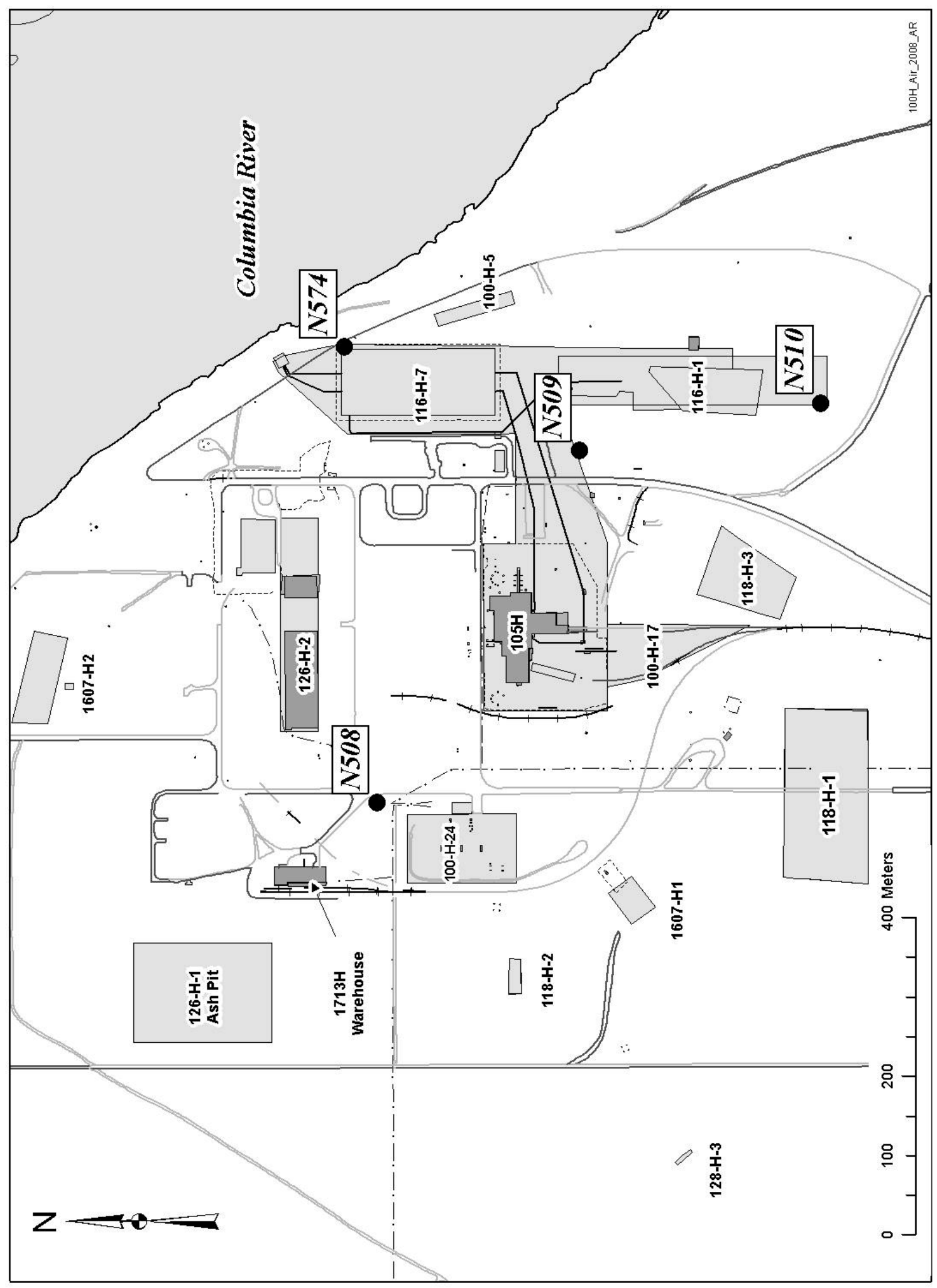


Figure 2-5. 100-K Area Air Sampler Locations.

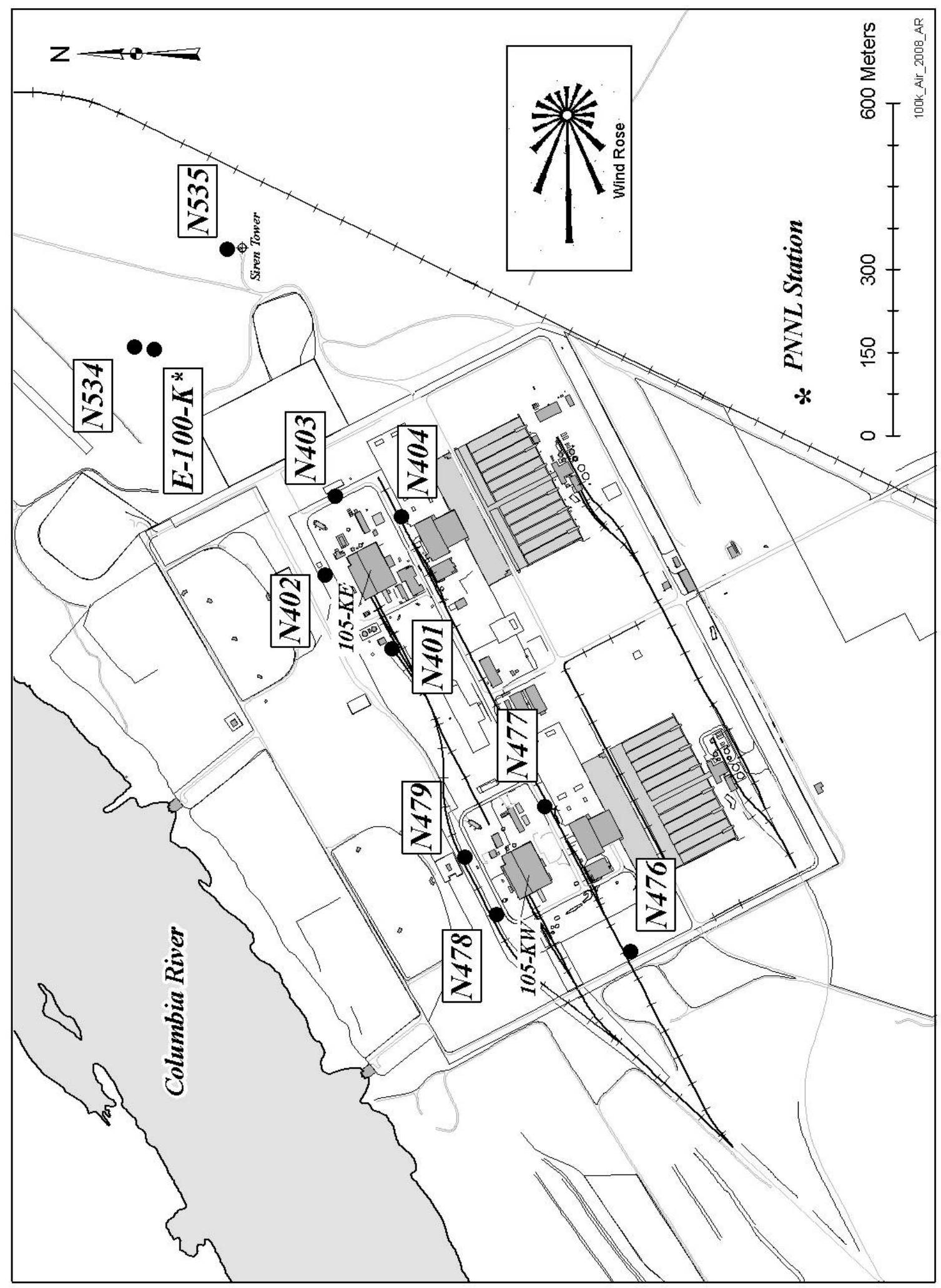


Figure 2-6. 100-N Air Sampler Locations.

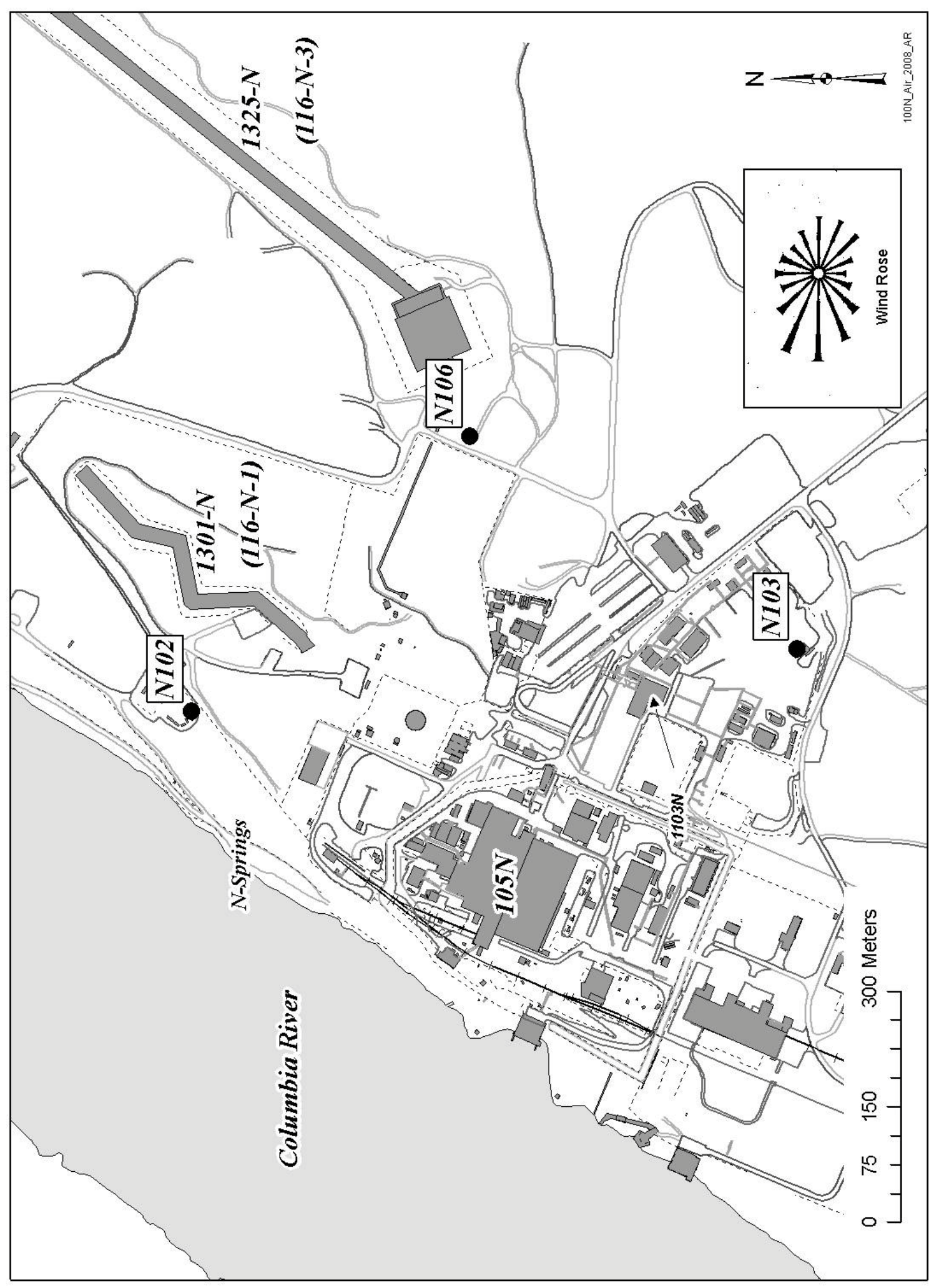


Figure 2-7. 100-IU2/6 Air Sampler Locations.

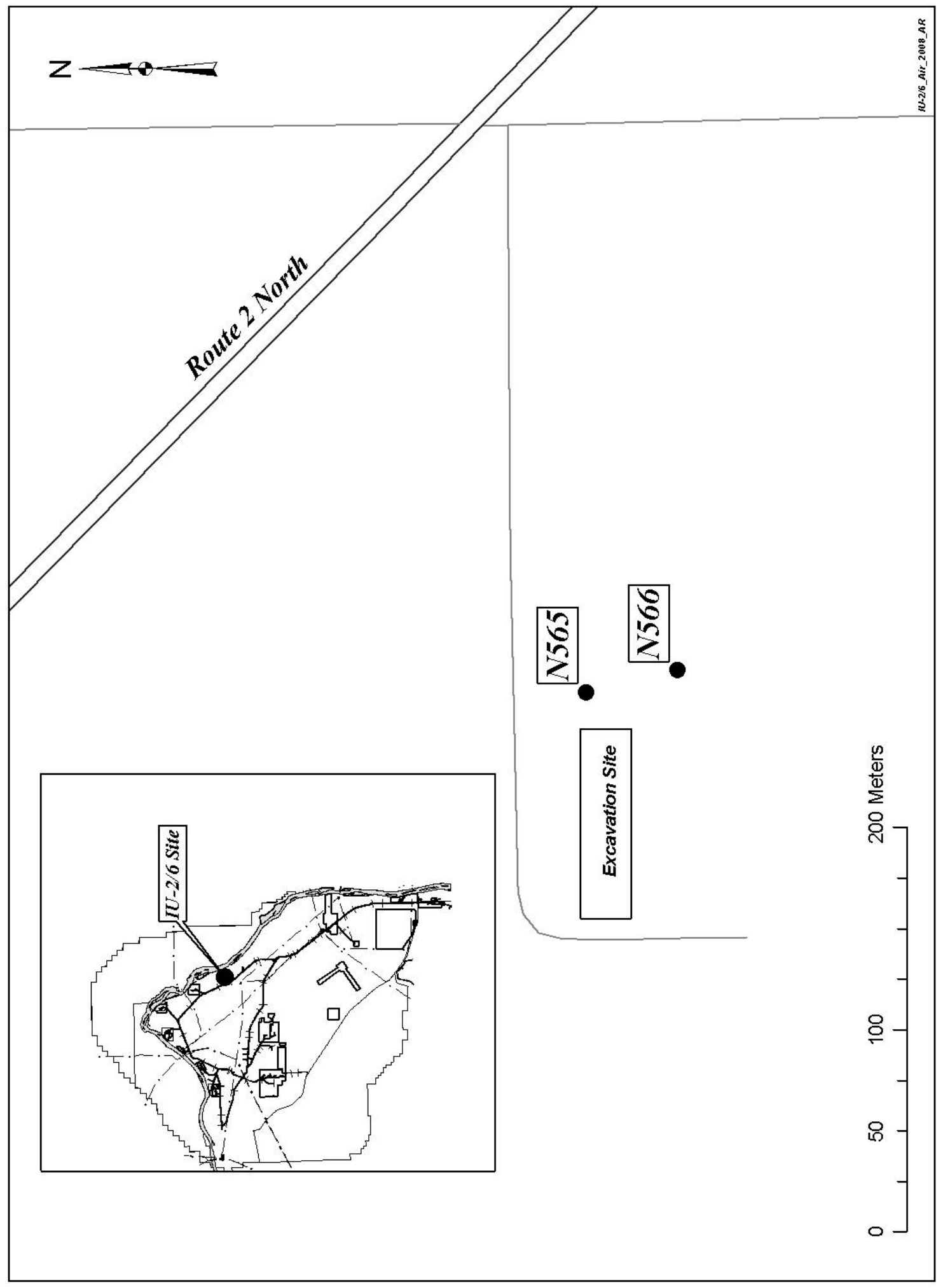


Figure 2-8. 200 East Air Sampler Locations.

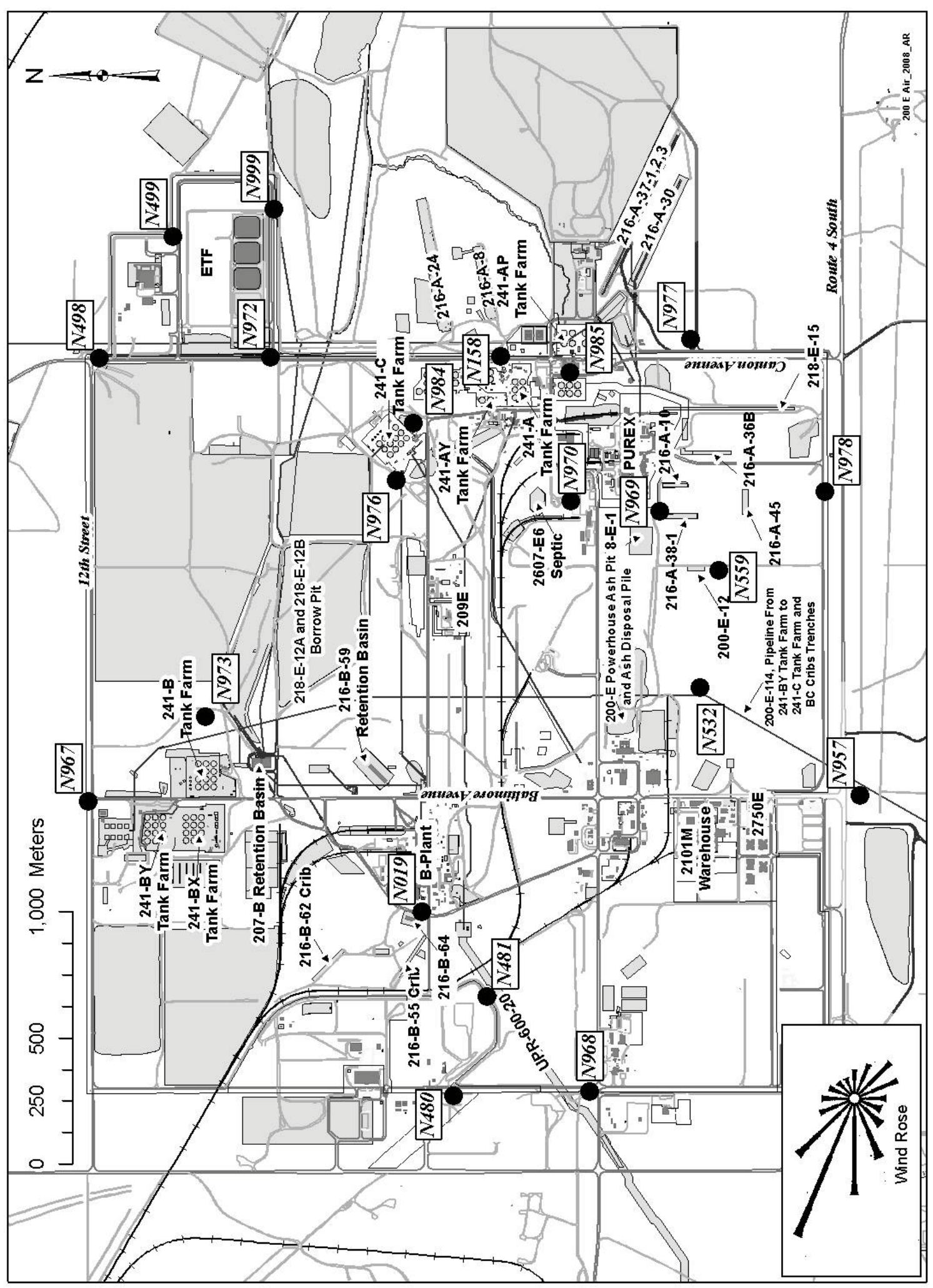


Figure 2-9. BC Controlled Area Air Sampler Locations.

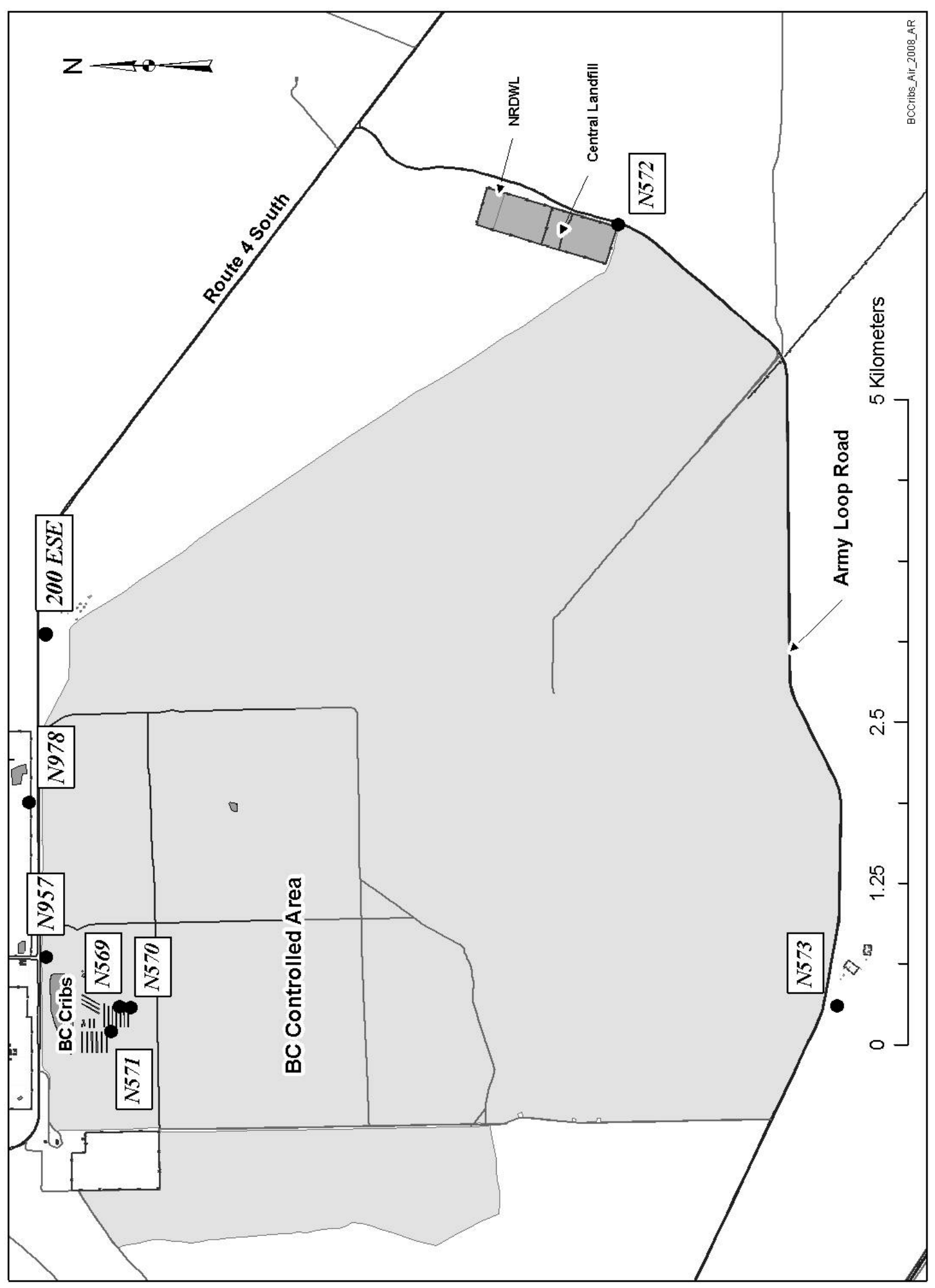


Figure 2-10. 200 West Area Air Sampler Locations.

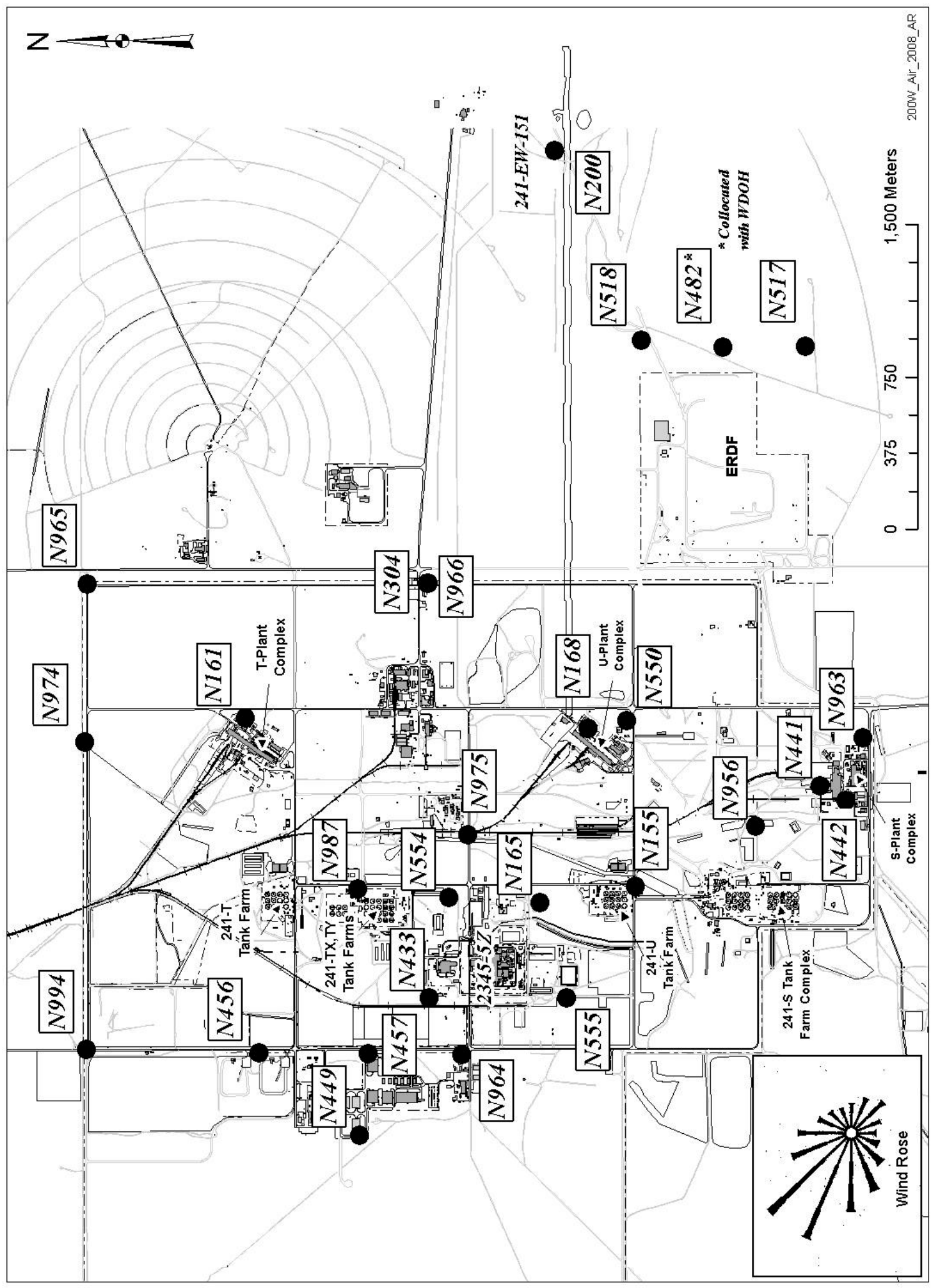


Figure 2-11. 300 Area Air Sampler Locations.

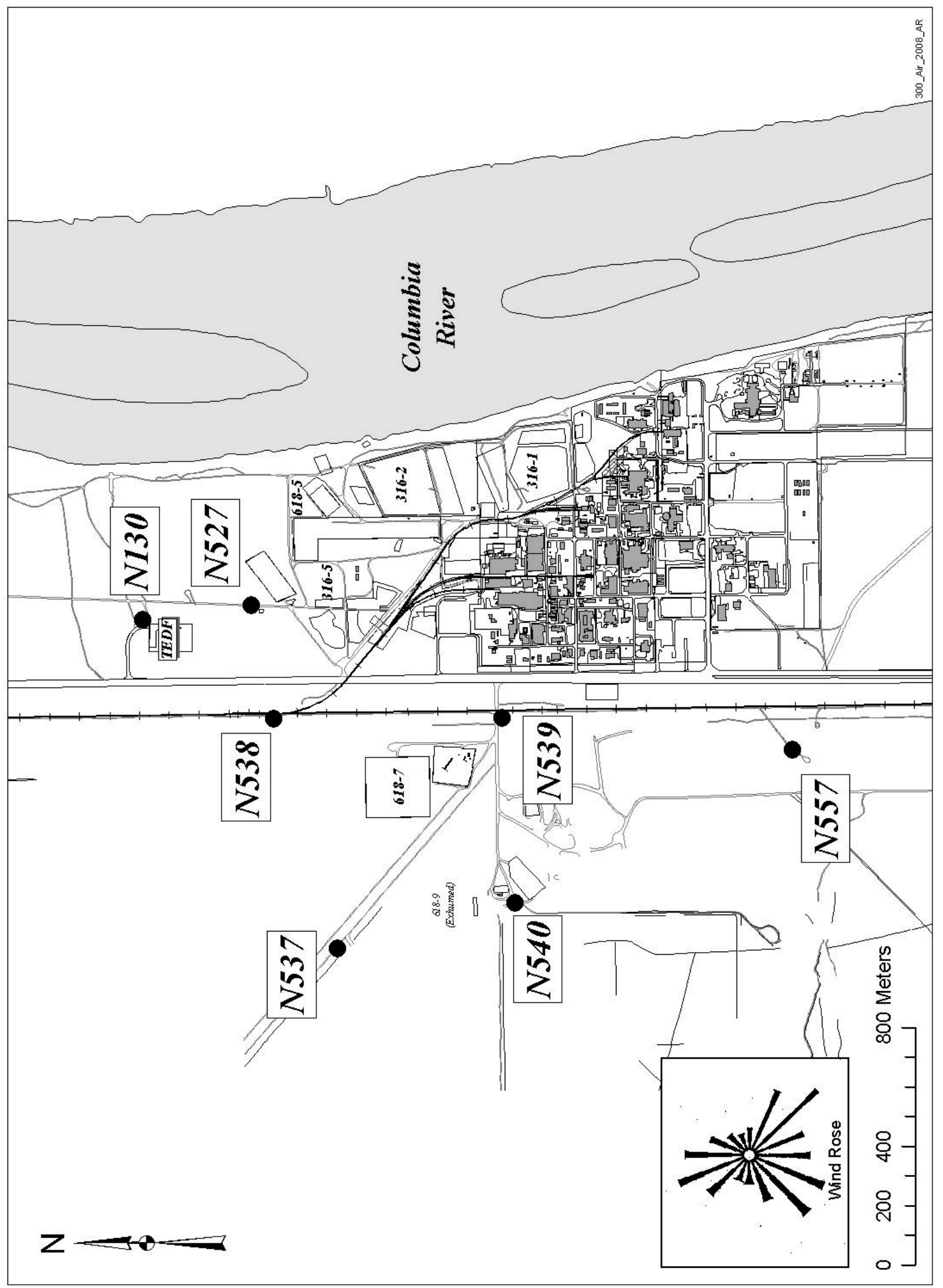


Figure 2-12. 600 Area Air Sampler Location.

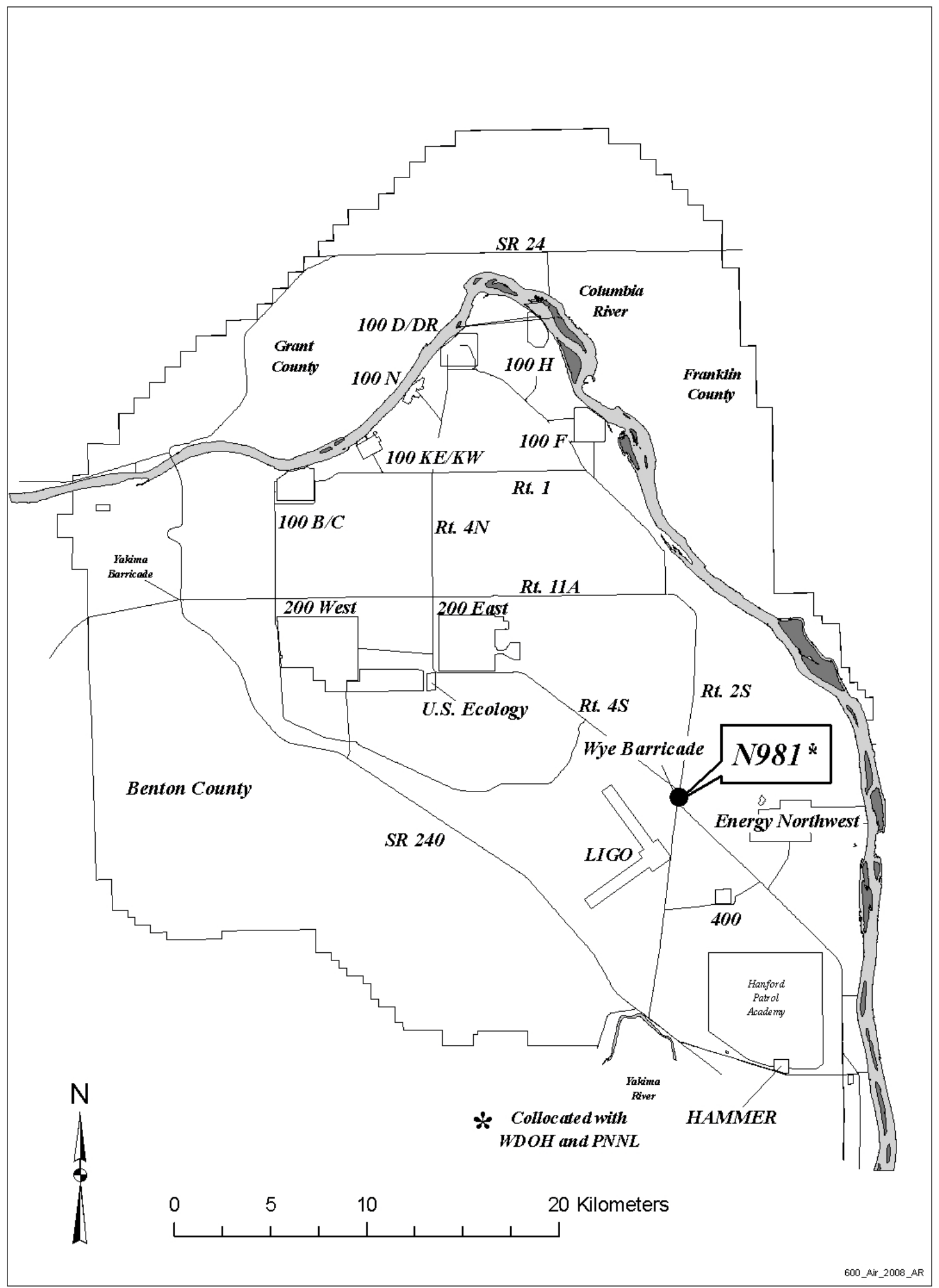


Figure 2-13. Annual Average Strontium-90 Concentrations in Air, 100-K Area.

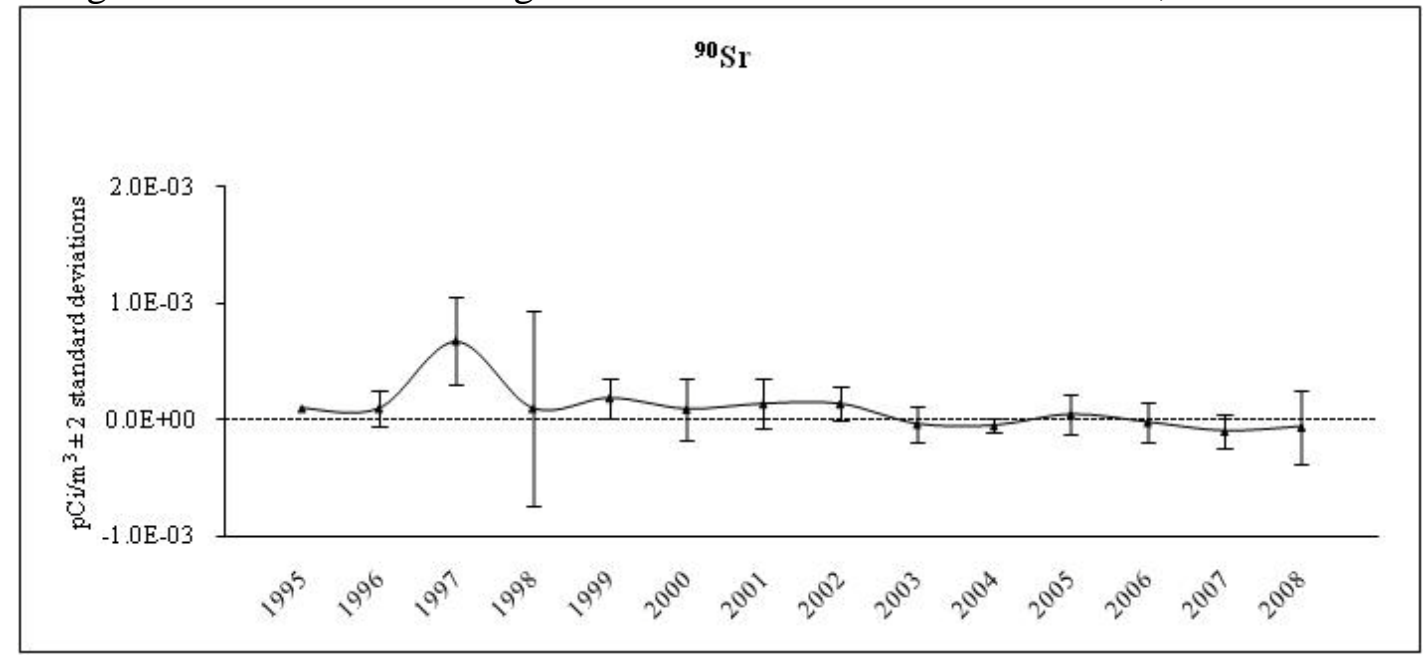

Figure 2-14. Annual Average Cesium-137 Concentrations in Air, 100-K Area.

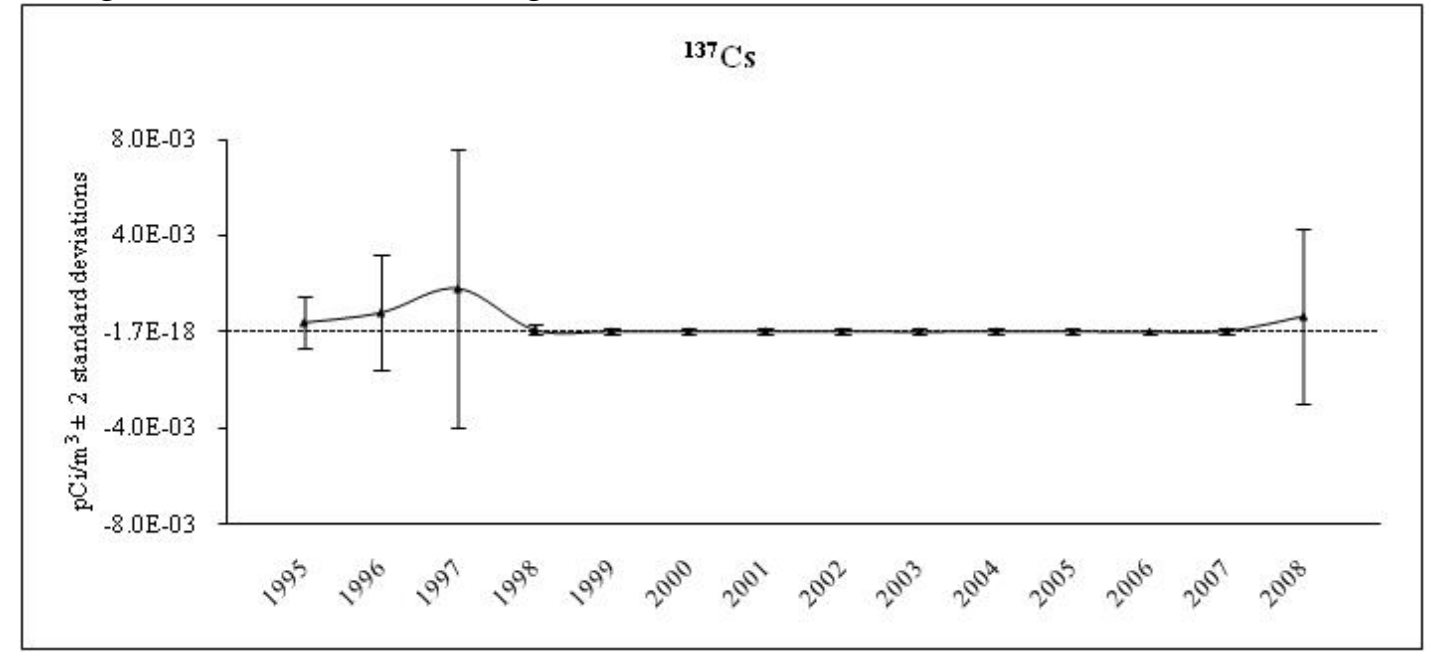

Figure 2-15. Annual Average Plutonium-239/240 Concentrations in Air, 100-K Area.

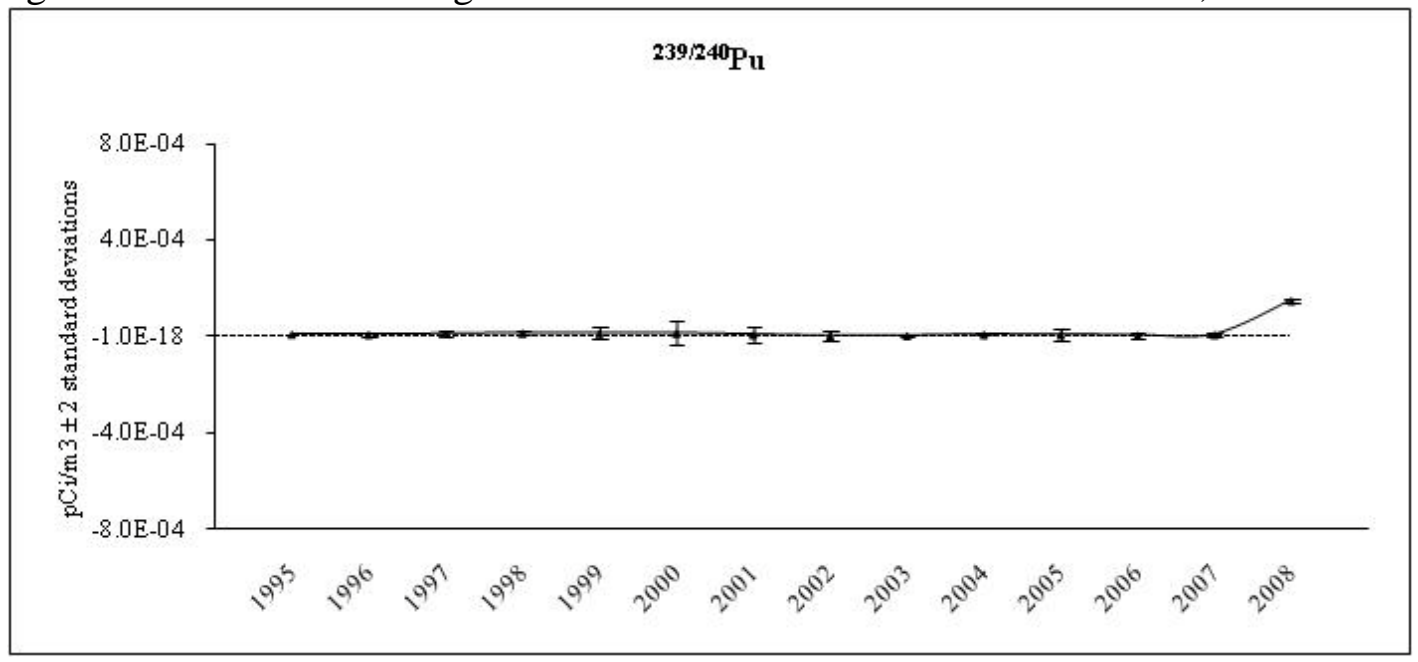


Figure 2-16. Annual Average Americium-241 Concentrations in Air, 100-K Area.

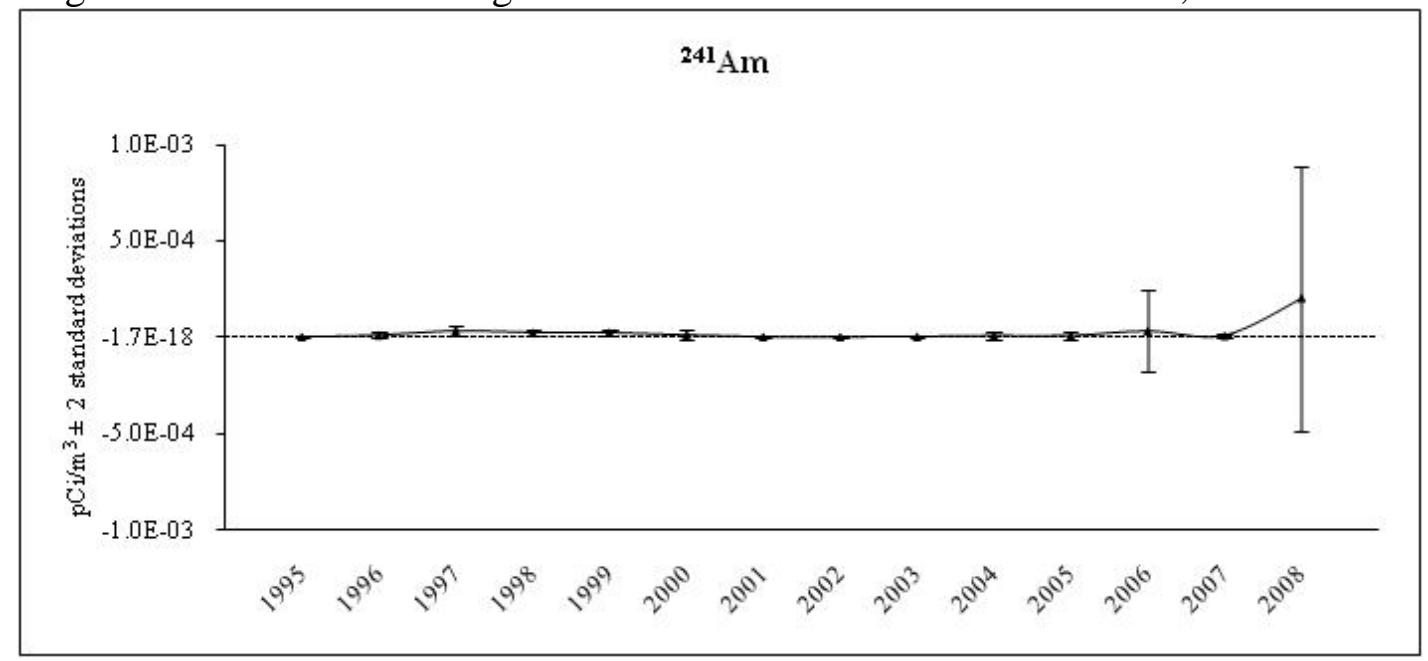

Figure 2-17. Annual Average Strontium-90 Concentrations in Air, 100-N.

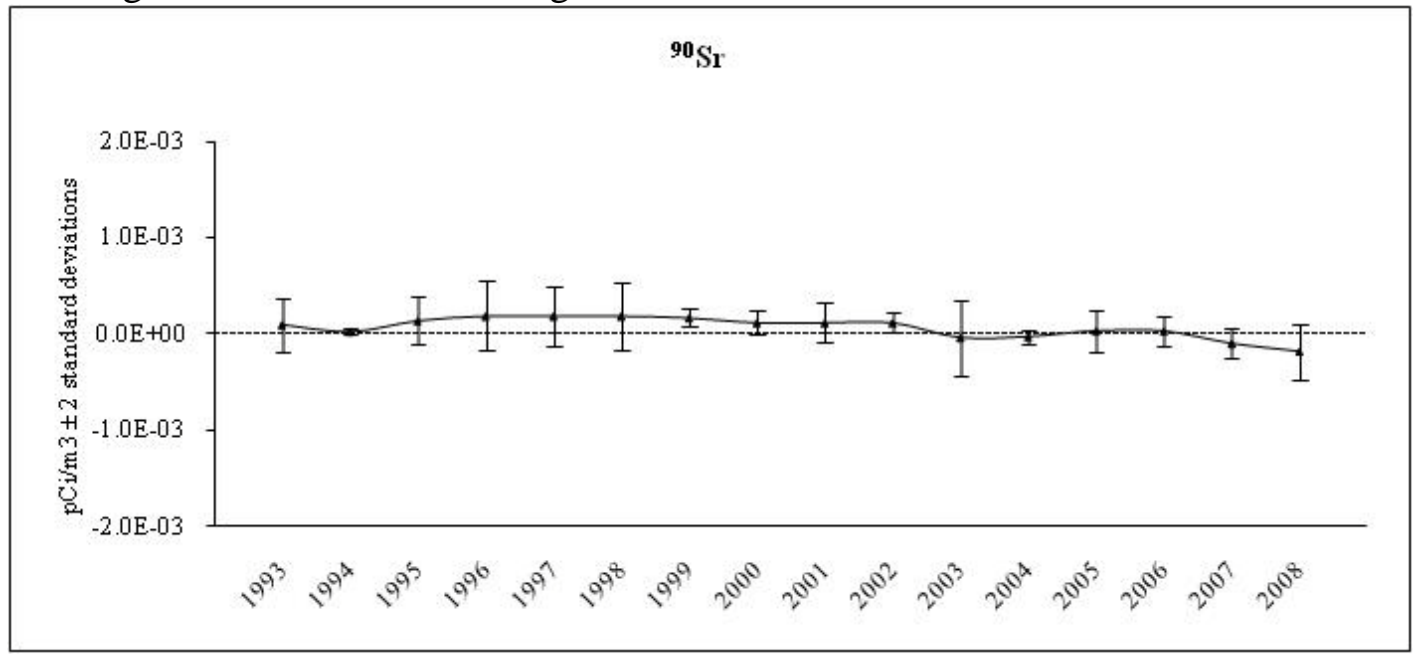

Figure 2-18. Annual Average Cesium-137 Concentrations in Air, 100-N.

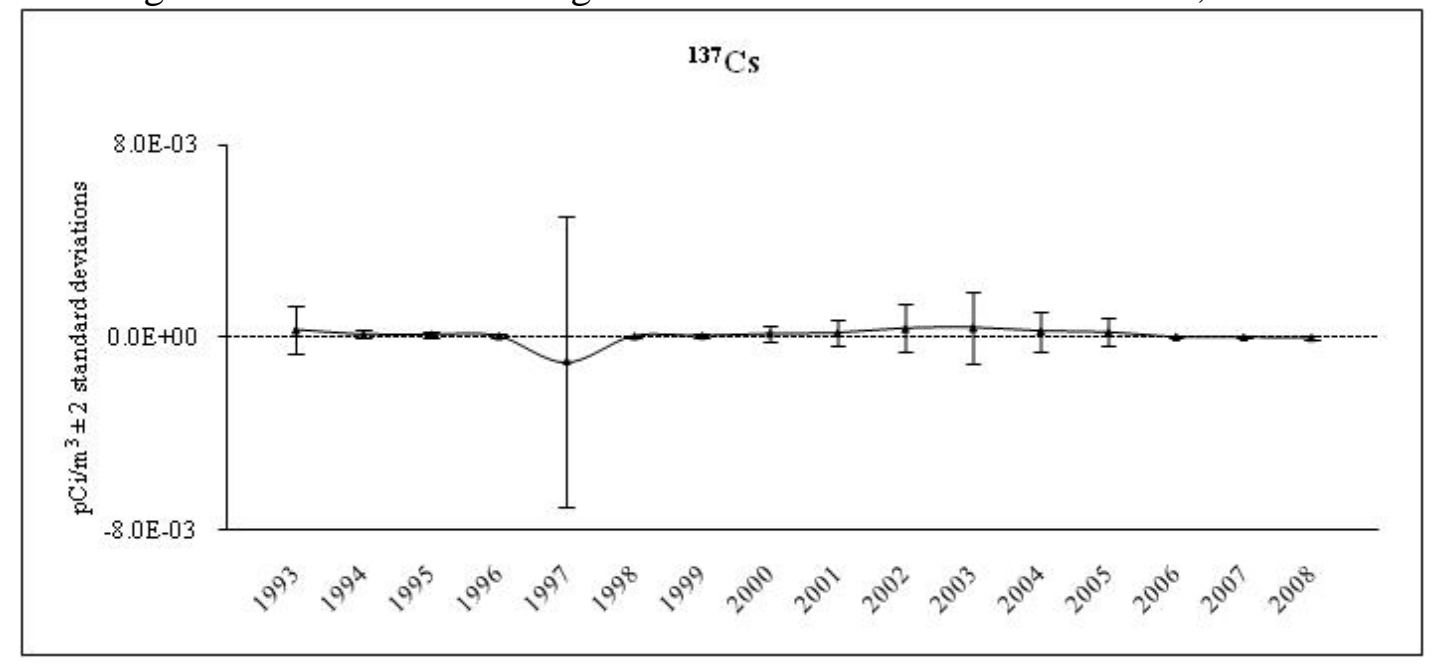


Figure 2-19. Annual Average Plutonium-239/240 Concentrations in Air, 100-N Area.

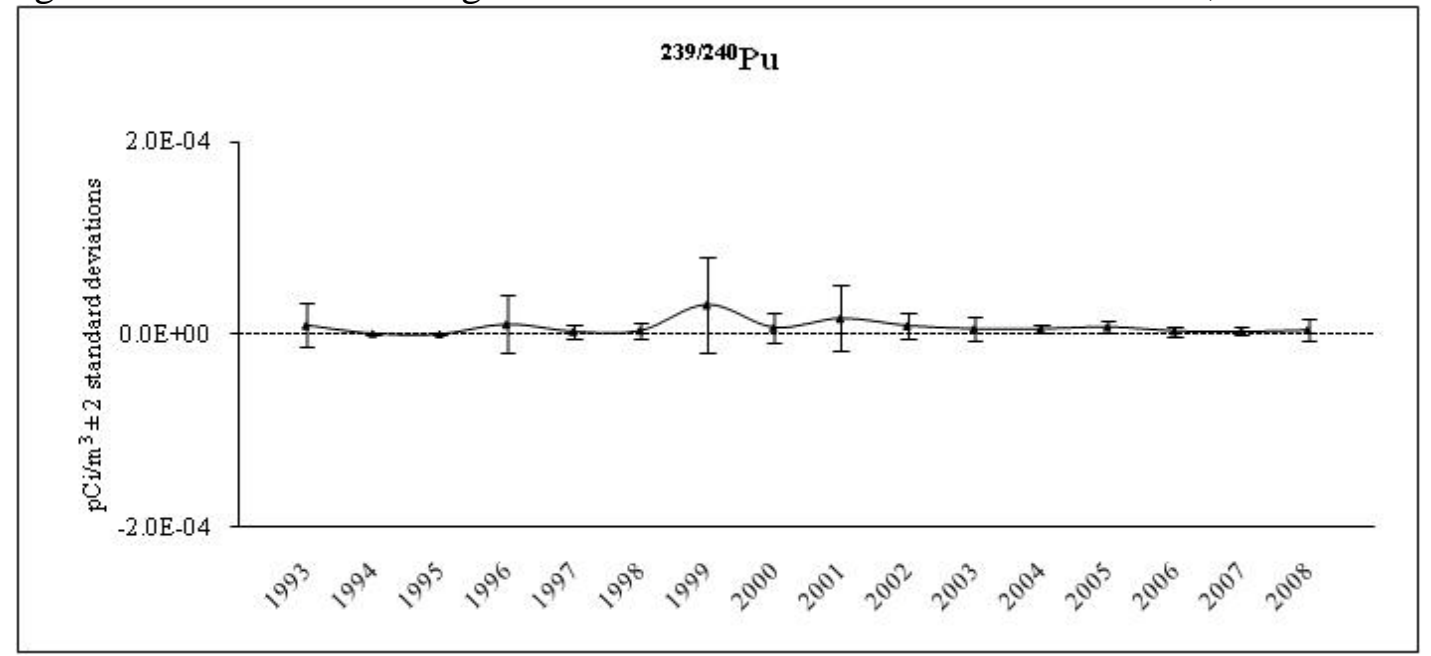

Figure 2-20. Annual Average Strontium-90 Concentrations in Air, 200 Areas.

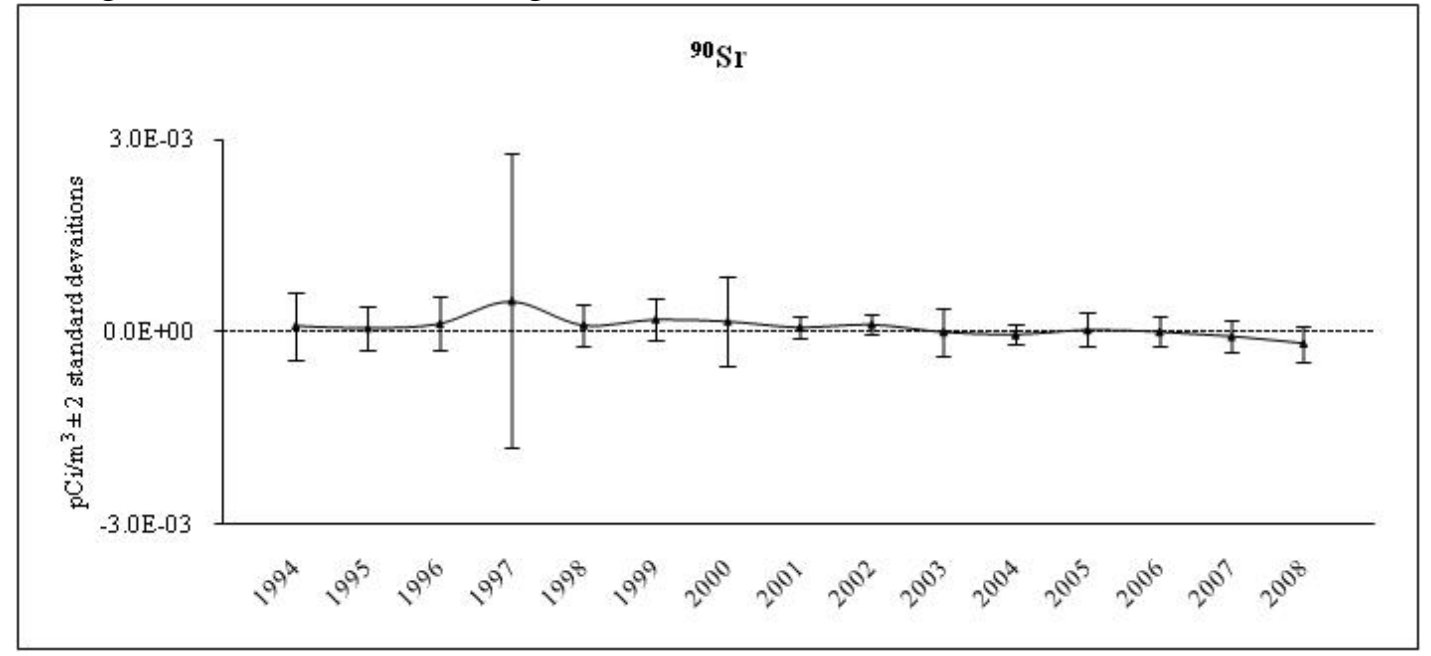

Figure 2-21. Annual Average Cesium-137 Concentrations in Air, 200 Areas.

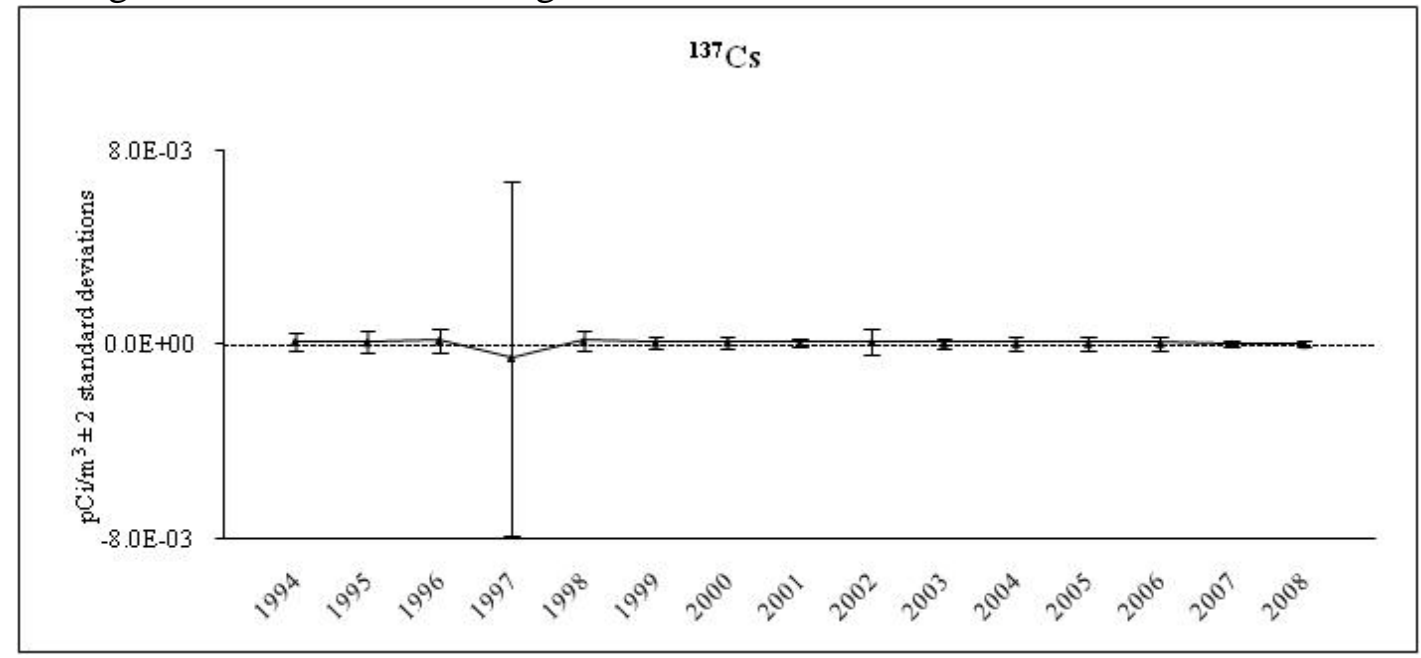


Figure 2-22. Annual Average Plutonium-239/240 Concentrations in Air, 200 Areas and N165.

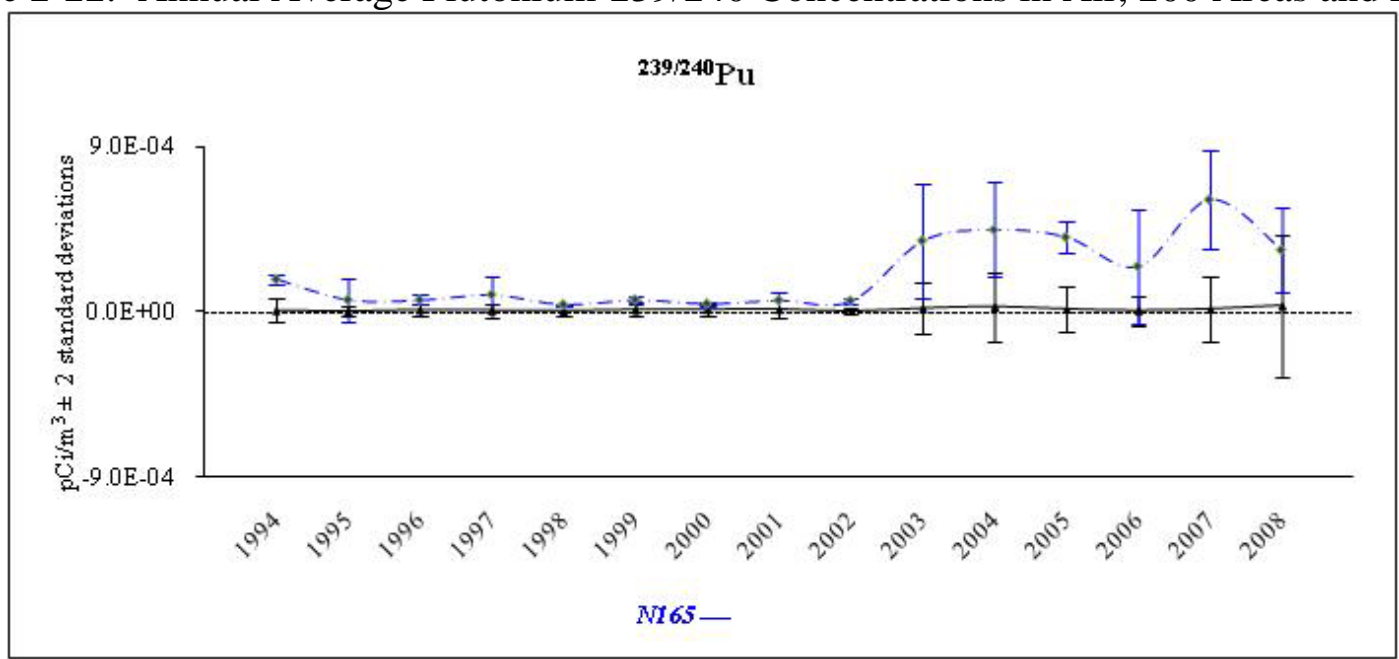

Figure 2-23. Annual Average Uranium-234 Concentrations in Air, 300 Area.

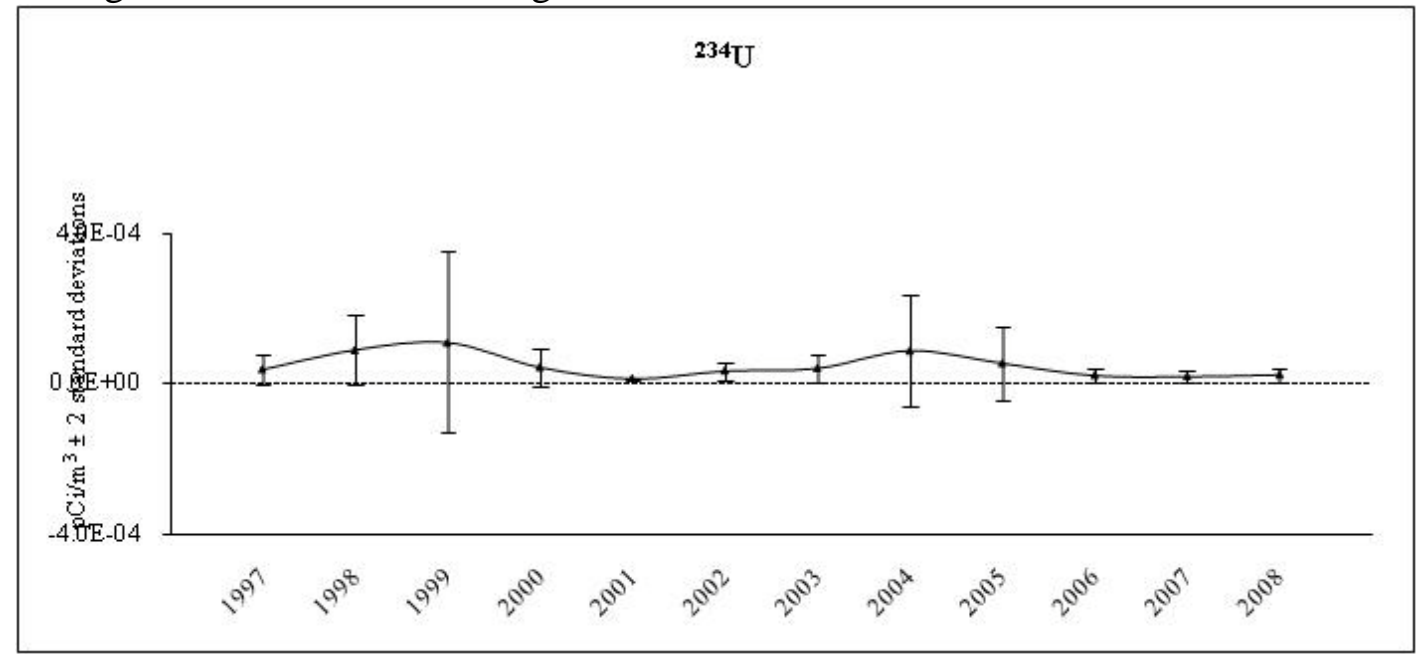

Figure 2-24. Annual Average Uranium-238 Concentrations in Air, 300 Area.

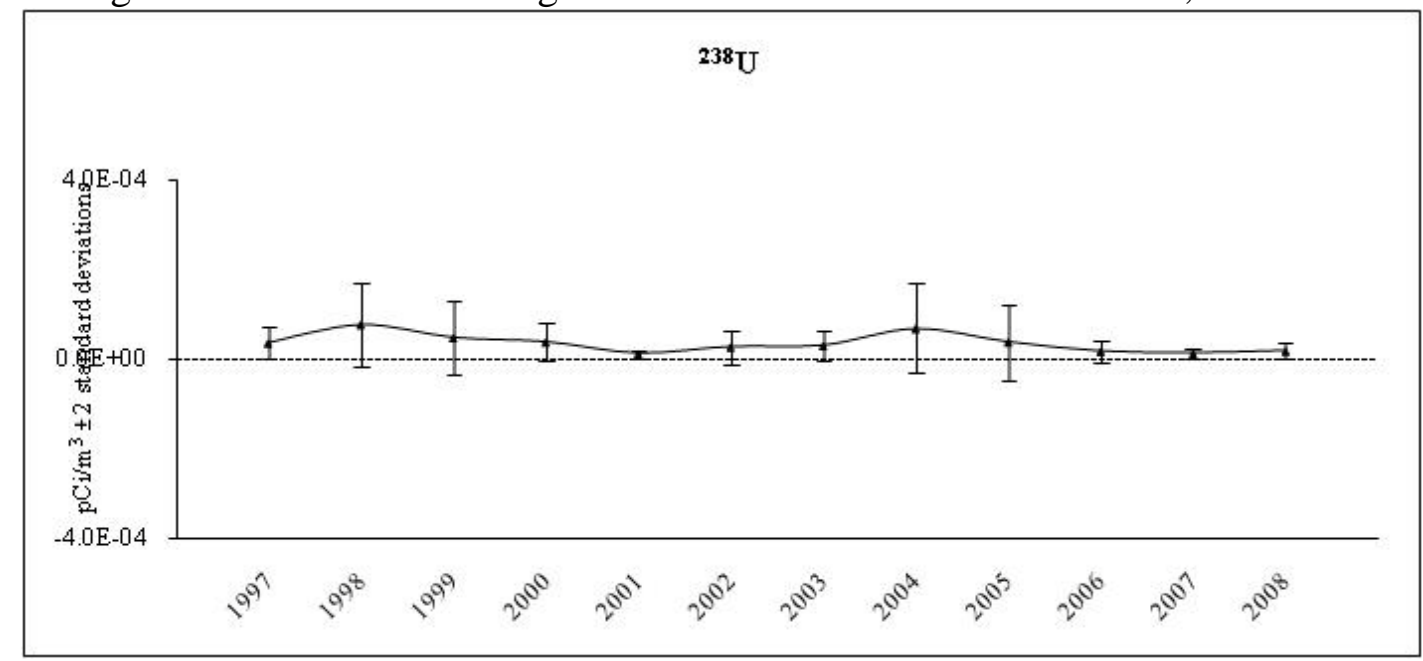


Table 2-3. Summary of Near-Facility Ambient Air Sampling Results (pCi/m³), 2008.

\begin{tabular}{|c|c|c|c|c|c|c|}
\hline \multirow[b]{2}{*}{ Isotope } & \multicolumn{2}{|c|}{ Number of } & \multirow[b]{2}{*}{ Mean $^{a}$} & \multirow[b]{2}{*}{ Maximum $^{\mathbf{b}}$} & \multirow[b]{2}{*}{ Location } & \multirow[b]{2}{*}{ Sampler } \\
\hline & Samples & Detects & & & & \\
\hline${ }^{241} \mathrm{Am}$ & 44 & 29 & $8.5 \mathrm{E}-05 \pm 4.6 \mathrm{E}-04$ & $1.2 \mathrm{E}-03 \pm 4.4 \mathrm{E}-04$ & 100-K East & N403 \\
\hline${ }^{60} \mathrm{Co}$ & 168 & 0 & $-1.0 \mathrm{E}-06 \pm 1.3 \mathrm{E}-04$ & $2.9 \mathrm{E}-04 \pm 6.1 \mathrm{E}-04$ & 100-B/C Area & N466 \\
\hline${ }^{134} \mathrm{Cs}$ & 168 & 0 & $5.9 \mathrm{E}-06 \pm 1.0 \mathrm{E}-04$ & $2.1 \mathrm{E}-04 \pm 3.0 \mathrm{E}-04$ & 100-IU2/6 & N565 \\
\hline${ }^{137} \mathrm{Cs}$ & 168 & 12 & $9.7 \mathrm{E}-05 \pm 1.2 \mathrm{E}-03$ & $7.4 \mathrm{E}-03 \pm 2.5 \mathrm{E}-03$ & 100-K East & N403 \\
\hline${ }^{152} \mathrm{Eu}$ & 168 & 0 & $4.5 \mathrm{E}-06 \pm 2.7 \mathrm{E}-04$ & $1.0 \mathrm{E}-03 \pm 1.4 \mathrm{E}-03$ & 100-B/C Area & N466 \\
\hline${ }^{154} \mathrm{Eu}$ & 168 & 1 & $-9.4 \mathrm{E}-06 \pm 3.0 \mathrm{E}-04$ & $4.0 \mathrm{E}-04 \pm 3.6 \mathrm{E}-04$ & 200 East Area & N999 \\
\hline${ }^{155} \mathrm{Eu}$ & 168 & 0 & $-4.8 \mathrm{E}-06 \pm 1.9 \mathrm{E}-04$ & $2.6 \mathrm{E}-04 \pm 1.9 \mathrm{E}-04$ & 200 East Area & N478 \\
\hline${ }^{238} \mathrm{Pu}$ & 167 & 9 & $4.4 \mathrm{E}-06 \pm 4.2 \mathrm{E}-05$ & $1.9 \mathrm{E}-04 \pm 6.8 \mathrm{E}-05$ & 200 East Area & N977 \\
\hline${ }^{239 / 240} \mathrm{Pu}$ & 168 & 35 & $3.3 \mathrm{E}-05 \pm 3.4 \mathrm{E}-04$ & $1.8 \mathrm{E}-03 \pm 6.5 \mathrm{E}-04$ & 200 East Area & N977 \\
\hline${ }^{241} \mathrm{Pu}$ & 28 & 5 & $9.1 \mathrm{E}-04 \pm 3.0 \mathrm{E}-03$ & $5.8 \mathrm{E}-03 \pm 1.8 \mathrm{E}-03$ & 100-K East & N403 \\
\hline${ }^{103} \mathrm{Ru}$ & 11 & 0 & $-3.5 \mathrm{E}-05 \pm 9.5 \mathrm{E}-05$ & $1.3 \mathrm{E}-05 \pm 6.0 \mathrm{E}-05$ & 200 East Area & N984 \\
\hline${ }^{106} \mathrm{Ru}$ & 168 & 0 & $-2.3 \mathrm{E}-05 \pm 8.7 \mathrm{E}-04$ & $1.8 \mathrm{E}-03 \pm 3.3 \mathrm{E}-03$ & 100-B/C Area & N496 \\
\hline${ }^{125} \mathrm{Sb}$ & 168 & 0 & $2.9 \mathrm{E}-06 \pm 2.1 \mathrm{E}-04$ & $3.2 \mathrm{E}-04 \pm 2.8 \mathrm{E}-04$ & 200 West Area & N456 \\
\hline${ }^{113} \mathrm{Sn}$ & 11 & 0 & $1.0 \mathrm{E}-05 \pm 8.4 \mathrm{E}-05$ & $6.9 \mathrm{E}-05 \pm 6.6 \mathrm{E}-05$ & 200 East Area & N158 \\
\hline${ }^{90} \mathrm{Sr}$ & 158 & 5 & $-1.8 \mathrm{E}-04 \pm 4.7 \mathrm{E}-04$ & $7.8 \mathrm{E}-04 \pm 3.0 \mathrm{E}-04$ & BC Controlled Area & N570 \\
\hline${ }^{234} \mathrm{U}$ & 168 & 163 & $1.7 \mathrm{E}-05 \pm 2.0 \mathrm{E}-05$ & $6.1 \mathrm{E}-05 \pm 3.7 \mathrm{E}-05$ & 100-B/C Area & N466 \\
\hline${ }^{235} \mathrm{U}$ & 168 & 41 & $3.3 \mathrm{E}-06 \pm 4.8 \mathrm{E}-06$ & $1.4 \mathrm{E}-05 \pm 1.2 \mathrm{E}-05$ & $100-\mathrm{IU} 2 / 6$ & N565 \\
\hline${ }^{238} \mathrm{U}$ & 168 & 151 & $1.2 \mathrm{E}-05 \pm 1.5 \mathrm{E}-05$ & $5.6 \mathrm{E}-05 \pm 3.6 \mathrm{E}-05$ & 100-B/C Area & N466 \\
\hline${ }^{65} \mathrm{Zn}$ & 10 & 0 & $-3.8 \mathrm{E}-05 \pm 2.3 \mathrm{E}-04$ & $1.4 \mathrm{E}-04 \pm 1.8 \mathrm{E}-04$ & 200 West Area & N457 \\
\hline gross $\alpha$ & 2020 & 1842 & $1.3 \mathrm{E}-03 \pm 1.7 \mathrm{E}-03$ & $1.5 \mathrm{E}-02 \pm 3.9 \mathrm{E}-03$ & 100-K East & N404 \\
\hline gross $\beta$ & 2020 & 2017 & $1.9 \mathrm{E}-02 \pm 2.6 \mathrm{E}-02$ & $2.2 \mathrm{E}-01 \pm 3.9 \mathrm{E}-02$ & 100-K East & N402 \\
\hline
\end{tabular}

\footnotetext{
a \pm 2 standard deviations

b \pm total analytical uncertainty
} 
Table 2-4. Near-Facility Air Sampling Results, 2008 (pCi/m³ \pm total analytical uncertainty). (Sheet 1 of 82)

\begin{tabular}{|c|c|c|c|c|c|c|c|c|c|}
\hline Location & Sample On & Sample Off & Isotope & Result \pm Uncertainty & Location & Composite Period & Isotope & Result \pm Uncertainty & $\mathbf{R Q *}$ \\
\hline N466 & $12 / 31 / 07$ & $01 / 14 / 08$ & gross $\alpha$ & $8.4 \mathrm{E}-04 \pm 5.6 \mathrm{E}-04$ & N466 & $12 / 31 / 07$ to $02 / 01 / 08$ & ${ }^{60} \mathrm{Co}$ & $2.9 \mathrm{E}-04 \pm 6.1 \mathrm{E}-04$ & $\bar{U}$ \\
\hline \multirow[t]{13}{*}{$(100-B / C)$} & $12 / 31 / 07$ & $01 / 14 / 08$ & gross $\beta$ & $8.7 \mathrm{E}-03 \pm 1.3 \mathrm{E}-03$ & & & ${ }^{134} \mathrm{Cs}$ & $1.9 \mathrm{E}-04 \pm 6.4 \mathrm{E}-04$ & $\mathrm{U}$ \\
\hline & $01 / 14 / 08$ & 01/29/08 & gross $\alpha$ & $1.8 \mathrm{E}-03 \pm 6.3 \mathrm{E}-04$ & & & ${ }^{137} \mathrm{Cs}$ & $9.0 \mathrm{E}-07 \pm 9.0 \mathrm{E}-06$ & $\mathrm{U}$ \\
\hline & $01 / 14 / 08$ & 01/29/08 & gross $\beta$ & $3.3 \mathrm{E}-02 \pm 3.3 \mathrm{E}-03$ & & & ${ }^{152} \mathrm{Eu}$ & $1.0 \mathrm{E}-03 \pm 1.4 \mathrm{E}-03$ & $\mathrm{U}$ \\
\hline & $01 / 29 / 08$ & 02/01/08 & gross $\alpha$ & $1.4 \mathrm{E}-03 \pm 2.0 \mathrm{E}-03$ & & & ${ }^{154} \mathrm{Eu}$ & $-6.2 \mathrm{E}-04 \pm 1.7 \mathrm{E}-03$ & $\mathrm{U}$ \\
\hline & $01 / 29 / 08$ & $02 / 01 / 08$ & gross $\beta$ & $3.0 \mathrm{E}-03 \pm 2.0 \mathrm{E}-03$ & & & ${ }^{155} \mathrm{Eu}$ & $-3.8 \mathrm{E}-05 \pm 3.8 \mathrm{E}-04$ & $\mathrm{U}$ \\
\hline & & & & & & & ${ }^{238} \mathrm{Pu}$ & $2.7 \mathrm{E}-05 \pm 5.5 \mathrm{E}-05$ & $\mathrm{U}$ \\
\hline & & & & & & & ${ }^{239 / 240} \mathrm{Pu}$ & $-9.0 \mathrm{E}-06 \pm 1.8 \mathrm{E}-05$ & $\mathrm{U}$ \\
\hline & & & & & & & ${ }^{106} \mathrm{Ru}$ & $-2.8 \mathrm{E}-04 \pm 2.8 \mathrm{E}-03$ & $\mathrm{U}$ \\
\hline & & & & & & & ${ }^{125} \mathrm{Sb}$ & $-1.2 \mathrm{E}-04 \pm 1.2 \mathrm{E}-03$ & $\mathrm{U}$ \\
\hline & & & & & & & ${ }^{90} \mathrm{Sr}$ & $-2.1 \mathrm{E}-03 \pm 2.1 \mathrm{E}-03$ & $\mathrm{U}$ \\
\hline & & & & & & & ${ }^{234} \mathrm{U}$ & $6.1 \mathrm{E}-05 \pm 3.7 \mathrm{E}-05$ & \\
\hline & & & & & & & ${ }^{235} \mathrm{U}$ & $1.4 \mathrm{E}-05 \pm 2.2 \mathrm{E}-05$ & $\mathrm{U}$ \\
\hline & & & & & & & ${ }^{238} \mathrm{U}$ & $5.6 \mathrm{E}-05 \pm 3.6 \mathrm{E}-05$ & \\
\hline \multirow{14}{*}{$\begin{array}{c}\text { N496 } \\
(100-B / C)\end{array}$} & $12 / 31 / 07$ & $01 / 14 / 08$ & $\operatorname{gross} \alpha$ & $8.4 \mathrm{E}-04 \pm 5.6 \mathrm{E}-04$ & $\mathrm{~N} 496$ & $12 / 31 / 07$ to $02 / 01 / 08$ & ${ }^{60} \mathrm{Co}$ & $6.7 \mathrm{E}-06 \pm 6.7 \mathrm{E}-05$ & U \\
\hline & $12 / 31 / 07$ & $01 / 14 / 08$ & gross $\beta$ & $1.3 \mathrm{E}-02 \pm 1.7 \mathrm{E}-03$ & & & ${ }^{134} \mathrm{Cs}$ & $4.2 \mathrm{E}-05 \pm 3.6 \mathrm{E}-04$ & $\mathrm{U}$ \\
\hline & $01 / 14 / 08$ & 01/29/08 & $\operatorname{gross} \alpha$ & $1.6 \mathrm{E}-03 \pm 5.9 \mathrm{E}-04$ & & & ${ }^{137} \mathrm{Cs}$ & $-4.9 \mathrm{E}-05 \pm 3.4 \mathrm{E}-04$ & $\mathrm{U}$ \\
\hline & $01 / 14 / 08$ & 01/29/08 & gross $\beta$ & $3.7 \mathrm{E}-02 \pm 3.6 \mathrm{E}-03$ & & & ${ }^{152} \mathrm{Eu}$ & $-1.1 \mathrm{E}-04 \pm 7.9 \mathrm{E}-04$ & U \\
\hline & 01/29/08 & 02/01/08 & gross $\alpha$ & $2.7 \mathrm{E}-04 \pm 1.4 \mathrm{E}-03$ & & & ${ }^{154} \mathrm{Eu}$ & $-3.0 \mathrm{E}-04 \pm 1.2 \mathrm{E}-03$ & $\mathrm{U}$ \\
\hline & 01/29/08 & 02/01/08 & gross $\beta$ & $4.7 \mathrm{E}-03 \pm 2.2 \mathrm{E}-03$ & & & ${ }^{155} \mathrm{Eu}$ & $-4.3 \mathrm{E}-05 \pm 4.3 \mathrm{E}-04$ & U \\
\hline & & & & & & & ${ }^{238} \mathrm{Pu}$ & $4.0 \mathrm{E}-06 \pm 2.9 \mathrm{E}-05$ & $\mathrm{U}$ \\
\hline & & & & & & & ${ }^{239 / 240} \mathrm{Pu}$ & $7.9 \mathrm{E}-06 \pm 1.1 \mathrm{E}-05$ & $\mathrm{U}$ \\
\hline & & & & & & & ${ }^{106} \mathrm{Ru}$ & $1.8 \mathrm{E}-03 \pm 3.3 \mathrm{E}-03$ & $\mathrm{U}$ \\
\hline & & & & & & & ${ }^{125} \mathrm{Sb}$ & $-2.1 \mathrm{E}-04 \pm 7.5 \mathrm{E}-04$ & $\mathrm{U}$ \\
\hline & & & & & & & ${ }^{90} \mathrm{Sr}$ & $-7.5 \mathrm{E}-04 \pm 7.6 \mathrm{E}-04$ & $\mathrm{U}$ \\
\hline & & & & & & & ${ }^{234} \mathrm{U}$ & $5.6 \mathrm{E}-05 \pm 3.5 \mathrm{E}-05$ & \\
\hline & & & & & & & ${ }^{235} \mathrm{U}$ & $4.0 \mathrm{E}-06 \pm 8.1 \mathrm{E}-07$ & $\mathrm{U}$ \\
\hline & & & & & & & ${ }^{238} \mathrm{U}$ & $2.8 \mathrm{E}-05 \pm 2.6 \mathrm{E}-05$ & $\mathrm{U}$ \\
\hline \multirow{14}{*}{$\begin{array}{c}\text { N497 } \\
(100-B / C)\end{array}$} & $12 / 31 / 07$ & $01 / 14 / 08$ & gross $\alpha$ & $5.2 \mathrm{E}-04 \pm 4.8 \mathrm{E}-04$ & N497 & $12 / 31 / 07$ to $02 / 01 / 08$ & ${ }^{60} \mathrm{Co}$ & $-3.2 \mathrm{E}-04 \pm 5.2 \mathrm{E}-04$ & U \\
\hline & $12 / 31 / 07$ & $01 / 14 / 08$ & gross $\beta$ & $6.3 \mathrm{E}-03 \pm 1.1 \mathrm{E}-03$ & & & ${ }^{134} \mathrm{Cs}$ & $-1.6 \mathrm{E}-04 \pm 3.9 \mathrm{E}-04$ & $\mathrm{U}$ \\
\hline & $01 / 14 / 08$ & 01/29/08 & gross $\alpha$ & $1.5 \mathrm{E}-03 \pm 5.6 \mathrm{E}-04$ & & & ${ }^{137} \mathrm{Cs}$ & $2.8 \mathrm{E}-04 \pm 3.6 \mathrm{E}-04$ & $\mathrm{U}$ \\
\hline & $01 / 14 / 08$ & 01/29/08 & gross $\beta$ & $3.8 \mathrm{E}-02 \pm 3.6 \mathrm{E}-03$ & & & ${ }^{152} \mathrm{Eu}$ & $2.2 \mathrm{E}-04 \pm 8.5 \mathrm{E}-04$ & $\mathrm{U}$ \\
\hline & 01/29/08 & 02/01/08 & gross $\alpha$ & $-2.9 \mathrm{E}-04 \pm 1.1 \mathrm{E}-03$ & & & ${ }^{154} \mathrm{Eu}$ & $-3.1 \mathrm{E}-04 \pm 1.3 \mathrm{E}-03$ & $\mathrm{U}$ \\
\hline & $01 / 29 / 08$ & $02 / 01 / 08$ & gross $\beta$ & $1.1 \mathrm{E}-03 \pm 1.9 \mathrm{E}-03$ & & & ${ }^{155} \mathrm{Eu}$ & $-2.0 \mathrm{E}-04 \pm 8.3 \mathrm{E}-04$ & $\mathrm{U}$ \\
\hline & & & & & & & ${ }^{238} \mathrm{Pu}$ & $3.1 \mathrm{E}-05 \pm 4.5 \mathrm{E}-05$ & $\mathrm{U}$ \\
\hline & & & & & & & ${ }^{239 / 240} \mathrm{Pu}$ & $2.4 \mathrm{E}-05 \pm 1.9 \mathrm{E}-05$ & \\
\hline & & & & & & & ${ }^{106} \mathrm{Ru}$ & $-1.4 \mathrm{E}-03 \pm 3.6 \mathrm{E}-03$ & $\mathrm{U}$ \\
\hline & & & & & & & ${ }^{125} \mathrm{Sb}$ & $-1.9 \mathrm{E}-04 \pm 8.4 \mathrm{E}-04$ & $\mathrm{U}$ \\
\hline & & & & & & & ${ }^{90} \mathrm{Sr}$ & $-8.6 \mathrm{E}-04 \pm 8.8 \mathrm{E}-04$ & $\mathrm{U}$ \\
\hline & & & & & & & ${ }^{234} \mathrm{U}$ & $5.9 \mathrm{E}-05 \pm 3.6 \mathrm{E}-05$ & \\
\hline & & & & & & & ${ }^{235} \mathrm{U}$ & $8.6 \mathrm{E}-06 \pm 1.2 \mathrm{E}-05$ & $\mathrm{U}$ \\
\hline & & & & & & & ${ }^{238} \mathrm{U}$ & $7.8 \mathrm{E}-06 \pm 1.1 \mathrm{E}-05$ & $\mathrm{U}$ \\
\hline
\end{tabular}


Table 2-4. Near-Facility Air Sampling Results, 2008 (pCi/m³ \pm total analytical uncertainty). (Sheet 2 of 82)

\begin{tabular}{|c|c|c|c|c|c|c|c|c|c|}
\hline Location & Sample On & Sample Off & Isotope & Result \pm Uncertainty & Location & Composite Period & Isotope & Result \pm Uncertainty & $\mathbf{R Q *}$ \\
\hline N467 & $01 / 02 / 08$ & $01 / 15 / 08$ & gross $\alpha$ & $3.0 \mathrm{E}-04 \pm 4.2 \mathrm{E}-04$ & N467 & $01 / 02 / 08$ to $07 / 01 / 08$ & ${ }^{241} \mathrm{Am}$ & $3.3 \mathrm{E}-06 \pm 3.7 \mathrm{E}-06$ & $\bar{U}$ \\
\hline \multirow[t]{51}{*}{$(100-D)$} & $01 / 02 / 08$ & $01 / 15 / 08$ & gross $\beta$ & $7.2 \mathrm{E}-03 \pm 1.2 \mathrm{E}-03$ & & & ${ }^{60} \mathrm{Co}$ & $-9.3 \mathrm{E}-05 \pm 9.6 \mathrm{E}-05$ & $\mathrm{U}$ \\
\hline & $01 / 15 / 08$ & 01/30/08 & gross $\alpha$ & $2.5 \mathrm{E}-03 \pm 7.3 \mathrm{E}-04$ & & & ${ }^{134} \mathrm{Cs}$ & $3.6 \mathrm{E}-05 \pm 7.3 \mathrm{E}-05$ & U \\
\hline & $01 / 15 / 08$ & 01/30/08 & gross $\beta$ & $3.4 \mathrm{E}-02 \pm 3.3 \mathrm{E}-03$ & & & ${ }^{137} \mathrm{Cs}$ & $-2.5 \mathrm{E}-05 \pm 6.9 \mathrm{E}-05$ & $\mathrm{U}$ \\
\hline & $01 / 30 / 08$ & 02/13/08 & gross $\alpha$ & $1.6 \mathrm{E}-04 \pm 3.5 \mathrm{E}-04$ & & & ${ }^{152} \mathrm{Eu}$ & $4.8 \mathrm{E}-05 \pm 1.7 \mathrm{E}-04$ & $\mathrm{U}$ \\
\hline & $01 / 30 / 08$ & $02 / 13 / 08$ & gross $\beta$ & $4.4 \mathrm{E}-03 \pm 9.3 \mathrm{E}-04$ & & & ${ }^{154} \mathrm{Eu}$ & $-8.7 \mathrm{E}-05 \pm 2.3 \mathrm{E}-04$ & $\mathrm{U}$ \\
\hline & $02 / 13 / 08$ & $02 / 27 / 08$ & gross $\alpha$ & $1.3 \mathrm{E}-03 \pm 5.6 \mathrm{E}-04$ & & & ${ }^{155} \mathrm{Eu}$ & $-5.1 \mathrm{E}-05 \pm 1.9 \mathrm{E}-04$ & $\mathrm{U}$ \\
\hline & $02 / 13 / 08$ & $02 / 27 / 08$ & gross $\beta$ & $3.5 \mathrm{E}-02 \pm 3.5 \mathrm{E}-03$ & & & ${ }^{238} \mathrm{Pu}$ & $-1.1 \mathrm{E}-06 \pm 7.4 \mathrm{E}-06$ & $\mathrm{U}$ \\
\hline & $02 / 27 / 08$ & 03/12/08 & gross $\alpha$ & $7.3 \mathrm{E}-04 \pm 5.4 \mathrm{E}-04$ & & & ${ }^{239 / 240} \mathrm{Pu}$ & $1.1 \mathrm{E}-06 \pm 1.1 \mathrm{E}-05$ & $\mathrm{U}$ \\
\hline & $02 / 27 / 08$ & $03 / 12 / 08$ & gross $\beta$ & $1.6 \mathrm{E}-02 \pm 2.0 \mathrm{E}-03$ & & & ${ }^{106} \mathrm{Ru}$ & $-9.9 \mathrm{E}-06 \pm 9.9 \mathrm{E}-05$ & $\mathrm{U}$ \\
\hline & 03/12/08 & 03/26/08 & gross $\alpha$ & $1.0 \mathrm{E}-03 \pm 6.2 \mathrm{E}-04$ & & & ${ }^{125} \mathrm{Sb}$ & $2.1 \mathrm{E}-05 \pm 1.6 \mathrm{E}-04$ & $\mathrm{U}$ \\
\hline & 03/12/08 & 03/26/08 & gross $\beta$ & $1.0 \mathrm{E}-02 \pm 1.5 \mathrm{E}-03$ & & & ${ }^{90} \mathrm{Sr}$ & $7.0 \mathrm{E}-06 \pm 7.0 \mathrm{E}-05$ & $\mathrm{U}$ \\
\hline & $03 / 26 / 08$ & $04 / 08 / 08$ & gross $\alpha$ & $6.6 \mathrm{E}-04 \pm 5.4 \mathrm{E}-04$ & & & ${ }^{234} \mathrm{U}$ & $1.0 \mathrm{E}-05 \pm 7.2 \mathrm{E}-06$ & \\
\hline & 03/26/08 & $04 / 08 / 08$ & gross $\beta$ & $8.7 \mathrm{E}-03 \pm 1.4 \mathrm{E}-03$ & & & ${ }^{235} \mathrm{U}$ & $2.2 \mathrm{E}-06 \pm 3.4 \mathrm{E}-06$ & U \\
\hline & $04 / 08 / 08$ & $04 / 23 / 08$ & gross $\alpha$ & $9.1 \mathrm{E}-04 \pm 5.7 \mathrm{E}-04$ & & & ${ }^{238} \mathrm{U}$ & $1.2 \mathrm{E}-05 \pm 7.7 \mathrm{E}-06$ & \\
\hline & $04 / 08 / 08$ & $04 / 23 / 08$ & gross $\beta$ & $1.1 \mathrm{E}-02 \pm 1.5 \mathrm{E}-03$ & & & & & \\
\hline & $04 / 23 / 08$ & 05/07/08 & gross $\alpha$ & $1.2 \mathrm{E}-03 \pm 5.1 \mathrm{E}-04$ & N467 & $07 / 01 / 08$ to $12 / 30 / 08$ & ${ }^{241} \mathrm{Am}$ & $5.6 \mathrm{E}-06 \pm 4.9 \mathrm{E}-06$ & $\mathrm{U}$ \\
\hline & $04 / 23 / 08$ & 05/07/08 & gross $\beta$ & $1.9 \mathrm{E}-02 \pm 2.2 \mathrm{E}-03$ & & & ${ }^{60} \mathrm{Co}$ & $3.7 \mathrm{E}-05 \pm 8.8 \mathrm{E}-05$ & $\mathrm{U}$ \\
\hline & 05/07/08 & $05 / 20 / 08$ & gross $\alpha$ & $1.8 \mathrm{E}-03 \pm 6.6 \mathrm{E}-04$ & & & ${ }^{134} \mathrm{Cs}$ & $5.6 \mathrm{E}-06 \pm 5.6 \mathrm{E}-05$ & U \\
\hline & 05/07/08 & 05/20/08 & gross $\beta$ & $9.2 \mathrm{E}-03 \pm 1.4 \mathrm{E}-03$ & & & ${ }^{137} \mathrm{Cs}$ & $6.5 \mathrm{E}-05 \pm 7.0 \mathrm{E}-05$ & $\mathrm{U}$ \\
\hline & 05/20/08 & $06 / 04 / 08$ & gross $\alpha$ & $7.3 \mathrm{E}-04 \pm 5.0 \mathrm{E}-04$ & & & ${ }^{152} \mathrm{Eu}$ & $-3.3 \mathrm{E}-05 \pm 1.8 \mathrm{E}-04$ & U \\
\hline & $05 / 20 / 08$ & $06 / 04 / 08$ & gross $\beta$ & $1.2 \mathrm{E}-02 \pm 1.6 \mathrm{E}-03$ & & & ${ }^{154} \mathrm{Eu}$ & $1.6 \mathrm{E}-04 \pm 2.6 \mathrm{E}-04$ & $\mathrm{U}$ \\
\hline & $06 / 04 / 08$ & $06 / 17 / 08$ & gross $\alpha$ & $2.9 \mathrm{E}-04 \pm 4.9 \mathrm{E}-04$ & & & ${ }^{155} \mathrm{Eu}$ & $1.5 \mathrm{E}-04 \pm 1.8 \mathrm{E}-04$ & $\mathrm{U}$ \\
\hline & $06 / 04 / 08$ & $06 / 17 / 08$ & gross $\beta$ & $1.2 \mathrm{E}-02 \pm 1.7 \mathrm{E}-03$ & & & ${ }^{238} \mathrm{Pu}$ & $-7.6 \mathrm{E}-07 \pm 7.7 \mathrm{E}-06$ & $\mathrm{U}$ \\
\hline & $06 / 17 / 08$ & 07/01/08 & gross $\alpha$ & $1.1 \mathrm{E}-03 \pm 5.2 \mathrm{E}-04$ & & & ${ }^{239 / 240} \mathrm{Pu}$ & $1.5 \mathrm{E}-06 \pm 3.8 \mathrm{E}-06$ & $\mathrm{U}$ \\
\hline & $06 / 17 / 08$ & 07/01/08 & gross $\beta$ & $1.5 \mathrm{E}-02 \pm 2.0 \mathrm{E}-03$ & & & ${ }^{106} \mathrm{Ru}$ & $6.9 \mathrm{E}-04 \pm 6.9 \mathrm{E}-04$ & U \\
\hline & $07 / 01 / 08$ & $07 / 15 / 08$ & gross $\alpha$ & $9.9 \mathrm{E}-04 \pm 7.0 \mathrm{E}-04$ & & & ${ }^{125} \mathrm{Sb}$ & $-2.8 \mathrm{E}-05 \pm 1.6 \mathrm{E}-04$ & $\mathrm{U}$ \\
\hline & 07/01/08 & 07/15/08 & gross $\beta$ & $1.5 \mathrm{E}-02 \pm 1.9 \mathrm{E}-03$ & & & ${ }^{90} \mathrm{Sr}$ & $-1.0 \mathrm{E}-04 \pm 1.0 \mathrm{E}-04$ & $\mathrm{U}$ \\
\hline & 07/15/08 & 07/29/08 & gross $\alpha$ & $9.4 \mathrm{E}-04 \pm 5.9 \mathrm{E}-04$ & & & ${ }^{234} \mathrm{U}$ & $1.5 \mathrm{E}-05 \pm 8.4 \mathrm{E}-06$ & \\
\hline & $07 / 15 / 08$ & 07/29/08 & gross $\beta$ & $1.8 \mathrm{E}-02 \pm 2.2 \mathrm{E}-03$ & & & ${ }^{235} \mathrm{U}$ & $3.7 \mathrm{E}-06 \pm 3.5 \mathrm{E}-06$ & \\
\hline & 07/29/08 & 08/12/08 & gross $\alpha$ & $6.8 \mathrm{E}-04 \pm 5.1 \mathrm{E}-04$ & & & ${ }^{238} \mathrm{U}$ & $1.0 \mathrm{E}-05 \pm 6.4 \mathrm{E}-06$ & \\
\hline & 07/29/08 & $08 / 12 / 08$ & gross $\beta$ & $1.7 \mathrm{E}-02 \pm 2.1 \mathrm{E}-03$ & & & & & \\
\hline & 08/12/08 & 08/26/08 & gross $\alpha$ & $6.8 \mathrm{E}-04 \pm 5.1 \mathrm{E}-04$ & & & & & \\
\hline & 08/12/08 & 08/26/08 & gross $\beta$ & $1.6 \mathrm{E}-02 \pm 2.0 \mathrm{E}-03$ & & & & & \\
\hline & $08 / 26 / 08$ & 09/09/08 & gross $\alpha$ & $7.1 \mathrm{E}-04 \pm 5.3 \mathrm{E}-04$ & & & & & \\
\hline & 08/26/08 & 09/09/08 & gross $\beta$ & $1.5 \mathrm{E}-02 \pm 2.0 \mathrm{E}-03$ & & & & & \\
\hline & 09/09/08 & 09/23/08 & gross $\alpha$ & $1.7 \mathrm{E}-03 \pm 6.4 \mathrm{E}-04$ & & & & & \\
\hline & 09/09/08 & 09/23/08 & gross $\beta$ & $2.6 \mathrm{E}-02 \pm 2.9 \mathrm{E}-03$ & & & & & \\
\hline & $09 / 23 / 08$ & $10 / 08 / 08$ & gross $\alpha$ & $1.1 \mathrm{E}-03 \pm 4.8 \mathrm{E}-04$ & & & & & \\
\hline & $09 / 23 / 08$ & $10 / 08 / 08$ & gross $\beta$ & $2.7 \mathrm{E}-02 \pm 2.8 \mathrm{E}-03$ & & & & & \\
\hline & $10 / 08 / 08$ & $10 / 21 / 08$ & gross $\alpha$ & $7.5 \mathrm{E}-04 \pm 5.6 \mathrm{E}-04$ & & & & & \\
\hline & $10 / 08 / 08$ & $10 / 21 / 08$ & gross $\beta$ & $2.4 \mathrm{E}-02 \pm 2.7 \mathrm{E}-03$ & & & & & \\
\hline & $10 / 21 / 08$ & $11 / 04 / 08$ & gross $\alpha$ & $2.3 \mathrm{E}-03 \pm 7.3 \mathrm{E}-04$ & & & & & \\
\hline & $10 / 21 / 08$ & $11 / 04 / 08$ & gross $\beta$ & $4.8 \mathrm{E}-02 \pm 4.5 \mathrm{E}-03$ & & & & & \\
\hline & $11 / 04 / 08$ & $11 / 18 / 08$ & gross $\alpha$ & $2.0 \mathrm{E}-03 \pm 7.1 \mathrm{E}-04$ & & & & & \\
\hline & $11 / 04 / 08$ & $11 / 18 / 08$ & gross $\beta$ & $2.6 \mathrm{E}-02 \pm 2.9 \mathrm{E}-03$ & & & & & \\
\hline & $11 / 18 / 08$ & $12 / 02 / 08$ & gross $\alpha$ & $9.5 \mathrm{E}-04 \pm 6.2 \mathrm{E}-04$ & & & & & \\
\hline & $11 / 18 / 08$ & $12 / 02 / 08$ & gross $\beta$ & $4.1 \mathrm{E}-02 \pm 4.0 \mathrm{E}-03$ & & & & & \\
\hline & $12 / 02 / 08$ & $12 / 16 / 08$ & gross $\alpha$ & $5.3 \mathrm{E}-04 \pm 4.8 \mathrm{E}-04$ & & & & & \\
\hline & $12 / 02 / 08$ & $12 / 16 / 08$ & gross $\beta$ & $2.6 \mathrm{E}-02 \pm 2.8 \mathrm{E}-03$ & & & & & \\
\hline & $12 / 16 / 08$ & $12 / 30 / 08$ & gross $\alpha$ & $1.8 \mathrm{E}-03 \pm 6.4 \mathrm{E}-04$ & & & & & \\
\hline & $12 / 16 / 08$ & $12 / 30 / 08$ & gross $\beta$ & $3.3 \mathrm{E}-02 \pm 3.2 \mathrm{E}-03$ & & & & & \\
\hline
\end{tabular}

$\overline{\mathrm{RQ}}=$ Result Qualifier. $\mathrm{U}=$ The analyte was analyzed for but not detected. 
Table 2-4. Near-Facility Air Sampling Results, 2008 (pCi/m³ \pm total analytical uncertainty). (Sheet 3 of 82)

\begin{tabular}{|c|c|c|c|c|c|c|c|c|c|}
\hline Location & Sample On & Sample Off & Isotope & Result \pm Uncertainty & Location & Composite Period & Isotope & Result \pm Uncertainty & $\mathbf{R Q *}$ \\
\hline N468 & $01 / 02 / 08$ & $01 / 15 / 08$ & gross $\alpha$ & $9.1 \mathrm{E}-04 \pm 6.1 \mathrm{E}-04$ & N468 & $01 / 02 / 08$ to $07 / 01 / 08$ & ${ }^{241} \mathrm{Am}$ & $5.8 \mathrm{E}-06 \pm 4.8 \mathrm{E}-06$ & \\
\hline \multirow[t]{51}{*}{$(100-D)$} & $01 / 02 / 08$ & $01 / 15 / 08$ & gross $\beta$ & $7.3 \mathrm{E}-03 \pm 1.3 \mathrm{E}-03$ & & & ${ }^{60} \mathrm{Co}$ & $3.4 \mathrm{E}-05 \pm 8.0 \mathrm{E}-05$ & $\mathrm{U}$ \\
\hline & $01 / 15 / 08$ & 01/30/08 & gross $\alpha$ & $1.5 \mathrm{E}-03 \pm 5.6 \mathrm{E}-04$ & & & ${ }^{134} \mathrm{Cs}$ & $-1.3 \mathrm{E}-05 \pm 6.8 \mathrm{E}-05$ & $\mathrm{U}$ \\
\hline & $01 / 15 / 08$ & 01/30/08 & gross $\beta$ & $3.5 \mathrm{E}-02 \pm 3.4 \mathrm{E}-03$ & & & ${ }^{137} \mathrm{Cs}$ & $3.6 \mathrm{E}-05 \pm 5.7 \mathrm{E}-05$ & $\mathrm{U}$ \\
\hline & 01/30/08 & $02 / 13 / 08$ & gross $\alpha$ & $2.7 \mathrm{E}-04 \pm 3.9 \mathrm{E}-04$ & & & ${ }^{152} \mathrm{Eu}$ & $3.2 \mathrm{E}-06 \pm 3.2 \mathrm{E}-05$ & $\mathrm{U}$ \\
\hline & $01 / 30 / 08$ & $02 / 13 / 08$ & gross $\beta$ & $3.8 \mathrm{E}-03 \pm 8.5 \mathrm{E}-04$ & & & ${ }^{154} \mathrm{Eu}$ & $7.0 \mathrm{E}-05 \pm 1.9 \mathrm{E}-04$ & $\mathrm{U}$ \\
\hline & $02 / 13 / 08$ & $02 / 27 / 08$ & gross $\alpha$ & $7.5 \mathrm{E}-04 \pm 5.5 \mathrm{E}-04$ & & & ${ }^{155} \mathrm{Eu}$ & $-2.6 \mathrm{E}-06 \pm 2.6 \mathrm{E}-05$ & $\mathrm{U}$ \\
\hline & $02 / 13 / 08$ & $02 / 27 / 08$ & gross $\beta$ & $2.9 \mathrm{E}-02 \pm 3.0 \mathrm{E}-03$ & & & ${ }^{238} \mathrm{Pu}$ & $5.2 \mathrm{E}-06 \pm 9.2 \mathrm{E}-06$ & $\mathrm{U}$ \\
\hline & $02 / 27 / 08$ & 03/12/08 & gross $\alpha$ & $9.6 \mathrm{E}-04 \pm 5.9 \mathrm{E}-04$ & & & ${ }^{239 / 240} \mathrm{Pu}$ & $3.0 \mathrm{E}-06 \pm 4.8 \mathrm{E}-06$ & $\mathrm{U}$ \\
\hline & $02 / 27 / 08$ & 03/12/08 & gross $\beta$ & $1.2 \mathrm{E}-02 \pm 1.6 \mathrm{E}-03$ & & & ${ }^{106} \mathrm{Ru}$ & $-4.0 \mathrm{E}-04 \pm 5.3 \mathrm{E}-04$ & $\mathrm{U}$ \\
\hline & 03/12/08 & 03/26/08 & gross $\alpha$ & $8.6 \mathrm{E}-04 \pm 5.8 \mathrm{E}-04$ & & & ${ }^{125} \mathrm{Sb}$ & $-3.2 \mathrm{E}-05 \pm 1.3 \mathrm{E}-04$ & $\mathrm{U}$ \\
\hline & 03/12/08 & 03/26/08 & gross $\beta$ & $8.5 \mathrm{E}-03 \pm 1.3 \mathrm{E}-03$ & & & ${ }^{90} \mathrm{Sr}$ & $-9.7 \mathrm{E}-05 \pm 1.0 \mathrm{E}-04$ & $\mathrm{U}$ \\
\hline & 03/26/08 & $04 / 08 / 08$ & gross $\alpha$ & $4.1 \mathrm{E}-04 \pm 4.6 \mathrm{E}-04$ & & & ${ }^{234} \mathrm{U}$ & $9.7 \mathrm{E}-06 \pm 6.4 \mathrm{E}-06$ & \\
\hline & 03/26/08 & $04 / 08 / 08$ & gross $\beta$ & $6.7 \mathrm{E}-03 \pm 1.2 \mathrm{E}-03$ & & & ${ }^{235} \mathrm{U}$ & $7.6 \mathrm{E}-07 \pm 2.6 \mathrm{E}-06$ & $\mathrm{U}$ \\
\hline & $04 / 08 / 08$ & $04 / 23 / 08$ & gross $\alpha$ & $8.7 \mathrm{E}-04 \pm 5.4 \mathrm{E}-04$ & & & ${ }^{238} \mathrm{U}$ & $7.7 \mathrm{E}-06 \pm 5.5 \mathrm{E}-06$ & \\
\hline & $04 / 08 / 08$ & $04 / 23 / 08$ & gross $\beta$ & $1.2 \mathrm{E}-02 \pm 1.6 \mathrm{E}-03$ & & & & & \\
\hline & $04 / 23 / 08$ & 05/07/08 & gross $\alpha$ & $9.9 \mathrm{E}-04 \pm 4.7 \mathrm{E}-04$ & N468 & $07 / 01 / 08$ to $12 / 30 / 08$ & ${ }^{241} \mathrm{Am}$ & $4.6 \mathrm{E}-06 \pm 4.1 \mathrm{E}-06$ & $\mathrm{U}$ \\
\hline & $04 / 23 / 08$ & 05/07/08 & gross $\beta$ & $1.9 \mathrm{E}-02 \pm 2.3 \mathrm{E}-03$ & & & ${ }^{60} \mathrm{Co}$ & $5.5 \mathrm{E}-06 \pm 5.6 \mathrm{E}-05$ & $\mathrm{U}$ \\
\hline & 05/07/08 & $05 / 20 / 08$ & gross $\alpha$ & $1.1 \mathrm{E}-03 \pm 5.2 \mathrm{E}-04$ & & & ${ }^{134} \mathrm{Cs}$ & $-2.2 \mathrm{E}-05 \pm 6.9 \mathrm{E}-05$ & $\mathrm{U}$ \\
\hline & 05/07/08 & 05/20/08 & gross $\beta$ & $9.2 \mathrm{E}-03 \pm 1.4 \mathrm{E}-03$ & & & ${ }^{137} \mathrm{Cs}$ & $5.1 \mathrm{E}-05 \pm 6.2 \mathrm{E}-05$ & $\mathrm{U}$ \\
\hline & $05 / 20 / 08$ & 06/04/08 & gross $\alpha$ & $1.2 \mathrm{E}-03 \pm 5.0 \mathrm{E}-04$ & & & ${ }^{152} \mathrm{Eu}$ & $-1.5 \mathrm{E}-05 \pm 1.5 \mathrm{E}-04$ & $\mathrm{U}$ \\
\hline & $05 / 20 / 08$ & $06 / 04 / 08$ & gross $\beta$ & $1.1 \mathrm{E}-02 \pm 1.5 \mathrm{E}-03$ & & & ${ }^{154} \mathrm{Eu}$ & $8.5 \mathrm{E}-05 \pm 2.0 \mathrm{E}-04$ & $\mathrm{U}$ \\
\hline & $06 / 04 / 08$ & $06 / 17 / 08$ & gross $\alpha$ & $8.0 \mathrm{E}-04 \pm 6.2 \mathrm{E}-04$ & & & ${ }^{155} \mathrm{Eu}$ & $-4.1 \mathrm{E}-05 \pm 1.4 \mathrm{E}-04$ & $\mathrm{U}$ \\
\hline & $06 / 04 / 08$ & 06/17/08 & gross $\beta$ & $8.8 \mathrm{E}-03 \pm 1.4 \mathrm{E}-03$ & & & ${ }^{238} \mathrm{Pu}$ & $-1.9 \mathrm{E}-06 \pm 9.9 \mathrm{E}-06$ & $\mathrm{U}$ \\
\hline & $06 / 17 / 08$ & 07/01/08 & gross $\alpha$ & $7.2 \mathrm{E}-04 \pm 5.4 \mathrm{E}-04$ & & & ${ }^{239 / 240} \mathrm{Pu}$ & $1.3 \mathrm{E}-06 \pm 1.9 \mathrm{E}-06$ & $\mathrm{U}$ \\
\hline & $06 / 17 / 08$ & 07/01/08 & gross $\beta$ & $1.5 \mathrm{E}-02 \pm 1.9 \mathrm{E}-03$ & & & ${ }^{106} \mathrm{Ru}$ & $-2.3 \mathrm{E}-04 \pm 5.8 \mathrm{E}-04$ & $\mathrm{U}$ \\
\hline & $07 / 01 / 08$ & $07 / 15 / 08$ & gross $\alpha$ & $1.4 \mathrm{E}-03 \pm 6.2 \mathrm{E}-04$ & & & ${ }^{125} \mathrm{Sb}$ & $-6.7 \mathrm{E}-05 \pm 1.5 \mathrm{E}-04$ & $\mathrm{U}$ \\
\hline & 07/01/08 & 07/15/08 & gross $\beta$ & $1.4 \mathrm{E}-02 \pm 1.8 \mathrm{E}-03$ & & & ${ }^{90} \mathrm{Sr}$ & $-4.1 \mathrm{E}-05 \pm 4.3 \mathrm{E}-05$ & $\mathrm{U}$ \\
\hline & 07/15/08 & $07 / 29 / 08$ & gross $\alpha$ & $8.9 \mathrm{E}-04 \pm 5.6 \mathrm{E}-04$ & & & ${ }^{234} \mathrm{U}$ & $1.3 \mathrm{E}-05 \pm 8.2 \mathrm{E}-06$ & \\
\hline & $07 / 15 / 08$ & 07/29/08 & gross $\beta$ & $1.2 \mathrm{E}-02 \pm 1.7 \mathrm{E}-03$ & & & ${ }^{235} \mathrm{U}$ & $2.7 \mathrm{E}-06 \pm 2.9 \mathrm{E}-06$ & \\
\hline & 07/29/08 & 08/12/08 & gross $\alpha$ & $7.8 \mathrm{E}-04 \pm 5.4 \mathrm{E}-04$ & & & ${ }^{238} \mathrm{U}$ & $8.7 \mathrm{E}-06 \pm$ 5.7E-06 & \\
\hline & 07/29/08 & 08/12/08 & gross $\beta$ & $1.6 \mathrm{E}-02 \pm 2.0 \mathrm{E}-03$ & & & & & \\
\hline & $08 / 12 / 08$ & 08/26/08 & gross $\alpha$ & $5.5 \mathrm{E}-04 \pm 4.6 \mathrm{E}-04$ & & & & & \\
\hline & 08/12/08 & 08/26/08 & gross $\beta$ & $1.6 \mathrm{E}-02 \pm 2.0 \mathrm{E}-03$ & & & & & \\
\hline & $08 / 26 / 08$ & 09/09/08 & gross $\alpha$ & $6.9 \mathrm{E}-04 \pm 5.2 \mathrm{E}-04$ & & & & & \\
\hline & 08/26/08 & 09/09/08 & gross $\beta$ & $1.1 \mathrm{E}-02 \pm 1.6 \mathrm{E}-03$ & & & & & \\
\hline & 09/09/08 & 09/23/08 & gross $\alpha$ & $1.7 \mathrm{E}-03 \pm 6.2 \mathrm{E}-04$ & & & & & \\
\hline & 09/09/08 & 09/23/08 & gross $\beta$ & $2.9 \mathrm{E}-02 \pm 3.0 \mathrm{E}-03$ & & & & & \\
\hline & 09/23/08 & $10 / 08 / 08$ & gross $\alpha$ & $1.5 \mathrm{E}-03 \pm 5.6 \mathrm{E}-04$ & & & & & \\
\hline & 09/23/08 & $10 / 08 / 08$ & gross $\beta$ & $2.4 \mathrm{E}-02 \pm 2.6 \mathrm{E}-03$ & & & & & \\
\hline & $10 / 08 / 08$ & $10 / 21 / 08$ & gross $\alpha$ & $1.3 \mathrm{E}-03 \pm 5.6 \mathrm{E}-04$ & & & & & \\
\hline & $10 / 08 / 08$ & $10 / 21 / 08$ & gross $\beta$ & $2.3 \mathrm{E}-02 \pm 2.6 \mathrm{E}-03$ & & & & & \\
\hline & $10 / 21 / 08$ & $11 / 04 / 08$ & gross $\alpha$ & $2.9 \mathrm{E}-03 \pm 8.2 \mathrm{E}-04$ & & & & & \\
\hline & $10 / 21 / 08$ & $11 / 04 / 08$ & gross $\beta$ & $4.9 \mathrm{E}-02 \pm 4.6 \mathrm{E}-03$ & & & & & \\
\hline & $11 / 04 / 08$ & $11 / 18 / 08$ & gross $\alpha$ & $2.7 \mathrm{E}-03 \pm 7.8 \mathrm{E}-04$ & & & & & \\
\hline & $11 / 04 / 08$ & $11 / 18 / 08$ & gross $\beta$ & $2.6 \mathrm{E}-02 \pm 2.8 \mathrm{E}-03$ & & & & & \\
\hline & $11 / 18 / 08$ & $12 / 02 / 08$ & gross $\alpha$ & $1.4 \mathrm{E}-03 \pm 5.6 \mathrm{E}-04$ & & & & & \\
\hline & $11 / 18 / 08$ & $12 / 02 / 08$ & gross $\beta$ & $3.2 \mathrm{E}-02 \pm 3.2 \mathrm{E}-03$ & & & & & \\
\hline & $12 / 02 / 08$ & $12 / 16 / 08$ & gross $\alpha$ & $1.4 \mathrm{E}-03 \pm 5.8 \mathrm{E}-04$ & & & & & \\
\hline & $12 / 02 / 08$ & $12 / 16 / 08$ & gross $\beta$ & $2.4 \mathrm{E}-02 \pm 2.6 \mathrm{E}-03$ & & & & & \\
\hline & $12 / 16 / 08$ & $12 / 30 / 08$ & gross $\alpha$ & $2.3 \mathrm{E}-03 \pm 7.2 \mathrm{E}-04$ & & & & & \\
\hline & $12 / 16 / 08$ & $12 / 30 / 08$ & gross $\beta$ & $2.5 \mathrm{E}-02 \pm 2.7 \mathrm{E}-03$ & & & & & \\
\hline
\end{tabular}

$\overline{\mathrm{RQ}}=$ Result Qualifier. $\mathrm{U}=$ The analyte was analyzed for but not detected. 
Table 2-4. Near-Facility Air Sampling Results, 2008 (pCi/m³ \pm total analytical uncertainty). (Sheet 4 of 82)

\begin{tabular}{|c|c|c|c|c|c|c|c|c|c|}
\hline Location & Sample On & Sample Off & Isotope & Result \pm Uncertainty & Location & Composite Period & Isotope & Result \pm Uncertainty & $\mathbf{R Q *}$ \\
\hline N514 & $01 / 02 / 08$ & $01 / 15 / 08$ & gross $\alpha$ & $7.4 \mathrm{E}-04 \pm 5.5 \mathrm{E}-04$ & N514 & $01 / 02 / 08$ to $07 / 01 / 08$ & ${ }^{241} \mathrm{Am}$ & $6.4 \mathrm{E}-06 \pm 5.5 \mathrm{E}-06$ & $\mathrm{U}$ \\
\hline \multirow[t]{51}{*}{$(100-D)$} & $01 / 02 / 08$ & $01 / 15 / 08$ & gross $\beta$ & $8.2 \mathrm{E}-03 \pm 1.3 \mathrm{E}-03$ & & & ${ }^{60} \mathrm{Co}$ & $-9.2 \mathrm{E}-05 \pm 1.4 \mathrm{E}-04$ & $\mathrm{U}$ \\
\hline & 01/15/08 & 01/30/08 & gross $\alpha$ & $1.8 \mathrm{E}-03 \pm 6.3 \mathrm{E}-04$ & & & ${ }^{134} \mathrm{Cs}$ & $7.7 \mathrm{E}-05 \pm 1.1 \mathrm{E}-04$ & $\mathrm{U}$ \\
\hline & $01 / 15 / 08$ & 01/30/08 & gross $\beta$ & $3.4 \mathrm{E}-02 \pm 3.3 \mathrm{E}-03$ & & & ${ }^{137} \mathrm{Cs}$ & $2.8 \mathrm{E}-06 \pm 2.8 \mathrm{E}-05$ & $\mathrm{U}$ \\
\hline & 01/30/08 & $02 / 13 / 08$ & gross $\alpha$ & $1.7 \mathrm{E}-04 \pm 3.5 \mathrm{E}-04$ & & & ${ }^{152} \mathrm{Eu}$ & $-5.9 \mathrm{E}-05 \pm 2.3 \mathrm{E}-04$ & $\mathrm{U}$ \\
\hline & $01 / 30 / 08$ & $02 / 13 / 08$ & gross $\beta$ & $3.3 \mathrm{E}-03 \pm 7.9 \mathrm{E}-04$ & & & ${ }^{154} \mathrm{Eu}$ & $-6.2 \mathrm{E}-05 \pm 3.7 \mathrm{E}-04$ & $\mathrm{U}$ \\
\hline & $02 / 13 / 08$ & $02 / 27 / 08$ & gross $\alpha$ & $1.6 \mathrm{E}-03 \pm 6.1 \mathrm{E}-04$ & & & ${ }^{155} \mathrm{Eu}$ & $-2.9 \mathrm{E}-05 \pm 2.0 \mathrm{E}-04$ & $\mathrm{U}$ \\
\hline & $02 / 13 / 08$ & $02 / 27 / 08$ & gross $\beta$ & $3.2 \mathrm{E}-02 \pm 3.2 \mathrm{E}-03$ & & & ${ }^{238} \mathrm{Pu}$ & $2.7 \mathrm{E}-06 \pm 1.5 \mathrm{E}-05$ & $\mathrm{U}$ \\
\hline & $02 / 27 / 08$ & 03/12/08 & gross $\alpha$ & $7.3 \mathrm{E}-04 \pm 5.4 \mathrm{E}-04$ & & & ${ }^{239 / 240} \mathrm{Pu}$ & $4.5 \mathrm{E}-06 \pm 6.6 \mathrm{E}-06$ & $\mathrm{U}$ \\
\hline & $02 / 27 / 08$ & 03/12/08 & gross $\beta$ & $1.3 \mathrm{E}-02 \pm 1.8 \mathrm{E}-03$ & & & ${ }^{106} \mathrm{Ru}$ & $1.2 \mathrm{E}-04 \pm 8.0 \mathrm{E}-04$ & $\mathrm{U}$ \\
\hline & 03/12/08 & 03/26/08 & gross $\alpha$ & $7.6 \mathrm{E}-04 \pm 5.6 \mathrm{E}-04$ & & & ${ }^{125} \mathrm{Sb}$ & $2.0 \mathrm{E}-04 \pm 2.5 \mathrm{E}-04$ & $\mathrm{U}$ \\
\hline & 03/12/08 & 03/26/08 & gross $\beta$ & $1.0 \mathrm{E}-02 \pm 1.5 \mathrm{E}-03$ & & & ${ }^{90} \mathrm{Sr}$ & $-1.9 \mathrm{E}-04 \pm 2.0 \mathrm{E}-04$ & $\mathrm{U}$ \\
\hline & 03/26/08 & $04 / 08 / 08$ & gross $\alpha$ & $3.0 \mathrm{E}-04 \pm 4.3 \mathrm{E}-04$ & & & ${ }^{234} \mathrm{U}$ & $1.7 \mathrm{E}-05 \pm 9.5 \mathrm{E}-06$ & \\
\hline & 03/26/08 & $04 / 08 / 08$ & gross $\beta$ & $7.4 \mathrm{E}-03 \pm 1.3 \mathrm{E}-03$ & & & ${ }^{235} \mathrm{U}$ & $3.0 \mathrm{E}-06 \pm 3.2 \mathrm{E}-06$ & \\
\hline & $04 / 08 / 08$ & $04 / 23 / 08$ & gross $\alpha$ & $1.1 \mathrm{E}-03 \pm 5.0 \mathrm{E}-04$ & & & ${ }^{238} \mathrm{U}$ & $6.2 \mathrm{E}-06 \pm 4.8 \mathrm{E}-06$ & \\
\hline & $04 / 08 / 08$ & $04 / 23 / 08$ & gross $\beta$ & $1.1 \mathrm{E}-02 \pm 1.5 \mathrm{E}-03$ & & & & & \\
\hline & $04 / 23 / 08$ & 05/07/08 & gross $\alpha$ & $1.9 \mathrm{E}-03 \pm 6.7 \mathrm{E}-04$ & N514 & $07 / 01 / 08$ to $12 / 30 / 08$ & ${ }^{241} \mathrm{Am}$ & $6.2 \mathrm{E}-06 \pm 4.6 \mathrm{E}-06$ & \\
\hline & $04 / 23 / 08$ & 05/07/08 & gross $\beta$ & $1.5 \mathrm{E}-02 \pm 1.9 \mathrm{E}-03$ & & & ${ }^{60} \mathrm{Co}$ & $2.8 \mathrm{E}-05 \pm 8.4 \mathrm{E}-05$ & $\mathrm{U}$ \\
\hline & 05/07/08 & $05 / 20 / 08$ & gross $\alpha$ & $1.3 \mathrm{E}-03 \pm 5.7 \mathrm{E}-04$ & & & ${ }^{134} \mathrm{Cs}$ & $-1.3 \mathrm{E}-05 \pm 7.5 \mathrm{E}-05$ & $\mathrm{U}$ \\
\hline & 05/07/08 & 05/20/08 & gross $\beta$ & $1.2 \mathrm{E}-02 \pm 1.6 \mathrm{E}-03$ & & & ${ }^{137} \mathrm{Cs}$ & $-1.9 \mathrm{E}-05 \pm 6.2 \mathrm{E}-05$ & $\mathrm{U}$ \\
\hline & $05 / 20 / 08$ & $06 / 04 / 08$ & gross $\alpha$ & $1.4 \mathrm{E}-03 \pm 5.5 \mathrm{E}-04$ & & & ${ }^{152} \mathrm{Eu}$ & $4.4 \mathrm{E}-06 \pm 4.4 \mathrm{E}-05$ & $\mathrm{U}$ \\
\hline & $05 / 20 / 08$ & $06 / 04 / 08$ & gross $\beta$ & $1.1 \mathrm{E}-02 \pm 1.5 \mathrm{E}-03$ & & & ${ }^{154} \mathrm{Eu}$ & $1.8 \mathrm{E}-04 \pm 2.3 \mathrm{E}-04$ & $\mathrm{U}$ \\
\hline & $06 / 04 / 08$ & 06/17/08 & gross $\alpha$ & $3.1 \mathrm{E}-04 \pm 4.2 \mathrm{E}-04$ & & & ${ }^{155} \mathrm{Eu}$ & $2.9 \mathrm{E}-06 \pm 2.9 \mathrm{E}-05$ & $\mathrm{U}$ \\
\hline & $06 / 04 / 08$ & 06/17/08 & gross $\beta$ & $8.1 \mathrm{E}-03 \pm 1.3 \mathrm{E}-03$ & & & ${ }^{238} \mathrm{Pu}$ & $6.7 \mathrm{E}-07 \pm 6.7 \mathrm{E}-06$ & $\mathrm{U}$ \\
\hline & $06 / 17 / 08$ & 07/01/08 & gross $\alpha$ & $1.5 \mathrm{E}-03 \pm 6.1 \mathrm{E}-04$ & & & ${ }^{239 / 240} \mathrm{Pu}$ & $2.0 \mathrm{E}-06 \pm 2.4 \mathrm{E}-06$ & \\
\hline & $06 / 17 / 08$ & 07/01/08 & gross $\beta$ & $1.6 \mathrm{E}-02 \pm 2.0 \mathrm{E}-03$ & & & ${ }^{106} \mathrm{Ru}$ & $6.2 \mathrm{E}-05 \pm 5.8 \mathrm{E}-04$ & $\mathrm{U}$ \\
\hline & $07 / 01 / 08$ & $07 / 15 / 08$ & gross $\alpha$ & $1.5 \mathrm{E}-03 \pm 6.0 \mathrm{E}-04$ & & & ${ }^{125} \mathrm{Sb}$ & $9.9 \mathrm{E}-06 \pm 9.9 \mathrm{E}-05$ & $\mathrm{U}$ \\
\hline & 07/01/08 & 07/15/08 & gross $\beta$ & $1.4 \mathrm{E}-02 \pm 1.8 \mathrm{E}-03$ & & & ${ }^{90} \mathrm{Sr}$ & $-4.3 \mathrm{E}-04 \pm 4.5 \mathrm{E}-04$ & $\mathrm{U}$ \\
\hline & 07/15/08 & 07/29/08 & gross $\alpha$ & $1.9 \mathrm{E}-03 \pm 6.5 \mathrm{E}-04$ & & & ${ }^{234} \mathrm{U}$ & $1.2 \mathrm{E}-05 \pm 7.6 \mathrm{E}-06$ & \\
\hline & $07 / 15 / 08$ & 07/29/08 & gross $\beta$ & $1.6 \mathrm{E}-02 \pm 1.9 \mathrm{E}-03$ & & & ${ }^{235} \mathrm{U}$ & $1.5 \mathrm{E}-06 \pm 2.2 \mathrm{E}-06$ & $\mathrm{U}$ \\
\hline & 07/29/08 & 08/12/08 & gross $\alpha$ & $5.3 \mathrm{E}-04 \pm 4.8 \mathrm{E}-04$ & & & ${ }^{238} \mathrm{U}$ & $1.0 \mathrm{E}-05 \pm 6.5 \mathrm{E}-06$ & \\
\hline & 07/29/08 & 08/12/08 & gross $\beta$ & $1.3 \mathrm{E}-02 \pm 1.8 \mathrm{E}-03$ & & & & & \\
\hline & $08 / 12 / 08$ & 08/26/08 & gross $\alpha$ & $7.4 \mathrm{E}-04 \pm 5.3 \mathrm{E}-04$ & & & & & \\
\hline & $08 / 12 / 08$ & 08/26/08 & gross $\beta$ & $1.2 \mathrm{E}-02 \pm 1.6 \mathrm{E}-03$ & & & & & \\
\hline & 08/26/08 & 09/09/08 & gross $\alpha$ & $8.7 \mathrm{E}-04 \pm 5.7 \mathrm{E}-04$ & & & & & \\
\hline & 08/26/08 & 09/09/08 & gross $\beta$ & $1.1 \mathrm{E}-02 \pm 1.5 \mathrm{E}-03$ & & & & & \\
\hline & 09/09/08 & 09/23/08 & gross $\alpha$ & $6.3 \mathrm{E}-04 \pm 5.0 \mathrm{E}-04$ & & & & & \\
\hline & 09/09/08 & 09/23/08 & gross $\beta$ & $2.4 \mathrm{E}-02 \pm 2.6 \mathrm{E}-03$ & & & & & \\
\hline & 09/23/08 & $10 / 08 / 08$ & gross $\alpha$ & $1.3 \mathrm{E}-03 \pm 5.5 \mathrm{E}-04$ & & & & & \\
\hline & 09/23/08 & $10 / 08 / 08$ & gross $\beta$ & $1.9 \mathrm{E}-02 \pm 2.2 \mathrm{E}-03$ & & & & & \\
\hline & $10 / 08 / 08$ & $10 / 21 / 08$ & gross $\alpha$ & $1.2 \mathrm{E}-03 \pm 5.5 \mathrm{E}-04$ & & & & & \\
\hline & $10 / 08 / 08$ & $10 / 21 / 08$ & gross $\beta$ & $1.9 \mathrm{E}-02 \pm 2.2 \mathrm{E}-03$ & & & & & \\
\hline & $10 / 21 / 08$ & $11 / 04 / 08$ & gross $\alpha$ & $3.1 \mathrm{E}-03 \pm 8.5 \mathrm{E}-04$ & & & & & \\
\hline & $10 / 21 / 08$ & $11 / 04 / 08$ & gross $\beta$ & $4.3 \mathrm{E}-02 \pm 4.1 \mathrm{E}-03$ & & & & & \\
\hline & $11 / 04 / 08$ & $11 / 18 / 08$ & gross $\alpha$ & $1.6 \mathrm{E}-03 \pm 6.4 \mathrm{E}-04$ & & & & & \\
\hline & $11 / 04 / 08$ & $11 / 18 / 08$ & gross $\beta$ & $2.5 \mathrm{E}-02 \pm 2.7 \mathrm{E}-03$ & & & & & \\
\hline & $11 / 18 / 08$ & $12 / 02 / 08$ & gross $\alpha$ & $1.5 \mathrm{E}-03 \pm 5.8 \mathrm{E}-04$ & & & & & \\
\hline & $11 / 18 / 08$ & $12 / 02 / 08$ & gross $\beta$ & $3.7 \mathrm{E}-02 \pm 3.7 \mathrm{E}-03$ & & & & & \\
\hline & $12 / 02 / 08$ & $12 / 16 / 08$ & gross $\alpha$ & $1.7 \mathrm{E}-03 \pm 6.3 \mathrm{E}-04$ & & & & & \\
\hline & $12 / 02 / 08$ & $12 / 16 / 08$ & gross $\beta$ & $2.3 \mathrm{E}-02 \pm 2.6 \mathrm{E}-03$ & & & & & \\
\hline & $12 / 16 / 08$ & $12 / 30 / 08$ & gross $\alpha$ & $1.5 \mathrm{E}-03 \pm 6.0 \mathrm{E}-04$ & & & & & \\
\hline & $12 / 16 / 08$ & $12 / 30 / 08$ & gross $\beta$ & $2.7 \mathrm{E}-02 \pm 2.8 \mathrm{E}-03$ & & & & & \\
\hline
\end{tabular}

$\overline{\mathrm{RQ}}=$ Result Qualifier. $\mathrm{U}=$ The analyte was analyzed for but not detected. 
Table 2-4. Near-Facility Air Sampling Results, 2008 (pCi/m³ \pm total analytical uncertainty). (Sheet 5 of 82)

\begin{tabular}{|c|c|c|c|c|c|c|c|c|c|}
\hline Location & Sample On & Sample Off & Isotope & Result \pm Uncertainty & Location & Composite Period & Isotope & Result \pm Uncertainty & $\mathbf{R Q}^{*}$ \\
\hline N515 & $01 / 02 / 08$ & $01 / 15 / 08$ & gross $\alpha$ & $5.2 \mathrm{E}-04 \pm 5.0 \mathrm{E}-04$ & N515 & $01 / 02 / 08$ to $07 / 01 / 08$ & ${ }^{241} \mathrm{Am}$ & $5.6 \mathrm{E}-06 \pm 4.1 \mathrm{E}-06$ & \\
\hline \multirow[t]{51}{*}{$(100-D)$} & $01 / 02 / 08$ & $01 / 15 / 08$ & gross $\beta$ & $9.9 \mathrm{E}-03 \pm 1.5 \mathrm{E}-03$ & & & ${ }^{60} \mathrm{Co}$ & $7.8 \mathrm{E}-05 \pm 8.6 \mathrm{E}-05$ & $\mathrm{U}$ \\
\hline & 01/15/08 & 01/30/08 & gross $\alpha$ & $2.0 \mathrm{E}-03 \pm 6.6 \mathrm{E}-04$ & & & ${ }^{134} \mathrm{Cs}$ & $-9.9 \mathrm{E}-06 \pm 7.7 \mathrm{E}-05$ & $\mathrm{U}$ \\
\hline & $01 / 15 / 08$ & 01/30/08 & gross $\beta$ & $4.4 \mathrm{E}-02 \pm 4.0 \mathrm{E}-03$ & & & ${ }^{137} \mathrm{Cs}$ & $-2.1 \mathrm{E}-05 \pm 6.3 \mathrm{E}-05$ & $\mathrm{U}$ \\
\hline & 01/30/08 & $02 / 13 / 08$ & gross $\alpha$ & $3.9 \mathrm{E}-04 \pm 4.4 \mathrm{E}-04$ & & & ${ }^{152} \mathrm{Eu}$ & $-2.1 \mathrm{E}-06 \pm 2.1 \mathrm{E}-05$ & $\mathrm{U}$ \\
\hline & 01/30/08 & $02 / 13 / 08$ & gross $\beta$ & $4.2 \mathrm{E}-03 \pm 8.9 \mathrm{E}-04$ & & & ${ }^{154} \mathrm{Eu}$ & $1.5 \mathrm{E}-04 \pm 2.1 \mathrm{E}-04$ & $\mathrm{U}$ \\
\hline & $02 / 13 / 08$ & $02 / 27 / 08$ & gross $\alpha$ & $2.1 \mathrm{E}-03 \pm 6.9 \mathrm{E}-04$ & & & ${ }^{155} \mathrm{Eu}$ & $-5.6 \mathrm{E}-06 \pm 5.7 \mathrm{E}-05$ & $\mathrm{U}$ \\
\hline & $02 / 13 / 08$ & $02 / 27 / 08$ & gross $\beta$ & $3.5 \mathrm{E}-02 \pm 3.5 \mathrm{E}-03$ & & & ${ }^{238} \mathrm{Pu}$ & $-1.2 \mathrm{E}-05 \pm 1.3 \mathrm{E}-05$ & $\mathrm{U}$ \\
\hline & $02 / 27 / 08$ & 03/12/08 & gross $\alpha$ & $6.0 \mathrm{E}-04 \pm 5.0 \mathrm{E}-04$ & & & ${ }^{239 / 240} \mathrm{Pu}$ & $7.6 \mathrm{E}-07 \pm 4.0 \mathrm{E}-06$ & $\mathrm{U}$ \\
\hline & $02 / 27 / 08$ & 03/12/08 & gross $\beta$ & $1.3 \mathrm{E}-02 \pm 1.7 \mathrm{E}-03$ & & & ${ }^{106} \mathrm{Ru}$ & $-2.1 \mathrm{E}-04 \pm 5.6 \mathrm{E}-04$ & $\mathrm{U}$ \\
\hline & 03/12/08 & 03/26/08 & gross $\alpha$ & $6.1 \mathrm{E}-04 \pm 5.0 \mathrm{E}-04$ & & & ${ }^{125} \mathrm{Sb}$ & $5.3 \mathrm{E}-05 \pm 1.4 \mathrm{E}-04$ & $\mathrm{U}$ \\
\hline & 03/12/08 & 03/26/08 & gross $\beta$ & $9.4 \mathrm{E}-03 \pm 1.4 \mathrm{E}-03$ & & & ${ }^{90} \mathrm{Sr}$ & $-2.8 \mathrm{E}-04 \pm 2.9 \mathrm{E}-04$ & $\mathrm{U}$ \\
\hline & 03/26/08 & $04 / 08 / 08$ & gross $\alpha$ & $7.8 \mathrm{E}-04 \pm 5.8 \mathrm{E}-04$ & & & ${ }^{234} \mathrm{U}$ & $1.5 \mathrm{E}-05 \pm 8.9 \mathrm{E}-06$ & \\
\hline & 03/26/08 & $04 / 08 / 08$ & gross $\beta$ & $6.4 \mathrm{E}-03 \pm 1.2 \mathrm{E}-03$ & & & ${ }^{235} \mathrm{U}$ & $2.8 \mathrm{E}-06 \pm 3.6 \mathrm{E}-06$ & $\mathrm{U}$ \\
\hline & $04 / 08 / 08$ & $04 / 23 / 08$ & gross $\alpha$ & $3.6 \mathrm{E}-04 \pm 4.1 \mathrm{E}-04$ & & & ${ }^{238} \mathrm{U}$ & $7.0 \mathrm{E}-06 \pm 5.0 \mathrm{E}-06$ & \\
\hline & $04 / 08 / 08$ & $04 / 23 / 08$ & gross $\beta$ & $1.2 \mathrm{E}-02 \pm 1.6 \mathrm{E}-03$ & & & & & \\
\hline & $04 / 23 / 08$ & 05/07/08 & gross $\alpha$ & $7.0 \mathrm{E}-04 \pm 5.2 \mathrm{E}-04$ & N515 & $07 / 01 / 08$ to $12 / 30 / 08$ & ${ }^{241} \mathrm{Am}$ & $6.9 \mathrm{E}-06 \pm 5.0 \mathrm{E}-06$ & \\
\hline & $04 / 23 / 08$ & 05/07/08 & gross $\beta$ & $1.7 \mathrm{E}-02 \pm 2.1 \mathrm{E}-03$ & & & ${ }^{60} \mathrm{Co}$ & $1.8 \mathrm{E}-06 \pm 1.8 \mathrm{E}-05$ & $\mathrm{U}$ \\
\hline & 05/07/08 & 05/20/08 & gross $\alpha$ & $9.2 \mathrm{E}-04 \pm 6.1 \mathrm{E}-04$ & & & ${ }^{134} \mathrm{Cs}$ & $2.2 \mathrm{E}-05 \pm 6.6 \mathrm{E}-05$ & $\mathrm{U}$ \\
\hline & 05/07/08 & $05 / 20 / 08$ & gross $\beta$ & $1.2 \mathrm{E}-02 \pm 1.7 \mathrm{E}-03$ & & & ${ }^{137} \mathrm{Cs}$ & $-1.1 \mathrm{E}-05 \pm 5.9 \mathrm{E}-05$ & $\mathrm{U}$ \\
\hline & $05 / 20 / 08$ & $06 / 04 / 08$ & gross $\alpha$ & $7.8 \mathrm{E}-04 \pm 5.1 \mathrm{E}-04$ & & & ${ }^{152} \mathrm{Eu}$ & $-9.0 \mathrm{E}-05 \pm 1.5 \mathrm{E}-04$ & $\mathrm{U}$ \\
\hline & $05 / 20 / 08$ & $06 / 04 / 08$ & gross $\beta$ & $1.2 \mathrm{E}-02 \pm 1.6 \mathrm{E}-03$ & & & ${ }^{154} \mathrm{Eu}$ & $9.5 \mathrm{E}-05 \pm 2.2 \mathrm{E}-04$ & $\mathrm{U}$ \\
\hline & $06 / 04 / 08$ & 06/17/08 & gross $\alpha$ & $6.4 \mathrm{E}-04 \pm 5.1 \mathrm{E}-04$ & & & ${ }^{155} \mathrm{Eu}$ & $-3.7 \mathrm{E}-06 \pm 3.7 \mathrm{E}-05$ & $\mathrm{U}$ \\
\hline & $06 / 04 / 08$ & 06/17/08 & gross $\beta$ & $9.0 \mathrm{E}-03 \pm 1.4 \mathrm{E}-03$ & & & ${ }^{238} \mathrm{Pu}$ & $-2.0 \mathrm{E}-05 \pm 2.1 \mathrm{E}-05$ & $\mathrm{U}$ \\
\hline & $06 / 17 / 08$ & 07/01/08 & gross $\alpha$ & $1.6 \mathrm{E}-03 \pm 6.2 \mathrm{E}-04$ & & & ${ }^{239 / 240} \mathrm{Pu}$ & $6.7 \mathrm{E}-07 \pm 3.0 \mathrm{E}-06$ & $\mathrm{U}$ \\
\hline & $06 / 17 / 08$ & 07/01/08 & gross $\beta$ & $1.2 \mathrm{E}-02 \pm 1.7 \mathrm{E}-03$ & & & ${ }^{106} \mathrm{Ru}$ & $-5.6 \mathrm{E}-04 \pm 5.9 \mathrm{E}-04$ & $\mathrm{U}$ \\
\hline & $07 / 01 / 08$ & $07 / 15 / 08$ & gross $\alpha$ & $1.5 \mathrm{E}-03 \pm 5.9 \mathrm{E}-04$ & & & ${ }^{125} \mathrm{Sb}$ & $-4.9 \mathrm{E}-05 \pm 1.3 \mathrm{E}-04$ & $\mathrm{U}$ \\
\hline & 07/01/08 & 07/15/08 & gross $\beta$ & $1.2 \mathrm{E}-02 \pm 1.6 \mathrm{E}-03$ & & & ${ }^{90} \mathrm{Sr}$ & $-9.7 \mathrm{E}-05 \pm 1.0 \mathrm{E}-04$ & $\mathrm{U}$ \\
\hline & 07/15/08 & 07/29/08 & gross $\alpha$ & $1.2 \mathrm{E}-03 \pm 5.1 \mathrm{E}-04$ & & & ${ }^{234} \mathrm{U}$ & $1.6 \mathrm{E}-05 \pm 9.3 \mathrm{E}-06$ & \\
\hline & $07 / 15 / 08$ & 07/29/08 & gross $\beta$ & $1.2 \mathrm{E}-02 \pm 1.6 \mathrm{E}-03$ & & & ${ }^{235} \mathrm{U}$ & $2.1 \mathrm{E}-06 \pm 2.6 \mathrm{E}-06$ & \\
\hline & 07/29/08 & 08/12/08 & gross $\alpha$ & $1.0 \mathrm{E}-03 \pm 4.8 \mathrm{E}-04$ & & & ${ }^{238} \mathrm{U}$ & $7.1 \mathrm{E}-06 \pm 5.0 \mathrm{E}-06$ & \\
\hline & 07/29/08 & 08/12/08 & gross $\beta$ & $1.5 \mathrm{E}-02 \pm 1.8 \mathrm{E}-03$ & & & & & \\
\hline & $08 / 12 / 08$ & 08/26/08 & gross $\alpha$ & $1.0 \mathrm{E}-03 \pm 4.8 \mathrm{E}-04$ & & & & & \\
\hline & $08 / 12 / 08$ & 08/26/08 & gross $\beta$ & $1.3 \mathrm{E}-02 \pm 1.7 \mathrm{E}-03$ & & & & & \\
\hline & $08 / 26 / 08$ & 09/09/08 & gross $\alpha$ & $8.6 \mathrm{E}-04 \pm 5.7 \mathrm{E}-04$ & & & & & \\
\hline & 08/26/08 & 09/09/08 & gross $\beta$ & $1.2 \mathrm{E}-02 \pm 1.6 \mathrm{E}-03$ & & & & & \\
\hline & 09/09/08 & 09/23/08 & gross $\alpha$ & $2.0 \mathrm{E}-03 \pm 6.7 \mathrm{E}-04$ & & & & & \\
\hline & 09/09/08 & 09/23/08 & gross $\beta$ & $2.3 \mathrm{E}-02 \pm 2.5 \mathrm{E}-03$ & & & & & \\
\hline & 09/23/08 & $10 / 08 / 08$ & gross $\alpha$ & $1.6 \mathrm{E}-03 \pm 5.8 \mathrm{E}-04$ & & & & & \\
\hline & $09 / 23 / 08$ & $10 / 08 / 08$ & gross $\beta$ & $2.2 \mathrm{E}-02 \pm 2.3 \mathrm{E}-03$ & & & & & \\
\hline & $10 / 08 / 08$ & $10 / 21 / 08$ & gross $\alpha$ & $4.3 \mathrm{E}-04 \pm 4.6 \mathrm{E}-04$ & & & & & \\
\hline & $10 / 08 / 08$ & $10 / 21 / 08$ & gross $\beta$ & $2.0 \mathrm{E}-02 \pm 2.3 \mathrm{E}-03$ & & & & & \\
\hline & $10 / 21 / 08$ & $11 / 04 / 08$ & gross $\alpha$ & $2.4 \mathrm{E}-03 \pm 7.5 \mathrm{E}-04$ & & & & & \\
\hline & $10 / 21 / 08$ & $11 / 04 / 08$ & gross $\beta$ & $4.3 \mathrm{E}-02 \pm 4.0 \mathrm{E}-03$ & & & & & \\
\hline & $11 / 04 / 08$ & $11 / 18 / 08$ & gross $\alpha$ & $1.4 \mathrm{E}-03 \pm 5.7 \mathrm{E}-04$ & & & & & \\
\hline & $11 / 04 / 08$ & $11 / 18 / 08$ & gross $\beta$ & $2.1 \mathrm{E}-02 \pm 2.4 \mathrm{E}-03$ & & & & & \\
\hline & $11 / 18 / 08$ & $12 / 02 / 08$ & gross $\alpha$ & $1.8 \mathrm{E}-03 \pm 6.4 \mathrm{E}-04$ & & & & & \\
\hline & $11 / 18 / 08$ & $12 / 02 / 08$ & gross $\beta$ & $3.9 \mathrm{E}-02 \pm 3.8 \mathrm{E}-03$ & & & & & \\
\hline & $12 / 02 / 08$ & $12 / 16 / 08$ & gross $\alpha$ & $2.0 \mathrm{E}-03 \pm 6.8 \mathrm{E}-04$ & & & & & \\
\hline & $12 / 02 / 08$ & $12 / 16 / 08$ & gross $\beta$ & $2.6 \mathrm{E}-02 \pm 2.8 \mathrm{E}-03$ & & & & & \\
\hline & $12 / 16 / 08$ & $12 / 30 / 08$ & gross $\alpha$ & $1.7 \mathrm{E}-03 \pm 6.3 \mathrm{E}-04$ & & & & & \\
\hline & $12 / 16 / 08$ & $12 / 30 / 08$ & gross $\beta$ & $2.6 \mathrm{E}-02 \pm 2.8 \mathrm{E}-03$ & & & & & \\
\hline
\end{tabular}

$\overline{\mathrm{RQ}}=$ Result Qualifier. $\mathrm{U}=$ The analyte was analyzed for but not detected. 
Table 2-4. Near-Facility Air Sampling Results, 2008 (pCi/m³ \pm total analytical uncertainty). (Sheet 6 of 82)

\begin{tabular}{|c|c|c|c|c|c|c|c|c|c|}
\hline Location & Sample On & Sample Off & Isotope & Result \pm Uncertainty & Location & Composite Period & Isotope & Result \pm Uncertainty & RQ* \\
\hline N519 & $01 / 02 / 08$ & $01 / 15 / 08$ & gross $\alpha$ & $5.2 \mathrm{E}-04 \pm 5.0 \mathrm{E}-04$ & N519 & $01 / 02 / 08$ to $04 / 08 / 08$ & ${ }^{60} \mathrm{Co}$ & $-4.3 \mathrm{E}-05 \pm 1.2 \mathrm{E}-04$ & $\mathrm{U}$ \\
\hline \multirow[t]{13}{*}{$(100-\mathrm{F})$} & $01 / 02 / 08$ & $01 / 15 / 08$ & gross $\beta$ & $9.4 \mathrm{E}-03 \pm 1.5 \mathrm{E}-03$ & & & ${ }^{134} \mathrm{Cs}$ & $2.8 \mathrm{E}-05 \pm 1.3 \mathrm{E}-04$ & $\mathrm{U}$ \\
\hline & $01 / 15 / 08$ & $01 / 30 / 08$ & gross $\alpha$ & $1.2 \mathrm{E}-03 \pm 5.1 \mathrm{E}-04$ & & & ${ }^{137} \mathrm{Cs}$ & $-3.4 \mathrm{E}-05 \pm 1.0 \mathrm{E}-04$ & $\mathrm{U}$ \\
\hline & $01 / 15 / 08$ & 01/30/08 & gross $\beta$ & $3.8 \mathrm{E}-02 \pm 3.7 \mathrm{E}-03$ & & & ${ }^{152} \mathrm{Eu}$ & $5.1 \mathrm{E}-05 \pm 2.5 \mathrm{E}-04$ & $\mathrm{U}$ \\
\hline & $01 / 30 / 08$ & 02/13/08 & gross $\alpha$ & $2.8 \mathrm{E}-04 \pm 4.0 \mathrm{E}-04$ & & & ${ }^{154} \mathrm{Eu}$ & $1.7 \mathrm{E}-04 \pm 3.2 \mathrm{E}-04$ & $\mathrm{U}$ \\
\hline & $01 / 30 / 08$ & $02 / 13 / 08$ & gross $\beta$ & $4.1 \mathrm{E}-03 \pm 8.8 \mathrm{E}-04$ & & & ${ }^{155} \mathrm{Eu}$ & $4.2 \mathrm{E}-05 \pm 2.7 \mathrm{E}-04$ & $\mathrm{U}$ \\
\hline & $02 / 13 / 08$ & $02 / 27 / 08$ & gross $\alpha$ & $2.1 \mathrm{E}-03 \pm 6.9 \mathrm{E}-04$ & & & ${ }^{238} \mathrm{Pu}$ & $1.1 \mathrm{E}-06 \pm 1.1 \mathrm{E}-06$ & $\mathrm{U}$ \\
\hline & $02 / 13 / 08$ & $02 / 27 / 08$ & gross $\beta$ & $2.4 \mathrm{E}-02 \pm 2.6 \mathrm{E}-03$ & & & ${ }^{239 / 240} \mathrm{Pu}$ & $1.1 \mathrm{E}-06 \pm 2.2 \mathrm{E}-06$ & $\mathrm{U}$ \\
\hline & $02 / 27 / 08$ & 03/12/08 & gross $\alpha$ & $6.2 \mathrm{E}-04 \pm 5.1 \mathrm{E}-04$ & & & ${ }^{106} \mathrm{Ru}$ & $7.6 \mathrm{E}-04 \pm 9.7 \mathrm{E}-04$ & $\mathrm{U}$ \\
\hline & $02 / 27 / 08$ & 03/12/08 & gross $\beta$ & $1.4 \mathrm{E}-02 \pm 1.9 \mathrm{E}-03$ & & & ${ }^{125} \mathrm{Sb}$ & $-2.2 \mathrm{E}-04 \pm 2.5 \mathrm{E}-04$ & $\mathrm{U}$ \\
\hline & 03/12/08 & 03/26/08 & gross $\alpha$ & $1.5 \mathrm{E}-03 \pm 6.0 \mathrm{E}-04$ & & & ${ }^{90} \mathrm{Sr}$ & $1.6 \mathrm{E}-05 \pm 1.6 \mathrm{E}-04$ & $\mathrm{U}$ \\
\hline & 03/12/08 & 03/26/08 & gross $\beta$ & $1.0 \mathrm{E}-02 \pm 1.5 \mathrm{E}-03$ & & & ${ }^{234} \mathrm{U}$ & $8.4 \mathrm{E}-06 \pm 9.2 \mathrm{E}-06$ & $\mathrm{U}$ \\
\hline & 03/26/08 & 04/08/08 & gross $\alpha$ & $1.0 \mathrm{E}-03 \pm 6.4 \mathrm{E}-04$ & & & ${ }^{235} \mathrm{U}$ & $7.9 \mathrm{E}-06 \pm 7.0 \mathrm{E}-06$ & \\
\hline & 03/26/08 & 04/08/08 & gross $\beta$ & $7.8 \mathrm{E}-03 \pm 1.3 \mathrm{E}-03$ & & & ${ }^{238} \mathrm{U}$ & $3.6 \mathrm{E}-06 \pm 4.4 \mathrm{E}-06$ & \\
\hline \multirow{14}{*}{$\begin{array}{c}\text { N520 } \\
(100-F)\end{array}$} & $01 / 02 / 08$ & $01 / 15 / 08$ & gross $\alpha$ & $4.2 \mathrm{E}-04 \pm 4.7 \mathrm{E}-04$ & N520 & $01 / 02 / 08$ to $04 / 08 / 08$ & ${ }^{60} \mathrm{Co}$ & $-1.0 \mathrm{E}-04 \pm 2.5 \mathrm{E}-04$ & $\mathrm{U}$ \\
\hline & $01 / 02 / 08$ & 01/15/08 & gross $\beta$ & $7.9 \mathrm{E}-03 \pm 1.3 \mathrm{E}-03$ & & & ${ }^{134} \mathrm{Cs}$ & $2.6 \mathrm{E}-05 \pm 1.9 \mathrm{E}-04$ & $\mathrm{U}$ \\
\hline & $01 / 15 / 08$ & 01/30/08 & gross $\alpha$ & $2.0 \mathrm{E}-03 \pm 6.6 \mathrm{E}-04$ & & & ${ }^{137} \mathrm{Cs}$ & $-4.7 \mathrm{E}-05 \pm 1.7 \mathrm{E}-04$ & $\mathrm{U}$ \\
\hline & $01 / 15 / 08$ & 01/30/08 & gross $\beta$ & $4.1 \mathrm{E}-02 \pm 3.8 \mathrm{E}-03$ & & & ${ }^{152} \mathrm{Eu}$ & $-1.1 \mathrm{E}-04 \pm 5.1 \mathrm{E}-04$ & $\mathrm{U}$ \\
\hline & $01 / 30 / 08$ & $02 / 13 / 08$ & gross $\alpha$ & $5.2 \mathrm{E}-04 \pm 4.8 \mathrm{E}-04$ & & & ${ }^{154} \mathrm{Eu}$ & $-3.0 \mathrm{E}-04 \pm 6.1 \mathrm{E}-04$ & $\mathrm{U}$ \\
\hline & $01 / 30 / 08$ & $02 / 13 / 08$ & gross $\beta$ & $3.8 \mathrm{E}-03 \pm 8.5 \mathrm{E}-04$ & & & ${ }^{155} \mathrm{Eu}$ & $-3.3 \mathrm{E}-05 \pm 3.3 \mathrm{E}-04$ & $\mathrm{U}$ \\
\hline & $02 / 13 / 08$ & $02 / 27 / 08$ & gross $\alpha$ & $9.5 \mathrm{E}-04 \pm 5.9 \mathrm{E}-04$ & & & ${ }^{238} \mathrm{Pu}$ & $2.5 \mathrm{E}-06 \pm 5.0 \mathrm{E}-06$ & $\mathrm{U}$ \\
\hline & $02 / 13 / 08$ & $02 / 27 / 08$ & gross $\beta$ & $3.3 \mathrm{E}-02 \pm 3.3 \mathrm{E}-03$ & & & ${ }^{239 / 240} \mathrm{Pu}$ & $-2.5 \mathrm{E}-06 \pm 3.6 \mathrm{E}-06$ & $\mathrm{U}$ \\
\hline & $02 / 27 / 08$ & 03/12/08 & gross $\alpha$ & $8.4 \mathrm{E}-04 \pm 5.7 \mathrm{E}-04$ & & & ${ }^{106} \mathrm{Ru}$ & $9.3 \mathrm{E}-05 \pm 9.3 \mathrm{E}-04$ & $\mathrm{U}$ \\
\hline & $02 / 27 / 08$ & 03/12/08 & gross $\beta$ & $1.3 \mathrm{E}-02 \pm 1.7 \mathrm{E}-03$ & & & ${ }^{125} \mathrm{Sb}$ & $-1.7 \mathrm{E}-04 \pm 4.4 \mathrm{E}-04$ & $\mathrm{U}$ \\
\hline & $03 / 12 / 08$ & $03 / 26 / 08$ & gross $\alpha$ & $6.2 \mathrm{E}-04 \pm 5.2 \mathrm{E}-04$ & & & ${ }^{90} \mathrm{Sr}$ & $-2.8 \mathrm{E}-04 \pm 2.9 \mathrm{E}-04$ & $\mathrm{U}$ \\
\hline & 03/12/08 & 03/26/08 & gross $\beta$ & $8.8 \mathrm{E}-03 \pm 1.4 \mathrm{E}-03$ & & & ${ }^{234} \mathrm{U}$ & $2.0 \mathrm{E}-05 \pm 1.5 \mathrm{E}-05$ & \\
\hline & $03 / 26 / 08$ & $04 / 08 / 08$ & gross $\alpha$ & $7.8 \mathrm{E}-04 \pm 5.8 \mathrm{E}-04$ & & & ${ }^{235} \mathrm{U}$ & $8.2 \mathrm{E}-06 \pm 7.3 \mathrm{E}-06$ & \\
\hline & 03/26/08 & 04/08/08 & gross $\beta$ & $6.4 \mathrm{E}-03 \pm 1.2 \mathrm{E}-03$ & & & ${ }^{238} \mathrm{U}$ & $1.4 \mathrm{E}-05 \pm 9.5 \mathrm{E}-06$ & \\
\hline \multirow{14}{*}{$\begin{array}{c}\text { N521 } \\
(100-F)\end{array}$} & $01 / 02 / 08$ & $01 / 15 / 08$ & gross $\alpha$ & $4.1 \mathrm{E}-04 \pm 4.6 \mathrm{E}-04$ & N521 & $01 / 02 / 08$ to $04 / 08 / 08$ & ${ }^{60} \mathrm{Co}$ & $2.4 \mathrm{E}-05 \pm 1.4 \mathrm{E}-04$ & $\mathrm{U}$ \\
\hline & $01 / 02 / 08$ & $01 / 15 / 08$ & gross $\beta$ & $9.1 \mathrm{E}-03 \pm 1.4 \mathrm{E}-03$ & & & ${ }^{134} \mathrm{Cs}$ & $-6.5 \mathrm{E}-05 \pm 1.3 \mathrm{E}-04$ & $\mathrm{U}$ \\
\hline & 01/15/08 & 01/30/08 & gross $\alpha$ & $1.7 \mathrm{E}-03 \pm 6.1 \mathrm{E}-04$ & & & ${ }^{137} \mathrm{Cs}$ & $-7.2 \mathrm{E}-05 \pm 1.2 \mathrm{E}-04$ & $\mathrm{U}$ \\
\hline & $01 / 15 / 08$ & 01/30/08 & gross $\beta$ & $3.8 \mathrm{E}-02 \pm 3.6 \mathrm{E}-03$ & & & ${ }^{152} \mathrm{Eu}$ & $9.4 \mathrm{E}-05 \pm 2.8 \mathrm{E}-04$ & $\mathrm{U}$ \\
\hline & $01 / 30 / 08$ & 02/13/08 & gross $\alpha$ & $4.0 \mathrm{E}-04 \pm 4.5 \mathrm{E}-04$ & & & ${ }^{154} \mathrm{Eu}$ & $1.3 \mathrm{E}-05 \pm 1.3 \mathrm{E}-04$ & $\mathrm{U}$ \\
\hline & $01 / 30 / 08$ & $02 / 13 / 08$ & gross $\beta$ & $3.6 \mathrm{E}-03 \pm 8.3 \mathrm{E}-04$ & & & ${ }^{155} \mathrm{Eu}$ & $1.0 \mathrm{E}-04 \pm 3.0 \mathrm{E}-04$ & $\mathrm{U}$ \\
\hline & $02 / 13 / 08$ & $02 / 27 / 08$ & gross $\alpha$ & $1.7 \mathrm{E}-03 \pm 6.3 \mathrm{E}-04$ & & & ${ }^{238} \mathrm{Pu}$ & $2.3 \mathrm{E}-06 \pm 5.8 \mathrm{E}-06$ & $\mathrm{U}$ \\
\hline & 02/13/08 & 02/27/08 & gross $\beta$ & $3.1 \mathrm{E}-02 \pm 3.1 \mathrm{E}-03$ & & & ${ }^{239 / 240} \mathrm{Pu}$ & $-1.2 \mathrm{E}-06 \pm 2.3 \mathrm{E}-06$ & U \\
\hline & $02 / 27 / 08$ & 03/12/08 & gross $\alpha$ & $1.2 \mathrm{E}-03 \pm 5.3 \mathrm{E}-04$ & & & ${ }^{106} \mathrm{Ru}$ & $2.1 \mathrm{E}-04 \pm 1.0 \mathrm{E}-03$ & $\mathrm{U}$ \\
\hline & $02 / 27 / 08$ & 03/12/08 & gross $\beta$ & $1.4 \mathrm{E}-02 \pm 1.8 \mathrm{E}-03$ & & & ${ }^{125} \mathrm{Sb}$ & $1.2 \mathrm{E}-04 \pm 2.5 \mathrm{E}-04$ & $\mathrm{U}$ \\
\hline & 03/12/08 & 03/26/08 & gross $\alpha$ & $4.0 \mathrm{E}-04 \pm 4.5 \mathrm{E}-04$ & & & ${ }^{90} \mathrm{Sr}$ & $-2.1 \mathrm{E}-04 \pm 2.2 \mathrm{E}-04$ & $\mathrm{U}$ \\
\hline & 03/12/08 & 03/26/08 & gross $\beta$ & $1.2 \mathrm{E}-02 \pm 1.7 \mathrm{E}-03$ & & & ${ }^{234} \mathrm{U}$ & $1.2 \mathrm{E}-05 \pm 1.1 \mathrm{E}-05$ & $\mathrm{U}$ \\
\hline & 03/26/08 & 04/08/08 & gross $\alpha$ & $1.7 \mathrm{E}-03 \pm 6.6 \mathrm{E}-04$ & & & ${ }^{235} \mathrm{U}$ & $4.0 \mathrm{E}-06 \pm 4.9 \mathrm{E}-06$ & \\
\hline & 03/26/08 & $04 / 08 / 08$ & gross $\beta$ & $6.8 \mathrm{E}-03 \pm 1.2 \mathrm{E}-03$ & & & ${ }^{238} \mathrm{U}$ & $3.7 \mathrm{E}-06 \pm 5.7 \mathrm{E}-06$ & $\mathrm{U}$ \\
\hline \multirow{14}{*}{$\begin{array}{c}\text { N552 } \\
(100-F)\end{array}$} & $01 / 02 / 08$ & 01/15/08 & gross $\alpha$ & $3.0 \mathrm{E}-04 \pm 4.3 \mathrm{E}-04$ & N552 & $01 / 02 / 08$ to $04 / 08 / 08$ & ${ }^{60} \mathrm{Co}$ & $-5.0 \mathrm{E}-05 \pm 1.2 \mathrm{E}-04$ & $\mathrm{U}$ \\
\hline & $01 / 02 / 08$ & $01 / 15 / 08$ & gross $\beta$ & $8.3 \mathrm{E}-03 \pm 1.4 \mathrm{E}-03$ & & & ${ }^{134} \mathrm{Cs}$ & $1.2 \mathrm{E}-04 \pm 1.2 \mathrm{E}-04$ & $\mathrm{U}$ \\
\hline & $01 / 15 / 08$ & 01/30/08 & gross $\alpha$ & $2.6 \mathrm{E}-03 \pm 7.6 \mathrm{E}-04$ & & & ${ }^{137} \mathrm{Cs}$ & $-7.7 \mathrm{E}-06 \pm 7.7 \mathrm{E}-05$ & $\mathrm{U}$ \\
\hline & $01 / 15 / 08$ & 01/30/08 & gross $\beta$ & $4.3 \mathrm{E}-02 \pm 4.5 \mathrm{E}-03$ & & & ${ }^{152} \mathrm{Eu}$ & $-1.5 \mathrm{E}-04 \pm 2.5 \mathrm{E}-04$ & $\mathrm{U}$ \\
\hline & $01 / 30 / 08$ & 02/13/08 & gross $\alpha$ & $1.6 \mathrm{E}-04 \pm 3.5 \mathrm{E}-04$ & & & ${ }^{154} \mathrm{Eu}$ & $1.5 \mathrm{E}-04 \pm 3.4 \mathrm{E}-04$ & $\mathrm{U}$ \\
\hline & $01 / 30 / 08$ & 02/13/08 & gross $\beta$ & $5.7 \mathrm{E}-03 \pm 1.1 \mathrm{E}-03$ & & & ${ }^{155} \mathrm{Eu}$ & $-4.9 \mathrm{E}-05 \pm 2.9 \mathrm{E}-04$ & $\mathrm{U}$ \\
\hline & 02/13/08 & 02/27/08 & gross $\alpha$ & $1.6 \mathrm{E}-03 \pm 6.2 \mathrm{E}-04$ & & & ${ }^{238} \mathrm{Pu}$ & $2.6 \mathrm{E}-06 \pm 1.8 \mathrm{E}-05$ & $\mathrm{U}$ \\
\hline & $02 / 13 / 08$ & $02 / 27 / 08$ & gross $\beta$ & $2.8 \mathrm{E}-02 \pm 3.2 \mathrm{E}-03$ & & & ${ }^{239 / 240} \mathrm{Pu}$ & $-1.3 \mathrm{E}-06 \pm 5.9 \mathrm{E}-06$ & $\mathrm{U}$ \\
\hline & $02 / 27 / 08$ & 03/12/08 & gross $\alpha$ & $5.3 \mathrm{E}-04 \pm 5.0 \mathrm{E}-04$ & & & ${ }^{106} \mathrm{Ru}$ & $1.8 \mathrm{E}-04 \pm 1.0 \mathrm{E}-03$ & $\mathrm{U}$ \\
\hline & $02 / 27 / 08$ & 03/12/08 & gross $\beta$ & $1.1 \mathrm{E}-02 \pm 1.7 \mathrm{E}-03$ & & & ${ }^{125} \mathrm{Sb}$ & $-2.2 \mathrm{E}-04 \pm 2.4 \mathrm{E}-04$ & $\mathrm{U}$ \\
\hline & 03/12/08 & 03/26/08 & gross $\alpha$ & $5.1 \mathrm{E}-04 \pm 4.8 \mathrm{E}-04$ & & & ${ }^{90} \mathrm{Sr}$ & $-4.0 \mathrm{E}-04 \pm 4.1 \mathrm{E}-04$ & $\mathrm{U}$ \\
\hline & 03/12/08 & 03/26/08 & gross $\beta$ & $8.5 \mathrm{E}-03 \pm 1.4 \mathrm{E}-03$ & & & ${ }^{234} \mathrm{U}$ & $1.9 \mathrm{E}-05 \pm 1.3 \mathrm{E}-05$ & \\
\hline & $03 / 26 / 08$ & $04 / 08 / 08$ & gross $\alpha$ & $8.0 \mathrm{E}-04 \pm 5.9 \mathrm{E}-04$ & & & ${ }^{235} \mathrm{U}$ & $1.4 \mathrm{E}-06 \pm 2.9 \mathrm{E}-06$ & $\mathrm{U}$ \\
\hline & $03 / 26 / 08$ & $04 / 08 / 08$ & gross $\beta$ & $6.8 \mathrm{E}-03 \pm 1.3 \mathrm{E}-03$ & & & ${ }^{238} \mathrm{U}$ & $1.3 \mathrm{E}-05 \pm 9.3 \mathrm{E}-06$ & \\
\hline
\end{tabular}

$\overline{\mathrm{RQ}}=$ Result Qualifier. $\mathrm{U}=$ The analyte was analyzed for but not detected. 
Table 2-4. Near-Facility Air Sampling Results, 2008 (pCi/m³ \pm total analytical uncertainty). (Sheet 7 of 82)

\begin{tabular}{|c|c|c|c|c|c|c|c|c|c|}
\hline Location & Sample On & Sample Off & Isotope & Result \pm Uncertainty & Location & Composite Period & Isotope & Result \pm Uncertainty & $\mathbf{R Q *}$ \\
\hline N553 & $01 / 02 / 08$ & $01 / 15 / 08$ & gross $\alpha$ & $4.3 \mathrm{E}-04 \pm 4.8 \mathrm{E}-04$ & N553 & $01 / 02 / 08$ to $04 / 08 / 08$ & ${ }^{60} \mathrm{Co}$ & $1.0 \mathrm{E}-04 \pm 1.9 \mathrm{E}-04$ & $\mathrm{U}$ \\
\hline \multirow[t]{13}{*}{$(100-F)$} & $01 / 02 / 08$ & 01/15/08 & gross $\beta$ & $8.8 \mathrm{E}-03 \pm 1.5 \mathrm{E}-03$ & & & ${ }^{134} \mathrm{Cs}$ & $-1.1 \mathrm{E}-05 \pm 1.1 \mathrm{E}-04$ & $\mathrm{U}$ \\
\hline & 01/15/08 & 01/30/08 & gross $\alpha$ & $1.4 \mathrm{E}-03 \pm 5.4 \mathrm{E}-04$ & & & ${ }^{137} \mathrm{Cs}$ & $-5.7 \mathrm{E}-05 \pm 2.0 \mathrm{E}-04$ & $\mathrm{U}$ \\
\hline & 01/15/08 & 01/30/08 & gross $\beta$ & $3.4 \mathrm{E}-02 \pm 3.7 \mathrm{E}-03$ & & & ${ }^{152} \mathrm{Eu}$ & $1.2 \mathrm{E}-05 \pm 1.2 \mathrm{E}-04$ & $\mathrm{U}$ \\
\hline & 01/30/08 & 02/13/08 & gross $\alpha$ & $1.6 \mathrm{E}-04 \pm 3.4 \mathrm{E}-04$ & & & ${ }^{154} \mathrm{Eu}$ & $-7.1 \mathrm{E}-05 \pm 5.4 \mathrm{E}-04$ & $\mathrm{U}$ \\
\hline & 01/30/08 & 02/13/08 & gross $\beta$ & $5.4 \mathrm{E}-03 \pm 1.1 \mathrm{E}-03$ & & & ${ }^{155} \mathrm{Eu}$ & $1.8 \mathrm{E}-04 \pm 3.5 \mathrm{E}-04$ & $\mathrm{U}$ \\
\hline & $02 / 13 / 08$ & $02 / 27 / 08$ & gross $\alpha$ & $2.2 \mathrm{E}-03 \pm 7.2 \mathrm{E}-04$ & & & ${ }^{238} \mathrm{Pu}$ & $-3.0 \mathrm{E}-06 \pm 1.8 \mathrm{E}-05$ & $\mathrm{U}$ \\
\hline & 02/13/08 & $02 / 27 / 08$ & gross $\beta$ & $2.7 \mathrm{E}-02 \pm 3.2 \mathrm{E}-03$ & & & ${ }^{239 / 240} \mathrm{Pu}$ & $1.5 \mathrm{E}-06 \pm 5.2 \mathrm{E}-06$ & $\mathrm{U}$ \\
\hline & 02/27/08 & 03/12/08 & gross $\alpha$ & $5.2 \mathrm{E}-04 \pm 4.9 \mathrm{E}-04$ & & & ${ }^{106} \mathrm{Ru}$ & $1.7 \mathrm{E}-03 \pm 1.7 \mathrm{E}-03$ & $\mathrm{U}$ \\
\hline & 02/27/08 & 03/12/08 & gross $\beta$ & $1.0 \mathrm{E}-02 \pm 1.6 \mathrm{E}-03$ & & & ${ }^{125} \mathrm{Sb}$ & $2.6 \mathrm{E}-04 \pm 4.5 \mathrm{E}-04$ & $\mathrm{U}$ \\
\hline & 03/12/08 & 03/26/08 & gross $\alpha$ & $1.2 \mathrm{E}-03 \pm 5.5 \mathrm{E}-04$ & & & ${ }^{90} \mathrm{Sr}$ & $-2.6 \mathrm{E}-04 \pm 2.7 \mathrm{E}-04$ & $\mathrm{U}$ \\
\hline & 03/12/08 & 03/26/08 & gross $\beta$ & $8.3 \mathrm{E}-03 \pm 1.4 \mathrm{E}-03$ & & & ${ }^{234} \mathrm{U}$ & $1.1 \mathrm{E}-05 \pm 9.9 \mathrm{E}-06$ & $\mathrm{U}$ \\
\hline & 03/26/08 & 04/08/08 & gross $\alpha$ & $1.3 \mathrm{E}-03 \pm 5.7 \mathrm{E}-04$ & & & ${ }^{235} \mathrm{U}$ & $4.1 \mathrm{E}-06 \pm 6.3 \mathrm{E}-06$ & $\mathrm{U}$ \\
\hline & 03/26/08 & 04/08/08 & gross $\beta$ & $9.4 \mathrm{E}-03 \pm 1.5 \mathrm{E}-03$ & & & ${ }^{238} \mathrm{U}$ & $1.3 \mathrm{E}-05 \pm 9.8 \mathrm{E}-06$ & \\
\hline \multirow{26}{*}{$\begin{array}{c}\text { N508 } \\
(100-H)\end{array}$} & 07/02/08 & 07/15/08 & gross $\alpha$ & $2.0 \mathrm{E}-03 \pm 7.1 \mathrm{E}-04$ & N508 & $07 / 02 / 08$ to $12 / 30 / 08$ & ${ }^{60} \mathrm{Co}$ & $5.0 \mathrm{E}-05 \pm 7.9 \mathrm{E}-05$ & $\mathrm{U}$ \\
\hline & $07 / 02 / 08$ & 07/15/08 & gross $\beta$ & $1.4 \mathrm{E}-02 \pm 1.9 \mathrm{E}-03$ & & & ${ }^{134} \mathrm{Cs}$ & $2.3 \mathrm{E}-05 \pm 7.2 \mathrm{E}-05$ & $\mathrm{U}$ \\
\hline & 07/15/08 & 07/29/08 & gross $\alpha$ & $1.6 \mathrm{E}-03 \pm 9.6 \mathrm{E}-04$ & & & ${ }^{137} \mathrm{Cs}$ & $-3.0 \mathrm{E}-06 \pm 3.0 \mathrm{E}-05$ & $\mathrm{U}$ \\
\hline & 07/15/08 & 07/29/08 & gross $\beta$ & $9.9 \mathrm{E}-03 \pm 1.8 \mathrm{E}-03$ & & & ${ }^{152} \mathrm{Eu}$ & $-1.3 \mathrm{E}-05 \pm 1.3 \mathrm{E}-04$ & $\mathrm{U}$ \\
\hline & 07/29/08 & 08/12/08 & gross $\alpha$ & $5.6 \mathrm{E}-04 \pm 5.1 \mathrm{E}-04$ & & & ${ }^{154} \mathrm{Eu}$ & $1.7 \mathrm{E}-04 \pm 2.1 \mathrm{E}-04$ & $\mathrm{U}$ \\
\hline & 07/29/08 & 08/12/08 & gross $\beta$ & $1.6 \mathrm{E}-02 \pm 2.0 \mathrm{E}-03$ & & & ${ }^{155} \mathrm{Eu}$ & $-6.8 \mathrm{E}-06 \pm 6.8 \mathrm{E}-05$ & $\mathrm{U}$ \\
\hline & 08/12/08 & 08/26/08 & gross $\alpha$ & $9.9 \mathrm{E}-04 \pm 6.1 \mathrm{E}-04$ & & & ${ }^{238} \mathrm{Pu}$ & $-4.2 \mathrm{E}-06 \pm 1.5 \mathrm{E}-05$ & $\mathrm{U}$ \\
\hline & 08/12/08 & 08/26/08 & gross $\beta$ & $1.2 \mathrm{E}-02 \pm 1.7 \mathrm{E}-03$ & & & ${ }^{239 / 240} \mathrm{Pu}$ & $-8.5 \mathrm{E}-07 \pm 4.5 \mathrm{E}-06$ & $\mathrm{U}$ \\
\hline & 08/26/08 & 09/09/08 & gross $\alpha$ & $1.8 \mathrm{E}-03 \pm 7.8 \mathrm{E}-04$ & & & ${ }^{106} \mathrm{Ru}$ & $8.5 \mathrm{E}-05 \pm 5.9 \mathrm{E}-04$ & $\mathrm{U}$ \\
\hline & $08 / 26 / 08$ & 09/09/08 & gross $\beta$ & $1.3 \mathrm{E}-02 \pm 2.0 \mathrm{E}-03$ & & & ${ }^{125} \mathrm{Sb}$ & $-2.8 \mathrm{E}-05 \pm 1.4 \mathrm{E}-04$ & $\mathrm{U}$ \\
\hline & 09/09/08 & 09/23/08 & gross $\alpha$ & $9.8 \mathrm{E}-04 \pm 6.0 \mathrm{E}-04$ & & & ${ }^{90} \mathrm{Sr}$ & $-2.3 \mathrm{E}-04 \pm 2.4 \mathrm{E}-04$ & \\
\hline & 09/09/08 & 09/23/08 & gross $\beta$ & $2.6 \mathrm{E}-02 \pm 2.8 \mathrm{E}-03$ & & & ${ }^{234} \mathrm{U}$ & $1.9 \mathrm{E}-05 \pm 1.2 \mathrm{E}-05$ & \\
\hline & 09/23/08 & $10 / 08 / 08$ & gross $\alpha$ & $1.9 \mathrm{E}-03 \pm 6.4 \mathrm{E}-04$ & & & ${ }^{235} \mathrm{U}$ & $2.9 \mathrm{E}-06 \pm 5.2 \mathrm{E}-06$ & $\mathrm{U}$ \\
\hline & 09/23/08 & $10 / 08 / 08$ & gross $\beta$ & $2.6 \mathrm{E}-02 \pm 2.7 \mathrm{E}-03$ & & & ${ }^{238} \mathrm{U}$ & $1.3 \mathrm{E}-05 \pm 8.9 \mathrm{E}-06$ & \\
\hline & $10 / 08 / 08$ & $10 / 21 / 08$ & gross $\alpha$ & $1.4 \mathrm{E}-03 \pm 5.8 \mathrm{E}-04$ & & & & & \\
\hline & $10 / 08 / 08$ & $10 / 21 / 08$ & gross $\beta$ & $2.0 \mathrm{E}-02 \pm 2.4 \mathrm{E}-03$ & & & & & \\
\hline & $10 / 21 / 08$ & $11 / 04 / 08$ & gross $\alpha$ & $1.9 \mathrm{E}-03 \pm 6.7 \mathrm{E}-04$ & & & & & \\
\hline & $10 / 21 / 08$ & $11 / 04 / 08$ & gross $\beta$ & $4.2 \mathrm{E}-02 \pm 4.0 \mathrm{E}-03$ & & & & & \\
\hline & $11 / 04 / 08$ & $11 / 18 / 08$ & gross $\alpha$ & $1.2 \mathrm{E}-03 \pm 5.4 \mathrm{E}-04$ & & & & & \\
\hline & $11 / 04 / 08$ & $11 / 18 / 08$ & gross $\beta$ & $2.1 \mathrm{E}-02 \pm 2.4 \mathrm{E}-03$ & & & & & \\
\hline & $11 / 18 / 08$ & $12 / 02 / 08$ & gross $\alpha$ & $1.5 \mathrm{E}-03 \pm 6.1 \mathrm{E}-04$ & & & & & \\
\hline & $11 / 18 / 08$ & $12 / 02 / 08$ & gross $\beta$ & $3.7 \mathrm{E}-02 \pm 3.7 \mathrm{E}-03$ & & & & & \\
\hline & $12 / 02 / 08$ & $12 / 16 / 08$ & gross $\alpha$ & $1.2 \mathrm{E}-03 \pm 5.2 \mathrm{E}-04$ & & & & & \\
\hline & $12 / 02 / 08$ & $12 / 16 / 08$ & gross $\beta$ & $2.7 \mathrm{E}-02 \pm 2.8 \mathrm{E}-03$ & & & & & \\
\hline & $12 / 16 / 08$ & $12 / 30 / 08$ & gross $\alpha$ & $2.1 \mathrm{E}-03 \pm 7.0 \mathrm{E}-04$ & & & & & \\
\hline & $12 / 16 / 08$ & $12 / 30 / 08$ & gross $\beta$ & $3.1 \mathrm{E}-02 \pm 3.2 \mathrm{E}-03$ & & & & & \\
\hline
\end{tabular}

$\overline{\mathrm{RQ}}=$ Result Qualifier. $\mathrm{U}=$ The analyte was analyzed for but not detected. 
Table 2-4. Near-Facility Air Sampling Results, 2008 (pCi/m³ \pm total analytical uncertainty). (Sheet 8 of 82)

\begin{tabular}{|c|c|c|c|c|c|c|c|c|c|}
\hline Location & Sample On & Sample Off & Isotope & Result \pm Uncertainty & Location & Composite Period & Isotope & Result \pm Uncertainty & $\mathbf{R Q *}$ \\
\hline N509 & $07 / 02 / 08$ & $07 / 15 / 08$ & gross $\alpha$ & $1.9 \mathrm{E}-03 \pm 6.8 \mathrm{E}-04$ & N509 & $07 / 02 / 08$ to $12 / 30 / 08$ & ${ }^{60} \mathrm{Co}$ & $-8.9 \mathrm{E}-07 \pm 8.9 \mathrm{E}-06$ & $\mathrm{U}$ \\
\hline \multirow[t]{25}{*}{$(100-\mathrm{H})$} & $07 / 02 / 08$ & $07 / 15 / 08$ & gross $\beta$ & $1.4 \mathrm{E}-02 \pm 1.9 \mathrm{E}-03$ & & & ${ }^{134} \mathrm{Cs}$ & $2.4 \mathrm{E}-06 \pm 2.4 \mathrm{E}-05$ & $\mathrm{U}$ \\
\hline & 07/15/08 & 07/29/08 & gross $\alpha$ & $1.4 \mathrm{E}-03 \pm 9.0 \mathrm{E}-04$ & & & ${ }^{137} \mathrm{Cs}$ & $-1.4 \mathrm{E}-05 \pm 6.1 \mathrm{E}-05$ & U \\
\hline & 07/15/08 & 07/29/08 & gross $\beta$ & $1.5 \mathrm{E}-02 \pm 2.3 \mathrm{E}-03$ & & & ${ }^{152} \mathrm{Eu}$ & $-7.8 \mathrm{E}-05 \pm 1.6 \mathrm{E}-04$ & $\mathrm{U}$ \\
\hline & 07/29/08 & 08/12/08 & gross $\alpha$ & $5.0 \mathrm{E}-04 \pm 4.6 \mathrm{E}-04$ & & & ${ }^{154} \mathrm{Eu}$ & $3.7 \mathrm{E}-05 \pm 2.1 \mathrm{E}-04$ & $\mathrm{U}$ \\
\hline & $07 / 29 / 08$ & 08/12/08 & gross $\beta$ & $1.6 \mathrm{E}-02 \pm 1.9 \mathrm{E}-03$ & & & ${ }^{155} \mathrm{Eu}$ & $1.7 \mathrm{E}-05 \pm 1.7 \mathrm{E}-04$ & $\mathrm{U}$ \\
\hline & 08/12/08 & 08/26/08 & gross $\alpha$ & $5.3 \mathrm{E}-04 \pm 4.9 \mathrm{E}-04$ & & & ${ }^{238} \mathrm{Pu}$ & $-3.6 \mathrm{E}-06 \pm 1.1 \mathrm{E}-05$ & $\mathrm{U}$ \\
\hline & $08 / 12 / 08$ & 08/26/08 & gross $\beta$ & $1.5 \mathrm{E}-02 \pm 1.9 \mathrm{E}-03$ & & & ${ }^{239 / 240} \mathrm{Pu}$ & $7.3 \mathrm{E}-07 \pm 3.3 \mathrm{E}-06$ & $\mathrm{U}$ \\
\hline & $08 / 26 / 08$ & 09/09/08 & gross $\alpha$ & $9.2 \mathrm{E}-04 \pm 7.4 \mathrm{E}-04$ & & & ${ }^{106} \mathrm{Ru}$ & $-1.3 \mathrm{E}-04 \pm 5.3 \mathrm{E}-04$ & $\mathrm{U}$ \\
\hline & 08/26/08 & 09/09/08 & gross $\beta$ & $1.6 \mathrm{E}-02 \pm 2.3 \mathrm{E}-03$ & & & ${ }^{125} \mathrm{Sb}$ & $7.1 \mathrm{E}-05 \pm 1.4 \mathrm{E}-04$ & $\mathrm{U}$ \\
\hline & 09/09/08 & 09/23/08 & gross $\alpha$ & $1.5 \mathrm{E}-03 \pm 5.8 \mathrm{E}-04$ & & & ${ }^{90} \mathrm{Sr}$ & $-2.8 \mathrm{E}-04 \pm 2.9 \mathrm{E}-04$ & $\mathrm{U}$ \\
\hline & 09/09/08 & 09/23/08 & gross $\beta$ & $2.3 \mathrm{E}-02 \pm 2.5 \mathrm{E}-03$ & & & ${ }^{234} \mathrm{U}$ & $2.2 \mathrm{E}-05 \pm 1.2 \mathrm{E}-05$ & \\
\hline & 09/23/08 & $10 / 08 / 08$ & gross $\alpha$ & $1.3 \mathrm{E}-03 \pm 5.3 \mathrm{E}-04$ & & & ${ }^{235} \mathrm{U}$ & $2.9 \mathrm{E}-06 \pm 3.5 \mathrm{E}-06$ & U \\
\hline & 09/23/08 & $10 / 08 / 08$ & gross $\beta$ & $2.4 \mathrm{E}-02 \pm 2.6 \mathrm{E}-03$ & & & ${ }^{238} \mathrm{U}$ & $1.6 \mathrm{E}-05 \pm 9.7 \mathrm{E}-06$ & \\
\hline & $10 / 08 / 08$ & $10 / 21 / 08$ & gross $\alpha$ & $1.5 \mathrm{E}-03 \pm 6.0 \mathrm{E}-04$ & & & & & \\
\hline & $10 / 08 / 08$ & $10 / 21 / 08$ & gross $\beta$ & $1.8 \mathrm{E}-02 \pm 2.2 \mathrm{E}-03$ & & & & & \\
\hline & $10 / 21 / 08$ & $11 / 04 / 08$ & gross $\alpha$ & $2.0 \mathrm{E}-03 \pm 6.7 \mathrm{E}-04$ & & & & & \\
\hline & $10 / 21 / 08$ & $11 / 04 / 08$ & gross $\beta$ & $4.3 \mathrm{E}-02 \pm 4.0 \mathrm{E}-03$ & & & & & \\
\hline & $11 / 04 / 08$ & $11 / 18 / 08$ & gross $\alpha$ & $1.8 \mathrm{E}-03 \pm 6.4 \mathrm{E}-04$ & & & & & \\
\hline & $11 / 04 / 08$ & $11 / 18 / 08$ & gross $\beta$ & $2.1 \mathrm{E}-02 \pm 2.4 \mathrm{E}-03$ & & & & & \\
\hline & $11 / 18 / 08$ & $12 / 02 / 08$ & gross $\alpha$ & $1.4 \mathrm{E}-03 \pm 5.5 \mathrm{E}-04$ & & & & & \\
\hline & $11 / 18 / 08$ & $12 / 02 / 08$ & gross $\beta$ & $3.9 \mathrm{E}-02 \pm 3.8 \mathrm{E}-03$ & & & & & \\
\hline & $12 / 02 / 08$ & $12 / 16 / 08$ & gross $\alpha$ & $1.3 \mathrm{E}-03 \pm 5.5 \mathrm{E}-04$ & & & & & \\
\hline & $12 / 02 / 08$ & $12 / 16 / 08$ & gross $\beta$ & $2.4 \mathrm{E}-02 \pm 2.6 \mathrm{E}-03$ & & & & & \\
\hline & $12 / 16 / 08$ & $12 / 30 / 08$ & gross $\alpha$ & $1.2 \mathrm{E}-03 \pm 5.4 \mathrm{E}-04$ & & & & & \\
\hline & $12 / 16 / 08$ & $12 / 30 / 08$ & gross $\beta$ & $2.8 \mathrm{E}-02 \pm 2.9 \mathrm{E}-03$ & & & & & \\
\hline \multirow{26}{*}{$\begin{array}{c}\text { N510 } \\
(100-\mathrm{H})\end{array}$} & 07/02/08 & 07/15/08 & gross $\alpha$ & $1.2 \mathrm{E}-03 \pm 5.7 \mathrm{E}-04$ & N510 & $07 / 02 / 08$ to $12 / 30 / 08$ & ${ }^{60} \mathrm{Co}$ & $1.1 \mathrm{E}-05 \pm 8.6 \mathrm{E}-05$ & $\mathrm{U}$ \\
\hline & 07/02/08 & 07/15/08 & gross $\beta$ & $1.2 \mathrm{E}-02 \pm 1.7 \mathrm{E}-03$ & & & ${ }^{134} \mathrm{Cs}$ & $-3.2 \mathrm{E}-06 \pm 3.2 \mathrm{E}-05$ & $\mathrm{U}$ \\
\hline & $07 / 15 / 08$ & 07/29/08 & gross $\alpha$ & $1.6 \mathrm{E}-03 \pm 9.5 \mathrm{E}-04$ & & & ${ }^{137} \mathrm{Cs}$ & $-3.8 \mathrm{E}-05 \pm 6.8 \mathrm{E}-05$ & $\mathrm{U}$ \\
\hline & 07/15/08 & 07/29/08 & gross $\beta$ & $1.2 \mathrm{E}-02 \pm 2.0 \mathrm{E}-03$ & & & ${ }^{152} \mathrm{Eu}$ & $-8.2 \mathrm{E}-05 \pm 1.7 \mathrm{E}-04$ & $\mathrm{U}$ \\
\hline & $07 / 29 / 08$ & $08 / 12 / 08$ & gross $\alpha$ & $7.2 \mathrm{E}-04 \pm 5.2 \mathrm{E}-04$ & & & ${ }^{154} \mathrm{Eu}$ & $-1.6 \mathrm{E}-04 \pm 2.4 \mathrm{E}-04$ & $\mathrm{U}$ \\
\hline & 07/29/08 & 08/12/08 & gross $\beta$ & $1.4 \mathrm{E}-02 \pm 1.8 \mathrm{E}-03$ & & & ${ }^{155} \mathrm{Eu}$ & $1.1 \mathrm{E}-04 \pm 1.9 \mathrm{E}-04$ & $\mathrm{U}$ \\
\hline & 08/12/08 & 08/26/08 & gross $\alpha$ & $1.3 \mathrm{E}-03 \pm 5.6 \mathrm{E}-04$ & & & ${ }^{238} \mathrm{Pu}$ & $-8.4 \mathrm{E}-07 \pm 8.4 \mathrm{E}-06$ & $\mathrm{U}$ \\
\hline & $08 / 12 / 08$ & 08/26/08 & gross $\beta$ & $1.6 \mathrm{E}-02 \pm 2.0 \mathrm{E}-03$ & & & ${ }^{239 / 240} \mathrm{Pu}$ & $-8.4 \mathrm{E}-07 \pm 8.4 \mathrm{E}-06$ & $\mathrm{U}$ \\
\hline & $08 / 26 / 08$ & 09/09/08 & gross $\alpha$ & $1.2 \mathrm{E}-03 \pm 8.8 \mathrm{E}-04$ & & & ${ }^{106} \mathrm{Ru}$ & $4.1 \mathrm{E}-04 \pm 5.9 \mathrm{E}-04$ & $\mathrm{U}$ \\
\hline & $08 / 26 / 08$ & 09/09/08 & gross $\beta$ & $1.2 \mathrm{E}-02 \pm 2.0 \mathrm{E}-03$ & & & ${ }^{125} \mathrm{Sb}$ & $6.6 \mathrm{E}-05 \pm 1.6 \mathrm{E}-04$ & $\mathrm{U}$ \\
\hline & 09/09/08 & 09/23/08 & gross $\alpha$ & $2.2 \mathrm{E}-03 \pm 7.1 \mathrm{E}-04$ & & & ${ }^{90} \mathrm{Sr}$ & $-2.1 \mathrm{E}-04 \pm 2.1 \mathrm{E}-04$ & $\mathrm{U}$ \\
\hline & 09/09/08 & 09/23/08 & gross $\beta$ & $2.4 \mathrm{E}-02 \pm 2.6 \mathrm{E}-03$ & & & ${ }^{234} \mathrm{U}$ & $4.0 \mathrm{E}-05 \pm 2.1 \mathrm{E}-05$ & \\
\hline & $09 / 23 / 08$ & $10 / 08 / 08$ & gross $\alpha$ & $1.1 \mathrm{E}-03 \pm 4.9 \mathrm{E}-04$ & & & ${ }^{235} \mathrm{U}$ & $1.4 \mathrm{E}-06 \pm 4.9 \mathrm{E}-06$ & $\mathrm{U}$ \\
\hline & $09 / 23 / 08$ & $10 / 08 / 08$ & gross $\beta$ & $2.3 \mathrm{E}-02 \pm 2.5 \mathrm{E}-03$ & & & ${ }^{238} \mathrm{U}$ & $2.4 \mathrm{E}-05 \pm 1.5 \mathrm{E}-05$ & \\
\hline & $10 / 08 / 08$ & $10 / 21 / 08$ & gross $\alpha$ & $9.0 \mathrm{E}-04 \pm 5.9 \mathrm{E}-04$ & & & & & \\
\hline & $10 / 08 / 08$ & $10 / 21 / 08$ & gross $\beta$ & $1.9 \mathrm{E}-02 \pm 2.2 \mathrm{E}-03$ & & & & & \\
\hline & $10 / 21 / 08$ & $11 / 04 / 08$ & gross $\alpha$ & $2.4 \mathrm{E}-03 \pm 7.5 \mathrm{E}-04$ & & & & & \\
\hline & $10 / 21 / 08$ & $11 / 04 / 08$ & gross $\beta$ & $4.4 \mathrm{E}-02 \pm 4.1 \mathrm{E}-03$ & & & & & \\
\hline & $11 / 04 / 08$ & $11 / 18 / 08$ & gross $\alpha$ & $9.9 \mathrm{E}-04 \pm 6.0 \mathrm{E}-04$ & & & & & \\
\hline & $11 / 04 / 08$ & $11 / 18 / 08$ & gross $\beta$ & $2.2 \mathrm{E}-02 \pm 2.5 \mathrm{E}-03$ & & & & & \\
\hline & $11 / 18 / 08$ & $12 / 02 / 08$ & gross $\alpha$ & $1.7 \mathrm{E}-03 \pm 7.0 \mathrm{E}-04$ & & & & & \\
\hline & $11 / 18 / 08$ & $12 / 02 / 08$ & gross $\beta$ & $5.1 \mathrm{E}-02 \pm 4.9 \mathrm{E}-03$ & & & & & \\
\hline & $12 / 02 / 08$ & $12 / 16 / 08$ & gross $\alpha$ & $1.1 \mathrm{E}-03 \pm 4.9 \mathrm{E}-04$ & & & & & \\
\hline & $12 / 02 / 08$ & $12 / 16 / 08$ & gross $\beta$ & $2.6 \mathrm{E}-02 \pm 2.7 \mathrm{E}-03$ & & & & & \\
\hline & $12 / 16 / 08$ & $12 / 30 / 08$ & gross $\alpha$ & $2.1 \mathrm{E}-03 \pm 6.9 \mathrm{E}-04$ & & & & & \\
\hline & $12 / 16 / 08$ & $12 / 30 / 08$ & gross $\beta$ & $2.7 \mathrm{E}-02 \pm 2.8 \mathrm{E}-03$ & & & & & \\
\hline
\end{tabular}

$\overline{\mathrm{RQ}}=$ Result Qualifier. $\mathrm{U}=$ The analyte was analyzed for but not detected. 
Table 2-4. Near-Facility Air Sampling Results, 2008 (pCi/m³ \pm total analytical uncertainty). (Sheet 9 of 82)

\begin{tabular}{cccll} 
Location & Sample On & Sample Off & Isotope & Result \pm Uncertainty \\
\hline N574 & $10 / 08 / 08$ & $10 / 21 / 08$ & gross $\alpha$ & $1.8 \mathrm{E}-03 \pm 6.7 \mathrm{E}-04$ \\
$(100-\mathrm{H})$ & $10 / 08 / 08$ & $10 / 21 / 08$ & gross $\beta$ & $2.1 \mathrm{E}-02 \pm 2.7 \mathrm{E}-03$ \\
& $10 / 21 / 08$ & $11 / 04 / 08$ & gross $\alpha$ & $2.8 \mathrm{E}-03 \pm 8.1 \mathrm{E}-04$ \\
& $10 / 21 / 08$ & $11 / 04 / 08$ & gross $\beta$ & $4.8 \mathrm{E}-02 \pm 5.1 \mathrm{E}-03$ \\
& $11 / 04 / 08$ & $11 / 18 / 08$ & gross $\alpha$ & $1.5 \mathrm{E}-03 \pm 5.8 \mathrm{E}-04$ \\
& $11 / 04 / 08$ & $11 / 18 / 08$ & gross $\beta$ & $2.4 \mathrm{E}-02 \pm 2.9 \mathrm{E}-03$ \\
& $11 / 18 / 08$ & $12 / 02 / 08$ & gross $\alpha$ & $1.4 \mathrm{E}-03 \pm 5.8 \mathrm{E}-04$ \\
& $11 / 18 / 08$ & $12 / 02 / 08$ & gross $\beta$ & $3.9 \mathrm{E}-02 \pm 4.2 \mathrm{E}-03$ \\
& $12 / 02 / 08$ & $12 / 16 / 08$ & gross $\alpha$ & $2.1 \mathrm{E}-03 \pm 7.0 \mathrm{E}-04$ \\
& $12 / 02 / 08$ & $12 / 16 / 08$ & gross $\beta$ & $2.9 \mathrm{E}-02 \pm 3.3 \mathrm{E}-03$ \\
& $12 / 16 / 08$ & $12 / 30 / 08$ & gross $\alpha$ & $1.8 \mathrm{E}-03 \pm 6.4 \mathrm{E}-04$ \\
& $12 / 16 / 08$ & $12 / 30 / 08$ & gross $\beta$ & $3.2 \mathrm{E}-02 \pm 3.6 \mathrm{E}-03$
\end{tabular}

\begin{tabular}{|c|c|c|c|c|}
\hline Location & Composite Period & Isotope & Result \pm Uncertainty & RQ* \\
\hline \multirow[t]{14}{*}{ N574 } & $10 / 08 / 08$ to $12 / 30 / 08$ & ${ }^{60} \mathrm{Co}$ & $1.5 \mathrm{E}-04 \pm 2.0 \mathrm{E}-04$ & $\overline{\mathrm{U}}$ \\
\hline & & ${ }^{134} \mathrm{Cs}$ & $-1.0 \mathrm{E}-04 \pm 1.4 \mathrm{E}-04$ & $\mathrm{U}$ \\
\hline & & ${ }^{137} \mathrm{Cs}$ & $-3.5 \mathrm{E}-05 \pm 1.3 \mathrm{E}-04$ & $\mathrm{U}$ \\
\hline & & ${ }^{152} \mathrm{Eu}$ & $-2.2 \mathrm{E}-04 \pm 3.2 \mathrm{E}-04$ & $\mathrm{U}$ \\
\hline & & ${ }^{154} \mathrm{Eu}$ & $-1.8 \mathrm{E}-04 \pm 4.7 \mathrm{E}-04$ & $\mathrm{U}$ \\
\hline & & ${ }^{155} \mathrm{Eu}$ & $3.9 \mathrm{E}-05 \pm 2.8 \mathrm{E}-04$ & $\mathrm{U}$ \\
\hline & & ${ }^{238} \mathrm{Pu}$ & $-1.5 \mathrm{E}-06 \pm 1.5 \mathrm{E}-05$ & $\mathrm{U}$ \\
\hline & & ${ }^{239 / 240} \mathrm{Pu}$ & $-7.8 \mathrm{E}-06 \pm 8.7 \mathrm{E}-06$ & $\mathrm{U}$ \\
\hline & & ${ }^{106} \mathrm{Ru}$ & $-6.4 \mathrm{E}-05 \pm 6.5 \mathrm{E}-04$ & $\mathrm{U}$ \\
\hline & & ${ }^{125} \mathrm{Sb}$ & $-1.2 \mathrm{E}-04 \pm 2.8 \mathrm{E}-04$ & $\mathrm{U}$ \\
\hline & & ${ }^{90} \mathrm{Sr}$ & $-2.9 \mathrm{E}-04 \pm 3.0 \mathrm{E}-04$ & $\mathrm{U}$ \\
\hline & & ${ }^{234} \mathrm{U}$ & $2.7 \mathrm{E}-05 \pm 1.9 \mathrm{E}-05$ & \\
\hline & & ${ }^{235} \mathrm{U}$ & $2.3 \mathrm{E}-06 \pm 7.9 \mathrm{E}-06$ & $\mathrm{U}$ \\
\hline & & ${ }^{238} \mathrm{U}$ & $2.1 \mathrm{E}-05 \pm 1.6 \mathrm{E}-05$ & \\
\hline
\end{tabular}

$\overline{\mathrm{RQ}}$ = Result Qualifier. U = The analyte was analyzed for but not detected. 
Table 2-4. Near-Facility Air Sampling Results, 2008 (pCi/m³ \pm total analytical uncertainty). (Sheet 10 of 82)

\begin{tabular}{|c|c|c|c|c|c|c|c|c|c|}
\hline Location & Sample On & Sample Off & Isotope & Result \pm Uncertainty & Location & Composite Period & Isotope & Result \pm Uncertainty & $\mathbf{R Q *}$ \\
\hline N401 & $01 / 02 / 08$ & $01 / 15 / 08$ & gross $\alpha$ & $1.4 \mathrm{E}-03 \pm 5.8 \mathrm{E}-04$ & N401 & $01 / 02 / 08$ to $07 / 01 / 08$ & ${ }^{241} \mathrm{Am}$ & $1.2 \mathrm{E}-05 \pm 6.8 \mathrm{E}-06$ & \\
\hline \multirow[t]{51}{*}{$(100-K)$} & $01 / 02 / 08$ & $01 / 15 / 08$ & gross $\beta$ & $9.6 \mathrm{E}-03 \pm 1.5 \mathrm{E}-03$ & & & ${ }^{60} \mathrm{Co}$ & $3.2 \mathrm{E}-05 \pm 1.4 \mathrm{E}-04$ & $\mathrm{U}$ \\
\hline & $01 / 15 / 08$ & 01/30/08 & gross $\alpha$ & $2.2 \mathrm{E}-03 \pm 7.0 \mathrm{E}-04$ & & & ${ }^{134} \mathrm{Cs}$ & $9.0 \mathrm{E}-05 \pm 1.1 \mathrm{E}-04$ & U \\
\hline & $01 / 15 / 08$ & 01/30/08 & gross $\beta$ & $3.4 \mathrm{E}-02 \pm 3.3 \mathrm{E}-03$ & & & ${ }^{137} \mathrm{Cs}$ & $-1.5 \mathrm{E}-05 \pm 9.4 \mathrm{E}-05$ & $\mathrm{U}$ \\
\hline & $01 / 30 / 08$ & 02/13/08 & gross $\alpha$ & $3.9 \mathrm{E}-04 \pm 4.4 \mathrm{E}-04$ & & & ${ }^{152} \mathrm{Eu}$ & $3.1 \mathrm{E}-05 \pm 2.3 \mathrm{E}-04$ & $\mathrm{U}$ \\
\hline & $01 / 30 / 08$ & $02 / 13 / 08$ & gross $\beta$ & $3.8 \mathrm{E}-03 \pm 8.5 \mathrm{E}-04$ & & & ${ }^{154} \mathrm{Eu}$ & $7.8 \mathrm{E}-05 \pm 3.4 \mathrm{E}-04$ & $\mathrm{U}$ \\
\hline & $02 / 13 / 08$ & $02 / 27 / 08$ & gross $\alpha$ & $1.4 \mathrm{E}-03 \pm 5.7 \mathrm{E}-04$ & & & ${ }^{155} \mathrm{Eu}$ & $-8.3 \mathrm{E}-05 \pm 1.8 \mathrm{E}-04$ & $\mathrm{U}$ \\
\hline & $02 / 13 / 08$ & $02 / 27 / 08$ & gross $\beta$ & $3.4 \mathrm{E}-02 \pm 3.4 \mathrm{E}-03$ & & & ${ }^{238} \mathrm{Pu}$ & $1.4 \mathrm{E}-06 \pm 1.4 \mathrm{E}-06$ & $\mathrm{U}$ \\
\hline & $02 / 27 / 08$ & 03/11/08 & gross $\alpha$ & $5.5 \mathrm{E}-04 \pm 5.2 \mathrm{E}-04$ & & & ${ }^{239 / 240} \mathrm{Pu}$ & $2.7 \mathrm{E}-06 \pm 4.0 \mathrm{E}-06$ & $\mathrm{U}$ \\
\hline & $02 / 27 / 08$ & 03/11/08 & gross $\beta$ & $1.3 \mathrm{E}-02 \pm 1.7 \mathrm{E}-03$ & & & ${ }^{241} \mathrm{Pu}$ & $2.7 \mathrm{E}-04 \pm 5.5 \mathrm{E}-04$ & $\mathrm{U}$ \\
\hline & 03/11/08 & $04 / 08 / 08$ & gross $\alpha$ & $9.3 \mathrm{E}-04 \pm 3.3 \mathrm{E}-04$ & & & ${ }^{106} \mathrm{Ru}$ & $-5.0 \mathrm{E}-04 \pm 8.9 \mathrm{E}-04$ & $\mathrm{U}$ \\
\hline & 03/11/08 & $04 / 08 / 08$ & gross $\beta$ & $7.3 \mathrm{E}-03 \pm 9.4 \mathrm{E}-04$ & & & ${ }^{125} \mathrm{Sb}$ & $1.4 \mathrm{E}-04 \pm 2.4 \mathrm{E}-04$ & $\mathrm{U}$ \\
\hline & $04 / 08 / 08$ & $04 / 21 / 08$ & gross $\alpha$ & $5.4 \mathrm{E}-04 \pm 5.1 \mathrm{E}-04$ & & & ${ }^{90} \mathrm{Sr}$ & $-7.7 \mathrm{E}-05 \pm 8.0 \mathrm{E}-05$ & $\mathrm{U}$ \\
\hline & $04 / 08 / 08$ & $04 / 21 / 08$ & gross $\beta$ & $1.3 \mathrm{E}-02 \pm 1.8 \mathrm{E}-03$ & & & ${ }^{234} \mathrm{U}$ & $2.4 \mathrm{E}-05 \pm 1.2 \mathrm{E}-05$ & \\
\hline & $04 / 21 / 08$ & 05/07/08 & gross $\alpha$ & $1.0 \mathrm{E}-03 \pm 4.5 \mathrm{E}-04$ & & & ${ }^{235} \mathrm{U}$ & $5.1 \mathrm{E}-06 \pm 4.3 \mathrm{E}-06$ & \\
\hline & $04 / 21 / 08$ & $05 / 07 / 08$ & gross $\beta$ & $1.4 \mathrm{E}-02 \pm 1.7 \mathrm{E}-03$ & & & ${ }^{238} \mathrm{U}$ & $2.7 \mathrm{E}-05 \pm 1.4 \mathrm{E}-05$ & \\
\hline & $05 / 07 / 08$ & $05 / 20 / 08$ & gross $\alpha$ & $6.5 \mathrm{E}-04 \pm 5.3 \mathrm{E}-04$ & & & & & \\
\hline & 05/07/08 & 05/20/08 & gross $\beta$ & $1.1 \mathrm{E}-02 \pm 1.6 \mathrm{E}-03$ & N401 & $07 / 01 / 08$ to $12 / 30 / 08$ & ${ }^{241} \mathrm{Am}$ & $4.8 \mathrm{E}-04 \pm 1.7 \mathrm{E}-04$ & \\
\hline & $05 / 20 / 08$ & 06/03/08 & gross $\alpha$ & $9.4 \mathrm{E}-04 \pm 5.7 \mathrm{E}-04$ & & & ${ }^{60} \mathrm{Co}$ & $3.4 \mathrm{E}-05 \pm 7.8 \mathrm{E}-05$ & U \\
\hline & $05 / 20 / 08$ & $06 / 03 / 08$ & gross $\beta$ & $1.1 \mathrm{E}-02 \pm 1.6 \mathrm{E}-03$ & & & ${ }^{134} \mathrm{Cs}$ & $4.0 \mathrm{E}-05 \pm 6.3 \mathrm{E}-05$ & $\mathrm{U}$ \\
\hline & 06/03/08 & $06 / 17 / 08$ & gross $\alpha$ & $4.9 \mathrm{E}-04 \pm 4.6 \mathrm{E}-04$ & & & ${ }^{137} \mathrm{Cs}$ & $3.6 \mathrm{E}-04 \pm 1.8 \mathrm{E}-04$ & \\
\hline & 06/03/08 & $06 / 17 / 08$ & gross $\beta$ & $8.1 \mathrm{E}-03 \pm 1.3 \mathrm{E}-03$ & & & ${ }^{152} \mathrm{Eu}$ & $9.1 \mathrm{E}-05 \pm 1.5 \mathrm{E}-04$ & $\mathrm{U}$ \\
\hline & $06 / 17 / 08$ & 07/01/08 & gross $\alpha$ & $1.4 \mathrm{E}-03 \pm 5.6 \mathrm{E}-04$ & & & ${ }^{154} \mathrm{Eu}$ & $1.9 \mathrm{E}-05 \pm 1.9 \mathrm{E}-04$ & $\mathrm{U}$ \\
\hline & $06 / 17 / 08$ & 07/01/08 & gross $\beta$ & $9.3 \mathrm{E}-03 \pm 1.4 \mathrm{E}-03$ & & & ${ }^{155} \mathrm{Eu}$ & $3.0 \mathrm{E}-05 \pm 1.3 \mathrm{E}-04$ & $\mathrm{U}$ \\
\hline & 07/01/08 & 07/15/08 & gross $\alpha$ & $1.6 \mathrm{E}-03 \pm 6.5 \mathrm{E}-04$ & & & ${ }^{238} \mathrm{Pu}$ & $6.8 \mathrm{E}-05 \pm 4.0 \mathrm{E}-05$ & \\
\hline & 07/01/08 & $07 / 15 / 08$ & gross $\beta$ & $1.3 \mathrm{E}-02 \pm 1.7 \mathrm{E}-03$ & & & ${ }^{239 / 240} \mathrm{Pu}$ & $3.0 \mathrm{E}-04 \pm 1.2 \mathrm{E}-04$ & \\
\hline & $07 / 15 / 08$ & $07 / 18 / 08$ & gross $\alpha$ & $5.7 \mathrm{E}-03 \pm 2.4 \mathrm{E}-03$ & & & ${ }^{241} \mathrm{Pu}$ & $2.6 \mathrm{E}-03 \pm 1.2 \mathrm{E}-03$ & \\
\hline & $07 / 15 / 08$ & 07/18/08 & gross $\beta$ & $1.8 \mathrm{E}-02 \pm 3.8 \mathrm{E}-03$ & & & ${ }^{106} \mathrm{Ru}$ & $-3.4 \mathrm{E}-04 \pm 5.7 \mathrm{E}-04$ & $\mathrm{U}$ \\
\hline & 07/18/08 & 07/29/08 & gross $\alpha$ & $7.9 \mathrm{E}-04 \pm 6.3 \mathrm{E}-04$ & & & ${ }^{125} \mathrm{Sb}$ & $5.7 \mathrm{E}-05 \pm 1.5 \mathrm{E}-04$ & $\mathrm{U}$ \\
\hline & 07/18/08 & 07/29/08 & gross $\beta$ & $1.0 \mathrm{E}-02 \pm 1.6 \mathrm{E}-03$ & & & ${ }^{90} \mathrm{Sr}$ & $-8.4 \mathrm{E}-05 \pm 8.7 \mathrm{E}-05$ & $\mathrm{U}$ \\
\hline & $07 / 29 / 08$ & $08 / 12 / 08$ & gross $\alpha$ & $3.2 \mathrm{E}-03 \pm 8.6 \mathrm{E}-04$ & & & ${ }^{234} \mathrm{U}$ & $1.4 \mathrm{E}-05 \pm 9.1 \mathrm{E}-06$ & \\
\hline & $07 / 29 / 08$ & 08/12/08 & gross $\beta$ & $1.2 \mathrm{E}-02 \pm 1.6 \mathrm{E}-03$ & & & ${ }^{235} \mathrm{U}$ & $8.6 \mathrm{E}-07 \pm 1.8 \mathrm{E}-06$ & $\mathrm{U}$ \\
\hline & $08 / 12 / 08$ & $08 / 26 / 08$ & gross $\alpha$ & $3.9 \mathrm{E}-03 \pm 9.5 \mathrm{E}-04$ & & & ${ }^{238} \mathrm{U}$ & $1.6 \mathrm{E}-06 \pm 3.9 \mathrm{E}-06$ & $\mathrm{U}$ \\
\hline & 08/12/08 & 08/26/08 & gross $\beta$ & $1.6 \mathrm{E}-02 \pm 2.0 \mathrm{E}-03$ & & & & & \\
\hline & $08 / 26 / 08$ & 09/09/08 & gross $\alpha$ & $1.7 \mathrm{E}-03 \pm 6.6 \mathrm{E}-04$ & & & & & \\
\hline & 08/26/08 & 09/09/08 & gross $\beta$ & $1.4 \mathrm{E}-02 \pm 1.9 \mathrm{E}-03$ & & & & & \\
\hline & 09/09/08 & 09/23/08 & gross $\alpha$ & $1.6 \mathrm{E}-03 \pm 5.9 \mathrm{E}-04$ & & & & & \\
\hline & 09/09/08 & 09/23/08 & gross $\beta$ & $2.7 \mathrm{E}-02 \pm 2.8 \mathrm{E}-03$ & & & & & \\
\hline & $09 / 23 / 08$ & $10 / 09 / 08$ & gross $\alpha$ & $2.1 \mathrm{E}-03 \pm 6.4 \mathrm{E}-04$ & & & & & \\
\hline & $09 / 23 / 08$ & $10 / 09 / 08$ & gross $\beta$ & $2.2 \mathrm{E}-02 \pm 2.4 \mathrm{E}-03$ & & & & & \\
\hline & $10 / 09 / 08$ & $10 / 21 / 08$ & gross $\alpha$ & $1.5 \mathrm{E}-03 \pm 6.2 \mathrm{E}-04$ & & & & & \\
\hline & $10 / 09 / 08$ & $10 / 21 / 08$ & gross $\beta$ & $1.6 \mathrm{E}-02 \pm 2.0 \mathrm{E}-03$ & & & & & \\
\hline & $10 / 21 / 08$ & $11 / 04 / 08$ & gross $\alpha$ & $3.4 \mathrm{E}-03 \pm 8.9 \mathrm{E}-04$ & & & & & \\
\hline & $10 / 21 / 08$ & $11 / 04 / 08$ & gross $\beta$ & $4.1 \mathrm{E}-02 \pm 3.8 \mathrm{E}-03$ & & & & & \\
\hline & $11 / 04 / 08$ & $11 / 18 / 08$ & gross $\alpha$ & $1.1 \mathrm{E}-03 \pm 5.0 \mathrm{E}-04$ & & & & & \\
\hline & $11 / 04 / 08$ & $11 / 18 / 08$ & gross $\beta$ & $2.1 \mathrm{E}-02 \pm 2.4 \mathrm{E}-03$ & & & & & \\
\hline & $11 / 18 / 08$ & $12 / 02 / 08$ & gross $\alpha$ & $1.5 \mathrm{E}-03 \pm 6.0 \mathrm{E}-04$ & & & & & \\
\hline & $11 / 18 / 08$ & $12 / 02 / 08$ & gross $\beta$ & $3.4 \mathrm{E}-02 \pm 3.4 \mathrm{E}-03$ & & & & & \\
\hline & $12 / 02 / 08$ & $12 / 16 / 08$ & gross $\alpha$ & $1.4 \mathrm{E}-03 \pm 5.7 \mathrm{E}-04$ & & & & & \\
\hline & $12 / 02 / 08$ & $12 / 16 / 08$ & gross $\beta$ & $2.4 \mathrm{E}-02 \pm 2.6 \mathrm{E}-03$ & & & & & \\
\hline & $12 / 16 / 08$ & $12 / 30 / 08$ & gross $\alpha$ & $9.4 \mathrm{E}-04 \pm 5.8 \mathrm{E}-04$ & & & & & \\
\hline & $12 / 16 / 08$ & $12 / 30 / 08$ & gross $\beta$ & $3.3 \mathrm{E}-02 \pm 3.3 \mathrm{E}-03$ & & & & & \\
\hline
\end{tabular}

$\overline{\mathrm{RQ}}=$ Result Qualifier. $\mathrm{U}=$ The analyte was analyzed for but not detected. 
Table 2-4. Near-Facility Air Sampling Results, 2008 (pCi/m³ \pm total analytical uncertainty). (Sheet 11 of 82)

\begin{tabular}{|c|c|c|c|c|c|c|c|c|c|}
\hline Location & Sample On & Sample Off & Isotope & Result \pm Uncertainty & Location & Composite Period & Isotope & Result \pm Uncertainty & $\mathbf{R Q}^{*}$ \\
\hline N402 & $01 / 02 / 08$ & $01 / 15 / 08$ & gross $\alpha$ & $1.0 \mathrm{E}-03 \pm 6.2 \mathrm{E}-04$ & N402 & $01 / 02 / 08$ to $07 / 01 / 08$ & ${ }^{241} \mathrm{Am}$ & $3.8 \mathrm{E}-05 \pm 1.7 \mathrm{E}-05$ & \\
\hline \multirow[t]{53}{*}{$(100-\mathrm{K})$} & $01 / 02 / 08$ & $01 / 15 / 08$ & gross $\beta$ & $9.5 \mathrm{E}-03 \pm 1.5 \mathrm{E}-03$ & & & ${ }^{60} \mathrm{Co}$ & $2.2 \mathrm{E}-05 \pm 9.0 \mathrm{E}-05$ & $\mathrm{U}$ \\
\hline & $01 / 15 / 08$ & 01/30/08 & gross $\alpha$ & $1.6 \mathrm{E}-03 \pm 5.9 \mathrm{E}-04$ & & & ${ }^{134} \mathrm{Cs}$ & $-2.7 \mathrm{E}-05 \pm 7.1 \mathrm{E}-05$ & U \\
\hline & $01 / 15 / 08$ & 01/30/08 & gross $\beta$ & $3.5 \mathrm{E}-02 \pm 3.4 \mathrm{E}-03$ & & & ${ }^{137} \mathrm{Cs}$ & $1.2 \mathrm{E}-04 \pm 9.0 \mathrm{E}-05$ & $\mathrm{U}$ \\
\hline & 01/30/08 & $02 / 13 / 08$ & gross $\alpha$ & $5.0 \mathrm{E}-04 \pm 4.7 \mathrm{E}-04$ & & & ${ }^{152} \mathrm{Eu}$ & $2.2 \mathrm{E}-05 \pm 1.6 \mathrm{E}-04$ & $\mathrm{U}$ \\
\hline & $01 / 30 / 08$ & $02 / 13 / 08$ & gross $\beta$ & $4.2 \mathrm{E}-03 \pm 9.0 \mathrm{E}-04$ & & & ${ }^{154} \mathrm{Eu}$ & $-6.4 \mathrm{E}-05 \pm 2.1 \mathrm{E}-04$ & $\mathrm{U}$ \\
\hline & $02 / 13 / 08$ & $02 / 27 / 08$ & gross $\alpha$ & $4.5 \mathrm{E}-03 \pm 1.3 \mathrm{E}-03$ & & & ${ }^{155} \mathrm{Eu}$ & $9.8 \mathrm{E}-05 \pm 1.8 \mathrm{E}-04$ & $\mathrm{U}$ \\
\hline & $02 / 13 / 08$ & $02 / 27 / 08$ & gross $\beta$ & $4.7 \mathrm{E}-02 \pm 5.0 \mathrm{E}-03$ & & & ${ }^{238} \mathrm{Pu}$ & $3.6 \mathrm{E}-05 \pm 2.8 \mathrm{E}-05$ & \\
\hline & $02 / 27 / 08$ & 03/11/08 & gross $\alpha$ & $5.5 \mathrm{E}-04 \pm 5.2 \mathrm{E}-04$ & & & ${ }^{239 / 240} \mathrm{Pu}$ & $3.0 \mathrm{E}-05 \pm 1.8 \mathrm{E}-05$ & \\
\hline & $02 / 27 / 08$ & 03/11/08 & gross $\beta$ & $1.4 \mathrm{E}-02 \pm 1.9 \mathrm{E}-03$ & & & ${ }^{241} \mathrm{Pu}$ & $5.5 \mathrm{E}-04 \pm 6.8 \mathrm{E}-04$ & $\mathrm{U}$ \\
\hline & 03/11/08 & 03/26/08 & gross $\alpha$ & $1.9 \mathrm{E}-03 \pm 6.4 \mathrm{E}-04$ & & & ${ }^{106} \mathrm{Ru}$ & $-4.2 \mathrm{E}-04 \pm 6.5 \mathrm{E}-04$ & $\mathrm{U}$ \\
\hline & 03/11/08 & 03/26/08 & gross $\beta$ & $1.6 \mathrm{E}-02 \pm 1.9 \mathrm{E}-03$ & & & ${ }^{125} \mathrm{Sb}$ & $7.3 \mathrm{E}-05 \pm 1.5 \mathrm{E}-04$ & $\mathrm{U}$ \\
\hline & $03 / 26 / 08$ & $04 / 08 / 08$ & gross $\alpha$ & $5.1 \mathrm{E}-03 \pm 1.1 \mathrm{E}-02$ & & & ${ }^{90} \mathrm{Sr}$ & $2.9 \mathrm{E}-04 \pm 1.7 \mathrm{E}-04$ & \\
\hline & 03/26/08 & $04 / 08 / 08$ & gross $\beta$ & $2.2 \mathrm{E}-01 \pm 3.9 \mathrm{E}-02$ & & & ${ }^{234} \mathrm{U}$ & $9.7 \mathrm{E}-06 \pm 7.1 \mathrm{E}-06$ & \\
\hline & $04 / 08 / 08$ & $04 / 23 / 08$ & gross $\alpha$ & $9.7 \mathrm{E}-04 \pm 4.5 \mathrm{E}-04$ & & & ${ }^{235} \mathrm{U}$ & $4.1 \mathrm{E}-06 \pm 3.9 \mathrm{E}-06$ & \\
\hline & $04 / 08 / 08$ & $04 / 23 / 08$ & gross $\beta$ & $1.1 \mathrm{E}-02 \pm 1.5 \mathrm{E}-03$ & & & ${ }^{238} \mathrm{U}$ & $7.5 \mathrm{E}-06 \pm 5.9 \mathrm{E}-06$ & \\
\hline & $04 / 23 / 08$ & $05 / 07 / 08$ & gross $\alpha$ & $1.7 \mathrm{E}-03 \pm 6.1 \mathrm{E}-04$ & & & & & \\
\hline & $04 / 23 / 08$ & 05/07/08 & gross $\beta$ & $1.5 \mathrm{E}-02 \pm 1.9 \mathrm{E}-03$ & N402 & $07 / 01 / 08$ to $12 / 30 / 08$ & ${ }^{241} \mathrm{Am}$ & $4.9 \mathrm{E}-04 \pm 1.8 \mathrm{E}-04$ & \\
\hline & 05/07/08 & $05 / 20 / 08$ & gross $\alpha$ & $1.2 \mathrm{E}-03 \pm 5.5 \mathrm{E}-04$ & & & ${ }^{60} \mathrm{Co}$ & $3.2 \mathrm{E}-06 \pm 3.2 \mathrm{E}-05$ & U \\
\hline & 05/07/08 & 05/20/08 & gross $\beta$ & $1.0 \mathrm{E}-02 \pm 1.6 \mathrm{E}-03$ & & & ${ }^{134} \mathrm{Cs}$ & $2.2 \mathrm{E}-05 \pm 6.6 \mathrm{E}-05$ & $\mathrm{U}$ \\
\hline & 05/20/08 & 06/03/08 & gross $\alpha$ & $5.0 \mathrm{E}-04 \pm 4.6 \mathrm{E}-04$ & & & ${ }^{137} \mathrm{Cs}$ & $4.4 \mathrm{E}-04 \pm 2.0 \mathrm{E}-04$ & \\
\hline & $05 / 20 / 08$ & $06 / 03 / 08$ & gross $\beta$ & $9.4 \mathrm{E}-03 \pm 1.4 \mathrm{E}-03$ & & & ${ }^{152} \mathrm{Eu}$ & $9.1 \mathrm{E}-05 \pm 1.6 \mathrm{E}-04$ & $\mathrm{U}$ \\
\hline & 06/03/08 & 06/17/08 & gross $\alpha$ & $3.8 \mathrm{E}-04 \pm 4.3 \mathrm{E}-04$ & & & ${ }^{154} \mathrm{Eu}$ & $2.4 \mathrm{E}-04 \pm 2.4 \mathrm{E}-04$ & $\mathrm{U}$ \\
\hline & 06/03/08 & $06 / 17 / 08$ & gross $\beta$ & $8.7 \mathrm{E}-03 \pm 1.4 \mathrm{E}-03$ & & & ${ }^{155} \mathrm{Eu}$ & $5.6 \mathrm{E}-05 \pm 1.6 \mathrm{E}-04$ & $\mathrm{U}$ \\
\hline & $06 / 17 / 08$ & 07/01/08 & gross $\alpha$ & $1.4 \mathrm{E}-03 \pm 5.7 \mathrm{E}-04$ & & & ${ }^{238} \mathrm{Pu}$ & $6.1 \mathrm{E}-05 \pm 4.1 \mathrm{E}-05$ & \\
\hline & $06 / 17 / 08$ & $07 / 01 / 08$ & gross $\beta$ & $1.7 \mathrm{E}-02 \pm 2.0 \mathrm{E}-03$ & & & ${ }^{239 / 240} \mathrm{Pu}$ & $3.6 \mathrm{E}-04 \pm 1.4 \mathrm{E}-04$ & \\
\hline & $07 / 01 / 08$ & $07 / 15 / 08$ & gross $\alpha$ & $2.2 \mathrm{E}-03 \pm 7.7 \mathrm{E}-04$ & & & ${ }^{241} \mathrm{Pu}$ & $4.0 \mathrm{E}-03 \pm 1.6 \mathrm{E}-03$ & \\
\hline & 07/01/08 & 07/15/08 & gross $\beta$ & $1.6 \mathrm{E}-02 \pm 2.0 \mathrm{E}-03$ & & & ${ }^{106} \mathrm{Ru}$ & $-1.3 \mathrm{E}-04 \pm 5.8 \mathrm{E}-04$ & $\mathrm{U}$ \\
\hline & 07/15/08 & 07/18/08 & gross $\alpha$ & $1.8 \mathrm{E}-03 \pm 1.9 \mathrm{E}-03$ & & & ${ }^{125} \mathrm{Sb}$ & $5.2 \mathrm{E}-05 \pm 1.6 \mathrm{E}-04$ & $\mathrm{U}$ \\
\hline & $07 / 15 / 08$ & 07/18/08 & gross $\beta$ & $1.5 \mathrm{E}-02 \pm 3.6 \mathrm{E}-03$ & & & ${ }^{90} \mathrm{Sr}$ & $2.1 \mathrm{E}-04 \pm 1.7 \mathrm{E}-04$ & $\mathrm{U}$ \\
\hline & 07/18/08 & $07 / 29 / 08$ & gross $\alpha$ & $1.6 \mathrm{E}-03 \pm 6.8 \mathrm{E}-04$ & & & ${ }^{234} \mathrm{U}$ & $2.1 \mathrm{E}-05 \pm 1.1 \mathrm{E}-05$ & \\
\hline & 07/18/08 & $07 / 29 / 08$ & gross $\beta$ & $1.6 \mathrm{E}-02 \pm 2.1 \mathrm{E}-03$ & & & ${ }^{235} \mathrm{U}$ & $4.3 \mathrm{E}-06 \pm 4.4 \mathrm{E}-06$ & $\mathrm{U}$ \\
\hline & 07/29/08 & $08 / 12 / 08$ & gross $\alpha$ & $5.5 \mathrm{E}-03 \pm 1.2 \mathrm{E}-03$ & & & ${ }^{238} \mathrm{U}$ & $9.9 \mathrm{E}-06 \pm 6.4 \mathrm{E}-06$ & \\
\hline & 07/29/08 & 08/12/08 & gross $\beta$ & $1.5 \mathrm{E}-02 \pm 1.9 \mathrm{E}-03$ & & & & & \\
\hline & $08 / 12 / 08$ & 08/26/08 & gross $\alpha$ & $2.2 \mathrm{E}-03 \pm 7.0 \mathrm{E}-04$ & & & & & \\
\hline & 08/12/08 & 08/26/08 & gross $\beta$ & $1.6 \mathrm{E}-02 \pm 1.9 \mathrm{E}-03$ & & & & & \\
\hline & 08/26/08 & 09/09/08 & gross $\alpha$ & $3.3 \mathrm{E}-03 \pm 8.7 \mathrm{E}-04$ & & & & & \\
\hline & $08 / 26 / 08$ & 09/09/08 & gross $\beta$ & $1.3 \mathrm{E}-02 \pm 1.7 \mathrm{E}-03$ & & & & & \\
\hline & 09/09/08 & 09/23/08 & gross $\alpha$ & $1.4 \mathrm{E}-03 \pm 5.5 \mathrm{E}-04$ & & & & & \\
\hline & 09/09/08 & 09/23/08 & gross $\beta$ & $2.5 \mathrm{E}-02 \pm 2.7 \mathrm{E}-03$ & & & & & \\
\hline & $09 / 23 / 08$ & $10 / 09 / 08$ & gross $\alpha$ & $2.4 \mathrm{E}-03 \pm 6.8 \mathrm{E}-04$ & & & & & \\
\hline & 09/23/08 & $10 / 09 / 08$ & gross $\beta$ & $2.5 \mathrm{E}-02 \pm 2.6 \mathrm{E}-03$ & & & & & \\
\hline & $10 / 09 / 08$ & $10 / 21 / 08$ & gross $\alpha$ & $1.8 \mathrm{E}-03 \pm 6.9 \mathrm{E}-04$ & & & & & \\
\hline & $10 / 09 / 08$ & $10 / 21 / 08$ & gross $\beta$ & $1.7 \mathrm{E}-02 \pm 2.2 \mathrm{E}-03$ & & & & & \\
\hline & $10 / 21 / 08$ & $11 / 04 / 08$ & gross $\alpha$ & $1.8 \mathrm{E}-03 \pm 6.3 \mathrm{E}-04$ & & & & & \\
\hline & $10 / 21 / 08$ & $11 / 04 / 08$ & gross $\beta$ & $4.3 \mathrm{E}-02 \pm 4.0 \mathrm{E}-03$ & & & & & \\
\hline & $11 / 04 / 08$ & $11 / 18 / 08$ & gross $\alpha$ & $2.0 \mathrm{E}-03 \pm 6.9 \mathrm{E}-04$ & & & & & \\
\hline & $11 / 04 / 08$ & $11 / 18 / 08$ & gross $\beta$ & $2.3 \mathrm{E}-02 \pm 2.5 \mathrm{E}-03$ & & & & & \\
\hline & $11 / 18 / 08$ & $12 / 02 / 08$ & gross $\alpha$ & $1.1 \mathrm{E}-03 \pm 5.2 \mathrm{E}-04$ & & & & & \\
\hline & $11 / 18 / 08$ & $12 / 02 / 08$ & gross $\beta$ & $3.7 \mathrm{E}-02 \pm 3.6 \mathrm{E}-03$ & & & & & \\
\hline & $12 / 02 / 08$ & $12 / 16 / 08$ & gross $\alpha$ & $1.7 \mathrm{E}-03 \pm 6.3 \mathrm{E}-04$ & & & & & \\
\hline & $12 / 02 / 08$ & $12 / 16 / 08$ & gross $\beta$ & $2.7 \mathrm{E}-02 \pm 2.9 \mathrm{E}-03$ & & & & & \\
\hline & $12 / 16 / 08$ & $12 / 30 / 08$ & gross $\alpha$ & $1.5 \mathrm{E}-03 \pm 6.0 \mathrm{E}-04$ & & & & & \\
\hline & $12 / 16 / 08$ & $12 / 30 / 08$ & gross $\beta$ & $3.4 \mathrm{E}-02 \pm 3.4 \mathrm{E}-03$ & & & & & \\
\hline
\end{tabular}

$\overline{\mathrm{RQ}}=$ Result Qualifier. $\mathrm{U}=$ The analyte was analyzed for but not detected. 
Table 2-4. Near-Facility Air Sampling Results, 2008 (pCi/m³ \pm total analytical uncertainty). (Sheet 12 of 82)

\begin{tabular}{|c|c|c|c|c|c|c|c|c|c|}
\hline Location & Sample On & Sample Off & Isotope & Result \pm Uncertainty & Location & Composite Period & Isotope & Result \pm Uncertainty & $\mathbf{R Q *}$ \\
\hline N403 & $01 / 02 / 08$ & $01 / 15 / 08$ & gross $\alpha$ & $4.1 \mathrm{E}-04 \pm 4.6 \mathrm{E}-04$ & N403 & $01 / 02 / 08$ to $07 / 01 / 08$ & ${ }^{241} \mathrm{Am}$ & $9.1 \mathrm{E}-06 \pm 5.8 \mathrm{E}-06$ & \\
\hline \multirow{53}{*}{$(100-\mathrm{K})$} & $01 / 02 / 08$ & $01 / 15 / 08$ & gross $\beta$ & $1.1 \mathrm{E}-02 \pm 1.6 \mathrm{E}-03$ & & & ${ }^{60} \mathrm{Co}$ & $-2.1 \mathrm{E}-05 \pm 8.2 \mathrm{E}-05$ & $\mathrm{U}$ \\
\hline & $01 / 15 / 08$ & $01 / 30 / 08$ & gross $\alpha$ & $1.6 \mathrm{E}-03 \pm 6.0 \mathrm{E}-04$ & & & ${ }^{134} \mathrm{Cs}$ & $-5.1 \mathrm{E}-05 \pm 8.3 \mathrm{E}-05$ & $\mathrm{U}$ \\
\hline & $01 / 15 / 08$ & 01/30/08 & gross $\beta$ & $3.5 \mathrm{E}-02 \pm 3.4 \mathrm{E}-03$ & & & ${ }^{137} \mathrm{Cs}$ & $3.5 \mathrm{E}-05 \pm 6.9 \mathrm{E}-05$ & $\mathrm{U}$ \\
\hline & 01/30/08 & $02 / 13 / 08$ & gross $\alpha$ & $7.3 \mathrm{E}-04 \pm 5.3 \mathrm{E}-04$ & & & ${ }^{152} \mathrm{Eu}$ & $-1.2 \mathrm{E}-04 \pm 1.7 \mathrm{E}-04$ & $\mathrm{U}$ \\
\hline & $01 / 30 / 08$ & $02 / 13 / 08$ & gross $\beta$ & $4.8 \mathrm{E}-03 \pm 9.6 \mathrm{E}-04$ & & & ${ }^{154} \mathrm{Eu}$ & $7.4 \mathrm{E}-05 \pm 2.3 \mathrm{E}-04$ & $\mathrm{U}$ \\
\hline & $02 / 13 / 08$ & $02 / 27 / 08$ & gross $\alpha$ & $1.7 \mathrm{E}-03 \pm 6.5 \mathrm{E}-04$ & & & ${ }^{155} \mathrm{Eu}$ & $-9.5 \mathrm{E}-07 \pm 9.5 \mathrm{E}-06$ & $\mathrm{U}$ \\
\hline & $02 / 13 / 08$ & $02 / 27 / 08$ & gross $\beta$ & $3.2 \mathrm{E}-02 \pm 3.3 \mathrm{E}-03$ & & & ${ }^{238} \mathrm{Pu}$ & $6.5 \mathrm{E}-06 \pm 2.0 \mathrm{E}-05$ & $\mathrm{U}$ \\
\hline & $02 / 27 / 08$ & 03/11/08 & gross $\alpha$ & $1.4 \mathrm{E}-03 \pm 6.0 \mathrm{E}-04$ & & & ${ }^{239 / 240} \mathrm{Pu}$ & $3.3 \mathrm{E}-06 \pm 8.1 \mathrm{E}-06$ & $\mathrm{U}$ \\
\hline & $02 / 27 / 08$ & 03/11/08 & gross $\beta$ & $1.4 \mathrm{E}-02 \pm 1.9 \mathrm{E}-03$ & & & ${ }^{241} \mathrm{Pu}$ & $5.2 \mathrm{E}-04 \pm 6.7 \mathrm{E}-04$ & $\mathrm{U}$ \\
\hline & 03/11/08 & 03/25/08 & gross $\alpha$ & $8.6 \mathrm{E}-04 \pm 5.8 \mathrm{E}-04$ & & & ${ }^{106} \mathrm{Ru}$ & $2.1 \mathrm{E}-04 \pm 6.5 \mathrm{E}-04$ & $\mathrm{U}$ \\
\hline & 03/11/08 & $03 / 25 / 08$ & gross $\beta$ & $3.7 \mathrm{E}-02 \pm 3.6 \mathrm{E}-03$ & & & ${ }^{125} \mathrm{Sb}$ & $-3.4 \mathrm{E}-05 \pm 1.5 \mathrm{E}-04$ & $\mathrm{U}$ \\
\hline & $03 / 25 / 08$ & $04 / 07 / 08$ & gross $\alpha$ & $1.0 \mathrm{E}-03 \pm 6.3 \mathrm{E}-04$ & & & ${ }^{90} \mathrm{Sr}$ & $-1.6 \mathrm{E}-04 \pm 1.6 \mathrm{E}-04$ & $\mathrm{U}$ \\
\hline & 03/25/08 & $04 / 07 / 08$ & gross $\beta$ & $7.0 \mathrm{E}-03 \pm 1.2 \mathrm{E}-03$ & & & ${ }^{234} \mathrm{U}$ & $1.3 \mathrm{E}-05 \pm 7.5 \mathrm{E}-06$ & \\
\hline & $04 / 07 / 08$ & $04 / 21 / 08$ & gross $\alpha$ & $4.0 \mathrm{E}-04 \pm 4.5 \mathrm{E}-04$ & & & ${ }^{235} \mathrm{U}$ & $3.5 \mathrm{E}-06 \pm 3.9 \mathrm{E}-06$ & $\mathrm{U}$ \\
\hline & $04 / 07 / 08$ & $04 / 21 / 08$ & gross $\beta$ & $1.1 \mathrm{E}-02 \pm 1.6 \mathrm{E}-03$ & & & ${ }^{238} \mathrm{U}$ & $1.1 \mathrm{E}-05 \pm 6.9 \mathrm{E}-06$ & \\
\hline & $04 / 21 / 08$ & 05/07/08 & gross $\alpha$ & $1.5 \mathrm{E}-03 \pm 5.6 \mathrm{E}-04$ & & & & & \\
\hline & $04 / 21 / 08$ & 05/07/08 & gross $\beta$ & $1.4 \mathrm{E}-02 \pm 1.7 \mathrm{E}-03$ & N403 & $07 / 01 / 08$ to $12 / 30 / 08$ & ${ }^{241} \mathrm{Am}$ & $1.2 \mathrm{E}-03 \pm 4.4 \mathrm{E}-04$ & \\
\hline & 05/07/08 & $05 / 20 / 08$ & gross $\alpha$ & $1.3 \mathrm{E}-03 \pm 5.8 \mathrm{E}-04$ & & & ${ }^{60} \mathrm{Co}$ & $2.3 \mathrm{E}-06 \pm 2.3 \mathrm{E}-05$ & $\mathrm{U}$ \\
\hline & 05/07/08 & 05/20/08 & gross $\beta$ & $1.0 \mathrm{E}-02 \pm 1.5 \mathrm{E}-03$ & & & ${ }^{134} \mathrm{Cs}$ & $2.2 \mathrm{E}-05 \pm 7.0 \mathrm{E}-05$ & $\mathrm{U}$ \\
\hline & 05/20/08 & 06/03/08 & gross $\alpha$ & $1.5 \mathrm{E}-03 \pm 5.8 \mathrm{E}-04$ & & & ${ }^{137} \mathrm{Cs}$ & $7.4 \mathrm{E}-03 \pm 2.5 \mathrm{E}-03$ & \\
\hline & $05 / 20 / 08$ & $06 / 03 / 08$ & gross $\beta$ & $1.0 \mathrm{E}-02 \pm 1.5 \mathrm{E}-03$ & & & ${ }^{152} \mathrm{Eu}$ & $5.7 \mathrm{E}-06 \pm 5.7 \mathrm{E}-05$ & $\mathrm{U}$ \\
\hline & 06/03/08 & 06/17/08 & gross $\alpha$ & $2.7 \mathrm{E}-04 \pm 4.0 \mathrm{E}-04$ & & & ${ }^{154} \mathrm{Eu}$ & $4.9 \mathrm{E}-05 \pm 2.3 \mathrm{E}-04$ & $\mathrm{U}$ \\
\hline & 06/03/08 & $06 / 17 / 08$ & gross $\beta$ & $7.6 \mathrm{E}-03 \pm 1.3 \mathrm{E}-03$ & & & ${ }^{155} \mathrm{Eu}$ & $1.0 \mathrm{E}-04 \pm 1.9 \mathrm{E}-04$ & $\mathrm{U}$ \\
\hline & $06 / 17 / 08$ & 07/01/08 & gross $\alpha$ & $6.6 \mathrm{E}-04 \pm 5.3 \mathrm{E}-04$ & & & ${ }^{238} \mathrm{Pu}$ & $1.1 \mathrm{E}-04 \pm 5.6 \mathrm{E}-05$ & \\
\hline & $06 / 17 / 08$ & $07 / 01 / 08$ & gross $\beta$ & $1.3 \mathrm{E}-02 \pm 1.7 \mathrm{E}-03$ & & & ${ }^{239 / 240} \mathrm{Pu}$ & $9.4 \mathrm{E}-04 \pm 3.6 \mathrm{E}-04$ & \\
\hline & $07 / 01 / 08$ & $07 / 15 / 08$ & gross $\alpha$ & $2.3 \mathrm{E}-03 \pm 8.1 \mathrm{E}-04$ & & & ${ }^{241} \mathrm{Pu}$ & $5.8 \mathrm{E}-03 \pm 1.8 \mathrm{E}-03$ & \\
\hline & 07/01/08 & 07/15/08 & gross $\beta$ & $1.3 \mathrm{E}-02 \pm 1.8 \mathrm{E}-03$ & & & ${ }^{106} \mathrm{Ru}$ & $-8.0 \mathrm{E}-04 \pm 8.3 \mathrm{E}-04$ & $\mathrm{U}$ \\
\hline & 07/15/08 & 07/18/08 & gross $\alpha$ & $9.4 \mathrm{E}-03 \pm 4.3 \mathrm{E}-03$ & & & ${ }^{125} \mathrm{Sb}$ & $4.6 \mathrm{E}-05 \pm 1.9 \mathrm{E}-04$ & $\mathrm{U}$ \\
\hline & $07 / 15 / 08$ & $07 / 18 / 08$ & gross $\beta$ & $1.9 \mathrm{E}-02 \pm 5.5 \mathrm{E}-03$ & & & ${ }^{90} \mathrm{Sr}$ & $1.4 \mathrm{E}-04 \pm 1.4 \mathrm{E}-04$ & \\
\hline & 07/18/08 & 07/29/08 & gross $\alpha$ & $3.4 \mathrm{E}-03 \pm 9.8 \mathrm{E}-04$ & & & ${ }^{234} \mathrm{U}$ & $1.5 \mathrm{E}-05 \pm 9.4 \mathrm{E}-06$ & \\
\hline & 07/18/08 & $07 / 29 / 08$ & gross $\beta$ & $1.8 \mathrm{E}-02 \pm 2.3 \mathrm{E}-03$ & & & ${ }^{235} \mathrm{U}$ & $3.7 \mathrm{E}-06 \pm 3.6 \mathrm{E}-06$ & \\
\hline & 07/29/08 & 08/12/08 & gross $\alpha$ & $4.2 \mathrm{E}-03 \pm 1.0 \mathrm{E}-03$ & & & ${ }^{238} \mathrm{U}$ & $2.4 \mathrm{E}-05 \pm 1.2 \mathrm{E}-05$ & \\
\hline & 07/29/08 & 08/12/08 & gross $\beta$ & $1.2 \mathrm{E}-01 \pm 9.3 \mathrm{E}-03$ & & & & & \\
\hline & $08 / 12 / 08$ & 08/26/08 & gross $\alpha$ & $6.6 \mathrm{E}-03 \pm 1.3 \mathrm{E}-03$ & & & & & \\
\hline & 08/12/08 & 08/26/08 & gross $\beta$ & $2.3 \mathrm{E}-02 \pm 2.5 \mathrm{E}-03$ & & & & & \\
\hline & 08/26/08 & 09/09/08 & gross $\alpha$ & $5.2 \mathrm{E}-03 \pm 1.1 \mathrm{E}-03$ & & & & & \\
\hline & $08 / 26 / 08$ & 09/09/08 & gross $\beta$ & $1.6 \mathrm{E}-02 \pm 2.0 \mathrm{E}-03$ & & & & & \\
\hline & 09/09/08 & 09/23/08 & gross $\alpha$ & $7.4 \mathrm{E}-04 \pm 5.3 \mathrm{E}-04$ & & & & & \\
\hline & 09/09/08 & 09/23/08 & gross $\beta$ & $1.3 \mathrm{E}-02 \pm 1.7 \mathrm{E}-03$ & & & & & \\
\hline & 09/23/08 & $10 / 09 / 08$ & gross $\alpha$ & $1.7 \mathrm{E}-03 \pm 5.7 \mathrm{E}-04$ & & & & & \\
\hline & 09/23/08 & $10 / 09 / 08$ & gross $\beta$ & $2.2 \mathrm{E}-02 \pm 2.4 \mathrm{E}-03$ & & & & & \\
\hline & $10 / 09 / 08$ & $10 / 21 / 08$ & gross $\alpha$ & $5.9 \mathrm{E}-04 \pm 5.4 \mathrm{E}-04$ & & & & & \\
\hline & $10 / 09 / 08$ & $10 / 21 / 08$ & gross $\beta$ & $2.2 \mathrm{E}-02 \pm 2.6 \mathrm{E}-03$ & & & & & \\
\hline & $10 / 21 / 08$ & $11 / 04 / 08$ & gross $\alpha$ & $1.9 \mathrm{E}-03 \pm 6.5 \mathrm{E}-04$ & & & & & \\
\hline & $10 / 21 / 08$ & $11 / 04 / 08$ & gross $\beta$ & $3.7 \mathrm{E}-02 \pm 3.6 \mathrm{E}-03$ & & & & & \\
\hline & $11 / 04 / 08$ & $11 / 18 / 08$ & gross $\alpha$ & $2.2 \mathrm{E}-03 \pm 7.3 \mathrm{E}-04$ & & & & & \\
\hline & $11 / 04 / 08$ & $11 / 18 / 08$ & gross $\beta$ & $2.2 \mathrm{E}-02 \pm 2.4 \mathrm{E}-03$ & & & & & \\
\hline & $11 / 18 / 08$ & $12 / 02 / 08$ & gross $\alpha$ & $1.5 \mathrm{E}-03 \pm 6.1 \mathrm{E}-04$ & & & & & \\
\hline & $11 / 18 / 08$ & $12 / 02 / 08$ & gross $\beta$ & $3.3 \mathrm{E}-02 \pm 3.3 \mathrm{E}-03$ & & & & & \\
\hline & $12 / 02 / 08$ & $12 / 16 / 08$ & gross $\alpha$ & $1.5 \mathrm{E}-03 \pm 6.1 \mathrm{E}-04$ & & & & & \\
\hline & $12 / 02 / 08$ & $12 / 16 / 08$ & gross $\beta$ & $2.4 \mathrm{E}-02 \pm 2.7 \mathrm{E}-03$ & & & & & \\
\hline & $12 / 16 / 08$ & $12 / 30 / 08$ & gross $\alpha$ & $2.9 \mathrm{E}-03 \pm 8.3 \mathrm{E}-04$ & & & & & \\
\hline & $12 / 16 / 08$ & $12 / 30 / 08$ & gross $\beta$ & $4.0 \mathrm{E}-02 \pm 3.8 \mathrm{E}-03$ & & & & & \\
\hline
\end{tabular}

$\overline{\mathrm{RQ}}=$ Result Qualifier. $\mathrm{U}=$ The analyte was analyzed for but not detected. 
Table 2-4. Near-Facility Air Sampling Results, 2008 (pCi/m³ \pm total analytical uncertainty). (Sheet 13 of 82)

\begin{tabular}{|c|c|c|c|c|c|c|c|c|c|}
\hline Location & Sample On & Sample Off & Isotope & Result \pm Uncertainty & Location & Composite Period & Isotope & Result \pm Uncertainty & $\mathbf{R Q *}$ \\
\hline N404 & $01 / 02 / 08$ & $01 / 15 / 08$ & gross $\alpha$ & $7.6 \mathrm{E}-04 \pm 5.6 \mathrm{E}-04$ & N404 & $01 / 02 / 08$ to $07 / 01 / 08$ & ${ }^{241} \mathrm{Am}$ & $4.3 \mathrm{E}-06 \pm 4.0 \mathrm{E}-06$ & $\bar{U}$ \\
\hline \multirow[t]{53}{*}{$(100-\mathrm{K})$} & $01 / 02 / 08$ & $01 / 15 / 08$ & gross $\beta$ & $9.5 \mathrm{E}-03 \pm 1.5 \mathrm{E}-03$ & & & ${ }^{60} \mathrm{Co}$ & $8.1 \mathrm{E}-05 \pm 8.2 \mathrm{E}-05$ & $\mathrm{U}$ \\
\hline & $01 / 15 / 08$ & 01/30/08 & gross $\alpha$ & $2.6 \mathrm{E}-03 \pm 8.0 \mathrm{E}-04$ & & & ${ }^{134} \mathrm{Cs}$ & $2.7 \mathrm{E}-05 \pm 6.8 \mathrm{E}-05$ & U \\
\hline & $01 / 15 / 08$ & 01/30/08 & gross $\beta$ & $3.4 \mathrm{E}-02 \pm 3.4 \mathrm{E}-03$ & & & ${ }^{137} \mathrm{Cs}$ & $5.7 \mathrm{E}-06 \pm 5.7 \mathrm{E}-05$ & $\mathrm{U}$ \\
\hline & $01 / 30 / 08$ & 02/13/08 & gross $\alpha$ & $7.3 \mathrm{E}-04 \pm 5.3 \mathrm{E}-04$ & & & ${ }^{152} \mathrm{Eu}$ & $-9.7 \mathrm{E}-05 \pm 1.3 \mathrm{E}-04$ & $\mathrm{U}$ \\
\hline & $01 / 30 / 08$ & $02 / 13 / 08$ & gross $\beta$ & $4.7 \mathrm{E}-03 \pm 9.5 \mathrm{E}-04$ & & & ${ }^{154} \mathrm{Eu}$ & $5.6 \mathrm{E}-05 \pm 2.1 \mathrm{E}-04$ & $\mathrm{U}$ \\
\hline & $02 / 13 / 08$ & $02 / 27 / 08$ & gross $\alpha$ & $2.1 \mathrm{E}-03 \pm 7.8 \mathrm{E}-04$ & & & ${ }^{155} \mathrm{Eu}$ & $-4.0 \mathrm{E}-05 \pm 1.4 \mathrm{E}-04$ & $\mathrm{U}$ \\
\hline & $02 / 13 / 08$ & $02 / 27 / 08$ & gross $\beta$ & $4.3 \mathrm{E}-02 \pm 4.2 \mathrm{E}-03$ & & & ${ }^{238} \mathrm{Pu}$ & $-1.7 \mathrm{E}-06 \pm 1.7 \mathrm{E}-05$ & $\mathrm{U}$ \\
\hline & $02 / 27 / 08$ & 03/11/08 & gross $\alpha$ & $1.1 \mathrm{E}-03 \pm 6.6 \mathrm{E}-04$ & & & ${ }^{239 / 240} \mathrm{Pu}$ & $1.3 \mathrm{E}-05 \pm 1.1 \mathrm{E}-05$ & \\
\hline & $02 / 27 / 08$ & 03/11/08 & gross $\beta$ & $1.5 \mathrm{E}-02 \pm 1.9 \mathrm{E}-03$ & & & ${ }^{241} \mathrm{Pu}$ & $3.7 \mathrm{E}-05 \pm 3.7 \mathrm{E}-04$ & U \\
\hline & 03/11/08 & 03/25/08 & gross $\alpha$ & $7.6 \mathrm{E}-04 \pm 5.6 \mathrm{E}-04$ & & & ${ }^{106} \mathrm{Ru}$ & $8.8 \mathrm{E}-05 \pm 5.3 \mathrm{E}-04$ & $\mathrm{U}$ \\
\hline & 03/11/08 & $03 / 25 / 08$ & gross $\beta$ & $1.2 \mathrm{E}-02 \pm 1.7 \mathrm{E}-03$ & & & ${ }^{125} \mathrm{Sb}$ & $-1.0 \mathrm{E}-04 \pm 1.4 \mathrm{E}-04$ & $\mathrm{U}$ \\
\hline & $03 / 25 / 08$ & $04 / 07 / 08$ & gross $\alpha$ & $9.2 \mathrm{E}-04 \pm 6.2 \mathrm{E}-04$ & & & ${ }^{90} \mathrm{Sr}$ & $8.9 \mathrm{E}-06 \pm 8.9 \mathrm{E}-05$ & $\mathrm{U}$ \\
\hline & 03/25/08 & $04 / 07 / 08$ & gross $\beta$ & $1.0 \mathrm{E}-02 \pm 1.6 \mathrm{E}-03$ & & & ${ }^{234} \mathrm{U}$ & $1.0 \mathrm{E}-05 \pm 8.0 \mathrm{E}-06$ & \\
\hline & $04 / 07 / 08$ & $04 / 21 / 08$ & gross $\alpha$ & $1.3 \mathrm{E}-03 \pm 5.6 \mathrm{E}-04$ & & & ${ }^{235} \mathrm{U}$ & $3.7 \mathrm{E}-06 \pm 4.2 \mathrm{E}-06$ & U \\
\hline & $04 / 07 / 08$ & $04 / 21 / 08$ & gross $\beta$ & $8.7 \mathrm{E}-03 \pm 1.4 \mathrm{E}-03$ & & & ${ }^{238} \mathrm{U}$ & $9.5 \mathrm{E}-06 \pm 6.8 \mathrm{E}-06$ & \\
\hline & $04 / 21 / 08$ & $05 / 07 / 08$ & gross $\alpha$ & $1.7 \mathrm{E}-03 \pm 5.9 \mathrm{E}-04$ & & & & & \\
\hline & $04 / 21 / 08$ & 05/07/08 & gross $\beta$ & $1.9 \mathrm{E}-02 \pm 2.2 \mathrm{E}-03$ & N404 & $07 / 01 / 08$ to $12 / 30 / 08$ & ${ }^{241} \mathrm{Am}$ & $7.2 \mathrm{E}-04 \pm 2.5 \mathrm{E}-04$ & \\
\hline & 05/07/08 & $05 / 20 / 08$ & gross $\alpha$ & $1.3 \mathrm{E}-03 \pm 6.0 \mathrm{E}-04$ & & & ${ }^{60} \mathrm{Co}$ & $5.1 \mathrm{E}-05 \pm 1.2 \mathrm{E}-04$ & U \\
\hline & 05/07/08 & $05 / 20 / 08$ & gross $\beta$ & $1.1 \mathrm{E}-02 \pm 1.7 \mathrm{E}-03$ & & & ${ }^{134} \mathrm{Cs}$ & $2.0 \mathrm{E}-05 \pm 1.3 \mathrm{E}-04$ & $\mathrm{U}$ \\
\hline & 05/20/08 & 06/03/08 & gross $\alpha$ & $1.9 \mathrm{E}-03 \pm 6.7 \mathrm{E}-04$ & & & ${ }^{137} \mathrm{Cs}$ & $1.9 \mathrm{E}-03 \pm 7.1 \mathrm{E}-04$ & \\
\hline & $05 / 20 / 08$ & $06 / 03 / 08$ & gross $\beta$ & $1.1 \mathrm{E}-02 \pm 1.5 \mathrm{E}-03$ & & & ${ }^{152} \mathrm{Eu}$ & $-9.4 \mathrm{E}-05 \pm 2.6 \mathrm{E}-04$ & $\mathrm{U}$ \\
\hline & 06/03/08 & 06/17/08 & gross $\alpha$ & $8.6 \mathrm{E}-04 \pm 5.8 \mathrm{E}-04$ & & & ${ }^{154} \mathrm{Eu}$ & $-1.5 \mathrm{E}-04 \pm 3.5 \mathrm{E}-04$ & $\mathrm{U}$ \\
\hline & 06/03/08 & $06 / 17 / 08$ & gross $\beta$ & $8.5 \mathrm{E}-03 \pm 1.4 \mathrm{E}-03$ & & & ${ }^{155} \mathrm{Eu}$ & $-2.1 \mathrm{E}-04 \pm 2.2 \mathrm{E}-04$ & $\mathrm{U}$ \\
\hline & $06 / 17 / 08$ & 07/01/08 & gross $\alpha$ & $7.0 \mathrm{E}-04 \pm 5.6 \mathrm{E}-04$ & & & ${ }^{238} \mathrm{Pu}$ & $8.4 \mathrm{E}-05 \pm 4.6 \mathrm{E}-05$ & \\
\hline & $06 / 17 / 08$ & $07 / 01 / 08$ & gross $\beta$ & $1.3 \mathrm{E}-02 \pm 1.8 \mathrm{E}-03$ & & & ${ }^{239 / 240} \mathrm{Pu}$ & $5.7 \mathrm{E}-04 \pm 2.2 \mathrm{E}-04$ & \\
\hline & $07 / 01 / 08$ & $07 / 15 / 08$ & gross $\alpha$ & $3.3 \mathrm{E}-03 \pm 1.0 \mathrm{E}-03$ & & & ${ }^{241} \mathrm{Pu}$ & $4.3 \mathrm{E}-03 \pm 1.5 \mathrm{E}-03$ & \\
\hline & 07/01/08 & 07/15/08 & gross $\beta$ & $2.3 \mathrm{E}-02 \pm 2.7 \mathrm{E}-03$ & & & ${ }^{106} \mathrm{Ru}$ & $7.3 \mathrm{E}-05 \pm 7.3 \mathrm{E}-04$ & $\mathrm{U}$ \\
\hline & 07/15/08 & 07/18/08 & gross $\alpha$ & $1.5 \mathrm{E}-02 \pm 3.9 \mathrm{E}-03$ & & & ${ }^{125} \mathrm{Sb}$ & $-9.4 \mathrm{E}-06 \pm 9.4 \mathrm{E}-05$ & U \\
\hline & $07 / 15 / 08$ & 07/18/08 & gross $\beta$ & $4.0 \mathrm{E}-02 \pm 6.1 \mathrm{E}-03$ & & & ${ }^{90} \mathrm{Sr}$ & $-2.2 \mathrm{E}-04 \pm 2.2 \mathrm{E}-04$ & $\mathrm{U}$ \\
\hline & 07/18/08 & $07 / 29 / 08$ & gross $\alpha$ & $1.7 \mathrm{E}-03 \pm 7.2 \mathrm{E}-04$ & & & ${ }^{234} \mathrm{U}$ & $1.7 \mathrm{E}-05 \pm 1.0 \mathrm{E}-05$ & \\
\hline & 07/18/08 & $07 / 29 / 08$ & gross $\beta$ & $1.2 \mathrm{E}-02 \pm 1.8 \mathrm{E}-03$ & & & ${ }^{235} \mathrm{U}$ & $1.8 \mathrm{E}-06 \pm 4.4 \mathrm{E}-06$ & $\mathrm{U}$ \\
\hline & 07/29/08 & 08/12/08 & gross $\alpha$ & $6.8 \mathrm{E}-04 \pm 5.5 \mathrm{E}-04$ & & & ${ }^{238} \mathrm{U}$ & $9.8 \mathrm{E}-06 \pm 7.1 \mathrm{E}-06$ & \\
\hline & 07/29/08 & 08/12/08 & gross $\beta$ & $7.0 \mathrm{E}-03 \pm 1.2 \mathrm{E}-03$ & & & & & \\
\hline & $08 / 12 / 08$ & 08/26/08 & gross $\alpha$ & $2.7 \mathrm{E}-03 \pm 8.3 \mathrm{E}-04$ & & & & & \\
\hline & 08/12/08 & 08/26/08 & gross $\beta$ & $1.5 \mathrm{E}-02 \pm 2.0 \mathrm{E}-03$ & & & & & \\
\hline & 08/26/08 & 09/09/08 & gross $\alpha$ & $1.7 \mathrm{E}-03 \pm 6.3 \mathrm{E}-04$ & & & & & \\
\hline & $08 / 26 / 08$ & 09/09/08 & gross $\beta$ & $1.5 \mathrm{E}-02 \pm 1.9 \mathrm{E}-03$ & & & & & \\
\hline & 09/09/08 & 09/23/08 & gross $\alpha$ & $7.7 \mathrm{E}-03 \pm 1.5 \mathrm{E}-03$ & & & & & \\
\hline & 09/09/08 & 09/23/08 & gross $\beta$ & $5.5 \mathrm{E}-02 \pm 5.0 \mathrm{E}-03$ & & & & & \\
\hline & $09 / 23 / 08$ & $10 / 09 / 08$ & gross $\alpha$ & $1.9 \mathrm{E}-03 \pm 6.2 \mathrm{E}-04$ & & & & & \\
\hline & 09/23/08 & $10 / 09 / 08$ & gross $\beta$ & $2.1 \mathrm{E}-02 \pm 2.4 \mathrm{E}-03$ & & & & & \\
\hline & $10 / 09 / 08$ & $10 / 21 / 08$ & gross $\alpha$ & $7.3 \mathrm{E}-04 \pm 5.9 \mathrm{E}-04$ & & & & & \\
\hline & $10 / 09 / 08$ & $10 / 21 / 08$ & gross $\beta$ & $2.1 \mathrm{E}-02 \pm 2.5 \mathrm{E}-03$ & & & & & \\
\hline & $10 / 21 / 08$ & $11 / 04 / 08$ & gross $\alpha$ & $2.8 \mathrm{E}-03 \pm 8.2 \mathrm{E}-04$ & & & & & \\
\hline & $10 / 21 / 08$ & $11 / 04 / 08$ & gross $\beta$ & $3.9 \mathrm{E}-02 \pm 3.8 \mathrm{E}-03$ & & & & & \\
\hline & $11 / 04 / 08$ & $11 / 18 / 08$ & gross $\alpha$ & $1.4 \mathrm{E}-03 \pm 6.0 \mathrm{E}-04$ & & & & & \\
\hline & $11 / 04 / 08$ & $11 / 18 / 08$ & gross $\beta$ & $3.0 \mathrm{E}-02 \pm 3.2 \mathrm{E}-03$ & & & & & \\
\hline & $11 / 18 / 08$ & $12 / 02 / 08$ & gross $\alpha$ & $1.6 \mathrm{E}-03 \pm 6.7 \mathrm{E}-04$ & & & & & \\
\hline & $11 / 18 / 08$ & $12 / 02 / 08$ & gross $\beta$ & 3.7E-02 \pm 3.7E-03 & & & & & \\
\hline & $12 / 02 / 08$ & $12 / 16 / 08$ & gross $\alpha$ & $1.2 \mathrm{E}-03 \pm 5.4 \mathrm{E}-04$ & & & & & \\
\hline & $12 / 02 / 08$ & $12 / 16 / 08$ & gross $\beta$ & $2.6 \mathrm{E}-02 \pm 2.9 \mathrm{E}-03$ & & & & & \\
\hline & $12 / 16 / 08$ & $12 / 30 / 08$ & gross $\alpha$ & $1.1 \mathrm{E}-03 \pm 4.9 \mathrm{E}-04$ & & & & & \\
\hline & $12 / 16 / 08$ & $12 / 30 / 08$ & gross $\beta$ & $3.1 \mathrm{E}-02 \pm 3.1 \mathrm{E}-03$ & & & & & \\
\hline
\end{tabular}

$\overline{\mathrm{RQ}}=$ Result Qualifier. $\mathrm{U}=$ The analyte was analyzed for but not detected. 
Table 2-4. Near-Facility Air Sampling Results, 2008 (pCi/m³ \pm total analytical uncertainty). (Sheet 14 of 82)

\begin{tabular}{|c|c|c|c|c|c|c|c|c|c|}
\hline Location & Sample On & Sample Off & Isotope & Result \pm Uncertainty & Location & Composite Period & Isotope & Result \pm Uncertainty & $\mathbf{R Q *}$ \\
\hline N476 & $01 / 02 / 08$ & $01 / 15 / 08$ & gross $\alpha$ & $1.2 \mathrm{E}-03 \pm 5.4 \mathrm{E}-04$ & N476 & $01 / 02 / 08$ to $07 / 01 / 08$ & ${ }^{241} \mathrm{Am}$ & $1.3 \mathrm{E}-05 \pm 9.0 \mathrm{E}-06$ & \\
\hline \multirow[t]{51}{*}{$(100-\mathrm{K})$} & $01 / 02 / 08$ & $01 / 15 / 08$ & gross $\beta$ & $9.8 \mathrm{E}-03 \pm 1.5 \mathrm{E}-03$ & & & ${ }^{60} \mathrm{Co}$ & $9.3 \mathrm{E}-05 \pm 7.1 \mathrm{E}-05$ & $\mathrm{U}$ \\
\hline & $01 / 15 / 08$ & 01/30/08 & gross $\alpha$ & $2.9 \mathrm{E}-03 \pm 8.3 \mathrm{E}-04$ & & & ${ }^{134} \mathrm{Cs}$ & $1.1 \mathrm{E}-04 \pm 7.7 \mathrm{E}-05$ & U \\
\hline & $01 / 15 / 08$ & 01/30/08 & gross $\beta$ & $3.6 \mathrm{E}-02 \pm 3.6 \mathrm{E}-03$ & & & ${ }^{137} \mathrm{Cs}$ & $1.2 \mathrm{E}-05 \pm 5.5 \mathrm{E}-05$ & $\mathrm{U}$ \\
\hline & $01 / 30 / 08$ & 02/13/08 & gross $\alpha$ & $3.9 \mathrm{E}-04 \pm 4.4 \mathrm{E}-04$ & & & ${ }^{152} \mathrm{Eu}$ & $-5.8 \mathrm{E}-05 \pm 1.3 \mathrm{E}-04$ & $\mathrm{U}$ \\
\hline & $01 / 30 / 08$ & $02 / 13 / 08$ & gross $\beta$ & $5.4 \mathrm{E}-03 \pm 1.0 \mathrm{E}-03$ & & & ${ }^{154} \mathrm{Eu}$ & $7.1 \mathrm{E}-06 \pm 7.2 \mathrm{E}-05$ & $\mathrm{U}$ \\
\hline & $02 / 13 / 08$ & $02 / 27 / 08$ & gross $\alpha$ & $1.4 \mathrm{E}-03 \pm 6.1 \mathrm{E}-04$ & & & ${ }^{155} \mathrm{Eu}$ & $8.5 \mathrm{E}-05 \pm 1.7 \mathrm{E}-04$ & $\mathrm{U}$ \\
\hline & $02 / 13 / 08$ & $02 / 27 / 08$ & gross $\beta$ & $3.3 \mathrm{E}-02 \pm 3.3 \mathrm{E}-03$ & & & ${ }^{238} \mathrm{Pu}$ & $1.2 \mathrm{E}-05 \pm 2.8 \mathrm{E}-05$ & $\mathrm{U}$ \\
\hline & $02 / 27 / 08$ & 03/11/08 & gross $\alpha$ & $9.6 \mathrm{E}-04 \pm 6.0 \mathrm{E}-04$ & & & ${ }^{239 / 240} \mathrm{Pu}$ & $3.4 \mathrm{E}-06 \pm 9.7 \mathrm{E}-06$ & $\mathrm{U}$ \\
\hline & $02 / 27 / 08$ & 03/11/08 & gross $\beta$ & $1.3 \mathrm{E}-02 \pm 1.7 \mathrm{E}-03$ & & & ${ }^{241} \mathrm{Pu}$ & $1.7 \mathrm{E}-04 \pm 7.6 \mathrm{E}-04$ & $\mathrm{U}$ \\
\hline & 03/11/08 & $03 / 25 / 08$ & gross $\alpha$ & $1.7 \mathrm{E}-04 \pm 3.5 \mathrm{E}-04$ & & & ${ }^{106} \mathrm{Ru}$ & $-2.2 \mathrm{E}-04 \pm 5.3 \mathrm{E}-04$ & $\mathrm{U}$ \\
\hline & 03/11/08 & $03 / 25 / 08$ & gross $\beta$ & $6.9 \mathrm{E}-03 \pm 1.2 \mathrm{E}-03$ & & & ${ }^{125} \mathrm{Sb}$ & $8.5 \mathrm{E}-05 \pm 1.4 \mathrm{E}-04$ & $\mathrm{U}$ \\
\hline & $03 / 25 / 08$ & $04 / 07 / 08$ & gross $\alpha$ & $9.5 \mathrm{E}-04 \pm 5.9 \mathrm{E}-04$ & & & ${ }^{90} \mathrm{Sr}$ & $-1.7 \mathrm{E}-04 \pm 1.7 \mathrm{E}-04$ & $\mathrm{U}$ \\
\hline & 03/25/08 & $04 / 07 / 08$ & gross $\beta$ & $8.0 \mathrm{E}-03 \pm 1.3 \mathrm{E}-03$ & & & ${ }^{234} \mathrm{U}$ & $1.6 \mathrm{E}-05 \pm 9.2 \mathrm{E}-06$ & \\
\hline & $04 / 07 / 08$ & $04 / 21 / 08$ & gross $\alpha$ & $7.4 \mathrm{E}-04 \pm 5.5 \mathrm{E}-04$ & & & ${ }^{235} \mathrm{U}$ & $3.3 \mathrm{E}-06 \pm 3.5 \mathrm{E}-06$ & \\
\hline & $04 / 07 / 08$ & $04 / 21 / 08$ & gross $\beta$ & $9.5 \mathrm{E}-03 \pm 1.5 \mathrm{E}-03$ & & & ${ }^{238} \mathrm{U}$ & $1.0 \mathrm{E}-05 \pm 6.8 \mathrm{E}-06$ & \\
\hline & $04 / 21 / 08$ & $05 / 07 / 08$ & gross $\alpha$ & $1.4 \mathrm{E}-03 \pm 5.3 \mathrm{E}-04$ & & & & & \\
\hline & $04 / 21 / 08$ & 05/07/08 & gross $\beta$ & $1.4 \mathrm{E}-02 \pm 1.8 \mathrm{E}-03$ & N476 & $07 / 01 / 08$ to $12 / 30 / 08$ & ${ }^{241} \mathrm{Am}$ & $2.2 \mathrm{E}-05 \pm 1.1 \mathrm{E}-05$ & \\
\hline & 05/07/08 & $05 / 20 / 08$ & gross $\alpha$ & $6.2 \mathrm{E}-04 \pm 5.7 \mathrm{E}-04$ & & & ${ }^{60} \mathrm{Co}$ & $-5.5 \mathrm{E}-06 \pm 5.5 \mathrm{E}-05$ & $\mathrm{U}$ \\
\hline & 05/07/08 & 05/20/08 & gross $\beta$ & $1.0 \mathrm{E}-02 \pm 1.6 \mathrm{E}-03$ & & & ${ }^{134} \mathrm{Cs}$ & $3.8 \mathrm{E}-05 \pm 7.0 \mathrm{E}-05$ & $\mathrm{U}$ \\
\hline & 05/20/08 & 06/03/08 & gross $\alpha$ & $6.9 \mathrm{E}-04 \pm 5.2 \mathrm{E}-04$ & & & ${ }^{137} \mathrm{Cs}$ & $-3.5 \mathrm{E}-05 \pm 5.7 \mathrm{E}-05$ & $\mathrm{U}$ \\
\hline & $05 / 20 / 08$ & $06 / 03 / 08$ & gross $\beta$ & $1.4 \mathrm{E}-02 \pm 1.9 \mathrm{E}-03$ & & & ${ }^{152} \mathrm{Eu}$ & $3.3 \mathrm{E}-05 \pm 1.6 \mathrm{E}-04$ & $\mathrm{U}$ \\
\hline & 06/03/08 & 06/17/08 & gross $\alpha$ & $1.5 \mathrm{E}-03 \pm 6.4 \mathrm{E}-04$ & & & ${ }^{154} \mathrm{Eu}$ & $-6.6 \mathrm{E}-05 \pm 2.1 \mathrm{E}-04$ & $\mathrm{U}$ \\
\hline & 06/03/08 & $06 / 17 / 08$ & gross $\beta$ & $7.5 \mathrm{E}-03 \pm 1.2 \mathrm{E}-03$ & & & ${ }^{155} \mathrm{Eu}$ & $1.5 \mathrm{E}-04 \pm 1.7 \mathrm{E}-04$ & $\mathrm{U}$ \\
\hline & $06 / 17 / 08$ & 07/01/08 & gross $\alpha$ & $1.3 \mathrm{E}-03 \pm 5.7 \mathrm{E}-04$ & & & ${ }^{238} \mathrm{Pu}$ & $6.1 \mathrm{E}-06 \pm 2.6 \mathrm{E}-05$ & $\mathrm{U}$ \\
\hline & $06 / 17 / 08$ & $07 / 01 / 08$ & gross $\beta$ & $1.7 \mathrm{E}-02 \pm 2.2 \mathrm{E}-03$ & & & ${ }^{239 / 240} \mathrm{Pu}$ & $9.1 \mathrm{E}-06 \pm 1.0 \mathrm{E}-05$ & $\mathrm{U}$ \\
\hline & $07 / 01 / 08$ & $07 / 15 / 08$ & gross $\alpha$ & $1.8 \mathrm{E}-03 \pm 7.3 \mathrm{E}-04$ & & & ${ }^{241} \mathrm{Pu}$ & $7.4 \mathrm{E}-05 \pm 7.4 \mathrm{E}-04$ & $\mathrm{U}$ \\
\hline & 07/01/08 & 07/15/08 & gross $\beta$ & $1.5 \mathrm{E}-02 \pm 1.9 \mathrm{E}-03$ & & & ${ }^{106} \mathrm{Ru}$ & $-2.6 \mathrm{E}-04 \pm 5.4 \mathrm{E}-04$ & $\mathrm{U}$ \\
\hline & 07/15/08 & 07/29/08 & gross $\alpha$ & $1.9 \mathrm{E}-03 \pm 6.7 \mathrm{E}-04$ & & & ${ }^{125} \mathrm{Sb}$ & $-5.5 \mathrm{E}-05 \pm 1.4 \mathrm{E}-04$ & $\mathrm{U}$ \\
\hline & $07 / 15 / 08$ & 07/29/08 & gross $\beta$ & $1.6 \mathrm{E}-02 \pm 2.1 \mathrm{E}-03$ & & & ${ }^{90} \mathrm{Sr}$ & $-4.1 \mathrm{E}-05 \pm 4.2 \mathrm{E}-05$ & $\mathrm{U}$ \\
\hline & $07 / 29 / 08$ & $08 / 12 / 08$ & gross $\alpha$ & $8.5 \mathrm{E}-04 \pm 5.8 \mathrm{E}-04$ & & & ${ }^{234} \mathrm{U}$ & $1.5 \mathrm{E}-05 \pm 9.1 \mathrm{E}-06$ & \\
\hline & $07 / 29 / 08$ & 08/12/08 & gross $\beta$ & $1.5 \mathrm{E}-02 \pm 2.0 \mathrm{E}-03$ & & & ${ }^{235} \mathrm{U}$ & $5.3 \mathrm{E}-06 \pm 4.8 \mathrm{E}-06$ & \\
\hline & $08 / 12 / 08$ & $08 / 26 / 08$ & gross $\alpha$ & $1.9 \mathrm{E}-03 \pm 6.6 \mathrm{E}-04$ & & & ${ }^{238} \mathrm{U}$ & $1.6 \mathrm{E}-05 \pm 9.4 \mathrm{E}-06$ & \\
\hline & 08/12/08 & 08/26/08 & gross $\beta$ & $1.4 \mathrm{E}-02 \pm 1.9 \mathrm{E}-03$ & & & & & \\
\hline & $08 / 26 / 08$ & 09/09/08 & gross $\alpha$ & $1.0 \mathrm{E}-03 \pm 5.0 \mathrm{E}-04$ & & & & & \\
\hline & $08 / 26 / 08$ & 09/09/08 & gross $\beta$ & $1.2 \mathrm{E}-02 \pm 1.7 \mathrm{E}-03$ & & & & & \\
\hline & 09/09/08 & 09/23/08 & gross $\alpha$ & $2.2 \mathrm{E}-03 \pm 7.3 \mathrm{E}-04$ & & & & & \\
\hline & 09/09/08 & 09/23/08 & gross $\beta$ & $2.9 \mathrm{E}-02 \pm 3.1 \mathrm{E}-03$ & & & & & \\
\hline & $09 / 23 / 08$ & $10 / 08 / 08$ & gross $\alpha$ & $1.6 \mathrm{E}-03 \pm 5.8 \mathrm{E}-04$ & & & & & \\
\hline & $09 / 23 / 08$ & $10 / 08 / 08$ & gross $\beta$ & $2.2 \mathrm{E}-02 \pm 2.5 \mathrm{E}-03$ & & & & & \\
\hline & $10 / 08 / 08$ & $10 / 21 / 08$ & gross $\alpha$ & $1.2 \mathrm{E}-03 \pm 5.5 \mathrm{E}-04$ & & & & & \\
\hline & $10 / 08 / 08$ & $10 / 21 / 08$ & gross $\beta$ & $1.0 \mathrm{E}-02 \pm 1.6 \mathrm{E}-03$ & & & & & \\
\hline & $10 / 21 / 08$ & $11 / 04 / 08$ & gross $\alpha$ & $2.9 \mathrm{E}-03 \pm 8.5 \mathrm{E}-04$ & & & & & \\
\hline & $10 / 21 / 08$ & $11 / 04 / 08$ & gross $\beta$ & $4.5 \mathrm{E}-02 \pm 4.3 \mathrm{E}-03$ & & & & & \\
\hline & $11 / 04 / 08$ & $11 / 18 / 08$ & gross $\alpha$ & $5.3 \mathrm{E}-04 \pm 5.1 \mathrm{E}-04$ & & & & & \\
\hline & $11 / 04 / 08$ & $11 / 18 / 08$ & gross $\beta$ & $2.0 \mathrm{E}-02 \pm 2.5 \mathrm{E}-03$ & & & & & \\
\hline & $11 / 18 / 08$ & $12 / 02 / 08$ & gross $\alpha$ & $1.4 \mathrm{E}-03 \pm 6.0 \mathrm{E}-04$ & & & & & \\
\hline & $11 / 18 / 08$ & $12 / 02 / 08$ & gross $\beta$ & $3.6 \mathrm{E}-02 \pm 3.6 \mathrm{E}-03$ & & & & & \\
\hline & $12 / 02 / 08$ & $12 / 16 / 08$ & gross $\alpha$ & $1.4 \mathrm{E}-03 \pm 5.8 \mathrm{E}-04$ & & & & & \\
\hline & $12 / 02 / 08$ & $12 / 16 / 08$ & gross $\beta$ & $2.3 \mathrm{E}-02 \pm 2.6 \mathrm{E}-03$ & & & & & \\
\hline & $12 / 16 / 08$ & $12 / 30 / 08$ & gross $\alpha$ & $1.8 \mathrm{E}-03 \pm 6.4 \mathrm{E}-04$ & & & & & \\
\hline & $12 / 16 / 08$ & $12 / 30 / 08$ & gross $\beta$ & $3.5 \mathrm{E}-02 \pm 3.4 \mathrm{E}-03$ & & & & & \\
\hline
\end{tabular}

$\overline{\mathrm{RQ}}=$ Result Qualifier. $\mathrm{U}=$ The analyte was analyzed for but not detected. 
Table 2-4. Near-Facility Air Sampling Results, 2008 (pCi/m³ \pm total analytical uncertainty). (Sheet 15 of 82)

\begin{tabular}{|c|c|c|c|c|c|c|c|c|c|}
\hline Location & Sample On & Sample Off & Isotope & Result \pm Uncertainty & Location & Composite Period & Isotope & Result \pm Uncertainty & $\mathbf{R Q *}$ \\
\hline N477 & $01 / 02 / 08$ & $01 / 15 / 08$ & gross $\alpha$ & $8.1 \mathrm{E}-04 \pm 5.9 \mathrm{E}-04$ & N477 & $01 / 02 / 08$ to $07 / 01 / 08$ & ${ }^{241} \mathrm{Am}$ & $1.1 \mathrm{E}-05 \pm 8.2 \mathrm{E}-06$ & \\
\hline \multirow[t]{51}{*}{$(100-\mathrm{K})$} & $01 / 02 / 08$ & $01 / 15 / 08$ & gross $\beta$ & $8.2 \mathrm{E}-03 \pm 1.4 \mathrm{E}-03$ & & & ${ }^{60} \mathrm{Co}$ & $4.1 \mathrm{E}-05 \pm 8.5 \mathrm{E}-05$ & $\mathrm{U}$ \\
\hline & 01/15/08 & 01/30/08 & gross $\alpha$ & $1.9 \mathrm{E}-03 \pm 6.8 \mathrm{E}-04$ & & & ${ }^{134} \mathrm{Cs}$ & $5.1 \mathrm{E}-06 \pm 5.1 \mathrm{E}-05$ & $\mathrm{U}$ \\
\hline & $01 / 15 / 08$ & 01/30/08 & gross $\beta$ & $3.4 \mathrm{E}-02 \pm 3.4 \mathrm{E}-03$ & & & ${ }^{137} \mathrm{Cs}$ & $-2.3 \mathrm{E}-05 \pm 6.5 \mathrm{E}-05$ & $\mathrm{U}$ \\
\hline & 01/30/08 & $02 / 13 / 08$ & gross $\alpha$ & $5.0 \mathrm{E}-04 \pm 4.7 \mathrm{E}-04$ & & & ${ }^{152} \mathrm{Eu}$ & $1.8 \mathrm{E}-04 \pm 1.6 \mathrm{E}-04$ & $\mathrm{U}$ \\
\hline & $01 / 30 / 08$ & $02 / 13 / 08$ & gross $\beta$ & $4.7 \mathrm{E}-03 \pm 9.7 \mathrm{E}-04$ & & & ${ }^{154} \mathrm{Eu}$ & $-1.3 \mathrm{E}-04 \pm 2.1 \mathrm{E}-04$ & $\mathrm{U}$ \\
\hline & $02 / 13 / 08$ & $02 / 27 / 08$ & gross $\alpha$ & $1.5 \mathrm{E}-03 \pm 5.6 \mathrm{E}-04$ & & & ${ }^{155} \mathrm{Eu}$ & $-8.9 \mathrm{E}-06 \pm 8.9 \mathrm{E}-05$ & $\mathrm{U}$ \\
\hline & $02 / 13 / 08$ & $02 / 27 / 08$ & gross $\beta$ & $3.9 \mathrm{E}-02 \pm 3.7 \mathrm{E}-03$ & & & ${ }^{238} \mathrm{Pu}$ & $-6.4 \mathrm{E}-06 \pm 3.6 \mathrm{E}-05$ & $\mathrm{U}$ \\
\hline & $02 / 27 / 08$ & 03/11/08 & gross $\alpha$ & $7.8 \mathrm{E}-04 \pm 5.8 \mathrm{E}-04$ & & & ${ }^{239 / 240} \mathrm{Pu}$ & $1.3 \mathrm{E}-05 \pm 1.2 \mathrm{E}-05$ & \\
\hline & $02 / 27 / 08$ & 03/11/08 & gross $\beta$ & $1.5 \mathrm{E}-02 \pm 2.0 \mathrm{E}-03$ & & & ${ }^{241} \mathrm{Pu}$ & $7.6 \mathrm{E}-04 \pm 1.0 \mathrm{E}-03$ & $\mathrm{U}$ \\
\hline & 03/11/08 & 03/25/08 & gross $\alpha$ & $1.3 \mathrm{E}-03 \pm 8.8 \mathrm{E}-04$ & & & ${ }^{106} \mathrm{Ru}$ & $-8.4 \mathrm{E}-05 \pm 6.1 \mathrm{E}-04$ & $\mathrm{U}$ \\
\hline & 03/11/08 & $03 / 25 / 08$ & gross $\beta$ & $1.4 \mathrm{E}-02 \pm 2.2 \mathrm{E}-03$ & & & ${ }^{125} \mathrm{Sb}$ & $7.2 \mathrm{E}-05 \pm 1.6 \mathrm{E}-04$ & $\mathrm{U}$ \\
\hline & 03/25/08 & $04 / 07 / 08$ & gross $\alpha$ & $1.7 \mathrm{E}-03 \pm 8.0 \mathrm{E}-04$ & & & ${ }^{90} \mathrm{Sr}$ & $-2.3 \mathrm{E}-04 \pm 2.4 \mathrm{E}-04$ & $\mathrm{U}$ \\
\hline & 03/25/08 & 04/07/08 & gross $\beta$ & $1.2 \mathrm{E}-02 \pm 2.0 \mathrm{E}-03$ & & & ${ }^{234} \mathrm{U}$ & $1.2 \mathrm{E}-05 \pm 8.3 \mathrm{E}-06$ & \\
\hline & $04 / 07 / 08$ & $04 / 21 / 08$ & gross $\alpha$ & $9.5 \mathrm{E}-04 \pm 5.9 \mathrm{E}-04$ & & & ${ }^{235} \mathrm{U}$ & $8.3 \mathrm{E}-07 \pm 2.5 \mathrm{E}-06$ & $\mathrm{U}$ \\
\hline & 04/07/08 & $04 / 21 / 08$ & gross $\beta$ & $1.0 \mathrm{E}-02 \pm 1.5 \mathrm{E}-03$ & & & ${ }^{238} \mathrm{U}$ & $6.4 \mathrm{E}-06 \pm 5.6 \mathrm{E}-06$ & \\
\hline & $04 / 21 / 08$ & 05/07/08 & gross $\alpha$ & $1.4 \mathrm{E}-03 \pm 5.5 \mathrm{E}-04$ & & & & & \\
\hline & $04 / 21 / 08$ & 05/07/08 & gross $\beta$ & $1.8 \mathrm{E}-02 \pm 2.2 \mathrm{E}-03$ & N477 & $07 / 01 / 08$ to $12 / 30 / 08$ & ${ }^{241} \mathrm{Am}$ & $3.3 \mathrm{E}-05 \pm 1.6 \mathrm{E}-05$ & \\
\hline & 05/07/08 & 05/20/08 & gross $\alpha$ & $1.5 \mathrm{E}-03 \pm 6.7 \mathrm{E}-04$ & & & ${ }^{60} \mathrm{Co}$ & $5.2 \mathrm{E}-06 \pm 5.2 \mathrm{E}-05$ & $\mathrm{U}$ \\
\hline & 05/07/08 & $05 / 20 / 08$ & gross $\beta$ & $9.8 \mathrm{E}-03 \pm 1.6 \mathrm{E}-03$ & & & ${ }^{134} \mathrm{Cs}$ & $-5.4 \mathrm{E}-05 \pm 1.3 \mathrm{E}-04$ & $\mathrm{U}$ \\
\hline & $05 / 20 / 08$ & 06/03/08 & gross $\alpha$ & $2.9 \mathrm{E}-03 \pm 8.1 \mathrm{E}-04$ & & & ${ }^{137} \mathrm{Cs}$ & $1.3 \mathrm{E}-04 \pm 1.0 \mathrm{E}-04$ & $\mathrm{U}$ \\
\hline & $05 / 20 / 08$ & $06 / 03 / 08$ & gross $\beta$ & $1.5 \mathrm{E}-02 \pm 1.9 \mathrm{E}-03$ & & & ${ }^{152} \mathrm{Eu}$ & $6.4 \mathrm{E}-05 \pm 2.4 \mathrm{E}-04$ & $\mathrm{U}$ \\
\hline & $06 / 03 / 08$ & 06/17/08 & gross $\alpha$ & $1.5 \mathrm{E}-04 \pm 4.0 \mathrm{E}-04$ & & & ${ }^{154} \mathrm{Eu}$ & $-1.4 \mathrm{E}-05 \pm 1.4 \mathrm{E}-04$ & $\mathrm{U}$ \\
\hline & 06/03/08 & 06/17/08 & gross $\beta$ & $7.0 \mathrm{E}-03 \pm 1.2 \mathrm{E}-03$ & & & ${ }^{155} \mathrm{Eu}$ & $1.9 \mathrm{E}-04 \pm 2.0 \mathrm{E}-04$ & $\mathrm{U}$ \\
\hline & $06 / 17 / 08$ & 07/01/08 & gross $\alpha$ & $2.3 \mathrm{E}-03 \pm 7.3 \mathrm{E}-04$ & & & ${ }^{238} \mathrm{Pu}$ & $-8.0 \mathrm{E}-06 \pm 2.5 \mathrm{E}-05$ & $\mathrm{U}$ \\
\hline & $06 / 17 / 08$ & 07/01/08 & gross $\beta$ & $1.4 \mathrm{E}-02 \pm 1.9 \mathrm{E}-03$ & & & ${ }^{239 / 240} \mathrm{Pu}$ & $1.6 \mathrm{E}-05 \pm 1.2 \mathrm{E}-05$ & \\
\hline & $07 / 01 / 08$ & $07 / 15 / 08$ & gross $\alpha$ & $1.7 \mathrm{E}-03 \pm 6.9 \mathrm{E}-04$ & & & ${ }^{241} \mathrm{Pu}$ & $2.8 \mathrm{E}-04 \pm 6.5 \mathrm{E}-04$ & $\mathrm{U}$ \\
\hline & 07/01/08 & 07/15/08 & gross $\beta$ & $4.5 \mathrm{E}-02 \pm 4.2 \mathrm{E}-03$ & & & ${ }^{106} \mathrm{Ru}$ & $5.4 \mathrm{E}-04 \pm 8.5 \mathrm{E}-04$ & $\mathrm{U}$ \\
\hline & 07/15/08 & 07/29/08 & gross $\alpha$ & $1.5 \mathrm{E}-03 \pm 5.8 \mathrm{E}-04$ & & & ${ }^{125} \mathrm{Sb}$ & $-3.9 \mathrm{E}-05 \pm 2.4 \mathrm{E}-04$ & $\mathrm{U}$ \\
\hline & $07 / 15 / 08$ & 07/29/08 & gross $\beta$ & $1.3 \mathrm{E}-02 \pm 1.8 \mathrm{E}-03$ & & & ${ }^{90} \mathrm{Sr}$ & $-7.8 \mathrm{E}-05 \pm 8.1 \mathrm{E}-05$ & $\mathrm{U}$ \\
\hline & $07 / 29 / 08$ & 08/12/08 & gross $\alpha$ & $1.6 \mathrm{E}-03 \pm 6.0 \mathrm{E}-04$ & & & ${ }^{234} \mathrm{U}$ & $1.8 \mathrm{E}-05 \pm 1.0 \mathrm{E}-05$ & \\
\hline & $07 / 29 / 08$ & 08/12/08 & gross $\beta$ & $1.6 \mathrm{E}-02 \pm 2.0 \mathrm{E}-03$ & & & ${ }^{235} \mathrm{U}$ & $4.6 \mathrm{E}-06 \pm 4.2 \mathrm{E}-06$ & \\
\hline & $08 / 12 / 08$ & $08 / 26 / 08$ & gross $\alpha$ & $1.3 \mathrm{E}-03 \pm 5.4 \mathrm{E}-04$ & & & ${ }^{238} \mathrm{U}$ & $8.5 \mathrm{E}-06 \pm 5.9 \mathrm{E}-06$ & \\
\hline & 08/12/08 & 08/26/08 & gross $\beta$ & $1.3 \mathrm{E}-02 \pm 1.8 \mathrm{E}-03$ & & & & & \\
\hline & $08 / 26 / 08$ & 09/09/08 & gross $\alpha$ & $1.5 \mathrm{E}-03 \pm 5.8 \mathrm{E}-04$ & & & & & \\
\hline & 08/26/08 & 09/09/08 & gross $\beta$ & $1.3 \mathrm{E}-02 \pm 1.8 \mathrm{E}-03$ & & & & & \\
\hline & 09/09/08 & 09/23/08 & gross $\alpha$ & $1.6 \mathrm{E}-03 \pm 6.0 \mathrm{E}-04$ & & & & & \\
\hline & 09/09/08 & 09/23/08 & gross $\beta$ & $2.6 \mathrm{E}-02 \pm 2.8 \mathrm{E}-03$ & & & & & \\
\hline & 09/23/08 & $10 / 09 / 08$ & gross $\alpha$ & $8.7 \mathrm{E}-04 \pm 4.1 \mathrm{E}-04$ & & & & & \\
\hline & $09 / 23 / 08$ & $10 / 09 / 08$ & gross $\beta$ & $2.3 \mathrm{E}-02 \pm 2.5 \mathrm{E}-03$ & & & & & \\
\hline & $10 / 09 / 08$ & $10 / 21 / 08$ & gross $\alpha$ & $1.4 \mathrm{E}-03 \pm 6.0 \mathrm{E}-04$ & & & & & \\
\hline & $10 / 09 / 08$ & $10 / 21 / 08$ & gross $\beta$ & $1.9 \mathrm{E}-02 \pm 2.4 \mathrm{E}-03$ & & & & & \\
\hline & $10 / 21 / 08$ & $11 / 04 / 08$ & gross $\alpha$ & $3.5 \mathrm{E}-03 \pm 9.1 \mathrm{E}-04$ & & & & & \\
\hline & $10 / 21 / 08$ & $11 / 04 / 08$ & gross $\beta$ & $4.4 \mathrm{E}-02 \pm 4.2 \mathrm{E}-03$ & & & & & \\
\hline & $11 / 04 / 08$ & $11 / 18 / 08$ & gross $\alpha$ & $9.6 \mathrm{E}-04 \pm 6.1 \mathrm{E}-04$ & & & & & \\
\hline & $11 / 04 / 08$ & $11 / 18 / 08$ & gross $\beta$ & $2.6 \mathrm{E}-02 \pm 2.8 \mathrm{E}-03$ & & & & & \\
\hline & $11 / 18 / 08$ & $12 / 02 / 08$ & gross $\alpha$ & $1.4 \mathrm{E}-03 \pm 5.8 \mathrm{E}-04$ & & & & & \\
\hline & $11 / 18 / 08$ & $12 / 02 / 08$ & gross $\beta$ & $3.9 \mathrm{E}-02 \pm 3.8 \mathrm{E}-03$ & & & & & \\
\hline & $12 / 02 / 08$ & $12 / 16 / 08$ & gross $\alpha$ & $1.6 \mathrm{E}-03 \pm 6.1 \mathrm{E}-04$ & & & & & \\
\hline & $12 / 02 / 08$ & $12 / 16 / 08$ & gross $\beta$ & $2.3 \mathrm{E}-02 \pm 2.5 \mathrm{E}-03$ & & & & & \\
\hline & $12 / 16 / 08$ & $12 / 30 / 08$ & gross $\alpha$ & $1.6 \mathrm{E}-03 \pm 5.9 \mathrm{E}-04$ & & & & & \\
\hline & $12 / 16 / 08$ & $12 / 30 / 08$ & gross $\beta$ & $4.4 \mathrm{E}-02 \pm 4.0 \mathrm{E}-03$ & & & & & \\
\hline
\end{tabular}

$\overline{\mathrm{RQ}}=$ Result Qualifier. $\mathrm{U}=$ The analyte was analyzed for but not detected. 
Table 2-4. Near-Facility Air Sampling Results, 2008 (pCi/m³ \pm total analytical uncertainty). (Sheet 16 of 82)

\begin{tabular}{|c|c|c|c|c|c|c|c|c|c|}
\hline Location & Sample On & Sample Off & Isotope & Result \pm Uncertainty & Location & Composite Period & Isotope & Result \pm Uncertainty & $\mathbf{R Q *}$ \\
\hline N478 & $01 / 02 / 08$ & $01 / 15 / 08$ & gross $\alpha$ & $1.4 \mathrm{E}-03 \pm 5.9 \mathrm{E}-04$ & N478 & $01 / 02 / 08$ to $07 / 01 / 08$ & ${ }^{241} \mathrm{Am}$ & $8.3 \mathrm{E}-06 \pm 5.7 \mathrm{E}-06$ & \\
\hline \multirow[t]{51}{*}{$(100-\mathrm{K})$} & $01 / 02 / 08$ & $01 / 15 / 08$ & gross $\beta$ & $9.1 \mathrm{E}-03 \pm 1.4 \mathrm{E}-03$ & & & ${ }^{60} \mathrm{Co}$ & $-1.2 \mathrm{E}-05 \pm 9.7 \mathrm{E}-05$ & $\mathrm{U}$ \\
\hline & 01/15/08 & 01/30/08 & gross $\alpha$ & $2.7 \mathrm{E}-03 \pm 7.6 \mathrm{E}-04$ & & & ${ }^{134} \mathrm{Cs}$ & $1.3 \mathrm{E}-04 \pm 1.2 \mathrm{E}-04$ & $\mathrm{U}$ \\
\hline & $01 / 15 / 08$ & 01/30/08 & gross $\beta$ & $3.8 \mathrm{E}-02 \pm 3.6 \mathrm{E}-03$ & & & ${ }^{137} \mathrm{Cs}$ & $1.4 \mathrm{E}-05 \pm 1.0 \mathrm{E}-04$ & $\mathrm{U}$ \\
\hline & 01/30/08 & $02 / 13 / 08$ & gross $\alpha$ & $7.1 \mathrm{E}-04 \pm 5.2 \mathrm{E}-04$ & & & ${ }^{152} \mathrm{Eu}$ & $-1.1 \mathrm{E}-04 \pm 2.5 \mathrm{E}-04$ & $\mathrm{U}$ \\
\hline & $01 / 30 / 08$ & $02 / 13 / 08$ & gross $\beta$ & $4.3 \mathrm{E}-03 \pm 9.1 \mathrm{E}-04$ & & & ${ }^{154} \mathrm{Eu}$ & $-2.5 \mathrm{E}-06 \pm 2.5 \mathrm{E}-05$ & $\mathrm{U}$ \\
\hline & $02 / 13 / 08$ & $02 / 27 / 08$ & gross $\alpha$ & $8.6 \mathrm{E}-04 \pm 5.8 \mathrm{E}-04$ & & & ${ }^{155} \mathrm{Eu}$ & $3.9 \mathrm{E}-05 \pm 1.9 \mathrm{E}-04$ & $\mathrm{U}$ \\
\hline & $02 / 13 / 08$ & $02 / 27 / 08$ & gross $\beta$ & $3.2 \mathrm{E}-02 \pm 3.3 \mathrm{E}-03$ & & & ${ }^{238} \mathrm{Pu}$ & $8.4 \mathrm{E}-06 \pm 3.9 \mathrm{E}-05$ & $\mathrm{U}$ \\
\hline & $02 / 27 / 08$ & 03/11/08 & gross $\alpha$ & $1.4 \mathrm{E}-03 \pm 5.8 \mathrm{E}-04$ & & & ${ }^{239 / 240} \mathrm{Pu}$ & $2.3 \mathrm{E}-05 \pm 1.8 \mathrm{E}-05$ & \\
\hline & $02 / 27 / 08$ & 03/11/08 & gross $\beta$ & $1.3 \mathrm{E}-02 \pm 1.8 \mathrm{E}-03$ & & & ${ }^{241} \mathrm{Pu}$ & $3.0 \mathrm{E}-04 \pm 9.2 \mathrm{E}-04$ & $\mathrm{U}$ \\
\hline & 03/11/08 & 03/26/08 & gross $\alpha$ & $3.8 \mathrm{E}-04 \pm 4.2 \mathrm{E}-04$ & & & ${ }^{106} \mathrm{Ru}$ & $6.6 \mathrm{E}-04 \pm 1.1 \mathrm{E}-03$ & $\mathrm{U}$ \\
\hline & 03/11/08 & 03/26/08 & gross $\beta$ & $7.5 \mathrm{E}-03 \pm 1.2 \mathrm{E}-03$ & & & ${ }^{125} \mathrm{Sb}$ & $1.4 \mathrm{E}-04 \pm 2.5 \mathrm{E}-04$ & $\mathrm{U}$ \\
\hline & 03/26/08 & $04 / 08 / 08$ & gross $\alpha$ & $9.0 \mathrm{E}-04 \pm 6.1 \mathrm{E}-04$ & & & ${ }^{90} \mathrm{Sr}$ & $-1.7 \mathrm{E}-04 \pm 1.8 \mathrm{E}-04$ & $\mathrm{U}$ \\
\hline & 03/26/08 & $04 / 08 / 08$ & gross $\beta$ & $7.0 \mathrm{E}-03 \pm 1.2 \mathrm{E}-03$ & & & ${ }^{234} \mathrm{U}$ & $1.3 \mathrm{E}-05 \pm 7.7 \mathrm{E}-06$ & \\
\hline & $04 / 08 / 08$ & $04 / 21 / 08$ & gross $\alpha$ & $1.7 \mathrm{E}-03 \pm 6.5 \mathrm{E}-04$ & & & ${ }^{235} \mathrm{U}$ & $2.3 \mathrm{E}-06 \pm 2.8 \mathrm{E}-06$ & \\
\hline & $04 / 08 / 08$ & $04 / 21 / 08$ & gross $\beta$ & $9.3 \mathrm{E}-03 \pm 1.5 \mathrm{E}-03$ & & & ${ }^{238} \mathrm{U}$ & $7.7 \mathrm{E}-06 \pm 5.8 \mathrm{E}-06$ & \\
\hline & $04 / 21 / 08$ & 05/07/08 & gross $\alpha$ & $1.1 \mathrm{E}-03 \pm 4.7 \mathrm{E}-04$ & & & & & \\
\hline & $04 / 21 / 08$ & 05/07/08 & gross $\beta$ & $1.5 \mathrm{E}-02 \pm 1.9 \mathrm{E}-03$ & N478 & $07 / 01 / 08$ to $12 / 30 / 08$ & ${ }^{241} \mathrm{Am}$ & $3.1 \mathrm{E}-05 \pm 1.5 \mathrm{E}-05$ & \\
\hline & 05/07/08 & $05 / 20 / 08$ & gross $\alpha$ & $2.1 \mathrm{E}-03 \pm 7.3 \mathrm{E}-04$ & & & ${ }^{60} \mathrm{Co}$ & $-3.8 \mathrm{E}-05 \pm 8.8 \mathrm{E}-05$ & $\mathrm{U}$ \\
\hline & 05/07/08 & $05 / 20 / 08$ & gross $\beta$ & $1.1 \mathrm{E}-02 \pm 1.6 \mathrm{E}-03$ & & & ${ }^{134} \mathrm{Cs}$ & $-2.4 \mathrm{E}-05 \pm 7.2 \mathrm{E}-05$ & $\mathrm{U}$ \\
\hline & $05 / 20 / 08$ & 06/03/08 & gross $\alpha$ & $1.4 \mathrm{E}-03 \pm 5.5 \mathrm{E}-04$ & & & ${ }^{137} \mathrm{Cs}$ & $3.6 \mathrm{E}-05 \pm 6.4 \mathrm{E}-05$ & $\mathrm{U}$ \\
\hline & $05 / 20 / 08$ & $06 / 03 / 08$ & gross $\beta$ & $1.2 \mathrm{E}-02 \pm 1.7 \mathrm{E}-03$ & & & ${ }^{152} \mathrm{Eu}$ & $3.1 \mathrm{E}-05 \pm 1.8 \mathrm{E}-04$ & $\mathrm{U}$ \\
\hline & $06 / 03 / 08$ & 06/17/08 & gross $\alpha$ & $9.2 \mathrm{E}-04 \pm 6.4 \mathrm{E}-04$ & & & ${ }^{154} \mathrm{Eu}$ & $2.2 \mathrm{E}-04 \pm 2.4 \mathrm{E}-04$ & $\mathrm{U}$ \\
\hline & 06/03/08 & 06/17/08 & gross $\beta$ & $1.1 \mathrm{E}-02 \pm 1.5 \mathrm{E}-03$ & & & ${ }^{155} \mathrm{Eu}$ & $2.6 \mathrm{E}-04 \pm 1.9 \mathrm{E}-04$ & $\mathrm{U}$ \\
\hline & $06 / 17 / 08$ & 07/01/08 & gross $\alpha$ & $1.6 \mathrm{E}-03 \pm 6.1 \mathrm{E}-04$ & & & ${ }^{238} \mathrm{Pu}$ & $-1.8 \mathrm{E}-05 \pm 2.5 \mathrm{E}-05$ & $\mathrm{U}$ \\
\hline & $06 / 17 / 08$ & 07/01/08 & gross $\beta$ & $1.4 \mathrm{E}-02 \pm 1.9 \mathrm{E}-03$ & & & ${ }^{239 / 240} \mathrm{Pu}$ & $1.2 \mathrm{E}-05 \pm 1.2 \mathrm{E}-05$ & $\mathrm{U}$ \\
\hline & $07 / 01 / 08$ & $07 / 15 / 08$ & gross $\alpha$ & $1.3 \mathrm{E}-03 \pm 6.1 \mathrm{E}-04$ & & & ${ }^{241} \mathrm{Pu}$ & $7.2 \mathrm{E}-06 \pm 7.2 \mathrm{E}-05$ & $\mathrm{U}$ \\
\hline & 07/01/08 & 07/15/08 & gross $\beta$ & $1.5 \mathrm{E}-02 \pm 1.9 \mathrm{E}-03$ & & & ${ }^{106} \mathrm{Ru}$ & $-2.0 \mathrm{E}-04 \pm 5.8 \mathrm{E}-04$ & $\mathrm{U}$ \\
\hline & 07/15/08 & 07/29/08 & gross $\alpha$ & $1.2 \mathrm{E}-03 \pm 5.2 \mathrm{E}-04$ & & & ${ }^{125} \mathrm{Sb}$ & $3.4 \mathrm{E}-05 \pm 1.5 \mathrm{E}-04$ & $\mathrm{U}$ \\
\hline & $07 / 15 / 08$ & 07/29/08 & gross $\beta$ & $1.5 \mathrm{E}-02 \pm 2.0 \mathrm{E}-03$ & & & ${ }^{90} \mathrm{Sr}$ & $-2.2 \mathrm{E}-04 \pm 2.2 \mathrm{E}-04$ & $\mathrm{U}$ \\
\hline & $07 / 29 / 08$ & 08/12/08 & gross $\alpha$ & $8.1 \mathrm{E}-04 \pm 5.5 \mathrm{E}-04$ & & & ${ }^{234} \mathrm{U}$ & $1.6 \mathrm{E}-05 \pm 9.1 \mathrm{E}-06$ & \\
\hline & $07 / 29 / 08$ & 08/12/08 & gross $\beta$ & $1.6 \mathrm{E}-02 \pm 2.1 \mathrm{E}-03$ & & & ${ }^{235} \mathrm{U}$ & $2.5 \mathrm{E}-06 \pm 3.0 \mathrm{E}-06$ & \\
\hline & $08 / 12 / 08$ & $08 / 26 / 08$ & gross $\alpha$ & $1.0 \mathrm{E}-03 \pm 4.8 \mathrm{E}-04$ & & & ${ }^{238} \mathrm{U}$ & $1.2 \mathrm{E}-05 \pm 7.4 \mathrm{E}-06$ & \\
\hline & 08/12/08 & 08/26/08 & gross $\beta$ & $1.5 \mathrm{E}-02 \pm 1.9 \mathrm{E}-03$ & & & & & \\
\hline & $08 / 26 / 08$ & 09/09/08 & gross $\alpha$ & $8.2 \mathrm{E}-04 \pm 5.6 \mathrm{E}-04$ & & & & & \\
\hline & 08/26/08 & 09/09/08 & gross $\beta$ & $1.3 \mathrm{E}-02 \pm 1.8 \mathrm{E}-03$ & & & & & \\
\hline & 09/09/08 & 09/23/08 & gross $\alpha$ & $1.8 \mathrm{E}-03 \pm 6.4 \mathrm{E}-04$ & & & & & \\
\hline & 09/09/08 & 09/23/08 & gross $\beta$ & $2.5 \mathrm{E}-02 \pm 2.8 \mathrm{E}-03$ & & & & & \\
\hline & 09/23/08 & $10 / 09 / 08$ & gross $\alpha$ & $1.2 \mathrm{E}-03 \pm 5.0 \mathrm{E}-04$ & & & & & \\
\hline & $09 / 23 / 08$ & $10 / 09 / 08$ & gross $\beta$ & $2.2 \mathrm{E}-02 \pm 2.5 \mathrm{E}-03$ & & & & & \\
\hline & $10 / 09 / 08$ & $10 / 21 / 08$ & gross $\alpha$ & $1.3 \mathrm{E}-03 \pm 5.8 \mathrm{E}-04$ & & & & & \\
\hline & $10 / 09 / 08$ & $10 / 21 / 08$ & gross $\beta$ & $2.0 \mathrm{E}-02 \pm 2.4 \mathrm{E}-03$ & & & & & \\
\hline & $10 / 21 / 08$ & $11 / 04 / 08$ & gross $\alpha$ & $4.1 \mathrm{E}-03 \pm 1.0 \mathrm{E}-03$ & & & & & \\
\hline & $10 / 21 / 08$ & $11 / 04 / 08$ & gross $\beta$ & $4.8 \mathrm{E}-02 \pm 4.5 \mathrm{E}-03$ & & & & & \\
\hline & $11 / 04 / 08$ & $11 / 18 / 08$ & gross $\alpha$ & $1.1 \mathrm{E}-03 \pm 5.3 \mathrm{E}-04$ & & & & & \\
\hline & $11 / 04 / 08$ & $11 / 18 / 08$ & gross $\beta$ & $2.6 \mathrm{E}-02 \pm 2.9 \mathrm{E}-03$ & & & & & \\
\hline & $11 / 18 / 08$ & $12 / 02 / 08$ & gross $\alpha$ & $2.0 \mathrm{E}-03 \pm 7.1 \mathrm{E}-04$ & & & & & \\
\hline & $11 / 18 / 08$ & $12 / 02 / 08$ & gross $\beta$ & $3.7 \mathrm{E}-02 \pm 3.6 \mathrm{E}-03$ & & & & & \\
\hline & $12 / 02 / 08$ & $12 / 16 / 08$ & gross $\alpha$ & $1.7 \mathrm{E}-03 \pm 6.3 \mathrm{E}-04$ & & & & & \\
\hline & $12 / 02 / 08$ & $12 / 16 / 08$ & gross $\beta$ & $2.4 \mathrm{E}-02 \pm 2.6 \mathrm{E}-03$ & & & & & \\
\hline & $12 / 16 / 08$ & $12 / 30 / 08$ & gross $\alpha$ & $1.2 \mathrm{E}-03 \pm 5.2 \mathrm{E}-04$ & & & & & \\
\hline & $12 / 16 / 08$ & $12 / 30 / 08$ & gross $\beta$ & $4.3 \mathrm{E}-02 \pm 4.0 \mathrm{E}-03$ & & & & & \\
\hline
\end{tabular}

$\overline{\mathrm{RQ}}=$ Result Qualifier. $\mathrm{U}=$ The analyte was analyzed for but not detected. 
Table 2-4. Near-Facility Air Sampling Results, 2008 (pCi/m³ \pm total analytical uncertainty). (Sheet 17 of 82)

\begin{tabular}{|c|c|c|c|c|c|c|c|c|c|}
\hline Location & Sample On & Sample Off & Isotope & Result \pm Uncertainty & Location & Composite Period & Isotope & Result \pm Uncertainty & $\mathbf{R Q *}$ \\
\hline N479 & $01 / 02 / 08$ & $01 / 15 / 08$ & gross $\alpha$ & $1.0 \mathrm{E}-03 \pm 6.4 \mathrm{E}-04$ & N479 & $01 / 02 / 08$ to $07 / 01 / 08$ & ${ }^{241} \mathrm{Am}$ & $8.5 \mathrm{E}-06 \pm 5.6 \mathrm{E}-06$ & \\
\hline \multirow[t]{51}{*}{$(100-\mathrm{K})$} & $01 / 02 / 08$ & $01 / 15 / 08$ & gross $\beta$ & $1.1 \mathrm{E}-02 \pm 1.6 \mathrm{E}-03$ & & & ${ }^{60} \mathrm{Co}$ & $1.0 \mathrm{E}-05 \pm 8.4 \mathrm{E}-05$ & $\mathrm{U}$ \\
\hline & 01/15/08 & 01/30/08 & gross $\alpha$ & $1.5 \mathrm{E}-03 \pm 5.7 \mathrm{E}-04$ & & & ${ }^{134} \mathrm{Cs}$ & $-4.5 \mathrm{E}-05 \pm 7.9 \mathrm{E}-05$ & $\mathrm{U}$ \\
\hline & $01 / 15 / 08$ & 01/30/08 & gross $\beta$ & $3.8 \mathrm{E}-02 \pm 3.6 \mathrm{E}-03$ & & & ${ }^{137} \mathrm{Cs}$ & $-3.8 \mathrm{E}-05 \pm 7.4 \mathrm{E}-05$ & $\mathrm{U}$ \\
\hline & 01/30/08 & $02 / 13 / 08$ & gross $\alpha$ & $1.8 \mathrm{E}-04 \pm 3.9 \mathrm{E}-04$ & & & ${ }^{152} \mathrm{Eu}$ & $4.2 \mathrm{E}-05 \pm 1.6 \mathrm{E}-04$ & $\mathrm{U}$ \\
\hline & $01 / 30 / 08$ & $02 / 13 / 08$ & gross $\beta$ & $6.7 \mathrm{E}-03 \pm 1.2 \mathrm{E}-03$ & & & ${ }^{154} \mathrm{Eu}$ & $1.5 \mathrm{E}-04 \pm 2.6 \mathrm{E}-04$ & $\mathrm{U}$ \\
\hline & $02 / 13 / 08$ & $02 / 27 / 08$ & gross $\alpha$ & $2.1 \mathrm{E}-03 \pm 7.8 \mathrm{E}-04$ & & & ${ }^{155} \mathrm{Eu}$ & $-2.1 \mathrm{E}-04 \pm 2.2 \mathrm{E}-04$ & $\mathrm{U}$ \\
\hline & $02 / 13 / 08$ & $02 / 27 / 08$ & gross $\beta$ & $4.3 \mathrm{E}-02 \pm 4.2 \mathrm{E}-03$ & & & ${ }^{238} \mathrm{Pu}$ & $-1.3 \mathrm{E}-05 \pm 3.3 \mathrm{E}-05$ & $\mathrm{U}$ \\
\hline & $02 / 27 / 08$ & 03/11/08 & gross $\alpha$ & $1.4 \mathrm{E}-03 \pm 5.8 \mathrm{E}-04$ & & & ${ }^{239 / 240} \mathrm{Pu}$ & $5.7 \mathrm{E}-06 \pm 1.0 \mathrm{E}-05$ & $\mathrm{U}$ \\
\hline & $02 / 27 / 08$ & 03/11/08 & gross $\beta$ & $1.3 \mathrm{E}-02 \pm 1.8 \mathrm{E}-03$ & & & ${ }^{241} \mathrm{Pu}$ & $1.3 \mathrm{E}-04 \pm 4.3 \mathrm{E}-04$ & $\mathrm{U}$ \\
\hline & 03/11/08 & 03/26/08 & gross $\alpha$ & $8.2 \mathrm{E}-04 \pm 5.5 \mathrm{E}-04$ & & & ${ }^{106} \mathrm{Ru}$ & $4.4 \mathrm{E}-04 \pm 6.4 \mathrm{E}-04$ & $\mathrm{U}$ \\
\hline & 03/11/08 & 03/26/08 & gross $\beta$ & $9.7 \mathrm{E}-03 \pm 1.4 \mathrm{E}-03$ & & & ${ }^{125} \mathrm{Sb}$ & $1.9 \mathrm{E}-05 \pm 1.5 \mathrm{E}-04$ & $\mathrm{U}$ \\
\hline & 03/26/08 & $04 / 08 / 08$ & gross $\alpha$ & $1.0 \mathrm{E}-03 \pm 6.2 \mathrm{E}-04$ & & & ${ }^{90} \mathrm{Sr}$ & $-3.8 \mathrm{E}-05 \pm 3.9 \mathrm{E}-05$ & $\mathrm{U}$ \\
\hline & 03/26/08 & $04 / 08 / 08$ & gross $\beta$ & $8.5 \mathrm{E}-03 \pm 1.4 \mathrm{E}-03$ & & & ${ }^{234} \mathrm{U}$ & $1.4 \mathrm{E}-05 \pm 8.5 \mathrm{E}-06$ & \\
\hline & $04 / 08 / 08$ & $04 / 21 / 08$ & gross $\alpha$ & $8.8 \mathrm{E}-04 \pm 6.0 \mathrm{E}-04$ & & & ${ }^{235} \mathrm{U}$ & $2.3 \mathrm{E}-06 \pm 2.7 \mathrm{E}-06$ & \\
\hline & $04 / 08 / 08$ & $04 / 21 / 08$ & gross $\beta$ & $1.2 \mathrm{E}-02 \pm 1.7 \mathrm{E}-03$ & & & ${ }^{238} \mathrm{U}$ & $8.3 \mathrm{E}-06 \pm 5.7 \mathrm{E}-06$ & \\
\hline & $04 / 21 / 08$ & 05/07/08 & gross $\alpha$ & $1.3 \mathrm{E}-03 \pm 5.0 \mathrm{E}-04$ & & & & & \\
\hline & $04 / 21 / 08$ & 05/07/08 & gross $\beta$ & $1.7 \mathrm{E}-02 \pm 2.0 \mathrm{E}-03$ & N479 & $07 / 01 / 08$ to $12 / 30 / 08$ & ${ }^{241} \mathrm{Am}$ & $3.9 \mathrm{E}-05 \pm 1.7 \mathrm{E}-05$ & \\
\hline & 05/07/08 & 05/20/08 & gross $\alpha$ & $9.3 \mathrm{E}-04 \pm 6.1 \mathrm{E}-04$ & & & ${ }^{60} \mathrm{Co}$ & $1.8 \mathrm{E}-05 \pm 9.0 \mathrm{E}-05$ & $\mathrm{U}$ \\
\hline & 05/07/08 & $05 / 20 / 08$ & gross $\beta$ & $1.1 \mathrm{E}-02 \pm 1.6 \mathrm{E}-03$ & & & ${ }^{134} \mathrm{Cs}$ & $-4.0 \mathrm{E}-06 \pm 4.0 \mathrm{E}-05$ & $\mathrm{U}$ \\
\hline & $05 / 20 / 08$ & 06/03/08 & gross $\alpha$ & $1.9 \mathrm{E}-03 \pm 7.1 \mathrm{E}-04$ & & & ${ }^{137} \mathrm{Cs}$ & $1.6 \mathrm{E}-05 \pm 7.1 \mathrm{E}-05$ & $\mathrm{U}$ \\
\hline & $05 / 20 / 08$ & $06 / 03 / 08$ & gross $\beta$ & $1.7 \mathrm{E}-02 \pm 2.2 \mathrm{E}-03$ & & & ${ }^{152} \mathrm{Eu}$ & $1.5 \mathrm{E}-05 \pm 1.5 \mathrm{E}-04$ & $\mathrm{U}$ \\
\hline & $06 / 03 / 08$ & 06/17/08 & gross $\alpha$ & $1.5 \mathrm{E}-03 \pm 6.2 \mathrm{E}-04$ & & & ${ }^{154} \mathrm{Eu}$ & $2.1 \mathrm{E}-04 \pm 2.5 \mathrm{E}-04$ & $\mathrm{U}$ \\
\hline & 06/03/08 & 06/17/08 & gross $\beta$ & $9.6 \mathrm{E}-03 \pm 1.4 \mathrm{E}-03$ & & & ${ }^{155} \mathrm{Eu}$ & $7.2 \mathrm{E}-05 \pm 1.7 \mathrm{E}-04$ & $\mathrm{U}$ \\
\hline & $06 / 17 / 08$ & 07/01/08 & gross $\alpha$ & $9.4 \mathrm{E}-04 \pm 5.9 \mathrm{E}-04$ & & & ${ }^{238} \mathrm{Pu}$ & $1.2 \mathrm{E}-05 \pm 2.0 \mathrm{E}-05$ & $\mathrm{U}$ \\
\hline & $06 / 17 / 08$ & 07/01/08 & gross $\beta$ & $1.6 \mathrm{E}-02 \pm 2.1 \mathrm{E}-03$ & & & ${ }^{239 / 240} \mathrm{Pu}$ & $2.6 \mathrm{E}-05 \pm 1.6 \mathrm{E}-05$ & \\
\hline & $07 / 01 / 08$ & $07 / 15 / 08$ & gross $\alpha$ & $2.9 \mathrm{E}-03 \pm 1.1 \mathrm{E}-03$ & & & ${ }^{241} \mathrm{Pu}$ & $5.1 \mathrm{E}-04 \pm 7.3 \mathrm{E}-04$ & $\mathrm{U}$ \\
\hline & 07/01/08 & 07/15/08 & gross $\beta$ & $2.5 \mathrm{E}-02 \pm 3.0 \mathrm{E}-03$ & & & ${ }^{106} \mathrm{Ru}$ & $-6.1 \mathrm{E}-04 \pm 6.7 \mathrm{E}-04$ & $\mathrm{U}$ \\
\hline & 07/15/08 & 07/29/08 & gross $\alpha$ & $1.2 \mathrm{E}-03 \pm 5.2 \mathrm{E}-04$ & & & ${ }^{125} \mathrm{Sb}$ & $-4.7 \mathrm{E}-05 \pm 1.5 \mathrm{E}-04$ & $\mathrm{U}$ \\
\hline & $07 / 15 / 08$ & 07/29/08 & gross $\beta$ & $1.4 \mathrm{E}-02 \pm 1.9 \mathrm{E}-03$ & & & ${ }^{90} \mathrm{Sr}$ & $-1.5 \mathrm{E}-04 \pm 1.5 \mathrm{E}-04$ & $\mathrm{U}$ \\
\hline & $07 / 29 / 08$ & 08/12/08 & gross $\alpha$ & $6.8 \mathrm{E}-04 \pm 5.1 \mathrm{E}-04$ & & & ${ }^{234} \mathrm{U}$ & $8.3 \mathrm{E}-06 \pm 6.3 \mathrm{E}-06$ & \\
\hline & $07 / 29 / 08$ & 08/12/08 & gross $\beta$ & $1.5 \mathrm{E}-02 \pm 2.0 \mathrm{E}-03$ & & & ${ }^{235} \mathrm{U}$ & $3.3 \mathrm{E}-06 \pm 3.5 \mathrm{E}-06$ & \\
\hline & $08 / 12 / 08$ & $08 / 26 / 08$ & gross $\alpha$ & $1.1 \mathrm{E}-03 \pm 5.0 \mathrm{E}-04$ & & & ${ }^{238} \mathrm{U}$ & $7.5 \mathrm{E}-06 \pm 6.0 \mathrm{E}-06$ & \\
\hline & 08/12/08 & 08/26/08 & gross $\beta$ & $1.6 \mathrm{E}-02 \pm 2.1 \mathrm{E}-03$ & & & & & \\
\hline & $08 / 26 / 08$ & 09/09/08 & gross $\alpha$ & $3.7 \mathrm{E}-04 \pm 4.3 \mathrm{E}-04$ & & & & & \\
\hline & 08/26/08 & 09/09/08 & gross $\beta$ & $1.3 \mathrm{E}-02 \pm 1.8 \mathrm{E}-03$ & & & & & \\
\hline & 09/09/08 & 09/23/08 & gross $\alpha$ & $2.3 \mathrm{E}-03 \pm 7.1 \mathrm{E}-04$ & & & & & \\
\hline & 09/09/08 & 09/23/08 & gross $\beta$ & $2.5 \mathrm{E}-02 \pm 2.7 \mathrm{E}-03$ & & & & & \\
\hline & 09/23/08 & $10 / 09 / 08$ & gross $\alpha$ & $2.0 \mathrm{E}-03 \pm 6.2 \mathrm{E}-04$ & & & & & \\
\hline & $09 / 23 / 08$ & $10 / 09 / 08$ & gross $\beta$ & $2.4 \mathrm{E}-02 \pm 2.6 \mathrm{E}-03$ & & & & & \\
\hline & $10 / 09 / 08$ & $10 / 21 / 08$ & gross $\alpha$ & $1.0 \mathrm{E}-03 \pm 6.6 \mathrm{E}-04$ & & & & & \\
\hline & $10 / 09 / 08$ & $10 / 21 / 08$ & gross $\beta$ & $2.0 \mathrm{E}-02 \pm 2.5 \mathrm{E}-03$ & & & & & \\
\hline & $10 / 21 / 08$ & $11 / 04 / 08$ & gross $\alpha$ & $3.0 \mathrm{E}-03 \pm 8.3 \mathrm{E}-04$ & & & & & \\
\hline & $10 / 21 / 08$ & $11 / 04 / 08$ & gross $\beta$ & $5.4 \mathrm{E}-02 \pm 4.9 \mathrm{E}-03$ & & & & & \\
\hline & $11 / 04 / 08$ & $11 / 18 / 08$ & gross $\alpha$ & $1.2 \mathrm{E}-03 \pm 5.3 \mathrm{E}-04$ & & & & & \\
\hline & $11 / 04 / 08$ & $11 / 18 / 08$ & gross $\beta$ & $2.6 \mathrm{E}-02 \pm 2.8 \mathrm{E}-03$ & & & & & \\
\hline & $11 / 18 / 08$ & $12 / 02 / 08$ & gross $\alpha$ & $1.1 \mathrm{E}-03 \pm 5.1 \mathrm{E}-04$ & & & & & \\
\hline & $11 / 18 / 08$ & $12 / 02 / 08$ & gross $\beta$ & $3.5 \mathrm{E}-02 \pm 3.5 \mathrm{E}-03$ & & & & & \\
\hline & $12 / 02 / 08$ & $12 / 16 / 08$ & gross $\alpha$ & $1.2 \mathrm{E}-03 \pm 5.1 \mathrm{E}-04$ & & & & & \\
\hline & $12 / 02 / 08$ & $12 / 16 / 08$ & gross $\beta$ & $2.3 \mathrm{E}-02 \pm 2.5 \mathrm{E}-03$ & & & & & \\
\hline & $12 / 16 / 08$ & $12 / 30 / 08$ & gross $\alpha$ & $2.1 \mathrm{E}-03 \pm 7.0 \mathrm{E}-04$ & & & & & \\
\hline & $12 / 16 / 08$ & $12 / 30 / 08$ & gross $\beta$ & $5.1 \mathrm{E}-02 \pm 4.6 \mathrm{E}-03$ & & & & & \\
\hline
\end{tabular}

$\overline{\mathrm{RQ}}=$ Result Qualifier. $\mathrm{U}=$ The analyte was analyzed for but not detected. 
Table 2-4. Near-Facility Air Sampling Results, 2008 (pCi/m³ \pm total analytical uncertainty). (Sheet 18 of 82)

\begin{tabular}{|c|c|c|c|c|c|c|c|c|c|}
\hline Location & Sample On & Sample Off & Isotope & Result \pm Uncertainty & Location & Composite Period & Isotope & Result \pm Uncertainty & $\mathbf{R Q *}$ \\
\hline N534 & $01 / 02 / 08$ & $01 / 15 / 08$ & gross $\alpha$ & $1.7 \mathrm{E}-04 \pm 3.6 \mathrm{E}-04$ & N534 & $01 / 02 / 08$ to $06 / 17 / 08$ & ${ }^{60} \mathrm{Co}$ & $-3.0 \mathrm{E}-06 \pm 3.0 \mathrm{E}-05$ & $\mathrm{U}$ \\
\hline \multirow[t]{23}{*}{$(100-K)$} & 01/02/08 & 01/15/08 & gross $\beta$ & $7.2 \mathrm{E}-03 \pm 1.3 \mathrm{E}-03$ & & & ${ }^{134} \mathrm{Cs}$ & $-1.1 \mathrm{E}-05 \pm 7.1 \mathrm{E}-05$ & $\mathrm{U}$ \\
\hline & 01/15/08 & 01/30/08 & gross $\alpha$ & $2.2 \mathrm{E}-03 \pm 7.1 \mathrm{E}-04$ & & & ${ }^{137} \mathrm{Cs}$ & $-3.3 \mathrm{E}-05 \pm 6.1 \mathrm{E}-05$ & $\mathrm{U}$ \\
\hline & 01/15/08 & 01/30/08 & gross $\beta$ & $3.6 \mathrm{E}-02 \pm 3.9 \mathrm{E}-03$ & & & ${ }^{152} \mathrm{Eu}$ & $-7.1 \mathrm{E}-05 \pm 1.3 \mathrm{E}-04$ & $\mathrm{U}$ \\
\hline & 01/30/08 & 02/13/08 & gross $\alpha$ & $4.0 \mathrm{E}-04 \pm 4.4 \mathrm{E}-04$ & & & ${ }^{154} \mathrm{Eu}$ & $-2.5 \mathrm{E}-06 \pm 2.5 \mathrm{E}-05$ & $\mathrm{U}$ \\
\hline & 01/30/08 & 02/13/08 & gross $\beta$ & $3.4 \mathrm{E}-03 \pm 8.2 \mathrm{E}-04$ & & & ${ }^{155} \mathrm{Eu}$ & $2.0 \mathrm{E}-05 \pm 1.4 \mathrm{E}-04$ & $\mathrm{U}$ \\
\hline & 02/13/08 & $02 / 27 / 08$ & gross $\alpha$ & $2.7 \mathrm{E}-03 \pm 7.9 \mathrm{E}-04$ & & & ${ }^{238} \mathrm{Pu}$ & $-6.0 \mathrm{E}-06 \pm 9.0 \mathrm{E}-06$ & $\mathrm{U}$ \\
\hline & 02/13/08 & $02 / 27 / 08$ & gross $\beta$ & $3.1 \mathrm{E}-02 \pm 3.5 \mathrm{E}-03$ & & & ${ }^{239 / 240} \mathrm{Pu}$ & $1.5 \mathrm{E}-06 \pm 3.1 \mathrm{E}-06$ & $\mathrm{U}$ \\
\hline & $02 / 27 / 08$ & 03/11/08 & gross $\alpha$ & $1.1 \mathrm{E}-03 \pm 5.3 \mathrm{E}-04$ & & & ${ }^{106} \mathrm{Ru}$ & $-4.9 \mathrm{E}-04 \pm 5.5 \mathrm{E}-04$ & $\mathrm{U}$ \\
\hline & 02/27/08 & 03/11/08 & gross $\beta$ & $9.3 \mathrm{E}-03 \pm 1.5 \mathrm{E}-03$ & & & ${ }^{125} \mathrm{Sb}$ & $-3.7 \mathrm{E}-05 \pm 1.3 \mathrm{E}-04$ & $\mathrm{U}$ \\
\hline & 03/11/08 & 03/25/08 & gross $\alpha$ & $1.2 \mathrm{E}-03 \pm 5.3 \mathrm{E}-04$ & & & ${ }^{90} \mathrm{Sr}$ & $-2.1 \mathrm{E}-04 \pm 2.2 \mathrm{E}-04$ & $\mathrm{U}$ \\
\hline & 03/11/08 & 03/25/08 & gross $\beta$ & $1.1 \mathrm{E}-02 \pm 1.7 \mathrm{E}-03$ & & & ${ }^{234} \mathrm{U}$ & $1.2 \mathrm{E}-05 \pm 7.9 \mathrm{E}-06$ & \\
\hline & 03/25/08 & 04/07/08 & gross $\alpha$ & $6.6 \mathrm{E}-04 \pm 5.4 \mathrm{E}-04$ & & & ${ }^{235} \mathrm{U}$ & $1.6 \mathrm{E}-06 \pm 2.4 \mathrm{E}-06$ & $\mathrm{U}$ \\
\hline & 03/25/08 & 04/07/08 & gross $\beta$ & $8.9 \mathrm{E}-03 \pm 1.5 \mathrm{E}-03$ & & & ${ }^{238} \mathrm{U}$ & $4.4 \mathrm{E}-06 \pm 4.5 \mathrm{E}-06$ & $\mathrm{U}$ \\
\hline & 04/07/08 & $04 / 21 / 08$ & gross $\alpha$ & $9.5 \mathrm{E}-04 \pm 5.9 \mathrm{E}-04$ & & & & & \\
\hline & 04/07/08 & 04/21/08 & gross $\beta$ & $9.5 \mathrm{E}-03 \pm 1.5 \mathrm{E}-03$ & & & & & \\
\hline & $04 / 21 / 08$ & 05/07/08 & gross $\alpha$ & $1.2 \mathrm{E}-03 \pm 4.9 \mathrm{E}-04$ & & & & & \\
\hline & $04 / 21 / 08$ & 05/07/08 & gross $\beta$ & $1.3 \mathrm{E}-02 \pm 1.8 \mathrm{E}-03$ & & & & & \\
\hline & 05/07/08 & 05/20/08 & gross $\alpha$ & $1.5 \mathrm{E}-03 \pm 6.2 \mathrm{E}-04$ & & & & & \\
\hline & 05/07/08 & $05 / 20 / 08$ & gross $\beta$ & $9.9 \mathrm{E}-03 \pm 1.6 \mathrm{E}-03$ & & & & & \\
\hline & $05 / 20 / 08$ & 06/04/08 & gross $\alpha$ & $8.0 \mathrm{E}-04 \pm 5.3 \mathrm{E}-04$ & & & & & \\
\hline & 05/20/08 & 06/04/08 & gross $\beta$ & $1.2 \mathrm{E}-02 \pm 1.7 \mathrm{E}-03$ & & & & & \\
\hline & 06/04/08 & 06/17/08 & gross $\alpha$ & $4.3 \mathrm{E}-04 \pm 4.7 \mathrm{E}-04$ & & & & & \\
\hline & 06/04/08 & 06/17/08 & gross $\beta$ & $8.5 \mathrm{E}-03 \pm 1.4 \mathrm{E}-03$ & & & & & \\
\hline \multirow{24}{*}{$\begin{array}{c}\text { N535 } \\
(100-K)\end{array}$} & 01/02/08 & 01/15/08 & gross $\alpha$ & $5.2 \mathrm{E}-04 \pm 5.0 \mathrm{E}-04$ & N535 & $01 / 02 / 08$ to $06 / 17 / 08$ & ${ }^{60} \mathrm{Co}$ & $1.0 \mathrm{E}-07 \pm 1.0 \mathrm{E}-06$ & $\mathrm{U}$ \\
\hline & 01/02/08 & 01/15/08 & gross $\beta$ & $9.4 \mathrm{E}-03 \pm 1.5 \mathrm{E}-03$ & & & ${ }^{134} \mathrm{Cs}$ & $-9.9 \mathrm{E}-07 \pm 9.9 \mathrm{E}-06$ & $\mathrm{U}$ \\
\hline & 01/15/08 & 01/30/08 & gross $\alpha$ & $1.3 \mathrm{E}-03 \pm 5.3 \mathrm{E}-04$ & & & ${ }^{137} \mathrm{Cs}$ & $1.3 \mathrm{E}-04 \pm 9.3 \mathrm{E}-05$ & \\
\hline & 01/15/08 & 01/30/08 & gross $\beta$ & $3.0 \mathrm{E}-02 \pm 3.3 \mathrm{E}-03$ & & & ${ }^{152} \mathrm{Eu}$ & $4.6 \mathrm{E}-07 \pm 4.8 \mathrm{E}-07$ & $\mathrm{U}$ \\
\hline & 01/30/08 & 02/13/08 & gross $\alpha$ & $4.0 \mathrm{E}-04 \pm 4.4 \mathrm{E}-04$ & & & ${ }^{154} \mathrm{Eu}$ & $-2.7 \mathrm{E}-04 \pm 2.8 \mathrm{E}-04$ & $\mathrm{U}$ \\
\hline & 01/30/08 & 02/13/08 & gross $\beta$ & $4.5 \mathrm{E}-03 \pm 9.6 \mathrm{E}-04$ & & & ${ }^{155} \mathrm{Eu}$ & $-3.4 \mathrm{E}-05 \pm 1.6 \mathrm{E}-04$ & $\mathrm{U}$ \\
\hline & 02/13/08 & 02/27/08 & gross $\alpha$ & $1.9 \mathrm{E}-03 \pm 6.6 \mathrm{E}-04$ & & & ${ }^{238} \mathrm{Pu}$ & $1.0 \mathrm{E}-05 \pm 1.5 \mathrm{E}-05$ & $\mathrm{U}$ \\
\hline & 02/13/08 & $02 / 27 / 08$ & gross $\beta$ & $3.4 \mathrm{E}-02 \pm 3.8 \mathrm{E}-03$ & & & ${ }^{239 / 240} \mathrm{Pu}$ & $5.9 \mathrm{E}-06 \pm 6.9 \mathrm{E}-06$ & $\mathrm{U}$ \\
\hline & $02 / 27 / 08$ & 03/11/08 & gross $\alpha$ & $7.5 \mathrm{E}-04 \pm 5.6 \mathrm{E}-04$ & & & ${ }^{106} \mathrm{Ru}$ & $-3.5 \mathrm{E}-04 \pm 5.9 \mathrm{E}-04$ & $\mathrm{U}$ \\
\hline & $02 / 27 / 08$ & 03/11/08 & gross $\beta$ & $1.2 \mathrm{E}-02 \pm 1.8 \mathrm{E}-03$ & & & ${ }^{125} \mathrm{Sb}$ & $-2.4 \mathrm{E}-05 \pm 1.4 \mathrm{E}-04$ & $\mathrm{U}$ \\
\hline & 03/11/08 & 03/25/08 & gross $\alpha$ & $5.0 \mathrm{E}-04 \pm 4.7 \mathrm{E}-04$ & & & ${ }^{90} \mathrm{Sr}$ & $-1.7 \mathrm{E}-04 \pm 1.7 \mathrm{E}-04$ & $\mathrm{U}$ \\
\hline & 03/11/08 & 03/25/08 & gross $\beta$ & $1.0 \mathrm{E}-02 \pm 1.6 \mathrm{E}-03$ & & & ${ }^{234} \mathrm{U}$ & $1.3 \mathrm{E}-05 \pm 7.9 \mathrm{E}-06$ & \\
\hline & 03/25/08 & 04/07/08 & gross $\alpha$ & $5.2 \mathrm{E}-04 \pm 5.0 \mathrm{E}-04$ & & & ${ }^{235} \mathrm{U}$ & $7.1 \mathrm{E}-07 \pm 2.2 \mathrm{E}-06$ & $\mathrm{U}$ \\
\hline & 03/25/08 & $04 / 07 / 08$ & gross $\beta$ & $5.9 \mathrm{E}-03 \pm 1.2 \mathrm{E}-03$ & & & ${ }^{238} \mathrm{U}$ & $9.9 \mathrm{E}-06 \pm 6.8 \mathrm{E}-06$ & \\
\hline & 04/07/08 & 04/21/08 & gross $\alpha$ & $1.6 \mathrm{E}-03 \pm 6.0 \mathrm{E}-04$ & & & & & \\
\hline & 04/07/08 & $04 / 21 / 08$ & gross $\beta$ & $8.8 \mathrm{E}-03 \pm 1.4 \mathrm{E}-03$ & & & & & \\
\hline & $04 / 21 / 08$ & 05/07/08 & gross $\alpha$ & $1.3 \mathrm{E}-03 \pm 5.1 \mathrm{E}-04$ & & & & & \\
\hline & $04 / 21 / 08$ & 05/07/08 & gross $\beta$ & $1.3 \mathrm{E}-02 \pm 1.8 \mathrm{E}-03$ & & & & & \\
\hline & 05/07/08 & 05/20/08 & gross $\alpha$ & $8.0 \mathrm{E}-04 \pm 5.8 \mathrm{E}-04$ & & & & & \\
\hline & 05/07/08 & 05/20/08 & gross $\beta$ & $8.6 \mathrm{E}-03 \pm 1.4 \mathrm{E}-03$ & & & & & \\
\hline & 05/20/08 & $06 / 04 / 08$ & gross $\alpha$ & $8.8 \mathrm{E}-04 \pm 5.4 \mathrm{E}-04$ & & & & & \\
\hline & $05 / 20 / 08$ & 06/04/08 & gross $\beta$ & $9.3 \mathrm{E}-03 \pm 1.4 \mathrm{E}-03$ & & & & & \\
\hline & 06/04/08 & 06/17/08 & gross $\alpha$ & $5.5 \mathrm{E}-04 \pm 5.1 \mathrm{E}-04$ & & & & & \\
\hline & 06/04/08 & 06/17/08 & gross $\beta$ & $6.8 \mathrm{E}-03 \pm 1.3 \mathrm{E}-03$ & & & & & \\
\hline
\end{tabular}

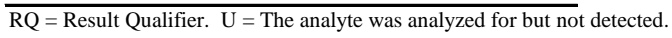


Table 2-4. Near-Facility Air Sampling Results, 2008 (pCi/m³ \pm total analytical uncertainty). (Sheet 19 of 82)

\begin{tabular}{|c|c|c|c|c|c|c|c|c|c|}
\hline Location & Sample On & Sample Off & Isotope & Result \pm Uncertainty & Location & Composite Period & Isotope & Result \pm Uncertainty & $\mathbf{R Q}^{*}$ \\
\hline N102 & $01 / 02 / 08$ & $01 / 15 / 08$ & gross $\alpha$ & $8.8 \mathrm{E}-04 \pm 5.9 \mathrm{E}-04$ & N102 & $01 / 02 / 08$ to $07 / 01 / 08$ & ${ }^{241} \mathrm{Am}$ & $7.6 \mathrm{E}-06 \pm 5.5 \mathrm{E}-06$ & \\
\hline \multirow[t]{51}{*}{$(100-\mathrm{N})$} & 01/02/08 & 01/15/08 & gross $\beta$ & $8.9 \mathrm{E}-03 \pm 1.5 \mathrm{E}-03$ & & & ${ }^{60} \mathrm{Co}$ & $5.1 \mathrm{E}-05 \pm 8.9 \mathrm{E}-05$ & $\mathrm{U}$ \\
\hline & $01 / 15 / 08$ & 01/30/08 & gross $\alpha$ & $2.0 \mathrm{E}-03 \pm 6.7 \mathrm{E}-04$ & & & ${ }^{134} \mathrm{Cs}$ & $-1.8 \mathrm{E}-05 \pm 7.2 \mathrm{E}-05$ & $\mathrm{U}$ \\
\hline & 01/15/08 & 01/30/08 & gross $\beta$ & $3.3 \mathrm{E}-02 \pm 3.7 \mathrm{E}-03$ & & & ${ }^{137} \mathrm{Cs}$ & $4.9 \mathrm{E}-05 \pm 7.1 \mathrm{E}-05$ & $\mathrm{U}$ \\
\hline & 01/30/08 & 02/13/08 & gross $\alpha$ & $5.3 \mathrm{E}-04 \pm 5.0 \mathrm{E}-04$ & & & ${ }^{152} \mathrm{Eu}$ & $-1.2 \mathrm{E}-04 \pm 1.7 \mathrm{E}-04$ & $\mathrm{U}$ \\
\hline & 01/30/08 & $02 / 13 / 08$ & gross $\beta$ & $4.2 \mathrm{E}-03 \pm 9.4 \mathrm{E}-04$ & & & ${ }^{154} \mathrm{Eu}$ & $1.8 \mathrm{E}-04 \pm 2.3 \mathrm{E}-04$ & $\mathrm{U}$ \\
\hline & 02/13/08 & $02 / 27 / 08$ & gross $\alpha$ & $2.2 \mathrm{E}-03 \pm 7.6 \mathrm{E}-04$ & & & ${ }^{155} \mathrm{Eu}$ & $5.3 \mathrm{E}-05 \pm 1.6 \mathrm{E}-04$ & $\mathrm{U}$ \\
\hline & 02/13/08 & $02 / 27 / 08$ & gross $\beta$ & $3.6 \mathrm{E}-02 \pm 4.1 \mathrm{E}-03$ & & & ${ }^{238} \mathrm{Pu}$ & $5.0 \mathrm{E}-06 \pm 1.5 \mathrm{E}-05$ & $\mathrm{U}$ \\
\hline & $02 / 27 / 08$ & 03/12/08 & gross $\alpha$ & $6.4 \mathrm{E}-04 \pm 5.2 \mathrm{E}-04$ & & & ${ }^{239 / 240} \mathrm{Pu}$ & $3.0 \mathrm{E}-06 \pm 3.6 \mathrm{E}-06$ & \\
\hline & $02 / 27 / 08$ & 03/12/08 & gross $\beta$ & $1.2 \mathrm{E}-02 \pm 1.8 \mathrm{E}-03$ & & & ${ }^{106} \mathrm{Ru}$ & $3.4 \mathrm{E}-04 \pm 7.0 \mathrm{E}-04$ & $\mathrm{U}$ \\
\hline & 03/12/08 & 03/26/08 & gross $\alpha$ & $8.5 \mathrm{E}-04 \pm 5.8 \mathrm{E}-04$ & & & ${ }^{125} \mathrm{Sb}$ & $-4.9 \mathrm{E}-05 \pm 1.5 \mathrm{E}-04$ & $\mathrm{U}$ \\
\hline & 03/12/08 & 03/26/08 & gross $\beta$ & $1.2 \mathrm{E}-02 \pm 1.8 \mathrm{E}-03$ & & & ${ }^{90} \mathrm{Sr}$ & $-1.3 \mathrm{E}-04 \pm 1.4 \mathrm{E}-04$ & $\mathrm{U}$ \\
\hline & 03/26/08 & 04/08/08 & gross $\alpha$ & $2.2 \mathrm{E}-03 \pm 7.5 \mathrm{E}-04$ & & & ${ }^{234} \mathrm{U}$ & $1.6 \mathrm{E}-05 \pm 9.2 \mathrm{E}-06$ & \\
\hline & 03/26/08 & 04/08/08 & gross $\beta$ & $1.1 \mathrm{E}-02 \pm 1.7 \mathrm{E}-03$ & & & ${ }^{235} \mathrm{U}$ & $3.9 \mathrm{E}-06 \pm 3.8 \mathrm{E}-06$ & \\
\hline & $04 / 08 / 08$ & $04 / 23 / 08$ & gross $\alpha$ & $1.1 \mathrm{E}-03 \pm 4.9 \mathrm{E}-04$ & & & ${ }^{238} U$ & $1.1 \mathrm{E}-05 \pm 7.9 \mathrm{E}-06$ & \\
\hline & $04 / 08 / 08$ & $04 / 23 / 08$ & gross $\beta$ & $1.0 \mathrm{E}-02 \pm 1.6 \mathrm{E}-03$ & & & & & \\
\hline & $04 / 23 / 08$ & 05/07/08 & gross $\alpha$ & $1.5 \mathrm{E}-03 \pm 5.8 \mathrm{E}-04$ & N102 & $07 / 01 / 08$ to $12 / 30 / 08$ & ${ }^{241} \mathrm{Am}$ & $5.5 \mathrm{E}-07 \pm 5.5 \mathrm{E}-06$ & $\mathrm{U}$ \\
\hline & $04 / 23 / 08$ & 05/07/08 & gross $\beta$ & $1.5 \mathrm{E}-02 \pm 2.0 \mathrm{E}-03$ & & & ${ }^{60} \mathrm{Co}$ & $-2.1 \mathrm{E}-05 \pm 7.1 \mathrm{E}-05$ & $\mathrm{U}$ \\
\hline & $05 / 07 / 08$ & $05 / 20 / 08$ & gross $\alpha$ & $1.7 \mathrm{E}-04 \pm 3.8 \mathrm{E}-04$ & & & ${ }^{134} \mathrm{Cs}$ & $1.8 \mathrm{E}-05 \pm 6.5 \mathrm{E}-05$ & $\mathrm{U}$ \\
\hline & 05/07/08 & 05/20/08 & gross $\beta$ & $9.3 \mathrm{E}-03 \pm 1.5 \mathrm{E}-03$ & & & ${ }^{137} \mathrm{Cs}$ & $-4.6 \mathrm{E}-05 \pm 6.5 \mathrm{E}-05$ & $\mathrm{U}$ \\
\hline & 05/20/08 & $06 / 04 / 08$ & gross $\alpha$ & $1.1 \mathrm{E}-03 \pm 4.8 \mathrm{E}-04$ & & & ${ }^{152} \mathrm{Eu}$ & $-5.7 \mathrm{E}-05 \pm 1.5 \mathrm{E}-04$ & $\mathrm{U}$ \\
\hline & $05 / 20 / 08$ & $06 / 04 / 08$ & gross $\beta$ & $1.2 \mathrm{E}-02 \pm 1.7 \mathrm{E}-03$ & & & ${ }^{154} \mathrm{Eu}$ & $1.2 \mathrm{E}-04 \pm 2.2 \mathrm{E}-04$ & $\mathrm{U}$ \\
\hline & $06 / 04 / 08$ & 06/17/08 & gross $\alpha$ & $5.4 \mathrm{E}-04 \pm 5.1 \mathrm{E}-04$ & & & ${ }^{155} \mathrm{Eu}$ & $-7.5 \mathrm{E}-05 \pm 1.4 \mathrm{E}-04$ & $\mathrm{U}$ \\
\hline & 06/04/08 & 06/17/08 & gross $\beta$ & $7.7 \mathrm{E}-03 \pm 1.4 \mathrm{E}-03$ & & & ${ }^{238} \mathrm{Pu}$ & $1.1 \mathrm{E}-05 \pm 1.1 \mathrm{E}-05$ & $\mathrm{U}$ \\
\hline & $06 / 17 / 08$ & 07/01/08 & gross $\alpha$ & $1.2 \mathrm{E}-03 \pm 9.9 \mathrm{E}-04$ & & & ${ }^{239 / 240} \mathrm{Pu}$ & $2.2 \mathrm{E}-06 \pm 2.6 \mathrm{E}-06$ & \\
\hline & 06/17/08 & 07/01/08 & gross $\beta$ & $9.2 \mathrm{E}-03 \pm 1.9 \mathrm{E}-03$ & & & ${ }^{106} \mathrm{Ru}$ & $-5.1 \mathrm{E}-04 \pm 5.7 \mathrm{E}-04$ & $\mathrm{U}$ \\
\hline & 07/01/08 & $07 / 15 / 08$ & gross $\alpha$ & $9.4 \mathrm{E}-04 \pm 6.1 \mathrm{E}-04$ & & & ${ }^{125} \mathrm{Sb}$ & $2.0 \mathrm{E}-05 \pm 1.3 \mathrm{E}-04$ & $\mathrm{U}$ \\
\hline & 07/01/08 & 07/15/08 & gross $\beta$ & $1.1 \mathrm{E}-02 \pm 1.6 \mathrm{E}-03$ & & & ${ }^{90} \mathrm{Sr}$ & $-1.3 \mathrm{E}-04 \pm 1.4 \mathrm{E}-04$ & $\mathrm{U}$ \\
\hline & 07/15/08 & 07/29/08 & gross $\alpha$ & $1.3 \mathrm{E}-03 \pm 5.6 \mathrm{E}-04$ & & & ${ }^{234} \mathrm{U}$ & $1.6 \mathrm{E}-05 \pm 9.2 \mathrm{E}-06$ & \\
\hline & 07/15/08 & 07/29/08 & gross $\beta$ & $1.3 \mathrm{E}-02 \pm 1.9 \mathrm{E}-03$ & & & ${ }^{235} \mathrm{U}$ & $3.9 \mathrm{E}-06 \pm 3.8 \mathrm{E}-06$ & \\
\hline & 07/29/08 & 08/12/08 & $\operatorname{gross} \alpha$ & $7.2 \mathrm{E}-04 \pm 5.2 \mathrm{E}-04$ & & & ${ }^{238} \mathrm{U}$ & $1.2 \mathrm{E}-05 \pm 7.2 \mathrm{E}-06$ & \\
\hline & $07 / 29 / 08$ & 08/12/08 & gross $\beta$ & $1.3 \mathrm{E}-02 \pm 1.8 \mathrm{E}-03$ & & & & & \\
\hline & 08/12/08 & $08 / 26 / 08$ & gross $\alpha$ & $9.6 \mathrm{E}-04 \pm 5.9 \mathrm{E}-04$ & & & & & \\
\hline & 08/12/08 & 08/26/08 & gross $\beta$ & $1.2 \mathrm{E}-02 \pm 1.7 \mathrm{E}-03$ & & & & & \\
\hline & $08 / 26 / 08$ & 09/09/08 & gross $\alpha$ & $1.1 \mathrm{E}-03 \pm 6.8 \mathrm{E}-04$ & & & & & \\
\hline & 08/26/08 & 09/09/08 & gross $\beta$ & $1.5 \mathrm{E}-02 \pm 2.2 \mathrm{E}-03$ & & & & & \\
\hline & 09/09/08 & 09/23/08 & gross $\alpha$ & $1.8 \mathrm{E}-03 \pm 6.9 \mathrm{E}-04$ & & & & & \\
\hline & 09/09/08 & 09/23/08 & gross $\beta$ & $2.6 \mathrm{E}-02 \pm 3.1 \mathrm{E}-03$ & & & & & \\
\hline & $09 / 23 / 08$ & $10 / 08 / 08$ & gross $\alpha$ & $1.7 \mathrm{E}-03 \pm 6.0 \mathrm{E}-04$ & & & & & \\
\hline & 09/23/08 & $10 / 08 / 08$ & gross $\beta$ & $2.5 \mathrm{E}-02 \pm 3.0 \mathrm{E}-03$ & & & & & \\
\hline & $10 / 08 / 08$ & $10 / 21 / 08$ & gross $\alpha$ & $1.0 \mathrm{E}-03 \pm 6.3 \mathrm{E}-04$ & & & & & \\
\hline & $10 / 08 / 08$ & $10 / 21 / 08$ & gross $\beta$ & $1.8 \mathrm{E}-02 \pm 2.4 \mathrm{E}-03$ & & & & & \\
\hline & $10 / 21 / 08$ & $11 / 04 / 08$ & gross $\alpha$ & $3.3 \mathrm{E}-03 \pm 9.0 \mathrm{E}-04$ & & & & & \\
\hline & $10 / 21 / 08$ & $11 / 04 / 08$ & gross $\beta$ & $4.1 \mathrm{E}-02 \pm 4.4 \mathrm{E}-03$ & & & & & \\
\hline & $11 / 04 / 08$ & $11 / 18 / 08$ & gross $\alpha$ & $1.8 \mathrm{E}-03 \pm 6.4 \mathrm{E}-04$ & & & & & \\
\hline & $11 / 04 / 08$ & $11 / 18 / 08$ & gross $\beta$ & $2.3 \mathrm{E}-02 \pm 2.8 \mathrm{E}-03$ & & & & & \\
\hline & $11 / 18 / 08$ & $12 / 02 / 08$ & gross $\alpha$ & $2.0 \mathrm{E}-03 \pm 7.0 \mathrm{E}-04$ & & & & & \\
\hline & $11 / 18 / 08$ & $12 / 02 / 08$ & gross $\beta$ & $3.5 \mathrm{E}-02 \pm 3.9 \mathrm{E}-03$ & & & & & \\
\hline & $12 / 02 / 08$ & $12 / 16 / 08$ & gross $\alpha$ & $8.4 \mathrm{E}-04 \pm 5.6 \mathrm{E}-04$ & & & & & \\
\hline & $12 / 02 / 08$ & $12 / 16 / 08$ & gross $\beta$ & $2.0 \mathrm{E}-02 \pm 2.5 \mathrm{E}-03$ & & & & & \\
\hline & $12 / 16 / 08$ & $12 / 30 / 08$ & $\operatorname{gross} \alpha$ & $1.2 \mathrm{E}-03 \pm 5.5 \mathrm{E}-04$ & & & & & \\
\hline & $12 / 16 / 08$ & $12 / 30 / 08$ & gross $\beta$ & $3.6 \mathrm{E}-02 \pm 4.0 \mathrm{E}-03$ & & & & & \\
\hline
\end{tabular}

$\overline{\mathrm{RQ}}=$ Result Qualifier. $\mathrm{U}=$ The analyte was analyzed for but not detected. 
Table 2-4. Near-Facility Air Sampling Results, 2008 (pCi/m \pm total analytical uncertainty). (Sheet 20 of 82)

\begin{tabular}{|c|c|c|c|c|c|c|c|c|c|}
\hline \multirow{2}{*}{$\begin{array}{c}\text { N103 } \\
(100-N)\end{array}$} & $01 / 02 / 08$ & 01/15/08 & gross $\alpha$ & $9.8 \mathrm{E}-04 \pm 6.1 \mathrm{E}-04$ & \multirow[t]{16}{*}{ N103 } & \multirow[t]{16}{*}{$01 / 02 / 08$ to $07 / 01 / 08$} & \multirow{2}{*}{$\begin{array}{l}{ }^{241} \mathrm{Am} \\
{ }^{60} \mathrm{Co}\end{array}$} & \multicolumn{2}{|l|}{$7.7 \mathrm{E}-06 \pm 5.1 \mathrm{E}-06$} \\
\hline & $01 / 02 / 08$ & 01/15/08 & gross $\beta$ & $1.1 \mathrm{E}-02 \pm 1.6 \mathrm{E}-03$ & & & & $-3.0 \mathrm{E}-05 \pm 7.3 \mathrm{E}-05$ & $\mathrm{U}$ \\
\hline & $01 / 15 / 08$ & 01/30/08 & gross $\alpha$ & $1.6 \mathrm{E}-03 \pm 6.0 \mathrm{E}-04$ & & & ${ }^{134} \mathrm{Cs}$ & $-4.4 \mathrm{E}-05 \pm 5.9 \mathrm{E}-05$ & $\mathrm{U}$ \\
\hline & 01/15/08 & 01/30/08 & gross $\beta$ & $3.1 \mathrm{E}-02 \pm 3.1 \mathrm{E}-03$ & & & ${ }^{137} \mathrm{Cs}$ & $-7.3 \mathrm{E}-06 \pm 5.4 \mathrm{E}-05$ & U \\
\hline & 01/30/08 & 02/13/08 & gross $\alpha$ & $2.9 \mathrm{E}-04 \pm 4.1 \mathrm{E}-04$ & & & ${ }^{152} \mathrm{Eu}$ & $-4.3 \mathrm{E}-05 \pm 1.3 \mathrm{E}-04$ & $\mathrm{U}$ \\
\hline & 01/30/08 & $02 / 13 / 08$ & gross $\beta$ & $3.6 \mathrm{E}-03 \pm 8.3 \mathrm{E}-04$ & & & ${ }^{154} \mathrm{Eu}$ & $9.5 \mathrm{E}-05 \pm 1.9 \mathrm{E}-04$ & $\mathrm{U}$ \\
\hline & 02/13/08 & $02 / 27 / 08$ & gross $\alpha$ & $1.3 \mathrm{E}-03 \pm 5.6 \mathrm{E}-04$ & & & ${ }^{155} \mathrm{Eu}$ & $4.3 \mathrm{E}-05 \pm 1.3 \mathrm{E}-04$ & $\mathrm{U}$ \\
\hline & $02 / 13 / 08$ & $02 / 27 / 08$ & gross $\beta$ & $3.1 \mathrm{E}-02 \pm 3.1 \mathrm{E}-03$ & & & ${ }^{238} \mathrm{Pu}$ & $2.0 \mathrm{E}-06 \pm 1.2 \mathrm{E}-05$ & $\mathrm{U}$ \\
\hline & $02 / 27 / 08$ & 03/12/08 & gross $\alpha$ & $6.2 \mathrm{E}-04 \pm 5.1 \mathrm{E}-04$ & & & ${ }^{239 / 240} \mathrm{Pu}$ & $2.0 \mathrm{E}-06 \pm 4.0 \mathrm{E}-06$ & $\mathrm{U}$ \\
\hline & $02 / 27 / 08$ & 03/12/08 & gross $\beta$ & $9.9 \mathrm{E}-03 \pm 1.4 \mathrm{E}-03$ & & & ${ }^{106} \mathrm{Ru}$ & $-1.2 \mathrm{E}-04 \pm 5.4 \mathrm{E}-04$ & $\mathrm{U}$ \\
\hline & 03/12/08 & 03/26/08 & gross $\alpha$ & $8.8 \mathrm{E}-04 \pm 5.9 \mathrm{E}-04$ & & & ${ }^{125} \mathrm{Sb}$ & $1.2 \mathrm{E}-04 \pm 1.3 \mathrm{E}-04$ & $\mathrm{U}$ \\
\hline & 03/12/08 & 03/26/08 & gross $\beta$ & $8.8 \mathrm{E}-03 \pm 1.4 \mathrm{E}-03$ & & & ${ }^{90} \mathrm{Sr}$ & $-2.3 \mathrm{E}-04 \pm 2.4 \mathrm{E}-04$ & $\mathrm{U}$ \\
\hline & 03/26/08 & 04/08/08 & gross $\alpha$ & $6.6 \mathrm{E}-04 \pm 5.4 \mathrm{E}-04$ & & & ${ }^{234} \mathrm{U}$ & $6.9 \mathrm{E}-06 \pm 5.8 \mathrm{E}-06$ & \\
\hline & 03/26/08 & $04 / 08 / 08$ & gross $\beta$ & $9.7 \mathrm{E}-03 \pm 1.5 \mathrm{E}-03$ & & & ${ }^{235} \mathrm{U}$ & $5.3 \mathrm{E}-06 \pm 4.5 \mathrm{E}-06$ & \\
\hline & $04 / 08 / 08$ & $04 / 23 / 08$ & gross $\alpha$ & $1.3 \mathrm{E}-03 \pm 5.7 \mathrm{E}-04$ & & & ${ }^{238} \mathrm{U}$ & $1.2 \mathrm{E}-05 \pm 7.5 \mathrm{E}-06$ & \\
\hline & 04/08/08 & $04 / 23 / 08$ & gross $\beta$ & $1.2 \mathrm{E}-02 \pm 1.7 \mathrm{E}-03$ & & & & & \\
\hline & $04 / 23 / 08$ & 05/07/08 & gross $\alpha$ & $3.2 \mathrm{E}-03 \pm 8.6 \mathrm{E}-04$ & N103 & $07 / 01 / 08$ to $12 / 30 / 08$ & ${ }^{241} \mathrm{Am}$ & $5.2 \mathrm{E}-06 \pm 5.4 \mathrm{E}-06$ & $\mathrm{U}$ \\
\hline & $04 / 23 / 08$ & 05/07/08 & gross $\beta$ & $1.8 \mathrm{E}-02 \pm 2.1 \mathrm{E}-03$ & & & ${ }^{60} \mathrm{Co}$ & $-3.0 \mathrm{E}-05 \pm 6.7 \mathrm{E}-05$ & $\mathrm{U}$ \\
\hline & 05/07/08 & 05/20/08 & gross $\alpha$ & $9.0 \mathrm{E}-04 \pm 6.1 \mathrm{E}-04$ & & & ${ }^{134} \mathrm{Cs}$ & $2.9 \mathrm{E}-06 \pm 2.9 \mathrm{E}-05$ & U \\
\hline & 05/07/08 & $05 / 20 / 08$ & gross $\beta$ & $1.4 \mathrm{E}-02 \pm 1.9 \mathrm{E}-03$ & & & ${ }^{137} \mathrm{Cs}$ & $2.9 \mathrm{E}-06 \pm 2.9 \mathrm{E}-05$ & $\mathrm{U}$ \\
\hline & $05 / 20 / 08$ & $06 / 04 / 08$ & gross $\alpha$ & $8.8 \mathrm{E}-04 \pm 5.4 \mathrm{E}-04$ & & & ${ }^{152} \mathrm{Eu}$ & $1.4 \mathrm{E}-04 \pm 1.6 \mathrm{E}-04$ & $\mathrm{U}$ \\
\hline & $05 / 20 / 08$ & $06 / 04 / 08$ & gross $\beta$ & $1.3 \mathrm{E}-02 \pm 1.6 \mathrm{E}-03$ & & & ${ }^{154} \mathrm{Eu}$ & $-1.6 \mathrm{E}-04 \pm 2.0 \mathrm{E}-04$ & $\mathrm{U}$ \\
\hline & $06 / 04 / 08$ & 06/17/08 & gross $\alpha$ & $5.1 \mathrm{E}-04 \pm 4.8 \mathrm{E}-04$ & & & ${ }^{155} \mathrm{Eu}$ & $2.4 \mathrm{E}-05 \pm 1.7 \mathrm{E}-04$ & $\mathrm{U}$ \\
\hline & $06 / 04 / 08$ & $06 / 17 / 08$ & gross $\beta$ & $8.1 \mathrm{E}-03 \pm 1.3 \mathrm{E}-03$ & & & ${ }^{238} \mathrm{Pu}$ & $-5.6 \mathrm{E}-06 \pm 1.4 \mathrm{E}-05$ & $\mathrm{U}$ \\
\hline & 06/17/08 & 07/01/08 & gross $\alpha$ & $9.9 \mathrm{E}-04 \pm 6.1 \mathrm{E}-04$ & & & ${ }^{239 / 240} \mathrm{Pu}$ & $1.8 \mathrm{E}-05 \pm 1.1 \mathrm{E}-05$ & \\
\hline & $06 / 17 / 08$ & 07/01/08 & gross $\beta$ & $1.4 \mathrm{E}-02 \pm 1.8 \mathrm{E}-03$ & & & ${ }^{106} \mathrm{Ru}$ & $-2.7 \mathrm{E}-05 \pm 2.7 \mathrm{E}-04$ & $\mathrm{U}$ \\
\hline & 07/01/08 & 07/15/08 & gross $\alpha$ & $8.0 \mathrm{E}-04 \pm 6.2 \mathrm{E}-04$ & & & ${ }^{125} \mathrm{Sb}$ & $1.1 \mathrm{E}-04 \pm 1.3 \mathrm{E}-04$ & $\mathrm{U}$ \\
\hline & 07/01/08 & 07/15/08 & gross $\beta$ & $1.3 \mathrm{E}-02 \pm 1.8 \mathrm{E}-03$ & & & ${ }^{90} \mathrm{Sr}$ & $-4.7 \mathrm{E}-04 \pm 4.9 \mathrm{E}-04$ & $\mathrm{U}$ \\
\hline & $07 / 15 / 08$ & 07/29/08 & gross $\alpha$ & $1.2 \mathrm{E}-03 \pm 5.2 \mathrm{E}-04$ & & & ${ }^{234} \mathrm{U}$ & $1.7 \mathrm{E}-05 \pm 1.0 \mathrm{E}-05$ & \\
\hline & 07/15/08 & 07/29/08 & gross $\beta$ & $1.3 \mathrm{E}-02 \pm 1.7 \mathrm{E}-03$ & & & ${ }^{235} \mathrm{U}$ & $5.2 \mathrm{E}-06 \pm 4.6 \mathrm{E}-06$ & \\
\hline & $07 / 29 / 08$ & 08/12/08 & gross $\alpha$ & $6.1 \mathrm{E}-04 \pm 4.9 \mathrm{E}-04$ & & & ${ }^{238} \mathrm{U}$ & $8.6 \mathrm{E}-06 \pm 6.5 \mathrm{E}-06$ & \\
\hline & $07 / 29 / 08$ & $08 / 12 / 08$ & gross $\beta$ & $1.3 \mathrm{E}-02 \pm 1.7 \mathrm{E}-03$ & & & & & \\
\hline & 08/12/08 & 08/26/08 & gross $\alpha$ & $9.6 \mathrm{E}-04 \pm 5.9 \mathrm{E}-04$ & & & & & \\
\hline & $08 / 12 / 08$ & $08 / 26 / 08$ & gross $\beta$ & $1.2 \mathrm{E}-02 \pm 1.6 \mathrm{E}-03$ & & & & & \\
\hline & 08/26/08 & 09/09/08 & gross $\alpha$ & $6.5 \mathrm{E}-04 \pm 5.2 \mathrm{E}-04$ & & & & & \\
\hline & 08/26/08 & 09/09/08 & gross $\beta$ & $1.2 \mathrm{E}-02 \pm 1.6 \mathrm{E}-03$ & & & & & \\
\hline & 09/09/08 & 09/23/08 & gross $\alpha$ & $1.2 \mathrm{E}-03 \pm 8.0 \mathrm{E}-04$ & & & & & \\
\hline & 09/09/08 & 09/23/08 & gross $\beta$ & $3.6 \mathrm{E}-02 \pm 3.8 \mathrm{E}-03$ & & & & & \\
\hline & 09/23/08 & $10 / 08 / 08$ & gross $\alpha$ & $1.7 \mathrm{E}-03 \pm 5.9 \mathrm{E}-04$ & & & & & \\
\hline & $09 / 23 / 08$ & $10 / 08 / 08$ & gross $\beta$ & $2.8 \mathrm{E}-02 \pm 2.9 \mathrm{E}-03$ & & & & & \\
\hline & $10 / 08 / 08$ & $10 / 21 / 08$ & gross $\alpha$ & $1.8 \mathrm{E}-03 \pm 7.2 \mathrm{E}-04$ & & & & & \\
\hline & $10 / 08 / 08$ & $10 / 21 / 08$ & gross $\beta$ & $1.9 \mathrm{E}-02 \pm 2.4 \mathrm{E}-03$ & & & & & \\
\hline & $10 / 21 / 08$ & $11 / 04 / 08$ & gross $\alpha$ & $2.7 \mathrm{E}-03 \pm 8.0 \mathrm{E}-04$ & & & & & \\
\hline & $10 / 21 / 08$ & $11 / 04 / 08$ & gross $\beta$ & $3.9 \mathrm{E}-02 \pm 3.7 \mathrm{E}-03$ & & & & & \\
\hline & $11 / 04 / 08$ & $11 / 18 / 08$ & gross $\alpha$ & $2.4 \mathrm{E}-03 \pm 7.7 \mathrm{E}-04$ & & & & & \\
\hline & $11 / 04 / 08$ & $11 / 18 / 08$ & gross $\beta$ & $2.3 \mathrm{E}-02 \pm 2.6 \mathrm{E}-03$ & & & & & \\
\hline & $11 / 18 / 08$ & $12 / 02 / 08$ & gross $\alpha$ & $2.2 \mathrm{E}-03 \pm 7.5 \mathrm{E}-04$ & & & & & \\
\hline & $11 / 18 / 08$ & $12 / 02 / 08$ & gross $\beta$ & $3.0 \mathrm{E}-02 \pm 3.1 \mathrm{E}-03$ & & & & & \\
\hline & $12 / 02 / 08$ & $12 / 16 / 08$ & gross $\alpha$ & $1.3 \mathrm{E}-03 \pm 5.6 \mathrm{E}-04$ & & & & & \\
\hline & $12 / 02 / 08$ & $12 / 16 / 08$ & gross $\beta$ & $2.1 \mathrm{E}-02 \pm 2.4 \mathrm{E}-03$ & & & & & \\
\hline & $12 / 16 / 08$ & $12 / 30 / 08$ & gross $\alpha$ & $2.3 \mathrm{E}-03 \pm 7.4 \mathrm{E}-04$ & & & & & \\
\hline & $12 / 16 / 08$ & $12 / 30 / 08$ & gross $\beta$ & $3.1 \mathrm{E}-02 \pm 3.2 \mathrm{E}-03$ & & & & & \\
\hline
\end{tabular}

$\overline{\mathrm{RQ}}=$ Result Qualifier. $\mathrm{U}=$ The analyte was analyzed for but not detected. 
Table 2-4. Near-Facility Air Sampling Results, 2008 (pCi/m³ \pm total analytical uncertainty). (Sheet 21 of 82)

\begin{tabular}{|c|c|c|c|c|c|c|c|c|c|c|}
\hline Location & Sample On & Sample Off & Isotope & Result \pm Uncertainty & Location & Composite & e Period & Isotope & Result \pm Uncertainty & $\mathbf{R Q}^{*}$ \\
\hline N106 & $01 / 02 / 08$ & $01 / 15 / 08$ & gross $\alpha$ & $7.4 \mathrm{E}-04 \pm 5.5 \mathrm{E}-04$ & N106 & $01 / 02 / 08$ to & $07 / 01 / 08$ & ${ }^{241} \mathrm{Am}$ & $4.9 \mathrm{E}-06 \pm 4.1 \mathrm{E}-06$ & \\
\hline \multirow[t]{51}{*}{$(100-\mathrm{N})$} & 01/02/08 & 01/15/08 & gross $\beta$ & $7.6 \mathrm{E}-03 \pm 1.3 \mathrm{E}-03$ & & & & ${ }^{60} \mathrm{Co}$ & $7.2 \mathrm{E}-06 \pm 6.3 \mathrm{E}-05$ & $\mathrm{U}$ \\
\hline & 01/15/08 & 01/30/08 & gross $\alpha$ & $2.4 \mathrm{E}-03 \pm 7.3 \mathrm{E}-04$ & & & & ${ }^{134} \mathrm{Cs}$ & $6.7 \mathrm{E}-06 \pm 6.2 \mathrm{E}-05$ & $\mathrm{U}$ \\
\hline & 01/15/08 & 01/30/08 & gross $\beta$ & $3.1 \mathrm{E}-02 \pm 3.1 \mathrm{E}-03$ & & & & ${ }^{137} \mathrm{Cs}$ & $-3.0 \mathrm{E}-05 \pm 5.6 \mathrm{E}-05$ & $\mathrm{U}$ \\
\hline & 01/30/08 & $02 / 13 / 08$ & gross $\alpha$ & $4.0 \mathrm{E}-04 \pm 4.5 \mathrm{E}-04$ & & & & ${ }^{152} \mathrm{Eu}$ & $5.2 \mathrm{E}-05 \pm 1.4 \mathrm{E}-04$ & $\mathrm{U}$ \\
\hline & 01/30/08 & $02 / 13 / 08$ & gross $\beta$ & $2.9 \mathrm{E}-03 \pm 7.5 \mathrm{E}-04$ & & & & ${ }^{154} \mathrm{Eu}$ & $-2.6 \mathrm{E}-05 \pm 1.9 \mathrm{E}-04$ & $\mathrm{U}$ \\
\hline & 02/13/08 & 02/27/08 & gross $\alpha$ & $1.9 \mathrm{E}-03 \pm 6.4 \mathrm{E}-04$ & & & & ${ }^{155} \mathrm{Eu}$ & $-4.7 \mathrm{E}-05 \pm 1.5 \mathrm{E}-04$ & $\mathrm{U}$ \\
\hline & 02/13/08 & $02 / 27 / 08$ & gross $\beta$ & $2.9 \mathrm{E}-02 \pm 3.0 \mathrm{E}-03$ & & & & ${ }^{238} \mathrm{Pu}$ & $7.9 \mathrm{E}-06 \pm 1.1 \mathrm{E}-05$ & $\mathrm{U}$ \\
\hline & $02 / 27 / 08$ & 03/12/08 & gross $\alpha$ & $1.6 \mathrm{E}-03 \pm 6.1 \mathrm{E}-04$ & & & & ${ }^{239 / 240} \mathrm{Pu}$ & $3.2 \mathrm{E}-06 \pm 4.6 \mathrm{E}-06$ & $\mathrm{U}$ \\
\hline & $02 / 27 / 08$ & 03/12/08 & gross $\beta$ & $1.0 \mathrm{E}-02 \pm 1.5 \mathrm{E}-03$ & & & & ${ }^{106} \mathrm{Ru}$ & $3.1 \mathrm{E}-04 \pm 5.2 \mathrm{E}-04$ & $\mathrm{U}$ \\
\hline & 03/12/08 & 03/26/08 & gross $\alpha$ & $1.2 \mathrm{E}-03 \pm 5.4 \mathrm{E}-04$ & & & & ${ }^{125} \mathrm{Sb}$ & $-5.2 \mathrm{E}-05 \pm 1.2 \mathrm{E}-04$ & $\mathrm{U}$ \\
\hline & 03/12/08 & 03/26/08 & gross $\beta$ & $9.2 \mathrm{E}-03 \pm 1.4 \mathrm{E}-03$ & & & & ${ }^{90} \mathrm{Sr}$ & $-9.8 \mathrm{E}-05 \pm 1.0 \mathrm{E}-04$ & $\mathrm{U}$ \\
\hline & 03/26/08 & 04/08/08 & gross $\alpha$ & $6.6 \mathrm{E}-04 \pm 5.4 \mathrm{E}-04$ & & & & ${ }^{234} \mathrm{U}$ & $1.0 \mathrm{E}-05 \pm 6.4 \mathrm{E}-06$ & \\
\hline & 03/26/08 & $04 / 08 / 08$ & gross $\beta$ & $6.1 \mathrm{E}-03 \pm 1.1 \mathrm{E}-03$ & & & & ${ }^{235} \mathrm{U}$ & $3.5 \mathrm{E}-06 \pm 4.0 \mathrm{E}-06$ & $\mathrm{U}$ \\
\hline & 04/08/08 & $04 / 23 / 08$ & gross $\alpha$ & $8.9 \mathrm{E}-04 \pm 5.5 \mathrm{E}-04$ & & & & ${ }^{238} \mathrm{U}$ & $9.1 \mathrm{E}-06 \pm 6.3 \mathrm{E}-06$ & \\
\hline & 04/08/08 & $04 / 23 / 08$ & gross $\beta$ & $9.7 \mathrm{E}-03 \pm 1.4 \mathrm{E}-03$ & & & & & & \\
\hline & 04/23/08 & 05/07/08 & gross $\alpha$ & $1.7 \mathrm{E}-03 \pm 6.1 \mathrm{E}-04$ & N106 & $07 / 01 / 08$ to & $12 / 30 / 08$ & ${ }^{241} \mathrm{Am}$ & $4.9 \mathrm{E}-06 \pm 4.3 \mathrm{E}-06$ & $\mathrm{U}$ \\
\hline & 04/23/08 & 05/07/08 & gross $\beta$ & $1.4 \mathrm{E}-02 \pm 1.8 \mathrm{E}-03$ & & & & ${ }^{60} \mathrm{Co}$ & $-7.8 \mathrm{E}-05 \pm 1.1 \mathrm{E}-04$ & $\mathrm{U}$ \\
\hline & 05/07/08 & $05 / 20 / 08$ & gross $\alpha$ & $1.0 \mathrm{E}-03 \pm 6.2 \mathrm{E}-04$ & & & & ${ }^{134} \mathrm{Cs}$ & $6.7 \mathrm{E}-05 \pm 1.0 \mathrm{E}-04$ & $\mathrm{U}$ \\
\hline & 05/07/08 & 05/20/08 & gross $\beta$ & $1.1 \mathrm{E}-02 \pm 1.6 \mathrm{E}-03$ & & & & ${ }^{137} \mathrm{Cs}$ & $1.2 \mathrm{E}-05 \pm 9.0 \mathrm{E}-05$ & $\mathrm{U}$ \\
\hline & 05/20/08 & $06 / 04 / 08$ & gross $\alpha$ & $1.1 \mathrm{E}-03 \pm 4.7 \mathrm{E}-04$ & & & & ${ }^{152} \mathrm{Eu}$ & $-4.2 \mathrm{E}-05 \pm 2.4 \mathrm{E}-04$ & $\mathrm{U}$ \\
\hline & 05/20/08 & $06 / 04 / 08$ & gross $\beta$ & $1.0 \mathrm{E}-02 \pm 1.4 \mathrm{E}-03$ & & & & ${ }^{154} \mathrm{Eu}$ & $3.0 \mathrm{E}-04 \pm 3.4 \mathrm{E}-04$ & $\mathrm{U}$ \\
\hline & $06 / 04 / 08$ & 06/17/08 & gross $\alpha$ & $5.2 \mathrm{E}-04 \pm 5.0 \mathrm{E}-04$ & & & & ${ }^{155} \mathrm{Eu}$ & $-5.5 \mathrm{E}-05 \pm 1.8 \mathrm{E}-04$ & $\mathrm{U}$ \\
\hline & 06/04/08 & 06/17/08 & gross $\beta$ & $6.7 \mathrm{E}-03 \pm 1.2 \mathrm{E}-03$ & & & & ${ }^{238} \mathrm{Pu}$ & $-7.0 \mathrm{E}-07 \pm 7.0 \mathrm{E}-06$ & $\mathrm{U}$ \\
\hline & 06/17/08 & 07/01/08 & gross $\alpha$ & $1.4 \mathrm{E}-03 \pm 5.8 \mathrm{E}-04$ & & & & ${ }^{239 / 240} \mathrm{Pu}$ & $2.1 \mathrm{E}-06 \pm 3.8 \mathrm{E}-06$ & $\mathrm{U}$ \\
\hline & 06/17/08 & 07/01/08 & gross $\beta$ & $1.2 \mathrm{E}-02 \pm 1.6 \mathrm{E}-03$ & & & & ${ }^{106} \mathrm{Ru}$ & $5.1 \mathrm{E}-04 \pm 8.3 \mathrm{E}-04$ & $\mathrm{U}$ \\
\hline & 07/01/08 & $07 / 15 / 08$ & gross $\alpha$ & $1.5 \mathrm{E}-03 \pm 6.3 \mathrm{E}-04$ & & & & ${ }^{125} \mathrm{Sb}$ & $-4.0 \mathrm{E}-05 \pm 2.3 \mathrm{E}-04$ & $\mathrm{U}$ \\
\hline & 07/01/08 & 07/15/08 & gross $\beta$ & $1.4 \mathrm{E}-02 \pm 1.8 \mathrm{E}-03$ & & & & ${ }^{90} \mathrm{Sr}$ & $-1.9 \mathrm{E}-05 \pm 2.0 \mathrm{E}-05$ & $\mathrm{U}$ \\
\hline & 07/15/08 & 07/29/08 & gross $\alpha$ & $7.2 \mathrm{E}-04 \pm 5.2 \mathrm{E}-04$ & & & & ${ }^{234} \mathrm{U}$ & $1.7 \mathrm{E}-05 \pm 9.5 \mathrm{E}-06$ & \\
\hline & 07/15/08 & 07/29/08 & gross $\beta$ & $1.1 \mathrm{E}-02 \pm 1.5 \mathrm{E}-03$ & & & & ${ }^{235} \mathrm{U}$ & $6.0 \mathrm{E}-06 \pm 4.8 \mathrm{E}-06$ & \\
\hline & 07/29/08 & 08/12/08 & gross $\alpha$ & $1.9 \mathrm{E}-03 \pm 6.5 \mathrm{E}-04$ & & & & ${ }^{238} \mathrm{U}$ & $1.6 \mathrm{E}-05 \pm 9.0 \mathrm{E}-06$ & \\
\hline & 07/29/08 & 08/12/08 & gross $\beta$ & $1.3 \mathrm{E}-02 \pm 1.7 \mathrm{E}-03$ & & & & & & \\
\hline & 08/12/08 & 08/26/08 & gross $\alpha$ & $6.3 \mathrm{E}-04 \pm 5.0 \mathrm{E}-04$ & & & & & & \\
\hline & 08/12/08 & 08/26/08 & gross $\beta$ & $1.3 \mathrm{E}-02 \pm 1.7 \mathrm{E}-03$ & & & & & & \\
\hline & 08/26/08 & 09/09/08 & gross $\alpha$ & $5.2 \mathrm{E}-04 \pm 4.7 \mathrm{E}-04$ & & & & & & \\
\hline & 08/26/08 & 09/09/08 & gross $\beta$ & $1.0 \mathrm{E}-02 \pm 1.5 \mathrm{E}-03$ & & & & & & \\
\hline & 09/09/08 & 09/23/08 & gross $\alpha$ & $1.4 \mathrm{E}-03 \pm 5.7 \mathrm{E}-04$ & & & & & & \\
\hline & 09/09/08 & 09/23/08 & gross $\beta$ & $2.4 \mathrm{E}-02 \pm 2.6 \mathrm{E}-03$ & & & & & & \\
\hline & 09/23/08 & $10 / 08 / 08$ & gross $\alpha$ & $1.5 \mathrm{E}-03 \pm 5.6 \mathrm{E}-04$ & & & & & & \\
\hline & 09/23/08 & $10 / 08 / 08$ & gross $\beta$ & $2.3 \mathrm{E}-02 \pm 2.6 \mathrm{E}-03$ & & & & & & \\
\hline & $10 / 08 / 08$ & $10 / 21 / 08$ & gross $\alpha$ & $1.7 \mathrm{E}-03 \pm 6.4 \mathrm{E}-04$ & & & & & & \\
\hline & $10 / 08 / 08$ & $10 / 21 / 08$ & gross $\beta$ & $2.0 \mathrm{E}-02 \pm 2.3 \mathrm{E}-03$ & & & & & & \\
\hline & $10 / 21 / 08$ & $11 / 04 / 08$ & gross $\alpha$ & $2.5 \mathrm{E}-03 \pm 7.7 \mathrm{E}-04$ & & & & & & \\
\hline & $10 / 21 / 08$ & $11 / 04 / 08$ & gross $\beta$ & $4.0 \mathrm{E}-02 \pm 3.8 \mathrm{E}-03$ & & & & & & \\
\hline & $11 / 04 / 08$ & $11 / 18 / 08$ & gross $\alpha$ & $1.6 \mathrm{E}-03 \pm 6.1 \mathrm{E}-04$ & & & & & & \\
\hline & $11 / 04 / 08$ & $11 / 18 / 08$ & gross $\beta$ & $2.3 \mathrm{E}-02 \pm 2.6 \mathrm{E}-03$ & & & & & & \\
\hline & $11 / 18 / 08$ & $12 / 02 / 08$ & gross $\alpha$ & $2.6 \mathrm{E}-03 \pm 7.9 \mathrm{E}-04$ & & & & & & \\
\hline & $11 / 18 / 08$ & $12 / 02 / 08$ & gross $\beta$ & $2.8 \mathrm{E}-02 \pm 2.9 \mathrm{E}-03$ & & & & & & \\
\hline & $12 / 02 / 08$ & $12 / 16 / 08$ & gross $\alpha$ & $1.3 \mathrm{E}-03 \pm 5.6 \mathrm{E}-04$ & & & & & & \\
\hline & $12 / 02 / 08$ & $12 / 16 / 08$ & gross $\beta$ & $2.9 \mathrm{E}-02 \pm 3.0 \mathrm{E}-03$ & & & & & & \\
\hline & $12 / 16 / 08$ & $12 / 30 / 08$ & gross $\alpha$ & $2.0 \mathrm{E}-03 \pm 6.9 \mathrm{E}-04$ & & & & & & \\
\hline & $12 / 16 / 08$ & $12 / 30 / 08$ & gross $\beta$ & $3.2 \mathrm{E}-02 \pm 3.2 \mathrm{E}-03$ & & & & & & \\
\hline
\end{tabular}

$\overline{\mathrm{RQ}}=$ Result Qualifier. $\mathrm{U}=$ The analyte was analyzed for but not detected. 
Table 2-4. Near-Facility Air Sampling Results, 2008 (pCi/m³ \pm total analytical uncertainty). (Sheet 22 of 82)

\begin{tabular}{ccclc} 
Location & Sample On & Sample Off & Isotope & Result \pm Uncertainty \\
\hline N565 & $01 / 24 / 08$ & $02 / 13 / 08$ & gross $\alpha$ & $7.8 \mathrm{E}-04 \pm 3.7 \mathrm{E}-04$ \\
$(100-\mathrm{IU} 2 / 6)$ & $01 / 24 / 08$ & $02 / 13 / 08$ & gross $\beta$ & $1.8 \mathrm{E}-02 \pm 2.2 \mathrm{E}-03$ \\
& $02 / 13 / 08$ & $02 / 27 / 08$ & gross $\alpha$ & $1.2 \mathrm{E}-03 \pm 5.2 \mathrm{E}-04$ \\
& $02 / 13 / 08$ & $02 / 27 / 08$ & gross $\beta$ & $2.5 \mathrm{E}-02 \pm 2.9 \mathrm{E}-03$ \\
& $02 / 27 / 08$ & $03 / 12 / 08$ & gross $\alpha$ & $1.2 \mathrm{E}-03 \pm 5.4 \mathrm{E}-04$ \\
& $02 / 27 / 08$ & $03 / 12 / 08$ & gross $\beta$ & $1.1 \mathrm{E}-02 \pm 1.7 \mathrm{E}-03$ \\
& $03 / 12 / 08$ & $03 / 26 / 08$ & gross $\alpha$ & $7.5 \mathrm{E}-04 \pm 5.5 \mathrm{E}-04$ \\
& $03 / 12 / 08$ & $03 / 26 / 08$ & gross $\beta$ & $8.3 \mathrm{E}-03 \pm 1.4 \mathrm{E}-03$ \\
& $03 / 26 / 08$ & $04 / 02 / 08$ & gross $\alpha$ & $9.3 \mathrm{E}-04 \pm 8.8 \mathrm{E}-04$ \\
& $03 / 26 / 08$ & $04 / 02 / 08$ & gross $\beta$ & $7.2 \mathrm{E}-03 \pm 1.7 \mathrm{E}-03$ \\
& & & & \\
N566 & & & & \\
$(100-\mathrm{IU} 2 / 6)$ & $01 / 24 / 08$ & $02 / 13 / 08$ & gross $\alpha$ & $1.6 \mathrm{E}-03 \pm 5.1 \mathrm{E}-04$ \\
& $01 / 24 / 08$ & $02 / 13 / 08$ & gross $\beta$ & $1.9 \mathrm{E}-02 \pm 2.2 \mathrm{E}-03$ \\
& $02 / 13 / 08$ & $02 / 27 / 08$ & gross $\alpha$ & $1.2 \mathrm{E}-03 \pm 5.2 \mathrm{E}-04$ \\
& $02 / 13 / 08$ & $02 / 27 / 08$ & gross $\beta$ & $2.4 \mathrm{E}-02 \pm 2.9 \mathrm{E}-03$ \\
& $02 / 27 / 08$ & $03 / 12 / 08$ & gross $\alpha$ & $1.2 \mathrm{E}-03 \pm 5.4 \mathrm{E}-04$ \\
& $02 / 27 / 08$ & $03 / 12 / 08$ & gross $\beta$ & $1.0 \mathrm{E}-02 \pm 1.6 \mathrm{E}-03$ \\
& $03 / 12 / 08$ & $03 / 26 / 08$ & gross $\alpha$ & $9.8 \mathrm{E}-04 \pm 6.1 \mathrm{E}-04$ \\
& $03 / 12 / 08$ & $03 / 26 / 08$ & gross $\beta$ & $7.5 \mathrm{E}-03 \pm 1.3 \mathrm{E}-03$ \\
& $03 / 26 / 08$ & $04 / 02 / 08$ & gross $\alpha$ & $3.0 \mathrm{E}-04 \pm 6.5 \mathrm{E}-04$ \\
& $03 / 26 / 08$ & $04 / 02 / 08$ & gross $\beta$ & $9.7 \mathrm{E}-03 \pm 2.0 \mathrm{E}-03$ \\
& & & &
\end{tabular}

\begin{tabular}{|c|c|c|c|c|}
\hline Location & Composite Period & Isotope & Result \pm Uncertainty & $\mathbf{R Q}^{*}$ \\
\hline \multirow[t]{15}{*}{ N565 } & $01 / 24 / 08$ to $04 / 02 / 08$ & ${ }^{241} \mathrm{Am}$ & $9.3 \mathrm{E}-06 \pm 1.0 \mathrm{E}-05$ & $\mathrm{U}$ \\
\hline & & ${ }^{60} \mathrm{Co}$ & $1.2 \mathrm{E}-04 \pm 2.6 \mathrm{E}-04$ & $\mathrm{U}$ \\
\hline & & ${ }^{134} \mathrm{Cs}$ & $2.1 \mathrm{E}-04 \pm 3.0 \mathrm{E}-04$ & $\mathrm{U}$ \\
\hline & & ${ }^{137} \mathrm{Cs}$ & $-1.6 \mathrm{E}-04 \pm 2.7 \mathrm{E}-04$ & $\mathrm{U}$ \\
\hline & & ${ }^{152} \mathrm{Eu}$ & $1.9 \mathrm{E}-04 \pm 6.3 \mathrm{E}-04$ & $\mathrm{U}$ \\
\hline & & ${ }^{154} \mathrm{Eu}$ & $-4.6 \mathrm{E}-04 \pm 8.0 \mathrm{E}-04$ & $\mathrm{U}$ \\
\hline & & ${ }^{155} \mathrm{Eu}$ & $1.0 \mathrm{E}-04 \pm 4.7 \mathrm{E}-04$ & $\mathrm{U}$ \\
\hline & & ${ }^{238} \mathrm{Pu}$ & $8.0 \mathrm{E}-06 \pm 2.3 \mathrm{E}-05$ & $\mathrm{U}$ \\
\hline & & ${ }^{239 / 240} \mathrm{Pu}$ & $4.0 \mathrm{E}-06 \pm 5.8 \mathrm{E}-06$ & $\mathrm{U}$ \\
\hline & & ${ }^{106} \mathrm{Ru}$ & $1.1 \mathrm{E}-04 \pm 1.1 \mathrm{E}-03$ & $\mathrm{U}$ \\
\hline & & ${ }^{125} \mathrm{Sb}$ & $-2.1 \mathrm{E}-04 \pm 6.3 \mathrm{E}-04$ & $\mathrm{U}$ \\
\hline & & ${ }^{90} \mathrm{Sr}$ & $-6.1 \mathrm{E}-04 \pm 6.3 \mathrm{E}-04$ & $\mathrm{U}$ \\
\hline & & ${ }^{234} \mathrm{U}$ & $6.5 \mathrm{E}-06 \pm 1.3 \mathrm{E}-05$ & $\mathrm{U}$ \\
\hline & & ${ }^{235} \mathrm{U}$ & $1.4 \mathrm{E}-05 \pm 1.2 \mathrm{E}-05$ & \\
\hline & & ${ }^{238} \mathrm{U}$ & $6.5 \mathrm{E}-06 \pm 9.5 \mathrm{E}-06$ & $\mathrm{U}$ \\
\hline \multirow[t]{15}{*}{ N566 } & $01 / 24 / 08$ to $04 / 02 / 08$ & ${ }^{241} \mathrm{Am}$ & $1.6 \mathrm{E}-05 \pm 1.2 \mathrm{E}-05$ & \\
\hline & & ${ }^{60} \mathrm{Co}$ & $1.7 \mathrm{E}-05 \pm 1.7 \mathrm{E}-04$ & $\mathrm{U}$ \\
\hline & & ${ }^{134} \mathrm{Cs}$ & $-2.5 \mathrm{E}-05 \pm 1.9 \mathrm{E}-04$ & $\mathrm{U}$ \\
\hline & & ${ }^{137} \mathrm{Cs}$ & $6.9 \mathrm{E}-05 \pm 1.6 \mathrm{E}-04$ & $\mathrm{U}$ \\
\hline & & ${ }^{152} \mathrm{Eu}$ & $-1.1 \mathrm{E}-04 \pm 4.0 \mathrm{E}-04$ & $\mathrm{U}$ \\
\hline & & ${ }^{154} \mathrm{Eu}$ & $-6.1 \mathrm{E}-05 \pm 5.0 \mathrm{E}-04$ & $\mathrm{U}$ \\
\hline & & ${ }^{155} \mathrm{Eu}$ & $1.7 \mathrm{E}-04 \pm 4.0 \mathrm{E}-04$ & $\mathrm{U}$ \\
\hline & & ${ }^{238} \mathrm{Pu}$ & $-2.3 \mathrm{E}-06 \pm 2.3 \mathrm{E}-05$ & $\mathrm{U}$ \\
\hline & & ${ }^{239 / 240} \mathrm{Pu}$ & $2.3 \mathrm{E}-06 \pm 4.7 \mathrm{E}-06$ & $\mathrm{U}$ \\
\hline & & ${ }^{106} \mathrm{Ru}$ & $1.1 \mathrm{E}-03 \pm 1.6 \mathrm{E}-03$ & $\mathrm{U}$ \\
\hline & & ${ }^{125} \mathrm{Sb}$ & $2.9 \mathrm{E}-04 \pm 3.6 \mathrm{E}-04$ & $\mathrm{U}$ \\
\hline & & ${ }^{90} \mathrm{Sr}$ & $-4.8 \mathrm{E}-04 \pm 4.9 \mathrm{E}-04$ & $\mathrm{U}$ \\
\hline & & ${ }^{234} \mathrm{U}$ & $2.4 \mathrm{E}-05 \pm 1.5 \mathrm{E}-05$ & \\
\hline & & ${ }^{235} \mathrm{U}$ & $7.4 \mathrm{E}-06 \pm 7.8 \mathrm{E}-06$ & \\
\hline & & ${ }^{238} \mathrm{U}$ & $1.7 \mathrm{E}-05 \pm 1.2 \mathrm{E}-05$ & \\
\hline
\end{tabular}

$\overline{\mathrm{RQ}}$ = Result Qualifier. $\mathrm{U}=$ The analyte was analyzed for but not detected. 
Table 2-4. Near-Facility Air Sampling Results, 2008 (pCi/m³ \pm total analytical uncertainty). (Sheet 23 of 82)

\begin{tabular}{|c|c|c|c|c|c|c|c|c|c|}
\hline Location & Sample On & Sample Off & Isotope & Result \pm Uncertainty & Location & Composite Period & Isotope & Result \pm Uncertainty & $\mathbf{R Q *}$ \\
\hline N019 & $12 / 31 / 07$ & $01 / 14 / 08$ & gross $\alpha$ & $1.1 \mathrm{E}-03 \pm 5.0 \mathrm{E}-04$ & N019 & $12 / 31 / 07$ to $06 / 30 / 08$ & ${ }^{60} \mathrm{Co}$ & $-5.4 \mathrm{E}-05 \pm 9.9 \mathrm{E}-05$ & $\mathrm{U}$ \\
\hline \multirow[t]{43}{*}{$(200-\mathrm{E})$} & $12 / 31 / 07$ & 01/14/08 & gross $\beta$ & $7.6 \mathrm{E}-03 \pm 1.2 \mathrm{E}-03$ & & & ${ }^{134} \mathrm{Cs}$ & $-4.2 \mathrm{E}-06 \pm 4.2 \mathrm{E}-05$ & $\mathrm{U}$ \\
\hline & 01/14/08 & $01 / 29 / 08$ & gross $\alpha$ & $1.7 \mathrm{E}-03 \pm 6.1 \mathrm{E}-04$ & & & ${ }^{137} \mathrm{Cs}$ & $1.9 \mathrm{E}-05 \pm 7.3 \mathrm{E}-05$ & $\mathrm{U}$ \\
\hline & $01 / 14 / 08$ & 01/29/08 & gross $\beta$ & $3.2 \mathrm{E}-02 \pm 3.2 \mathrm{E}-03$ & & & ${ }^{152} \mathrm{Eu}$ & $2.2 \mathrm{E}-05 \pm 1.5 \mathrm{E}-04$ & $\mathrm{U}$ \\
\hline & $01 / 29 / 08$ & $02 / 12 / 08$ & gross $\alpha$ & $5.7 \mathrm{E}-05 \pm 3.0 \mathrm{E}-04$ & & & ${ }^{154} \mathrm{Eu}$ & $8.8 \mathrm{E}-05 \pm 1.9 \mathrm{E}-04$ & $\mathrm{U}$ \\
\hline & $01 / 29 / 08$ & $02 / 12 / 08$ & gross $\beta$ & $4.7 \mathrm{E}-03 \pm 9.6 \mathrm{E}-04$ & & & ${ }^{155} \mathrm{Eu}$ & $-1.1 \mathrm{E}-04 \pm 1.7 \mathrm{E}-04$ & $\mathrm{U}$ \\
\hline & $02 / 12 / 08$ & $02 / 25 / 08$ & gross $\alpha$ & $7.8 \mathrm{E}-04 \pm 5.7 \mathrm{E}-04$ & & & ${ }^{238} \mathrm{Pu}$ & $-4.6 \mathrm{E}-06 \pm 9.1 \mathrm{E}-06$ & $\mathrm{U}$ \\
\hline & $02 / 12 / 08$ & $02 / 25 / 08$ & gross $\beta$ & $2.9 \mathrm{E}-02 \pm 3.1 \mathrm{E}-03$ & & & ${ }^{239 / 240} \mathrm{Pu}$ & $6.6 \mathrm{E}-07 \pm 6.6 \mathrm{E}-06$ & $\mathrm{U}$ \\
\hline & $02 / 25 / 08$ & 03/11/08 & gross $\alpha$ & $1.0 \mathrm{E}-03 \pm 4.8 \mathrm{E}-04$ & & & ${ }^{106} \mathrm{Ru}$ & $-2.4 \mathrm{E}-04 \pm 5.8 \mathrm{E}-04$ & $\mathrm{U}$ \\
\hline & $02 / 25 / 08$ & 03/11/08 & gross $\beta$ & $1.5 \mathrm{E}-02 \pm 1.9 \mathrm{E}-03$ & & & ${ }^{125} \mathrm{Sb}$ & $-6.0 \mathrm{E}-05 \pm 1.4 \mathrm{E}-04$ & $\mathrm{U}$ \\
\hline & 03/11/08 & 03/25/08 & gross $\alpha$ & $1.3 \mathrm{E}-03 \pm 5.5 \mathrm{E}-04$ & & & ${ }^{90} \mathrm{Sr}$ & $-2.3 \mathrm{E}-04 \pm 2.4 \mathrm{E}-04$ & $\mathrm{U}$ \\
\hline & 03/11/08 & $03 / 25 / 08$ & gross $\beta$ & $9.9 \mathrm{E}-03 \pm 1.5 \mathrm{E}-03$ & & & ${ }^{234} \mathrm{U}$ & $1.3 \mathrm{E}-05 \pm 8.8 \mathrm{E}-06$ & \\
\hline & 03/25/08 & $04 / 07 / 08$ & gross $\alpha$ & $1.4 \mathrm{E}-03 \pm 5.9 \mathrm{E}-04$ & & & ${ }^{235} \mathrm{U}$ & $6.8 \mathrm{E}-06 \pm 5.2 \mathrm{E}-06$ & \\
\hline & $03 / 25 / 08$ & $04 / 07 / 08$ & gross $\beta$ & $1.3 \mathrm{E}-02 \pm 1.8 \mathrm{E}-03$ & & & ${ }^{238} \mathrm{U}$ & $8.9 \mathrm{E}-06 \pm 6.0 \mathrm{E}-06$ & \\
\hline & 04/07/08 & $04 / 21 / 08$ & gross $\alpha$ & $1.5 \mathrm{E}-03 \pm 5.9 \mathrm{E}-04$ & & & & & \\
\hline & $04 / 07 / 08$ & $04 / 21 / 08$ & gross $\beta$ & $1.0 \mathrm{E}-02 \pm 1.5 \mathrm{E}-03$ & N019 & $06 / 30 / 08$ to $12 / 30 / 08$ & ${ }^{60} \mathrm{Co}$ & $-6.4 \mathrm{E}-05 \pm 1.3 \mathrm{E}-04$ & $\mathrm{U}$ \\
\hline & $04 / 21 / 08$ & 05/06/08 & gross $\alpha$ & $1.7 \mathrm{E}-03 \pm 6.0 \mathrm{E}-04$ & & & ${ }^{134} \mathrm{Cs}$ & $6.0 \mathrm{E}-05 \pm 1.1 \mathrm{E}-04$ & $\mathrm{U}$ \\
\hline & $04 / 21 / 08$ & 05/06/08 & gross $\beta$ & $1.6 \mathrm{E}-02 \pm 1.9 \mathrm{E}-03$ & & & ${ }^{137} \mathrm{Cs}$ & $1.6 \mathrm{E}-04 \pm 1.7 \mathrm{E}-04$ & $\mathrm{U}$ \\
\hline & 05/06/08 & 05/19/08 & gross $\alpha$ & $4.2 \mathrm{E}-04 \pm 4.7 \mathrm{E}-04$ & & & ${ }^{152} \mathrm{Eu}$ & $-9.2 \mathrm{E}-05 \pm 2.6 \mathrm{E}-04$ & U \\
\hline & 05/06/08 & 05/19/08 & gross $\beta$ & $1.2 \mathrm{E}-02 \pm 1.7 \mathrm{E}-03$ & & & ${ }^{154} \mathrm{Eu}$ & $8.0 \mathrm{E}-05 \pm 3.6 \mathrm{E}-04$ & $\mathrm{U}$ \\
\hline & 05/19/08 & $06 / 02 / 08$ & gross $\alpha$ & $1.2 \mathrm{E}-03 \pm 5.1 \mathrm{E}-04$ & & & ${ }^{155} \mathrm{Eu}$ & $2.0 \mathrm{E}-05 \pm 2.0 \mathrm{E}-04$ & $\mathrm{U}$ \\
\hline & 05/19/08 & $06 / 02 / 08$ & gross $\beta$ & $1.4 \mathrm{E}-02 \pm 1.8 \mathrm{E}-03$ & & & ${ }^{238} \mathrm{Pu}$ & $8.2 \mathrm{E}-06 \pm 2.2 \mathrm{E}-05$ & $\mathrm{U}$ \\
\hline & $06 / 02 / 08$ & $06 / 16 / 08$ & gross $\alpha$ & $9.7 \mathrm{E}-04 \pm 6.0 \mathrm{E}-04$ & & & ${ }^{239 / 240} \mathrm{Pu}$ & $4.1 \mathrm{E}-06 \pm 6.3 \mathrm{E}-06$ & $\mathrm{U}$ \\
\hline & $06 / 02 / 08$ & 06/16/08 & gross $\beta$ & $8.2 \mathrm{E}-03 \pm 1.3 \mathrm{E}-03$ & & & ${ }^{106} \mathrm{Ru}$ & $5.7 \mathrm{E}-04 \pm 9.4 \mathrm{E}-04$ & $\mathrm{U}$ \\
\hline & $06 / 16 / 08$ & 06/30/08 & gross $\alpha$ & $1.6 \mathrm{E}-03 \pm 6.0 \mathrm{E}-04$ & & & ${ }^{125} \mathrm{Sb}$ & $-1.1 \mathrm{E}-04 \pm 2.3 \mathrm{E}-04$ & $\mathrm{U}$ \\
\hline & $06 / 16 / 08$ & 06/30/08 & gross $\beta$ & $1.5 \mathrm{E}-02 \pm 1.9 \mathrm{E}-03$ & & & ${ }^{90} \mathrm{Sr}$ & $-4.1 \mathrm{E}-04 \pm 4.2 \mathrm{E}-04$ & $\mathrm{U}$ \\
\hline & $06 / 30 / 08$ & 07/14/08 & gross $\alpha$ & $2.4 \mathrm{E}-03 \pm 8.3 \mathrm{E}-04$ & & & ${ }^{234} \mathrm{U}$ & $3.2 \mathrm{E}-05 \pm 1.7 \mathrm{E}-05$ & \\
\hline & $06 / 30 / 08$ & 07/14/08 & gross $\beta$ & $1.6 \mathrm{E}-02 \pm 2.0 \mathrm{E}-03$ & & & ${ }^{235} \mathrm{U}$ & $3.6 \mathrm{E}-06 \pm 6.4 \mathrm{E}-06$ & $\mathrm{U}$ \\
\hline & $07 / 14 / 08$ & 07/28/08 & gross $\alpha$ & $1.1 \mathrm{E}-03 \pm 5.0 \mathrm{E}-04$ & & & ${ }^{238} \mathrm{U}$ & $6.6 \mathrm{E}-06 \pm 5.9 \mathrm{E}-06$ & \\
\hline & $07 / 14 / 08$ & $07 / 28 / 08$ & gross $\beta$ & $1.3 \mathrm{E}-02 \pm 1.7 \mathrm{E}-03$ & & & & & \\
\hline & 07/28/08 & 08/11/08 & gross $\alpha$ & 7.4E-04 $\pm 5.3 \mathrm{E}-04$ & & & & & \\
\hline & 07/28/08 & 08/11/08 & gross $\beta$ & $1.5 \mathrm{E}-02 \pm 1.9 \mathrm{E}-03$ & & & & & \\
\hline & $08 / 11 / 08$ & $08 / 25 / 08$ & gross $\alpha$ & $7.2 \mathrm{E}-04 \pm 5.2 \mathrm{E}-04$ & & & & & \\
\hline & 08/11/08 & $08 / 25 / 08$ & gross $\beta$ & $1.3 \mathrm{E}-02 \pm 1.7 \mathrm{E}-03$ & & & & & \\
\hline & $08 / 25 / 08$ & 09/08/08 & gross $\alpha$ & $1.2 \mathrm{E}-03 \pm 5.2 \mathrm{E}-04$ & & & & & \\
\hline & $08 / 25 / 08$ & 09/08/08 & gross $\beta$ & $1.3 \mathrm{E}-02 \pm 1.7 \mathrm{E}-03$ & & & & & \\
\hline & 09/08/08 & 09/22/08 & gross $\alpha$ & $4.8 \mathrm{E}-03 \pm 1.5 \mathrm{E}-03$ & & & & & \\
\hline & 09/08/08 & 09/22/08 & gross $\beta$ & $2.5 \mathrm{E}-02 \pm 3.3 \mathrm{E}-03$ & & & & & \\
\hline & $11 / 17 / 08$ & $12 / 01 / 08$ & gross $\alpha$ & $1.4 \mathrm{E}-03 \pm 6.0 \mathrm{E}-04$ & & & & & \\
\hline & $11 / 17 / 08$ & $12 / 01 / 08$ & gross $\beta$ & $2.6 \mathrm{E}-02 \pm 2.8 \mathrm{E}-03$ & & & & & \\
\hline & $12 / 01 / 08$ & $12 / 15 / 08$ & gross $\alpha$ & $1.5 \mathrm{E}-03 \pm 6.0 \mathrm{E}-04$ & & & & & \\
\hline & $12 / 01 / 08$ & $12 / 15 / 08$ & gross $\beta$ & $1.4 \mathrm{E}-02 \pm 1.9 \mathrm{E}-03$ & & & & & \\
\hline & $12 / 15 / 08$ & $12 / 30 / 08$ & gross $\alpha$ & $1.8 \mathrm{E}-03 \pm 6.5 \mathrm{E}-04$ & & & & & \\
\hline & $12 / 15 / 08$ & $12 / 30 / 08$ & gross $\beta$ & $2.9 \mathrm{E}-02 \pm 3.0 \mathrm{E}-03$ & & & & & \\
\hline
\end{tabular}

$\overline{\mathrm{RQ}}=$ Result Qualifier. $\mathrm{U}=$ The analyte was analyzed for but not detected. 
Table 2-4. Near-Facility Air Sampling Results, 2008 (pCi/m³ \pm total analytical uncertainty). (Sheet 24 of 82)

\begin{tabular}{|c|c|c|c|c|c|c|c|c|c|c|}
\hline Location & Sample On & Sample Off & Isotope & Result \pm Uncertainty & Location & Composite & e Period & Isotope & Result \pm Uncertainty & $\mathbf{R Q *}$ \\
\hline N158 & $12 / 31 / 07$ & $01 / 14 / 08$ & gross $\alpha$ & $1.2 \mathrm{E}-03 \pm 5.2 \mathrm{E}-04$ & N158 & $12 / 31 / 07$ to & $06 / 30 / 08$ & ${ }^{60} \mathrm{Co}$ & $-1.1 \mathrm{E}-04 \pm 1.1 \mathrm{E}-04$ & $\mathrm{U}$ \\
\hline \multirow[t]{51}{*}{$(200-E)$} & $12 / 31 / 07$ & 01/14/08 & gross $\beta$ & $9.6 \mathrm{E}-03 \pm 1.4 \mathrm{E}-03$ & & & & ${ }^{134} \mathrm{Cs}$ & $6.9 \mathrm{E}-05 \pm 8.2 \mathrm{E}-05$ & $\mathrm{U}$ \\
\hline & $01 / 14 / 08$ & 01/29/08 & gross $\alpha$ & $1.0 \mathrm{E}-03 \pm 4.9 \mathrm{E}-04$ & & & & ${ }^{137} \mathrm{Cs}$ & $1.2 \mathrm{E}-04 \pm 1.3 \mathrm{E}-04$ & \\
\hline & $01 / 14 / 08$ & 01/29/08 & gross $\beta$ & $4.0 \mathrm{E}-02 \pm 3.8 \mathrm{E}-03$ & & & & ${ }^{152} \mathrm{Eu}$ & $2.8 \mathrm{E}-05 \pm 1.6 \mathrm{E}-04$ & $\mathrm{U}$ \\
\hline & 01/29/08 & $02 / 12 / 08$ & gross $\alpha$ & $4.0 \mathrm{E}-04 \pm 4.5 \mathrm{E}-04$ & & & & ${ }^{154} \mathrm{Eu}$ & $1.2 \mathrm{E}-04 \pm 2.2 \mathrm{E}-04$ & $\mathrm{U}$ \\
\hline & $01 / 29 / 08$ & $02 / 12 / 08$ & gross $\beta$ & $6.6 \mathrm{E}-03 \pm 1.2 \mathrm{E}-03$ & & & & ${ }^{155} \mathrm{Eu}$ & $-8.2 \mathrm{E}-07 \pm 8.2 \mathrm{E}-06$ & U \\
\hline & $02 / 12 / 08$ & $02 / 25 / 08$ & gross $\alpha$ & $1.1 \mathrm{E}-03 \pm 5.4 \mathrm{E}-04$ & & & & ${ }^{238} \mathrm{Pu}$ & $8.2 \mathrm{E}-06 \pm 1.4 \mathrm{E}-05$ & $\mathrm{U}$ \\
\hline & $02 / 12 / 08$ & $02 / 25 / 08$ & gross $\beta$ & $2.8 \mathrm{E}-02 \pm 3.0 \mathrm{E}-03$ & & & & ${ }^{239 / 240} \mathrm{Pu}$ & $7.4 \mathrm{E}-07 \pm 2.1 \mathrm{E}-06$ & $\mathrm{U}$ \\
\hline & $02 / 25 / 08$ & 03/11/08 & gross $\alpha$ & $1.0 \mathrm{E}-03 \pm 4.8 \mathrm{E}-04$ & & & & ${ }^{106} \mathrm{Ru}$ & $6.0 \mathrm{E}-05 \pm 6.0 \mathrm{E}-04$ & $\mathrm{U}$ \\
\hline & $02 / 25 / 08$ & 03/11/08 & gross $\beta$ & $1.4 \mathrm{E}-02 \pm 1.8 \mathrm{E}-03$ & & & & ${ }^{125} \mathrm{Sb}$ & $-1.3 \mathrm{E}-04 \pm 1.6 \mathrm{E}-04$ & $\mathrm{U}$ \\
\hline & 03/11/08 & 03/25/08 & gross $\alpha$ & $7.4 \mathrm{E}-04 \pm 5.5 \mathrm{E}-04$ & & & & ${ }^{90} \mathrm{Sr}$ & $-2.1 \mathrm{E}-04 \pm 2.1 \mathrm{E}-04$ & $\mathrm{U}$ \\
\hline & 03/11/08 & $03 / 25 / 08$ & gross $\beta$ & $6.9 \mathrm{E}-03 \pm 1.2 \mathrm{E}-03$ & & & & ${ }^{234} \mathrm{U}$ & $8.1 \mathrm{E}-06 \pm 5.6 \mathrm{E}-06$ & \\
\hline & 03/25/08 & $04 / 07 / 08$ & gross $\alpha$ & $9.5 \mathrm{E}-04 \pm 6.4 \mathrm{E}-04$ & & & & ${ }^{235} \mathrm{U}$ & $3.0 \mathrm{E}-06 \pm 4.3 \mathrm{E}-06$ & $\mathrm{U}$ \\
\hline & 03/25/08 & $04 / 07 / 08$ & gross $\beta$ & $9.4 \mathrm{E}-03 \pm 1.5 \mathrm{E}-03$ & & & & ${ }^{238} \mathrm{U}$ & $7.4 \mathrm{E}-06 \pm 5.6 \mathrm{E}-06$ & \\
\hline & 04/07/08 & $04 / 21 / 08$ & gross $\alpha$ & $7.6 \mathrm{E}-04 \pm 5.6 \mathrm{E}-04$ & & & & & & \\
\hline & $04 / 07 / 08$ & $04 / 21 / 08$ & gross $\beta$ & $9.7 \mathrm{E}-03 \pm 1.5 \mathrm{E}-03$ & N158 & $06 / 30 / 08$ to & $12 / 30 / 08$ & ${ }^{60} \mathrm{Co}$ & $4.3 \mathrm{E}-06 \pm 4.3 \mathrm{E}-05$ & $\mathrm{U}$ \\
\hline & $04 / 21 / 08$ & 05/06/08 & gross $\alpha$ & $1.3 \mathrm{E}-03 \pm 5.3 \mathrm{E}-04$ & & & & ${ }^{134} \mathrm{Cs}$ & $5.9 \mathrm{E}-06 \pm 5.9 \mathrm{E}-05$ & $\mathrm{U}$ \\
\hline & $04 / 21 / 08$ & 05/06/08 & gross $\beta$ & $1.3 \mathrm{E}-02 \pm 1.6 \mathrm{E}-03$ & & & & ${ }^{137} \mathrm{Cs}$ & $8.2 \mathrm{E}-05 \pm 7.3 \mathrm{E}-05$ & $\mathrm{U}$ \\
\hline & 05/06/08 & 05/19/08 & gross $\alpha$ & $8.2 \mathrm{E}-04 \pm 6.1 \mathrm{E}-04$ & & & & ${ }^{152} \mathrm{Eu}$ & $3.9 \mathrm{E}-05 \pm 1.6 \mathrm{E}-04$ & U \\
\hline & 05/06/08 & 05/19/08 & gross $\beta$ & $1.1 \mathrm{E}-02 \pm 1.6 \mathrm{E}-03$ & & & & ${ }^{154} \mathrm{Eu}$ & $1.7 \mathrm{E}-05 \pm 1.7 \mathrm{E}-04$ & $\mathrm{U}$ \\
\hline & 05/19/08 & 06/02/08 & gross $\alpha$ & $8.8 \mathrm{E}-04 \pm 5.8 \mathrm{E}-04$ & & & & ${ }^{155} \mathrm{Eu}$ & $6.6 \mathrm{E}-05 \pm 1.4 \mathrm{E}-04$ & U \\
\hline & 05/19/08 & $06 / 02 / 08$ & gross $\beta$ & $1.0 \mathrm{E}-02 \pm 1.5 \mathrm{E}-03$ & & & & ${ }^{238} \mathrm{Pu}$ & $1.0 \mathrm{E}-05 \pm 1.4 \mathrm{E}-05$ & $\mathrm{U}$ \\
\hline & $06 / 02 / 08$ & 06/16/08 & gross $\alpha$ & $1.2 \mathrm{E}-03 \pm 5.5 \mathrm{E}-04$ & & & & ${ }^{239 / 240} \mathrm{Pu}$ & $3.5 \mathrm{E}-06 \pm 4.4 \mathrm{E}-06$ & $\mathrm{U}$ \\
\hline & $06 / 02 / 08$ & 06/16/08 & gross $\beta$ & $8.1 \mathrm{E}-03 \pm 1.3 \mathrm{E}-03$ & & & & ${ }^{103} \mathrm{Ru}$ & $1.2 \mathrm{E}-05 \pm 5.8 \mathrm{E}-05$ & $\mathrm{U}$ \\
\hline & 06/16/08 & 06/30/08 & gross $\alpha$ & $5.9 \mathrm{E}-04 \pm 6.3 \mathrm{E}-04$ & & & & ${ }^{106} \mathrm{Ru}$ & $-6.3 \mathrm{E}-04 \pm 6.5 \mathrm{E}-04$ & $\mathrm{U}$ \\
\hline & $06 / 16 / 08$ & 06/30/08 & gross $\beta$ & $1.1 \mathrm{E}-02 \pm 1.8 \mathrm{E}-03$ & & & & ${ }^{125} \mathrm{Sb}$ & $-4.1 \mathrm{E}-05 \pm 1.4 \mathrm{E}-04$ & $\mathrm{U}$ \\
\hline & $06 / 30 / 08$ & $07 / 14 / 08$ & gross $\alpha$ & $1.4 \mathrm{E}-03 \pm 9.0 \mathrm{E}-04$ & & & & ${ }^{113} \mathrm{Sn}$ & $6.9 \mathrm{E}-05 \pm 6.6 \mathrm{E}-05$ & $\mathrm{U}$ \\
\hline & $06 / 30 / 08$ & $07 / 14 / 08$ & gross $\beta$ & $1.2 \mathrm{E}-02 \pm 1.9 \mathrm{E}-03$ & & & & ${ }^{90} \mathrm{Sr}$ & $-1.0 \mathrm{E}-04 \pm 1.1 \mathrm{E}-04$ & $\mathrm{U}$ \\
\hline & 07/14/08 & 07/28/08 & gross $\alpha$ & $7.6 \mathrm{E}-04 \pm 5.5 \mathrm{E}-04$ & & & & ${ }^{234} \mathrm{U}$ & $1.7 \mathrm{E}-05 \pm 1.0 \mathrm{E}-05$ & \\
\hline & $07 / 14 / 08$ & $07 / 28 / 08$ & gross $\beta$ & $1.4 \mathrm{E}-02 \pm 1.8 \mathrm{E}-03$ & & & & ${ }^{235} \mathrm{U}$ & $3.9 \mathrm{E}-06 \pm 3.8 \mathrm{E}-06$ & \\
\hline & $07 / 28 / 08$ & 08/11/08 & gross $\alpha$ & $8.8 \mathrm{E}-04 \pm 5.8 \mathrm{E}-04$ & & & & ${ }^{238} \mathrm{U}$ & $7.1 \mathrm{E}-06 \pm 5.6 \mathrm{E}-06$ & \\
\hline & $07 / 28 / 08$ & 08/11/08 & gross $\beta$ & $1.4 \mathrm{E}-02 \pm 1.9 \mathrm{E}-03$ & & & & ${ }^{65} \mathrm{Zn}$ & $1.1 \mathrm{E}-04 \pm 1.6 \mathrm{E}-04$ & $\mathrm{U}$ \\
\hline & $08 / 11 / 08$ & $08 / 25 / 08$ & gross $\alpha$ & $7.5 \mathrm{E}-04 \pm 6.0 \mathrm{E}-04$ & & & & & & \\
\hline & 08/11/08 & 08/25/08 & gross $\beta$ & $1.3 \mathrm{E}-02 \pm 1.9 \mathrm{E}-03$ & & & & & & \\
\hline & $08 / 25 / 08$ & 09/08/08 & gross $\alpha$ & $4.0 \mathrm{E}-04 \pm 4.4 \mathrm{E}-04$ & & & & & & \\
\hline & $08 / 25 / 08$ & 09/08/08 & gross $\beta$ & $1.0 \mathrm{E}-02 \pm 1.5 \mathrm{E}-03$ & & & & & & \\
\hline & 09/08/08 & 09/22/08 & gross $\alpha$ & $1.9 \mathrm{E}-03 \pm 6.5 \mathrm{E}-04$ & & & & & & \\
\hline & 09/08/08 & $09 / 22 / 08$ & gross $\beta$ & $2.5 \mathrm{E}-02 \pm 2.7 \mathrm{E}-03$ & & & & & & \\
\hline & $09 / 22 / 08$ & $10 / 07 / 08$ & gross $\alpha$ & $2.2 \mathrm{E}-03 \pm 6.8 \mathrm{E}-04$ & & & & & & \\
\hline & $09 / 22 / 08$ & $10 / 07 / 08$ & gross $\beta$ & $2.8 \mathrm{E}-02 \pm 2.9 \mathrm{E}-03$ & & & & & & \\
\hline & $10 / 07 / 08$ & $10 / 20 / 08$ & gross $\alpha$ & $1.0 \mathrm{E}-03 \pm 6.3 \mathrm{E}-04$ & & & & & & \\
\hline & $10 / 07 / 08$ & $10 / 20 / 08$ & gross $\beta$ & $1.8 \mathrm{E}-02 \pm 2.2 \mathrm{E}-03$ & & & & & & \\
\hline & $10 / 20 / 08$ & $11 / 03 / 08$ & gross $\alpha$ & $2.8 \mathrm{E}-03 \pm 8.3 \mathrm{E}-04$ & & & & & & \\
\hline & $10 / 20 / 08$ & $11 / 03 / 08$ & gross $\beta$ & 4.3E-02 $\pm 4.1 \mathrm{E}-03$ & & & & & & \\
\hline & $11 / 03 / 08$ & $11 / 17 / 08$ & gross $\alpha$ & $1.1 \mathrm{E}-03 \pm 5.0 \mathrm{E}-04$ & & & & & & \\
\hline & $11 / 03 / 08$ & $11 / 17 / 08$ & gross $\beta$ & $1.8 \mathrm{E}-02 \pm 2.1 \mathrm{E}-03$ & & & & & & \\
\hline & $11 / 17 / 08$ & $12 / 01 / 08$ & gross $\alpha$ & $2.8 \mathrm{E}-03 \pm 8.9 \mathrm{E}-04$ & & & & & & \\
\hline & $11 / 17 / 08$ & $12 / 01 / 08$ & gross $\beta$ & $3.6 \mathrm{E}-02 \pm 3.7 \mathrm{E}-03$ & & & & & & \\
\hline & $12 / 01 / 08$ & $12 / 15 / 08$ & gross $\alpha$ & $1.9 \mathrm{E}-03 \pm 6.7 \mathrm{E}-04$ & & & & & & \\
\hline & $12 / 01 / 08$ & $12 / 15 / 08$ & gross $\beta$ & $2.3 \mathrm{E}-02 \pm 2.5 \mathrm{E}-03$ & & & & & & \\
\hline & $12 / 15 / 08$ & $12 / 30 / 08$ & gross $\alpha$ & $1.3 \mathrm{E}-03 \pm 5.4 \mathrm{E}-04$ & & & & & & \\
\hline & $12 / 15 / 08$ & $12 / 30 / 08$ & gross $\beta$ & $2.9 \mathrm{E}-02 \pm 3.0 \mathrm{E}-03$ & & & & & & \\
\hline
\end{tabular}

$\overline{\mathrm{RQ}}=$ Result Qualifier. $\mathrm{U}=$ The analyte was analyzed for but not detected. 
Table 2-4. Near-Facility Air Sampling Results, 2008 (pCi/m³ \pm total analytical uncertainty). (Sheet 25 of 82)

\begin{tabular}{|c|c|c|c|c|c|c|c|c|c|}
\hline Location & Sample On & Sample Off & Isotope & Result \pm Uncertainty & Location & Composite Period & Isotope & Result \pm Uncertainty & $\mathbf{R Q *}$ \\
\hline N480 & $12 / 31 / 07$ & $01 / 14 / 08$ & gross $\alpha$ & $1.2 \mathrm{E}-03 \pm 5.4 \mathrm{E}-04$ & N480 & $12 / 31 / 07$ to $06 / 30 / 08$ & ${ }^{241} \mathrm{Am}$ & $1.0 \mathrm{E}-05 \pm 6.1 \mathrm{E}-06$ & \\
\hline \multirow[t]{51}{*}{$(200-E)$} & $12 / 31 / 07$ & $01 / 14 / 08$ & gross $\beta$ & $7.2 \mathrm{E}-03 \pm 1.2 \mathrm{E}-03$ & & & ${ }^{60} \mathrm{Co}$ & $-2.4 \mathrm{E}-05 \pm 7.5 \mathrm{E}-05$ & $\mathrm{U}$ \\
\hline & 01/14/08 & $01 / 29 / 08$ & gross $\alpha$ & $1.1 \mathrm{E}-03 \pm 4.9 \mathrm{E}-04$ & & & ${ }^{134} \mathrm{Cs}$ & $2.9 \mathrm{E}-05 \pm 6.5 \mathrm{E}-05$ & $\mathrm{U}$ \\
\hline & $01 / 14 / 08$ & 01/29/08 & gross $\beta$ & $3.6 \mathrm{E}-02 \pm 3.5 \mathrm{E}-03$ & & & ${ }^{137} \mathrm{Cs}$ & $-1.3 \mathrm{E}-05 \pm 5.3 \mathrm{E}-05$ & $\mathrm{U}$ \\
\hline & $01 / 29 / 08$ & $02 / 12 / 08$ & gross $\alpha$ & $2.7 \mathrm{E}-04 \pm 4.0 \mathrm{E}-04$ & & & ${ }^{152} \mathrm{Eu}$ & $8.9 \mathrm{E}-05 \pm 1.2 \mathrm{E}-04$ & $\mathrm{U}$ \\
\hline & $01 / 29 / 08$ & $02 / 12 / 08$ & gross $\beta$ & $4.1 \mathrm{E}-03 \pm 9.0 \mathrm{E}-04$ & & & ${ }^{154} \mathrm{Eu}$ & $-1.2 \mathrm{E}-04 \pm 2.0 \mathrm{E}-04$ & $\mathrm{U}$ \\
\hline & $02 / 12 / 08$ & $02 / 25 / 08$ & gross $\alpha$ & $1.2 \mathrm{E}-03 \pm 5.5 \mathrm{E}-04$ & & & ${ }^{155} \mathrm{Eu}$ & $2.6 \mathrm{E}-06 \pm 2.6 \mathrm{E}-05$ & $\mathrm{U}$ \\
\hline & $02 / 12 / 08$ & $02 / 25 / 08$ & gross $\beta$ & $3.2 \mathrm{E}-02 \pm 3.3 \mathrm{E}-03$ & & & ${ }^{238} \mathrm{Pu}$ & $-1.8 \mathrm{E}-06 \pm 1.8 \mathrm{E}-05$ & $\mathrm{U}$ \\
\hline & $02 / 25 / 08$ & 03/11/08 & gross $\alpha$ & $6.8 \mathrm{E}-04 \pm 5.0 \mathrm{E}-04$ & & & ${ }^{239 / 240} \mathrm{Pu}$ & $1.8 \mathrm{E}-06 \pm 9.4 \mathrm{E}-06$ & $\mathrm{U}$ \\
\hline & $02 / 25 / 08$ & 03/11/08 & gross $\beta$ & $1.5 \mathrm{E}-02 \pm 1.9 \mathrm{E}-03$ & & & ${ }^{241} \mathrm{Pu}$ & $7.7 \mathrm{E}-04 \pm 8.4 \mathrm{E}-04$ & $\mathrm{U}$ \\
\hline & 03/11/08 & 03/25/08 & gross $\alpha$ & $8.6 \mathrm{E}-04 \pm 5.8 \mathrm{E}-04$ & & & ${ }^{106} \mathrm{Ru}$ & $-3.8 \mathrm{E}-05 \pm 3.8 \mathrm{E}-04$ & $\mathrm{U}$ \\
\hline & 03/11/08 & $03 / 25 / 08$ & gross $\beta$ & $7.5 \mathrm{E}-03 \pm 1.2 \mathrm{E}-03$ & & & ${ }^{125} \mathrm{Sb}$ & $3.6 \mathrm{E}-05 \pm 1.4 \mathrm{E}-04$ & $\mathrm{U}$ \\
\hline & 03/25/08 & $04 / 07 / 08$ & gross $\alpha$ & $4.2 \mathrm{E}-04 \pm 4.7 \mathrm{E}-04$ & & & ${ }^{90} \mathrm{Sr}$ & $-6.8 \mathrm{E}-06 \pm 7.0 \mathrm{E}-06$ & $\mathrm{U}$ \\
\hline & 03/25/08 & $04 / 07 / 08$ & gross $\beta$ & $7.4 \mathrm{E}-03 \pm 1.3 \mathrm{E}-03$ & & & ${ }^{234} \mathrm{U}$ & $1.4 \mathrm{E}-05 \pm 8.1 \mathrm{E}-06$ & \\
\hline & $04 / 07 / 08$ & $04 / 21 / 08$ & gross $\alpha$ & $7.2 \mathrm{E}-04 \pm 5.3 \mathrm{E}-04$ & & & ${ }^{235} \mathrm{U}$ & $7.0 \mathrm{E}-07 \pm 4.2 \mathrm{E}-06$ & $\mathrm{U}$ \\
\hline & 04/07/08 & $04 / 21 / 08$ & gross $\beta$ & $1.1 \mathrm{E}-02 \pm 1.6 \mathrm{E}-03$ & & & ${ }^{238} \mathrm{U}$ & $4.5 \mathrm{E}-06 \pm 4.2 \mathrm{E}-06$ & $\mathrm{U}$ \\
\hline & $04 / 21 / 08$ & 05/06/08 & gross $\alpha$ & $1.6 \mathrm{E}-03 \pm 5.7 \mathrm{E}-04$ & & & & & \\
\hline & $04 / 21 / 08$ & 05/06/08 & gross $\beta$ & $1.8 \mathrm{E}-02 \pm 2.1 \mathrm{E}-03$ & N480 & $06 / 30 / 08$ to $12 / 30 / 08$ & ${ }^{241} \mathrm{Am}$ & $9.7 \mathrm{E}-06 \pm 6.8 \mathrm{E}-06$ & \\
\hline & 05/06/08 & 05/19/08 & gross $\alpha$ & $1.2 \mathrm{E}-03 \pm 5.5 \mathrm{E}-04$ & & & ${ }^{60} \mathrm{Co}$ & $-1.4 \mathrm{E}-05 \pm 9.1 \mathrm{E}-05$ & $\mathrm{U}$ \\
\hline & 05/06/08 & 05/19/08 & gross $\beta$ & $1.0 \mathrm{E}-02 \pm 1.5 \mathrm{E}-03$ & & & ${ }^{134} \mathrm{Cs}$ & $2.6 \mathrm{E}-05 \pm 6.7 \mathrm{E}-05$ & $\mathrm{U}$ \\
\hline & 05/19/08 & $06 / 02 / 08$ & gross $\alpha$ & $9.1 \mathrm{E}-04 \pm 5.8 \mathrm{E}-04$ & & & ${ }^{137} \mathrm{Cs}$ & $-6.5 \mathrm{E}-06 \pm 6.5 \mathrm{E}-05$ & $\mathrm{U}$ \\
\hline & 05/19/08 & $06 / 02 / 08$ & gross $\beta$ & $1.5 \mathrm{E}-02 \pm 1.9 \mathrm{E}-03$ & & & ${ }^{152} \mathrm{Eu}$ & $1.8 \mathrm{E}-05 \pm 1.4 \mathrm{E}-04$ & $\mathrm{U}$ \\
\hline & $06 / 02 / 08$ & $06 / 16 / 08$ & gross $\alpha$ & $6.3 \mathrm{E}-04 \pm 5.5 \mathrm{E}-04$ & & & ${ }^{154} \mathrm{Eu}$ & $-2.0 \mathrm{E}-05 \pm 2.0 \mathrm{E}-04$ & $\mathrm{U}$ \\
\hline & $06 / 02 / 08$ & 06/16/08 & gross $\beta$ & $7.0 \mathrm{E}-03 \pm 1.2 \mathrm{E}-03$ & & & ${ }^{155} \mathrm{Eu}$ & $-1.2 \mathrm{E}-04 \pm 1.5 \mathrm{E}-04$ & $\mathrm{U}$ \\
\hline & $06 / 16 / 08$ & 06/30/08 & gross $\alpha$ & $9.4 \mathrm{E}-04 \pm 5.9 \mathrm{E}-04$ & & & ${ }^{238} \mathrm{Pu}$ & $6.8 \mathrm{E}-06 \pm 1.8 \mathrm{E}-05$ & $\mathrm{U}$ \\
\hline & $06 / 16 / 08$ & 06/30/08 & gross $\beta$ & $1.3 \mathrm{E}-02 \pm 1.8 \mathrm{E}-03$ & & & ${ }^{239 / 240} \mathrm{Pu}$ & $4.1 \mathrm{E}-06 \pm 4.9 \mathrm{E}-06$ & \\
\hline & $06 / 30 / 08$ & $07 / 14 / 08$ & gross $\alpha$ & $1.9 \mathrm{E}-03 \pm 7.2 \mathrm{E}-04$ & & & ${ }^{241} \mathrm{Pu}$ & $1.5 \mathrm{E}-04 \pm 6.5 \mathrm{E}-04$ & $\mathrm{U}$ \\
\hline & $06 / 30 / 08$ & 07/14/08 & gross $\beta$ & $1.6 \mathrm{E}-02 \pm 2.0 \mathrm{E}-03$ & & & ${ }^{106} \mathrm{Ru}$ & $-3.2 \mathrm{E}-04 \pm 5.8 \mathrm{E}-04$ & $\mathrm{U}$ \\
\hline & $07 / 14 / 08$ & 07/28/08 & gross $\alpha$ & $2.1 \mathrm{E}-03 \pm 6.8 \mathrm{E}-04$ & & & ${ }^{125} \mathrm{Sb}$ & $1.6 \mathrm{E}-05 \pm 1.4 \mathrm{E}-04$ & $\mathrm{U}$ \\
\hline & $07 / 14 / 08$ & $07 / 28 / 08$ & gross $\beta$ & $4.6 \mathrm{E}-02 \pm 4.3 \mathrm{E}-03$ & & & ${ }^{90} \mathrm{Sr}$ & $-3.0 \mathrm{E}-04 \pm 3.1 \mathrm{E}-04$ & \\
\hline & $07 / 28 / 08$ & 08/11/08 & gross $\alpha$ & $1.4 \mathrm{E}-03 \pm 5.5 \mathrm{E}-04$ & & & ${ }^{234} \mathrm{U}$ & $1.4 \mathrm{E}-05 \pm 8.1 \mathrm{E}-06$ & \\
\hline & $07 / 28 / 08$ & 08/11/08 & gross $\beta$ & $1.7 \mathrm{E}-02 \pm 2.1 \mathrm{E}-03$ & & & ${ }^{235} \mathrm{U}$ & $4.9 \mathrm{E}-06 \pm 4.2 \mathrm{E}-06$ & \\
\hline & 08/11/08 & $08 / 25 / 08$ & gross $\alpha$ & $6.8 \mathrm{E}-04 \pm 5.1 \mathrm{E}-04$ & & & ${ }^{238} \mathrm{U}$ & $1.1 \mathrm{E}-05 \pm 6.8 \mathrm{E}-06$ & \\
\hline & 08/11/08 & 08/25/08 & gross $\beta$ & $1.3 \mathrm{E}-02 \pm 1.8 \mathrm{E}-03$ & & & & & \\
\hline & $08 / 25 / 08$ & 09/08/08 & gross $\alpha$ & $1.1 \mathrm{E}-03 \pm 5.0 \mathrm{E}-04$ & & & & & \\
\hline & $08 / 25 / 08$ & 09/08/08 & gross $\beta$ & $1.0 \mathrm{E}-02 \pm 1.5 \mathrm{E}-03$ & & & & & \\
\hline & 09/08/08 & 09/22/08 & gross $\alpha$ & $1.2 \mathrm{E}-03 \pm 8.3 \mathrm{E}-04$ & & & & & \\
\hline & 09/08/08 & 09/22/08 & gross $\beta$ & $2.4 \mathrm{E}-02 \pm 3.0 \mathrm{E}-03$ & & & & & \\
\hline & $09 / 22 / 08$ & $10 / 07 / 08$ & gross $\alpha$ & $1.4 \mathrm{E}-03 \pm 5.4 \mathrm{E}-04$ & & & & & \\
\hline & $09 / 22 / 08$ & $10 / 07 / 08$ & gross $\beta$ & $2.4 \mathrm{E}-02 \pm 2.6 \mathrm{E}-03$ & & & & & \\
\hline & $10 / 07 / 08$ & $10 / 20 / 08$ & gross $\alpha$ & $1.1 \mathrm{E}-03 \pm 5.2 \mathrm{E}-04$ & & & & & \\
\hline & $10 / 07 / 08$ & $10 / 20 / 08$ & gross $\beta$ & $1.9 \mathrm{E}-02 \pm 2.3 \mathrm{E}-03$ & & & & & \\
\hline & $10 / 20 / 08$ & $11 / 03 / 08$ & gross $\alpha$ & $2.7 \mathrm{E}-03 \pm 7.8 \mathrm{E}-04$ & & & & & \\
\hline & $10 / 20 / 08$ & $11 / 03 / 08$ & gross $\beta$ & $4.3 \mathrm{E}-02 \pm 4.1 \mathrm{E}-03$ & & & & & \\
\hline & $11 / 03 / 08$ & $11 / 17 / 08$ & gross $\alpha$ & $1.9 \mathrm{E}-03 \pm 6.6 \mathrm{E}-04$ & & & & & \\
\hline & $11 / 03 / 08$ & $11 / 17 / 08$ & gross $\beta$ & $1.7 \mathrm{E}-02 \pm 2.1 \mathrm{E}-03$ & & & & & \\
\hline & $11 / 17 / 08$ & $12 / 01 / 08$ & gross $\alpha$ & $2.7 \mathrm{E}-03 \pm 8.3 \mathrm{E}-04$ & & & & & \\
\hline & $11 / 17 / 08$ & $12 / 01 / 08$ & gross $\beta$ & $4.1 \mathrm{E}-02 \pm 3.9 \mathrm{E}-03$ & & & & & \\
\hline & $12 / 01 / 08$ & $12 / 15 / 08$ & gross $\alpha$ & $9.5 \mathrm{E}-04 \pm 5.9 \mathrm{E}-04$ & & & & & \\
\hline & $12 / 01 / 08$ & $12 / 15 / 08$ & gross $\beta$ & $1.9 \mathrm{E}-02 \pm 2.2 \mathrm{E}-03$ & & & & & \\
\hline & $12 / 15 / 08$ & $12 / 30 / 08$ & gross $\alpha$ & $1.8 \mathrm{E}-03 \pm 6.2 \mathrm{E}-04$ & & & & & \\
\hline & $12 / 15 / 08$ & $12 / 30 / 08$ & gross $\beta$ & $3.9 \mathrm{E}-02 \pm 3.7 \mathrm{E}-03$ & & & & & \\
\hline
\end{tabular}

$\overline{\mathrm{RQ}}=$ Result Qualifier. $\mathrm{U}=$ The analyte was analyzed for but not detected. 
Table 2-4. Near-Facility Air Sampling Results, 2008 (pCi/m³ \pm total analytical uncertainty). (Sheet 26 of 82)

\begin{tabular}{|c|c|c|c|c|c|c|c|c|c|}
\hline Location & Sample On & Sample Off & Isotope & Result \pm Uncertainty & Location & Composite Period & Isotope & Result \pm Uncertainty & $\mathbf{R Q *}$ \\
\hline N481 & $12 / 31 / 07$ & $01 / 14 / 08$ & gross $\alpha$ & $1.2 \mathrm{E}-03 \pm 5.2 \mathrm{E}-04$ & N481 & $12 / 31 / 07$ to $06 / 30 / 08$ & ${ }^{241} \mathrm{Am}$ & $8.0 \mathrm{E}-06 \pm 6.3 \mathrm{E}-06$ & \\
\hline \multirow[t]{51}{*}{$(200-E)$} & $12 / 31 / 07$ & $01 / 14 / 08$ & gross $\beta$ & $8.0 \mathrm{E}-03 \pm 1.3 \mathrm{E}-03$ & & & ${ }^{60} \mathrm{Co}$ & $8.4 \mathrm{E}-06 \pm 5.7 \mathrm{E}-05$ & $\mathrm{U}$ \\
\hline & 01/14/08 & $01 / 29 / 08$ & gross $\alpha$ & $1.6 \mathrm{E}-03 \pm 5.8 \mathrm{E}-04$ & & & ${ }^{134} \mathrm{Cs}$ & $-7.7 \mathrm{E}-06 \pm 6.2 \mathrm{E}-05$ & $\mathrm{U}$ \\
\hline & $01 / 14 / 08$ & 01/29/08 & gross $\beta$ & $3.4 \mathrm{E}-02 \pm 3.3 \mathrm{E}-03$ & & & ${ }^{137} \mathrm{Cs}$ & $4.0 \mathrm{E}-05 \pm 6.0 \mathrm{E}-05$ & $\mathrm{U}$ \\
\hline & $01 / 29 / 08$ & $02 / 12 / 08$ & gross $\alpha$ & $7.2 \mathrm{E}-04 \pm 5.3 \mathrm{E}-04$ & & & ${ }^{152} \mathrm{Eu}$ & $-5.3 \mathrm{E}-05 \pm 1.3 \mathrm{E}-04$ & $\mathrm{U}$ \\
\hline & $01 / 29 / 08$ & $02 / 12 / 08$ & gross $\beta$ & $4.3 \mathrm{E}-03 \pm 9.2 \mathrm{E}-04$ & & & ${ }^{154} \mathrm{Eu}$ & $1.5 \mathrm{E}-05 \pm 1.5 \mathrm{E}-04$ & $\mathrm{U}$ \\
\hline & $02 / 12 / 08$ & $02 / 25 / 08$ & gross $\alpha$ & $1.5 \mathrm{E}-03 \pm 6.3 \mathrm{E}-04$ & & & ${ }^{155} \mathrm{Eu}$ & $-4.6 \mathrm{E}-05 \pm 1.5 \mathrm{E}-04$ & $\mathrm{U}$ \\
\hline & $02 / 12 / 08$ & $02 / 25 / 08$ & gross $\beta$ & $2.9 \mathrm{E}-02 \pm 3.1 \mathrm{E}-03$ & & & ${ }^{238} \mathrm{Pu}$ & $6.0 \mathrm{E}-06 \pm 2.6 \mathrm{E}-05$ & $\mathrm{U}$ \\
\hline & $02 / 25 / 08$ & 03/11/08 & gross $\alpha$ & $2.4 \mathrm{E}-03 \pm 7.2 \mathrm{E}-04$ & & & ${ }^{239 / 240} \mathrm{Pu}$ & $3.0 \mathrm{E}-06 \pm 9.6 \mathrm{E}-06$ & $\mathrm{U}$ \\
\hline & $02 / 25 / 08$ & 03/11/08 & gross $\beta$ & $1.4 \mathrm{E}-02 \pm 1.8 \mathrm{E}-03$ & & & ${ }^{241} \mathrm{Pu}$ & $3.7 \mathrm{E}-04 \pm 6.2 \mathrm{E}-04$ & $\mathrm{U}$ \\
\hline & 03/11/08 & 03/25/08 & gross $\alpha$ & $5.3 \mathrm{E}-04 \pm 5.0 \mathrm{E}-04$ & & & ${ }^{106} \mathrm{Ru}$ & $-1.4 \mathrm{E}-05 \pm 1.4 \mathrm{E}-04$ & $\mathrm{U}$ \\
\hline & 03/11/08 & $03 / 25 / 08$ & gross $\beta$ & $8.2 \mathrm{E}-03 \pm 1.3 \mathrm{E}-03$ & & & ${ }^{125} \mathrm{Sb}$ & $5.0 \mathrm{E}-05 \pm 1.2 \mathrm{E}-04$ & $\mathrm{U}$ \\
\hline & 03/25/08 & $04 / 07 / 08$ & gross $\alpha$ & $1.1 \mathrm{E}-03 \pm 5.4 \mathrm{E}-04$ & & & ${ }^{90} \mathrm{Sr}$ & $-3.3 \mathrm{E}-04 \pm 3.4 \mathrm{E}-04$ & $\mathrm{U}$ \\
\hline & 03/25/08 & $04 / 07 / 08$ & gross $\beta$ & $6.9 \mathrm{E}-03 \pm 1.2 \mathrm{E}-03$ & & & ${ }^{234} \mathrm{U}$ & $1.0 \mathrm{E}-05 \pm 7.0 \mathrm{E}-06$ & \\
\hline & $04 / 07 / 08$ & $04 / 21 / 08$ & gross $\alpha$ & $2.8 \mathrm{E}-04 \pm 4.1 \mathrm{E}-04$ & & & ${ }^{235} \mathrm{U}$ & $4.7 \mathrm{E}-06 \pm 4.3 \mathrm{E}-06$ & \\
\hline & 04/07/08 & $04 / 21 / 08$ & gross $\beta$ & $8.4 \mathrm{E}-03 \pm 1.4 \mathrm{E}-03$ & & & ${ }^{238} \mathrm{U}$ & $1.0 \mathrm{E}-05 \pm 6.6 \mathrm{E}-06$ & \\
\hline & $04 / 21 / 08$ & 05/06/08 & gross $\alpha$ & $9.3 \mathrm{E}-04 \pm 4.4 \mathrm{E}-04$ & & & & & \\
\hline & $04 / 21 / 08$ & 05/06/08 & gross $\beta$ & $1.6 \mathrm{E}-02 \pm 2.0 \mathrm{E}-03$ & N481 & $06 / 30 / 08$ to $12 / 30 / 08$ & ${ }^{241} \mathrm{Am}$ & $2.1 \mathrm{E}-06 \pm 1.1 \mathrm{E}-05$ & $\mathrm{U}$ \\
\hline & 05/06/08 & 05/19/08 & gross $\alpha$ & $9.7 \mathrm{E}-04 \pm 6.4 \mathrm{E}-04$ & & & ${ }^{60} \mathrm{Co}$ & $2.8 \mathrm{E}-05 \pm 6.3 \mathrm{E}-05$ & $\mathrm{U}$ \\
\hline & 05/06/08 & 05/19/08 & gross $\beta$ & $1.1 \mathrm{E}-02 \pm 1.6 \mathrm{E}-03$ & & & ${ }^{134} \mathrm{Cs}$ & $2.7 \mathrm{E}-05 \pm 6.2 \mathrm{E}-05$ & $\mathrm{U}$ \\
\hline & 05/19/08 & $06 / 02 / 08$ & gross $\alpha$ & $9.8 \mathrm{E}-04 \pm 4.6 \mathrm{E}-04$ & & & ${ }^{137} \mathrm{Cs}$ & $-2.2 \mathrm{E}-05 \pm 5.9 \mathrm{E}-05$ & $\mathrm{U}$ \\
\hline & 05/19/08 & $06 / 02 / 08$ & gross $\beta$ & $1.2 \mathrm{E}-02 \pm 1.7 \mathrm{E}-03$ & & & ${ }^{152} \mathrm{Eu}$ & $-5.9 \mathrm{E}-06 \pm 5.9 \mathrm{E}-05$ & $\mathrm{U}$ \\
\hline & $06 / 02 / 08$ & $06 / 16 / 08$ & gross $\alpha$ & $5.4 \mathrm{E}-04 \pm 5.5 \mathrm{E}-04$ & & & ${ }^{154} \mathrm{Eu}$ & $-1.4 \mathrm{E}-04 \pm 1.9 \mathrm{E}-04$ & $\mathrm{U}$ \\
\hline & $06 / 02 / 08$ & 06/16/08 & gross $\beta$ & $8.0 \mathrm{E}-03 \pm 1.3 \mathrm{E}-03$ & & & ${ }^{155} \mathrm{Eu}$ & $7.7 \mathrm{E}-05 \pm 1.9 \mathrm{E}-04$ & $\mathrm{U}$ \\
\hline & 06/16/08 & 06/30/08 & gross $\alpha$ & $1.0 \mathrm{E}-03 \pm 5.0 \mathrm{E}-04$ & & & ${ }^{238} \mathrm{Pu}$ & $-2.8 \mathrm{E}-05 \pm 3.9 \mathrm{E}-05$ & $\mathrm{U}$ \\
\hline & $06 / 16 / 08$ & 06/30/08 & gross $\beta$ & $1.5 \mathrm{E}-02 \pm 2.0 \mathrm{E}-03$ & & & ${ }^{239 / 240} \mathrm{Pu}$ & $-1.8 \mathrm{E}-06 \pm 6.4 \mathrm{E}-06$ & $\mathrm{U}$ \\
\hline & $06 / 30 / 08$ & $07 / 14 / 08$ & gross $\alpha$ & $2.0 \mathrm{E}-03 \pm 7.4 \mathrm{E}-04$ & & & ${ }^{241} \mathrm{Pu}$ & $-5.3 \mathrm{E}-04 \pm 5.5 \mathrm{E}-04$ & $\mathrm{U}$ \\
\hline & $06 / 30 / 08$ & 07/14/08 & gross $\beta$ & $1.4 \mathrm{E}-02 \pm 1.8 \mathrm{E}-03$ & & & ${ }^{106} \mathrm{Ru}$ & $-9.5 \mathrm{E}-05 \pm 5.4 \mathrm{E}-04$ & $\mathrm{U}$ \\
\hline & $07 / 14 / 08$ & 07/28/08 & gross $\alpha$ & $1.2 \mathrm{E}-03 \pm 5.4 \mathrm{E}-04$ & & & ${ }^{125} \mathrm{Sb}$ & $-1.2 \mathrm{E}-04 \pm 1.3 \mathrm{E}-04$ & $\mathrm{U}$ \\
\hline & $07 / 14 / 08$ & $07 / 28 / 08$ & gross $\beta$ & $1.7 \mathrm{E}-02 \pm 2.2 \mathrm{E}-03$ & & & ${ }^{90} \mathrm{Sr}$ & $-1.5 \mathrm{E}-04 \pm 1.5 \mathrm{E}-03$ & $\mathrm{U}$ \\
\hline & $07 / 28 / 08$ & 08/11/08 & gross $\alpha$ & $6.9 \mathrm{E}-04 \pm 5.2 \mathrm{E}-04$ & & & ${ }^{234} \mathrm{U}$ & $1.5 \mathrm{E}-05 \pm 8.7 \mathrm{E}-06$ & \\
\hline & $07 / 28 / 08$ & 08/11/08 & gross $\beta$ & $1.8 \mathrm{E}-02 \pm 2.2 \mathrm{E}-03$ & & & ${ }^{235} \mathrm{U}$ & $-7.4 \mathrm{E}-07 \pm 7.4 \mathrm{E}-06$ & $\mathrm{U}$ \\
\hline & 08/11/08 & $08 / 25 / 08$ & gross $\alpha$ & $1.6 \mathrm{E}-03 \pm 6.1 \mathrm{E}-04$ & & & ${ }^{238} \mathrm{U}$ & $1.5 \mathrm{E}-05 \pm 9.0 \mathrm{E}-06$ & \\
\hline & 08/11/08 & 08/25/08 & gross $\beta$ & $1.8 \mathrm{E}-02 \pm 2.2 \mathrm{E}-03$ & & & & & \\
\hline & $08 / 25 / 08$ & 09/08/08 & gross $\alpha$ & $7.0 \mathrm{E}-04 \pm 5.2 \mathrm{E}-04$ & & & & & \\
\hline & $08 / 25 / 08$ & 09/08/08 & gross $\beta$ & $1.2 \mathrm{E}-02 \pm 1.7 \mathrm{E}-03$ & & & & & \\
\hline & 09/08/08 & 09/22/08 & gross $\alpha$ & $1.9 \mathrm{E}-03 \pm 6.6 \mathrm{E}-04$ & & & & & \\
\hline & 09/08/08 & 09/22/08 & gross $\beta$ & $2.7 \mathrm{E}-02 \pm 2.9 \mathrm{E}-03$ & & & & & \\
\hline & $09 / 22 / 08$ & $10 / 07 / 08$ & gross $\alpha$ & $1.9 \mathrm{E}-03 \pm 6.3 \mathrm{E}-04$ & & & & & \\
\hline & $09 / 22 / 08$ & $10 / 07 / 08$ & gross $\beta$ & $2.5 \mathrm{E}-02 \pm 2.7 \mathrm{E}-03$ & & & & & \\
\hline & $10 / 07 / 08$ & $10 / 20 / 08$ & gross $\alpha$ & $9.9 \mathrm{E}-04 \pm 6.2 \mathrm{E}-04$ & & & & & \\
\hline & $10 / 07 / 08$ & $10 / 20 / 08$ & gross $\beta$ & $2.2 \mathrm{E}-02 \pm 2.5 \mathrm{E}-03$ & & & & & \\
\hline & $10 / 20 / 08$ & $11 / 03 / 08$ & gross $\alpha$ & $2.6 \mathrm{E}-03 \pm 7.6 \mathrm{E}-04$ & & & & & \\
\hline & $10 / 20 / 08$ & $11 / 03 / 08$ & gross $\beta$ & $4.7 \mathrm{E}-02 \pm 4.4 \mathrm{E}-03$ & & & & & \\
\hline & $11 / 03 / 08$ & $11 / 17 / 08$ & gross $\alpha$ & $9.1 \mathrm{E}-04 \pm 5.8 \mathrm{E}-04$ & & & & & \\
\hline & $11 / 03 / 08$ & $11 / 17 / 08$ & gross $\beta$ & $1.8 \mathrm{E}-02 \pm 2.2 \mathrm{E}-03$ & & & & & \\
\hline & $11 / 17 / 08$ & $12 / 01 / 08$ & gross $\alpha$ & $1.9 \mathrm{E}-03 \pm 6.9 \mathrm{E}-04$ & & & & & \\
\hline & $11 / 17 / 08$ & $12 / 01 / 08$ & gross $\beta$ & $4.1 \mathrm{E}-02 \pm 4.0 \mathrm{E}-03$ & & & & & \\
\hline & $12 / 01 / 08$ & $12 / 15 / 08$ & gross $\alpha$ & $1.4 \mathrm{E}-03 \pm 5.6 \mathrm{E}-04$ & & & & & \\
\hline & $12 / 01 / 08$ & $12 / 15 / 08$ & gross $\beta$ & $2.0 \mathrm{E}-02 \pm 2.3 \mathrm{E}-03$ & & & & & \\
\hline & $12 / 15 / 08$ & $12 / 30 / 08$ & gross $\alpha$ & $1.7 \mathrm{E}-03 \pm 6.1 \mathrm{E}-04$ & & & & & \\
\hline & $12 / 15 / 08$ & $12 / 30 / 08$ & gross $\beta$ & $4.5 \mathrm{E}-02 \pm 4.1 \mathrm{E}-03$ & & & & & \\
\hline
\end{tabular}

$\overline{\mathrm{RQ}}=$ Result Qualifier. $\mathrm{U}=$ The analyte was analyzed for but not detected. 
Table 2-4. Near-Facility Air Sampling Results, 2008 (pCi/m³ \pm total analytical uncertainty). (Sheet 27 of 82)

\begin{tabular}{|c|c|c|c|c|c|c|c|c|c|}
\hline Location & Sample On & Sample Off & Isotope & Result \pm Uncertainty & Location & Composite Period & Isotope & Result \pm Uncertainty & $\mathbf{R Q *}$ \\
\hline N498 & $12 / 31 / 07$ & $01 / 14 / 08$ & gross $\alpha$ & $7.1 \mathrm{E}-04 \pm 5.2 \mathrm{E}-04$ & N498 & $12 / 31 / 07$ to $06 / 30 / 08$ & ${ }^{60} \mathrm{Co}$ & $-2.9 \mathrm{E}-05 \pm 7.0 \mathrm{E}-05$ & $\mathrm{U}$ \\
\hline \multirow[t]{51}{*}{$(200-E)$} & $12 / 31 / 07$ & 01/14/08 & gross $\beta$ & $1.0 \mathrm{E}-02 \pm 1.5 \mathrm{E}-03$ & & & ${ }^{134} \mathrm{Cs}$ & $6.8 \mathrm{E}-05 \pm 6.8 \mathrm{E}-05$ & $\mathrm{U}$ \\
\hline & 01/14/08 & $01 / 29 / 08$ & gross $\alpha$ & $2.1 \mathrm{E}-03 \pm 6.7 \mathrm{E}-04$ & & & ${ }^{137} \mathrm{Cs}$ & $1.1 \mathrm{E}-05 \pm 5.7 \mathrm{E}-05$ & $\mathrm{U}$ \\
\hline & $01 / 14 / 08$ & 01/29/08 & gross $\beta$ & $3.5 \mathrm{E}-02 \pm 3.4 \mathrm{E}-03$ & & & ${ }^{152} \mathrm{Eu}$ & $3.9 \mathrm{E}-05 \pm 1.3 \mathrm{E}-04$ & $\mathrm{U}$ \\
\hline & $01 / 29 / 08$ & $02 / 12 / 08$ & gross $\alpha$ & $3.8 \mathrm{E}-04 \pm 4.3 \mathrm{E}-04$ & & & ${ }^{154} \mathrm{Eu}$ & $3.3 \mathrm{E}-05 \pm 2.0 \mathrm{E}-04$ & $\mathrm{U}$ \\
\hline & $01 / 29 / 08$ & $02 / 12 / 08$ & gross $\beta$ & $4.0 \mathrm{E}-03 \pm 8.8 \mathrm{E}-04$ & & & ${ }^{155} \mathrm{Eu}$ & $-6.7 \mathrm{E}-05 \pm 1.2 \mathrm{E}-04$ & $\mathrm{U}$ \\
\hline & $02 / 12 / 08$ & $02 / 25 / 08$ & gross $\alpha$ & $1.4 \mathrm{E}-03 \pm 6.0 \mathrm{E}-04$ & & & ${ }^{238} \mathrm{Pu}$ & $1.3 \mathrm{E}-05 \pm 1.0 \mathrm{E}-05$ & \\
\hline & $02 / 12 / 08$ & $02 / 25 / 08$ & gross $\beta$ & $2.7 \mathrm{E}-02 \pm 2.9 \mathrm{E}-03$ & & & ${ }^{239 / 240} \mathrm{Pu}$ & $-6.3 \mathrm{E}-07 \pm 2.2 \mathrm{E}-06$ & $\mathrm{U}$ \\
\hline & $02 / 25 / 08$ & 03/11/08 & gross $\alpha$ & $1.3 \mathrm{E}-03 \pm 5.3 \mathrm{E}-04$ & & & ${ }^{103} \mathrm{Ru}$ & $-4.8 \mathrm{E}-06 \pm 4.8 \mathrm{E}-05$ & $\mathrm{U}$ \\
\hline & $02 / 25 / 08$ & 03/11/08 & gross $\beta$ & $1.5 \mathrm{E}-02 \pm 1.8 \mathrm{E}-03$ & & & ${ }^{106} \mathrm{Ru}$ & $7.0 \mathrm{E}-05 \pm 5.0 \mathrm{E}-04$ & $\mathrm{U}$ \\
\hline & 03/11/08 & 03/25/08 & gross $\alpha$ & $2.9 \mathrm{E}-04 \pm 4.1 \mathrm{E}-04$ & & & ${ }^{125} \mathrm{Sb}$ & $-9.9 \mathrm{E}-05 \pm 1.4 \mathrm{E}-04$ & $\mathrm{U}$ \\
\hline & 03/11/08 & $03 / 25 / 08$ & gross $\beta$ & $8.6 \mathrm{E}-03 \pm 1.3 \mathrm{E}-03$ & & & ${ }^{113} \mathrm{Sn}$ & $-4.6 \mathrm{E}-05 \pm 6.1 \mathrm{E}-05$ & $\mathrm{U}$ \\
\hline & 03/25/08 & $04 / 07 / 08$ & gross $\alpha$ & $7.8 \mathrm{E}-04 \pm 5.8 \mathrm{E}-04$ & & & ${ }^{90} \mathrm{Sr}$ & $-1.7 \mathrm{E}-04 \pm 1.8 \mathrm{E}-04$ & $\mathrm{U}$ \\
\hline & 03/25/08 & $04 / 07 / 08$ & gross $\beta$ & $8.6 \mathrm{E}-03 \pm 1.4 \mathrm{E}-03$ & & & ${ }^{234} \mathrm{U}$ & $1.1 \mathrm{E}-05 \pm 7.2 \mathrm{E}-06$ & \\
\hline & $04 / 07 / 08$ & $04 / 21 / 08$ & gross $\alpha$ & $7.2 \mathrm{E}-04 \pm 5.3 \mathrm{E}-04$ & & & ${ }^{235} \mathrm{U}$ & $3.6 \mathrm{E}-06 \pm 3.5 \mathrm{E}-06$ & \\
\hline & $04 / 07 / 08$ & $04 / 21 / 08$ & gross $\beta$ & $1.0 \mathrm{E}-02 \pm 1.5 \mathrm{E}-03$ & & & ${ }^{238} \mathrm{U}$ & $6.6 \mathrm{E}-07 \pm 2.3 \mathrm{E}-06$ & $\mathrm{U}$ \\
\hline & $04 / 21 / 08$ & 05/06/08 & gross $\alpha$ & $1.5 \mathrm{E}-03 \pm 5.7 \mathrm{E}-04$ & & & ${ }^{65} \mathrm{Zn}$ & $-9.2 \mathrm{E}-05 \pm 1.5 \mathrm{E}-04$ & $\mathrm{U}$ \\
\hline & $04 / 21 / 08$ & $05 / 06 / 08$ & gross $\beta$ & $1.4 \mathrm{E}-02 \pm 1.8 \mathrm{E}-03$ & & & & & \\
\hline & 05/06/08 & 05/19/08 & gross $\alpha$ & $9.2 \mathrm{E}-04 \pm 6.1 \mathrm{E}-04$ & N498 & $06 / 30 / 08$ to $12 / 30 / 08$ & ${ }^{60} \mathrm{Co}$ & $-1.8 \mathrm{E}-05 \pm 7.5 \mathrm{E}-05$ & $\mathrm{U}$ \\
\hline & 05/06/08 & 05/19/08 & gross $\beta$ & $9.3 \mathrm{E}-03 \pm 1.4 \mathrm{E}-03$ & & & ${ }^{134} \mathrm{Cs}$ & $-2.3 \mathrm{E}-05 \pm 6.7 \mathrm{E}-05$ & $\mathrm{U}$ \\
\hline & 05/19/08 & $06 / 02 / 08$ & gross $\alpha$ & $1.1 \mathrm{E}-03 \pm 5.0 \mathrm{E}-04$ & & & ${ }^{137} \mathrm{Cs}$ & $5.5 \mathrm{E}-05 \pm 6.7 \mathrm{E}-05$ & $\mathrm{U}$ \\
\hline & 05/19/08 & $06 / 02 / 08$ & gross $\beta$ & $1.4 \mathrm{E}-02 \pm 1.9 \mathrm{E}-03$ & & & ${ }^{152} \mathrm{Eu}$ & $1.4 \mathrm{E}-04 \pm 1.7 \mathrm{E}-04$ & $\mathrm{U}$ \\
\hline & $06 / 02 / 08$ & $06 / 16 / 08$ & gross $\alpha$ & $6.4 \mathrm{E}-04 \pm 5.2 \mathrm{E}-04$ & & & ${ }^{154} \mathrm{Eu}$ & $6.8 \mathrm{E}-05 \pm 2.0 \mathrm{E}-04$ & $\mathrm{U}$ \\
\hline & $06 / 02 / 08$ & 06/16/08 & gross $\beta$ & $9.3 \mathrm{E}-03 \pm 1.4 \mathrm{E}-03$ & & & ${ }^{155} \mathrm{Eu}$ & $-1.7 \mathrm{E}-05 \pm 1.7 \mathrm{E}-04$ & $\mathrm{U}$ \\
\hline & 06/16/08 & 06/30/08 & gross $\alpha$ & $1.1 \mathrm{E}-03 \pm 5.3 \mathrm{E}-04$ & & & ${ }^{238} \mathrm{Pu}$ & $-9.5 \mathrm{E}-07 \pm 9.5 \mathrm{E}-06$ & $\mathrm{U}$ \\
\hline & $06 / 16 / 08$ & 06/30/08 & gross $\beta$ & $1.4 \mathrm{E}-02 \pm 1.8 \mathrm{E}-03$ & & & ${ }^{239 / 240} \mathrm{Pu}$ & $-9.5 \mathrm{E}-07 \pm 4.2 \mathrm{E}-06$ & $\mathrm{U}$ \\
\hline & $06 / 30 / 08$ & $07 / 14 / 08$ & gross $\alpha$ & $4.2 \mathrm{E}-04 \pm 5.2 \mathrm{E}-04$ & & & ${ }^{106} \mathrm{Ru}$ & $2.3 \mathrm{E}-04 \pm 5.7 \mathrm{E}-04$ & $\mathrm{U}$ \\
\hline & $06 / 30 / 08$ & 07/14/08 & gross $\beta$ & $1.8 \mathrm{E}-02 \pm 2.2 \mathrm{E}-03$ & & & ${ }^{125} \mathrm{Sb}$ & $-1.3 \mathrm{E}-06 \pm 1.3 \mathrm{E}-05$ & $\mathrm{U}$ \\
\hline & $07 / 14 / 08$ & 07/28/08 & gross $\alpha$ & $1.1 \mathrm{E}-03 \pm 5.1 \mathrm{E}-04$ & & & ${ }^{90} \mathrm{Sr}$ & $-1.1 \mathrm{E}-04 \pm 1.1 \mathrm{E}-04$ & $\mathrm{U}$ \\
\hline & $07 / 14 / 08$ & $07 / 28 / 08$ & gross $\beta$ & $1.3 \mathrm{E}-02 \pm 1.8 \mathrm{E}-03$ & & & ${ }^{234} \mathrm{U}$ & $1.5 \mathrm{E}-05 \pm 8.1 \mathrm{E}-06$ & \\
\hline & $07 / 28 / 08$ & 08/11/08 & gross $\alpha$ & $1.4 \mathrm{E}-03 \pm 5.9 \mathrm{E}-04$ & & & ${ }^{235} \mathrm{U}$ & $2.2 \mathrm{E}-06 \pm 2.6 \mathrm{E}-06$ & \\
\hline & $07 / 28 / 08$ & 08/11/08 & gross $\beta$ & $1.5 \mathrm{E}-02 \pm 2.0 \mathrm{E}-03$ & & & ${ }^{238} \mathrm{U}$ & $8.8 \mathrm{E}-06 \pm 5.5 \mathrm{E}-06$ & \\
\hline & $08 / 11 / 08$ & $08 / 25 / 08$ & gross $\alpha$ & $1.3 \mathrm{E}-03 \pm 5.6 \mathrm{E}-04$ & & & & & \\
\hline & 08/11/08 & 08/25/08 & gross $\beta$ & $1.3 \mathrm{E}-02 \pm 1.8 \mathrm{E}-03$ & & & & & \\
\hline & $08 / 25 / 08$ & 09/08/08 & gross $\alpha$ & $4.5 \mathrm{E}-04 \pm 4.4 \mathrm{E}-04$ & & & & & \\
\hline & $08 / 25 / 08$ & 09/08/08 & gross $\beta$ & $1.0 \mathrm{E}-02 \pm 1.5 \mathrm{E}-03$ & & & & & \\
\hline & 09/08/08 & 09/22/08 & gross $\alpha$ & $1.2 \mathrm{E}-03 \pm 5.2 \mathrm{E}-04$ & & & & & \\
\hline & 09/08/08 & 09/22/08 & gross $\beta$ & $2.6 \mathrm{E}-02 \pm 2.8 \mathrm{E}-03$ & & & & & \\
\hline & 09/22/08 & $10 / 07 / 08$ & gross $\alpha$ & $1.3 \mathrm{E}-03 \pm 5.2 \mathrm{E}-04$ & & & & & \\
\hline & $09 / 22 / 08$ & $10 / 07 / 08$ & gross $\beta$ & $2.3 \mathrm{E}-02 \pm 2.5 \mathrm{E}-03$ & & & & & \\
\hline & $10 / 07 / 08$ & $10 / 20 / 08$ & gross $\alpha$ & $1.8 \mathrm{E}-03 \pm 6.5 \mathrm{E}-04$ & & & & & \\
\hline & $10 / 07 / 08$ & $10 / 20 / 08$ & gross $\beta$ & $2.0 \mathrm{E}-02 \pm 2.4 \mathrm{E}-03$ & & & & & \\
\hline & $10 / 20 / 08$ & $11 / 03 / 08$ & gross $\alpha$ & $2.6 \mathrm{E}-03 \pm 7.6 \mathrm{E}-04$ & & & & & \\
\hline & $10 / 20 / 08$ & $11 / 03 / 08$ & gross $\beta$ & $4.4 \mathrm{E}-02 \pm 4.2 \mathrm{E}-03$ & & & & & \\
\hline & $11 / 03 / 08$ & $11 / 17 / 08$ & gross $\alpha$ & $6.9 \mathrm{E}-04 \pm 5.2 \mathrm{E}-04$ & & & & & \\
\hline & $11 / 03 / 08$ & $11 / 17 / 08$ & gross $\beta$ & $1.8 \mathrm{E}-02 \pm 2.2 \mathrm{E}-03$ & & & & & \\
\hline & $11 / 17 / 08$ & $12 / 01 / 08$ & gross $\alpha$ & $2.7 \mathrm{E}-03 \pm 8.1 \mathrm{E}-04$ & & & & & \\
\hline & $11 / 17 / 08$ & $12 / 01 / 08$ & gross $\beta$ & $5.2 \mathrm{E}-02 \pm 4.9 \mathrm{E}-03$ & & & & & \\
\hline & $12 / 01 / 08$ & $12 / 15 / 08$ & gross $\alpha$ & $1.6 \mathrm{E}-03 \pm 6.1 \mathrm{E}-04$ & & & & & \\
\hline & $12 / 01 / 08$ & $12 / 15 / 08$ & gross $\beta$ & $2.0 \mathrm{E}-02 \pm 2.3 \mathrm{E}-03$ & & & & & \\
\hline & $12 / 15 / 08$ & $12 / 30 / 08$ & gross $\alpha$ & $1.4 \mathrm{E}-03 \pm 5.6 \mathrm{E}-04$ & & & & & \\
\hline & $12 / 15 / 08$ & $12 / 30 / 08$ & gross $\beta$ & $4.0 \mathrm{E}-02 \pm 3.7 \mathrm{E}-03$ & & & & & \\
\hline
\end{tabular}

$\overline{\mathrm{RQ}}=$ Result Qualifier. $\mathrm{U}=$ The analyte was analyzed for but not detected. 
Table 2-4. Near-Facility Air Sampling Results, 2008 (pCi/m³ \pm total analytical uncertainty). (Sheet 28 of 82)

\begin{tabular}{|c|c|c|c|c|c|c|c|c|c|c|}
\hline Location & Sample On & Sample Off & Isotope & Result \pm Uncertainty & Location & Composite & e Period & Isotope & Result \pm Uncertainty & $\mathbf{R Q *}$ \\
\hline N499 & $12 / 31 / 07$ & $01 / 14 / 08$ & gross $\alpha$ & $4.9 \mathrm{E}-04 \pm 4.6 \mathrm{E}-04$ & N499 & $12 / 31 / 07$ to & $06 / 30 / 08$ & ${ }^{60} \mathrm{Co}$ & $-1.9 \mathrm{E}-05 \pm 6.0 \mathrm{E}-05$ & $\mathrm{U}$ \\
\hline \multirow[t]{51}{*}{$(200-E)$} & $12 / 31 / 07$ & 01/14/08 & gross $\beta$ & $1.1 \mathrm{E}-02 \pm 1.6 \mathrm{E}-03$ & & & & ${ }^{134} \mathrm{Cs}$ & $3.5 \mathrm{E}-05 \pm 6.1 \mathrm{E}-05$ & $\mathrm{U}$ \\
\hline & $01 / 14 / 08$ & 01/29/08 & gross $\alpha$ & $1.7 \mathrm{E}-03 \pm 6.1 \mathrm{E}-04$ & & & & ${ }^{137} \mathrm{Cs}$ & $-2.3 \mathrm{E}-05 \pm 5.5 \mathrm{E}-05$ & U \\
\hline & $01 / 14 / 08$ & 01/29/08 & gross $\beta$ & $3.0 \mathrm{E}-02 \pm 3.0 \mathrm{E}-03$ & & & & ${ }^{152} \mathrm{Eu}$ & $9.2 \mathrm{E}-05 \pm 1.3 \mathrm{E}-04$ & $\mathrm{U}$ \\
\hline & $01 / 29 / 08$ & $02 / 12 / 08$ & gross $\alpha$ & $-6.1 \mathrm{E}-05 \pm 2.0 \mathrm{E}-04$ & & & & ${ }^{154} \mathrm{Eu}$ & $4.0 \mathrm{E}-05 \pm 1.8 \mathrm{E}-04$ & $\mathrm{U}$ \\
\hline & $01 / 29 / 08$ & $02 / 12 / 08$ & gross $\beta$ & $3.8 \mathrm{E}-03 \pm 8.5 \mathrm{E}-04$ & & & & ${ }^{155} \mathrm{Eu}$ & $-9.0 \mathrm{E}-05 \pm 1.6 \mathrm{E}-04$ & $\mathrm{U}$ \\
\hline & $02 / 12 / 08$ & $02 / 25 / 08$ & gross $\alpha$ & $1.7 \mathrm{E}-03 \pm 6.5 \mathrm{E}-04$ & & & & ${ }^{238} \mathrm{Pu}$ & $-3.1 \mathrm{E}-06 \pm 3.9 \mathrm{E}-06$ & $\mathrm{U}$ \\
\hline & $02 / 12 / 08$ & $02 / 25 / 08$ & gross $\beta$ & $3.2 \mathrm{E}-02 \pm 3.3 \mathrm{E}-03$ & & & & ${ }^{239 / 240} \mathrm{Pu}$ & $6.2 \mathrm{E}-07 \pm 1.3 \mathrm{E}-06$ & $\mathrm{U}$ \\
\hline & $02 / 25 / 08$ & 03/11/08 & gross $\alpha$ & $5.7 \mathrm{E}-04 \pm 4.7 \mathrm{E}-04$ & & & & ${ }^{103} \mathrm{Ru}$ & $-4.0 \mathrm{E}-06 \pm 4.0 \mathrm{E}-05$ & $\mathrm{U}$ \\
\hline & $02 / 25 / 08$ & 03/11/08 & gross $\beta$ & $1.4 \mathrm{E}-02 \pm 1.8 \mathrm{E}-03$ & & & & ${ }^{106} \mathrm{Ru}$ & $4.6 \mathrm{E}-04 \pm 5.2 \mathrm{E}-04$ & $\mathrm{U}$ \\
\hline & 03/11/08 & $03 / 25 / 08$ & gross $\alpha$ & $2.9 \mathrm{E}-04 \pm 4.1 \mathrm{E}-04$ & & & & ${ }^{125} \mathrm{Sb}$ & $-8.1 \mathrm{E}-07 \pm 8.1 \mathrm{E}-06$ & $\mathrm{U}$ \\
\hline & 03/11/08 & $03 / 25 / 08$ & gross $\beta$ & $7.1 \mathrm{E}-03 \pm 1.2 \mathrm{E}-03$ & & & & ${ }^{113} \mathrm{Sn}$ & $1.6 \mathrm{E}-05 \pm 5.6 \mathrm{E}-05$ & $\mathrm{U}$ \\
\hline & $03 / 25 / 08$ & $04 / 07 / 08$ & gross $\alpha$ & $1.0 \mathrm{E}-03 \pm 6.3 \mathrm{E}-04$ & & & & ${ }^{90} \mathrm{Sr}$ & $-2.2 \mathrm{E}-04 \pm 2.3 \mathrm{E}-04$ & $\mathrm{U}$ \\
\hline & 03/25/08 & $04 / 07 / 08$ & gross $\beta$ & $8.3 \mathrm{E}-03 \pm 1.4 \mathrm{E}-03$ & & & & ${ }^{234} \mathrm{U}$ & $1.5 \mathrm{E}-05 \pm 9.2 \mathrm{E}-06$ & \\
\hline & $04 / 07 / 08$ & $04 / 21 / 08$ & gross $\alpha$ & $9.5 \mathrm{E}-04 \pm 5.9 \mathrm{E}-04$ & & & & ${ }^{235} \mathrm{U}$ & $6.6 \mathrm{E}-07 \pm 6.9 \mathrm{E}-07$ & $\mathrm{U}$ \\
\hline & $04 / 07 / 08$ & $04 / 21 / 08$ & gross $\beta$ & $1.0 \mathrm{E}-02 \pm 1.5 \mathrm{E}-03$ & & & & ${ }^{238} \mathrm{U}$ & $1.1 \mathrm{E}-05 \pm 7.5 \mathrm{E}-06$ & \\
\hline & $04 / 21 / 08$ & $05 / 06 / 08$ & gross $\alpha$ & $1.9 \mathrm{E}-03 \pm 6.4 \mathrm{E}-04$ & & & & ${ }^{65} \mathrm{Zn}$ & $-1.2 \mathrm{E}-04 \pm 1.5 \mathrm{E}-04$ & $\mathrm{U}$ \\
\hline & $04 / 21 / 08$ & 05/06/08 & gross $\beta$ & $1.3 \mathrm{E}-02 \pm 1.7 \mathrm{E}-03$ & & & & & & \\
\hline & 05/06/08 & 05/19/08 & gross $\alpha$ & $4.4 \mathrm{E}-04 \pm 4.7 \mathrm{E}-04$ & N499 & $06 / 30 / 08$ to & $12 / 30 / 08$ & ${ }^{60} \mathrm{Co}$ & $9.3 \mathrm{E}-05 \pm 1.1 \mathrm{E}-04$ & U \\
\hline & 05/06/08 & $05 / 19 / 08$ & gross $\beta$ & $1.0 \mathrm{E}-02 \pm 1.5 \mathrm{E}-03$ & & & & ${ }^{134} \mathrm{Cs}$ & $2.9 \mathrm{E}-05 \pm 7.9 \mathrm{E}-05$ & $\mathrm{U}$ \\
\hline & 05/19/08 & $06 / 02 / 08$ & gross $\alpha$ & $7.2 \mathrm{E}-04 \pm 5.2 \mathrm{E}-04$ & & & & ${ }^{137} \mathrm{Cs}$ & $5.2 \mathrm{E}-05 \pm 7.0 \mathrm{E}-05$ & U \\
\hline & 05/19/08 & $06 / 02 / 08$ & gross $\beta$ & $1.0 \mathrm{E}-02 \pm 1.5 \mathrm{E}-03$ & & & & ${ }^{152} \mathrm{Eu}$ & $3.3 \mathrm{E}-05 \pm 1.7 \mathrm{E}-04$ & $\mathrm{U}$ \\
\hline & $06 / 02 / 08$ & $06 / 16 / 08$ & gross $\alpha$ & $7.2 \mathrm{E}-04 \pm 5.2 \mathrm{E}-04$ & & & & ${ }^{154} \mathrm{Eu}$ & $1.1 \mathrm{E}-04 \pm 2.5 \mathrm{E}-04$ & $\mathrm{U}$ \\
\hline & $06 / 02 / 08$ & 06/16/08 & gross $\beta$ & $7.0 \mathrm{E}-03 \pm 1.2 \mathrm{E}-03$ & & & & ${ }^{155} \mathrm{Eu}$ & $-5.2 \mathrm{E}-05 \pm 1.6 \mathrm{E}-04$ & $\mathrm{U}$ \\
\hline & 06/16/08 & 06/30/08 & gross $\alpha$ & $1.2 \mathrm{E}-03 \pm 5.2 \mathrm{E}-04$ & & & & ${ }^{238} \mathrm{Pu}$ & $-2.8 \mathrm{E}-06 \pm 1.1 \mathrm{E}-05$ & $\mathrm{U}$ \\
\hline & $06 / 16 / 08$ & 06/30/08 & gross $\beta$ & $1.4 \mathrm{E}-02 \pm 1.8 \mathrm{E}-03$ & & & & ${ }^{239 / 240} \mathrm{Pu}$ & $-6.9 \mathrm{E}-07 \pm 6.9 \mathrm{E}-06$ & $\mathrm{U}$ \\
\hline & $06 / 30 / 08$ & $07 / 14 / 08$ & gross $\alpha$ & $1.4 \mathrm{E}-03 \pm 5.5 \mathrm{E}-04$ & & & & ${ }^{106} \mathrm{Ru}$ & $1.6 \mathrm{E}-04 \pm 6.0 \mathrm{E}-04$ & $\mathrm{U}$ \\
\hline & 06/30/08 & 07/14/08 & gross $\beta$ & $1.3 \mathrm{E}-02 \pm 1.7 \mathrm{E}-03$ & & & & ${ }^{125} \mathrm{Sb}$ & $-1.7 \mathrm{E}-05 \pm 1.6 \mathrm{E}-04$ & $\mathrm{U}$ \\
\hline & 07/14/08 & 07/28/08 & gross $\alpha$ & $1.6 \mathrm{E}-03 \pm 6.0 \mathrm{E}-04$ & & & & ${ }^{90} \mathrm{Sr}$ & $-2.4 \mathrm{E}-04 \pm 2.5 \mathrm{E}-04$ & \\
\hline & $07 / 14 / 08$ & $07 / 28 / 08$ & gross $\beta$ & $1.7 \mathrm{E}-02 \pm 2.1 \mathrm{E}-03$ & & & & ${ }^{234} \mathrm{U}$ & $1.2 \mathrm{E}-05 \pm 8.0 \mathrm{E}-06$ & \\
\hline & $07 / 28 / 08$ & 08/11/08 & gross $\alpha$ & $1.4 \mathrm{E}-03 \pm 5.6 \mathrm{E}-04$ & & & & ${ }^{235} \mathrm{U}$ & $1.9 \mathrm{E}-06 \pm 2.8 \mathrm{E}-06$ & $\mathrm{U}$ \\
\hline & $07 / 28 / 08$ & 08/11/08 & gross $\beta$ & $9.9 \mathrm{E}-03 \pm 1.5 \mathrm{E}-03$ & & & & ${ }^{238} \mathrm{U}$ & $1.4 \mathrm{E}-05 \pm 8.8 \mathrm{E}-06$ & \\
\hline & $08 / 11 / 08$ & $08 / 25 / 08$ & gross $\alpha$ & $1.7 \mathrm{E}-03 \pm 6.2 \mathrm{E}-04$ & & & & & & \\
\hline & 08/11/08 & $08 / 25 / 08$ & gross $\beta$ & $1.6 \mathrm{E}-02 \pm 1.9 \mathrm{E}-03$ & & & & & & \\
\hline & $08 / 25 / 08$ & 09/08/08 & gross $\alpha$ & $8.3 \mathrm{E}-04 \pm 5.5 \mathrm{E}-04$ & & & & & & \\
\hline & $08 / 25 / 08$ & 09/08/08 & gross $\beta$ & $8.6 \mathrm{E}-03 \pm 1.3 \mathrm{E}-03$ & & & & & & \\
\hline & 09/08/08 & 09/22/08 & gross $\alpha$ & $1.8 \mathrm{E}-03 \pm 6.3 \mathrm{E}-04$ & & & & & & \\
\hline & 09/08/08 & $09 / 22 / 08$ & gross $\beta$ & $2.5 \mathrm{E}-02 \pm 2.6 \mathrm{E}-03$ & & & & & & \\
\hline & $09 / 22 / 08$ & $10 / 07 / 08$ & gross $\alpha$ & $1.4 \mathrm{E}-03 \pm 5.4 \mathrm{E}-04$ & & & & & & \\
\hline & $09 / 22 / 08$ & $10 / 07 / 08$ & gross $\beta$ & $2.2 \mathrm{E}-02 \pm 2.4 \mathrm{E}-03$ & & & & & & \\
\hline & $10 / 07 / 08$ & $10 / 20 / 08$ & gross $\alpha$ & $9.7 \mathrm{E}-04 \pm 6.1 \mathrm{E}-04$ & & & & & & \\
\hline & $10 / 07 / 08$ & $10 / 20 / 08$ & gross $\beta$ & $1.8 \mathrm{E}-02 \pm 2.2 \mathrm{E}-03$ & & & & & & \\
\hline & $10 / 20 / 08$ & $11 / 03 / 08$ & gross $\alpha$ & $2.3 \mathrm{E}-03 \pm 7.3 \mathrm{E}-04$ & & & & & & \\
\hline & $10 / 20 / 08$ & $11 / 03 / 08$ & gross $\beta$ & $4.5 \mathrm{E}-02 \pm 4.2 \mathrm{E}-03$ & & & & & & \\
\hline & $11 / 03 / 08$ & $11 / 17 / 08$ & gross $\alpha$ & $4.9 \mathrm{E}-04 \pm 4.5 \mathrm{E}-04$ & & & & & & \\
\hline & $11 / 03 / 08$ & $11 / 17 / 08$ & gross $\beta$ & $8.4 \mathrm{E}-03 \pm 1.3 \mathrm{E}-03$ & & & & & & \\
\hline & $11 / 17 / 08$ & $12 / 01 / 08$ & gross $\alpha$ & $1.7 \mathrm{E}-03 \pm 6.3 \mathrm{E}-04$ & & & & & & \\
\hline & $11 / 17 / 08$ & $12 / 01 / 08$ & gross $\beta$ & $4.6 \mathrm{E}-02 \pm 4.4 \mathrm{E}-03$ & & & & & & \\
\hline & $12 / 01 / 08$ & $12 / 15 / 08$ & gross $\alpha$ & $1.1 \mathrm{E}-03 \pm 5.1 \mathrm{E}-04$ & & & & & & \\
\hline & $12 / 01 / 08$ & $12 / 15 / 08$ & gross $\beta$ & $2.2 \mathrm{E}-02 \pm 2.4 \mathrm{E}-03$ & & & & & & \\
\hline & $12 / 15 / 08$ & $12 / 30 / 08$ & gross $\alpha$ & $1.3 \mathrm{E}-03 \pm 5.4 \mathrm{E}-04$ & & & & & & \\
\hline & $12 / 15 / 08$ & $12 / 30 / 08$ & gross $\beta$ & $4.1 \mathrm{E}-02 \pm 3.8 \mathrm{E}-03$ & & & & & & \\
\hline
\end{tabular}

$\overline{\mathrm{RQ}}=$ Result Qualifier. $\mathrm{U}=$ The analyte was analyzed for but not detected. 
Table 2-4. Near-Facility Air Sampling Results, 2008 (pCi/m³ \pm total analytical uncertainty). (Sheet 29 of 82)

\begin{tabular}{|c|c|c|c|c|c|c|c|c|c|}
\hline Location & Sample On & Sample Off & Isotope & Result \pm Uncertainty & Location & Composite Period & Isotope & Result \pm Uncertainty & $\mathbf{R Q *}$ \\
\hline N532 & $01 / 02 / 08$ & $01 / 16 / 08$ & gross $\alpha$ & $9.5 \mathrm{E}-04 \pm 5.9 \mathrm{E}-04$ & N532 & $01 / 02 / 08$ to $07 / 02 / 08$ & ${ }^{60} \mathrm{Co}$ & $-5.2 \mathrm{E}-05 \pm 8.3 \mathrm{E}-05$ & $\mathrm{U}$ \\
\hline \multirow[t]{51}{*}{$(200-E)$} & $01 / 02 / 08$ & 01/16/08 & gross $\beta$ & $9.5 \mathrm{E}-03 \pm 1.5 \mathrm{E}-03$ & & & ${ }^{134} \mathrm{Cs}$ & $-3.4 \mathrm{E}-05 \pm 7.3 \mathrm{E}-05$ & $\mathrm{U}$ \\
\hline & $01 / 16 / 08$ & 01/30/08 & gross $\alpha$ & $1.4 \mathrm{E}-03 \pm 5.7 \mathrm{E}-04$ & & & ${ }^{137} \mathrm{Cs}$ & $-5.5 \mathrm{E}-06 \pm 5.5 \mathrm{E}-05$ & U \\
\hline & $01 / 16 / 08$ & 01/30/08 & gross $\beta$ & $4.0 \mathrm{E}-02 \pm 4.3 \mathrm{E}-03$ & & & ${ }^{152} \mathrm{Eu}$ & $-4.3 \mathrm{E}-05 \pm 1.5 \mathrm{E}-04$ & U \\
\hline & 01/30/08 & 02/13/08 & gross $\alpha$ & $6.2 \mathrm{E}-04 \pm 5.1 \mathrm{E}-04$ & & & ${ }^{154} \mathrm{Eu}$ & $-6.6 \mathrm{E}-05 \pm 2.3 \mathrm{E}-04$ & $\mathrm{U}$ \\
\hline & 01/30/08 & $02 / 13 / 08$ & gross $\beta$ & $4.8 \mathrm{E}-03 \pm 9.9 \mathrm{E}-04$ & & & ${ }^{155} \mathrm{Eu}$ & $-4.5 \mathrm{E}-05 \pm 1.6 \mathrm{E}-04$ & U \\
\hline & $02 / 13 / 08$ & $02 / 27 / 08$ & gross $\alpha$ & $1.1 \mathrm{E}-03 \pm 6.8 \mathrm{E}-04$ & & & ${ }^{238} \mathrm{Pu}$ & $-7.2 \mathrm{E}-07 \pm 7.2 \mathrm{E}-06$ & $\mathrm{U}$ \\
\hline & $02 / 13 / 08$ & $02 / 27 / 08$ & gross $\beta$ & $3.3 \mathrm{E}-02 \pm 3.8 \mathrm{E}-03$ & & & ${ }^{239 / 240} \mathrm{Pu}$ & $4.3 \mathrm{E}-06 \pm 4.8 \mathrm{E}-06$ & $\mathrm{U}$ \\
\hline & $02 / 27 / 08$ & 03/12/08 & gross $\alpha$ & $9.5 \mathrm{E}-04 \pm 5.9 \mathrm{E}-04$ & & & ${ }^{106} \mathrm{Ru}$ & $1.8 \mathrm{E}-04 \pm 5.9 \mathrm{E}-04$ & $\mathrm{U}$ \\
\hline & $02 / 27 / 08$ & $03 / 12 / 08$ & gross $\beta$ & $9.9 \mathrm{E}-03 \pm 1.5 \mathrm{E}-03$ & & & ${ }^{125} \mathrm{Sb}$ & $1.1 \mathrm{E}-04 \pm 1.5 \mathrm{E}-04$ & $\mathrm{U}$ \\
\hline & 03/12/08 & 03/26/08 & gross $\alpha$ & $3.0 \mathrm{E}-04 \pm 4.3 \mathrm{E}-04$ & & & ${ }^{90} \mathrm{Sr}$ & $-1.9 \mathrm{E}-04 \pm 2.0 \mathrm{E}-04$ & $\mathrm{U}$ \\
\hline & 03/12/08 & 03/26/08 & gross $\beta$ & $1.0 \mathrm{E}-02 \pm 1.6 \mathrm{E}-03$ & & & ${ }^{234} \mathrm{U}$ & $4.7 \mathrm{E}-06 \pm 5.1 \mathrm{E}-06$ & $\mathrm{U}$ \\
\hline & $03 / 26 / 08$ & $04 / 09 / 08$ & gross $\alpha$ & $3.7 \mathrm{E}-04 \pm 4.2 \mathrm{E}-04$ & & & ${ }^{235} \mathrm{U}$ & $2.9 \mathrm{E}-06 \pm 3.1 \mathrm{E}-06$ & \\
\hline & 03/26/08 & 04/09/08 & gross $\beta$ & $6.5 \mathrm{E}-03 \pm 1.2 \mathrm{E}-03$ & & & ${ }^{238} \mathrm{U}$ & $5.3 \mathrm{E}-06 \pm 4.7 \mathrm{E}-06$ & \\
\hline & 04/09/08 & 04/23/08 & gross $\alpha$ & $1.1 \mathrm{E}-03 \pm 5.1 \mathrm{E}-04$ & & & & & \\
\hline & $04 / 09 / 08$ & $04 / 23 / 08$ & gross $\beta$ & $1.0 \mathrm{E}-02 \pm 1.6 \mathrm{E}-03$ & N532 & $07 / 02 / 08$ to $12 / 30 / 08$ & ${ }^{60} \mathrm{Co}$ & $-8.1 \mathrm{E}-06 \pm 7.8 \mathrm{E}-05$ & $\mathrm{U}$ \\
\hline & $04 / 23 / 08$ & 05/07/08 & gross $\alpha$ & $7.0 \mathrm{E}-04 \pm 5.2 \mathrm{E}-04$ & & & ${ }^{134} \mathrm{Cs}$ & $-7.2 \mathrm{E}-05 \pm 7.9 \mathrm{E}-05$ & $\mathrm{U}$ \\
\hline & $04 / 23 / 08$ & 05/07/08 & gross $\beta$ & $1.9 \mathrm{E}-02 \pm 2.4 \mathrm{E}-03$ & & & ${ }^{137} \mathrm{Cs}$ & $1.1 \mathrm{E}-05 \pm 6.3 \mathrm{E}-05$ & $\mathrm{U}$ \\
\hline & 05/07/08 & 05/21/08 & gross $\alpha$ & $1.5 \mathrm{E}-03 \pm 5.8 \mathrm{E}-04$ & & & ${ }^{152} \mathrm{Eu}$ & $-2.6 \mathrm{E}-05 \pm 1.8 \mathrm{E}-04$ & U \\
\hline & 05/07/08 & 05/21/08 & gross $\beta$ & $1.2 \mathrm{E}-02 \pm 1.7 \mathrm{E}-03$ & & & ${ }^{154} \mathrm{Eu}$ & $-1.4 \mathrm{E}-04 \pm 2.5 \mathrm{E}-04$ & $\mathrm{U}$ \\
\hline & 05/21/08 & 06/04/08 & gross $\alpha$ & $2.8 \mathrm{E}-04 \pm 3.9 \mathrm{E}-04$ & & & ${ }^{155} \mathrm{Eu}$ & $-3.9 \mathrm{E}-05 \pm 1.8 \mathrm{E}-04$ & U \\
\hline & $05 / 21 / 08$ & $06 / 04 / 08$ & gross $\beta$ & $8.3 \mathrm{E}-03 \pm 1.4 \mathrm{E}-03$ & & & ${ }^{238} \mathrm{Pu}$ & $1.0 \mathrm{E}-05 \pm 1.1 \mathrm{E}-05$ & $\mathrm{U}$ \\
\hline & $06 / 04 / 08$ & 06/18/08 & gross $\alpha$ & $8.3 \mathrm{E}-04 \pm 5.5 \mathrm{E}-04$ & & & ${ }^{239 / 240} \mathrm{Pu}$ & $7.9 \mathrm{E}-07 \pm 8.2 \mathrm{E}-07$ & $\mathrm{U}$ \\
\hline & $06 / 04 / 08$ & 06/18/08 & gross $\beta$ & $7.1 \mathrm{E}-03 \pm 1.2 \mathrm{E}-03$ & & & ${ }^{106} \mathrm{Ru}$ & $1.2 \mathrm{E}-04 \pm 6.4 \mathrm{E}-04$ & $\mathrm{U}$ \\
\hline & 06/18/08 & $07 / 02 / 08$ & gross $\alpha$ & $1.0 \mathrm{E}-03 \pm 5.0 \mathrm{E}-04$ & & & ${ }^{125} \mathrm{Sb}$ & $3.4 \mathrm{E}-05 \pm 1.5 \mathrm{E}-04$ & $\mathrm{U}$ \\
\hline & $06 / 18 / 08$ & $07 / 02 / 08$ & gross $\beta$ & $1.2 \mathrm{E}-02 \pm 1.8 \mathrm{E}-03$ & & & ${ }^{90} \mathrm{Sr}$ & $-9.3 \mathrm{E}-05 \pm 9.7 \mathrm{E}-05$ & $\mathrm{U}$ \\
\hline & $07 / 02 / 08$ & $07 / 16 / 08$ & gross $\alpha$ & $1.8 \mathrm{E}-03 \pm 6.4 \mathrm{E}-04$ & & & ${ }^{234} \mathrm{U}$ & $1.2 \mathrm{E}-05 \pm 8.1 \mathrm{E}-06$ & \\
\hline & $07 / 02 / 08$ & 07/16/08 & gross $\beta$ & $1.4 \mathrm{E}-02 \pm 1.9 \mathrm{E}-03$ & & & ${ }^{235} \mathrm{U}$ & $4.2 \mathrm{E}-06 \pm 4.1 \mathrm{E}-06$ & \\
\hline & 07/16/08 & 07/30/08 & gross $\alpha$ & $6.1 \mathrm{E}-04 \pm 4.9 \mathrm{E}-04$ & & & ${ }^{238} \mathrm{U}$ & $6.2 \mathrm{E}-06 \pm 5.4 \mathrm{E}-06$ & \\
\hline & $07 / 16 / 08$ & 07/30/08 & gross $\beta$ & $1.1 \mathrm{E}-02 \pm 1.6 \mathrm{E}-03$ & & & & & \\
\hline & 07/30/08 & 08/13/08 & gross $\alpha$ & $1.2 \mathrm{E}-03 \pm 5.1 \mathrm{E}-04$ & & & & & \\
\hline & 07/30/08 & $08 / 13 / 08$ & gross $\beta$ & $1.4 \mathrm{E}-02 \pm 1.9 \mathrm{E}-03$ & & & & & \\
\hline & 08/13/08 & $08 / 27 / 08$ & gross $\alpha$ & $1.0 \mathrm{E}-03 \pm 4.9 \mathrm{E}-04$ & & & & & \\
\hline & 08/13/08 & 08/27/08 & gross $\beta$ & $1.1 \mathrm{E}-02 \pm 1.6 \mathrm{E}-03$ & & & & & \\
\hline & $08 / 27 / 08$ & 09/10/08 & gross $\alpha$ & $7.2 \mathrm{E}-04 \pm 5.2 \mathrm{E}-04$ & & & & & \\
\hline & 08/27/08 & 09/10/08 & gross $\beta$ & $1.0 \mathrm{E}-02 \pm 1.6 \mathrm{E}-03$ & & & & & \\
\hline & 09/10/08 & 09/24/08 & gross $\alpha$ & $1.2 \mathrm{E}-03 \pm 5.1 \mathrm{E}-04$ & & & & & \\
\hline & 09/10/08 & 09/24/08 & gross $\beta$ & $2.4 \mathrm{E}-02 \pm 2.8 \mathrm{E}-03$ & & & & & \\
\hline & $09 / 24 / 08$ & $10 / 08 / 08$ & gross $\alpha$ & $2.3 \mathrm{E}-03 \pm 7.3 \mathrm{E}-04$ & & & & & \\
\hline & $09 / 24 / 08$ & $10 / 08 / 08$ & gross $\beta$ & $2.2 \mathrm{E}-02 \pm 2.6 \mathrm{E}-03$ & & & & & \\
\hline & $10 / 08 / 08$ & $10 / 22 / 08$ & gross $\alpha$ & $6.2 \mathrm{E}-04 \pm 5.0 \mathrm{E}-04$ & & & & & \\
\hline & $10 / 08 / 08$ & $10 / 22 / 08$ & gross $\beta$ & $1.6 \mathrm{E}-02 \pm 2.1 \mathrm{E}-03$ & & & & & \\
\hline & $10 / 22 / 08$ & $11 / 05 / 08$ & gross $\alpha$ & $2.1 \mathrm{E}-03 \pm 7.0 \mathrm{E}-04$ & & & & & \\
\hline & $10 / 22 / 08$ & $11 / 05 / 08$ & gross $\beta$ & $4.0 \mathrm{E}-02 \pm 4.2 \mathrm{E}-03$ & & & & & \\
\hline & $11 / 05 / 08$ & $11 / 18 / 08$ & gross $\alpha$ & $7.7 \mathrm{E}-04 \pm 5.5 \mathrm{E}-04$ & & & & & \\
\hline & $11 / 05 / 08$ & $11 / 18 / 08$ & gross $\beta$ & $2.3 \mathrm{E}-02 \pm 2.8 \mathrm{E}-03$ & & & & & \\
\hline & $11 / 18 / 08$ & $12 / 03 / 08$ & gross $\alpha$ & $1.6 \mathrm{E}-03 \pm 1.0 \mathrm{E}-03$ & & & & & \\
\hline & $11 / 18 / 08$ & $12 / 03 / 08$ & gross $\beta$ & $2.9 \mathrm{E}-02 \pm 3.9 \mathrm{E}-03$ & & & & & \\
\hline & $12 / 03 / 08$ & $12 / 17 / 08$ & gross $\alpha$ & $9.8 \mathrm{E}-04 \pm 6.1 \mathrm{E}-04$ & & & & & \\
\hline & $12 / 03 / 08$ & $12 / 17 / 08$ & gross $\beta$ & $1.9 \mathrm{E}-02 \pm 2.5 \mathrm{E}-03$ & & & & & \\
\hline & $12 / 17 / 08$ & $12 / 30 / 08$ & gross $\alpha$ & $2.2 \mathrm{E}-03 \pm 7.5 \mathrm{E}-04$ & & & & & \\
\hline & $12 / 17 / 08$ & $12 / 30 / 08$ & gross $\beta$ & $4.1 \mathrm{E}-02 \pm 4.4 \mathrm{E}-03$ & & & & & \\
\hline
\end{tabular}

$\overline{\mathrm{RQ}}=$ Result Qualifier. $\mathrm{U}=$ The analyte was analyzed for but not detected. 
Table 2-4. Near-Facility Air Sampling Results, 2008 (pCi/m³ \pm total analytical uncertainty). (Sheet 30 of 82)

\begin{tabular}{|c|c|c|c|c|c|c|c|c|c|}
\hline Location & Sample On & Sample Off & Isotope & Result \pm Uncertainty & Location & Composite Period & Isotope & Result \pm Uncertainty & $\mathbf{R Q *}$ \\
\hline N559 & $01 / 02 / 08$ & $01 / 16 / 08$ & gross $\alpha$ & $8.4 \mathrm{E}-04 \pm 5.6 \mathrm{E}-04$ & N559 & $01 / 02 / 08$ to $07 / 02 / 08$ & ${ }^{60} \mathrm{Co}$ & $2.9 \mathrm{E}-05 \pm 7.0 \mathrm{E}-05$ & $\mathrm{U}$ \\
\hline \multirow[t]{51}{*}{$(200-E)$} & $01 / 02 / 08$ & 01/16/08 & gross $\beta$ & $8.1 \mathrm{E}-03 \pm 1.4 \mathrm{E}-03$ & & & ${ }^{134} \mathrm{Cs}$ & $-5.9 \mathrm{E}-05 \pm 6.6 \mathrm{E}-05$ & $\mathrm{U}$ \\
\hline & $01 / 16 / 08$ & 01/30/08 & gross $\alpha$ & $2.2 \mathrm{E}-03 \pm 7.2 \mathrm{E}-04$ & & & ${ }^{137} \mathrm{Cs}$ & $1.6 \mathrm{E}-05 \pm 5.8 \mathrm{E}-05$ & U \\
\hline & $01 / 16 / 08$ & 01/30/08 & gross $\beta$ & $3.6 \mathrm{E}-02 \pm 4.0 \mathrm{E}-03$ & & & ${ }^{152} \mathrm{Eu}$ & $6.5 \mathrm{E}-05 \pm 1.4 \mathrm{E}-04$ & U \\
\hline & 01/30/08 & 02/13/08 & gross $\alpha$ & $1.6 \mathrm{E}-04 \pm 3.4 \mathrm{E}-04$ & & & ${ }^{154} \mathrm{Eu}$ & $5.9 \mathrm{E}-05 \pm 1.8 \mathrm{E}-04$ & $\mathrm{U}$ \\
\hline & 01/30/08 & 02/13/08 & gross $\beta$ & $3.0 \mathrm{E}-03 \pm 7.7 \mathrm{E}-04$ & & & ${ }^{155} \mathrm{Eu}$ & $-4.4 \mathrm{E}-05 \pm 1.6 \mathrm{E}-04$ & U \\
\hline & $02 / 13 / 08$ & $02 / 27 / 08$ & gross $\alpha$ & $1.2 \mathrm{E}-03 \pm 5.4 \mathrm{E}-04$ & & & ${ }^{238} \mathrm{Pu}$ & $-5.9 \mathrm{E}-06 \pm 1.1 \mathrm{E}-05$ & $\mathrm{U}$ \\
\hline & $02 / 13 / 08$ & $02 / 27 / 08$ & gross $\beta$ & $3.1 \mathrm{E}-02 \pm 3.5 \mathrm{E}-03$ & & & ${ }^{239 / 240} \mathrm{Pu}$ & $1.7 \mathrm{E}-06 \pm 2.4 \mathrm{E}-06$ & $\mathrm{U}$ \\
\hline & $02 / 27 / 08$ & 03/12/08 & gross $\alpha$ & $5.1 \mathrm{E}-04 \pm 4.8 \mathrm{E}-04$ & & & ${ }^{106} \mathrm{Ru}$ & $-2.4 \mathrm{E}-05 \pm 2.4 \mathrm{E}-04$ & $\mathrm{U}$ \\
\hline & $02 / 27 / 08$ & $03 / 12 / 08$ & gross $\beta$ & $1.2 \mathrm{E}-02 \pm 1.7 \mathrm{E}-03$ & & & ${ }^{125} \mathrm{Sb}$ & $-7.9 \mathrm{E}-05 \pm 1.4 \mathrm{E}-04$ & $\mathrm{U}$ \\
\hline & 03/12/08 & 03/26/08 & gross $\alpha$ & $8.4 \mathrm{E}-04 \pm 5.6 \mathrm{E}-04$ & & & ${ }^{90} \mathrm{Sr}$ & $9.0 \mathrm{E}-05 \pm 1.9 \mathrm{E}-04$ & $\mathrm{U}$ \\
\hline & 03/12/08 & 03/26/08 & gross $\beta$ & $9.6 \mathrm{E}-03 \pm 1.5 \mathrm{E}-03$ & & & ${ }^{234} \mathrm{U}$ & $1.4 \mathrm{E}-05 \pm 9.0 \mathrm{E}-06$ & \\
\hline & 03/26/08 & $04 / 09 / 08$ & gross $\alpha$ & $6.0 \mathrm{E}-04 \pm 4.9 \mathrm{E}-04$ & & & ${ }^{235} \mathrm{U}$ & $3.7 \mathrm{E}-06 \pm 3.6 \mathrm{E}-06$ & \\
\hline & 03/26/08 & 04/09/08 & gross $\beta$ & $5.8 \mathrm{E}-03 \pm 1.1 \mathrm{E}-03$ & & & ${ }^{238} \mathrm{U}$ & $8.8 \mathrm{E}-06 \pm 5.9 \mathrm{E}-06$ & \\
\hline & 04/09/08 & 04/23/08 & gross $\alpha$ & $1.5 \mathrm{E}-03 \pm 5.8 \mathrm{E}-04$ & & & & & \\
\hline & $04 / 09 / 08$ & $04 / 23 / 08$ & gross $\beta$ & $9.6 \mathrm{E}-03 \pm 1.5 \mathrm{E}-03$ & N559 & $07 / 02 / 08$ to $12 / 30 / 08$ & ${ }^{60} \mathrm{Co}$ & $-5.0 \mathrm{E}-06 \pm 5.0 \mathrm{E}-05$ & $\mathrm{U}$ \\
\hline & $04 / 23 / 08$ & 05/07/08 & gross $\alpha$ & $1.4 \mathrm{E}-03 \pm 5.9 \mathrm{E}-04$ & & & ${ }^{134} \mathrm{Cs}$ & $1.9 \mathrm{E}-05 \pm 1.0 \mathrm{E}-04$ & $\mathrm{U}$ \\
\hline & $04 / 23 / 08$ & 05/07/08 & gross $\beta$ & $1.5 \mathrm{E}-02 \pm 2.0 \mathrm{E}-03$ & & & ${ }^{137} \mathrm{Cs}$ & $-3.2 \mathrm{E}-05 \pm 9.6 \mathrm{E}-05$ & $\mathrm{U}$ \\
\hline & 05/07/08 & 05/21/08 & gross $\alpha$ & $1.3 \mathrm{E}-03 \pm 5.5 \mathrm{E}-04$ & & & ${ }^{152} \mathrm{Eu}$ & $-3.0 \mathrm{E}-04 \pm 3.2 \mathrm{E}-04$ & U \\
\hline & 05/07/08 & 05/21/08 & gross $\beta$ & $1.2 \mathrm{E}-02 \pm 1.7 \mathrm{E}-03$ & & & ${ }^{154} \mathrm{Eu}$ & $5.5 \mathrm{E}-05 \pm 3.2 \mathrm{E}-04$ & $\mathrm{U}$ \\
\hline & 05/21/08 & 06/04/08 & gross $\alpha$ & $8.9 \mathrm{E}-04 \pm 5.6 \mathrm{E}-04$ & & & ${ }^{155} \mathrm{Eu}$ & $1.1 \mathrm{E}-06 \pm 1.1 \mathrm{E}-05$ & U \\
\hline & $05 / 21 / 08$ & $06 / 04 / 08$ & gross $\beta$ & $1.4 \mathrm{E}-02 \pm 2.0 \mathrm{E}-03$ & & & ${ }^{238} \mathrm{Pu}$ & $6.5 \mathrm{E}-07 \pm 6.5 \mathrm{E}-06$ & $\mathrm{U}$ \\
\hline & $06 / 04 / 08$ & 06/18/08 & gross $\alpha$ & $9.5 \mathrm{E}-04 \pm 5.9 \mathrm{E}-04$ & & & ${ }^{239 / 240} \mathrm{Pu}$ & $6.5 \mathrm{E}-07 \pm 3.2 \mathrm{E}-06$ & $\mathrm{U}$ \\
\hline & $06 / 04 / 08$ & 06/18/08 & gross $\beta$ & $9.1 \mathrm{E}-03 \pm 1.5 \mathrm{E}-03$ & & & ${ }^{106} \mathrm{Ru}$ & $2.7 \mathrm{E}-05 \pm 2.7 \mathrm{E}-04$ & $\mathrm{U}$ \\
\hline & 06/18/08 & $07 / 02 / 08$ & gross $\alpha$ & $8.0 \mathrm{E}-04 \pm 6.2 \mathrm{E}-04$ & & & ${ }^{125} \mathrm{Sb}$ & $-1.0 \mathrm{E}-04 \pm 2.4 \mathrm{E}-04$ & $\mathrm{U}$ \\
\hline & $06 / 18 / 08$ & $07 / 02 / 08$ & gross $\beta$ & $1.5 \mathrm{E}-02 \pm 2.0 \mathrm{E}-03$ & & & ${ }^{90} \mathrm{Sr}$ & $-4.6 \mathrm{E}-04 \pm 4.8 \mathrm{E}-04$ & $\mathrm{U}$ \\
\hline & $07 / 02 / 08$ & $07 / 16 / 08$ & gross $\alpha$ & $1.4 \mathrm{E}-03 \pm 6.1 \mathrm{E}-04$ & & & ${ }^{234} \mathrm{U}$ & $1.7 \mathrm{E}-05 \pm 9.4 \mathrm{E}-06$ & \\
\hline & $07 / 02 / 08$ & 07/16/08 & gross $\beta$ & $1.3 \mathrm{E}-02 \pm 1.9 \mathrm{E}-03$ & & & ${ }^{235} \mathrm{U}$ & $2.1 \mathrm{E}-06 \pm 2.5 \mathrm{E}-06$ & \\
\hline & 07/16/08 & 07/30/08 & gross $\alpha$ & $1.2 \mathrm{E}-03 \pm 5.2 \mathrm{E}-04$ & & & ${ }^{238} \mathrm{U}$ & $7.6 \mathrm{E}-06 \pm 5.2 \mathrm{E}-06$ & \\
\hline & $07 / 16 / 08$ & 07/30/08 & gross $\beta$ & $1.4 \mathrm{E}-02 \pm 2.0 \mathrm{E}-03$ & & & & & \\
\hline & 07/30/08 & 08/13/08 & gross $\alpha$ & $1.0 \mathrm{E}-03 \pm 4.8 \mathrm{E}-04$ & & & & & \\
\hline & 07/30/08 & $08 / 13 / 08$ & gross $\beta$ & $1.7 \mathrm{E}-02 \pm 2.3 \mathrm{E}-03$ & & & & & \\
\hline & 08/13/08 & $08 / 27 / 08$ & gross $\alpha$ & $1.0 \mathrm{E}-03 \pm 4.8 \mathrm{E}-04$ & & & & & \\
\hline & 08/13/08 & 08/27/08 & gross $\beta$ & $1.5 \mathrm{E}-02 \pm 2.1 \mathrm{E}-03$ & & & & & \\
\hline & $08 / 27 / 08$ & 09/10/08 & gross $\alpha$ & $1.6 \mathrm{E}-03 \pm 6.1 \mathrm{E}-04$ & & & & & \\
\hline & 08/27/08 & 09/10/08 & gross $\beta$ & $1.4 \mathrm{E}-02 \pm 2.0 \mathrm{E}-03$ & & & & & \\
\hline & 09/10/08 & 09/24/08 & gross $\alpha$ & $1.5 \mathrm{E}-03 \pm 5.9 \mathrm{E}-04$ & & & & & \\
\hline & 09/10/08 & 09/24/08 & gross $\beta$ & $2.7 \mathrm{E}-02 \pm 3.2 \mathrm{E}-03$ & & & & & \\
\hline & $09 / 24 / 08$ & $10 / 08 / 08$ & gross $\alpha$ & $1.4 \mathrm{E}-03 \pm 5.7 \mathrm{E}-04$ & & & & & \\
\hline & $09 / 24 / 08$ & $10 / 08 / 08$ & gross $\beta$ & $2.7 \mathrm{E}-02 \pm 3.1 \mathrm{E}-03$ & & & & & \\
\hline & $10 / 08 / 08$ & $10 / 22 / 08$ & gross $\alpha$ & $2.0 \mathrm{E}-03 \pm 6.7 \mathrm{E}-04$ & & & & & \\
\hline & $10 / 08 / 08$ & $10 / 22 / 08$ & gross $\beta$ & $1.8 \mathrm{E}-02 \pm 2.4 \mathrm{E}-03$ & & & & & \\
\hline & $10 / 22 / 08$ & $11 / 05 / 08$ & gross $\alpha$ & $2.7 \mathrm{E}-03 \pm 7.9 \mathrm{E}-04$ & & & & & \\
\hline & $10 / 22 / 08$ & $11 / 05 / 08$ & gross $\beta$ & $4.2 \mathrm{E}-02 \pm 4.5 \mathrm{E}-03$ & & & & & \\
\hline & $11 / 05 / 08$ & $11 / 18 / 08$ & gross $\alpha$ & $1.5 \mathrm{E}-03 \pm 6.0 \mathrm{E}-04$ & & & & & \\
\hline & $11 / 05 / 08$ & $11 / 18 / 08$ & gross $\beta$ & $2.3 \mathrm{E}-02 \pm 2.8 \mathrm{E}-03$ & & & & & \\
\hline & $11 / 18 / 08$ & $12 / 03 / 08$ & gross $\alpha$ & $1.4 \mathrm{E}-03 \pm 5.6 \mathrm{E}-04$ & & & & & \\
\hline & $11 / 18 / 08$ & $12 / 03 / 08$ & gross $\beta$ & $3.3 \mathrm{E}-02 \pm 3.6 \mathrm{E}-03$ & & & & & \\
\hline & $12 / 03 / 08$ & $12 / 17 / 08$ & gross $\alpha$ & $1.4 \mathrm{E}-03 \pm 5.6 \mathrm{E}-04$ & & & & & \\
\hline & $12 / 03 / 08$ & $12 / 17 / 08$ & gross $\beta$ & $2.1 \mathrm{E}-02 \pm 2.5 \mathrm{E}-03$ & & & & & \\
\hline & $12 / 17 / 08$ & $12 / 30 / 08$ & gross $\alpha$ & $1.5 \mathrm{E}-03 \pm 6.0 \mathrm{E}-04$ & & & & & \\
\hline & $12 / 17 / 08$ & $12 / 30 / 08$ & gross $\beta$ & $3.0 \mathrm{E}-02 \pm 3.4 \mathrm{E}-03$ & & & & & \\
\hline
\end{tabular}

$\overline{\mathrm{RQ}}=$ Result Qualifier. $\mathrm{U}=$ The analyte was analyzed for but not detected. 
Table 2-4. Near-Facility Air Sampling Results, 2008 (pCi/m³ \pm total analytical uncertainty). (Sheet 31 of 82)

\begin{tabular}{|c|c|c|c|c|c|c|c|c|c|}
\hline Location & Sample On & Sample Off & Isotope & Result \pm Uncertainty & Location & Composite Period & Isotope & Result \pm Uncertainty & $\mathbf{R Q *}$ \\
\hline N957 & $12 / 31 / 07$ & $01 / 14 / 08$ & gross $\alpha$ & $1.2 \mathrm{E}-03 \pm 5.7 \mathrm{E}-04$ & N957 & $12 / 31 / 07$ to $06 / 30 / 08$ & ${ }^{60} \mathrm{Co}$ & $-1.6 \mathrm{E}-04 \pm 1.6 \mathrm{E}-04$ & $\mathrm{U}$ \\
\hline \multirow[t]{51}{*}{$(200-E)$} & $12 / 31 / 07$ & 01/14/08 & gross $\beta$ & $7.3 \mathrm{E}-03 \pm 1.3 \mathrm{E}-03$ & & & ${ }^{134} \mathrm{Cs}$ & $6.6 \mathrm{E}-05 \pm 1.1 \mathrm{E}-04$ & $\mathrm{U}$ \\
\hline & 01/14/08 & $01 / 29 / 08$ & gross $\alpha$ & $1.7 \mathrm{E}-03 \pm 6.1 \mathrm{E}-04$ & & & ${ }^{137} \mathrm{Cs}$ & $-6.0 \mathrm{E}-05 \pm 1.0 \mathrm{E}-04$ & $\mathrm{U}$ \\
\hline & $01 / 14 / 08$ & 01/29/08 & gross $\beta$ & $3.0 \mathrm{E}-02 \pm 3.0 \mathrm{E}-03$ & & & ${ }^{152} \mathrm{Eu}$ & $1.8 \mathrm{E}-05 \pm 1.8 \mathrm{E}-04$ & $\mathrm{U}$ \\
\hline & $01 / 29 / 08$ & $02 / 12 / 08$ & gross $\alpha$ & $6.1 \mathrm{E}-04 \pm 5.0 \mathrm{E}-04$ & & & ${ }^{154} \mathrm{Eu}$ & $-5.5 \mathrm{E}-05 \pm 3.5 \mathrm{E}-04$ & $\mathrm{U}$ \\
\hline & $01 / 29 / 08$ & $02 / 12 / 08$ & gross $\beta$ & $4.4 \mathrm{E}-03 \pm 9.3 \mathrm{E}-04$ & & & ${ }^{155} \mathrm{Eu}$ & $-5.2 \mathrm{E}-05 \pm 1.8 \mathrm{E}-04$ & $\mathrm{U}$ \\
\hline & $02 / 12 / 08$ & $02 / 25 / 08$ & gross $\alpha$ & $2.0 \mathrm{E}-03 \pm 7.2 \mathrm{E}-04$ & & & ${ }^{238} \mathrm{Pu}$ & $5.7 \mathrm{E}-07 \pm 5.7 \mathrm{E}-06$ & \\
\hline & $02 / 12 / 08$ & $02 / 25 / 08$ & gross $\beta$ & $3.1 \mathrm{E}-02 \pm 3.2 \mathrm{E}-03$ & & & ${ }^{239 / 240} \mathrm{Pu}$ & $-5.7 \mathrm{E}-07 \pm 1.2 \mathrm{E}-06$ & $\mathrm{U}$ \\
\hline & $02 / 25 / 08$ & 03/11/08 & $\operatorname{gross} \alpha$ & $1.0 \mathrm{E}-03 \pm 4.9 \mathrm{E}-04$ & & & ${ }^{106} \mathrm{Ru}$ & $-5.2 \mathrm{E}-04 \pm 9.3 \mathrm{E}-04$ & $\mathrm{U}$ \\
\hline & $02 / 25 / 08$ & 03/11/08 & gross $\beta$ & $1.4 \mathrm{E}-02 \pm 1.8 \mathrm{E}-03$ & & & ${ }^{125} \mathrm{Sb}$ & $-2.8 \mathrm{E}-05 \pm 2.4 \mathrm{E}-04$ & $\mathrm{U}$ \\
\hline & 03/11/08 & 03/25/08 & gross $\alpha$ & $5.3 \mathrm{E}-04 \pm 5.0 \mathrm{E}-04$ & & & ${ }^{90} \mathrm{Sr}$ & $-1.4 \mathrm{E}-04 \pm 1.5 \mathrm{E}-04$ & $\mathrm{U}$ \\
\hline & 03/11/08 & $03 / 25 / 08$ & gross $\beta$ & $7.8 \mathrm{E}-03 \pm 1.3 \mathrm{E}-03$ & & & ${ }^{234} \mathrm{U}$ & $1.1 \mathrm{E}-05 \pm 6.7 \mathrm{E}-06$ & \\
\hline & 03/25/08 & $04 / 07 / 08$ & gross $\alpha$ & $8.0 \mathrm{E}-04 \pm 5.9 \mathrm{E}-04$ & & & ${ }^{235} \mathrm{U}$ & $7.3 \mathrm{E}-07 \pm 3.3 \mathrm{E}-06$ & $\mathrm{U}$ \\
\hline & 03/25/08 & 04/07/08 & gross $\beta$ & $8.8 \mathrm{E}-03 \pm 1.4 \mathrm{E}-03$ & & & ${ }^{238} \mathrm{U}$ & $8.0 \mathrm{E}-06 \pm 5.8 \mathrm{E}-06$ & \\
\hline & 04/07/08 & $04 / 21 / 08$ & gross $\alpha$ & $1.3 \mathrm{E}-03 \pm 5.6 \mathrm{E}-04$ & & & & & \\
\hline & $04 / 07 / 08$ & $04 / 21 / 08$ & gross $\beta$ & $1.0 \mathrm{E}-02 \pm 1.5 \mathrm{E}-03$ & N957 & $06 / 30 / 08$ to $12 / 30 / 08$ & ${ }^{60} \mathrm{Co}$ & $9.1 \mathrm{E}-05 \pm 5.7 \mathrm{E}-05$ & $\mathrm{U}$ \\
\hline & $04 / 21 / 08$ & 05/07/08 & gross $\alpha$ & $1.2 \mathrm{E}-03 \pm 4.9 \mathrm{E}-04$ & & & ${ }^{134} \mathrm{Cs}$ & $2.9 \mathrm{E}-06 \pm 2.9 \mathrm{E}-05$ & $\mathrm{U}$ \\
\hline & $04 / 21 / 08$ & 05/07/08 & gross $\beta$ & $1.4 \mathrm{E}-02 \pm 1.8 \mathrm{E}-03$ & & & ${ }^{137} \mathrm{Cs}$ & $-5.8 \mathrm{E}-05 \pm 6.7 \mathrm{E}-05$ & $\mathrm{U}$ \\
\hline & 05/07/08 & 05/19/08 & gross $\alpha$ & $9.4 \mathrm{E}-04 \pm 6.5 \mathrm{E}-04$ & & & ${ }^{152} \mathrm{Eu}$ & $-5.6 \mathrm{E}-05 \pm 1.5 \mathrm{E}-04$ & $\mathrm{U}$ \\
\hline & 05/07/08 & 05/19/08 & gross $\beta$ & $1.5 \mathrm{E}-02 \pm 2.1 \mathrm{E}-03$ & & & ${ }^{154} \mathrm{Eu}$ & $3.8 \mathrm{E}-05 \pm 2.3 \mathrm{E}-04$ & $\mathrm{U}$ \\
\hline & 05/19/08 & $06 / 02 / 08$ & gross $\alpha$ & $7.9 \mathrm{E}-04 \pm 5.4 \mathrm{E}-04$ & & & ${ }^{155} \mathrm{Eu}$ & $-6.2 \mathrm{E}-05 \pm 1.6 \mathrm{E}-04$ & U \\
\hline & 05/19/08 & $06 / 02 / 08$ & gross $\beta$ & $8.8 \mathrm{E}-03 \pm 1.4 \mathrm{E}-03$ & & & ${ }^{238} \mathrm{Pu}$ & $1.1 \mathrm{E}-05 \pm 1.1 \mathrm{E}-05$ & $\mathrm{U}$ \\
\hline & $06 / 02 / 08$ & $06 / 16 / 08$ & gross $\alpha$ & $8.0 \mathrm{E}-04 \pm 5.4 \mathrm{E}-04$ & & & ${ }^{239 / 240} \mathrm{Pu}$ & $-1.6 \mathrm{E}-06 \pm 3.2 \mathrm{E}-06$ & $\mathrm{U}$ \\
\hline & $06 / 02 / 08$ & 06/16/08 & gross $\beta$ & $7.6 \mathrm{E}-03 \pm 1.2 \mathrm{E}-03$ & & & ${ }^{106} \mathrm{Ru}$ & $2.6 \mathrm{E}-04 \pm 5.9 \mathrm{E}-04$ & $\mathrm{U}$ \\
\hline & $06 / 16 / 08$ & 06/30/08 & gross $\alpha$ & $1.4 \mathrm{E}-03 \pm 6.2 \mathrm{E}-04$ & & & ${ }^{125} \mathrm{Sb}$ & $-2.5 \mathrm{E}-05 \pm 1.4 \mathrm{E}-04$ & $\mathrm{U}$ \\
\hline & $06 / 16 / 08$ & 06/30/08 & gross $\beta$ & $1.5 \mathrm{E}-02 \pm 1.9 \mathrm{E}-03$ & & & ${ }^{90} \mathrm{Sr}$ & $-2.4 \mathrm{E}-04 \pm 2.5 \mathrm{E}-04$ & $\mathrm{U}$ \\
\hline & $06 / 30 / 08$ & 07/11/08 & gross $\alpha$ & $2.2 \mathrm{E}-03 \pm 8.7 \mathrm{E}-04$ & & & ${ }^{234} \mathrm{U}$ & $1.0 \mathrm{E}-05 \pm 7.5 \mathrm{E}-06$ & \\
\hline & $06 / 30 / 08$ & 07/11/08 & gross $\beta$ & $1.4 \mathrm{E}-02 \pm 2.0 \mathrm{E}-03$ & & & ${ }^{235} \mathrm{U}$ & $1.4 \mathrm{E}-06 \pm 2.1 \mathrm{E}-06$ & $\mathrm{U}$ \\
\hline & 07/11/08 & 07/28/08 & gross $\alpha$ & $1.3 \mathrm{E}-03 \pm 4.9 \mathrm{E}-04$ & & & ${ }^{238} \mathrm{U}$ & $2.0 \mathrm{E}-06 \pm 2.4 \mathrm{E}-06$ & \\
\hline & 07/11/08 & $07 / 28 / 08$ & gross $\beta$ & $1.5 \mathrm{E}-02 \pm 1.9 \mathrm{E}-03$ & & & & & \\
\hline & 07/28/08 & 08/11/08 & gross $\alpha$ & $1.5 \mathrm{E}-03 \pm 5.9 \mathrm{E}-04$ & & & & & \\
\hline & 07/28/08 & 08/11/08 & gross $\beta$ & $1.1 \mathrm{E}-02 \pm 1.6 \mathrm{E}-03$ & & & & & \\
\hline & $08 / 11 / 08$ & $08 / 25 / 08$ & gross $\alpha$ & $1.0 \mathrm{E}-03 \pm 4.8 \mathrm{E}-04$ & & & & & \\
\hline & 08/11/08 & $08 / 25 / 08$ & gross $\beta$ & $1.4 \mathrm{E}-02 \pm 1.9 \mathrm{E}-03$ & & & & & \\
\hline & $08 / 25 / 08$ & 09/08/08 & gross $\alpha$ & $1.1 \mathrm{E}-03 \pm 4.9 \mathrm{E}-04$ & & & & & \\
\hline & $08 / 25 / 08$ & 09/08/08 & gross $\beta$ & $1.2 \mathrm{E}-02 \pm 1.7 \mathrm{E}-03$ & & & & & \\
\hline & 09/08/08 & 09/22/08 & gross $\alpha$ & $1.5 \mathrm{E}-03 \pm 5.8 \mathrm{E}-04$ & & & & & \\
\hline & 09/08/08 & 09/22/08 & gross $\beta$ & $2.5 \mathrm{E}-02 \pm 2.7 \mathrm{E}-03$ & & & & & \\
\hline & 09/22/08 & $10 / 07 / 08$ & gross $\alpha$ & $1.1 \mathrm{E}-03 \pm 4.7 \mathrm{E}-04$ & & & & & \\
\hline & $09 / 22 / 08$ & $10 / 07 / 08$ & gross $\beta$ & $1.9 \mathrm{E}-02 \pm 2.2 \mathrm{E}-03$ & & & & & \\
\hline & $10 / 07 / 08$ & $10 / 20 / 08$ & gross $\alpha$ & $2.2 \mathrm{E}-03 \pm 7.4 \mathrm{E}-04$ & & & & & \\
\hline & $10 / 07 / 08$ & $10 / 20 / 08$ & gross $\beta$ & $1.9 \mathrm{E}-02 \pm 2.3 \mathrm{E}-03$ & & & & & \\
\hline & $10 / 20 / 08$ & $11 / 03 / 08$ & gross $\alpha$ & $2.0 \mathrm{E}-03 \pm 6.8 \mathrm{E}-04$ & & & & & \\
\hline & $10 / 20 / 08$ & $11 / 03 / 08$ & gross $\beta$ & $4.3 \mathrm{E}-02 \pm 4.1 \mathrm{E}-03$ & & & & & \\
\hline & $11 / 03 / 08$ & $11 / 17 / 08$ & gross $\alpha$ & $8.1 \mathrm{E}-04 \pm 5.5 \mathrm{E}-04$ & & & & & \\
\hline & $11 / 03 / 08$ & $11 / 17 / 08$ & gross $\beta$ & $1.9 \mathrm{E}-02 \pm 2.3 \mathrm{E}-03$ & & & & & \\
\hline & $11 / 17 / 08$ & $12 / 01 / 08$ & gross $\alpha$ & $2.0 \mathrm{E}-03 \pm 7.0 \mathrm{E}-04$ & & & & & \\
\hline & $11 / 17 / 08$ & $12 / 01 / 08$ & gross $\beta$ & $3.7 \mathrm{E}-02 \pm 3.6 \mathrm{E}-03$ & & & & & \\
\hline & $12 / 01 / 08$ & $12 / 15 / 08$ & gross $\alpha$ & $1.2 \mathrm{E}-03 \pm 5.2 \mathrm{E}-04$ & & & & & \\
\hline & $12 / 01 / 08$ & $12 / 15 / 08$ & gross $\beta$ & $1.8 \mathrm{E}-02 \pm 2.1 \mathrm{E}-03$ & & & & & \\
\hline & $12 / 15 / 08$ & $12 / 30 / 08$ & gross $\alpha$ & $1.5 \mathrm{E}-03 \pm 5.7 \mathrm{E}-04$ & & & & & \\
\hline & $12 / 15 / 08$ & $12 / 30 / 08$ & gross $\beta$ & $3.5 \mathrm{E}-02 \pm 3.4 \mathrm{E}-03$ & & & & & \\
\hline
\end{tabular}

$\overline{\mathrm{RQ}}=$ Result Qualifier. $\mathrm{U}=$ The analyte was analyzed for but not detected. 
Table 2-4. Near-Facility Air Sampling Results, 2008 (pCi/m³ \pm total analytical uncertainty). (Sheet 32 of 82)

\begin{tabular}{|c|c|c|c|c|c|c|c|c|c|c|}
\hline Location & Sample On & Sample Off & Isotope & Result \pm Uncertainty & Location & Composite & e Period & Isotope & Result \pm Uncertainty & $\mathbf{R Q *}$ \\
\hline N967 & $12 / 31 / 07$ & $01 / 14 / 08$ & gross $\alpha$ & $7.5 \mathrm{E}-04 \pm 5.5 \mathrm{E}-04$ & N967 & $12 / 31 / 07$ to & $06 / 30 / 08$ & ${ }^{60} \mathrm{Co}$ & $-1.0 \mathrm{E}-04 \pm 1.4 \mathrm{E}-04$ & $\mathrm{U}$ \\
\hline \multirow[t]{51}{*}{$(200-E)$} & $12 / 31 / 07$ & 01/14/08 & gross $\beta$ & $1.1 \mathrm{E}-02 \pm 1.6 \mathrm{E}-03$ & & & & ${ }^{134} \mathrm{Cs}$ & $-3.7 \mathrm{E}-05 \pm 9.8 \mathrm{E}-05$ & $\mathrm{U}$ \\
\hline & $01 / 14 / 08$ & 01/29/08 & gross $\alpha$ & $1.6 \mathrm{E}-03 \pm 5.9 \mathrm{E}-04$ & & & & ${ }^{137} \mathrm{Cs}$ & $6.6 \mathrm{E}-06 \pm 6.6 \mathrm{E}-05$ & U \\
\hline & $01 / 14 / 08$ & 01/29/08 & gross $\beta$ & $3.0 \mathrm{E}-02 \pm 3.0 \mathrm{E}-03$ & & & & ${ }^{152} \mathrm{Eu}$ & $-1.2 \mathrm{E}-04 \pm 2.4 \mathrm{E}-04$ & U \\
\hline & 01/29/08 & $02 / 12 / 08$ & gross $\alpha$ & $5.0 \mathrm{E}-05 \pm 2.9 \mathrm{E}-04$ & & & & ${ }^{154} \mathrm{Eu}$ & $1.0 \mathrm{E}-04 \pm 3.2 \mathrm{E}-04$ & $\mathrm{U}$ \\
\hline & $01 / 29 / 08$ & 02/12/08 & gross $\beta$ & $5.0 \mathrm{E}-03 \pm 1.0 \mathrm{E}-03$ & & & & ${ }^{155} \mathrm{Eu}$ & $-1.2 \mathrm{E}-04 \pm 1.9 \mathrm{E}-04$ & U \\
\hline & $02 / 12 / 08$ & $02 / 25 / 08$ & gross $\alpha$ & $9.3 \mathrm{E}-04 \pm 6.2 \mathrm{E}-04$ & & & & ${ }^{238} \mathrm{Pu}$ & $-5.6 \mathrm{E}-07 \pm 3.0 \mathrm{E}-06$ & $\mathrm{U}$ \\
\hline & $02 / 12 / 08$ & $02 / 25 / 08$ & gross $\beta$ & $2.8 \mathrm{E}-02 \pm 3.0 \mathrm{E}-03$ & & & & ${ }^{239 / 240} \mathrm{Pu}$ & $5.6 \mathrm{E}-07 \pm 1.1 \mathrm{E}-06$ & U \\
\hline & $02 / 25 / 08$ & 03/11/08 & gross $\alpha$ & $1.6 \mathrm{E}-03 \pm 6.0 \mathrm{E}-04$ & & & & ${ }^{106} \mathrm{Ru}$ & $-1.4 \mathrm{E}-04 \pm 8.5 \mathrm{E}-04$ & $\mathrm{U}$ \\
\hline & $02 / 25 / 08$ & 03/11/08 & gross $\beta$ & $1.6 \mathrm{E}-02 \pm 1.9 \mathrm{E}-03$ & & & & ${ }^{125} \mathrm{Sb}$ & $3.9 \mathrm{E}-05 \pm 2.3 \mathrm{E}-04$ & $\mathrm{U}$ \\
\hline & 03/11/08 & 03/25/08 & gross $\alpha$ & $3.0 \mathrm{E}-04 \pm 4.3 \mathrm{E}-04$ & & & & ${ }^{90} \mathrm{Sr}$ & $9.3 \mathrm{E}-05 \pm 1.5 \mathrm{E}-04$ & $\mathrm{U}$ \\
\hline & 03/11/08 & $03 / 25 / 08$ & gross $\beta$ & $9.2 \mathrm{E}-03 \pm 1.4 \mathrm{E}-03$ & & & & ${ }^{234} \mathrm{U}$ & $9.2 \mathrm{E}-06 \pm 6.3 \mathrm{E}-06$ & \\
\hline & 03/25/08 & $04 / 07 / 08$ & gross $\alpha$ & $8.3 \mathrm{E}-04 \pm 6.1 \mathrm{E}-04$ & & & & ${ }^{235} \mathrm{U}$ & $1.4 \mathrm{E}-06 \pm 2.1 \mathrm{E}-06$ & $\mathrm{U}$ \\
\hline & 03/25/08 & $04 / 07 / 08$ & gross $\beta$ & $9.0 \mathrm{E}-03 \pm 1.4 \mathrm{E}-03$ & & & & ${ }^{238} \mathrm{U}$ & $9.2 \mathrm{E}-06 \pm 6.0 \mathrm{E}-06$ & \\
\hline & 04/07/08 & $04 / 21 / 08$ & gross $\alpha$ & $1.6 \mathrm{E}-03 \pm 6.1 \mathrm{E}-04$ & & & & & & \\
\hline & $04 / 07 / 08$ & $04 / 21 / 08$ & gross $\beta$ & $1.2 \mathrm{E}-02 \pm 1.6 \mathrm{E}-03$ & N967 & $06 / 30 / 08$ to & $12 / 30 / 08$ & ${ }^{60} \mathrm{Co}$ & $-4.2 \mathrm{E}-05 \pm 7.5 \mathrm{E}-05$ & $\mathrm{U}$ \\
\hline & $04 / 21 / 08$ & 05/06/08 & gross $\alpha$ & $1.4 \mathrm{E}-03 \pm 5.6 \mathrm{E}-04$ & & & & ${ }^{134} \mathrm{Cs}$ & $-2.0 \mathrm{E}-05 \pm 6.3 \mathrm{E}-05$ & $\mathrm{U}$ \\
\hline & $04 / 21 / 08$ & 05/06/08 & gross $\beta$ & $1.4 \mathrm{E}-02 \pm 1.8 \mathrm{E}-03$ & & & & ${ }^{137} \mathrm{Cs}$ & $-3.5 \mathrm{E}-05 \pm 6.2 \mathrm{E}-05$ & $\mathrm{U}$ \\
\hline & 05/06/08 & 05/19/08 & gross $\alpha$ & $7.7 \mathrm{E}-04 \pm 5.8 \mathrm{E}-04$ & & & & ${ }^{152} \mathrm{Eu}$ & $-1.3 \mathrm{E}-04 \pm 1.5 \mathrm{E}-04$ & U \\
\hline & 05/06/08 & 05/19/08 & gross $\beta$ & $1.4 \mathrm{E}-02 \pm 1.9 \mathrm{E}-03$ & & & & ${ }^{154} \mathrm{Eu}$ & $2.6 \mathrm{E}-04 \pm 2.3 \mathrm{E}-04$ & $\mathrm{U}$ \\
\hline & 05/19/08 & 06/02/08 & gross $\alpha$ & $1.5 \mathrm{E}-03 \pm 5.8 \mathrm{E}-04$ & & & & ${ }^{155} \mathrm{Eu}$ & $4.9 \mathrm{E}-06 \pm 4.9 \mathrm{E}-05$ & U \\
\hline & 05/19/08 & $06 / 02 / 08$ & gross $\beta$ & $1.3 \mathrm{E}-02 \pm 1.8 \mathrm{E}-03$ & & & & ${ }^{238} \mathrm{Pu}$ & $9.8 \mathrm{E}-06 \pm 1.4 \mathrm{E}-05$ & $\mathrm{U}$ \\
\hline & $06 / 02 / 08$ & $06 / 16 / 08$ & gross $\alpha$ & $8.8 \mathrm{E}-04 \pm 5.9 \mathrm{E}-04$ & & & & ${ }^{239 / 240} \mathrm{Pu}$ & $8.2 \mathrm{E}-07 \pm 2.9 \mathrm{E}-06$ & $\mathrm{U}$ \\
\hline & $06 / 02 / 08$ & 06/16/08 & gross $\beta$ & $8.2 \mathrm{E}-03 \pm 1.3 \mathrm{E}-03$ & & & & ${ }^{106} \mathrm{Ru}$ & $-9.9 \mathrm{E}-06 \pm 9.9 \mathrm{E}-05$ & $\mathrm{U}$ \\
\hline & $06 / 16 / 08$ & 06/30/08 & gross $\alpha$ & $9.9 \mathrm{E}-04 \pm 7.0 \mathrm{E}-04$ & & & & ${ }^{125} \mathrm{Sb}$ & $-3.6 \mathrm{E}-05 \pm 1.5 \mathrm{E}-04$ & $\mathrm{U}$ \\
\hline & $06 / 16 / 08$ & $06 / 30 / 08$ & gross $\beta$ & $1.3 \mathrm{E}-02 \pm 1.8 \mathrm{E}-03$ & & & & ${ }^{90} \mathrm{Sr}$ & $-4.2 \mathrm{E}-04 \pm 4.4 \mathrm{E}-04$ & $\mathrm{U}$ \\
\hline & $06 / 30 / 08$ & $07 / 14 / 08$ & gross $\alpha$ & $1.9 \mathrm{E}-03 \pm 7.5 \mathrm{E}-04$ & & & & ${ }^{234} \mathrm{U}$ & $9.9 \mathrm{E}-06 \pm 6.6 \mathrm{E}-06$ & \\
\hline & $06 / 30 / 08$ & 07/14/08 & gross $\beta$ & $1.4 \mathrm{E}-02 \pm 1.9 \mathrm{E}-03$ & & & & ${ }^{235} \mathrm{U}$ & $2.2 \mathrm{E}-06 \pm 3.3 \mathrm{E}-06$ & $\mathrm{U}$ \\
\hline & $07 / 14 / 08$ & 07/28/08 & gross $\alpha$ & $2.4 \mathrm{E}-03 \pm 7.8 \mathrm{E}-04$ & & & & ${ }^{238} \mathrm{U}$ & $5.9 \mathrm{E}-06 \pm 4.9 \mathrm{E}-06$ & \\
\hline & $07 / 14 / 08$ & $07 / 28 / 08$ & gross $\beta$ & $1.5 \mathrm{E}-02 \pm 2.0 \mathrm{E}-03$ & & & & & & \\
\hline & 07/28/08 & 08/11/08 & gross $\alpha$ & $6.9 \mathrm{E}-04 \pm 5.2 \mathrm{E}-04$ & & & & & & \\
\hline & $07 / 28 / 08$ & $08 / 11 / 08$ & gross $\beta$ & $1.1 \mathrm{E}-02 \pm 1.6 \mathrm{E}-03$ & & & & & & \\
\hline & $08 / 11 / 08$ & $08 / 25 / 08$ & gross $\alpha$ & $1.2 \mathrm{E}-03 \pm 5.4 \mathrm{E}-04$ & & & & & & \\
\hline & 08/11/08 & 08/25/08 & gross $\beta$ & $1.3 \mathrm{E}-02 \pm 1.8 \mathrm{E}-03$ & & & & & & \\
\hline & $08 / 25 / 08$ & 09/08/08 & gross $\alpha$ & $4.8 \mathrm{E}-04 \pm 4.6 \mathrm{E}-04$ & & & & & & \\
\hline & $08 / 25 / 08$ & 09/08/08 & gross $\beta$ & $1.1 \mathrm{E}-02 \pm 1.6 \mathrm{E}-03$ & & & & & & \\
\hline & 09/08/08 & 09/22/08 & gross $\alpha$ & $1.2 \mathrm{E}-03 \pm 5.4 \mathrm{E}-04$ & & & & & & \\
\hline & 09/08/08 & $09 / 22 / 08$ & gross $\beta$ & $2.9 \mathrm{E}-02 \pm 3.1 \mathrm{E}-03$ & & & & & & \\
\hline & $09 / 22 / 08$ & $10 / 07 / 08$ & gross $\alpha$ & $1.9 \mathrm{E}-03 \pm 6.4 \mathrm{E}-04$ & & & & & & \\
\hline & $09 / 22 / 08$ & $10 / 07 / 08$ & gross $\beta$ & $2.0 \mathrm{E}-02 \pm 2.3 \mathrm{E}-03$ & & & & & & \\
\hline & $10 / 07 / 08$ & $10 / 20 / 08$ & gross $\alpha$ & $1.2 \mathrm{E}-03 \pm 5.5 \mathrm{E}-04$ & & & & & & \\
\hline & $10 / 07 / 08$ & $10 / 20 / 08$ & gross $\beta$ & $1.8 \mathrm{E}-02 \pm 2.2 \mathrm{E}-03$ & & & & & & \\
\hline & $10 / 20 / 08$ & $11 / 03 / 08$ & gross $\alpha$ & $2.5 \mathrm{E}-03 \pm 7.5 \mathrm{E}-04$ & & & & & & \\
\hline & $10 / 20 / 08$ & $11 / 03 / 08$ & gross $\beta$ & $4.9 \mathrm{E}-02 \pm 4.6 \mathrm{E}-03$ & & & & & & \\
\hline & $11 / 03 / 08$ & $11 / 17 / 08$ & gross $\alpha$ & $1.1 \mathrm{E}-03 \pm 5.3 \mathrm{E}-04$ & & & & & & \\
\hline & $11 / 03 / 08$ & $11 / 17 / 08$ & gross $\beta$ & $1.7 \mathrm{E}-02 \pm 2.2 \mathrm{E}-03$ & & & & & & \\
\hline & $11 / 17 / 08$ & $12 / 01 / 08$ & gross $\alpha$ & $1.3 \mathrm{E}-03 \pm 5.8 \mathrm{E}-04$ & & & & & & \\
\hline & $11 / 17 / 08$ & $12 / 01 / 08$ & gross $\beta$ & $3.5 \mathrm{E}-02 \pm 3.5 \mathrm{E}-03$ & & & & & & \\
\hline & $12 / 01 / 08$ & $12 / 15 / 08$ & gross $\alpha$ & $2.1 \mathrm{E}-03 \pm 7.0 \mathrm{E}-04$ & & & & & & \\
\hline & $12 / 01 / 08$ & $12 / 15 / 08$ & gross $\beta$ & $2.6 \mathrm{E}-02 \pm 2.8 \mathrm{E}-03$ & & & & & & \\
\hline & $12 / 15 / 08$ & $12 / 30 / 08$ & gross $\alpha$ & $2.0 \mathrm{E}-03 \pm 6.7 \mathrm{E}-04$ & & & & & & \\
\hline & $12 / 15 / 08$ & $12 / 30 / 08$ & gross $\beta$ & $3.9 \mathrm{E}-02 \pm 3.7 \mathrm{E}-03$ & & & & & & \\
\hline
\end{tabular}

$\overline{\mathrm{RQ}}=$ Result Qualifier. $\mathrm{U}=$ The analyte was analyzed for but not detected. 
Table 2-4. Near-Facility Air Sampling Results, 2008 (pCi/m³ \pm total analytical uncertainty). (Sheet 33 of 82)

\begin{tabular}{|c|c|c|c|c|c|c|c|c|c|c|}
\hline Location & Sample On & Sample Off & Isotope & Result \pm Uncertainty & Location & Composite & e Period & Isotope & Result \pm Uncertainty & $\mathbf{R Q *}$ \\
\hline N968 & $12 / 31 / 07$ & $01 / 14 / 08$ & gross $\alpha$ & $6.0 \mathrm{E}-04 \pm 4.9 \mathrm{E}-04$ & N968 & $12 / 31 / 07$ to & $06 / 30 / 08$ & ${ }^{60} \mathrm{Co}$ & $-1.0 \mathrm{E}-05 \pm 6.9 \mathrm{E}-05$ & $\mathrm{U}$ \\
\hline \multirow[t]{51}{*}{$(200-E)$} & $12 / 31 / 07$ & 01/14/08 & gross $\beta$ & $8.8 \mathrm{E}-03 \pm 1.4 \mathrm{E}-03$ & & & & ${ }^{134} \mathrm{Cs}$ & $8.0 \mathrm{E}-06 \pm 6.7 \mathrm{E}-05$ & $\mathrm{U}$ \\
\hline & $01 / 14 / 08$ & 01/29/08 & gross $\alpha$ & $1.2 \mathrm{E}-03 \pm 5.2 \mathrm{E}-04$ & & & & ${ }^{137} \mathrm{Cs}$ & $-3.4 \mathrm{E}-05 \pm 5.9 \mathrm{E}-05$ & U \\
\hline & $01 / 14 / 08$ & 01/29/08 & gross $\beta$ & $3.2 \mathrm{E}-02 \pm 3.1 \mathrm{E}-03$ & & & & ${ }^{152} \mathrm{Eu}$ & $-1.8 \mathrm{E}-05 \pm 1.4 \mathrm{E}-04$ & U \\
\hline & 01/29/08 & $02 / 12 / 08$ & gross $\alpha$ & $6.2 \mathrm{E}-04 \pm 5.0 \mathrm{E}-04$ & & & & ${ }^{154} \mathrm{Eu}$ & $-1.3 \mathrm{E}-04 \pm 1.7 \mathrm{E}-04$ & $\mathrm{U}$ \\
\hline & $01 / 29 / 08$ & 02/12/08 & gross $\beta$ & $3.5 \mathrm{E}-03 \pm 8.1 \mathrm{E}-04$ & & & & ${ }^{155} \mathrm{Eu}$ & $-2.5 \mathrm{E}-06 \pm 2.5 \mathrm{E}-05$ & U \\
\hline & $02 / 12 / 08$ & $02 / 25 / 08$ & gross $\alpha$ & $1.0 \mathrm{E}-03 \pm 6.5 \mathrm{E}-04$ & & & & ${ }^{238} \mathrm{Pu}$ & $5.4 \mathrm{E}-07 \pm 2.4 \mathrm{E}-06$ & $\mathrm{U}$ \\
\hline & $02 / 12 / 08$ & $02 / 25 / 08$ & gross $\beta$ & $2.8 \mathrm{E}-02 \pm 3.0 \mathrm{E}-03$ & & & & ${ }^{239 / 240} \mathrm{Pu}$ & $2.2 \mathrm{E}-06 \pm 2.3 \mathrm{E}-06$ & \\
\hline & $02 / 25 / 08$ & 03/11/08 & gross $\alpha$ & $1.3 \mathrm{E}-03 \pm 5.3 \mathrm{E}-04$ & & & & ${ }^{106} \mathrm{Ru}$ & $-2.1 \mathrm{E}-04 \pm 5.1 \mathrm{E}-04$ & $\mathrm{U}$ \\
\hline & $02 / 25 / 08$ & 03/11/08 & gross $\beta$ & $1.5 \mathrm{E}-02 \pm 1.8 \mathrm{E}-03$ & & & & ${ }^{125} \mathrm{Sb}$ & $8.5 \mathrm{E}-05 \pm 1.4 \mathrm{E}-04$ & $\mathrm{U}$ \\
\hline & 03/11/08 & 03/25/08 & gross $\alpha$ & $3.9 \mathrm{E}-04 \pm 4.4 \mathrm{E}-04$ & & & & ${ }^{90} \mathrm{Sr}$ & $-2.0 \mathrm{E}-04 \pm 2.0 \mathrm{E}-04$ & $\mathrm{U}$ \\
\hline & 03/11/08 & $03 / 25 / 08$ & gross $\beta$ & $7.1 \mathrm{E}-03 \pm 1.2 \mathrm{E}-03$ & & & & ${ }^{234} \mathrm{U}$ & $9.6 \mathrm{E}-06 \pm 7.2 \mathrm{E}-06$ & \\
\hline & 03/25/08 & $04 / 07 / 08$ & gross $\alpha$ & $9.3 \mathrm{E}-04 \pm 6.2 \mathrm{E}-04$ & & & & ${ }^{235} \mathrm{U}$ & $2.8 \mathrm{E}-06 \pm 3.0 \mathrm{E}-06$ & \\
\hline & 03/25/08 & $04 / 07 / 08$ & gross $\beta$ & $7.6 \mathrm{E}-03 \pm 1.3 \mathrm{E}-03$ & & & & ${ }^{238} \mathrm{U}$ & $9.6 \mathrm{E}-06 \pm 6.2 \mathrm{E}-06$ & \\
\hline & 04/07/08 & $04 / 21 / 08$ & gross $\alpha$ & $1.3 \mathrm{E}-03 \pm 5.5 \mathrm{E}-04$ & & & & & & \\
\hline & $04 / 07 / 08$ & $04 / 21 / 08$ & gross $\beta$ & $8.4 \mathrm{E}-03 \pm 1.3 \mathrm{E}-03$ & N968 & $06 / 30 / 08$ to & $12 / 30 / 08$ & ${ }^{60} \mathrm{Co}$ & $-3.7 \mathrm{E}-05 \pm 6.5 \mathrm{E}-05$ & $\mathrm{U}$ \\
\hline & $04 / 21 / 08$ & 05/06/08 & gross $\alpha$ & $1.2 \mathrm{E}-03 \pm 5.2 \mathrm{E}-04$ & & & & ${ }^{134} \mathrm{Cs}$ & $4.7 \mathrm{E}-05 \pm 6.1 \mathrm{E}-05$ & $\mathrm{U}$ \\
\hline & $04 / 21 / 08$ & 05/06/08 & gross $\beta$ & $1.1 \mathrm{E}-02 \pm 1.5 \mathrm{E}-03$ & & & & ${ }^{137} \mathrm{Cs}$ & $1.9 \mathrm{E}-05 \pm 5.7 \mathrm{E}-05$ & $\mathrm{U}$ \\
\hline & 05/06/08 & 05/19/08 & gross $\alpha$ & $1.5 \mathrm{E}-03 \pm 6.0 \mathrm{E}-04$ & & & & ${ }^{152} \mathrm{Eu}$ & $-1.3 \mathrm{E}-05 \pm 1.3 \mathrm{E}-04$ & U \\
\hline & 05/06/08 & 05/19/08 & gross $\beta$ & $1.2 \mathrm{E}-02 \pm 1.8 \mathrm{E}-03$ & & & & ${ }^{154} \mathrm{Eu}$ & $-5.7 \mathrm{E}-06 \pm 5.7 \mathrm{E}-05$ & $\mathrm{U}$ \\
\hline & 05/19/08 & 06/02/08 & gross $\alpha$ & $8.1 \mathrm{E}-04 \pm 5.5 \mathrm{E}-04$ & & & & ${ }^{155} \mathrm{Eu}$ & $1.3 \mathrm{E}-04 \pm 1.6 \mathrm{E}-04$ & U \\
\hline & 05/19/08 & $06 / 02 / 08$ & gross $\beta$ & $1.2 \mathrm{E}-02 \pm 1.7 \mathrm{E}-03$ & & & & ${ }^{238} \mathrm{Pu}$ & $-1.6 \mathrm{E}-06 \pm 1.1 \mathrm{E}-05$ & $\mathrm{U}$ \\
\hline & $06 / 02 / 08$ & 06/16/08 & gross $\alpha$ & $6.3 \mathrm{E}-04 \pm 5.2 \mathrm{E}-04$ & & & & ${ }^{239 / 240} \mathrm{Pu}$ & $3.2 \mathrm{E}-06 \pm 4.0 \mathrm{E}-06$ & $\mathrm{U}$ \\
\hline & $06 / 02 / 08$ & 06/16/08 & gross $\beta$ & $8.2 \mathrm{E}-03 \pm 1.3 \mathrm{E}-03$ & & & & ${ }^{106} \mathrm{Ru}$ & $-3.6 \mathrm{E}-04 \pm 5.1 \mathrm{E}-04$ & $\mathrm{U}$ \\
\hline & $06 / 16 / 08$ & 06/30/08 & gross $\alpha$ & $1.7 \mathrm{E}-03 \pm 6.7 \mathrm{E}-04$ & & & & ${ }^{125} \mathrm{Sb}$ & $1.1 \mathrm{E}-05 \pm 1.1 \mathrm{E}-04$ & $\mathrm{U}$ \\
\hline & $06 / 16 / 08$ & 06/30/08 & gross $\beta$ & $1.1 \mathrm{E}-02 \pm 1.6 \mathrm{E}-03$ & & & & ${ }^{90} \mathrm{Sr}$ & $-1.4 \mathrm{E}-04 \pm 1.5 \mathrm{E}-04$ & $\mathrm{U}$ \\
\hline & $06 / 30 / 08$ & $07 / 14 / 08$ & gross $\alpha$ & $1.8 \mathrm{E}-03 \pm 7.0 \mathrm{E}-04$ & & & & ${ }^{234} \mathrm{U}$ & $1.7 \mathrm{E}-05 \pm 9.4 \mathrm{E}-06$ & \\
\hline & $06 / 30 / 08$ & 07/14/08 & gross $\beta$ & $1.4 \mathrm{E}-02 \pm 1.8 \mathrm{E}-03$ & & & & ${ }^{235} \mathrm{U}$ & $2.2 \mathrm{E}-06 \pm 2.6 \mathrm{E}-06$ & \\
\hline & $07 / 14 / 08$ & 07/28/08 & gross $\alpha$ & $1.5 \mathrm{E}-03 \pm 5.8 \mathrm{E}-04$ & & & & ${ }^{238} \mathrm{U}$ & $1.2 \mathrm{E}-05 \pm 7.2 \mathrm{E}-06$ & \\
\hline & $07 / 14 / 08$ & $07 / 28 / 08$ & gross $\beta$ & $1.7 \mathrm{E}-02 \pm 2.1 \mathrm{E}-03$ & & & & & & \\
\hline & 07/28/08 & 08/11/08 & gross $\alpha$ & $6.9 \mathrm{E}-04 \pm 5.2 \mathrm{E}-04$ & & & & & & \\
\hline & $07 / 28 / 08$ & $08 / 11 / 08$ & gross $\beta$ & $1.1 \mathrm{E}-02 \pm 1.6 \mathrm{E}-03$ & & & & & & \\
\hline & $08 / 11 / 08$ & $08 / 25 / 08$ & gross $\alpha$ & $1.4 \mathrm{E}-03 \pm 5.7 \mathrm{E}-04$ & & & & & & \\
\hline & 08/11/08 & 08/25/08 & gross $\beta$ & $1.2 \mathrm{E}-02 \pm 1.6 \mathrm{E}-03$ & & & & & & \\
\hline & $08 / 25 / 08$ & 09/08/08 & gross $\alpha$ & $7.4 \mathrm{E}-04 \pm 5.3 \mathrm{E}-04$ & & & & & & \\
\hline & $08 / 25 / 08$ & 09/08/08 & gross $\beta$ & $9.4 \mathrm{E}-03 \pm 1.4 \mathrm{E}-03$ & & & & & & \\
\hline & 09/08/08 & $09 / 22 / 08$ & gross $\alpha$ & $9.7 \mathrm{E}-04 \pm 5.9 \mathrm{E}-04$ & & & & & & \\
\hline & 09/08/08 & $09 / 22 / 08$ & gross $\beta$ & $2.3 \mathrm{E}-02 \pm 2.6 \mathrm{E}-03$ & & & & & & \\
\hline & $09 / 22 / 08$ & $10 / 07 / 08$ & gross $\alpha$ & $1.2 \mathrm{E}-03 \pm 5.0 \mathrm{E}-04$ & & & & & & \\
\hline & $09 / 22 / 08$ & $10 / 07 / 08$ & gross $\beta$ & $2.1 \mathrm{E}-02 \pm 2.3 \mathrm{E}-03$ & & & & & & \\
\hline & $10 / 07 / 08$ & $10 / 20 / 08$ & gross $\alpha$ & $2.1 \mathrm{E}-03 \pm 7.3 \mathrm{E}-04$ & & & & & & \\
\hline & $10 / 07 / 08$ & $10 / 20 / 08$ & gross $\beta$ & $1.6 \mathrm{E}-02 \pm 2.1 \mathrm{E}-03$ & & & & & & \\
\hline & $10 / 20 / 08$ & $11 / 03 / 08$ & gross $\alpha$ & $3.0 \mathrm{E}-03 \pm 8.3 \mathrm{E}-04$ & & & & & & \\
\hline & $10 / 20 / 08$ & $11 / 03 / 08$ & gross $\beta$ & $4.1 \mathrm{E}-02 \pm 4.0 \mathrm{E}-03$ & & & & & & \\
\hline & $11 / 03 / 08$ & $11 / 17 / 08$ & gross $\alpha$ & $1.1 \mathrm{E}-03 \pm 5.1 \mathrm{E}-04$ & & & & & & \\
\hline & $11 / 03 / 08$ & $11 / 17 / 08$ & gross $\beta$ & $1.9 \mathrm{E}-02 \pm 2.3 \mathrm{E}-03$ & & & & & & \\
\hline & $11 / 17 / 08$ & $12 / 01 / 08$ & gross $\alpha$ & $2.5 \mathrm{E}-03 \pm 7.7 \mathrm{E}-04$ & & & & & & \\
\hline & $11 / 17 / 08$ & $12 / 01 / 08$ & gross $\beta$ & $3.2 \mathrm{E}-02 \pm 3.2 \mathrm{E}-03$ & & & & & & \\
\hline & $12 / 01 / 08$ & $12 / 15 / 08$ & gross $\alpha$ & $2.0 \mathrm{E}-03 \pm 6.7 \mathrm{E}-04$ & & & & & & \\
\hline & $12 / 01 / 08$ & $12 / 15 / 08$ & gross $\beta$ & $2.2 \mathrm{E}-02 \pm 2.4 \mathrm{E}-03$ & & & & & & \\
\hline & $12 / 15 / 08$ & $12 / 30 / 08$ & gross $\alpha$ & $1.8 \mathrm{E}-03 \pm 6.2 \mathrm{E}-04$ & & & & & & \\
\hline & $12 / 15 / 08$ & $12 / 30 / 08$ & gross $\beta$ & $3.7 \mathrm{E}-02 \pm 3.5 \mathrm{E}-03$ & & & & & & \\
\hline
\end{tabular}

$\overline{\mathrm{RQ}}=$ Result Qualifier. $\mathrm{U}=$ The analyte was analyzed for but not detected. 
Table 2-4. Near-Facility Air Sampling Results, 2008 (pCi/m³ \pm total analytical uncertainty). (Sheet 34 of 82)

\begin{tabular}{|c|c|c|c|c|c|c|c|c|c|c|}
\hline Location & Sample On & Sample Off & Isotope & Result \pm Uncertainty & Location & Composite & e Period & Isotope & Result \pm Uncertainty & $\mathbf{R Q *}$ \\
\hline N969 & $12 / 31 / 07$ & $01 / 14 / 08$ & gross $\alpha$ & $8.1 \mathrm{E}-04 \pm 5.5 \mathrm{E}-04$ & N969 & $12 / 31 / 07$ to & $06 / 30 / 08$ & ${ }^{60} \mathrm{Co}$ & $-9.9 \mathrm{E}-06 \pm 8.8 \mathrm{E}-05$ & $\mathrm{U}$ \\
\hline \multirow[t]{51}{*}{$(200-E)$} & $12 / 31 / 07$ & 01/14/08 & gross $\beta$ & $7.3 \mathrm{E}-03 \pm 1.2 \mathrm{E}-03$ & & & & ${ }^{134} \mathrm{Cs}$ & $-3.3 \mathrm{E}-06 \pm 3.3 \mathrm{E}-05$ & $\mathrm{U}$ \\
\hline & $01 / 14 / 08$ & 01/29/08 & gross $\alpha$ & $1.0 \mathrm{E}-03 \pm 4.9 \mathrm{E}-04$ & & & & ${ }^{137} \mathrm{Cs}$ & $-9.0 \mathrm{E}-07 \pm 9.0 \mathrm{E}-06$ & U \\
\hline & $01 / 14 / 08$ & 01/29/08 & gross $\beta$ & $3.2 \mathrm{E}-02 \pm 3.2 \mathrm{E}-03$ & & & & ${ }^{152} \mathrm{Eu}$ & $5.9 \mathrm{E}-05 \pm 1.8 \mathrm{E}-04$ & U \\
\hline & $01 / 29 / 08$ & $02 / 12 / 08$ & gross $\alpha$ & $8.6 \mathrm{E}-04 \pm 5.8 \mathrm{E}-04$ & & & & ${ }^{154} \mathrm{Eu}$ & $-3.2 \mathrm{E}-04 \pm 3.3 \mathrm{E}-04$ & $\mathrm{U}$ \\
\hline & $01 / 29 / 08$ & 02/12/08 & gross $\beta$ & $4.2 \mathrm{E}-03 \pm 9.0 \mathrm{E}-04$ & & & & ${ }^{155} \mathrm{Eu}$ & $-1.2 \mathrm{E}-04 \pm 1.7 \mathrm{E}-04$ & U \\
\hline & $02 / 12 / 08$ & $02 / 25 / 08$ & gross $\alpha$ & $1.5 \mathrm{E}-03 \pm 5.9 \mathrm{E}-04$ & & & & ${ }^{238} \mathrm{Pu}$ & $1.1 \mathrm{E}-06 \pm 2.7 \mathrm{E}-06$ & $\mathrm{U}$ \\
\hline & $02 / 12 / 08$ & $02 / 25 / 08$ & gross $\beta$ & $1.9 \mathrm{E}-02 \pm 2.2 \mathrm{E}-03$ & & & & ${ }^{239 / 240} \mathrm{Pu}$ & $5.4 \mathrm{E}-07 \pm 1.9 \mathrm{E}-06$ & $\mathrm{U}$ \\
\hline & $02 / 25 / 08$ & 03/11/08 & gross $\alpha$ & $9.4 \mathrm{E}-04 \pm 4.4 \mathrm{E}-04$ & & & & ${ }^{106} \mathrm{Ru}$ & $8.9 \mathrm{E}-05 \pm 6.3 \mathrm{E}-04$ & $\mathrm{U}$ \\
\hline & $02 / 25 / 08$ & 03/11/08 & gross $\beta$ & $1.8 \mathrm{E}-02 \pm 2.1 \mathrm{E}-03$ & & & & ${ }^{125} \mathrm{Sb}$ & $9.3 \mathrm{E}-06 \pm 9.3 \mathrm{E}-05$ & $\mathrm{U}$ \\
\hline & 03/11/08 & 03/25/08 & gross $\alpha$ & $1.1 \mathrm{E}-03 \pm 5.0 \mathrm{E}-04$ & & & & ${ }^{90} \mathrm{Sr}$ & $-9.0 \mathrm{E}-06 \pm 9.4 \mathrm{E}-06$ & $\mathrm{U}$ \\
\hline & 03/11/08 & $03 / 25 / 08$ & gross $\beta$ & $1.0 \mathrm{E}-02 \pm 1.5 \mathrm{E}-03$ & & & & ${ }^{234} \mathrm{U}$ & $1.0 \mathrm{E}-05 \pm 6.7 \mathrm{E}-06$ & \\
\hline & 03/25/08 & $04 / 07 / 08$ & gross $\alpha$ & $9.3 \mathrm{E}-04 \pm 6.2 \mathrm{E}-04$ & & & & ${ }^{235} \mathrm{U}$ & $1.5 \mathrm{E}-06 \pm 3.1 \mathrm{E}-06$ & $\mathrm{U}$ \\
\hline & 03/25/08 & $04 / 07 / 08$ & gross $\beta$ & $7.9 \mathrm{E}-03 \pm 1.3 \mathrm{E}-03$ & & & & ${ }^{238} \mathrm{U}$ & $6.9 \mathrm{E}-06 \pm 5.1 \mathrm{E}-06$ & \\
\hline & 04/07/08 & $04 / 21 / 08$ & gross $\alpha$ & $1.1 \mathrm{E}-03 \pm 5.0 \mathrm{E}-04$ & & & & & & \\
\hline & $04 / 07 / 08$ & $04 / 21 / 08$ & gross $\beta$ & $1.0 \mathrm{E}-02 \pm 1.5 \mathrm{E}-03$ & N969 & $06 / 30 / 08$ to & $12 / 30 / 08$ & ${ }^{60} \mathrm{Co}$ & $1.5 \mathrm{E}-05 \pm 7.8 \mathrm{E}-05$ & $\mathrm{U}$ \\
\hline & $04 / 21 / 08$ & 05/07/08 & gross $\alpha$ & $1.8 \mathrm{E}-03 \pm 6.1 \mathrm{E}-04$ & & & & ${ }^{134} \mathrm{Cs}$ & $2.4 \mathrm{E}-05 \pm 6.3 \mathrm{E}-05$ & $\mathrm{U}$ \\
\hline & $04 / 21 / 08$ & 05/07/08 & gross $\beta$ & $1.7 \mathrm{E}-02 \pm 2.0 \mathrm{E}-03$ & & & & ${ }^{137} \mathrm{Cs}$ & $5.1 \mathrm{E}-05 \pm 6.3 \mathrm{E}-05$ & $\mathrm{U}$ \\
\hline & 05/07/08 & 05/19/08 & gross $\alpha$ & $1.1 \mathrm{E}-03 \pm 6.9 \mathrm{E}-04$ & & & & ${ }^{152} \mathrm{Eu}$ & $-8.3 \mathrm{E}-05 \pm 1.5 \mathrm{E}-04$ & U \\
\hline & 05/07/08 & 05/19/08 & gross $\beta$ & $1.2 \mathrm{E}-02 \pm 1.8 \mathrm{E}-03$ & & & & ${ }^{154} \mathrm{Eu}$ & $7.1 \mathrm{E}-05 \pm 2.1 \mathrm{E}-04$ & $\mathrm{U}$ \\
\hline & 05/19/08 & 06/02/08 & gross $\alpha$ & $9.5 \mathrm{E}-04 \pm 5.9 \mathrm{E}-04$ & & & & ${ }^{155} \mathrm{Eu}$ & $2.4 \mathrm{E}-05 \pm 1.7 \mathrm{E}-04$ & U \\
\hline & 05/19/08 & $06 / 02 / 08$ & gross $\beta$ & $1.1 \mathrm{E}-02 \pm 1.6 \mathrm{E}-03$ & & & & ${ }^{238} \mathrm{Pu}$ & $2.4 \mathrm{E}-06 \pm 1.5 \mathrm{E}-05$ & $\mathrm{U}$ \\
\hline & 06/02/08 & 06/16/08 & gross $\alpha$ & $2.3 \mathrm{E}-03 \pm 9.7 \mathrm{E}-04$ & & & & ${ }^{239 / 240} \mathrm{Pu}$ & $4.9 \mathrm{E}-06 \pm 5.2 \mathrm{E}-06$ & \\
\hline & $06 / 02 / 08$ & 06/16/08 & gross $\beta$ & $1.5 \mathrm{E}-02 \pm 2.2 \mathrm{E}-03$ & & & & ${ }^{106} \mathrm{Ru}$ & $-6.1 \mathrm{E}-05 \pm 5.3 \mathrm{E}-04$ & $\mathrm{U}$ \\
\hline & $06 / 16 / 08$ & 06/30/08 & gross $\alpha$ & $1.2 \mathrm{E}-03 \pm 5.7 \mathrm{E}-04$ & & & & ${ }^{125} \mathrm{Sb}$ & $3.5 \mathrm{E}-05 \pm 1.4 \mathrm{E}-04$ & $\mathrm{U}$ \\
\hline & $06 / 16 / 08$ & $06 / 30 / 08$ & gross $\beta$ & $1.2 \mathrm{E}-02 \pm 1.6 \mathrm{E}-03$ & & & & ${ }^{90} \mathrm{Sr}$ & $-8.4 \mathrm{E}-05 \pm 8.7 \mathrm{E}-05$ & $\mathrm{U}$ \\
\hline & $06 / 30 / 08$ & $07 / 14 / 08$ & gross $\alpha$ & $6.4 \mathrm{E}-04 \pm 5.2 \mathrm{E}-04$ & & & & ${ }^{234} \mathrm{U}$ & $1.3 \mathrm{E}-05 \pm 8.1 \mathrm{E}-06$ & \\
\hline & $06 / 30 / 08$ & 07/14/08 & gross $\beta$ & $1.2 \mathrm{E}-02 \pm 1.6 \mathrm{E}-03$ & & & & ${ }^{235} \mathrm{U}$ & $2.9 \mathrm{E}-06 \pm 3.0 \mathrm{E}-06$ & \\
\hline & $07 / 14 / 08$ & 07/28/08 & gross $\alpha$ & $1.3 \mathrm{E}-03 \pm 5.5 \mathrm{E}-04$ & & & & ${ }^{238} \mathrm{U}$ & $7.8 \mathrm{E}-06 \pm 5.7 \mathrm{E}-06$ & \\
\hline & $07 / 14 / 08$ & $07 / 28 / 08$ & gross $\beta$ & $1.3 \mathrm{E}-02 \pm 1.7 \mathrm{E}-03$ & & & & & & \\
\hline & 07/28/08 & 08/11/08 & gross $\alpha$ & 7.4E-04 $\pm 5.3 \mathrm{E}-04$ & & & & & & \\
\hline & $07 / 28 / 08$ & $08 / 11 / 08$ & gross $\beta$ & $1.4 \mathrm{E}-02 \pm 1.8 \mathrm{E}-03$ & & & & & & \\
\hline & $08 / 11 / 08$ & $08 / 25 / 08$ & gross $\alpha$ & $1.2 \mathrm{E}-03 \pm 5.2 \mathrm{E}-04$ & & & & & & \\
\hline & 08/11/08 & 08/25/08 & gross $\beta$ & $1.3 \mathrm{E}-02 \pm 1.7 \mathrm{E}-03$ & & & & & & \\
\hline & $08 / 25 / 08$ & 09/08/08 & gross $\alpha$ & $4.0 \mathrm{E}-04 \pm 4.4 \mathrm{E}-04$ & & & & & & \\
\hline & $08 / 25 / 08$ & 09/08/08 & gross $\beta$ & $1.1 \mathrm{E}-02 \pm 1.6 \mathrm{E}-03$ & & & & & & \\
\hline & 09/08/08 & 09/22/08 & gross $\alpha$ & $1.5 \mathrm{E}-03 \pm 5.9 \mathrm{E}-04$ & & & & & & \\
\hline & 09/08/08 & $09 / 22 / 08$ & gross $\beta$ & $2.6 \mathrm{E}-02 \pm 2.8 \mathrm{E}-03$ & & & & & & \\
\hline & $09 / 22 / 08$ & $10 / 07 / 08$ & gross $\alpha$ & $1.5 \mathrm{E}-03 \pm 5.6 \mathrm{E}-04$ & & & & & & \\
\hline & $09 / 22 / 08$ & $10 / 07 / 08$ & gross $\beta$ & $2.2 \mathrm{E}-02 \pm 2.5 \mathrm{E}-03$ & & & & & & \\
\hline & $10 / 07 / 08$ & $10 / 20 / 08$ & gross $\alpha$ & $8.1 \mathrm{E}-04 \pm 6.0 \mathrm{E}-04$ & & & & & & \\
\hline & $10 / 07 / 08$ & $10 / 20 / 08$ & gross $\beta$ & $1.6 \mathrm{E}-02 \pm 2.1 \mathrm{E}-03$ & & & & & & \\
\hline & $10 / 20 / 08$ & $11 / 03 / 08$ & gross $\alpha$ & $4.0 \mathrm{E}-03 \pm 9.8 \mathrm{E}-04$ & & & & & & \\
\hline & $10 / 20 / 08$ & $11 / 03 / 08$ & gross $\beta$ & $4.2 \mathrm{E}-02 \pm 4.0 \mathrm{E}-03$ & & & & & & \\
\hline & $11 / 03 / 08$ & $11 / 17 / 08$ & gross $\alpha$ & $1.3 \mathrm{E}-03 \pm 5.4 \mathrm{E}-04$ & & & & & & \\
\hline & $11 / 03 / 08$ & $11 / 17 / 08$ & gross $\beta$ & $1.9 \mathrm{E}-02 \pm 2.2 \mathrm{E}-03$ & & & & & & \\
\hline & $11 / 17 / 08$ & $12 / 01 / 08$ & gross $\alpha$ & $2.3 \mathrm{E}-03 \pm 7.4 \mathrm{E}-04$ & & & & & & \\
\hline & $11 / 17 / 08$ & $12 / 01 / 08$ & gross $\beta$ & $3.6 \mathrm{E}-02 \pm 3.5 \mathrm{E}-03$ & & & & & & \\
\hline & $12 / 01 / 08$ & $12 / 15 / 08$ & gross $\alpha$ & $1.7 \mathrm{E}-03 \pm 6.3 \mathrm{E}-04$ & & & & & & \\
\hline & $12 / 01 / 08$ & $12 / 15 / 08$ & gross $\beta$ & $2.0 \mathrm{E}-02 \pm 2.3 \mathrm{E}-03$ & & & & & & \\
\hline & $12 / 15 / 08$ & $12 / 30 / 08$ & gross $\alpha$ & $2.3 \mathrm{E}-03 \pm 7.0 \mathrm{E}-04$ & & & & & & \\
\hline & $12 / 15 / 08$ & $12 / 30 / 08$ & gross $\beta$ & $3.9 \mathrm{E}-02 \pm 3.7 \mathrm{E}-03$ & & & & & & \\
\hline
\end{tabular}

$\overline{\mathrm{RQ}}=$ Result Qualifier. $\mathrm{U}=$ The analyte was analyzed for but not detected. 
Table 2-4. Near-Facility Air Sampling Results, 2008 (pCi/m³ \pm total analytical uncertainty). (Sheet 35 of 82)

\begin{tabular}{|c|c|c|c|c|c|c|c|c|c|}
\hline Location & Sample On & Sample Off & Isotope & Result \pm Uncertainty & Location & Composite Period & Isotope & Result \pm Uncertainty & $\mathbf{R Q *}$ \\
\hline N970 & $12 / 31 / 07$ & $01 / 14 / 08$ & gross $\alpha$ & $9.5 \mathrm{E}-04 \pm 5.9 \mathrm{E}-04$ & N970 & $12 / 31 / 07$ to $06 / 30 / 08$ & ${ }^{60} \mathrm{Co}$ & $-2.3 \mathrm{E}-05 \pm 6.6 \mathrm{E}-05$ & $\mathrm{U}$ \\
\hline \multirow[t]{51}{*}{$(200-E)$} & $12 / 31 / 07$ & 01/14/08 & gross $\beta$ & $7.2 \mathrm{E}-03 \pm 1.2 \mathrm{E}-03$ & & & ${ }^{134} \mathrm{Cs}$ & $4.0 \mathrm{E}-05 \pm 6.0 \mathrm{E}-05$ & $\mathrm{U}$ \\
\hline & 01/14/08 & $01 / 29 / 08$ & gross $\alpha$ & $2.2 \mathrm{E}-03 \pm 7.0 \mathrm{E}-04$ & & & ${ }^{137} \mathrm{Cs}$ & $1.1 \mathrm{E}-05 \pm 5.7 \mathrm{E}-05$ & $\mathrm{U}$ \\
\hline & $01 / 14 / 08$ & 01/29/08 & gross $\beta$ & $3.7 \mathrm{E}-02 \pm 3.5 \mathrm{E}-03$ & & & ${ }^{152} \mathrm{Eu}$ & $-3.9 \mathrm{E}-05 \pm 1.4 \mathrm{E}-04$ & $\mathrm{U}$ \\
\hline & $01 / 29 / 08$ & $02 / 12 / 08$ & gross $\alpha$ & $3.9 \mathrm{E}-04 \pm 4.4 \mathrm{E}-04$ & & & ${ }^{154} \mathrm{Eu}$ & $1.9 \mathrm{E}-05 \pm 1.7 \mathrm{E}-04$ & $\mathrm{U}$ \\
\hline & $01 / 29 / 08$ & $02 / 12 / 08$ & gross $\beta$ & $2.7 \mathrm{E}-03 \pm 7.1 \mathrm{E}-04$ & & & ${ }^{155} \mathrm{Eu}$ & $1.0 \mathrm{E}-04 \pm 1.3 \mathrm{E}-04$ & $\mathrm{U}$ \\
\hline & $02 / 12 / 08$ & $02 / 25 / 08$ & gross $\alpha$ & $2.3 \mathrm{E}-03 \pm 7.5 \mathrm{E}-04$ & & & ${ }^{238} \mathrm{Pu}$ & $5.3 \mathrm{E}-07 \pm 5.5 \mathrm{E}-07$ & $\mathrm{U}$ \\
\hline & $02 / 12 / 08$ & $02 / 25 / 08$ & gross $\beta$ & $3.2 \mathrm{E}-02 \pm 3.3 \mathrm{E}-03$ & & & ${ }^{239 / 240} \mathrm{Pu}$ & $5.3 \mathrm{E}-07 \pm 1.9 \mathrm{E}-06$ & $\mathrm{U}$ \\
\hline & $02 / 25 / 08$ & 03/11/08 & gross $\alpha$ & $6.6 \mathrm{E}-04 \pm 4.9 \mathrm{E}-04$ & & & ${ }^{106} \mathrm{Ru}$ & $-2.0 \mathrm{E}-04 \pm 5.2 \mathrm{E}-04$ & $\mathrm{U}$ \\
\hline & $02 / 25 / 08$ & 03/11/08 & gross $\beta$ & $1.4 \mathrm{E}-02 \pm 1.8 \mathrm{E}-03$ & & & ${ }^{125} \mathrm{Sb}$ & $-3.0 \mathrm{E}-05 \pm 1.3 \mathrm{E}-04$ & $\mathrm{U}$ \\
\hline & 03/11/08 & 03/25/08 & gross $\alpha$ & $5.0 \mathrm{E}-04 \pm 4.7 \mathrm{E}-04$ & & & ${ }^{90} \mathrm{Sr}$ & $-1.5 \mathrm{E}-04 \pm 1.6 \mathrm{E}-04$ & $\mathrm{U}$ \\
\hline & 03/11/08 & $03 / 25 / 08$ & gross $\beta$ & $1.2 \mathrm{E}-02 \pm 1.7 \mathrm{E}-03$ & & & ${ }^{234} \mathrm{U}$ & $7.3 \mathrm{E}-06 \pm 6.1 \mathrm{E}-06$ & \\
\hline & 03/25/08 & $04 / 07 / 08$ & gross $\alpha$ & $9.3 \mathrm{E}-04 \pm 6.2 \mathrm{E}-04$ & & & ${ }^{235} \mathrm{U}$ & $1.5 \mathrm{E}-06 \pm 2.1 \mathrm{E}-06$ & $\mathrm{U}$ \\
\hline & $03 / 25 / 08$ & $04 / 07 / 08$ & gross $\beta$ & $7.1 \mathrm{E}-03 \pm 1.2 \mathrm{E}-03$ & & & ${ }^{238} \mathrm{U}$ & $6.6 \mathrm{E}-06 \pm 4.9 \mathrm{E}-06$ & \\
\hline & 04/07/08 & $04 / 21 / 08$ & gross $\alpha$ & $9.8 \mathrm{E}-04 \pm 6.1 \mathrm{E}-04$ & & & & & \\
\hline & $04 / 07 / 08$ & $04 / 21 / 08$ & gross $\beta$ & $1.0 \mathrm{E}-02 \pm 1.5 \mathrm{E}-03$ & N970 & $06 / 30 / 08$ to $12 / 30 / 08$ & ${ }^{60} \mathrm{Co}$ & $-3.4 \mathrm{E}-05 \pm 8.8 \mathrm{E}-05$ & $\mathrm{U}$ \\
\hline & $04 / 21 / 08$ & 05/07/08 & gross $\alpha$ & $1.2 \mathrm{E}-03 \pm 4.9 \mathrm{E}-04$ & & & ${ }^{134} \mathrm{Cs}$ & $2.8 \mathrm{E}-05 \pm 6.3 \mathrm{E}-05$ & $\mathrm{U}$ \\
\hline & $04 / 21 / 08$ & 05/07/08 & gross $\beta$ & $1.0 \mathrm{E}-02 \pm 1.4 \mathrm{E}-03$ & & & ${ }^{137} \mathrm{Cs}$ & $1.8 \mathrm{E}-05 \pm 5.8 \mathrm{E}-05$ & $\mathrm{U}$ \\
\hline & 05/07/08 & 05/19/08 & gross $\alpha$ & $1.8 \mathrm{E}-04 \pm 4.0 \mathrm{E}-04$ & & & ${ }^{152} \mathrm{Eu}$ & $-4.4 \mathrm{E}-05 \pm 1.5 \mathrm{E}-04$ & $\mathrm{U}$ \\
\hline & 05/07/08 & 05/19/08 & gross $\beta$ & $1.0 \mathrm{E}-02 \pm 1.6 \mathrm{E}-03$ & & & ${ }^{154} \mathrm{Eu}$ & $-7.1 \mathrm{E}-05 \pm 2.1 \mathrm{E}-04$ & $\mathrm{U}$ \\
\hline & 05/19/08 & $06 / 02 / 08$ & gross $\alpha$ & $8.2 \mathrm{E}-04 \pm 5.5 \mathrm{E}-04$ & & & ${ }^{155} \mathrm{Eu}$ & $5.4 \mathrm{E}-05 \pm 1.4 \mathrm{E}-04$ & U \\
\hline & 05/19/08 & $06 / 02 / 08$ & gross $\beta$ & $7.4 \mathrm{E}-03 \pm 1.2 \mathrm{E}-03$ & & & ${ }^{238} \mathrm{Pu}$ & $-6.5 \mathrm{E}-07 \pm 6.5 \mathrm{E}-06$ & $\mathrm{U}$ \\
\hline & $06 / 02 / 08$ & $06 / 16 / 08$ & gross $\alpha$ & $8.0 \mathrm{E}-04 \pm 6.2 \mathrm{E}-04$ & & & ${ }^{239 / 240} \mathrm{Pu}$ & $6.5 \mathrm{E}-07 \pm 3.2 \mathrm{E}-06$ & $\mathrm{U}$ \\
\hline & $06 / 02 / 08$ & 06/16/08 & gross $\beta$ & $8.5 \mathrm{E}-03 \pm 1.3 \mathrm{E}-03$ & & & ${ }^{106} \mathrm{Ru}$ & $1.6 \mathrm{E}-04 \pm 4.9 \mathrm{E}-04$ & $\mathrm{U}$ \\
\hline & $06 / 16 / 08$ & 06/30/08 & gross $\alpha$ & $1.2 \mathrm{E}-03 \pm 5.8 \mathrm{E}-04$ & & & ${ }^{125} \mathrm{Sb}$ & $-3.9 \mathrm{E}-05 \pm 1.3 \mathrm{E}-04$ & $\mathrm{U}$ \\
\hline & $06 / 16 / 08$ & 06/30/08 & gross $\beta$ & $1.2 \mathrm{E}-02 \pm 1.6 \mathrm{E}-03$ & & & ${ }^{90} \mathrm{Sr}$ & $-6.9 \mathrm{E}-05 \pm 7.2 \mathrm{E}-05$ & $\mathrm{U}$ \\
\hline & $06 / 30 / 08$ & $07 / 14 / 08$ & gross $\alpha$ & $2.2 \mathrm{E}-03 \pm 7.3 \mathrm{E}-04$ & & & ${ }^{234} \mathrm{U}$ & $1.2 \mathrm{E}-05 \pm 7.9 \mathrm{E}-06$ & \\
\hline & $06 / 30 / 08$ & 07/14/08 & gross $\beta$ & $1.5 \mathrm{E}-02 \pm 1.9 \mathrm{E}-03$ & & & ${ }^{235} \mathrm{U}$ & $4.3 \mathrm{E}-06 \pm 3.9 \mathrm{E}-06$ & \\
\hline & $07 / 14 / 08$ & 07/28/08 & gross $\alpha$ & $1.6 \mathrm{E}-03 \pm 6.0 \mathrm{E}-04$ & & & ${ }^{238} \mathrm{U}$ & $1.1 \mathrm{E}-05 \pm 6.9 \mathrm{E}-06$ & \\
\hline & $07 / 14 / 08$ & $07 / 28 / 08$ & gross $\beta$ & $1.1 \mathrm{E}-02 \pm 1.5 \mathrm{E}-03$ & & & & & \\
\hline & 07/28/08 & 08/11/08 & gross $\alpha$ & $1.1 \mathrm{E}-03 \pm 5.0 \mathrm{E}-04$ & & & & & \\
\hline & 07/28/08 & 08/11/08 & gross $\beta$ & $1.3 \mathrm{E}-02 \pm 1.7 \mathrm{E}-03$ & & & & & \\
\hline & $08 / 11 / 08$ & $08 / 25 / 08$ & gross $\alpha$ & $1.6 \mathrm{E}-03 \pm 5.9 \mathrm{E}-04$ & & & & & \\
\hline & 08/11/08 & $08 / 25 / 08$ & gross $\beta$ & $1.0 \mathrm{E}-02 \pm 1.5 \mathrm{E}-03$ & & & & & \\
\hline & $08 / 25 / 08$ & 09/08/08 & gross $\alpha$ & $7.2 \mathrm{E}-04 \pm 5.2 \mathrm{E}-04$ & & & & & \\
\hline & $08 / 25 / 08$ & 09/08/08 & gross $\beta$ & $1.3 \mathrm{E}-02 \pm 1.7 \mathrm{E}-03$ & & & & & \\
\hline & 09/08/08 & 09/22/08 & gross $\alpha$ & $8.5 \mathrm{E}-04 \pm 5.6 \mathrm{E}-04$ & & & & & \\
\hline & 09/08/08 & 09/22/08 & gross $\beta$ & $2.6 \mathrm{E}-02 \pm 2.8 \mathrm{E}-03$ & & & & & \\
\hline & 09/22/08 & $10 / 07 / 08$ & gross $\alpha$ & $1.4 \mathrm{E}-03 \pm 5.4 \mathrm{E}-04$ & & & & & \\
\hline & $09 / 22 / 08$ & $10 / 07 / 08$ & gross $\beta$ & $2.4 \mathrm{E}-02 \pm 2.7 \mathrm{E}-03$ & & & & & \\
\hline & $10 / 07 / 08$ & $10 / 20 / 08$ & gross $\alpha$ & $9.4 \mathrm{E}-04 \pm 6.3 \mathrm{E}-04$ & & & & & \\
\hline & $10 / 07 / 08$ & $10 / 20 / 08$ & gross $\beta$ & $6.8 \mathrm{E}-03 \pm 1.2 \mathrm{E}-03$ & & & & & \\
\hline & $10 / 20 / 08$ & $11 / 03 / 08$ & gross $\alpha$ & $3.6 \mathrm{E}-03 \pm 9.2 \mathrm{E}-04$ & & & & & \\
\hline & $10 / 20 / 08$ & $11 / 03 / 08$ & gross $\beta$ & $4.0 \mathrm{E}-02 \pm 3.8 \mathrm{E}-03$ & & & & & \\
\hline & $11 / 03 / 08$ & $11 / 17 / 08$ & gross $\alpha$ & $2.7 \mathrm{E}-03 \pm 8.0 \mathrm{E}-04$ & & & & & \\
\hline & $11 / 03 / 08$ & $11 / 17 / 08$ & gross $\beta$ & $1.7 \mathrm{E}-02 \pm 2.0 \mathrm{E}-03$ & & & & & \\
\hline & $11 / 17 / 08$ & $12 / 01 / 08$ & gross $\alpha$ & $1.8 \mathrm{E}-03 \pm 6.8 \mathrm{E}-04$ & & & & & \\
\hline & $11 / 17 / 08$ & $12 / 01 / 08$ & gross $\beta$ & $4.3 \mathrm{E}-02 \pm 4.1 \mathrm{E}-03$ & & & & & \\
\hline & $12 / 01 / 08$ & $12 / 15 / 08$ & gross $\alpha$ & $1.9 \mathrm{E}-03 \pm 6.7 \mathrm{E}-04$ & & & & & \\
\hline & $12 / 01 / 08$ & $12 / 15 / 08$ & gross $\beta$ & $1.5 \mathrm{E}-02 \pm 1.9 \mathrm{E}-03$ & & & & & \\
\hline & $12 / 15 / 08$ & $12 / 30 / 08$ & gross $\alpha$ & $1.9 \mathrm{E}-03 \pm 6.5 \mathrm{E}-04$ & & & & & \\
\hline & $12 / 15 / 08$ & $12 / 30 / 08$ & gross $\beta$ & $4.0 \mathrm{E}-02 \pm 3.7 \mathrm{E}-03$ & & & & & \\
\hline
\end{tabular}

$\overline{\mathrm{RQ}}=$ Result Qualifier. $\mathrm{U}=$ The analyte was analyzed for but not detected. 
Table 2-4. Near-Facility Air Sampling Results, 2008 (pCi/m³ \pm total analytical uncertainty). (Sheet 36 of 82)

\begin{tabular}{|c|c|c|c|c|c|c|c|c|c|c|}
\hline Location & Sample On & Sample Off & Isotope & Result \pm Uncertainty & Location & Composite & e Period & Isotope & Result \pm Uncertainty & $\mathbf{R Q *}$ \\
\hline N972 & $12 / 31 / 07$ & $01 / 14 / 08$ & gross $\alpha$ & $1.2 \mathrm{E}-03 \pm 5.2 \mathrm{E}-04$ & N972 & $12 / 31 / 07$ to & $06 / 30 / 08$ & ${ }^{60} \mathrm{Co}$ & $-6.9 \mathrm{E}-06 \pm 6.5 \mathrm{E}-05$ & $\mathrm{U}$ \\
\hline \multirow[t]{51}{*}{$(200-E)$} & $12 / 31 / 07$ & 01/14/08 & gross $\beta$ & $1.1 \mathrm{E}-02 \pm 1.6 \mathrm{E}-03$ & & & & ${ }^{134} \mathrm{Cs}$ & $5.2 \mathrm{E}-05 \pm 6.5 \mathrm{E}-05$ & $\mathrm{U}$ \\
\hline & $01 / 14 / 08$ & 01/29/08 & gross $\alpha$ & $1.8 \mathrm{E}-03 \pm 6.3 \mathrm{E}-04$ & & & & ${ }^{137} \mathrm{Cs}$ & $8.4 \mathrm{E}-05 \pm 8.1 \mathrm{E}-05$ & U \\
\hline & $01 / 14 / 08$ & 01/29/08 & gross $\beta$ & $3.4 \mathrm{E}-02 \pm 3.3 \mathrm{E}-03$ & & & & ${ }^{152} \mathrm{Eu}$ & $-5.4 \mathrm{E}-05 \pm 1.5 \mathrm{E}-04$ & U \\
\hline & 01/29/08 & $02 / 12 / 08$ & gross $\alpha$ & $1.7 \mathrm{E}-04 \pm 3.5 \mathrm{E}-04$ & & & & ${ }^{154} \mathrm{Eu}$ & $-6.8 \mathrm{E}-05 \pm 2.0 \mathrm{E}-04$ & $\mathrm{U}$ \\
\hline & $01 / 29 / 08$ & 02/12/08 & gross $\beta$ & $3.3 \mathrm{E}-03 \pm 7.8 \mathrm{E}-04$ & & & & ${ }^{155} \mathrm{Eu}$ & $7.8 \mathrm{E}-05 \pm 1.6 \mathrm{E}-04$ & U \\
\hline & $02 / 12 / 08$ & $02 / 25 / 08$ & gross $\alpha$ & $1.5 \mathrm{E}-03 \pm 6.1 \mathrm{E}-04$ & & & & ${ }^{238} \mathrm{Pu}$ & $-5.2 \mathrm{E}-07 \pm 5.0 \mathrm{E}-06$ & $\mathrm{U}$ \\
\hline & $02 / 12 / 08$ & $02 / 25 / 08$ & gross $\beta$ & $3.0 \mathrm{E}-02 \pm 3.1 \mathrm{E}-03$ & & & & ${ }^{239 / 240} \mathrm{Pu}$ & $5.2 \mathrm{E}-07 \pm 1.0 \mathrm{E}-06$ & $\mathrm{U}$ \\
\hline & $02 / 25 / 08$ & 03/11/08 & gross $\alpha$ & $6.8 \mathrm{E}-04 \pm 5.0 \mathrm{E}-04$ & & & & ${ }^{106} \mathrm{Ru}$ & $-1.5 \mathrm{E}-04 \pm 5.4 \mathrm{E}-04$ & $\mathrm{U}$ \\
\hline & $02 / 25 / 08$ & 03/11/08 & gross $\beta$ & $1.5 \mathrm{E}-02 \pm 1.9 \mathrm{E}-03$ & & & & ${ }^{125} \mathrm{Sb}$ & $-4.6 \mathrm{E}-06 \pm 4.6 \mathrm{E}-05$ & $\mathrm{U}$ \\
\hline & 03/11/08 & $03 / 25 / 08$ & gross $\alpha$ & $8.3 \mathrm{E}-04 \pm 5.6 \mathrm{E}-04$ & & & & ${ }^{90} \mathrm{Sr}$ & $-6.4 \mathrm{E}-05 \pm 6.6 \mathrm{E}-05$ & $\mathrm{U}$ \\
\hline & 03/11/08 & $03 / 25 / 08$ & gross $\beta$ & $8.9 \mathrm{E}-03 \pm 1.4 \mathrm{E}-03$ & & & & ${ }^{234} \mathrm{U}$ & $8.1 \mathrm{E}-06 \pm 6.2 \mathrm{E}-06$ & \\
\hline & 03/25/08 & $04 / 07 / 08$ & gross $\alpha$ & $6.8 \mathrm{E}-04 \pm 5.6 \mathrm{E}-04$ & & & & ${ }^{235} \mathrm{U}$ & $-7.4 \mathrm{E}-07 \pm 3.3 \mathrm{E}-06$ & $\mathrm{U}$ \\
\hline & 03/25/08 & $04 / 07 / 08$ & gross $\beta$ & $9.2 \mathrm{E}-03 \pm 1.4 \mathrm{E}-03$ & & & & ${ }^{238} \mathrm{U}$ & $1.0 \mathrm{E}-05 \pm 6.6 \mathrm{E}-06$ & \\
\hline & 04/07/08 & $04 / 21 / 08$ & gross $\alpha$ & $5.2 \mathrm{E}-04 \pm 4.9 \mathrm{E}-04$ & & & & & & \\
\hline & $04 / 07 / 08$ & $04 / 21 / 08$ & gross $\beta$ & $1.0 \mathrm{E}-02 \pm 1.5 \mathrm{E}-03$ & N972 & $06 / 30 / 08$ to & $12 / 30 / 08$ & ${ }^{60} \mathrm{Co}$ & $3.7 \mathrm{E}-05 \pm 6.4 \mathrm{E}-05$ & $\mathrm{U}$ \\
\hline & $04 / 21 / 08$ & 05/06/08 & gross $\alpha$ & $7.8 \mathrm{E}-04 \pm 5.2 \mathrm{E}-04$ & & & & ${ }^{134} \mathrm{Cs}$ & $2.2 \mathrm{E}-05 \pm 6.1 \mathrm{E}-05$ & $\mathrm{U}$ \\
\hline & $04 / 21 / 08$ & 05/06/08 & gross $\beta$ & $1.4 \mathrm{E}-02 \pm 1.8 \mathrm{E}-03$ & & & & ${ }^{137} \mathrm{Cs}$ & $3.7 \mathrm{E}-05 \pm 5.3 \mathrm{E}-05$ & $\mathrm{U}$ \\
\hline & 05/06/08 & 05/19/08 & gross $\alpha$ & $1.4 \mathrm{E}-03 \pm 5.9 \mathrm{E}-04$ & & & & ${ }^{152} \mathrm{Eu}$ & $-1.5 \mathrm{E}-04 \pm 1.6 \mathrm{E}-04$ & U \\
\hline & 05/06/08 & 05/19/08 & gross $\beta$ & $1.1 \mathrm{E}-02 \pm 1.7 \mathrm{E}-03$ & & & & ${ }^{154} \mathrm{Eu}$ & $2.7 \mathrm{E}-05 \pm 1.6 \mathrm{E}-04$ & $\mathrm{U}$ \\
\hline & 05/19/08 & 06/02/08 & gross $\alpha$ & $6.1 \mathrm{E}-04 \pm 5.1 \mathrm{E}-04$ & & & & ${ }^{155} \mathrm{Eu}$ & $1.0 \mathrm{E}-04 \pm 1.6 \mathrm{E}-04$ & U \\
\hline & 05/19/08 & $06 / 02 / 08$ & gross $\beta$ & $1.2 \mathrm{E}-02 \pm 1.6 \mathrm{E}-03$ & & & & ${ }^{238} \mathrm{Pu}$ & $-4.9 \mathrm{E}-06 \pm 8.7 \mathrm{E}-06$ & $\mathrm{U}$ \\
\hline & 06/02/08 & 06/16/08 & gross $\alpha$ & $6.4 \mathrm{E}-04 \pm 5.6 \mathrm{E}-04$ & & & & ${ }^{239 / 240} \mathrm{Pu}$ & $3.5 \mathrm{E}-06 \pm 3.4 \mathrm{E}-06$ & \\
\hline & $06 / 02 / 08$ & 06/16/08 & gross $\beta$ & $7.2 \mathrm{E}-03 \pm 1.2 \mathrm{E}-03$ & & & & ${ }^{106} \mathrm{Ru}$ & $-8.1 \mathrm{E}-05 \pm 4.8 \mathrm{E}-04$ & $\mathrm{U}$ \\
\hline & $06 / 16 / 08$ & 06/30/08 & gross $\alpha$ & $1.3 \mathrm{E}-03 \pm 6.0 \mathrm{E}-04$ & & & & ${ }^{125} \mathrm{Sb}$ & $-6.6 \mathrm{E}-05 \pm 1.3 \mathrm{E}-04$ & $\mathrm{U}$ \\
\hline & $06 / 16 / 08$ & $06 / 30 / 08$ & gross $\beta$ & $1.5 \mathrm{E}-02 \pm 1.9 \mathrm{E}-03$ & & & & ${ }^{90} \mathrm{Sr}$ & $-5.5 \mathrm{E}-05 \pm 5.7 \mathrm{E}-05$ & $\mathrm{U}$ \\
\hline & $06 / 30 / 08$ & $07 / 14 / 08$ & gross $\alpha$ & $1.4 \mathrm{E}-03 \pm 5.6 \mathrm{E}-04$ & & & & ${ }^{234} \mathrm{U}$ & $8.8 \mathrm{E}-06 \pm 6.0 \mathrm{E}-06$ & \\
\hline & $06 / 30 / 08$ & 07/14/08 & gross $\beta$ & $1.5 \mathrm{E}-02 \pm 1.9 \mathrm{E}-03$ & & & & ${ }^{235} \mathrm{U}$ & $2.7 \mathrm{E}-06 \pm 2.9 \mathrm{E}-06$ & \\
\hline & $07 / 14 / 08$ & 07/28/08 & gross $\alpha$ & $1.2 \mathrm{E}-03 \pm 5.2 \mathrm{E}-04$ & & & & ${ }^{238} \mathrm{U}$ & $1.6 \mathrm{E}-05 \pm 8.5 \mathrm{E}-06$ & \\
\hline & $07 / 14 / 08$ & $07 / 28 / 08$ & gross $\beta$ & $1.3 \mathrm{E}-02 \pm 1.7 \mathrm{E}-03$ & & & & & & \\
\hline & 07/28/08 & 08/11/08 & gross $\alpha$ & $1.3 \mathrm{E}-03 \pm 5.5 \mathrm{E}-04$ & & & & & & \\
\hline & $07 / 28 / 08$ & $08 / 11 / 08$ & gross $\beta$ & $1.3 \mathrm{E}-02 \pm 1.7 \mathrm{E}-03$ & & & & & & \\
\hline & $08 / 11 / 08$ & $08 / 25 / 08$ & gross $\alpha$ & $1.1 \mathrm{E}-03 \pm 5.0 \mathrm{E}-04$ & & & & & & \\
\hline & 08/11/08 & 08/25/08 & gross $\beta$ & $1.5 \mathrm{E}-02 \pm 1.9 \mathrm{E}-03$ & & & & & & \\
\hline & $08 / 25 / 08$ & 09/08/08 & gross $\alpha$ & $1.8 \mathrm{E}-04 \pm 3.4 \mathrm{E}-04$ & & & & & & \\
\hline & $08 / 25 / 08$ & 09/08/08 & gross $\beta$ & $1.0 \mathrm{E}-02 \pm 1.5 \mathrm{E}-03$ & & & & & & \\
\hline & 09/08/08 & 09/22/08 & gross $\alpha$ & $1.8 \mathrm{E}-03 \pm 6.3 \mathrm{E}-04$ & & & & & & \\
\hline & 09/08/08 & $09 / 22 / 08$ & gross $\beta$ & $2.3 \mathrm{E}-02 \pm 2.5 \mathrm{E}-03$ & & & & & & \\
\hline & $09 / 22 / 08$ & $10 / 07 / 08$ & gross $\alpha$ & $1.8 \mathrm{E}-03 \pm 6.1 \mathrm{E}-04$ & & & & & & \\
\hline & $09 / 22 / 08$ & $10 / 07 / 08$ & gross $\beta$ & $2.3 \mathrm{E}-02 \pm 2.5 \mathrm{E}-03$ & & & & & & \\
\hline & $10 / 07 / 08$ & $10 / 20 / 08$ & gross $\alpha$ & $8.1 \mathrm{E}-04 \pm 6.0 \mathrm{E}-04$ & & & & & & \\
\hline & $10 / 07 / 08$ & $10 / 20 / 08$ & gross $\beta$ & $1.5 \mathrm{E}-02 \pm 1.9 \mathrm{E}-03$ & & & & & & \\
\hline & $10 / 20 / 08$ & $11 / 03 / 08$ & gross $\alpha$ & $2.8 \mathrm{E}-03 \pm 8.3 \mathrm{E}-04$ & & & & & & \\
\hline & $10 / 20 / 08$ & $11 / 03 / 08$ & gross $\beta$ & $4.1 \mathrm{E}-02 \pm 3.9 \mathrm{E}-03$ & & & & & & \\
\hline & $11 / 03 / 08$ & $11 / 17 / 08$ & gross $\alpha$ & $5.2 \mathrm{E}-04 \pm 4.8 \mathrm{E}-04$ & & & & & & \\
\hline & $11 / 03 / 08$ & $11 / 17 / 08$ & gross $\beta$ & $1.5 \mathrm{E}-02 \pm 1.9 \mathrm{E}-03$ & & & & & & \\
\hline & $11 / 17 / 08$ & $12 / 01 / 08$ & gross $\alpha$ & $1.6 \mathrm{E}-03 \pm 6.1 \mathrm{E}-04$ & & & & & & \\
\hline & $11 / 17 / 08$ & $12 / 01 / 08$ & gross $\beta$ & $4.1 \mathrm{E}-02 \pm 3.9 \mathrm{E}-03$ & & & & & & \\
\hline & $12 / 01 / 08$ & $12 / 15 / 08$ & gross $\alpha$ & $1.9 \mathrm{E}-03 \pm 6.7 \mathrm{E}-04$ & & & & & & \\
\hline & $12 / 01 / 08$ & $12 / 15 / 08$ & gross $\beta$ & $2.0 \mathrm{E}-02 \pm 2.3 \mathrm{E}-03$ & & & & & & \\
\hline & $12 / 15 / 08$ & $12 / 30 / 08$ & gross $\alpha$ & $2.3 \mathrm{E}-03 \pm 7.4 \mathrm{E}-04$ & & & & & & \\
\hline & $12 / 15 / 08$ & $12 / 30 / 08$ & gross $\beta$ & $4.2 \mathrm{E}-02 \pm 3.9 \mathrm{E}-03$ & & & & & & \\
\hline
\end{tabular}

$\overline{\mathrm{RQ}}=$ Result Qualifier. $\mathrm{U}=$ The analyte was analyzed for but not detected. 
Table 2-4. Near-Facility Air Sampling Results, 2008 (pCi/m³ \pm total analytical uncertainty). (Sheet 37 of 82)

\begin{tabular}{|c|c|c|c|c|c|c|c|c|c|c|}
\hline Location & Sample On & Sample Off & Isotope & Result \pm Uncertainty & Location & Composite & e Period & Isotope & Result \pm Uncertainty & $\mathbf{R Q *}$ \\
\hline N973 & $12 / 31 / 07$ & $01 / 14 / 08$ & gross $\alpha$ & $1.1 \mathrm{E}-03 \pm 5.1 \mathrm{E}-04$ & N973 & $12 / 31 / 07$ to & $06 / 30 / 08$ & ${ }^{60} \mathrm{Co}$ & $-1.3 \mathrm{E}-04 \pm 1.3 \mathrm{E}-04$ & $\mathrm{U}$ \\
\hline \multirow[t]{51}{*}{$(200-E)$} & $12 / 31 / 07$ & 01/14/08 & gross $\beta$ & $9.9 \mathrm{E}-03 \pm 1.5 \mathrm{E}-03$ & & & & ${ }^{134} \mathrm{Cs}$ & $-3.5 \mathrm{E}-05 \pm 1.2 \mathrm{E}-04$ & $\mathrm{U}$ \\
\hline & $01 / 14 / 08$ & 01/29/08 & gross $\alpha$ & $1.9 \mathrm{E}-03 \pm 6.6 \mathrm{E}-04$ & & & & ${ }^{137} \mathrm{Cs}$ & $-9.5 \mathrm{E}-05 \pm 1.0 \mathrm{E}-04$ & U \\
\hline & $01 / 14 / 08$ & 01/29/08 & gross $\beta$ & $3.7 \mathrm{E}-02 \pm 3.5 \mathrm{E}-03$ & & & & ${ }^{152} \mathrm{Eu}$ & $3.7 \mathrm{E}-05 \pm 2.4 \mathrm{E}-04$ & $\mathrm{U}$ \\
\hline & $01 / 29 / 08$ & $02 / 12 / 08$ & gross $\alpha$ & $3.9 \mathrm{E}-04 \pm 4.4 \mathrm{E}-04$ & & & & ${ }^{154} \mathrm{Eu}$ & $-1.5 \mathrm{E}-04 \pm 3.7 \mathrm{E}-04$ & $\mathrm{U}$ \\
\hline & $01 / 29 / 08$ & $02 / 12 / 08$ & gross $\beta$ & $5.6 \mathrm{E}-03 \pm 1.0 \mathrm{E}-03$ & & & & ${ }^{155} \mathrm{Eu}$ & $-8.8 \mathrm{E}-05 \pm 1.8 \mathrm{E}-04$ & $\mathrm{U}$ \\
\hline & $02 / 12 / 08$ & $02 / 25 / 08$ & gross $\alpha$ & $6.6 \mathrm{E}-04 \pm 5.4 \mathrm{E}-04$ & & & & ${ }^{239 / 240} \mathrm{Pu}$ & $-1.0 \mathrm{E}-06 \pm 1.5 \mathrm{E}-06$ & $\mathrm{U}$ \\
\hline & $02 / 12 / 08$ & $02 / 25 / 08$ & gross $\beta$ & $3.0 \mathrm{E}-02 \pm 3.2 \mathrm{E}-03$ & & & & ${ }^{106} \mathrm{Ru}$ & $-2.8 \mathrm{E}-04 \pm 9.0 \mathrm{E}-04$ & $\mathrm{U}$ \\
\hline & $02 / 25 / 08$ & 03/11/08 & gross $\alpha$ & $4.7 \mathrm{E}-04 \pm 4.4 \mathrm{E}-04$ & & & & ${ }^{125} \mathrm{Sb}$ & $1.1 \mathrm{E}-04 \pm 2.3 \mathrm{E}-04$ & $\mathrm{U}$ \\
\hline & $02 / 25 / 08$ & 03/11/08 & gross $\beta$ & $1.8 \mathrm{E}-02 \pm 2.1 \mathrm{E}-03$ & & & & ${ }^{90} \mathrm{Sr}$ & $-2.3 \mathrm{E}-05 \pm 2.3 \mathrm{E}-05$ & U \\
\hline & 03/11/08 & $03 / 25 / 08$ & gross $\alpha$ & $6.1 \mathrm{E}-04 \pm 5.0 \mathrm{E}-04$ & & & & ${ }^{234} \mathrm{U}$ & $8.6 \mathrm{E}-06 \pm 6.1 \mathrm{E}-06$ & \\
\hline & 03/11/08 & $03 / 25 / 08$ & gross $\beta$ & $8.6 \mathrm{E}-03 \pm 1.4 \mathrm{E}-03$ & & & & ${ }^{235} \mathrm{U}$ & $7.2 \mathrm{E}-07 \pm 1.5 \mathrm{E}-06$ & $\mathrm{U}$ \\
\hline & 03/25/08 & 04/07/08 & gross $\alpha$ & $1.2 \mathrm{E}-03 \pm 5.5 \mathrm{E}-04$ & & & & ${ }^{238} \mathrm{U}$ & $7.9 \mathrm{E}-06 \pm 5.7 \mathrm{E}-06$ & \\
\hline & $03 / 25 / 08$ & $04 / 07 / 08$ & gross $\beta$ & $7.9 \mathrm{E}-03 \pm 1.3 \mathrm{E}-03$ & & & & & & \\
\hline & $04 / 07 / 08$ & $04 / 21 / 08$ & gross $\alpha$ & $8.6 \mathrm{E}-04 \pm 5.8 \mathrm{E}-04$ & N973 & $06 / 30 / 08$ to & $12 / 30 / 08$ & ${ }^{60} \mathrm{Co}$ & $-5.5 \mathrm{E}-05 \pm 1.0 \mathrm{E}-04$ & $\mathrm{U}$ \\
\hline & $04 / 07 / 08$ & $04 / 21 / 08$ & gross $\beta$ & $1.0 \mathrm{E}-02 \pm 1.5 \mathrm{E}-03$ & & & & ${ }^{134} \mathrm{Cs}$ & $-5.0 \mathrm{E}-06 \pm 5.0 \mathrm{E}-05$ & $\mathrm{U}$ \\
\hline & $04 / 21 / 08$ & 05/06/08 & gross $\alpha$ & $1.7 \mathrm{E}-03 \pm 6.1 \mathrm{E}-04$ & & & & ${ }^{137} \mathrm{Cs}$ & $-2.0 \mathrm{E}-05 \pm 1.0 \mathrm{E}-04$ & $\mathrm{U}$ \\
\hline & $04 / 21 / 08$ & 05/06/08 & gross $\beta$ & $1.5 \mathrm{E}-02 \pm 1.9 \mathrm{E}-03$ & & & & ${ }^{152} \mathrm{Eu}$ & $-2.7 \mathrm{E}-04 \pm 2.8 \mathrm{E}-04$ & $\mathrm{U}$ \\
\hline & 05/06/08 & 05/19/08 & gross $\alpha$ & $9.3 \mathrm{E}-04 \pm 6.2 \mathrm{E}-04$ & & & & ${ }^{154} \mathrm{Eu}$ & $-1.3 \mathrm{E}-04 \pm 3.0 \mathrm{E}-04$ & U \\
\hline & 05/06/08 & 05/19/08 & gross $\beta$ & $1.1 \mathrm{E}-02 \pm 1.7 \mathrm{E}-03$ & & & & ${ }^{155} \mathrm{Eu}$ & $-1.0 \mathrm{E}-04 \pm 1.9 \mathrm{E}-04$ & $\mathrm{U}$ \\
\hline & 05/19/08 & $06 / 02 / 08$ & gross $\alpha$ & $8.4 \mathrm{E}-04 \pm 5.7 \mathrm{E}-04$ & & & & ${ }^{238} \mathrm{Pu}$ & $1.7 \mathrm{E}-06 \pm 1.1 \mathrm{E}-05$ & U \\
\hline & 05/19/08 & $06 / 02 / 08$ & gross $\beta$ & $9.9 \mathrm{E}-03 \pm 1.5 \mathrm{E}-03$ & & & & ${ }^{239 / 240} \mathrm{Pu}$ & $8.4 \mathrm{E}-07 \pm 1.7 \mathrm{E}-06$ & $\mathrm{U}$ \\
\hline & $06 / 02 / 08$ & $06 / 16 / 08$ & gross $\alpha$ & $9.4 \mathrm{E}-04 \pm 6.6 \mathrm{E}-04$ & & & & ${ }^{106} \mathrm{Ru}$ & $-7.4 \mathrm{E}-04 \pm 8.9 \mathrm{E}-04$ & $\mathrm{U}$ \\
\hline & $06 / 02 / 08$ & 06/16/08 & gross $\beta$ & $7.2 \mathrm{E}-03 \pm 1.2 \mathrm{E}-03$ & & & & ${ }^{125} \mathrm{Sb}$ & $2.5 \mathrm{E}-05 \pm 2.5 \mathrm{E}-04$ & $\mathrm{U}$ \\
\hline & $06 / 16 / 08$ & 06/30/08 & gross $\alpha$ & $1.8 \mathrm{E}-03 \pm 7.1 \mathrm{E}-04$ & & & & ${ }^{90} \mathrm{Sr}$ & $-2.7 \mathrm{E}-05 \pm 2.8 \mathrm{E}-05$ & $\mathrm{U}$ \\
\hline & $06 / 16 / 08$ & $06 / 30 / 08$ & gross $\beta$ & $1.5 \mathrm{E}-02 \pm 1.9 \mathrm{E}-03$ & & & & ${ }^{234} \mathrm{U}$ & $1.1 \mathrm{E}-05 \pm 8.2 \mathrm{E}-06$ & \\
\hline & $06 / 30 / 08$ & $07 / 14 / 08$ & gross $\alpha$ & $1.8 \mathrm{E}-03 \pm 6.8 \mathrm{E}-04$ & & & & ${ }^{235} \mathrm{U}$ & $2.4 \mathrm{E}-06 \pm 4.3 \mathrm{E}-06$ & $\mathrm{U}$ \\
\hline & $06 / 30 / 08$ & 07/14/08 & gross $\beta$ & $1.3 \mathrm{E}-02 \pm 1.8 \mathrm{E}-03$ & & & & ${ }^{238} \mathrm{U}$ & $9.5 \mathrm{E}-06 \pm 6.7 \mathrm{E}-06$ & \\
\hline & 07/14/08 & 07/28/08 & gross $\alpha$ & $1.1 \mathrm{E}-03 \pm 5.0 \mathrm{E}-04$ & & & & & & \\
\hline & $07 / 14 / 08$ & $07 / 28 / 08$ & gross $\beta$ & $1.4 \mathrm{E}-02 \pm 1.8 \mathrm{E}-03$ & & & & & & \\
\hline & 07/28/08 & 08/11/08 & gross $\alpha$ & $1.0 \mathrm{E}-03 \pm 4.8 \mathrm{E}-04$ & & & & & & \\
\hline & $07 / 28 / 08$ & $08 / 11 / 08$ & gross $\beta$ & $1.4 \mathrm{E}-02 \pm 1.8 \mathrm{E}-03$ & & & & & & \\
\hline & $08 / 11 / 08$ & $08 / 25 / 08$ & gross $\alpha$ & $8.5 \mathrm{E}-04 \pm 5.6 \mathrm{E}-04$ & & & & & & \\
\hline & 08/11/08 & $08 / 25 / 08$ & gross $\beta$ & $1.0 \mathrm{E}-02 \pm 1.5 \mathrm{E}-03$ & & & & & & \\
\hline & $08 / 25 / 08$ & 09/08/08 & gross $\alpha$ & $6.3 \mathrm{E}-04 \pm 5.0 \mathrm{E}-04$ & & & & & & \\
\hline & $08 / 25 / 08$ & 09/08/08 & gross $\beta$ & $1.1 \mathrm{E}-02 \pm 1.5 \mathrm{E}-03$ & & & & & & \\
\hline & 09/08/08 & 09/22/08 & gross $\alpha$ & $1.5 \mathrm{E}-03 \pm 6.2 \mathrm{E}-04$ & & & & & & \\
\hline & 09/08/08 & $09 / 22 / 08$ & gross $\beta$ & $2.6 \mathrm{E}-02 \pm 2.9 \mathrm{E}-03$ & & & & & & \\
\hline & $09 / 22 / 08$ & $10 / 07 / 08$ & gross $\alpha$ & $1.6 \mathrm{E}-03 \pm 5.8 \mathrm{E}-04$ & & & & & & \\
\hline & $09 / 22 / 08$ & $10 / 07 / 08$ & gross $\beta$ & $2.1 \mathrm{E}-02 \pm 2.4 \mathrm{E}-03$ & & & & & & \\
\hline & $10 / 07 / 08$ & $10 / 20 / 08$ & gross $\alpha$ & $3.1 \mathrm{E}-04 \pm 4.4 \mathrm{E}-04$ & & & & & & \\
\hline & $10 / 07 / 08$ & $10 / 20 / 08$ & gross $\beta$ & $1.5 \mathrm{E}-02 \pm 2.0 \mathrm{E}-03$ & & & & & & \\
\hline & $10 / 20 / 08$ & $11 / 03 / 08$ & gross $\alpha$ & $2.9 \mathrm{E}-03 \pm 8.7 \mathrm{E}-04$ & & & & & & \\
\hline & $10 / 20 / 08$ & $11 / 03 / 08$ & gross $\beta$ & $3.9 \mathrm{E}-02 \pm 3.8 \mathrm{E}-03$ & & & & & & \\
\hline & $11 / 03 / 08$ & $11 / 17 / 08$ & gross $\alpha$ & $1.4 \mathrm{E}-03 \pm 5.9 \mathrm{E}-04$ & & & & & & \\
\hline & $11 / 03 / 08$ & $11 / 17 / 08$ & gross $\beta$ & $2.0 \mathrm{E}-02 \pm 2.3 \mathrm{E}-03$ & & & & & & \\
\hline & $11 / 17 / 08$ & $12 / 01 / 08$ & gross $\alpha$ & $1.4 \mathrm{E}-03 \pm 6.4 \mathrm{E}-04$ & & & & & & \\
\hline & $11 / 17 / 08$ & $12 / 01 / 08$ & gross $\beta$ & $4.2 \mathrm{E}-02 \pm 4.1 \mathrm{E}-03$ & & & & & & \\
\hline & $12 / 01 / 08$ & $12 / 15 / 08$ & gross $\alpha$ & $1.8 \mathrm{E}-03 \pm 6.6 \mathrm{E}-04$ & & & & & & \\
\hline & $12 / 01 / 08$ & $12 / 15 / 08$ & gross $\beta$ & $2.2 \mathrm{E}-02 \pm 2.5 \mathrm{E}-03$ & & & & & & \\
\hline & $12 / 15 / 08$ & $12 / 30 / 08$ & gross $\alpha$ & $2.6 \mathrm{E}-03 \pm 7.8 \mathrm{E}-04$ & & & & & & \\
\hline & $12 / 15 / 08$ & $12 / 30 / 08$ & gross $\beta$ & $3.6 \mathrm{E}-02 \pm 3.5 \mathrm{E}-03$ & & & & & & \\
\hline
\end{tabular}

$\overline{\mathrm{RQ}}=$ Result Qualifier. $\mathrm{U}=$ The analyte was analyzed for but not detected. 
Table 2-4. Near-Facility Air Sampling Results, 2008 (pCi/m³ \pm total analytical uncertainty). (Sheet 38 of 82)

\begin{tabular}{|c|c|c|c|c|c|c|c|c|c|}
\hline Location & Sample On & Sample Off & Isotope & Result \pm Uncertainty & Location & Composite Period & Isotope & Result \pm Uncertainty & $\mathbf{R Q *}$ \\
\hline N976 & $12 / 31 / 07$ & $01 / 14 / 08$ & gross $\alpha$ & $9.5 \mathrm{E}-04 \pm 5.9 \mathrm{E}-04$ & N976 & $12 / 31 / 07$ to $06 / 30 / 08$ & ${ }^{60} \mathrm{Co}$ & $2.4 \mathrm{E}-06 \pm 2.4 \mathrm{E}-05$ & $\mathrm{U}$ \\
\hline \multirow[t]{51}{*}{$(200-E)$} & $12 / 31 / 07$ & 01/14/08 & gross $\beta$ & $1.4 \mathrm{E}-02 \pm 1.8 \mathrm{E}-03$ & & & ${ }^{134} \mathrm{Cs}$ & $-6.2 \mathrm{E}-05 \pm 6.6 \mathrm{E}-05$ & $\mathrm{U}$ \\
\hline & $01 / 14 / 08$ & 01/29/08 & gross $\alpha$ & $1.4 \mathrm{E}-03 \pm 5.3 \mathrm{E}-04$ & & & ${ }^{137} \mathrm{Cs}$ & $5.1 \mathrm{E}-05 \pm 6.7 \mathrm{E}-05$ & U \\
\hline & $01 / 14 / 08$ & 01/29/08 & gross $\beta$ & $2.5 \mathrm{E}-02 \pm 2.6 \mathrm{E}-03$ & & & ${ }^{152} \mathrm{Eu}$ & $-5.7 \mathrm{E}-05 \pm 1.3 \mathrm{E}-04$ & U \\
\hline & 01/29/08 & $02 / 12 / 08$ & gross $\alpha$ & $4.1 \mathrm{E}-04 \pm 4.6 \mathrm{E}-04$ & & & ${ }^{154} \mathrm{Eu}$ & $-2.9 \mathrm{E}-05 \pm 1.7 \mathrm{E}-04$ & $\mathrm{U}$ \\
\hline & $01 / 29 / 08$ & 02/12/08 & gross $\beta$ & $3.0 \mathrm{E}-03 \pm 7.7 \mathrm{E}-04$ & & & ${ }^{155} \mathrm{Eu}$ & $-1.2 \mathrm{E}-04 \pm 1.6 \mathrm{E}-04$ & U \\
\hline & $02 / 12 / 08$ & $02 / 25 / 08$ & gross $\alpha$ & $1.4 \mathrm{E}-03 \pm 5.7 \mathrm{E}-04$ & & & ${ }^{238} \mathrm{Pu}$ & $-5.6 \mathrm{E}-07 \pm 3.0 \mathrm{E}-06$ & $\mathrm{U}$ \\
\hline & $02 / 12 / 08$ & $02 / 25 / 08$ & gross $\beta$ & $3.0 \mathrm{E}-02 \pm 3.1 \mathrm{E}-03$ & & & ${ }^{239 / 240} \mathrm{Pu}$ & $5.6 \mathrm{E}-07 \pm 1.1 \mathrm{E}-06$ & $\mathrm{U}$ \\
\hline & $02 / 25 / 08$ & 03/11/08 & gross $\alpha$ & $1.5 \mathrm{E}-03 \pm 6.0 \mathrm{E}-04$ & & & ${ }^{106} \mathrm{Ru}$ & $-2.7 \mathrm{E}-04 \pm 5.7 \mathrm{E}-04$ & $\mathrm{U}$ \\
\hline & $02 / 25 / 08$ & 03/11/08 & gross $\beta$ & $1.4 \mathrm{E}-02 \pm 1.9 \mathrm{E}-03$ & & & ${ }^{125} \mathrm{Sb}$ & $-3.3 \mathrm{E}-06 \pm 3.3 \mathrm{E}-05$ & $\mathrm{U}$ \\
\hline & 03/11/08 & $03 / 25 / 08$ & gross $\alpha$ & $7.6 \mathrm{E}-04 \pm 5.6 \mathrm{E}-04$ & & & ${ }^{90} \mathrm{Sr}$ & $3.2 \mathrm{E}-05 \pm 1.4 \mathrm{E}-04$ & $\mathrm{U}$ \\
\hline & 03/11/08 & $03 / 25 / 08$ & gross $\beta$ & $7.7 \mathrm{E}-03 \pm 1.3 \mathrm{E}-03$ & & & ${ }^{234} \mathrm{U}$ & $1.9 \mathrm{E}-05 \pm 1.0 \mathrm{E}-05$ & \\
\hline & 03/25/08 & $04 / 07 / 08$ & gross $\alpha$ & $4.1 \mathrm{E}-04 \pm 4.6 \mathrm{E}-04$ & & & ${ }^{235} \mathrm{U}$ & $2.2 \mathrm{E}-06 \pm 2.6 \mathrm{E}-06$ & \\
\hline & 03/25/08 & $04 / 07 / 08$ & gross $\beta$ & $6.7 \mathrm{E}-03 \pm 1.2 \mathrm{E}-03$ & & & ${ }^{238} \mathrm{U}$ & $8.0 \mathrm{E}-06 \pm 5.5 \mathrm{E}-06$ & \\
\hline & 04/07/08 & $04 / 21 / 08$ & gross $\alpha$ & $4.5 \mathrm{E}-04 \pm 5.0 \mathrm{E}-04$ & & & & & \\
\hline & $04 / 07 / 08$ & $04 / 21 / 08$ & gross $\beta$ & $7.9 \mathrm{E}-03 \pm 1.3 \mathrm{E}-03$ & N976 & $06 / 30 / 08$ to $12 / 30 / 08$ & ${ }^{60} \mathrm{Co}$ & $-1.3 \mathrm{E}-05 \pm 7.8 \mathrm{E}-05$ & $\mathrm{U}$ \\
\hline & $04 / 21 / 08$ & 05/06/08 & gross $\alpha$ & $1.1 \mathrm{E}-03 \pm 4.7 \mathrm{E}-04$ & & & ${ }^{134} \mathrm{Cs}$ & $-1.6 \mathrm{E}-05 \pm 7.2 \mathrm{E}-05$ & $\mathrm{U}$ \\
\hline & $04 / 21 / 08$ & 05/06/08 & gross $\beta$ & $1.2 \mathrm{E}-02 \pm 1.6 \mathrm{E}-03$ & & & ${ }^{137} \mathrm{Cs}$ & $2.0 \mathrm{E}-04 \pm 1.3 \mathrm{E}-04$ & \\
\hline & 05/06/08 & 05/19/08 & gross $\alpha$ & $1.3 \mathrm{E}-03 \pm 5.6 \mathrm{E}-04$ & & & ${ }^{152} \mathrm{Eu}$ & $3.1 \mathrm{E}-06 \pm 3.1 \mathrm{E}-05$ & U \\
\hline & 05/06/08 & 05/19/08 & gross $\beta$ & $1.0 \mathrm{E}-02 \pm 1.6 \mathrm{E}-03$ & & & ${ }^{154} \mathrm{Eu}$ & $-1.5 \mathrm{E}-06 \pm 1.5 \mathrm{E}-05$ & $\mathrm{U}$ \\
\hline & 05/19/08 & 06/02/08 & gross $\alpha$ & $7.9 \mathrm{E}-04 \pm 5.8 \mathrm{E}-04$ & & & ${ }^{155} \mathrm{Eu}$ & $1.1 \mathrm{E}-05 \pm 1.1 \mathrm{E}-04$ & U \\
\hline & 05/19/08 & $06 / 02 / 08$ & gross $\beta$ & $7.7 \mathrm{E}-03 \pm 1.3 \mathrm{E}-03$ & & & ${ }^{238} \mathrm{Pu}$ & $-3.6 \mathrm{E}-06 \pm 1.2 \mathrm{E}-05$ & $\mathrm{U}$ \\
\hline & $06 / 02 / 08$ & 06/16/08 & gross $\alpha$ & $1.4 \mathrm{E}-03 \pm 6.3 \mathrm{E}-04$ & & & ${ }^{239 / 240} \mathrm{Pu}$ & $1.5 \mathrm{E}-06 \pm 3.6 \mathrm{E}-06$ & $\mathrm{U}$ \\
\hline & $06 / 02 / 08$ & 06/16/08 & gross $\beta$ & $9.3 \mathrm{E}-03 \pm 1.4 \mathrm{E}-03$ & & & ${ }^{106} \mathrm{Ru}$ & $-3.3 \mathrm{E}-04 \pm 5.6 \mathrm{E}-04$ & $\mathrm{U}$ \\
\hline & $06 / 16 / 08$ & 06/30/08 & gross $\alpha$ & $8.7 \mathrm{E}-04 \pm 6.8 \mathrm{E}-04$ & & & ${ }^{125} \mathrm{Sb}$ & $2.7 \mathrm{E}-05 \pm 1.4 \mathrm{E}-04$ & $\mathrm{U}$ \\
\hline & $06 / 16 / 08$ & $06 / 30 / 08$ & gross $\beta$ & $6.7 \mathrm{E}-03 \pm 1.2 \mathrm{E}-03$ & & & ${ }^{90} \mathrm{Sr}$ & $-5.9 \mathrm{E}-05 \pm 6.1 \mathrm{E}-05$ & $\mathrm{U}$ \\
\hline & $06 / 30 / 08$ & $07 / 14 / 08$ & gross $\alpha$ & $5.6 \mathrm{E}-04 \pm 5.1 \mathrm{E}-04$ & & & ${ }^{234} \mathrm{U}$ & $1.3 \mathrm{E}-05 \pm 8.2 \mathrm{E}-06$ & \\
\hline & $06 / 30 / 08$ & 07/14/08 & gross $\beta$ & $1.2 \mathrm{E}-02 \pm 1.7 \mathrm{E}-03$ & & & ${ }^{235} \mathrm{U}$ & $2.3 \mathrm{E}-06 \pm 3.5 \mathrm{E}-06$ & $\mathrm{U}$ \\
\hline & $07 / 14 / 08$ & 07/28/08 & gross $\alpha$ & $1.2 \mathrm{E}-03 \pm 5.4 \mathrm{E}-04$ & & & ${ }^{238} \mathrm{U}$ & $1.3 \mathrm{E}-05 \pm 8.2 \mathrm{E}-06$ & \\
\hline & $07 / 14 / 08$ & $07 / 28 / 08$ & gross $\beta$ & $1.3 \mathrm{E}-02 \pm 1.7 \mathrm{E}-03$ & & & & & \\
\hline & 07/28/08 & 08/11/08 & gross $\alpha$ & $1.3 \mathrm{E}-03 \pm 5.7 \mathrm{E}-04$ & & & & & \\
\hline & $07 / 28 / 08$ & $08 / 11 / 08$ & gross $\beta$ & $1.2 \mathrm{E}-02 \pm 1.6 \mathrm{E}-03$ & & & & & \\
\hline & $08 / 11 / 08$ & $08 / 25 / 08$ & gross $\alpha$ & $9.0 \mathrm{E}-04 \pm 5.9 \mathrm{E}-04$ & & & & & \\
\hline & 08/11/08 & 08/25/08 & gross $\beta$ & $1.2 \mathrm{E}-02 \pm 1.7 \mathrm{E}-03$ & & & & & \\
\hline & $08 / 25 / 08$ & 09/08/08 & gross $\alpha$ & $9.7 \mathrm{E}-04 \pm 5.9 \mathrm{E}-04$ & & & & & \\
\hline & $08 / 25 / 08$ & 09/08/08 & gross $\beta$ & $6.2 \mathrm{E}-03 \pm 1.1 \mathrm{E}-03$ & & & & & \\
\hline & 09/08/08 & 09/22/08 & gross $\alpha$ & $4.4 \mathrm{E}-04 \pm 4.7 \mathrm{E}-04$ & & & & & \\
\hline & 09/08/08 & $09 / 22 / 08$ & gross $\beta$ & $1.6 \mathrm{E}-02 \pm 2.0 \mathrm{E}-03$ & & & & & \\
\hline & $09 / 22 / 08$ & $10 / 07 / 08$ & gross $\alpha$ & $1.3 \mathrm{E}-03 \pm 5.4 \mathrm{E}-04$ & & & & & \\
\hline & $09 / 22 / 08$ & $10 / 07 / 08$ & gross $\beta$ & $2.5 \mathrm{E}-02 \pm 2.7 \mathrm{E}-03$ & & & & & \\
\hline & $10 / 07 / 08$ & $10 / 20 / 08$ & gross $\alpha$ & $1.7 \mathrm{E}-03 \pm 6.6 \mathrm{E}-04$ & & & & & \\
\hline & $10 / 07 / 08$ & $10 / 20 / 08$ & gross $\beta$ & $2.0 \mathrm{E}-02 \pm 2.4 \mathrm{E}-03$ & & & & & \\
\hline & $10 / 20 / 08$ & $11 / 03 / 08$ & gross $\alpha$ & $1.9 \mathrm{E}-03 \pm 7.0 \mathrm{E}-04$ & & & & & \\
\hline & $10 / 20 / 08$ & $11 / 03 / 08$ & gross $\beta$ & $3.8 \mathrm{E}-02 \pm 3.7 \mathrm{E}-03$ & & & & & \\
\hline & $11 / 03 / 08$ & $11 / 17 / 08$ & gross $\alpha$ & $7.5 \mathrm{E}-04 \pm 5.5 \mathrm{E}-04$ & & & & & \\
\hline & $11 / 03 / 08$ & $11 / 17 / 08$ & gross $\beta$ & $1.4 \mathrm{E}-02 \pm 1.8 \mathrm{E}-03$ & & & & & \\
\hline & $11 / 17 / 08$ & $12 / 01 / 08$ & gross $\alpha$ & $2.5 \mathrm{E}-03 \pm 7.9 \mathrm{E}-04$ & & & & & \\
\hline & $11 / 17 / 08$ & $12 / 01 / 08$ & gross $\beta$ & $3.7 \mathrm{E}-02 \pm 3.7 \mathrm{E}-03$ & & & & & \\
\hline & $12 / 01 / 08$ & $12 / 15 / 08$ & gross $\alpha$ & $9.7 \mathrm{E}-04 \pm 6.0 \mathrm{E}-04$ & & & & & \\
\hline & $12 / 01 / 08$ & $12 / 15 / 08$ & gross $\beta$ & $2.2 \mathrm{E}-02 \pm 2.5 \mathrm{E}-03$ & & & & & \\
\hline & $12 / 15 / 08$ & $12 / 30 / 08$ & gross $\alpha$ & $2.8 \mathrm{E}-03 \pm 7.9 \mathrm{E}-04$ & & & & & \\
\hline & $12 / 15 / 08$ & $12 / 30 / 08$ & gross $\beta$ & $3.5 \mathrm{E}-02 \pm 3.4 \mathrm{E}-03$ & & & & & \\
\hline
\end{tabular}

$\overline{\mathrm{RQ}}=$ Result Qualifier. $\mathrm{U}=$ The analyte was analyzed for but not detected. 
Table 2-4. Near-Facility Air Sampling Results, 2008 (pCi/m³ \pm total analytical uncertainty). (Sheet 39 of 82)

\begin{tabular}{|c|c|c|c|c|c|c|c|c|c|c|}
\hline Location & Sample On & Sample Off & Isotope & Result \pm Uncertainty & Location & Composite & e Period & Isotope & Result \pm Uncertainty & $\mathbf{R Q *}$ \\
\hline N977 & $12 / 31 / 07$ & $01 / 14 / 08$ & gross $\alpha$ & $9.3 \mathrm{E}-04 \pm 5.7 \mathrm{E}-04$ & N977 & $12 / 31 / 07$ to & $06 / 30 / 08$ & ${ }^{60} \mathrm{Co}$ & $1.4 \mathrm{E}-04 \pm 1.7 \mathrm{E}-04$ & $\mathrm{U}$ \\
\hline \multirow[t]{51}{*}{$(200-E)$} & $12 / 31 / 07$ & 01/14/08 & gross $\beta$ & $7.5 \mathrm{E}-03 \pm 1.2 \mathrm{E}-03$ & & & & ${ }^{134} \mathrm{Cs}$ & $2.8 \mathrm{E}-05 \pm 1.1 \mathrm{E}-04$ & $\mathrm{U}$ \\
\hline & $01 / 14 / 08$ & $01 / 29 / 08$ & gross $\alpha$ & $2.0 \mathrm{E}-03 \pm 6.7 \mathrm{E}-04$ & & & & ${ }^{137} \mathrm{Cs}$ & $1.5 \mathrm{E}-05 \pm 9.9 \mathrm{E}-05$ & U \\
\hline & $01 / 14 / 08$ & 01/29/08 & gross $\beta$ & $3.3 \mathrm{E}-02 \pm 3.3 \mathrm{E}-03$ & & & & ${ }^{152} \mathrm{Eu}$ & $-1.0 \mathrm{E}-04 \pm 2.4 \mathrm{E}-04$ & $\mathrm{U}$ \\
\hline & $01 / 29 / 08$ & $02 / 12 / 08$ & gross $\alpha$ & $2.9 \mathrm{E}-04 \pm 4.1 \mathrm{E}-04$ & & & & ${ }^{154} \mathrm{Eu}$ & $-5.9 \mathrm{E}-05 \pm 3.5 \mathrm{E}-04$ & $\mathrm{U}$ \\
\hline & $01 / 29 / 08$ & $02 / 12 / 08$ & gross $\beta$ & $3.8 \mathrm{E}-03 \pm 8.5 \mathrm{E}-04$ & & & & ${ }^{155} \mathrm{Eu}$ & $-1.2 \mathrm{E}-04 \pm 1.9 \mathrm{E}-04$ & $\mathrm{U}$ \\
\hline & $02 / 12 / 08$ & $02 / 25 / 08$ & gross $\alpha$ & $1.5 \mathrm{E}-03 \pm 6.1 \mathrm{E}-04$ & & & & ${ }^{238} \mathrm{Pu}$ & $1.9 \mathrm{E}-04 \pm 6.8 \mathrm{E}-05$ & \\
\hline & $02 / 12 / 08$ & $02 / 25 / 08$ & gross $\beta$ & $3.1 \mathrm{E}-02 \pm 3.2 \mathrm{E}-03$ & & & & ${ }^{239 / 240} \mathrm{Pu}$ & $1.8 \mathrm{E}-03 \pm 6.5 \mathrm{E}-04$ & \\
\hline & $02 / 25 / 08$ & 03/11/08 & $\operatorname{gross} \alpha$ & $1.7 \mathrm{E}-03 \pm 6.1 \mathrm{E}-04$ & & & & ${ }^{106} \mathrm{Ru}$ & $-6.3 \mathrm{E}-04 \pm 8.5 \mathrm{E}-04$ & $\mathrm{U}$ \\
\hline & $02 / 25 / 08$ & 03/11/08 & gross $\beta$ & $1.4 \mathrm{E}-02 \pm 1.8 \mathrm{E}-03$ & & & & ${ }^{125} \mathrm{Sb}$ & $-8.4 \mathrm{E}-05 \pm 2.4 \mathrm{E}-04$ & U \\
\hline & 03/11/08 & 03/25/08 & gross $\alpha$ & $1.6 \mathrm{E}-04 \pm 3.5 \mathrm{E}-04$ & & & & ${ }^{90} \mathrm{Sr}$ & $2.1 \mathrm{E}-04 \pm 1.5 \mathrm{E}-04$ & \\
\hline & 03/11/08 & $03 / 25 / 08$ & gross $\beta$ & $7.5 \mathrm{E}-03 \pm 1.2 \mathrm{E}-03$ & & & & ${ }^{234} \mathrm{U}$ & $8.2 \mathrm{E}-06 \pm 6.0 \mathrm{E}-06$ & \\
\hline & $03 / 25 / 08$ & $04 / 07 / 08$ & gross $\alpha$ & $5.6 \mathrm{E}-04 \pm 5.2 \mathrm{E}-04$ & & & & ${ }^{235} \mathrm{U}$ & $2.1 \mathrm{E}-06 \pm 2.5 \mathrm{E}-06$ & \\
\hline & 03/25/08 & $04 / 07 / 08$ & gross $\beta$ & $7.6 \mathrm{E}-03 \pm 1.3 \mathrm{E}-03$ & & & & ${ }^{238} \mathrm{U}$ & $1.0 \mathrm{E}-05 \pm 6.3 \mathrm{E}-06$ & \\
\hline & 04/07/08 & $04 / 21 / 08$ & gross $\alpha$ & $8.6 \mathrm{E}-04 \pm 5.8 \mathrm{E}-04$ & & & & & & \\
\hline & $04 / 07 / 08$ & $04 / 21 / 08$ & gross $\beta$ & $1.1 \mathrm{E}-02 \pm 1.6 \mathrm{E}-03$ & N977 & $06 / 30 / 08$ to & $12 / 30 / 08$ & ${ }^{60} \mathrm{Co}$ & $1.6 \mathrm{E}-05 \pm 6.4 \mathrm{E}-05$ & $\mathrm{U}$ \\
\hline & $04 / 21 / 08$ & 05/07/08 & gross $\alpha$ & $1.0 \mathrm{E}-03 \pm 4.6 \mathrm{E}-04$ & & & & ${ }^{134} \mathrm{Cs}$ & $1.1 \mathrm{E}-05 \pm 5.9 \mathrm{E}-05$ & $\mathrm{U}$ \\
\hline & $04 / 21 / 08$ & 05/07/08 & gross $\beta$ & $1.4 \mathrm{E}-02 \pm 1.8 \mathrm{E}-03$ & & & & ${ }^{137} \mathrm{Cs}$ & $-2.8 \mathrm{E}-05 \pm 6.3 \mathrm{E}-05$ & $\mathrm{U}$ \\
\hline & 05/07/08 & 05/19/08 & gross $\alpha$ & $9.4 \mathrm{E}-04 \pm 6.3 \mathrm{E}-04$ & & & & ${ }^{152} \mathrm{Eu}$ & $3.8 \mathrm{E}-05 \pm 1.6 \mathrm{E}-04$ & U \\
\hline & 05/07/08 & 05/19/08 & gross $\beta$ & $1.3 \mathrm{E}-02 \pm 1.8 \mathrm{E}-03$ & & & & ${ }^{154} \mathrm{Eu}$ & $-1.6 \mathrm{E}-04 \pm 1.9 \mathrm{E}-04$ & $\mathrm{U}$ \\
\hline & 05/19/08 & 06/02/08 & gross $\alpha$ & $9.5 \mathrm{E}-04 \pm 5.9 \mathrm{E}-04$ & & & & ${ }^{155} \mathrm{Eu}$ & $1.4 \mathrm{E}-05 \pm 1.4 \mathrm{E}-04$ & U \\
\hline & 05/19/08 & $06 / 02 / 08$ & gross $\beta$ & $1.0 \mathrm{E}-02 \pm 1.5 \mathrm{E}-03$ & & & & ${ }^{238} \mathrm{Pu}$ & $-3.7 \mathrm{E}-06 \pm 1.4 \mathrm{E}-05$ & $\mathrm{U}$ \\
\hline & $06 / 02 / 08$ & 06/16/08 & $\operatorname{gross} \alpha$ & $1.8 \mathrm{E}-03 \pm 7.0 \mathrm{E}-04$ & & & & ${ }^{239 / 240} \mathrm{Pu}$ & $1.5 \mathrm{E}-06 \pm 3.1 \mathrm{E}-06$ & $\mathrm{U}$ \\
\hline & $06 / 02 / 08$ & 06/16/08 & gross $\beta$ & $9.1 \mathrm{E}-03 \pm 1.4 \mathrm{E}-03$ & & & & ${ }^{106} \mathrm{Ru}$ & $1.8 \mathrm{E}-04 \pm 5.2 \mathrm{E}-04$ & $\mathrm{U}$ \\
\hline & 06/16/08 & 06/30/08 & gross $\alpha$ & $9.1 \mathrm{E}-04 \pm 6.4 \mathrm{E}-04$ & & & & ${ }^{125} \mathrm{Sb}$ & $2.1 \mathrm{E}-05 \pm 1.2 \mathrm{E}-04$ & $\mathrm{U}$ \\
\hline & $06 / 16 / 08$ & $06 / 30 / 08$ & gross $\beta$ & $1.2 \mathrm{E}-02 \pm 1.6 \mathrm{E}-03$ & & & & ${ }^{90} \mathrm{Sr}$ & $-1.3 \mathrm{E}-04 \pm 1.3 \mathrm{E}-04$ & $\mathrm{U}$ \\
\hline & $06 / 30 / 08$ & $07 / 14 / 08$ & gross $\alpha$ & $1.1 \mathrm{E}-03 \pm 5.1 \mathrm{E}-04$ & & & & ${ }^{234} \mathrm{U}$ & $1.0 \mathrm{E}-05 \pm 6.8 \mathrm{E}-06$ & \\
\hline & $06 / 30 / 08$ & 07/14/08 & gross $\beta$ & $1.4 \mathrm{E}-02 \pm 1.8 \mathrm{E}-03$ & & & & ${ }^{235} \mathrm{U}$ & $1.5 \mathrm{E}-06 \pm 2.2 \mathrm{E}-06$ & $\mathrm{U}$ \\
\hline & $07 / 14 / 08$ & 07/28/08 & gross $\alpha$ & $8.5 \mathrm{E}-04 \pm 5.6 \mathrm{E}-04$ & & & & ${ }^{238} \mathrm{U}$ & $9.5 \mathrm{E}-06 \pm 6.2 \mathrm{E}-06$ & \\
\hline & $07 / 14 / 08$ & $07 / 28 / 08$ & gross $\beta$ & $1.2 \mathrm{E}-02 \pm 1.7 \mathrm{E}-03$ & & & & & & \\
\hline & 07/28/08 & 08/11/08 & gross $\alpha$ & $1.2 \mathrm{E}-03 \pm 5.4 \mathrm{E}-04$ & & & & & & \\
\hline & $07 / 28 / 08$ & 08/11/08 & gross $\beta$ & $1.7 \mathrm{E}-02 \pm 2.1 \mathrm{E}-03$ & & & & & & \\
\hline & $08 / 11 / 08$ & $08 / 25 / 08$ & gross $\alpha$ & $8.5 \mathrm{E}-04 \pm 5.6 \mathrm{E}-04$ & & & & & & \\
\hline & 08/11/08 & 08/25/08 & gross $\beta$ & $1.4 \mathrm{E}-02 \pm 1.8 \mathrm{E}-03$ & & & & & & \\
\hline & $08 / 25 / 08$ & 09/08/08 & gross $\alpha$ & $7.4 \mathrm{E}-04 \pm 5.3 \mathrm{E}-04$ & & & & & & \\
\hline & $08 / 25 / 08$ & 09/08/08 & gross $\beta$ & $8.0 \mathrm{E}-03 \pm 1.3 \mathrm{E}-03$ & & & & & & \\
\hline & 09/08/08 & 09/22/08 & gross $\alpha$ & $1.9 \mathrm{E}-03 \pm 6.5 \mathrm{E}-04$ & & & & & & \\
\hline & 09/08/08 & 09/22/08 & gross $\beta$ & $2.9 \mathrm{E}-02 \pm 3.0 \mathrm{E}-03$ & & & & & & \\
\hline & $09 / 22 / 08$ & $10 / 07 / 08$ & gross $\alpha$ & $1.5 \mathrm{E}-03 \pm 5.6 \mathrm{E}-04$ & & & & & & \\
\hline & $09 / 22 / 08$ & $10 / 07 / 08$ & gross $\beta$ & $2.4 \mathrm{E}-02 \pm 2.6 \mathrm{E}-03$ & & & & & & \\
\hline & $10 / 07 / 08$ & $10 / 20 / 08$ & gross $\alpha$ & $1.4 \mathrm{E}-03 \pm 5.9 \mathrm{E}-04$ & & & & & & \\
\hline & $10 / 07 / 08$ & $10 / 20 / 08$ & gross $\beta$ & $1.6 \mathrm{E}-02 \pm 2.1 \mathrm{E}-03$ & & & & & & \\
\hline & $10 / 20 / 08$ & $11 / 03 / 08$ & gross $\alpha$ & $3.5 \mathrm{E}-03 \pm 9.2 \mathrm{E}-04$ & & & & & & \\
\hline & $10 / 20 / 08$ & $11 / 03 / 08$ & gross $\beta$ & $3.5 \mathrm{E}-02 \pm 3.4 \mathrm{E}-03$ & & & & & & \\
\hline & $11 / 03 / 08$ & $11 / 17 / 08$ & gross $\alpha$ & $1.2 \mathrm{E}-03 \pm 5.4 \mathrm{E}-04$ & & & & & & \\
\hline & $11 / 03 / 08$ & $11 / 17 / 08$ & gross $\beta$ & $1.6 \mathrm{E}-02 \pm 2.0 \mathrm{E}-03$ & & & & & & \\
\hline & $11 / 17 / 08$ & $12 / 01 / 08$ & gross $\alpha$ & $1.3 \mathrm{E}-03 \pm 5.7 \mathrm{E}-04$ & & & & & & \\
\hline & $11 / 17 / 08$ & $12 / 01 / 08$ & gross $\beta$ & $4.0 \mathrm{E}-02 \pm 3.9 \mathrm{E}-03$ & & & & & & \\
\hline & $12 / 01 / 08$ & $12 / 15 / 08$ & gross $\alpha$ & $1.8 \mathrm{E}-03 \pm 6.6 \mathrm{E}-04$ & & & & & & \\
\hline & $12 / 01 / 08$ & $12 / 15 / 08$ & gross $\beta$ & $1.8 \mathrm{E}-02 \pm 2.2 \mathrm{E}-03$ & & & & & & \\
\hline & $12 / 15 / 08$ & $12 / 30 / 08$ & gross $\alpha$ & $2.1 \mathrm{E}-03 \pm 6.9 \mathrm{E}-04$ & & & & & & \\
\hline & $12 / 15 / 08$ & $12 / 30 / 08$ & gross $\beta$ & $3.6 \mathrm{E}-02 \pm 3.5 \mathrm{E}-03$ & & & & & & \\
\hline
\end{tabular}

$\overline{\mathrm{RQ}}=$ Result Qualifier. $\mathrm{U}=$ The analyte was analyzed for but not detected. 
Table 2-4. Near-Facility Air Sampling Results, 2008 (pCi/m³ \pm total analytical uncertainty). (Sheet 40 of 82)

\begin{tabular}{|c|c|c|c|c|c|c|c|c|c|c|}
\hline Location & Sample On & Sample Off & Isotope & Result \pm Uncertainty & Location & Composite & e Period & Isotope & Result \pm Uncertainty & $\mathbf{R Q *}$ \\
\hline N978 & $12 / 31 / 07$ & $01 / 14 / 08$ & gross $\alpha$ & $2.1 \mathrm{E}-03 \pm 6.9 \mathrm{E}-04$ & N978 & $12 / 31 / 07$ to & $06 / 30 / 08$ & ${ }^{60} \mathrm{Co}$ & $7.6 \mathrm{E}-05 \pm 8.1 \mathrm{E}-05$ & $\mathrm{U}$ \\
\hline \multirow[t]{51}{*}{$(200-E)$} & $12 / 31 / 07$ & 01/14/08 & gross $\beta$ & $6.4 \mathrm{E}-03 \pm 1.1 \mathrm{E}-03$ & & & & ${ }^{134} \mathrm{Cs}$ & $-1.8 \mathrm{E}-05 \pm 7.5 \mathrm{E}-05$ & $\mathrm{U}$ \\
\hline & $01 / 14 / 08$ & 01/29/08 & gross $\alpha$ & $1.4 \mathrm{E}-03 \pm 5.7 \mathrm{E}-04$ & & & & ${ }^{137} \mathrm{Cs}$ & $6.0 \mathrm{E}-06 \pm 6.0 \mathrm{E}-05$ & U \\
\hline & 01/14/08 & 01/29/08 & gross $\beta$ & $2.9 \mathrm{E}-02 \pm 2.9 \mathrm{E}-03$ & & & & ${ }^{152} \mathrm{Eu}$ & $-9.6 \mathrm{E}-06 \pm 9.6 \mathrm{E}-05$ & U \\
\hline & 01/29/08 & $02 / 12 / 08$ & gross $\alpha$ & $6.2 \mathrm{E}-04 \pm 5.0 \mathrm{E}-04$ & & & & ${ }^{154} \mathrm{Eu}$ & $-2.2 \mathrm{E}-04 \pm 2.3 \mathrm{E}-04$ & $\mathrm{U}$ \\
\hline & 01/29/08 & 02/12/08 & gross $\beta$ & $4.2 \mathrm{E}-03 \pm 8.9 \mathrm{E}-04$ & & & & ${ }^{155} \mathrm{Eu}$ & $-1.2 \mathrm{E}-04 \pm 1.6 \mathrm{E}-04$ & U \\
\hline & $02 / 12 / 08$ & $02 / 25 / 08$ & gross $\alpha$ & $1.7 \mathrm{E}-03 \pm 6.6 \mathrm{E}-04$ & & & & ${ }^{238} \mathrm{Pu}$ & $1.5 \mathrm{E}-06 \pm 2.8 \mathrm{E}-06$ & $\mathrm{U}$ \\
\hline & $02 / 12 / 08$ & $02 / 25 / 08$ & gross $\beta$ & $3.0 \mathrm{E}-02 \pm 3.1 \mathrm{E}-03$ & & & & ${ }^{239 / 240} \mathrm{Pu}$ & $1.0 \mathrm{E}-06 \pm 2.1 \mathrm{E}-06$ & $\mathrm{U}$ \\
\hline & $02 / 25 / 08$ & 03/11/08 & gross $\alpha$ & $1.5 \mathrm{E}-03 \pm 5.8 \mathrm{E}-04$ & & & & ${ }^{106} \mathrm{Ru}$ & $-2.7 \mathrm{E}-04 \pm 6.6 \mathrm{E}-04$ & $\mathrm{U}$ \\
\hline & $02 / 25 / 08$ & 03/11/08 & gross $\beta$ & $1.7 \mathrm{E}-02 \pm 2.0 \mathrm{E}-03$ & & & & ${ }^{125} \mathrm{Sb}$ & $1.2 \mathrm{E}-05 \pm 1.2 \mathrm{E}-04$ & $\mathrm{U}$ \\
\hline & 03/11/08 & 03/25/08 & gross $\alpha$ & $5.0 \mathrm{E}-04 \pm 4.8 \mathrm{E}-04$ & & & & ${ }^{90} \mathrm{Sr}$ & $-2.6 \mathrm{E}-04 \pm 2.7 \mathrm{E}-04$ & $\mathrm{U}$ \\
\hline & 03/11/08 & $03 / 25 / 08$ & gross $\beta$ & $1.0 \mathrm{E}-02 \pm 1.5 \mathrm{E}-03$ & & & & ${ }^{234} \mathrm{U}$ & $1.2 \mathrm{E}-05 \pm 7.1 \mathrm{E}-06$ & \\
\hline & 03/25/08 & $04 / 07 / 08$ & gross $\alpha$ & $1.9 \mathrm{E}-03 \pm 7.0 \mathrm{E}-04$ & & & & ${ }^{235} \mathrm{U}$ & $7.5 \mathrm{E}-07 \pm 2.6 \mathrm{E}-06$ & $\mathrm{U}$ \\
\hline & 03/25/08 & $04 / 07 / 08$ & gross $\beta$ & $8.4 \mathrm{E}-03 \pm 1.4 \mathrm{E}-03$ & & & & ${ }^{238} \mathrm{U}$ & $6.8 \mathrm{E}-06 \pm 5.0 \mathrm{E}-06$ & \\
\hline & 04/07/08 & $04 / 21 / 08$ & gross $\alpha$ & $7.5 \mathrm{E}-04 \pm 5.5 \mathrm{E}-04$ & & & & & & \\
\hline & $04 / 07 / 08$ & $04 / 21 / 08$ & gross $\beta$ & $8.4 \mathrm{E}-03 \pm 1.3 \mathrm{E}-03$ & N978 & $06 / 30 / 08$ to & $12 / 30 / 08$ & ${ }^{60} \mathrm{Co}$ & $-2.6 \mathrm{E}-05 \pm 1.1 \mathrm{E}-04$ & $\mathrm{U}$ \\
\hline & $04 / 21 / 08$ & 05/07/08 & gross $\alpha$ & $1.7 \mathrm{E}-03 \pm 6.0 \mathrm{E}-04$ & & & & & $-1.9 \mathrm{E}-05 \pm 1.2 \mathrm{E}-04$ & $\mathrm{U}$ \\
\hline & $04 / 21 / 08$ & 05/07/08 & gross $\beta$ & $1.4 \mathrm{E}-02 \pm 1.8 \mathrm{E}-03$ & & & & & $7.1 \mathrm{E}-06 \pm 7.1 \mathrm{E}-05$ & $\mathrm{U}$ \\
\hline & 05/07/08 & 05/19/08 & gross $\alpha$ & $1.1 \mathrm{E}-03 \pm 6.9 \mathrm{E}-04$ & & & & & $1.4 \mathrm{E}-04 \pm 2.9 \mathrm{E}-04$ & $\mathrm{U}$ \\
\hline & 05/07/08 & $05 / 19 / 08$ & gross $\beta$ & $1.1 \mathrm{E}-02 \pm 1.7 \mathrm{E}-03$ & & & & & $-7.3 \mathrm{E}-05 \pm 3.0 \mathrm{E}-04$ & $\mathrm{U}$ \\
\hline & 05/19/08 & $06 / 02 / 08$ & gross $\alpha$ & $6.1 \mathrm{E}-04 \pm 5.1 \mathrm{E}-04$ & & & & & $-1.2 \mathrm{E}-04 \pm 2.0 \mathrm{E}-04$ & $\mathrm{U}$ \\
\hline & 05/19/08 & $06 / 02 / 08$ & gross $\beta$ & $1.0 \mathrm{E}-02 \pm 1.5 \mathrm{E}-03$ & & & & & $-1.3 \mathrm{E}-05 \pm 1.3 \mathrm{E}-05$ & $\mathrm{U}$ \\
\hline & $06 / 02 / 08$ & $06 / 16 / 08$ & gross $\alpha$ & $5.4 \mathrm{E}-04 \pm 5.5 \mathrm{E}-04$ & & & & & $-1.5 \mathrm{E}-06 \pm 3.0 \mathrm{E}-06$ & $\mathrm{U}$ \\
\hline & $06 / 02 / 08$ & 06/16/08 & gross $\beta$ & $7.3 \mathrm{E}-03 \pm 1.2 \mathrm{E}-03$ & & & & & $5.7 \mathrm{E}-04 \pm 9.0 \mathrm{E}-04$ & $\mathrm{U}$ \\
\hline & $06 / 16 / 08$ & 06/30/08 & gross $\alpha$ & $1.5 \mathrm{E}-03 \pm 6.4 \mathrm{E}-04$ & & & & & $2.2 \mathrm{E}-04 \pm 2.6 \mathrm{E}-04$ & $\mathrm{U}$ \\
\hline & $06 / 16 / 08$ & 06/30/08 & gross $\beta$ & $1.6 \mathrm{E}-02 \pm 2.0 \mathrm{E}-03$ & & & & & $-1.8 \mathrm{E}-04 \pm 1.9 \mathrm{E}-04$ & $\mathrm{U}$ \\
\hline & 06/30/08 & $07 / 14 / 08$ & gross $\alpha$ & $2.1 \mathrm{E}-03 \pm 7.1 \mathrm{E}-04$ & & & & & $1.5 \mathrm{E}-05 \pm 8.6 \mathrm{E}-06$ & \\
\hline & $06 / 30 / 08$ & $07 / 14 / 08$ & gross $\beta$ & $1.1 \mathrm{E}-02 \pm 1.6 \mathrm{E}-03$ & & & & & $3.5 \mathrm{E}-06 \pm 3.4 \mathrm{E}-06$ & \\
\hline & 07/14/08 & 07/28/08 & gross $\alpha$ & $1.2 \mathrm{E}-03 \pm 5.2 \mathrm{E}-04$ & & & & & $7.7 \mathrm{E}-06 \pm 5.6 \mathrm{E}-06$ & \\
\hline & $07 / 14 / 08$ & $07 / 28 / 08$ & gross $\beta$ & $1.2 \mathrm{E}-02 \pm 1.6 \mathrm{E}-03$ & & & & & & \\
\hline & 07/28/08 & 08/11/08 & gross $\alpha$ & $1.1 \mathrm{E}-03 \pm 5.0 \mathrm{E}-04$ & & & & & & \\
\hline & $07 / 28 / 08$ & $08 / 11 / 08$ & gross $\beta$ & $1.3 \mathrm{E}-02 \pm 1.7 \mathrm{E}-03$ & & & & & & \\
\hline & $08 / 11 / 08$ & $08 / 25 / 08$ & gross $\alpha$ & $7.4 \mathrm{E}-04 \pm 5.3 \mathrm{E}-04$ & & & & & & \\
\hline & 08/11/08 & 08/25/08 & gross $\beta$ & $1.2 \mathrm{E}-02 \pm 1.6 \mathrm{E}-03$ & & & & & & \\
\hline & $08 / 25 / 08$ & 09/08/08 & gross $\alpha$ & $2.9 \mathrm{E}-04 \pm 4.1 \mathrm{E}-04$ & & & & & & \\
\hline & $08 / 25 / 08$ & 09/08/08 & gross $\beta$ & $1.1 \mathrm{E}-02 \pm 1.5 \mathrm{E}-03$ & & & & & & \\
\hline & $09 / 08 / 08$ & $09 / 22 / 08$ & gross $\alpha$ & $2.1 \mathrm{E}-03 \pm 6.9 \mathrm{E}-04$ & & & & & & \\
\hline & 09/08/08 & $09 / 22 / 08$ & gross $\beta$ & $2.3 \mathrm{E}-02 \pm 2.5 \mathrm{E}-03$ & & & & & & \\
\hline & $09 / 22 / 08$ & $10 / 07 / 08$ & gross $\alpha$ & $1.5 \mathrm{E}-03 \pm 5.6 \mathrm{E}-04$ & & & & & & \\
\hline & $09 / 22 / 08$ & $10 / 07 / 08$ & gross $\beta$ & $2.3 \mathrm{E}-02 \pm 2.6 \mathrm{E}-03$ & & & & & & \\
\hline & $10 / 07 / 08$ & $10 / 20 / 08$ & gross $\alpha$ & $6.7 \mathrm{E}-04 \pm 5.5 \mathrm{E}-04$ & & & & & & \\
\hline & $10 / 07 / 08$ & $10 / 20 / 08$ & gross $\beta$ & $1.5 \mathrm{E}-02 \pm 2.0 \mathrm{E}-03$ & & & & & & \\
\hline & $10 / 20 / 08$ & $11 / 03 / 08$ & gross $\alpha$ & $2.6 \mathrm{E}-03 \pm 8.2 \mathrm{E}-04$ & & & & & & \\
\hline & $10 / 20 / 08$ & $11 / 03 / 08$ & gross $\beta$ & $3.9 \mathrm{E}-02 \pm 3.8 \mathrm{E}-03$ & & & & & & \\
\hline & $11 / 03 / 08$ & $11 / 17 / 08$ & gross $\alpha$ & $1.4 \mathrm{E}-03 \pm 5.8 \mathrm{E}-04$ & & & & & & \\
\hline & $11 / 03 / 08$ & $11 / 17 / 08$ & gross $\beta$ & $1.4 \mathrm{E}-02 \pm 1.8 \mathrm{E}-03$ & & & & & & \\
\hline & $11 / 17 / 08$ & $12 / 01 / 08$ & gross $\alpha$ & $1.9 \mathrm{E}-03 \pm 6.8 \mathrm{E}-04$ & & & & & & \\
\hline & $11 / 17 / 08$ & $12 / 01 / 08$ & gross $\beta$ & $3.3 \mathrm{E}-02 \pm 3.4 \mathrm{E}-03$ & & & & & & \\
\hline & $12 / 01 / 08$ & $12 / 15 / 08$ & gross $\alpha$ & $1.1 \mathrm{E}-03 \pm 5.1 \mathrm{E}-04$ & & & & & & \\
\hline & $12 / 01 / 08$ & $12 / 15 / 08$ & gross $\beta$ & $1.4 \mathrm{E}-02 \pm 1.8 \mathrm{E}-03$ & & & & & & \\
\hline & $12 / 15 / 08$ & $12 / 30 / 08$ & gross $\alpha$ & $2.6 \mathrm{E}-03 \pm 8.9 \mathrm{E}-04$ & & & & & & \\
\hline & $12 / 15 / 08$ & $12 / 30 / 08$ & gross $\beta$ & $5.1 \mathrm{E}-02 \pm 4.8 \mathrm{E}-03$ & & & & & & \\
\hline
\end{tabular}

$\overline{\mathrm{RQ}}=$ Result Qualifier. $\mathrm{U}=$ The analyte was analyzed for but not detected. 
Table 2-4. Near-Facility Air Sampling Results, 2008 (pCi/m³ \pm total analytical uncertainty). (Sheet 41 of 82)

\begin{tabular}{|c|c|c|c|c|c|c|c|c|c|c|}
\hline Location & Sample On & Sample Off & Isotope & Result \pm Uncertainty & Location & Composite & e Period & Isotope & Result \pm Uncertainty & $\mathbf{R Q *}$ \\
\hline N984 & $12 / 31 / 07$ & $01 / 14 / 08$ & gross $\alpha$ & $9.3 \mathrm{E}-04 \pm 5.7 \mathrm{E}-04$ & N984 & $12 / 31 / 07$ to & $06 / 30 / 08$ & ${ }^{60} \mathrm{Co}$ & $-5.7 \mathrm{E}-05 \pm 6.9 \mathrm{E}-05$ & $\mathrm{U}$ \\
\hline \multirow[t]{51}{*}{$(200-E)$} & $12 / 31 / 07$ & 01/14/08 & gross $\beta$ & $9.8 \mathrm{E}-03 \pm 1.5 \mathrm{E}-03$ & & & & ${ }^{134} \mathrm{Cs}$ & $5.5 \mathrm{E}-05 \pm 6.4 \mathrm{E}-05$ & $\mathrm{U}$ \\
\hline & $01 / 14 / 08$ & 01/29/08 & gross $\alpha$ & $2.0 \mathrm{E}-03 \pm 6.7 \mathrm{E}-04$ & & & & ${ }^{137} \mathrm{Cs}$ & $1.5 \mathrm{E}-04 \pm 9.7 \mathrm{E}-05$ & \\
\hline & $01 / 14 / 08$ & 01/29/08 & gross $\beta$ & $3.1 \mathrm{E}-02 \pm 3.1 \mathrm{E}-03$ & & & & ${ }^{152} \mathrm{Eu}$ & $7.4 \mathrm{E}-06 \pm 7.4 \mathrm{E}-05$ & $\mathrm{U}$ \\
\hline & $01 / 29 / 08$ & $02 / 12 / 08$ & gross $\alpha$ & $2.8 \mathrm{E}-04 \pm 4.0 \mathrm{E}-04$ & & & & ${ }^{154} \mathrm{Eu}$ & $1.2 \mathrm{E}-04 \pm 1.9 \mathrm{E}-04$ & $\mathrm{U}$ \\
\hline & $01 / 29 / 08$ & $02 / 12 / 08$ & gross $\beta$ & $3.6 \mathrm{E}-03 \pm 8.2 \mathrm{E}-04$ & & & & ${ }^{155} \mathrm{Eu}$ & $-2.8 \mathrm{E}-05 \pm 1.5 \mathrm{E}-04$ & U \\
\hline & $02 / 12 / 08$ & $02 / 25 / 08$ & gross $\alpha$ & $2.6 \mathrm{E}-03 \pm 8.1 \mathrm{E}-04$ & & & & ${ }^{238} \mathrm{Pu}$ & $1.8 \mathrm{E}-05 \pm 1.4 \mathrm{E}-05$ & $\mathrm{U}$ \\
\hline & $02 / 12 / 08$ & $02 / 25 / 08$ & gross $\beta$ & $2.7 \mathrm{E}-02 \pm 2.9 \mathrm{E}-03$ & & & & ${ }^{239 / 240} \mathrm{Pu}$ & $2.3 \mathrm{E}-06 \pm 4.8 \mathrm{E}-06$ & $\mathrm{U}$ \\
\hline & $02 / 25 / 08$ & 03/11/08 & gross $\alpha$ & $1.4 \mathrm{E}-03 \pm 5.4 \mathrm{E}-04$ & & & & ${ }^{103} \mathrm{Ru}$ & $1.3 \mathrm{E}-05 \pm 6.0 \mathrm{E}-05$ & $\mathrm{U}$ \\
\hline & $02 / 25 / 08$ & 03/11/08 & gross $\beta$ & $1.6 \mathrm{E}-02 \pm 1.9 \mathrm{E}-03$ & & & & ${ }^{106} \mathrm{Ru}$ & $-1.3 \mathrm{E}-04 \pm 5.3 \mathrm{E}-04$ & $\mathrm{U}$ \\
\hline & 03/11/08 & $03 / 25 / 08$ & gross $\alpha$ & $5.0 \mathrm{E}-04 \pm 4.7 \mathrm{E}-04$ & & & & ${ }^{125} \mathrm{Sb}$ & $4.6 \mathrm{E}-05 \pm 1.3 \mathrm{E}-04$ & $\mathrm{U}$ \\
\hline & 03/11/08 & $03 / 25 / 08$ & gross $\beta$ & $9.8 \mathrm{E}-03 \pm 1.5 \mathrm{E}-03$ & & & & ${ }^{113} \mathrm{Sn}$ & $5.2 \mathrm{E}-05 \pm 6.7 \mathrm{E}-05$ & $\mathrm{U}$ \\
\hline & $03 / 25 / 08$ & $04 / 07 / 08$ & gross $\alpha$ & $6.7 \mathrm{E}-04 \pm 5.4 \mathrm{E}-04$ & & & & ${ }^{90} \mathrm{Sr}$ & $-1.3 \mathrm{E}-04 \pm 1.3 \mathrm{E}-04$ & $\mathrm{U}$ \\
\hline & 03/25/08 & $04 / 07 / 08$ & gross $\beta$ & $7.1 \mathrm{E}-03 \pm 1.2 \mathrm{E}-03$ & & & & ${ }^{234} \mathrm{U}$ & $2.0 \mathrm{E}-05 \pm 1.1 \mathrm{E}-05$ & \\
\hline & $04 / 07 / 08$ & $04 / 21 / 08$ & gross $\alpha$ & $1.1 \mathrm{E}-03 \pm 5.1 \mathrm{E}-04$ & & & & ${ }^{235} \mathrm{U}$ & $4.7 \mathrm{E}-06 \pm 4.3 \mathrm{E}-06$ & \\
\hline & $04 / 07 / 08$ & $04 / 21 / 08$ & gross $\beta$ & $8.6 \mathrm{E}-03 \pm 1.3 \mathrm{E}-03$ & & & & ${ }^{238} \mathrm{U}$ & $2.0 \mathrm{E}-05 \pm 1.1 \mathrm{E}-05$ & \\
\hline & $04 / 21 / 08$ & 05/06/08 & gross $\alpha$ & $5.7 \mathrm{E}-04 \pm 4.7 \mathrm{E}-04$ & & & & ${ }^{65} \mathrm{Zn}$ & $-1.0 \mathrm{E}-04 \pm 1.3 \mathrm{E}-04$ & $\mathrm{U}$ \\
\hline & $04 / 21 / 08$ & 05/06/08 & gross $\beta$ & $1.5 \mathrm{E}-02 \pm 1.9 \mathrm{E}-03$ & & & & & & \\
\hline & 05/06/08 & 05/19/08 & gross $\alpha$ & $6.6 \mathrm{E}-04 \pm 5.4 \mathrm{E}-04$ & N984 & $06 / 30 / 08$ to & $12 / 30 / 08$ & ${ }^{60} \mathrm{Co}$ & $1.8 \mathrm{E}-05 \pm 7.5 \mathrm{E}-05$ & U \\
\hline & 05/06/08 & $05 / 19 / 08$ & gross $\beta$ & $1.4 \mathrm{E}-02 \pm 1.9 \mathrm{E}-03$ & & & & ${ }^{134} \mathrm{Cs}$ & $8.5 \mathrm{E}-06 \pm 6.8 \mathrm{E}-05$ & $\mathrm{U}$ \\
\hline & 05/19/08 & 06/02/08 & gross $\alpha$ & $9.5 \mathrm{E}-04 \pm 5.9 \mathrm{E}-04$ & & & & ${ }^{137} \mathrm{Cs}$ & $1.6 \mathrm{E}-04 \pm 1.1 \mathrm{E}-04$ & \\
\hline & 05/19/08 & $06 / 02 / 08$ & gross $\beta$ & $1.2 \mathrm{E}-02 \pm 1.7 \mathrm{E}-03$ & & & & ${ }^{152} \mathrm{Eu}$ & $-6.1 \mathrm{E}-05 \pm 1.6 \mathrm{E}-04$ & $\mathrm{U}$ \\
\hline & $06 / 02 / 08$ & $06 / 16 / 08$ & gross $\alpha$ & $1.2 \mathrm{E}-03 \pm 5.7 \mathrm{E}-04$ & & & & ${ }^{154} \mathrm{Eu}$ & $9.1 \mathrm{E}-06 \pm 9.1 \mathrm{E}-05$ & $\mathrm{U}$ \\
\hline & $06 / 02 / 08$ & 06/16/08 & gross $\beta$ & $1.1 \mathrm{E}-02 \pm 1.6 \mathrm{E}-03$ & & & & ${ }^{155} \mathrm{Eu}$ & $5.1 \mathrm{E}-05 \pm 1.6 \mathrm{E}-04$ & $\mathrm{U}$ \\
\hline & $06 / 16 / 08$ & 06/30/08 & gross $\alpha$ & $2.1 \mathrm{E}-03 \pm 7.7 \mathrm{E}-04$ & & & & ${ }^{238} \mathrm{Pu}$ & $-7.0 \mathrm{E}-07 \pm 7.0 \mathrm{E}-06$ & $\mathrm{U}$ \\
\hline & $06 / 16 / 08$ & $06 / 30 / 08$ & gross $\beta$ & $1.4 \mathrm{E}-02 \pm 1.8 \mathrm{E}-03$ & & & & ${ }^{239 / 240} \mathrm{Pu}$ & $2.8 \mathrm{E}-06 \pm 3.0 \mathrm{E}-06$ & \\
\hline & $06 / 30 / 08$ & $07 / 14 / 08$ & gross $\alpha$ & $1.5 \mathrm{E}-03 \pm 6.0 \mathrm{E}-04$ & & & & ${ }^{103} \mathrm{Ru}$ & $-3.9 \mathrm{E}-05 \pm 6.4 \mathrm{E}-05$ & $\mathrm{U}$ \\
\hline & $06 / 30 / 08$ & $07 / 14 / 08$ & gross $\beta$ & $1.3 \mathrm{E}-02 \pm 1.7 \mathrm{E}-03$ & & & & ${ }^{106} \mathrm{Ru}$ & $4.5 \mathrm{E}-04 \pm 5.9 \mathrm{E}-04$ & $\mathrm{U}$ \\
\hline & 07/14/08 & 07/28/08 & gross $\alpha$ & $2.2 \mathrm{E}-03 \pm 7.1 \mathrm{E}-04$ & & & & ${ }^{125} \mathrm{Sb}$ & $-7.6 \mathrm{E}-05 \pm 1.4 \mathrm{E}-04$ & U \\
\hline & $07 / 14 / 08$ & $07 / 28 / 08$ & gross $\beta$ & $1.1 \mathrm{E}-02 \pm 1.5 \mathrm{E}-03$ & & & & ${ }^{113} \mathrm{Sn}$ & $3.6 \mathrm{E}-06 \pm 3.6 \mathrm{E}-05$ & $\mathrm{U}$ \\
\hline & $07 / 28 / 08$ & 08/11/08 & gross $\alpha$ & $7.4 \mathrm{E}-04 \pm 5.3 \mathrm{E}-04$ & & & & ${ }^{90} \mathrm{Sr}$ & $-2.3 \mathrm{E}-04 \pm 2.4 \mathrm{E}-04$ & $\mathrm{U}$ \\
\hline & $07 / 28 / 08$ & 08/11/08 & gross $\beta$ & $1.7 \mathrm{E}-02 \pm 2.1 \mathrm{E}-03$ & & & & ${ }^{234} \mathrm{U}$ & $1.3 \mathrm{E}-05 \pm 7.8 \mathrm{E}-06$ & \\
\hline & 08/11/08 & $08 / 25 / 08$ & gross $\alpha$ & $8.5 \mathrm{E}-04 \pm 5.6 \mathrm{E}-04$ & & & & ${ }^{235} \mathrm{U}$ & $2.9 \mathrm{E}-06 \pm 3.0 \mathrm{E}-06$ & \\
\hline & $08 / 11 / 08$ & $08 / 25 / 08$ & gross $\beta$ & $1.3 \mathrm{E}-02 \pm 1.7 \mathrm{E}-03$ & & & & ${ }^{238} \mathrm{U}$ & $1.5 \mathrm{E}-05 \pm 8.3 \mathrm{E}-06$ & \\
\hline & $08 / 25 / 08$ & 09/08/08 & gross $\alpha$ & $9.4 \mathrm{E}-04 \pm 5.8 \mathrm{E}-04$ & & & & ${ }^{65} \mathrm{Zn}$ & $2.4 \mathrm{E}-06 \pm 2.4 \mathrm{E}-05$ & $\mathrm{U}$ \\
\hline & $08 / 25 / 08$ & 09/08/08 & gross $\beta$ & $1.0 \mathrm{E}-02 \pm 1.5 \mathrm{E}-03$ & & & & & & \\
\hline & 09/08/08 & 09/22/08 & gross $\alpha$ & $8.5 \mathrm{E}-04 \pm 5.6 \mathrm{E}-04$ & & & & & & \\
\hline & 09/08/08 & $09 / 22 / 08$ & gross $\beta$ & $2.5 \mathrm{E}-02 \pm 2.7 \mathrm{E}-03$ & & & & & & \\
\hline & $09 / 22 / 08$ & $10 / 07 / 08$ & gross $\alpha$ & $1.4 \mathrm{E}-03 \pm 5.3 \mathrm{E}-04$ & & & & & & \\
\hline & $09 / 22 / 08$ & $10 / 07 / 08$ & gross $\beta$ & $2.7 \mathrm{E}-02 \pm 2.8 \mathrm{E}-03$ & & & & & & \\
\hline & $10 / 10 / 08$ & $10 / 20 / 08$ & gross $\alpha$ & $1.2 \mathrm{E}-03 \pm 8.0 \mathrm{E}-04$ & & & & & & \\
\hline & $10 / 10 / 08$ & $10 / 20 / 08$ & gross $\beta$ & $1.9 \mathrm{E}-02 \pm 2.5 \mathrm{E}-03$ & & & & & & \\
\hline & $10 / 20 / 08$ & $11 / 03 / 08$ & gross $\alpha$ & $2.9 \mathrm{E}-03 \pm 8.2 \mathrm{E}-04$ & & & & & & \\
\hline & $10 / 20 / 08$ & $11 / 03 / 08$ & gross $\beta$ & $4.0 \mathrm{E}-02 \pm 3.8 \mathrm{E}-03$ & & & & & & \\
\hline & $11 / 03 / 08$ & $11 / 17 / 08$ & gross $\alpha$ & $1.4 \mathrm{E}-03 \pm 5.9 \mathrm{E}-04$ & & & & & & \\
\hline & $11 / 03 / 08$ & $11 / 17 / 08$ & gross $\beta$ & $1.6 \mathrm{E}-02 \pm 2.0 \mathrm{E}-03$ & & & & & & \\
\hline & $11 / 17 / 08$ & $12 / 01 / 08$ & gross $\alpha$ & $1.9 \mathrm{E}-03 \pm 7.1 \mathrm{E}-04$ & & & & & & \\
\hline & $11 / 17 / 08$ & $12 / 01 / 08$ & gross $\beta$ & $4.1 \mathrm{E}-02 \pm 3.9 \mathrm{E}-03$ & & & & & & \\
\hline & $12 / 01 / 08$ & $12 / 15 / 08$ & gross $\alpha$ & $1.8 \mathrm{E}-03 \pm 6.6 \mathrm{E}-04$ & & & & & & \\
\hline & $12 / 01 / 08$ & $12 / 15 / 08$ & gross $\beta$ & $2.2 \mathrm{E}-02 \pm 2.4 \mathrm{E}-03$ & & & & & & \\
\hline & $12 / 15 / 08$ & $12 / 30 / 08$ & gross $\alpha$ & $1.6 \mathrm{E}-03 \pm 6.0 \mathrm{E}-04$ & & & & & & \\
\hline & $12 / 15 / 08$ & $12 / 30 / 08$ & gross $\beta$ & $3.5 \mathrm{E}-02 \pm 3.4 \mathrm{E}-03$ & & & & & & \\
\hline
\end{tabular}

$\overline{\mathrm{RQ}}=$ Result Qualifier. $\mathrm{U}=$ The analyte was analyzed for but not detected. 
Table 2-4. Near-Facility Air Sampling Results, 2008 (pCi/m³ \pm total analytical uncertainty). (Sheet 42 of 82)

\begin{tabular}{|c|c|c|c|c|c|c|c|c|c|}
\hline Location & Sample On & Sample Off & Isotope & Result \pm Uncertainty & Location & Composite Period & Isotope & Result \pm Uncertainty & $\mathbf{R Q *}$ \\
\hline N985 & $12 / 31 / 07$ & $01 / 14 / 08$ & gross $\alpha$ & $1.3 \mathrm{E}-03 \pm 5.3 \mathrm{E}-04$ & N985 & $12 / 31 / 07$ to $06 / 30 / 08$ & ${ }^{60} \mathrm{Co}$ & $8.3 \mathrm{E}-05 \pm 8.5 \mathrm{E}-05$ & $\mathrm{U}$ \\
\hline \multirow[t]{51}{*}{$(200-E)$} & $12 / 31 / 07$ & 01/14/08 & gross $\beta$ & $9.7 \mathrm{E}-03 \pm 1.4 \mathrm{E}-03$ & & & ${ }^{134} \mathrm{Cs}$ & $-4.9 \mathrm{E}-05 \pm 7.9 \mathrm{E}-05$ & $\mathrm{U}$ \\
\hline & $01 / 14 / 08$ & 01/29/08 & gross $\alpha$ & $1.7 \mathrm{E}-03 \pm 6.4 \mathrm{E}-04$ & & & ${ }^{137} \mathrm{Cs}$ & $4.4 \mathrm{E}-06 \pm 4.4 \mathrm{E}-05$ & U \\
\hline & $01 / 14 / 08$ & 01/29/08 & gross $\beta$ & $2.9 \mathrm{E}-02 \pm 3.0 \mathrm{E}-03$ & & & ${ }^{152} \mathrm{Eu}$ & $3.1 \mathrm{E}-05 \pm 1.8 \mathrm{E}-04$ & U \\
\hline & 01/29/08 & $02 / 12 / 08$ & gross $\alpha$ & $5.7 \mathrm{E}-05 \pm 3.0 \mathrm{E}-04$ & & & ${ }^{154} \mathrm{Eu}$ & $3.3 \mathrm{E}-05 \pm 2.0 \mathrm{E}-04$ & $\mathrm{U}$ \\
\hline & $01 / 29 / 08$ & $02 / 12 / 08$ & gross $\beta$ & $2.8 \mathrm{E}-03 \pm 7.4 \mathrm{E}-04$ & & & ${ }^{155} \mathrm{Eu}$ & $-6.6 \mathrm{E}-06 \pm 6.6 \mathrm{E}-05$ & U \\
\hline & $02 / 12 / 08$ & $02 / 25 / 08$ & gross $\alpha$ & $9.0 \mathrm{E}-04 \pm 6.1 \mathrm{E}-04$ & & & ${ }^{238} \mathrm{Pu}$ & $8.6 \mathrm{E}-07 \pm 8.6 \mathrm{E}-06$ & $\mathrm{U}$ \\
\hline & $02 / 12 / 08$ & $02 / 25 / 08$ & gross $\beta$ & $3.1 \mathrm{E}-02 \pm 3.2 \mathrm{E}-03$ & & & ${ }^{239 / 240} \mathrm{Pu}$ & $-8.5 \mathrm{E}-07 \pm 2.9 \mathrm{E}-06$ & $\mathrm{U}$ \\
\hline & $02 / 25 / 08$ & 03/11/08 & gross $\alpha$ & $1.4 \mathrm{E}-03 \pm 5.5 \mathrm{E}-04$ & & & ${ }^{106} \mathrm{Ru}$ & $5.7 \mathrm{E}-05 \pm 5.7 \mathrm{E}-04$ & $\mathrm{U}$ \\
\hline & $02 / 25 / 08$ & 03/11/08 & gross $\beta$ & $1.3 \mathrm{E}-02 \pm 1.7 \mathrm{E}-03$ & & & ${ }^{125} \mathrm{Sb}$ & $2.3 \mathrm{E}-05 \pm 1.6 \mathrm{E}-04$ & U \\
\hline & 03/11/08 & $03 / 25 / 08$ & gross $\alpha$ & $1.3 \mathrm{E}-03 \pm 5.6 \mathrm{E}-04$ & & & ${ }^{90} \mathrm{Sr}$ & $-1.9 \mathrm{E}-04 \pm 2.0 \mathrm{E}-04$ & $\mathrm{U}$ \\
\hline & 03/11/08 & $03 / 25 / 08$ & gross $\beta$ & $1.5 \mathrm{E}-02 \pm 1.9 \mathrm{E}-03$ & & & ${ }^{234} \mathrm{U}$ & $7.5 \mathrm{E}-06 \pm 5.9 \mathrm{E}-06$ & \\
\hline & 03/25/08 & $04 / 07 / 08$ & gross $\alpha$ & $5.6 \mathrm{E}-04 \pm 5.2 \mathrm{E}-04$ & & & ${ }^{235} \mathrm{U}$ & $2.4 \mathrm{E}-06 \pm 3.0 \mathrm{E}-06$ & \\
\hline & 03/25/08 & $04 / 07 / 08$ & gross $\beta$ & $1.7 \mathrm{E}-02 \pm 2.1 \mathrm{E}-03$ & & & ${ }^{238} \mathrm{U}$ & $1.7 \mathrm{E}-05 \pm 9.6 \mathrm{E}-06$ & \\
\hline & 04/07/08 & $04 / 21 / 08$ & gross $\alpha$ & $1.4 \mathrm{E}-03 \pm 5.8 \mathrm{E}-04$ & & & & & \\
\hline & $04 / 07 / 08$ & $04 / 21 / 08$ & gross $\beta$ & $1.1 \mathrm{E}-02 \pm 1.6 \mathrm{E}-03$ & N985 & $06 / 30 / 08$ to $12 / 30 / 08$ & ${ }^{60} \mathrm{Co}$ & $-3.5 \mathrm{E}-05 \pm 7.9 \mathrm{E}-05$ & $\mathrm{U}$ \\
\hline & $04 / 21 / 08$ & 05/06/08 & gross $\alpha$ & $1.8 \mathrm{E}-03 \pm 6.2 \mathrm{E}-04$ & & & ${ }^{134} \mathrm{Cs}$ & $9.7 \mathrm{E}-06 \pm 8.0 \mathrm{E}-05$ & $\mathrm{U}$ \\
\hline & $04 / 21 / 08$ & 05/06/08 & gross $\beta$ & $1.6 \mathrm{E}-02 \pm 1.9 \mathrm{E}-03$ & & & ${ }^{137} \mathrm{Cs}$ & $4.9 \mathrm{E}-05 \pm 7.3 \mathrm{E}-05$ & $\mathrm{U}$ \\
\hline & 05/06/08 & 05/19/08 & gross $\alpha$ & $1.3 \mathrm{E}-03 \pm 5.8 \mathrm{E}-04$ & & & ${ }^{152} \mathrm{Eu}$ & $-5.9 \mathrm{E}-05 \pm 1.7 \mathrm{E}-04$ & U \\
\hline & 05/06/08 & 05/19/08 & gross $\beta$ & $1.4 \mathrm{E}-02 \pm 1.9 \mathrm{E}-03$ & & & ${ }^{154} \mathrm{Eu}$ & $8.7 \mathrm{E}-05 \pm 2.0 \mathrm{E}-04$ & $\mathrm{U}$ \\
\hline & 05/19/08 & 06/02/08 & gross $\alpha$ & $1.3 \mathrm{E}-03 \pm 5.6 \mathrm{E}-04$ & & & ${ }^{155} \mathrm{Eu}$ & $-1.1 \mathrm{E}-04 \pm 1.6 \mathrm{E}-04$ & U \\
\hline & 05/19/08 & $06 / 02 / 08$ & gross $\beta$ & $1.0 \mathrm{E}-02 \pm 1.5 \mathrm{E}-03$ & & & ${ }^{238} \mathrm{Pu}$ & $-9.1 \mathrm{E}-07 \pm 9.1 \mathrm{E}-06$ & $\mathrm{U}$ \\
\hline & $06 / 02 / 08$ & $06 / 16 / 08$ & gross $\alpha$ & $1.4 \mathrm{E}-03 \pm 6.3 \mathrm{E}-04$ & & & ${ }^{239 / 240} \mathrm{Pu}$ & $9.1 \mathrm{E}-07 \pm 4.8 \mathrm{E}-06$ & $\mathrm{U}$ \\
\hline & $06 / 02 / 08$ & 06/16/08 & gross $\beta$ & $7.6 \mathrm{E}-03 \pm 1.3 \mathrm{E}-03$ & & & ${ }^{103} \mathrm{Ru}$ & $-3.4 \mathrm{E}-05 \pm 6.2 \mathrm{E}-05$ & $\mathrm{U}$ \\
\hline & 06/16/08 & 06/30/08 & gross $\alpha$ & $1.6 \mathrm{E}-03 \pm 6.9 \mathrm{E}-04$ & & & ${ }^{106} \mathrm{Ru}$ & $3.7 \mathrm{E}-04 \pm 5.8 \mathrm{E}-04$ & $\mathrm{U}$ \\
\hline & $06 / 16 / 08$ & 06/30/08 & gross $\beta$ & $1.2 \mathrm{E}-02 \pm 1.7 \mathrm{E}-03$ & & & ${ }^{125} \mathrm{Sb}$ & $-8.6 \mathrm{E}-06 \pm 8.6 \mathrm{E}-05$ & $\mathrm{U}$ \\
\hline & $06 / 30 / 08$ & $07 / 14 / 08$ & gross $\alpha$ & $1.3 \mathrm{E}-03 \pm 5.6 \mathrm{E}-04$ & & & ${ }^{113} \mathrm{Sn}$ & $1.7 \mathrm{E}-05 \pm 6.7 \mathrm{E}-05$ & $\mathrm{U}$ \\
\hline & $06 / 30 / 08$ & $07 / 14 / 08$ & gross $\beta$ & $1.2 \mathrm{E}-02 \pm 1.6 \mathrm{E}-03$ & & & ${ }^{90} \mathrm{Sr}$ & $-3.7 \mathrm{E}-04 \pm 3.8 \mathrm{E}-04$ & $\mathrm{U}$ \\
\hline & 07/14/08 & 07/28/08 & gross $\alpha$ & $1.5 \mathrm{E}-03 \pm 6.0 \mathrm{E}-04$ & & & ${ }^{234} \mathrm{U}$ & $1.9 \mathrm{E}-05 \pm 1.0 \mathrm{E}-05$ & \\
\hline & $07 / 14 / 08$ & $07 / 28 / 08$ & gross $\beta$ & $1.5 \mathrm{E}-02 \pm 1.9 \mathrm{E}-03$ & & & ${ }^{235} \mathrm{U}$ & $2.3 \mathrm{E}-06 \pm 3.5 \mathrm{E}-06$ & $\mathrm{U}$ \\
\hline & $07 / 28 / 08$ & 08/11/08 & gross $\alpha$ & $1.8 \mathrm{E}-03 \pm 6.7 \mathrm{E}-04$ & & & ${ }^{238} \mathrm{U}$ & $1.3 \mathrm{E}-05 \pm 8.1 \mathrm{E}-06$ & \\
\hline & $07 / 28 / 08$ & 08/11/08 & gross $\beta$ & $1.3 \mathrm{E}-02 \pm 1.7 \mathrm{E}-03$ & & & ${ }^{65} \mathrm{Zn}$ & $-2.1 \mathrm{E}-04 \pm 2.2 \mathrm{E}-04$ & $\mathrm{U}$ \\
\hline & $08 / 11 / 08$ & $08 / 25 / 08$ & gross $\alpha$ & $1.2 \mathrm{E}-03 \pm 9.8 \mathrm{E}-04$ & & & & & \\
\hline & 08/11/08 & 08/25/08 & gross $\beta$ & $2.2 \mathrm{E}-02 \pm 3.1 \mathrm{E}-03$ & & & & & \\
\hline & $08 / 25 / 08$ & 09/08/08 & gross $\alpha$ & $5.0 \mathrm{E}-04 \pm 6.7 \mathrm{E}-04$ & & & & & \\
\hline & $08 / 25 / 08$ & 09/08/08 & gross $\beta$ & $1.3 \mathrm{E}-02 \pm 2.1 \mathrm{E}-03$ & & & & & \\
\hline & 09/08/08 & 09/22/08 & gross $\alpha$ & $1.4 \mathrm{E}-03 \pm 5.6 \mathrm{E}-04$ & & & & & \\
\hline & 09/08/08 & $09 / 22 / 08$ & gross $\beta$ & $2.4 \mathrm{E}-02 \pm 2.6 \mathrm{E}-03$ & & & & & \\
\hline & $09 / 22 / 08$ & $10 / 07 / 08$ & gross $\alpha$ & $1.8 \mathrm{E}-03 \pm 6.1 \mathrm{E}-04$ & & & & & \\
\hline & $09 / 22 / 08$ & $10 / 07 / 08$ & gross $\beta$ & $2.5 \mathrm{E}-02 \pm 2.7 \mathrm{E}-03$ & & & & & \\
\hline & $10 / 07 / 08$ & $10 / 20 / 08$ & gross $\alpha$ & $1.2 \mathrm{E}-03 \pm 5.4 \mathrm{E}-04$ & & & & & \\
\hline & $10 / 07 / 08$ & $10 / 20 / 08$ & gross $\beta$ & $1.8 \mathrm{E}-02 \pm 2.1 \mathrm{E}-03$ & & & & & \\
\hline & $10 / 20 / 08$ & $11 / 03 / 08$ & gross $\alpha$ & $1.2 \mathrm{E}-03 \pm 5.4 \mathrm{E}-04$ & & & & & \\
\hline & $10 / 20 / 08$ & $11 / 03 / 08$ & gross $\beta$ & $4.0 \mathrm{E}-02 \pm 3.9 \mathrm{E}-03$ & & & & & \\
\hline & $11 / 03 / 08$ & $11 / 17 / 08$ & gross $\alpha$ & $1.2 \mathrm{E}-03 \pm 5.4 \mathrm{E}-04$ & & & & & \\
\hline & $11 / 03 / 08$ & $11 / 17 / 08$ & gross $\beta$ & $1.5 \mathrm{E}-02 \pm 1.9 \mathrm{E}-03$ & & & & & \\
\hline & $11 / 17 / 08$ & $12 / 01 / 08$ & gross $\alpha$ & $2.1 \mathrm{E}-03 \pm 7.3 \mathrm{E}-04$ & & & & & \\
\hline & $11 / 17 / 08$ & $12 / 01 / 08$ & gross $\beta$ & $4.1 \mathrm{E}-02 \pm 4.0 \mathrm{E}-03$ & & & & & \\
\hline & $12 / 01 / 08$ & $12 / 15 / 08$ & gross $\alpha$ & $2.1 \mathrm{E}-03 \pm 7.1 \mathrm{E}-04$ & & & & & \\
\hline & $12 / 01 / 08$ & $12 / 15 / 08$ & gross $\beta$ & $2.1 \mathrm{E}-02 \pm 2.4 \mathrm{E}-03$ & & & & & \\
\hline & $12 / 15 / 08$ & $12 / 30 / 08$ & gross $\alpha$ & $1.5 \mathrm{E}-03 \pm 5.7 \mathrm{E}-04$ & & & & & \\
\hline & $12 / 15 / 08$ & $12 / 30 / 08$ & gross $\beta$ & $3.9 \mathrm{E}-02 \pm 3.6 \mathrm{E}-03$ & & & & & \\
\hline
\end{tabular}

$\overline{\mathrm{RQ}}=$ Result Qualifier. $\mathrm{U}=$ The analyte was analyzed for but not detected. 
Table 2-4. Near-Facility Air Sampling Results, 2008 (pCi/m³ \pm total analytical uncertainty). (Sheet 43 of 82)

\begin{tabular}{|c|c|c|c|c|c|c|c|c|c|}
\hline Location & Sample On & Sample Off & Isotope & Result \pm Uncertainty & Location & Composite Period & Isotope & Result \pm Uncertainty & $\mathbf{R Q *}$ \\
\hline N999 & $12 / 31 / 07$ & $01 / 14 / 08$ & gross $\alpha$ & $9.5 \mathrm{E}-04 \pm 5.9 \mathrm{E}-04$ & N999 & $12 / 31 / 07$ to $06 / 30 / 08$ & ${ }^{60} \mathrm{Co}$ & $6.4 \mathrm{E}-05 \pm 9.0 \mathrm{E}-05$ & $\mathrm{U}$ \\
\hline \multirow[t]{51}{*}{$(200-E)$} & $12 / 31 / 07$ & 01/14/08 & gross $\beta$ & $1.4 \mathrm{E}-02 \pm 1.8 \mathrm{E}-03$ & & & ${ }^{134} \mathrm{Cs}$ & $3.3 \mathrm{E}-06 \pm 3.3 \mathrm{E}-05$ & $\mathrm{U}$ \\
\hline & $01 / 14 / 08$ & 01/29/08 & gross $\alpha$ & $1.1 \mathrm{E}-03 \pm 4.9 \mathrm{E}-04$ & & & ${ }^{137} \mathrm{Cs}$ & $4.6 \mathrm{E}-05 \pm 1.1 \mathrm{E}-04$ & U \\
\hline & $01 / 14 / 08$ & 01/29/08 & gross $\beta$ & $3.2 \mathrm{E}-02 \pm 3.2 \mathrm{E}-03$ & & & ${ }^{152} \mathrm{Eu}$ & $-2.1 \mathrm{E}-05 \pm 2.1 \mathrm{E}-04$ & U \\
\hline & $01 / 29 / 08$ & $02 / 12 / 08$ & gross $\alpha$ & $6.3 \mathrm{E}-04 \pm 5.2 \mathrm{E}-04$ & & & ${ }^{154} \mathrm{Eu}$ & $-3.0 \mathrm{E}-05 \pm 3.0 \mathrm{E}-04$ & $\mathrm{U}$ \\
\hline & $01 / 29 / 08$ & 02/12/08 & gross $\beta$ & $3.4 \mathrm{E}-03 \pm 8.1 \mathrm{E}-04$ & & & ${ }^{155} \mathrm{Eu}$ & $-1.1 \mathrm{E}-04 \pm 1.9 \mathrm{E}-04$ & U \\
\hline & $02 / 12 / 08$ & $02 / 25 / 08$ & gross $\alpha$ & $7.6 \mathrm{E}-04 \pm 5.6 \mathrm{E}-04$ & & & ${ }^{238} \mathrm{Pu}$ & $-1.4 \mathrm{E}-06 \pm 9.2 \mathrm{E}-06$ & $\mathrm{U}$ \\
\hline & $02 / 12 / 08$ & $02 / 25 / 08$ & gross $\beta$ & $2.8 \mathrm{E}-02 \pm 3.0 \mathrm{E}-03$ & & & ${ }^{239 / 240} \mathrm{Pu}$ & $1.4 \mathrm{E}-06 \pm 2.8 \mathrm{E}-06$ & $\mathrm{U}$ \\
\hline & $02 / 25 / 08$ & 03/11/08 & gross $\alpha$ & $1.8 \mathrm{E}-03 \pm 6.3 \mathrm{E}-04$ & & & ${ }^{106} \mathrm{Ru}$ & $-4.4 \mathrm{E}-04 \pm 9.3 \mathrm{E}-04$ & $\mathrm{U}$ \\
\hline & $02 / 25 / 08$ & 03/11/08 & gross $\beta$ & $1.3 \mathrm{E}-02 \pm 1.7 \mathrm{E}-03$ & & & ${ }^{125} \mathrm{Sb}$ & $-6.8 \mathrm{E}-05 \pm 2.5 \mathrm{E}-04$ & $\mathrm{U}$ \\
\hline & 03/11/08 & $03 / 25 / 08$ & gross $\alpha$ & $8.3 \mathrm{E}-04 \pm 5.6 \mathrm{E}-04$ & & & ${ }^{90} \mathrm{Sr}$ & $-2.5 \mathrm{E}-04 \pm 2.6 \mathrm{E}-04$ & $\mathrm{U}$ \\
\hline & 03/11/08 & $03 / 25 / 08$ & gross $\beta$ & $8.6 \mathrm{E}-03 \pm 1.4 \mathrm{E}-03$ & & & ${ }^{234} \mathrm{U}$ & $1.1 \mathrm{E}-05 \pm 7.4 \mathrm{E}-06$ & \\
\hline & $03 / 25 / 08$ & $04 / 07 / 08$ & gross $\alpha$ & $8.1 \mathrm{E}-04 \pm 5.9 \mathrm{E}-04$ & & & ${ }^{235} \mathrm{U}$ & $3.2 \mathrm{E}-06 \pm 3.4 \mathrm{E}-06$ & \\
\hline & 03/25/08 & $04 / 07 / 08$ & gross $\beta$ & $7.6 \mathrm{E}-03 \pm 1.3 \mathrm{E}-03$ & & & ${ }^{238} \mathrm{U}$ & $8.8 \mathrm{E}-06 \pm 6.1 \mathrm{E}-06$ & \\
\hline & 04/07/08 & $04 / 21 / 08$ & gross $\alpha$ & $8.9 \mathrm{E}-04 \pm 5.9 \mathrm{E}-04$ & & & & & \\
\hline & $04 / 07 / 08$ & $04 / 21 / 08$ & gross $\beta$ & $8.9 \mathrm{E}-03 \pm 1.4 \mathrm{E}-03$ & N999 & $06 / 30 / 08$ to $12 / 30 / 08$ & ${ }^{60} \mathrm{Co}$ & $5.0 \mathrm{E}-05 \pm 6.6 \mathrm{E}-05$ & $\mathrm{U}$ \\
\hline & $04 / 21 / 08$ & 05/06/08 & gross $\alpha$ & $2.0 \mathrm{E}-03 \pm 6.8 \mathrm{E}-04$ & & & ${ }^{134} \mathrm{Cs}$ & $1.6 \mathrm{E}-06 \pm 1.7 \mathrm{E}-05$ & $\mathrm{U}$ \\
\hline & $04 / 21 / 08$ & 05/06/08 & gross $\beta$ & $1.6 \mathrm{E}-02 \pm 2.0 \mathrm{E}-03$ & & & ${ }^{137} \mathrm{Cs}$ & $2.6 \mathrm{E}-05 \pm 5.8 \mathrm{E}-05$ & $\mathrm{U}$ \\
\hline & 05/06/08 & 05/19/08 & gross $\alpha$ & $1.5 \mathrm{E}-03 \pm 6.9 \mathrm{E}-04$ & & & ${ }^{152} \mathrm{Eu}$ & $-4.3 \mathrm{E}-05 \pm 1.5 \mathrm{E}-04$ & U \\
\hline & 05/06/08 & 05/19/08 & gross $\beta$ & $1.2 \mathrm{E}-02 \pm 1.8 \mathrm{E}-03$ & & & ${ }^{154} \mathrm{Eu}$ & $4.0 \mathrm{E}-04 \pm 3.6 \mathrm{E}-04$ & \\
\hline & 05/19/08 & 06/02/08 & gross $\alpha$ & $1.8 \mathrm{E}-03 \pm 6.7 \mathrm{E}-04$ & & & ${ }^{155} \mathrm{Eu}$ & $6.5 \mathrm{E}-05 \pm 1.6 \mathrm{E}-04$ & U \\
\hline & 05/19/08 & $06 / 02 / 08$ & gross $\beta$ & $1.4 \mathrm{E}-02 \pm 1.9 \mathrm{E}-03$ & & & ${ }^{238} \mathrm{Pu}$ & $-1.3 \mathrm{E}-05 \pm 1.6 \mathrm{E}-05$ & $\mathrm{U}$ \\
\hline & $06 / 02 / 08$ & 06/16/08 & gross $\alpha$ & $9.9 \mathrm{E}-04 \pm 7.7 \mathrm{E}-04$ & & & ${ }^{239 / 240} \mathrm{Pu}$ & $-1.1 \mathrm{E}-06 \pm 3.7 \mathrm{E}-06$ & $\mathrm{U}$ \\
\hline & $06 / 02 / 08$ & 06/16/08 & gross $\beta$ & $8.5 \mathrm{E}-03 \pm 1.5 \mathrm{E}-03$ & & & ${ }^{106} \mathrm{Ru}$ & $4.3 \mathrm{E}-04 \pm 5.3 \mathrm{E}-04$ & $\mathrm{U}$ \\
\hline & $06 / 16 / 08$ & 06/30/08 & gross $\alpha$ & $1.7 \mathrm{E}-03 \pm 6.6 \mathrm{E}-04$ & & & ${ }^{125} \mathrm{Sb}$ & $-5.5 \mathrm{E}-05 \pm 1.3 \mathrm{E}-04$ & $\mathrm{U}$ \\
\hline & $06 / 16 / 08$ & $06 / 30 / 08$ & gross $\beta$ & $1.3 \mathrm{E}-02 \pm 1.7 \mathrm{E}-03$ & & & ${ }^{90} \mathrm{Sr}$ & $-1.8 \mathrm{E}-04 \pm 1.9 \mathrm{E}-04$ & $\mathrm{U}$ \\
\hline & $06 / 30 / 08$ & $07 / 14 / 08$ & gross $\alpha$ & $9.6 \mathrm{E}-04 \pm 6.9 \mathrm{E}-04$ & & & ${ }^{234} \mathrm{U}$ & $1.5 \mathrm{E}-05 \pm 8.7 \mathrm{E}-06$ & \\
\hline & $06 / 30 / 08$ & 07/14/08 & gross $\beta$ & $1.1 \mathrm{E}-02 \pm 1.7 \mathrm{E}-03$ & & & ${ }^{235} \mathrm{U}$ & $1.6 \mathrm{E}-06 \pm 2.3 \mathrm{E}-06$ & $\mathrm{U}$ \\
\hline & $07 / 14 / 08$ & 07/28/08 & gross $\alpha$ & $7.2 \mathrm{E}-04 \pm 5.2 \mathrm{E}-04$ & & & ${ }^{238} \mathrm{U}$ & $8.6 \mathrm{E}-06 \pm 6.6 \mathrm{E}-06$ & \\
\hline & $07 / 14 / 08$ & $07 / 28 / 08$ & gross $\beta$ & $1.2 \mathrm{E}-02 \pm 1.6 \mathrm{E}-03$ & & & & & \\
\hline & 07/28/08 & 08/11/08 & gross $\alpha$ & $8.3 \mathrm{E}-04 \pm 5.5 \mathrm{E}-04$ & & & & & \\
\hline & $07 / 28 / 08$ & $08 / 11 / 08$ & gross $\beta$ & $1.0 \mathrm{E}-02 \pm 1.5 \mathrm{E}-03$ & & & & & \\
\hline & $08 / 11 / 08$ & $08 / 25 / 08$ & gross $\alpha$ & $5.0 \mathrm{E}-04 \pm 4.6 \mathrm{E}-04$ & & & & & \\
\hline & 08/11/08 & 08/25/08 & gross $\beta$ & $1.2 \mathrm{E}-02 \pm 1.6 \mathrm{E}-03$ & & & & & \\
\hline & $08 / 25 / 08$ & 09/08/08 & gross $\alpha$ & $9.4 \mathrm{E}-04 \pm 5.8 \mathrm{E}-04$ & & & & & \\
\hline & $08 / 25 / 08$ & 09/08/08 & gross $\beta$ & $9.8 \mathrm{E}-03 \pm 1.4 \mathrm{E}-03$ & & & & & \\
\hline & 09/08/08 & 09/22/08 & gross $\alpha$ & $1.4 \mathrm{E}-03 \pm 5.6 \mathrm{E}-04$ & & & & & \\
\hline & 09/08/08 & $09 / 22 / 08$ & gross $\beta$ & $2.7 \mathrm{E}-02 \pm 2.8 \mathrm{E}-03$ & & & & & \\
\hline & $09 / 22 / 08$ & $10 / 07 / 08$ & gross $\alpha$ & $2.1 \mathrm{E}-03 \pm 6.6 \mathrm{E}-04$ & & & & & \\
\hline & $09 / 22 / 08$ & $10 / 07 / 08$ & gross $\beta$ & $2.2 \mathrm{E}-02 \pm 2.5 \mathrm{E}-03$ & & & & & \\
\hline & $10 / 07 / 08$ & $10 / 20 / 08$ & gross $\alpha$ & $1.1 \mathrm{E}-03 \pm 6.6 \mathrm{E}-04$ & & & & & \\
\hline & $10 / 07 / 08$ & $10 / 20 / 08$ & gross $\beta$ & $1.8 \mathrm{E}-02 \pm 2.2 \mathrm{E}-03$ & & & & & \\
\hline & $10 / 20 / 08$ & $11 / 03 / 08$ & gross $\alpha$ & $1.7 \mathrm{E}-03 \pm 6.3 \mathrm{E}-04$ & & & & & \\
\hline & $10 / 20 / 08$ & $11 / 03 / 08$ & gross $\beta$ & $4.2 \mathrm{E}-02 \pm 4.0 \mathrm{E}-03$ & & & & & \\
\hline & $11 / 03 / 08$ & $11 / 17 / 08$ & gross $\alpha$ & $1.2 \mathrm{E}-03 \pm 5.4 \mathrm{E}-04$ & & & & & \\
\hline & $11 / 03 / 08$ & $11 / 17 / 08$ & gross $\beta$ & $1.8 \mathrm{E}-02 \pm 2.1 \mathrm{E}-03$ & & & & & \\
\hline & $11 / 17 / 08$ & $12 / 01 / 08$ & gross $\alpha$ & $3.5 \mathrm{E}-04 \pm 6.8 \mathrm{E}-04$ & & & & & \\
\hline & $11 / 17 / 08$ & $12 / 01 / 08$ & gross $\beta$ & $1.1 \mathrm{E}-02 \pm 2.1 \mathrm{E}-03$ & & & & & \\
\hline & $12 / 01 / 08$ & $12 / 15 / 08$ & gross $\alpha$ & $2.2 \mathrm{E}-03 \pm 1.0 \mathrm{E}-03$ & & & & & \\
\hline & $12 / 01 / 08$ & $12 / 15 / 08$ & gross $\beta$ & $4.1 \mathrm{E}-02 \pm 4.7 \mathrm{E}-03$ & & & & & \\
\hline & $12 / 15 / 08$ & $12 / 30 / 08$ & gross $\alpha$ & $2.1 \mathrm{E}-03 \pm 6.7 \mathrm{E}-04$ & & & & & \\
\hline & $12 / 15 / 08$ & $12 / 30 / 08$ & gross $\beta$ & $3.6 \mathrm{E}-02 \pm 3.5 \mathrm{E}-03$ & & & & & \\
\hline
\end{tabular}

$\overline{\mathrm{RQ}}=$ Result Qualifier. $\mathrm{U}=$ The analyte was analyzed for but not detected. 
Table 2-4. Near-Facility Air Sampling Results, 2008 (pCi/m³ \pm total analytical uncertainty). (Sheet 44 of 82)

\begin{tabular}{|c|c|c|c|c|}
\hline Location & Sample On & Sample Off & Isotope & Result \pm Uncertainty \\
\hline N569 & 04/25/08 & 05/08/08 & gross $\alpha$ & $1.3 \mathrm{E}-03 \pm 5.9 \mathrm{E}-04$ \\
\hline \multirow[t]{27}{*}{ (BC CA) } & $04 / 25 / 08$ & 05/08/08 & gross $\beta$ & $1.3 \mathrm{E}-02 \pm 2.0 \mathrm{E}-03$ \\
\hline & 05/08/08 & 05/20/08 & gross $\alpha$ & $1.3 \mathrm{E}-03 \pm 5.8 \mathrm{E}-04$ \\
\hline & 05/08/08 & 05/20/08 & gross $\beta$ & $9.5 \mathrm{E}-03 \pm 1.6 \mathrm{E}-03$ \\
\hline & 05/20/08 & $06 / 04 / 08$ & gross $\alpha$ & $1.1 \mathrm{E}-03 \pm 4.9 \mathrm{E}-04$ \\
\hline & 05/20/08 & 06/04/08 & gross $\beta$ & $1.1 \mathrm{E}-02 \pm 1.6 \mathrm{E}-03$ \\
\hline & 06/04/08 & 06/17/08 & gross $\alpha$ & $1.4 \mathrm{E}-03 \pm 6.1 \mathrm{E}-04$ \\
\hline & 06/04/08 & 06/17/08 & gross $\beta$ & $8.0 \mathrm{E}-03 \pm 1.4 \mathrm{E}-03$ \\
\hline & 06/17/08 & 07/01/08 & gross $\alpha$ & $1.1 \mathrm{E}-03 \pm 7.1 \mathrm{E}-04$ \\
\hline & 06/17/08 & 07/01/08 & gross $\beta$ & $1.5 \mathrm{E}-02 \pm 2.1 \mathrm{E}-03$ \\
\hline & 07/01/08 & 07/11/08 & gross $\alpha$ & $1.8 \mathrm{E}-03 \pm 8.8 \mathrm{E}-04$ \\
\hline & 07/01/08 & 07/11/08 & gross $\beta$ & $1.8 \mathrm{E}-02 \pm 2.6 \mathrm{E}-03$ \\
\hline & 07/11/08 & 07/30/08 & gross $\alpha$ & $1.3 \mathrm{E}-03 \pm 4.7 \mathrm{E}-04$ \\
\hline & 07/11/08 & 07/30/08 & gross $\beta$ & $1.8 \mathrm{E}-02 \pm 2.2 \mathrm{E}-03$ \\
\hline & 07/30/08 & 08/12/08 & gross $\alpha$ & $4.5 \mathrm{E}-04 \pm 1.1 \mathrm{E}-03$ \\
\hline & 07/30/08 & 08/12/08 & gross $\beta$ & $8.8 \mathrm{E}-03 \pm 2.4 \mathrm{E}-03$ \\
\hline & 08/12/08 & 08/26/08 & gross $\alpha$ & $1.7 \mathrm{E}-03 \pm 7.8 \mathrm{E}-04$ \\
\hline & 08/12/08 & 08/26/08 & gross $\beta$ & $1.5 \mathrm{E}-02 \pm 2.4 \mathrm{E}-03$ \\
\hline & 08/26/08 & 09/09/08 & gross $\alpha$ & $4.9 \mathrm{E}-04 \pm 4.7 \mathrm{E}-04$ \\
\hline & 08/26/08 & 09/09/08 & gross $\beta$ & $1.4 \mathrm{E}-02 \pm 2.0 \mathrm{E}-03$ \\
\hline & 09/09/08 & 09/23/08 & gross $\alpha$ & $6.3 \mathrm{E}-04 \pm 5.3 \mathrm{E}-04$ \\
\hline & 09/09/08 & 09/23/08 & gross $\beta$ & $2.8 \mathrm{E}-02 \pm 3.3 \mathrm{E}-03$ \\
\hline & 09/23/08 & $10 / 08 / 08$ & gross $\alpha$ & $1.5 \mathrm{E}-03 \pm 6.0 \mathrm{E}-04$ \\
\hline & 09/23/08 & $10 / 08 / 08$ & gross $\beta$ & $1.5 \mathrm{E}-02 \pm 2.0 \mathrm{E}-03$ \\
\hline & $10 / 08 / 08$ & $10 / 21 / 08$ & gross $\alpha$ & $1.1 \mathrm{E}-03 \pm 6.7 \mathrm{E}-04$ \\
\hline & $10 / 08 / 08$ & $10 / 21 / 08$ & gross $\beta$ & $1.8 \mathrm{E}-02 \pm 2.5 \mathrm{E}-03$ \\
\hline & $10 / 21 / 08$ & $10 / 23 / 08$ & gross $\alpha$ & $1.0 \mathrm{E}-03 \pm 2.3 \mathrm{E}-03$ \\
\hline & $10 / 21 / 08$ & $10 / 23 / 08$ & gross $\beta$ & $1.8 \mathrm{E}-02 \pm 5.2 \mathrm{E}-03$ \\
\hline
\end{tabular}

\begin{tabular}{|c|c|c|c|c|}
\hline Location & Composite Period & Isotope & Result \pm Uncertainty & $\mathbf{R Q}^{*}$ \\
\hline \multirow[t]{16}{*}{ N569 } & $04 / 25 / 08$ to $07 / 01 / 08$ & ${ }^{241} \mathrm{Am}$ & $2.1 \mathrm{E}-05 \pm 1.5 \mathrm{E}-05$ & \\
\hline & & ${ }^{60} \mathrm{Co}$ & $-1.2 \mathrm{E}-04 \pm 2.3 \mathrm{E}-04$ & $\mathrm{U}$ \\
\hline & & ${ }^{134} \mathrm{Cs}$ & $-7.5 \mathrm{E}-05 \pm 2.2 \mathrm{E}-04$ & $\mathrm{U}$ \\
\hline & & ${ }^{137} \mathrm{Cs}$ & $1.2 \mathrm{E}-05 \pm 1.2 \mathrm{E}-04$ & $\mathrm{U}$ \\
\hline & & ${ }^{152} \mathrm{Eu}$ & $1.1 \mathrm{E}-04 \pm 4.4 \mathrm{E}-04$ & $\mathrm{U}$ \\
\hline & & ${ }^{154} \mathrm{Eu}$ & $-3.4 \mathrm{E}-04 \pm 6.3 \mathrm{E}-04$ & $\mathrm{U}$ \\
\hline & & ${ }^{155} \mathrm{Eu}$ & $2.6 \mathrm{E}-04 \pm 5.1 \mathrm{E}-04$ & $\mathrm{U}$ \\
\hline & & ${ }^{238} \mathrm{Pu}$ & $-2.3 \mathrm{E}-05 \pm 4.9 \mathrm{E}-05$ & $\mathrm{U}$ \\
\hline & & ${ }^{239 / 240} \mathrm{Pu}$ & $1.4 \mathrm{E}-05 \pm 1.6 \mathrm{E}-05$ & \\
\hline & & ${ }^{241} \mathrm{Pu}$ & $8.5 \mathrm{E}-04 \pm 1.9 \mathrm{E}-03$ & $\mathrm{U}$ \\
\hline & & ${ }^{106} \mathrm{Ru}$ & $-4.7 \mathrm{E}-04 \pm 1.9 \mathrm{E}-03$ & $\mathrm{U}$ \\
\hline & & ${ }^{125} \mathrm{Sb}$ & $-1.4 \mathrm{E}-04 \pm 4.1 \mathrm{E}-04$ & $\mathrm{U}$ \\
\hline & & ${ }^{90} \mathrm{Sr}$ & $-6.9 \mathrm{E}-06 \pm 7.1 \mathrm{E}-06$ & $\mathrm{U}$ \\
\hline & & ${ }^{234} \mathrm{U}$ & $3.8 \mathrm{E}-05 \pm 2.2 \mathrm{E}-05$ & \\
\hline & & ${ }^{235} \mathrm{U}$ & $4.4 \mathrm{E}-06 \pm 6.4 \mathrm{E}-06$ & $\mathrm{U}$ \\
\hline & & ${ }^{238} \mathrm{U}$ & $2.2 \mathrm{E}-05 \pm 1.5 \mathrm{E}-05$ & \\
\hline \multirow[t]{16}{*}{ N569 } & $07 / 01 / 08$ to $10 / 23 / 08$ & ${ }^{241} \mathrm{Am}$ & $8.1 \mathrm{E}-06 \pm 1.6 \mathrm{E}-05$ & $\mathrm{U}$ \\
\hline & & ${ }^{60} \mathrm{Co}$ & $5.9 \mathrm{E}-05 \pm 1.9 \mathrm{E}-04$ & $\mathrm{U}$ \\
\hline & & ${ }^{134} \mathrm{Cs}$ & $-6.9 \mathrm{E}-05 \pm 2.0 \mathrm{E}-04$ & $\mathrm{U}$ \\
\hline & & ${ }^{137} \mathrm{Cs}$ & $5.7 \mathrm{E}-04 \pm 2.9 \mathrm{E}-04$ & $\mathrm{U}$ \\
\hline & & ${ }^{152} \mathrm{Eu}$ & $1.5 \mathrm{E}-04 \pm 4.9 \mathrm{E}-04$ & $\mathrm{U}$ \\
\hline & & ${ }^{154} \mathrm{Eu}$ & $2.0 \mathrm{E}-04 \pm 6.0 \mathrm{E}-04$ & $\mathrm{U}$ \\
\hline & & ${ }^{155} \mathrm{Eu}$ & $-1.7 \mathrm{E}-05 \pm 1.7 \mathrm{E}-04$ & $\mathrm{U}$ \\
\hline & & ${ }^{238} \mathrm{Pu}$ & $-1.3 \mathrm{E}-05 \pm 5.4 \mathrm{E}-05$ & $\mathrm{U}$ \\
\hline & & ${ }^{239 / 240} \mathrm{Pu}$ & $3.2 \mathrm{E}-06 \pm 3.2 \mathrm{E}-05$ & $\mathrm{U}$ \\
\hline & & ${ }^{241} \mathrm{Pu}$ & $-4.0 \mathrm{E}-04 \pm 4.1 \mathrm{E}-04$ & $\mathrm{U}$ \\
\hline & & ${ }^{106} \mathrm{Ru}$ & $-1.1 \mathrm{E}-04 \pm 1.1 \mathrm{E}-03$ & $\mathrm{U}$ \\
\hline & & ${ }^{125} \mathrm{Sb}$ & $3.1 \mathrm{E}-04 \pm 4.6 \mathrm{E}-04$ & $\mathrm{U}$ \\
\hline & & ${ }^{90} \mathrm{Sr}$ & $3.5 \mathrm{E}-04 \pm 3.0 \mathrm{E}-04$ & \\
\hline & & ${ }^{234} \mathrm{U}$ & $3.1 \mathrm{E}-05 \pm 1.7 \mathrm{E}-05$ & \\
\hline & & ${ }^{235} \mathrm{U}$ & $2.9 \mathrm{E}-06 \pm 4.3 \mathrm{E}-06$ & $\mathrm{U}$ \\
\hline & & ${ }^{238} \mathrm{U}$ & $1.7 \mathrm{E}-05 \pm 1.2 \mathrm{E}-05$ & \\
\hline
\end{tabular}


Table 2-4. Near-Facility Air Sampling Results, 2008 (pCi/m³ \pm total analytical uncertainty). (Sheet 45 of 82)

\begin{tabular}{cccll} 
Location & Sample On & Sample Off & Isotope & Result \pm Uncertainty \\
\hline N570 & $04 / 25 / 08$ & $05 / 08 / 08$ & gross $\alpha$ & $1.2 \mathrm{E}-03 \pm 7.7 \mathrm{E}-04$ \\
(BC CA) & $04 / 25 / 08$ & $05 / 08 / 08$ & gross $\beta$ & $1.3 \mathrm{E}-02 \pm 2.1 \mathrm{E}-03$ \\
& $05 / 08 / 08$ & $05 / 20 / 08$ & gross $\alpha$ & $1.3 \mathrm{E}-03 \pm 6.2 \mathrm{E}-04$ \\
& $05 / 08 / 08$ & $05 / 20 / 08$ & gross $\beta$ & $7.4 \mathrm{E}-03 \pm 1.5 \mathrm{E}-03$ \\
& $05 / 20 / 08$ & $06 / 04 / 08$ & gross $\alpha$ & $1.6 \mathrm{E}-03 \pm 6.8 \mathrm{E}-04$ \\
& $05 / 20 / 08$ & $06 / 04 / 08$ & gross $\beta$ & $9.0 \mathrm{E}-03 \pm 1.6 \mathrm{E}-03$ \\
& $06 / 04 / 08$ & $06 / 17 / 08$ & gross $\alpha$ & $6.1 \mathrm{E}-04 \pm 5.8 \mathrm{E}-04$ \\
& $06 / 04 / 08$ & $06 / 17 / 08$ & gross $\beta$ & $8.5 \mathrm{E}-03 \pm 1.5 \mathrm{E}-03$ \\
& $06 / 17 / 08$ & $07 / 01 / 08$ & gross $\alpha$ & $1.0 \mathrm{E}-03 \pm 7.2 \mathrm{E}-04$ \\
& $06 / 17 / 08$ & $07 / 01 / 08$ & gross $\beta$ & $4.3 \mathrm{E}-02 \pm 4.7 \mathrm{E}-03$ \\
& $07 / 01 / 08$ & $07 / 11 / 08$ & gross $\alpha$ & $2.3 \mathrm{E}-03 \pm 1.0 \mathrm{E}-03$ \\
& $07 / 01 / 08$ & $07 / 11 / 08$ & gross $\beta$ & $5.0 \mathrm{E}-02 \pm 5.7 \mathrm{E}-03$ \\
& $07 / 11 / 08$ & $07 / 30 / 08$ & gross $\alpha$ & $1.4 \mathrm{E}-03 \pm 5.1 \mathrm{E}-04$ \\
& $07 / 11 / 08$ & $07 / 30 / 08$ & gross $\beta$ & $3.4 \mathrm{E}-02 \pm 3.7 \mathrm{E}-03$ \\
& $07 / 30 / 08$ & $08 / 12 / 08$ & gross $\alpha$ & $1.1 \mathrm{E}-03 \pm 5.1 \mathrm{E}-04$ \\
& $07 / 30 / 08$ & $08 / 12 / 08$ & gross $\beta$ & $1.5 \mathrm{E}-02 \pm 2.1 \mathrm{E}-03$ \\
& $08 / 12 / 08$ & $08 / 26 / 08$ & gross $\alpha$ & $6.1 \mathrm{E}-04 \pm 7.1 \mathrm{E}-04$ \\
& $08 / 12 / 08$ & $08 / 26 / 08$ & gross $\beta$ & $1.5 \mathrm{E}-02 \pm 2.5 \mathrm{E}-03$ \\
$08 / 26 / 08$ & $09 / 09 / 08$ & gross $\alpha$ & $7.8 \mathrm{E}-04 \pm 5.4 \mathrm{E}-04$ \\
& $08 / 26 / 08$ & $09 / 09 / 08$ & gross $\beta$ & $6.9 \mathrm{E}-03 \pm 1.3 \mathrm{E}-03$ \\
$09 / 09 / 08$ & $09 / 23 / 08$ & gross $\alpha$ & $1.6 \mathrm{E}-03 \pm 6.0 \mathrm{E}-04$ \\
$09 / 09 / 08$ & $09 / 23 / 08$ & gross $\beta$ & $2.4 \mathrm{E}-02 \pm 3.0 \mathrm{E}-03$ \\
$09 / 23 / 08$ & $10 / 08 / 08$ & gross $\alpha$ & $8.2 \mathrm{E}-04 \pm 5.4 \mathrm{E}-04$ \\
$09 / 23 / 08$ & $10 / 08 / 08$ & gross $\beta$ & $2.2 \mathrm{E}-02 \pm 2.6 \mathrm{E}-03$ \\
$10 / 08 / 08$ & $10 / 21 / 08$ & gross $\alpha$ & $1.9 \mathrm{E}-03 \pm 6.9 \mathrm{E}-04$ \\
$10 / 08 / 08$ & $10 / 21 / 08$ & gross $\beta$ & $2.0 \mathrm{E}-02 \pm 2.6 \mathrm{E}-03$ \\
$10 / 21 / 08$ & $10 / 23 / 08$ & gross $\alpha$ & $4.7 \mathrm{E}-03 \pm 3.6 \mathrm{E}-03$ \\
& $10 / 21 / 08$ & $10 / 23 / 08$ & gross $\beta$ & $1.3 \mathrm{E}-02 \pm 4.5 \mathrm{E}-03$ \\
& & & &
\end{tabular}

\begin{tabular}{|c|c|c|c|c|}
\hline Location & Composite Period & Isotope & Result \pm Uncertainty & $\mathbf{R Q}^{*}$ \\
\hline \multirow[t]{16}{*}{ N570 } & $04 / 25 / 08$ to $07 / 01 / 08$ & ${ }^{241} \mathrm{Am}$ & $2.4 \mathrm{E}-05 \pm 2.7 \mathrm{E}-05$ & $\mathrm{U}$ \\
\hline & & ${ }^{60} \mathrm{Co}$ & $-4.2 \mathrm{E}-05 \pm 2.5 \mathrm{E}-04$ & $\mathrm{U}$ \\
\hline & & ${ }^{134} \mathrm{Cs}$ & $2.4 \mathrm{E}-05 \pm 2.0 \mathrm{E}-04$ & $\mathrm{U}$ \\
\hline & & ${ }^{137} \mathrm{Cs}$ & $1.1 \mathrm{E}-04 \pm 1.9 \mathrm{E}-04$ & $\mathrm{U}$ \\
\hline & & ${ }^{152} \mathrm{Eu}$ & $5.0 \mathrm{E}-05 \pm 3.9 \mathrm{E}-04$ & $\mathrm{U}$ \\
\hline & & ${ }^{154} \mathrm{Eu}$ & $1.6 \mathrm{E}-04 \pm 5.8 \mathrm{E}-04$ & $\mathrm{U}$ \\
\hline & & ${ }^{155} \mathrm{Eu}$ & $-1.7 \mathrm{E}-05 \pm 1.7 \mathrm{E}-04$ & $\mathrm{U}$ \\
\hline & & ${ }^{238} \mathrm{Pu}$ & $2.7 \mathrm{E}-05 \pm 7.6 \mathrm{E}-05$ & $\mathrm{U}$ \\
\hline & & ${ }^{239 / 240} \mathrm{Pu}$ & $-4.7 \mathrm{E}-06 \pm 1.6 \mathrm{E}-05$ & $\mathrm{U}$ \\
\hline & & ${ }^{241} \mathrm{Pu}$ & $1.2 \mathrm{E}-03 \pm 2.1 \mathrm{E}-03$ & $\mathrm{U}$ \\
\hline & & ${ }^{106} \mathrm{Ru}$ & $3.6 \mathrm{E}-04 \pm 1.7 \mathrm{E}-03$ & $\mathrm{U}$ \\
\hline & & ${ }^{125} \mathrm{Sb}$ & $3.2 \mathrm{E}-05 \pm 3.2 \mathrm{E}-04$ & $\mathrm{U}$ \\
\hline & & ${ }^{90} \mathrm{Sr}$ & $1.3 \mathrm{E}-04 \pm 3.8 \mathrm{E}-04$ & $\mathrm{U}$ \\
\hline & & ${ }^{234} \mathrm{U}$ & $3.2 \mathrm{E}-05 \pm 2.0 \mathrm{E}-05$ & \\
\hline & & ${ }^{235} \mathrm{U}$ & $2.1 \mathrm{E}-06 \pm 6.6 \mathrm{E}-06$ & $\mathrm{U}$ \\
\hline & & ${ }^{238} \mathrm{U}$ & $1.3 \mathrm{E}-05 \pm 1.3 \mathrm{E}-05$ & $\mathrm{U}$ \\
\hline \multirow[t]{16}{*}{ N570 } & $07 / 01 / 08$ to $10 / 23 / 08$ & ${ }^{241} \mathrm{Am}$ & $9.3 \mathrm{E}-06 \pm 9.3 \mathrm{E}-05$ & \\
\hline & & ${ }^{60} \mathrm{Co}$ & $-6.7 \mathrm{E}-05 \pm 1.4 \mathrm{E}-04$ & $\mathrm{U}$ \\
\hline & & ${ }^{134} \mathrm{Cs}$ & $2.4 \mathrm{E}-05 \pm 1.2 \mathrm{E}-04$ & $\mathrm{U}$ \\
\hline & & ${ }^{137} \mathrm{Cs}$ & $2.5 \mathrm{E}-03 \pm 8.1 \mathrm{E}-04$ & \\
\hline & & ${ }^{152} \mathrm{Eu}$ & $-2.5 \mathrm{E}-04 \pm 2.8 \mathrm{E}-04$ & $\mathrm{U}$ \\
\hline & & ${ }^{154} \mathrm{Eu}$ & $3.3 \mathrm{E}-04 \pm 3.8 \mathrm{E}-04$ & $\mathrm{U}$ \\
\hline & & ${ }^{155} \mathrm{Eu}$ & $-1.1 \mathrm{E}-04 \pm 2.9 \mathrm{E}-04$ & $\mathrm{U}$ \\
\hline & & ${ }^{238} \mathrm{Pu}$ & $4.8 \mathrm{E}-06 \pm 4.2 \mathrm{E}-05$ & $\mathrm{U}$ \\
\hline & & ${ }^{239 / 240} \mathrm{Pu}$ & $-2.4 \mathrm{E}-06 \pm 1.1 \mathrm{E}-05$ & $\mathrm{U}$ \\
\hline & & ${ }^{241} \mathrm{Pu}$ & $-5.8 \mathrm{E}-04 \pm 6.0 \mathrm{E}-04$ & $\mathrm{U}$ \\
\hline & & ${ }^{106} \mathrm{Ru}$ & $-2.9 \mathrm{E}-04 \pm 9.2 \mathrm{E}-04$ & $\mathrm{U}$ \\
\hline & & ${ }^{125} \mathrm{Sb}$ & $5.2 \mathrm{E}-05 \pm 2.5 \mathrm{E}-04$ & $\mathrm{U}$ \\
\hline & & ${ }^{90} \mathrm{Sr}$ & $7.8 \mathrm{E}-04 \pm 3.0 \mathrm{E}-04$ & \\
\hline & & ${ }^{234} \mathrm{U}$ & $3.2 \mathrm{E}-05 \pm 1.7 \mathrm{E}-05$ & \\
\hline & & ${ }^{235} \mathrm{U}$ & $4.9 \mathrm{E}-06 \pm 5.2 \mathrm{E}-06$ & \\
\hline & & ${ }^{238} \mathrm{U}$ & $1.5 \mathrm{E}-05 \pm 9.6 \mathrm{E}-06$ & \\
\hline
\end{tabular}


Table 2-4. Near-Facility Air Sampling Results, 2008 (pCi/m³ \pm total analytical uncertainty). (Sheet 46 of 82)

\begin{tabular}{|c|c|c|c|c|c|c|c|c|c|}
\hline Location & Sample On & Sample Off & Isotope & Result \pm Uncertainty & Location & Composite Period & Isotope & Result \pm Uncertainty & RQ* \\
\hline N571 & $04 / 26 / 08$ & $05 / 08 / 08$ & gross $\alpha$ & $1.4 \mathrm{E}-03 \pm 6.7 \mathrm{E}-04$ & N571 & $04 / 26 / 08$ to $07 / 01 / 08$ & ${ }^{241} \mathrm{Am}$ & $3.0 \mathrm{E}-05 \pm 1.9 \mathrm{E}-05$ & \\
\hline \multirow[t]{32}{*}{ (BC CA) } & $04 / 26 / 08$ & 05/08/08 & gross $\beta$ & $1.0 \mathrm{E}-02 \pm 1.8 \mathrm{E}-03$ & & & ${ }^{60} \mathrm{Co}$ & $1.1 \mathrm{E}-04 \pm 1.8 \mathrm{E}-04$ & $\mathrm{U}$ \\
\hline & 05/08/08 & $05 / 20 / 08$ & gross $\alpha$ & $1.7 \mathrm{E}-03 \pm 7.2 \mathrm{E}-04$ & & & ${ }^{134} \mathrm{Cs}$ & $-8.4 \mathrm{E}-05 \pm 2.0 \mathrm{E}-04$ & $\mathrm{U}$ \\
\hline & 05/08/08 & $05 / 20 / 08$ & gross $\beta$ & $9.7 \mathrm{E}-03 \pm 1.7 \mathrm{E}-03$ & & & ${ }^{137} \mathrm{Cs}$ & $-2.8 \mathrm{E}-05 \pm 1.7 \mathrm{E}-04$ & $\mathrm{U}$ \\
\hline & 05/20/08 & $06 / 04 / 08$ & gross $\alpha$ & $7.2 \mathrm{E}-04 \pm 5.4 \mathrm{E}-04$ & & & ${ }^{152} \mathrm{Eu}$ & $-6.7 \mathrm{E}-05 \pm 3.9 \mathrm{E}-04$ & $\mathrm{U}$ \\
\hline & 05/20/08 & $06 / 04 / 08$ & gross $\beta$ & $6.5 \mathrm{E}-03 \pm 1.3 \mathrm{E}-03$ & & & ${ }^{154} \mathrm{Eu}$ & $-2.4 \mathrm{E}-04 \pm 5.4 \mathrm{E}-04$ & $\mathrm{U}$ \\
\hline & $06 / 04 / 08$ & $06 / 17 / 08$ & gross $\alpha$ & $4.5 \mathrm{E}-04 \pm 5.1 \mathrm{E}-04$ & & & ${ }^{155} \mathrm{Eu}$ & $-1.4 \mathrm{E}-05 \pm 1.4 \mathrm{E}-04$ & $\mathrm{U}$ \\
\hline & 06/04/08 & 06/17/08 & gross $\beta$ & $8.2 \mathrm{E}-03 \pm 1.5 \mathrm{E}-03$ & & & ${ }^{238} \mathrm{Pu}$ & $5.6 \mathrm{E}-05 \pm 9.4 \mathrm{E}-05$ & $\mathrm{U}$ \\
\hline & 06/17/08 & 07/01/08 & $\operatorname{gross} \alpha$ & $9.0 \mathrm{E}-04 \pm 6.3 \mathrm{E}-04$ & & & ${ }^{239 / 240} \mathrm{Pu}$ & $1.0 \mathrm{E}-05 \pm 2.1 \mathrm{E}-05$ & $\mathrm{U}$ \\
\hline & 06/17/08 & 07/01/08 & gross $\beta$ & $1.0 \mathrm{E}-02 \pm 1.6 \mathrm{E}-03$ & & & ${ }^{241} \mathrm{Pu}$ & $-1.3 \mathrm{E}-04 \pm 1.3 \mathrm{E}-03$ & $\mathrm{U}$ \\
\hline & 07/01/08 & 07/11/08 & gross $\alpha$ & $7.6 \mathrm{E}-04 \pm 7.7 \mathrm{E}-04$ & & & ${ }^{106} \mathrm{Ru}$ & $9.9 \mathrm{E}-04 \pm 1.6 \mathrm{E}-03$ & $\mathrm{U}$ \\
\hline & 07/01/08 & 07/11/08 & gross $\beta$ & $1.3 \mathrm{E}-02 \pm 2.1 \mathrm{E}-03$ & & & ${ }^{125} \mathrm{Sb}$ & $1.1 \mathrm{E}-04 \pm 4.0 \mathrm{E}-04$ & $\mathrm{U}$ \\
\hline & 07/11/08 & 07/30/08 & gross $\alpha$ & $3.2 \mathrm{E}-03 \pm 1.1 \mathrm{E}-03$ & & & ${ }^{90} \mathrm{Sr}$ & $-3.8 \mathrm{E}-04 \pm 3.8 \mathrm{E}-04$ & $\mathrm{U}$ \\
\hline & 07/11/08 & 07/30/08 & gross $\beta$ & $2.8 \mathrm{E}-02 \pm 3.9 \mathrm{E}-03$ & & & ${ }^{234} \mathrm{U}$ & $3.9 \mathrm{E}-05 \pm 2.7 \mathrm{E}-05$ & \\
\hline & 07/30/08 & 08/12/08 & gross $\alpha$ & $9.2 \mathrm{E}-04 \pm 6.3 \mathrm{E}-04$ & & & ${ }^{235} \mathrm{U}$ & $9.5 \mathrm{E}-06 \pm 1.2 \mathrm{E}-05$ & $\mathrm{U}$ \\
\hline & 07/30/08 & 08/12/08 & gross $\beta$ & $1.6 \mathrm{E}-02 \pm 2.3 \mathrm{E}-03$ & & & ${ }^{238} \mathrm{U}$ & $1.5 \mathrm{E}-05 \pm 1.5 \mathrm{E}-05$ & $\mathrm{U}$ \\
\hline & 08/12/08 & 08/26/08 & gross $\alpha$ & $7.2 \mathrm{E}-04 \pm 5.4 \mathrm{E}-04$ & & & & & \\
\hline & 08/12/08 & 08/26/08 & gross $\beta$ & $1.5 \mathrm{E}-02 \pm 2.1 \mathrm{E}-03$ & N571 & $07 / 01 / 08$ to $10 / 23 / 08$ & ${ }^{241} \mathrm{Am}$ & $4.1 \mathrm{E}-06 \pm 1.7 \mathrm{E}-05$ & $\mathrm{U}$ \\
\hline & 08/26/08 & 09/09/08 & gross $\alpha$ & $5.2 \mathrm{E}-04 \pm 5.0 \mathrm{E}-04$ & & & ${ }^{60} \mathrm{Co}$ & $1.9 \mathrm{E}-05 \pm 1.9 \mathrm{E}-04$ & $\mathrm{U}$ \\
\hline & 08/26/08 & 09/09/08 & gross $\beta$ & $1.0 \mathrm{E}-02 \pm 1.7 \mathrm{E}-03$ & & & ${ }^{134} \mathrm{Cs}$ & $-1.7 \mathrm{E}-04 \pm 2.4 \mathrm{E}-04$ & $\mathrm{U}$ \\
\hline & 09/09/08 & 09/23/08 & gross $\alpha$ & $1.2 \mathrm{E}-03 \pm 5.3 \mathrm{E}-04$ & & & ${ }^{137} \mathrm{Cs}$ & $3.4 \mathrm{E}-05 \pm 1.9 \mathrm{E}-04$ & $\mathrm{U}$ \\
\hline & 09/09/08 & 09/23/08 & gross $\beta$ & $2.0 \mathrm{E}-02 \pm 2.6 \mathrm{E}-03$ & & & ${ }^{152} \mathrm{Eu}$ & $9.7 \mathrm{E}-05 \pm 4.3 \mathrm{E}-04$ & $\mathrm{U}$ \\
\hline & 09/23/08 & $10 / 08 / 08$ & gross $\alpha$ & $1.2 \mathrm{E}-03 \pm 5.2 \mathrm{E}-04$ & & & ${ }^{154} \mathrm{Eu}$ & $4.0 \mathrm{E}-04 \pm 4.9 \mathrm{E}-04$ & $\mathrm{U}$ \\
\hline & 09/23/08 & $10 / 08 / 08$ & gross $\beta$ & $2.1 \mathrm{E}-02 \pm 2.6 \mathrm{E}-03$ & & & ${ }^{155} \mathrm{Eu}$ & $1.1 \mathrm{E}-04 \pm 3.4 \mathrm{E}-04$ & $\mathrm{U}$ \\
\hline & $10 / 08 / 08$ & $10 / 21 / 08$ & gross $\alpha$ & $1.6 \mathrm{E}-03 \pm 6.6 \mathrm{E}-04$ & & & ${ }^{238} \mathrm{Pu}$ & $-1.3 \mathrm{E}-05 \pm 4.8 \mathrm{E}-05$ & $\mathrm{U}$ \\
\hline & $10 / 08 / 08$ & $10 / 21 / 08$ & gross $\beta$ & $1.8 \mathrm{E}-02 \pm 2.5 \mathrm{E}-03$ & & & ${ }^{239 / 240} \mathrm{Pu}$ & $7.9 \mathrm{E}-06 \pm 1.2 \mathrm{E}-05$ & $\mathrm{U}$ \\
\hline & $10 / 21 / 08$ & $10 / 23 / 08$ & gross $\alpha$ & $2.6 \mathrm{E}-03 \pm 3.0 \mathrm{E}-03$ & & & ${ }^{241} \mathrm{Pu}$ & $7.9 \mathrm{E}-04 \pm 1.7 \mathrm{E}-03$ & $\mathrm{U}$ \\
\hline & $10 / 21 / 08$ & $10 / 23 / 08$ & gross $\beta$ & $1.6 \mathrm{E}-02 \pm 5.0 \mathrm{E}-03$ & & & ${ }^{106} \mathrm{Ru}$ & $-3.8 \mathrm{E}-04 \pm 1.6 \mathrm{E}-03$ & $\mathrm{U}$ \\
\hline & & & & & & & ${ }^{125} \mathrm{Sb}$ & $1.4 \mathrm{E}-05 \pm 1.4 \mathrm{E}-04$ & $\mathrm{U}$ \\
\hline & & & & & & & ${ }^{90} \mathrm{Sr}$ & $8.8 \mathrm{E}-05 \pm 2.7 \mathrm{E}-04$ & $\mathrm{U}$ \\
\hline & & & & & & & ${ }^{234} \mathrm{U}$ & $2.8 \mathrm{E}-05 \pm 1.7 \mathrm{E}-05$ & \\
\hline & & & & & & & ${ }^{235} \mathrm{U}$ & $2.8 \mathrm{E}-06 \pm 5.8 \mathrm{E}-06$ & $\mathrm{U}$ \\
\hline & & & & & & & ${ }^{238} \mathrm{U}$ & $1.6 \mathrm{E}-05 \pm 1.1 \mathrm{E}-05$ & \\
\hline N572 & 07/21/08 & 07/29/08 & gross $\alpha$ & $1.1 \mathrm{E}-03 \pm 9.0 \mathrm{E}-04$ & N572 & $07 / 21 / 08$ to $11 / 04 / 08$ & ${ }^{60} \mathrm{Co}$ & $-1.5 \mathrm{E}-06 \pm 1.5 \mathrm{E}-05$ & $\mathrm{U}$ \\
\hline \multirow[t]{15}{*}{ (BC CA) } & 07/21/08 & 07/29/08 & gross $\beta$ & $1.3 \mathrm{E}-02 \pm 2.4 \mathrm{E}-03$ & & & ${ }^{134} \mathrm{Cs}$ & $5.9 \mathrm{E}-05 \pm 1.0 \mathrm{E}-04$ & $\mathrm{U}$ \\
\hline & 07/29/08 & 08/12/08 & $\operatorname{gross} \alpha$ & $5.2 \mathrm{E}-04 \pm 5.0 \mathrm{E}-04$ & & & ${ }^{137} \mathrm{Cs}$ & $3.3 \mathrm{E}-05 \pm 9.7 \mathrm{E}-05$ & $\mathrm{U}$ \\
\hline & 07/29/08 & 08/12/08 & gross $\beta$ & $1.3 \mathrm{E}-02 \pm 1.9 \mathrm{E}-03$ & & & ${ }^{152} \mathrm{Eu}$ & $-1.6 \mathrm{E}-05 \pm 1.6 \mathrm{E}-04$ & $\mathrm{U}$ \\
\hline & 08/12/08 & 08/26/08 & gross $\alpha$ & $9.7 \mathrm{E}-04 \pm 6.1 \mathrm{E}-04$ & & & ${ }^{154} \mathrm{Eu}$ & $-1.2 \mathrm{E}-05 \pm 1.2 \mathrm{E}-04$ & $\mathrm{U}$ \\
\hline & 08/12/08 & 08/26/08 & gross $\beta$ & $1.2 \mathrm{E}-02 \pm 1.8 \mathrm{E}-03$ & & & ${ }^{155} \mathrm{Eu}$ & $-2.3 \mathrm{E}-05 \pm 2.3 \mathrm{E}-04$ & $\mathrm{U}$ \\
\hline & $08 / 26 / 08$ & 09/09/08 & gross $\alpha$ & $7.1 \mathrm{E}-04 \pm 5.3 \mathrm{E}-04$ & & & ${ }^{238} \mathrm{Pu}$ & $-1.4 \mathrm{E}-05 \pm 2.1 \mathrm{E}-05$ & $\mathrm{U}$ \\
\hline & 08/26/08 & 09/09/08 & gross $\beta$ & $1.1 \mathrm{E}-02 \pm 1.7 \mathrm{E}-03$ & & & ${ }^{239 / 240} \mathrm{Pu}$ & $1.4 \mathrm{E}-06 \pm 6.3 \mathrm{E}-06$ & $\mathrm{U}$ \\
\hline & 09/09/08 & 09/23/08 & gross $\alpha$ & $2.4 \mathrm{E}-03 \pm 7.6 \mathrm{E}-04$ & & & ${ }^{106} \mathrm{Ru}$ & $-5.3 \mathrm{E}-04 \pm 8.7 \mathrm{E}-04$ & $\mathrm{U}$ \\
\hline & 09/09/08 & 09/23/08 & gross $\beta$ & $3.2 \mathrm{E}-02 \pm 3.6 \mathrm{E}-03$ & & & ${ }^{125} \mathrm{Sb}$ & $2.9 \mathrm{E}-05 \pm 2.1 \mathrm{E}-04$ & $\mathrm{U}$ \\
\hline & 09/23/08 & $10 / 08 / 08$ & gross $\alpha$ & $1.5 \mathrm{E}-03 \pm 5.7 \mathrm{E}-04$ & & & ${ }^{90} \mathrm{Sr}$ & $8.9 \mathrm{E}-06 \pm 8.9 \mathrm{E}-05$ & $\mathrm{U}$ \\
\hline & 09/23/08 & $10 / 08 / 08$ & gross $\beta$ & $2.3 \mathrm{E}-02 \pm 2.7 \mathrm{E}-03$ & & & ${ }^{234} \mathrm{U}$ & $2.4 \mathrm{E}-05 \pm 1.4 \mathrm{E}-05$ & \\
\hline & $10 / 08 / 08$ & $10 / 21 / 08$ & gross $\alpha$ & $1.3 \mathrm{E}-03 \pm 5.7 \mathrm{E}-04$ & & & ${ }^{235} \mathrm{U}$ & $2.8 \mathrm{E}-06 \pm 4.0 \mathrm{E}-06$ & $\mathrm{U}$ \\
\hline & $10 / 08 / 08$ & $10 / 21 / 08$ & gross $\beta$ & $2.1 \mathrm{E}-02 \pm 2.7 \mathrm{E}-03$ & & & ${ }^{238} \mathrm{U}$ & $1.5 \mathrm{E}-05 \pm 1.1 \mathrm{E}-05$ & \\
\hline & $10 / 21 / 08$ & $11 / 04 / 08$ & gross $\alpha$ & $3.2 \mathrm{E}-03 \pm 9.8 \mathrm{E}-04$ & & & & & \\
\hline & $10 / 21 / 08$ & $11 / 04 / 08$ & gross $\beta$ & $4.4 \mathrm{E}-02 \pm 5.0 \mathrm{E}-03$ & & & & & \\
\hline
\end{tabular}


Table 2-4. Near-Facility Air Sampling Results, 2008 (pCi/m³ \pm total analytical uncertainty). (Sheet 47 of 82)

\begin{tabular}{ccclc} 
Location & Sample On & Sample Off & Isotope & Result \pm Uncertainty \\
\hline N573 & $07 / 21 / 08$ & $07 / 29 / 08$ & gross $\alpha$ & $4.7 \mathrm{E}-04 \pm 7.1 \mathrm{E}-04$ \\
(BC CA $)$ & $07 / 21 / 08$ & $07 / 29 / 08$ & gross $\beta$ & $1.7 \mathrm{E}-02 \pm 2.8 \mathrm{E}-03$ \\
& $07 / 29 / 08$ & $08 / 12 / 08$ & gross $\alpha$ & $1.4 \mathrm{E}-03 \pm 5.6 \mathrm{E}-04$ \\
& $07 / 29 / 08$ & $08 / 12 / 08$ & gross $\beta$ & $1.5 \mathrm{E}-02 \pm 2.1 \mathrm{E}-03$ \\
& $08 / 12 / 08$ & $08 / 26 / 08$ & gross $\alpha$ & $1.1 \mathrm{E}-03 \pm 5.2 \mathrm{E}-04$ \\
& $08 / 12 / 08$ & $08 / 26 / 08$ & gross $\beta$ & $1.6 \mathrm{E}-02 \pm 2.2 \mathrm{E}-03$ \\
& $08 / 26 / 08$ & $09 / 09 / 08$ & gross $\alpha$ & $5.8 \mathrm{E}-04 \pm 4.9 \mathrm{E}-04$ \\
& $08 / 26 / 08$ & $09 / 09 / 08$ & gross $\beta$ & $1.4 \mathrm{E}-02 \pm 2.0 \mathrm{E}-03$ \\
& $09 / 09 / 08$ & $09 / 23 / 08$ & gross $\alpha$ & $1.9 \mathrm{E}-03 \pm 6.8 \mathrm{E}-04$ \\
& $09 / 09 / 08$ & $09 / 23 / 08$ & gross $\beta$ & $2.9 \mathrm{E}-02 \pm 3.4 \mathrm{E}-03$ \\
& $09 / 23 / 08$ & $10 / 08 / 08$ & gross $\alpha$ & $2.0 \mathrm{E}-03 \pm 6.7 \mathrm{E}-04$ \\
& $09 / 23 / 08$ & $10 / 08 / 08$ & gross $\beta$ & $2.1 \mathrm{E}-02 \pm 2.6 \mathrm{E}-03$ \\
& $10 / 08 / 08$ & $10 / 21 / 08$ & gross $\alpha$ & $1.8 \mathrm{E}-03 \pm 6.7 \mathrm{E}-04$ \\
& $10 / 08 / 08$ & $10 / 21 / 08$ & gross $\beta$ & $2.5 \mathrm{E}-02 \pm 3.1 \mathrm{E}-03$ \\
& $10 / 21 / 08$ & $11 / 04 / 08$ & gross $\alpha$ & $2.8 \mathrm{E}-03 \pm 8.3 \mathrm{E}-04$ \\
& $10 / 21 / 08$ & $11 / 04 / 08$ & gross $\beta$ & $4.3 \mathrm{E}-02 \pm 4.6 \mathrm{E}-03$
\end{tabular}

\begin{tabular}{ccccc} 
Location & Composite Period & Isotope & Result \pm Uncertainty & RQ* \\
\hline N573 & $07 / 21 / 08$ to $11 / 04 / 08$ & ${ }^{60} \mathrm{Co}$ & $-5.5 \mathrm{E}-05 \pm 1.3 \mathrm{E}-04$ & $\mathrm{U}$ \\
& ${ }^{134} \mathrm{Cs}$ & $1.3 \mathrm{E}-04 \pm 1.4 \mathrm{E}-04$ & $\mathrm{U}$ \\
& ${ }^{137} \mathrm{Cs}$ & $-7.6 \mathrm{E}-05 \pm 1.1 \mathrm{E}-04$ & $\mathrm{U}$ \\
& ${ }^{152} \mathrm{Eu}$ & $-1.1 \mathrm{E}-04 \pm 2.9 \mathrm{E}-04$ & $\mathrm{U}$ \\
& ${ }^{154} \mathrm{Eu}$ & $6.2 \mathrm{E}-05 \pm 4.1 \mathrm{E}-04$ & $\mathrm{U}$ \\
& ${ }^{155} \mathrm{Eu}$ & $1.5 \mathrm{E}-05 \pm 1.5 \mathrm{E}-04$ & $\mathrm{U}$ \\
& ${ }^{238} \mathrm{Pu}$ & $9.9 \mathrm{E}-06 \pm 2.3 \mathrm{E}-05$ & $\mathrm{U}$ \\
& ${ }^{239 / 240} \mathrm{Pu}$ & $4.2 \mathrm{E}-06 \pm 6.5 \mathrm{E}-06$ & $\mathrm{U}$ \\
& ${ }^{106} \mathrm{Ru}$ & $-2.2 \mathrm{E}-04 \pm 9.9 \mathrm{E}-04$ & $\mathrm{U}$ \\
& ${ }^{125} \mathrm{Sb}$ & $2.6 \mathrm{E}-05 \pm 2.4 \mathrm{E}-04$ & $\mathrm{U}$ \\
& ${ }^{90} \mathrm{Sr}$ & $-2.5 \mathrm{E}-04 \pm 2.5 \mathrm{E}-04$ & $\mathrm{U}$ \\
& ${ }^{234} \mathrm{U}$ & $1.5 \mathrm{E}-05 \pm 1.1 \mathrm{E}-05$ & \\
& ${ }^{235} \mathrm{U}$ & $5.5 \mathrm{E}-06 \pm 6.9 \mathrm{E}-06$ & $\mathrm{U}$
\end{tabular}


Table 2-4. Near-Facility Air Sampling Results, 2008 (pCi/m³ \pm total analytical uncertainty). (Sheet 48 of 82)

\begin{tabular}{|c|c|c|c|c|c|c|c|c|c|c|}
\hline Location & Sample On & Sample Off & Isotope & Result \pm Uncertainty & Location & Composite & e Period & Isotope & Result \pm Uncertainty & $\mathbf{R Q *}$ \\
\hline N155 & $12 / 31 / 07$ & $01 / 14 / 08$ & gross $\alpha$ & $8.1 \mathrm{E}-04 \pm 5.5 \mathrm{E}-04$ & N155 & $12 / 31 / 07$ to & $06 / 30 / 08$ & ${ }^{60} \mathrm{Co}$ & $-5.6 \mathrm{E}-05 \pm 7.7 \mathrm{E}-05$ & $\mathrm{U}$ \\
\hline \multirow[t]{51}{*}{$(200-W)$} & $12 / 31 / 07$ & 01/14/08 & gross $\beta$ & $8.7 \mathrm{E}-03 \pm 1.4 \mathrm{E}-03$ & & & & ${ }^{134} \mathrm{Cs}$ & $-3.0 \mathrm{E}-05 \pm 7.3 \mathrm{E}-05$ & $\mathrm{U}$ \\
\hline & $01 / 14 / 08$ & 01/29/08 & gross $\alpha$ & $8.5 \mathrm{E}-04 \pm 5.7 \mathrm{E}-04$ & & & & ${ }^{137} \mathrm{Cs}$ & $1.1 \mathrm{E}-05 \pm 6.8 \mathrm{E}-05$ & U \\
\hline & $01 / 14 / 08$ & 01/29/08 & gross $\beta$ & $2.0 \mathrm{E}-02 \pm 2.3 \mathrm{E}-03$ & & & & ${ }^{152} \mathrm{Eu}$ & $8.5 \mathrm{E}-05 \pm 1.6 \mathrm{E}-04$ & U \\
\hline & 01/29/08 & $02 / 12 / 08$ & gross $\alpha$ & $1.8 \mathrm{E}-04 \pm 3.7 \mathrm{E}-04$ & & & & ${ }^{154} \mathrm{Eu}$ & $-5.7 \mathrm{E}-05 \pm 2.2 \mathrm{E}-04$ & $\mathrm{U}$ \\
\hline & $01 / 29 / 08$ & $02 / 12 / 08$ & gross $\beta$ & $4.3 \mathrm{E}-03 \pm 9.3 \mathrm{E}-04$ & & & & ${ }^{155} \mathrm{Eu}$ & $1.4 \mathrm{E}-05 \pm 1.4 \mathrm{E}-04$ & U \\
\hline & $02 / 12 / 08$ & $02 / 25 / 08$ & gross $\alpha$ & $1.4 \mathrm{E}-03 \pm 5.7 \mathrm{E}-04$ & & & & ${ }^{238} \mathrm{Pu}$ & $4.9 \mathrm{E}-06 \pm 9.3 \mathrm{E}-06$ & $\mathrm{U}$ \\
\hline & $02 / 12 / 08$ & $02 / 25 / 08$ & gross $\beta$ & $2.8 \mathrm{E}-02 \pm 2.9 \mathrm{E}-03$ & & & & ${ }^{239 / 240} \mathrm{Pu}$ & $1.1 \mathrm{E}-05 \pm 6.9 \mathrm{E}-06$ & \\
\hline & $02 / 25 / 08$ & 03/11/08 & gross $\alpha$ & $7.0 \mathrm{E}-04 \pm 5.1 \mathrm{E}-04$ & & & & ${ }^{106} \mathrm{Ru}$ & $2.0 \mathrm{E}-05 \pm 2.0 \mathrm{E}-04$ & $\mathrm{U}$ \\
\hline & $02 / 25 / 08$ & 03/11/08 & gross $\beta$ & $1.8 \mathrm{E}-02 \pm 2.1 \mathrm{E}-03$ & & & & ${ }^{125} \mathrm{Sb}$ & $-7.1 \mathrm{E}-05 \pm 1.3 \mathrm{E}-04$ & U \\
\hline & 03/11/08 & 03/25/08 & gross $\alpha$ & $3.9 \mathrm{E}-04 \pm 4.4 \mathrm{E}-04$ & & & & ${ }^{90} \mathrm{Sr}$ & $-1.6 \mathrm{E}-04 \pm 1.6 \mathrm{E}-04$ & $\mathrm{U}$ \\
\hline & 03/11/08 & $03 / 25 / 08$ & gross $\beta$ & $7.7 \mathrm{E}-03 \pm 1.3 \mathrm{E}-03$ & & & & ${ }^{234} \mathrm{U}$ & $9.1 \mathrm{E}-06 \pm 7.0 \mathrm{E}-06$ & \\
\hline & 03/25/08 & $04 / 07 / 08$ & gross $\alpha$ & $3.0 \mathrm{E}-04 \pm 4.3 \mathrm{E}-04$ & & & & ${ }^{235} \mathrm{U}$ & $1.5 \mathrm{E}-06 \pm 2.2 \mathrm{E}-06$ & $\mathrm{U}$ \\
\hline & 03/25/08 & $04 / 07 / 08$ & gross $\beta$ & $7.0 \mathrm{E}-03 \pm 1.2 \mathrm{E}-03$ & & & & ${ }^{238} \mathrm{U}$ & $9.8 \mathrm{E}-06 \pm 6.4 \mathrm{E}-06$ & \\
\hline & 04/07/08 & $04 / 21 / 08$ & gross $\alpha$ & $8.6 \mathrm{E}-04 \pm 5.8 \mathrm{E}-04$ & & & & & & \\
\hline & $04 / 07 / 08$ & $04 / 21 / 08$ & gross $\beta$ & $1.0 \mathrm{E}-02 \pm 1.5 \mathrm{E}-03$ & N155 & $06 / 30 / 08$ to & $12 / 29 / 08$ & ${ }^{60} \mathrm{Co}$ & $-2.8 \mathrm{E}-07 \pm 2.8 \mathrm{E}-06$ & $\mathrm{U}$ \\
\hline & $04 / 21 / 08$ & 05/06/08 & gross $\alpha$ & $5.9 \mathrm{E}-04 \pm 4.7 \mathrm{E}-04$ & & & & ${ }^{134} \mathrm{Cs}$ & $-2.1 \mathrm{E}-05 \pm 7.8 \mathrm{E}-05$ & $\mathrm{U}$ \\
\hline & $04 / 21 / 08$ & 05/06/08 & gross $\beta$ & $1.3 \mathrm{E}-02 \pm 1.6 \mathrm{E}-03$ & & & & ${ }^{137} \mathrm{Cs}$ & $1.0 \mathrm{E}-04 \pm 9.0 \mathrm{E}-05$ & $\mathrm{U}$ \\
\hline & 05/06/08 & 05/19/08 & gross $\alpha$ & $1.6 \mathrm{E}-03 \pm 6.4 \mathrm{E}-04$ & & & & ${ }^{152} \mathrm{Eu}$ & $3.4 \mathrm{E}-05 \pm 1.7 \mathrm{E}-04$ & U \\
\hline & 05/06/08 & 05/19/08 & gross $\beta$ & $1.2 \mathrm{E}-02 \pm 1.7 \mathrm{E}-03$ & & & & ${ }^{154} \mathrm{Eu}$ & $-2.1 \mathrm{E}-04 \pm 2.4 \mathrm{E}-04$ & $\mathrm{U}$ \\
\hline & 05/19/08 & 06/02/08 & gross $\alpha$ & $7.4 \mathrm{E}-04 \pm 5.3 \mathrm{E}-04$ & & & & ${ }^{155} \mathrm{Eu}$ & $3.3 \mathrm{E}-05 \pm 1.7 \mathrm{E}-04$ & U \\
\hline & 05/19/08 & $06 / 02 / 08$ & gross $\beta$ & $8.2 \mathrm{E}-03 \pm 1.3 \mathrm{E}-03$ & & & & ${ }^{238} \mathrm{Pu}$ & $6.2 \mathrm{E}-06 \pm 1.1 \mathrm{E}-05$ & $\mathrm{U}$ \\
\hline & $06 / 02 / 08$ & 06/16/08 & gross $\alpha$ & $5.1 \mathrm{E}-04 \pm 4.9 \mathrm{E}-04$ & & & & ${ }^{239 / 240} \mathrm{Pu}$ & $3.9 \mathrm{E}-06 \pm 4.4 \mathrm{E}-06$ & $\mathrm{U}$ \\
\hline & $06 / 02 / 08$ & 06/16/08 & gross $\beta$ & $8.2 \mathrm{E}-03 \pm 1.3 \mathrm{E}-03$ & & & & ${ }^{106} \mathrm{Ru}$ & $-3.8 \mathrm{E}-04 \pm 5.7 \mathrm{E}-04$ & $\mathrm{U}$ \\
\hline & $06 / 16 / 08$ & 06/30/08 & gross $\alpha$ & $1.4 \mathrm{E}-03 \pm 5.8 \mathrm{E}-04$ & & & & ${ }^{125} \mathrm{Sb}$ & $1.7 \mathrm{E}-04 \pm 1.6 \mathrm{E}-04$ & $\mathrm{U}$ \\
\hline & $06 / 16 / 08$ & $06 / 30 / 08$ & gross $\beta$ & $1.2 \mathrm{E}-02 \pm 1.6 \mathrm{E}-03$ & & & & ${ }^{90} \mathrm{Sr}$ & $-1.2 \mathrm{E}-04 \pm 1.3 \mathrm{E}-04$ & $\mathrm{U}$ \\
\hline & $06 / 30 / 08$ & $07 / 14 / 08$ & gross $\alpha$ & $1.5 \mathrm{E}-03 \pm 6.6 \mathrm{E}-04$ & & & & ${ }^{234} \mathrm{U}$ & $1.2 \mathrm{E}-05 \pm 8.2 \mathrm{E}-06$ & \\
\hline & $06 / 30 / 08$ & 07/14/08 & gross $\beta$ & $1.6 \mathrm{E}-02 \pm 2.0 \mathrm{E}-03$ & & & & ${ }^{235} \mathrm{U}$ & $3.7 \mathrm{E}-06 \pm 4.2 \mathrm{E}-06$ & $\mathrm{U}$ \\
\hline & $07 / 14 / 08$ & 07/28/08 & gross $\alpha$ & $7.4 \mathrm{E}-04 \pm 5.3 \mathrm{E}-04$ & & & & ${ }^{238} \mathrm{U}$ & $1.6 \mathrm{E}-05 \pm 8.9 \mathrm{E}-06$ & \\
\hline & $07 / 14 / 08$ & $07 / 28 / 08$ & gross $\beta$ & $1.4 \mathrm{E}-02 \pm 1.8 \mathrm{E}-03$ & & & & & & \\
\hline & 07/28/08 & 08/11/08 & gross $\alpha$ & $7.2 \mathrm{E}-04 \pm 5.2 \mathrm{E}-04$ & & & & & & \\
\hline & $07 / 28 / 08$ & $08 / 11 / 08$ & gross $\beta$ & $1.2 \mathrm{E}-02 \pm 1.6 \mathrm{E}-03$ & & & & & & \\
\hline & $08 / 11 / 08$ & $08 / 25 / 08$ & gross $\alpha$ & $8.3 \mathrm{E}-04 \pm 5.5 \mathrm{E}-04$ & & & & & & \\
\hline & 08/11/08 & 08/25/08 & gross $\beta$ & $1.2 \mathrm{E}-02 \pm 1.6 \mathrm{E}-03$ & & & & & & \\
\hline & $08 / 25 / 08$ & 09/08/08 & gross $\alpha$ & $5.2 \mathrm{E}-04 \pm 4.7 \mathrm{E}-04$ & & & & & & \\
\hline & $08 / 25 / 08$ & 09/08/08 & gross $\beta$ & $1.0 \mathrm{E}-02 \pm 1.5 \mathrm{E}-03$ & & & & & & \\
\hline & 09/08/08 & 09/22/08 & gross $\alpha$ & $1.2 \mathrm{E}-03 \pm 5.2 \mathrm{E}-04$ & & & & & & \\
\hline & 09/08/08 & $09 / 22 / 08$ & gross $\beta$ & $2.3 \mathrm{E}-02 \pm 2.5 \mathrm{E}-03$ & & & & & & \\
\hline & $09 / 22 / 08$ & $10 / 07 / 08$ & gross $\alpha$ & $1.3 \mathrm{E}-03 \pm 5.2 \mathrm{E}-04$ & & & & & & \\
\hline & $09 / 22 / 08$ & $10 / 07 / 08$ & gross $\beta$ & $2.6 \mathrm{E}-02 \pm 2.7 \mathrm{E}-03$ & & & & & & \\
\hline & $10 / 07 / 08$ & $10 / 20 / 08$ & gross $\alpha$ & $1.5 \mathrm{E}-03 \pm 6.0 \mathrm{E}-04$ & & & & & & \\
\hline & $10 / 07 / 08$ & $10 / 20 / 08$ & gross $\beta$ & $1.8 \mathrm{E}-02 \pm 2.2 \mathrm{E}-03$ & & & & & & \\
\hline & $10 / 20 / 08$ & $11 / 03 / 08$ & gross $\alpha$ & $1.5 \mathrm{E}-03 \pm 6.0 \mathrm{E}-04$ & & & & & & \\
\hline & $10 / 20 / 08$ & $11 / 03 / 08$ & gross $\beta$ & $3.8 \mathrm{E}-02 \pm 3.7 \mathrm{E}-03$ & & & & & & \\
\hline & $11 / 03 / 08$ & $11 / 17 / 08$ & gross $\alpha$ & $1.9 \mathrm{E}-03 \pm 6.8 \mathrm{E}-04$ & & & & & & \\
\hline & $11 / 03 / 08$ & $11 / 17 / 08$ & gross $\beta$ & $1.6 \mathrm{E}-02 \pm 2.0 \mathrm{E}-03$ & & & & & & \\
\hline & $11 / 17 / 08$ & $12 / 01 / 08$ & gross $\alpha$ & $1.6 \mathrm{E}-03 \pm 6.5 \mathrm{E}-04$ & & & & & & \\
\hline & $11 / 17 / 08$ & $12 / 01 / 08$ & gross $\beta$ & $3.7 \mathrm{E}-02 \pm 3.7 \mathrm{E}-03$ & & & & & & \\
\hline & $12 / 01 / 08$ & $12 / 15 / 08$ & gross $\alpha$ & $1.6 \mathrm{E}-03 \pm 6.1 \mathrm{E}-04$ & & & & & & \\
\hline & $12 / 01 / 08$ & $12 / 15 / 08$ & gross $\beta$ & $1.8 \mathrm{E}-02 \pm 2.1 \mathrm{E}-03$ & & & & & & \\
\hline & $12 / 15 / 08$ & $12 / 29 / 08$ & gross $\alpha$ & $1.5 \mathrm{E}-03 \pm 5.9 \mathrm{E}-04$ & & & & & & \\
\hline & $12 / 15 / 08$ & $12 / 29 / 08$ & gross $\beta$ & $3.9 \mathrm{E}-02 \pm 3.7 \mathrm{E}-03$ & & & & & & \\
\hline
\end{tabular}

$\overline{\mathrm{RQ}}=$ Result Qualifier. $\mathrm{U}=$ The analyte was analyzed for but not detected. 
Table 2-4. Near-Facility Air Sampling Results, 2008 (pCi/m³ \pm total analytical uncertainty). (Sheet 49 of 82)

\begin{tabular}{|c|c|c|c|c|c|c|c|c|c|c|}
\hline Location & Sample On & Sample Off & Isotope & Result \pm Uncertainty & Location & Composite & e Period & Isotope & Result \pm Uncertainty & RQ* \\
\hline N161 & $12 / 31 / 07$ & $01 / 16 / 08$ & gross $\alpha$ & $7.1 \mathrm{E}-04 \pm 4.8 \mathrm{E}-04$ & N161 & $12 / 31 / 07$ to & $06 / 30 / 08$ & ${ }^{60} \mathrm{Co}$ & $6.9 \mathrm{E}-05 \pm 6.6 \mathrm{E}-05$ & $\bar{U}$ \\
\hline \multirow[t]{51}{*}{$(200-W)$} & $12 / 31 / 07$ & 01/16/08 & gross $\beta$ & $1.1 \mathrm{E}-02 \pm 1.5 \mathrm{E}-03$ & & & & ${ }^{134} \mathrm{Cs}$ & $-8.8 \mathrm{E}-06 \pm 6.7 \mathrm{E}-05$ & $\mathrm{U}$ \\
\hline & $01 / 16 / 08$ & 01/29/08 & gross $\alpha$ & $3.0 \mathrm{E}-03 \pm 8.9 \mathrm{E}-04$ & & & & ${ }^{137} \mathrm{Cs}$ & $1.6 \mathrm{E}-06 \pm 1.6 \mathrm{E}-05$ & U \\
\hline & $01 / 16 / 08$ & 01/29/08 & gross $\beta$ & $3.6 \mathrm{E}-02 \pm 3.6 \mathrm{E}-03$ & & & & ${ }^{152} \mathrm{Eu}$ & $1.0 \mathrm{E}-04 \pm 1.4 \mathrm{E}-04$ & U \\
\hline & $01 / 29 / 08$ & $02 / 12 / 08$ & gross $\alpha$ & $2.8 \mathrm{E}-04 \pm 4.0 \mathrm{E}-04$ & & & & ${ }^{154} \mathrm{Eu}$ & $3.6 \mathrm{E}-05 \pm 2.0 \mathrm{E}-04$ & $\mathrm{U}$ \\
\hline & $01 / 29 / 08$ & $02 / 12 / 08$ & gross $\beta$ & $3.9 \mathrm{E}-03 \pm 8.6 \mathrm{E}-04$ & & & & ${ }^{155} \mathrm{Eu}$ & $6.6 \mathrm{E}-05 \pm 1.3 \mathrm{E}-04$ & U \\
\hline & $02 / 12 / 08$ & $02 / 25 / 08$ & gross $\alpha$ & $2.0 \mathrm{E}-03 \pm 7.1 \mathrm{E}-04$ & & & & ${ }^{238} \mathrm{Pu}$ & $-3.8 \mathrm{E}-06 \pm 1.1 \mathrm{E}-05$ & $\mathrm{U}$ \\
\hline & $02 / 12 / 08$ & $02 / 25 / 08$ & gross $\beta$ & $2.6 \mathrm{E}-02 \pm 2.9 \mathrm{E}-03$ & & & & ${ }^{239 / 240} \mathrm{Pu}$ & $4.5 \mathrm{E}-06 \pm 4.2 \mathrm{E}-06$ & $\mathrm{U}$ \\
\hline & $02 / 25 / 08$ & 03/11/08 & gross $\alpha$ & $1.0 \mathrm{E}-03 \pm 4.7 \mathrm{E}-04$ & & & & ${ }^{106} \mathrm{Ru}$ & $1.3 \mathrm{E}-04 \pm 5.0 \mathrm{E}-04$ & $\mathrm{U}$ \\
\hline & $02 / 25 / 08$ & 03/11/08 & gross $\beta$ & $1.6 \mathrm{E}-02 \pm 1.9 \mathrm{E}-03$ & & & & ${ }^{125} \mathrm{Sb}$ & $-8.1 \mathrm{E}-05 \pm 1.4 \mathrm{E}-04$ & $\mathrm{U}$ \\
\hline & 03/11/08 & $03 / 25 / 08$ & gross $\alpha$ & $5.0 \mathrm{E}-04 \pm 4.7 \mathrm{E}-04$ & & & & ${ }^{90} \mathrm{Sr}$ & $-2.5 \mathrm{E}-04 \pm 2.6 \mathrm{E}-04$ & \\
\hline & 03/11/08 & $03 / 25 / 08$ & gross $\beta$ & $8.6 \mathrm{E}-03 \pm 1.4 \mathrm{E}-03$ & & & & ${ }^{234} \mathrm{U}$ & $1.4 \mathrm{E}-05 \pm 8.2 \mathrm{E}-06$ & \\
\hline & $03 / 25 / 08$ & $04 / 07 / 08$ & gross $\alpha$ & $4.2 \mathrm{E}-04 \pm 4.7 \mathrm{E}-04$ & & & & ${ }^{235} \mathrm{U}$ & $1.4 \mathrm{E}-06 \pm 2.9 \mathrm{E}-06$ & U \\
\hline & 03/25/08 & $04 / 07 / 08$ & gross $\beta$ & $7.4 \mathrm{E}-03 \pm 1.3 \mathrm{E}-03$ & & & & ${ }^{238} \mathrm{U}$ & $9.8 \mathrm{E}-06 \pm 6.5 \mathrm{E}-06$ & \\
\hline & 04/07/08 & $04 / 21 / 08$ & gross $\alpha$ & $1.5 \mathrm{E}-03 \pm 5.9 \mathrm{E}-04$ & & & & & & \\
\hline & $04 / 07 / 08$ & $04 / 21 / 08$ & gross $\beta$ & $8.1 \mathrm{E}-03 \pm 1.3 \mathrm{E}-03$ & N161 & $06 / 30 / 08$ to & $12 / 29 / 08$ & ${ }^{60} \mathrm{Co}$ & $-5.0 \mathrm{E}-06 \pm 5.0 \mathrm{E}-05$ & $\mathrm{U}$ \\
\hline & $04 / 21 / 08$ & $05 / 05 / 08$ & gross $\alpha$ & $2.1 \mathrm{E}-03 \pm 6.8 \mathrm{E}-04$ & & & & ${ }^{134} \mathrm{Cs}$ & $1.8 \mathrm{E}-06 \pm 1.8 \mathrm{E}-05$ & $\mathrm{U}$ \\
\hline & $04 / 21 / 08$ & 05/05/08 & gross $\beta$ & $1.4 \mathrm{E}-02 \pm 1.8 \mathrm{E}-03$ & & & & ${ }^{137} \mathrm{Cs}$ & $4.4 \mathrm{E}-05 \pm 6.7 \mathrm{E}-05$ & $\mathrm{U}$ \\
\hline & 05/05/08 & 05/19/08 & gross $\alpha$ & $1.1 \mathrm{E}-03 \pm 5.0 \mathrm{E}-04$ & & & & ${ }^{152} \mathrm{Eu}$ & $6.9 \mathrm{E}-05 \pm 1.6 \mathrm{E}-04$ & U \\
\hline & 05/05/08 & 05/19/08 & gross $\beta$ & $1.2 \mathrm{E}-02 \pm 1.6 \mathrm{E}-03$ & & & & ${ }^{154} \mathrm{Eu}$ & $-3.5 \mathrm{E}-05 \pm 2.0 \mathrm{E}-04$ & $\mathrm{U}$ \\
\hline & 05/19/08 & 06/02/08 & gross $\alpha$ & $1.4 \mathrm{E}-03 \pm 6.5 \mathrm{E}-04$ & & & & ${ }^{155} \mathrm{Eu}$ & $-1.2 \mathrm{E}-04 \pm 1.9 \mathrm{E}-04$ & U \\
\hline & 05/19/08 & $06 / 02 / 08$ & gross $\beta$ & $1.4 \mathrm{E}-02 \pm 2.0 \mathrm{E}-03$ & & & & ${ }^{238} \mathrm{Pu}$ & $8.4 \mathrm{E}-06 \pm 1.2 \mathrm{E}-05$ & $\mathrm{U}$ \\
\hline & $06 / 02 / 08$ & $06 / 16 / 08$ & gross $\alpha$ & $7.4 \mathrm{E}-04 \pm 5.5 \mathrm{E}-04$ & & & & ${ }^{239 / 240} \mathrm{Pu}$ & $4.2 \mathrm{E}-06 \pm 5.8 \mathrm{E}-06$ & $\mathrm{U}$ \\
\hline & $06 / 02 / 08$ & 06/16/08 & gross $\beta$ & $7.4 \mathrm{E}-03 \pm 1.3 \mathrm{E}-03$ & & & & ${ }^{103} \mathrm{Ru}$ & $-3.4 \mathrm{E}-05 \pm 5.7 \mathrm{E}-05$ & $\mathrm{U}$ \\
\hline & $06 / 16 / 08$ & 06/30/08 & gross $\alpha$ & $1.4 \mathrm{E}-03 \pm 5.8 \mathrm{E}-04$ & & & & ${ }^{106} \mathrm{Ru}$ & $-4.0 \mathrm{E}-04 \pm 5.6 \mathrm{E}-04$ & $\mathrm{U}$ \\
\hline & $06 / 16 / 08$ & 06/30/08 & gross $\beta$ & $1.2 \mathrm{E}-02 \pm 1.6 \mathrm{E}-03$ & & & & ${ }^{125} \mathrm{Sb}$ & $1.3 \mathrm{E}-04 \pm 1.5 \mathrm{E}-04$ & $\mathrm{U}$ \\
\hline & $06 / 30 / 08$ & $07 / 14 / 08$ & gross $\alpha$ & $1.1 \mathrm{E}-03 \pm 7.1 \mathrm{E}-04$ & & & & ${ }^{113} \mathrm{Sn}$ & $3.2 \mathrm{E}-06 \pm 3.2 \mathrm{E}-05$ & $\mathrm{U}$ \\
\hline & $06 / 30 / 08$ & 07/14/08 & gross $\beta$ & $1.3 \mathrm{E}-02 \pm 1.8 \mathrm{E}-03$ & & & & ${ }^{90} \mathrm{Sr}$ & $-4.0 \mathrm{E}-05 \pm 4.1 \mathrm{E}-05$ & $\mathrm{U}$ \\
\hline & 07/14/08 & 07/28/08 & gross $\alpha$ & $1.1 \mathrm{E}-03 \pm 5.0 \mathrm{E}-04$ & & & & ${ }^{234} \mathrm{U}$ & $2.1 \mathrm{E}-05 \pm 1.1 \mathrm{E}-05$ & \\
\hline & $07 / 14 / 08$ & $07 / 28 / 08$ & gross $\beta$ & $1.5 \mathrm{E}-02 \pm 1.9 \mathrm{E}-03$ & & & & ${ }^{235} \mathrm{U}$ & $5.1 \mathrm{E}-06 \pm 4.6 \mathrm{E}-06$ & \\
\hline & $07 / 28 / 08$ & 08/11/08 & gross $\alpha$ & $1.0 \mathrm{E}-03 \pm 6.7 \mathrm{E}-04$ & & & & ${ }^{238} \mathrm{U}$ & $1.1 \mathrm{E}-05 \pm 7.4 \mathrm{E}-06$ & \\
\hline & $07 / 28 / 08$ & 08/11/08 & gross $\beta$ & $1.4 \mathrm{E}-02 \pm 1.9 \mathrm{E}-03$ & & & & ${ }^{65} \mathrm{Zn}$ & $3.4 \mathrm{E}-05 \pm 1.5 \mathrm{E}-04$ & $\mathrm{U}$ \\
\hline & $08 / 11 / 08$ & $08 / 25 / 08$ & gross $\alpha$ & $7.5 \mathrm{E}-04 \pm 8.1 \mathrm{E}-04$ & & & & & & \\
\hline & 08/11/08 & 08/25/08 & gross $\beta$ & $3.1 \mathrm{E}-02 \pm 3.7 \mathrm{E}-03$ & & & & & & \\
\hline & $08 / 25 / 08$ & 09/08/08 & gross $\alpha$ & $5.1 \mathrm{E}-04 \pm 4.6 \mathrm{E}-04$ & & & & & & \\
\hline & $08 / 25 / 08$ & 09/08/08 & gross $\beta$ & $1.0 \mathrm{E}-02 \pm 1.5 \mathrm{E}-03$ & & & & & & \\
\hline & 09/08/08 & 09/22/08 & gross $\alpha$ & $1.2 \mathrm{E}-03 \pm 7.1 \mathrm{E}-04$ & & & & & & \\
\hline & 09/08/08 & $09 / 22 / 08$ & gross $\beta$ & $2.5 \mathrm{E}-02 \pm 2.9 \mathrm{E}-03$ & & & & & & \\
\hline & $09 / 22 / 08$ & $10 / 07 / 08$ & gross $\alpha$ & $1.9 \mathrm{E}-03 \pm 7.1 \mathrm{E}-04$ & & & & & & \\
\hline & $09 / 22 / 08$ & $10 / 07 / 08$ & gross $\beta$ & $2.9 \mathrm{E}-02 \pm 3.2 \mathrm{E}-03$ & & & & & & \\
\hline & $10 / 07 / 08$ & $10 / 20 / 08$ & gross $\alpha$ & $2.2 \mathrm{E}-03 \pm 1.3 \mathrm{E}-03$ & & & & & & \\
\hline & $10 / 07 / 08$ & $10 / 20 / 08$ & gross $\beta$ & $3.6 \mathrm{E}-02 \pm 4.4 \mathrm{E}-03$ & & & & & & \\
\hline & $10 / 20 / 08$ & $11 / 03 / 08$ & gross $\alpha$ & $2.6 \mathrm{E}-03 \pm 7.8 \mathrm{E}-04$ & & & & & & \\
\hline & $10 / 20 / 08$ & $11 / 03 / 08$ & gross $\beta$ & $3.6 \mathrm{E}-02 \pm 3.5 \mathrm{E}-03$ & & & & & & \\
\hline & $11 / 03 / 08$ & $11 / 17 / 08$ & gross $\alpha$ & $1.4 \mathrm{E}-03 \pm 5.7 \mathrm{E}-04$ & & & & & & \\
\hline & $11 / 03 / 08$ & $11 / 17 / 08$ & gross $\beta$ & $1.6 \mathrm{E}-02 \pm 2.0 \mathrm{E}-03$ & & & & & & \\
\hline & $11 / 17 / 08$ & $12 / 01 / 08$ & gross $\alpha$ & $1.3 \mathrm{E}-03 \pm 5.8 \mathrm{E}-04$ & & & & & & \\
\hline & $11 / 17 / 08$ & $12 / 01 / 08$ & gross $\beta$ & $3.2 \mathrm{E}-02 \pm 3.3 \mathrm{E}-03$ & & & & & & \\
\hline & $12 / 01 / 08$ & $12 / 15 / 08$ & gross $\alpha$ & $1.8 \mathrm{E}-03 \pm 6.7 \mathrm{E}-04$ & & & & & & \\
\hline & $12 / 01 / 08$ & $12 / 15 / 08$ & gross $\beta$ & $1.9 \mathrm{E}-02 \pm 2.3 \mathrm{E}-03$ & & & & & & \\
\hline & $12 / 15 / 08$ & $12 / 29 / 08$ & gross $\alpha$ & $1.4 \mathrm{E}-03 \pm 5.7 \mathrm{E}-04$ & & & & & & \\
\hline & $12 / 15 / 08$ & $12 / 29 / 08$ & gross $\beta$ & $4.0 \mathrm{E}-02 \pm 3.8 \mathrm{E}-03$ & & & & & & \\
\hline
\end{tabular}

$\overline{\mathrm{RQ}}=$ Result Qualifier. $\mathrm{U}=$ The analyte was analyzed for but not detected. 
Table 2-4. Near-Facility Air Sampling Results, 2008 (pCi/m³ \pm total analytical uncertainty). (Sheet 50 of 82)

\begin{tabular}{|c|c|c|c|c|c|c|c|c|c|}
\hline Location & Sample On & Sample Off & Isotope & Result \pm Uncertainty & Location & Composite Period & Isotope & Result \pm Uncertainty & RQ* \\
\hline N165 & $12 / 31 / 07$ & $01 / 14 / 08$ & gross $\alpha$ & $1.3 \mathrm{E}-03 \pm 5.4 \mathrm{E}-04$ & N165 & $12 / 31 / 07$ to $06 / 30 / 08$ & ${ }^{241} \mathrm{Am}$ & $3.1 \mathrm{E}-04 \pm 1.1 \mathrm{E}-04$ & \\
\hline \multirow[t]{47}{*}{$(200-W)$} & $12 / 31 / 07$ & 01/14/08 & gross $\beta$ & $9.5 \mathrm{E}-03 \pm 1.4 \mathrm{E}-03$ & & & ${ }^{60} \mathrm{Co}$ & $-6.3 \mathrm{E}-06 \pm 6.3 \mathrm{E}-05$ & $\mathrm{U}$ \\
\hline & $01 / 14 / 08$ & 01/29/08 & gross $\alpha$ & $1.9 \mathrm{E}-03 \pm 7.6 \mathrm{E}-04$ & & & ${ }^{134} \mathrm{Cs}$ & $-5.0 \mathrm{E}-05 \pm 8.8 \mathrm{E}-05$ & U \\
\hline & $01 / 14 / 08$ & 01/29/08 & gross $\beta$ & $3.6 \mathrm{E}-02 \pm 3.7 \mathrm{E}-03$ & & & ${ }^{137} \mathrm{Cs}$ & $-1.4 \mathrm{E}-05 \pm 8.8 \mathrm{E}-05$ & $\mathrm{U}$ \\
\hline & $01 / 29 / 08$ & $02 / 12 / 08$ & gross $\alpha$ & $5.5 \mathrm{E}-04 \pm 5.1 \mathrm{E}-04$ & & & ${ }^{152} \mathrm{Eu}$ & $1.4 \mathrm{E}-04 \pm 2.2 \mathrm{E}-04$ & $\mathrm{U}$ \\
\hline & $01 / 29 / 08$ & $02 / 12 / 08$ & gross $\beta$ & $4.5 \mathrm{E}-03 \pm 9.6 \mathrm{E}-04$ & & & ${ }^{154} \mathrm{Eu}$ & $-5.5 \mathrm{E}-05 \pm 2.7 \mathrm{E}-04$ & $\mathrm{U}$ \\
\hline & $02 / 12 / 08$ & $02 / 25 / 08$ & gross $\alpha$ & $2.7 \mathrm{E}-03 \pm 8.7 \mathrm{E}-04$ & & & ${ }^{155} \mathrm{Eu}$ & $1.2 \mathrm{E}-04 \pm 2.3 \mathrm{E}-04$ & $\mathrm{U}$ \\
\hline & $02 / 12 / 08$ & $02 / 25 / 08$ & gross $\beta$ & $3.1 \mathrm{E}-02 \pm 3.3 \mathrm{E}-03$ & & & ${ }^{238} \mathrm{Pu}$ & $-1.0 \mathrm{E}-05 \pm 2.4 \mathrm{E}-05$ & $\mathrm{U}$ \\
\hline & $02 / 25 / 08$ & 03/11/08 & gross $\alpha$ & $1.2 \mathrm{E}-03 \pm 5.3 \mathrm{E}-04$ & & & ${ }^{239 / 240} \mathrm{Pu}$ & $4.6 \mathrm{E}-04 \pm 1.8 \mathrm{E}-04$ & \\
\hline & $02 / 25 / 08$ & 03/11/08 & gross $\beta$ & $1.5 \mathrm{E}-02 \pm 1.9 \mathrm{E}-03$ & & & ${ }^{241} \mathrm{Pu}$ & $1.1 \mathrm{E}-03 \pm 6.6 \mathrm{E}-04$ & \\
\hline & 03/11/08 & $03 / 25 / 08$ & gross $\alpha$ & $1.2 \mathrm{E}-03 \pm 7.3 \mathrm{E}-04$ & & & ${ }^{106} \mathrm{Ru}$ & $-7.0 \mathrm{E}-04 \pm 8.0 \mathrm{E}-04$ & U \\
\hline & 03/11/08 & $03 / 25 / 08$ & gross $\beta$ & $8.7 \mathrm{E}-03 \pm 1.5 \mathrm{E}-03$ & & & ${ }^{125} \mathrm{Sb}$ & $-1.4 \mathrm{E}-04 \pm 2.0 \mathrm{E}-04$ & $\mathrm{U}$ \\
\hline & 03/25/08 & $04 / 07 / 08$ & gross $\alpha$ & $1.0 \mathrm{E}-03 \pm 6.7 \mathrm{E}-04$ & & & ${ }^{90} \mathrm{Sr}$ & $-2.1 \mathrm{E}-04 \pm 2.1 \mathrm{E}-04$ & $\mathrm{U}$ \\
\hline & 03/25/08 & $04 / 07 / 08$ & gross $\beta$ & $9.3 \mathrm{E}-03 \pm 1.5 \mathrm{E}-03$ & & & ${ }^{234} \mathrm{U}$ & $1.4 \mathrm{E}-05 \pm 8.7 \mathrm{E}-06$ & \\
\hline & $04 / 07 / 08$ & $04 / 21 / 08$ & gross $\alpha$ & $1.4 \mathrm{E}-03 \pm 5.9 \mathrm{E}-04$ & & & ${ }^{235} \mathrm{U}$ & $1.6 \mathrm{E}-06 \pm 3.2 \mathrm{E}-06$ & $\mathrm{U}$ \\
\hline & $04 / 07 / 08$ & $04 / 21 / 08$ & gross $\beta$ & $1.2 \mathrm{E}-02 \pm 1.6 \mathrm{E}-03$ & & & ${ }^{238} \mathrm{U}$ & $1.1 \mathrm{E}-05 \pm 6.8 \mathrm{E}-06$ & \\
\hline & $04 / 21 / 08$ & $05 / 05 / 08$ & gross $\alpha$ & $1.2 \mathrm{E}-03 \pm 5.3 \mathrm{E}-04$ & & & ${ }^{241} \mathrm{Am}$ & $2.4 \mathrm{E}-05 \pm 2.0 \mathrm{E}-05$ & $\mathrm{U}$ \\
\hline & $04 / 21 / 08$ & $05 / 05 / 08$ & gross $\beta$ & $1.5 \mathrm{E}-02 \pm 1.9 \mathrm{E}-03$ & & & & & \\
\hline & 05/05/08 & 05/19/08 & gross $\alpha$ & $2.8 \mathrm{E}-03 \pm 8.4 \mathrm{E}-04$ & N165 & $06 / 30 / 08$ to $12 / 29 / 08$ & ${ }^{60} \mathrm{Co}$ & $-1.1 \mathrm{E}-04 \pm 2.1 \mathrm{E}-04$ & U \\
\hline & 05/05/08 & 05/19/08 & gross $\beta$ & $1.2 \mathrm{E}-02 \pm 1.7 \mathrm{E}-03$ & & & ${ }^{134} \mathrm{Cs}$ & $2.7 \mathrm{E}-05 \pm 2.3 \mathrm{E}-04$ & $\mathrm{U}$ \\
\hline & 05/19/08 & $06 / 02 / 08$ & gross $\alpha$ & $3.2 \mathrm{E}-03 \pm 8.8 \mathrm{E}-04$ & & & ${ }^{137} \mathrm{Cs}$ & $-1.4 \mathrm{E}-04 \pm 2.0 \mathrm{E}-04$ & $\mathrm{U}$ \\
\hline & 05/19/08 & $06 / 02 / 08$ & gross $\beta$ & $9.8 \mathrm{E}-03 \pm 1.5 \mathrm{E}-03$ & & & ${ }^{152} \mathrm{Eu}$ & $6.9 \mathrm{E}-04 \pm 5.9 \mathrm{E}-04$ & $\mathrm{U}$ \\
\hline & $06 / 02 / 08$ & 06/16/08 & gross $\alpha$ & $1.5 \mathrm{E}-03 \pm 6.1 \mathrm{E}-04$ & & & ${ }^{154} \mathrm{Eu}$ & $-1.8 \mathrm{E}-04 \pm 6.0 \mathrm{E}-04$ & $\mathrm{U}$ \\
\hline & $06 / 02 / 08$ & 06/16/08 & gross $\beta$ & $8.5 \mathrm{E}-03 \pm 1.4 \mathrm{E}-03$ & & & ${ }^{155} \mathrm{Eu}$ & $-6.6 \mathrm{E}-05 \pm 3.8 \mathrm{E}-04$ & $\mathrm{U}$ \\
\hline & 06/16/08 & 06/30/08 & gross $\alpha$ & $1.9 \mathrm{E}-03 \pm 6.8 \mathrm{E}-04$ & & & ${ }^{238} \mathrm{Pu}$ & $-1.2 \mathrm{E}-05 \pm 5.1 \mathrm{E}-05$ & $\mathrm{U}$ \\
\hline & $06 / 16 / 08$ & 06/30/08 & gross $\beta$ & $1.2 \mathrm{E}-02 \pm 1.7 \mathrm{E}-03$ & & & ${ }^{239 / 240} \mathrm{Pu}$ & $2.2 \mathrm{E}-04 \pm 9.3 \mathrm{E}-05$ & \\
\hline & $06 / 30 / 08$ & $07 / 14 / 08$ & gross $\alpha$ & $1.2 \mathrm{E}-02 \pm 4.6 \mathrm{E}-03$ & & & ${ }^{241} \mathrm{Pu}$ & $1.7 \mathrm{E}-03 \pm 1.5 \mathrm{E}-03$ & $\mathrm{U}$ \\
\hline & 06/30/08 & 07/14/08 & gross $\beta$ & $5.8 \mathrm{E}-02 \pm 8.8 \mathrm{E}-03$ & & & ${ }^{103} \mathrm{Ru}$ & $-1.7 \mathrm{E}-04 \pm 1.9 \mathrm{E}-04$ & $\mathrm{U}$ \\
\hline & $08 / 20 / 08$ & 08/25/08 & gross $\alpha$ & $8.0 \mathrm{E}-04 \pm 1.1 \mathrm{E}-03$ & & & ${ }^{106} \mathrm{Ru}$ & $8.9 \mathrm{E}-04 \pm 1.7 \mathrm{E}-03$ & $\mathrm{U}$ \\
\hline & $08 / 20 / 08$ & $08 / 25 / 08$ & gross $\beta$ & $2.3 \mathrm{E}-02 \pm 3.6 \mathrm{E}-03$ & & & ${ }^{125} \mathrm{Sb}$ & $-3.7 \mathrm{E}-04 \pm 5.6 \mathrm{E}-04$ & $\mathrm{U}$ \\
\hline & $08 / 25 / 08$ & 09/08/08 & gross $\alpha$ & $1.7 \mathrm{E}-03 \pm 1.4 \mathrm{E}-03$ & & & ${ }^{113} \mathrm{Sn}$ & $6.4 \mathrm{E}-05 \pm 2.7 \mathrm{E}-04$ & $\mathrm{U}$ \\
\hline & $08 / 25 / 08$ & 09/08/08 & gross $\beta$ & $1.3 \mathrm{E}-02 \pm 2.6 \mathrm{E}-03$ & & & ${ }^{90} \mathrm{Sr}$ & $-2.2 \mathrm{E}-04 \pm 2.2 \mathrm{E}-04$ & $\mathrm{U}$ \\
\hline & 09/08/08 & 09/22/08 & gross $\alpha$ & $7.0 \mathrm{E}-03 \pm 4.3 \mathrm{E}-03$ & & & ${ }^{234} \mathrm{U}$ & $1.4 \mathrm{E}-05 \pm 1.1 \mathrm{E}-05$ & \\
\hline & 09/08/08 & 09/22/08 & gross $\beta$ & $1.2 \mathrm{E}-01 \pm 1.5 \mathrm{E}-02$ & & & ${ }^{235} \mathrm{U}$ & $1.1 \mathrm{E}-05 \pm 8.8 \mathrm{E}-06$ & \\
\hline & $09 / 22 / 08$ & $10 / 07 / 08$ & gross $\alpha$ & $6.4 \mathrm{E}-03 \pm 4.4 \mathrm{E}-03$ & & & ${ }^{238} \mathrm{U}$ & $1.5 \mathrm{E}-05 \pm 1.1 \mathrm{E}-05$ & \\
\hline & $09 / 22 / 08$ & $10 / 07 / 08$ & gross $\beta$ & $1.7 \mathrm{E}-01 \pm 2.0 \mathrm{E}-02$ & & & ${ }^{65} \mathrm{Zn}$ & $5.4 \mathrm{E}-05 \pm 4.9 \mathrm{E}-04$ & $\mathrm{U}$ \\
\hline & $10 / 07 / 08$ & $10 / 20 / 08$ & gross $\alpha$ & $1.9 \mathrm{E}-03 \pm 8.6 \mathrm{E}-04$ & & & & & \\
\hline & $10 / 07 / 08$ & $10 / 20 / 08$ & gross $\beta$ & $3.1 \mathrm{E}-02 \pm 3.7 \mathrm{E}-03$ & & & & & \\
\hline & $10 / 20 / 08$ & $11 / 03 / 08$ & gross $\alpha$ & $3.7 \mathrm{E}-03 \pm 1.1 \mathrm{E}-03$ & & & & & \\
\hline & $10 / 20 / 08$ & $11 / 03 / 08$ & gross $\beta$ & $5.2 \mathrm{E}-02 \pm 5.1 \mathrm{E}-03$ & & & & & \\
\hline & $11 / 03 / 08$ & $11 / 17 / 08$ & gross $\alpha$ & $1.2 \mathrm{E}-03 \pm 5.1 \mathrm{E}-04$ & & & & & \\
\hline & $11 / 03 / 08$ & $11 / 17 / 08$ & gross $\beta$ & $1.8 \mathrm{E}-02 \pm 2.1 \mathrm{E}-03$ & & & & & \\
\hline & $11 / 17 / 08$ & $12 / 01 / 08$ & gross $\alpha$ & $1.0 \mathrm{E}-03 \pm 6.8 \mathrm{E}-04$ & & & & & \\
\hline & $11 / 17 / 08$ & $12 / 01 / 08$ & gross $\beta$ & $4.4 \mathrm{E}-02 \pm 4.2 \mathrm{E}-03$ & & & & & \\
\hline & $12 / 01 / 08$ & $12 / 15 / 08$ & gross $\alpha$ & $1.3 \mathrm{E}-03 \pm 5.5 \mathrm{E}-04$ & & & & & \\
\hline & $12 / 01 / 08$ & $12 / 15 / 08$ & gross $\beta$ & $2.4 \mathrm{E}-02 \pm 2.6 \mathrm{E}-03$ & & & & & \\
\hline & $12 / 15 / 08$ & $12 / 29 / 08$ & gross $\alpha$ & $2.2 \mathrm{E}-03 \pm 7.2 \mathrm{E}-04$ & & & & & \\
\hline & $12 / 15 / 08$ & $12 / 29 / 08$ & gross $\beta$ & $4.6 \mathrm{E}-02 \pm 4.2 \mathrm{E}-03$ & & & & & \\
\hline
\end{tabular}

$\overline{\mathrm{RQ}}=$ Result Qualifier. $\mathrm{U}=$ The analyte was analyzed for but not detected. 
Table 2-4. Near-Facility Air Sampling Results, 2008 (pCi/m³ \pm total analytical uncertainty). (Sheet 51 of 82)

\begin{tabular}{|c|c|c|c|c|c|c|c|c|c|c|}
\hline Location & Sample On & Sample Off & Isotope & Result \pm Uncertainty & Location & Composite & e Period & Isotope & Result \pm Uncertainty & RQ* \\
\hline N168 & $12 / 31 / 07$ & $01 / 14 / 08$ & gross $\alpha$ & $9.3 \mathrm{E}-04 \pm 5.7 \mathrm{E}-04$ & N168 & $12 / 31 / 07$ to & $06 / 30 / 08$ & ${ }^{60} \mathrm{Co}$ & $-8.0 \mathrm{E}-05 \pm 9.7 \mathrm{E}-05$ & $\bar{U}$ \\
\hline \multirow[t]{51}{*}{$(200-W)$} & $12 / 31 / 07$ & 01/14/08 & gross $\beta$ & $8.8 \mathrm{E}-03 \pm 1.4 \mathrm{E}-03$ & & & & ${ }^{134} \mathrm{Cs}$ & $-1.0 \mathrm{E}-05 \pm 8.8 \mathrm{E}-05$ & $\mathrm{U}$ \\
\hline & $01 / 14 / 08$ & 01/29/08 & gross $\alpha$ & $1.2 \mathrm{E}-03 \pm 5.2 \mathrm{E}-04$ & & & & ${ }^{137} \mathrm{Cs}$ & $4.6 \mathrm{E}-05 \pm 8.1 \mathrm{E}-05$ & U \\
\hline & $01 / 14 / 08$ & 01/29/08 & gross $\beta$ & $3.2 \mathrm{E}-02 \pm 3.2 \mathrm{E}-03$ & & & & ${ }^{152} \mathrm{Eu}$ & $-1.0 \mathrm{E}-05 \pm 1.0 \mathrm{E}-04$ & U \\
\hline & $01 / 29 / 08$ & $02 / 12 / 08$ & gross $\alpha$ & $-5.9 \mathrm{E}-05 \pm 2.2 \mathrm{E}-04$ & & & & ${ }^{154} \mathrm{Eu}$ & $6.0 \mathrm{E}-05 \pm 2.8 \mathrm{E}-04$ & $\mathrm{U}$ \\
\hline & $01 / 29 / 08$ & $02 / 12 / 08$ & gross $\beta$ & $3.7 \mathrm{E}-03 \pm 8.5 \mathrm{E}-04$ & & & & ${ }^{155} \mathrm{Eu}$ & $2.2 \mathrm{E}-04 \pm 1.9 \mathrm{E}-04$ & U \\
\hline & $02 / 12 / 08$ & $02 / 25 / 08$ & gross $\alpha$ & $1.7 \mathrm{E}-03 \pm 6.6 \mathrm{E}-04$ & & & & ${ }^{238} \mathrm{Pu}$ & $8.4 \mathrm{E}-06 \pm 1.4 \mathrm{E}-05$ & $\mathrm{U}$ \\
\hline & $02 / 12 / 08$ & $02 / 25 / 08$ & gross $\beta$ & $3.2 \mathrm{E}-02 \pm 3.3 \mathrm{E}-03$ & & & & ${ }^{239 / 240} \mathrm{Pu}$ & $5.8 \mathrm{E}-06 \pm 5.9 \mathrm{E}-06$ & $\mathrm{U}$ \\
\hline & $02 / 25 / 08$ & 03/11/08 & gross $\alpha$ & $5.0 \mathrm{E}-04 \pm 7.2 \mathrm{E}-04$ & & & & ${ }^{103} \mathrm{Ru}$ & $-4.6 \mathrm{E}-05 \pm 9.4 \mathrm{E}-05$ & $\mathrm{U}$ \\
\hline & $02 / 25 / 08$ & 03/11/08 & gross $\beta$ & $1.8 \mathrm{E}-02 \pm 2.6 \mathrm{E}-03$ & & & & ${ }^{106} \mathrm{Ru}$ & $1.7 \mathrm{E}-04 \pm 7.4 \mathrm{E}-04$ & U \\
\hline & 03/11/08 & $03 / 25 / 08$ & gross $\alpha$ & $1.7 \mathrm{E}-04 \pm 9.9 \mathrm{E}-04$ & & & & ${ }^{125} \mathrm{Sb}$ & $1.1 \mathrm{E}-04 \pm 1.8 \mathrm{E}-04$ & $\mathrm{U}$ \\
\hline & 03/11/08 & $03 / 25 / 08$ & gross $\beta$ & $6.9 \mathrm{E}-03 \pm 2.2 \mathrm{E}-03$ & & & & ${ }^{113} \mathrm{Sn}$ & $2.2 \mathrm{E}-05 \pm 9.2 \mathrm{E}-05$ & $\mathrm{U}$ \\
\hline & $03 / 25 / 08$ & $04 / 07 / 08$ & gross $\alpha$ & $2.9 \mathrm{E}-04 \pm 4.2 \mathrm{E}-04$ & & & & ${ }^{90} \mathrm{Sr}$ & $-2.8 \mathrm{E}-04 \pm 2.9 \mathrm{E}-04$ & $\mathrm{U}$ \\
\hline & 03/25/08 & $04 / 07 / 08$ & gross $\beta$ & $9.3 \mathrm{E}-03 \pm 1.5 \mathrm{E}-03$ & & & & ${ }^{234} \mathrm{U}$ & $1.6 \mathrm{E}-05 \pm 1.1 \mathrm{E}-05$ & \\
\hline & $04 / 07 / 08$ & $04 / 21 / 08$ & gross $\alpha$ & $1.5 \mathrm{E}-03 \pm 5.9 \mathrm{E}-04$ & & & & ${ }^{235} \mathrm{U}$ & $2.7 \mathrm{E}-06 \pm 3.2 \mathrm{E}-06$ & \\
\hline & $04 / 07 / 08$ & $04 / 21 / 08$ & gross $\beta$ & $1.1 \mathrm{E}-02 \pm 1.5 \mathrm{E}-03$ & & & & ${ }^{238} \mathrm{U}$ & $1.2 \mathrm{E}-05 \pm 8.4 \mathrm{E}-06$ & \\
\hline & $04 / 21 / 08$ & $05 / 06 / 08$ & gross $\alpha$ & $1.4 \mathrm{E}-03 \pm 5.5 \mathrm{E}-04$ & & & & & & \\
\hline & $04 / 21 / 08$ & 05/06/08 & gross $\beta$ & $1.3 \mathrm{E}-02 \pm 1.7 \mathrm{E}-03$ & N168 & $06 / 30 / 08$ to & $12 / 29 / 08$ & ${ }^{60} \mathrm{Co}$ & $4.6 \mathrm{E}-06 \pm 4.6 \mathrm{E}-05$ & $\mathrm{U}$ \\
\hline & 05/06/08 & 05/19/08 & gross $\alpha$ & $1.2 \mathrm{E}-03 \pm 5.4 \mathrm{E}-04$ & & & & ${ }^{134} \mathrm{Cs}$ & $6.3 \mathrm{E}-05 \pm 8.1 \mathrm{E}-05$ & U \\
\hline & 05/06/08 & $05 / 19 / 08$ & gross $\beta$ & $1.0 \mathrm{E}-02 \pm 1.5 \mathrm{E}-03$ & & & & ${ }^{137} \mathrm{Cs}$ & $-2.1 \mathrm{E}-05 \pm 6.7 \mathrm{E}-05$ & $\mathrm{U}$ \\
\hline & 05/19/08 & 06/02/08 & gross $\alpha$ & $1.8 \mathrm{E}-03 \pm 6.5 \mathrm{E}-04$ & & & & ${ }^{152} \mathrm{Eu}$ & $-5.4 \mathrm{E}-06 \pm 5.4 \mathrm{E}-05$ & U \\
\hline & 05/19/08 & $06 / 02 / 08$ & gross $\beta$ & $9.9 \mathrm{E}-03 \pm 1.5 \mathrm{E}-03$ & & & & ${ }^{154} \mathrm{Eu}$ & $6.1 \mathrm{E}-05 \pm 2.3 \mathrm{E}-04$ & $\mathrm{U}$ \\
\hline & $06 / 02 / 08$ & $06 / 16 / 08$ & gross $\alpha$ & $8.9 \mathrm{E}-04 \pm 6.0 \mathrm{E}-04$ & & & & ${ }^{155} \mathrm{Eu}$ & $-4.8 \mathrm{E}-05 \pm 1.8 \mathrm{E}-04$ & $\mathrm{U}$ \\
\hline & $06 / 02 / 08$ & 06/16/08 & gross $\beta$ & $8.3 \mathrm{E}-03 \pm 1.4 \mathrm{E}-03$ & & & & ${ }^{238} \mathrm{Pu}$ & $1.7 \mathrm{E}-06 \pm 1.4 \mathrm{E}-05$ & $\mathrm{U}$ \\
\hline & $06 / 16 / 08$ & 06/30/08 & gross $\alpha$ & $9.6 \mathrm{E}-04 \pm 6.3 \mathrm{E}-04$ & & & & ${ }^{239 / 240} \mathrm{Pu}$ & $1.7 \mathrm{E}-06 \pm 4.9 \mathrm{E}-06$ & $\mathrm{U}$ \\
\hline & $06 / 16 / 08$ & 06/30/08 & gross $\beta$ & $1.1 \mathrm{E}-02 \pm 1.6 \mathrm{E}-03$ & & & & ${ }^{103} \mathrm{Ru}$ & $-2.2 \mathrm{E}-05 \pm 6.1 \mathrm{E}-05$ & $\mathrm{U}$ \\
\hline & $06 / 30 / 08$ & $07 / 14 / 08$ & gross $\alpha$ & $1.7 \mathrm{E}-03 \pm 7.6 \mathrm{E}-04$ & & & & ${ }^{106} \mathrm{Ru}$ & $5.8 \mathrm{E}-05 \pm 5.8 \mathrm{E}-04$ & $\mathrm{U}$ \\
\hline & $06 / 30 / 08$ & $07 / 14 / 08$ & gross $\beta$ & $4.5 \mathrm{E}-02 \pm 4.3 \mathrm{E}-03$ & & & & ${ }^{125} \mathrm{Sb}$ & $5.2 \mathrm{E}-05 \pm 1.6 \mathrm{E}-04$ & $\mathrm{U}$ \\
\hline & 07/14/08 & 07/28/08 & gross $\alpha$ & $3.2 \mathrm{E}-03 \pm 2.0 \mathrm{E}-03$ & & & & ${ }^{113} \mathrm{Sn}$ & $-7.5 \mathrm{E}-05 \pm 7.8 \mathrm{E}-05$ & $\mathrm{U}$ \\
\hline & $07 / 14 / 08$ & $07 / 28 / 08$ & gross $\beta$ & $2.6 \mathrm{E}-02 \pm 4.2 \mathrm{E}-03$ & & & & ${ }^{90} \mathrm{Sr}$ & $-2.6 \mathrm{E}-04 \pm 2.7 \mathrm{E}-04$ & $\mathrm{U}$ \\
\hline & $07 / 28 / 08$ & 08/11/08 & gross $\alpha$ & $1.3 \mathrm{E}-03 \pm 5.3 \mathrm{E}-04$ & & & & ${ }^{234} \mathrm{U}$ & $2.3 \mathrm{E}-05 \pm 1.2 \mathrm{E}-05$ & \\
\hline & $07 / 28 / 08$ & 08/11/08 & gross $\beta$ & $1.6 \mathrm{E}-02 \pm 2.0 \mathrm{E}-03$ & & & & ${ }^{235} \mathrm{U}$ & $7.2 \mathrm{E}-06 \pm 5.5 \mathrm{E}-06$ & \\
\hline & 08/11/08 & 08/25/08 & gross $\alpha$ & $9.8 \mathrm{E}-04 \pm 6.0 \mathrm{E}-04$ & & & & ${ }^{238} \mathrm{U}$ & $1.2 \mathrm{E}-05 \pm 7.9 \mathrm{E}-06$ & \\
\hline & $08 / 11 / 08$ & $08 / 25 / 08$ & gross $\beta$ & $1.3 \mathrm{E}-02 \pm 1.8 \mathrm{E}-03$ & & & & ${ }^{65} \mathrm{Zn}$ & $-1.9 \mathrm{E}-04 \pm 2.0 \mathrm{E}-04$ & $\mathrm{U}$ \\
\hline & $08 / 25 / 08$ & 09/08/08 & gross $\alpha$ & $4.0 \mathrm{E}-04 \pm 4.4 \mathrm{E}-04$ & & & & & & \\
\hline & $08 / 25 / 08$ & 09/08/08 & gross $\beta$ & $1.0 \mathrm{E}-02 \pm 1.5 \mathrm{E}-03$ & & & & & & \\
\hline & 09/08/08 & 09/22/08 & gross $\alpha$ & $1.8 \mathrm{E}-03 \pm 6.4 \mathrm{E}-04$ & & & & & & \\
\hline & 09/08/08 & $09 / 22 / 08$ & gross $\beta$ & $2.4 \mathrm{E}-02 \pm 2.6 \mathrm{E}-03$ & & & & & & \\
\hline & $09 / 22 / 08$ & $10 / 07 / 08$ & gross $\alpha$ & $1.9 \mathrm{E}-03 \pm 6.3 \mathrm{E}-04$ & & & & & & \\
\hline & $09 / 22 / 08$ & $10 / 07 / 08$ & gross $\beta$ & $2.3 \mathrm{E}-02 \pm 2.5 \mathrm{E}-03$ & & & & & & \\
\hline & $10 / 07 / 08$ & $10 / 20 / 08$ & gross $\alpha$ & $9.2 \mathrm{E}-04 \pm 6.1 \mathrm{E}-04$ & & & & & & \\
\hline & $10 / 07 / 08$ & $10 / 20 / 08$ & gross $\beta$ & $1.7 \mathrm{E}-02 \pm 2.1 \mathrm{E}-03$ & & & & & & \\
\hline & $10 / 20 / 08$ & $11 / 03 / 08$ & gross $\alpha$ & $2.8 \mathrm{E}-03 \pm 8.2 \mathrm{E}-04$ & & & & & & \\
\hline & $10 / 20 / 08$ & $11 / 03 / 08$ & gross $\beta$ & $3.9 \mathrm{E}-02 \pm 3.8 \mathrm{E}-03$ & & & & & & \\
\hline & $11 / 03 / 08$ & $11 / 17 / 08$ & gross $\alpha$ & $1.2 \mathrm{E}-03 \pm 5.4 \mathrm{E}-04$ & & & & & & \\
\hline & $11 / 03 / 08$ & $11 / 17 / 08$ & gross $\beta$ & $1.5 \mathrm{E}-02 \pm 1.9 \mathrm{E}-03$ & & & & & & \\
\hline & $11 / 17 / 08$ & $12 / 01 / 08$ & gross $\alpha$ & $2.1 \mathrm{E}-03 \pm 7.3 \mathrm{E}-04$ & & & & & & \\
\hline & $11 / 17 / 08$ & $12 / 01 / 08$ & gross $\beta$ & $3.8 \mathrm{E}-02 \pm 3.8 \mathrm{E}-03$ & & & & & & \\
\hline & $12 / 01 / 08$ & $12 / 15 / 08$ & gross $\alpha$ & $2.2 \mathrm{E}-03 \pm 7.3 \mathrm{E}-04$ & & & & & & \\
\hline & $12 / 01 / 08$ & $12 / 15 / 08$ & gross $\beta$ & $2.2 \mathrm{E}-02 \pm 2.5 \mathrm{E}-03$ & & & & & & \\
\hline & $12 / 15 / 08$ & $12 / 29 / 08$ & gross $\alpha$ & $1.5 \mathrm{E}-03 \pm 6.0 \mathrm{E}-04$ & & & & & & \\
\hline & $12 / 15 / 08$ & $12 / 29 / 08$ & gross $\beta$ & $3.8 \mathrm{E}-02 \pm 3.6 \mathrm{E}-03$ & & & & & & \\
\hline
\end{tabular}

$\overline{\mathrm{RQ}}=$ Result Qualifier. $\mathrm{U}=$ The analyte was analyzed for but not detected. 
Table 2-4. Near-Facility Air Sampling Results, 2008 (pCi/m³ \pm total analytical uncertainty). (Sheet 52 of 82)

\begin{tabular}{|c|c|c|c|c|c|c|c|c|c|}
\hline Location & Sample On & Sample Off & Isotope & Result \pm Uncertainty & Location & Composite Period & Isotope & Result \pm Uncertainty & RQ* \\
\hline N200 & $01 / 02 / 08$ & $01 / 15 / 08$ & gross $\alpha$ & $1.6 \mathrm{E}-03 \pm 6.4 \mathrm{E}-04$ & N200 & $01 / 02 / 08$ to $07 / 01 / 08$ & ${ }^{60} \mathrm{Co}$ & $5.6 \mathrm{E}-05 \pm 6.5 \mathrm{E}-05$ & $\bar{U}$ \\
\hline \multirow[t]{43}{*}{$(200-W)$} & $01 / 02 / 08$ & $01 / 15 / 08$ & gross $\beta$ & $8.2 \mathrm{E}-03 \pm 1.4 \mathrm{E}-03$ & & & ${ }^{134} \mathrm{Cs}$ & $-8.1 \mathrm{E}-06 \pm 5.5 \mathrm{E}-05$ & $\mathrm{U}$ \\
\hline & $01 / 15 / 08$ & 01/30/08 & gross $\alpha$ & $1.7 \mathrm{E}-03 \pm 6.1 \mathrm{E}-04$ & & & ${ }^{137} \mathrm{Cs}$ & $5.7 \mathrm{E}-05 \pm 6.0 \mathrm{E}-05$ & U \\
\hline & $01 / 15 / 08$ & 01/30/08 & gross $\beta$ & $2.9 \mathrm{E}-02 \pm 2.9 \mathrm{E}-03$ & & & ${ }^{152} \mathrm{Eu}$ & $1.2 \mathrm{E}-05 \pm 1.2 \mathrm{E}-04$ & $\mathrm{U}$ \\
\hline & $01 / 30 / 08$ & $02 / 13 / 08$ & gross $\alpha$ & $5.6 \mathrm{E}-05 \pm 3.0 \mathrm{E}-04$ & & & ${ }^{154} \mathrm{Eu}$ & $4.2 \mathrm{E}-05 \pm 1.9 \mathrm{E}-04$ & $\mathrm{U}$ \\
\hline & 01/30/08 & 02/13/08 & gross $\beta$ & $4.9 \mathrm{E}-03 \pm 9.8 \mathrm{E}-04$ & & & ${ }^{155} \mathrm{Eu}$ & $4.5 \mathrm{E}-05 \pm 1.3 \mathrm{E}-04$ & U \\
\hline & $02 / 13 / 08$ & $02 / 27 / 08$ & gross $\alpha$ & $1.6 \mathrm{E}-03 \pm 6.2 \mathrm{E}-04$ & & & ${ }^{238} \mathrm{Pu}$ & $1.4 \mathrm{E}-06 \pm 9.8 \mathrm{E}-06$ & $\mathrm{U}$ \\
\hline & $02 / 13 / 08$ & $02 / 27 / 08$ & gross $\beta$ & $2.9 \mathrm{E}-02 \pm 3.0 \mathrm{E}-03$ & & & ${ }^{239 / 240} \mathrm{Pu}$ & $7.1 \mathrm{E}-07 \pm 7.3 \mathrm{E}-07$ & U \\
\hline & $02 / 27 / 08$ & 03/12/08 & gross $\alpha$ & $7.4 \mathrm{E}-04 \pm 5.4 \mathrm{E}-04$ & & & ${ }^{106} \mathrm{Ru}$ & $-1.7 \mathrm{E}-04 \pm 5.0 \mathrm{E}-04$ & $\mathrm{U}$ \\
\hline & $02 / 27 / 08$ & 03/12/08 & gross $\beta$ & $9.4 \mathrm{E}-03 \pm 1.4 \mathrm{E}-03$ & & & ${ }^{125} \mathrm{Sb}$ & $5.6 \mathrm{E}-05 \pm 1.3 \mathrm{E}-04$ & $\mathrm{U}$ \\
\hline & 03/12/08 & $03 / 26 / 08$ & gross $\alpha$ & $2.7 \mathrm{E}-04 \pm 4.0 \mathrm{E}-04$ & & & ${ }^{90} \mathrm{Sr}$ & $-1.5 \mathrm{E}-04 \pm 1.5 \mathrm{E}-04$ & $\mathrm{U}$ \\
\hline & 03/12/08 & 03/26/08 & gross $\beta$ & $7.8 \mathrm{E}-03 \pm 1.3 \mathrm{E}-03$ & & & ${ }^{234} \mathrm{U}$ & $1.8 \mathrm{E}-05 \pm 1.0 \mathrm{E}-05$ & \\
\hline & $03 / 26 / 08$ & $04 / 08 / 08$ & gross $\alpha$ & $4.2 \mathrm{E}-04 \pm 4.7 \mathrm{E}-04$ & & & ${ }^{235} \mathrm{U}$ & $6.6 \mathrm{E}-07 \pm 6.6 \mathrm{E}-06$ & $\mathrm{U}$ \\
\hline & 03/26/08 & $04 / 08 / 08$ & gross $\beta$ & $5.9 \mathrm{E}-03 \pm 1.1 \mathrm{E}-03$ & & & ${ }^{238} \mathrm{U}$ & $1.3 \mathrm{E}-05 \pm 7.6 \mathrm{E}-06$ & \\
\hline & 04/08/08 & 04/23/08 & gross $\alpha$ & $1.6 \mathrm{E}-03 \pm 5.8 \mathrm{E}-04$ & & & & & \\
\hline & $04 / 08 / 08$ & $04 / 23 / 08$ & gross $\beta$ & $9.6 \mathrm{E}-03 \pm 1.4 \mathrm{E}-03$ & N200 & $07 / 01 / 08$ to $12 / 31 / 08$ & ${ }^{60} \mathrm{Co}$ & $6.7 \mathrm{E}-05 \pm 1.3 \mathrm{E}-04$ & $\mathrm{U}$ \\
\hline & $04 / 23 / 08$ & $05 / 08 / 08$ & gross $\alpha$ & $1.5 \mathrm{E}-03 \pm 5.7 \mathrm{E}-04$ & & & ${ }^{134} \mathrm{Cs}$ & $-1.7 \mathrm{E}-04 \pm 1.8 \mathrm{E}-04$ & $\mathrm{U}$ \\
\hline & $04 / 23 / 08$ & $05 / 08 / 08$ & gross $\beta$ & $1.5 \mathrm{E}-02 \pm 1.8 \mathrm{E}-03$ & & & ${ }^{137} \mathrm{Cs}$ & $3.5 \mathrm{E}-05 \pm 1.2 \mathrm{E}-04$ & $\mathrm{U}$ \\
\hline & 05/08/08 & 05/20/08 & gross $\alpha$ & $9.7 \mathrm{E}-04 \pm 6.5 \mathrm{E}-04$ & & & ${ }^{152} \mathrm{Eu}$ & $-4.2 \mathrm{E}-05 \pm 2.9 \mathrm{E}-04$ & U \\
\hline & 05/08/08 & $05 / 20 / 08$ & gross $\beta$ & $1.1 \mathrm{E}-02 \pm 1.7 \mathrm{E}-03$ & & & ${ }^{154} \mathrm{Eu}$ & $3.4 \mathrm{E}-05 \pm 3.4 \mathrm{E}-04$ & $\mathrm{U}$ \\
\hline & $05 / 20 / 08$ & $06 / 04 / 08$ & gross $\alpha$ & $3.7 \mathrm{E}-04 \pm 4.0 \mathrm{E}-04$ & & & ${ }^{155} \mathrm{Eu}$ & $-1.2 \mathrm{E}-04 \pm 2.6 \mathrm{E}-04$ & U \\
\hline & $05 / 20 / 08$ & $06 / 04 / 08$ & gross $\beta$ & $8.6 \mathrm{E}-03 \pm 1.3 \mathrm{E}-03$ & & & ${ }^{238} \mathrm{Pu}$ & $1.7 \mathrm{E}-06 \pm 1.7 \mathrm{E}-05$ & $\mathrm{U}$ \\
\hline & $06 / 04 / 08$ & $06 / 17 / 08$ & gross $\alpha$ & $5.3 \mathrm{E}-05 \pm 3.2 \mathrm{E}-04$ & & & ${ }^{239 / 240} \mathrm{Pu}$ & $5.1 \mathrm{E}-06 \pm 6.1 \mathrm{E}-06$ & \\
\hline & $06 / 04 / 08$ & $06 / 17 / 08$ & gross $\beta$ & $5.3 \mathrm{E}-03 \pm 1.1 \mathrm{E}-03$ & & & ${ }^{106} \mathrm{Ru}$ & $1.3 \mathrm{E}-04 \pm 1.1 \mathrm{E}-03$ & $\mathrm{U}$ \\
\hline & $06 / 17 / 08$ & 07/01/08 & gross $\alpha$ & $4.2 \mathrm{E}-04 \pm 4.5 \mathrm{E}-04$ & & & ${ }^{125} \mathrm{Sb}$ & $-3.9 \mathrm{E}-05 \pm 2.4 \mathrm{E}-04$ & $\mathrm{U}$ \\
\hline & $06 / 17 / 08$ & 07/01/08 & gross $\beta$ & $1.2 \mathrm{E}-02 \pm 1.6 \mathrm{E}-03$ & & & ${ }^{90} \mathrm{Sr}$ & $-1.9 \mathrm{E}-04 \pm 2.0 \mathrm{E}-04$ & $\mathrm{U}$ \\
\hline & $07 / 01 / 08$ & 07/15/08 & gross $\alpha$ & $1.6 \mathrm{E}-03 \pm 6.5 \mathrm{E}-04$ & & & ${ }^{234} \mathrm{U}$ & $2.4 \mathrm{E}-05 \pm 1.4 \mathrm{E}-05$ & \\
\hline & 07/01/08 & 07/15/08 & gross $\beta$ & $1.3 \mathrm{E}-02 \pm 1.7 \mathrm{E}-03$ & & & ${ }^{235} \mathrm{U}$ & $4.3 \mathrm{E}-06 \pm 7.7 \mathrm{E}-06$ & $\mathrm{U}$ \\
\hline & 07/15/08 & 07/30/08 & gross $\alpha$ & $1.3 \mathrm{E}-03 \pm 5.4 \mathrm{E}-04$ & & & ${ }^{238} \mathrm{U}$ & $6.5 \mathrm{E}-06 \pm 6.3 \mathrm{E}-06$ & \\
\hline & $07 / 15 / 08$ & 07/30/08 & gross $\beta$ & $1.2 \mathrm{E}-02 \pm 1.6 \mathrm{E}-03$ & & & & & \\
\hline & 07/30/08 & 08/12/08 & gross $\alpha$ & $2.0 \mathrm{E}-03 \pm 6.9 \mathrm{E}-04$ & & & & & \\
\hline & 07/30/08 & $08 / 12 / 08$ & gross $\beta$ & $8.6 \mathrm{E}-03 \pm 1.4 \mathrm{E}-03$ & & & & & \\
\hline & $08 / 12 / 08$ & $08 / 26 / 08$ & gross $\alpha$ & $8.1 \mathrm{E}-04 \pm 5.4 \mathrm{E}-04$ & & & & & \\
\hline & 08/12/08 & $08 / 26 / 08$ & gross $\beta$ & $1.1 \mathrm{E}-02 \pm 1.5 \mathrm{E}-03$ & & & & & \\
\hline & $08 / 26 / 08$ & 09/09/08 & gross $\alpha$ & $2.9 \mathrm{E}-04 \pm 3.9 \mathrm{E}-04$ & & & & & \\
\hline & $08 / 26 / 08$ & 09/09/08 & gross $\beta$ & $1.2 \mathrm{E}-02 \pm 1.6 \mathrm{E}-03$ & & & & & \\
\hline & 09/09/08 & $09 / 23 / 08$ & gross $\alpha$ & $1.3 \mathrm{E}-03 \pm 5.5 \mathrm{E}-04$ & & & & & \\
\hline & 09/09/08 & 09/23/08 & gross $\beta$ & $2.4 \mathrm{E}-02 \pm 2.6 \mathrm{E}-03$ & & & & & \\
\hline & $09 / 23 / 08$ & $10 / 08 / 08$ & gross $\alpha$ & $2.4 \mathrm{E}-03 \pm 5.6 \mathrm{E}-03$ & & & & & \\
\hline & 09/23/08 & $10 / 08 / 08$ & gross $\beta$ & $2.7 \mathrm{E}-02 \pm 9.8 \mathrm{E}-03$ & & & & & \\
\hline & $12 / 02 / 08$ & $12 / 16 / 08$ & gross $\alpha$ & $4.7 \mathrm{E}-04 \pm 9.8 \mathrm{E}-04$ & & & & & \\
\hline & $12 / 02 / 08$ & $12 / 16 / 08$ & gross $\beta$ & $1.3 \mathrm{E}-03 \pm 1.2 \mathrm{E}-03$ & & & & & \\
\hline & $12 / 16 / 08$ & $12 / 31 / 08$ & gross $\alpha$ & $1.2 \mathrm{E}-03 \pm 5.2 \mathrm{E}-04$ & & & & & \\
\hline & $12 / 16 / 08$ & $12 / 31 / 08$ & gross $\beta$ & $3.4 \mathrm{E}-02 \pm 3.3 \mathrm{E}-03$ & & & & & \\
\hline
\end{tabular}

$\overline{\mathrm{RQ}}=$ Result Qualifier. $\mathrm{U}=$ The analyte was analyzed for but not detected. 
Table 2-4. Near-Facility Air Sampling Results, 2008 (pCi/m³ \pm total analytical uncertainty). (Sheet 53 of 82)

\begin{tabular}{|c|c|c|c|c|c|c|c|c|c|}
\hline Location & Sample On & Sample Off & Isotope & Result \pm Uncertainty & Location & Composite Period & Isotope & Result \pm Uncertainty & RQ* \\
\hline N304 & $12 / 31 / 07$ & $01 / 14 / 08$ & gross $\alpha$ & $7.2 \mathrm{E}-04 \pm 5.3 \mathrm{E}-04$ & N304 & $12 / 31 / 07$ to $06 / 30 / 08$ & ${ }^{60} \mathrm{Co}$ & $2.3 \mathrm{E}-05 \pm 7.1 \mathrm{E}-05$ & $\bar{U}$ \\
\hline \multirow[t]{51}{*}{$(200-W)$} & $12 / 31 / 07$ & 01/14/08 & gross $\beta$ & $1.0 \mathrm{E}-02 \pm 1.5 \mathrm{E}-03$ & & & ${ }^{134} \mathrm{Cs}$ & $-6.0 \mathrm{E}-06 \pm 6.0 \mathrm{E}-05$ & $\mathrm{U}$ \\
\hline & $01 / 14 / 08$ & 01/29/08 & gross $\alpha$ & $1.6 \mathrm{E}-03 \pm 5.9 \mathrm{E}-04$ & & & ${ }^{137} \mathrm{Cs}$ & $2.1 \mathrm{E}-05 \pm 5.3 \mathrm{E}-05$ & U \\
\hline & 01/14/08 & 01/29/08 & gross $\beta$ & $2.9 \mathrm{E}-02 \pm 3.0 \mathrm{E}-03$ & & & ${ }^{152} \mathrm{Eu}$ & $7.6 \mathrm{E}-05 \pm 1.3 \mathrm{E}-04$ & U \\
\hline & $01 / 29 / 08$ & $02 / 12 / 08$ & gross $\alpha$ & $1.7 \mathrm{E}-04 \pm 3.6 \mathrm{E}-04$ & & & ${ }^{154} \mathrm{Eu}$ & $3.5 \mathrm{E}-05 \pm 1.9 \mathrm{E}-04$ & $\mathrm{U}$ \\
\hline & $01 / 29 / 08$ & 02/12/08 & gross $\beta$ & $3.4 \mathrm{E}-03 \pm 8.1 \mathrm{E}-04$ & & & ${ }^{155} \mathrm{Eu}$ & $-1.1 \mathrm{E}-04 \pm 1.5 \mathrm{E}-04$ & U \\
\hline & $02 / 12 / 08$ & $02 / 25 / 08$ & gross $\alpha$ & $1.9 \mathrm{E}-03 \pm 6.9 \mathrm{E}-04$ & & & ${ }^{238} \mathrm{Pu}$ & $7.3 \mathrm{E}-06 \pm 1.0 \mathrm{E}-05$ & $\mathrm{U}$ \\
\hline & $02 / 12 / 08$ & $02 / 25 / 08$ & gross $\beta$ & $2.7 \mathrm{E}-02 \pm 2.9 \mathrm{E}-03$ & & & ${ }^{239 / 240} \mathrm{Pu}$ & $1.2 \mathrm{E}-05 \pm 8.0 \mathrm{E}-06$ & \\
\hline & $02 / 25 / 08$ & 03/11/08 & gross $\alpha$ & $1.0 \mathrm{E}-03 \pm 4.8 \mathrm{E}-04$ & & & ${ }^{106} \mathrm{Ru}$ & $3.5 \mathrm{E}-04 \pm 4.4 \mathrm{E}-04$ & $\mathrm{U}$ \\
\hline & $02 / 25 / 08$ & 03/11/08 & gross $\beta$ & $1.6 \mathrm{E}-02 \pm 1.9 \mathrm{E}-03$ & & & ${ }^{125} \mathrm{Sb}$ & $-8.1 \mathrm{E}-06 \pm 8.1 \mathrm{E}-05$ & $\mathrm{U}$ \\
\hline & 03/11/08 & 03/25/08 & gross $\alpha$ & $4.9 \mathrm{E}-04 \pm 4.6 \mathrm{E}-04$ & & & ${ }^{90} \mathrm{Sr}$ & $9.7 \mathrm{E}-05 \pm 1.6 \mathrm{E}-04$ & $\mathrm{U}$ \\
\hline & 03/11/08 & $03 / 25 / 08$ & gross $\beta$ & $8.1 \mathrm{E}-03 \pm 1.3 \mathrm{E}-03$ & & & ${ }^{234} \mathrm{U}$ & $7.1 \mathrm{E}-06 \pm 5.6 \mathrm{E}-06$ & \\
\hline & $03 / 25 / 08$ & $04 / 07 / 08$ & gross $\alpha$ & $4.2 \mathrm{E}-04 \pm 4.7 \mathrm{E}-04$ & & & ${ }^{235} \mathrm{U}$ & $3.9 \mathrm{E}-06 \pm 3.7 \mathrm{E}-06$ & \\
\hline & $03 / 25 / 08$ & $04 / 07 / 08$ & gross $\beta$ & $8.5 \mathrm{E}-03 \pm 1.4 \mathrm{E}-03$ & & & ${ }^{238} \mathrm{U}$ & $1.3 \mathrm{E}-05 \pm 8.0 \mathrm{E}-06$ & \\
\hline & 04/07/08 & $04 / 21 / 08$ & gross $\alpha$ & $1.4 \mathrm{E}-03 \pm 5.5 \mathrm{E}-04$ & & & & & \\
\hline & $04 / 07 / 08$ & $04 / 21 / 08$ & gross $\beta$ & $8.7 \mathrm{E}-03 \pm 1.4 \mathrm{E}-03$ & N304 & $06 / 30 / 08$ to $12 / 29 / 08$ & ${ }^{60} \mathrm{Co}$ & $-4.9 \mathrm{E}-05 \pm 6.8 \mathrm{E}-05$ & $\mathrm{U}$ \\
\hline & $04 / 21 / 08$ & 05/06/08 & gross $\alpha$ & $1.2 \mathrm{E}-03 \pm 4.9 \mathrm{E}-04$ & & & ${ }^{134} \mathrm{Cs}$ & $7.4 \mathrm{E}-06 \pm 6.7 \mathrm{E}-05$ & $\mathrm{U}$ \\
\hline & $04 / 21 / 08$ & 05/06/08 & gross $\beta$ & $1.5 \mathrm{E}-02 \pm 1.8 \mathrm{E}-03$ & & & ${ }^{137} \mathrm{Cs}$ & $2.4 \mathrm{E}-05 \pm 6.1 \mathrm{E}-05$ & $\mathrm{U}$ \\
\hline & 05/06/08 & 05/19/08 & gross $\alpha$ & $1.0 \mathrm{E}-03 \pm 6.2 \mathrm{E}-04$ & & & ${ }^{152} \mathrm{Eu}$ & $5.6 \mathrm{E}-07 \pm 5.6 \mathrm{E}-06$ & U \\
\hline & 05/06/08 & 05/19/08 & gross $\beta$ & $8.8 \mathrm{E}-03 \pm 1.4 \mathrm{E}-03$ & & & ${ }^{154} \mathrm{Eu}$ & $1.3 \mathrm{E}-04 \pm 1.8 \mathrm{E}-04$ & $\mathrm{U}$ \\
\hline & 05/19/08 & $06 / 02 / 08$ & gross $\alpha$ & $1.0 \mathrm{E}-03 \pm 4.8 \mathrm{E}-04$ & & & ${ }^{155} \mathrm{Eu}$ & $3.2 \mathrm{E}-05 \pm 1.9 \mathrm{E}-04$ & $\mathrm{U}$ \\
\hline & 05/19/08 & $06 / 02 / 08$ & gross $\beta$ & $1.2 \mathrm{E}-02 \pm 1.7 \mathrm{E}-03$ & & & ${ }^{238} \mathrm{Pu}$ & $-1.5 \mathrm{E}-06 \pm 1.1 \mathrm{E}-05$ & $\mathrm{U}$ \\
\hline & $06 / 02 / 08$ & $06 / 16 / 08$ & gross $\alpha$ & $3.8 \mathrm{E}-04 \pm 4.3 \mathrm{E}-04$ & & & ${ }^{239 / 240} \mathrm{Pu}$ & $1.5 \mathrm{E}-06 \pm 3.8 \mathrm{E}-06$ & $\mathrm{U}$ \\
\hline & $06 / 02 / 08$ & 06/16/08 & gross $\beta$ & $4.4 \mathrm{E}-03 \pm 9.3 \mathrm{E}-04$ & & & ${ }^{106} \mathrm{Ru}$ & $-1.5 \mathrm{E}-04 \pm 5.5 \mathrm{E}-04$ & $\mathrm{U}$ \\
\hline & $06 / 16 / 08$ & 06/30/08 & gross $\alpha$ & $1.7 \mathrm{E}-03 \pm 6.2 \mathrm{E}-04$ & & & ${ }^{125} \mathrm{Sb}$ & $3.6 \mathrm{E}-05 \pm 1.4 \mathrm{E}-04$ & $\mathrm{U}$ \\
\hline & $06 / 16 / 08$ & 06/30/08 & gross $\beta$ & $1.3 \mathrm{E}-02 \pm 1.7 \mathrm{E}-03$ & & & ${ }^{90} \mathrm{Sr}$ & $-1.3 \mathrm{E}-04 \pm 1.3 \mathrm{E}-04$ & $\mathrm{U}$ \\
\hline & $06 / 30 / 08$ & $07 / 14 / 08$ & gross $\alpha$ & $1.9 \mathrm{E}-03 \pm 7.2 \mathrm{E}-04$ & & & ${ }^{234} \mathrm{U}$ & $9.7 \mathrm{E}-06 \pm 6.9 \mathrm{E}-06$ & \\
\hline & $06 / 30 / 08$ & 07/14/08 & gross $\beta$ & $1.9 \mathrm{E}-02 \pm 2.2 \mathrm{E}-03$ & & & ${ }^{235} \mathrm{U}$ & $1.5 \mathrm{E}-06 \pm 2.2 \mathrm{E}-06$ & $\mathrm{U}$ \\
\hline & $07 / 14 / 08$ & 07/28/08 & gross $\alpha$ & $8.5 \mathrm{E}-04 \pm 5.6 \mathrm{E}-04$ & & & ${ }^{238} \mathrm{U}$ & $1.4 \mathrm{E}-05 \pm 8.0 \mathrm{E}-06$ & \\
\hline & $07 / 14 / 08$ & $07 / 28 / 08$ & gross $\beta$ & $1.3 \mathrm{E}-02 \pm 1.7 \mathrm{E}-03$ & & & & & \\
\hline & 07/28/08 & 08/11/08 & gross $\alpha$ & $1.0 \mathrm{E}-03 \pm 4.8 \mathrm{E}-04$ & & & & & \\
\hline & $07 / 28 / 08$ & $08 / 11 / 08$ & gross $\beta$ & $1.4 \mathrm{E}-02 \pm 1.8 \mathrm{E}-03$ & & & & & \\
\hline & $08 / 11 / 08$ & $08 / 25 / 08$ & gross $\alpha$ & $1.1 \mathrm{E}-03 \pm 5.0 \mathrm{E}-04$ & & & & & \\
\hline & 08/11/08 & 08/25/08 & gross $\beta$ & $1.3 \mathrm{E}-02 \pm 1.7 \mathrm{E}-03$ & & & & & \\
\hline & $08 / 25 / 08$ & 09/08/08 & gross $\alpha$ & $9.4 \mathrm{E}-04 \pm 5.8 \mathrm{E}-04$ & & & & & \\
\hline & $08 / 25 / 08$ & 09/08/08 & gross $\beta$ & $1.1 \mathrm{E}-02 \pm 1.5 \mathrm{E}-03$ & & & & & \\
\hline & $09 / 08 / 08$ & $09 / 22 / 08$ & gross $\alpha$ & $1.5 \mathrm{E}-03 \pm 5.9 \mathrm{E}-04$ & & & & & \\
\hline & 09/08/08 & $09 / 22 / 08$ & gross $\beta$ & $2.3 \mathrm{E}-02 \pm 2.5 \mathrm{E}-03$ & & & & & \\
\hline & $09 / 22 / 08$ & $10 / 07 / 08$ & gross $\alpha$ & $1.7 \mathrm{E}-03 \pm 6.0 \mathrm{E}-04$ & & & & & \\
\hline & $09 / 22 / 08$ & $10 / 07 / 08$ & gross $\beta$ & $2.4 \mathrm{E}-02 \pm 2.6 \mathrm{E}-03$ & & & & & \\
\hline & $10 / 07 / 08$ & $10 / 20 / 08$ & gross $\alpha$ & $1.5 \mathrm{E}-03 \pm 6.2 \mathrm{E}-04$ & & & & & \\
\hline & $10 / 07 / 08$ & $10 / 20 / 08$ & gross $\beta$ & $1.9 \mathrm{E}-02 \pm 2.3 \mathrm{E}-03$ & & & & & \\
\hline & $10 / 20 / 08$ & $11 / 03 / 08$ & gross $\alpha$ & $2.6 \mathrm{E}-03 \pm 7.8 \mathrm{E}-04$ & & & & & \\
\hline & $10 / 20 / 08$ & $11 / 03 / 08$ & gross $\beta$ & $4.1 \mathrm{E}-02 \pm 3.9 \mathrm{E}-03$ & & & & & \\
\hline & $11 / 03 / 08$ & $11 / 17 / 08$ & gross $\alpha$ & $1.4 \mathrm{E}-03 \pm 5.7 \mathrm{E}-04$ & & & & & \\
\hline & $11 / 03 / 08$ & $11 / 17 / 08$ & gross $\beta$ & $1.6 \mathrm{E}-02 \pm 2.0 \mathrm{E}-03$ & & & & & \\
\hline & $11 / 17 / 08$ & $12 / 01 / 08$ & gross $\alpha$ & $1.5 \mathrm{E}-03 \pm 6.1 \mathrm{E}-04$ & & & & & \\
\hline & $11 / 17 / 08$ & $12 / 01 / 08$ & gross $\beta$ & $3.5 \mathrm{E}-02 \pm 3.5 \mathrm{E}-03$ & & & & & \\
\hline & $12 / 01 / 08$ & $12 / 15 / 08$ & gross $\alpha$ & $2.0 \mathrm{E}-03 \pm 6.9 \mathrm{E}-04$ & & & & & \\
\hline & $12 / 01 / 08$ & $12 / 15 / 08$ & gross $\beta$ & $2.3 \mathrm{E}-02 \pm 2.6 \mathrm{E}-03$ & & & & & \\
\hline & $12 / 15 / 08$ & $12 / 29 / 08$ & gross $\alpha$ & $2.8 \mathrm{E}-03 \pm 8.1 \mathrm{E}-04$ & & & & & \\
\hline & $12 / 15 / 08$ & $12 / 29 / 08$ & gross $\beta$ & $3.6 \mathrm{E}-02 \pm 3.5 \mathrm{E}-03$ & & & & & \\
\hline
\end{tabular}

$\overline{\mathrm{RQ}}=$ Result Qualifier. $\mathrm{U}=$ The analyte was analyzed for but not detected. 
Table 2-4. Near-Facility Air Sampling Results, 2008 (pCi/m³ \pm total analytical uncertainty). (Sheet 54 of 82)

\begin{tabular}{|c|c|c|c|c|c|c|c|c|c|c|}
\hline Location & Sample On & Sample Off & Isotope & Result \pm Uncertainty & Location & Composite & e Period & Isotope & Result \pm Uncertainty & RQ* \\
\hline N433 & $12 / 31 / 07$ & $01 / 14 / 08$ & gross $\alpha$ & $1.2 \mathrm{E}-03 \pm 7.3 \mathrm{E}-04$ & N433 & $12 / 31 / 07$ to & $06 / 30 / 08$ & ${ }^{60} \mathrm{Co}$ & $-8.4 \mathrm{E}-06 \pm 6.1 \mathrm{E}-05$ & $\bar{U}$ \\
\hline \multirow[t]{51}{*}{$(200-W)$} & $12 / 31 / 07$ & 01/14/08 & gross $\beta$ & $1.5 \mathrm{E}-02 \pm 2.1 \mathrm{E}-03$ & & & & ${ }^{134} \mathrm{Cs}$ & $3.0 \mathrm{E}-05 \pm 7.9 \mathrm{E}-05$ & $\mathrm{U}$ \\
\hline & $01 / 14 / 08$ & 01/29/08 & gross $\alpha$ & $1.6 \mathrm{E}-03 \pm 6.3 \mathrm{E}-04$ & & & & ${ }^{137} \mathrm{Cs}$ & $8.3 \mathrm{E}-06 \pm 6.1 \mathrm{E}-05$ & U \\
\hline & $01 / 14 / 08$ & 01/29/08 & gross $\beta$ & $3.2 \mathrm{E}-02 \pm 3.3 \mathrm{E}-03$ & & & & ${ }^{152} \mathrm{Eu}$ & $-3.7 \mathrm{E}-05 \pm 1.4 \mathrm{E}-04$ & $\mathrm{U}$ \\
\hline & $01 / 29 / 08$ & $02 / 12 / 08$ & gross $\alpha$ & $1.8 \mathrm{E}-04 \pm 3.7 \mathrm{E}-04$ & & & & ${ }^{154} \mathrm{Eu}$ & $4.0 \mathrm{E}-05 \pm 2.0 \mathrm{E}-04$ & $\mathrm{U}$ \\
\hline & $01 / 29 / 08$ & 02/12/08 & gross $\beta$ & $4.0 \mathrm{E}-03 \pm 9.0 \mathrm{E}-04$ & & & & ${ }^{155} \mathrm{Eu}$ & $-7.2 \mathrm{E}-05 \pm 1.8 \mathrm{E}-04$ & U \\
\hline & $02 / 12 / 08$ & $02 / 25 / 08$ & gross $\alpha$ & $2.1 \mathrm{E}-03 \pm 7.8 \mathrm{E}-04$ & & & & ${ }^{238} \mathrm{Pu}$ & $-3.2 \mathrm{E}-06 \pm 1.0 \mathrm{E}-05$ & $\mathrm{U}$ \\
\hline & $02 / 12 / 08$ & $02 / 25 / 08$ & gross $\beta$ & $4.0 \mathrm{E}-02 \pm 4.0 \mathrm{E}-03$ & & & & ${ }^{239 / 240} \mathrm{Pu}$ & $7.1 \mathrm{E}-05 \pm 3.0 \mathrm{E}-05$ & \\
\hline & $02 / 25 / 08$ & 03/11/08 & gross $\alpha$ & $8.3 \mathrm{E}-04 \pm 5.6 \mathrm{E}-04$ & & & & ${ }^{106} \mathrm{Ru}$ & $3.7 \mathrm{E}-04 \pm 5.5 \mathrm{E}-04$ & $\mathrm{U}$ \\
\hline & $02 / 25 / 08$ & 03/11/08 & gross $\beta$ & $1.4 \mathrm{E}-02 \pm 1.8 \mathrm{E}-03$ & & & & ${ }^{125} \mathrm{Sb}$ & $-2.7 \mathrm{E}-05 \pm 1.5 \mathrm{E}-04$ & $\mathrm{U}$ \\
\hline & 03/11/08 & 03/25/08 & gross $\alpha$ & $5.6 \mathrm{E}-04 \pm 5.3 \mathrm{E}-04$ & & & & ${ }^{90} \mathrm{Sr}$ & $-2.2 \mathrm{E}-04 \pm 2.3 \mathrm{E}-04$ & $\mathrm{U}$ \\
\hline & 03/11/08 & $03 / 25 / 08$ & gross $\beta$ & $9.2 \mathrm{E}-03 \pm 1.5 \mathrm{E}-03$ & & & & ${ }^{234} \mathrm{U}$ & $1.9 \mathrm{E}-05 \pm 1.1 \mathrm{E}-05$ & \\
\hline & $03 / 25 / 08$ & $04 / 07 / 08$ & gross $\alpha$ & $4.5 \mathrm{E}-04 \pm 5.1 \mathrm{E}-04$ & & & & ${ }^{235} \mathrm{U}$ & $3.5 \mathrm{E}-06 \pm 3.7 \mathrm{E}-06$ & \\
\hline & 03/25/08 & $04 / 07 / 08$ & gross $\beta$ & $7.1 \mathrm{E}-03 \pm 1.3 \mathrm{E}-03$ & & & & ${ }^{238} \mathrm{U}$ & $7.1 \mathrm{E}-06 \pm 6.3 \mathrm{E}-06$ & $\mathrm{U}$ \\
\hline & 04/07/08 & $04 / 21 / 08$ & gross $\alpha$ & $9.1 \mathrm{E}-04 \pm 6.1 \mathrm{E}-04$ & & & & & & \\
\hline & $04 / 07 / 08$ & $04 / 21 / 08$ & gross $\beta$ & $1.1 \mathrm{E}-02 \pm 1.6 \mathrm{E}-03$ & N433 & $06 / 30 / 08$ to & $12 / 29 / 08$ & ${ }^{60} \mathrm{Co}$ & $-8.4 \mathrm{E}-05 \pm 8.7 \mathrm{E}-05$ & U \\
\hline & $04 / 21 / 08$ & $05 / 05 / 08$ & gross $\alpha$ & $1.7 \mathrm{E}-03 \pm 6.3 \mathrm{E}-04$ & & & & ${ }^{134} \mathrm{Cs}$ & $3.9 \mathrm{E}-05 \pm 7.7 \mathrm{E}-05$ & $\mathrm{U}$ \\
\hline & $04 / 21 / 08$ & 05/05/08 & gross $\beta$ & $1.3 \mathrm{E}-02 \pm 1.8 \mathrm{E}-03$ & & & & ${ }^{137} \mathrm{Cs}$ & $1.8 \mathrm{E}-04 \pm 1.2 \mathrm{E}-04$ & \\
\hline & 05/05/08 & 05/19/08 & gross $\alpha$ & $1.3 \mathrm{E}-03 \pm 5.9 \mathrm{E}-04$ & & & & ${ }^{152} \mathrm{Eu}$ & $-6.5 \mathrm{E}-06 \pm 6.5 \mathrm{E}-05$ & $\mathrm{U}$ \\
\hline & 05/05/08 & 05/19/08 & gross $\beta$ & $1.1 \mathrm{E}-02 \pm 1.7 \mathrm{E}-03$ & & & & ${ }^{154} \mathrm{Eu}$ & $6.5 \mathrm{E}-05 \pm 2.3 \mathrm{E}-04$ & $\mathrm{U}$ \\
\hline & 05/19/08 & $06 / 02 / 08$ & gross $\alpha$ & $2.4 \mathrm{E}-03 \pm 8.0 \mathrm{E}-04$ & & & & ${ }^{155} \mathrm{Eu}$ & $1.7 \mathrm{E}-04 \pm 2.0 \mathrm{E}-04$ & $\mathrm{U}$ \\
\hline & 05/19/08 & $06 / 02 / 08$ & gross $\beta$ & $1.6 \mathrm{E}-02 \pm 2.1 \mathrm{E}-03$ & & & & ${ }^{238} \mathrm{Pu}$ & $-6.8 \mathrm{E}-06 \pm 1.4 \mathrm{E}-05$ & $\mathrm{U}$ \\
\hline & $06 / 02 / 08$ & $06 / 16 / 08$ & gross $\alpha$ & $9.3 \mathrm{E}-04 \pm 7.2 \mathrm{E}-04$ & & & & ${ }^{239 / 240} \mathrm{Pu}$ & $1.1 \mathrm{E}-05 \pm 8.7 \mathrm{E}-06$ & \\
\hline & $06 / 02 / 08$ & 06/16/08 & gross $\beta$ & $1.2 \mathrm{E}-02 \pm 1.8 \mathrm{E}-03$ & & & & ${ }^{106} \mathrm{Ru}$ & $-4.1 \mathrm{E}-04 \pm 6.2 \mathrm{E}-04$ & $\mathrm{U}$ \\
\hline & $06 / 16 / 08$ & 06/30/08 & gross $\alpha$ & $2.2 \mathrm{E}-03 \pm 7.7 \mathrm{E}-04$ & & & & ${ }^{125} \mathrm{Sb}$ & $4.1 \mathrm{E}-05 \pm 1.5 \mathrm{E}-04$ & $\mathrm{U}$ \\
\hline & $06 / 16 / 08$ & 06/30/08 & gross $\beta$ & $1.4 \mathrm{E}-02 \pm 2.0 \mathrm{E}-03$ & & & & ${ }^{90} \mathrm{Sr}$ & $-1.3 \mathrm{E}-04 \pm 1.3 \mathrm{E}-04$ & $\mathrm{U}$ \\
\hline & $06 / 30 / 08$ & 07/14/08 & gross $\alpha$ & $1.4 \mathrm{E}-03 \pm 6.7 \mathrm{E}-04$ & & & & ${ }^{234} \mathrm{U}$ & $1.5 \mathrm{E}-05 \pm 8.9 \mathrm{E}-06$ & \\
\hline & 06/30/08 & 07/14/08 & gross $\beta$ & $1.6 \mathrm{E}-02 \pm 2.1 \mathrm{E}-03$ & & & & ${ }^{235} \mathrm{U}$ & $6.3 \mathrm{E}-06 \pm 5.0 \mathrm{E}-06$ & \\
\hline & $07 / 14 / 08$ & 07/28/08 & gross $\alpha$ & $1.0 \mathrm{E}-03 \pm 6.4 \mathrm{E}-04$ & & & & ${ }^{238} \mathrm{U}$ & $8.6 \mathrm{E}-06 \pm 6.2 \mathrm{E}-06$ & \\
\hline & $07 / 14 / 08$ & $07 / 28 / 08$ & gross $\beta$ & $1.8 \mathrm{E}-02 \pm 2.2 \mathrm{E}-03$ & & & & & & \\
\hline & 07/28/08 & 08/11/08 & gross $\alpha$ & $1.1 \mathrm{E}-03 \pm 5.0 \mathrm{E}-04$ & & & & & & \\
\hline & $07 / 28 / 08$ & $08 / 11 / 08$ & gross $\beta$ & $1.2 \mathrm{E}-02 \pm 1.6 \mathrm{E}-03$ & & & & & & \\
\hline & $08 / 11 / 08$ & $08 / 25 / 08$ & gross $\alpha$ & $1.9 \mathrm{E}-03 \pm 7.3 \mathrm{E}-04$ & & & & & & \\
\hline & 08/11/08 & 08/25/08 & gross $\beta$ & $2.0 \mathrm{E}-02 \pm 2.5 \mathrm{E}-03$ & & & & & & \\
\hline & $08 / 25 / 08$ & 09/08/08 & gross $\alpha$ & $1.8 \mathrm{E}-03 \pm 6.7 \mathrm{E}-04$ & & & & & & \\
\hline & $08 / 25 / 08$ & 09/08/08 & gross $\beta$ & $1.4 \mathrm{E}-02 \pm 1.9 \mathrm{E}-03$ & & & & & & \\
\hline & $09 / 08 / 08$ & $09 / 22 / 08$ & gross $\alpha$ & $3.7 \mathrm{E}-03 \pm 9.7 \mathrm{E}-04$ & & & & & & \\
\hline & 09/08/08 & $09 / 22 / 08$ & gross $\beta$ & $3.4 \mathrm{E}-02 \pm 3.5 \mathrm{E}-03$ & & & & & & \\
\hline & $09 / 22 / 08$ & $10 / 07 / 08$ & gross $\alpha$ & $2.5 \mathrm{E}-03 \pm 7.3 \mathrm{E}-04$ & & & & & & \\
\hline & $09 / 22 / 08$ & $10 / 07 / 08$ & gross $\beta$ & $2.6 \mathrm{E}-02 \pm 2.8 \mathrm{E}-03$ & & & & & & \\
\hline & $10 / 07 / 08$ & $10 / 20 / 08$ & gross $\alpha$ & $1.3 \mathrm{E}-03 \pm 5.8 \mathrm{E}-04$ & & & & & & \\
\hline & $10 / 07 / 08$ & $10 / 20 / 08$ & gross $\beta$ & $1.6 \mathrm{E}-02 \pm 2.1 \mathrm{E}-03$ & & & & & & \\
\hline & $10 / 20 / 08$ & $11 / 03 / 08$ & gross $\alpha$ & $2.0 \mathrm{E}-03 \pm 7.1 \mathrm{E}-04$ & & & & & & \\
\hline & $10 / 20 / 08$ & $11 / 03 / 08$ & gross $\beta$ & $3.6 \mathrm{E}-02 \pm 3.6 \mathrm{E}-03$ & & & & & & \\
\hline & $11 / 03 / 08$ & $11 / 17 / 08$ & gross $\alpha$ & $1.3 \mathrm{E}-03 \pm 5.6 \mathrm{E}-04$ & & & & & & \\
\hline & $11 / 03 / 08$ & $11 / 17 / 08$ & gross $\beta$ & $1.5 \mathrm{E}-02 \pm 2.0 \mathrm{E}-03$ & & & & & & \\
\hline & $11 / 17 / 08$ & $12 / 01 / 08$ & gross $\alpha$ & $3.0 \mathrm{E}-03 \pm 9.0 \mathrm{E}-04$ & & & & & & \\
\hline & $11 / 17 / 08$ & $12 / 01 / 08$ & gross $\beta$ & $4.3 \mathrm{E}-02 \pm 4.1 \mathrm{E}-03$ & & & & & & \\
\hline & $12 / 01 / 08$ & $12 / 15 / 08$ & gross $\alpha$ & $1.2 \mathrm{E}-03 \pm 5.5 \mathrm{E}-04$ & & & & & & \\
\hline & $12 / 01 / 08$ & $12 / 15 / 08$ & gross $\beta$ & $2.3 \mathrm{E}-02 \pm 2.6 \mathrm{E}-03$ & & & & & & \\
\hline & $12 / 15 / 08$ & $12 / 29 / 08$ & gross $\alpha$ & $2.2 \mathrm{E}-03 \pm 7.1 \mathrm{E}-04$ & & & & & & \\
\hline & $12 / 15 / 08$ & $12 / 29 / 08$ & gross $\beta$ & $3.7 \mathrm{E}-02 \pm 3.6 \mathrm{E}-03$ & & & & & & \\
\hline
\end{tabular}

$\overline{\mathrm{RQ}}=$ Result Qualifier. $\mathrm{U}=$ The analyte was analyzed for but not detected. 
Table 2-4. Near-Facility Air Sampling Results, 2008 (pCi/m³ \pm total analytical uncertainty). (Sheet 55 of 82)

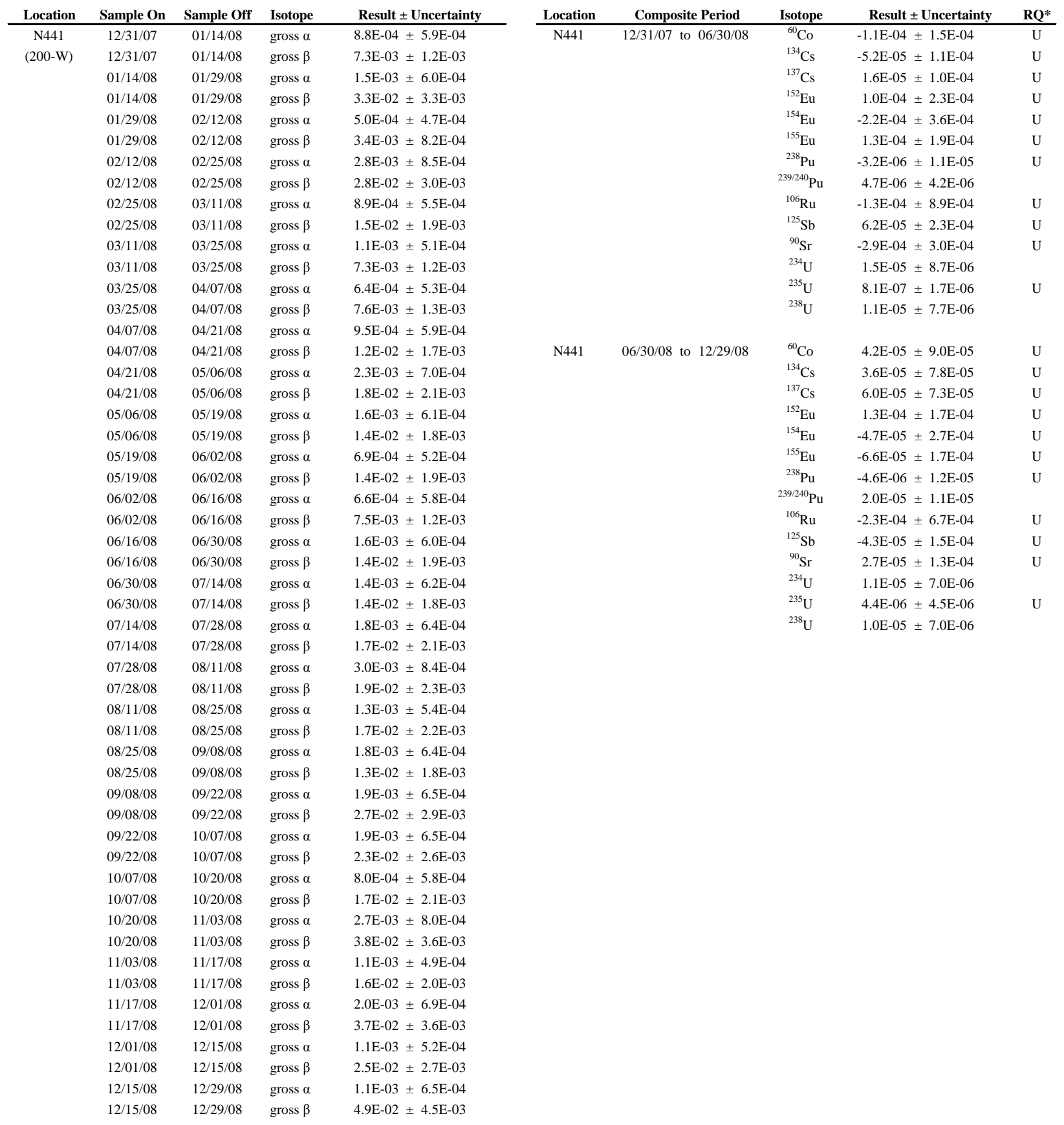

$\overline{\mathrm{RQ}}=$ Result Qualifier. $\mathrm{U}=$ The analyte was analyzed for but not detected. 
Table 2-4. Near-Facility Air Sampling Results, 2008 (pCi/m³ \pm total analytical uncertainty). (Sheet 56 of 82)

\begin{tabular}{|c|c|c|c|c|c|c|c|c|c|c|}
\hline Location & Sample On & Sample Off & Isotope & Result \pm Uncertainty & Location & Composite & e Period & Isotope & Result \pm Uncertainty & RQ* \\
\hline N442 & $12 / 31 / 07$ & $01 / 14 / 08$ & gross $\alpha$ & $1.4 \mathrm{E}-03 \pm 5.8 \mathrm{E}-04$ & N442 & $12 / 31 / 07$ to & $06 / 30 / 08$ & ${ }^{60} \mathrm{Co}$ & $-1.3 \mathrm{E}-05 \pm 7.1 \mathrm{E}-05$ & $\bar{U}$ \\
\hline \multirow[t]{51}{*}{$(200-W)$} & $12 / 31 / 07$ & 01/14/08 & gross $\beta$ & $1.0 \mathrm{E}-02 \pm 1.5 \mathrm{E}-03$ & & & & ${ }^{134} \mathrm{Cs}$ & $4.6 \mathrm{E}-05 \pm 7.2 \mathrm{E}-05$ & $\mathrm{U}$ \\
\hline & $01 / 14 / 08$ & 01/29/08 & gross $\alpha$ & $8.7 \mathrm{E}-04 \pm 5.4 \mathrm{E}-04$ & & & & ${ }^{137} \mathrm{Cs}$ & $9.7 \mathrm{E}-05 \pm 7.5 \mathrm{E}-05$ & U \\
\hline & $01 / 14 / 08$ & 01/29/08 & gross $\beta$ & $3.0 \mathrm{E}-02 \pm 3.0 \mathrm{E}-03$ & & & & ${ }^{152} \mathrm{Eu}$ & $1.1 \mathrm{E}-05 \pm 1.1 \mathrm{E}-04$ & $\mathrm{U}$ \\
\hline & $01 / 29 / 08$ & $02 / 12 / 08$ & gross $\alpha$ & $1.1 \mathrm{E}-03 \pm 5.1 \mathrm{E}-04$ & & & & ${ }^{154} \mathrm{Eu}$ & $-9.8 \mathrm{E}-05 \pm 2.3 \mathrm{E}-04$ & $\mathrm{U}$ \\
\hline & $01 / 29 / 08$ & 02/12/08 & gross $\beta$ & $4.6 \mathrm{E}-03 \pm 9.5 \mathrm{E}-04$ & & & & ${ }^{155} \mathrm{Eu}$ & $-4.8 \mathrm{E}-05 \pm 1.7 \mathrm{E}-04$ & U \\
\hline & $02 / 12 / 08$ & $02 / 25 / 08$ & gross $\alpha$ & $2.2 \mathrm{E}-03 \pm 7.4 \mathrm{E}-04$ & & & & ${ }^{238} \mathrm{Pu}$ & $2.3 \mathrm{E}-06 \pm 1.0 \mathrm{E}-05$ & $\mathrm{U}$ \\
\hline & $02 / 12 / 08$ & $02 / 25 / 08$ & gross $\beta$ & $3.0 \mathrm{E}-02 \pm 3.1 \mathrm{E}-03$ & & & & ${ }^{239 / 240} \mathrm{Pu}$ & $3.8 \mathrm{E}-06 \pm 4.3 \mathrm{E}-06$ & $\mathrm{U}$ \\
\hline & $02 / 25 / 08$ & 03/11/08 & gross $\alpha$ & $1.7 \mathrm{E}-03 \pm 6.0 \mathrm{E}-04$ & & & & ${ }^{106} \mathrm{Ru}$ & $-1.2 \mathrm{E}-04 \pm 5.6 \mathrm{E}-04$ & $\mathrm{U}$ \\
\hline & $02 / 25 / 08$ & 03/11/08 & gross $\beta$ & $1.8 \mathrm{E}-02 \pm 2.1 \mathrm{E}-03$ & & & & ${ }^{125} \mathrm{Sb}$ & $2.7 \mathrm{E}-06 \pm 2.7 \mathrm{E}-05$ & $\mathrm{U}$ \\
\hline & 03/11/08 & 03/25/08 & gross $\alpha$ & $8.6 \mathrm{E}-04 \pm 5.8 \mathrm{E}-04$ & & & & ${ }^{90} \mathrm{Sr}$ & $-3.4 \mathrm{E}-04 \pm 3.6 \mathrm{E}-04$ & $\mathrm{U}$ \\
\hline & 03/11/08 & $03 / 25 / 08$ & gross $\beta$ & $1.0 \mathrm{E}-02 \pm 1.5 \mathrm{E}-03$ & & & & ${ }^{234} \mathrm{U}$ & $1.0 \mathrm{E}-05 \pm 6.5 \mathrm{E}-06$ & \\
\hline & $03 / 25 / 08$ & $04 / 07 / 08$ & gross $\alpha$ & $4.2 \mathrm{E}-04 \pm 4.7 \mathrm{E}-04$ & & & & ${ }^{235} \mathrm{U}$ & $2.1 \mathrm{E}-06 \pm 2.6 \mathrm{E}-06$ & \\
\hline & 03/25/08 & $04 / 07 / 08$ & gross $\beta$ & $8.2 \mathrm{E}-03 \pm 1.4 \mathrm{E}-03$ & & & & ${ }^{238} \mathrm{U}$ & $5.8 \mathrm{E}-06 \pm 4.4 \mathrm{E}-06$ & \\
\hline & 04/07/08 & $04 / 21 / 08$ & gross $\alpha$ & $9.5 \mathrm{E}-04 \pm 5.9 \mathrm{E}-04$ & & & & & & \\
\hline & $04 / 07 / 08$ & $04 / 21 / 08$ & gross $\beta$ & $1.0 \mathrm{E}-02 \pm 1.5 \mathrm{E}-03$ & $\mathrm{~N} 442$ & $06 / 30 / 08$ to & $12 / 29 / 08$ & ${ }^{60} \mathrm{Co}$ & $-1.4 \mathrm{E}-05 \pm 6.5 \mathrm{E}-05$ & U \\
\hline & $04 / 21 / 08$ & 05/06/08 & gross $\alpha$ & $1.6 \mathrm{E}-03 \pm 5.8 \mathrm{E}-04$ & & & & ${ }^{134} \mathrm{Cs}$ & $3.3 \mathrm{E}-05 \pm 6.2 \mathrm{E}-05$ & $\mathrm{U}$ \\
\hline & $04 / 21 / 08$ & 05/06/08 & gross $\beta$ & $1.8 \mathrm{E}-02 \pm 2.2 \mathrm{E}-03$ & & & & ${ }^{137} \mathrm{Cs}$ & $1.3 \mathrm{E}-04 \pm 1.2 \mathrm{E}-04$ & \\
\hline & 05/06/08 & 05/19/08 & gross $\alpha$ & $1.0 \mathrm{E}-03 \pm 6.3 \mathrm{E}-04$ & & & & ${ }^{152} \mathrm{Eu}$ & $7.5 \mathrm{E}-05 \pm 1.4 \mathrm{E}-04$ & $\mathrm{U}$ \\
\hline & 05/06/08 & 05/19/08 & gross $\beta$ & $9.4 \mathrm{E}-03 \pm 1.4 \mathrm{E}-03$ & & & & ${ }^{154} \mathrm{Eu}$ & $-1.9 \mathrm{E}-05 \pm 1.9 \mathrm{E}-04$ & $\mathrm{U}$ \\
\hline & 05/19/08 & $06 / 02 / 08$ & gross $\alpha$ & $2.8 \mathrm{E}-03 \pm 8.0 \mathrm{E}-04$ & & & & ${ }^{155} \mathrm{Eu}$ & $6.1 \mathrm{E}-05 \pm 1.7 \mathrm{E}-04$ & $\mathrm{U}$ \\
\hline & 05/19/08 & $06 / 02 / 08$ & gross $\beta$ & $1.4 \mathrm{E}-02 \pm 1.9 \mathrm{E}-03$ & & & & ${ }^{238} \mathrm{Pu}$ & $-2.0 \mathrm{E}-06 \pm 1.0 \mathrm{E}-05$ & $\mathrm{U}$ \\
\hline & $06 / 02 / 08$ & $06 / 16 / 08$ & gross $\alpha$ & $1.3 \mathrm{E}-03 \pm 6.0 \mathrm{E}-04$ & & & & ${ }^{239 / 240} \mathrm{Pu}$ & $2.4 \mathrm{E}-05 \pm 1.2 \mathrm{E}-05$ & \\
\hline & $06 / 02 / 08$ & 06/16/08 & gross $\beta$ & $8.3 \mathrm{E}-03 \pm 1.3 \mathrm{E}-03$ & & & & ${ }^{106} \mathrm{Ru}$ & $-1.7 \mathrm{E}-04 \pm 5.0 \mathrm{E}-04$ & $\mathrm{U}$ \\
\hline & $06 / 16 / 08$ & 06/30/08 & gross $\alpha$ & $1.7 \mathrm{E}-03 \pm 6.3 \mathrm{E}-04$ & & & & ${ }^{125} \mathrm{Sb}$ & $1.6 \mathrm{E}-06 \pm 1.6 \mathrm{E}-05$ & $\mathrm{U}$ \\
\hline & $06 / 16 / 08$ & 06/30/08 & gross $\beta$ & $1.5 \mathrm{E}-02 \pm 2.0 \mathrm{E}-03$ & & & & ${ }^{90} \mathrm{Sr}$ & $-1.3 \mathrm{E}-04 \pm 1.3 \mathrm{E}-04$ & $\mathrm{U}$ \\
\hline & $06 / 30 / 08$ & $07 / 14 / 08$ & gross $\alpha$ & $1.6 \mathrm{E}-03 \pm 6.8 \mathrm{E}-04$ & & & & ${ }^{234} \mathrm{U}$ & $1.4 \mathrm{E}-05 \pm 8.4 \mathrm{E}-06$ & \\
\hline & 06/30/08 & 07/14/08 & gross $\beta$ & $1.6 \mathrm{E}-02 \pm 2.0 \mathrm{E}-03$ & & & & ${ }^{235} \mathrm{U}$ & $5.8 \mathrm{E}-06 \pm 4.6 \mathrm{E}-06$ & \\
\hline & $07 / 14 / 08$ & 07/28/08 & gross $\alpha$ & $2.4 \mathrm{E}-03 \pm 7.8 \mathrm{E}-04$ & & & & ${ }^{238} \mathrm{U}$ & $1.2 \mathrm{E}-05 \pm 7.2 \mathrm{E}-06$ & \\
\hline & $07 / 14 / 08$ & $07 / 28 / 08$ & gross $\beta$ & $1.6 \mathrm{E}-02 \pm 2.1 \mathrm{E}-03$ & & & & & & \\
\hline & 07/28/08 & 08/11/08 & gross $\alpha$ & $1.2 \mathrm{E}-03 \pm 5.2 \mathrm{E}-04$ & & & & & & \\
\hline & $07 / 28 / 08$ & $08 / 11 / 08$ & gross $\beta$ & $1.2 \mathrm{E}-02 \pm 1.7 \mathrm{E}-03$ & & & & & & \\
\hline & $08 / 11 / 08$ & $08 / 25 / 08$ & gross $\alpha$ & $6.8 \mathrm{E}-04 \pm 5.1 \mathrm{E}-04$ & & & & & & \\
\hline & 08/11/08 & 08/25/08 & gross $\beta$ & $1.5 \mathrm{E}-02 \pm 1.9 \mathrm{E}-03$ & & & & & & \\
\hline & $08 / 25 / 08$ & 09/08/08 & gross $\alpha$ & $4.6 \mathrm{E}-04 \pm 4.5 \mathrm{E}-04$ & & & & & & \\
\hline & $08 / 25 / 08$ & 09/08/08 & gross $\beta$ & $1.4 \mathrm{E}-02 \pm 1.8 \mathrm{E}-03$ & & & & & & \\
\hline & $09 / 08 / 08$ & $09 / 22 / 08$ & gross $\alpha$ & $2.4 \mathrm{E}-03 \pm 7.4 \mathrm{E}-04$ & & & & & & \\
\hline & 09/08/08 & $09 / 22 / 08$ & gross $\beta$ & $3.2 \mathrm{E}-02 \pm 3.3 \mathrm{E}-03$ & & & & & & \\
\hline & $09 / 22 / 08$ & $10 / 07 / 08$ & gross $\alpha$ & $1.3 \mathrm{E}-03 \pm 5.2 \mathrm{E}-04$ & & & & & & \\
\hline & $09 / 22 / 08$ & $10 / 07 / 08$ & gross $\beta$ & $2.6 \mathrm{E}-02 \pm 2.8 \mathrm{E}-03$ & & & & & & \\
\hline & $10 / 07 / 08$ & $10 / 20 / 08$ & gross $\alpha$ & $2.1 \mathrm{E}-03 \pm 7.1 \mathrm{E}-04$ & & & & & & \\
\hline & $10 / 07 / 08$ & $10 / 20 / 08$ & gross $\beta$ & $2.7 \mathrm{E}-02 \pm 3.0 \mathrm{E}-03$ & & & & & & \\
\hline & $10 / 20 / 08$ & $11 / 03 / 08$ & gross $\alpha$ & $3.1 \mathrm{E}-03 \pm 8.4 \mathrm{E}-04$ & & & & & & \\
\hline & $10 / 20 / 08$ & $11 / 03 / 08$ & gross $\beta$ & $5.0 \mathrm{E}-02 \pm 4.6 \mathrm{E}-03$ & & & & & & \\
\hline & $11 / 03 / 08$ & $11 / 17 / 08$ & gross $\alpha$ & $1.1 \mathrm{E}-03 \pm 5.0 \mathrm{E}-04$ & & & & & & \\
\hline & $11 / 03 / 08$ & $11 / 17 / 08$ & gross $\beta$ & $2.2 \mathrm{E}-02 \pm 2.5 \mathrm{E}-03$ & & & & & & \\
\hline & $11 / 17 / 08$ & $12 / 01 / 08$ & gross $\alpha$ & $1.2 \mathrm{E}-03 \pm 5.5 \mathrm{E}-04$ & & & & & & \\
\hline & $11 / 17 / 08$ & $12 / 01 / 08$ & gross $\beta$ & $4.2 \mathrm{E}-02 \pm 4.0 \mathrm{E}-03$ & & & & & & \\
\hline & $12 / 01 / 08$ & $12 / 15 / 08$ & gross $\alpha$ & $1.6 \mathrm{E}-03 \pm 5.9 \mathrm{E}-04$ & & & & & & \\
\hline & $12 / 01 / 08$ & $12 / 15 / 08$ & gross $\beta$ & $2.3 \mathrm{E}-02 \pm 2.5 \mathrm{E}-03$ & & & & & & \\
\hline & $12 / 15 / 08$ & $12 / 29 / 08$ & gross $\alpha$ & $2.7 \mathrm{E}-03 \pm 7.7 \mathrm{E}-04$ & & & & & & \\
\hline & $12 / 15 / 08$ & $12 / 29 / 08$ & gross $\beta$ & $4.2 \mathrm{E}-02 \pm 3.9 \mathrm{E}-03$ & & & & & & \\
\hline
\end{tabular}

$\overline{\mathrm{RQ}}=$ Result Qualifier. $\mathrm{U}=$ The analyte was analyzed for but not detected. 
Table 2-4. Near-Facility Air Sampling Results, 2008 (pCi/m³ \pm total analytical uncertainty). (Sheet 57 of 82)

\begin{tabular}{|c|c|c|c|c|c|c|c|c|c|c|}
\hline Location & Sample On & Sample Off & Isotope & Result \pm Uncertainty & Location & Composite & e Period & Isotope & Result \pm Uncertainty & RQ* \\
\hline N449 & $12 / 31 / 07$ & $01 / 14 / 08$ & gross $\alpha$ & $7.7 \mathrm{E}-04 \pm 5.6 \mathrm{E}-04$ & N449 & $12 / 31 / 07$ to & $06 / 30 / 08$ & ${ }^{60} \mathrm{Co}$ & $-1.9 \mathrm{E}-05 \pm 8.3 \mathrm{E}-05$ & $\bar{U}$ \\
\hline \multirow[t]{51}{*}{$(200-W)$} & $12 / 31 / 07$ & 01/14/08 & gross $\beta$ & $9.0 \mathrm{E}-03 \pm 1.4 \mathrm{E}-03$ & & & & ${ }^{134} \mathrm{Cs}$ & $-4.8 \mathrm{E}-05 \pm 8.4 \mathrm{E}-05$ & $\mathrm{U}$ \\
\hline & $01 / 14 / 08$ & 01/29/08 & gross $\alpha$ & $2.1 \mathrm{E}-03 \pm 6.8 \mathrm{E}-04$ & & & & ${ }^{137} \mathrm{Cs}$ & $-9.4 \mathrm{E}-06 \pm 7.2 \mathrm{E}-05$ & U \\
\hline & $01 / 14 / 08$ & 01/29/08 & gross $\beta$ & $2.6 \mathrm{E}-02 \pm 2.7 \mathrm{E}-03$ & & & & ${ }^{152} \mathrm{Eu}$ & $-6.1 \mathrm{E}-06 \pm 6.1 \mathrm{E}-05$ & $\mathrm{U}$ \\
\hline & $01 / 29 / 08$ & $02 / 12 / 08$ & gross $\alpha$ & $1.1 \mathrm{E}-03 \pm 5.0 \mathrm{E}-04$ & & & & ${ }^{154} \mathrm{Eu}$ & $1.8 \mathrm{E}-05 \pm 1.8 \mathrm{E}-04$ & $\mathrm{U}$ \\
\hline & $01 / 29 / 08$ & 02/12/08 & gross $\beta$ & $3.9 \mathrm{E}-03 \pm 8.7 \mathrm{E}-04$ & & & & ${ }^{155} \mathrm{Eu}$ & $-8.0 \mathrm{E}-05 \pm 1.8 \mathrm{E}-04$ & U \\
\hline & $02 / 12 / 08$ & $02 / 25 / 08$ & gross $\alpha$ & $9.5 \mathrm{E}-04 \pm 6.4 \mathrm{E}-04$ & & & & ${ }^{238} \mathrm{Pu}$ & $-7.4 \mathrm{E}-07 \pm 7.4 \mathrm{E}-06$ & $\mathrm{U}$ \\
\hline & $02 / 12 / 08$ & $02 / 25 / 08$ & gross $\beta$ & $2.8 \mathrm{E}-02 \pm 3.0 \mathrm{E}-03$ & & & & ${ }^{239 / 240} \mathrm{Pu}$ & $1.5 \mathrm{E}-06 \pm 2.2 \mathrm{E}-06$ & $\mathrm{U}$ \\
\hline & $02 / 25 / 08$ & 03/11/08 & gross $\alpha$ & $7.4 \mathrm{E}-04 \pm 5.4 \mathrm{E}-04$ & & & & ${ }^{106} \mathrm{Ru}$ & $1.0 \mathrm{E}-03 \pm 7.6 \mathrm{E}-04$ & $\mathrm{U}$ \\
\hline & $02 / 25 / 08$ & 03/11/08 & gross $\beta$ & $1.3 \mathrm{E}-02 \pm 1.7 \mathrm{E}-03$ & & & & ${ }^{125} \mathrm{Sb}$ & $6.7 \mathrm{E}-05 \pm 1.6 \mathrm{E}-04$ & $\mathrm{U}$ \\
\hline & 03/11/08 & 03/25/08 & gross $\alpha$ & $1.2 \mathrm{E}-03 \pm 5.4 \mathrm{E}-04$ & & & & ${ }^{90} \mathrm{Sr}$ & $-2.5 \mathrm{E}-04 \pm 2.6 \mathrm{E}-04$ & $\mathrm{U}$ \\
\hline & 03/11/08 & $03 / 25 / 08$ & gross $\beta$ & $7.7 \mathrm{E}-03 \pm 1.3 \mathrm{E}-03$ & & & & ${ }^{234} \mathrm{U}$ & $1.8 \mathrm{E}-05 \pm 9.7 \mathrm{E}-06$ & \\
\hline & $03 / 25 / 08$ & $04 / 07 / 08$ & gross $\alpha$ & $8.4 \mathrm{E}-04 \pm 6.2 \mathrm{E}-04$ & & & & ${ }^{235} \mathrm{U}$ & $2.3 \mathrm{E}-06 \pm 2.8 \mathrm{E}-06$ & \\
\hline & $03 / 25 / 08$ & $04 / 07 / 08$ & gross $\beta$ & $8.2 \mathrm{E}-03 \pm 1.4 \mathrm{E}-03$ & & & & ${ }^{238} \mathrm{U}$ & $7.7 \mathrm{E}-06 \pm 5.5 \mathrm{E}-06$ & \\
\hline & 04/07/08 & $04 / 21 / 08$ & gross $\alpha$ & $1.6 \mathrm{E}-03 \pm 6.2 \mathrm{E}-04$ & & & & & & \\
\hline & $04 / 07 / 08$ & $04 / 21 / 08$ & gross $\beta$ & $1.2 \mathrm{E}-02 \pm 1.7 \mathrm{E}-03$ & N449 & $06 / 30 / 08$ to & $12 / 29 / 08$ & ${ }^{60} \mathrm{Co}$ & $4.5 \mathrm{E}-05 \pm 8.0 \mathrm{E}-05$ & U \\
\hline & $04 / 21 / 08$ & 05/06/08 & gross $\alpha$ & $1.5 \mathrm{E}-03 \pm 5.8 \mathrm{E}-04$ & & & & ${ }^{134} \mathrm{Cs}$ & $1.6 \mathrm{E}-05 \pm 6.8 \mathrm{E}-05$ & $\mathrm{U}$ \\
\hline & $04 / 21 / 08$ & 05/06/08 & gross $\beta$ & $1.4 \mathrm{E}-02 \pm 1.9 \mathrm{E}-03$ & & & & ${ }^{137} \mathrm{Cs}$ & $2.6 \mathrm{E}-05 \pm 6.4 \mathrm{E}-05$ & $\mathrm{U}$ \\
\hline & 05/06/08 & 05/19/08 & gross $\alpha$ & $3.5 \mathrm{E}-04 \pm 4.7 \mathrm{E}-04$ & & & & ${ }^{152} \mathrm{Eu}$ & $-1.2 \mathrm{E}-04 \pm 1.6 \mathrm{E}-04$ & U \\
\hline & 05/06/08 & 05/19/08 & gross $\beta$ & $9.7 \mathrm{E}-03 \pm 1.5 \mathrm{E}-03$ & & & & ${ }^{154} \mathrm{Eu}$ & $1.7 \mathrm{E}-04 \pm 2.4 \mathrm{E}-04$ & $\mathrm{U}$ \\
\hline & 05/19/08 & $06 / 02 / 08$ & gross $\alpha$ & $1.1 \mathrm{E}-03 \pm 5.1 \mathrm{E}-04$ & & & & ${ }^{155} \mathrm{Eu}$ & $1.7 \mathrm{E}-05 \pm 1.4 \mathrm{E}-04$ & $\mathrm{U}$ \\
\hline & 05/19/08 & $06 / 02 / 08$ & gross $\beta$ & $1.4 \mathrm{E}-02 \pm 1.9 \mathrm{E}-03$ & & & & ${ }^{238} \mathrm{Pu}$ & $-3.2 \mathrm{E}-06 \pm 1.5 \mathrm{E}-05$ & $\mathrm{U}$ \\
\hline & 06/02/08 & 06/16/08 & gross $\alpha$ & $1.4 \mathrm{E}-03 \pm 6.5 \mathrm{E}-04$ & & & & ${ }^{239 / 240} \mathrm{Pu}$ & $1.6 \mathrm{E}-06 \pm 2.4 \mathrm{E}-06$ & $\mathrm{U}$ \\
\hline & $06 / 02 / 08$ & 06/16/08 & gross $\beta$ & $7.9 \mathrm{E}-03 \pm 1.3 \mathrm{E}-03$ & & & & ${ }^{106} \mathrm{Ru}$ & $-6.2 \mathrm{E}-04 \pm 6.4 \mathrm{E}-04$ & $\mathrm{U}$ \\
\hline & $06 / 16 / 08$ & 06/30/08 & gross $\alpha$ & $1.3 \mathrm{E}-03 \pm 5.7 \mathrm{E}-04$ & & & & ${ }^{125} \mathrm{Sb}$ & $1.2 \mathrm{E}-04 \pm 1.5 \mathrm{E}-04$ & $\mathrm{U}$ \\
\hline & $06 / 16 / 08$ & 06/30/08 & gross $\beta$ & $1.5 \mathrm{E}-02 \pm 2.0 \mathrm{E}-03$ & & & & ${ }^{90} \mathrm{Sr}$ & $-3.3 \mathrm{E}-04 \pm 3.5 \mathrm{E}-04$ & $\mathrm{U}$ \\
\hline & $06 / 30 / 08$ & $07 / 14 / 08$ & gross $\alpha$ & $2.6 \mathrm{E}-03 \pm 8.8 \mathrm{E}-04$ & & & & ${ }^{234} \mathrm{U}$ & $1.4 \mathrm{E}-05 \pm 8.4 \mathrm{E}-06$ & \\
\hline & $06 / 30 / 08$ & 07/14/08 & gross $\beta$ & $1.1 \mathrm{E}-02 \pm 1.6 \mathrm{E}-03$ & & & & ${ }^{235} \mathrm{U}$ & $5.2 \mathrm{E}-06 \pm 4.9 \mathrm{E}-06$ & $\mathrm{U}$ \\
\hline & $07 / 14 / 08$ & 07/28/08 & gross $\alpha$ & $1.6 \mathrm{E}-03 \pm 6.4 \mathrm{E}-04$ & & & & ${ }^{238} \mathrm{U}$ & $1.1 \mathrm{E}-05 \pm 7.1 \mathrm{E}-06$ & \\
\hline & $07 / 14 / 08$ & $07 / 28 / 08$ & gross $\beta$ & $1.6 \mathrm{E}-02 \pm 2.1 \mathrm{E}-03$ & & & & & & \\
\hline & 07/28/08 & 08/11/08 & gross $\alpha$ & $1.6 \mathrm{E}-03 \pm 6.2 \mathrm{E}-04$ & & & & & & \\
\hline & 07/28/08 & 08/11/08 & gross $\beta$ & $2.0 \mathrm{E}-02 \pm 2.4 \mathrm{E}-03$ & & & & & & \\
\hline & $08 / 11 / 08$ & $08 / 25 / 08$ & gross $\alpha$ & $1.3 \mathrm{E}-03 \pm 5.6 \mathrm{E}-04$ & & & & & & \\
\hline & 08/11/08 & 08/25/08 & gross $\beta$ & $1.5 \mathrm{E}-02 \pm 2.0 \mathrm{E}-03$ & & & & & & \\
\hline & $08 / 25 / 08$ & 09/08/08 & gross $\alpha$ & $7.0 \mathrm{E}-04 \pm 5.2 \mathrm{E}-04$ & & & & & & \\
\hline & $08 / 25 / 08$ & 09/08/08 & gross $\beta$ & $1.2 \mathrm{E}-02 \pm 1.7 \mathrm{E}-03$ & & & & & & \\
\hline & $09 / 08 / 08$ & $09 / 22 / 08$ & gross $\alpha$ & $2.5 \mathrm{E}-03 \pm 7.9 \mathrm{E}-04$ & & & & & & \\
\hline & 09/08/08 & $09 / 22 / 08$ & gross $\beta$ & $3.2 \mathrm{E}-02 \pm 3.4 \mathrm{E}-03$ & & & & & & \\
\hline & $09 / 22 / 08$ & $10 / 07 / 08$ & gross $\alpha$ & $1.8 \mathrm{E}-03 \pm 6.3 \mathrm{E}-04$ & & & & & & \\
\hline & $09 / 22 / 08$ & $10 / 07 / 08$ & gross $\beta$ & $2.4 \mathrm{E}-02 \pm 2.7 \mathrm{E}-03$ & & & & & & \\
\hline & $10 / 07 / 08$ & $10 / 20 / 08$ & gross $\alpha$ & $1.9 \mathrm{E}-03 \pm 6.9 \mathrm{E}-04$ & & & & & & \\
\hline & $10 / 07 / 08$ & $10 / 20 / 08$ & gross $\beta$ & $2.3 \mathrm{E}-02 \pm 2.7 \mathrm{E}-03$ & & & & & & \\
\hline & $10 / 20 / 08$ & $11 / 03 / 08$ & gross $\alpha$ & $3.3 \mathrm{E}-03 \pm 8.9 \mathrm{E}-04$ & & & & & & \\
\hline & $10 / 20 / 08$ & $11 / 03 / 08$ & gross $\beta$ & $3.9 \mathrm{E}-02 \pm 3.9 \mathrm{E}-03$ & & & & & & \\
\hline & $11 / 03 / 08$ & $11 / 17 / 08$ & gross $\alpha$ & $1.2 \mathrm{E}-03 \pm 5.4 \mathrm{E}-04$ & & & & & & \\
\hline & $11 / 03 / 08$ & $11 / 17 / 08$ & gross $\beta$ & $1.8 \mathrm{E}-02 \pm 2.2 \mathrm{E}-03$ & & & & & & \\
\hline & $11 / 17 / 08$ & $12 / 01 / 08$ & gross $\alpha$ & $1.6 \mathrm{E}-03 \pm 6.1 \mathrm{E}-04$ & & & & & & \\
\hline & $11 / 17 / 08$ & $12 / 01 / 08$ & gross $\beta$ & $3.1 \mathrm{E}-02 \pm 3.1 \mathrm{E}-03$ & & & & & & \\
\hline & $12 / 01 / 08$ & $12 / 15 / 08$ & gross $\alpha$ & $1.1 \mathrm{E}-03 \pm 5.1 \mathrm{E}-04$ & & & & & & \\
\hline & $12 / 01 / 08$ & $12 / 15 / 08$ & gross $\beta$ & $2.2 \mathrm{E}-02 \pm 2.5 \mathrm{E}-03$ & & & & & & \\
\hline & $12 / 15 / 08$ & $12 / 29 / 08$ & gross $\alpha$ & $1.9 \mathrm{E}-03 \pm 6.8 \mathrm{E}-04$ & & & & & & \\
\hline & $12 / 15 / 08$ & $12 / 29 / 08$ & gross $\beta$ & $4.7 \mathrm{E}-02 \pm 4.4 \mathrm{E}-03$ & & & & & & \\
\hline
\end{tabular}

$\overline{\mathrm{RQ}}=$ Result Qualifier. $\mathrm{U}=$ The analyte was analyzed for but not detected. 
Table 2-4. Near-Facility Air Sampling Results, 2008 (pCi/m³ \pm total analytical uncertainty). (Sheet 58 of 82)

\begin{tabular}{|c|c|c|c|c|c|c|c|c|c|c|}
\hline Location & Sample On & Sample Off & Isotope & Result \pm Uncertainty & Location & Composite & e Period & Isotope & Result \pm Uncertainty & RQ* \\
\hline N456 & $12 / 31 / 07$ & $01 / 14 / 08$ & gross $\alpha$ & $1.5 \mathrm{E}-03 \pm 5.9 \mathrm{E}-04$ & N456 & $12 / 31 / 07$ to & $06 / 30 / 08$ & ${ }^{60} \mathrm{Co}$ & $-9.1 \mathrm{E}-06 \pm 8.9 \mathrm{E}-05$ & $\bar{U}$ \\
\hline \multirow[t]{51}{*}{$(200-W)$} & $12 / 31 / 07$ & 01/14/08 & gross $\beta$ & $9.5 \mathrm{E}-03 \pm 1.4 \mathrm{E}-03$ & & & & ${ }^{134} \mathrm{Cs}$ & $1.9 \mathrm{E}-05 \pm 8.3 \mathrm{E}-05$ & $\mathrm{U}$ \\
\hline & $01 / 14 / 08$ & 01/29/08 & gross $\alpha$ & $1.9 \mathrm{E}-03 \pm 6.4 \mathrm{E}-04$ & & & & ${ }^{137} \mathrm{Cs}$ & $8.3 \mathrm{E}-06 \pm 7.6 \mathrm{E}-05$ & U \\
\hline & $01 / 14 / 08$ & 01/29/08 & gross $\beta$ & $3.4 \mathrm{E}-02 \pm 3.4 \mathrm{E}-03$ & & & & ${ }^{152} \mathrm{Eu}$ & $7.0 \mathrm{E}-05 \pm 1.8 \mathrm{E}-04$ & $\mathrm{U}$ \\
\hline & $01 / 29 / 08$ & $02 / 12 / 08$ & gross $\alpha$ & $3.9 \mathrm{E}-04 \pm 4.4 \mathrm{E}-04$ & & & & ${ }^{154} \mathrm{Eu}$ & $1.2 \mathrm{E}-05 \pm 1.2 \mathrm{E}-04$ & $\mathrm{U}$ \\
\hline & $01 / 29 / 08$ & 02/12/08 & gross $\beta$ & $3.9 \mathrm{E}-03 \pm 8.7 \mathrm{E}-04$ & & & & ${ }^{155} \mathrm{Eu}$ & $-3.0 \mathrm{E}-05 \pm 2.0 \mathrm{E}-04$ & U \\
\hline & $02 / 12 / 08$ & $02 / 25 / 08$ & gross $\alpha$ & $1.2 \mathrm{E}-03 \pm 5.5 \mathrm{E}-04$ & & & & ${ }^{238} \mathrm{Pu}$ & $3.3 \mathrm{E}-06 \pm 1.4 \mathrm{E}-05$ & $\mathrm{U}$ \\
\hline & $02 / 12 / 08$ & $02 / 25 / 08$ & gross $\beta$ & $2.7 \mathrm{E}-02 \pm 2.9 \mathrm{E}-03$ & & & & ${ }^{239 / 240} \mathrm{Pu}$ & $3.3 \mathrm{E}-06 \pm 5.8 \mathrm{E}-06$ & $\mathrm{U}$ \\
\hline & $02 / 25 / 08$ & 03/11/08 & gross $\alpha$ & $1.1 \mathrm{E}-03 \pm 4.9 \mathrm{E}-04$ & & & & ${ }^{106} \mathrm{Ru}$ & $-3.4 \mathrm{E}-04 \pm 7.5 \mathrm{E}-04$ & $\mathrm{U}$ \\
\hline & $02 / 25 / 08$ & 03/11/08 & gross $\beta$ & $1.6 \mathrm{E}-02 \pm 2.0 \mathrm{E}-03$ & & & & ${ }^{125} \mathrm{Sb}$ & $7.3 \mathrm{E}-06 \pm 7.3 \mathrm{E}-05$ & $\mathrm{U}$ \\
\hline & 03/11/08 & 03/25/08 & gross $\alpha$ & $1.0 \mathrm{E}-03 \pm 6.2 \mathrm{E}-04$ & & & & ${ }^{90} \mathrm{Sr}$ & $-2.3 \mathrm{E}-04 \pm 2.4 \mathrm{E}-04$ & $\mathrm{U}$ \\
\hline & 03/11/08 & $03 / 25 / 08$ & gross $\beta$ & $9.4 \mathrm{E}-03 \pm 1.4 \mathrm{E}-03$ & & & & ${ }^{234} \mathrm{U}$ & $1.2 \mathrm{E}-05 \pm 7.6 \mathrm{E}-06$ & \\
\hline & $03 / 25 / 08$ & $04 / 07 / 08$ & gross $\alpha$ & $4.3 \mathrm{E}-04 \pm 4.8 \mathrm{E}-04$ & & & & ${ }^{235} \mathrm{U}$ & $2.4 \mathrm{E}-06 \pm 2.9 \mathrm{E}-06$ & \\
\hline & 03/25/08 & $04 / 07 / 08$ & gross $\beta$ & $7.2 \mathrm{E}-03 \pm 1.3 \mathrm{E}-03$ & & & & ${ }^{238} \mathrm{U}$ & $8.8 \mathrm{E}-06 \pm 6.0 \mathrm{E}-06$ & \\
\hline & 04/07/08 & $04 / 21 / 08$ & gross $\alpha$ & $7.4 \mathrm{E}-04 \pm 5.5 \mathrm{E}-04$ & & & & & & \\
\hline & $04 / 07 / 08$ & $04 / 21 / 08$ & gross $\beta$ & $1.0 \mathrm{E}-02 \pm 1.5 \mathrm{E}-03$ & N456 & $06 / 30 / 08$ to & $12 / 29 / 08$ & ${ }^{60} \mathrm{Co}$ & $1.1 \mathrm{E}-04 \pm 1.3 \mathrm{E}-04$ & U \\
\hline & $04 / 21 / 08$ & $05 / 05 / 08$ & gross $\alpha$ & $1.2 \mathrm{E}-03 \pm 9.8 \mathrm{E}-04$ & & & & ${ }^{134} \mathrm{Cs}$ & $-1.9 \mathrm{E}-05 \pm 1.1 \mathrm{E}-04$ & $\mathrm{U}$ \\
\hline & $04 / 21 / 08$ & 05/05/08 & gross $\beta$ & $7.3 \mathrm{E}-03 \pm 1.7 \mathrm{E}-03$ & & & & ${ }^{137} \mathrm{Cs}$ & $9.3 \mathrm{E}-05 \pm 1.1 \mathrm{E}-04$ & $\mathrm{U}$ \\
\hline & 05/05/08 & 05/19/08 & gross $\alpha$ & $-2.2 \mathrm{E}-04 \pm 1.0 \mathrm{E}-03$ & & & & ${ }^{152} \mathrm{Eu}$ & $5.8 \mathrm{E}-05 \pm 2.7 \mathrm{E}-04$ & U \\
\hline & 05/05/08 & 05/19/08 & gross $\beta$ & $7.1 \mathrm{E}-04 \pm 1.7 \mathrm{E}-03$ & & & & ${ }^{154} \mathrm{Eu}$ & $-5.6 \mathrm{E}-05 \pm 3.4 \mathrm{E}-04$ & $\mathrm{U}$ \\
\hline & 05/19/08 & $06 / 02 / 08$ & gross $\alpha$ & $1.5 \mathrm{E}-03 \pm 6.9 \mathrm{E}-04$ & & & & ${ }^{155} \mathrm{Eu}$ & $1.6 \mathrm{E}-04 \pm 2.0 \mathrm{E}-04$ & $\mathrm{U}$ \\
\hline & 05/19/08 & $06 / 02 / 08$ & gross $\beta$ & $1.5 \mathrm{E}-02 \pm 2.2 \mathrm{E}-03$ & & & & ${ }^{238} \mathrm{Pu}$ & $-1.5 \mathrm{E}-06 \pm 1.2 \mathrm{E}-05$ & $\mathrm{U}$ \\
\hline & $06 / 02 / 08$ & $06 / 16 / 08$ & gross $\alpha$ & $9.1 \mathrm{E}-04 \pm 6.4 \mathrm{E}-04$ & & & & ${ }^{239 / 240} \mathrm{Pu}$ & $2.3 \mathrm{E}-06 \pm 2.8 \mathrm{E}-06$ & \\
\hline & $06 / 02 / 08$ & 06/16/08 & gross $\beta$ & $6.6 \mathrm{E}-03 \pm 1.2 \mathrm{E}-03$ & & & & ${ }^{106} \mathrm{Ru}$ & $-2.1 \mathrm{E}-04 \pm 8.6 \mathrm{E}-04$ & $\mathrm{U}$ \\
\hline & $06 / 16 / 08$ & 06/30/08 & gross $\alpha$ & $1.4 \mathrm{E}-03 \pm 5.7 \mathrm{E}-04$ & & & & ${ }^{125} \mathrm{Sb}$ & $3.2 \mathrm{E}-04 \pm 2.8 \mathrm{E}-04$ & $\mathrm{U}$ \\
\hline & $06 / 16 / 08$ & 06/30/08 & gross $\beta$ & $4.6 \mathrm{E}-02 \pm 4.3 \mathrm{E}-03$ & & & & ${ }^{90} \mathrm{Sr}$ & $-4.0 \mathrm{E}-04 \pm 4.2 \mathrm{E}-04$ & $\mathrm{U}$ \\
\hline & $06 / 30 / 08$ & $07 / 14 / 08$ & gross $\alpha$ & $1.2 \mathrm{E}-03 \pm 6.0 \mathrm{E}-04$ & & & & ${ }^{234} \mathrm{U}$ & $1.6 \mathrm{E}-05 \pm 9.2 \mathrm{E}-06$ & \\
\hline & $06 / 30 / 08$ & 07/14/08 & gross $\beta$ & $1.2 \mathrm{E}-02 \pm 1.7 \mathrm{E}-03$ & & & & ${ }^{235} \mathrm{U}$ & $3.9 \mathrm{E}-06 \pm 4.8 \mathrm{E}-06$ & $\mathrm{U}$ \\
\hline & $07 / 14 / 08$ & 07/28/08 & gross $\alpha$ & $2.3 \mathrm{E}-03 \pm 7.3 \mathrm{E}-04$ & & & & ${ }^{238} \mathrm{U}$ & $1.2 \mathrm{E}-05 \pm 7.4 \mathrm{E}-06$ & \\
\hline & $07 / 14 / 08$ & $07 / 28 / 08$ & gross $\beta$ & $1.5 \mathrm{E}-02 \pm 2.0 \mathrm{E}-03$ & & & & & & \\
\hline & 07/28/08 & 08/11/08 & gross $\alpha$ & $8.9 \mathrm{E}-04 \pm 5.6 \mathrm{E}-04$ & & & & & & \\
\hline & 07/28/08 & 08/11/08 & gross $\beta$ & $1.7 \mathrm{E}-02 \pm 2.1 \mathrm{E}-03$ & & & & & & \\
\hline & $08 / 11 / 08$ & $08 / 25 / 08$ & gross $\alpha$ & $2.6 \mathrm{E}-04 \pm 3.9 \mathrm{E}-04$ & & & & & & \\
\hline & 08/11/08 & 08/25/08 & gross $\beta$ & $1.2 \mathrm{E}-02 \pm 1.7 \mathrm{E}-03$ & & & & & & \\
\hline & $08 / 25 / 08$ & 09/08/08 & gross $\alpha$ & $7.0 \mathrm{E}-04 \pm 5.2 \mathrm{E}-04$ & & & & & & \\
\hline & $08 / 25 / 08$ & 09/08/08 & gross $\beta$ & $1.0 \mathrm{E}-02 \pm 1.6 \mathrm{E}-03$ & & & & & & \\
\hline & $09 / 08 / 08$ & $09 / 22 / 08$ & gross $\alpha$ & $1.5 \mathrm{E}-03 \pm 5.8 \mathrm{E}-04$ & & & & & & \\
\hline & 09/08/08 & $09 / 22 / 08$ & gross $\beta$ & $3.0 \mathrm{E}-02 \pm 3.2 \mathrm{E}-03$ & & & & & & \\
\hline & $09 / 22 / 08$ & $10 / 07 / 08$ & gross $\alpha$ & $1.2 \mathrm{E}-03 \pm 5.0 \mathrm{E}-04$ & & & & & & \\
\hline & $09 / 22 / 08$ & $10 / 07 / 08$ & gross $\beta$ & $2.5 \mathrm{E}-02 \pm 2.7 \mathrm{E}-03$ & & & & & & \\
\hline & $10 / 07 / 08$ & $10 / 20 / 08$ & gross $\alpha$ & $1.6 \mathrm{E}-03 \pm 6.2 \mathrm{E}-04$ & & & & & & \\
\hline & $10 / 07 / 08$ & $10 / 20 / 08$ & gross $\beta$ & $2.1 \mathrm{E}-02 \pm 2.5 \mathrm{E}-03$ & & & & & & \\
\hline & $10 / 20 / 08$ & $11 / 03 / 08$ & gross $\alpha$ & $1.1 \mathrm{E}-03 \pm 5.1 \mathrm{E}-04$ & & & & & & \\
\hline & $10 / 20 / 08$ & $11 / 03 / 08$ & gross $\beta$ & $4.3 \mathrm{E}-02 \pm 4.1 \mathrm{E}-03$ & & & & & & \\
\hline & $11 / 03 / 08$ & $11 / 17 / 08$ & gross $\alpha$ & $4.2 \mathrm{E}-03 \pm 1.5 \mathrm{E}-03$ & & & & & & \\
\hline & $11 / 03 / 08$ & $11 / 17 / 08$ & gross $\beta$ & $4.8 \mathrm{E}-02 \pm 5.7 \mathrm{E}-03$ & & & & & & \\
\hline & $11 / 17 / 08$ & $12 / 01 / 08$ & gross $\alpha$ & $1.5 \mathrm{E}-03 \pm 6.1 \mathrm{E}-04$ & & & & & & \\
\hline & $11 / 17 / 08$ & $12 / 01 / 08$ & gross $\beta$ & $3.8 \mathrm{E}-02 \pm 3.7 \mathrm{E}-03$ & & & & & & \\
\hline & $12 / 01 / 08$ & $12 / 15 / 08$ & gross $\alpha$ & $1.2 \mathrm{E}-03 \pm 5.2 \mathrm{E}-04$ & & & & & & \\
\hline & $12 / 01 / 08$ & $12 / 15 / 08$ & gross $\beta$ & $2.5 \mathrm{E}-02 \pm 2.7 \mathrm{E}-03$ & & & & & & \\
\hline & $12 / 15 / 08$ & $12 / 29 / 08$ & gross $\alpha$ & $2.7 \mathrm{E}-03 \pm 8.0 \mathrm{E}-04$ & & & & & & \\
\hline & $12 / 15 / 08$ & $12 / 29 / 08$ & gross $\beta$ & 4.7E-02 $\pm 4.3 \mathrm{E}-03$ & & & & & & \\
\hline
\end{tabular}

$\overline{\mathrm{RQ}}=$ Result Qualifier. $\mathrm{U}=$ The analyte was analyzed for but not detected. 
Table 2-4. Near-Facility Air Sampling Results, 2008 (pCi/m³ \pm total analytical uncertainty). (Sheet 59 of 82)

\begin{tabular}{|c|c|c|c|c|c|c|c|c|c|c|}
\hline Location & Sample On & Sample Off & Isotope & Result \pm Uncertainty & Location & Composite & e Period & Isotope & Result \pm Uncertainty & $\mathbf{R Q}^{*}$ \\
\hline N457 & $12 / 31 / 07$ & $01 / 14 / 08$ & gross $\alpha$ & $1.1 \mathrm{E}-03 \pm 5.0 \mathrm{E}-04$ & N457 & $12 / 31 / 07$ to & $06 / 30 / 08$ & ${ }^{60} \mathrm{Co}$ & $1.0 \mathrm{E}-05 \pm 8.7 \mathrm{E}-05$ & $\mathrm{U}$ \\
\hline \multirow[t]{51}{*}{$(200-W)$} & $12 / 31 / 07$ & 01/14/08 & gross $\beta$ & $8.0 \mathrm{E}-03 \pm 1.3 \mathrm{E}-03$ & & & & ${ }^{134} \mathrm{Cs}$ & $-6.1 \mathrm{E}-05 \pm 6.6 \mathrm{E}-05$ & $\mathrm{U}$ \\
\hline & $01 / 14 / 08$ & 01/29/08 & gross $\alpha$ & $1.7 \mathrm{E}-03 \pm 6.1 \mathrm{E}-04$ & & & & ${ }^{137} \mathrm{Cs}$ & $-1.1 \mathrm{E}-06 \pm 1.1 \mathrm{E}-05$ & U \\
\hline & $01 / 14 / 08$ & 01/29/08 & gross $\beta$ & $2.9 \mathrm{E}-02 \pm 3.0 \mathrm{E}-03$ & & & & ${ }^{152} \mathrm{Eu}$ & $5.2 \mathrm{E}-06 \pm 5.2 \mathrm{E}-05$ & U \\
\hline & $01 / 29 / 08$ & $02 / 12 / 08$ & gross $\alpha$ & $2.7 \mathrm{E}-04 \pm 3.9 \mathrm{E}-04$ & & & & ${ }^{154} \mathrm{Eu}$ & $3.3 \mathrm{E}-05 \pm 2.1 \mathrm{E}-04$ & $\mathrm{U}$ \\
\hline & $01 / 29 / 08$ & 02/12/08 & gross $\beta$ & $3.4 \mathrm{E}-03 \pm 8.2 \mathrm{E}-04$ & & & & ${ }^{155} \mathrm{Eu}$ & $3.0 \mathrm{E}-05 \pm 1.2 \mathrm{E}-04$ & U \\
\hline & $02 / 12 / 08$ & $02 / 25 / 08$ & gross $\alpha$ & $1.5 \mathrm{E}-03 \pm 6.3 \mathrm{E}-04$ & & & & ${ }^{238} \mathrm{Pu}$ & $-9.6 \mathrm{E}-06 \pm 1.4 \mathrm{E}-05$ & $\mathrm{U}$ \\
\hline & $02 / 12 / 08$ & $02 / 25 / 08$ & gross $\beta$ & $2.6 \mathrm{E}-02 \pm 2.8 \mathrm{E}-03$ & & & & ${ }^{239 / 240} \mathrm{Pu}$ & $4.0 \mathrm{E}-06 \pm 5.5 \mathrm{E}-06$ & $\mathrm{U}$ \\
\hline & $02 / 25 / 08$ & 03/11/08 & gross $\alpha$ & $9.9 \mathrm{E}-04 \pm 4.7 \mathrm{E}-04$ & & & & ${ }^{103} \mathrm{Ru}$ & $-5.7 \mathrm{E}-05 \pm 8.7 \mathrm{E}-05$ & $\mathrm{U}$ \\
\hline & $02 / 25 / 08$ & 03/11/08 & gross $\beta$ & $1.7 \mathrm{E}-02 \pm 2.0 \mathrm{E}-03$ & & & & ${ }^{106} \mathrm{Ru}$ & $4.0 \mathrm{E}-04 \pm 5.3 \mathrm{E}-04$ & U \\
\hline & 03/11/08 & 03/25/08 & gross $\alpha$ & $2.9 \mathrm{E}-04 \pm 4.2 \mathrm{E}-04$ & & & & ${ }^{125} \mathrm{Sb}$ & $7.1 \mathrm{E}-05 \pm 1.3 \mathrm{E}-04$ & $\mathrm{U}$ \\
\hline & 03/11/08 & $03 / 25 / 08$ & gross $\beta$ & $7.7 \mathrm{E}-03 \pm 1.3 \mathrm{E}-03$ & & & & ${ }^{113} \mathrm{Sn}$ & $-1.2 \mathrm{E}-05 \pm 7.0 \mathrm{E}-05$ & $\mathrm{U}$ \\
\hline & $03 / 25 / 08$ & $04 / 07 / 08$ & gross $\alpha$ & $1.0 \mathrm{E}-03 \pm 6.3 \mathrm{E}-04$ & & & & ${ }^{90} \mathrm{Sr}$ & $-1.3 \mathrm{E}-04 \pm 1.4 \mathrm{E}-04$ & $\mathrm{U}$ \\
\hline & 03/25/08 & $04 / 07 / 08$ & gross $\beta$ & $1.1 \mathrm{E}-02 \pm 1.6 \mathrm{E}-03$ & & & & ${ }^{234} \mathrm{U}$ & $2.0 \mathrm{E}-05 \pm 1.0 \mathrm{E}-05$ & \\
\hline & $04 / 07 / 08$ & $04 / 21 / 08$ & gross $\alpha$ & $3.9 \mathrm{E}-04 \pm 4.4 \mathrm{E}-04$ & & & & ${ }^{235} \mathrm{U}$ & $7.5 \mathrm{E}-07 \pm 3.3 \mathrm{E}-06$ & $\mathrm{U}$ \\
\hline & $04 / 07 / 08$ & $04 / 21 / 08$ & gross $\beta$ & $9.4 \mathrm{E}-03 \pm 1.4 \mathrm{E}-03$ & & & & ${ }^{238} \mathrm{U}$ & $1.1 \mathrm{E}-05 \pm 7.1 \mathrm{E}-06$ & \\
\hline & $04 / 21 / 08$ & $05 / 05 / 08$ & gross $\alpha$ & $1.1 \mathrm{E}-03 \pm 4.9 \mathrm{E}-04$ & & & & ${ }^{65} \mathrm{Zn}$ & $1.4 \mathrm{E}-04 \pm 1.8 \mathrm{E}-04$ & $\mathrm{U}$ \\
\hline & $04 / 21 / 08$ & $05 / 05 / 08$ & gross $\beta$ & $1.6 \mathrm{E}-02 \pm 2.0 \mathrm{E}-03$ & & & & & & \\
\hline & 05/05/08 & 05/19/08 & gross $\alpha$ & $7.7 \mathrm{E}-04 \pm 5.5 \mathrm{E}-04$ & N457 & $06 / 30 / 08$ to & $12 / 29 / 08$ & ${ }^{60} \mathrm{Co}$ & $1.8 \mathrm{E}-05 \pm 7.7 \mathrm{E}-05$ & U \\
\hline & 05/05/08 & $05 / 19 / 08$ & gross $\beta$ & $1.2 \mathrm{E}-02 \pm 1.6 \mathrm{E}-03$ & & & & ${ }^{134} \mathrm{Cs}$ & $-1.1 \mathrm{E}-05 \pm 7.6 \mathrm{E}-05$ & $\mathrm{U}$ \\
\hline & 05/19/08 & $06 / 02 / 08$ & gross $\alpha$ & $9.1 \mathrm{E}-04 \pm 5.8 \mathrm{E}-04$ & & & & ${ }^{137} \mathrm{Cs}$ & $1.8 \mathrm{E}-05 \pm 6.9 \mathrm{E}-05$ & $\mathrm{U}$ \\
\hline & 05/19/08 & $06 / 02 / 08$ & gross $\beta$ & $1.3 \mathrm{E}-02 \pm 1.8 \mathrm{E}-03$ & & & & ${ }^{152} \mathrm{Eu}$ & $-1.5 \mathrm{E}-04 \pm 2.1 \mathrm{E}-04$ & $\mathrm{U}$ \\
\hline & $06 / 02 / 08$ & $06 / 16 / 08$ & gross $\alpha$ & $2.7 \mathrm{E}-04 \pm 4.5 \mathrm{E}-04$ & & & & ${ }^{154} \mathrm{Eu}$ & $-2.9 \mathrm{E}-06 \pm 2.9 \mathrm{E}-05$ & $\mathrm{U}$ \\
\hline & $06 / 02 / 08$ & 06/16/08 & gross $\beta$ & $9.3 \mathrm{E}-03 \pm 1.4 \mathrm{E}-03$ & & & & ${ }^{155} \mathrm{Eu}$ & $-4.2 \mathrm{E}-05 \pm 1.8 \mathrm{E}-04$ & $\mathrm{U}$ \\
\hline & $06 / 16 / 08$ & 06/30/08 & gross $\alpha$ & $1.7 \mathrm{E}-03 \pm 6.2 \mathrm{E}-04$ & & & & ${ }^{238} \mathrm{Pu}$ & $-3.6 \mathrm{E}-06 \pm 1.2 \mathrm{E}-05$ & $\mathrm{U}$ \\
\hline & $06 / 16 / 08$ & 06/30/08 & gross $\beta$ & $1.4 \mathrm{E}-02 \pm 1.9 \mathrm{E}-03$ & & & & ${ }^{239 / 240} \mathrm{Pu}$ & $5.0 \mathrm{E}-06 \pm 5.5 \mathrm{E}-06$ & $\mathrm{U}$ \\
\hline & $06 / 30 / 08$ & $07 / 14 / 08$ & gross $\alpha$ & $1.1 \mathrm{E}-03 \pm 7.1 \mathrm{E}-04$ & & & & ${ }^{106} \mathrm{Ru}$ & $-2.4 \mathrm{E}-04 \pm 5.8 \mathrm{E}-04$ & $\mathrm{U}$ \\
\hline & 06/30/08 & 07/14/08 & gross $\beta$ & $1.5 \mathrm{E}-02 \pm 1.9 \mathrm{E}-03$ & & & & ${ }^{125} \mathrm{Sb}$ & $6.6 \mathrm{E}-05 \pm 1.6 \mathrm{E}-04$ & $\mathrm{U}$ \\
\hline & 07/14/08 & 07/28/08 & gross $\alpha$ & $1.1 \mathrm{E}-03 \pm 5.3 \mathrm{E}-04$ & & & & ${ }^{90} \mathrm{Sr}$ & $-2.6 \mathrm{E}-04 \pm 2.7 \mathrm{E}-04$ & U \\
\hline & $07 / 14 / 08$ & $07 / 28 / 08$ & gross $\beta$ & $1.8 \mathrm{E}-02 \pm 2.3 \mathrm{E}-03$ & & & & ${ }^{234} \mathrm{U}$ & $1.4 \mathrm{E}-05 \pm 8.6 \mathrm{E}-06$ & \\
\hline & $07 / 28 / 08$ & 08/11/08 & gross $\alpha$ & $1.2 \mathrm{E}-03 \pm 5.3 \mathrm{E}-04$ & & & & ${ }^{235} \mathrm{U}$ & $2.3 \mathrm{E}-06 \pm 2.8 \mathrm{E}-06$ & \\
\hline & $07 / 28 / 08$ & 08/11/08 & gross $\beta$ & $1.2 \mathrm{E}-02 \pm 1.7 \mathrm{E}-03$ & & & & ${ }^{238} \mathrm{U}$ & $1.1 \mathrm{E}-05 \pm 7.1 \mathrm{E}-06$ & \\
\hline & $08 / 11 / 08$ & $08 / 25 / 08$ & gross $\alpha$ & $4.7 \mathrm{E}-04 \pm 4.6 \mathrm{E}-04$ & & & & & & \\
\hline & 08/11/08 & 08/25/08 & gross $\beta$ & $1.3 \mathrm{E}-02 \pm 1.8 \mathrm{E}-03$ & & & & & & \\
\hline & $08 / 25 / 08$ & 09/08/08 & gross $\alpha$ & $8.1 \mathrm{E}-04 \pm 5.5 \mathrm{E}-04$ & & & & & & \\
\hline & $08 / 25 / 08$ & 09/08/08 & gross $\beta$ & $1.0 \mathrm{E}-02 \pm 1.5 \mathrm{E}-03$ & & & & & & \\
\hline & $09 / 08 / 08$ & $09 / 22 / 08$ & gross $\alpha$ & $1.4 \mathrm{E}-03 \pm 5.5 \mathrm{E}-04$ & & & & & & \\
\hline & 09/08/08 & $09 / 22 / 08$ & gross $\beta$ & $2.6 \mathrm{E}-02 \pm 2.8 \mathrm{E}-03$ & & & & & & \\
\hline & $09 / 22 / 08$ & $10 / 07 / 08$ & gross $\alpha$ & $1.8 \mathrm{E}-03 \pm 6.1 \mathrm{E}-04$ & & & & & & \\
\hline & $09 / 22 / 08$ & $10 / 07 / 08$ & gross $\beta$ & $2.4 \mathrm{E}-02 \pm 2.6 \mathrm{E}-03$ & & & & & & \\
\hline & $10 / 07 / 08$ & $10 / 20 / 08$ & gross $\alpha$ & $1.6 \mathrm{E}-03 \pm 6.2 \mathrm{E}-04$ & & & & & & \\
\hline & $10 / 07 / 08$ & $10 / 20 / 08$ & gross $\beta$ & $2.4 \mathrm{E}-02 \pm 2.8 \mathrm{E}-03$ & & & & & & \\
\hline & $10 / 20 / 08$ & $11 / 03 / 08$ & gross $\alpha$ & $2.5 \mathrm{E}-03 \pm 7.7 \mathrm{E}-04$ & & & & & & \\
\hline & $10 / 20 / 08$ & $11 / 03 / 08$ & gross $\beta$ & 4.7E-02 $\pm 4.4 \mathrm{E}-03$ & & & & & & \\
\hline & $11 / 03 / 08$ & $11 / 17 / 08$ & gross $\alpha$ & $7.9 \mathrm{E}-05 \pm 6.0 \mathrm{E}-04$ & & & & & & \\
\hline & $11 / 03 / 08$ & $11 / 17 / 08$ & gross $\beta$ & $2.0 \mathrm{E}-02 \pm 3.1 \mathrm{E}-03$ & & & & & & \\
\hline & $11 / 17 / 08$ & $12 / 01 / 08$ & gross $\alpha$ & $1.8 \mathrm{E}-03 \pm 6.6 \mathrm{E}-04$ & & & & & & \\
\hline & $11 / 17 / 08$ & $12 / 01 / 08$ & gross $\beta$ & $3.4 \mathrm{E}-02 \pm 3.4 \mathrm{E}-03$ & & & & & & \\
\hline & $12 / 01 / 08$ & $12 / 15 / 08$ & gross $\alpha$ & $1.2 \mathrm{E}-03 \pm 5.2 \mathrm{E}-04$ & & & & & & \\
\hline & $12 / 01 / 08$ & $12 / 15 / 08$ & gross $\beta$ & $3.1 \mathrm{E}-02 \pm 3.1 \mathrm{E}-03$ & & & & & & \\
\hline & $12 / 15 / 08$ & $12 / 29 / 08$ & gross $\alpha$ & $2.0 \mathrm{E}-03 \pm 6.7 \mathrm{E}-04$ & & & & & & \\
\hline & $12 / 15 / 08$ & $12 / 29 / 08$ & gross $\beta$ & $4.5 \mathrm{E}-02 \pm 4.2 \mathrm{E}-03$ & & & & & & \\
\hline
\end{tabular}

$\overline{\mathrm{RQ}}=$ Result Qualifier. $\mathrm{U}=$ The analyte was analyzed for but not detected. 
Table 2-4. Near-Facility Air Sampling Results, 2008 (pCi/m³ \pm total analytical uncertainty). (Sheet 60 of 82)

\begin{tabular}{|c|c|c|c|c|c|c|c|c|c|c|}
\hline Location & Sample On & Sample Off & Isotope & Result \pm Uncertainty & Location & Composite & e Period & Isotope & Result \pm Uncertainty & RQ* \\
\hline N550 & $12 / 31 / 07$ & $01 / 14 / 08$ & gross $\alpha$ & $9.8 \mathrm{E}-04 \pm 6.1 \mathrm{E}-04$ & N550 & $12 / 31 / 07$ to & $06 / 30 / 08$ & ${ }^{60} \mathrm{Co}$ & $2.9 \mathrm{E}-05 \pm 7.1 \mathrm{E}-05$ & $\bar{U}$ \\
\hline \multirow[t]{51}{*}{$(200-W)$} & $12 / 31 / 07$ & 01/14/08 & gross $\beta$ & $8.6 \mathrm{E}-03 \pm 1.4 \mathrm{E}-03$ & & & & ${ }^{134} \mathrm{Cs}$ & $2.9 \mathrm{E}-05 \pm 6.6 \mathrm{E}-05$ & $\mathrm{U}$ \\
\hline & $01 / 14 / 08$ & 01/29/08 & gross $\alpha$ & $2.0 \mathrm{E}-03 \pm 6.7 \mathrm{E}-04$ & & & & ${ }^{137} \mathrm{Cs}$ & $2.7 \mathrm{E}-05 \pm 6.2 \mathrm{E}-05$ & U \\
\hline & $01 / 14 / 08$ & 01/29/08 & gross $\beta$ & $3.2 \mathrm{E}-02 \pm 3.6 \mathrm{E}-03$ & & & & ${ }^{152} \mathrm{Eu}$ & $-1.2 \mathrm{E}-04 \pm 1.3 \mathrm{E}-04$ & $\mathrm{U}$ \\
\hline & $01 / 29 / 08$ & $02 / 12 / 08$ & gross $\alpha$ & $7.5 \mathrm{E}-04 \pm 5.5 \mathrm{E}-04$ & & & & ${ }^{154} \mathrm{Eu}$ & $9.2 \mathrm{E}-05 \pm 2.3 \mathrm{E}-04$ & $\mathrm{U}$ \\
\hline & $01 / 29 / 08$ & 02/12/08 & gross $\beta$ & $4.0 \mathrm{E}-03 \pm 9.1 \mathrm{E}-04$ & & & & ${ }^{155} \mathrm{Eu}$ & $-9.7 \mathrm{E}-06 \pm 9.7 \mathrm{E}-05$ & U \\
\hline & $02 / 12 / 08$ & $02 / 25 / 08$ & gross $\alpha$ & $1.4 \mathrm{E}-03 \pm 5.9 \mathrm{E}-04$ & & & & ${ }^{238} \mathrm{Pu}$ & $1.0 \mathrm{E}-05 \pm 1.2 \mathrm{E}-05$ & $\mathrm{U}$ \\
\hline & $02 / 12 / 08$ & $02 / 25 / 08$ & gross $\beta$ & $3.2 \mathrm{E}-02 \pm 3.7 \mathrm{E}-03$ & & & & ${ }^{239 / 240} \mathrm{Pu}$ & $6.5 \mathrm{E}-06 \pm 6.1 \mathrm{E}-06$ & $\mathrm{U}$ \\
\hline & $02 / 25 / 08$ & 03/11/08 & gross $\alpha$ & $9.7 \mathrm{E}-04 \pm 6.0 \mathrm{E}-04$ & & & & ${ }^{106} \mathrm{Ru}$ & $1.0 \mathrm{E}-04 \pm 5.1 \mathrm{E}-04$ & $\mathrm{U}$ \\
\hline & $02 / 25 / 08$ & 03/11/08 & gross $\beta$ & $1.3 \mathrm{E}-02 \pm 1.9 \mathrm{E}-03$ & & & & ${ }^{125} \mathrm{Sb}$ & $-5.1 \mathrm{E}-05 \pm 1.4 \mathrm{E}-04$ & $\mathrm{U}$ \\
\hline & 03/11/08 & 03/25/08 & gross $\alpha$ & $5.0 \mathrm{E}-04 \pm 4.7 \mathrm{E}-04$ & & & & ${ }^{90} \mathrm{Sr}$ & $-1.0 \mathrm{E}-04 \pm 1.0 \mathrm{E}-04$ & $\mathrm{U}$ \\
\hline & 03/11/08 & $03 / 25 / 08$ & gross $\beta$ & $9.8 \mathrm{E}-03 \pm 1.5 \mathrm{E}-03$ & & & & ${ }^{234} \mathrm{U}$ & $2.4 \mathrm{E}-05 \pm 1.3 \mathrm{E}-05$ & \\
\hline & $03 / 25 / 08$ & $04 / 07 / 08$ & gross $\alpha$ & $1.0 \mathrm{E}-03 \pm 6.3 \mathrm{E}-04$ & & & & ${ }^{235} \mathrm{U}$ & $5.2 \mathrm{E}-06 \pm 4.4 \mathrm{E}-06$ & \\
\hline & $03 / 25 / 08$ & $04 / 07 / 08$ & gross $\beta$ & $8.5 \mathrm{E}-03 \pm 1.5 \mathrm{E}-03$ & & & & ${ }^{238} \mathrm{U}$ & $2.3 \mathrm{E}-05 \pm 1.2 \mathrm{E}-05$ & \\
\hline & 04/07/08 & $04 / 21 / 08$ & gross $\alpha$ & $1.1 \mathrm{E}-03 \pm 5.0 \mathrm{E}-04$ & & & & & & \\
\hline & $04 / 07 / 08$ & $04 / 21 / 08$ & gross $\beta$ & $8.4 \mathrm{E}-03 \pm 1.4 \mathrm{E}-03$ & N550 & $06 / 30 / 08$ to & $12 / 29 / 08$ & ${ }^{60} \mathrm{Co}$ & $7.4 \mathrm{E}-06 \pm 7.4 \mathrm{E}-05$ & U \\
\hline & $04 / 21 / 08$ & 05/06/08 & gross $\alpha$ & $1.7 \mathrm{E}-03 \pm 6.2 \mathrm{E}-04$ & & & & ${ }^{134} \mathrm{Cs}$ & $4.1 \mathrm{E}-05 \pm 7.2 \mathrm{E}-05$ & $\mathrm{U}$ \\
\hline & $04 / 21 / 08$ & 05/06/08 & gross $\beta$ & $1.2 \mathrm{E}-02 \pm 1.7 \mathrm{E}-03$ & & & & ${ }^{137} \mathrm{Cs}$ & $-7.2 \mathrm{E}-06 \pm 6.3 \mathrm{E}-05$ & $\mathrm{U}$ \\
\hline & 05/06/08 & 05/19/08 & gross $\alpha$ & $1.4 \mathrm{E}-03 \pm 6.0 \mathrm{E}-04$ & & & & ${ }^{152} \mathrm{Eu}$ & $-1.1 \mathrm{E}-04 \pm 1.5 \mathrm{E}-04$ & U \\
\hline & 05/06/08 & 05/19/08 & gross $\beta$ & $1.2 \mathrm{E}-02 \pm 1.8 \mathrm{E}-03$ & & & & ${ }^{154} \mathrm{Eu}$ & $1.1 \mathrm{E}-04 \pm 2.2 \mathrm{E}-04$ & $\mathrm{U}$ \\
\hline & 05/19/08 & $06 / 02 / 08$ & gross $\alpha$ & $5.2 \mathrm{E}-04 \pm 4.7 \mathrm{E}-04$ & & & & ${ }^{155} \mathrm{Eu}$ & $-8.7 \mathrm{E}-05 \pm 1.4 \mathrm{E}-04$ & $\mathrm{U}$ \\
\hline & 05/19/08 & $06 / 02 / 08$ & gross $\beta$ & $9.6 \mathrm{E}-03 \pm 1.5 \mathrm{E}-03$ & & & & ${ }^{238} \mathrm{Pu}$ & $1.5 \mathrm{E}-06 \pm 9.3 \mathrm{E}-06$ & $\mathrm{U}$ \\
\hline & $06 / 02 / 08$ & $06 / 16 / 08$ & gross $\alpha$ & $7.6 \mathrm{E}-04 \pm 5.5 \mathrm{E}-04$ & & & & ${ }^{239 / 240} \mathrm{Pu}$ & $2.2 \mathrm{E}-06 \pm 3.4 \mathrm{E}-06$ & $\mathrm{U}$ \\
\hline & $06 / 02 / 08$ & 06/16/08 & gross $\beta$ & $6.1 \mathrm{E}-03 \pm 1.1 \mathrm{E}-03$ & & & & ${ }^{106} \mathrm{Ru}$ & $-1.7 \mathrm{E}-04 \pm 5.3 \mathrm{E}-04$ & $\mathrm{U}$ \\
\hline & $06 / 16 / 08$ & 06/30/08 & gross $\alpha$ & $1.2 \mathrm{E}-03 \pm 5.5 \mathrm{E}-04$ & & & & ${ }^{125} \mathrm{Sb}$ & $-1.2 \mathrm{E}-04 \pm 1.5 \mathrm{E}-04$ & $\mathrm{U}$ \\
\hline & $06 / 16 / 08$ & 06/30/08 & gross $\beta$ & $1.2 \mathrm{E}-02 \pm 1.8 \mathrm{E}-03$ & & & & ${ }^{90} \mathrm{Sr}$ & $-2.4 \mathrm{E}-04 \pm 2.5 \mathrm{E}-04$ & $\mathrm{U}$ \\
\hline & $06 / 30 / 08$ & $07 / 14 / 08$ & gross $\alpha$ & $1.7 \mathrm{E}-03 \pm 6.3 \mathrm{E}-04$ & & & & ${ }^{234} \mathrm{U}$ & $2.2 \mathrm{E}-05 \pm 1.1 \mathrm{E}-05$ & \\
\hline & $06 / 30 / 08$ & 07/14/08 & gross $\beta$ & $1.4 \mathrm{E}-02 \pm 2.0 \mathrm{E}-03$ & & & & ${ }^{235} \mathrm{U}$ & $7.4 \mathrm{E}-07 \pm 2.6 \mathrm{E}-06$ & $\mathrm{U}$ \\
\hline & $07 / 14 / 08$ & 07/28/08 & gross $\alpha$ & $1.3 \mathrm{E}-03 \pm 5.6 \mathrm{E}-04$ & & & & ${ }^{238} \mathrm{U}$ & $2.0 \mathrm{E}-05 \pm 1.1 \mathrm{E}-05$ & \\
\hline & $07 / 14 / 08$ & $07 / 28 / 08$ & gross $\beta$ & $1.5 \mathrm{E}-02 \pm 2.0 \mathrm{E}-03$ & & & & & & \\
\hline & 07/28/08 & 08/11/08 & gross $\alpha$ & $1.1 \mathrm{E}-03 \pm 5.1 \mathrm{E}-04$ & & & & & & \\
\hline & $07 / 28 / 08$ & $08 / 11 / 08$ & gross $\beta$ & $1.2 \mathrm{E}-02 \pm 1.8 \mathrm{E}-03$ & & & & & & \\
\hline & $08 / 11 / 08$ & $08 / 25 / 08$ & gross $\alpha$ & $7.4 \mathrm{E}-04 \pm 5.3 \mathrm{E}-04$ & & & & & & \\
\hline & 08/11/08 & 08/25/08 & gross $\beta$ & $1.2 \mathrm{E}-02 \pm 1.8 \mathrm{E}-03$ & & & & & & \\
\hline & $08 / 25 / 08$ & 09/08/08 & gross $\alpha$ & $6.1 \mathrm{E}-04 \pm 4.9 \mathrm{E}-04$ & & & & & & \\
\hline & $08 / 25 / 08$ & 09/08/08 & gross $\beta$ & $8.8 \mathrm{E}-03 \pm 1.4 \mathrm{E}-03$ & & & & & & \\
\hline & $09 / 08 / 08$ & $09 / 22 / 08$ & gross $\alpha$ & $1.2 \mathrm{E}-03 \pm 5.2 \mathrm{E}-04$ & & & & & & \\
\hline & 09/08/08 & $09 / 22 / 08$ & gross $\beta$ & $2.4 \mathrm{E}-02 \pm 2.8 \mathrm{E}-03$ & & & & & & \\
\hline & $09 / 22 / 08$ & $10 / 07 / 08$ & gross $\alpha$ & $1.3 \mathrm{E}-03 \pm 5.4 \mathrm{E}-04$ & & & & & & \\
\hline & $09 / 22 / 08$ & $10 / 07 / 08$ & gross $\beta$ & $2.0 \mathrm{E}-02 \pm 2.4 \mathrm{E}-03$ & & & & & & \\
\hline & $10 / 07 / 08$ & $10 / 20 / 08$ & gross $\alpha$ & $9.2 \mathrm{E}-04 \pm 6.1 \mathrm{E}-04$ & & & & & & \\
\hline & $10 / 07 / 08$ & $10 / 20 / 08$ & gross $\beta$ & $1.9 \mathrm{E}-02 \pm 2.4 \mathrm{E}-03$ & & & & & & \\
\hline & $10 / 20 / 08$ & $11 / 03 / 08$ & gross $\alpha$ & $2.0 \mathrm{E}-03 \pm 7.0 \mathrm{E}-04$ & & & & & & \\
\hline & $10 / 20 / 08$ & $11 / 03 / 08$ & gross $\beta$ & $3.8 \mathrm{E}-02 \pm 4.2 \mathrm{E}-03$ & & & & & & \\
\hline & $11 / 03 / 08$ & $11 / 17 / 08$ & gross $\alpha$ & $1.2 \mathrm{E}-03 \pm 5.4 \mathrm{E}-04$ & & & & & & \\
\hline & $11 / 03 / 08$ & $11 / 17 / 08$ & gross $\beta$ & $1.7 \mathrm{E}-02 \pm 2.2 \mathrm{E}-03$ & & & & & & \\
\hline & $11 / 17 / 08$ & $12 / 01 / 08$ & gross $\alpha$ & $1.8 \mathrm{E}-03 \pm 6.9 \mathrm{E}-04$ & & & & & & \\
\hline & $11 / 17 / 08$ & $12 / 01 / 08$ & gross $\beta$ & $3.7 \mathrm{E}-02 \pm 4.2 \mathrm{E}-03$ & & & & & & \\
\hline & $12 / 01 / 08$ & $12 / 15 / 08$ & gross $\alpha$ & $1.9 \mathrm{E}-03 \pm 6.6 \mathrm{E}-04$ & & & & & & \\
\hline & $12 / 01 / 08$ & $12 / 15 / 08$ & gross $\beta$ & $1.9 \mathrm{E}-02 \pm 2.4 \mathrm{E}-03$ & & & & & & \\
\hline & $12 / 15 / 08$ & $12 / 29 / 08$ & gross $\alpha$ & $2.3 \mathrm{E}-03 \pm 7.3 \mathrm{E}-04$ & & & & & & \\
\hline & $12 / 15 / 08$ & $12 / 29 / 08$ & gross $\beta$ & $4.1 \mathrm{E}-02 \pm 4.4 \mathrm{E}-03$ & & & & & & \\
\hline
\end{tabular}

$\overline{\mathrm{RQ}}=$ Result Qualifier. $\mathrm{U}=$ The analyte was analyzed for but not detected. 
Table 2-4. Near-Facility Air Sampling Results, 2008 (pCi/m³ \pm total analytical uncertainty). (Sheet 61 of 82)

\begin{tabular}{|c|c|c|c|c|c|c|c|c|c|c|}
\hline Location & Sample On & Sample Off & Isotope & Result \pm Uncertainty & Location & Composite & e Period & Isotope & Result \pm Uncertainty & RQ* \\
\hline N554 & $12 / 31 / 07$ & $01 / 14 / 08$ & gross $\alpha$ & $5.2 \mathrm{E}-04 \pm 4.9 \mathrm{E}-04$ & N554 & $12 / 31 / 07$ to & $06 / 30 / 08$ & ${ }^{60} \mathrm{Co}$ & $4.5 \mathrm{E}-05 \pm 7.9 \mathrm{E}-05$ & $\bar{U}$ \\
\hline \multirow[t]{51}{*}{$(200-W)$} & $12 / 31 / 07$ & 01/14/08 & gross $\beta$ & $9.6 \mathrm{E}-03 \pm 1.5 \mathrm{E}-03$ & & & & ${ }^{134} \mathrm{Cs}$ & $-6.1 \mathrm{E}-05 \pm 7.4 \mathrm{E}-05$ & $\mathrm{U}$ \\
\hline & $01 / 14 / 08$ & 01/29/08 & gross $\alpha$ & $8.9 \mathrm{E}-04 \pm 5.5 \mathrm{E}-04$ & & & & ${ }^{137} \mathrm{Cs}$ & $6.2 \mathrm{E}-05 \pm 6.9 \mathrm{E}-05$ & U \\
\hline & 01/14/08 & 01/29/08 & gross $\beta$ & $3.6 \mathrm{E}-02 \pm 3.9 \mathrm{E}-03$ & & & & ${ }^{152} \mathrm{Eu}$ & $-4.2 \mathrm{E}-05 \pm 1.6 \mathrm{E}-04$ & U \\
\hline & $01 / 29 / 08$ & $02 / 12 / 08$ & gross $\alpha$ & $3.9 \mathrm{E}-04 \pm 4.4 \mathrm{E}-04$ & & & & ${ }^{154} \mathrm{Eu}$ & $-5.0 \mathrm{E}-05 \pm 1.9 \mathrm{E}-04$ & $\mathrm{U}$ \\
\hline & 01/29/08 & 02/12/08 & gross $\beta$ & $2.5 \mathrm{E}-03 \pm 7.2 \mathrm{E}-04$ & & & & ${ }^{155} \mathrm{Eu}$ & $-6.5 \mathrm{E}-05 \pm 1.7 \mathrm{E}-04$ & U \\
\hline & $02 / 12 / 08$ & $02 / 25 / 08$ & gross $\alpha$ & $2.1 \mathrm{E}-03 \pm 7.5 \mathrm{E}-04$ & & & & ${ }^{238} \mathrm{Pu}$ & $-2.0 \mathrm{E}-06 \pm 5.6 \mathrm{E}-06$ & $\mathrm{U}$ \\
\hline & $02 / 12 / 08$ & $02 / 25 / 08$ & gross $\beta$ & $3.1 \mathrm{E}-02 \pm 3.6 \mathrm{E}-03$ & & & & ${ }^{239 / 240} \mathrm{Pu}$ & $6.8 \mathrm{E}-07 \pm 3.0 \mathrm{E}-06$ & U \\
\hline & $02 / 25 / 08$ & 03/11/08 & gross $\alpha$ & $7.0 \mathrm{E}-04 \pm 5.1 \mathrm{E}-04$ & & & & ${ }^{106} \mathrm{Ru}$ & $4.7 \mathrm{E}-05 \pm 4.7 \mathrm{E}-04$ & $\mathrm{U}$ \\
\hline & $02 / 25 / 08$ & 03/11/08 & gross $\beta$ & $1.2 \mathrm{E}-02 \pm 1.7 \mathrm{E}-03$ & & & & ${ }^{125} \mathrm{Sb}$ & $1.1 \mathrm{E}-04 \pm 1.6 \mathrm{E}-04$ & $\mathrm{U}$ \\
\hline & 03/11/08 & 03/25/08 & gross $\alpha$ & $7.7 \mathrm{E}-04 \pm 5.6 \mathrm{E}-04$ & & & & ${ }^{90} \mathrm{Sr}$ & $-9.3 \mathrm{E}-05 \pm 9.7 \mathrm{E}-05$ & $\mathrm{U}$ \\
\hline & 03/11/08 & $03 / 25 / 08$ & gross $\beta$ & $9.7 \mathrm{E}-03 \pm 1.5 \mathrm{E}-03$ & & & & ${ }^{234} \mathrm{U}$ & $9.3 \mathrm{E}-06 \pm 6.8 \mathrm{E}-06$ & \\
\hline & $03 / 25 / 08$ & $04 / 07 / 08$ & gross $\alpha$ & $9.0 \mathrm{E}-04 \pm 6.1 \mathrm{E}-04$ & & & & ${ }^{235} \mathrm{U}$ & $1.6 \mathrm{E}-06 \pm 3.2 \mathrm{E}-06$ & U \\
\hline & 03/25/08 & $04 / 07 / 08$ & gross $\beta$ & $7.3 \mathrm{E}-03 \pm 1.3 \mathrm{E}-03$ & & & & ${ }^{238} \mathrm{U}$ & $1.4 \mathrm{E}-05 \pm 8.8 \mathrm{E}-06$ & \\
\hline & 04/07/08 & $04 / 21 / 08$ & gross $\alpha$ & $8.4 \mathrm{E}-04 \pm 5.6 \mathrm{E}-04$ & & & & & & \\
\hline & $04 / 07 / 08$ & $04 / 21 / 08$ & gross $\beta$ & $1.1 \mathrm{E}-02 \pm 1.6 \mathrm{E}-03$ & N554 & $06 / 30 / 08$ to & $12 / 29 / 08$ & ${ }^{60} \mathrm{Co}$ & $-8.2 \mathrm{E}-05 \pm 8.6 \mathrm{E}-05$ & $\mathrm{U}$ \\
\hline & $04 / 21 / 08$ & $05 / 05 / 08$ & gross $\alpha$ & $7.4 \mathrm{E}-04 \pm 5.4 \mathrm{E}-04$ & & & & ${ }^{134} \mathrm{Cs}$ & $1.6 \mathrm{E}-06 \pm 1.6 \mathrm{E}-05$ & $\mathrm{U}$ \\
\hline & $04 / 21 / 08$ & 05/05/08 & gross $\beta$ & $1.1 \mathrm{E}-02 \pm 1.6 \mathrm{E}-03$ & & & & ${ }^{137} \mathrm{Cs}$ & $4.4 \mathrm{E}-05 \pm 6.4 \mathrm{E}-05$ & $\mathrm{U}$ \\
\hline & 05/05/08 & 05/19/08 & gross $\alpha$ & $1.4 \mathrm{E}-03 \pm 5.7 \mathrm{E}-04$ & & & & ${ }^{152} \mathrm{Eu}$ & $6.5 \mathrm{E}-05 \pm 1.5 \mathrm{E}-04$ & U \\
\hline & 05/05/08 & 05/19/08 & gross $\beta$ & $1.4 \mathrm{E}-02 \pm 1.9 \mathrm{E}-03$ & & & & ${ }^{154} \mathrm{Eu}$ & $-1.3 \mathrm{E}-04 \pm 2.4 \mathrm{E}-04$ & $\mathrm{U}$ \\
\hline & 05/19/08 & $06 / 02 / 08$ & gross $\alpha$ & $1.2 \mathrm{E}-03 \pm 5.3 \mathrm{E}-04$ & & & & ${ }^{155} \mathrm{Eu}$ & $-1.4 \mathrm{E}-04 \pm 1.6 \mathrm{E}-04$ & $\mathrm{U}$ \\
\hline & 05/19/08 & $06 / 02 / 08$ & gross $\beta$ & $1.2 \mathrm{E}-02 \pm 1.7 \mathrm{E}-03$ & & & & ${ }^{238} \mathrm{Pu}$ & $8.4 \mathrm{E}-07 \pm 8.5 \mathrm{E}-06$ & $\mathrm{U}$ \\
\hline & $06 / 02 / 08$ & $06 / 16 / 08$ & gross $\alpha$ & $1.2 \mathrm{E}-03 \pm 5.6 \mathrm{E}-04$ & & & & ${ }^{239 / 240} \mathrm{Pu}$ & $2.5 \mathrm{E}-06 \pm 3.0 \mathrm{E}-06$ & \\
\hline & $06 / 02 / 08$ & 06/16/08 & gross $\beta$ & $6.8 \mathrm{E}-03 \pm 1.3 \mathrm{E}-03$ & & & & ${ }^{106} \mathrm{Ru}$ & $1.0 \mathrm{E}-04 \pm 5.9 \mathrm{E}-04$ & U \\
\hline & $06 / 16 / 08$ & 06/30/08 & gross $\alpha$ & $1.2 \mathrm{E}-03 \pm 5.4 \mathrm{E}-04$ & & & & ${ }^{125} \mathrm{Sb}$ & $1.0 \mathrm{E}-04 \pm 1.6 \mathrm{E}-04$ & $\mathrm{U}$ \\
\hline & $06 / 16 / 08$ & 06/30/08 & gross $\beta$ & $1.5 \mathrm{E}-02 \pm 2.1 \mathrm{E}-03$ & & & & ${ }^{90} \mathrm{Sr}$ & $-4.1 \mathrm{E}-04 \pm 4.3 \mathrm{E}-04$ & $\mathrm{U}$ \\
\hline & $06 / 30 / 08$ & $07 / 14 / 08$ & gross $\alpha$ & $2.7 \mathrm{E}-03 \pm 1.1 \mathrm{E}-03$ & & & & ${ }^{234} \mathrm{U}$ & $1.0 \mathrm{E}-05 \pm 7.6 \mathrm{E}-06$ & \\
\hline & $06 / 30 / 08$ & 07/14/08 & gross $\beta$ & $1.6 \mathrm{E}-02 \pm 2.7 \mathrm{E}-03$ & & & & ${ }^{235} \mathrm{U}$ & $7.3 \mathrm{E}-07 \pm 7.3 \mathrm{E}-06$ & $\mathrm{U}$ \\
\hline & $07 / 14 / 08$ & 07/28/08 & gross $\alpha$ & $6.4 \mathrm{E}-04 \pm 8.6 \mathrm{E}-04$ & & & & ${ }^{238} \mathrm{U}$ & $1.2 \mathrm{E}-05 \pm 7.6 \mathrm{E}-06$ & \\
\hline & $07 / 14 / 08$ & $07 / 28 / 08$ & gross $\beta$ & $2.3 \mathrm{E}-02 \pm 3.5 \mathrm{E}-03$ & & & & & & \\
\hline & 07/28/08 & 08/11/08 & gross $\alpha$ & 7.4E-04 $\pm 5.3 \mathrm{E}-04$ & & & & & & \\
\hline & $07 / 28 / 08$ & $08 / 11 / 08$ & gross $\beta$ & $1.4 \mathrm{E}-02 \pm 1.9 \mathrm{E}-03$ & & & & & & \\
\hline & $08 / 11 / 08$ & $08 / 25 / 08$ & gross $\alpha$ & $1.1 \mathrm{E}-03 \pm 5.1 \mathrm{E}-04$ & & & & & & \\
\hline & 08/11/08 & 08/25/08 & gross $\beta$ & $1.1 \mathrm{E}-02 \pm 1.7 \mathrm{E}-03$ & & & & & & \\
\hline & $08 / 25 / 08$ & 09/08/08 & gross $\alpha$ & $4.2 \mathrm{E}-04 \pm 4.5 \mathrm{E}-04$ & & & & & & \\
\hline & $08 / 25 / 08$ & 09/08/08 & gross $\beta$ & $1.1 \mathrm{E}-02 \pm 1.6 \mathrm{E}-03$ & & & & & & \\
\hline & $09 / 08 / 08$ & $09 / 22 / 08$ & gross $\alpha$ & $6.2 \mathrm{E}-04 \pm 5.0 \mathrm{E}-04$ & & & & & & \\
\hline & 09/08/08 & $09 / 22 / 08$ & gross $\beta$ & $2.2 \mathrm{E}-02 \pm 2.7 \mathrm{E}-03$ & & & & & & \\
\hline & $09 / 22 / 08$ & $10 / 07 / 08$ & gross $\alpha$ & $1.4 \mathrm{E}-03 \pm 5.6 \mathrm{E}-04$ & & & & & & \\
\hline & $09 / 22 / 08$ & $10 / 07 / 08$ & gross $\beta$ & $2.1 \mathrm{E}-02 \pm 2.6 \mathrm{E}-03$ & & & & & & \\
\hline & $10 / 07 / 08$ & $10 / 20 / 08$ & gross $\alpha$ & $1.3 \mathrm{E}-03 \pm 5.7 \mathrm{E}-04$ & & & & & & \\
\hline & $10 / 07 / 08$ & $10 / 20 / 08$ & gross $\beta$ & $1.5 \mathrm{E}-02 \pm 2.1 \mathrm{E}-03$ & & & & & & \\
\hline & $10 / 20 / 08$ & $11 / 03 / 08$ & gross $\alpha$ & $1.4 \mathrm{E}-03 \pm 5.7 \mathrm{E}-04$ & & & & & & \\
\hline & $10 / 20 / 08$ & $11 / 03 / 08$ & gross $\beta$ & $3.8 \mathrm{E}-02 \pm 4.1 \mathrm{E}-03$ & & & & & & \\
\hline & $11 / 03 / 08$ & $11 / 17 / 08$ & gross $\alpha$ & $1.3 \mathrm{E}-03 \pm 5.5 \mathrm{E}-04$ & & & & & & \\
\hline & $11 / 03 / 08$ & $11 / 17 / 08$ & gross $\beta$ & $1.7 \mathrm{E}-02 \pm 2.3 \mathrm{E}-03$ & & & & & & \\
\hline & $11 / 17 / 08$ & $12 / 01 / 08$ & gross $\alpha$ & $1.6 \mathrm{E}-03 \pm 6.5 \mathrm{E}-04$ & & & & & & \\
\hline & $11 / 17 / 08$ & $12 / 01 / 08$ & gross $\beta$ & $4.0 \mathrm{E}-02 \pm 4.4 \mathrm{E}-03$ & & & & & & \\
\hline & $12 / 01 / 08$ & $12 / 15 / 08$ & gross $\alpha$ & $1.9 \mathrm{E}-03 \pm 6.6 \mathrm{E}-04$ & & & & & & \\
\hline & $12 / 01 / 08$ & $12 / 15 / 08$ & gross $\beta$ & $2.3 \mathrm{E}-02 \pm 2.8 \mathrm{E}-03$ & & & & & & \\
\hline & $12 / 15 / 08$ & $12 / 29 / 08$ & gross $\alpha$ & $2.7 \mathrm{E}-03 \pm 8.1 \mathrm{E}-04$ & & & & & & \\
\hline & $12 / 15 / 08$ & $12 / 29 / 08$ & gross $\beta$ & $5.2 \mathrm{E}-02 \pm 5.4 \mathrm{E}-03$ & & & & & & \\
\hline
\end{tabular}

$\overline{\mathrm{RQ}}=$ Result Qualifier. $\mathrm{U}=$ The analyte was analyzed for but not detected. 
Table 2-4. Near-Facility Air Sampling Results, 2008 (pCi/m³ \pm total analytical uncertainty). (Sheet 62 of 82)

\begin{tabular}{|c|c|c|c|c|c|c|c|c|c|c|}
\hline Location & Sample On & Sample Off & Isotope & Result \pm Uncertainty & Location & Composite & e Period & Isotope & Result \pm Uncertainty & $\mathbf{R Q *}$ \\
\hline N555 & $12 / 31 / 07$ & $01 / 14 / 08$ & gross $\alpha$ & $3.8 \mathrm{E}-04 \pm 4.3 \mathrm{E}-04$ & N555 & $12 / 31 / 07$ to & $06 / 30 / 08$ & ${ }^{60} \mathrm{Co}$ & $-5.0 \mathrm{E}-05 \pm 8.9 \mathrm{E}-05$ & $\mathrm{U}$ \\
\hline \multirow[t]{51}{*}{$(200-W)$} & $12 / 31 / 07$ & 01/14/08 & gross $\beta$ & $8.1 \mathrm{E}-03 \pm 1.3 \mathrm{E}-03$ & & & & ${ }^{134} \mathrm{Cs}$ & $4.7 \mathrm{E}-05 \pm 7.2 \mathrm{E}-05$ & $\mathrm{U}$ \\
\hline & $01 / 14 / 08$ & 01/29/08 & gross $\alpha$ & $1.8 \mathrm{E}-03 \pm 6.4 \mathrm{E}-04$ & & & & ${ }^{137} \mathrm{Cs}$ & $1.2 \mathrm{E}-05 \pm 7.0 \mathrm{E}-05$ & U \\
\hline & $01 / 14 / 08$ & 01/29/08 & gross $\beta$ & $3.5 \mathrm{E}-02 \pm 3.8 \mathrm{E}-03$ & & & & ${ }^{152} \mathrm{Eu}$ & $2.9 \mathrm{E}-05 \pm 1.7 \mathrm{E}-04$ & U \\
\hline & 01/29/08 & $02 / 12 / 08$ & gross $\alpha$ & $5.0 \mathrm{E}-04 \pm 4.7 \mathrm{E}-04$ & & & & ${ }^{154} \mathrm{Eu}$ & $-6.3 \mathrm{E}-05 \pm 2.3 \mathrm{E}-04$ & $\mathrm{U}$ \\
\hline & $01 / 29 / 08$ & $02 / 12 / 08$ & gross $\beta$ & $3.7 \mathrm{E}-03 \pm 8.8 \mathrm{E}-04$ & & & & ${ }^{155} \mathrm{Eu}$ & $-1.8 \mathrm{E}-04 \pm 2.0 \mathrm{E}-04$ & U \\
\hline & $02 / 12 / 08$ & $02 / 25 / 08$ & gross $\alpha$ & $1.2 \mathrm{E}-03 \pm 5.7 \mathrm{E}-04$ & & & & ${ }^{238} \mathrm{Pu}$ & $-1.8 \mathrm{E}-06 \pm 1.2 \mathrm{E}-05$ & $\mathrm{U}$ \\
\hline & $02 / 12 / 08$ & $02 / 25 / 08$ & gross $\beta$ & $3.0 \mathrm{E}-02 \pm 3.5 \mathrm{E}-03$ & & & & ${ }^{239 / 240} \mathrm{Pu}$ & $4.6 \mathrm{E}-06 \pm 5.8 \mathrm{E}-06$ & $\mathrm{U}$ \\
\hline & $02 / 25 / 08$ & 03/11/08 & gross $\alpha$ & $1.0 \mathrm{E}-03 \pm 4.8 \mathrm{E}-04$ & & & & ${ }^{106} \mathrm{Ru}$ & $3.7 \mathrm{E}-04 \pm 7.1 \mathrm{E}-04$ & $\mathrm{U}$ \\
\hline & $02 / 25 / 08$ & 03/11/08 & gross $\beta$ & $1.4 \mathrm{E}-02 \pm 1.9 \mathrm{E}-03$ & & & & ${ }^{125} \mathrm{Sb}$ & $4.3 \mathrm{E}-05 \pm 1.6 \mathrm{E}-04$ & U \\
\hline & 03/11/08 & $03 / 25 / 08$ & gross $\alpha$ & $1.0 \mathrm{E}-03 \pm 6.2 \mathrm{E}-04$ & & & & ${ }^{90} \mathrm{Sr}$ & $-3.2 \mathrm{E}-04 \pm 3.3 \mathrm{E}-04$ & $\mathrm{U}$ \\
\hline & 03/11/08 & $03 / 25 / 08$ & gross $\beta$ & $8.0 \mathrm{E}-03 \pm 1.4 \mathrm{E}-03$ & & & & ${ }^{234} \mathrm{U}$ & $1.2 \mathrm{E}-05 \pm 7.5 \mathrm{E}-06$ & \\
\hline & $03 / 25 / 08$ & $04 / 07 / 08$ & gross $\alpha$ & $1.2 \mathrm{E}-03 \pm 5.4 \mathrm{E}-04$ & & & & ${ }^{235} \mathrm{U}$ & $2.3 \mathrm{E}-06 \pm 2.8 \mathrm{E}-06$ & \\
\hline & 03/25/08 & $04 / 07 / 08$ & gross $\beta$ & $8.0 \mathrm{E}-03 \pm 1.4 \mathrm{E}-03$ & & & & ${ }^{238} \mathrm{U}$ & $5.6 \mathrm{E}-06 \pm 4.4 \mathrm{E}-06$ & \\
\hline & 04/07/08 & $04 / 21 / 08$ & gross $\alpha$ & $9.7 \mathrm{E}-04 \pm 6.1 \mathrm{E}-04$ & & & & & & \\
\hline & $04 / 07 / 08$ & $04 / 21 / 08$ & gross $\beta$ & $9.9 \mathrm{E}-03 \pm 1.6 \mathrm{E}-03$ & N555 & $06 / 30 / 08$ to & $12 / 29 / 08$ & ${ }^{60} \mathrm{Co}$ & $7.4 \mathrm{E}-05 \pm 7.3 \mathrm{E}-05$ & $\mathrm{U}$ \\
\hline & $04 / 21 / 08$ & 05/06/08 & gross $\alpha$ & $1.2 \mathrm{E}-03 \pm 5.3 \mathrm{E}-04$ & & & & ${ }^{134} \mathrm{Cs}$ & $-8.2 \mathrm{E}-07 \pm 8.2 \mathrm{E}-06$ & $\mathrm{U}$ \\
\hline & $04 / 21 / 08$ & 05/06/08 & gross $\beta$ & $1.2 \mathrm{E}-02 \pm 1.7 \mathrm{E}-03$ & & & & ${ }^{137} \mathrm{Cs}$ & $-1.3 \mathrm{E}-05 \pm 6.0 \mathrm{E}-05$ & $\mathrm{U}$ \\
\hline & 05/06/08 & 05/19/08 & gross $\alpha$ & $1.2 \mathrm{E}-03 \pm 5.5 \mathrm{E}-04$ & & & & ${ }^{152} \mathrm{Eu}$ & $-6.9 \mathrm{E}-05 \pm 1.4 \mathrm{E}-04$ & U \\
\hline & 05/06/08 & 05/19/08 & gross $\beta$ & $1.0 \mathrm{E}-02 \pm 1.7 \mathrm{E}-03$ & & & & ${ }^{154} \mathrm{Eu}$ & $-8.1 \mathrm{E}-06 \pm 8.1 \mathrm{E}-05$ & $\mathrm{U}$ \\
\hline & 05/19/08 & 06/02/08 & gross $\alpha$ & $1.0 \mathrm{E}-03 \pm 5.0 \mathrm{E}-04$ & & & & ${ }^{155} \mathrm{Eu}$ & $-7.6 \mathrm{E}-05 \pm 1.6 \mathrm{E}-04$ & U \\
\hline & 05/19/08 & $06 / 02 / 08$ & gross $\beta$ & $1.2 \mathrm{E}-02 \pm 1.8 \mathrm{E}-03$ & & & & ${ }^{238} \mathrm{Pu}$ & $1.2 \mathrm{E}-06 \pm 4.0 \mathrm{E}-06$ & $\mathrm{U}$ \\
\hline & $06 / 02 / 08$ & 06/16/08 & gross $\alpha$ & $6.3 \mathrm{E}-04 \pm 5.2 \mathrm{E}-04$ & & & & ${ }^{239 / 240} \mathrm{Pu}$ & $5.0 \mathrm{E}-06 \pm 3.9 \mathrm{E}-06$ & \\
\hline & $06 / 02 / 08$ & 06/16/08 & gross $\beta$ & $6.9 \mathrm{E}-03 \pm 1.2 \mathrm{E}-03$ & & & & ${ }^{106} \mathrm{Ru}$ & $-1.7 \mathrm{E}-04 \pm 5.1 \mathrm{E}-04$ & $\mathrm{U}$ \\
\hline & $06 / 16 / 08$ & 06/30/08 & gross $\alpha$ & $2.4 \mathrm{E}-03 \pm 8.5 \mathrm{E}-04$ & & & & ${ }^{125} \mathrm{Sb}$ & $4.6 \mathrm{E}-05 \pm 1.4 \mathrm{E}-04$ & $\mathrm{U}$ \\
\hline & $06 / 16 / 08$ & $06 / 30 / 08$ & gross $\beta$ & $1.5 \mathrm{E}-02 \pm 2.2 \mathrm{E}-03$ & & & & ${ }^{90} \mathrm{Sr}$ & $-2.7 \mathrm{E}-04 \pm 2.8 \mathrm{E}-04$ & $\mathrm{U}$ \\
\hline & $06 / 30 / 08$ & $07 / 14 / 08$ & gross $\alpha$ & $8.9 \mathrm{E}-04 \pm 6.3 \mathrm{E}-04$ & & & & ${ }^{234} \mathrm{U}$ & $1.2 \mathrm{E}-05 \pm 7.3 \mathrm{E}-06$ & \\
\hline & $06 / 30 / 08$ & 07/14/08 & gross $\beta$ & $1.2 \mathrm{E}-02 \pm 1.7 \mathrm{E}-03$ & & & & ${ }^{235} \mathrm{U}$ & $4.4 \mathrm{E}-06 \pm 4.0 \mathrm{E}-06$ & \\
\hline & $07 / 14 / 08$ & 07/28/08 & gross $\alpha$ & $2.2 \mathrm{E}-03 \pm 7.2 \mathrm{E}-04$ & & & & ${ }^{238} \mathrm{U}$ & $1.2 \mathrm{E}-05 \pm 7.1 \mathrm{E}-06$ & \\
\hline & $07 / 14 / 08$ & $07 / 28 / 08$ & gross $\beta$ & $1.4 \mathrm{E}-02 \pm 1.9 \mathrm{E}-03$ & & & & & & \\
\hline & 07/28/08 & 08/11/08 & gross $\alpha$ & $9.4 \mathrm{E}-04 \pm 5.8 \mathrm{E}-04$ & & & & & & \\
\hline & $07 / 28 / 08$ & $08 / 11 / 08$ & gross $\beta$ & $1.2 \mathrm{E}-02 \pm 1.8 \mathrm{E}-03$ & & & & & & \\
\hline & $08 / 11 / 08$ & $08 / 25 / 08$ & gross $\alpha$ & $2.1 \mathrm{E}-03 \pm 7.0 \mathrm{E}-04$ & & & & & & \\
\hline & 08/11/08 & 08/25/08 & gross $\beta$ & $1.9 \mathrm{E}-02 \pm 2.5 \mathrm{E}-03$ & & & & & & \\
\hline & $08 / 25 / 08$ & 09/08/08 & gross $\alpha$ & $4.8 \mathrm{E}-04 \pm 5.6 \mathrm{E}-04$ & & & & & & \\
\hline & $08 / 25 / 08$ & 09/08/08 & gross $\beta$ & $9.9 \mathrm{E}-03 \pm 1.8 \mathrm{E}-03$ & & & & & & \\
\hline & 09/08/08 & 09/22/08 & gross $\alpha$ & $2.1 \mathrm{E}-03 \pm 6.8 \mathrm{E}-04$ & & & & & & \\
\hline & 09/08/08 & $09 / 22 / 08$ & gross $\beta$ & $2.5 \mathrm{E}-02 \pm 3.0 \mathrm{E}-03$ & & & & & & \\
\hline & $09 / 22 / 08$ & $10 / 07 / 08$ & gross $\alpha$ & $1.3 \mathrm{E}-03 \pm 5.4 \mathrm{E}-04$ & & & & & & \\
\hline & $09 / 22 / 08$ & $10 / 07 / 08$ & gross $\beta$ & $2.2 \mathrm{E}-02 \pm 2.6 \mathrm{E}-03$ & & & & & & \\
\hline & $10 / 07 / 08$ & $10 / 20 / 08$ & gross $\alpha$ & $1.7 \mathrm{E}-03 \pm 6.5 \mathrm{E}-04$ & & & & & & \\
\hline & $10 / 07 / 08$ & $10 / 20 / 08$ & gross $\beta$ & $1.6 \mathrm{E}-02 \pm 2.2 \mathrm{E}-03$ & & & & & & \\
\hline & $10 / 20 / 08$ & $11 / 03 / 08$ & gross $\alpha$ & $2.7 \mathrm{E}-03 \pm 7.9 \mathrm{E}-04$ & & & & & & \\
\hline & $10 / 20 / 08$ & $11 / 03 / 08$ & gross $\beta$ & $4.2 \mathrm{E}-02 \pm 4.6 \mathrm{E}-03$ & & & & & & \\
\hline & $11 / 03 / 08$ & $11 / 17 / 08$ & gross $\alpha$ & $1.9 \mathrm{E}-03 \pm 6.6 \mathrm{E}-04$ & & & & & & \\
\hline & $11 / 03 / 08$ & $11 / 17 / 08$ & gross $\beta$ & $2.1 \mathrm{E}-02 \pm 2.6 \mathrm{E}-03$ & & & & & & \\
\hline & $11 / 17 / 08$ & $12 / 01 / 08$ & gross $\alpha$ & $2.1 \mathrm{E}-03 \pm 7.3 \mathrm{E}-04$ & & & & & & \\
\hline & $11 / 17 / 08$ & $12 / 01 / 08$ & gross $\beta$ & $3.3 \mathrm{E}-02 \pm 3.7 \mathrm{E}-03$ & & & & & & \\
\hline & $12 / 01 / 08$ & $12 / 15 / 08$ & gross $\alpha$ & $8.3 \mathrm{E}-04 \pm 5.5 \mathrm{E}-04$ & & & & & & \\
\hline & $12 / 01 / 08$ & $12 / 15 / 08$ & gross $\beta$ & $1.8 \mathrm{E}-02 \pm 2.3 \mathrm{E}-03$ & & & & & & \\
\hline & $12 / 15 / 08$ & $12 / 29 / 08$ & gross $\alpha$ & $3.8 \mathrm{E}-03 \pm 1.3 \mathrm{E}-03$ & & & & & & \\
\hline & $12 / 15 / 08$ & $12 / 29 / 08$ & gross $\beta$ & $9.2 \mathrm{E}-02 \pm 9.7 \mathrm{E}-03$ & & & & & & \\
\hline
\end{tabular}

$\overline{\mathrm{RQ}}=$ Result Qualifier. $\mathrm{U}=$ The analyte was analyzed for but not detected. 
Table 2-4. Near-Facility Air Sampling Results, 2008 (pCi/m³ \pm total analytical uncertainty). (Sheet 63 of 82)

\begin{tabular}{|c|c|c|c|c|c|c|c|c|c|c|}
\hline Location & Sample On & Sample Off & Isotope & Result \pm Uncertainty & Location & Composite & e Period & Isotope & Result \pm Uncertainty & RQ* \\
\hline N956 & $12 / 31 / 07$ & $01 / 14 / 08$ & gross $\alpha$ & $1.1 \mathrm{E}-03 \pm 5.1 \mathrm{E}-04$ & N956 & $12 / 31 / 07$ to & $06 / 30 / 08$ & ${ }^{60} \mathrm{Co}$ & $2.2 \mathrm{E}-05 \pm 6.8 \mathrm{E}-05$ & $\bar{U}$ \\
\hline \multirow[t]{51}{*}{$(200-W)$} & $12 / 31 / 07$ & $01 / 14 / 08$ & gross $\beta$ & $9.5 \mathrm{E}-03 \pm 1.4 \mathrm{E}-03$ & & & & ${ }^{134} \mathrm{Cs}$ & $4.4 \mathrm{E}-05 \pm 6.3 \mathrm{E}-05$ & $\mathrm{U}$ \\
\hline & 01/14/08 & $01 / 29 / 08$ & gross $\alpha$ & $1.5 \mathrm{E}-03 \pm 5.9 \mathrm{E}-04$ & & & & ${ }^{137} \mathrm{Cs}$ & $1.3 \mathrm{E}-05 \pm 5.9 \mathrm{E}-05$ & $\mathrm{U}$ \\
\hline & $01 / 14 / 08$ & 01/29/08 & gross $\beta$ & $3.2 \mathrm{E}-02 \pm 3.3 \mathrm{E}-03$ & & & & ${ }^{152} \mathrm{Eu}$ & $-4.4 \mathrm{E}-05 \pm 1.4 \mathrm{E}-04$ & U \\
\hline & $01 / 29 / 08$ & $02 / 12 / 08$ & gross $\alpha$ & $6.0 \mathrm{E}-04 \pm 4.9 \mathrm{E}-04$ & & & & ${ }^{154} \mathrm{Eu}$ & $1.3 \mathrm{E}-04 \pm 2.0 \mathrm{E}-04$ & $\mathrm{U}$ \\
\hline & $01 / 29 / 08$ & $02 / 12 / 08$ & gross $\beta$ & $3.8 \mathrm{E}-03 \pm 8.5 \mathrm{E}-04$ & & & & ${ }^{155} \mathrm{Eu}$ & $3.6 \mathrm{E}-07 \pm 3.6 \mathrm{E}-06$ & U \\
\hline & $02 / 12 / 08$ & $02 / 25 / 08$ & gross $\alpha$ & $1.4 \mathrm{E}-03 \pm 6.1 \mathrm{E}-04$ & & & & ${ }^{238} \mathrm{Pu}$ & $-7.8 \mathrm{E}-07 \pm 3.5 \mathrm{E}-06$ & $\mathrm{U}$ \\
\hline & $02 / 12 / 08$ & $02 / 25 / 08$ & gross $\beta$ & $3.3 \mathrm{E}-02 \pm 3.4 \mathrm{E}-03$ & & & & ${ }^{239 / 240} \mathrm{Pu}$ & $5.5 \mathrm{E}-06 \pm 5.0 \mathrm{E}-06$ & $\mathrm{U}$ \\
\hline & $02 / 25 / 08$ & 03/11/08 & $\operatorname{gross} \alpha$ & $1.9 \mathrm{E}-03 \pm 7.0 \mathrm{E}-04$ & & & & ${ }^{106} \mathrm{Ru}$ & $-7.5 \mathrm{E}-07 \pm 7.5 \mathrm{E}-06$ & $\mathrm{U}$ \\
\hline & $02 / 25 / 08$ & 03/11/08 & gross $\beta$ & $1.3 \mathrm{E}-02 \pm 1.8 \mathrm{E}-03$ & & & & ${ }^{125} \mathrm{Sb}$ & $-9.9 \mathrm{E}-05 \pm 1.3 \mathrm{E}-04$ & $\mathrm{U}$ \\
\hline & 03/11/08 & 03/25/08 & gross $\alpha$ & $8.3 \mathrm{E}-04 \pm 6.1 \mathrm{E}-04$ & & & & ${ }^{90} \mathrm{Sr}$ & $-7.3 \mathrm{E}-05 \pm 7.5 \mathrm{E}-05$ & $\mathrm{U}$ \\
\hline & 03/11/08 & $03 / 25 / 08$ & gross $\beta$ & $9.4 \mathrm{E}-03 \pm 1.5 \mathrm{E}-03$ & & & & ${ }^{234} \mathrm{U}$ & $1.5 \mathrm{E}-05 \pm 8.7 \mathrm{E}-06$ & \\
\hline & $03 / 25 / 08$ & $04 / 07 / 08$ & gross $\alpha$ & $9.5 \mathrm{E}-04 \pm 5.9 \mathrm{E}-04$ & & & & ${ }^{235} \mathrm{U}$ & $7.0 \mathrm{E}-07 \pm 7.2 \mathrm{E}-07$ & $\mathrm{U}$ \\
\hline & 03/25/08 & $04 / 07 / 08$ & gross $\beta$ & $9.4 \mathrm{E}-03 \pm 1.4 \mathrm{E}-03$ & & & & ${ }^{238} \mathrm{U}$ & $9.7 \mathrm{E}-06 \pm 6.3 \mathrm{E}-06$ & \\
\hline & 04/07/08 & $04 / 21 / 08$ & gross $\alpha$ & $9.8 \mathrm{E}-04 \pm 6.1 \mathrm{E}-04$ & & & & & & \\
\hline & $04 / 07 / 08$ & $04 / 21 / 08$ & gross $\beta$ & $1.2 \mathrm{E}-02 \pm 1.7 \mathrm{E}-03$ & N956 & $06 / 30 / 08$ to & $12 / 29 / 08$ & ${ }^{60} \mathrm{Co}$ & $1.1 \mathrm{E}-05 \pm 1.0 \mathrm{E}-04$ & $\mathrm{U}$ \\
\hline & $04 / 21 / 08$ & 05/06/08 & gross $\alpha$ & $2.6 \mathrm{E}-03 \pm 7.7 \mathrm{E}-04$ & & & & ${ }^{134} \mathrm{Cs}$ & $4.0 \mathrm{E}-05 \pm 1.2 \mathrm{E}-04$ & $\mathrm{U}$ \\
\hline & $04 / 21 / 08$ & 05/06/08 & gross $\beta$ & $1.0 \mathrm{E}-02 \pm 1.5 \mathrm{E}-03$ & & & & ${ }^{137} \mathrm{Cs}$ & $1.2 \mathrm{E}-04 \pm 1.3 \mathrm{E}-04$ & $\mathrm{U}$ \\
\hline & 05/06/08 & 05/19/08 & gross $\alpha$ & $1.2 \mathrm{E}-03 \pm 5.6 \mathrm{E}-04$ & & & & ${ }^{152} \mathrm{Eu}$ & $1.7 \mathrm{E}-04 \pm 3.0 \mathrm{E}-04$ & U \\
\hline & 05/06/08 & 05/19/08 & gross $\beta$ & $1.2 \mathrm{E}-02 \pm 1.8 \mathrm{E}-03$ & & & & ${ }^{154} \mathrm{Eu}$ & $-2.0 \mathrm{E}-04 \pm 3.7 \mathrm{E}-04$ & $\mathrm{U}$ \\
\hline & 05/19/08 & 06/02/08 & gross $\alpha$ & $1.6 \mathrm{E}-03 \pm 6.0 \mathrm{E}-04$ & & & & ${ }^{155} \mathrm{Eu}$ & $-4.3 \mathrm{E}-05 \pm 2.1 \mathrm{E}-04$ & U \\
\hline & 05/19/08 & $06 / 02 / 08$ & gross $\beta$ & $1.1 \mathrm{E}-02 \pm 1.6 \mathrm{E}-03$ & & & & ${ }^{238} \mathrm{Pu}$ & $1.2 \mathrm{E}-05 \pm 1.1 \mathrm{E}-05$ & $\mathrm{U}$ \\
\hline & $06 / 02 / 08$ & $06 / 16 / 08$ & gross $\alpha$ & $4.0 \mathrm{E}-04 \pm 4.5 \mathrm{E}-04$ & & & & ${ }^{239 / 240} \mathrm{Pu}$ & $9.1 \mathrm{E}-05 \pm 3.8 \mathrm{E}-05$ & \\
\hline & $06 / 02 / 08$ & 06/16/08 & gross $\beta$ & $6.7 \mathrm{E}-03 \pm 1.2 \mathrm{E}-03$ & & & & ${ }^{106} \mathrm{Ru}$ & $1.4 \mathrm{E}-04 \pm 9.9 \mathrm{E}-04$ & U \\
\hline & $06 / 16 / 08$ & 06/30/08 & gross $\alpha$ & $1.3 \mathrm{E}-03 \pm 6.1 \mathrm{E}-04$ & & & & ${ }^{125} \mathrm{Sb}$ & $-1.4 \mathrm{E}-05 \pm 1.4 \mathrm{E}-04$ & $\mathrm{U}$ \\
\hline & $06 / 16 / 08$ & 06/30/08 & gross $\beta$ & $1.5 \mathrm{E}-02 \pm 1.9 \mathrm{E}-03$ & & & & ${ }^{90} \mathrm{Sr}$ & $-5.1 \mathrm{E}-04 \pm 5.3 \mathrm{E}-04$ & $\mathrm{U}$ \\
\hline & $06 / 30 / 08$ & $07 / 14 / 08$ & gross $\alpha$ & $5.4 \mathrm{E}-04 \pm 5.5 \mathrm{E}-04$ & & & & ${ }^{234} \mathrm{U}$ & $1.3 \mathrm{E}-05 \pm 8.6 \mathrm{E}-06$ & \\
\hline & $06 / 30 / 08$ & 07/14/08 & gross $\beta$ & $1.5 \mathrm{E}-02 \pm 1.9 \mathrm{E}-03$ & & & & ${ }^{235} \mathrm{U}$ & $3.5 \mathrm{E}-06 \pm 3.7 \mathrm{E}-06$ & \\
\hline & $07 / 14 / 08$ & 07/28/08 & gross $\alpha$ & $1.8 \mathrm{E}-03 \pm 6.4 \mathrm{E}-04$ & & & & ${ }^{238} \mathrm{U}$ & $1.1 \mathrm{E}-05 \pm 7.6 \mathrm{E}-06$ & \\
\hline & $07 / 14 / 08$ & $07 / 28 / 08$ & gross $\beta$ & $1.9 \mathrm{E}-02 \pm 2.3 \mathrm{E}-03$ & & & & & & \\
\hline & 07/28/08 & 08/11/08 & gross $\alpha$ & $6.0 \mathrm{E}-04 \pm 5.1 \mathrm{E}-04$ & & & & & & \\
\hline & $07 / 28 / 08$ & $08 / 11 / 08$ & gross $\beta$ & $1.6 \mathrm{E}-02 \pm 2.1 \mathrm{E}-03$ & & & & & & \\
\hline & $08 / 11 / 08$ & $08 / 25 / 08$ & gross $\alpha$ & $9.4 \mathrm{E}-04 \pm 5.9 \mathrm{E}-04$ & & & & & & \\
\hline & 08/11/08 & 08/25/08 & gross $\beta$ & $1.6 \mathrm{E}-02 \pm 2.0 \mathrm{E}-03$ & & & & & & \\
\hline & $08 / 25 / 08$ & 09/08/08 & gross $\alpha$ & $4.8 \mathrm{E}-04 \pm 4.6 \mathrm{E}-04$ & & & & & & \\
\hline & $08 / 25 / 08$ & 09/08/08 & gross $\beta$ & $1.2 \mathrm{E}-02 \pm 1.7 \mathrm{E}-03$ & & & & & & \\
\hline & $09 / 08 / 08$ & $09 / 22 / 08$ & gross $\alpha$ & $1.7 \mathrm{E}-03 \pm 6.3 \mathrm{E}-04$ & & & & & & \\
\hline & 09/08/08 & $09 / 22 / 08$ & gross $\beta$ & $2.8 \mathrm{E}-02 \pm 3.0 \mathrm{E}-03$ & & & & & & \\
\hline & $09 / 22 / 08$ & $10 / 07 / 08$ & gross $\alpha$ & $2.0 \mathrm{E}-03 \pm 6.6 \mathrm{E}-04$ & & & & & & \\
\hline & $09 / 22 / 08$ & $10 / 07 / 08$ & gross $\beta$ & $2.0 \mathrm{E}-02 \pm 2.3 \mathrm{E}-03$ & & & & & & \\
\hline & $10 / 07 / 08$ & $10 / 20 / 08$ & gross $\alpha$ & $1.6 \mathrm{E}-03 \pm 6.5 \mathrm{E}-04$ & & & & & & \\
\hline & $10 / 07 / 08$ & $10 / 20 / 08$ & gross $\beta$ & $2.0 \mathrm{E}-02 \pm 2.4 \mathrm{E}-03$ & & & & & & \\
\hline & $10 / 20 / 08$ & $11 / 03 / 08$ & gross $\alpha$ & $2.0 \mathrm{E}-03 \pm 4.6 \mathrm{E}-03$ & & & & & & \\
\hline & $10 / 20 / 08$ & $11 / 03 / 08$ & gross $\beta$ & $1.9 \mathrm{E}-02 \pm 7.6 \mathrm{E}-03$ & & & & & & \\
\hline & $11 / 03 / 08$ & $11 / 17 / 08$ & gross $\alpha$ & $8.8 \mathrm{E}-04 \pm 1.0 \mathrm{E}-03$ & & & & & & \\
\hline & $11 / 03 / 08$ & $11 / 17 / 08$ & gross $\beta$ & $2.4 \mathrm{E}-02 \pm 3.6 \mathrm{E}-03$ & & & & & & \\
\hline & $11 / 17 / 08$ & $12 / 01 / 08$ & gross $\alpha$ & $1.7 \mathrm{E}-03 \pm 6.6 \mathrm{E}-04$ & & & & & & \\
\hline & $11 / 17 / 08$ & $12 / 01 / 08$ & gross $\beta$ & $3.7 \mathrm{E}-02 \pm 3.6 \mathrm{E}-03$ & & & & & & \\
\hline & $12 / 01 / 08$ & $12 / 15 / 08$ & gross $\alpha$ & $2.1 \mathrm{E}-03 \pm 6.9 \mathrm{E}-04$ & & & & & & \\
\hline & $12 / 01 / 08$ & $12 / 15 / 08$ & gross $\beta$ & $1.6 \mathrm{E}-02 \pm 2.0 \mathrm{E}-03$ & & & & & & \\
\hline & $12 / 15 / 08$ & $12 / 29 / 08$ & gross $\alpha$ & $1.2 \mathrm{E}-03 \pm 5.0 \mathrm{E}-04$ & & & & & & \\
\hline & $12 / 15 / 08$ & $12 / 29 / 08$ & gross $\beta$ & $3.5 \mathrm{E}-02 \pm 3.4 \mathrm{E}-03$ & & & & & & \\
\hline
\end{tabular}

$\overline{\mathrm{RQ}}=$ Result Qualifier. $\mathrm{U}=$ The analyte was analyzed for but not detected. 
Table 2-4. Near-Facility Air Sampling Results, 2008 (pCi/m³ \pm total analytical uncertainty). (Sheet 64 of 82)

\begin{tabular}{|c|c|c|c|c|c|c|c|c|c|c|}
\hline Location & Sample On & Sample Off & Isotope & Result \pm Uncertainty & Location & Composite & e Period & Isotope & Result \pm Uncertainty & RQ* \\
\hline N963 & $12 / 31 / 07$ & $01 / 14 / 08$ & gross $\alpha$ & $5.2 \mathrm{E}-04 \pm 4.8 \mathrm{E}-04$ & N963 & $12 / 31 / 07$ to & $06 / 30 / 08$ & ${ }^{60} \mathrm{Co}$ & $2.5 \mathrm{E}-05 \pm 7.7 \mathrm{E}-05$ & $\bar{U}$ \\
\hline \multirow[t]{51}{*}{$(200-W)$} & $12 / 31 / 07$ & 01/14/08 & gross $\beta$ & $1.0 \mathrm{E}-02 \pm 1.5 \mathrm{E}-03$ & & & & ${ }^{134} \mathrm{Cs}$ & $-6.1 \mathrm{E}-06 \pm 6.1 \mathrm{E}-05$ & $\mathrm{U}$ \\
\hline & $01 / 14 / 08$ & 01/29/08 & gross $\alpha$ & $1.6 \mathrm{E}-03 \pm 5.8 \mathrm{E}-04$ & & & & ${ }^{137} \mathrm{Cs}$ & $-1.0 \mathrm{E}-05 \pm 6.3 \mathrm{E}-05$ & U \\
\hline & $01 / 14 / 08$ & 01/29/08 & gross $\beta$ & $3.0 \mathrm{E}-02 \pm 3.0 \mathrm{E}-03$ & & & & ${ }^{152} \mathrm{Eu}$ & $-4.6 \mathrm{E}-05 \pm 1.5 \mathrm{E}-04$ & $\mathrm{U}$ \\
\hline & $01 / 29 / 08$ & $02 / 12 / 08$ & gross $\alpha$ & $4.9 \mathrm{E}-04 \pm 4.6 \mathrm{E}-04$ & & & & ${ }^{154} \mathrm{Eu}$ & $-1.3 \mathrm{E}-04 \pm 2.1 \mathrm{E}-04$ & $\mathrm{U}$ \\
\hline & $01 / 29 / 08$ & 02/12/08 & gross $\beta$ & $4.3 \mathrm{E}-03 \pm 9.1 \mathrm{E}-04$ & & & & ${ }^{155} \mathrm{Eu}$ & $-1.6 \mathrm{E}-04 \pm 1.8 \mathrm{E}-04$ & U \\
\hline & $02 / 12 / 08$ & $02 / 25 / 08$ & gross $\alpha$ & $1.3 \mathrm{E}-03 \pm 5.8 \mathrm{E}-04$ & & & & ${ }^{238} \mathrm{Pu}$ & $2.8 \mathrm{E}-06 \pm 6.0 \mathrm{E}-06$ & $\mathrm{U}$ \\
\hline & $02 / 12 / 08$ & $02 / 25 / 08$ & gross $\beta$ & $2.6 \mathrm{E}-02 \pm 2.9 \mathrm{E}-03$ & & & & ${ }^{239 / 240} \mathrm{Pu}$ & $3.3 \mathrm{E}-06 \pm 2.9 \mathrm{E}-06$ & \\
\hline & $02 / 25 / 08$ & 03/11/08 & gross $\alpha$ & $1.6 \mathrm{E}-03 \pm 5.9 \mathrm{E}-04$ & & & & ${ }^{106} \mathrm{Ru}$ & $5.3 \mathrm{E}-05 \pm 5.3 \mathrm{E}-04$ & $\mathrm{U}$ \\
\hline & $02 / 25 / 08$ & 03/11/08 & gross $\beta$ & $1.3 \mathrm{E}-02 \pm 1.7 \mathrm{E}-03$ & & & & ${ }^{125} \mathrm{Sb}$ & $8.4 \mathrm{E}-06 \pm 8.4 \mathrm{E}-05$ & $\mathrm{U}$ \\
\hline & 03/11/08 & 03/25/08 & gross $\alpha$ & $5.2 \mathrm{E}-04 \pm 4.8 \mathrm{E}-04$ & & & & ${ }^{90} \mathrm{Sr}$ & $-7.7 \mathrm{E}-05 \pm 8.0 \mathrm{E}-05$ & $\mathrm{U}$ \\
\hline & 03/11/08 & $03 / 25 / 08$ & gross $\beta$ & $6.9 \mathrm{E}-03 \pm 1.2 \mathrm{E}-03$ & & & & ${ }^{234} \mathrm{U}$ & $8.7 \mathrm{E}-06 \pm 6.4 \mathrm{E}-06$ & \\
\hline & $03 / 25 / 08$ & $04 / 07 / 08$ & gross $\alpha$ & $1.3 \mathrm{E}-03 \pm 5.6 \mathrm{E}-04$ & & & & ${ }^{235} \mathrm{U}$ & $2.2 \mathrm{E}-06 \pm 2.7 \mathrm{E}-06$ & \\
\hline & $03 / 25 / 08$ & $04 / 07 / 08$ & gross $\beta$ & $6.3 \mathrm{E}-03 \pm 1.2 \mathrm{E}-03$ & & & & ${ }^{238} \mathrm{U}$ & $9.4 \mathrm{E}-06 \pm 6.1 \mathrm{E}-06$ & \\
\hline & 04/07/08 & $04 / 21 / 08$ & gross $\alpha$ & $1.5 \mathrm{E}-03 \pm 5.9 \mathrm{E}-04$ & & & & & & \\
\hline & $04 / 07 / 08$ & $04 / 21 / 08$ & gross $\beta$ & $1.1 \mathrm{E}-02 \pm 1.6 \mathrm{E}-03$ & N963 & $06 / 30 / 08$ to & $12 / 29 / 08$ & ${ }^{60} \mathrm{Co}$ & $5.1 \mathrm{E}-05 \pm 8.7 \mathrm{E}-05$ & U \\
\hline & $04 / 21 / 08$ & 05/06/08 & gross $\alpha$ & $2.1 \mathrm{E}-03 \pm 6.7 \mathrm{E}-04$ & & & & ${ }^{134} \mathrm{Cs}$ & $1.4 \mathrm{E}-05 \pm 8.1 \mathrm{E}-05$ & $\mathrm{U}$ \\
\hline & $04 / 21 / 08$ & 05/06/08 & gross $\beta$ & $1.4 \mathrm{E}-02 \pm 1.8 \mathrm{E}-03$ & & & & ${ }^{137} \mathrm{Cs}$ & $8.1 \mathrm{E}-05 \pm 6.9 \mathrm{E}-05$ & $\mathrm{U}$ \\
\hline & 05/06/08 & 05/19/08 & gross $\alpha$ & $5.1 \mathrm{E}-04 \pm 4.9 \mathrm{E}-04$ & & & & ${ }^{152} \mathrm{Eu}$ & $9.8 \mathrm{E}-06 \pm 9.8 \mathrm{E}-05$ & U \\
\hline & 05/06/08 & 05/19/08 & gross $\beta$ & $1.4 \mathrm{E}-02 \pm 1.9 \mathrm{E}-03$ & & & & ${ }^{154} \mathrm{Eu}$ & $-7.5 \mathrm{E}-05 \pm 2.1 \mathrm{E}-04$ & $\mathrm{U}$ \\
\hline & 05/19/08 & $06 / 02 / 08$ & gross $\alpha$ & $1.1 \mathrm{E}-03 \pm 5.1 \mathrm{E}-04$ & & & & ${ }^{155} \mathrm{Eu}$ & $-2.4 \mathrm{E}-05 \pm 1.6 \mathrm{E}-04$ & $\mathrm{U}$ \\
\hline & 05/19/08 & $06 / 02 / 08$ & gross $\beta$ & $1.2 \mathrm{E}-02 \pm 1.7 \mathrm{E}-03$ & & & & ${ }^{238} \mathrm{Pu}$ & $2.9 \mathrm{E}-06 \pm 4.3 \mathrm{E}-06$ & $\mathrm{U}$ \\
\hline & $06 / 02 / 08$ & $06 / 16 / 08$ & gross $\alpha$ & $9.5 \mathrm{E}-04 \pm 5.9 \mathrm{E}-04$ & & & & ${ }^{239 / 240} \mathrm{Pu}$ & $5.7 \mathrm{E}-07 \pm 3.0 \mathrm{E}-06$ & $\mathrm{U}$ \\
\hline & $06 / 02 / 08$ & 06/16/08 & gross $\beta$ & $6.8 \mathrm{E}-03 \pm 1.2 \mathrm{E}-03$ & & & & ${ }^{106} \mathrm{Ru}$ & $1.1 \mathrm{E}-04 \pm 6.0 \mathrm{E}-04$ & $\mathrm{U}$ \\
\hline & $06 / 16 / 08$ & 06/30/08 & gross $\alpha$ & $1.6 \mathrm{E}-03 \pm 6.5 \mathrm{E}-04$ & & & & ${ }^{125} \mathrm{Sb}$ & $4.7 \mathrm{E}-05 \pm 1.5 \mathrm{E}-04$ & $\mathrm{U}$ \\
\hline & $06 / 16 / 08$ & 06/30/08 & gross $\beta$ & $1.4 \mathrm{E}-02 \pm 1.8 \mathrm{E}-03$ & & & & ${ }^{90} \mathrm{Sr}$ & $-2.3 \mathrm{E}-04 \pm 2.4 \mathrm{E}-04$ & $\mathrm{U}$ \\
\hline & $06 / 30 / 08$ & $07 / 14 / 08$ & gross $\alpha$ & $1.3 \mathrm{E}-03 \pm 5.8 \mathrm{E}-04$ & & & & ${ }^{234} \mathrm{U}$ & $1.2 \mathrm{E}-05 \pm 7.4 \mathrm{E}-06$ & \\
\hline & 06/30/08 & 07/14/08 & gross $\beta$ & $4.5 \mathrm{E}-02 \pm 4.2 \mathrm{E}-03$ & & & & ${ }^{235} \mathrm{U}$ & $3.0 \mathrm{E}-06 \pm 3.2 \mathrm{E}-06$ & \\
\hline & $07 / 14 / 08$ & 07/28/08 & gross $\alpha$ & $1.4 \mathrm{E}-03 \pm 5.6 \mathrm{E}-04$ & & & & ${ }^{238} \mathrm{U}$ & $6.2 \mathrm{E}-06 \pm 4.8 \mathrm{E}-06$ & \\
\hline & $07 / 14 / 08$ & $07 / 28 / 08$ & gross $\beta$ & $1.8 \mathrm{E}-02 \pm 2.1 \mathrm{E}-03$ & & & & & & \\
\hline & 07/28/08 & 08/11/08 & gross $\alpha$ & $1.0 \mathrm{E}-03 \pm 4.7 \mathrm{E}-04$ & & & & & & \\
\hline & $07 / 28 / 08$ & $08 / 11 / 08$ & gross $\beta$ & $1.2 \mathrm{E}-02 \pm 1.7 \mathrm{E}-03$ & & & & & & \\
\hline & $08 / 11 / 08$ & $08 / 25 / 08$ & gross $\alpha$ & $2.3 \mathrm{E}-03 \pm 7.1 \mathrm{E}-04$ & & & & & & \\
\hline & 08/11/08 & 08/25/08 & gross $\beta$ & $1.6 \mathrm{E}-02 \pm 2.0 \mathrm{E}-03$ & & & & & & \\
\hline & $08 / 25 / 08$ & 09/08/08 & gross $\alpha$ & $2.5 \mathrm{E}-04 \pm 3.8 \mathrm{E}-04$ & & & & & & \\
\hline & $08 / 25 / 08$ & 09/08/08 & gross $\beta$ & $1.1 \mathrm{E}-02 \pm 1.6 \mathrm{E}-03$ & & & & & & \\
\hline & $09 / 08 / 08$ & $09 / 22 / 08$ & gross $\alpha$ & $2.1 \mathrm{E}-03 \pm 6.7 \mathrm{E}-04$ & & & & & & \\
\hline & 09/08/08 & $09 / 22 / 08$ & gross $\beta$ & $3.0 \mathrm{E}-02 \pm 3.1 \mathrm{E}-03$ & & & & & & \\
\hline & $09 / 22 / 08$ & $10 / 07 / 08$ & gross $\alpha$ & $1.9 \mathrm{E}-03 \pm 6.5 \mathrm{E}-04$ & & & & & & \\
\hline & $09 / 22 / 08$ & $10 / 07 / 08$ & gross $\beta$ & $2.1 \mathrm{E}-02 \pm 2.3 \mathrm{E}-03$ & & & & & & \\
\hline & $10 / 07 / 08$ & $10 / 20 / 08$ & gross $\alpha$ & $1.7 \mathrm{E}-03 \pm 6.5 \mathrm{E}-04$ & & & & & & \\
\hline & $10 / 07 / 08$ & $10 / 20 / 08$ & gross $\beta$ & $2.1 \mathrm{E}-02 \pm 2.5 \mathrm{E}-03$ & & & & & & \\
\hline & $10 / 20 / 08$ & $11 / 03 / 08$ & gross $\alpha$ & $2.8 \mathrm{E}-03 \pm 8.0 \mathrm{E}-04$ & & & & & & \\
\hline & $10 / 20 / 08$ & $11 / 03 / 08$ & gross $\beta$ & $4.9 \mathrm{E}-02 \pm 4.5 \mathrm{E}-03$ & & & & & & \\
\hline & $11 / 03 / 08$ & $11 / 17 / 08$ & gross $\alpha$ & $1.2 \mathrm{E}-03 \pm 5.2 \mathrm{E}-04$ & & & & & & \\
\hline & $11 / 03 / 08$ & $11 / 17 / 08$ & gross $\beta$ & $2.0 \mathrm{E}-02 \pm 2.3 \mathrm{E}-03$ & & & & & & \\
\hline & $11 / 17 / 08$ & $12 / 01 / 08$ & gross $\alpha$ & $2.0 \mathrm{E}-03 \pm 6.9 \mathrm{E}-04$ & & & & & & \\
\hline & $11 / 17 / 08$ & $12 / 01 / 08$ & gross $\beta$ & $4.0 \mathrm{E}-02 \pm 3.8 \mathrm{E}-03$ & & & & & & \\
\hline & $12 / 01 / 08$ & $12 / 15 / 08$ & gross $\alpha$ & $8.7 \mathrm{E}-04 \pm 5.7 \mathrm{E}-04$ & & & & & & \\
\hline & $12 / 01 / 08$ & $12 / 15 / 08$ & gross $\beta$ & $2.0 \mathrm{E}-02 \pm 2.3 \mathrm{E}-03$ & & & & & & \\
\hline & $12 / 15 / 08$ & $12 / 29 / 08$ & gross $\alpha$ & $2.2 \mathrm{E}-03 \pm 7.0 \mathrm{E}-04$ & & & & & & \\
\hline & $12 / 15 / 08$ & $12 / 29 / 08$ & gross $\beta$ & $4.9 \mathrm{E}-02 \pm 4.4 \mathrm{E}-03$ & & & & & & \\
\hline
\end{tabular}

$\overline{\mathrm{RQ}}=$ Result Qualifier. $\mathrm{U}=$ The analyte was analyzed for but not detected. 
Table 2-4. Near-Facility Air Sampling Results, 2008 (pCi/m³ \pm total analytical uncertainty). (Sheet 65 of 82)

\begin{tabular}{|c|c|c|c|c|c|c|c|c|c|}
\hline Location & Sample On & Sample Off & Isotope & Result \pm Uncertainty & Location & Composite Period & Isotope & Result \pm Uncertainty & $\mathbf{R Q *}$ \\
\hline N964 & $12 / 31 / 07$ & $01 / 14 / 08$ & gross $\alpha$ & $1.2 \mathrm{E}-03 \pm 5.2 \mathrm{E}-04$ & N964 & $12 / 31 / 07$ to $06 / 30 / 08$ & ${ }^{60} \mathrm{Co}$ & $1.1 \mathrm{E}-05 \pm 7.5 \mathrm{E}-05$ & $\mathrm{U}$ \\
\hline \multirow[t]{51}{*}{$(200-W)$} & $12 / 31 / 07$ & 01/14/08 & gross $\beta$ & $9.2 \mathrm{E}-03 \pm 1.4 \mathrm{E}-03$ & & & ${ }^{134} \mathrm{Cs}$ & $1.3 \mathrm{E}-06 \pm 1.3 \mathrm{E}-05$ & $\mathrm{U}$ \\
\hline & $01 / 14 / 08$ & 01/29/08 & gross $\alpha$ & $1.2 \mathrm{E}-03 \pm 5.1 \mathrm{E}-04$ & & & ${ }^{137} \mathrm{Cs}$ & $1.4 \mathrm{E}-05 \pm 6.9 \mathrm{E}-05$ & U \\
\hline & $01 / 14 / 08$ & 01/29/08 & gross $\beta$ & $2.8 \mathrm{E}-02 \pm 2.9 \mathrm{E}-03$ & & & ${ }^{152} \mathrm{Eu}$ & $-2.4 \mathrm{E}-06 \pm 2.4 \mathrm{E}-05$ & $\mathrm{U}$ \\
\hline & 01/29/08 & $02 / 12 / 08$ & gross $\alpha$ & $6.1 \mathrm{E}-04 \pm 5.1 \mathrm{E}-04$ & & & ${ }^{154} \mathrm{Eu}$ & $3.6 \mathrm{E}-05 \pm 2.3 \mathrm{E}-04$ & $\mathrm{U}$ \\
\hline & $01 / 29 / 08$ & $02 / 12 / 08$ & gross $\beta$ & $3.5 \mathrm{E}-03 \pm 8.4 \mathrm{E}-04$ & & & ${ }^{155} \mathrm{Eu}$ & $-1.5 \mathrm{E}-05 \pm 1.6 \mathrm{E}-04$ & $\mathrm{U}$ \\
\hline & $02 / 12 / 08$ & $02 / 25 / 08$ & gross $\alpha$ & $1.5 \mathrm{E}-03 \pm 6.3 \mathrm{E}-04$ & & & ${ }^{238} \mathrm{Pu}$ & $-5.1 \mathrm{E}-07 \pm 3.1 \mathrm{E}-06$ & $\mathrm{U}$ \\
\hline & $02 / 12 / 08$ & $02 / 25 / 08$ & gross $\beta$ & $2.8 \mathrm{E}-02 \pm 3.0 \mathrm{E}-03$ & & & ${ }^{239 / 240} \mathrm{Pu}$ & $5.1 \mathrm{E}-07 \pm 5.3 \mathrm{E}-07$ & $\mathrm{U}$ \\
\hline & $02 / 25 / 08$ & 03/11/08 & gross $\alpha$ & $7.0 \mathrm{E}-04 \pm 5.1 \mathrm{E}-04$ & & & ${ }^{106} \mathrm{Ru}$ & $3.1 \mathrm{E}-04 \pm 6.4 \mathrm{E}-04$ & $\mathrm{U}$ \\
\hline & $02 / 25 / 08$ & 03/11/08 & gross $\beta$ & $1.5 \mathrm{E}-02 \pm 1.9 \mathrm{E}-03$ & & & ${ }^{125} \mathrm{Sb}$ & $1.1 \mathrm{E}-04 \pm 1.7 \mathrm{E}-04$ & U \\
\hline & 03/11/08 & $03 / 25 / 08$ & gross $\alpha$ & $8.6 \mathrm{E}-04 \pm 5.8 \mathrm{E}-04$ & & & ${ }^{90} \mathrm{Sr}$ & $-1.3 \mathrm{E}-04 \pm 1.4 \mathrm{E}-04$ & $\mathrm{U}$ \\
\hline & 03/11/08 & $03 / 25 / 08$ & gross $\beta$ & $8.8 \mathrm{E}-03 \pm 1.4 \mathrm{E}-03$ & & & ${ }^{234} \mathrm{U}$ & $1.2 \mathrm{E}-05 \pm 7.4 \mathrm{E}-06$ & \\
\hline & $03 / 25 / 08$ & $04 / 07 / 08$ & gross $\alpha$ & $1.0 \mathrm{E}-03 \pm 6.3 \mathrm{E}-04$ & & & ${ }^{235} \mathrm{U}$ & $1.4 \mathrm{E}-06 \pm 2.1 \mathrm{E}-06$ & U \\
\hline & 03/25/08 & $04 / 07 / 08$ & gross $\beta$ & $5.4 \mathrm{E}-03 \pm 1.1 \mathrm{E}-03$ & & & ${ }^{238} \mathrm{U}$ & $7.2 \mathrm{E}-06 \pm 5.2 \mathrm{E}-06$ & \\
\hline & $04 / 07 / 08$ & $04 / 21 / 08$ & gross $\alpha$ & $1.2 \mathrm{E}-03 \pm 5.2 \mathrm{E}-04$ & & & ${ }^{60} \mathrm{Co}$ & $-3.7 \mathrm{E}-05 \pm 7.5 \mathrm{E}-05$ & $\mathrm{U}$ \\
\hline & $04 / 07 / 08$ & $04 / 21 / 08$ & gross $\beta$ & $9.3 \mathrm{E}-03 \pm 1.4 \mathrm{E}-03$ & & & & & \\
\hline & $04 / 21 / 08$ & $05 / 05 / 08$ & gross $\alpha$ & $1.7 \mathrm{E}-03 \pm 6.2 \mathrm{E}-04$ & N964 & $06 / 30 / 08$ to $12 / 29 / 08$ & ${ }^{134} \mathrm{Cs}$ & $-2.4 \mathrm{E}-05 \pm 6.2 \mathrm{E}-05$ & $\mathrm{U}$ \\
\hline & $04 / 21 / 08$ & 05/05/08 & gross $\beta$ & $1.4 \mathrm{E}-02 \pm 1.8 \mathrm{E}-03$ & & & ${ }^{137} \mathrm{Cs}$ & $1.8 \mathrm{E}-05 \pm 5.8 \mathrm{E}-05$ & $\mathrm{U}$ \\
\hline & 05/05/08 & 05/19/08 & gross $\alpha$ & $1.5 \mathrm{E}-03 \pm 5.8 \mathrm{E}-04$ & & & ${ }^{152} \mathrm{Eu}$ & $-5.1 \mathrm{E}-05 \pm 1.5 \mathrm{E}-04$ & U \\
\hline & $05 / 05 / 08$ & 05/19/08 & gross $\beta$ & $1.1 \mathrm{E}-02 \pm 1.6 \mathrm{E}-03$ & & & ${ }^{154} \mathrm{Eu}$ & $-2.1 \mathrm{E}-04 \pm 2.1 \mathrm{E}-04$ & $\mathrm{U}$ \\
\hline & 05/19/08 & 06/02/08 & gross $\alpha$ & $8.9 \mathrm{E}-04 \pm 5.6 \mathrm{E}-04$ & & & ${ }^{155} \mathrm{Eu}$ & $2.8 \mathrm{E}-05 \pm 1.3 \mathrm{E}-04$ & U \\
\hline & 05/19/08 & $06 / 02 / 08$ & gross $\beta$ & $1.3 \mathrm{E}-02 \pm 1.8 \mathrm{E}-03$ & & & ${ }^{238} \mathrm{Pu}$ & $3.7 \mathrm{E}-06 \pm 7.0 \mathrm{E}-06$ & $\mathrm{U}$ \\
\hline & $06 / 02 / 08$ & 06/16/08 & gross $\alpha$ & $1.0 \mathrm{E}-03 \pm 7.1 \mathrm{E}-04$ & & & ${ }^{239 / 240} \mathrm{Pu}$ & $3.1 \mathrm{E}-06 \pm 2.9 \mathrm{E}-06$ & \\
\hline & $06 / 02 / 08$ & 06/16/08 & gross $\beta$ & $9.0 \mathrm{E}-03 \pm 1.5 \mathrm{E}-03$ & & & ${ }^{106} \mathrm{Ru}$ & $1.9 \mathrm{E}-04 \pm 5.5 \mathrm{E}-04$ & $\mathrm{U}$ \\
\hline & $06 / 16 / 08$ & 06/30/08 & gross $\alpha$ & $1.4 \mathrm{E}-03 \pm 6.2 \mathrm{E}-04$ & & & ${ }^{125} \mathrm{Sb}$ & $-8.1 \mathrm{E}-05 \pm 1.4 \mathrm{E}-04$ & $\mathrm{U}$ \\
\hline & $06 / 16 / 08$ & $06 / 30 / 08$ & gross $\beta$ & $1.3 \mathrm{E}-02 \pm 1.7 \mathrm{E}-03$ & & & ${ }^{90} \mathrm{Sr}$ & $-3.0 \mathrm{E}-04 \pm 3.2 \mathrm{E}-04$ & $\mathrm{U}$ \\
\hline & $06 / 30 / 08$ & $07 / 14 / 08$ & gross $\alpha$ & $9.1 \mathrm{E}-04 \pm 6.4 \mathrm{E}-04$ & & & ${ }^{234} \mathrm{U}$ & $1.3 \mathrm{E}-05 \pm 8.6 \mathrm{E}-06$ & \\
\hline & $06 / 30 / 08$ & 07/14/08 & gross $\beta$ & $1.3 \mathrm{E}-02 \pm 1.7 \mathrm{E}-03$ & & & ${ }^{235} \mathrm{U}$ & $2.3 \mathrm{E}-06 \pm 4.1 \mathrm{E}-06$ & $\mathrm{U}$ \\
\hline & $07 / 14 / 08$ & 07/28/08 & gross $\alpha$ & $8.1 \mathrm{E}-04 \pm 5.5 \mathrm{E}-04$ & & & ${ }^{238} \mathrm{U}$ & $1.6 \mathrm{E}-05 \pm 9.3 \mathrm{E}-06$ & \\
\hline & $07 / 14 / 08$ & $07 / 28 / 08$ & gross $\beta$ & $1.1 \mathrm{E}-02 \pm 1.6 \mathrm{E}-03$ & & & & & \\
\hline & 07/28/08 & 08/11/08 & gross $\alpha$ & $1.1 \mathrm{E}-03 \pm 7.0 \mathrm{E}-04$ & & & & & \\
\hline & $07 / 28 / 08$ & $08 / 11 / 08$ & gross $\beta$ & $1.8 \mathrm{E}-02 \pm 2.3 \mathrm{E}-03$ & & & & & \\
\hline & 08/11/08 & $08 / 25 / 08$ & gross $\alpha$ & $4.4 \mathrm{E}-04 \pm 4.3 \mathrm{E}-04$ & & & & & \\
\hline & 08/11/08 & 08/25/08 & gross $\beta$ & $1.3 \mathrm{E}-02 \pm 1.8 \mathrm{E}-03$ & & & & & \\
\hline & $08 / 25 / 08$ & 09/08/08 & gross $\alpha$ & $7.0 \mathrm{E}-04 \pm 5.2 \mathrm{E}-04$ & & & & & \\
\hline & $08 / 25 / 08$ & 09/08/08 & gross $\beta$ & $8.3 \mathrm{E}-03 \pm 1.4 \mathrm{E}-03$ & & & & & \\
\hline & 09/08/08 & 09/22/08 & gross $\alpha$ & $5.8 \mathrm{E}-04 \pm 4.9 \mathrm{E}-04$ & & & & & \\
\hline & 09/08/08 & $09 / 22 / 08$ & gross $\beta$ & $2.8 \mathrm{E}-02 \pm 3.0 \mathrm{E}-03$ & & & & & \\
\hline & $09 / 22 / 08$ & $10 / 07 / 08$ & gross $\alpha$ & $1.7 \mathrm{E}-03 \pm 6.1 \mathrm{E}-04$ & & & & & \\
\hline & $09 / 22 / 08$ & $10 / 07 / 08$ & gross $\beta$ & $2.1 \mathrm{E}-02 \pm 2.3 \mathrm{E}-03$ & & & & & \\
\hline & $10 / 07 / 08$ & $10 / 20 / 08$ & gross $\alpha$ & $2.2 \mathrm{E}-03 \pm 7.3 \mathrm{E}-04$ & & & & & \\
\hline & $10 / 07 / 08$ & $10 / 20 / 08$ & gross $\beta$ & $1.9 \mathrm{E}-02 \pm 2.3 \mathrm{E}-03$ & & & & & \\
\hline & $10 / 20 / 08$ & $11 / 03 / 08$ & gross $\alpha$ & $2.6 \mathrm{E}-03 \pm 7.6 \mathrm{E}-04$ & & & & & \\
\hline & $10 / 20 / 08$ & $11 / 03 / 08$ & gross $\beta$ & $4.2 \mathrm{E}-02 \pm 4.0 \mathrm{E}-03$ & & & & & \\
\hline & $11 / 03 / 08$ & $11 / 17 / 08$ & gross $\alpha$ & $2.9 \mathrm{E}-03 \pm 8.2 \mathrm{E}-04$ & & & & & \\
\hline & $11 / 03 / 08$ & $11 / 17 / 08$ & gross $\beta$ & $1.7 \mathrm{E}-02 \pm 2.1 \mathrm{E}-03$ & & & & & \\
\hline & $11 / 17 / 08$ & $12 / 01 / 08$ & gross $\alpha$ & $1.8 \mathrm{E}-03 \pm 6.7 \mathrm{E}-04$ & & & & & \\
\hline & $11 / 17 / 08$ & $12 / 01 / 08$ & gross $\beta$ & $3.4 \mathrm{E}-02 \pm 3.4 \mathrm{E}-03$ & & & & & \\
\hline & $12 / 01 / 08$ & $12 / 15 / 08$ & gross $\alpha$ & $2.1 \mathrm{E}-03 \pm 6.9 \mathrm{E}-04$ & & & & & \\
\hline & $12 / 01 / 08$ & $12 / 15 / 08$ & gross $\beta$ & $2.1 \mathrm{E}-02 \pm 2.4 \mathrm{E}-03$ & & & & & \\
\hline & $12 / 15 / 08$ & $12 / 29 / 08$ & gross $\alpha$ & $2.1 \mathrm{E}-03 \pm 6.9 \mathrm{E}-04$ & & & & & \\
\hline & $12 / 15 / 08$ & $12 / 29 / 08$ & gross $\beta$ & $4.3 \mathrm{E}-02 \pm 4.0 \mathrm{E}-03$ & & & & & \\
\hline
\end{tabular}

$\overline{\mathrm{RQ}}=$ Result Qualifier. $\mathrm{U}=$ The analyte was analyzed for but not detected. 
Table 2-4. Near-Facility Air Sampling Results, 2008 (pCi/m³ \pm total analytical uncertainty). (Sheet 66 of 82)

\begin{tabular}{|c|c|c|c|c|c|c|c|c|c|c|}
\hline Location & Sample On & Sample Off & Isotope & Result \pm Uncertainty & Location & Composite & e Period & Isotope & Result \pm Uncertainty & RQ* \\
\hline N965 & $12 / 31 / 07$ & $01 / 14 / 08$ & gross $\alpha$ & $2.6 \mathrm{E}-03 \pm 7.9 \mathrm{E}-04$ & N965 & $12 / 31 / 07$ to & $06 / 30 / 08$ & ${ }^{60} \mathrm{Co}$ & $1.9 \mathrm{E}-05 \pm 7.2 \mathrm{E}-05$ & $\bar{U}$ \\
\hline \multirow[t]{51}{*}{$(200-W)$} & $12 / 31 / 07$ & 01/14/08 & gross $\beta$ & $6.9 \mathrm{E}-03 \pm 1.2 \mathrm{E}-03$ & & & & ${ }^{134} \mathrm{Cs}$ & $1.1 \mathrm{E}-05 \pm 5.8 \mathrm{E}-05$ & $\mathrm{U}$ \\
\hline & $01 / 14 / 08$ & 01/29/08 & gross $\alpha$ & $1.9 \mathrm{E}-03 \pm 6.3 \mathrm{E}-04$ & & & & ${ }^{137} \mathrm{Cs}$ & $-2.9 \mathrm{E}-05 \pm 5.7 \mathrm{E}-05$ & U \\
\hline & $01 / 14 / 08$ & 01/29/08 & gross $\beta$ & $3.1 \mathrm{E}-02 \pm 3.1 \mathrm{E}-03$ & & & & ${ }^{152} \mathrm{Eu}$ & $4.5 \mathrm{E}-05 \pm 1.2 \mathrm{E}-04$ & $\mathrm{U}$ \\
\hline & $01 / 29 / 08$ & $02 / 12 / 08$ & gross $\alpha$ & $3.8 \mathrm{E}-04 \pm 4.3 \mathrm{E}-04$ & & & & ${ }^{154} \mathrm{Eu}$ & $-5.7 \mathrm{E}-05 \pm 1.9 \mathrm{E}-04$ & $\mathrm{U}$ \\
\hline & $01 / 29 / 08$ & 02/12/08 & gross $\beta$ & $5.8 \mathrm{E}-03 \pm 1.1 \mathrm{E}-03$ & & & & ${ }^{155} \mathrm{Eu}$ & $-1.5 \mathrm{E}-05 \pm 1.3 \mathrm{E}-04$ & U \\
\hline & $02 / 12 / 08$ & $02 / 25 / 08$ & gross $\alpha$ & $1.7 \mathrm{E}-03 \pm 6.5 \mathrm{E}-04$ & & & & ${ }^{238} \mathrm{Pu}$ & $1.7 \mathrm{E}-06 \pm 3.4 \mathrm{E}-06$ & $\mathrm{U}$ \\
\hline & $02 / 12 / 08$ & $02 / 25 / 08$ & gross $\beta$ & $3.0 \mathrm{E}-02 \pm 3.1 \mathrm{E}-03$ & & & & ${ }^{239 / 240} \mathrm{Pu}$ & $5.0 \mathrm{E}-06 \pm 4.4 \mathrm{E}-06$ & $\mathrm{U}$ \\
\hline & $02 / 25 / 08$ & 03/11/08 & gross $\alpha$ & $8.1 \mathrm{E}-04 \pm 5.4 \mathrm{E}-04$ & & & & ${ }^{106} \mathrm{Ru}$ & $-4.1 \mathrm{E}-04 \pm 5.2 \mathrm{E}-04$ & $\mathrm{U}$ \\
\hline & $02 / 25 / 08$ & 03/11/08 & gross $\beta$ & $1.7 \mathrm{E}-02 \pm 2.0 \mathrm{E}-03$ & & & & ${ }^{125} \mathrm{Sb}$ & $1.8 \mathrm{E}-05 \pm 1.3 \mathrm{E}-04$ & $\mathrm{U}$ \\
\hline & 03/11/08 & 03/25/08 & gross $\alpha$ & $7.5 \mathrm{E}-04 \pm 5.5 \mathrm{E}-04$ & & & & ${ }^{90} \mathrm{Sr}$ & $-3.4 \mathrm{E}-05 \pm 3.6 \mathrm{E}-05$ & $\mathrm{U}$ \\
\hline & 03/11/08 & $03 / 25 / 08$ & gross $\beta$ & $7.2 \mathrm{E}-03 \pm 1.2 \mathrm{E}-03$ & & & & ${ }^{234} \mathrm{U}$ & $7.1 \mathrm{E}-06 \pm 5.7 \mathrm{E}-06$ & \\
\hline & $03 / 25 / 08$ & $04 / 07 / 08$ & gross $\alpha$ & $9.0 \mathrm{E}-04 \pm 6.1 \mathrm{E}-04$ & & & & ${ }^{235} \mathrm{U}$ & $2.1 \mathrm{E}-06 \pm 3.2 \mathrm{E}-06$ & $\mathrm{U}$ \\
\hline & $03 / 25 / 08$ & $04 / 07 / 08$ & gross $\beta$ & $8.0 \mathrm{E}-03 \pm 1.3 \mathrm{E}-03$ & & & & ${ }^{238} \mathrm{U}$ & $1.4 \mathrm{E}-05 \pm 8.0 \mathrm{E}-06$ & \\
\hline & 04/07/08 & $04 / 21 / 08$ & gross $\alpha$ & $1.3 \mathrm{E}-03 \pm 5.5 \mathrm{E}-04$ & & & & & & \\
\hline & $04 / 07 / 08$ & $04 / 21 / 08$ & gross $\beta$ & $1.1 \mathrm{E}-02 \pm 1.5 \mathrm{E}-03$ & N965 & $06 / 30 / 08$ to & $12 / 29 / 08$ & ${ }^{60} \mathrm{Co}$ & $-1.6 \mathrm{E}-05 \pm 7.3 \mathrm{E}-05$ & U \\
\hline & $04 / 21 / 08$ & $05 / 05 / 08$ & gross $\alpha$ & $1.5 \mathrm{E}-03 \pm 6.0 \mathrm{E}-04$ & & & & ${ }^{134} \mathrm{Cs}$ & $-1.8 \mathrm{E}-05 \pm 5.9 \mathrm{E}-05$ & $\mathrm{U}$ \\
\hline & $04 / 21 / 08$ & 05/05/08 & gross $\beta$ & $1.3 \mathrm{E}-02 \pm 1.8 \mathrm{E}-03$ & & & & ${ }^{137} \mathrm{Cs}$ & $5.1 \mathrm{E}-05 \pm 6.5 \mathrm{E}-05$ & $\mathrm{U}$ \\
\hline & 05/05/08 & 05/19/08 & gross $\alpha$ & $6.1 \mathrm{E}-04 \pm 5.1 \mathrm{E}-04$ & & & & ${ }^{152} \mathrm{Eu}$ & $3.3 \mathrm{E}-05 \pm 1.4 \mathrm{E}-04$ & U \\
\hline & 05/05/08 & 05/19/08 & gross $\beta$ & $1.2 \mathrm{E}-02 \pm 1.7 \mathrm{E}-03$ & & & & ${ }^{154} \mathrm{Eu}$ & $-8.4 \mathrm{E}-05 \pm 2.0 \mathrm{E}-04$ & $\mathrm{U}$ \\
\hline & 05/19/08 & $06 / 02 / 08$ & gross $\alpha$ & $1.4 \mathrm{E}-03 \pm 5.6 \mathrm{E}-04$ & & & & ${ }^{155} \mathrm{Eu}$ & $-1.0 \mathrm{E}-04 \pm 1.8 \mathrm{E}-04$ & $\mathrm{U}$ \\
\hline & 05/19/08 & $06 / 02 / 08$ & gross $\beta$ & $1.3 \mathrm{E}-02 \pm 1.8 \mathrm{E}-03$ & & & & ${ }^{238} \mathrm{Pu}$ & $9.7 \mathrm{E}-07 \pm 9.7 \mathrm{E}-06$ & $\mathrm{U}$ \\
\hline & $06 / 02 / 08$ & $06 / 16 / 08$ & gross $\alpha$ & $7.2 \mathrm{E}-04 \pm 5.3 \mathrm{E}-04$ & & & & ${ }^{239 / 240} \mathrm{Pu}$ & $9.8 \mathrm{E}-07 \pm 5.2 \mathrm{E}-06$ & $\mathrm{U}$ \\
\hline & $06 / 02 / 08$ & 06/16/08 & gross $\beta$ & $7.2 \mathrm{E}-03 \pm 1.2 \mathrm{E}-03$ & & & & ${ }^{106} \mathrm{Ru}$ & $-2.7 \mathrm{E}-04 \pm 5.1 \mathrm{E}-04$ & $\mathrm{U}$ \\
\hline & $06 / 16 / 08$ & 06/30/08 & gross $\alpha$ & $2.1 \mathrm{E}-03 \pm 7.5 \mathrm{E}-04$ & & & & ${ }^{125} \mathrm{Sb}$ & $4.3 \mathrm{E}-05 \pm 1.5 \mathrm{E}-04$ & $\mathrm{U}$ \\
\hline & $06 / 16 / 08$ & 06/30/08 & gross $\beta$ & $1.5 \mathrm{E}-02 \pm 1.9 \mathrm{E}-03$ & & & & ${ }^{90} \mathrm{Sr}$ & $-5.3 \mathrm{E}-04 \pm 5.5 \mathrm{E}-04$ & $\mathrm{U}$ \\
\hline & $06 / 30 / 08$ & $07 / 14 / 08$ & gross $\alpha$ & $2.0 \mathrm{E}-03 \pm 7.4 \mathrm{E}-04$ & & & & ${ }^{234} \mathrm{U}$ & $1.5 \mathrm{E}-05 \pm 8.8 \mathrm{E}-06$ & \\
\hline & $06 / 30 / 08$ & 07/14/08 & gross $\beta$ & $1.9 \mathrm{E}-02 \pm 2.2 \mathrm{E}-03$ & & & & ${ }^{235} \mathrm{U}$ & $3.3 \mathrm{E}-06 \pm 3.5 \mathrm{E}-06$ & \\
\hline & $07 / 14 / 08$ & 07/28/08 & gross $\alpha$ & $1.9 \mathrm{E}-03 \pm 6.6 \mathrm{E}-04$ & & & & ${ }^{238} \mathrm{U}$ & $6.9 \mathrm{E}-06 \pm 5.6 \mathrm{E}-06$ & \\
\hline & $07 / 14 / 08$ & $07 / 28 / 08$ & gross $\beta$ & $1.7 \mathrm{E}-02 \pm 2.1 \mathrm{E}-03$ & & & & & & \\
\hline & 07/28/08 & 08/11/08 & gross $\alpha$ & $1.8 \mathrm{E}-03 \pm 6.9 \mathrm{E}-04$ & & & & & & \\
\hline & $07 / 28 / 08$ & $08 / 11 / 08$ & gross $\beta$ & $1.8 \mathrm{E}-02 \pm 2.4 \mathrm{E}-03$ & & & & & & \\
\hline & $08 / 11 / 08$ & $08 / 25 / 08$ & gross $\alpha$ & $2.0 \mathrm{E}-03 \pm 6.7 \mathrm{E}-04$ & & & & & & \\
\hline & 08/11/08 & 08/25/08 & gross $\beta$ & $1.7 \mathrm{E}-02 \pm 2.1 \mathrm{E}-03$ & & & & & & \\
\hline & $08 / 25 / 08$ & 09/08/08 & gross $\alpha$ & $6.6 \mathrm{E}-04 \pm 5.0 \mathrm{E}-04$ & & & & & & \\
\hline & $08 / 25 / 08$ & 09/08/08 & gross $\beta$ & $1.0 \mathrm{E}-02 \pm 1.5 \mathrm{E}-03$ & & & & & & \\
\hline & $09 / 08 / 08$ & $09 / 22 / 08$ & gross $\alpha$ & $2.0 \mathrm{E}-03 \pm 6.7 \mathrm{E}-04$ & & & & & & \\
\hline & 09/08/08 & $09 / 22 / 08$ & gross $\beta$ & $2.7 \mathrm{E}-02 \pm 2.9 \mathrm{E}-03$ & & & & & & \\
\hline & $09 / 22 / 08$ & $10 / 07 / 08$ & gross $\alpha$ & $9.8 \mathrm{E}-04 \pm 4.5 \mathrm{E}-04$ & & & & & & \\
\hline & $09 / 22 / 08$ & $10 / 07 / 08$ & gross $\beta$ & $1.3 \mathrm{E}-02 \pm 1.7 \mathrm{E}-03$ & & & & & & \\
\hline & $10 / 14 / 08$ & $10 / 20 / 08$ & gross $\alpha$ & $2.4 \mathrm{E}-03 \pm 1.1 \mathrm{E}-03$ & & & & & & \\
\hline & $10 / 14 / 08$ & $10 / 20 / 08$ & gross $\beta$ & $2.4 \mathrm{E}-02 \pm 3.4 \mathrm{E}-03$ & & & & & & \\
\hline & $10 / 20 / 08$ & $11 / 03 / 08$ & gross $\alpha$ & $3.9 \mathrm{E}-03 \pm 9.4 \mathrm{E}-04$ & & & & & & \\
\hline & $10 / 20 / 08$ & $11 / 03 / 08$ & gross $\beta$ & $4.4 \mathrm{E}-02 \pm 4.2 \mathrm{E}-03$ & & & & & & \\
\hline & $11 / 03 / 08$ & $11 / 17 / 08$ & gross $\alpha$ & $7.3 \mathrm{E}-04 \pm 5.5 \mathrm{E}-04$ & & & & & & \\
\hline & $11 / 03 / 08$ & $11 / 17 / 08$ & gross $\beta$ & $1.9 \mathrm{E}-02 \pm 2.3 \mathrm{E}-03$ & & & & & & \\
\hline & $11 / 17 / 08$ & $12 / 01 / 08$ & gross $\alpha$ & $1.2 \mathrm{E}-03 \pm 5.5 \mathrm{E}-04$ & & & & & & \\
\hline & $11 / 17 / 08$ & $12 / 01 / 08$ & gross $\beta$ & $3.6 \mathrm{E}-02 \pm 3.6 \mathrm{E}-03$ & & & & & & \\
\hline & $12 / 01 / 08$ & $12 / 15 / 08$ & gross $\alpha$ & $3.2 \mathrm{E}-03 \pm 1.2 \mathrm{E}-03$ & & & & & & \\
\hline & $12 / 01 / 08$ & $12 / 15 / 08$ & gross $\beta$ & $3.4 \mathrm{E}-02 \pm 4.1 \mathrm{E}-03$ & & & & & & \\
\hline & $12 / 15 / 08$ & $12 / 29 / 08$ & gross $\alpha$ & $5.0 \mathrm{E}-03 \pm 1.5 \mathrm{E}-03$ & & & & & & \\
\hline & $12 / 15 / 08$ & $12 / 29 / 08$ & gross $\beta$ & $9.8 \mathrm{E}-02 \pm 9.0 \mathrm{E}-03$ & & & & & & \\
\hline
\end{tabular}

$\overline{\mathrm{RQ}}=$ Result Qualifier. $\mathrm{U}=$ The analyte was analyzed for but not detected. 
Table 2-4. Near-Facility Air Sampling Results, 2008 (pCi/m³ \pm total analytical uncertainty). (Sheet 67 of 82)

\begin{tabular}{|c|c|c|c|c|c|c|c|c|c|c|}
\hline Location & Sample On & Sample Off & Isotope & Result \pm Uncertainty & Location & Composite & e Period & Isotope & Result \pm Uncertainty & $\mathbf{R Q *}$ \\
\hline N966 & $12 / 31 / 07$ & $01 / 14 / 08$ & gross $\alpha$ & $4.0 \mathrm{E}-04 \pm 4.5 \mathrm{E}-04$ & N966 & $12 / 31 / 07$ to & $06 / 30 / 08$ & ${ }^{60} \mathrm{Co}$ & $-6.3 \mathrm{E}-06 \pm 6.2 \mathrm{E}-05$ & $\mathrm{U}$ \\
\hline \multirow[t]{51}{*}{$(200-W)$} & $12 / 31 / 07$ & 01/14/08 & gross $\beta$ & $8.9 \mathrm{E}-03 \pm 1.4 \mathrm{E}-03$ & & & & ${ }^{134} \mathrm{Cs}$ & $-1.5 \mathrm{E}-05 \pm 6.2 \mathrm{E}-05$ & $\mathrm{U}$ \\
\hline & $01 / 14 / 08$ & 01/29/08 & gross $\alpha$ & $1.7 \mathrm{E}-03 \pm 6.1 \mathrm{E}-04$ & & & & ${ }^{137} \mathrm{Cs}$ & $2.4 \mathrm{E}-07 \pm 2.4 \mathrm{E}-06$ & U \\
\hline & $01 / 14 / 08$ & 01/29/08 & gross $\beta$ & $3.1 \mathrm{E}-02 \pm 3.1 \mathrm{E}-03$ & & & & ${ }^{152} \mathrm{Eu}$ & $7.9 \mathrm{E}-05 \pm 1.4 \mathrm{E}-04$ & U \\
\hline & 01/29/08 & $02 / 12 / 08$ & gross $\alpha$ & $4.0 \mathrm{E}-04 \pm 4.5 \mathrm{E}-04$ & & & & ${ }^{154} \mathrm{Eu}$ & $-2.0 \mathrm{E}-05 \pm 1.6 \mathrm{E}-04$ & $\mathrm{U}$ \\
\hline & $01 / 29 / 08$ & $02 / 12 / 08$ & gross $\beta$ & $5.3 \mathrm{E}-03 \pm 1.0 \mathrm{E}-03$ & & & & ${ }^{155} \mathrm{Eu}$ & $-6.6 \mathrm{E}-05 \pm 1.6 \mathrm{E}-04$ & U \\
\hline & $02 / 12 / 08$ & $02 / 25 / 08$ & gross $\alpha$ & $1.5 \mathrm{E}-03 \pm 6.3 \mathrm{E}-04$ & & & & ${ }^{238} \mathrm{Pu}$ & $4.1 \mathrm{E}-06 \pm 3.8 \mathrm{E}-06$ & $\mathrm{U}$ \\
\hline & $02 / 12 / 08$ & $02 / 25 / 08$ & gross $\beta$ & $3.4 \mathrm{E}-02 \pm 3.5 \mathrm{E}-03$ & & & & ${ }^{239 / 240} \mathrm{Pu}$ & $5.3 \mathrm{E}-06 \pm 4.0 \mathrm{E}-06$ & \\
\hline & $02 / 25 / 08$ & 03/11/08 & gross $\alpha$ & $1.2 \mathrm{E}-03 \pm 5.2 \mathrm{E}-04$ & & & & ${ }^{106} \mathrm{Ru}$ & $-5.4 \mathrm{E}-04 \pm 5.6 \mathrm{E}-04$ & $\mathrm{U}$ \\
\hline & $02 / 25 / 08$ & 03/11/08 & gross $\beta$ & $1.7 \mathrm{E}-02 \pm 2.0 \mathrm{E}-03$ & & & & ${ }^{125} \mathrm{Sb}$ & $2.3 \mathrm{E}-05 \pm 1.2 \mathrm{E}-04$ & U \\
\hline & 03/11/08 & 03/25/08 & gross $\alpha$ & $9.8 \mathrm{E}-04 \pm 6.0 \mathrm{E}-04$ & & & & ${ }^{90} \mathrm{Sr}$ & $7.0 \mathrm{E}-05 \pm 1.5 \mathrm{E}-04$ & $\mathrm{U}$ \\
\hline & 03/11/08 & $03 / 25 / 08$ & gross $\beta$ & $8.8 \mathrm{E}-03 \pm 1.4 \mathrm{E}-03$ & & & & ${ }^{234} \mathrm{U}$ & $1.3 \mathrm{E}-05 \pm 8.1 \mathrm{E}-06$ & \\
\hline & 03/25/08 & $04 / 07 / 08$ & gross $\alpha$ & $1.0 \mathrm{E}-03 \pm 6.3 \mathrm{E}-04$ & & & & ${ }^{235} \mathrm{U}$ & $1.5 \mathrm{E}-06 \pm 3.1 \mathrm{E}-06$ & $\mathrm{U}$ \\
\hline & 03/25/08 & $04 / 07 / 08$ & gross $\beta$ & $1.1 \mathrm{E}-02 \pm 1.6 \mathrm{E}-03$ & & & & ${ }^{238} \mathrm{U}$ & $9.7 \mathrm{E}-06 \pm 7.0 \mathrm{E}-06$ & \\
\hline & 04/07/08 & $04 / 21 / 08$ & gross $\alpha$ & $1.6 \mathrm{E}-03 \pm 6.1 \mathrm{E}-04$ & & & & & & \\
\hline & $04 / 07 / 08$ & $04 / 21 / 08$ & gross $\beta$ & $1.1 \mathrm{E}-02 \pm 1.6 \mathrm{E}-03$ & N966 & $06 / 30 / 08$ to & $12 / 29 / 08$ & ${ }^{60} \mathrm{Co}$ & $2.7 \mathrm{E}-05 \pm 8.7 \mathrm{E}-05$ & $\mathrm{U}$ \\
\hline & $04 / 21 / 08$ & 05/06/08 & gross $\alpha$ & $1.2 \mathrm{E}-03 \pm 5.1 \mathrm{E}-04$ & & & & ${ }^{134} \mathrm{Cs}$ & $4.8 \mathrm{E}-05 \pm 7.9 \mathrm{E}-05$ & $\mathrm{U}$ \\
\hline & $04 / 21 / 08$ & 05/06/08 & gross $\beta$ & $1.4 \mathrm{E}-02 \pm 1.8 \mathrm{E}-03$ & & & & ${ }^{137} \mathrm{Cs}$ & $-5.4 \mathrm{E}-05 \pm 6.6 \mathrm{E}-05$ & $\mathrm{U}$ \\
\hline & 05/06/08 & 05/19/08 & gross $\alpha$ & $5.1 \mathrm{E}-04 \pm 5.0 \mathrm{E}-04$ & & & & ${ }^{152} \mathrm{Eu}$ & $1.1 \mathrm{E}-04 \pm 1.7 \mathrm{E}-04$ & U \\
\hline & 05/06/08 & 05/19/08 & gross $\beta$ & $1.2 \mathrm{E}-02 \pm 1.8 \mathrm{E}-03$ & & & & ${ }^{154} \mathrm{Eu}$ & $1.3 \mathrm{E}-04 \pm 2.4 \mathrm{E}-04$ & $\mathrm{U}$ \\
\hline & 05/19/08 & 06/02/08 & gross $\alpha$ & $6.9 \mathrm{E}-04 \pm 5.2 \mathrm{E}-04$ & & & & ${ }^{155} \mathrm{Eu}$ & $2.8 \mathrm{E}-05 \pm 1.6 \mathrm{E}-04$ & U \\
\hline & 05/19/08 & $06 / 02 / 08$ & gross $\beta$ & $1.2 \mathrm{E}-02 \pm 1.7 \mathrm{E}-03$ & & & & ${ }^{238} \mathrm{Pu}$ & $8.3 \mathrm{E}-07 \pm 8.3 \mathrm{E}-06$ & $\mathrm{U}$ \\
\hline & $06 / 02 / 08$ & 06/16/08 & gross $\alpha$ & $9.5 \mathrm{E}-04 \pm 5.9 \mathrm{E}-04$ & & & & ${ }^{239 / 240} \mathrm{Pu}$ & $1.6 \mathrm{E}-06 \pm 4.1 \mathrm{E}-06$ & $\mathrm{U}$ \\
\hline & $06 / 02 / 08$ & 06/16/08 & gross $\beta$ & $8.6 \mathrm{E}-03 \pm 1.4 \mathrm{E}-03$ & & & & ${ }^{106} \mathrm{Ru}$ & $2.3 \mathrm{E}-05 \pm 2.3 \mathrm{E}-04$ & $\mathrm{U}$ \\
\hline & $06 / 16 / 08$ & 06/30/08 & gross $\alpha$ & $1.4 \mathrm{E}-03 \pm 6.3 \mathrm{E}-04$ & & & & ${ }^{125} \mathrm{Sb}$ & $1.0 \mathrm{E}-04 \pm 1.5 \mathrm{E}-04$ & $\mathrm{U}$ \\
\hline & $06 / 16 / 08$ & $06 / 30 / 08$ & gross $\beta$ & $1.3 \mathrm{E}-02 \pm 1.7 \mathrm{E}-03$ & & & & ${ }^{90} \mathrm{Sr}$ & $-3.2 \mathrm{E}-04 \pm 3.3 \mathrm{E}-04$ & $\mathrm{U}$ \\
\hline & $06 / 30 / 08$ & $07 / 14 / 08$ & gross $\alpha$ & $1.1 \mathrm{E}-03 \pm 6.9 \mathrm{E}-04$ & & & & ${ }^{234} \mathrm{U}$ & $6.2 \mathrm{E}-06 \pm 5.1 \mathrm{E}-06$ & \\
\hline & $06 / 30 / 08$ & 07/14/08 & gross $\beta$ & $1.3 \mathrm{E}-02 \pm 1.8 \mathrm{E}-03$ & & & & ${ }^{235} \mathrm{U}$ & $3.0 \mathrm{E}-06 \pm 3.8 \mathrm{E}-06$ & $\mathrm{U}$ \\
\hline & $07 / 14 / 08$ & 07/28/08 & gross $\alpha$ & $1.4 \mathrm{E}-03 \pm 5.6 \mathrm{E}-04$ & & & & ${ }^{238} \mathrm{U}$ & $1.0 \mathrm{E}-05 \pm 6.6 \mathrm{E}-06$ & \\
\hline & $07 / 14 / 08$ & $07 / 28 / 08$ & gross $\beta$ & $1.5 \mathrm{E}-02 \pm 2.0 \mathrm{E}-03$ & & & & & & \\
\hline & 07/28/08 & 08/11/08 & gross $\alpha$ & $1.6 \mathrm{E}-03 \pm 6.0 \mathrm{E}-04$ & & & & & & \\
\hline & $07 / 28 / 08$ & $08 / 11 / 08$ & gross $\beta$ & $1.6 \mathrm{E}-02 \pm 2.0 \mathrm{E}-03$ & & & & & & \\
\hline & $08 / 11 / 08$ & $08 / 25 / 08$ & gross $\alpha$ & $1.1 \mathrm{E}-03 \pm 5.1 \mathrm{E}-04$ & & & & & & \\
\hline & 08/11/08 & 08/25/08 & gross $\beta$ & $1.6 \mathrm{E}-02 \pm 2.0 \mathrm{E}-03$ & & & & & & \\
\hline & $08 / 25 / 08$ & 09/08/08 & gross $\alpha$ & $4.8 \mathrm{E}-04 \pm 4.6 \mathrm{E}-04$ & & & & & & \\
\hline & $08 / 25 / 08$ & 09/08/08 & gross $\beta$ & $1.2 \mathrm{E}-02 \pm 1.7 \mathrm{E}-03$ & & & & & & \\
\hline & 09/08/08 & 09/22/08 & gross $\alpha$ & $1.1 \mathrm{E}-03 \pm 5.1 \mathrm{E}-04$ & & & & & & \\
\hline & 09/08/08 & $09 / 22 / 08$ & gross $\beta$ & $2.8 \mathrm{E}-02 \pm 3.0 \mathrm{E}-03$ & & & & & & \\
\hline & $09 / 22 / 08$ & $10 / 07 / 08$ & gross $\alpha$ & $1.2 \mathrm{E}-03 \pm 5.3 \mathrm{E}-04$ & & & & & & \\
\hline & $09 / 22 / 08$ & $10 / 07 / 08$ & gross $\beta$ & $2.3 \mathrm{E}-02 \pm 2.5 \mathrm{E}-03$ & & & & & & \\
\hline & $10 / 07 / 08$ & $10 / 20 / 08$ & gross $\alpha$ & $1.3 \mathrm{E}-03 \pm 5.8 \mathrm{E}-04$ & & & & & & \\
\hline & $10 / 07 / 08$ & $10 / 20 / 08$ & gross $\beta$ & $2.0 \mathrm{E}-02 \pm 2.3 \mathrm{E}-03$ & & & & & & \\
\hline & $10 / 20 / 08$ & $11 / 03 / 08$ & gross $\alpha$ & $2.5 \mathrm{E}-03 \pm 7.5 \mathrm{E}-04$ & & & & & & \\
\hline & $10 / 20 / 08$ & $11 / 03 / 08$ & gross $\beta$ & $5.0 \mathrm{E}-02 \pm 4.6 \mathrm{E}-03$ & & & & & & \\
\hline & $11 / 03 / 08$ & $11 / 17 / 08$ & gross $\alpha$ & $1.5 \mathrm{E}-03 \pm 6.1 \mathrm{E}-04$ & & & & & & \\
\hline & $11 / 03 / 08$ & $11 / 17 / 08$ & gross $\beta$ & $2.0 \mathrm{E}-02 \pm 2.4 \mathrm{E}-03$ & & & & & & \\
\hline & $11 / 17 / 08$ & $12 / 01 / 08$ & gross $\alpha$ & $1.4 \mathrm{E}-03 \pm 6.0 \mathrm{E}-04$ & & & & & & \\
\hline & $11 / 17 / 08$ & $12 / 01 / 08$ & gross $\beta$ & $4.2 \mathrm{E}-02 \pm 4.1 \mathrm{E}-03$ & & & & & & \\
\hline & $12 / 01 / 08$ & $12 / 15 / 08$ & gross $\alpha$ & $1.4 \mathrm{E}-03 \pm 5.7 \mathrm{E}-04$ & & & & & & \\
\hline & $12 / 01 / 08$ & $12 / 15 / 08$ & gross $\beta$ & $2.2 \mathrm{E}-02 \pm 2.4 \mathrm{E}-03$ & & & & & & \\
\hline & $12 / 15 / 08$ & $12 / 29 / 08$ & gross $\alpha$ & $1.9 \mathrm{E}-03 \pm 6.6 \mathrm{E}-04$ & & & & & & \\
\hline & $12 / 15 / 08$ & $12 / 29 / 08$ & gross $\beta$ & $3.7 \mathrm{E}-02 \pm 3.6 \mathrm{E}-03$ & & & & & & \\
\hline
\end{tabular}

$\overline{\mathrm{RQ}}=$ Result Qualifier. $\mathrm{U}=$ The analyte was analyzed for but not detected. 
Table 2-4. Near-Facility Air Sampling Results, 2008 (pCi/m³ \pm total analytical uncertainty). (Sheet 68 of 82)

\begin{tabular}{|c|c|c|c|c|c|c|c|c|c|c|}
\hline Location & Sample On & Sample Off & Isotope & Result \pm Uncertainty & Location & Composite & e Period & Isotope & Result \pm Uncertainty & RQ* \\
\hline N974 & $12 / 31 / 07$ & $01 / 14 / 08$ & gross $\alpha$ & $5.3 \mathrm{E}-04 \pm 5.0 \mathrm{E}-04$ & N974 & $12 / 31 / 07$ to & $06 / 30 / 08$ & ${ }^{60} \mathrm{Co}$ & $-4.9 \mathrm{E}-05 \pm 8.6 \mathrm{E}-05$ & $\bar{U}$ \\
\hline \multirow[t]{51}{*}{$(200-W)$} & $12 / 31 / 07$ & 01/14/08 & gross $\beta$ & $1.0 \mathrm{E}-02 \pm 1.5 \mathrm{E}-03$ & & & & ${ }^{134} \mathrm{Cs}$ & $5.0 \mathrm{E}-05 \pm 7.8 \mathrm{E}-05$ & $\mathrm{U}$ \\
\hline & $01 / 14 / 08$ & 01/29/08 & gross $\alpha$ & $5.4 \mathrm{E}-04 \pm 5.0 \mathrm{E}-04$ & & & & ${ }^{137} \mathrm{Cs}$ & $-2.9 \mathrm{E}-07 \pm 2.9 \mathrm{E}-06$ & U \\
\hline & 01/14/08 & 01/29/08 & gross $\beta$ & $3.7 \mathrm{E}-02 \pm 3.6 \mathrm{E}-03$ & & & & ${ }^{152} \mathrm{Eu}$ & $-3.1 \mathrm{E}-05 \pm 1.8 \mathrm{E}-04$ & U \\
\hline & $01 / 29 / 08$ & $02 / 12 / 08$ & gross $\alpha$ & $7.3 \mathrm{E}-04 \pm 5.4 \mathrm{E}-04$ & & & & ${ }^{154} \mathrm{Eu}$ & $-8.2 \mathrm{E}-05 \pm 2.6 \mathrm{E}-04$ & $\mathrm{U}$ \\
\hline & $01 / 29 / 08$ & 02/12/08 & gross $\beta$ & $6.4 \mathrm{E}-03 \pm 1.1 \mathrm{E}-03$ & & & & ${ }^{155} \mathrm{Eu}$ & $-1.8 \mathrm{E}-04 \pm 1.9 \mathrm{E}-04$ & U \\
\hline & $02 / 12 / 08$ & $02 / 25 / 08$ & gross $\alpha$ & $1.3 \mathrm{E}-03 \pm 5.9 \mathrm{E}-04$ & & & & ${ }^{238} \mathrm{Pu}$ & $2.0 \mathrm{E}-06 \pm 4.1 \mathrm{E}-06$ & $\mathrm{U}$ \\
\hline & $02 / 12 / 08$ & $02 / 25 / 08$ & gross $\beta$ & $2.8 \mathrm{E}-02 \pm 3.1 \mathrm{E}-03$ & & & & ${ }^{239 / 240} \mathrm{Pu}$ & $6.8 \mathrm{E}-06 \pm 5.6 \mathrm{E}-06$ & \\
\hline & $02 / 25 / 08$ & 03/11/08 & gross $\alpha$ & $6.9 \mathrm{E}-04 \pm 5.1 \mathrm{E}-04$ & & & & ${ }^{106} \mathrm{Ru}$ & $1.5 \mathrm{E}-04 \pm 6.7 \mathrm{E}-04$ & $\mathrm{U}$ \\
\hline & $02 / 25 / 08$ & 03/11/08 & gross $\beta$ & $1.6 \mathrm{E}-02 \pm 2.0 \mathrm{E}-03$ & & & & ${ }^{125} \mathrm{Sb}$ & $1.3 \mathrm{E}-04 \pm 1.6 \mathrm{E}-04$ & $\mathrm{U}$ \\
\hline & 03/11/08 & 03/25/08 & gross $\alpha$ & $1.2 \mathrm{E}-03 \pm 5.5 \mathrm{E}-04$ & & & & ${ }^{90} \mathrm{Sr}$ & $-1.7 \mathrm{E}-04 \pm 1.7 \mathrm{E}-04$ & $\mathrm{U}$ \\
\hline & 03/11/08 & $03 / 25 / 08$ & gross $\beta$ & $8.8 \mathrm{E}-03 \pm 1.4 \mathrm{E}-03$ & & & & ${ }^{234} \mathrm{U}$ & $9.4 \mathrm{E}-06 \pm 6.7 \mathrm{E}-06$ & \\
\hline & $03 / 25 / 08$ & $04 / 07 / 08$ & gross $\alpha$ & $1.4 \mathrm{E}-03 \pm 6.4 \mathrm{E}-04$ & & & & ${ }^{235} \mathrm{U}$ & $3.7 \mathrm{E}-06 \pm 3.5 \mathrm{E}-06$ & \\
\hline & 03/25/08 & $04 / 07 / 08$ & gross $\beta$ & $7.9 \mathrm{E}-03 \pm 1.4 \mathrm{E}-03$ & & & & ${ }^{238} \mathrm{U}$ & $8.7 \mathrm{E}-06 \pm 6.4 \mathrm{E}-06$ & \\
\hline & 04/07/08 & $04 / 21 / 08$ & gross $\alpha$ & $9.3 \mathrm{E}-04 \pm 6.3 \mathrm{E}-04$ & & & & & & \\
\hline & $04 / 07 / 08$ & $04 / 21 / 08$ & gross $\beta$ & $8.5 \mathrm{E}-03 \pm 1.4 \mathrm{E}-03$ & N974 & $06 / 30 / 08$ to & $12 / 29 / 08$ & ${ }^{60} \mathrm{Co}$ & $5.1 \mathrm{E}-05 \pm 7.8 \mathrm{E}-05$ & $\mathrm{U}$ \\
\hline & $04 / 21 / 08$ & $05 / 05 / 08$ & gross $\alpha$ & $1.2 \mathrm{E}-03 \pm 5.4 \mathrm{E}-04$ & & & & ${ }^{134} \mathrm{Cs}$ & $-5.1 \mathrm{E}-06 \pm 5.1 \mathrm{E}-05$ & $\mathrm{U}$ \\
\hline & $04 / 21 / 08$ & 05/05/08 & gross $\beta$ & $1.5 \mathrm{E}-02 \pm 1.9 \mathrm{E}-03$ & & & & ${ }^{137} \mathrm{Cs}$ & $-3.4 \mathrm{E}-05 \pm 6.6 \mathrm{E}-05$ & $\mathrm{U}$ \\
\hline & 05/05/08 & 05/19/08 & gross $\alpha$ & $1.4 \mathrm{E}-03 \pm 6.3 \mathrm{E}-04$ & & & & ${ }^{152} \mathrm{Eu}$ & $-9.6 \mathrm{E}-05 \pm 1.6 \mathrm{E}-04$ & U \\
\hline & 05/05/08 & 05/19/08 & gross $\beta$ & $1.2 \mathrm{E}-02 \pm 1.8 \mathrm{E}-03$ & & & & ${ }^{154} \mathrm{Eu}$ & $-3.5 \mathrm{E}-05 \pm 2.3 \mathrm{E}-04$ & $\mathrm{U}$ \\
\hline & 05/19/08 & 06/02/08 & gross $\alpha$ & $2.1 \mathrm{E}-03 \pm 7.5 \mathrm{E}-04$ & & & & ${ }^{155} \mathrm{Eu}$ & $-7.5 \mathrm{E}-06 \pm 7.5 \mathrm{E}-05$ & U \\
\hline & 05/19/08 & $06 / 02 / 08$ & gross $\beta$ & $1.3 \mathrm{E}-02 \pm 1.8 \mathrm{E}-03$ & & & & ${ }^{238} \mathrm{Pu}$ & $8.7 \mathrm{E}-06 \pm 1.4 \mathrm{E}-05$ & $\mathrm{U}$ \\
\hline & $06 / 02 / 08$ & $06 / 16 / 08$ & gross $\alpha$ & $8.8 \mathrm{E}-04 \pm 6.8 \mathrm{E}-04$ & & & & ${ }^{239 / 240} \mathrm{Pu}$ & $4.4 \mathrm{E}-06 \pm 4.9 \mathrm{E}-06$ & $\mathrm{U}$ \\
\hline & $06 / 02 / 08$ & 06/16/08 & gross $\beta$ & $8.1 \mathrm{E}-03 \pm 1.4 \mathrm{E}-03$ & & & & ${ }^{106} \mathrm{Ru}$ & $3.4 \mathrm{E}-04 \pm 6.0 \mathrm{E}-04$ & $\mathrm{U}$ \\
\hline & $06 / 16 / 08$ & 06/30/08 & gross $\alpha$ & $5.9 \mathrm{E}-04 \pm 6.0 \mathrm{E}-04$ & & & & ${ }^{125} \mathrm{Sb}$ & $4.9 \mathrm{E}-05 \pm 1.6 \mathrm{E}-04$ & $\mathrm{U}$ \\
\hline & $06 / 16 / 08$ & 06/30/08 & gross $\beta$ & $1.1 \mathrm{E}-02 \pm 1.6 \mathrm{E}-03$ & & & & ${ }^{90} \mathrm{Sr}$ & $-3.1 \mathrm{E}-04 \pm 3.2 \mathrm{E}-04$ & $\mathrm{U}$ \\
\hline & $06 / 30 / 08$ & $07 / 14 / 08$ & gross $\alpha$ & $9.5 \mathrm{E}-04 \pm 6.2 \mathrm{E}-04$ & & & & ${ }^{234} \mathrm{U}$ & $9.9 \mathrm{E}-06 \pm 7.0 \mathrm{E}-06$ & \\
\hline & 06/30/08 & 07/14/08 & gross $\beta$ & $1.5 \mathrm{E}-02 \pm 2.0 \mathrm{E}-03$ & & & & ${ }^{235} \mathrm{U}$ & $3.4 \mathrm{E}-06 \pm 3.6 \mathrm{E}-06$ & \\
\hline & $07 / 14 / 08$ & 07/28/08 & gross $\alpha$ & $1.0 \mathrm{E}-03 \pm 6.2 \mathrm{E}-04$ & & & & ${ }^{238} \mathrm{U}$ & $4.6 \mathrm{E}-06 \pm 4.7 \mathrm{E}-06$ & $\mathrm{U}$ \\
\hline & $07 / 14 / 08$ & $07 / 28 / 08$ & gross $\beta$ & $1.6 \mathrm{E}-02 \pm 2.0 \mathrm{E}-03$ & & & & & & \\
\hline & 07/28/08 & 08/11/08 & gross $\alpha$ & $1.9 \mathrm{E}-03 \pm 6.7 \mathrm{E}-04$ & & & & & & \\
\hline & $07 / 28 / 08$ & $08 / 11 / 08$ & gross $\beta$ & $7.6 \mathrm{E}-03 \pm 1.3 \mathrm{E}-03$ & & & & & & \\
\hline & $08 / 11 / 08$ & $08 / 25 / 08$ & gross $\alpha$ & $8.5 \mathrm{E}-04 \pm 5.6 \mathrm{E}-04$ & & & & & & \\
\hline & 08/11/08 & 08/25/08 & gross $\beta$ & $1.2 \mathrm{E}-02 \pm 1.6 \mathrm{E}-03$ & & & & & & \\
\hline & $08 / 25 / 08$ & 09/08/08 & gross $\alpha$ & $6.8 \mathrm{E}-04 \pm 5.5 \mathrm{E}-04$ & & & & & & \\
\hline & $08 / 25 / 08$ & 09/08/08 & gross $\beta$ & $1.3 \mathrm{E}-02 \pm 1.7 \mathrm{E}-03$ & & & & & & \\
\hline & $09 / 08 / 08$ & $09 / 22 / 08$ & gross $\alpha$ & $1.2 \mathrm{E}-03 \pm 5.5 \mathrm{E}-04$ & & & & & & \\
\hline & 09/08/08 & $09 / 22 / 08$ & gross $\beta$ & $2.9 \mathrm{E}-02 \pm 3.0 \mathrm{E}-03$ & & & & & & \\
\hline & $09 / 22 / 08$ & $10 / 07 / 08$ & gross $\alpha$ & $2.0 \mathrm{E}-03 \pm 6.5 \mathrm{E}-04$ & & & & & & \\
\hline & $09 / 22 / 08$ & $10 / 07 / 08$ & gross $\beta$ & $2.4 \mathrm{E}-02 \pm 2.7 \mathrm{E}-03$ & & & & & & \\
\hline & $10 / 07 / 08$ & $10 / 20 / 08$ & gross $\alpha$ & $1.4 \mathrm{E}-03 \pm 6.0 \mathrm{E}-04$ & & & & & & \\
\hline & $10 / 07 / 08$ & $10 / 20 / 08$ & gross $\beta$ & $1.7 \mathrm{E}-02 \pm 2.1 \mathrm{E}-03$ & & & & & & \\
\hline & $10 / 20 / 08$ & $11 / 03 / 08$ & gross $\alpha$ & $1.7 \mathrm{E}-03 \pm 6.6 \mathrm{E}-04$ & & & & & & \\
\hline & $10 / 20 / 08$ & $11 / 03 / 08$ & gross $\beta$ & $4.0 \mathrm{E}-02 \pm 3.9 \mathrm{E}-03$ & & & & & & \\
\hline & $11 / 03 / 08$ & $11 / 17 / 08$ & gross $\alpha$ & $1.7 \mathrm{E}-03 \pm 6.6 \mathrm{E}-04$ & & & & & & \\
\hline & $11 / 03 / 08$ & $11 / 17 / 08$ & gross $\beta$ & $1.6 \mathrm{E}-02 \pm 2.0 \mathrm{E}-03$ & & & & & & \\
\hline & $11 / 17 / 08$ & $12 / 01 / 08$ & gross $\alpha$ & $2.2 \mathrm{E}-03 \pm 7.7 \mathrm{E}-04$ & & & & & & \\
\hline & $11 / 17 / 08$ & $12 / 01 / 08$ & gross $\beta$ & $3.9 \mathrm{E}-02 \pm 3.8 \mathrm{E}-03$ & & & & & & \\
\hline & $12 / 01 / 08$ & $12 / 15 / 08$ & gross $\alpha$ & $1.4 \mathrm{E}-03 \pm 6.0 \mathrm{E}-04$ & & & & & & \\
\hline & $12 / 01 / 08$ & $12 / 15 / 08$ & gross $\beta$ & $2.8 \mathrm{E}-02 \pm 3.0 \mathrm{E}-03$ & & & & & & \\
\hline & $12 / 15 / 08$ & $12 / 29 / 08$ & gross $\alpha$ & $1.8 \mathrm{E}-03 \pm 6.8 \mathrm{E}-04$ & & & & & & \\
\hline & $12 / 15 / 08$ & $12 / 29 / 08$ & gross $\beta$ & $4.4 \mathrm{E}-02 \pm 4.2 \mathrm{E}-03$ & & & & & & \\
\hline
\end{tabular}

$\overline{\mathrm{RQ}}=$ Result Qualifier. $\mathrm{U}=$ The analyte was analyzed for but not detected. 
Table 2-4. Near-Facility Air Sampling Results, 2008 (pCi/m³ \pm total analytical uncertainty). (Sheet 69 of 82)

\begin{tabular}{|c|c|c|c|c|c|c|c|c|c|c|}
\hline Location & Sample On & Sample Off & Isotope & Result \pm Uncertainty & Location & Composite & e Period & Isotope & Result \pm Uncertainty & RQ* \\
\hline N975 & $12 / 31 / 07$ & $01 / 14 / 08$ & gross $\alpha$ & $1.3 \mathrm{E}-03 \pm 5.3 \mathrm{E}-04$ & N975 & $12 / 31 / 07$ to & $06 / 30 / 08$ & ${ }^{60} \mathrm{Co}$ & $-2.0 \mathrm{E}-05 \pm 6.6 \mathrm{E}-05$ & $\bar{U}$ \\
\hline \multirow[t]{51}{*}{$(200-W)$} & $12 / 31 / 07$ & 01/14/08 & gross $\beta$ & $9.2 \mathrm{E}-03 \pm 1.4 \mathrm{E}-03$ & & & & ${ }^{134} \mathrm{Cs}$ & $-1.7 \mathrm{E}-05 \pm 6.4 \mathrm{E}-05$ & $\mathrm{U}$ \\
\hline & $01 / 14 / 08$ & 01/29/08 & gross $\alpha$ & $8.7 \mathrm{E}-04 \pm 5.8 \mathrm{E}-04$ & & & & ${ }^{137} \mathrm{Cs}$ & $-2.3 \mathrm{E}-05 \pm 5.8 \mathrm{E}-05$ & U \\
\hline & 01/14/08 & 01/29/08 & gross $\beta$ & $3.1 \mathrm{E}-02 \pm 3.2 \mathrm{E}-03$ & & & & ${ }^{152} \mathrm{Eu}$ & $1.3 \mathrm{E}-05 \pm 1.3 \mathrm{E}-04$ & U \\
\hline & $01 / 29 / 08$ & $02 / 12 / 08$ & gross $\alpha$ & $4.0 \mathrm{E}-04 \pm 4.5 \mathrm{E}-04$ & & & & ${ }^{154} \mathrm{Eu}$ & $-1.1 \mathrm{E}-04 \pm 2.2 \mathrm{E}-04$ & $\mathrm{U}$ \\
\hline & $01 / 29 / 08$ & 02/12/08 & gross $\beta$ & $3.3 \mathrm{E}-03 \pm 7.9 \mathrm{E}-04$ & & & & ${ }^{155} \mathrm{Eu}$ & $-1.1 \mathrm{E}-06 \pm 1.1 \mathrm{E}-05$ & U \\
\hline & $02 / 12 / 08$ & $02 / 25 / 08$ & gross $\alpha$ & $1.6 \mathrm{E}-03 \pm 6.4 \mathrm{E}-04$ & & & & ${ }^{238} \mathrm{Pu}$ & $-5.6 \mathrm{E}-07 \pm 1.1 \mathrm{E}-06$ & $\mathrm{U}$ \\
\hline & $02 / 12 / 08$ & $02 / 25 / 08$ & gross $\beta$ & $2.8 \mathrm{E}-02 \pm 3.0 \mathrm{E}-03$ & & & & ${ }^{239 / 240} \mathrm{Pu}$ & $1.9 \mathrm{E}-05 \pm 9.2 \mathrm{E}-06$ & \\
\hline & $02 / 25 / 08$ & 03/11/08 & gross $\alpha$ & $8.2 \mathrm{E}-04 \pm 5.6 \mathrm{E}-04$ & & & & ${ }^{106} \mathrm{Ru}$ & $6.6 \mathrm{E}-05 \pm 4.9 \mathrm{E}-04$ & $\mathrm{U}$ \\
\hline & $02 / 25 / 08$ & 03/11/08 & gross $\beta$ & $1.6 \mathrm{E}-02 \pm 2.0 \mathrm{E}-03$ & & & & ${ }^{125} \mathrm{Sb}$ & $2.2 \mathrm{E}-04 \pm 2.6 \mathrm{E}-04$ & $\mathrm{U}$ \\
\hline & 03/11/08 & 03/25/08 & gross $\alpha$ & $2.8 \mathrm{E}-04 \pm 4.1 \mathrm{E}-04$ & & & & ${ }^{90} \mathrm{Sr}$ & $3.9 \mathrm{E}-05 \pm 1.6 \mathrm{E}-04$ & $\mathrm{U}$ \\
\hline & 03/11/08 & $03 / 25 / 08$ & gross $\beta$ & $6.1 \mathrm{E}-03 \pm 1.1 \mathrm{E}-03$ & & & & ${ }^{234} \mathrm{U}$ & $1.3 \mathrm{E}-05 \pm 7.8 \mathrm{E}-06$ & \\
\hline & $03 / 25 / 08$ & $04 / 07 / 08$ & gross $\alpha$ & $8.3 \mathrm{E}-04 \pm 6.1 \mathrm{E}-04$ & & & & ${ }^{235} \mathrm{U}$ & $7.1 \mathrm{E}-07 \pm 2.1 \mathrm{E}-07$ & U \\
\hline & 03/25/08 & $04 / 07 / 08$ & gross $\beta$ & $6.2 \mathrm{E}-03 \pm 1.2 \mathrm{E}-03$ & & & & ${ }^{238} \mathrm{U}$ & $9.9 \mathrm{E}-06 \pm 6.8 \mathrm{E}-06$ & \\
\hline & 04/07/08 & $04 / 21 / 08$ & gross $\alpha$ & $7.9 \mathrm{E}-04 \pm 5.8 \mathrm{E}-04$ & & & & & & \\
\hline & $04 / 07 / 08$ & $04 / 21 / 08$ & gross $\beta$ & $9.3 \mathrm{E}-03 \pm 1.4 \mathrm{E}-03$ & N975 & $06 / 30 / 08$ to & $12 / 29 / 08$ & ${ }^{60} \mathrm{Co}$ & $5.6 \mathrm{E}-05 \pm 9.2 \mathrm{E}-05$ & $\mathrm{U}$ \\
\hline & $04 / 21 / 08$ & $05 / 05 / 08$ & gross $\alpha$ & $2.0 \mathrm{E}-03 \pm 6.8 \mathrm{E}-04$ & & & & ${ }^{134} \mathrm{Cs}$ & $3.3 \mathrm{E}-05 \pm 7.8 \mathrm{E}-05$ & $\mathrm{U}$ \\
\hline & $04 / 21 / 08$ & $05 / 05 / 08$ & gross $\beta$ & $1.4 \mathrm{E}-02 \pm 1.9 \mathrm{E}-03$ & & & & ${ }^{137} \mathrm{Cs}$ & $-4.6 \mathrm{E}-05 \pm 6.9 \mathrm{E}-05$ & $\mathrm{U}$ \\
\hline & 05/05/08 & 05/19/08 & gross $\alpha$ & $7.9 \mathrm{E}-04 \pm 5.9 \mathrm{E}-04$ & & & & ${ }^{152} \mathrm{Eu}$ & $-1.2 \mathrm{E}-04 \pm 1.8 \mathrm{E}-04$ & U \\
\hline & 05/05/08 & 05/19/08 & gross $\beta$ & $1.0 \mathrm{E}-02 \pm 1.6 \mathrm{E}-03$ & & & & ${ }^{154} \mathrm{Eu}$ & $2.0 \mathrm{E}-05 \pm 2.0 \mathrm{E}-04$ & $\mathrm{U}$ \\
\hline & 05/19/08 & 06/02/08 & gross $\alpha$ & $9.8 \mathrm{E}-04 \pm 6.1 \mathrm{E}-04$ & & & & ${ }^{155} \mathrm{Eu}$ & $1.8 \mathrm{E}-04 \pm 2.1 \mathrm{E}-04$ & U \\
\hline & 05/19/08 & $06 / 02 / 08$ & gross $\beta$ & $9.6 \mathrm{E}-03 \pm 1.5 \mathrm{E}-03$ & & & & ${ }^{238} \mathrm{Pu}$ & $-2.9 \mathrm{E}-06 \pm 1.1 \mathrm{E}-05$ & $\mathrm{U}$ \\
\hline & $06 / 02 / 08$ & $06 / 16 / 08$ & gross $\alpha$ & $1.4 \mathrm{E}-03 \pm 6.3 \mathrm{E}-04$ & & & & ${ }^{239 / 240} \mathrm{Pu}$ & $1.2 \mathrm{E}-05 \pm 7.7 \mathrm{E}-06$ & \\
\hline & $06 / 02 / 08$ & 06/16/08 & gross $\beta$ & $9.6 \mathrm{E}-03 \pm 1.5 \mathrm{E}-03$ & & & & ${ }^{106} \mathrm{Ru}$ & $-9.7 \mathrm{E}-05 \pm 6.9 \mathrm{E}-04$ & U \\
\hline & $06 / 16 / 08$ & 06/30/08 & gross $\alpha$ & $1.2 \mathrm{E}-03 \pm 6.0 \mathrm{E}-04$ & & & & ${ }^{125} \mathrm{Sb}$ & $-9.2 \mathrm{E}-06 \pm 9.2 \mathrm{E}-05$ & $\mathrm{U}$ \\
\hline & $06 / 16 / 08$ & 06/30/08 & gross $\beta$ & $1.2 \mathrm{E}-02 \pm 1.7 \mathrm{E}-03$ & & & & ${ }^{90} \mathrm{Sr}$ & $-2.1 \mathrm{E}-04 \pm 2.2 \mathrm{E}-04$ & $\mathrm{U}$ \\
\hline & $06 / 30 / 08$ & 07/14/08 & gross $\alpha$ & $1.1 \mathrm{E}-03 \pm 6.6 \mathrm{E}-04$ & & & & ${ }^{234} \mathrm{U}$ & $1.4 \mathrm{E}-05 \pm 8.4 \mathrm{E}-06$ & \\
\hline & $06 / 30 / 08$ & 07/14/08 & gross $\beta$ & $1.3 \mathrm{E}-02 \pm 1.8 \mathrm{E}-03$ & & & & ${ }^{235} \mathrm{U}$ & $5.4 \mathrm{E}-06 \pm 5.1 \mathrm{E}-06$ & $\mathrm{U}$ \\
\hline & $07 / 14 / 08$ & 07/28/08 & gross $\alpha$ & $1.1 \mathrm{E}-03 \pm 5.1 \mathrm{E}-04$ & & & & ${ }^{238} \mathrm{U}$ & $1.5 \mathrm{E}-05 \pm 8.6 \mathrm{E}-06$ & \\
\hline & $07 / 14 / 08$ & $07 / 28 / 08$ & gross $\beta$ & $1.3 \mathrm{E}-02 \pm 1.7 \mathrm{E}-03$ & & & & & & \\
\hline & 07/28/08 & 08/11/08 & gross $\alpha$ & $7.6 \mathrm{E}-04 \pm 5.5 \mathrm{E}-04$ & & & & & & \\
\hline & $07 / 28 / 08$ & $08 / 11 / 08$ & gross $\beta$ & $1.4 \mathrm{E}-02 \pm 1.8 \mathrm{E}-03$ & & & & & & \\
\hline & $08 / 11 / 08$ & $08 / 25 / 08$ & gross $\alpha$ & $1.3 \mathrm{E}-03 \pm 5.6 \mathrm{E}-04$ & & & & & & \\
\hline & 08/11/08 & 08/25/08 & gross $\beta$ & $1.4 \mathrm{E}-02 \pm 1.8 \mathrm{E}-03$ & & & & & & \\
\hline & $08 / 25 / 08$ & 09/08/08 & gross $\alpha$ & $7.4 \mathrm{E}-04 \pm 5.3 \mathrm{E}-04$ & & & & & & \\
\hline & $08 / 25 / 08$ & 09/08/08 & gross $\beta$ & $1.1 \mathrm{E}-02 \pm 1.5 \mathrm{E}-03$ & & & & & & \\
\hline & $09 / 08 / 08$ & $09 / 22 / 08$ & gross $\alpha$ & $1.2 \mathrm{E}-03 \pm 5.3 \mathrm{E}-04$ & & & & & & \\
\hline & 09/08/08 & $09 / 22 / 08$ & gross $\beta$ & $2.5 \mathrm{E}-02 \pm 2.7 \mathrm{E}-03$ & & & & & & \\
\hline & $09 / 22 / 08$ & $10 / 07 / 08$ & gross $\alpha$ & $1.4 \mathrm{E}-03 \pm 5.4 \mathrm{E}-04$ & & & & & & \\
\hline & $09 / 22 / 08$ & $10 / 07 / 08$ & gross $\beta$ & $2.4 \mathrm{E}-02 \pm 2.6 \mathrm{E}-03$ & & & & & & \\
\hline & $10 / 07 / 08$ & $10 / 20 / 08$ & gross $\alpha$ & $1.1 \mathrm{E}-03 \pm 6.6 \mathrm{E}-04$ & & & & & & \\
\hline & $10 / 07 / 08$ & $10 / 20 / 08$ & gross $\beta$ & $1.9 \mathrm{E}-02 \pm 2.3 \mathrm{E}-03$ & & & & & & \\
\hline & $10 / 20 / 08$ & $11 / 03 / 08$ & gross $\alpha$ & $3.5 \mathrm{E}-03 \pm 9.2 \mathrm{E}-04$ & & & & & & \\
\hline & $10 / 20 / 08$ & $11 / 03 / 08$ & gross $\beta$ & $3.8 \mathrm{E}-02 \pm 3.7 \mathrm{E}-03$ & & & & & & \\
\hline & $11 / 03 / 08$ & $11 / 17 / 08$ & gross $\alpha$ & $1.7 \mathrm{E}-03 \pm 6.5 \mathrm{E}-04$ & & & & & & \\
\hline & $11 / 03 / 08$ & $11 / 17 / 08$ & gross $\beta$ & $1.7 \mathrm{E}-02 \pm 2.0 \mathrm{E}-03$ & & & & & & \\
\hline & $11 / 17 / 08$ & $12 / 01 / 08$ & gross $\alpha$ & $2.2 \mathrm{E}-03 \pm 7.5 \mathrm{E}-04$ & & & & & & \\
\hline & $11 / 17 / 08$ & $12 / 01 / 08$ & gross $\beta$ & $3.6 \mathrm{E}-02 \pm 3.6 \mathrm{E}-03$ & & & & & & \\
\hline & $12 / 01 / 08$ & $12 / 15 / 08$ & gross $\alpha$ & $1.9 \mathrm{E}-03 \pm 6.7 \mathrm{E}-04$ & & & & & & \\
\hline & $12 / 01 / 08$ & $12 / 15 / 08$ & gross $\beta$ & $1.8 \mathrm{E}-02 \pm 2.1 \mathrm{E}-03$ & & & & & & \\
\hline & $12 / 15 / 08$ & $12 / 29 / 08$ & gross $\alpha$ & $2.7 \mathrm{E}-03 \pm 8.0 \mathrm{E}-04$ & & & & & & \\
\hline & $12 / 15 / 08$ & $12 / 29 / 08$ & gross $\beta$ & $4.1 \mathrm{E}-02 \pm 3.9 \mathrm{E}-03$ & & & & & & \\
\hline
\end{tabular}

$\overline{\mathrm{RQ}}=$ Result Qualifier. $\mathrm{U}=$ The analyte was analyzed for but not detected. 
Table 2-4. Near-Facility Air Sampling Results, 2008 (pCi/m³ \pm total analytical uncertainty). (Sheet 70 of 82)

\begin{tabular}{|c|c|c|c|c|c|c|c|c|c|c|}
\hline Location & Sample On & Sample Off & Isotope & Result \pm Uncertainty & Location & Composite & e Period & Isotope & Result \pm Uncertainty & RQ* \\
\hline N987 & $12 / 31 / 07$ & $01 / 14 / 08$ & gross $\alpha$ & $1.7 \mathrm{E}-03 \pm 6.4 \mathrm{E}-04$ & N987 & $12 / 31 / 07$ to & $06 / 30 / 08$ & ${ }^{60} \mathrm{Co}$ & $-6.1 \mathrm{E}-05 \pm 1.4 \mathrm{E}-04$ & $\bar{U}$ \\
\hline \multirow[t]{51}{*}{$(200-W)$} & $12 / 31 / 07$ & 01/14/08 & gross $\beta$ & $9.1 \mathrm{E}-03 \pm 1.4 \mathrm{E}-03$ & & & & ${ }^{134} \mathrm{Cs}$ & $-3.0 \mathrm{E}-05 \pm 1.1 \mathrm{E}-04$ & $\mathrm{U}$ \\
\hline & $01 / 14 / 08$ & 01/29/08 & gross $\alpha$ & $2.1 \mathrm{E}-03 \pm 7.2 \mathrm{E}-04$ & & & & ${ }^{137} \mathrm{Cs}$ & $8.5 \mathrm{E}-05 \pm 1.1 \mathrm{E}-04$ & U \\
\hline & $01 / 14 / 08$ & 01/29/08 & gross $\beta$ & $3.4 \mathrm{E}-02 \pm 3.4 \mathrm{E}-03$ & & & & ${ }^{152} \mathrm{Eu}$ & $1.8 \mathrm{E}-04 \pm 2.5 \mathrm{E}-04$ & $\mathrm{U}$ \\
\hline & $01 / 29 / 08$ & $02 / 12 / 08$ & gross $\alpha$ & $5.2 \mathrm{E}-04 \pm 4.9 \mathrm{E}-04$ & & & & ${ }^{154} \mathrm{Eu}$ & $-1.3 \mathrm{E}-04 \pm 3.7 \mathrm{E}-04$ & $\mathrm{U}$ \\
\hline & $01 / 29 / 08$ & 02/12/08 & gross $\beta$ & $4.6 \mathrm{E}-03 \pm 9.5 \mathrm{E}-04$ & & & & ${ }^{155} \mathrm{Eu}$ & $-9.1 \mathrm{E}-07 \pm 9.1 \mathrm{E}-06$ & U \\
\hline & $02 / 12 / 08$ & $02 / 25 / 08$ & gross $\alpha$ & $2.3 \mathrm{E}-03 \pm 7.9 \mathrm{E}-04$ & & & & ${ }^{238} \mathrm{Pu}$ & $2.0 \mathrm{E}-05 \pm 1.5 \mathrm{E}-05$ & \\
\hline & $02 / 12 / 08$ & $02 / 25 / 08$ & gross $\beta$ & $2.9 \mathrm{E}-02 \pm 3.1 \mathrm{E}-03$ & & & & ${ }^{239 / 240} \mathrm{Pu}$ & $1.5 \mathrm{E}-05 \pm 8.5 \mathrm{E}-06$ & \\
\hline & $02 / 25 / 08$ & 03/11/08 & gross $\alpha$ & $8.0 \mathrm{E}-04 \pm 5.4 \mathrm{E}-04$ & & & & ${ }^{106} \mathrm{Ru}$ & $-2.4 \mathrm{E}-04 \pm 9.5 \mathrm{E}-04$ & $\mathrm{U}$ \\
\hline & $02 / 25 / 08$ & 03/11/08 & gross $\beta$ & $1.6 \mathrm{E}-02 \pm 1.9 \mathrm{E}-03$ & & & & ${ }^{125} \mathrm{Sb}$ & $3.8 \mathrm{E}-05 \pm 2.4 \mathrm{E}-04$ & $\mathrm{U}$ \\
\hline & 03/11/08 & 03/25/08 & gross $\alpha$ & $5.3 \mathrm{E}-04 \pm 5.0 \mathrm{E}-04$ & & & & ${ }^{90} \mathrm{Sr}$ & $-2.8 \mathrm{E}-04 \pm 2.9 \mathrm{E}-04$ & $\mathrm{U}$ \\
\hline & 03/11/08 & $03 / 25 / 08$ & gross $\beta$ & $3.8 \mathrm{E}-02 \pm 3.7 \mathrm{E}-03$ & & & & ${ }^{234} \mathrm{U}$ & $1.0 \mathrm{E}-05 \pm 7.2 \mathrm{E}-06$ & \\
\hline & $03 / 25 / 08$ & $04 / 07 / 08$ & gross $\alpha$ & $1.1 \mathrm{E}-03 \pm 6.7 \mathrm{E}-04$ & & & & ${ }^{235} \mathrm{U}$ & $7.9 \mathrm{E}-06 \pm 6.2 \mathrm{E}-06$ & \\
\hline & 03/25/08 & $04 / 07 / 08$ & gross $\beta$ & $8.4 \mathrm{E}-03 \pm 1.4 \mathrm{E}-03$ & & & & ${ }^{238} \mathrm{U}$ & $5.1 \mathrm{E}-06 \pm 4.8 \mathrm{E}-06$ & $\mathrm{U}$ \\
\hline & 04/07/08 & $04 / 21 / 08$ & gross $\alpha$ & $1.2 \mathrm{E}-03 \pm 5.5 \mathrm{E}-04$ & & & & & & \\
\hline & $04 / 07 / 08$ & $04 / 21 / 08$ & gross $\beta$ & $9.5 \mathrm{E}-03 \pm 1.4 \mathrm{E}-03$ & N987 & $06 / 30 / 08$ to & $12 / 29 / 08$ & ${ }^{60} \mathrm{Co}$ & $8.3 \mathrm{E}-05 \pm 8.2 \mathrm{E}-05$ & U \\
\hline & $04 / 21 / 08$ & $05 / 05 / 08$ & gross $\alpha$ & $1.4 \mathrm{E}-03 \pm 5.6 \mathrm{E}-04$ & & & & ${ }^{134} \mathrm{Cs}$ & $-9.7 \mathrm{E}-06 \pm 7.5 \mathrm{E}-05$ & $\mathrm{U}$ \\
\hline & $04 / 21 / 08$ & 05/05/08 & gross $\beta$ & $1.4 \mathrm{E}-02 \pm 1.8 \mathrm{E}-03$ & & & & ${ }^{137} \mathrm{Cs}$ & $7.1 \mathrm{E}-05 \pm 7.0 \mathrm{E}-05$ & $\mathrm{U}$ \\
\hline & 05/05/08 & 05/19/08 & gross $\alpha$ & $6.9 \mathrm{E}-04 \pm 5.7 \mathrm{E}-04$ & & & & ${ }^{152} \mathrm{Eu}$ & $-1.1 \mathrm{E}-04 \pm 1.7 \mathrm{E}-04$ & U \\
\hline & 05/05/08 & 05/19/08 & gross $\beta$ & $6.4 \mathrm{E}-03 \pm 1.2 \mathrm{E}-03$ & & & & ${ }^{154} \mathrm{Eu}$ & $1.8 \mathrm{E}-04 \pm 2.1 \mathrm{E}-04$ & $\mathrm{U}$ \\
\hline & 05/19/08 & 06/02/08 & gross $\alpha$ & $3.0 \mathrm{E}-04 \pm 4.3 \mathrm{E}-04$ & & & & ${ }^{155} \mathrm{Eu}$ & $2.7 \mathrm{E}-06 \pm 2.7 \mathrm{E}-05$ & U \\
\hline & 05/19/08 & $06 / 02 / 08$ & gross $\beta$ & $9.7 \mathrm{E}-03 \pm 1.5 \mathrm{E}-03$ & & & & ${ }^{238} \mathrm{Pu}$ & $2.3 \mathrm{E}-06 \pm 9.3 \mathrm{E}-06$ & $\mathrm{U}$ \\
\hline & 06/02/08 & 06/16/08 & gross $\alpha$ & $1.1 \mathrm{E}-03 \pm 7.1 \mathrm{E}-04$ & & & & ${ }^{239 / 240} \mathrm{Pu}$ & $7.8 \mathrm{E}-07 \pm 3.5 \mathrm{E}-06$ & $\mathrm{U}$ \\
\hline & $06 / 02 / 08$ & 06/16/08 & gross $\beta$ & $8.1 \mathrm{E}-03 \pm 1.3 \mathrm{E}-03$ & & & & ${ }^{106} \mathrm{Ru}$ & $3.4 \mathrm{E}-04 \pm 6.6 \mathrm{E}-04$ & $\mathrm{U}$ \\
\hline & $06 / 16 / 08$ & 06/30/08 & gross $\alpha$ & $8.3 \mathrm{E}-04 \pm 6.4 \mathrm{E}-04$ & & & & ${ }^{125} \mathrm{Sb}$ & $5.6 \mathrm{E}-05 \pm 1.6 \mathrm{E}-04$ & $\mathrm{U}$ \\
\hline & $06 / 16 / 08$ & 06/30/08 & gross $\beta$ & $1.2 \mathrm{E}-02 \pm 1.6 \mathrm{E}-03$ & & & & ${ }^{90} \mathrm{Sr}$ & $-1.3 \mathrm{E}-04 \pm 1.4 \mathrm{E}-04$ & $\mathrm{U}$ \\
\hline & $06 / 30 / 08$ & $07 / 14 / 08$ & gross $\alpha$ & $1.3 \mathrm{E}-03 \pm 5.6 \mathrm{E}-04$ & & & & ${ }^{234} \mathrm{U}$ & $2.2 \mathrm{E}-05 \pm 1.2 \mathrm{E}-05$ & \\
\hline & 06/30/08 & 07/14/08 & gross $\beta$ & $1.1 \mathrm{E}-02 \pm 1.6 \mathrm{E}-03$ & & & & ${ }^{235} \mathrm{U}$ & $4.6 \mathrm{E}-06 \pm 4.1 \mathrm{E}-06$ & \\
\hline & $07 / 14 / 08$ & 07/28/08 & gross $\alpha$ & $1.0 \mathrm{E}-03 \pm 6.2 \mathrm{E}-04$ & & & & ${ }^{238} \mathrm{U}$ & $2.1 \mathrm{E}-05 \pm 1.1 \mathrm{E}-05$ & \\
\hline & $07 / 14 / 08$ & $07 / 28 / 08$ & gross $\beta$ & $1.3 \mathrm{E}-02 \pm 1.8 \mathrm{E}-03$ & & & & & & \\
\hline & 07/28/08 & 08/11/08 & gross $\alpha$ & $7.8 \mathrm{E}-04 \pm 5.6 \mathrm{E}-04$ & & & & & & \\
\hline & $07 / 28 / 08$ & $08 / 11 / 08$ & gross $\beta$ & $1.3 \mathrm{E}-02 \pm 1.8 \mathrm{E}-03$ & & & & & & \\
\hline & $08 / 11 / 08$ & $08 / 25 / 08$ & gross $\alpha$ & $1.3 \mathrm{E}-03 \pm 6.1 \mathrm{E}-04$ & & & & & & \\
\hline & 08/11/08 & 08/25/08 & gross $\beta$ & $1.3 \mathrm{E}-02 \pm 1.9 \mathrm{E}-03$ & & & & & & \\
\hline & $08 / 25 / 08$ & 09/08/08 & gross $\alpha$ & $1.4 \mathrm{E}-03 \pm 5.5 \mathrm{E}-04$ & & & & & & \\
\hline & $08 / 25 / 08$ & 09/08/08 & gross $\beta$ & $7.8 \mathrm{E}-03 \pm 1.2 \mathrm{E}-03$ & & & & & & \\
\hline & $09 / 08 / 08$ & $09 / 22 / 08$ & gross $\alpha$ & $1.2 \mathrm{E}-03 \pm 5.5 \mathrm{E}-04$ & & & & & & \\
\hline & 09/08/08 & $09 / 22 / 08$ & gross $\beta$ & $2.3 \mathrm{E}-02 \pm 2.6 \mathrm{E}-03$ & & & & & & \\
\hline & $09 / 22 / 08$ & $10 / 07 / 08$ & gross $\alpha$ & $1.1 \mathrm{E}-03 \pm 4.9 \mathrm{E}-04$ & & & & & & \\
\hline & $09 / 22 / 08$ & $10 / 07 / 08$ & gross $\beta$ & $2.8 \mathrm{E}-02 \pm 3.0 \mathrm{E}-03$ & & & & & & \\
\hline & $10 / 07 / 08$ & $10 / 20 / 08$ & gross $\alpha$ & $1.1 \mathrm{E}-03 \pm 6.9 \mathrm{E}-04$ & & & & & & \\
\hline & $10 / 07 / 08$ & $10 / 20 / 08$ & gross $\beta$ & $1.6 \mathrm{E}-02 \pm 2.1 \mathrm{E}-03$ & & & & & & \\
\hline & $10 / 20 / 08$ & $11 / 03 / 08$ & gross $\alpha$ & $3.3 \mathrm{E}-03 \pm 9.2 \mathrm{E}-04$ & & & & & & \\
\hline & $10 / 20 / 08$ & $11 / 03 / 08$ & gross $\beta$ & $4.0 \mathrm{E}-02 \pm 3.8 \mathrm{E}-03$ & & & & & & \\
\hline & $11 / 03 / 08$ & $11 / 17 / 08$ & gross $\alpha$ & $7.7 \mathrm{E}-04 \pm 5.6 \mathrm{E}-04$ & & & & & & \\
\hline & $11 / 03 / 08$ & $11 / 17 / 08$ & gross $\beta$ & $1.5 \mathrm{E}-02 \pm 1.9 \mathrm{E}-03$ & & & & & & \\
\hline & $11 / 17 / 08$ & $12 / 01 / 08$ & gross $\alpha$ & $1.9 \mathrm{E}-03 \pm 6.8 \mathrm{E}-04$ & & & & & & \\
\hline & $11 / 17 / 08$ & $12 / 01 / 08$ & gross $\beta$ & $3.4 \mathrm{E}-02 \pm 3.4 \mathrm{E}-03$ & & & & & & \\
\hline & $12 / 01 / 08$ & $12 / 15 / 08$ & gross $\alpha$ & $1.9 \mathrm{E}-03 \pm 6.9 \mathrm{E}-04$ & & & & & & \\
\hline & $12 / 01 / 08$ & $12 / 15 / 08$ & gross $\beta$ & $2.3 \mathrm{E}-02 \pm 2.6 \mathrm{E}-03$ & & & & & & \\
\hline & $12 / 15 / 08$ & $12 / 29 / 08$ & gross $\alpha$ & $1.7 \mathrm{E}-03 \pm 6.2 \mathrm{E}-04$ & & & & & & \\
\hline & $12 / 15 / 08$ & $12 / 29 / 08$ & gross $\beta$ & $3.6 \mathrm{E}-02 \pm 3.5 \mathrm{E}-03$ & & & & & & \\
\hline
\end{tabular}

$\overline{\mathrm{RQ}}=$ Result Qualifier. $\mathrm{U}=$ The analyte was analyzed for but not detected. 
Table 2-4. Near-Facility Air Sampling Results, 2008 (pCi/m³ \pm total analytical uncertainty). (Sheet 71 of 82)

\begin{tabular}{|c|c|c|c|c|c|c|c|c|c|}
\hline Location & Sample On & Sample Off & Isotope & Result \pm Uncertainty & Location & Composite Period & Isotope & Result \pm Uncertainty & $\mathbf{R Q *}$ \\
\hline N994 & $12 / 31 / 07$ & $01 / 14 / 08$ & gross $\alpha$ & $8.8 \mathrm{E}-04 \pm 5.9 \mathrm{E}-04$ & N994 & $12 / 31 / 07$ to $06 / 30 / 08$ & ${ }^{60} \mathrm{Co}$ & $-4.8 \mathrm{E}-05 \pm 8.0 \mathrm{E}-05$ & $\mathrm{U}$ \\
\hline \multirow[t]{51}{*}{$(200-W)$} & $12 / 31 / 07$ & 01/14/08 & gross $\beta$ & $6.6 \mathrm{E}-03 \pm 1.2 \mathrm{E}-03$ & & & ${ }^{134} \mathrm{Cs}$ & $-6.0 \mathrm{E}-05 \pm 7.5 \mathrm{E}-05$ & $\mathrm{U}$ \\
\hline & $01 / 14 / 08$ & 01/29/08 & gross $\alpha$ & $1.6 \mathrm{E}-03 \pm 6.0 \mathrm{E}-04$ & & & ${ }^{137} \mathrm{Cs}$ & $4.6 \mathrm{E}-05 \pm 6.4 \mathrm{E}-05$ & $\mathrm{U}$ \\
\hline & 01/14/08 & 01/29/08 & gross $\beta$ & $3.2 \mathrm{E}-02 \pm 3.2 \mathrm{E}-03$ & & & ${ }^{152} \mathrm{Eu}$ & $-9.7 \mathrm{E}-06 \pm 9.7 \mathrm{E}-05$ & $\mathrm{U}$ \\
\hline & 01/29/08 & $02 / 12 / 08$ & gross $\alpha$ & $1.7 \mathrm{E}-04 \pm 3.6 \mathrm{E}-04$ & & & ${ }^{154} \mathrm{Eu}$ & $2.0 \mathrm{E}-05 \pm 2.0 \mathrm{E}-04$ & $\mathrm{U}$ \\
\hline & 01/29/08 & $02 / 12 / 08$ & gross $\beta$ & $2.9 \mathrm{E}-03 \pm 7.5 \mathrm{E}-04$ & & & ${ }^{155} \mathrm{Eu}$ & $5.5 \mathrm{E}-06 \pm 5.5 \mathrm{E}-05$ & U \\
\hline & 02/12/08 & $02 / 25 / 08$ & gross $\alpha$ & $9.5 \mathrm{E}-04 \pm 6.4 \mathrm{E}-04$ & & & ${ }^{238} \mathrm{Pu}$ & $-3.5 \mathrm{E}-06 \pm 8.4 \mathrm{E}-06$ & $\mathrm{U}$ \\
\hline & 02/12/08 & 02/25/08 & gross $\beta$ & $3.0 \mathrm{E}-02 \pm 3.2 \mathrm{E}-03$ & & & ${ }^{239 / 240} \mathrm{Pu}$ & $1.0 \mathrm{E}-05 \pm 7.0 \mathrm{E}-06$ & \\
\hline & 02/25/08 & 03/11/08 & gross $\alpha$ & $9.4 \mathrm{E}-04 \pm 5.8 \mathrm{E}-04$ & & & ${ }^{106} \mathrm{Ru}$ & $5.8 \mathrm{E}-05 \pm 5.4 \mathrm{E}-04$ & $\mathrm{U}$ \\
\hline & $02 / 25 / 08$ & 03/11/08 & gross $\beta$ & $1.2 \mathrm{E}-02 \pm 1.7 \mathrm{E}-03$ & & & ${ }^{125} \mathrm{Sb}$ & $-1.9 \mathrm{E}-05 \pm 1.5 \mathrm{E}-04$ & $\mathrm{U}$ \\
\hline & 03/11/08 & 03/25/08 & gross $\alpha$ & $5.1 \mathrm{E}-04 \pm 4.9 \mathrm{E}-04$ & & & ${ }^{90} \mathrm{Sr}$ & $-2.5 \mathrm{E}-04 \pm 2.6 \mathrm{E}-04$ & $\mathrm{U}$ \\
\hline & 03/11/08 & 03/25/08 & gross $\beta$ & $5.5 \mathrm{E}-03 \pm 1.1 \mathrm{E}-03$ & & & ${ }^{234} \mathrm{U}$ & $1.0 \mathrm{E}-05 \pm 7.1 \mathrm{E}-06$ & \\
\hline & 03/25/08 & 04/07/08 & gross $\alpha$ & $1.3 \mathrm{E}-03 \pm 5.9 \mathrm{E}-04$ & & & ${ }^{235} \mathrm{U}$ & $2.3 \mathrm{E}-06 \pm 3.6 \mathrm{E}-06$ & $\mathrm{U}$ \\
\hline & 03/25/08 & 04/07/08 & gross $\beta$ & $5.8 \mathrm{E}-03 \pm 1.1 \mathrm{E}-03$ & & & ${ }^{238} \mathrm{U}$ & $2.8 \mathrm{E}-06 \pm 4.2 \mathrm{E}-06$ & $\mathrm{U}$ \\
\hline & 04/07/08 & 04/21/08 & gross $\alpha$ & $5.3 \mathrm{E}-04 \pm 5.0 \mathrm{E}-04$ & & & & & \\
\hline & 04/07/08 & $04 / 21 / 08$ & gross $\beta$ & $8.4 \mathrm{E}-03 \pm 1.3 \mathrm{E}-03$ & N994 & $06 / 30 / 08$ to $12 / 29 / 08$ & ${ }^{60} \mathrm{Co}$ & $-3.8 \mathrm{E}-05 \pm 8.2 \mathrm{E}-05$ & $\mathrm{U}$ \\
\hline & $04 / 21 / 08$ & 05/05/08 & gross $\alpha$ & $1.6 \mathrm{E}-03 \pm 6.2 \mathrm{E}-04$ & & & ${ }^{134} \mathrm{Cs}$ & $-9.4 \mathrm{E}-06 \pm 7.1 \mathrm{E}-05$ & $\mathrm{U}$ \\
\hline & $04 / 21 / 08$ & 05/05/08 & gross $\beta$ & $1.2 \mathrm{E}-02 \pm 1.7 \mathrm{E}-03$ & & & ${ }^{137} \mathrm{Cs}$ & $4.9 \mathrm{E}-05 \pm 6.7 \mathrm{E}-05$ & $\mathrm{U}$ \\
\hline & 05/05/08 & 05/19/08 & gross $\alpha$ & $1.2 \mathrm{E}-03 \pm 5.6 \mathrm{E}-04$ & & & ${ }^{152} \mathrm{Eu}$ & $5.5 \mathrm{E}-05 \pm 1.6 \mathrm{E}-04$ & $\mathrm{U}$ \\
\hline & $05 / 05 / 08$ & 05/19/08 & gross $\beta$ & $1.1 \mathrm{E}-02 \pm 1.6 \mathrm{E}-03$ & & & ${ }^{154} \mathrm{Eu}$ & $-2.4 \mathrm{E}-04 \pm 2.5 \mathrm{E}-04$ & $\mathrm{U}$ \\
\hline & 05/19/08 & 06/02/08 & gross $\alpha$ & $8.4 \mathrm{E}-04 \pm 5.6 \mathrm{E}-04$ & & & ${ }^{155} \mathrm{Eu}$ & $4.1 \mathrm{E}-05 \pm 1.6 \mathrm{E}-04$ & $\mathrm{U}$ \\
\hline & 05/19/08 & $06 / 02 / 08$ & gross $\beta$ & $1.1 \mathrm{E}-02 \pm 1.6 \mathrm{E}-03$ & & & ${ }^{238} \mathrm{Pu}$ & $2.3 \mathrm{E}-06 \pm 8.9 \mathrm{E}-06$ & $\mathrm{U}$ \\
\hline & 06/02/08 & 06/16/08 & gross $\alpha$ & $1.2 \mathrm{E}-03 \pm 5.8 \mathrm{E}-04$ & & & ${ }^{239 / 240} \mathrm{Pu}$ & $-7.5 \mathrm{E}-07 \pm 2.6 \mathrm{E}-06$ & $\mathrm{U}$ \\
\hline & 06/02/08 & 06/16/08 & gross $\beta$ & $8.9 \mathrm{E}-03 \pm 1.4 \mathrm{E}-03$ & & & ${ }^{106} \mathrm{Ru}$ & $-7.7 \mathrm{E}-05 \pm 5.7 \mathrm{E}-04$ & $\mathrm{U}$ \\
\hline & 06/16/08 & 06/30/08 & gross $\alpha$ & $8.3 \mathrm{E}-04 \pm 6.4 \mathrm{E}-04$ & & & ${ }^{125} \mathrm{Sb}$ & $-2.6 \mathrm{E}-05 \pm 1.4 \mathrm{E}-04$ & $\mathrm{U}$ \\
\hline & 06/16/08 & 06/30/08 & gross $\beta$ & $1.3 \mathrm{E}-02 \pm 1.7 \mathrm{E}-03$ & & & ${ }^{90} \mathrm{Sr}$ & $-3.1 \mathrm{E}-04 \pm 3.2 \mathrm{E}-04$ & $\mathrm{U}$ \\
\hline & 06/30/08 & 07/14/08 & gross $\alpha$ & $1.7 \mathrm{E}-03 \pm 6.4 \mathrm{E}-04$ & & & ${ }^{234} \mathrm{U}$ & $1.8 \mathrm{E}-05 \pm 9.8 \mathrm{E}-06$ & \\
\hline & 06/30/08 & 07/14/08 & gross $\beta$ & $1.2 \mathrm{E}-02 \pm 1.6 \mathrm{E}-03$ & & & ${ }^{235} \mathrm{U}$ & $3.1 \mathrm{E}-06 \pm 4.5 \mathrm{E}-06$ & $\mathrm{U}$ \\
\hline & 07/14/08 & 07/28/08 & gross $\alpha$ & $8.5 \mathrm{E}-04 \pm 5.6 \mathrm{E}-04$ & & & ${ }^{238} \mathrm{U}$ & $8.4 \mathrm{E}-06 \pm 6.0 \mathrm{E}-06$ & \\
\hline & 07/14/08 & 07/28/08 & gross $\beta$ & $1.3 \mathrm{E}-02 \pm 1.7 \mathrm{E}-03$ & & & & & \\
\hline & 07/28/08 & 08/11/08 & gross $\alpha$ & $8.8 \mathrm{E}-04 \pm 5.8 \mathrm{E}-04$ & & & & & \\
\hline & 07/28/08 & 08/11/08 & gross $\beta$ & $1.3 \mathrm{E}-02 \pm 1.7 \mathrm{E}-03$ & & & & & \\
\hline & 08/11/08 & 08/25/08 & gross $\alpha$ & $8.5 \mathrm{E}-04 \pm 5.6 \mathrm{E}-04$ & & & & & \\
\hline & 08/11/08 & 08/25/08 & gross $\beta$ & $1.1 \mathrm{E}-02 \pm 1.5 \mathrm{E}-03$ & & & & & \\
\hline & 08/25/08 & 09/08/08 & gross $\alpha$ & $7.2 \mathrm{E}-04 \pm 5.2 \mathrm{E}-04$ & & & & & \\
\hline & 08/25/08 & 09/08/08 & gross $\beta$ & $8.7 \mathrm{E}-03 \pm 1.3 \mathrm{E}-03$ & & & & & \\
\hline & 09/08/08 & 09/22/08 & gross $\alpha$ & $9.5 \mathrm{E}-04 \pm 5.9 \mathrm{E}-04$ & & & & & \\
\hline & 09/08/08 & 09/22/08 & gross $\beta$ & $2.6 \mathrm{E}-02 \pm 2.8 \mathrm{E}-03$ & & & & & \\
\hline & 09/22/08 & $10 / 07 / 08$ & gross $\alpha$ & $2.7 \mathrm{E}-03 \pm 1.1 \mathrm{E}-03$ & & & & & \\
\hline & 09/22/08 & $10 / 07 / 08$ & gross $\beta$ & $3.6 \mathrm{E}-02 \pm 4.2 \mathrm{E}-03$ & & & & & \\
\hline & $10 / 14 / 08$ & $10 / 20 / 08$ & gross $\alpha$ & $1.4 \mathrm{E}-03 \pm 1.2 \mathrm{E}-03$ & & & & & \\
\hline & $10 / 14 / 08$ & $10 / 20 / 08$ & gross $\beta$ & $2.3 \mathrm{E}-02 \pm 3.4 \mathrm{E}-03$ & & & & & \\
\hline & $10 / 20 / 08$ & $11 / 03 / 08$ & gross $\alpha$ & $3.2 \mathrm{E}-03 \pm 8.7 \mathrm{E}-04$ & & & & & \\
\hline & $10 / 20 / 08$ & $11 / 03 / 08$ & gross $\beta$ & $3.9 \mathrm{E}-02 \pm 3.8 \mathrm{E}-03$ & & & & & \\
\hline & $11 / 03 / 08$ & $11 / 17 / 08$ & gross $\alpha$ & $3.6 \mathrm{E}-04 \pm 7.5 \mathrm{E}-04$ & & & & & \\
\hline & $11 / 03 / 08$ & $11 / 17 / 08$ & gross $\beta$ & $1.4 \mathrm{E}-02 \pm 2.5 \mathrm{E}-03$ & & & & & \\
\hline & $11 / 17 / 08$ & $12 / 01 / 08$ & gross $\alpha$ & $1.9 \mathrm{E}-03 \pm 6.7 \mathrm{E}-04$ & & & & & \\
\hline & $11 / 17 / 08$ & $12 / 01 / 08$ & gross $\beta$ & $3.4 \mathrm{E}-02 \pm 3.4 \mathrm{E}-03$ & & & & & \\
\hline & $12 / 01 / 08$ & $12 / 15 / 08$ & gross $\alpha$ & $2.0 \mathrm{E}-03 \pm 6.8 \mathrm{E}-04$ & & & & & \\
\hline & $12 / 01 / 08$ & $12 / 15 / 08$ & gross $\beta$ & $2.2 \mathrm{E}-02 \pm 2.5 \mathrm{E}-03$ & & & & & \\
\hline & $12 / 15 / 08$ & $12 / 29 / 08$ & gross $\alpha$ & $1.5 \mathrm{E}-03 \pm 5.9 \mathrm{E}-04$ & & & & & \\
\hline & $12 / 15 / 08$ & $12 / 29 / 08$ & gross $\beta$ & $3.6 \mathrm{E}-02 \pm 3.5 \mathrm{E}-03$ & & & & & \\
\hline
\end{tabular}

$\overline{\mathrm{RQ}}=$ Result Qualifier. $\mathrm{U}=$ The analyte was analyzed for but not detected. 
Table 2-4. Near-Facility Air Sampling Results, 2008 (pCi/m³ \pm total analytical uncertainty). (Sheet 72 of 82)

\begin{tabular}{|c|c|c|c|c|}
\hline Location & Sample On & Sample Off & Isotope & Result \pm Uncertainty \\
\hline N557 & $01 / 02 / 08$ & $01 / 15 / 08$ & gross $\alpha$ & $4.2 \mathrm{E}-04 \pm 4.7 \mathrm{E}-04$ \\
\hline \multirow[t]{51}{*}{ (300 Area) } & 01/02/08 & 01/15/08 & gross $\beta$ & $8.1 \mathrm{E}-03 \pm 1.4 \mathrm{E}-03$ \\
\hline & 01/15/08 & 01/30/08 & gross $\alpha$ & $1.5 \mathrm{E}-03 \pm 5.6 \mathrm{E}-04$ \\
\hline & $01 / 15 / 08$ & 01/30/08 & gross $\beta$ & $3.3 \mathrm{E}-02 \pm 3.7 \mathrm{E}-03$ \\
\hline & 01/30/08 & $02 / 13 / 08$ & gross $\alpha$ & $1.6 \mathrm{E}-04 \pm 3.4 \mathrm{E}-04$ \\
\hline & 01/30/08 & 02/13/08 & gross $\beta$ & $3.2 \mathrm{E}-03 \pm 8.0 \mathrm{E}-04$ \\
\hline & 02/13/08 & $02 / 27 / 08$ & gross $\alpha$ & $1.9 \mathrm{E}-03 \pm 6.9 \mathrm{E}-04$ \\
\hline & 02/13/08 & $02 / 27 / 08$ & gross $\beta$ & $3.6 \mathrm{E}-02 \pm 4.0 \mathrm{E}-03$ \\
\hline & $02 / 27 / 08$ & 03/12/08 & gross $\alpha$ & $7.3 \mathrm{E}-04 \pm 5.4 \mathrm{E}-04$ \\
\hline & $02 / 27 / 08$ & 03/12/08 & gross $\beta$ & $1.2 \mathrm{E}-02 \pm 1.8 \mathrm{E}-03$ \\
\hline & 03/12/08 & 03/26/08 & gross $\alpha$ & $7.5 \mathrm{E}-04 \pm 5.5 \mathrm{E}-04$ \\
\hline & 03/12/08 & 03/26/08 & gross $\beta$ & $1.0 \mathrm{E}-02 \pm 1.6 \mathrm{E}-03$ \\
\hline & 03/26/08 & 04/08/08 & gross $\alpha$ & $1.0 \mathrm{E}-03 \pm 6.3 \mathrm{E}-04$ \\
\hline & 03/26/08 & 04/08/08 & gross $\beta$ & $8.0 \mathrm{E}-03 \pm 1.4 \mathrm{E}-03$ \\
\hline & $04 / 08 / 08$ & $04 / 23 / 08$ & gross $\alpha$ & $1.2 \mathrm{E}-03 \pm 5.2 \mathrm{E}-04$ \\
\hline & 04/08/08 & $04 / 23 / 08$ & gross $\beta$ & $1.1 \mathrm{E}-02 \pm 1.6 \mathrm{E}-03$ \\
\hline & 04/23/08 & 05/08/08 & gross $\alpha$ & $1.1 \mathrm{E}-03 \pm 5.1 \mathrm{E}-04$ \\
\hline & $04 / 23 / 08$ & 05/08/08 & gross $\beta$ & $1.8 \mathrm{E}-02 \pm 2.3 \mathrm{E}-03$ \\
\hline & 05/08/08 & $05 / 20 / 08$ & gross $\alpha$ & $4.7 \mathrm{E}-04 \pm 5.5 \mathrm{E}-04$ \\
\hline & 05/08/08 & $05 / 20 / 08$ & gross $\beta$ & $1.3 \mathrm{E}-02 \pm 2.0 \mathrm{E}-03$ \\
\hline & 05/20/08 & 06/03/08 & gross $\alpha$ & $1.0 \mathrm{E}-03 \pm 4.8 \mathrm{E}-04$ \\
\hline & 05/20/08 & 06/03/08 & gross $\beta$ & $1.1 \mathrm{E}-02 \pm 1.7 \mathrm{E}-03$ \\
\hline & 06/03/08 & 06/23/08 & gross $\alpha$ & $1.3 \mathrm{E}-03 \pm 4.9 \mathrm{E}-04$ \\
\hline & 06/03/08 & 06/23/08 & gross $\beta$ & $9.5 \mathrm{E}-03 \pm 1.3 \mathrm{E}-03$ \\
\hline & $06 / 23 / 08$ & $07 / 02 / 08$ & gross $\alpha$ & $2.0 \mathrm{E}-03 \pm 9.6 \mathrm{E}-04$ \\
\hline & 06/23/08 & 07/02/08 & gross $\beta$ & $1.7 \mathrm{E}-02 \pm 2.6 \mathrm{E}-03$ \\
\hline & $07 / 02 / 08$ & 07/16/08 & gross $\alpha$ & $1.5 \mathrm{E}-03 \pm 6.8 \mathrm{E}-04$ \\
\hline & 07/02/08 & 07/16/08 & gross $\beta$ & $1.4 \mathrm{E}-02 \pm 2.0 \mathrm{E}-03$ \\
\hline & $07 / 16 / 08$ & 07/30/08 & gross $\alpha$ & $1.0 \mathrm{E}-03 \pm 4.9 \mathrm{E}-04$ \\
\hline & 07/16/08 & 07/30/08 & gross $\beta$ & $1.4 \mathrm{E}-02 \pm 2.0 \mathrm{E}-03$ \\
\hline & 07/30/08 & 08/13/08 & gross $\alpha$ & $1.2 \mathrm{E}-03 \pm 5.4 \mathrm{E}-04$ \\
\hline & 07/30/08 & 08/13/08 & gross $\beta$ & $1.4 \mathrm{E}-02 \pm 2.0 \mathrm{E}-03$ \\
\hline & 08/13/08 & $08 / 25 / 08$ & gross $\alpha$ & $1.9 \mathrm{E}-03 \pm 7.2 \mathrm{E}-04$ \\
\hline & 08/13/08 & $08 / 25 / 08$ & gross $\beta$ & $1.7 \mathrm{E}-02 \pm 2.5 \mathrm{E}-03$ \\
\hline & 08/25/08 & 09/09/08 & gross $\alpha$ & $7.8 \mathrm{E}-04 \pm 5.4 \mathrm{E}-04$ \\
\hline & $08 / 25 / 08$ & 09/09/08 & gross $\beta$ & $1.5 \mathrm{E}-02 \pm 2.1 \mathrm{E}-03$ \\
\hline & 09/09/08 & 09/23/08 & gross $\alpha$ & $1.1 \mathrm{E}-03 \pm 5.1 \mathrm{E}-04$ \\
\hline & 09/09/08 & 09/23/08 & gross $\beta$ & $3.0 \mathrm{E}-02 \pm 3.5 \mathrm{E}-03$ \\
\hline & $09 / 23 / 08$ & $10 / 08 / 08$ & gross $\alpha$ & $6.0 \mathrm{E}-04 \pm 4.8 \mathrm{E}-04$ \\
\hline & 09/23/08 & $10 / 08 / 08$ & gross $\beta$ & $2.0 \mathrm{E}-02 \pm 2.5 \mathrm{E}-03$ \\
\hline & $10 / 08 / 08$ & $10 / 21 / 08$ & gross $\alpha$ & $1.4 \mathrm{E}-03 \pm 6.1 \mathrm{E}-04$ \\
\hline & $10 / 08 / 08$ & $10 / 21 / 08$ & gross $\beta$ & $1.6 \mathrm{E}-02 \pm 2.2 \mathrm{E}-03$ \\
\hline & $10 / 21 / 08$ & $11 / 04 / 08$ & gross $\alpha$ & $2.8 \mathrm{E}-03 \pm 8.3 \mathrm{E}-04$ \\
\hline & $10 / 21 / 08$ & $11 / 04 / 08$ & gross $\beta$ & $4.4 \mathrm{E}-02 \pm 4.8 \mathrm{E}-03$ \\
\hline & $11 / 04 / 08$ & $11 / 18 / 08$ & gross $\alpha$ & $1.3 \mathrm{E}-03 \pm 6.1 \mathrm{E}-04$ \\
\hline & $11 / 04 / 08$ & $11 / 18 / 08$ & gross $\beta$ & $2.8 \mathrm{E}-02 \pm 3.4 \mathrm{E}-03$ \\
\hline & $11 / 18 / 08$ & $12 / 02 / 08$ & gross $\alpha$ & $2.3 \mathrm{E}-03 \pm 8.5 \mathrm{E}-04$ \\
\hline & $11 / 18 / 08$ & $12 / 02 / 08$ & gross $\beta$ & $4.1 \mathrm{E}-02 \pm 4.6 \mathrm{E}-03$ \\
\hline & $12 / 02 / 08$ & $12 / 16 / 08$ & gross $\alpha$ & $7.8 \mathrm{E}-04 \pm 5.6 \mathrm{E}-04$ \\
\hline & $12 / 02 / 08$ & $12 / 16 / 08$ & gross $\beta$ & $1.7 \mathrm{E}-02 \pm 2.2 \mathrm{E}-03$ \\
\hline & $12 / 16 / 08$ & $12 / 31 / 08$ & gross $\alpha$ & $2.3 \mathrm{E}-03 \pm 7.5 \mathrm{E}-04$ \\
\hline & $12 / 16 / 08$ & $12 / 31 / 08$ & gross $\beta$ & $4.4 \mathrm{E}-02 \pm 4.7 \mathrm{E}-03$ \\
\hline
\end{tabular}

\begin{tabular}{|c|c|c|c|c|}
\hline Location & Composite Period & Isotope & Result \pm Uncertainty & RQ* \\
\hline \multirow[t]{14}{*}{ N557 } & $01 / 02 / 08$ to $03 / 26 / 08$ & ${ }^{60} \mathrm{Co}$ & $-1.9 \mathrm{E}-05 \pm 1.3 \mathrm{E}-04$ & $\mathrm{U}$ \\
\hline & & ${ }^{134} \mathrm{Cs}$ & $4.3 \mathrm{E}-05 \pm 1.4 \mathrm{E}-04$ & $\mathrm{U}$ \\
\hline & & ${ }^{137} \mathrm{Cs}$ & $-5.2 \mathrm{E}-06 \pm 5.2 \mathrm{E}-05$ & $\mathrm{U}$ \\
\hline & & ${ }^{152} \mathrm{Eu}$ & $-7.7 \mathrm{E}-05 \pm 3.0 \mathrm{E}-04$ & $\mathrm{U}$ \\
\hline & & ${ }^{154} \mathrm{Eu}$ & $-2.0 \mathrm{E}-04 \pm 4.3 \mathrm{E}-04$ & $\mathrm{U}$ \\
\hline & & ${ }^{155} \mathrm{Eu}$ & $-1.8 \mathrm{E}-04 \pm 3.4 \mathrm{E}-04$ & $\mathrm{U}$ \\
\hline & & ${ }^{238} \mathrm{Pu}$ & $-2.6 \mathrm{E}-06 \pm 9.0 \mathrm{E}-06$ & $\mathrm{U}$ \\
\hline & & ${ }^{239 / 240} \mathrm{Pu}$ & $3.1 \mathrm{E}-05 \pm 2.0 \mathrm{E}-05$ & \\
\hline & & ${ }^{106} \mathrm{Ru}$ & $-1.0 \mathrm{E}-03 \pm 1.2 \mathrm{E}-03$ & $\mathrm{U}$ \\
\hline & & ${ }^{125} \mathrm{Sb}$ & $1.1 \mathrm{E}-04 \pm 2.9 \mathrm{E}-04$ & $\mathrm{U}$ \\
\hline & & ${ }^{90} \mathrm{Sr}$ & $8.4 \mathrm{E}-05 \pm 3.0 \mathrm{E}-04$ & $\mathrm{U}$ \\
\hline & & ${ }^{234} \mathrm{U}$ & $1.7 \mathrm{E}-05 \pm 1.3 \mathrm{E}-05$ & \\
\hline & & ${ }^{235} \mathrm{U}$ & $1.8 \mathrm{E}-06 \pm 3.7 \mathrm{E}-06$ & $\mathrm{U}$ \\
\hline & & ${ }^{238} \mathrm{U}$ & $6.6 \mathrm{E}-06 \pm 8.4 \mathrm{E}-06$ & $\mathrm{U}$ \\
\hline \multirow[t]{14}{*}{ N557 } & $03 / 26 / 08$ to $07 / 02 / 08$ & ${ }^{60} \mathrm{Co}$ & $8.3 \mathrm{E}-05 \pm 1.5 \mathrm{E}-04$ & $\mathrm{U}$ \\
\hline & & ${ }^{134} \mathrm{Cs}$ & $-1.4 \mathrm{E}-05 \pm 1.3 \mathrm{E}-04$ & $\mathrm{U}$ \\
\hline & & ${ }^{137} \mathrm{Cs}$ & $-1.1 \mathrm{E}-05 \pm 1.1 \mathrm{E}-04$ & $\mathrm{U}$ \\
\hline & & ${ }^{152} \mathrm{Eu}$ & $8.7 \mathrm{E}-05 \pm 2.9 \mathrm{E}-04$ & $\mathrm{U}$ \\
\hline & & ${ }^{154} \mathrm{Eu}$ & $3.0 \mathrm{E}-04 \pm 3.5 \mathrm{E}-04$ & $\mathrm{U}$ \\
\hline & & ${ }^{155} \mathrm{Eu}$ & $-9.0 \mathrm{E}-05 \pm 3.2 \mathrm{E}-04$ & $\mathrm{U}$ \\
\hline & & ${ }^{238} \mathrm{Pu}$ & $1.8 \mathrm{E}-05 \pm 1.1 \mathrm{E}-05$ & \\
\hline & & ${ }^{239 / 240} \mathrm{Pu}$ & $1.2 \mathrm{E}-06 \pm 2.4 \mathrm{E}-06$ & $\mathrm{U}$ \\
\hline & & ${ }^{106} \mathrm{Ru}$ & $-1.1 \mathrm{E}-04 \pm 1.0 \mathrm{E}-03$ & $\mathrm{U}$ \\
\hline & & ${ }^{125} \mathrm{Sb}$ & $1.9 \mathrm{E}-04 \pm 3.0 \mathrm{E}-04$ & $\mathrm{U}$ \\
\hline & & ${ }^{90} \mathrm{Sr}$ & $-1.2 \mathrm{E}-04 \pm 1.3 \mathrm{E}-04$ & $\mathrm{U}$ \\
\hline & & ${ }^{234} \mathrm{U}$ & $1.4 \mathrm{E}-05 \pm 8.9 \mathrm{E}-06$ & \\
\hline & & ${ }^{235} \mathrm{U}$ & $1.2 \mathrm{E}-06 \pm 2.5 \mathrm{E}-06$ & \\
\hline & & ${ }^{238} \mathrm{U}$ & $1.3 \mathrm{E}-05 \pm 8.5 \mathrm{E}-06$ & \\
\hline \multirow[t]{14}{*}{ N557 } & $07 / 02 / 08$ to $09 / 23 / 08$ & ${ }^{60} \mathrm{Co}$ & $-3.5 \mathrm{E}-05 \pm 2.0 \mathrm{E}-04$ & $\mathrm{U}$ \\
\hline & & ${ }^{134} \mathrm{Cs}$ & $6.8 \mathrm{E}-05 \pm 1.7 \mathrm{E}-04$ & $\mathrm{U}$ \\
\hline & & ${ }^{137} \mathrm{Cs}$ & $-1.5 \mathrm{E}-05 \pm 1.5 \mathrm{E}-04$ & $\mathrm{U}$ \\
\hline & & ${ }^{152} \mathrm{Eu}$ & $3.3 \mathrm{E}-04 \pm 3.9 \mathrm{E}-04$ & $\mathrm{U}$ \\
\hline & & ${ }^{154} \mathrm{Eu}$ & $7.7 \mathrm{E}-05 \pm 4.9 \mathrm{E}-04$ & $\mathrm{U}$ \\
\hline & & ${ }^{155} \mathrm{Eu}$ & $2.6 \mathrm{E}-04 \pm 3.9 \mathrm{E}-04$ & $\mathrm{U}$ \\
\hline & & ${ }^{238} \mathrm{Pu}$ & $-1.1 \mathrm{E}-05 \pm 2.1 \mathrm{E}-05$ & \\
\hline & & ${ }^{239 / 240} \mathrm{Pu}$ & $3.6 \mathrm{E}-06 \pm 7.3 \mathrm{E}-06$ & \\
\hline & & ${ }^{106} \mathrm{Ru}$ & $-5.1 \mathrm{E}-04 \pm 1.4 \mathrm{E}-03$ & $\mathrm{U}$ \\
\hline & & ${ }^{125} \mathrm{Sb}$ & $-2.1 \mathrm{E}-04 \pm 4.0 \mathrm{E}-04$ & $\mathrm{U}$ \\
\hline & & ${ }^{90} \mathrm{Sr}$ & $-1.1 \mathrm{E}-04 \pm 1.1 \mathrm{E}-04$ & $\mathrm{U}$ \\
\hline & & ${ }^{234} \mathrm{U}$ & $3.0 \mathrm{E}-05 \pm 1.7 \mathrm{E}-05$ & \\
\hline & & ${ }^{235} \mathrm{U}$ & $-1.6 \mathrm{E}-06 \pm 3.3 \mathrm{E}-06$ & $\mathrm{U}$ \\
\hline & & ${ }^{238} \mathrm{U}$ & $1.8 \mathrm{E}-05 \pm 1.3 \mathrm{E}-05$ & \\
\hline \multirow[t]{14}{*}{ N557 } & $09 / 23 / 08$ to $12 / 31 / 08$ & ${ }^{60} \mathrm{Co}$ & $-7.3 \mathrm{E}-05 \pm 1.8 \mathrm{E}-04$ & $\mathrm{U}$ \\
\hline & & ${ }^{134} \mathrm{Cs}$ & $1.4 \mathrm{E}-05 \pm 1.4 \mathrm{E}-04$ & $\mathrm{U}$ \\
\hline & & ${ }^{137} \mathrm{Cs}$ & $-4.0 \mathrm{E}-05 \pm 1.3 \mathrm{E}-04$ & $\mathrm{U}$ \\
\hline & & ${ }^{152} \mathrm{Eu}$ & $4.3 \mathrm{E}-05 \pm 3.3 \mathrm{E}-04$ & $\mathrm{U}$ \\
\hline & & ${ }^{154} \mathrm{Eu}$ & $-3.0 \mathrm{E}-04 \pm 4.8 \mathrm{E}-04$ & $\mathrm{U}$ \\
\hline & & ${ }^{155} \mathrm{Eu}$ & $-3.9 \mathrm{E}-04 \pm 4.0 \mathrm{E}-04$ & $\mathrm{U}$ \\
\hline & & ${ }^{238} \mathrm{Pu}$ & $-1.2 \mathrm{E}-05 \pm 2.0 \mathrm{E}-05$ & $\mathrm{U}$ \\
\hline & & ${ }^{239 / 240} \mathrm{Pu}$ & $1.4 \mathrm{E}-06 \pm 4.8 \mathrm{E}-06$ & $\mathrm{U}$ \\
\hline & & ${ }^{106} \mathrm{Ru}$ & $3.5 \mathrm{E}-04 \pm 1.2 \mathrm{E}-03$ & $\mathrm{U}$ \\
\hline & & ${ }^{125} \mathrm{Sb}$ & $-7.3 \mathrm{E}-05 \pm 3.1 \mathrm{E}-04$ & $\mathrm{U}$ \\
\hline & & ${ }^{90} \mathrm{Sr}$ & $-2.0 \mathrm{E}-04 \pm 2.0 \mathrm{E}-04$ & $\mathrm{U}$ \\
\hline & & ${ }^{234} \mathrm{U}$ & $2.2 \mathrm{E}-05 \pm 1.4 \mathrm{E}-05$ & \\
\hline & & ${ }^{235} \mathrm{U}$ & $2.9 \mathrm{E}-06 \pm 4.3 \mathrm{E}-06$ & $\mathrm{U}$ \\
\hline & & ${ }^{238} \mathrm{U}$ & $1.1 \mathrm{E}-05 \pm 9.3 \mathrm{E}-06$ & \\
\hline
\end{tabular}

$\overline{\mathrm{RQ}}=$ Result Qualifier. $\mathrm{U}=$ The analyte was analyzed for but not detected. 
Table 2-4. Near-Facility Air Sampling Results, 2008 (pCi/m³ \pm total analytical uncertainty). (Sheet 73 of 82)

\begin{tabular}{|c|c|c|c|c|c|c|c|c|c|}
\hline Location & Sample On & Sample Off & Isotope & Result \pm Uncertainty & Location & Composite Period & Isotope & Result \pm Uncertainty & RQ* \\
\hline N527 & $01 / 02 / 08$ & $01 / 15 / 08$ & gross $\alpha$ & $5.1 \mathrm{E}-04 \pm 4.9 \mathrm{E}-04$ & N527 & $01 / 02 / 08$ to $07 / 01 / 08$ & ${ }^{60} \mathrm{Co}$ & $-8.1 \mathrm{E}-05 \pm 1.1 \mathrm{E}-04$ & $\bar{U}$ \\
\hline \multirow[t]{51}{*}{ (300 Area) } & $01 / 02 / 08$ & $01 / 15 / 08$ & gross $\beta$ & $1.1 \mathrm{E}-02 \pm 1.6 \mathrm{E}-03$ & & & ${ }^{134} \mathrm{Cs}$ & $-3.0 \mathrm{E}-05 \pm 7.3 \mathrm{E}-05$ & $\mathrm{U}$ \\
\hline & $01 / 15 / 08$ & 01/30/08 & gross $\alpha$ & $2.2 \mathrm{E}-03 \pm 6.8 \mathrm{E}-04$ & & & ${ }^{137} \mathrm{Cs}$ & $-8.8 \mathrm{E}-06 \pm 6.1 \mathrm{E}-05$ & U \\
\hline & $01 / 15 / 08$ & 01/30/08 & gross $\beta$ & $3.4 \mathrm{E}-02 \pm 3.3 \mathrm{E}-03$ & & & ${ }^{152} \mathrm{Eu}$ & $-6.4 \mathrm{E}-06 \pm 6.4 \mathrm{E}-05$ & U \\
\hline & 01/30/08 & 02/13/08 & gross $\alpha$ & $3.8 \mathrm{E}-04 \pm 4.3 \mathrm{E}-04$ & & & ${ }^{154} \mathrm{Eu}$ & $2.2 \mathrm{E}-04 \pm 2.6 \mathrm{E}-04$ & $\mathrm{U}$ \\
\hline & 01/30/08 & 02/13/08 & gross $\beta$ & $3.5 \mathrm{E}-03 \pm 8.0 \mathrm{E}-04$ & & & ${ }^{155} \mathrm{Eu}$ & $-2.3 \mathrm{E}-05 \pm 1.5 \mathrm{E}-04$ & U \\
\hline & $02 / 13 / 08$ & $02 / 27 / 08$ & gross $\alpha$ & $1.4 \mathrm{E}-03 \pm 5.7 \mathrm{E}-04$ & & & ${ }^{238} \mathrm{Pu}$ & $6.2 \mathrm{E}-07 \pm 6.2 \mathrm{E}-06$ & $\mathrm{U}$ \\
\hline & $02 / 13 / 08$ & $02 / 27 / 08$ & gross $\beta$ & $3.3 \mathrm{E}-02 \pm 3.3 \mathrm{E}-03$ & & & ${ }^{239 / 240} \mathrm{Pu}$ & $-1.2 \mathrm{E}-06 \pm 1.8 \mathrm{E}-06$ & $\mathrm{U}$ \\
\hline & $02 / 27 / 08$ & 03/12/08 & gross $\alpha$ & $8.2 \mathrm{E}-04 \pm 5.5 \mathrm{E}-04$ & & & ${ }^{106} \mathrm{Ru}$ & $-5.6 \mathrm{E}-04 \pm 6.8 \mathrm{E}-04$ & $\mathrm{U}$ \\
\hline & $02 / 27 / 08$ & $03 / 12 / 08$ & gross $\beta$ & $9.8 \mathrm{E}-03 \pm 1.5 \mathrm{E}-03$ & & & ${ }^{125} \mathrm{Sb}$ & $-6.7 \mathrm{E}-05 \pm 1.6 \mathrm{E}-04$ & $\mathrm{U}$ \\
\hline & 03/12/08 & 03/26/08 & gross $\alpha$ & $4.9 \mathrm{E}-04 \pm 4.6 \mathrm{E}-04$ & & & ${ }^{234} \mathrm{U}$ & $1.3 \mathrm{E}-05 \pm 7.9 \mathrm{E}-06$ & \\
\hline & 03/12/08 & 03/26/08 & gross $\beta$ & $8.8 \mathrm{E}-03 \pm 1.4 \mathrm{E}-03$ & & & ${ }^{235} \mathrm{U}$ & $3.6 \mathrm{E}-06 \pm 3.5 \mathrm{E}-06$ & \\
\hline & 03/26/08 & 04/08/08 & gross $\alpha$ & $7.6 \mathrm{E}-04 \pm 5.6 \mathrm{E}-04$ & & & ${ }^{238} \mathrm{U}$ & $1.2 \mathrm{E}-05 \pm 7.5 \mathrm{E}-06$ & \\
\hline & $03 / 26 / 08$ & $04 / 08 / 08$ & gross $\beta$ & $8.4 \mathrm{E}-03 \pm 1.4 \mathrm{E}-03$ & & & & & \\
\hline & $04 / 08 / 08$ & $04 / 23 / 08$ & gross $\alpha$ & $6.6 \mathrm{E}-04 \pm 4.9 \mathrm{E}-04$ & N527 & $07 / 01 / 08$ to $12 / 31 / 08$ & ${ }^{60} \mathrm{Co}$ & $-7.2 \mathrm{E}-05 \pm 7.9 \mathrm{E}-05$ & $\mathrm{U}$ \\
\hline & $04 / 08 / 08$ & $04 / 23 / 08$ & gross $\beta$ & $1.1 \mathrm{E}-02 \pm 1.5 \mathrm{E}-03$ & & & ${ }^{134} \mathrm{Cs}$ & $-2.8 \mathrm{E}-05 \pm 7.0 \mathrm{E}-05$ & $\mathrm{U}$ \\
\hline & $04 / 23 / 08$ & $05 / 08 / 08$ & gross $\alpha$ & $1.3 \mathrm{E}-03 \pm 5.2 \mathrm{E}-04$ & & & ${ }^{137} \mathrm{Cs}$ & $7.6 \mathrm{E}-06 \pm 6.0 \mathrm{E}-05$ & $\mathrm{U}$ \\
\hline & $04 / 23 / 08$ & 05/08/08 & gross $\beta$ & $1.4 \mathrm{E}-02 \pm 1.8 \mathrm{E}-03$ & & & ${ }^{152} \mathrm{Eu}$ & $1.8 \mathrm{E}-05 \pm 1.5 \mathrm{E}-04$ & $\mathrm{U}$ \\
\hline & 05/08/08 & 05/20/08 & gross $\alpha$ & $4.6 \mathrm{E}-04 \pm 5.0 \mathrm{E}-04$ & & & ${ }^{154} \mathrm{Eu}$ & $-6.6 \mathrm{E}-05 \pm 2.5 \mathrm{E}-04$ & U \\
\hline & 05/08/08 & $05 / 20 / 08$ & gross $\beta$ & $1.3 \mathrm{E}-02 \pm 1.8 \mathrm{E}-03$ & & & ${ }^{155} \mathrm{Eu}$ & $9.3 \mathrm{E}-05 \pm 1.7 \mathrm{E}-04$ & $\mathrm{U}$ \\
\hline & $05 / 20 / 08$ & $06 / 03 / 08$ & gross $\alpha$ & $1.1 \mathrm{E}-03 \pm 4.9 \mathrm{E}-04$ & & & ${ }^{238} \mathrm{Pu}$ & $4.4 \mathrm{E}-06 \pm 1.1 \mathrm{E}-05$ & U \\
\hline & $05 / 20 / 08$ & $06 / 03 / 08$ & gross $\beta$ & $9.6 \mathrm{E}-03 \pm 1.4 \mathrm{E}-03$ & & & ${ }^{239 / 240} \mathrm{Pu}$ & $1.5 \mathrm{E}-06 \pm 3.0 \mathrm{E}-06$ & $\mathrm{U}$ \\
\hline & $06 / 03 / 08$ & $06 / 17 / 08$ & gross $\alpha$ & $6.2 \mathrm{E}-04 \pm 5.0 \mathrm{E}-04$ & & & ${ }^{106} \mathrm{Ru}$ & $-2.5 \mathrm{E}-04 \pm 5.4 \mathrm{E}-04$ & $\mathrm{U}$ \\
\hline & 06/03/08 & $06 / 17 / 08$ & gross $\beta$ & $6.6 \mathrm{E}-03 \pm 1.1 \mathrm{E}-03$ & & & ${ }^{125} \mathrm{Sb}$ & $-6.0 \mathrm{E}-05 \pm 1.4 \mathrm{E}-04$ & $\mathrm{U}$ \\
\hline & $06 / 17 / 08$ & 07/01/08 & gross $\alpha$ & $6.3 \mathrm{E}-04 \pm 5.2 \mathrm{E}-04$ & & & ${ }^{234} \mathrm{U}$ & $2.1 \mathrm{E}-05 \pm 1.2 \mathrm{E}-05$ & \\
\hline & $06 / 17 / 08$ & 07/01/08 & gross $\beta$ & $1.2 \mathrm{E}-02 \pm 1.7 \mathrm{E}-03$ & & & ${ }^{235} \mathrm{U}$ & $7.6 \mathrm{E}-07 \pm 7.7 \mathrm{E}-06$ & $\mathrm{U}$ \\
\hline & $07 / 01 / 08$ & $07 / 15 / 08$ & gross $\alpha$ & $9.5 \mathrm{E}-04 \pm 5.8 \mathrm{E}-04$ & & & ${ }^{238} \mathrm{U}$ & $2.3 \mathrm{E}-05 \pm 1.2 \mathrm{E}-05$ & \\
\hline & 07/01/08 & $07 / 15 / 08$ & gross $\beta$ & $1.2 \mathrm{E}-02 \pm 1.7 \mathrm{E}-03$ & & & & & \\
\hline & 07/15/08 & 07/29/08 & gross $\alpha$ & $6.1 \mathrm{E}-04 \pm 4.9 \mathrm{E}-04$ & & & & & \\
\hline & $07 / 15 / 08$ & 07/29/08 & gross $\beta$ & $1.2 \mathrm{E}-02 \pm 1.6 \mathrm{E}-03$ & & & & & \\
\hline & 07/29/08 & 08/12/08 & gross $\alpha$ & 7.4E-04 $\pm 5.3 \mathrm{E}-04$ & & & & & \\
\hline & 07/29/08 & $08 / 12 / 08$ & gross $\beta$ & $1.4 \mathrm{E}-02 \pm 1.8 \mathrm{E}-03$ & & & & & \\
\hline & $08 / 12 / 08$ & $08 / 25 / 08$ & gross $\alpha$ & $9.3 \mathrm{E}-04 \pm 6.1 \mathrm{E}-04$ & & & & & \\
\hline & 08/12/08 & 08/25/08 & gross $\beta$ & $1.4 \mathrm{E}-02 \pm 1.9 \mathrm{E}-03$ & & & & & \\
\hline & $08 / 25 / 08$ & 09/09/08 & gross $\alpha$ & $9.8 \mathrm{E}-04 \pm 4.5 \mathrm{E}-04$ & & & & & \\
\hline & $08 / 25 / 08$ & 09/09/08 & gross $\beta$ & $1.1 \mathrm{E}-02 \pm 1.5 \mathrm{E}-03$ & & & & & \\
\hline & 09/09/08 & 09/23/08 & gross $\alpha$ & $1.0 \mathrm{E}-03 \pm 6.4 \mathrm{E}-04$ & & & & & \\
\hline & 09/09/08 & 09/23/08 & gross $\beta$ & $2.0 \mathrm{E}-02 \pm 2.3 \mathrm{E}-03$ & & & & & \\
\hline & $09 / 23 / 08$ & $10 / 08 / 08$ & gross $\alpha$ & $1.1 \mathrm{E}-03 \pm 4.7 \mathrm{E}-04$ & & & & & \\
\hline & 09/23/08 & $10 / 08 / 08$ & gross $\beta$ & $2.2 \mathrm{E}-02 \pm 2.4 \mathrm{E}-03$ & & & & & \\
\hline & $10 / 08 / 08$ & $10 / 21 / 08$ & gross $\alpha$ & $1.6 \mathrm{E}-03 \pm 6.2 \mathrm{E}-04$ & & & & & \\
\hline & $10 / 08 / 08$ & $10 / 21 / 08$ & gross $\beta$ & $1.8 \mathrm{E}-02 \pm 2.2 \mathrm{E}-03$ & & & & & \\
\hline & $10 / 21 / 08$ & $11 / 04 / 08$ & gross $\alpha$ & $2.7 \mathrm{E}-03 \pm 7.8 \mathrm{E}-04$ & & & & & \\
\hline & $10 / 21 / 08$ & $11 / 04 / 08$ & gross $\beta$ & $4.1 \mathrm{E}-02 \pm 3.9 \mathrm{E}-03$ & & & & & \\
\hline & $11 / 04 / 08$ & $11 / 18 / 08$ & gross $\alpha$ & $6.3 \mathrm{E}-04 \pm 5.0 \mathrm{E}-04$ & & & & & \\
\hline & $11 / 04 / 08$ & $11 / 18 / 08$ & gross $\beta$ & $2.1 \mathrm{E}-02 \pm 2.3 \mathrm{E}-03$ & & & & & \\
\hline & $11 / 18 / 08$ & $12 / 02 / 08$ & gross $\alpha$ & $1.2 \mathrm{E}-03 \pm 5.2 \mathrm{E}-04$ & & & & & \\
\hline & $11 / 18 / 08$ & $12 / 02 / 08$ & gross $\beta$ & $4.4 \mathrm{E}-02 \pm 4.2 \mathrm{E}-03$ & & & & & \\
\hline & $12 / 02 / 08$ & $12 / 16 / 08$ & gross $\alpha$ & $1.7 \mathrm{E}-03 \pm 6.4 \mathrm{E}-04$ & & & & & \\
\hline & $12 / 02 / 08$ & $12 / 16 / 08$ & gross $\beta$ & $2.2 \mathrm{E}-02 \pm 2.5 \mathrm{E}-03$ & & & & & \\
\hline & $12 / 16 / 08$ & $12 / 31 / 08$ & gross $\alpha$ & $2.3 \mathrm{E}-03 \pm 7.0 \mathrm{E}-04$ & & & & & \\
\hline & $12 / 16 / 08$ & $12 / 31 / 08$ & gross $\beta$ & $3.8 \mathrm{E}-02 \pm 3.6 \mathrm{E}-03$ & & & & & \\
\hline
\end{tabular}

$\overline{\mathrm{RQ}}=$ Result Qualifier. $\mathrm{U}=$ The analyte was analyzed for but not detected. 
Table 2-4. Near-Facility Air Sampling Results, 2008 (pCi/m³ \pm total analytical uncertainty). (Sheet 74 of 82)

\begin{tabular}{|c|c|c|c|c|c|c|c|c|c|}
\hline Location & Sample On & Sample Off & Isotope & Result \pm Uncertainty & Location & Composite Period & Isotope & Result \pm Uncertainty & RQ* \\
\hline N537 & $01 / 02 / 08$ & $01 / 15 / 08$ & gross $\alpha$ & $1.0 \mathrm{E}-03 \pm 6.2 \mathrm{E}-04$ & N537 & $01 / 02 / 08$ to $07 / 01 / 08$ & ${ }^{60} \mathrm{Co}$ & $3.1 \mathrm{E}-05 \pm 9.4 \mathrm{E}-05$ & $\bar{U}$ \\
\hline \multirow[t]{51}{*}{ (300 Area) } & $01 / 02 / 08$ & $01 / 15 / 08$ & gross $\beta$ & $9.6 \mathrm{E}-03 \pm 1.6 \mathrm{E}-03$ & & & ${ }^{134} \mathrm{Cs}$ & $-3.2 \mathrm{E}-05 \pm 1.1 \mathrm{E}-04$ & $\mathrm{U}$ \\
\hline & $01 / 15 / 08$ & 01/30/08 & gross $\alpha$ & $1.6 \mathrm{E}-03 \pm 6.0 \mathrm{E}-04$ & & & ${ }^{137} \mathrm{Cs}$ & $-4.4 \mathrm{E}-05 \pm 1.0 \mathrm{E}-04$ & U \\
\hline & $01 / 15 / 08$ & 01/30/08 & gross $\beta$ & $3.8 \mathrm{E}-02 \pm 4.1 \mathrm{E}-03$ & & & ${ }^{152} \mathrm{Eu}$ & $4.5 \mathrm{E}-05 \pm 2.5 \mathrm{E}-04$ & U \\
\hline & 01/30/08 & 02/13/08 & gross $\alpha$ & $1.7 \mathrm{E}-04 \pm 3.6 \mathrm{E}-04$ & & & ${ }^{154} \mathrm{Eu}$ & $9.9 \mathrm{E}-06 \pm 9.9 \mathrm{E}-05$ & $\mathrm{U}$ \\
\hline & 01/30/08 & 02/13/08 & gross $\beta$ & $2.6 \mathrm{E}-03 \pm 7.2 \mathrm{E}-04$ & & & ${ }^{155} \mathrm{Eu}$ & $2.3 \mathrm{E}-04 \pm 2.7 \mathrm{E}-04$ & U \\
\hline & $02 / 13 / 08$ & $02 / 27 / 08$ & gross $\alpha$ & $1.5 \mathrm{E}-03 \pm 6.0 \mathrm{E}-04$ & & & ${ }^{238} \mathrm{Pu}$ & $4.5 \mathrm{E}-06 \pm 1.4 \mathrm{E}-05$ & $\mathrm{U}$ \\
\hline & $02 / 13 / 08$ & $02 / 27 / 08$ & gross $\beta$ & $3.2 \mathrm{E}-02 \pm 3.6 \mathrm{E}-03$ & & & ${ }^{239 / 240} \mathrm{Pu}$ & $7.5 \mathrm{E}-07 \pm 1.5 \mathrm{E}-06$ & $\mathrm{U}$ \\
\hline & $02 / 27 / 08$ & 03/12/08 & gross $\alpha$ & $1.4 \mathrm{E}-03 \pm 5.8 \mathrm{E}-04$ & & & ${ }^{106} \mathrm{Ru}$ & $-4.3 \mathrm{E}-04 \pm 9.9 \mathrm{E}-04$ & $\mathrm{U}$ \\
\hline & $02 / 27 / 08$ & 03/12/08 & gross $\beta$ & $1.3 \mathrm{E}-02 \pm 1.8 \mathrm{E}-03$ & & & ${ }^{125} \mathrm{Sb}$ & $2.3 \mathrm{E}-04 \pm 2.5 \mathrm{E}-04$ & $\mathrm{U}$ \\
\hline & 03/12/08 & 03/26/08 & gross $\alpha$ & $1.8 \mathrm{E}-03 \pm 6.5 \mathrm{E}-04$ & & & ${ }^{234} \mathrm{U}$ & $1.1 \mathrm{E}-05 \pm 7.5 \mathrm{E}-06$ & \\
\hline & 03/12/08 & 03/26/08 & gross $\beta$ & $8.9 \mathrm{E}-03 \pm 1.4 \mathrm{E}-03$ & & & ${ }^{235} \mathrm{U}$ & $6.8 \mathrm{E}-07 \pm 7.0 \mathrm{E}-07$ & $\mathrm{U}$ \\
\hline & 03/26/08 & 04/08/08 & gross $\alpha$ & $4.3 \mathrm{E}-04 \pm 4.8 \mathrm{E}-04$ & & & ${ }^{238} \mathrm{U}$ & $8.8 \mathrm{E}-06 \pm 6.8 \mathrm{E}-06$ & \\
\hline & $03 / 26 / 08$ & $04 / 08 / 08$ & gross $\beta$ & $6.8 \mathrm{E}-03 \pm 1.3 \mathrm{E}-03$ & & & & & \\
\hline & $04 / 08 / 08$ & $04 / 23 / 08$ & gross $\alpha$ & $6.9 \mathrm{E}-04 \pm 5.1 \mathrm{E}-04$ & N537 & $07 / 01 / 08$ to $12 / 31 / 08$ & ${ }^{60} \mathrm{Co}$ & $-1.0 \mathrm{E}-05 \pm 7.1 \mathrm{E}-05$ & $\mathrm{U}$ \\
\hline & $04 / 08 / 08$ & $04 / 23 / 08$ & gross $\beta$ & $1.0 \mathrm{E}-02 \pm 1.6 \mathrm{E}-03$ & & & ${ }^{134} \mathrm{Cs}$ & $1.2 \mathrm{E}-05 \pm 6.9 \mathrm{E}-05$ & $\mathrm{U}$ \\
\hline & $04 / 23 / 08$ & $05 / 08 / 08$ & gross $\alpha$ & $1.5 \mathrm{E}-03 \pm 6.5 \mathrm{E}-04$ & & & ${ }^{137} \mathrm{Cs}$ & $-5.7 \mathrm{E}-07 \pm 5.7 \mathrm{E}-06$ & $\mathrm{U}$ \\
\hline & $04 / 23 / 08$ & 05/08/08 & gross $\beta$ & $1.5 \mathrm{E}-02 \pm 2.2 \mathrm{E}-03$ & & & ${ }^{152} \mathrm{Eu}$ & $-4.0 \mathrm{E}-05 \pm 1.6 \mathrm{E}-04$ & $\mathrm{U}$ \\
\hline & 05/08/08 & 05/20/08 & gross $\alpha$ & $1.3 \mathrm{E}-03 \pm 5.9 \mathrm{E}-04$ & & & ${ }^{154} \mathrm{Eu}$ & $-1.6 \mathrm{E}-04 \pm 2.2 \mathrm{E}-04$ & U \\
\hline & 05/08/08 & $05 / 20 / 08$ & gross $\beta$ & $1.1 \mathrm{E}-02 \pm 1.8 \mathrm{E}-03$ & & & ${ }^{155} \mathrm{Eu}$ & $6.1 \mathrm{E}-05 \pm 1.5 \mathrm{E}-04$ & $\mathrm{U}$ \\
\hline & $05 / 20 / 08$ & $06 / 03 / 08$ & gross $\alpha$ & $9.4 \mathrm{E}-04 \pm 5.8 \mathrm{E}-04$ & & & ${ }^{238} \mathrm{Pu}$ & $-3.9 \mathrm{E}-06 \pm 1.3 \mathrm{E}-05$ & $\mathrm{U}$ \\
\hline & $05 / 20 / 08$ & $06 / 03 / 08$ & gross $\beta$ & $9.5 \mathrm{E}-03 \pm 1.5 \mathrm{E}-03$ & & & ${ }^{239 / 240} \mathrm{Pu}$ & $3.9 \mathrm{E}-06 \pm 4.4 \mathrm{E}-06$ & $\mathrm{U}$ \\
\hline & $06 / 03 / 08$ & $06 / 17 / 08$ & gross $\alpha$ & $9.5 \mathrm{E}-04 \pm 5.9 \mathrm{E}-04$ & & & ${ }^{106} \mathrm{Ru}$ & $1.6 \mathrm{E}-04 \pm 5.4 \mathrm{E}-04$ & $\mathrm{U}$ \\
\hline & 06/03/08 & $06 / 17 / 08$ & gross $\beta$ & $7.9 \mathrm{E}-03 \pm 1.3 \mathrm{E}-03$ & & & ${ }^{125} \mathrm{Sb}$ & $1.3 \mathrm{E}-05 \pm 1.3 \mathrm{E}-04$ & $\mathrm{U}$ \\
\hline & $06 / 17 / 08$ & 07/01/08 & gross $\alpha$ & $1.5 \mathrm{E}-03 \pm 6.1 \mathrm{E}-04$ & & & ${ }^{234} \mathrm{U}$ & $2.8 \mathrm{E}-05 \pm 1.5 \mathrm{E}-05$ & \\
\hline & $06 / 17 / 08$ & 07/01/08 & gross $\beta$ & $1.4 \mathrm{E}-02 \pm 2.0 \mathrm{E}-03$ & & & ${ }^{235} \mathrm{U}$ & $7.1 \mathrm{E}-06 \pm 6.0 \mathrm{E}-06$ & \\
\hline & $07 / 01 / 08$ & $07 / 15 / 08$ & gross $\alpha$ & $7.5 \mathrm{E}-04 \pm 5.5 \mathrm{E}-04$ & & & ${ }^{238} \mathrm{U}$ & $1.5 \mathrm{E}-05 \pm 9.2 \mathrm{E}-06$ & \\
\hline & 07/01/08 & $07 / 15 / 08$ & gross $\beta$ & $1.1 \mathrm{E}-02 \pm 1.6 \mathrm{E}-03$ & & & & & \\
\hline & 07/15/08 & 07/29/08 & gross $\alpha$ & $7.4 \mathrm{E}-04 \pm 5.4 \mathrm{E}-04$ & & & & & \\
\hline & $07 / 15 / 08$ & $07 / 29 / 08$ & gross $\beta$ & $1.2 \mathrm{E}-02 \pm 1.8 \mathrm{E}-03$ & & & & & \\
\hline & 07/29/08 & 08/12/08 & gross $\alpha$ & 7.4E-04 $\pm 5.3 \mathrm{E}-04$ & & & & & \\
\hline & 07/29/08 & $08 / 12 / 08$ & gross $\beta$ & $1.3 \mathrm{E}-02 \pm 1.8 \mathrm{E}-03$ & & & & & \\
\hline & $08 / 12 / 08$ & $08 / 25 / 08$ & gross $\alpha$ & $1.3 \mathrm{E}-03 \pm 5.6 \mathrm{E}-04$ & & & & & \\
\hline & 08/12/08 & 08/25/08 & gross $\beta$ & $1.5 \mathrm{E}-02 \pm 2.1 \mathrm{E}-03$ & & & & & \\
\hline & $08 / 25 / 08$ & 09/09/08 & gross $\alpha$ & $6.8 \mathrm{E}-04 \pm 4.9 \mathrm{E}-04$ & & & & & \\
\hline & $08 / 25 / 08$ & 09/09/08 & gross $\beta$ & $1.2 \mathrm{E}-02 \pm 1.7 \mathrm{E}-03$ & & & & & \\
\hline & 09/09/08 & 09/23/08 & gross $\alpha$ & $6.3 \mathrm{E}-04 \pm 5.0 \mathrm{E}-04$ & & & & & \\
\hline & 09/09/08 & 09/23/08 & gross $\beta$ & $2.9 \mathrm{E}-02 \pm 3.3 \mathrm{E}-03$ & & & & & \\
\hline & $09 / 23 / 08$ & $10 / 08 / 08$ & gross $\alpha$ & $1.2 \mathrm{E}-03 \pm 5.1 \mathrm{E}-04$ & & & & & \\
\hline & 09/23/08 & $10 / 08 / 08$ & gross $\beta$ & $2.2 \mathrm{E}-02 \pm 2.6 \mathrm{E}-03$ & & & & & \\
\hline & $10 / 08 / 08$ & $10 / 21 / 08$ & gross $\alpha$ & $1.5 \mathrm{E}-03 \pm 6.2 \mathrm{E}-04$ & & & & & \\
\hline & $10 / 08 / 08$ & $10 / 21 / 08$ & gross $\beta$ & $1.9 \mathrm{E}-02 \pm 2.4 \mathrm{E}-03$ & & & & & \\
\hline & $10 / 21 / 08$ & $11 / 04 / 08$ & gross $\alpha$ & $1.9 \mathrm{E}-03 \pm 6.8 \mathrm{E}-04$ & & & & & \\
\hline & $10 / 21 / 08$ & $11 / 04 / 08$ & gross $\beta$ & $4.4 \mathrm{E}-02 \pm 4.7 \mathrm{E}-03$ & & & & & \\
\hline & $11 / 04 / 08$ & $11 / 18 / 08$ & gross $\alpha$ & $1.4 \mathrm{E}-03 \pm 5.9 \mathrm{E}-04$ & & & & & \\
\hline & $11 / 04 / 08$ & $11 / 18 / 08$ & gross $\beta$ & $2.0 \mathrm{E}-02 \pm 2.5 \mathrm{E}-03$ & & & & & \\
\hline & $11 / 18 / 08$ & $12 / 02 / 08$ & gross $\alpha$ & $2.3 \mathrm{E}-03 \pm 7.6 \mathrm{E}-04$ & & & & & \\
\hline & $11 / 18 / 08$ & $12 / 02 / 08$ & gross $\beta$ & $4.7 \mathrm{E}-02 \pm 5.1 \mathrm{E}-03$ & & & & & \\
\hline & $12 / 02 / 08$ & $12 / 16 / 08$ & gross $\alpha$ & $1.3 \mathrm{E}-03 \pm 5.7 \mathrm{E}-04$ & & & & & \\
\hline & $12 / 02 / 08$ & $12 / 16 / 08$ & gross $\beta$ & $2.7 \mathrm{E}-02 \pm 3.1 \mathrm{E}-03$ & & & & & \\
\hline & $12 / 16 / 08$ & $12 / 31 / 08$ & gross $\alpha$ & $1.8 \mathrm{E}-03 \pm 6.6 \mathrm{E}-04$ & & & & & \\
\hline & $12 / 16 / 08$ & $12 / 31 / 08$ & gross $\beta$ & $4.1 \mathrm{E}-02 \pm 4.4 \mathrm{E}-03$ & & & & & \\
\hline
\end{tabular}

$\overline{\mathrm{RQ}}=$ Result Qualifier. $\mathrm{U}=$ The analyte was analyzed for but not detected. 
Table 2-4. Near-Facility Air Sampling Results, 2008 (pCi/m³ \pm total analytical uncertainty). (Sheet 75 of 82)

\begin{tabular}{|c|c|c|c|c|c|c|c|c|c|}
\hline Location & Sample On & Sample Off & Isotope & Result \pm Uncertainty & Location & Composite Period & Isotope & Result \pm Uncertainty & RQ* \\
\hline N538 & $01 / 02 / 08$ & $01 / 15 / 08$ & gross $\alpha$ & $8.8 \mathrm{E}-04 \pm 5.9 \mathrm{E}-04$ & N538 & $01 / 02 / 08$ to $07 / 01 / 08$ & ${ }^{60} \mathrm{Co}$ & $2.6 \mathrm{E}-05 \pm 7.9 \mathrm{E}-05$ & $\bar{U}$ \\
\hline \multirow[t]{51}{*}{ (300 Area) } & $01 / 02 / 08$ & $01 / 15 / 08$ & gross $\beta$ & $1.2 \mathrm{E}-02 \pm 1.8 \mathrm{E}-03$ & & & ${ }^{134} \mathrm{Cs}$ & $4.0 \mathrm{E}-05 \pm 7.4 \mathrm{E}-05$ & $\mathrm{U}$ \\
\hline & $01 / 15 / 08$ & 01/30/08 & gross $\alpha$ & $1.2 \mathrm{E}-03 \pm 5.0 \mathrm{E}-04$ & & & ${ }^{137} \mathrm{Cs}$ & $1.1 \mathrm{E}-06 \pm 1.1 \mathrm{E}-06$ & U \\
\hline & $01 / 15 / 08$ & 01/30/08 & gross $\beta$ & $3.4 \mathrm{E}-02 \pm 3.7 \mathrm{E}-03$ & & & ${ }^{152} \mathrm{Eu}$ & $-8.4 \mathrm{E}-05 \pm 1.6 \mathrm{E}-04$ & U \\
\hline & 01/30/08 & 02/13/08 & gross $\alpha$ & $-5.9 \mathrm{E}-05 \pm 2.1 \mathrm{E}-04$ & & & ${ }^{154} \mathrm{Eu}$ & $1.2 \mathrm{E}-04 \pm 2.0 \mathrm{E}-04$ & $\mathrm{U}$ \\
\hline & 01/30/08 & 02/13/08 & gross $\beta$ & $4.0 \mathrm{E}-03 \pm 9.0 \mathrm{E}-04$ & & & ${ }^{155} \mathrm{Eu}$ & $-1.8 \mathrm{E}-05 \pm 1.7 \mathrm{E}-04$ & U \\
\hline & $02 / 13 / 08$ & $02 / 27 / 08$ & gross $\alpha$ & $2.1 \mathrm{E}-03 \pm 7.2 \mathrm{E}-04$ & & & ${ }^{238} \mathrm{Pu}$ & $-3.4 \mathrm{E}-06 \pm 1.5 \mathrm{E}-05$ & $\mathrm{U}$ \\
\hline & $02 / 13 / 08$ & $02 / 27 / 08$ & gross $\beta$ & $2.7 \mathrm{E}-02 \pm 3.2 \mathrm{E}-03$ & & & ${ }^{239 / 240} \mathrm{Pu}$ & $8.5 \mathrm{E}-07 \pm 4.5 \mathrm{E}-06$ & $\mathrm{U}$ \\
\hline & $02 / 27 / 08$ & 03/12/08 & gross $\alpha$ & $1.3 \mathrm{E}-03 \pm 5.6 \mathrm{E}-04$ & & & ${ }^{106} \mathrm{Ru}$ & $6.1 \mathrm{E}-04 \pm 6.6 \mathrm{E}-04$ & $\mathrm{U}$ \\
\hline & $02 / 27 / 08$ & 03/12/08 & gross $\beta$ & $1.1 \mathrm{E}-02 \pm 1.7 \mathrm{E}-03$ & & & ${ }^{125} \mathrm{Sb}$ & $1.2 \mathrm{E}-04 \pm 1.4 \mathrm{E}-04$ & $\mathrm{U}$ \\
\hline & 03/12/08 & 03/26/08 & gross $\alpha$ & $9.3 \mathrm{E}-04 \pm 5.8 \mathrm{E}-04$ & & & ${ }^{234} \mathrm{U}$ & $3.3 \mathrm{E}-05 \pm 1.6 \mathrm{E}-05$ & \\
\hline & 03/12/08 & 03/26/08 & gross $\beta$ & $8.2 \mathrm{E}-03 \pm 1.4 \mathrm{E}-03$ & & & ${ }^{235} \mathrm{U}$ & $-7.3 \mathrm{E}-07 \pm 3.3 \mathrm{E}-06$ & $\mathrm{U}$ \\
\hline & 03/26/08 & $04 / 08 / 08$ & gross $\alpha$ & $8.3 \mathrm{E}-04 \pm 6.1 \mathrm{E}-04$ & & & ${ }^{238} \mathrm{U}$ & $2.0 \mathrm{E}-05 \pm 1.0 \mathrm{E}-05$ & \\
\hline & $03 / 26 / 08$ & $04 / 08 / 08$ & gross $\beta$ & $8.3 \mathrm{E}-03 \pm 1.5 \mathrm{E}-03$ & & & & & \\
\hline & $04 / 08 / 08$ & $04 / 23 / 08$ & gross $\alpha$ & $9.2 \mathrm{E}-04 \pm 5.7 \mathrm{E}-04$ & N538 & $07 / 01 / 08$ to $12 / 31 / 08$ & ${ }^{60} \mathrm{Co}$ & $1.3 \mathrm{E}-05 \pm 7.0 \mathrm{E}-05$ & $\mathrm{U}$ \\
\hline & $04 / 08 / 08$ & $04 / 23 / 08$ & gross $\beta$ & $1.2 \mathrm{E}-02 \pm 1.7 \mathrm{E}-03$ & & & ${ }^{134} \mathrm{Cs}$ & $1.2 \mathrm{E}-05 \pm 6.0 \mathrm{E}-05$ & $\mathrm{U}$ \\
\hline & $04 / 23 / 08$ & $05 / 08 / 08$ & gross $\alpha$ & $1.4 \mathrm{E}-03 \pm 6.0 \mathrm{E}-04$ & & & ${ }^{137} \mathrm{Cs}$ & $1.0 \mathrm{E}-04 \pm 7.7 \mathrm{E}-05$ & $\mathrm{U}$ \\
\hline & $04 / 23 / 08$ & 05/08/08 & gross $\beta$ & $1.7 \mathrm{E}-02 \pm 2.3 \mathrm{E}-03$ & & & ${ }^{152} \mathrm{Eu}$ & $-6.6 \mathrm{E}-05 \pm 1.4 \mathrm{E}-04$ & $\mathrm{U}$ \\
\hline & 05/08/08 & 05/20/08 & gross $\alpha$ & $8.1 \mathrm{E}-04 \pm 5.9 \mathrm{E}-04$ & & & ${ }^{154} \mathrm{Eu}$ & $3.7 \mathrm{E}-05 \pm 1.9 \mathrm{E}-04$ & U \\
\hline & 05/08/08 & $05 / 20 / 08$ & gross $\beta$ & $7.3 \mathrm{E}-03 \pm 1.3 \mathrm{E}-03$ & & & ${ }^{155} \mathrm{Eu}$ & $-3.7 \mathrm{E}-05 \pm 1.6 \mathrm{E}-04$ & $\mathrm{U}$ \\
\hline & $05 / 20 / 08$ & $06 / 03 / 08$ & gross $\alpha$ & $6.3 \mathrm{E}-04 \pm 5.1 \mathrm{E}-04$ & & & ${ }^{238} \mathrm{Pu}$ & $7.7 \mathrm{E}-06 \pm 1.1 \mathrm{E}-05$ & $\mathrm{U}$ \\
\hline & $05 / 20 / 08$ & $06 / 03 / 08$ & gross $\beta$ & $9.1 \mathrm{E}-03 \pm 1.4 \mathrm{E}-03$ & & & ${ }^{239 / 240} \mathrm{Pu}$ & $1.5 \mathrm{E}-06 \pm 2.3 \mathrm{E}-06$ & $\mathrm{U}$ \\
\hline & $06 / 03 / 08$ & $06 / 17 / 08$ & gross $\alpha$ & $1.2 \mathrm{E}-03 \pm 5.4 \mathrm{E}-04$ & & & ${ }^{106} \mathrm{Ru}$ & $-6.3 \mathrm{E}-04 \pm 6.6 \mathrm{E}-04$ & $\mathrm{U}$ \\
\hline & 06/03/08 & $06 / 17 / 08$ & gross $\beta$ & $1.1 \mathrm{E}-02 \pm 1.7 \mathrm{E}-03$ & & & ${ }^{125} \mathrm{Sb}$ & $1.4 \mathrm{E}-04 \pm 1.4 \mathrm{E}-04$ & $\mathrm{U}$ \\
\hline & $06 / 17 / 08$ & 07/01/08 & gross $\alpha$ & $1.5 \mathrm{E}-03 \pm 6.1 \mathrm{E}-04$ & & & ${ }^{234} \mathrm{U}$ & $3.2 \mathrm{E}-05 \pm 1.7 \mathrm{E}-05$ & \\
\hline & $06 / 17 / 08$ & $07 / 01 / 08$ & gross $\beta$ & $1.4 \mathrm{E}-02 \pm 1.9 \mathrm{E}-03$ & & & ${ }^{235} \mathrm{U}$ & $1.0 \mathrm{E}-06 \pm 2.1 \mathrm{E}-06$ & U \\
\hline & $07 / 01 / 08$ & $07 / 15 / 08$ & gross $\alpha$ & $1.5 \mathrm{E}-03 \pm 6.0 \mathrm{E}-04$ & & & ${ }^{238} \mathrm{U}$ & $3.6 \mathrm{E}-05 \pm 1.8 \mathrm{E}-05$ & \\
\hline & 07/01/08 & $07 / 15 / 08$ & gross $\beta$ & $1.4 \mathrm{E}-02 \pm 1.9 \mathrm{E}-03$ & & & & & \\
\hline & 07/15/08 & $07 / 29 / 08$ & gross $\alpha$ & $1.5 \mathrm{E}-03 \pm 6.2 \mathrm{E}-04$ & & & & & \\
\hline & $07 / 15 / 08$ & $07 / 29 / 08$ & gross $\beta$ & $1.5 \mathrm{E}-02 \pm 2.1 \mathrm{E}-03$ & & & & & \\
\hline & 07/29/08 & 08/12/08 & gross $\alpha$ & $8.5 \mathrm{E}-04 \pm 5.6 \mathrm{E}-04$ & & & & & \\
\hline & 07/29/08 & $08 / 12 / 08$ & gross $\beta$ & $1.3 \mathrm{E}-02 \pm 1.8 \mathrm{E}-03$ & & & & & \\
\hline & $08 / 12 / 08$ & $08 / 25 / 08$ & gross $\alpha$ & $1.3 \mathrm{E}-03 \pm 5.9 \mathrm{E}-04$ & & & & & \\
\hline & 08/12/08 & 08/25/08 & gross $\beta$ & $1.5 \mathrm{E}-02 \pm 2.1 \mathrm{E}-03$ & & & & & \\
\hline & $08 / 25 / 08$ & 09/09/08 & gross $\alpha$ & $1.1 \mathrm{E}-03 \pm 4.9 \mathrm{E}-04$ & & & & & \\
\hline & $08 / 25 / 08$ & 09/09/08 & gross $\beta$ & $9.7 \mathrm{E}-03 \pm 1.5 \mathrm{E}-03$ & & & & & \\
\hline & 09/09/08 & 09/23/08 & gross $\alpha$ & $1.9 \mathrm{E}-03 \pm 6.7 \mathrm{E}-04$ & & & & & \\
\hline & 09/09/08 & 09/23/08 & gross $\beta$ & $2.4 \mathrm{E}-02 \pm 2.9 \mathrm{E}-03$ & & & & & \\
\hline & $09 / 23 / 08$ & $10 / 08 / 08$ & gross $\alpha$ & $1.7 \mathrm{E}-03 \pm 6.1 \mathrm{E}-04$ & & & & & \\
\hline & 09/23/08 & $10 / 08 / 08$ & gross $\beta$ & $2.0 \mathrm{E}-02 \pm 2.5 \mathrm{E}-03$ & & & & & \\
\hline & $10 / 08 / 08$ & $10 / 21 / 08$ & gross $\alpha$ & $1.2 \mathrm{E}-03 \pm 5.5 \mathrm{E}-04$ & & & & & \\
\hline & $10 / 08 / 08$ & $10 / 21 / 08$ & gross $\beta$ & $1.9 \mathrm{E}-02 \pm 2.5 \mathrm{E}-03$ & & & & & \\
\hline & $10 / 21 / 08$ & $11 / 04 / 08$ & gross $\alpha$ & $3.0 \mathrm{E}-03 \pm 8.5 \mathrm{E}-04$ & & & & & \\
\hline & $10 / 21 / 08$ & $11 / 04 / 08$ & gross $\beta$ & $4.1 \mathrm{E}-02 \pm 4.4 \mathrm{E}-03$ & & & & & \\
\hline & $11 / 04 / 08$ & $11 / 18 / 08$ & gross $\alpha$ & $1.1 \mathrm{E}-03 \pm 5.3 \mathrm{E}-04$ & & & & & \\
\hline & $11 / 04 / 08$ & $11 / 18 / 08$ & gross $\beta$ & $2.2 \mathrm{E}-02 \pm 2.7 \mathrm{E}-03$ & & & & & \\
\hline & $11 / 18 / 08$ & $12 / 02 / 08$ & gross $\alpha$ & $1.9 \mathrm{E}-03 \pm 6.9 \mathrm{E}-04$ & & & & & \\
\hline & $11 / 18 / 08$ & $12 / 02 / 08$ & gross $\beta$ & $4.6 \mathrm{E}-02 \pm 5.0 \mathrm{E}-03$ & & & & & \\
\hline & $12 / 02 / 08$ & $12 / 16 / 08$ & gross $\alpha$ & $1.7 \mathrm{E}-03 \pm 6.3 \mathrm{E}-04$ & & & & & \\
\hline & $12 / 02 / 08$ & $12 / 16 / 08$ & gross $\beta$ & $2.3 \mathrm{E}-02 \pm 2.8 \mathrm{E}-03$ & & & & & \\
\hline & $12 / 16 / 08$ & $12 / 31 / 08$ & gross $\alpha$ & $1.3 \mathrm{E}-03 \pm 5.5 \mathrm{E}-04$ & & & & & \\
\hline & $12 / 16 / 08$ & $12 / 31 / 08$ & gross $\beta$ & $3.8 \mathrm{E}-02 \pm 4.1 \mathrm{E}-03$ & & & & & \\
\hline
\end{tabular}

$\overline{\mathrm{RQ}}=$ Result Qualifier. $\mathrm{U}=$ The analyte was analyzed for but not detected. 
Table 2-4. Near-Facility Air Sampling Results, 2008 (pCi/m³ \pm total analytical uncertainty). (Sheet 76 of 82)

\begin{tabular}{|c|c|c|c|c|c|c|c|c|c|}
\hline Location & Sample On & Sample Off & Isotope & Result \pm Uncertainty & Location & Composite Period & Isotope & Result \pm Uncertainty & RQ* \\
\hline N539 & $01 / 02 / 08$ & $01 / 15 / 08$ & gross $\alpha$ & $1.1 \mathrm{E}-03 \pm 5.4 \mathrm{E}-04$ & N539 & $01 / 02 / 08$ to $07 / 01 / 08$ & ${ }^{60} \mathrm{Co}$ & $-9.5 \mathrm{E}-05 \pm 1.4 \mathrm{E}-04$ & $\bar{U}$ \\
\hline \multirow[t]{51}{*}{ (300 Area) } & $01 / 02 / 08$ & $01 / 15 / 08$ & gross $\beta$ & $1.6 \mathrm{E}-02 \pm 2.2 \mathrm{E}-03$ & & & ${ }^{134} \mathrm{Cs}$ & $8.8 \mathrm{E}-06 \pm 8.8 \mathrm{E}-05$ & $\mathrm{U}$ \\
\hline & $01 / 15 / 08$ & 01/30/08 & gross $\alpha$ & $1.9 \mathrm{E}-03 \pm 6.5 \mathrm{E}-04$ & & & ${ }^{137} \mathrm{Cs}$ & $5.8 \mathrm{E}-05 \pm 1.1 \mathrm{E}-04$ & U \\
\hline & $01 / 15 / 08$ & 01/30/08 & gross $\beta$ & $3.6 \mathrm{E}-02 \pm 3.9 \mathrm{E}-03$ & & & ${ }^{152} \mathrm{Eu}$ & $-3.2 \mathrm{E}-05 \pm 2.9 \mathrm{E}-04$ & U \\
\hline & $01 / 30 / 08$ & $02 / 13 / 08$ & gross $\alpha$ & $2.9 \mathrm{E}-04 \pm 4.1 \mathrm{E}-04$ & & & ${ }^{154} \mathrm{Eu}$ & $-7.9 \mathrm{E}-05 \pm 3.2 \mathrm{E}-04$ & $\mathrm{U}$ \\
\hline & 01/30/08 & 02/13/08 & gross $\beta$ & $2.1 \mathrm{E}-03 \pm 6.4 \mathrm{E}-04$ & & & ${ }^{155} \mathrm{Eu}$ & $-3.8 \mathrm{E}-05 \pm 2.0 \mathrm{E}-04$ & U \\
\hline & $02 / 13 / 08$ & $02 / 27 / 08$ & gross $\alpha$ & $2.4 \mathrm{E}-03 \pm 7.6 \mathrm{E}-04$ & & & ${ }^{238} \mathrm{Pu}$ & $8.7 \mathrm{E}-06 \pm 1.3 \mathrm{E}-05$ & $\mathrm{U}$ \\
\hline & $02 / 13 / 08$ & $02 / 27 / 08$ & gross $\beta$ & $3.0 \mathrm{E}-02 \pm 3.4 \mathrm{E}-03$ & & & ${ }^{239 / 240} \mathrm{Pu}$ & $2.4 \mathrm{E}-06 \pm 4.3 \mathrm{E}-06$ & $\mathrm{U}$ \\
\hline & $02 / 27 / 08$ & 03/12/08 & gross $\alpha$ & $1.2 \mathrm{E}-03 \pm 5.3 \mathrm{E}-04$ & & & ${ }^{106} \mathrm{Ru}$ & $8.2 \mathrm{E}-04 \pm 9.9 \mathrm{E}-04$ & $\mathrm{U}$ \\
\hline & $02 / 27 / 08$ & 03/12/08 & gross $\beta$ & $1.3 \mathrm{E}-02 \pm 1.9 \mathrm{E}-03$ & & & ${ }^{125} \mathrm{Sb}$ & $-3.5 \mathrm{E}-05 \pm 2.6 \mathrm{E}-04$ & $\mathrm{U}$ \\
\hline & 03/12/08 & 03/26/08 & gross $\alpha$ & $1.1 \mathrm{E}-03 \pm 5.3 \mathrm{E}-04$ & & & ${ }^{234} \mathrm{U}$ & $2.1 \mathrm{E}-05 \pm 1.1 \mathrm{E}-05$ & \\
\hline & 03/12/08 & 03/26/08 & gross $\beta$ & $1.5 \mathrm{E}-02 \pm 2.1 \mathrm{E}-03$ & & & ${ }^{235} \mathrm{U}$ & $1.5 \mathrm{E}-06 \pm 2.2 \mathrm{E}-06$ & $\mathrm{U}$ \\
\hline & 03/26/08 & 04/08/08 & gross $\alpha$ & $1.2 \mathrm{E}-03 \pm 7.8 \mathrm{E}-04$ & & & ${ }^{238} \mathrm{U}$ & $1.9 \mathrm{E}-05 \pm 1.0 \mathrm{E}-05$ & \\
\hline & $03 / 26 / 08$ & $04 / 08 / 08$ & gross $\beta$ & $8.5 \mathrm{E}-03 \pm 1.6 \mathrm{E}-03$ & & & & & \\
\hline & $04 / 08 / 08$ & $04 / 23 / 08$ & gross $\alpha$ & $1.6 \mathrm{E}-03 \pm 6.3 \mathrm{E}-04$ & N539 & $07 / 01 / 08$ to $12 / 31 / 08$ & ${ }^{60} \mathrm{Co}$ & $7.1 \mathrm{E}-05 \pm 1.0 \mathrm{E}-04$ & $\mathrm{U}$ \\
\hline & $04 / 08 / 08$ & $04 / 23 / 08$ & gross $\beta$ & $1.0 \mathrm{E}-02 \pm 1.6 \mathrm{E}-03$ & & & ${ }^{134} \mathrm{Cs}$ & $1.3 \mathrm{E}-04 \pm 1.2 \mathrm{E}-04$ & $\mathrm{U}$ \\
\hline & $04 / 23 / 08$ & $05 / 08 / 08$ & gross $\alpha$ & $1.5 \mathrm{E}-03 \pm 6.1 \mathrm{E}-04$ & & & ${ }^{137} \mathrm{Cs}$ & $1.9 \mathrm{E}-05 \pm 1.1 \mathrm{E}-04$ & $\mathrm{U}$ \\
\hline & $04 / 23 / 08$ & 05/08/08 & gross $\beta$ & $1.6 \mathrm{E}-02 \pm 2.1 \mathrm{E}-03$ & & & ${ }^{152} \mathrm{Eu}$ & $-1.1 \mathrm{E}-04 \pm 2.7 \mathrm{E}-04$ & $\mathrm{U}$ \\
\hline & 05/08/08 & 05/20/08 & gross $\alpha$ & $1.2 \mathrm{E}-03 \pm 7.2 \mathrm{E}-04$ & & & ${ }^{154} \mathrm{Eu}$ & $-6.8 \mathrm{E}-05 \pm 3.4 \mathrm{E}-04$ & U \\
\hline & 05/08/08 & $05 / 20 / 08$ & gross $\beta$ & $1.2 \mathrm{E}-02 \pm 1.9 \mathrm{E}-03$ & & & ${ }^{155} \mathrm{Eu}$ & $2.4 \mathrm{E}-05 \pm 2.0 \mathrm{E}-04$ & $\mathrm{U}$ \\
\hline & $05 / 20 / 08$ & $06 / 03 / 08$ & gross $\alpha$ & $6.5 \mathrm{E}-04 \pm 5.2 \mathrm{E}-04$ & & & ${ }^{238} \mathrm{Pu}$ & $4.7 \mathrm{E}-06 \pm 1.2 \mathrm{E}-05$ & $\mathrm{U}$ \\
\hline & $05 / 20 / 08$ & $06 / 03 / 08$ & gross $\beta$ & $1.1 \mathrm{E}-02 \pm 1.7 \mathrm{E}-03$ & & & ${ }^{239 / 240} \mathrm{Pu}$ & $7.5 \mathrm{E}-07 \pm 2.2 \mathrm{E}-06$ & $\mathrm{U}$ \\
\hline & $06 / 03 / 08$ & $06 / 17 / 08$ & gross $\alpha$ & $5.5 \mathrm{E}-04 \pm 5.1 \mathrm{E}-04$ & & & ${ }^{106} \mathrm{Ru}$ & $3.4 \mathrm{E}-04 \pm 9.4 \mathrm{E}-04$ & $\mathrm{U}$ \\
\hline & 06/03/08 & $06 / 17 / 08$ & gross $\beta$ & $7.2 \mathrm{E}-03 \pm 1.3 \mathrm{E}-03$ & & & ${ }^{125} \mathrm{Sb}$ & $-1.4 \mathrm{E}-04 \pm 2.5 \mathrm{E}-04$ & $\mathrm{U}$ \\
\hline & $06 / 17 / 08$ & 07/01/08 & gross $\alpha$ & $1.1 \mathrm{E}-03 \pm 6.6 \mathrm{E}-04$ & & & ${ }^{234} \mathrm{U}$ & $4.7 \mathrm{E}-05 \pm 2.1 \mathrm{E}-05$ & \\
\hline & $06 / 17 / 08$ & 07/01/08 & gross $\beta$ & $1.0 \mathrm{E}-02 \pm 1.6 \mathrm{E}-03$ & & & ${ }^{235} \mathrm{U}$ & $7.8 \mathrm{E}-06 \pm 5.7 \mathrm{E}-06$ & \\
\hline & $07 / 01 / 08$ & 07/15/08 & gross $\alpha$ & $1.4 \mathrm{E}-03 \pm 6.1 \mathrm{E}-04$ & & & ${ }^{238} \mathrm{U}$ & $3.8 \mathrm{E}-05 \pm 1.8 \mathrm{E}-05$ & \\
\hline & 07/01/08 & $07 / 15 / 08$ & gross $\beta$ & $1.5 \mathrm{E}-02 \pm 2.1 \mathrm{E}-03$ & & & & & \\
\hline & 07/15/08 & $07 / 29 / 08$ & gross $\alpha$ & $1.0 \mathrm{E}-03 \pm 6.2 \mathrm{E}-04$ & & & & & \\
\hline & $07 / 15 / 08$ & 07/29/08 & gross $\beta$ & $1.3 \mathrm{E}-02 \pm 1.9 \mathrm{E}-03$ & & & & & \\
\hline & 07/29/08 & 08/12/08 & gross $\alpha$ & $8.0 \mathrm{E}-04 \pm 5.8 \mathrm{E}-04$ & & & & & \\
\hline & 07/29/08 & $08 / 12 / 08$ & gross $\beta$ & $1.4 \mathrm{E}-02 \pm 1.9 \mathrm{E}-03$ & & & & & \\
\hline & $08 / 12 / 08$ & $08 / 25 / 08$ & gross $\alpha$ & $1.3 \mathrm{E}-03 \pm 5.9 \mathrm{E}-04$ & & & & & \\
\hline & 08/12/08 & 08/25/08 & gross $\beta$ & $1.6 \mathrm{E}-02 \pm 2.3 \mathrm{E}-03$ & & & & & \\
\hline & $08 / 25 / 08$ & 09/09/08 & gross $\alpha$ & $6.0 \mathrm{E}-04 \pm 4.8 \mathrm{E}-04$ & & & & & \\
\hline & $08 / 25 / 08$ & 09/09/08 & gross $\beta$ & $1.3 \mathrm{E}-02 \pm 1.8 \mathrm{E}-03$ & & & & & \\
\hline & 09/09/08 & 09/23/08 & gross $\alpha$ & $1.4 \mathrm{E}-03 \pm 5.8 \mathrm{E}-04$ & & & & & \\
\hline & 09/09/08 & 09/23/08 & gross $\beta$ & $2.9 \mathrm{E}-02 \pm 3.4 \mathrm{E}-03$ & & & & & \\
\hline & $09 / 23 / 08$ & $10 / 08 / 08$ & gross $\alpha$ & $1.4 \mathrm{E}-03 \pm 5.5 \mathrm{E}-04$ & & & & & \\
\hline & 09/23/08 & $10 / 08 / 08$ & gross $\beta$ & $2.1 \mathrm{E}-02 \pm 2.5 \mathrm{E}-03$ & & & & & \\
\hline & $10 / 08 / 08$ & $10 / 21 / 08$ & gross $\alpha$ & $7.2 \mathrm{E}-04 \pm 5.8 \mathrm{E}-04$ & & & & & \\
\hline & $10 / 08 / 08$ & $10 / 21 / 08$ & gross $\beta$ & $1.7 \mathrm{E}-02 \pm 2.3 \mathrm{E}-03$ & & & & & \\
\hline & $10 / 21 / 08$ & $11 / 04 / 08$ & gross $\alpha$ & $1.8 \mathrm{E}-03 \pm 6.5 \mathrm{E}-04$ & & & & & \\
\hline & $10 / 21 / 08$ & $11 / 04 / 08$ & gross $\beta$ & 4.3E-02 $\pm 4.6 \mathrm{E}-03$ & & & & & \\
\hline & $11 / 04 / 08$ & $11 / 18 / 08$ & gross $\alpha$ & $2.4 \mathrm{E}-03 \pm 8.0 \mathrm{E}-04$ & & & & & \\
\hline & $11 / 04 / 08$ & $11 / 18 / 08$ & gross $\beta$ & $2.1 \mathrm{E}-02 \pm 2.7 \mathrm{E}-03$ & & & & & \\
\hline & $11 / 18 / 08$ & $12 / 02 / 08$ & gross $\alpha$ & $1.3 \mathrm{E}-03 \pm 6.3 \mathrm{E}-04$ & & & & & \\
\hline & $11 / 18 / 08$ & $12 / 02 / 08$ & gross $\beta$ & $5.3 \mathrm{E}-02 \pm 5.8 \mathrm{E}-03$ & & & & & \\
\hline & $12 / 02 / 08$ & $12 / 16 / 08$ & gross $\alpha$ & $1.4 \mathrm{E}-03 \pm 5.9 \mathrm{E}-04$ & & & & & \\
\hline & $12 / 02 / 08$ & $12 / 16 / 08$ & gross $\beta$ & $2.3 \mathrm{E}-02 \pm 2.8 \mathrm{E}-03$ & & & & & \\
\hline & $12 / 16 / 08$ & $12 / 31 / 08$ & gross $\alpha$ & $1.7 \mathrm{E}-03 \pm 6.4 \mathrm{E}-04$ & & & & & \\
\hline & $12 / 16 / 08$ & $12 / 31 / 08$ & gross $\beta$ & $4.4 \mathrm{E}-02 \pm 4.7 \mathrm{E}-03$ & & & & & \\
\hline
\end{tabular}

$\overline{\mathrm{RQ}}=$ Result Qualifier. $\mathrm{U}=$ The analyte was analyzed for but not detected. 
Table 2-4. Near-Facility Air Sampling Results, 2008 (pCi/m³ \pm total analytical uncertainty). (Sheet 77 of 82)

\begin{tabular}{|c|c|c|c|c|c|c|c|c|c|}
\hline Location & Sample On & Sample Off & Isotope & Result \pm Uncertainty & Location & Composite Period & Isotope & Result \pm Uncertainty & $\mathbf{R Q}^{*}$ \\
\hline N540 & $01 / 02 / 08$ & $01 / 15 / 08$ & gross $\alpha$ & $9.1 \mathrm{E}-04 \pm 6.1 \mathrm{E}-04$ & N540 & $01 / 02 / 08$ to $07 / 01 / 08$ & ${ }^{60} \mathrm{Co}$ & $1.6 \mathrm{E}-05 \pm 8.1 \mathrm{E}-05$ & $\bar{U}$ \\
\hline \multirow[t]{51}{*}{ (300 Area) } & $01 / 02 / 08$ & $01 / 15 / 08$ & gross $\beta$ & $1.2 \mathrm{E}-02 \pm 1.8 \mathrm{E}-03$ & & & ${ }^{134} \mathrm{Cs}$ & $-2.3 \mathrm{E}-06 \pm 2.3 \mathrm{E}-05$ & $\mathrm{U}$ \\
\hline & $01 / 15 / 08$ & 01/30/08 & gross $\alpha$ & $1.3 \mathrm{E}-03 \pm 5.2 \mathrm{E}-04$ & & & ${ }^{137} \mathrm{Cs}$ & $-1.8 \mathrm{E}-05 \pm 6.7 \mathrm{E}-05$ & U \\
\hline & $01 / 15 / 08$ & 01/30/08 & gross $\beta$ & $3.8 \mathrm{E}-02 \pm 4.1 \mathrm{E}-03$ & & & ${ }^{152} \mathrm{Eu}$ & $-6.2 \mathrm{E}-05 \pm 1.7 \mathrm{E}-04$ & U \\
\hline & $01 / 30 / 08$ & $02 / 13 / 08$ & gross $\alpha$ & $1.7 \mathrm{E}-04 \pm 3.5 \mathrm{E}-04$ & & & ${ }^{154} \mathrm{Eu}$ & $-1.1 \mathrm{E}-04 \pm 2.1 \mathrm{E}-04$ & $\mathrm{U}$ \\
\hline & 01/30/08 & 02/13/08 & gross $\beta$ & $4.0 \mathrm{E}-03 \pm 8.9 \mathrm{E}-04$ & & & ${ }^{155} \mathrm{Eu}$ & $-4.8 \mathrm{E}-05 \pm 1.9 \mathrm{E}-04$ & U \\
\hline & $02 / 13 / 08$ & $02 / 27 / 08$ & gross $\alpha$ & $1.5 \mathrm{E}-03 \pm 6.0 \mathrm{E}-04$ & & & ${ }^{238} \mathrm{Pu}$ & $-1.7 \mathrm{E}-06 \pm 1.4 \mathrm{E}-05$ & $\mathrm{U}$ \\
\hline & $02 / 13 / 08$ & $02 / 27 / 08$ & gross $\beta$ & $3.4 \mathrm{E}-02 \pm 3.7 \mathrm{E}-03$ & & & ${ }^{239 / 240} \mathrm{Pu}$ & $4.3 \mathrm{E}-06 \pm 4.9 \mathrm{E}-06$ & $\mathrm{U}$ \\
\hline & $02 / 27 / 08$ & 03/12/08 & gross $\alpha$ & $1.1 \mathrm{E}-03 \pm 5.1 \mathrm{E}-04$ & & & ${ }^{106} \mathrm{Ru}$ & $-8.6 \mathrm{E}-04 \pm 8.9 \mathrm{E}-04$ & $\mathrm{U}$ \\
\hline & $02 / 27 / 08$ & 03/12/08 & gross $\beta$ & $1.4 \mathrm{E}-02 \pm 1.9 \mathrm{E}-03$ & & & ${ }^{125} \mathrm{Sb}$ & $-1.3 \mathrm{E}-04 \pm 1.6 \mathrm{E}-04$ & $\mathrm{U}$ \\
\hline & 03/12/08 & 03/26/08 & gross $\alpha$ & $5.0 \mathrm{E}-04 \pm 4.7 \mathrm{E}-04$ & & & ${ }^{234} \mathrm{U}$ & $1.7 \mathrm{E}-05 \pm 9.6 \mathrm{E}-06$ & \\
\hline & 03/12/08 & 03/26/08 & gross $\beta$ & $4.3 \mathrm{E}-02 \pm 4.6 \mathrm{E}-03$ & & & ${ }^{235} \mathrm{U}$ & $2.3 \mathrm{E}-06 \pm 2.7 \mathrm{E}-06$ & \\
\hline & $03 / 26 / 08$ & $04 / 08 / 08$ & gross $\alpha$ & $1.0 \mathrm{E}-03 \pm 6.3 \mathrm{E}-04$ & & & ${ }^{238} \mathrm{U}$ & $8.3 \mathrm{E}-06 \pm 6.4 \mathrm{E}-06$ & \\
\hline & $03 / 26 / 08$ & $04 / 08 / 08$ & gross $\beta$ & $7.2 \mathrm{E}-03 \pm 1.3 \mathrm{E}-03$ & & & & & \\
\hline & $04 / 08 / 08$ & $04 / 23 / 08$ & gross $\alpha$ & $8.0 \mathrm{E}-04 \pm 5.4 \mathrm{E}-04$ & N540 & $07 / 01 / 08$ to $12 / 31 / 08$ & ${ }^{60} \mathrm{Co}$ & $5.0 \mathrm{E}-05 \pm 7.1 \mathrm{E}-05$ & $\mathrm{U}$ \\
\hline & $04 / 08 / 08$ & $04 / 23 / 08$ & gross $\beta$ & $9.8 \mathrm{E}-03 \pm 1.5 \mathrm{E}-03$ & & & ${ }^{134} \mathrm{Cs}$ & $2.4 \mathrm{E}-05 \pm 7.0 \mathrm{E}-05$ & $\mathrm{U}$ \\
\hline & $04 / 23 / 08$ & $05 / 08 / 08$ & gross $\alpha$ & $1.5 \mathrm{E}-03 \pm 5.8 \mathrm{E}-04$ & & & ${ }^{137} \mathrm{Cs}$ & $5.3 \mathrm{E}-05 \pm 6.6 \mathrm{E}-05$ & $\mathrm{U}$ \\
\hline & $04 / 23 / 08$ & 05/08/08 & gross $\beta$ & $1.4 \mathrm{E}-02 \pm 1.9 \mathrm{E}-03$ & & & ${ }^{152} \mathrm{Eu}$ & $-3.2 \mathrm{E}-05 \pm 1.7 \mathrm{E}-04$ & $\mathrm{U}$ \\
\hline & 05/08/08 & 05/20/08 & gross $\alpha$ & $6.0 \mathrm{E}-04 \pm 5.5 \mathrm{E}-04$ & & & ${ }^{154} \mathrm{Eu}$ & $-7.0 \mathrm{E}-06 \pm 7.0 \mathrm{E}-05$ & U \\
\hline & 05/08/08 & $05 / 20 / 08$ & gross $\beta$ & $1.1 \mathrm{E}-02 \pm 1.7 \mathrm{E}-03$ & & & ${ }^{155} \mathrm{Eu}$ & $7.3 \mathrm{E}-05 \pm 1.7 \mathrm{E}-04$ & $\mathrm{U}$ \\
\hline & $05 / 20 / 08$ & $06 / 03 / 08$ & gross $\alpha$ & $9.4 \mathrm{E}-04 \pm 5.8 \mathrm{E}-04$ & & & ${ }^{238} \mathrm{Pu}$ & $4.3 \mathrm{E}-06 \pm 1.2 \mathrm{E}-05$ & $\mathrm{U}$ \\
\hline & $05 / 20 / 08$ & $06 / 03 / 08$ & gross $\beta$ & $1.3 \mathrm{E}-02 \pm 1.8 \mathrm{E}-03$ & & & ${ }^{239 / 240} \mathrm{Pu}$ & $3.5 \mathrm{E}-06 \pm 4.4 \mathrm{E}-06$ & $\mathrm{U}$ \\
\hline & $06 / 03 / 08$ & $06 / 17 / 08$ & gross $\alpha$ & $7.2 \mathrm{E}-04 \pm 5.2 \mathrm{E}-04$ & & & ${ }^{106} \mathrm{Ru}$ & $-1.9 \mathrm{E}-04 \pm 6.0 \mathrm{E}-04$ & $\mathrm{U}$ \\
\hline & 06/03/08 & $06 / 17 / 08$ & gross $\beta$ & $5.7 \mathrm{E}-03 \pm 1.1 \mathrm{E}-03$ & & & ${ }^{125} \mathrm{Sb}$ & $9.6 \mathrm{E}-05 \pm 1.5 \mathrm{E}-04$ & $\mathrm{U}$ \\
\hline & $06 / 17 / 08$ & 07/01/08 & gross $\alpha$ & $7.3 \mathrm{E}-04 \pm 5.4 \mathrm{E}-04$ & & & ${ }^{234} \mathrm{U}$ & $2.5 \mathrm{E}-05 \pm 1.3 \mathrm{E}-05$ & \\
\hline & $06 / 17 / 08$ & $07 / 01 / 08$ & gross $\beta$ & $1.3 \mathrm{E}-02 \pm 1.8 \mathrm{E}-03$ & & & ${ }^{235} \mathrm{U}$ & $2.2 \mathrm{E}-06 \pm 3.4 \mathrm{E}-06$ & U \\
\hline & $07 / 01 / 08$ & $07 / 15 / 08$ & gross $\alpha$ & $1.1 \mathrm{E}-03 \pm 4.9 \mathrm{E}-04$ & & & ${ }^{238} \mathrm{U}$ & $1.6 \mathrm{E}-05 \pm 8.8 \mathrm{E}-06$ & \\
\hline & 07/01/08 & $07 / 15 / 08$ & gross $\beta$ & $1.1 \mathrm{E}-02 \pm 1.7 \mathrm{E}-03$ & & & & & \\
\hline & 07/15/08 & 07/29/08 & gross $\alpha$ & $1.1 \mathrm{E}-03 \pm 5.0 \mathrm{E}-04$ & & & & & \\
\hline & $07 / 15 / 08$ & $07 / 29 / 08$ & gross $\beta$ & $1.1 \mathrm{E}-02 \pm 1.7 \mathrm{E}-03$ & & & & & \\
\hline & 07/29/08 & 08/12/08 & gross $\alpha$ & $4.0 \mathrm{E}-04 \pm 4.4 \mathrm{E}-04$ & & & & & \\
\hline & 07/29/08 & $08 / 12 / 08$ & gross $\beta$ & $1.6 \mathrm{E}-02 \pm 2.1 \mathrm{E}-03$ & & & & & \\
\hline & $08 / 12 / 08$ & $08 / 25 / 08$ & gross $\alpha$ & $5.6 \mathrm{E}-04 \pm 5.2 \mathrm{E}-04$ & & & & & \\
\hline & 08/12/08 & 08/25/08 & gross $\beta$ & $1.5 \mathrm{E}-02 \pm 2.0 \mathrm{E}-03$ & & & & & \\
\hline & $08 / 25 / 08$ & 09/09/08 & gross $\alpha$ & $1.2 \mathrm{E}-03 \pm 5.1 \mathrm{E}-04$ & & & & & \\
\hline & $08 / 25 / 08$ & 09/09/08 & gross $\beta$ & $1.1 \mathrm{E}-02 \pm 1.6 \mathrm{E}-03$ & & & & & \\
\hline & 09/09/08 & 09/23/08 & gross $\alpha$ & $1.1 \mathrm{E}-03 \pm 5.0 \mathrm{E}-04$ & & & & & \\
\hline & 09/09/08 & 09/23/08 & gross $\beta$ & $2.6 \mathrm{E}-02 \pm 3.0 \mathrm{E}-03$ & & & & & \\
\hline & $09 / 23 / 08$ & $10 / 08 / 08$ & gross $\alpha$ & $1.1 \mathrm{E}-03 \pm 4.9 \mathrm{E}-04$ & & & & & \\
\hline & 09/23/08 & $10 / 08 / 08$ & gross $\beta$ & $2.2 \mathrm{E}-02 \pm 2.7 \mathrm{E}-03$ & & & & & \\
\hline & $10 / 08 / 08$ & $10 / 21 / 08$ & gross $\alpha$ & $1.4 \mathrm{E}-03 \pm 5.8 \mathrm{E}-04$ & & & & & \\
\hline & $10 / 08 / 08$ & $10 / 21 / 08$ & gross $\beta$ & $1.9 \mathrm{E}-02 \pm 2.4 \mathrm{E}-03$ & & & & & \\
\hline & $10 / 21 / 08$ & $11 / 04 / 08$ & gross $\alpha$ & $2.6 \mathrm{E}-03 \pm 7.8 \mathrm{E}-04$ & & & & & \\
\hline & $10 / 21 / 08$ & $11 / 04 / 08$ & gross $\beta$ & $4.2 \mathrm{E}-02 \pm 4.5 \mathrm{E}-03$ & & & & & \\
\hline & $11 / 04 / 08$ & $11 / 18 / 08$ & gross $\alpha$ & $1.1 \mathrm{E}-03 \pm 5.1 \mathrm{E}-04$ & & & & & \\
\hline & $11 / 04 / 08$ & $11 / 18 / 08$ & gross $\beta$ & $1.9 \mathrm{E}-02 \pm 2.4 \mathrm{E}-03$ & & & & & \\
\hline & $11 / 18 / 08$ & $12 / 02 / 08$ & gross $\alpha$ & $2.2 \mathrm{E}-03 \pm 7.6 \mathrm{E}-04$ & & & & & \\
\hline & $11 / 18 / 08$ & $12 / 02 / 08$ & gross $\beta$ & $4.5 \mathrm{E}-02 \pm 4.9 \mathrm{E}-03$ & & & & & \\
\hline & $12 / 02 / 08$ & $12 / 16 / 08$ & gross $\alpha$ & $1.6 \mathrm{E}-03 \pm 6.5 \mathrm{E}-04$ & & & & & \\
\hline & $12 / 02 / 08$ & $12 / 16 / 08$ & gross $\beta$ & $2.6 \mathrm{E}-02 \pm 3.1 \mathrm{E}-03$ & & & & & \\
\hline & $12 / 16 / 08$ & $12 / 31 / 08$ & gross $\alpha$ & $2.3 \mathrm{E}-03 \pm 7.5 \mathrm{E}-04$ & & & & & \\
\hline & $12 / 16 / 08$ & $12 / 31 / 08$ & gross $\beta$ & $4.5 \mathrm{E}-02 \pm 4.8 \mathrm{E}-03$ & & & & & \\
\hline
\end{tabular}

$\overline{\mathrm{RQ}}=$ Result Qualifier. $\mathrm{U}=$ The analyte was analyzed for but not detected. 
Table 2-4. Near-Facility Air Sampling Results, 2008 (pCi/m³ \pm total analytical uncertainty). (Sheet 78 of 82)

\begin{tabular}{|c|c|c|c|c|c|c|c|c|c|}
\hline Location & Sample On & Sample Off & Isotope & Result \pm Uncertainty & Location & Composite Period & Isotope & Result \pm Uncertainty & $\mathbf{R Q}^{*}$ \\
\hline N130 & $01 / 02 / 08$ & $01 / 15 / 08$ & gross $\alpha$ & $4.1 \mathrm{E}-04 \pm 4.6 \mathrm{E}-04$ & N130 & $01 / 02 / 08$ to $07 / 01 / 08$ & ${ }^{60} \mathrm{Co}$ & $-1.9 \mathrm{E}-05 \pm 1.3 \mathrm{E}-04$ & $\bar{U}$ \\
\hline \multirow[t]{51}{*}{ (300 Area) } & 01/02/08 & 01/15/08 & gross $\beta$ & $9.9 \mathrm{E}-03 \pm 1.5 \mathrm{E}-03$ & & & ${ }^{134} \mathrm{Cs}$ & $-4.8 \mathrm{E}-05 \pm 1.1 \mathrm{E}-04$ & $\mathrm{U}$ \\
\hline & 01/15/08 & 01/30/08 & gross $\alpha$ & $1.1 \mathrm{E}-03 \pm 4.9 \mathrm{E}-04$ & & & ${ }^{137} \mathrm{Cs}$ & $-2.3 \mathrm{E}-05 \pm 9.5 \mathrm{E}-05$ & $\mathrm{U}$ \\
\hline & 01/15/08 & 01/30/08 & gross $\beta$ & $3.3 \mathrm{E}-02 \pm 3.3 \mathrm{E}-03$ & & & ${ }^{152} \mathrm{Eu}$ & $1.7 \mathrm{E}-04 \pm 2.5 \mathrm{E}-04$ & $\mathrm{U}$ \\
\hline & 01/30/08 & 02/13/08 & gross $\alpha$ & $3.9 \mathrm{E}-04 \pm 4.4 \mathrm{E}-04$ & & & ${ }^{154} \mathrm{Eu}$ & $-1.4 \mathrm{E}-04 \pm 3.7 \mathrm{E}-04$ & $\mathrm{U}$ \\
\hline & 01/30/08 & 02/13/08 & gross $\beta$ & $3.1 \mathrm{E}-03 \pm 7.6 \mathrm{E}-04$ & & & ${ }^{155} \mathrm{Eu}$ & $-1.4 \mathrm{E}-05 \pm 1.4 \mathrm{E}-04$ & $\mathrm{U}$ \\
\hline & 02/13/08 & $02 / 27 / 08$ & gross $\alpha$ & $2.2 \mathrm{E}-03 \pm 7.1 \mathrm{E}-04$ & & & ${ }^{238} \mathrm{Pu}$ & $4.8 \mathrm{E}-06 \pm 8.1 \mathrm{E}-06$ & $\mathrm{U}$ \\
\hline & 02/13/08 & $02 / 27 / 08$ & gross $\beta$ & $3.1 \mathrm{E}-02 \pm 3.1 \mathrm{E}-03$ & & & ${ }^{239 / 240} \mathrm{Pu}$ & $2.1 \mathrm{E}-06 \pm 4.7 \mathrm{E}-06$ & $\mathrm{U}$ \\
\hline & $02 / 27 / 08$ & 03/12/08 & gross $\alpha$ & $7.5 \mathrm{E}-04 \pm 5.5 \mathrm{E}-04$ & & & ${ }^{106} \mathrm{Ru}$ & $9.1 \mathrm{E}-05 \pm 8.8 \mathrm{E}-04$ & $\mathrm{U}$ \\
\hline & $02 / 27 / 08$ & 03/12/08 & gross $\beta$ & $1.0 \mathrm{E}-02 \pm 1.5 \mathrm{E}-03$ & & & ${ }^{125} \mathrm{Sb}$ & $-2.8 \mathrm{E}-04 \pm 2.9 \mathrm{E}-04$ & $\mathrm{U}$ \\
\hline & 03/12/08 & 03/26/08 & gross $\alpha$ & $5.0 \mathrm{E}-04 \pm 4.7 \mathrm{E}-04$ & & & ${ }^{90} \mathrm{Sr}$ & $-2.0 \mathrm{E}-04 \pm 2.1 \mathrm{E}-04$ & $\mathrm{U}$ \\
\hline & 03/12/08 & 03/26/08 & gross $\beta$ & $9.9 \mathrm{E}-03 \pm 1.5 \mathrm{E}-03$ & & & ${ }^{234} \mathrm{U}$ & $2.2 \mathrm{E}-05 \pm 1.1 \mathrm{E}-05$ & \\
\hline & 03/26/08 & 04/08/08 & gross $\alpha$ & $5.4 \mathrm{E}-04 \pm 5.1 \mathrm{E}-04$ & & & ${ }^{235} \mathrm{U}$ & $1.5 \mathrm{E}-06 \pm 3.0 \mathrm{E}-06$ & $\mathrm{U}$ \\
\hline & 03/26/08 & 04/08/08 & gross $\beta$ & $5.4 \mathrm{E}-03 \pm 1.1 \mathrm{E}-03$ & & & ${ }^{238} \mathrm{U}$ & $1.6 \mathrm{E}-05 \pm 8.9 \mathrm{E}-06$ & \\
\hline & 04/08/08 & 04/23/08 & gross $\alpha$ & $1.1 \mathrm{E}-03 \pm 4.9 \mathrm{E}-04$ & & & & & \\
\hline & 04/08/08 & $04 / 23 / 08$ & gross $\beta$ & $8.5 \mathrm{E}-03 \pm 1.3 \mathrm{E}-03$ & N130 & $07 / 01 / 08$ to $12 / 31 / 08$ & ${ }^{60} \mathrm{Co}$ & $-1.6 \mathrm{E}-05 \pm 7.8 \mathrm{E}-05$ & $\mathrm{U}$ \\
\hline & $04 / 23 / 08$ & 05/08/08 & gross $\alpha$ & $9.0 \mathrm{E}-04 \pm 5.5 \mathrm{E}-04$ & & & ${ }^{134} \mathrm{Cs}$ & $4.2 \mathrm{E}-05 \pm 7.0 \mathrm{E}-05$ & $\mathrm{U}$ \\
\hline & 04/23/08 & 05/08/08 & gross $\beta$ & $1.5 \mathrm{E}-02 \pm 1.9 \mathrm{E}-03$ & & & ${ }^{137} \mathrm{Cs}$ & $3.2 \mathrm{E}-06 \pm 3.2 \mathrm{E}-05$ & $\mathrm{U}$ \\
\hline & 05/08/08 & $05 / 20 / 08$ & gross $\alpha$ & $1.7 \mathrm{E}-03 \pm 6.6 \mathrm{E}-04$ & & & ${ }^{152} \mathrm{Eu}$ & $1.2 \mathrm{E}-05 \pm 1.2 \mathrm{E}-04$ & $\mathrm{U}$ \\
\hline & 05/08/08 & 05/20/08 & gross $\beta$ & $8.6 \mathrm{E}-03 \pm 1.4 \mathrm{E}-03$ & & & ${ }^{154} \mathrm{Eu}$ & $1.3 \mathrm{E}-04 \pm 2.1 \mathrm{E}-04$ & $\mathrm{U}$ \\
\hline & 05/20/08 & 06/03/08 & gross $\alpha$ & $7.2 \mathrm{E}-04 \pm 5.2 \mathrm{E}-04$ & & & ${ }^{155} \mathrm{Eu}$ & $-1.4 \mathrm{E}-04 \pm 1.7 \mathrm{E}-04$ & $\mathrm{U}$ \\
\hline & 05/20/08 & 06/03/08 & gross $\beta$ & $9.8 \mathrm{E}-03 \pm 1.4 \mathrm{E}-03$ & & & ${ }^{238} \mathrm{Pu}$ & $6.0 \mathrm{E}-06 \pm 1.1 \mathrm{E}-05$ & $\mathrm{U}$ \\
\hline & 06/03/08 & $06 / 17 / 08$ & gross $\alpha$ & $1.0 \mathrm{E}-03 \pm 4.8 \mathrm{E}-04$ & & & ${ }^{239 / 240} \mathrm{Pu}$ & $7.8 \mathrm{E}-07 \pm 3.0 \mathrm{E}-06$ & $\mathrm{U}$ \\
\hline & 06/03/08 & 06/17/08 & gross $\beta$ & $8.9 \mathrm{E}-03 \pm 1.4 \mathrm{E}-03$ & & & ${ }^{106} \mathrm{Ru}$ & $3.3 \mathrm{E}-04 \pm 5.4 \mathrm{E}-04$ & $\mathrm{U}$ \\
\hline & 06/17/08 & 07/01/08 & gross $\alpha$ & $1.3 \mathrm{E}-03 \pm 5.5 \mathrm{E}-04$ & & & ${ }^{125} \mathrm{Sb}$ & $-1.3 \mathrm{E}-04 \pm 1.5 \mathrm{E}-04$ & $\mathrm{U}$ \\
\hline & 06/17/08 & 07/01/08 & gross $\beta$ & $1.3 \mathrm{E}-02 \pm 1.7 \mathrm{E}-03$ & & & ${ }^{90} \mathrm{Sr}$ & $-2.5 \mathrm{E}-04 \pm 2.6 \mathrm{E}-04$ & \\
\hline & 07/01/08 & 07/15/08 & gross $\alpha$ & $9.1 \mathrm{E}-04 \pm 6.4 \mathrm{E}-04$ & & & ${ }^{234} \mathrm{U}$ & $1.9 \mathrm{E}-05 \pm 9.8 \mathrm{E}-06$ & \\
\hline & 07/01/08 & 07/15/08 & gross $\beta$ & $1.3 \mathrm{E}-02 \pm 1.7 \mathrm{E}-03$ & & & ${ }^{235} \mathrm{U}$ & $3.5 \mathrm{E}-06 \pm 3.4 \mathrm{E}-06$ & \\
\hline & 07/15/08 & 07/29/08 & gross $\alpha$ & $8.3 \mathrm{E}-04 \pm 5.5 \mathrm{E}-04$ & & & ${ }^{238} \mathrm{U}$ & $1.5 \mathrm{E}-05 \pm 8.5 \mathrm{E}-06$ & \\
\hline & 07/15/08 & 07/29/08 & gross $\beta$ & $1.4 \mathrm{E}-02 \pm 1.8 \mathrm{E}-03$ & & & & & \\
\hline & 07/29/08 & 08/12/08 & gross $\alpha$ & $1.1 \mathrm{E}-03 \pm 5.0 \mathrm{E}-04$ & & & & & \\
\hline & 07/29/08 & 08/12/08 & gross $\beta$ & $1.6 \mathrm{E}-02 \pm 1.9 \mathrm{E}-03$ & & & & & \\
\hline & 08/12/08 & 08/25/08 & gross $\alpha$ & $4.4 \mathrm{E}-04 \pm 4.7 \mathrm{E}-04$ & & & & & \\
\hline & 08/12/08 & 08/25/08 & gross $\beta$ & $1.3 \mathrm{E}-02 \pm 1.8 \mathrm{E}-03$ & & & & & \\
\hline & 08/25/08 & 09/09/08 & gross $\alpha$ & $1.0 \mathrm{E}-03 \pm 4.6 \mathrm{E}-04$ & & & & & \\
\hline & 08/25/08 & 09/09/08 & gross $\beta$ & $1.1 \mathrm{E}-02 \pm 1.5 \mathrm{E}-03$ & & & & & \\
\hline & 09/09/08 & 09/23/08 & gross $\alpha$ & $1.7 \mathrm{E}-03 \pm 6.3 \mathrm{E}-04$ & & & & & \\
\hline & 09/09/08 & 09/23/08 & gross $\beta$ & $2.6 \mathrm{E}-02 \pm 2.7 \mathrm{E}-03$ & & & & & \\
\hline & 09/23/08 & $10 / 08 / 08$ & gross $\alpha$ & $2.3 \mathrm{E}-03 \pm 6.9 \mathrm{E}-04$ & & & & & \\
\hline & 09/23/08 & $10 / 08 / 08$ & gross $\beta$ & $2.2 \mathrm{E}-02 \pm 2.5 \mathrm{E}-03$ & & & & & \\
\hline & $10 / 08 / 08$ & $10 / 21 / 08$ & gross $\alpha$ & $1.6 \mathrm{E}-03 \pm 6.2 \mathrm{E}-04$ & & & & & \\
\hline & $10 / 08 / 08$ & $10 / 21 / 08$ & gross $\beta$ & $1.8 \mathrm{E}-02 \pm 2.2 \mathrm{E}-03$ & & & & & \\
\hline & $10 / 21 / 08$ & $11 / 04 / 08$ & gross $\alpha$ & $2.2 \mathrm{E}-03 \pm 7.1 \mathrm{E}-04$ & & & & & \\
\hline & $10 / 21 / 08$ & $11 / 04 / 08$ & gross $\beta$ & $3.9 \mathrm{E}-02 \pm 3.8 \mathrm{E}-03$ & & & & & \\
\hline & $11 / 04 / 08$ & $11 / 18 / 08$ & gross $\alpha$ & $1.6 \mathrm{E}-03 \pm 6.0 \mathrm{E}-04$ & & & & & \\
\hline & $11 / 04 / 08$ & 11/18/08 & gross $\beta$ & $2.1 \mathrm{E}-02 \pm 2.4 \mathrm{E}-03$ & & & & & \\
\hline & $11 / 18 / 08$ & $12 / 02 / 08$ & gross $\alpha$ & $1.5 \mathrm{E}-03 \pm 6.2 \mathrm{E}-04$ & & & & & \\
\hline & $11 / 18 / 08$ & $12 / 02 / 08$ & gross $\beta$ & $3.7 \mathrm{E}-02 \pm 3.7 \mathrm{E}-03$ & & & & & \\
\hline & $12 / 02 / 08$ & $12 / 16 / 08$ & gross $\alpha$ & $1.7 \mathrm{E}-03 \pm 6.3 \mathrm{E}-04$ & & & & & \\
\hline & $12 / 02 / 08$ & $12 / 16 / 08$ & gross $\beta$ & $2.7 \mathrm{E}-02 \pm 2.9 \mathrm{E}-03$ & & & & & \\
\hline & $12 / 16 / 08$ & $12 / 31 / 08$ & gross $\alpha$ & $1.6 \mathrm{E}-03 \pm 6.2 \mathrm{E}-04$ & & & & & \\
\hline & $12 / 16 / 08$ & $12 / 31 / 08$ & gross $\beta$ & $3.6 \mathrm{E}-02 \pm 3.5 \mathrm{E}-03$ & & & & & \\
\hline
\end{tabular}

$\overline{\mathrm{RQ}}=$ Result Qualifier. $\mathrm{U}=$ The analyte was analyzed for but not detected. 
Table 2-4. Near-Facility Air Sampling Results, 2008 (pCi/m³ \pm total analytical uncertainty). (Sheet 79 of 82)

\begin{tabular}{|c|c|c|c|c|c|c|c|c|c|}
\hline Location & Sample On & Sample Off & Isotope & Result \pm Uncertainty & Location & Composite Period & Isotope & Result \pm Uncertainty & $\mathbf{R Q *}$ \\
\hline N482 & $01 / 03 / 08$ & $01 / 15 / 08$ & gross $\alpha$ & $1.6 \mathrm{E}-03 \pm 6.5 \mathrm{E}-04$ & N482 & $01 / 03 / 08$ to $07 / 01 / 08$ & ${ }^{60} \mathrm{Co}$ & $-6.4 \mathrm{E}-05 \pm 1.4 \mathrm{E}-04$ & $\mathrm{U}$ \\
\hline \multirow[t]{51}{*}{ (ERDF) } & 01/03/08 & $01 / 15 / 08$ & gross $\beta$ & $5.4 \mathrm{E}-03 \pm 1.1 \mathrm{E}-03$ & & & ${ }^{134} \mathrm{Cs}$ & $1.5 \mathrm{E}-05 \pm 1.1 \mathrm{E}-04$ & $\mathrm{U}$ \\
\hline & $01 / 15 / 08$ & 01/30/08 & gross $\alpha$ & $1.7 \mathrm{E}-03 \pm 6.1 \mathrm{E}-04$ & & & ${ }^{137} \mathrm{Cs}$ & $-6.6 \mathrm{E}-05 \pm 1.1 \mathrm{E}-04$ & U \\
\hline & $01 / 15 / 08$ & 01/30/08 & gross $\beta$ & $2.0 \mathrm{E}-02 \pm 2.3 \mathrm{E}-03$ & & & ${ }^{152} \mathrm{Eu}$ & $3.0 \mathrm{E}-05 \pm 2.5 \mathrm{E}-04$ & U \\
\hline & 01/30/08 & $02 / 14 / 08$ & gross $\alpha$ & $7.2 \mathrm{E}-04 \pm 5.3 \mathrm{E}-04$ & & & ${ }^{154} \mathrm{Eu}$ & $-1.1 \mathrm{E}-04 \pm 4.4 \mathrm{E}-04$ & $\mathrm{U}$ \\
\hline & 01/30/08 & $02 / 14 / 08$ & gross $\beta$ & $3.3 \mathrm{E}-03 \pm 8.1 \mathrm{E}-04$ & & & ${ }^{155} \mathrm{Eu}$ & $2.4 \mathrm{E}-05 \pm 2.3 \mathrm{E}-04$ & U \\
\hline & $02 / 14 / 08$ & $02 / 28 / 08$ & gross $\alpha$ & $8.6 \mathrm{E}-04 \pm 5.8 \mathrm{E}-04$ & & & ${ }^{238} \mathrm{Pu}$ & $-1.2 \mathrm{E}-05 \pm 1.1 \mathrm{E}-05$ & $\mathrm{U}$ \\
\hline & $02 / 14 / 08$ & $02 / 28 / 08$ & gross $\beta$ & $2.8 \mathrm{E}-02 \pm 3.0 \mathrm{E}-03$ & & & ${ }^{239 / 240} \mathrm{Pu}$ & $4.3 \mathrm{E}-06 \pm 4.8 \mathrm{E}-06$ & $\mathrm{U}$ \\
\hline & $02 / 28 / 08$ & 03/12/08 & gross $\alpha$ & $9.9 \mathrm{E}-04 \pm 6.7 \mathrm{E}-04$ & & & ${ }^{106} \mathrm{Ru}$ & $-6.6 \mathrm{E}-04 \pm 1.0 \mathrm{E}-03$ & $\mathrm{U}$ \\
\hline & $02 / 28 / 08$ & $03 / 12 / 08$ & gross $\beta$ & $7.8 \mathrm{E}-03 \pm 1.4 \mathrm{E}-03$ & & & ${ }^{125} \mathrm{Sb}$ & $-9.6 \mathrm{E}-05 \pm 2.5 \mathrm{E}-04$ & $\mathrm{U}$ \\
\hline & 03/12/08 & 03/26/08 & gross $\alpha$ & $4.6 \mathrm{E}-04 \pm 5.1 \mathrm{E}-04$ & & & ${ }^{90} \mathrm{Sr}$ & $-2.3 \mathrm{E}-05 \pm 2.4 \mathrm{E}-05$ & $\mathrm{U}$ \\
\hline & 03/12/08 & 03/26/08 & gross $\beta$ & $7.7 \mathrm{E}-03 \pm 1.3 \mathrm{E}-03$ & & & ${ }^{234} \mathrm{U}$ & $4.0 \mathrm{E}-05 \pm 1.8 \mathrm{E}-05$ & \\
\hline & 03/26/08 & $04 / 08 / 08$ & gross $\alpha$ & $8.9 \mathrm{E}-04 \pm 6.6 \mathrm{E}-04$ & & & ${ }^{235} \mathrm{U}$ & $2.3 \mathrm{E}-06 \pm 2.8 \mathrm{E}-06$ & \\
\hline & 03/26/08 & $04 / 08 / 08$ & gross $\beta$ & $7.2 \mathrm{E}-03 \pm 1.3 \mathrm{E}-03$ & & & ${ }^{238} \mathrm{U}$ & $3.7 \mathrm{E}-05 \pm 1.7 \mathrm{E}-05$ & \\
\hline & 04/08/08 & 04/23/08 & gross $\alpha$ & $1.3 \mathrm{E}-03 \pm 5.8 \mathrm{E}-04$ & & & & & \\
\hline & $04 / 08 / 08$ & $04 / 23 / 08$ & gross $\beta$ & $8.4 \mathrm{E}-03 \pm 1.4 \mathrm{E}-03$ & N482 & $07 / 01 / 08$ to $12 / 31 / 08$ & ${ }^{60} \mathrm{Co}$ & $7.1 \mathrm{E}-05 \pm 1.1 \mathrm{E}-04$ & $\mathrm{U}$ \\
\hline & $04 / 23 / 08$ & $05 / 08 / 08$ & gross $\alpha$ & $1.3 \mathrm{E}-03 \pm 6.1 \mathrm{E}-04$ & & & ${ }^{134} \mathrm{Cs}$ & $-8.7 \mathrm{E}-06 \pm 8.7 \mathrm{E}-05$ & $\mathrm{U}$ \\
\hline & $04 / 23 / 08$ & 05/08/08 & gross $\beta$ & $8.1 \mathrm{E}-03 \pm 1.4 \mathrm{E}-03$ & & & ${ }^{137} \mathrm{Cs}$ & $9.4 \mathrm{E}-05 \pm 1.2 \mathrm{E}-04$ & $\mathrm{U}$ \\
\hline & 05/08/08 & 05/20/08 & gross $\alpha$ & $8.6 \mathrm{E}-04 \pm 6.9 \mathrm{E}-04$ & & & ${ }^{152} \mathrm{Eu}$ & $2.0 \mathrm{E}-04 \pm 2.9 \mathrm{E}-04$ & U \\
\hline & 05/08/08 & 05/20/08 & gross $\beta$ & $9.9 \mathrm{E}-03 \pm 1.6 \mathrm{E}-03$ & & & ${ }^{154} \mathrm{Eu}$ & $-5.0 \mathrm{E}-05 \pm 3.3 \mathrm{E}-04$ & $\mathrm{U}$ \\
\hline & 05/20/08 & 06/05/08 & gross $\alpha$ & $1.0 \mathrm{E}-03 \pm 6.6 \mathrm{E}-04$ & & & ${ }^{155} \mathrm{Eu}$ & $4.2 \mathrm{E}-05 \pm 1.9 \mathrm{E}-04$ & U \\
\hline & $05 / 20 / 08$ & $06 / 05 / 08$ & gross $\beta$ & $7.3 \mathrm{E}-03 \pm 1.3 \mathrm{E}-03$ & & & ${ }^{238} \mathrm{Pu}$ & $-3.2 \mathrm{E}-06 \pm 1.4 \mathrm{E}-05$ & $\mathrm{U}$ \\
\hline & $06 / 05 / 08$ & 06/18/08 & gross $\alpha$ & $5.5 \mathrm{E}-04 \pm 6.8 \mathrm{E}-04$ & & & ${ }^{239 / 240} \mathrm{Pu}$ & $1.6 \mathrm{E}-06 \pm 3.2 \mathrm{E}-06$ & $\mathrm{U}$ \\
\hline & $06 / 05 / 08$ & 06/18/08 & gross $\beta$ & $5.6 \mathrm{E}-03 \pm 1.2 \mathrm{E}-03$ & & & ${ }^{106} \mathrm{Ru}$ & $-2.2 \mathrm{E}-04 \pm 8.6 \mathrm{E}-04$ & $\mathrm{U}$ \\
\hline & 06/18/08 & 07/01/08 & gross $\alpha$ & $8.4 \mathrm{E}-04 \pm 6.3 \mathrm{E}-04$ & & & ${ }^{125} \mathrm{Sb}$ & $-1.2 \mathrm{E}-04 \pm 2.6 \mathrm{E}-04$ & $\mathrm{U}$ \\
\hline & $06 / 18 / 08$ & $07 / 01 / 08$ & gross $\beta$ & $1.3 \mathrm{E}-02 \pm 1.9 \mathrm{E}-03$ & & & ${ }^{90} \mathrm{Sr}$ & $9.0 \mathrm{E}-06 \pm 9.0 \mathrm{E}-05$ & \\
\hline & $07 / 01 / 08$ & $07 / 16 / 08$ & gross $\alpha$ & $1.5 \mathrm{E}-03 \pm 6.7 \mathrm{E}-04$ & & & ${ }^{234} \mathrm{U}$ & $3.2 \mathrm{E}-05 \pm 1.5 \mathrm{E}-05$ & \\
\hline & 07/01/08 & 07/16/08 & gross $\beta$ & $1.0 \mathrm{E}-02 \pm 1.5 \mathrm{E}-03$ & & & ${ }^{235} \mathrm{U}$ & $3.9 \mathrm{E}-06 \pm 4.4 \mathrm{E}-06$ & $\mathrm{U}$ \\
\hline & 07/16/08 & 07/30/08 & gross $\alpha$ & $1.1 \mathrm{E}-03 \pm 5.4 \mathrm{E}-04$ & & & ${ }^{238} \mathrm{U}$ & $2.4 \mathrm{E}-05 \pm 1.2 \mathrm{E}-05$ & \\
\hline & $07 / 16 / 08$ & 07/30/08 & gross $\beta$ & $1.9 \mathrm{E}-02 \pm 2.3 \mathrm{E}-03$ & & & & & \\
\hline & 07/30/08 & 08/13/08 & gross $\alpha$ & $6.7 \mathrm{E}-04 \pm 5.7 \mathrm{E}-04$ & & & & & \\
\hline & 07/30/08 & $08 / 13 / 08$ & gross $\beta$ & $1.4 \mathrm{E}-02 \pm 2.0 \mathrm{E}-03$ & & & & & \\
\hline & 08/13/08 & $08 / 26 / 08$ & gross $\alpha$ & $6.0 \mathrm{E}-04 \pm 5.8 \mathrm{E}-04$ & & & & & \\
\hline & 08/13/08 & 08/26/08 & gross $\beta$ & $1.2 \mathrm{E}-02 \pm 1.9 \mathrm{E}-03$ & & & & & \\
\hline & $08 / 26 / 08$ & 09/09/08 & gross $\alpha$ & $1.0 \mathrm{E}-03 \pm 5.0 \mathrm{E}-04$ & & & & & \\
\hline & 08/26/08 & 09/09/08 & gross $\beta$ & $9.4 \mathrm{E}-03 \pm 1.5 \mathrm{E}-03$ & & & & & \\
\hline & 09/09/08 & 09/23/08 & gross $\alpha$ & $9.3 \mathrm{E}-04 \pm 5.9 \mathrm{E}-04$ & & & & & \\
\hline & 09/09/08 & 09/23/08 & gross $\beta$ & $2.1 \mathrm{E}-02 \pm 2.5 \mathrm{E}-03$ & & & & & \\
\hline & $09 / 23 / 08$ & $10 / 08 / 08$ & gross $\alpha$ & $8.1 \mathrm{E}-04 \pm 5.5 \mathrm{E}-04$ & & & & & \\
\hline & 09/23/08 & $10 / 08 / 08$ & gross $\beta$ & $1.8 \mathrm{E}-02 \pm 2.2 \mathrm{E}-03$ & & & & & \\
\hline & $10 / 08 / 08$ & $10 / 21 / 08$ & gross $\alpha$ & $1.6 \mathrm{E}-03 \pm 6.4 \mathrm{E}-04$ & & & & & \\
\hline & $10 / 08 / 08$ & $10 / 21 / 08$ & gross $\beta$ & $1.9 \mathrm{E}-02 \pm 2.4 \mathrm{E}-03$ & & & & & \\
\hline & $10 / 21 / 08$ & $11 / 04 / 08$ & gross $\alpha$ & $3.0 \mathrm{E}-03 \pm 8.3 \mathrm{E}-04$ & & & & & \\
\hline & $10 / 21 / 08$ & $11 / 04 / 08$ & gross $\beta$ & 4.3E-02 $\pm 4.1 \mathrm{E}-03$ & & & & & \\
\hline & $11 / 04 / 08$ & $11 / 18 / 08$ & gross $\alpha$ & $1.9 \mathrm{E}-03 \pm 6.7 \mathrm{E}-04$ & & & & & \\
\hline & $11 / 04 / 08$ & $11 / 18 / 08$ & gross $\beta$ & $2.5 \mathrm{E}-02 \pm 2.8 \mathrm{E}-03$ & & & & & \\
\hline & $11 / 18 / 08$ & $12 / 02 / 08$ & gross $\alpha$ & $7.8 \mathrm{E}-04 \pm 5.6 \mathrm{E}-04$ & & & & & \\
\hline & $11 / 18 / 08$ & $12 / 02 / 08$ & gross $\beta$ & $3.1 \mathrm{E}-02 \pm 3.2 \mathrm{E}-03$ & & & & & \\
\hline & $12 / 02 / 08$ & $12 / 16 / 08$ & gross $\alpha$ & $1.4 \mathrm{E}-03 \pm 5.8 \mathrm{E}-04$ & & & & & \\
\hline & $12 / 02 / 08$ & $12 / 16 / 08$ & gross $\beta$ & $1.7 \mathrm{E}-02 \pm 2.1 \mathrm{E}-03$ & & & & & \\
\hline & $12 / 16 / 08$ & $12 / 31 / 08$ & gross $\alpha$ & $1.3 \mathrm{E}-03 \pm 5.3 \mathrm{E}-04$ & & & & & \\
\hline & $12 / 16 / 08$ & $12 / 31 / 08$ & gross $\beta$ & $3.5 \mathrm{E}-02 \pm 3.4 \mathrm{E}-03$ & & & & & \\
\hline
\end{tabular}

$\overline{\mathrm{RQ}}=$ Result Qualifier. $\mathrm{U}=$ The analyte was analyzed for but not detected. 
Table 2-4. Near-Facility Air Sampling Results, 2008 (pCi/m³ \pm total analytical uncertainty). (Sheet 80 of 82)

\begin{tabular}{|c|c|c|c|c|c|c|c|c|c|}
\hline Location & Sample On & Sample Off & Isotope & Result \pm Uncertainty & Location & Composite Period & Isotope & Result \pm Uncertainty & $\mathbf{R Q *}$ \\
\hline N517 & $01 / 03 / 08$ & $01 / 15 / 08$ & gross $\alpha$ & $5.6 \mathrm{E}-04 \pm 5.3 \mathrm{E}-04$ & N517 & $01 / 03 / 08$ to $07 / 01 / 08$ & ${ }^{60} \mathrm{Co}$ & $-4.4 \mathrm{E}-05 \pm 1.2 \mathrm{E}-04$ & $\mathrm{U}$ \\
\hline \multirow[t]{51}{*}{ (ERDF) } & 01/03/08 & $01 / 15 / 08$ & gross $\beta$ & $6.9 \mathrm{E}-03 \pm 1.3 \mathrm{E}-03$ & & & ${ }^{134} \mathrm{Cs}$ & $-2.1 \mathrm{E}-05 \pm 9.6 \mathrm{E}-05$ & $\mathrm{U}$ \\
\hline & $01 / 15 / 08$ & 01/30/08 & gross $\alpha$ & $1.4 \mathrm{E}-03 \pm 5.7 \mathrm{E}-04$ & & & ${ }^{137} \mathrm{Cs}$ & $1.5 \mathrm{E}-04 \pm 1.1 \mathrm{E}-04$ & U \\
\hline & $01 / 15 / 08$ & 01/30/08 & gross $\beta$ & $2.6 \mathrm{E}-02 \pm 2.7 \mathrm{E}-03$ & & & ${ }^{152} \mathrm{Eu}$ & $1.1 \mathrm{E}-04 \pm 2.3 \mathrm{E}-04$ & $\mathrm{U}$ \\
\hline & 01/30/08 & $02 / 14 / 08$ & gross $\alpha$ & $6.4 \mathrm{E}-04 \pm 7.1 \mathrm{E}-04$ & & & ${ }^{154} \mathrm{Eu}$ & $1.3 \mathrm{E}-04 \pm 3.1 \mathrm{E}-04$ & $\mathrm{U}$ \\
\hline & $01 / 30 / 08$ & $02 / 14 / 08$ & gross $\beta$ & $4.5 \mathrm{E}-03 \pm 1.2 \mathrm{E}-03$ & & & ${ }^{155} \mathrm{Eu}$ & $8.0 \mathrm{E}-05 \pm 2.1 \mathrm{E}-04$ & $\mathrm{U}$ \\
\hline & $02 / 14 / 08$ & $02 / 28 / 08$ & gross $\alpha$ & $1.2 \mathrm{E}-03 \pm 5.5 \mathrm{E}-04$ & & & ${ }^{238} \mathrm{Pu}$ & $7.6 \mathrm{E}-07 \pm 3.4 \mathrm{E}-06$ & $\mathrm{U}$ \\
\hline & $02 / 14 / 08$ & $02 / 28 / 08$ & gross $\beta$ & $3.0 \mathrm{E}-02 \pm 3.1 \mathrm{E}-03$ & & & ${ }^{239 / 240} \mathrm{Pu}$ & $6.8 \mathrm{E}-06 \pm 5.5 \mathrm{E}-06$ & \\
\hline & $02 / 28 / 08$ & 03/12/08 & gross $\alpha$ & $1.0 \mathrm{E}-03 \pm 7.1 \mathrm{E}-04$ & & & ${ }^{106} \mathrm{Ru}$ & $-2.0 \mathrm{E}-04 \pm 8.6 \mathrm{E}-04$ & $\mathrm{U}$ \\
\hline & $02 / 28 / 08$ & 03/12/08 & gross $\beta$ & $8.0 \mathrm{E}-03 \pm 1.4 \mathrm{E}-03$ & & & ${ }^{125} \mathrm{Sb}$ & $-1.2 \mathrm{E}-04 \pm 2.0 \mathrm{E}-04$ & $\mathrm{U}$ \\
\hline & 03/12/08 & 03/26/08 & gross $\alpha$ & $1.6 \mathrm{E}-03 \pm 6.7 \mathrm{E}-04$ & & & ${ }^{90} \mathrm{Sr}$ & $-2.1 \mathrm{E}-04 \pm 2.1 \mathrm{E}-04$ & $\mathrm{U}$ \\
\hline & 03/12/08 & 03/26/08 & gross $\beta$ & $5.4 \mathrm{E}-03 \pm 1.1 \mathrm{E}-03$ & & & ${ }^{234} \mathrm{U}$ & $4.9 \mathrm{E}-05 \pm 2.2 \mathrm{E}-05$ & \\
\hline & $03 / 26 / 08$ & $04 / 08 / 08$ & gross $\alpha$ & $1.1 \mathrm{E}-03 \pm 7.4 \mathrm{E}-04$ & & & ${ }^{235} \mathrm{U}$ & $1.0 \mathrm{E}-05 \pm 7.4 \mathrm{E}-06$ & \\
\hline & 03/26/08 & $04 / 08 / 08$ & gross $\beta$ & $7.2 \mathrm{E}-03 \pm 1.4 \mathrm{E}-03$ & & & ${ }^{238} \mathrm{U}$ & $2.5 \mathrm{E}-05 \pm 1.3 \mathrm{E}-05$ & \\
\hline & 04/08/08 & 04/23/08 & gross $\alpha$ & $1.2 \mathrm{E}-03 \pm 5.9 \mathrm{E}-04$ & & & & & \\
\hline & $04 / 08 / 08$ & $04 / 23 / 08$ & gross $\beta$ & $5.8 \mathrm{E}-03 \pm 1.2 \mathrm{E}-03$ & N517 & $07 / 01 / 08$ to $12 / 31 / 08$ & ${ }^{60} \mathrm{Co}$ & $1.3 \mathrm{E}-05 \pm 5.9 \mathrm{E}-05$ & $\mathrm{U}$ \\
\hline & $04 / 23 / 08$ & $05 / 08 / 08$ & gross $\alpha$ & $9.4 \mathrm{E}-04 \pm 7.0 \mathrm{E}-04$ & & & ${ }^{134} \mathrm{Cs}$ & $1.3 \mathrm{E}-05 \pm 6.4 \mathrm{E}-05$ & $\mathrm{U}$ \\
\hline & $04 / 23 / 08$ & 05/08/08 & gross $\beta$ & $5.6 \mathrm{E}-03 \pm 1.2 \mathrm{E}-03$ & & & ${ }^{137} \mathrm{Cs}$ & $1.1 \mathrm{E}-04 \pm 8.6 \mathrm{E}-05$ & \\
\hline & 05/08/08 & $05 / 20 / 08$ & gross $\alpha$ & $7.4 \mathrm{E}-04 \pm 1.0 \mathrm{E}-03$ & & & ${ }^{152} \mathrm{Eu}$ & $-1.3 \mathrm{E}-04 \pm 1.5 \mathrm{E}-04$ & $\mathrm{U}$ \\
\hline & 05/08/08 & 05/20/08 & gross $\beta$ & $1.1 \mathrm{E}-02 \pm 2.3 \mathrm{E}-03$ & & & ${ }^{154} \mathrm{Eu}$ & $-1.1 \mathrm{E}-04 \pm 1.9 \mathrm{E}-04$ & $\mathrm{U}$ \\
\hline & 05/20/08 & $06 / 05 / 08$ & gross $\alpha$ & $-5.4 \mathrm{E}-05 \pm 2.6 \mathrm{E}-04$ & & & ${ }^{155} \mathrm{Eu}$ & $-7.7 \mathrm{E}-05 \pm 1.6 \mathrm{E}-04$ & U \\
\hline & $05 / 20 / 08$ & $06 / 05 / 08$ & gross $\beta$ & $8.0 \mathrm{E}-03 \pm 1.4 \mathrm{E}-03$ & & & ${ }^{238} \mathrm{Pu}$ & $-2.3 \mathrm{E}-06 \pm 1.1 \mathrm{E}-05$ & $\mathrm{U}$ \\
\hline & $06 / 05 / 08$ & 06/18/08 & gross $\alpha$ & $8.5 \mathrm{E}-04 \pm 9.2 \mathrm{E}-04$ & & & ${ }^{239 / 240} \mathrm{Pu}$ & $3.9 \mathrm{E}-06 \pm 5.3 \mathrm{E}-06$ & $\mathrm{U}$ \\
\hline & $06 / 05 / 08$ & 06/18/08 & gross $\beta$ & $4.7 \mathrm{E}-04 \pm 7.8 \mathrm{E}-04$ & & & ${ }^{106} \mathrm{Ru}$ & $-1.9 \mathrm{E}-04 \pm 5.1 \mathrm{E}-04$ & $\mathrm{U}$ \\
\hline & 06/18/08 & 07/01/08 & gross $\alpha$ & $3.5 \mathrm{E}-04 \pm 7.6 \mathrm{E}-04$ & & & ${ }^{125} \mathrm{Sb}$ & $-1.5 \mathrm{E}-05 \pm 1.4 \mathrm{E}-04$ & $\mathrm{U}$ \\
\hline & $06 / 18 / 08$ & $07 / 01 / 08$ & gross $\beta$ & $1.6 \mathrm{E}-02 \pm 2.7 \mathrm{E}-03$ & & & ${ }^{90} \mathrm{Sr}$ & $-1.4 \mathrm{E}-04 \pm 1.5 \mathrm{E}-04$ & $\mathrm{U}$ \\
\hline & $07 / 01 / 08$ & $07 / 16 / 08$ & gross $\alpha$ & $1.3 \mathrm{E}-03 \pm 5.6 \mathrm{E}-04$ & & & ${ }^{234} \mathrm{U}$ & $2.4 \mathrm{E}-05 \pm 1.4 \mathrm{E}-05$ & \\
\hline & 07/01/08 & $07 / 16 / 08$ & gross $\beta$ & $1.1 \mathrm{E}-02 \pm 1.6 \mathrm{E}-03$ & & & ${ }^{235} \mathrm{U}$ & $5.1 \mathrm{E}-06 \pm 5.8 \mathrm{E}-06$ & $\mathrm{U}$ \\
\hline & 07/16/08 & 07/30/08 & gross $\alpha$ & $6.4 \mathrm{E}-04 \pm 5.2 \mathrm{E}-04$ & & & ${ }^{238} \mathrm{U}$ & $3.4 \mathrm{E}-05 \pm 1.7 \mathrm{E}-05$ & \\
\hline & $07 / 16 / 08$ & 07/30/08 & gross $\beta$ & $1.2 \mathrm{E}-02 \pm 1.7 \mathrm{E}-03$ & & & & & \\
\hline & 07/30/08 & 08/13/08 & gross $\alpha$ & $5.8 \mathrm{E}-04 \pm 5.3 \mathrm{E}-04$ & & & & & \\
\hline & 07/30/08 & $08 / 13 / 08$ & gross $\beta$ & $7.0 \mathrm{E}-03 \pm 1.2 \mathrm{E}-03$ & & & & & \\
\hline & 08/13/08 & $08 / 26 / 08$ & gross $\alpha$ & $6.7 \mathrm{E}-04 \pm 6.1 \mathrm{E}-04$ & & & & & \\
\hline & 08/13/08 & 08/26/08 & gross $\beta$ & $1.1 \mathrm{E}-02 \pm 1.7 \mathrm{E}-03$ & & & & & \\
\hline & $08 / 26 / 08$ & 09/09/08 & gross $\alpha$ & $3.2 \mathrm{E}-04 \pm 4.3 \mathrm{E}-04$ & & & & & \\
\hline & 08/26/08 & 09/09/08 & gross $\beta$ & $5.3 \mathrm{E}-03 \pm 1.1 \mathrm{E}-03$ & & & & & \\
\hline & 09/09/08 & 09/23/08 & gross $\alpha$ & $9.2 \mathrm{E}-04 \pm 6.1 \mathrm{E}-04$ & & & & & \\
\hline & 09/09/08 & 09/23/08 & gross $\beta$ & $1.4 \mathrm{E}-02 \pm 1.9 \mathrm{E}-03$ & & & & & \\
\hline & $09 / 23 / 08$ & $10 / 08 / 08$ & gross $\alpha$ & $3.0 \mathrm{E}-04 \pm 4.0 \mathrm{E}-04$ & & & & & \\
\hline & 09/23/08 & $10 / 08 / 08$ & gross $\beta$ & $1.2 \mathrm{E}-02 \pm 1.6 \mathrm{E}-03$ & & & & & \\
\hline & $10 / 08 / 08$ & $10 / 21 / 08$ & gross $\alpha$ & $7.4 \mathrm{E}-04 \pm 5.9 \mathrm{E}-04$ & & & & & \\
\hline & $10 / 08 / 08$ & $10 / 21 / 08$ & gross $\beta$ & $1.2 \mathrm{E}-02 \pm 1.7 \mathrm{E}-03$ & & & & & \\
\hline & $10 / 21 / 08$ & $11 / 04 / 08$ & gross $\alpha$ & $1.3 \mathrm{E}-03 \pm 5.3 \mathrm{E}-04$ & & & & & \\
\hline & $10 / 21 / 08$ & $11 / 04 / 08$ & gross $\beta$ & $3.7 \mathrm{E}-02 \pm 3.5 \mathrm{E}-03$ & & & & & \\
\hline & $11 / 04 / 08$ & $11 / 18 / 08$ & gross $\alpha$ & $4.4 \mathrm{E}-04 \pm 4.7 \mathrm{E}-04$ & & & & & \\
\hline & $11 / 04 / 08$ & $11 / 18 / 08$ & gross $\beta$ & $1.8 \mathrm{E}-02 \pm 2.2 \mathrm{E}-03$ & & & & & \\
\hline & $11 / 18 / 08$ & $12 / 02 / 08$ & gross $\alpha$ & $1.8 \mathrm{E}-03 \pm 6.4 \mathrm{E}-04$ & & & & & \\
\hline & $11 / 18 / 08$ & $12 / 02 / 08$ & gross $\beta$ & $3.1 \mathrm{E}-02 \pm 3.2 \mathrm{E}-03$ & & & & & \\
\hline & $12 / 02 / 08$ & $12 / 16 / 08$ & gross $\alpha$ & $7.3 \mathrm{E}-04 \pm 5.4 \mathrm{E}-04$ & & & & & \\
\hline & $12 / 02 / 08$ & $12 / 16 / 08$ & gross $\beta$ & $1.6 \mathrm{E}-02 \pm 2.0 \mathrm{E}-03$ & & & & & \\
\hline & $12 / 16 / 08$ & $12 / 31 / 08$ & gross $\alpha$ & $1.5 \mathrm{E}-03 \pm 5.7 \mathrm{E}-04$ & & & & & \\
\hline & $12 / 16 / 08$ & $12 / 31 / 08$ & gross $\beta$ & $3.6 \mathrm{E}-02 \pm 3.4 \mathrm{E}-03$ & & & & & \\
\hline
\end{tabular}

$\overline{\mathrm{RQ}}=$ Result Qualifier. $\mathrm{U}=$ The analyte was analyzed for but not detected. 
Table 2-4. Near-Facility Air Sampling Results, 2008 (pCi/m³ \pm total analytical uncertainty). (Sheet 81 of 82 )

\begin{tabular}{|c|c|c|c|c|c|c|c|c|c|}
\hline Location & Sample On & Sample Off & Isotope & Result \pm Uncertainty & Location & Composite Period & Isotope & Result \pm Uncertainty & $\mathbf{R Q *}$ \\
\hline N518 & $01 / 03 / 08$ & $01 / 15 / 08$ & gross $\alpha$ & $3.1 \mathrm{E}-04 \pm 4.4 \mathrm{E}-04$ & N518 & $01 / 03 / 08$ to $07 / 01 / 08$ & ${ }^{60} \mathrm{Co}$ & $-2.1 \mathrm{E}-05 \pm 6.9 \mathrm{E}-05$ & $\mathrm{U}$ \\
\hline \multirow[t]{51}{*}{ (ERDF) } & 01/03/08 & $01 / 15 / 08$ & gross $\beta$ & $7.1 \mathrm{E}-03 \pm 1.3 \mathrm{E}-03$ & & & ${ }^{134} \mathrm{Cs}$ & $3.6 \mathrm{E}-06 \pm 3.6 \mathrm{E}-05$ & $\mathrm{U}$ \\
\hline & 01/15/08 & 01/30/08 & gross $\alpha$ & $2.2 \mathrm{E}-03 \pm 7.0 \mathrm{E}-04$ & & & ${ }^{137} \mathrm{Cs}$ & $3.7 \mathrm{E}-07 \pm 3.7 \mathrm{E}-06$ & $\mathrm{U}$ \\
\hline & $01 / 15 / 08$ & 01/30/08 & gross $\beta$ & $3.2 \mathrm{E}-02 \pm 3.2 \mathrm{E}-03$ & & & ${ }^{152} \mathrm{Eu}$ & $-1.4 \mathrm{E}-04 \pm 1.5 \mathrm{E}-04$ & $\mathrm{U}$ \\
\hline & 01/30/08 & $02 / 14 / 08$ & gross $\alpha$ & $4.2 \mathrm{E}-04 \pm 5.9 \mathrm{E}-04$ & & & ${ }^{154} \mathrm{Eu}$ & $1.6 \mathrm{E}-06 \pm 1.6 \mathrm{E}-05$ & $\mathrm{U}$ \\
\hline & $01 / 30 / 08$ & $02 / 14 / 08$ & gross $\beta$ & $4.5 \mathrm{E}-03 \pm 1.1 \mathrm{E}-03$ & & & ${ }^{155} \mathrm{Eu}$ & $-2.8 \mathrm{E}-05 \pm 1.3 \mathrm{E}-04$ & $\mathrm{U}$ \\
\hline & $02 / 14 / 08$ & $02 / 28 / 08$ & gross $\alpha$ & $8.1 \mathrm{E}-04 \pm 5.5 \mathrm{E}-04$ & & & ${ }^{238} \mathrm{Pu}$ & $6.1 \mathrm{E}-07 \pm 1.2 \mathrm{E}-06$ & $\mathrm{U}$ \\
\hline & $02 / 14 / 08$ & $02 / 28 / 08$ & gross $\beta$ & $2.9 \mathrm{E}-02 \pm 3.0 \mathrm{E}-03$ & & & ${ }^{239 / 240} \mathrm{Pu}$ & $1.2 \mathrm{E}-06 \pm 1.8 \mathrm{E}-06$ & $\mathrm{U}$ \\
\hline & $02 / 28 / 08$ & 03/12/08 & gross $\alpha$ & $8.9 \mathrm{E}-04 \pm 6.0 \mathrm{E}-04$ & & & ${ }^{106} \mathrm{Ru}$ & $-8.1 \mathrm{E}-05 \pm 5.5 \mathrm{E}-04$ & $\mathrm{U}$ \\
\hline & $02 / 28 / 08$ & 03/12/08 & gross $\beta$ & $1.0 \mathrm{E}-02 \pm 1.5 \mathrm{E}-03$ & & & ${ }^{125} \mathrm{Sb}$ & $-6.3 \mathrm{E}-05 \pm 1.3 \mathrm{E}-04$ & $\mathrm{U}$ \\
\hline & 03/12/08 & 03/26/08 & gross $\alpha$ & $6.3 \mathrm{E}-04 \pm 5.2 \mathrm{E}-04$ & & & ${ }^{90} \mathrm{Sr}$ & $-1.7 \mathrm{E}-04 \pm 1.8 \mathrm{E}-04$ & $\mathrm{U}$ \\
\hline & 03/12/08 & 03/26/08 & gross $\beta$ & $5.4 \mathrm{E}-03 \pm 1.1 \mathrm{E}-03$ & & & ${ }^{234} \mathrm{U}$ & $2.0 \mathrm{E}-05 \pm 1.1 \mathrm{E}-05$ & \\
\hline & 03/26/08 & $04 / 08 / 08$ & gross $\alpha$ & $9.0 \mathrm{E}-04 \pm 6.1 \mathrm{E}-04$ & & & ${ }^{235} \mathrm{U}$ & $5.4 \mathrm{E}-06 \pm 5.5 \mathrm{E}-06$ & $\mathrm{U}$ \\
\hline & $03 / 26 / 08$ & $04 / 08 / 08$ & gross $\beta$ & $5.8 \mathrm{E}-03 \pm 1.1 \mathrm{E}-03$ & & & ${ }^{238} \mathrm{U}$ & $1.9 \mathrm{E}-05 \pm 1.1 \mathrm{E}-05$ & \\
\hline & 04/08/08 & 04/23/08 & gross $\alpha$ & $8.9 \mathrm{E}-04 \pm 5.5 \mathrm{E}-04$ & & & & & \\
\hline & $04 / 08 / 08$ & $04 / 23 / 08$ & gross $\beta$ & $9.9 \mathrm{E}-03 \pm 1.4 \mathrm{E}-03$ & N518 & $07 / 01 / 08$ to $12 / 31 / 08$ & ${ }^{60} \mathrm{Co}$ & $-2.5 \mathrm{E}-05 \pm 9.6 \mathrm{E}-05$ & $\mathrm{U}$ \\
\hline & $04 / 23 / 08$ & $05 / 08 / 08$ & gross $\alpha$ & $7.8 \mathrm{E}-04 \pm 5.3 \mathrm{E}-04$ & & & ${ }^{134} \mathrm{Cs}$ & $-5.4 \mathrm{E}-05 \pm 1.0 \mathrm{E}-04$ & $\mathrm{U}$ \\
\hline & $04 / 23 / 08$ & 05/08/08 & gross $\beta$ & $6.8 \mathrm{E}-03 \pm 1.1 \mathrm{E}-03$ & & & ${ }^{137} \mathrm{Cs}$ & $6.3 \mathrm{E}-05 \pm 1.0 \mathrm{E}-04$ & $\mathrm{U}$ \\
\hline & 05/08/08 & 05/20/08 & gross $\alpha$ & $9.9 \mathrm{E}-04 \pm 6.5 \mathrm{E}-04$ & & & ${ }^{152} \mathrm{Eu}$ & $-7.6 \mathrm{E}-05 \pm 2.3 \mathrm{E}-04$ & $\mathrm{U}$ \\
\hline & 05/08/08 & $05 / 20 / 08$ & gross $\beta$ & $6.1 \mathrm{E}-03 \pm 1.2 \mathrm{E}-03$ & & & ${ }^{154} \mathrm{Eu}$ & $-1.9 \mathrm{E}-05 \pm 2.0 \mathrm{E}-04$ & $\mathrm{U}$ \\
\hline & $05 / 20 / 08$ & $06 / 05 / 08$ & gross $\alpha$ & $4.5 \mathrm{E}-04 \pm 4.1 \mathrm{E}-04$ & & & ${ }^{155} \mathrm{Eu}$ & $-2.8 \mathrm{E}-06 \pm 2.8 \mathrm{E}-05$ & U \\
\hline & $05 / 20 / 08$ & $06 / 05 / 08$ & gross $\beta$ & $8.4 \mathrm{E}-03 \pm 1.3 \mathrm{E}-03$ & & & ${ }^{238} \mathrm{Pu}$ & $-2.9 \mathrm{E}-06 \pm 1.5 \mathrm{E}-05$ & $\mathrm{U}$ \\
\hline & $06 / 05 / 08$ & $06 / 18 / 08$ & gross $\alpha$ & $5.6 \mathrm{E}-04 \pm 5.1 \mathrm{E}-04$ & & & ${ }^{239 / 240} \mathrm{Pu}$ & $2.9 \mathrm{E}-06 \pm 3.1 \mathrm{E}-06$ & \\
\hline & $06 / 05 / 08$ & 06/18/08 & gross $\beta$ & $2.8 \mathrm{E}-03 \pm 7.6 \mathrm{E}-04$ & & & ${ }^{106} \mathrm{Ru}$ & $-3.0 \mathrm{E}-04 \pm 9.1 \mathrm{E}-04$ & $\mathrm{U}$ \\
\hline & 06/18/08 & 07/01/08 & gross $\alpha$ & $4.6 \mathrm{E}-04 \pm 5.2 \mathrm{E}-04$ & & & ${ }^{125} \mathrm{Sb}$ & $2.0 \mathrm{E}-05 \pm 2.0 \mathrm{E}-04$ & $\mathrm{U}$ \\
\hline & $06 / 18 / 08$ & 07/01/08 & gross $\beta$ & $9.7 \mathrm{E}-03 \pm 1.6 \mathrm{E}-03$ & & & ${ }^{90} \mathrm{Sr}$ & $-1.7 \mathrm{E}-04 \pm 1.7 \mathrm{E}-04$ & $\mathrm{U}$ \\
\hline & $07 / 01 / 08$ & $07 / 16 / 08$ & gross $\alpha$ & $1.1 \mathrm{E}-03 \pm 4.9 \mathrm{E}-04$ & & & ${ }^{234} \mathrm{U}$ & $5.4 \mathrm{E}-05 \pm 3.0 \mathrm{E}-05$ & \\
\hline & 07/01/08 & 07/16/08 & gross $\beta$ & $1.1 \mathrm{E}-02 \pm 1.5 \mathrm{E}-03$ & & & ${ }^{235} \mathrm{U}$ & $8.4 \mathrm{E}-06 \pm 9.0 \mathrm{E}-06$ & \\
\hline & 07/16/08 & 07/30/08 & gross $\alpha$ & $7.4 \mathrm{E}-04 \pm 5.3 \mathrm{E}-04$ & & & ${ }^{238} \mathrm{U}$ & $3.5 \mathrm{E}-05 \pm 2.1 \mathrm{E}-05$ & \\
\hline & $07 / 16 / 08$ & 07/30/08 & gross $\beta$ & $1.3 \mathrm{E}-02 \pm 1.7 \mathrm{E}-03$ & & & & & \\
\hline & 07/30/08 & 08/13/08 & gross $\alpha$ & $6.8 \mathrm{E}-04 \pm 5.5 \mathrm{E}-04$ & & & & & \\
\hline & 07/30/08 & 08/13/08 & gross $\beta$ & $1.3 \mathrm{E}-02 \pm 1.7 \mathrm{E}-03$ & & & & & \\
\hline & 08/13/08 & $08 / 26 / 08$ & gross $\alpha$ & $1.6 \mathrm{E}-03 \pm 6.1 \mathrm{E}-04$ & & & & & \\
\hline & 08/13/08 & 08/26/08 & gross $\beta$ & $1.2 \mathrm{E}-02 \pm 1.7 \mathrm{E}-03$ & & & & & \\
\hline & $08 / 26 / 08$ & 09/09/08 & gross $\alpha$ & $3.9 \mathrm{E}-04 \pm 4.2 \mathrm{E}-04$ & & & & & \\
\hline & 08/26/08 & 09/09/08 & gross $\beta$ & $1.0 \mathrm{E}-02 \pm 1.5 \mathrm{E}-03$ & & & & & \\
\hline & 09/09/08 & 09/23/08 & gross $\alpha$ & $1.3 \mathrm{E}-03 \pm 5.3 \mathrm{E}-04$ & & & & & \\
\hline & 09/09/08 & 09/23/08 & gross $\beta$ & $2.1 \mathrm{E}-02 \pm 2.4 \mathrm{E}-03$ & & & & & \\
\hline & 09/23/08 & $10 / 08 / 08$ & gross $\alpha$ & $5.8 \mathrm{E}-04 \pm 4.7 \mathrm{E}-04$ & & & & & \\
\hline & 09/23/08 & $10 / 08 / 08$ & gross $\beta$ & $1.8 \mathrm{E}-02 \pm 2.0 \mathrm{E}-03$ & & & & & \\
\hline & $10 / 08 / 08$ & $10 / 21 / 08$ & gross $\alpha$ & $1.3 \mathrm{E}-03 \pm 5.5 \mathrm{E}-04$ & & & & & \\
\hline & $10 / 08 / 08$ & $10 / 21 / 08$ & gross $\beta$ & $1.4 \mathrm{E}-02 \pm 1.8 \mathrm{E}-03$ & & & & & \\
\hline & $10 / 21 / 08$ & $11 / 04 / 08$ & gross $\alpha$ & $2.5 \mathrm{E}-03 \pm 7.7 \mathrm{E}-04$ & & & & & \\
\hline & $10 / 21 / 08$ & $11 / 04 / 08$ & gross $\beta$ & $3.7 \mathrm{E}-02 \pm 3.6 \mathrm{E}-03$ & & & & & \\
\hline & $11 / 04 / 08$ & $11 / 18 / 08$ & gross $\alpha$ & $2.8 \mathrm{E}-04 \pm 3.9 \mathrm{E}-04$ & & & & & \\
\hline & $11 / 04 / 08$ & $11 / 18 / 08$ & gross $\beta$ & $1.8 \mathrm{E}-02 \pm 2.1 \mathrm{E}-03$ & & & & & \\
\hline & $11 / 18 / 08$ & $12 / 02 / 08$ & gross $\alpha$ & $2.2 \mathrm{E}-03 \pm 6.9 \mathrm{E}-04$ & & & & & \\
\hline & $11 / 18 / 08$ & $12 / 02 / 08$ & gross $\beta$ & 3.3E-02 \pm 3.3E-03 & & & & & \\
\hline & $12 / 02 / 08$ & $12 / 16 / 08$ & gross $\alpha$ & $1.2 \mathrm{E}-03 \pm 5.2 \mathrm{E}-04$ & & & & & \\
\hline & $12 / 02 / 08$ & $12 / 16 / 08$ & gross $\beta$ & $2.1 \mathrm{E}-02 \pm 2.4 \mathrm{E}-03$ & & & & & \\
\hline & $12 / 16 / 08$ & $12 / 31 / 08$ & gross $\alpha$ & $2.4 \mathrm{E}-03 \pm 7.2 \mathrm{E}-04$ & & & & & \\
\hline & $12 / 16 / 08$ & $12 / 31 / 08$ & gross $\beta$ & $4.2 \mathrm{E}-02 \pm 3.9 \mathrm{E}-03$ & & & & & \\
\hline
\end{tabular}

$\overline{\mathrm{RQ}}=$ Result Qualifier. $\mathrm{U}=$ The analyte was analyzed for but not detected. 
Table 2-4. Near-Facility Air Sampling Results, 2008 (pCi/m³ \pm total analytical uncertainty). (Sheet 82 of 82)

\begin{tabular}{|c|c|c|c|c|c|c|c|c|c|}
\hline Location & Sample On & Sample Off & Isotope & Result \pm Uncertainty & Location & Composite Period & Isotope & Result \pm Uncertainty & $\mathbf{R Q}^{*}$ \\
\hline N981 & $01 / 02 / 08$ & $01 / 15 / 08$ & gross $\alpha$ & $1.0 \mathrm{E}-03 \pm 6.2 \mathrm{E}-04$ & N981 & $01 / 02 / 08$ to $07 / 01 / 08$ & ${ }^{60} \mathrm{Co}$ & $3.7 \mathrm{E}-05 \pm 6.9 \mathrm{E}-05$ & $\bar{U}$ \\
\hline \multirow[t]{51}{*}{ (600 Area) } & $01 / 02 / 08$ & $01 / 15 / 08$ & gross $\beta$ & $9.3 \mathrm{E}-03 \pm 1.4 \mathrm{E}-03$ & & & ${ }^{134} \mathrm{Cs}$ & $1.7 \mathrm{E}-05 \pm 6.3 \mathrm{E}-05$ & $\mathrm{U}$ \\
\hline & $01 / 15 / 08$ & 01/30/08 & gross $\alpha$ & $1.6 \mathrm{E}-03 \pm 5.9 \mathrm{E}-04$ & & & ${ }^{137} \mathrm{Cs}$ & $-1.2 \mathrm{E}-05 \pm 5.7 \mathrm{E}-05$ & U \\
\hline & $01 / 15 / 08$ & 01/30/08 & gross $\beta$ & $3.3 \mathrm{E}-02 \pm 3.2 \mathrm{E}-03$ & & & ${ }^{152} \mathrm{Eu}$ & $-1.6 \mathrm{E}-04 \pm 1.6 \mathrm{E}-04$ & U \\
\hline & 01/30/08 & 02/13/08 & gross $\alpha$ & $5.2 \mathrm{E}-04 \pm 4.8 \mathrm{E}-04$ & & & ${ }^{154} \mathrm{Eu}$ & $4.1 \mathrm{E}-05 \pm 2.2 \mathrm{E}-04$ & $\mathrm{U}$ \\
\hline & 01/30/08 & 02/13/08 & gross $\beta$ & $4.6 \mathrm{E}-03 \pm 9.5 \mathrm{E}-04$ & & & ${ }^{155} \mathrm{Eu}$ & $6.1 \mathrm{E}-05 \pm 1.3 \mathrm{E}-04$ & U \\
\hline & $02 / 13 / 08$ & $02 / 27 / 08$ & gross $\alpha$ & $3.0 \mathrm{E}-03 \pm 8.3 \mathrm{E}-04$ & & & ${ }^{238} \mathrm{Pu}$ & $2.8 \mathrm{E}-06 \pm 4.4 \mathrm{E}-06$ & $\mathrm{U}$ \\
\hline & $02 / 13 / 08$ & $02 / 27 / 08$ & gross $\beta$ & $3.2 \mathrm{E}-02 \pm 3.3 \mathrm{E}-03$ & & & ${ }^{239 / 240} \mathrm{Pu}$ & $5.5 \mathrm{E}-07 \pm 1.9 \mathrm{E}-06$ & $\mathrm{U}$ \\
\hline & $02 / 27 / 08$ & 03/12/08 & gross $\alpha$ & $6.2 \mathrm{E}-04 \pm 5.1 \mathrm{E}-04$ & & & ${ }^{106} \mathrm{Ru}$ & $4.6 \mathrm{E}-04 \pm 6.1 \mathrm{E}-04$ & $\mathrm{U}$ \\
\hline & $02 / 27 / 08$ & 03/12/08 & gross $\beta$ & $1.0 \mathrm{E}-02 \pm 1.5 \mathrm{E}-03$ & & & ${ }^{125} \mathrm{Sb}$ & $-2.1 \mathrm{E}-05 \pm 1.4 \mathrm{E}-04$ & $\mathrm{U}$ \\
\hline & 03/12/08 & 03/26/08 & gross $\alpha$ & $1.2 \mathrm{E}-03 \pm 5.2 \mathrm{E}-04$ & & & ${ }^{90} \mathrm{Sr}$ & $-1.4 \mathrm{E}-04 \pm 1.5 \mathrm{E}-04$ & $\mathrm{U}$ \\
\hline & 03/12/08 & 03/26/08 & gross $\beta$ & $8.5 \mathrm{E}-03 \pm 1.3 \mathrm{E}-03$ & & & ${ }^{234} \mathrm{U}$ & $7.5 \mathrm{E}-06 \pm 6.3 \mathrm{E}-06$ & \\
\hline & $03 / 26 / 08$ & $04 / 08 / 08$ & gross $\alpha$ & $9.3 \mathrm{E}-04 \pm 6.2 \mathrm{E}-04$ & & & ${ }^{235} \mathrm{U}$ & $6.8 \mathrm{E}-07 \pm 2.0 \mathrm{E}-07$ & $\mathrm{U}$ \\
\hline & 03/26/08 & $04 / 08 / 08$ & gross $\beta$ & $8.2 \mathrm{E}-03 \pm 1.3 \mathrm{E}-03$ & & & ${ }^{238} \mathrm{U}$ & $1.3 \mathrm{E}-05 \pm 7.7 \mathrm{E}-06$ & \\
\hline & 04/08/08 & 04/23/08 & gross $\alpha$ & $1.4 \mathrm{E}-03 \pm 5.7 \mathrm{E}-04$ & & & & & \\
\hline & $04 / 08 / 08$ & $04 / 23 / 08$ & gross $\beta$ & $1.0 \mathrm{E}-02 \pm 1.5 \mathrm{E}-03$ & N981 & $07 / 01 / 08$ to $12 / 31 / 08$ & ${ }^{60} \mathrm{Co}$ & $-1.1 \mathrm{E}-05 \pm 8.6 \mathrm{E}-05$ & $\mathrm{U}$ \\
\hline & $04 / 23 / 08$ & $05 / 08 / 08$ & gross $\alpha$ & $1.9 \mathrm{E}-03 \pm 6.5 \mathrm{E}-04$ & & & ${ }^{134} \mathrm{Cs}$ & $2.3 \mathrm{E}-05 \pm 7.4 \mathrm{E}-05$ & $\mathrm{U}$ \\
\hline & $04 / 23 / 08$ & 05/08/08 & gross $\beta$ & $1.6 \mathrm{E}-02 \pm 2.0 \mathrm{E}-03$ & & & ${ }^{137} \mathrm{Cs}$ & $1.2 \mathrm{E}-05 \pm 7.0 \mathrm{E}-05$ & $\mathrm{U}$ \\
\hline & 05/08/08 & 05/20/08 & gross $\alpha$ & $9.0 \mathrm{E}-04 \pm 6.7 \mathrm{E}-04$ & & & ${ }^{152} \mathrm{Eu}$ & $-7.4 \mathrm{E}-05 \pm 1.8 \mathrm{E}-04$ & U \\
\hline & 05/08/08 & $05 / 20 / 08$ & gross $\beta$ & $1.7 \mathrm{E}-02 \pm 2.3 \mathrm{E}-03$ & & & ${ }^{154} \mathrm{Eu}$ & $-1.2 \mathrm{E}-04 \pm 2.7 \mathrm{E}-04$ & $\mathrm{U}$ \\
\hline & $05 / 20 / 08$ & $06 / 03 / 08$ & gross $\alpha$ & $6.2 \mathrm{E}-04 \pm 5.1 \mathrm{E}-04$ & & & ${ }^{155} \mathrm{Eu}$ & $-4.0 \mathrm{E}-05 \pm 1.7 \mathrm{E}-04$ & $\mathrm{U}$ \\
\hline & $05 / 20 / 08$ & $06 / 03 / 08$ & gross $\beta$ & $1.1 \mathrm{E}-02 \pm 1.6 \mathrm{E}-03$ & & & ${ }^{238} \mathrm{Pu}$ & $1.1 \mathrm{E}-05 \pm 1.0 \mathrm{E}-05$ & $\mathrm{U}$ \\
\hline & $06 / 03 / 08$ & $06 / 17 / 08$ & gross $\alpha$ & $1.8 \mathrm{E}-03 \pm 7.4 \mathrm{E}-04$ & & & ${ }^{239 / 240} \mathrm{Pu}$ & $2.0 \mathrm{E}-06 \pm 3.0 \mathrm{E}-06$ & $\mathrm{U}$ \\
\hline & 06/03/08 & $06 / 17 / 08$ & gross $\beta$ & $9.5 \mathrm{E}-03 \pm 1.5 \mathrm{E}-03$ & & & ${ }^{106} \mathrm{Ru}$ & $-2.6 \mathrm{E}-04 \pm 6.1 \mathrm{E}-04$ & $\mathrm{U}$ \\
\hline & $06 / 17 / 08$ & 07/01/08 & gross $\alpha$ & $2.4 \mathrm{E}-03 \pm 8.6 \mathrm{E}-04$ & & & ${ }^{125} \mathrm{Sb}$ & $-7.8 \mathrm{E}-05 \pm 1.6 \mathrm{E}-04$ & $\mathrm{U}$ \\
\hline & $06 / 17 / 08$ & 07/01/08 & gross $\beta$ & $1.9 \mathrm{E}-02 \pm 2.3 \mathrm{E}-03$ & & & ${ }^{90} \mathrm{Sr}$ & $-1.4 \mathrm{E}-04 \pm 1.4 \mathrm{E}-04$ & $\mathrm{U}$ \\
\hline & $07 / 01 / 08$ & $07 / 15 / 08$ & gross $\alpha$ & $1.4 \mathrm{E}-03 \pm 6.0 \mathrm{E}-04$ & & & ${ }^{234} \mathrm{U}$ & $2.0 \mathrm{E}-05 \pm 1.1 \mathrm{E}-05$ & \\
\hline & 07/01/08 & 07/15/08 & gross $\beta$ & $1.7 \mathrm{E}-02 \pm 2.1 \mathrm{E}-03$ & & & ${ }^{235} \mathrm{U}$ & $5.7 \mathrm{E}-06 \pm 4.6 \mathrm{E}-06$ & \\
\hline & 07/15/08 & 07/29/08 & gross $\alpha$ & $7.6 \mathrm{E}-04 \pm 5.5 \mathrm{E}-04$ & & & ${ }^{238} \mathrm{U}$ & $8.5 \mathrm{E}-06 \pm 5.7 \mathrm{E}-06$ & \\
\hline & $07 / 15 / 08$ & 07/29/08 & gross $\beta$ & $1.5 \mathrm{E}-02 \pm 1.9 \mathrm{E}-03$ & & & & & \\
\hline & 07/29/08 & 08/12/08 & gross $\alpha$ & $1.3 \mathrm{E}-03 \pm 5.7 \mathrm{E}-04$ & & & & & \\
\hline & 07/29/08 & $08 / 12 / 08$ & gross $\beta$ & $1.6 \mathrm{E}-02 \pm 2.1 \mathrm{E}-03$ & & & & & \\
\hline & $08 / 12 / 08$ & $08 / 25 / 08$ & gross $\alpha$ & $8.1 \mathrm{E}-04 \pm 5.8 \mathrm{E}-04$ & & & & & \\
\hline & 08/12/08 & 08/25/08 & gross $\beta$ & $1.4 \mathrm{E}-02 \pm 1.9 \mathrm{E}-03$ & & & & & \\
\hline & $08 / 25 / 08$ & 09/09/08 & gross $\alpha$ & $3.8 \mathrm{E}-04 \pm 4.1 \mathrm{E}-04$ & & & & & \\
\hline & $08 / 25 / 08$ & 09/09/08 & gross $\beta$ & $1.1 \mathrm{E}-02 \pm 1.5 \mathrm{E}-03$ & & & & & \\
\hline & 09/09/08 & $09 / 23 / 08$ & gross $\alpha$ & $1.7 \mathrm{E}-03 \pm 6.3 \mathrm{E}-04$ & & & & & \\
\hline & 09/09/08 & 09/23/08 & gross $\beta$ & $2.8 \mathrm{E}-02 \pm 2.9 \mathrm{E}-03$ & & & & & \\
\hline & $09 / 23 / 08$ & $10 / 08 / 08$ & gross $\alpha$ & $1.7 \mathrm{E}-03 \pm 5.9 \mathrm{E}-04$ & & & & & \\
\hline & 09/23/08 & $10 / 08 / 08$ & gross $\beta$ & $2.5 \mathrm{E}-02 \pm 2.7 \mathrm{E}-03$ & & & & & \\
\hline & $10 / 08 / 08$ & $10 / 21 / 08$ & gross $\alpha$ & $2.2 \mathrm{E}-03 \pm 7.7 \mathrm{E}-04$ & & & & & \\
\hline & $10 / 08 / 08$ & $10 / 21 / 08$ & gross $\beta$ & $1.9 \mathrm{E}-02 \pm 2.3 \mathrm{E}-03$ & & & & & \\
\hline & $10 / 21 / 08$ & $11 / 04 / 08$ & gross $\alpha$ & $3.3 \mathrm{E}-03 \pm 9.2 \mathrm{E}-04$ & & & & & \\
\hline & $10 / 21 / 08$ & $11 / 04 / 08$ & gross $\beta$ & $4.1 \mathrm{E}-02 \pm 3.9 \mathrm{E}-03$ & & & & & \\
\hline & $11 / 04 / 08$ & $11 / 18 / 08$ & gross $\alpha$ & $1.6 \mathrm{E}-03 \pm 6.5 \mathrm{E}-04$ & & & & & \\
\hline & $11 / 04 / 08$ & $11 / 18 / 08$ & gross $\beta$ & $1.9 \mathrm{E}-02 \pm 2.3 \mathrm{E}-03$ & & & & & \\
\hline & $11 / 18 / 08$ & $12 / 02 / 08$ & gross $\alpha$ & $2.4 \mathrm{E}-03 \pm 7.7 \mathrm{E}-04$ & & & & & \\
\hline & $11 / 18 / 08$ & $12 / 02 / 08$ & gross $\beta$ & $3.0 \mathrm{E}-02 \pm 3.2 \mathrm{E}-03$ & & & & & \\
\hline & $12 / 02 / 08$ & $12 / 16 / 08$ & gross $\alpha$ & $2.3 \mathrm{E}-03 \pm 7.7 \mathrm{E}-04$ & & & & & \\
\hline & $12 / 02 / 08$ & $12 / 16 / 08$ & gross $\beta$ & $2.0 \mathrm{E}-02 \pm 2.3 \mathrm{E}-03$ & & & & & \\
\hline & $12 / 16 / 08$ & $12 / 31 / 08$ & gross $\alpha$ & $1.5 \mathrm{E}-03 \pm 5.8 \mathrm{E}-04$ & & & & & \\
\hline & $12 / 16 / 08$ & $12 / 31 / 08$ & gross $\beta$ & $2.6 \mathrm{E}-02 \pm 2.7 \mathrm{E}-03$ & & & & & \\
\hline
\end{tabular}

$\overline{\mathrm{RQ}}=$ Result Qualifier. $\mathrm{U}=$ The analyte was analyzed for but not detected. 
This page intentionally left blank. 


\subsection{SOIL MONITORING}

The radionuclide content of soil was measured to evaluate long-term trends in environmental accumulation of radioactivity in the 100, 200/600, and 300/400 Areas. Soil samples were collected on or near facilities that store, handle, or dispose of radioactive waste. The number of soil samples collected in 2008 and their locations are shown in Table 3-1.

Table 3-1. Soil Samples Collected during 2008.

\begin{tabular}{|c|c|c|c|c|c|c|c|c|c|c|}
\hline \multirow{2}{*}{$\begin{array}{c}\text { Number of } \\
\text { Samples }\end{array}$} & \multicolumn{10}{|c|}{ Operational Area } \\
\hline & $100-B / C$ & 100-D & $100-F$ & $100-\mathrm{H}$ & 200-West ${ }^{\mathrm{a}}$ & 200 -East $^{\mathrm{a}}$ & $600^{a, b}$ & $300^{\mathrm{a}}$ & 400 & ERDF $^{c}$ \\
\hline 95 & 3 & 4 & 5 & 4 & 27 & 15 & 18 & 17 & 1 & 1 \\
\hline
\end{tabular}

${ }^{a}$ Number of samples includes one or more Replicate Samples.

${ }^{\mathrm{b}}$ Includes soil sample at 100-IU2/6.

${ }^{\mathrm{c}}$ Environmental Restoration Disposal Facility in the 200 West Area.

Soil sampling locations are illustrated in Figures 3-1 through 3-10. Radionuclide analyses indicated that strontium-90, cesium-137, plutonium-239/240, and uranium were detectable in soil samples in 2008. Historically, the predominant radionuclides observed in soil samples were activation and fission products in the 100 Areas, fission products in the 200 Areas, and uranium in the 300 Area.

A summary of near-facility soil sampling results for selected radionuclides collected during 2008 is presented in Table 3-2. Historical soil sampling results for the 100, 200/600, and 300/400 Areas are displayed in Table 3-3. The 2008 soil sampling results for all areas are provided in Table 3-4.

Additional discussion of the 2008 soil sampling results can be found in Section 10.9.1 of PNNL-18427. 
Figure 3-1. 2008 Soil Sampling Locations, 100-B/C Area.

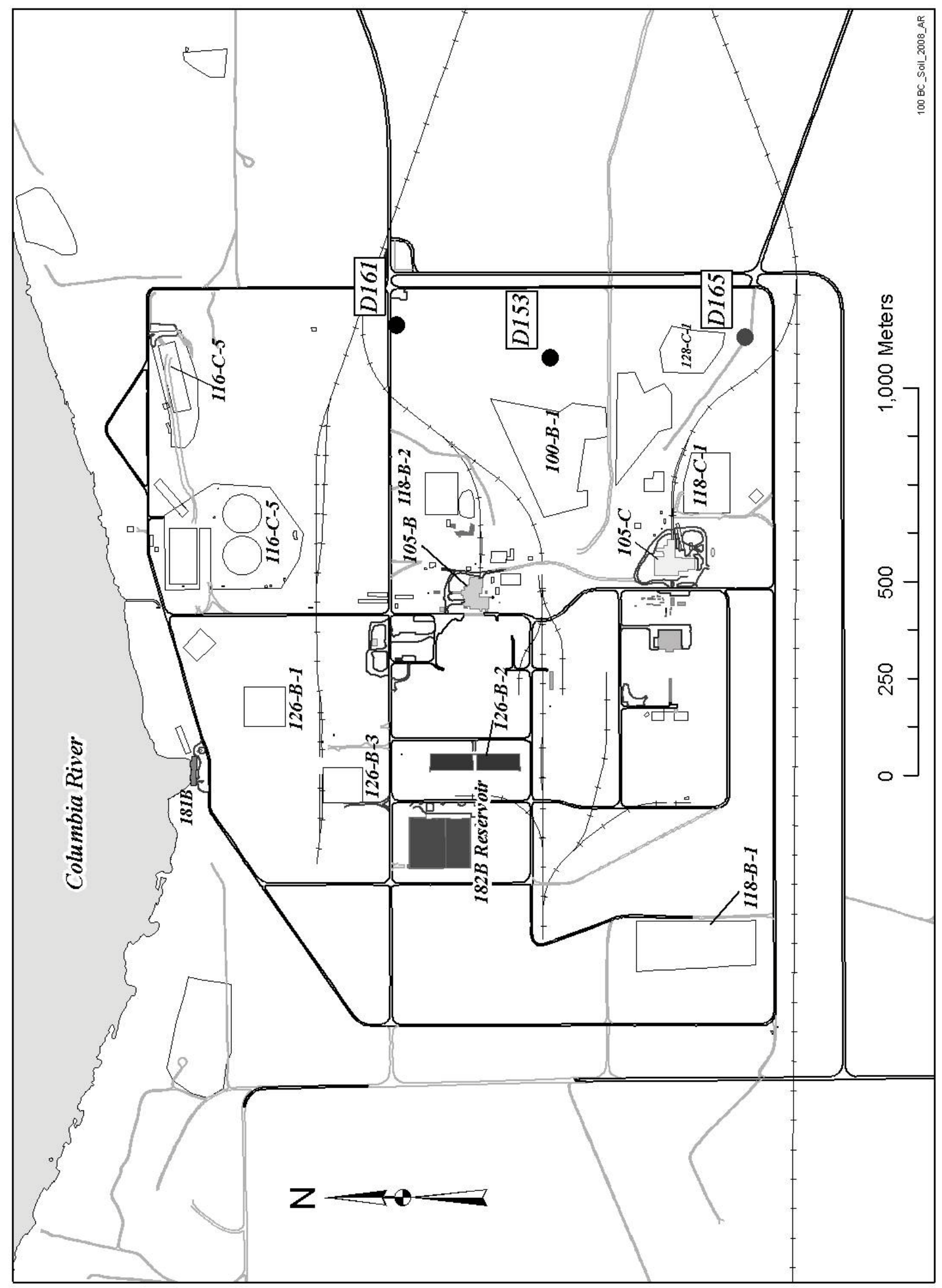


Figure 3-2. 2008 Soil Sampling Locations, 100-D Area.

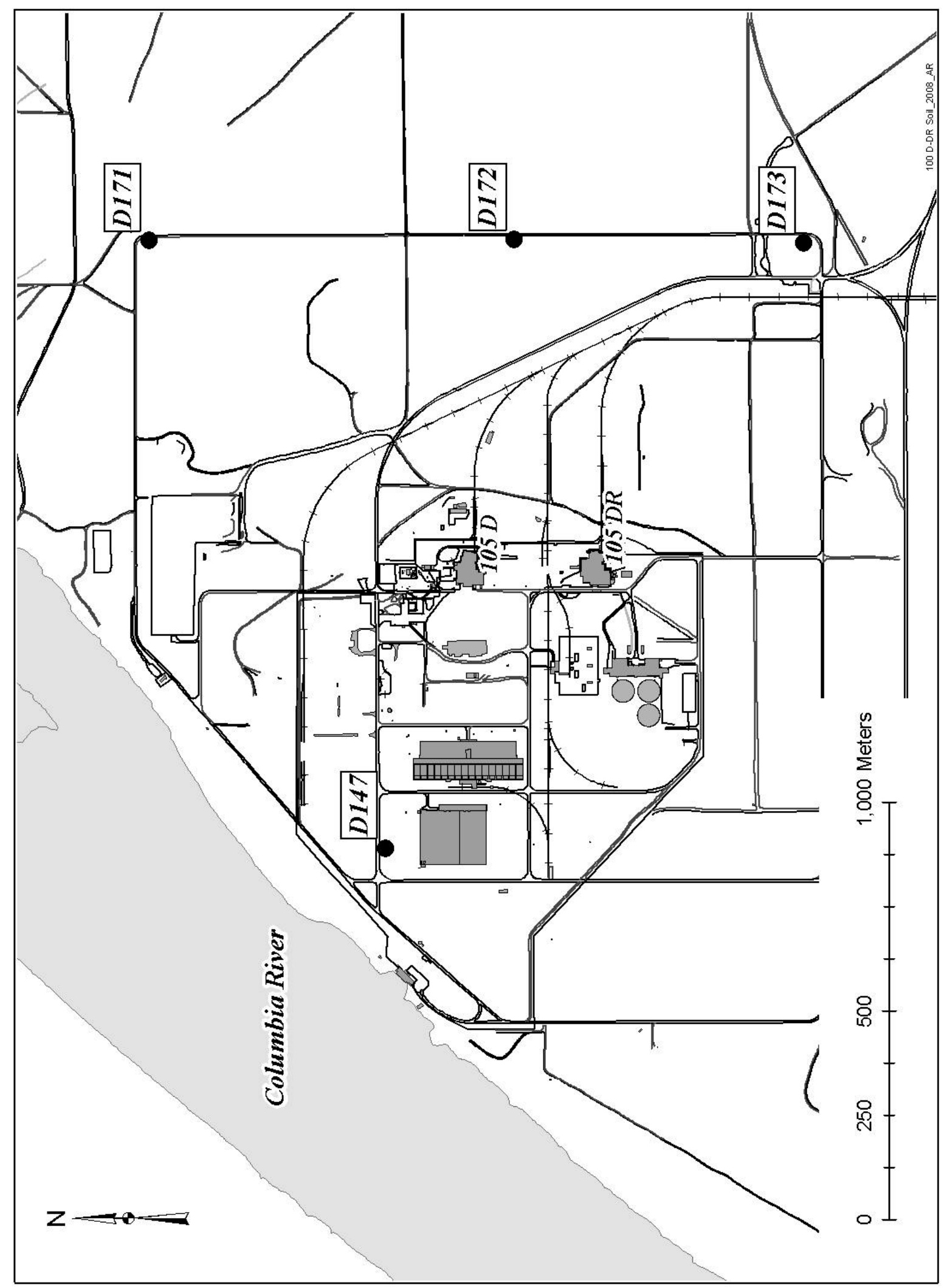


Figure 3-3. 2008 Soil Sampling Locations, 100-F Area.

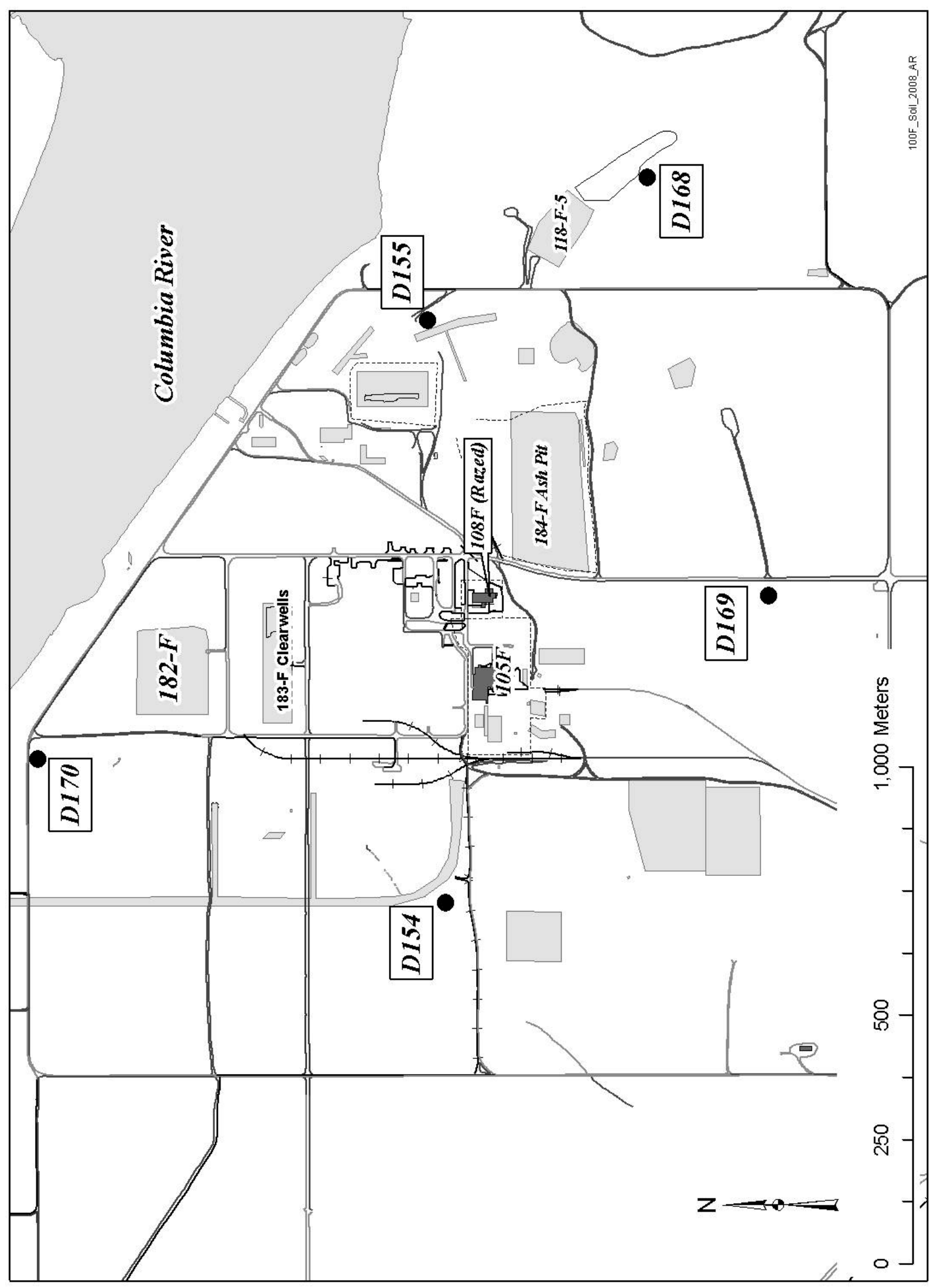


Figure 3-4. 2008 Soil Sampling Locations, 100-H Area.

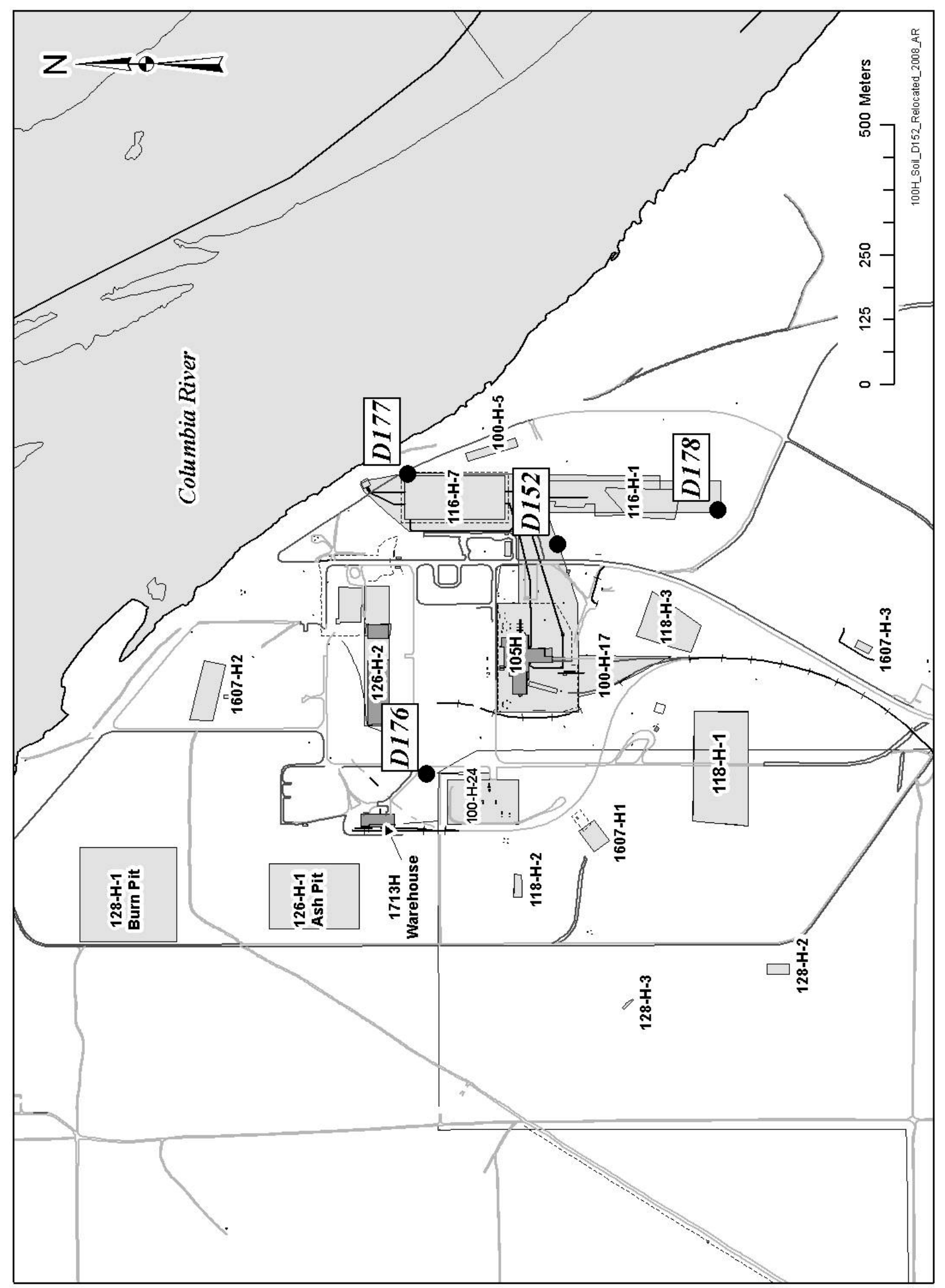


Figure 3-5. 2008 Soil Sampling Locations, 200 East Area.

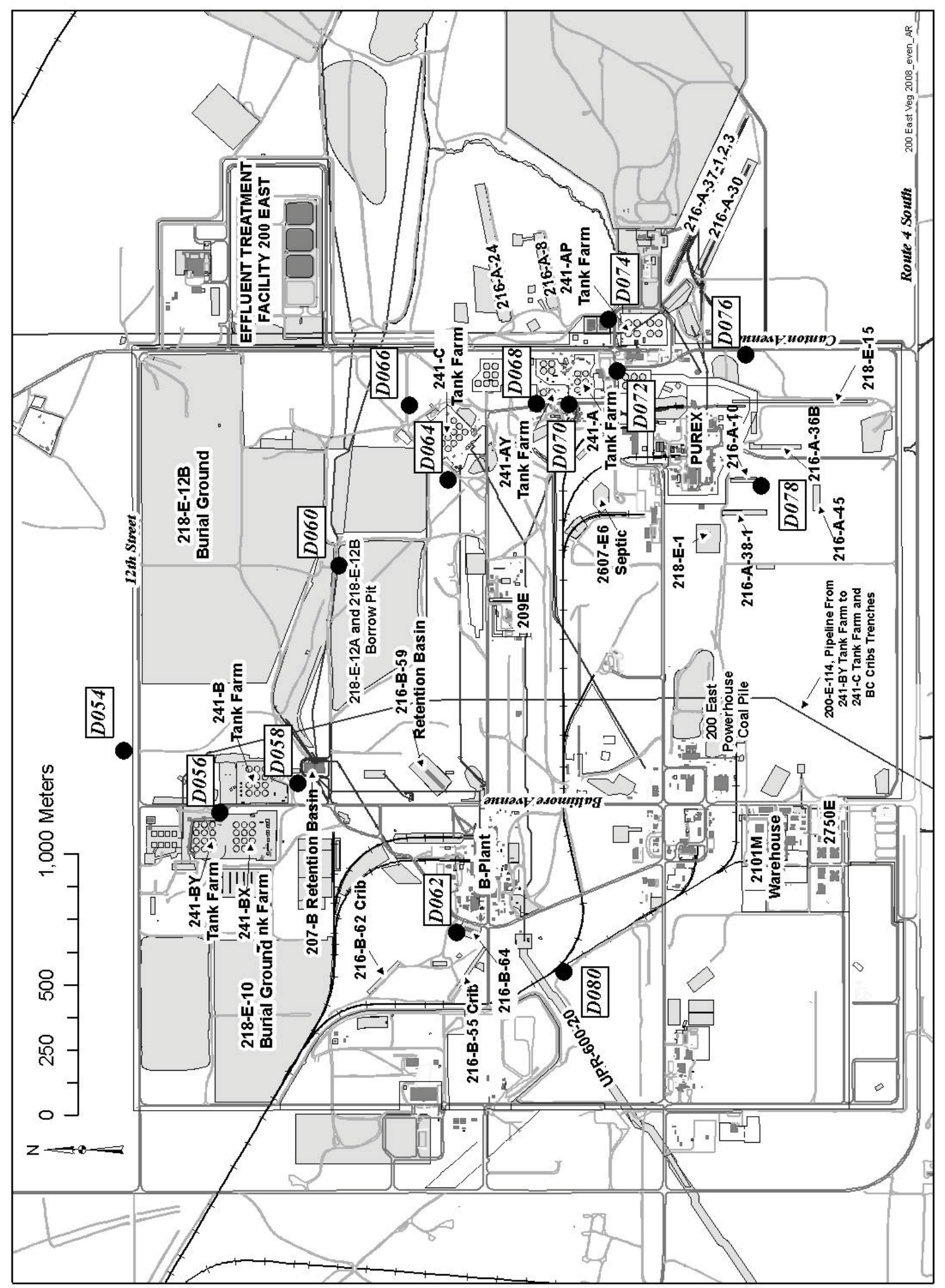


Figure 3-6. 2008 Soil Sampling Locations, 200 West Area.

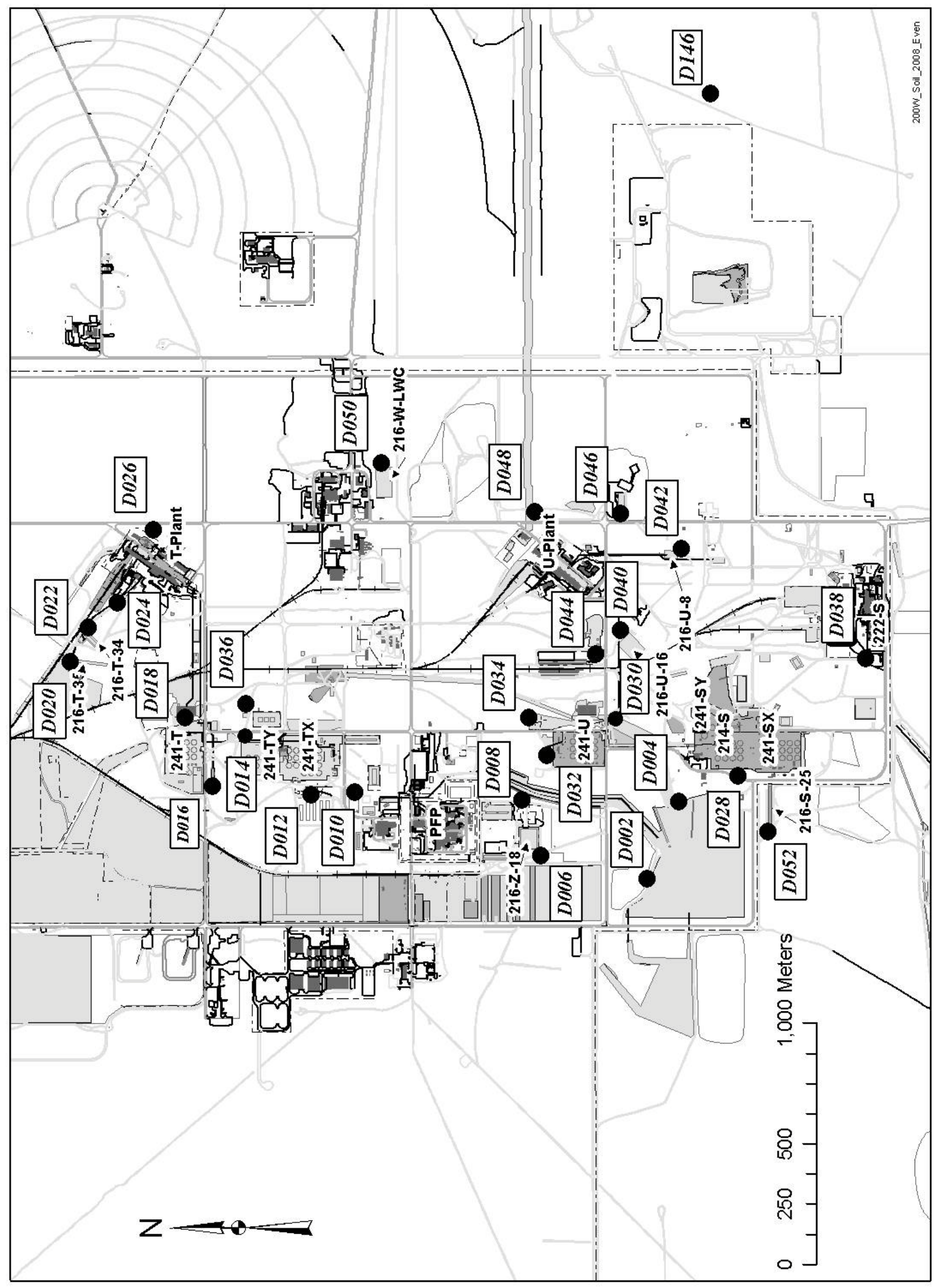


Figure 3-7. 2008 Soil Sampling Locations, 300 Area.

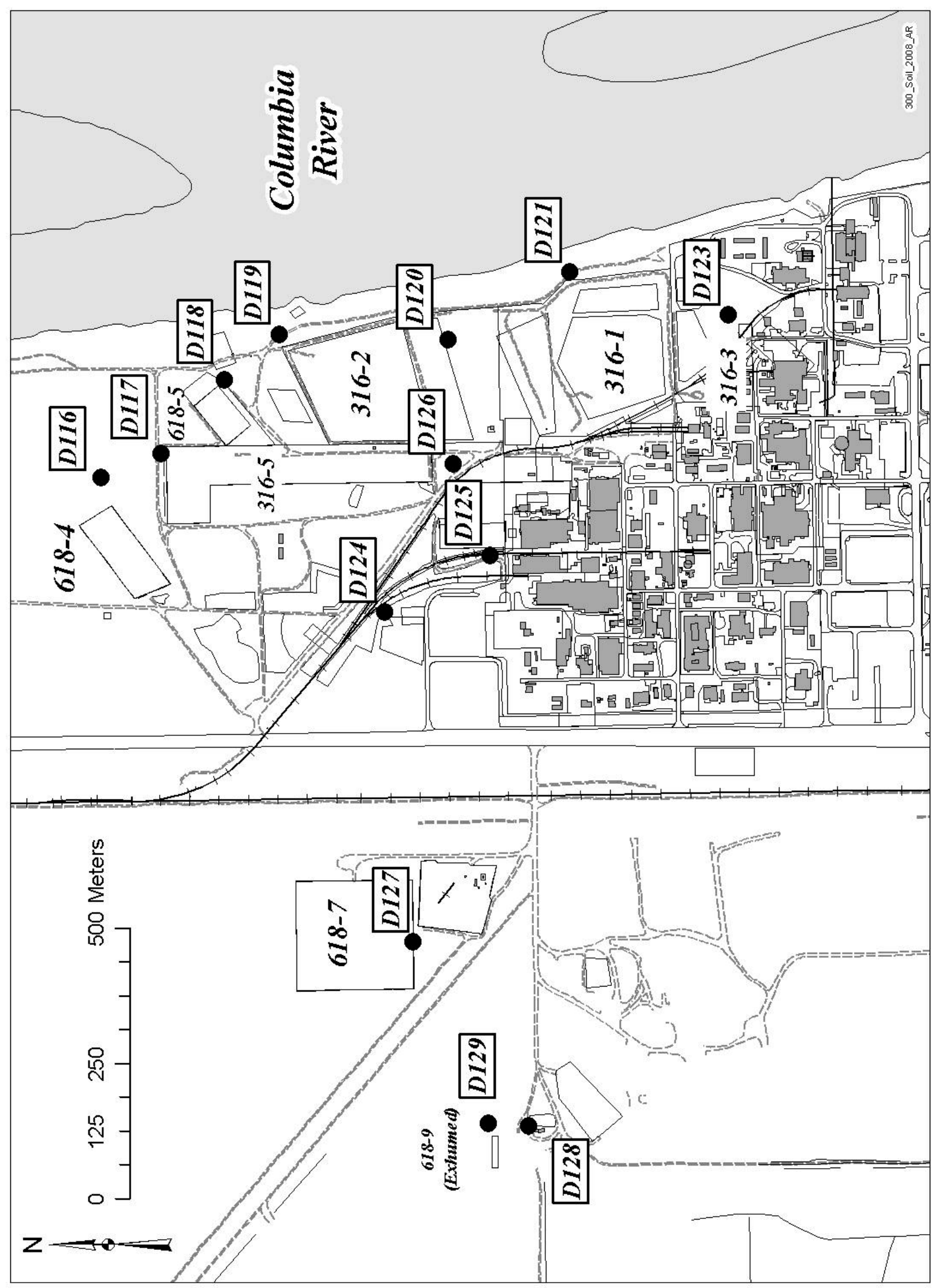


Figure 3-8. 2008 Soil Sampling Locations, 400 Area.

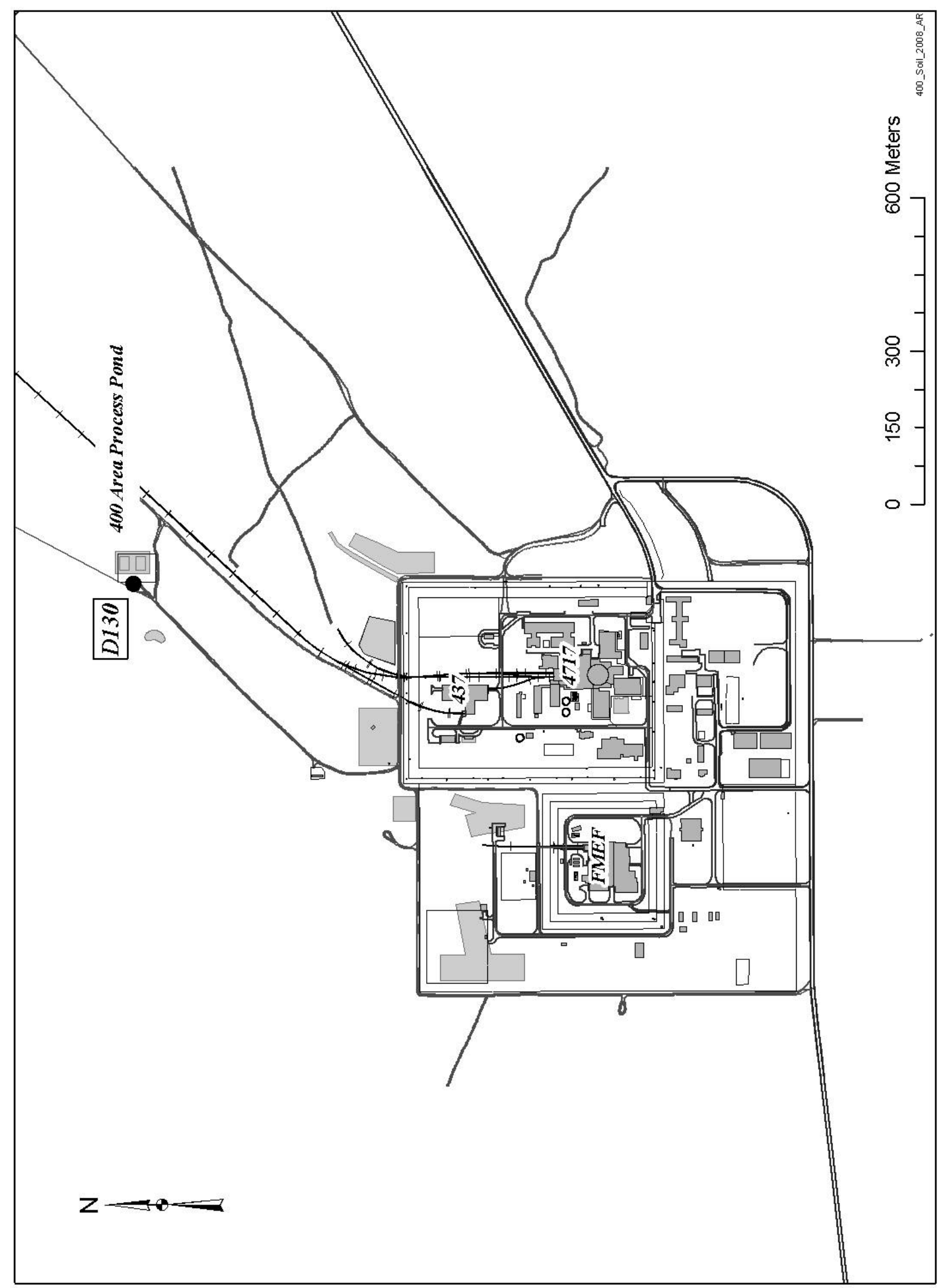


Figure 3-9. 2008 Soil Sampling Locations, 600 Area.

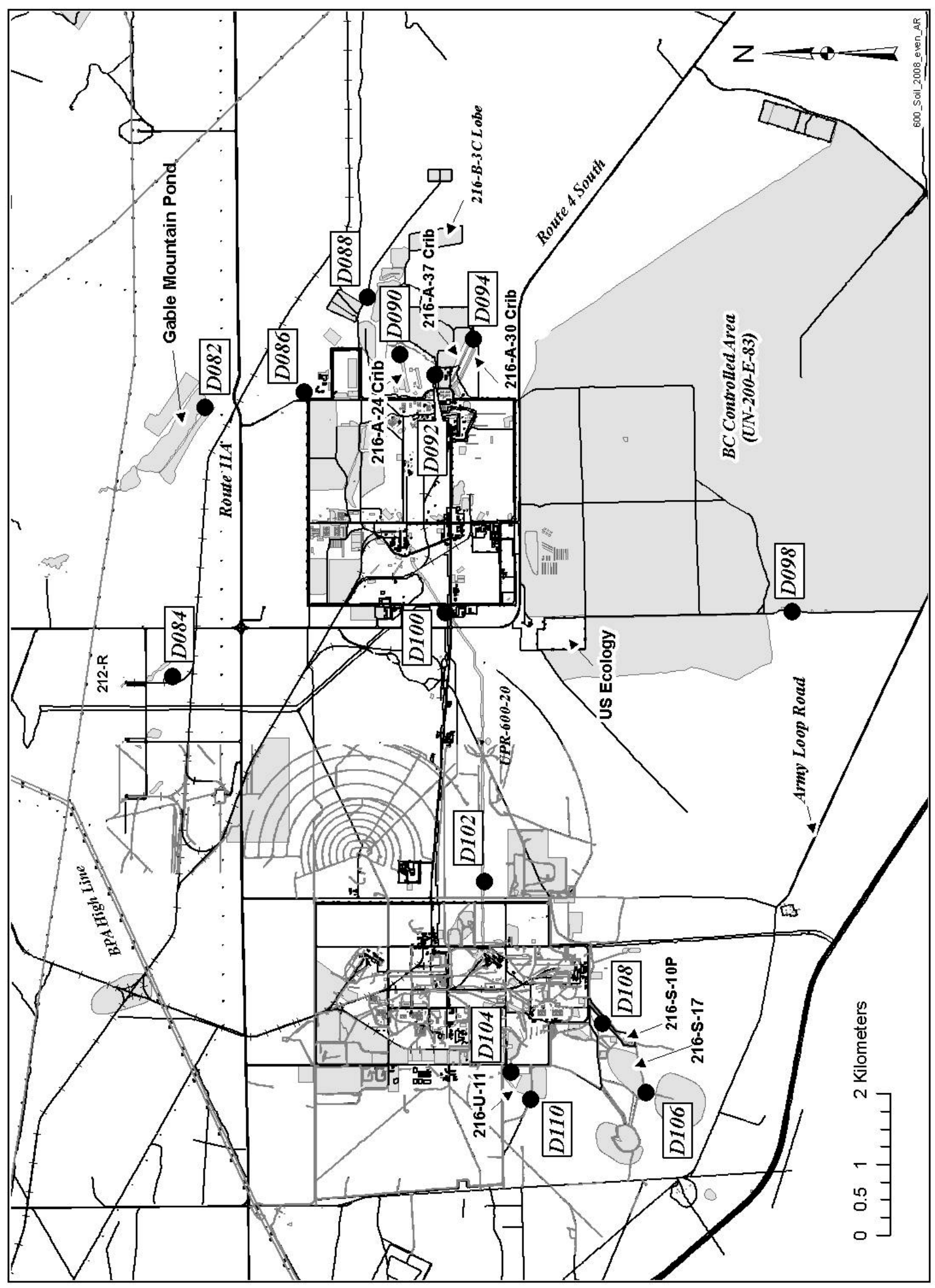


Figure 3-10. 2008 Soil Sampling Location, 100-IU2/6.

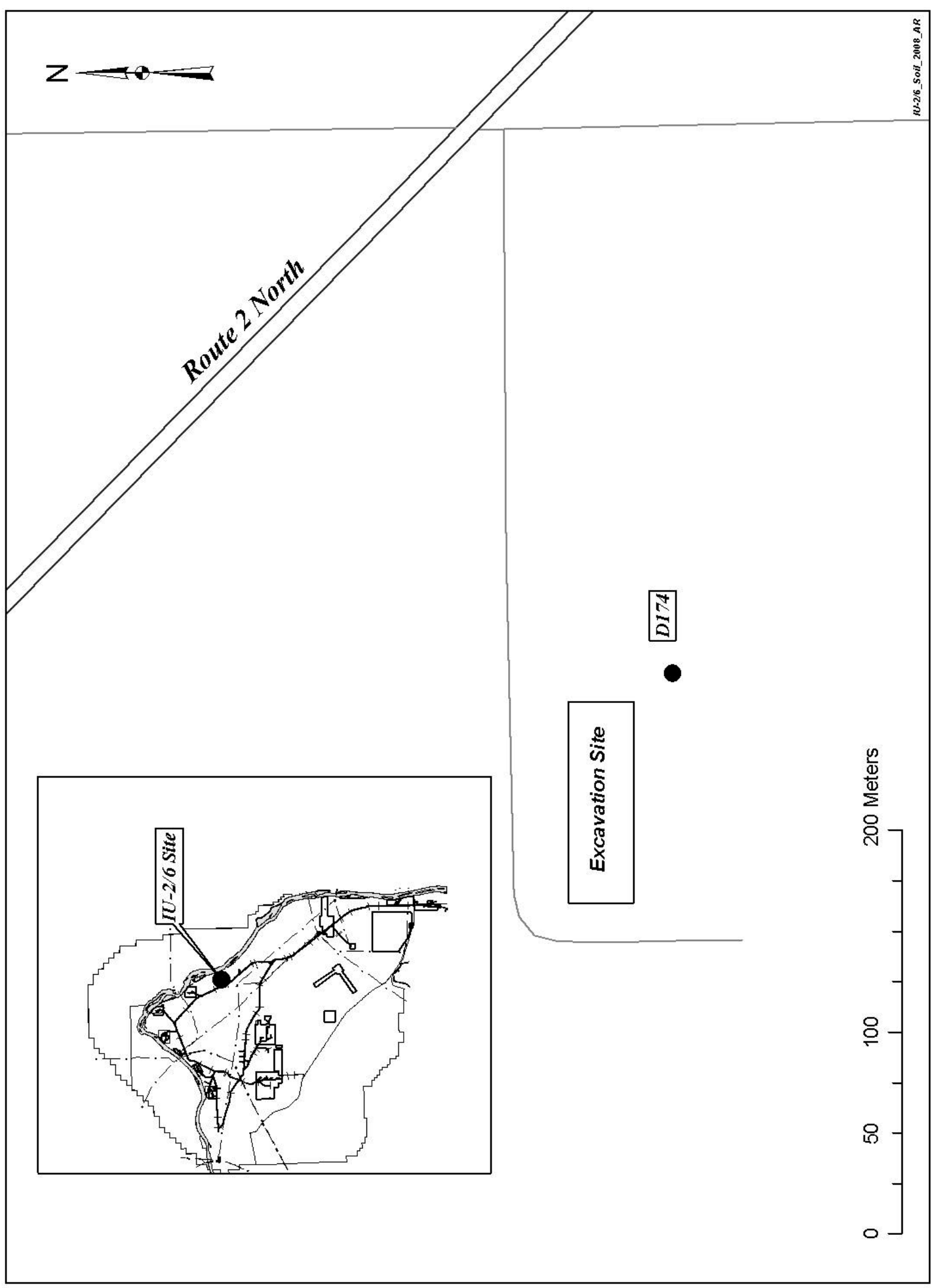


Table 3-2. Summary of Near-Facility Soil Sampling Results (pCi/g) ${ }^{a}$ for Selected Radionuclides, 2008.

\begin{tabular}{|c|c|c|c|c|c|c|}
\hline \multirow[b]{2}{*}{ Isotope } & \multicolumn{2}{|c|}{ Number of } & \multirow[b]{2}{*}{ Average $^{c}$} & \multirow[b]{2}{*}{ Maximum $^{d}$} & \multicolumn{2}{|c|}{ Location } \\
\hline & Samples ${ }^{\text {b }}$ & Detects & & & Area & Site ID \\
\hline${ }^{144} \mathrm{Ce}$ & 95 & 0 & $1.5 \mathrm{E}-01 \pm 2.7 \mathrm{E}+00$ & $1.3 \mathrm{E}+01 \pm 8.7 \mathrm{E}+01^{\mathrm{e}}$ & 600 Area & D174 \\
\hline${ }^{60} \mathrm{Co}$ & 95 & 1 & $-1.4 \mathrm{E}-02 \pm 2.6 \mathrm{E}-01$ & $1.3 \mathrm{E}-02 \pm 9.6 \mathrm{E}-03^{\mathrm{e}}$ & $100-\mathrm{F}$ & D155 \\
\hline${ }^{134} \mathrm{Cs}$ & 95 & 89 & $3.4 \mathrm{E}-01 \pm 5.9 \mathrm{E}+00$ & $2.9 \mathrm{E}+01 \pm 9.9 \mathrm{E}+00$ & 600 Area & D174 \\
\hline${ }^{137} \mathrm{Cs}$ & 95 & 90 & $1.7 \mathrm{E}+00 \pm 1.9 \mathrm{E}+01$ & $9.4 \mathrm{E}+01 \pm 1.7 \mathrm{E}+01$ & 600 Area & D174 \\
\hline${ }^{152} \mathrm{Eu}$ & 95 & 4 & $-7.5 \mathrm{E}-02 \pm 1.5 \mathrm{E}+00$ & $1.7 \mathrm{E}-01 \pm 3.1 \mathrm{E}-02$ & $100-\mathrm{F}$ & D155 \\
\hline${ }^{154} \mathrm{Eu}$ & 95 & 0 & $-1.4 \mathrm{E}-01 \pm 2.6 \mathrm{E}+00$ & $3.9 \mathrm{E}-02 \pm 2.9 \mathrm{E}-02^{\mathrm{e}}$ & 200 West & D052 \\
\hline${ }^{155} \mathrm{Eu}$ & 95 & 24 & $1.8 \mathrm{E}-01 \pm 2.7 \mathrm{E}+00$ & $1.4 \mathrm{E}+01 \pm 2.1 \mathrm{E}+01^{\mathrm{e}}$ & 600 Area & D174 \\
\hline${ }^{238} \mathrm{Pu}$ & 95 & 7 & $9.0 \mathrm{E}-03 \pm 6.7 \mathrm{E}-02$ & $2.1 \mathrm{E}-01 \pm 5.9 \mathrm{E}-02$ & 200 West & D028 \\
\hline${ }^{239 / 240} \mathrm{Pu}$ & 95 & 36 & $8.4 \mathrm{E}-02 \pm 5.9 \mathrm{E}-01$ & $2.4 \mathrm{E}+00 \pm 6.2 \mathrm{E}-01$ & 200 West & D008 \\
\hline${ }^{103} \mathrm{Ru}$ & 95 & 0 & $1.9 \mathrm{E}-02 \pm 3.6 \mathrm{E}-01$ & $1.8 \mathrm{E}+00 \pm 4.4 \mathrm{E}+00^{\mathrm{e}}$ & 600 Area & D174 \\
\hline${ }^{106} \mathrm{Ru}$ & 95 & 0 & $1.8 \mathrm{E}-01 \pm 3.5 \mathrm{E}+00$ & $1.7 \mathrm{E}+01 \pm 3.6 \mathrm{E}+01^{\mathrm{e}}$ & 600 Area & D174 \\
\hline${ }^{125} \mathrm{Sb}$ & 95 & 0 & $-3.9 \mathrm{E}-02 \pm 8.0 \mathrm{E}-01$ & $3.4 \mathrm{E}-02 \pm 3.4 \mathrm{E}-02^{\mathrm{e}}$ & $100-\mathrm{D}$ & D171 \\
\hline${ }^{113} \mathrm{Sn}$ & 95 & 0 & $-3.9 \mathrm{E}-02 \pm 6.9 \mathrm{E}-01$ & $1.7 \mathrm{E}-02 \pm 1.8 \mathrm{E}-02^{\mathrm{e}}$ & $100-\mathrm{F}$ & D170 \\
\hline${ }^{90} \mathrm{Sr}$ & 95 & 3 & $-3.2 \mathrm{E}-01 \pm 7.8 \mathrm{E}-01$ & $1.2 \mathrm{E}+00 \pm 5.0 \mathrm{E}-01$ & 600 Area & D094 \\
\hline${ }^{234} \mathrm{U}$ & 95 & 95 & $2.4 \mathrm{E}-01 \pm 6.1 \mathrm{E}-01$ & $2.2 \mathrm{E}+00 \pm 5.9 \mathrm{E}-01$ & 300 Area & D126 \\
\hline${ }^{235} \mathrm{U}$ & 95 & 57 & $1.9 \mathrm{E}-02 \pm 5.2 \mathrm{E}-02$ & $2.5 \mathrm{E}-01 \pm 8.0 \mathrm{E}-02$ & 300 Area & D126 \\
\hline${ }^{238} \mathrm{U}$ & 95 & 95 & $2.2 \mathrm{E}-01 \pm 5.3 \mathrm{E}-01$ & $1.6 \mathrm{E}+00 \pm 4.3 \mathrm{E}-01$ & 300 Area & D119 \\
\hline${ }^{65} \mathrm{Zn}$ & 95 & 11 & $8.9 \mathrm{E}-02 \pm 1.6 \mathrm{E}+00$ & $7.6 \mathrm{E}+00 \pm 1.2 \mathrm{E}+01^{\mathrm{e}}$ & 600 Area & D174 \\
\hline
\end{tabular}

${ }^{\mathrm{a}} 1 \mathrm{pCi}=0.037 \mathrm{~Bq}$.

${ }^{\mathrm{b}}$ Includes replicate samples and/or multiple samples collected at some locations

${ }^{\mathrm{c}}$ Average \pm two standard deviations

${ }^{\mathrm{d}}$ Maximum \pm analytical uncertainty

${ }^{\mathrm{e}}$ Maximum value reported is a non detect 
Table 3-3. Average Radionuclide Concentrations (pCi/g) ${ }^{\mathrm{a}}$ in Hanford Soils, 1998 through 2008.

\begin{tabular}{ccccccc}
\multicolumn{5}{c}{$\mathbf{1 0 0}$ Areas } \\
Year & ${ }^{60} \mathbf{C o}$ & ${ }^{90} \mathbf{S r}$ & ${ }^{137} \mathbf{C s}$ & ${ }^{234} \mathbf{U}$ & ${ }^{238} \mathbf{U}$ & ${ }^{239,240} \mathbf{P u}$ \\
\hline 1998 & $4.9 \mathrm{E}+00 \pm 7.7 \mathrm{E}+00$ & $1.2 \mathrm{E}+00 \pm 1.1 \mathrm{E}+00$ & $3.1 \mathrm{E}+00 \pm 4.1 \mathrm{E}+00$ & $2.1 \mathrm{E}-01 \pm 6.0 \mathrm{E}-02$ & $1.7 \mathrm{E}-01 \pm 3.0 \mathrm{E}-02$ & $1.5 \mathrm{E}-01 \pm 1.3 \mathrm{E}-01$ \\
1999 & $1.6 \mathrm{E}+00 \pm 2.1 \mathrm{E}+00$ & $2.0 \mathrm{E}+00 \pm 2.0 \mathrm{E}+00$ & $8.4 \mathrm{E}-01 \pm 8.1 \mathrm{E}-01$ & $2.2 \mathrm{E}-01 \pm 3.0 \mathrm{E}-02$ & $2.0 \mathrm{E}-01 \pm 3.0 \mathrm{E}-02$ & $2.9 \mathrm{E}-02 \pm 2.3 \mathrm{E}-02$ \\
2000 & $3.1 \mathrm{E}+00 \pm 3.0 \mathrm{E}+00$ & $8.4 \mathrm{E}-01 \pm 4.5 \mathrm{E}-01$ & $2.5 \mathrm{E}+00 \pm 2.3 \mathrm{E}+00$ & $2.2 \mathrm{E}-01 \pm 8.7 \mathrm{E}-02$ & $2.2 \mathrm{E}-01 \pm 3.2 \mathrm{E}-02$ & $5.8 \mathrm{E}-02 \pm 3.3 \mathrm{E}-02$ \\
2001 & $4.0 \mathrm{E}-01 \pm 3.4 \mathrm{E}-01$ & $4.8 \mathrm{E}-01 \pm 3.0 \mathrm{E}-01$ & $3.9 \mathrm{E}-01 \pm 1.6 \mathrm{E}-01$ & $2.4 \mathrm{E}-01 \pm 3.6 \mathrm{E}-02$ & $2.5 \mathrm{E}-01 \pm 2.7 \mathrm{E}-02$ & $3.1 \mathrm{E}-02 \pm 2.0 \mathrm{E}-02$ \\
2002 & $3.0 \mathrm{E}-01 \pm 1.1 \mathrm{E}+00$ & $1.5 \mathrm{E}-01 \pm 4.7 \mathrm{E}-01$ & $2.6 \mathrm{E}-01 \pm 5.1 \mathrm{E}-01$ & $1.3 \mathrm{E}-01 \pm 4.7 \mathrm{E}-02$ & $1.1 \mathrm{E}-01 \pm 3.9 \mathrm{E}-02$ & $6.1 \mathrm{E}-03 \pm 6.1 \mathrm{E}-03$ \\
2003 & $1.8 \mathrm{E}-01 \pm 2.1 \mathrm{E}-02$ & $-8.2 \mathrm{E}-02 \pm 2.4 \mathrm{E}-01$ & $2.1 \mathrm{E}-01 \pm 3.6 \mathrm{E}-02$ & $1.4 \mathrm{E}-01 \pm 4.8 \mathrm{E}-02$ & $1.5 \mathrm{E}-01 \pm 5.1 \mathrm{E}-02$ & $1.8 \mathrm{E}-03 \pm 6.3 \mathrm{E}-03$ \\
2004 & $3.9 \mathrm{E}-01 \pm 2.0 \mathrm{E}+00$ & $-1.3 \mathrm{E}-01 \pm 5.7 \mathrm{E}-01$ & $3.8 \mathrm{E}-01 \pm 1.1 \mathrm{E}+00$ & $1.3 \mathrm{E}-01 \pm 5.9 \mathrm{E}-02$ & $1.4 \mathrm{E}-01 \pm 6.4 \mathrm{E}-02$ & $1.1 \mathrm{E}-01 \pm 6.0 \mathrm{E}-01$ \\
2005 & $3.5 \mathrm{E}-02 \pm 1.8 \mathrm{E}-01$ & $-4.3 \mathrm{E}-02 \pm 6.1 \mathrm{E}-01$ & $3.2 \mathrm{E}-01 \pm 1.2 \mathrm{E}+00$ & $1.3 \mathrm{E}-01 \pm 6.5 \mathrm{E}-02$ & $1.3 \mathrm{E}-01 \pm 5.8 \mathrm{E}-02$ & $1.1 \mathrm{E}-02 \pm 4.3 \mathrm{E}-02$ \\
2006 & $7.3 \mathrm{E}-01 \pm 6.8 \mathrm{E}+00$ & Not Detected & $7.0 \mathrm{E}+00 \pm 6.0 \mathrm{E}+01$ & $1.3 \mathrm{E}-01 \pm 9.5 \mathrm{E}-02$ & $1.3 \mathrm{E}-01 \pm 8.6 \mathrm{E}-02$ & $1.1 \mathrm{E}-02 \pm 2.0 \mathrm{E}-02$ \\
2007 & & \multicolumn{7}{c}{ No Soil Samples Collected in 100 Areas During 2007 } \\
2008 & Not Detected & $-3.7 \mathrm{E}-01 \pm 8.4 \mathrm{E}-01$ & $2.0 \mathrm{E}-01 \pm 4.0 \mathrm{E}-01$ & $1.5 \mathrm{E}-01 \pm 9.4 \mathrm{E}-02$ & $1.2 \mathrm{E}-01 \pm 7.9 \mathrm{E}-02$ & Not Detected
\end{tabular}

\section{0/600 Areas}

\begin{tabular}{lcccccc} 
Year & ${ }^{60} \mathbf{C o}$ & ${ }^{90} \mathbf{S r}$ & ${ }^{137} \mathbf{C s}$ & ${ }^{234} \mathbf{U}$ & ${ }^{238} \mathbf{U}$ & ${ }^{239,240} \mathbf{P u}$ \\
\hline 1998 & $1.9 \mathrm{E}-02 \pm 6.0 \mathrm{E}-03$ & $5.0 \mathrm{E}-01 \pm 1.4 \mathrm{E}-01$ & $1.1 \mathrm{E}+00 \pm 4.0 \mathrm{E}-01$ & $1.9 \mathrm{E}-01 \pm 1.0 \mathrm{E}-02$ & $1.9 \mathrm{E}-01 \pm 1.0 \mathrm{E}-02$ & $1.3 \mathrm{E}-01 \pm 1.0 \mathrm{E}-02$ \\
1999 & Not Detected & $1.1 \mathrm{E}+00 \pm 5.0 \mathrm{E}-01$ & $1.4 \mathrm{E}+00 \pm 5.0 \mathrm{E}-01$ & $2.3 \mathrm{E}-01 \pm 2.0 \mathrm{E}-02$ & $2.2 \mathrm{E}-01 \pm 2.0 \mathrm{E}-02$ & $1.0 \mathrm{E}-01 \pm 5.0 \mathrm{E}-02$ \\
2000 & $6.0 \mathrm{E}-03 \pm 6.0 \mathrm{E}-03$ & $1.1 \mathrm{E}+00 \pm 2.0 \mathrm{E}-01$ & $1.4 \mathrm{E}+00 \pm 5.0 \mathrm{E}-01$ & $2.3 \mathrm{E}-01 \pm 3.0 \mathrm{E}-02$ & $2.3 \mathrm{E}-01 \pm 3.0 \mathrm{E}-02$ & $4.1 \mathrm{E}-01 \pm 4.2 \mathrm{E}-01$ \\
2001 & Not Detected & $5.5 \mathrm{E}-01 \pm 2.3 \mathrm{E}-01$ & $1.5 \mathrm{E}+00 \pm 5.4 \mathrm{E}-01$ & $2.2 \mathrm{E}-01 \pm 1.4 \mathrm{E}-02$ & $2.2 \mathrm{E}-01 \pm 1.4 \mathrm{E}-02$ & $1.3 \mathrm{E}-01 \pm 6.2 \mathrm{E}-02$ \\
2002 & Not Detected & $2.7 \mathrm{E}-01 \pm 6.6 \mathrm{E}-01$ & $1.4 \mathrm{E}+00 \pm 4.3 \mathrm{E}+00$ & $1.7 \mathrm{E}-01 \pm 1.0 \mathrm{E}-01$ & $1.7 \mathrm{E}-01 \pm 1.1 \mathrm{E}-01$ & $1.2 \mathrm{E}-01 \pm 7.2 \mathrm{E}-01$ \\
2003 & $2.4 \mathrm{E}-03 \pm 1.3 \mathrm{E}-02$ & $8.4 \mathrm{E}-02 \pm 6.3 \mathrm{E}-01$ & $1.8 \mathrm{E}+00 \pm 6.3 \mathrm{E}-01$ & $1.6 \mathrm{E}-01 \pm 9.6 \mathrm{E}-02$ & $1.7 \mathrm{E}-01 \pm 1.0 \mathrm{E}-01$ & $9.3 \mathrm{E}-02 \pm 5.0 \mathrm{E}-01$ \\
2004 & $8.1 \mathrm{E}-04 \pm 1.1 \mathrm{E}-02$ & $1.3 \mathrm{E}-01 \pm 7.8 \mathrm{E}-01$ & $2.8 \mathrm{E}+00 \pm 1.7 \mathrm{E}+01$ & $1.7 \mathrm{E}-01 \pm 1.9 \mathrm{E}-01$ & $1.7 \mathrm{E}-01 \pm 1.5 \mathrm{E}-01$ & $3.5 \mathrm{E}-01 \pm 3.2 \mathrm{E}+00$ \\
2005 & Not Detected & $2.7 \mathrm{E}-02 \pm 5.4 \mathrm{E}-01$ & $1.5 \mathrm{E}+00 \pm 5.1 \mathrm{E}+00$ & $1.6 \mathrm{E}-01 \pm 9.6 \mathrm{E}-02$ & $1.5 \mathrm{E}-01 \pm 8.8 \mathrm{E}-02$ & $8.0 \mathrm{E}-02 \pm 4.6 \mathrm{E}-01$ \\
2006 & Not Detected & $2.6 \mathrm{E}-01 \pm 2.1 \mathrm{E}+00$ & $1.3 \mathrm{E}+00 \pm 4.3 \mathrm{E}+00$ & $1.7 \mathrm{E}-01 \pm 2.3 \mathrm{E}-01$ & $1.7 \mathrm{E}-01 \pm 2.2 \mathrm{E}-01$ & $9.3 \mathrm{E}-02 \pm 5.2 \mathrm{E}-01$ \\
2007 & Not Detected & $1.5 \mathrm{E}+00 \pm 1.5 \mathrm{E}+01$ & $1.4 \mathrm{E}+00 \pm 4.7 \mathrm{E}+00$ & $1.6 \mathrm{E}-01 \pm 1.1 \mathrm{E}-01$ & $1.6 \mathrm{E}-01 \pm 1.3 \mathrm{E}-01$ & $1.8 \mathrm{E}-01 \pm 1.6 \mathrm{E}+00$ \\
2008 & $2.2 \mathrm{E}-04 \pm 8.5 \mathrm{E}-03$ & $-3.7 \mathrm{E}-01 \pm 8.1 \mathrm{E}-01$ & $1.0 \mathrm{E}+00 \pm 3.9 \mathrm{E}+00$ & $1.6 \mathrm{E}-01 \pm 7.4 \mathrm{E}-02$ & $1.6 \mathrm{E}-01 \pm 7.1 \mathrm{E}-02$ & $1.3 \mathrm{E}-01 \pm 7.3 \mathrm{E}-01$
\end{tabular}

\begin{tabular}{|c|c|c|c|c|c|c|}
\hline \multicolumn{7}{|c|}{ 300/400 Areas } \\
\hline Year & ${ }^{60} \mathrm{Co}$ & ${ }^{90} \mathrm{Sr}$ & ${ }^{137} \mathrm{Cs}$ & ${ }^{234} \mathbf{U}$ & ${ }^{238} \mathbf{U}$ & ${ }^{239,240} \mathbf{P u}$ \\
\hline 1998 & Not Detected & $2.4 \mathrm{E}-01 \pm 1.2 \mathrm{E}-01$ & $9.0 \mathrm{E}-02 \pm 8.0 \mathrm{E}-02$ & $8.5 \mathrm{E}-01 \pm 9.8 \mathrm{E}-01$ & $8.2 \mathrm{E}-01 \pm 9.8 \mathrm{E}-01$ & $4.5 \mathrm{E}-02 \pm 5.7 \mathrm{E}-02$ \\
\hline 1999 & Not Detected & $8.7 \mathrm{E}-01 \pm 1.9 \mathrm{E}-01$ & $9.0 \mathrm{E}-02 \pm 3.0 \mathrm{E}-02$ & $7.5 \mathrm{E}-01 \pm 5.4 \mathrm{E}-01$ & 7.1E-01 $\pm 5.3 \mathrm{E}-01$ & $4.0 \mathrm{E}-02 \pm 2.0 \mathrm{E}-02$ \\
\hline 2000 & Not Detected & $5.9 \mathrm{E}-01 \pm 1.8 \mathrm{E}-01$ & $1.4 \mathrm{E}-01 \pm 6.0 \mathrm{E}-02$ & $5.4 \mathrm{E}+00 \pm 5.6 \mathrm{E}+00$ & $5.4 \mathrm{E}+00 \pm 5.7 \mathrm{E}+00$ & $1.7 \mathrm{E}-01 \pm 8.0 \mathrm{E}-02$ \\
\hline 2001 & Not Detected & Not Detected & $5.0 \mathrm{E}-02 \pm 2.1 \mathrm{E}-02$ & $9.4 \mathrm{E}-01 \pm 7.1 \mathrm{E}-01$ & $9.5 \mathrm{E}-01 \pm 7.3 \mathrm{E}-01$ & $4.1 \mathrm{E}-02 \pm 2.6 \mathrm{E}-02$ \\
\hline 2002 & Not Detected & $2.8 \mathrm{E}-02 \pm 2.9 \mathrm{E}-02$ & $7.4 \mathrm{E}-02 \pm 1.3 \mathrm{E}-01$ & $1.5 \mathrm{E}+00 \pm 6.4 \mathrm{E}+00$ & $1.5 \mathrm{E}+00 \pm 6.4 \mathrm{E}+00$ & $2.4 \mathrm{E}-02 \pm 9.9 \mathrm{E}-02$ \\
\hline 2003 & Not Detected & $5.6 \mathrm{E}-02 \pm 7.3 \mathrm{E}-02$ & $8.1 \mathrm{E}-02 \pm 1.4 \mathrm{E}-01$ & $1.3 \mathrm{E}+00 \pm 5.1 \mathrm{E}+00$ & $1.3 \mathrm{E}+00 \pm 5.2 \mathrm{E}+00$ & $7.5 \mathrm{E}-02 \pm 3.8 \mathrm{E}-01$ \\
\hline 2004 & Not Detected & Not Detected & $9.2 \mathrm{E}-02 \pm 1.4 \mathrm{E}-01$ & $9.6 \mathrm{E}-01 \pm 2.9 \mathrm{E}+00$ & $9.7 \mathrm{E}-01 \pm 3.0 \mathrm{E}+00$ & $2.8 \mathrm{E}-02 \pm 6.7 \mathrm{E}-02$ \\
\hline 2005 & Not Detected & Not Detected & $5.0 \mathrm{E}-02 \pm 1.1 \mathrm{E}-01$ & $5.6 \mathrm{E}-01 \pm 1.6 \mathrm{E}+00$ & $5.6 \mathrm{E}-01 \pm 1.6 \mathrm{E}+00$ & $1.4 \mathrm{E}-02 \pm 3.5 \mathrm{E}-02$ \\
\hline 2006 & Not Detected & $6.5 \mathrm{E}-02 \pm \quad 5.6 \mathrm{E}-01$ & $9.4 \mathrm{E}-02 \pm 1.4 \mathrm{E}-01$ & $1.2 \mathrm{E}+00 \pm 3.3 \mathrm{E}+00$ & $1.2 \mathrm{E}+00 \pm 3.4 \mathrm{E}+00$ & $1.8 \mathrm{E}-02 \pm 4.9 \mathrm{E}-02$ \\
\hline 2007 & Not Detected & Not Detected & $9.1 \mathrm{E}-02 \pm 2.1 \mathrm{E}-02$ & $8.2 \mathrm{E}-01 \pm 2.2 \mathrm{E}+00$ & $8.0 \mathrm{E}-01 \pm 2.2 \mathrm{E}+00$ & $1.7 \mathrm{E}-02 \pm 4.6 \mathrm{E}-02$ \\
\hline 2008 & Not Detected & $-1.4 \mathrm{E}-01 \pm 5.5 \mathrm{E}-01$ & $3.8 \mathrm{E}-02 \pm 8.6 \mathrm{E}-02$ & $5.5 \mathrm{E}-01 \pm 1.2 \mathrm{E}+00$ & $5.2 \mathrm{E}-01 \pm 1.0 \mathrm{E}+00$ & $8.2 \mathrm{E}-03 \pm 1.6 \mathrm{E}-02$ \\
\hline
\end{tabular}


Table 3-4. 2008 Soil Sampling Results (pCi/g \pm total analytical uncertainty). (Sheet 1 of 24)

\begin{tabular}{|c|c|c|c|c|c|c|c|}
\hline Location & Isotope & Result \pm Error & $\mathbf{R Q}^{*}$ & Location & Isotope & Result \pm Error & $\mathbf{R Q}^{*}$ \\
\hline D153 & ${ }^{144} \mathrm{Ce}$ & $-7.6 \mathrm{E}-02 \pm 1.3 \mathrm{E}-01$ & $\mathrm{U}$ & D161 & ${ }^{144} \mathrm{Ce}$ & $8.3 \mathrm{E}-03 \pm 8.3 \mathrm{E}-02$ & $\mathrm{U}$ \\
\hline \multirow[t]{17}{*}{$(100-B / C)$} & ${ }^{60} \mathrm{Co}$ & $-5.5 \mathrm{E}-03 \pm 8.3 \mathrm{E}-03$ & $\mathrm{U}$ & $(100-B / C)$ & ${ }^{60} \mathrm{Co}$ & $4.4 \mathrm{E}-03 \pm 7.8 \mathrm{E}-03$ & $\mathrm{U}$ \\
\hline & ${ }^{134} \mathrm{Cs}$ & $3.1 \mathrm{E}-02 \pm 1.2 \mathrm{E}-02$ & $\mathrm{U}$ & & ${ }^{134} \mathrm{Cs}$ & $3.5 \mathrm{E}-02 \pm 1.2 \mathrm{E}-02$ & $\mathrm{U}$ \\
\hline & ${ }^{137} \mathrm{Cs}$ & $2.9 \mathrm{E}-01 \pm 5.1 \mathrm{E}-02$ & & & ${ }^{137} \mathrm{Cs}$ & $8.2 \mathrm{E}-02 \pm 1.9 \mathrm{E}-02$ & \\
\hline & ${ }^{152} \mathrm{Eu}$ & $6.3 \mathrm{E}-03 \pm 2.7 \mathrm{E}-02$ & $\mathrm{U}$ & & ${ }^{152} \mathrm{Eu}$ & $3.7 \mathrm{E}-02 \pm 2.1 \mathrm{E}-02$ & U \\
\hline & ${ }^{154} \mathrm{Eu}$ & $-4.5 \mathrm{E}-02 \pm 4.5 \mathrm{E}-02$ & $\mathrm{U}$ & & ${ }^{154} \mathrm{Eu}$ & $-5.2 \mathrm{E}-03 \pm 2.6 \mathrm{E}-02$ & $\mathrm{U}$ \\
\hline & ${ }^{155} \mathrm{Eu}$ & $4.0 \mathrm{E}-02 \pm 4.2 \mathrm{E}-02$ & $\mathrm{U}$ & & ${ }^{155} \mathrm{Eu}$ & $6.4 \mathrm{E}-02 \pm 4.2 \mathrm{E}-02$ & $\mathrm{U}$ \\
\hline & ${ }^{238} \mathrm{Pu}$ & $2.0 \mathrm{E}-03 \pm 2.0 \mathrm{E}-02$ & $\mathrm{U}$ & & ${ }^{238} \mathrm{Pu}$ & $-4.2 \mathrm{E}-03 \pm 3.5 \mathrm{E}-02$ & $\mathrm{U}$ \\
\hline & ${ }^{239 / 240} \mathrm{Pu}$ & $1.2 \mathrm{E}-02 \pm 1.3 \mathrm{E}-02$ & $\mathrm{U}$ & & ${ }^{239 / 240} \mathrm{Pu}$ & $4.2 \mathrm{E}-03 \pm 1.0 \mathrm{E}-02$ & $\mathrm{U}$ \\
\hline & ${ }^{103} \mathrm{Ru}$ & $-4.9 \mathrm{E}-03 \pm 7.6 \mathrm{E}-03$ & $\mathrm{U}$ & & ${ }^{103} \mathrm{Ru}$ & $5.6 \mathrm{E}-03 \pm 7.3 \mathrm{E}-03$ & $\mathrm{U}$ \\
\hline & ${ }^{106} \mathrm{Ru}$ & $-1.9 \mathrm{E}-02 \pm 7.3 \mathrm{E}-02$ & $\mathrm{U}$ & & ${ }^{106} \mathrm{Ru}$ & $2.3 \mathrm{E}-02 \pm 6.9 \mathrm{E}-02$ & $\mathrm{U}$ \\
\hline & ${ }^{125} \mathrm{Sb}$ & $1.2 \mathrm{E}-02 \pm 2.1 \mathrm{E}-02$ & $\mathrm{U}$ & & ${ }^{125} \mathrm{Sb}$ & $-5.8 \mathrm{E}-03 \pm 2.1 \mathrm{E}-02$ & $\mathrm{U}$ \\
\hline & ${ }^{113} \mathrm{Sn}$ & $-2.9 \mathrm{E}-03 \pm 9.9 \mathrm{E}-03$ & $\mathrm{U}$ & & ${ }^{113} \mathrm{Sn}$ & $5.0 \mathrm{E}-03 \pm 9.5 \mathrm{E}-03$ & $\mathrm{U}$ \\
\hline & ${ }^{90} \mathrm{Sr}$ & $-4.5 \mathrm{E}-01 \pm 4.5 \mathrm{E}-01$ & $\mathrm{U}$ & & ${ }^{90} \mathrm{Sr}$ & $3.4 \mathrm{E}-01 \pm 4.1 \mathrm{E}-01$ & $\mathrm{U}$ \\
\hline & ${ }^{234} \mathrm{U}$ & $1.7 \mathrm{E}-01 \pm 5.9 \mathrm{E}-02$ & & & ${ }^{234} \mathrm{U}$ & $1.7 \mathrm{E}-01 \pm 5.9 \mathrm{E}-02$ & \\
\hline & ${ }^{235} \mathrm{U}$ & $1.5 \mathrm{E}-02 \pm 1.3 \mathrm{E}-02$ & & & ${ }^{235} \mathrm{U}$ & $1.9 \mathrm{E}-02 \pm 1.4 \mathrm{E}-02$ & \\
\hline & ${ }^{238} \mathrm{U}$ & $1.4 \mathrm{E}-01 \pm 5.0 \mathrm{E}-02$ & & & ${ }^{238} \mathrm{U}$ & $1.8 \mathrm{E}-01 \pm 6.1 \mathrm{E}-02$ & \\
\hline & ${ }^{65} \mathrm{Zn}$ & $2.8 \mathrm{E}-02 \pm 2.4 \mathrm{E}-02$ & $\mathrm{U}$ & & ${ }^{65} \mathrm{Zn}$ & $2.0 \mathrm{E}-02 \pm 2.2 \mathrm{E}-02$ & $\mathrm{U}$ \\
\hline \multirow{18}{*}{$\begin{array}{c}\text { D165 } \\
(100-B / C)\end{array}$} & ${ }^{144} \mathrm{Ce}$ & $6.6 \mathrm{E}-03 \pm 6.6 \mathrm{E}-02$ & $\mathrm{U}$ & D147 & ${ }^{144} \mathrm{Ce}$ & $-2.2 \mathrm{E}-02 \pm 8.7 \mathrm{E}-02$ & $\mathrm{U}$ \\
\hline & ${ }^{60} \mathrm{Co}$ & $-6.8 \mathrm{E}-04 \pm 6.8 \mathrm{E}-03$ & $\mathrm{U}$ & (100-D) & ${ }^{60} \mathrm{Co}$ & $-2.7 \mathrm{E}-03 \pm 4.6 \mathrm{E}-03$ & $\mathrm{U}$ \\
\hline & ${ }^{134} \mathrm{Cs}$ & $3.9 \mathrm{E}-02 \pm 1.3 \mathrm{E}-02$ & $\mathrm{U}$ & & ${ }^{134} \mathrm{Cs}$ & $2.4 \mathrm{E}-02 \pm 7.2 \mathrm{E}-03$ & \\
\hline & ${ }^{137} \mathrm{Cs}$ & $1.2 \mathrm{E}-01 \pm 2.4 \mathrm{E}-02$ & & & ${ }^{137} \mathrm{Cs}$ & 1.9E-01 $\pm 3.1 \mathrm{E}-02$ & \\
\hline & ${ }^{152} \mathrm{Eu}$ & $-1.0 \mathrm{E}-02 \pm 2.5 \mathrm{E}-02$ & U & & ${ }^{152} \mathrm{Eu}$ & $2.7 \mathrm{E}-02 \pm 1.8 \mathrm{E}-02$ & \\
\hline & ${ }^{154} \mathrm{Eu}$ & $4.2 \mathrm{E}-03 \pm 2.1 \mathrm{E}-02$ & $\mathrm{U}$ & & ${ }^{154} \mathrm{Eu}$ & $-2.0 \mathrm{E}-02 \pm 2.0 \mathrm{E}-02$ & $\mathrm{U}$ \\
\hline & ${ }^{155} \mathrm{Eu}$ & $4.0 \mathrm{E}-02 \pm 3.5 \mathrm{E}-02$ & $\mathrm{U}$ & & ${ }^{155} \mathrm{Eu}$ & $6.8 \mathrm{E}-03 \pm 2.1 \mathrm{E}-02$ & $\mathrm{U}$ \\
\hline & ${ }^{238} \mathrm{Pu}$ & $-2.8 \mathrm{E}-02 \pm 3.1 \mathrm{E}-02$ & $\mathrm{U}$ & & ${ }^{238} \mathrm{Pu}$ & 4.3E-03 $\pm 3.5 \mathrm{E}-02$ & $\mathrm{U}$ \\
\hline & ${ }^{239 / 240} \mathrm{Pu}$ & $2.0 \mathrm{E}-03 \pm 9.0 \mathrm{E}-03$ & $\mathrm{U}$ & & ${ }^{239 / 240} \mathrm{Pu}$ & $1.3 \mathrm{E}-02 \pm 1.3 \mathrm{E}-02$ & $\mathrm{U}$ \\
\hline & ${ }^{103} \mathrm{Ru}$ & $6.8 \mathrm{E}-04 \pm 6.3 \mathrm{E}-03$ & $\mathrm{U}$ & & ${ }^{103} \mathrm{Ru}$ & $2.1 \mathrm{E}-03 \pm 5.8 \mathrm{E}-03$ & $\mathrm{U}$ \\
\hline & ${ }^{106} \mathrm{Ru}$ & $-1.0 \mathrm{E}-02 \pm 6.1 \mathrm{E}-02$ & $\mathrm{U}$ & & ${ }^{106} \mathrm{Ru}$ & $4.7 \mathrm{E}-03 \pm 4.2 \mathrm{E}-02$ & $\mathrm{U}$ \\
\hline & ${ }^{125} \mathrm{Sb}$ & $-8.9 \mathrm{E}-03 \pm 1.9 \mathrm{E}-02$ & $\mathrm{U}$ & & ${ }^{125} \mathrm{Sb}$ & $4.2 \mathrm{E}-03 \pm 1.4 \mathrm{E}-02$ & $\mathrm{U}$ \\
\hline & ${ }^{113} \mathrm{Sn}$ & $-1.4 \mathrm{E}-02 \pm 1.4 \mathrm{E}-02$ & $\mathrm{U}$ & & ${ }^{113} \mathrm{Sn}$ & $-2.4 \mathrm{E}-03 \pm 6.8 \mathrm{E}-03$ & $\mathrm{U}$ \\
\hline & ${ }^{90} \mathrm{Sr}$ & $-3.9 \mathrm{E}-01 \pm 3.9 \mathrm{E}-01$ & $\mathrm{U}$ & & ${ }^{90} \mathrm{Sr}$ & $-1.2 \mathrm{E}-01 \pm 4.0 \mathrm{E}-01$ & $\mathrm{U}$ \\
\hline & ${ }^{234} \mathrm{U}$ & $1.3 \mathrm{E}-01 \pm 4.7 \mathrm{E}-02$ & & & ${ }^{234} \mathrm{U}$ & $1.6 \mathrm{E}-01 \pm 5.9 \mathrm{E}-02$ & \\
\hline & ${ }^{235} \mathrm{U}$ & $2.1 \mathrm{E}-02 \pm 1.4 \mathrm{E}-02$ & & & ${ }^{235} \mathrm{U}$ & $5.6 \mathrm{E}-03 \pm 1.8 \mathrm{E}-02$ & $\mathrm{U}$ \\
\hline & ${ }^{238} \mathrm{U}$ & $1.0 \mathrm{E}-01 \pm 3.8 \mathrm{E}-02$ & & & ${ }^{238} \mathrm{U}$ & $1.4 \mathrm{E}-01 \pm 5.3 \mathrm{E}-02$ & \\
\hline & ${ }^{65} \mathrm{Zn}$ & $-5.5 \mathrm{E}-04 \pm 5.5 \mathrm{E}-03$ & $\mathrm{U}$ & & ${ }^{65} \mathrm{Zn}$ & $-7.4 \mathrm{E}-03 \pm 1.3 \mathrm{E}-02$ & $\mathrm{U}$ \\
\hline
\end{tabular}

$\overline{\mathrm{RQ}}=$ Result Qualifier. $\mathrm{U}=$ The analyte was analyzed for but not detected. 
Table 3-4. 2008 Soil Sampling Results (pCi/g \pm total analytical uncertainty). (Sheet 2 of 24)

\begin{tabular}{|c|c|c|c|c|c|c|c|}
\hline Location & Isotope & Result \pm Error & $\mathbf{R Q}^{*}$ & Location & Isotope & Result \pm Error & $\mathbf{R Q}^{*}$ \\
\hline D171 & ${ }^{144} \mathrm{Ce}$ & $7.3 \mathrm{E}-02 \pm 2.0 \mathrm{E}-01$ & $\mathrm{U}$ & D172 & ${ }^{144} \mathrm{Ce}$ & $-1.5 \mathrm{E}-02 \pm 1.5 \mathrm{E}-01$ & $\mathrm{U}$ \\
\hline \multirow[t]{17}{*}{$(100-\mathrm{D})$} & ${ }^{60} \mathrm{Co}$ & $-3.4 \mathrm{E}-03 \pm 1.1 \mathrm{E}-02$ & $\mathrm{U}$ & $(100-D)$ & ${ }^{60} \mathrm{Co}$ & $-1.3 \mathrm{E}-03 \pm 8.2 \mathrm{E}-03$ & $\mathrm{U}$ \\
\hline & ${ }^{134} \mathrm{Cs}$ & $2.4 \mathrm{E}-02 \pm 1.9 \mathrm{E}-02$ & $\mathrm{U}$ & & ${ }^{134} \mathrm{Cs}$ & $3.6 \mathrm{E}-02 \pm 1.5 \mathrm{E}-02$ & \\
\hline & ${ }^{137} \mathrm{Cs}$ & $4.4 \mathrm{E}-01 \pm 7.2 \mathrm{E}-02$ & & & ${ }^{137} \mathrm{Cs}$ & $1.4 \mathrm{E}-01 \pm 3.0 \mathrm{E}-02$ & \\
\hline & ${ }^{152} \mathrm{Eu}$ & $6.2 \mathrm{E}-02 \pm 5.1 \mathrm{E}-02$ & & & ${ }^{152} \mathrm{Eu}$ & $-1.5 \mathrm{E}-02 \pm 3.2 \mathrm{E}-02$ & $\mathrm{U}$ \\
\hline & ${ }^{154} \mathrm{Eu}$ & $-2.8 \mathrm{E}-02 \pm 4.3 \mathrm{E}-02$ & $\mathrm{U}$ & & ${ }^{154} \mathrm{Eu}$ & $-3.1 \mathrm{E}-02 \pm 3.1 \mathrm{E}-02$ & $\mathrm{U}$ \\
\hline & ${ }^{155} \mathrm{Eu}$ & $4.0 \mathrm{E}-02 \pm 4.8 \mathrm{E}-02$ & $\mathrm{U}$ & & ${ }^{155} \mathrm{Eu}$ & $-2.4 \mathrm{E}-05 \pm 2.4 \mathrm{E}-04$ & $\mathrm{U}$ \\
\hline & ${ }^{238} \mathrm{Pu}$ & $-6.4 \mathrm{E}-03 \pm 3.2 \mathrm{E}-02$ & $\mathrm{U}$ & & ${ }^{238} \mathrm{Pu}$ & $1.7 \mathrm{E}-02 \pm 3.2 \mathrm{E}-02$ & $\mathrm{U}$ \\
\hline & ${ }^{239 / 240} \mathrm{Pu}$ & $6.4 \mathrm{E}-03 \pm 1.1 \mathrm{E}-02$ & $\mathrm{U}$ & & ${ }^{239 / 240} \mathrm{Pu}$ & $8.3 \mathrm{E}-03 \pm 1.2 \mathrm{E}-02$ & $\mathrm{U}$ \\
\hline & ${ }^{103} \mathrm{Ru}$ & $5.4 \mathrm{E}-03 \pm 1.5 \mathrm{E}-02$ & $\mathrm{U}$ & & ${ }^{103} \mathrm{Ru}$ & $2.2 \mathrm{E}-03 \pm 9.3 \mathrm{E}-03$ & $\mathrm{U}$ \\
\hline & ${ }^{106} \mathrm{Ru}$ & $2.8 \mathrm{E}-02 \pm 1.1 \mathrm{E}-01$ & $\mathrm{U}$ & & ${ }^{106} \mathrm{Ru}$ & $4.0 \mathrm{E}-02 \pm 7.3 \mathrm{E}-02$ & $\mathrm{U}$ \\
\hline & ${ }^{125} \mathrm{Sb}$ & $3.4 \mathrm{E}-02 \pm 3.4 \mathrm{E}-02$ & $\mathrm{U}$ & & ${ }^{125} \mathrm{Sb}$ & $1.9 \mathrm{E}-02 \pm 2.6 \mathrm{E}-02$ & $\mathrm{U}$ \\
\hline & ${ }^{113} \mathrm{Sn}$ & $1.6 \mathrm{E}-02 \pm 1.9 \mathrm{E}-02$ & $\mathrm{U}$ & & ${ }^{113} \mathrm{Sn}$ & $-7.1 \mathrm{E}-03 \pm 1.1 \mathrm{E}-02$ & $\mathrm{U}$ \\
\hline & ${ }^{90} \mathrm{Sr}$ & $1.1 \mathrm{E}-02 \pm 1.1 \mathrm{E}-01$ & $\mathrm{U}$ & & ${ }^{90} \mathrm{Sr}$ & $-4.7 \mathrm{E}-01 \pm 4.7 \mathrm{E}-01$ & $\mathrm{U}$ \\
\hline & ${ }^{234} \mathrm{U}$ & $1.5 \mathrm{E}-01 \pm 5.6 \mathrm{E}-02$ & & & ${ }^{234} \mathrm{U}$ & $1.2 \mathrm{E}-01 \pm 4.6 \mathrm{E}-02$ & \\
\hline & ${ }^{235} \mathrm{U}$ & $1.5 \mathrm{E}-02 \pm 1.4 \mathrm{E}-02$ & & & ${ }^{235} \mathrm{U}$ & $1.3 \mathrm{E}-02 \pm 1.2 \mathrm{E}-02$ & \\
\hline & ${ }^{238} \mathrm{U}$ & $1.2 \mathrm{E}-01 \pm 4.8 \mathrm{E}-02$ & & & ${ }^{238} \mathrm{U}$ & $1.2 \mathrm{E}-01 \pm 4.6 \mathrm{E}-02$ & \\
\hline & ${ }^{65} \mathrm{Zn}$ & $1.8 \mathrm{E}-03 \pm 1.8 \mathrm{E}-02$ & $\mathrm{U}$ & & ${ }^{65} \mathrm{Zn}$ & $6.3 \mathrm{E}-03 \pm 2.2 \mathrm{E}-02$ & $\mathrm{U}$ \\
\hline \multirow{18}{*}{$\begin{array}{c}\text { D173 } \\
(100-\mathrm{D})\end{array}$} & ${ }^{144} \mathrm{Ce}$ & $1.0 \mathrm{E}-02 \pm 1.0 \mathrm{E}-01$ & $\mathrm{U}$ & D154 & ${ }^{144} \mathrm{Ce}$ & $2.1 \mathrm{E}-02 \pm 2.0 \mathrm{E}-01$ & $\mathrm{U}$ \\
\hline & ${ }^{60} \mathrm{Co}$ & $-1.0 \mathrm{E}-03 \pm 5.5 \mathrm{E}-03$ & $\mathrm{U}$ & $(100-F)$ & ${ }^{60} \mathrm{Co}$ & $-4.3 \mathrm{E}-03 \pm 9.1 \mathrm{E}-03$ & $\mathrm{U}$ \\
\hline & ${ }^{134} \mathrm{Cs}$ & $3.4 \mathrm{E}-02 \pm 1.2 \mathrm{E}-02$ & & & ${ }^{134} \mathrm{Cs}$ & $5.7 \mathrm{E}-02 \pm 1.9 \mathrm{E}-02$ & \\
\hline & ${ }^{137} \mathrm{Cs}$ & $6.3 \mathrm{E}-02 \pm 1.3 \mathrm{E}-02$ & & & ${ }^{137} \mathrm{Cs}$ & $1.3 \mathrm{E}-01 \pm 3.3 \mathrm{E}-02$ & \\
\hline & ${ }^{152} \mathrm{Eu}$ & $3.8 \mathrm{E}-03 \pm 2.2 \mathrm{E}-02$ & $\mathrm{U}$ & & ${ }^{152} \mathrm{Eu}$ & $-1.2 \mathrm{E}-02 \pm 4.4 \mathrm{E}-02$ & $\mathrm{U}$ \\
\hline & ${ }^{154} \mathrm{Eu}$ & $-2.0 \mathrm{E}-04 \pm 2.0 \mathrm{E}-03$ & $\mathrm{U}$ & & ${ }^{154} \mathrm{Eu}$ & $-2.4 \mathrm{E}-02 \pm 2.7 \mathrm{E}-02$ & $\mathrm{U}$ \\
\hline & ${ }^{155} \mathrm{Eu}$ & $4.0 \mathrm{E}-02 \pm 3.6 \mathrm{E}-02$ & $\mathrm{U}$ & & ${ }^{155} \mathrm{Eu}$ & $8.9 \mathrm{E}-02 \pm 6.4 \mathrm{E}-02$ & \\
\hline & ${ }^{238} \mathrm{Pu}$ & $-2.3 \mathrm{E}-02 \pm 3.4 \mathrm{E}-02$ & $\mathrm{U}$ & & ${ }^{238} \mathrm{Pu}$ & $-1.1 \mathrm{E}-02 \pm 4.8 \mathrm{E}-02$ & $\mathrm{U}$ \\
\hline & ${ }^{239 / 240} \mathrm{Pu}$ & $4.2 \mathrm{E}-03 \pm 6.0 \mathrm{E}-03$ & $\mathrm{U}$ & & ${ }^{239 / 240} \mathrm{Pu}$ & $5.6 \mathrm{E}-03 \pm 1.1 \mathrm{E}-02$ & $\mathrm{U}$ \\
\hline & ${ }^{103} \mathrm{Ru}$ & $-3.8 \mathrm{E}-03 \pm 6.1 \mathrm{E}-03$ & $\mathrm{U}$ & & ${ }^{103} \mathrm{Ru}$ & $7.1 \mathrm{E}-03 \pm 9.6 \mathrm{E}-03$ & $\mathrm{U}$ \\
\hline & ${ }^{106} \mathrm{Ru}$ & $5.2 \mathrm{E}-02 \pm 4.9 \mathrm{E}-02$ & $\mathrm{U}$ & & ${ }^{106} \mathrm{Ru}$ & $-7.6 \mathrm{E}-04 \pm 7.6 \mathrm{E}-03$ & $\mathrm{U}$ \\
\hline & ${ }^{125} \mathrm{Sb}$ & $-2.3 \mathrm{E}-03 \pm 1.6 \mathrm{E}-02$ & $\mathrm{U}$ & & ${ }^{125} \mathrm{Sb}$ & $-1.4 \mathrm{E}-02 \pm 2.8 \mathrm{E}-02$ & $\mathrm{U}$ \\
\hline & ${ }^{113} \mathrm{Sn}$ & $-2.6 \mathrm{E}-03 \pm 7.6 \mathrm{E}-03$ & $\mathrm{U}$ & & ${ }^{113} \mathrm{Sn}$ & $-1.2 \mathrm{E}-03 \pm 1.2 \mathrm{E}-02$ & $\mathrm{U}$ \\
\hline & ${ }^{90} \mathrm{Sr}$ & $-4.7 \mathrm{E}-01 \pm 4.7 \mathrm{E}-01$ & $\mathrm{U}$ & & ${ }^{90} \mathrm{Sr}$ & $-1.9 \mathrm{E}-01 \pm 3.9 \mathrm{E}-01$ & $\mathrm{U}$ \\
\hline & ${ }^{234} \mathrm{U}$ & $1.1 \mathrm{E}-01 \pm 4.4 \mathrm{E}-02$ & & & ${ }^{234} \mathrm{U}$ & $3.1 \mathrm{E}-01 \pm 8.1 \mathrm{E}-02$ & \\
\hline & ${ }^{235} \mathrm{U}$ & $1.2 \mathrm{E}-02 \pm 1.1 \mathrm{E}-02$ & & & ${ }^{235} \mathrm{U}$ & $2.2 \mathrm{E}-03 \pm 4.4 \mathrm{E}-03$ & $\mathrm{U}$ \\
\hline & ${ }^{238} \mathrm{U}$ & 8.8E-02 \pm 3.7E-02 & & & ${ }^{238} \mathrm{U}$ & $2.0 \mathrm{E}-01 \pm 5.8 \mathrm{E}-02$ & \\
\hline & ${ }^{65} \mathrm{Zn}$ & $5.6 \mathrm{E}-03 \pm 1.3 \mathrm{E}-02$ & $\mathrm{U}$ & & ${ }^{65} \mathrm{Zn}$ & $-5.6 \mathrm{E}-03 \pm 2.4 \mathrm{E}-02$ & $\mathrm{U}$ \\
\hline
\end{tabular}

$\overline{\mathrm{RQ}}=$ Result Qualifier. $\mathrm{U}=$ The analyte was analyzed for but not detected. 
Table 3-4. 2008 Soil Sampling Results (pCi/g \pm total analytical uncertainty). (Sheet 3 of 24)

\begin{tabular}{|c|c|c|c|c|c|c|c|}
\hline Location & Isotope & Result \pm Error & $\mathbf{R Q}^{*}$ & Location & Isotope & Result \pm Error & $\mathbf{R Q}^{*}$ \\
\hline D155 & ${ }^{144} \mathrm{Ce}$ & $-9.4 \mathrm{E}-02 \pm 1.8 \mathrm{E}-01$ & $\mathrm{U}$ & D168 & ${ }^{144} \mathrm{Ce}$ & $2.1 \mathrm{E}-02 \pm 1.2 \mathrm{E}-01$ & $\mathrm{U}$ \\
\hline \multirow[t]{17}{*}{$(100-F)$} & ${ }^{60} \mathrm{Co}$ & $1.3 \mathrm{E}-02 \pm 9.6 \mathrm{E}-03$ & $\mathrm{U}$ & $(100-F)$ & ${ }^{60} \mathrm{Co}$ & $-2.5 \mathrm{E}-03 \pm 7.0 \mathrm{E}-03$ & $\mathrm{U}$ \\
\hline & ${ }^{134} \mathrm{Cs}$ & $4.1 \mathrm{E}-02 \pm 1.5 \mathrm{E}-02$ & & & ${ }^{134} \mathrm{Cs}$ & $3.1 \mathrm{E}-02 \pm 1.2 \mathrm{E}-02$ & \\
\hline & ${ }^{137} \mathrm{Cs}$ & $3.2 \mathrm{E}-01 \pm 6.1 \mathrm{E}-02$ & & & ${ }^{137} \mathrm{Cs}$ & $6.7 \mathrm{E}-02 \pm 1.9 \mathrm{E}-02$ & \\
\hline & ${ }^{152} \mathrm{Eu}$ & $1.7 \mathrm{E}-01 \pm 3.1 \mathrm{E}-02$ & & & ${ }^{152} \mathrm{Eu}$ & $1.8 \mathrm{E}-02 \pm 2.4 \mathrm{E}-02$ & $\mathrm{U}$ \\
\hline & ${ }^{154} \mathrm{Eu}$ & $2.8 \mathrm{E}-02 \pm 3.3 \mathrm{E}-02$ & $\mathrm{U}$ & & ${ }^{154} \mathrm{Eu}$ & $1.9 \mathrm{E}-02 \pm 2.6 \mathrm{E}-02$ & $\mathrm{U}$ \\
\hline & ${ }^{155} \mathrm{Eu}$ & 4.7E-02 $\pm 4.7 \mathrm{E}-02$ & $\mathrm{U}$ & & ${ }^{155} \mathrm{Eu}$ & $3.0 \mathrm{E}-02 \pm 3.0 \mathrm{E}-02$ & $\mathrm{U}$ \\
\hline & ${ }^{238} \mathrm{Pu}$ & $1.8 \mathrm{E}-03 \pm 1.8 \mathrm{E}-02$ & $\mathrm{U}$ & & ${ }^{238} \mathrm{Pu}$ & $2.4 \mathrm{E}-02 \pm 4.2 \mathrm{E}-02$ & $\mathrm{U}$ \\
\hline & ${ }^{239 / 240} \mathrm{Pu}$ & $8.9 \mathrm{E}-03 \pm 9.7 \mathrm{E}-03$ & $\mathrm{U}$ & & ${ }^{239 / 240} \mathrm{Pu}$ & $2.2 \mathrm{E}-03 \pm 1.5 \mathrm{E}-02$ & $\mathrm{U}$ \\
\hline & ${ }^{103} \mathrm{Ru}$ & $-4.4 \mathrm{E}-03 \pm 8.5 \mathrm{E}-03$ & $\mathrm{U}$ & & ${ }^{103} \mathrm{Ru}$ & $-6.6 \mathrm{E}-04 \pm 6.3 \mathrm{E}-03$ & $\mathrm{U}$ \\
\hline & ${ }^{106} \mathrm{Ru}$ & $-3.4 \mathrm{E}-02 \pm 7.9 \mathrm{E}-02$ & $\mathrm{U}$ & & ${ }^{106} \mathrm{Ru}$ & $-5.6 \mathrm{E}-02 \pm 6.3 \mathrm{E}-02$ & $\mathrm{U}$ \\
\hline & ${ }^{125} \mathrm{Sb}$ & $-1.1 \mathrm{E}-02 \pm 2.4 \mathrm{E}-02$ & $\mathrm{U}$ & & ${ }^{125} \mathrm{Sb}$ & $-6.5 \mathrm{E}-04 \pm 6.5 \mathrm{E}-03$ & $\mathrm{U}$ \\
\hline & ${ }^{113} \mathrm{Sn}$ & $-5.0 \mathrm{E}-03 \pm 1.1 \mathrm{E}-02$ & $\mathrm{U}$ & & ${ }^{113} \mathrm{Sn}$ & $-1.6 \mathrm{E}-03 \pm 8.8 \mathrm{E}-03$ & $\mathrm{U}$ \\
\hline & ${ }^{90} \mathrm{Sr}$ & $-2.8 \mathrm{E}-01 \pm 4.1 \mathrm{E}-01$ & $\mathrm{U}$ & & ${ }^{90} \mathrm{Sr}$ & $-7.6 \mathrm{E}-01 \pm 7.6 \mathrm{E}-01$ & $\mathrm{U}$ \\
\hline & ${ }^{234} \mathrm{U}$ & $1.1 \mathrm{E}-01 \pm 3.6 \mathrm{E}-02$ & & & ${ }^{234} \mathrm{U}$ & $1.4 \mathrm{E}-01 \pm 4.3 \mathrm{E}-02$ & \\
\hline & ${ }^{235} \mathrm{U}$ & $1.0 \mathrm{E}-02 \pm 9.2 \mathrm{E}-03$ & & & ${ }^{235} \mathrm{U}$ & $1.1 \mathrm{E}-02 \pm 1.0 \mathrm{E}-02$ & \\
\hline & ${ }^{238} \mathrm{U}$ & $7.0 \mathrm{E}-02 \pm 2.7 \mathrm{E}-02$ & & & ${ }^{238} \mathrm{U}$ & $1.1 \mathrm{E}-01 \pm 3.7 \mathrm{E}-02$ & \\
\hline & ${ }^{65} \mathrm{Zn}$ & $6.5 \mathrm{E}-02 \pm 3.6 \mathrm{E}-02$ & & & ${ }^{65} \mathrm{Zn}$ & $1.2 \mathrm{E}-03 \pm 1.2 \mathrm{E}-02$ & $\mathrm{U}$ \\
\hline \multirow{18}{*}{$\begin{array}{c}\text { D169 } \\
(100-F)\end{array}$} & ${ }^{144} \mathrm{Ce}$ & $-2.9 \mathrm{E}-03 \pm 2.9 \mathrm{E}-02$ & $\mathrm{U}$ & D170 & ${ }^{144} \mathrm{Ce}$ & $3.4 \mathrm{E}-03 \pm 3.4 \mathrm{E}-02$ & $\mathrm{U}$ \\
\hline & ${ }^{60} \mathrm{Co}$ & $-2.7 \mathrm{E}-03 \pm 7.6 \mathrm{E}-03$ & $\mathrm{U}$ & $(100-F)$ & ${ }^{60} \mathrm{Co}$ & $-1.4 \mathrm{E}-02 \pm 1.4 \mathrm{E}-02$ & $\mathrm{U}$ \\
\hline & ${ }^{134} \mathrm{Cs}$ & $2.9 \mathrm{E}-02 \pm 1.1 \mathrm{E}-02$ & & & ${ }^{134} \mathrm{Cs}$ & $5.5 \mathrm{E}-02 \pm 2.3 \mathrm{E}-02$ & \\
\hline & ${ }^{137} \mathrm{Cs}$ & $4.5 \mathrm{E}-02 \pm 1.4 \mathrm{E}-02$ & & & ${ }^{137} \mathrm{Cs}$ & $4.8 \mathrm{E}-02 \pm 2.1 \mathrm{E}-02$ & \\
\hline & ${ }^{152} \mathrm{Eu}$ & $-1.6 \mathrm{E}-02 \pm 2.2 \mathrm{E}-02$ & U & & ${ }^{152} \mathrm{Eu}$ & $-2.6 \mathrm{E}-02 \pm 4.9 \mathrm{E}-02$ & $\mathrm{U}$ \\
\hline & ${ }^{154} \mathrm{Eu}$ & $1.8 \mathrm{E}-02 \pm 2.8 \mathrm{E}-02$ & $\mathrm{U}$ & & ${ }^{154} \mathrm{Eu}$ & $-2.8 \mathrm{E}-02 \pm 4.1 \mathrm{E}-02$ & $\mathrm{U}$ \\
\hline & ${ }^{155} \mathrm{Eu}$ & $5.3 \mathrm{E}-02 \pm 3.6 \mathrm{E}-02$ & & & ${ }^{155} \mathrm{Eu}$ & $6.1 \mathrm{E}-02 \pm 5.3 \mathrm{E}-02$ & $\mathrm{U}$ \\
\hline & ${ }^{238} \mathrm{Pu}$ & $8.1 \mathrm{E}-03 \pm 3.0 \mathrm{E}-02$ & $\mathrm{U}$ & & ${ }^{238} \mathrm{Pu}$ & $-2.8 \mathrm{E}-02 \pm 3.8 \mathrm{E}-02$ & $\mathrm{U}$ \\
\hline & ${ }^{239 / 240} \mathrm{Pu}$ & $8.1 \mathrm{E}-03 \pm 1.0 \mathrm{E}-02$ & $\mathrm{U}$ & & ${ }^{239 / 240} \mathrm{Pu}$ & $2.4 \mathrm{E}-03 \pm 2.4 \mathrm{E}-02$ & $\mathrm{U}$ \\
\hline & ${ }^{103} \mathrm{Ru}$ & $2.1 \mathrm{E}-03 \pm 6.5 \mathrm{E}-03$ & $\mathrm{U}$ & & ${ }^{103} \mathrm{Ru}$ & $-6.8 \mathrm{E}-03 \pm 1.2 \mathrm{E}-02$ & $\mathrm{U}$ \\
\hline & ${ }^{106} \mathrm{Ru}$ & $-1.0 \mathrm{E}-02 \pm 6.2 \mathrm{E}-02$ & $\mathrm{U}$ & & ${ }^{106} \mathrm{Ru}$ & $1.1 \mathrm{E}-01 \pm 1.2 \mathrm{E}-01$ & $\mathrm{U}$ \\
\hline & ${ }^{125} \mathrm{Sb}$ & $-6.9 \mathrm{E}-03 \pm 1.8 \mathrm{E}-02$ & $\mathrm{U}$ & & ${ }^{125} \mathrm{Sb}$ & $-4.9 \mathrm{E}-03 \pm 3.5 \mathrm{E}-02$ & $\mathrm{U}$ \\
\hline & ${ }^{113} \mathrm{Sn}$ & $-4.6 \mathrm{E}-03 \pm 8.6 \mathrm{E}-03$ & $\mathrm{U}$ & & ${ }^{113} \mathrm{Sn}$ & $1.7 \mathrm{E}-02 \pm 1.8 \mathrm{E}-02$ & $\mathrm{U}$ \\
\hline & ${ }^{90} \mathrm{Sr}$ & $1.8 \mathrm{E}-01 \pm 3.9 \mathrm{E}-01$ & $\mathrm{U}$ & & ${ }^{90} \mathrm{Sr}$ & $1.4 \mathrm{E}-01 \pm 4.3 \mathrm{E}-01$ & $\mathrm{U}$ \\
\hline & ${ }^{234} \mathrm{U}$ & 1.0E-01 \pm 3.7E-02 & & & ${ }^{234} \mathrm{U}$ & $1.5 \mathrm{E}-01 \pm 4.7 \mathrm{E}-02$ & \\
\hline & ${ }^{235} \mathrm{U}$ & $1.3 \mathrm{E}-02 \pm 1.0 \mathrm{E}-02$ & & & ${ }^{235} \mathrm{U}$ & $4.8 \mathrm{E}-03 \pm 6.9 \mathrm{E}-03$ & $\mathrm{U}$ \\
\hline & ${ }^{238} \mathrm{U}$ & 8.6E-02 $\pm 3.3 \mathrm{E}-02$ & & & ${ }^{238} \mathrm{U}$ & $1.0 \mathrm{E}-01 \pm 3.5 \mathrm{E}-02$ & \\
\hline & ${ }^{65} \mathrm{Zn}$ & $7.6 \mathrm{E}-03 \pm 2.1 \mathrm{E}-02$ & $\mathrm{U}$ & & ${ }^{65} \mathrm{Zn}$ & $-1.1 \mathrm{E}-03 \pm 1.1 \mathrm{E}-02$ & $\mathrm{U}$ \\
\hline
\end{tabular}

$\overline{\mathrm{RQ}}=$ Result Qualifier. $\mathrm{U}=$ The analyte was analyzed for but not detected. 
Table 3-4. 2008 Soil Sampling Results (pCi/g \pm total analytical uncertainty). (Sheet 4 of 24)

\begin{tabular}{|c|c|c|c|c|c|c|c|}
\hline Location & Isotope & Result \pm Error & $\mathbf{R Q}^{*}$ & Location & Isotope & Result \pm Error & $\mathbf{R Q}^{*}$ \\
\hline D152 & ${ }^{144} \mathrm{Ce}$ & $-1.8 \mathrm{E}-02 \pm 1.2 \mathrm{E}-01$ & $\mathrm{U}$ & D176 & ${ }^{144} \mathrm{Ce}$ & $6.4 \mathrm{E}-02 \pm 1.7 \mathrm{E}-01$ & $\mathrm{U}$ \\
\hline \multirow[t]{17}{*}{$(100-\mathrm{H})$} & ${ }^{60} \mathrm{Co}$ & $4.4 \mathrm{E}-03 \pm 5.9 \mathrm{E}-03$ & $\mathrm{U}$ & $(100-\mathrm{H})$ & ${ }^{60} \mathrm{Co}$ & $6.5 \mathrm{E}-04 \pm 6.5 \mathrm{E}-03$ & $\mathrm{U}$ \\
\hline & ${ }^{134} \mathrm{Cs}$ & $2.0 \mathrm{E}-02 \pm 9.8 \mathrm{E}-03$ & & & ${ }^{134} \mathrm{Cs}$ & $2.8 \mathrm{E}-02 \pm 1.3 \mathrm{E}-02$ & \\
\hline & ${ }^{137} \mathrm{Cs}$ & $3.7 \mathrm{E}-01 \pm 6.1 \mathrm{E}-02$ & & & ${ }^{137} \mathrm{Cs}$ & 7.9E-01 $\pm 1.4 \mathrm{E}-01$ & \\
\hline & ${ }^{152} \mathrm{Eu}$ & $7.7 \mathrm{E}-02 \pm 2.9 \mathrm{E}-02$ & & & ${ }^{152} \mathrm{Eu}$ & $1.2 \mathrm{E}-02 \pm 3.6 \mathrm{E}-02$ & $\mathrm{U}$ \\
\hline & ${ }^{154} \mathrm{Eu}$ & $2.5 \mathrm{E}-02 \pm 2.2 \mathrm{E}-02$ & $\mathrm{U}$ & & ${ }^{154} \mathrm{Eu}$ & $-8.3 \mathrm{E}-03 \pm 2.7 \mathrm{E}-02$ & $\mathrm{U}$ \\
\hline & ${ }^{155} \mathrm{Eu}$ & $9.8 \mathrm{E}-03 \pm 2.8 \mathrm{E}-02$ & $\mathrm{U}$ & & ${ }^{155} \mathrm{Eu}$ & $4.3 \mathrm{E}-02 \pm 4.7 \mathrm{E}-02$ & $\mathrm{U}$ \\
\hline & ${ }^{238} \mathrm{Pu}$ & $2.0 \mathrm{E}-03 \pm 2.0 \mathrm{E}-02$ & $\mathrm{U}$ & & ${ }^{238} \mathrm{Pu}$ & $-4.4 \mathrm{E}-03 \pm 3.4 \mathrm{E}-02$ & $\mathrm{U}$ \\
\hline & ${ }^{239 / 240} \mathrm{Pu}$ & $1.0 \mathrm{E}-02 \pm 1.2 \mathrm{E}-02$ & $\mathrm{U}$ & & ${ }^{239 / 240} \mathrm{Pu}$ & $2.9 \mathrm{E}-02 \pm 2.3 \mathrm{E}-02$ & $\mathrm{U}$ \\
\hline & ${ }^{103} \mathrm{Ru}$ & $1.7 \mathrm{E}-03 \pm 5.9 \mathrm{E}-03$ & $\mathrm{U}$ & & ${ }^{103} \mathrm{Ru}$ & $-2.4 \mathrm{E}-03 \pm 9.3 \mathrm{E}-03$ & $\mathrm{U}$ \\
\hline & ${ }^{106} \mathrm{Ru}$ & $3.6 \mathrm{E}-02 \pm 5.0 \mathrm{E}-02$ & $\mathrm{U}$ & & ${ }^{106} \mathrm{Ru}$ & $1.8 \mathrm{E}-02 \pm 7.2 \mathrm{E}-02$ & $\mathrm{U}$ \\
\hline & ${ }^{125} \mathrm{Sb}$ & $1.1 \mathrm{E}-02 \pm 1.8 \mathrm{E}-02$ & $\mathrm{U}$ & & ${ }^{125} \mathrm{Sb}$ & $1.7 \mathrm{E}-02 \pm 2.4 \mathrm{E}-02$ & $\mathrm{U}$ \\
\hline & ${ }^{113} \mathrm{Sn}$ & $-4.8 \mathrm{E}-03 \pm 8.0 \mathrm{E}-03$ & $\mathrm{U}$ & & ${ }^{113} \mathrm{Sn}$ & $1.2 \mathrm{E}-03 \pm 1.1 \mathrm{E}-02$ & $\mathrm{U}$ \\
\hline & ${ }^{90} \mathrm{Sr}$ & $-4.6 \mathrm{E}-01 \pm 4.7 \mathrm{E}-01$ & $\mathrm{U}$ & & ${ }^{90} \mathrm{Sr}$ & $-6.7 \mathrm{E}-01 \pm 6.7 \mathrm{E}-01$ & $\mathrm{U}$ \\
\hline & ${ }^{234} \mathrm{U}$ & $1.4 \mathrm{E}-01 \pm 4.9 \mathrm{E}-02$ & & & ${ }^{234} \mathrm{U}$ & $1.2 \mathrm{E}-01 \pm 4.3 \mathrm{E}-02$ & \\
\hline & ${ }^{235} \mathrm{U}$ & $8.0 \mathrm{E}-03 \pm 1.2 \mathrm{E}-02$ & $\mathrm{U}$ & & ${ }^{235} \mathrm{U}$ & $4.4 \mathrm{E}-03 \pm 8.9 \mathrm{E}-03$ & $\mathrm{U}$ \\
\hline & ${ }^{238} \mathrm{U}$ & $1.2 \mathrm{E}-01 \pm 4.3 \mathrm{E}-02$ & & & ${ }^{238} \mathrm{U}$ & $1.2 \mathrm{E}-01 \pm 4.4 \mathrm{E}-02$ & \\
\hline & ${ }^{65} \mathrm{Zn}$ & $-3.2 \mathrm{E}-02 \pm 3.2 \mathrm{E}-02$ & $\mathrm{U}$ & & ${ }^{65} \mathrm{Zn}$ & $-5.3 \mathrm{E}-03 \pm 2.1 \mathrm{E}-02$ & $\mathrm{U}$ \\
\hline \multirow{18}{*}{$\begin{array}{c}\text { D177 } \\
(100-\mathrm{H})\end{array}$} & ${ }^{144} \mathrm{Ce}$ & $1.1 \mathrm{E}-01 \pm 1.3 \mathrm{E}-01$ & $\mathrm{U}$ & D178 & ${ }^{144} \mathrm{Ce}$ & $-2.6 \mathrm{E}-02 \pm 1.1 \mathrm{E}-01$ & $\mathrm{U}$ \\
\hline & ${ }^{60} \mathrm{Co}$ & $-2.0 \mathrm{E}-03 \pm 8.3 \mathrm{E}-03$ & $\mathrm{U}$ & $(100-\mathrm{H})$ & ${ }^{60} \mathrm{Co}$ & $1.6 \mathrm{E}-03 \pm 7.3 \mathrm{E}-03$ & $\mathrm{U}$ \\
\hline & ${ }^{134} \mathrm{Cs}$ & $2.1 \mathrm{E}-02 \pm 1.1 \mathrm{E}-02$ & & & ${ }^{134} \mathrm{Cs}$ & $3.4 \mathrm{E}-02 \pm 1.0 \mathrm{E}-02$ & \\
\hline & ${ }^{137} \mathrm{Cs}$ & $2.1 \mathrm{E}-02 \pm 1.3 \mathrm{E}-02$ & & & ${ }^{137} \mathrm{Cs}$ & $3.8 \mathrm{E}-02 \pm 1.2 \mathrm{E}-02$ & \\
\hline & ${ }^{152} \mathrm{Eu}$ & $6.3 \mathrm{E}-03 \pm 2.8 \mathrm{E}-02$ & U & & ${ }^{152} \mathrm{Eu}$ & $1.5 \mathrm{E}-02 \pm 2.1 \mathrm{E}-02$ & $\mathrm{U}$ \\
\hline & ${ }^{154} \mathrm{Eu}$ & $-1.5 \mathrm{E}-02 \pm 3.0 \mathrm{E}-02$ & $\mathrm{U}$ & & ${ }^{154} \mathrm{Eu}$ & $-3.6 \mathrm{E}-03 \pm 2.4 \mathrm{E}-02$ & $\mathrm{U}$ \\
\hline & ${ }^{155} \mathrm{Eu}$ & $4.4 \mathrm{E}-02 \pm 4.0 \mathrm{E}-02$ & $\mathrm{U}$ & & ${ }^{155} \mathrm{Eu}$ & $2.0 \mathrm{E}-02 \pm 2.8 \mathrm{E}-02$ & $\mathrm{U}$ \\
\hline & ${ }^{238} \mathrm{Pu}$ & $-3.0 \mathrm{E}-02 \pm 3.2 \mathrm{E}-02$ & $\mathrm{U}$ & & ${ }^{238} \mathrm{Pu}$ & $1.0 \mathrm{E}-02 \pm 2.6 \mathrm{E}-02$ & $\mathrm{U}$ \\
\hline & ${ }^{239 / 240} \mathrm{Pu}$ & $1.1 \mathrm{E}-02 \pm 1.2 \mathrm{E}-02$ & $\mathrm{U}$ & & ${ }^{239 / 240} \mathrm{Pu}$ & $6.0 \mathrm{E}-03 \pm 7.1 \mathrm{E}-03$ & \\
\hline & ${ }^{103} \mathrm{Ru}$ & $-3.8 \mathrm{E}-03 \pm 8.1 \mathrm{E}-03$ & $\mathrm{U}$ & & ${ }^{103} \mathrm{Ru}$ & $1.1 \mathrm{E}-02 \pm 7.7 \mathrm{E}-03$ & $\mathrm{U}$ \\
\hline & ${ }^{106} \mathrm{Ru}$ & $-2.3 \mathrm{E}-02 \pm 7.0 \mathrm{E}-02$ & $\mathrm{U}$ & & ${ }^{106} \mathrm{Ru}$ & $-3.6 \mathrm{E}-02 \pm 6.1 \mathrm{E}-02$ & $\mathrm{U}$ \\
\hline & ${ }^{125} \mathrm{Sb}$ & $1.4 \mathrm{E}-02 \pm 2.0 \mathrm{E}-02$ & $\mathrm{U}$ & & ${ }^{125} \mathrm{Sb}$ & $5.4 \mathrm{E}-03 \pm 1.8 \mathrm{E}-02$ & $\mathrm{U}$ \\
\hline & ${ }^{113} \mathrm{Sn}$ & $-5.0 \mathrm{E}-03 \pm 1.0 \mathrm{E}-02$ & $\mathrm{U}$ & & ${ }^{113} \mathrm{Sn}$ & $-1.1 \mathrm{E}-02 \pm 1.1 \mathrm{E}-02$ & $\mathrm{U}$ \\
\hline & ${ }^{90} \mathrm{Sr}$ & $-9.7 \mathrm{E}-01 \pm 9.7 \mathrm{E}-01$ & $\mathrm{U}$ & & ${ }^{90} \mathrm{Sr}$ & $-1.3 \mathrm{E}+00 \pm 1.3 \mathrm{E}+00$ & $\mathrm{U}$ \\
\hline & ${ }^{234} \mathrm{U}$ & $1.2 \mathrm{E}-01 \pm 4.3 \mathrm{E}-02$ & & & ${ }^{234} \mathrm{U}$ & $1.5 \mathrm{E}-01 \pm 5.1 \mathrm{E}-02$ & \\
\hline & ${ }^{235} \mathrm{U}$ & $1.4 \mathrm{E}-02 \pm 1.1 \mathrm{E}-02$ & & & ${ }^{235} \mathrm{U}$ & $2.2 \mathrm{E}-02 \pm 1.5 \mathrm{E}-02$ & \\
\hline & ${ }^{238} \mathrm{U}$ & $9.6 \mathrm{E}-02 \pm 3.6 \mathrm{E}-02$ & & & ${ }^{238} \mathrm{U}$ & $2.1 \mathrm{E}-01 \pm 6.7 \mathrm{E}-02$ & \\
\hline & ${ }^{65} \mathrm{Zn}$ & $3.5 \mathrm{E}-02 \pm 2.3 \mathrm{E}-02$ & $\mathrm{U}$ & & ${ }^{65} \mathrm{Zn}$ & $4.4 \mathrm{E}-03 \pm 3.2 \mathrm{E}-02$ & $\mathrm{U}$ \\
\hline
\end{tabular}

$\overline{\mathrm{RQ}}=$ Result Qualifier. $\mathrm{U}=$ The analyte was analyzed for but not detected. 
Table 3-4. 2008 Soil Sampling Results (pCi/g \pm total analytical uncertainty). (Sheet 5 of 24)

\begin{tabular}{|c|c|c|c|c|c|c|c|}
\hline Location & Isotope & Result \pm Error & $\mathbf{R Q}^{*}$ & Location & Isotope & Result \pm Error & $\mathbf{R Q}^{*}$ \\
\hline D002 & ${ }^{144} \mathrm{Ce}$ & $-1.2 \mathrm{E}-01 \pm 1.6 \mathrm{E}-01$ & $\mathrm{U}$ & D004 & ${ }^{144} \mathrm{Ce}$ & $5.4 \mathrm{E}-03 \pm 5.4 \mathrm{E}-02$ & $\mathrm{U}$ \\
\hline \multirow[t]{17}{*}{$(200-W)$} & ${ }^{60} \mathrm{Co}$ & $-7.3 \mathrm{E}-03 \pm 8.2 \mathrm{E}-03$ & $\mathrm{U}$ & $(200-W)$ & ${ }^{60} \mathrm{Co}$ & $-1.9 \mathrm{E}-03 \pm 8.7 \mathrm{E}-03$ & $\mathrm{U}$ \\
\hline & ${ }^{134} \mathrm{Cs}$ & $2.7 \mathrm{E}-02 \pm 1.1 \mathrm{E}-02$ & & & ${ }^{134} \mathrm{Cs}$ & $4.7 \mathrm{E}-02 \pm 1.7 \mathrm{E}-02$ & \\
\hline & ${ }^{137} \mathrm{Cs}$ & $1.1 \mathrm{E}-01 \pm 2.2 \mathrm{E}-02$ & & & ${ }^{137} \mathrm{Cs}$ & $3.5 \mathrm{E}+00 \pm 5.6 \mathrm{E}-01$ & \\
\hline & ${ }^{152} \mathrm{Eu}$ & $-3.9 \mathrm{E}-02 \pm 3.9 \mathrm{E}-02$ & $\mathrm{U}$ & & ${ }^{152} \mathrm{Eu}$ & $-1.4 \mathrm{E}-02 \pm 3.1 \mathrm{E}-02$ & $\mathrm{U}$ \\
\hline & ${ }^{154} \mathrm{Eu}$ & $-3.0 \mathrm{E}-02 \pm 3.0 \mathrm{E}-02$ & $\mathrm{U}$ & & ${ }^{154} \mathrm{Eu}$ & $-3.4 \mathrm{E}-03 \pm 2.8 \mathrm{E}-02$ & $\mathrm{U}$ \\
\hline & ${ }^{155} \mathrm{Eu}$ & $2.2 \mathrm{E}-02 \pm 3.6 \mathrm{E}-02$ & $\mathrm{U}$ & & ${ }^{155} \mathrm{Eu}$ & $3.3 \mathrm{E}-02 \pm 3.7 \mathrm{E}-02$ & $\mathrm{U}$ \\
\hline & ${ }^{238} \mathrm{Pu}$ & 4.2E-02 $\pm 3.6 \mathrm{E}-02$ & $\mathrm{U}$ & & ${ }^{238} \mathrm{Pu}$ & $-1.9 \mathrm{E}-02 \pm 5.2 \mathrm{E}-02$ & $\mathrm{U}$ \\
\hline & ${ }^{239 / 240} \mathrm{Pu}$ & $2.5 \mathrm{E}-02 \pm 1.8 \mathrm{E}-02$ & & & ${ }^{239 / 240} \mathrm{Pu}$ & $1.3 \mathrm{E}-02 \pm 2.3 \mathrm{E}-02$ & $\mathrm{U}$ \\
\hline & ${ }^{103} \mathrm{Ru}$ & $4.1 \mathrm{E}-04 \pm 4.1 \mathrm{E}-03$ & $\mathrm{U}$ & & ${ }^{103} \mathrm{Ru}$ & $1.2 \mathrm{E}-02 \pm 1.5 \mathrm{E}-02$ & $\mathrm{U}$ \\
\hline & ${ }^{106} \mathrm{Ru}$ & $-4.1 \mathrm{E}-03 \pm 4.1 \mathrm{E}-02$ & $\mathrm{U}$ & & ${ }^{106} \mathrm{Ru}$ & $-5.1 \mathrm{E}-02 \pm 8.5 \mathrm{E}-02$ & $\mathrm{U}$ \\
\hline & ${ }^{125} \mathrm{Sb}$ & $-5.7 \mathrm{E}-03 \pm 2.2 \mathrm{E}-02$ & $\mathrm{U}$ & & ${ }^{125} \mathrm{Sb}$ & $-3.8 \mathrm{E}-03 \pm 3.0 \mathrm{E}-02$ & $\mathrm{U}$ \\
\hline & ${ }^{113} \mathrm{Sn}$ & $-3.8 \mathrm{E}-04 \pm 3.7 \mathrm{E}-03$ & $\mathrm{U}$ & & ${ }^{113} \mathrm{Sn}$ & $-1.9 \mathrm{E}-03 \pm 1.5 \mathrm{E}-02$ & $\mathrm{U}$ \\
\hline & ${ }^{90} \mathrm{Sr}$ & $-5.2 \mathrm{E}-01 \pm 5.2 \mathrm{E}-01$ & $\mathrm{U}$ & & ${ }^{90} \mathrm{Sr}$ & $-3.5 \mathrm{E}-01 \pm 4.2 \mathrm{E}-01$ & $\mathrm{U}$ \\
\hline & ${ }^{234} \mathrm{U}$ & $1.8 \mathrm{E}-01 \pm 6.7 \mathrm{E}-02$ & & & ${ }^{234} \mathrm{U}$ & $1.2 \mathrm{E}-01 \pm 4.8 \mathrm{E}-02$ & \\
\hline & ${ }^{235} \mathrm{U}$ & $1.3 \mathrm{E}-02 \pm 1.6 \mathrm{E}-02$ & $\mathrm{U}$ & & ${ }^{235} \mathrm{U}$ & $1.2 \mathrm{E}-02 \pm 1.2 \mathrm{E}-02$ & \\
\hline & ${ }^{238} \mathrm{U}$ & $1.7 \mathrm{E}-01 \pm 6.3 \mathrm{E}-02$ & & & ${ }^{238} \mathrm{U}$ & $1.4 \mathrm{E}-01 \pm 5.3 \mathrm{E}-02$ & \\
\hline & ${ }^{65} \mathrm{Zn}$ & 3.3E-02 $\pm 2.4 \mathrm{E}-02$ & $\mathrm{U}$ & & ${ }^{65} \mathrm{Zn}$ & $-7.7 \mathrm{E}-04 \pm 7.7 \mathrm{E}-03$ & $\mathrm{U}$ \\
\hline \multirow{18}{*}{$\begin{array}{c}\text { D006 } \\
(200-W)\end{array}$} & ${ }^{144} \mathrm{Ce}$ & $-4.0 \mathrm{E}-02 \pm 1.3 \mathrm{E}-01$ & $\mathrm{U}$ & D008 & ${ }^{144} \mathrm{Ce}$ & $-1.5 \mathrm{E}-02 \pm 1.5 \mathrm{E}-01$ & $\mathrm{U}$ \\
\hline & ${ }^{60} \mathrm{Co}$ & $2.3 \mathrm{E}-03 \pm 5.4 \mathrm{E}-03$ & $\mathrm{U}$ & $(200-W)$ & ${ }^{60} \mathrm{Co}$ & $1.6 \mathrm{E}-03 \pm 7.0 \mathrm{E}-03$ & $\mathrm{U}$ \\
\hline & ${ }^{134} \mathrm{Cs}$ & $3.5 \mathrm{E}-02 \pm 1.5 \mathrm{E}-02$ & & & ${ }^{134} \mathrm{Cs}$ & $2.2 \mathrm{E}-02 \pm 1.6 \mathrm{E}-02$ & \\
\hline & ${ }^{137} \mathrm{Cs}$ & $8.8 \mathrm{E}-02 \pm 1.9 \mathrm{E}-02$ & & & ${ }^{137} \mathrm{Cs}$ & $1.7 \mathrm{E}-01 \pm 3.5 \mathrm{E}-02$ & \\
\hline & ${ }^{152} \mathrm{Eu}$ & $-2.6 \mathrm{E}-02 \pm 2.6 \mathrm{E}-02$ & $\mathrm{U}$ & & ${ }^{152} \mathrm{Eu}$ & $-1.6 \mathrm{E}-02 \pm 3.3 \mathrm{E}-02$ & $\mathrm{U}$ \\
\hline & ${ }^{154} \mathrm{Eu}$ & $-3.0 \mathrm{E}-03 \pm 2.1 \mathrm{E}-02$ & $\mathrm{U}$ & & ${ }^{154} \mathrm{Eu}$ & $-7.7 \mathrm{E}-03 \pm 2.7 \mathrm{E}-02$ & $\mathrm{U}$ \\
\hline & ${ }^{155} \mathrm{Eu}$ & $5.9 \mathrm{E}-02 \pm 4.1 \mathrm{E}-02$ & & & ${ }^{155} \mathrm{Eu}$ & 4.3E-02 \pm 3.7E-02 & $\mathrm{U}$ \\
\hline & ${ }^{238} \mathrm{Pu}$ & $2.2 \mathrm{E}-03 \pm 1.6 \mathrm{E}-02$ & $\mathrm{U}$ & & ${ }^{238} \mathrm{Pu}$ & $4.6 \mathrm{E}-02 \pm 3.5 \mathrm{E}-02$ & $\mathrm{U}$ \\
\hline & ${ }^{239 / 240} \mathrm{Pu}$ & $1.3 \mathrm{E}-02 \pm 1.1 \mathrm{E}-02$ & & & ${ }^{239 / 240} \mathrm{Pu}$ & $2.4 \mathrm{E}+00 \pm 6.2 \mathrm{E}-01$ & \\
\hline & ${ }^{103} \mathrm{Ru}$ & $-3.8 \mathrm{E}-03 \pm 8.1 \mathrm{E}-03$ & $\mathrm{U}$ & & ${ }^{103} \mathrm{Ru}$ & $1.5 \mathrm{E}-03 \pm 9.6 \mathrm{E}-03$ & $\mathrm{U}$ \\
\hline & ${ }^{106} \mathrm{Ru}$ & $1.8 \mathrm{E}-02 \pm 5.4 \mathrm{E}-02$ & $\mathrm{U}$ & & ${ }^{106} \mathrm{Ru}$ & $-1.8 \mathrm{E}-02 \pm 6.5 \mathrm{E}-02$ & $\mathrm{U}$ \\
\hline & ${ }^{125} \mathrm{Sb}$ & $-7.8 \mathrm{E}-03 \pm 1.7 \mathrm{E}-02$ & $\mathrm{U}$ & & ${ }^{125} \mathrm{Sb}$ & $2.3 \mathrm{E}-03 \pm 2.0 \mathrm{E}-02$ & $\mathrm{U}$ \\
\hline & ${ }^{113} \mathrm{Sn}$ & $2.9 \mathrm{E}-03 \pm 8.5 \mathrm{E}-03$ & $\mathrm{U}$ & & ${ }^{113} \mathrm{Sn}$ & $-6.5 \mathrm{E}-03 \pm 1.1 \mathrm{E}-02$ & $\mathrm{U}$ \\
\hline & ${ }^{90} \mathrm{Sr}$ & $-5.6 \mathrm{E}-01 \pm 5.6 \mathrm{E}-01$ & $\mathrm{U}$ & & ${ }^{90} \mathrm{Sr}$ & $-5.9 \mathrm{E}-01 \pm 5.9 \mathrm{E}-01$ & $\mathrm{U}$ \\
\hline & ${ }^{234} \mathrm{U}$ & $1.5 \mathrm{E}-01 \pm 5.3 \mathrm{E}-02$ & & & ${ }^{234} \mathrm{U}$ & $1.0 \mathrm{E}-01 \pm 4.2 \mathrm{E}-02$ & \\
\hline & ${ }^{235} \mathrm{U}$ & $7.1 \mathrm{E}-03 \pm 8.4 \mathrm{E}-03$ & & & ${ }^{235} \mathrm{U}$ & $1.1 \mathrm{E}-02 \pm 1.1 \mathrm{E}-02$ & \\
\hline & ${ }^{238} \mathrm{U}$ & $1.5 \mathrm{E}-01 \pm 5.3 \mathrm{E}-02$ & & & ${ }^{238} \mathrm{U}$ & $1.1 \mathrm{E}-01 \pm 4.4 \mathrm{E}-02$ & \\
\hline & ${ }^{65} \mathrm{Zn}$ & $-1.1 \mathrm{E}-02 \pm 1.6 \mathrm{E}-02$ & $\mathrm{U}$ & & ${ }^{65} \mathrm{Zn}$ & $1.0 \mathrm{E}-02 \pm 2.0 \mathrm{E}-02$ & $\mathrm{U}$ \\
\hline
\end{tabular}

$\overline{\mathrm{RQ}}=$ Result Qualifier. U = The analyte was analyzed for but not detected. 
Table 3-4. 2008 Soil Sampling Results (pCi/g \pm total analytical uncertainty). (Sheet 6 of 24)

\begin{tabular}{|c|c|c|c|c|c|c|c|}
\hline Location & Isotope & Result \pm Error & $\mathbf{R Q}^{*}$ & Location & Isotope & Result \pm Error & $\mathbf{R Q}^{*}$ \\
\hline D010 & ${ }^{144} \mathrm{Ce}$ & $9.2 \mathrm{E}-02 \pm 2.2 \mathrm{E}-01$ & $\mathrm{U}$ & D012 & ${ }^{144} \mathrm{Ce}$ & $8.7 \mathrm{E}-03 \pm 8.7 \mathrm{E}-02$ & $\mathrm{U}$ \\
\hline \multirow[t]{17}{*}{$(200-W)$} & ${ }^{60} \mathrm{Co}$ & $-3.6 \mathrm{E}-03 \pm 1.2 \mathrm{E}-02$ & $\mathrm{U}$ & $(200-W)$ & ${ }^{60} \mathrm{Co}$ & $8.2 \mathrm{E}-03 \pm 6.9 \mathrm{E}-03$ & $\mathrm{U}$ \\
\hline & ${ }^{134} \mathrm{Cs}$ & $4.9 \mathrm{E}-02 \pm 2.6 \mathrm{E}-02$ & & & ${ }^{134} \mathrm{Cs}$ & $4.1 \mathrm{E}-02 \pm 1.6 \mathrm{E}-02$ & \\
\hline & ${ }^{137} \mathrm{Cs}$ & $2.3 \mathrm{E}+00 \pm 3.5 \mathrm{E}-01$ & & & ${ }^{137} \mathrm{Cs}$ & $2.2 \mathrm{E}+00 \pm 3.5 \mathrm{E}-01$ & \\
\hline & ${ }^{152} \mathrm{Eu}$ & $-2.6 \mathrm{E}-02 \pm 5.6 \mathrm{E}-02$ & $\mathrm{U}$ & & ${ }^{152} \mathrm{Eu}$ & $-8.7 \mathrm{E}-03 \pm 2.8 \mathrm{E}-02$ & $\mathrm{U}$ \\
\hline & ${ }^{154} \mathrm{Eu}$ & 3.7E-03 \pm 3.7E-02 & $\mathrm{U}$ & & ${ }^{154} \mathrm{Eu}$ & $7.3 \mathrm{E}-03 \pm 2.2 \mathrm{E}-02$ & $\mathrm{U}$ \\
\hline & ${ }^{155} \mathrm{Eu}$ & $6.0 \mathrm{E}-02 \pm 4.9 \mathrm{E}-02$ & $\mathrm{U}$ & & ${ }^{155} \mathrm{Eu}$ & $6.4 \mathrm{E}-02 \pm 4.7 \mathrm{E}-02$ & \\
\hline & ${ }^{238} \mathrm{Pu}$ & $1.1 \mathrm{E}-02 \pm 4.0 \mathrm{E}-02$ & $\mathrm{U}$ & & ${ }^{238} \mathrm{Pu}$ & $-2.0 \mathrm{E}-02 \pm 5.4 \mathrm{E}-02$ & $\mathrm{U}$ \\
\hline & ${ }^{239 / 240} \mathrm{Pu}$ & $8.4 \mathrm{E}-01 \pm 2.4 \mathrm{E}-01$ & & & ${ }^{239 / 240} \mathrm{Pu}$ & $4.2 \mathrm{E}-02 \pm 3.3 \mathrm{E}-02$ & $\mathrm{U}$ \\
\hline & ${ }^{103} \mathrm{Ru}$ & $-1.8 \mathrm{E}-02 \pm 2.0 \mathrm{E}-02$ & $\mathrm{U}$ & & ${ }^{103} \mathrm{Ru}$ & $2.9 \mathrm{E}-03 \pm 1.2 \mathrm{E}-02$ & $\mathrm{U}$ \\
\hline & ${ }^{106} \mathrm{Ru}$ & $2.0 \mathrm{E}-02 \pm 1.2 \mathrm{E}-01$ & $\mathrm{U}$ & & ${ }^{106} \mathrm{Ru}$ & $-1.9 \mathrm{E}-04 \pm 1.9 \mathrm{E}-03$ & $\mathrm{U}$ \\
\hline & ${ }^{125} \mathrm{Sb}$ & $-1.9 \mathrm{E}-02 \pm 4.2 \mathrm{E}-02$ & $\mathrm{U}$ & & ${ }^{125} \mathrm{Sb}$ & $-1.1 \mathrm{E}-02 \pm 2.6 \mathrm{E}-02$ & $\mathrm{U}$ \\
\hline & ${ }^{113} \mathrm{Sn}$ & $-6.6 \mathrm{E}-03 \pm 2.1 \mathrm{E}-02$ & $\mathrm{U}$ & & ${ }^{113} \mathrm{Sn}$ & $-9.5 \mathrm{E}-03 \pm 1.3 \mathrm{E}-02$ & $\mathrm{U}$ \\
\hline & ${ }^{90} \mathrm{Sr}$ & $-6.1 \mathrm{E}-01 \pm 6.1 \mathrm{E}-01$ & $\mathrm{U}$ & & ${ }^{90} \mathrm{Sr}$ & $-4.3 \mathrm{E}-01 \pm 4.3 \mathrm{E}-01$ & $\mathrm{U}$ \\
\hline & ${ }^{234} \mathrm{U}$ & $1.1 \mathrm{E}-01 \pm 5.1 \mathrm{E}-02$ & & & ${ }^{234} \mathrm{U}$ & $1.2 \mathrm{E}-01 \pm 5.2 \mathrm{E}-02$ & \\
\hline & ${ }^{235} \mathrm{U}$ & $7.2 \mathrm{E}-03 \pm 1.0 \mathrm{E}-02$ & $\mathrm{U}$ & & ${ }^{235} \mathrm{U}$ & $3.2 \mathrm{E}-02 \pm 2.3 \mathrm{E}-02$ & \\
\hline & ${ }^{238} \mathrm{U}$ & $1.2 \mathrm{E}-01 \pm 5.3 \mathrm{E}-02$ & & & ${ }^{238} \mathrm{U}$ & $1.7 \mathrm{E}-01 \pm 6.5 \mathrm{E}-02$ & \\
\hline & ${ }^{65} \mathrm{Zn}$ & $-2.7 \mathrm{E}-02 \pm 3.4 \mathrm{E}-02$ & $\mathrm{U}$ & & ${ }^{65} \mathrm{Zn}$ & $-2.4 \mathrm{E}-03 \pm 2.0 \mathrm{E}-02$ & $\mathrm{U}$ \\
\hline \multirow{18}{*}{$\begin{array}{c}\text { D014 } \\
(200-W)\end{array}$} & ${ }^{144} \mathrm{Ce}$ & $2.6 \mathrm{E}-02 \pm 1.3 \mathrm{E}-01$ & $\mathrm{U}$ & D016 & ${ }^{144} \mathrm{Ce}$ & $7.5 \mathrm{E}-02 \pm 1.5 \mathrm{E}-01$ & $\mathrm{U}$ \\
\hline & ${ }^{60} \mathrm{Co}$ & $-2.6 \mathrm{E}-03 \pm 6.4 \mathrm{E}-03$ & $\mathrm{U}$ & $(200-W)$ & ${ }^{60} \mathrm{Co}$ & $3.1 \mathrm{E}-06 \pm 3.1 \mathrm{E}-05$ & $\mathrm{U}$ \\
\hline & ${ }^{134} \mathrm{Cs}$ & $3.6 \mathrm{E}-02 \pm 1.2 \mathrm{E}-02$ & & & ${ }^{134} \mathrm{Cs}$ & $3.1 \mathrm{E}-02 \pm 1.1 \mathrm{E}-02$ & \\
\hline & ${ }^{137} \mathrm{Cs}$ & $4.2 \mathrm{E}-02 \pm 1.3 \mathrm{E}-02$ & & & ${ }^{137} \mathrm{Cs}$ & $4.3 \mathrm{E}-01 \pm 8.1 \mathrm{E}-02$ & \\
\hline & ${ }^{152} \mathrm{Eu}$ & $6.9 \mathrm{E}-03 \pm 2.3 \mathrm{E}-02$ & $\mathrm{U}$ & & ${ }^{152} \mathrm{Eu}$ & $2.3 \mathrm{E}-02 \pm 3.9 \mathrm{E}-02$ & $\mathrm{U}$ \\
\hline & ${ }^{154} \mathrm{Eu}$ & $3.9 \mathrm{E}-03 \pm 2.3 \mathrm{E}-02$ & $\mathrm{U}$ & & ${ }^{154} \mathrm{Eu}$ & $4.3 \mathrm{E}-03 \pm 2.6 \mathrm{E}-02$ & $\mathrm{U}$ \\
\hline & ${ }^{155} \mathrm{Eu}$ & $6.3 \mathrm{E}-03 \pm 3.1 \mathrm{E}-02$ & $\mathrm{U}$ & & ${ }^{155} \mathrm{Eu}$ & $3.2 \mathrm{E}-02 \pm 3.5 \mathrm{E}-02$ & $\mathrm{U}$ \\
\hline & ${ }^{238} \mathrm{Pu}$ & $1.2 \mathrm{E}-02 \pm 3.3 \mathrm{E}-02$ & $\mathrm{U}$ & & ${ }^{238} \mathrm{Pu}$ & $2.3 \mathrm{E}-02 \pm 2.7 \mathrm{E}-02$ & $\mathrm{U}$ \\
\hline & ${ }^{239 / 240} \mathrm{Pu}$ & $-5.9 \mathrm{E}-03 \pm 1.1 \mathrm{E}-02$ & $\mathrm{U}$ & & ${ }^{239 / 240} \mathrm{Pu}$ & $9.2 \mathrm{E}-03 \pm 1.3 \mathrm{E}-02$ & $\mathrm{U}$ \\
\hline & ${ }^{103} \mathrm{Ru}$ & $2.2 \mathrm{E}-03 \pm 8.5 \mathrm{E}-03$ & $\mathrm{U}$ & & ${ }^{103} \mathrm{Ru}$ & $-4.3 \mathrm{E}-03 \pm 9.9 \mathrm{E}-03$ & $\mathrm{U}$ \\
\hline & ${ }^{106} \mathrm{Ru}$ & 8.3E-02 \pm 5.9E-02 & $\mathrm{U}$ & & ${ }^{106} \mathrm{Ru}$ & $3.8 \mathrm{E}-03 \pm 3.8 \mathrm{E}-02$ & $\mathrm{U}$ \\
\hline & ${ }^{125} \mathrm{Sb}$ & $7.5 \mathrm{E}-03 \pm 1.7 \mathrm{E}-02$ & $\mathrm{U}$ & & ${ }^{125} \mathrm{Sb}$ & $-9.2 \mathrm{E}-03 \pm 2.0 \mathrm{E}-02$ & $\mathrm{U}$ \\
\hline & ${ }^{113} \mathrm{Sn}$ & $-4.9 \mathrm{E}-03 \pm 9.0 \mathrm{E}-03$ & $\mathrm{U}$ & & ${ }^{113} \mathrm{Sn}$ & $-8.5 \mathrm{E}-03 \pm 1.1 \mathrm{E}-02$ & $\mathrm{U}$ \\
\hline & ${ }^{90} \mathrm{Sr}$ & $-3.5 \mathrm{E}-01 \pm 3.9 \mathrm{E}-01$ & $\mathrm{U}$ & & ${ }^{90} \mathrm{Sr}$ & $3.7 \mathrm{E}-01 \pm 4.5 \mathrm{E}-01$ & $\mathrm{U}$ \\
\hline & ${ }^{234} \mathrm{U}$ & $1.5 \mathrm{E}-01 \pm 5.1 \mathrm{E}-02$ & & & ${ }^{234} \mathrm{U}$ & $1.3 \mathrm{E}-01 \pm 4.9 \mathrm{E}-02$ & \\
\hline & ${ }^{235} \mathrm{U}$ & $1.7 \mathrm{E}-02 \pm 1.4 \mathrm{E}-02$ & & & ${ }^{235} \mathrm{U}$ & $-2.6 \mathrm{E}-03 \pm 2.6 \mathrm{E}-03$ & $\mathrm{U}$ \\
\hline & ${ }^{238} \mathrm{U}$ & $1.4 \mathrm{E}-01 \pm 4.9 \mathrm{E}-02$ & & & ${ }^{238} \mathrm{U}$ & $1.3 \mathrm{E}-01 \pm 4.9 \mathrm{E}-02$ & \\
\hline & ${ }^{65} \mathrm{Zn}$ & $-7.4 \mathrm{E}-04 \pm 7.4 \mathrm{E}-03$ & $\mathrm{U}$ & & ${ }^{65} \mathrm{Zn}$ & $2.4 \mathrm{E}-02 \pm 2.0 \mathrm{E}-02$ & $\mathrm{U}$ \\
\hline
\end{tabular}

$\overline{\mathrm{RQ}}=$ Result Qualifier. $\mathrm{U}=$ The analyte was analyzed for but not detected. 
Table 3-4. 2008 Soil Sampling Results (pCi/g \pm total analytical uncertainty). (Sheet 7 of 24)

\begin{tabular}{|c|c|c|c|c|c|c|c|}
\hline Location & Isotope & Result \pm Error & $\mathbf{R Q}^{*}$ & Location & Isotope & Result \pm Error & $\mathbf{R Q}^{*}$ \\
\hline D018 & ${ }^{144} \mathrm{Ce}$ & $1.1 \mathrm{E}-01 \pm 1.9 \mathrm{E}-01$ & $\mathrm{U}$ & D020 & ${ }^{144} \mathrm{Ce}$ & $7.0 \mathrm{E}-02 \pm 1.7 \mathrm{E}-01$ & $\mathrm{U}$ \\
\hline \multirow[t]{17}{*}{$(200-W)$} & ${ }^{60} \mathrm{Co}$ & $-1.3 \mathrm{E}-04 \pm 1.3 \mathrm{E}-03$ & $\mathrm{U}$ & $(200-W)$ & ${ }^{60} \mathrm{Co}$ & $8.1 \mathrm{E}-03 \pm 9.3 \mathrm{E}-03$ & $\mathrm{U}$ \\
\hline & ${ }^{134} \mathrm{Cs}$ & $3.1 \mathrm{E}-02 \pm 2.1 \mathrm{E}-02$ & & & ${ }^{134} \mathrm{Cs}$ & $4.2 \mathrm{E}-02 \pm 1.3 \mathrm{E}-02$ & \\
\hline & ${ }^{137} \mathrm{Cs}$ & $4.2 \mathrm{E}-01 \pm 6.9 \mathrm{E}-02$ & & & ${ }^{137} \mathrm{Cs}$ & $3.4 \mathrm{E}-01 \pm 6.7 \mathrm{E}-02$ & \\
\hline & ${ }^{152} \mathrm{Eu}$ & $-1.1 \mathrm{E}-02 \pm 4.3 \mathrm{E}-02$ & $\mathrm{U}$ & & ${ }^{152} \mathrm{Eu}$ & $9.2 \mathrm{E}-03 \pm 4.8 \mathrm{E}-02$ & $\mathrm{U}$ \\
\hline & ${ }^{154} \mathrm{Eu}$ & $-1.2 \mathrm{E}-02 \pm 3.4 \mathrm{E}-02$ & $\mathrm{U}$ & & ${ }^{154} \mathrm{Eu}$ & $1.2 \mathrm{E}-02 \pm 3.1 \mathrm{E}-02$ & $\mathrm{U}$ \\
\hline & ${ }^{155} \mathrm{Eu}$ & $4.5 \mathrm{E}-02 \pm 5.2 \mathrm{E}-02$ & $\mathrm{U}$ & & ${ }^{155} \mathrm{Eu}$ & $2.8 \mathrm{E}-02 \pm 4.2 \mathrm{E}-02$ & $\mathrm{U}$ \\
\hline & ${ }^{238} \mathrm{Pu}$ & $-4.4 \mathrm{E}-03 \pm 3.1 \mathrm{E}-02$ & $\mathrm{U}$ & & ${ }^{238} \mathrm{Pu}$ & $1.7 \mathrm{E}-03 \pm 5.9 \mathrm{E}-03$ & $\mathrm{U}$ \\
\hline & ${ }^{239 / 240} \mathrm{Pu}$ & $6.7 \mathrm{E}-03 \pm 7.9 \mathrm{E}-03$ & & & ${ }^{239 / 240} \mathrm{Pu}$ & $1.9 \mathrm{E}-02 \pm 1.2 \mathrm{E}-02$ & \\
\hline & ${ }^{103} \mathrm{Ru}$ & $4.7 \mathrm{E}-03 \pm 1.6 \mathrm{E}-02$ & $\mathrm{U}$ & & ${ }^{103} \mathrm{Ru}$ & $-1.1 \mathrm{E}-03 \pm 1.0 \mathrm{E}-02$ & $\mathrm{U}$ \\
\hline & ${ }^{106} \mathrm{Ru}$ & $3.9 \mathrm{E}-02 \pm 1.0 \mathrm{E}-01$ & $\mathrm{U}$ & & ${ }^{106} \mathrm{Ru}$ & $2.1 \mathrm{E}-02 \pm 7.6 \mathrm{E}-02$ & $\mathrm{U}$ \\
\hline & ${ }^{125} \mathrm{Sb}$ & $2.9 \mathrm{E}-03 \pm 2.9 \mathrm{E}-02$ & $\mathrm{U}$ & & ${ }^{125} \mathrm{Sb}$ & $1.2 \mathrm{E}-02 \pm 2.4 \mathrm{E}-02$ & $\mathrm{U}$ \\
\hline & ${ }^{113} \mathrm{Sn}$ & $-1.2 \mathrm{E}-02 \pm 1.6 \mathrm{E}-02$ & $\mathrm{U}$ & & ${ }^{113} \mathrm{Sn}$ & $3.8 \mathrm{E}-03 \pm 1.2 \mathrm{E}-02$ & $\mathrm{U}$ \\
\hline & ${ }^{90} \mathrm{Sr}$ & $-4.2 \mathrm{E}-01 \pm 4.2 \mathrm{E}-01$ & $\mathrm{U}$ & & ${ }^{90} \mathrm{Sr}$ & $-6.8 \mathrm{E}-01 \pm 6.8 \mathrm{E}-01$ & $\mathrm{U}$ \\
\hline & ${ }^{234} \mathrm{U}$ & $1.3 \mathrm{E}-01 \pm 4.8 \mathrm{E}-02$ & & & ${ }^{234} \mathrm{U}$ & $1.3 \mathrm{E}-01 \pm 4.7 \mathrm{E}-02$ & \\
\hline & ${ }^{235} \mathrm{U}$ & $-2.4 \mathrm{E}-03 \pm 4.8 \mathrm{E}-03$ & $\mathrm{U}$ & & ${ }^{235} \mathrm{U}$ & $4.2 \mathrm{E}-03 \pm 1.0 \mathrm{E}-02$ & $\mathrm{U}$ \\
\hline & ${ }^{238} \mathrm{U}$ & $1.2 \mathrm{E}-01 \pm 4.6 \mathrm{E}-02$ & & & ${ }^{238} \mathrm{U}$ & $1.2 \mathrm{E}-01 \pm 4.3 \mathrm{E}-02$ & \\
\hline & ${ }^{65} \mathrm{Zn}$ & $1.9 \mathrm{E}-02 \pm 3.1 \mathrm{E}-02$ & $\mathrm{U}$ & & ${ }^{65} \mathrm{Zn}$ & $1.3 \mathrm{E}-02 \pm 2.4 \mathrm{E}-02$ & $\mathrm{U}$ \\
\hline \multirow{18}{*}{$\begin{array}{c}\text { D022 } \\
(200-W)\end{array}$} & ${ }^{144} \mathrm{Ce}$ & $-3.6 \mathrm{E}-03 \pm 3.6 \mathrm{E}-02$ & $\mathrm{U}$ & D024 & ${ }^{144} \mathrm{Ce}$ & $-1.6 \mathrm{E}-01 \pm 1.6 \mathrm{E}-01$ & $\mathrm{U}$ \\
\hline & ${ }^{60} \mathrm{Co}$ & $9.1 \mathrm{E}-03 \pm 5.1 \mathrm{E}-03$ & & $(200-W)$ & ${ }^{60} \mathrm{Co}$ & $-1.6 \mathrm{E}-03 \pm 8.5 \mathrm{E}-03$ & $\mathrm{U}$ \\
\hline & ${ }^{134} \mathrm{Cs}$ & $1.6 \mathrm{E}-02 \pm 4.8 \mathrm{E}-03$ & & & ${ }^{134} \mathrm{Cs}$ & $5.5 \mathrm{E}-02 \pm 1.8 \mathrm{E}-02$ & \\
\hline & ${ }^{137} \mathrm{Cs}$ & 7.3E-01 $\pm 1.2 \mathrm{E}-01$ & & & ${ }^{137} \mathrm{Cs}$ & $5.4 \mathrm{E}-01 \pm 9.1 \mathrm{E}-02$ & \\
\hline & ${ }^{152} \mathrm{Eu}$ & $-5.9 \mathrm{E}-03 \pm 1.0 \mathrm{E}-02$ & $\mathrm{U}$ & & ${ }^{152} \mathrm{Eu}$ & $-1.3 \mathrm{E}-02 \pm 2.7 \mathrm{E}-02$ & $\mathrm{U}$ \\
\hline & ${ }^{154} \mathrm{Eu}$ & $-8.9 \mathrm{E}-03 \pm 1.2 \mathrm{E}-02$ & $\mathrm{U}$ & & ${ }^{154} \mathrm{Eu}$ & $-4.6 \mathrm{E}-03 \pm 3.0 \mathrm{E}-02$ & $\mathrm{U}$ \\
\hline & ${ }^{155} \mathrm{Eu}$ & $2.0 \mathrm{E}-02 \pm 1.5 \mathrm{E}-02$ & & & ${ }^{155} \mathrm{Eu}$ & $5.8 \mathrm{E}-02 \pm 3.4 \mathrm{E}-02$ & $\mathrm{U}$ \\
\hline & ${ }^{238} \mathrm{Pu}$ & $-1.1 \mathrm{E}-02 \pm 4.2 \mathrm{E}-02$ & $\mathrm{U}$ & & ${ }^{238} \mathrm{Pu}$ & $1.8 \mathrm{E}-02 \pm 3.9 \mathrm{E}-02$ & $\mathrm{U}$ \\
\hline & ${ }^{239 / 240} \mathrm{Pu}$ & $5.2 \mathrm{E}-02 \pm 2.8 \mathrm{E}-02$ & & & ${ }^{239 / 240} \mathrm{Pu}$ & $5.8 \mathrm{E}-02 \pm 2.7 \mathrm{E}-02$ & \\
\hline & ${ }^{103} \mathrm{Ru}$ & $-1.2 \mathrm{E}-03 \pm 4.2 \mathrm{E}-03$ & $\mathrm{U}$ & & ${ }^{103} \mathrm{Ru}$ & $-4.1 \mathrm{E}-03 \pm 1.1 \mathrm{E}-02$ & $\mathrm{U}$ \\
\hline & ${ }^{106} \mathrm{Ru}$ & $1.6 \mathrm{E}-02 \pm 2.9 \mathrm{E}-02$ & $\mathrm{U}$ & & ${ }^{106} \mathrm{Ru}$ & $-9.4 \mathrm{E}-02 \pm 9.4 \mathrm{E}-02$ & $\mathrm{U}$ \\
\hline & ${ }^{125} \mathrm{Sb}$ & 3.7E-04 \pm 3.7E-03 & $\mathrm{U}$ & & ${ }^{125} \mathrm{Sb}$ & $1.6 \mathrm{E}-02 \pm 2.4 \mathrm{E}-02$ & $\mathrm{U}$ \\
\hline & ${ }^{113} \mathrm{Sn}$ & $-2.3 \mathrm{E}-03 \pm 4.3 \mathrm{E}-03$ & $\mathrm{U}$ & & ${ }^{113} \mathrm{Sn}$ & $-2.1 \mathrm{E}-02 \pm 2.1 \mathrm{E}-02$ & $\mathrm{U}$ \\
\hline & ${ }^{90} \mathrm{Sr}$ & $-1.7 \mathrm{E}-01 \pm 4.2 \mathrm{E}-01$ & $\mathrm{U}$ & & ${ }^{90} \mathrm{Sr}$ & $-6.9 \mathrm{E}-01 \pm 6.9 \mathrm{E}-01$ & $\mathrm{U}$ \\
\hline & ${ }^{234} \mathrm{U}$ & $2.3 \mathrm{E}-01 \pm 7.6 \mathrm{E}-02$ & & & ${ }^{234} \mathrm{U}$ & $1.7 \mathrm{E}-01 \pm 5.8 \mathrm{E}-02$ & \\
\hline & ${ }^{235} \mathrm{U}$ & $2.1 \mathrm{E}-02 \pm 1.6 \mathrm{E}-02$ & & & ${ }^{235} \mathrm{U}$ & $1.2 \mathrm{E}-02 \pm 1.1 \mathrm{E}-02$ & \\
\hline & ${ }^{238} \mathrm{U}$ & $1.4 \mathrm{E}-01 \pm 5.0 \mathrm{E}-02$ & & & ${ }^{238} \mathrm{U}$ & $1.6 \mathrm{E}-01 \pm 5.4 \mathrm{E}-02$ & \\
\hline & ${ }^{65} \mathrm{Zn}$ & $1.9 \mathrm{E}-02 \pm 9.5 \mathrm{E}-03$ & & & ${ }^{65} \mathrm{Zn}$ & $2.6 \mathrm{E}-03 \pm 2.5 \mathrm{E}-02$ & $\mathrm{U}$ \\
\hline
\end{tabular}

$\overline{\mathrm{RQ}}=$ Result Qualifier. $\mathrm{U}=$ The analyte was analyzed for but not detected. 
Table 3-4. 2008 Soil Sampling Results (pCi/g \pm total analytical uncertainty). (Sheet 8 of 24)

\begin{tabular}{|c|c|c|c|c|c|c|c|}
\hline Location & Isotope & Result \pm Error & $\mathbf{R Q}^{*}$ & Location & Isotope & Result \pm Error & $\mathbf{R Q}^{*}$ \\
\hline D026 & ${ }^{144} \mathrm{Ce}$ & $1.9 \mathrm{E}-03 \pm 1.9 \mathrm{E}-02$ & $\mathrm{U}$ & D028 & ${ }^{144} \mathrm{Ce}$ & $1.3 \mathrm{E}-03 \pm 1.3 \mathrm{E}-02$ & $\mathrm{U}$ \\
\hline \multirow[t]{17}{*}{$(200-W)$} & ${ }^{60} \mathrm{Co}$ & $-2.8 \mathrm{E}-03 \pm 7.0 \mathrm{E}-03$ & $\mathrm{U}$ & $(200-W)$ & ${ }^{60} \mathrm{Co}$ & $-4.4 \mathrm{E}-03 \pm 5.6 \mathrm{E}-03$ & $\mathrm{U}$ \\
\hline & ${ }^{134} \mathrm{Cs}$ & $4.9 \mathrm{E}-02 \pm 1.5 \mathrm{E}-02$ & & & ${ }^{134} \mathrm{Cs}$ & $3.2 \mathrm{E}-02 \pm 1.1 \mathrm{E}-02$ & \\
\hline & ${ }^{137} \mathrm{Cs}$ & $2.0 \mathrm{E}+00 \pm 3.5 \mathrm{E}-01$ & & & ${ }^{137} \mathrm{Cs}$ & $3.4 \mathrm{E}-01 \pm 5.6 \mathrm{E}-02$ & \\
\hline & ${ }^{152} \mathrm{Eu}$ & $-2.8 \mathrm{E}-02 \pm 3.2 \mathrm{E}-02$ & $\mathrm{U}$ & & ${ }^{152} \mathrm{Eu}$ & $-1.4 \mathrm{E}-02 \pm 2.0 \mathrm{E}-02$ & $\mathrm{U}$ \\
\hline & ${ }^{154} \mathrm{Eu}$ & $-2.1 \mathrm{E}-02 \pm 2.2 \mathrm{E}-02$ & $\mathrm{U}$ & & ${ }^{154} \mathrm{Eu}$ & $-1.5 \mathrm{E}-02 \pm 2.0 \mathrm{E}-02$ & $\mathrm{U}$ \\
\hline & ${ }^{155} \mathrm{Eu}$ & $1.7 \mathrm{E}-02 \pm 3.9 \mathrm{E}-02$ & $\mathrm{U}$ & & ${ }^{155} \mathrm{Eu}$ & $3.8 \mathrm{E}-02 \pm 3.9 \mathrm{E}-02$ & $\mathrm{U}$ \\
\hline & ${ }^{238} \mathrm{Pu}$ & $1.4 \mathrm{E}-02 \pm 4.2 \mathrm{E}-02$ & $\mathrm{U}$ & & ${ }^{238} \mathrm{Pu}$ & $2.1 \mathrm{E}-01 \pm 5.9 \mathrm{E}-02$ & \\
\hline & ${ }^{239 / 240} \mathrm{Pu}$ & $6.2 \mathrm{E}-01 \pm 1.7 \mathrm{E}-01$ & & & ${ }^{239 / 240} \mathrm{Pu}$ & $1.0 \mathrm{E}+00 \pm 2.3 \mathrm{E}-01$ & \\
\hline & ${ }^{103} \mathrm{Ru}$ & $1.5 \mathrm{E}-03 \pm 9.4 \mathrm{E}-03$ & $\mathrm{U}$ & & ${ }^{103} \mathrm{Ru}$ & $1.1 \mathrm{E}-03 \pm 6.8 \mathrm{E}-03$ & $\mathrm{U}$ \\
\hline & ${ }^{106} \mathrm{Ru}$ & $-2.0 \mathrm{E}-02 \pm 7.0 \mathrm{E}-02$ & $\mathrm{U}$ & & ${ }^{106} \mathrm{Ru}$ & $-3.4 \mathrm{E}-02 \pm 5.2 \mathrm{E}-02$ & $\mathrm{U}$ \\
\hline & ${ }^{125} \mathrm{Sb}$ & $8.7 \mathrm{E}-03 \pm 2.5 \mathrm{E}-02$ & $\mathrm{U}$ & & ${ }^{125} \mathrm{Sb}$ & $6.7 \mathrm{E}-03 \pm 1.7 \mathrm{E}-02$ & $\mathrm{U}$ \\
\hline & ${ }^{113} \mathrm{Sn}$ & $-4.2 \mathrm{E}-04 \pm 4.2 \mathrm{E}-03$ & $\mathrm{U}$ & & ${ }^{113} \mathrm{Sn}$ & $-5.0 \mathrm{E}-03 \pm 8.2 \mathrm{E}-03$ & $\mathrm{U}$ \\
\hline & ${ }^{90} \mathrm{Sr}$ & $1.5 \mathrm{E}-01 \pm 4.5 \mathrm{E}-01$ & $\mathrm{U}$ & & ${ }^{90} \mathrm{Sr}$ & $-5.2 \mathrm{E}-02 \pm 4.4 \mathrm{E}-01$ & $\mathrm{U}$ \\
\hline & ${ }^{234} \mathrm{U}$ & $1.8 \mathrm{E}-01 \pm 6.1 \mathrm{E}-02$ & & & ${ }^{234} \mathrm{U}$ & $1.4 \mathrm{E}-01 \pm 5.0 \mathrm{E}-02$ & \\
\hline & ${ }^{235} \mathrm{U}$ & $9.7 \mathrm{E}-03 \pm 1.0 \mathrm{E}-02$ & & & ${ }^{235} \mathrm{U}$ & $4.7 \mathrm{E}-03 \pm 9.5 \mathrm{E}-03$ & $\mathrm{U}$ \\
\hline & ${ }^{238} \mathrm{U}$ & $1.6 \mathrm{E}-01 \pm 5.6 \mathrm{E}-02$ & & & ${ }^{238} \mathrm{U}$ & $1.1 \mathrm{E}-01 \pm 4.3 \mathrm{E}-02$ & \\
\hline & ${ }^{65} \mathrm{Zn}$ & $-1.9 \mathrm{E}-02 \pm 1.9 \mathrm{E}-02$ & $\mathrm{U}$ & & ${ }^{65} \mathrm{Zn}$ & $7.8 \mathrm{E}-03 \pm 1.5 \mathrm{E}-02$ & $\mathrm{U}$ \\
\hline \multirow{18}{*}{$\begin{array}{c}\text { D030 } \\
(200-W)\end{array}$} & ${ }^{144} \mathrm{Ce}$ & $1.8 \mathrm{E}-01 \pm 2.3 \mathrm{E}-01$ & $\mathrm{U}$ & D032 & ${ }^{144} \mathrm{Ce}$ & $-1.5 \mathrm{E}-02 \pm 1.2 \mathrm{E}-01$ & $\mathrm{U}$ \\
\hline & ${ }^{60} \mathrm{Co}$ & $3.5 \mathrm{E}-03 \pm 1.3 \mathrm{E}-02$ & $\mathrm{U}$ & $(200-W)$ & ${ }^{60} \mathrm{Co}$ & $3.3 \mathrm{E}-03 \pm 5.8 \mathrm{E}-03$ & $\mathrm{U}$ \\
\hline & ${ }^{134} \mathrm{Cs}$ & $4.1 \mathrm{E}-02 \pm 1.9 \mathrm{E}-02$ & & & ${ }^{134} \mathrm{Cs}$ & $3.7 \mathrm{E}-02 \pm 1.2 \mathrm{E}-02$ & \\
\hline & ${ }^{137} \mathrm{Cs}$ & $4.1 \mathrm{E}-01 \pm 6.8 \mathrm{E}-02$ & & & ${ }^{137} \mathrm{Cs}$ & $6.3 \mathrm{E}-01 \pm 1.0 \mathrm{E}-01$ & \\
\hline & ${ }^{152} \mathrm{Eu}$ & $-2.6 \mathrm{E}-02 \pm 5.6 \mathrm{E}-02$ & $\mathrm{U}$ & & ${ }^{152} \mathrm{Eu}$ & $1.0 \mathrm{E}-02 \pm 2.2 \mathrm{E}-02$ & $\mathrm{U}$ \\
\hline & ${ }^{154} \mathrm{Eu}$ & $-2.9 \mathrm{E}-02 \pm 4.4 \mathrm{E}-02$ & $\mathrm{U}$ & & ${ }^{154} \mathrm{Eu}$ & $-6.3 \mathrm{E}-03 \pm 1.9 \mathrm{E}-02$ & $\mathrm{U}$ \\
\hline & ${ }^{155} \mathrm{Eu}$ & $6.0 \mathrm{E}-02 \pm 5.9 \mathrm{E}-02$ & $\mathrm{U}$ & & ${ }^{155} \mathrm{Eu}$ & $3.2 \mathrm{E}-02 \pm 2.8 \mathrm{E}-02$ & $\mathrm{U}$ \\
\hline & ${ }^{238} \mathrm{Pu}$ & $1.8 \mathrm{E}-03 \pm 1.8 \mathrm{E}-03$ & $\mathrm{U}$ & & ${ }^{238} \mathrm{Pu}$ & $3.8 \mathrm{E}-02 \pm 1.8 \mathrm{E}-02$ & \\
\hline & ${ }^{239 / 240} \mathrm{Pu}$ & $2.1 \mathrm{E}-02 \pm 1.3 \mathrm{E}-02$ & & & ${ }^{239 / 240} \mathrm{Pu}$ & $1.7 \mathrm{E}-01 \pm 4.9 \mathrm{E}-02$ & \\
\hline & ${ }^{103} \mathrm{Ru}$ & $6.4 \mathrm{E}-03 \pm 1.7 \mathrm{E}-02$ & $\mathrm{U}$ & & ${ }^{103} \mathrm{Ru}$ & $3.1 \mathrm{E}-03 \pm 7.2 \mathrm{E}-03$ & $\mathrm{U}$ \\
\hline & ${ }^{106} \mathrm{Ru}$ & $1.3 \mathrm{E}-01 \pm 1.3 \mathrm{E}-01$ & $\mathrm{U}$ & & ${ }^{106} \mathrm{Ru}$ & $-1.5 \mathrm{E}-02 \pm 5.4 \mathrm{E}-02$ & $\mathrm{U}$ \\
\hline & ${ }^{125} \mathrm{Sb}$ & $5.8 \mathrm{E}-03 \pm 4.0 \mathrm{E}-02$ & $\mathrm{U}$ & & ${ }^{125} \mathrm{Sb}$ & $-8.4 \mathrm{E}-03 \pm 1.8 \mathrm{E}-02$ & $\mathrm{U}$ \\
\hline & ${ }^{113} \mathrm{Sn}$ & $6.3 \mathrm{E}-03 \pm 1.9 \mathrm{E}-02$ & $\mathrm{U}$ & & ${ }^{113} \mathrm{Sn}$ & $-1.3 \mathrm{E}-02 \pm 1.3 \mathrm{E}-02$ & $\mathrm{U}$ \\
\hline & ${ }^{90} \mathrm{Sr}$ & $-2.1 \mathrm{E}-01 \pm 4.1 \mathrm{E}-01$ & $\mathrm{U}$ & & ${ }^{90} \mathrm{Sr}$ & $-7.9 \mathrm{E}-01 \pm 7.9 \mathrm{E}-01$ & $\mathrm{U}$ \\
\hline & ${ }^{234} \mathrm{U}$ & $1.3 \mathrm{E}-01 \pm 4.7 \mathrm{E}-02$ & & & ${ }^{234} \mathrm{U}$ & $1.3 \mathrm{E}-01 \pm 4.9 \mathrm{E}-02$ & \\
\hline & ${ }^{235} \mathrm{U}$ & $1.1 \mathrm{E}-02 \pm 1.0 \mathrm{E}-02$ & & & ${ }^{235} \mathrm{U}$ & $1.6 \mathrm{E}-02 \pm 1.6 \mathrm{E}-02$ & $\mathrm{U}$ \\
\hline & ${ }^{238} \mathrm{U}$ & $1.7 \mathrm{E}-01 \pm 5.8 \mathrm{E}-02$ & & & ${ }^{238} \mathrm{U}$ & $1.3 \mathrm{E}-01 \pm 4.9 \mathrm{E}-02$ & \\
\hline & ${ }^{65} \mathrm{Zn}$ & $3.2 \mathrm{E}-02 \pm 3.6 \mathrm{E}-02$ & $\mathrm{U}$ & & ${ }^{65} \mathrm{Zn}$ & $2.3 \mathrm{E}-03 \pm 2.3 \mathrm{E}-02$ & $\mathrm{U}$ \\
\hline
\end{tabular}

$\overline{\mathrm{RQ}}=$ Result Qualifier. $\mathrm{U}=$ The analyte was analyzed for but not detected. 
Table 3-4. 2008 Soil Sampling Results (pCi/g \pm total analytical uncertainty). (Sheet 9 of 24)

\begin{tabular}{|c|c|c|c|c|c|c|c|}
\hline Location & Isotope & Result \pm Error & $\mathbf{R Q}^{*}$ & Location & Isotope & Result \pm Error & RQ* \\
\hline D034 & ${ }^{144} \mathrm{Ce}$ & $-3.2 \mathrm{E}-02 \pm 1.6 \mathrm{E}-01$ & $\mathrm{U}$ & D038 & ${ }^{144} \mathrm{Ce}$ & $-1.4 \mathrm{E}-01 \pm 1.7 \mathrm{E}-01$ & $\mathrm{U}$ \\
\hline \multirow[t]{17}{*}{$(200-W)$} & ${ }^{60} \mathrm{Co}$ & $-4.8 \mathrm{E}-04 \pm 4.8 \mathrm{E}-03$ & U & $(200-W)$ & ${ }^{60} \mathrm{Co}$ & $-3.6 \mathrm{E}-03 \pm 8.3 \mathrm{E}-03$ & U \\
\hline & ${ }^{134} \mathrm{Cs}$ & $3.1 \mathrm{E}-02 \pm 1.2 \mathrm{E}-02$ & & & ${ }^{134} \mathrm{Cs}$ & $4.5 \mathrm{E}-02 \pm 1.5 \mathrm{E}-02$ & \\
\hline & ${ }^{137} \mathrm{Cs}$ & $1.9 \mathrm{E}+00 \pm 3.1 \mathrm{E}-01$ & & & ${ }^{137} \mathrm{Cs}$ & $3.7 \mathrm{E}-01 \pm 6.9 \mathrm{E}-02$ & \\
\hline & ${ }^{152} \mathrm{Eu}$ & $-2.4 \mathrm{E}-02 \pm 3.3 \mathrm{E}-02$ & $\mathrm{U}$ & & ${ }^{152} \mathrm{Eu}$ & $7.4 \mathrm{E}-03 \pm 3.9 \mathrm{E}-02$ & $\mathrm{U}$ \\
\hline & ${ }^{154} \mathrm{Eu}$ & $5.5 \mathrm{E}-04 \pm 5.5 \mathrm{E}-03$ & U & & ${ }^{154} \mathrm{Eu}$ & $-1.2 \mathrm{E}-02 \pm 3.2 \mathrm{E}-02$ & $\mathrm{U}$ \\
\hline & ${ }^{155} \mathrm{Eu}$ & $9.6 \mathrm{E}-02 \pm 5.6 \mathrm{E}-02$ & & & ${ }^{155} \mathrm{Eu}$ & $2.3 \mathrm{E}-02 \pm 4.3 \mathrm{E}-02$ & $\mathrm{U}$ \\
\hline & ${ }^{238} \mathrm{Pu}$ & $7.8 \mathrm{E}-02 \pm 3.1 \mathrm{E}-02$ & & & ${ }^{238} \mathrm{Pu}$ & $2.6 \mathrm{E}-02 \pm 2.7 \mathrm{E}-02$ & $\mathrm{U}$ \\
\hline & ${ }^{239 / 240} \mathrm{Pu}$ & $8.1 \mathrm{E}-01 \pm 1.9 \mathrm{E}-01$ & & & ${ }^{239 / 240} \mathrm{Pu}$ & $4.0 \mathrm{E}-02 \pm 2.1 \mathrm{E}-02$ & \\
\hline & ${ }^{103} \mathrm{Ru}$ & $-2.7 \mathrm{E}-03 \pm 1.0 \mathrm{E}-02$ & U & & ${ }^{103} \mathrm{Ru}$ & $4.4 \mathrm{E}-03 \pm 9.1 \mathrm{E}-03$ & U \\
\hline & ${ }^{106} \mathrm{Ru}$ & $2.7 \mathrm{E}-02 \pm 7.9 \mathrm{E}-02$ & $\mathrm{U}$ & & ${ }^{106} \mathrm{Ru}$ & $2.3 \mathrm{E}-03 \pm 2.3 \mathrm{E}-02$ & U \\
\hline & ${ }^{125} \mathrm{Sb}$ & $-1.3 \mathrm{E}-02 \pm 2.7 \mathrm{E}-02$ & U & & ${ }^{125} \mathrm{Sb}$ & $-6.8 \mathrm{E}-03 \pm 2.7 \mathrm{E}-02$ & U \\
\hline & ${ }^{113} \mathrm{Sn}$ & $-1.2 \mathrm{E}-02 \pm 1.2 \mathrm{E}-02$ & U & & ${ }^{113} \mathrm{Sn}$ & $-1.4 \mathrm{E}-02 \pm 1.2 \mathrm{E}-02$ & $\mathrm{U}$ \\
\hline & ${ }^{90} \mathrm{Sr}$ & $-1.0 \mathrm{E}+00 \pm 1.0 \mathrm{E}+00$ & $\mathrm{U}$ & & ${ }^{90} \mathrm{Sr}$ & $-5.2 \mathrm{E}-01 \pm 5.2 \mathrm{E}-01$ & $\mathrm{U}$ \\
\hline & ${ }^{234} \mathrm{U}$ & $1.3 \mathrm{E}-01 \pm 4.7 \mathrm{E}-02$ & & & ${ }^{234} \mathrm{U}$ & $1.6 \mathrm{E}-01 \pm 5.6 \mathrm{E}-02$ & \\
\hline & ${ }^{235} \mathrm{U}$ & $1.3 \mathrm{E}-02 \pm 1.1 \mathrm{E}-02$ & & & ${ }^{235} \mathrm{U}$ & $9.8 \mathrm{E}-03 \pm 1.0 \mathrm{E}-02$ & \\
\hline & ${ }^{238} \mathrm{U}$ & $1.1 \mathrm{E}-01 \pm 4.3 \mathrm{E}-02$ & & & ${ }^{238} \mathrm{U}$ & $1.7 \mathrm{E}-01 \pm 5.9 \mathrm{E}-02$ & \\
\hline & ${ }^{65} \mathrm{Zn}$ & $3.2 \mathrm{E}-02 \pm 2.3 \mathrm{E}-02$ & $\mathrm{U}$ & & ${ }^{65} \mathrm{Zn}$ & $1.4 \mathrm{E}-02 \pm 2.4 \mathrm{E}-02$ & $\mathrm{U}$ \\
\hline \multirow{18}{*}{$\begin{array}{c}\text { D040 } \\
(200-W)\end{array}$} & ${ }^{144} \mathrm{Ce}$ & $7.1 \mathrm{E}-02 \pm 2.1 \mathrm{E}-01$ & $\mathrm{U}$ & D042 & ${ }^{144} \mathrm{Ce}$ & $-6.5 \mathrm{E}-02 \pm 1.6 \mathrm{E}-01$ & $\mathrm{U}$ \\
\hline & ${ }^{60} \mathrm{Co}$ & $2.3 \mathrm{E}-03 \pm 1.2 \mathrm{E}-02$ & $\mathrm{U}$ & $(200-W)$ & ${ }^{60} \mathrm{Co}$ & $5.5 \mathrm{E}-03 \pm 9.1 \mathrm{E}-03$ & $\mathrm{U}$ \\
\hline & ${ }^{134} \mathrm{Cs}$ & $4.9 \mathrm{E}-02 \pm 2.5 \mathrm{E}-02$ & & & ${ }^{134} \mathrm{Cs}$ & $5.0 \mathrm{E}-02 \pm 1.4 \mathrm{E}-02$ & \\
\hline & ${ }^{137} \mathrm{Cs}$ & $1.1 \mathrm{E}+00 \pm 1.7 \mathrm{E}-01$ & & & ${ }^{137} \mathrm{Cs}$ & $3.0 \mathrm{E}-01 \pm 5.5 \mathrm{E}-02$ & \\
\hline & ${ }^{152} \mathrm{Eu}$ & $7.4 \mathrm{E}-03 \pm 5.6 \mathrm{E}-02$ & $\mathrm{U}$ & & ${ }^{152} \mathrm{Eu}$ & $1.5 \mathrm{E}-02 \pm 4.3 \mathrm{E}-02$ & $\mathrm{U}$ \\
\hline & ${ }^{154} \mathrm{Eu}$ & $3.3 \mathrm{E}-05 \pm 3.3 \mathrm{E}-04$ & $\mathrm{U}$ & & ${ }^{154} \mathrm{Eu}$ & $-3.5 \mathrm{E}-03 \pm 2.9 \mathrm{E}-02$ & $\mathrm{U}$ \\
\hline & ${ }^{155} \mathrm{Eu}$ & $1.0 \mathrm{E}-01 \pm 6.4 \mathrm{E}-02$ & & & ${ }^{155} \mathrm{Eu}$ & $1.5 \mathrm{E}-02 \pm 3.8 \mathrm{E}-02$ & $\mathrm{U}$ \\
\hline & ${ }^{238} \mathrm{Pu}$ & $1.2 \mathrm{E}-02 \pm 1.2 \mathrm{E}-02$ & $\mathrm{U}$ & & ${ }^{238} \mathrm{Pu}$ & $1.5 \mathrm{E}-01 \pm 6.5 \mathrm{E}-02$ & \\
\hline & ${ }^{239 / 240} \mathrm{Pu}$ & $5.9 \mathrm{E}-02 \pm 2.5 \mathrm{E}-02$ & & & ${ }^{239 / 240} \mathrm{Pu}$ & $5.2 \mathrm{E}-01 \pm 1.5 \mathrm{E}-01$ & \\
\hline & ${ }^{103} \mathrm{Ru}$ & $2.2 \mathrm{E}-03 \pm 1.5 \mathrm{E}-02$ & $\mathrm{U}$ & & ${ }^{103} \mathrm{Ru}$ & $-1.8 \mathrm{E}-03 \pm 9.5 \mathrm{E}-03$ & $\mathrm{U}$ \\
\hline & ${ }^{106} \mathrm{Ru}$ & $4.6 \mathrm{E}-02 \pm 1.2 \mathrm{E}-01$ & $\mathrm{U}$ & & ${ }^{106} \mathrm{Ru}$ & $1.6 \mathrm{E}-03 \pm 1.6 \mathrm{E}-02$ & $\mathrm{U}$ \\
\hline & ${ }^{125} \mathrm{Sb}$ & $7.5 \mathrm{E}-03 \pm 3.7 \mathrm{E}-02$ & $\mathrm{U}$ & & ${ }^{125} \mathrm{Sb}$ & $-1.4 \mathrm{E}-02 \pm 2.4 \mathrm{E}-02$ & $\mathrm{U}$ \\
\hline & ${ }^{113} \mathrm{Sn}$ & $-1.1 \mathrm{E}-02 \pm 1.7 \mathrm{E}-02$ & $\mathrm{U}$ & & ${ }^{113} \mathrm{Sn}$ & $-2.9 \mathrm{E}-03 \pm 1.1 \mathrm{E}-02$ & $\mathrm{U}$ \\
\hline & ${ }^{90} \mathrm{Sr}$ & $-5.0 \mathrm{E}-01 \pm 5.0 \mathrm{E}-01$ & $\mathrm{U}$ & & ${ }^{90} \mathrm{Sr}$ & $-5.6 \mathrm{E}-01 \pm 5.6 \mathrm{E}-01$ & $\mathrm{U}$ \\
\hline & ${ }^{234} \mathrm{U}$ & $1.5 \mathrm{E}-01 \pm 5.6 \mathrm{E}-02$ & & & ${ }^{234} \mathrm{U}$ & $1.4 \mathrm{E}-01 \pm 5.3 \mathrm{E}-02$ & \\
\hline & ${ }^{235} \mathrm{U}$ & $2.4 \mathrm{E}-02 \pm 1.9 \mathrm{E}-02$ & & & ${ }^{235} \mathrm{U}$ & $1.1 \mathrm{E}-02 \pm 1.1 \mathrm{E}-02$ & \\
\hline & ${ }^{238} \mathrm{U}$ & $1.4 \mathrm{E}-01 \pm 5.3 \mathrm{E}-02$ & & & ${ }^{238} \mathrm{U}$ & $1.8 \mathrm{E}-01 \pm 6.3 \mathrm{E}-02$ & \\
\hline & ${ }^{65} \mathrm{Zn}$ & $6.3 \mathrm{E}-03 \pm 3.2 \mathrm{E}-02$ & U & & ${ }^{65} \mathrm{Zn}$ & $6.4 \mathrm{E}-02 \pm 2.6 \mathrm{E}-02$ & \\
\hline
\end{tabular}

$\overline{\mathrm{RQ}}=$ Result Qualifier. $\mathrm{U}=$ The analyte was analyzed for but not detected. 
Table 3-4. 2008 Soil Sampling Results (pCi/g \pm total analytical uncertainty). (Sheet 10 of 24)

\begin{tabular}{|c|c|c|c|c|c|c|c|}
\hline Location & Isotope & Result \pm Error & $\mathbf{R Q}^{*}$ & Location & Isotope & Result \pm Error & RQ* \\
\hline \multirow{18}{*}{$\begin{array}{c}\text { D044 } \\
(200-W)\end{array}$} & ${ }^{144} \mathrm{Ce}$ & $3.7 \mathrm{E}-02 \pm 2.3 \mathrm{E}-01$ & $\mathrm{U}$ & \multirow{18}{*}{$\begin{array}{c}\text { D046 } \\
(200-W)\end{array}$} & ${ }^{144} \mathrm{Ce}$ & $-2.7 \mathrm{E}-02 \pm 1.8 \mathrm{E}-01$ & $\mathrm{U}$ \\
\hline & ${ }^{60} \mathrm{Co}$ & $1.1 \mathrm{E}-02 \pm 9.6 \mathrm{E}-03$ & $\mathrm{U}$ & & ${ }^{60} \mathrm{Co}$ & $-9.8 \mathrm{E}-04 \pm 9.8 \mathrm{E}-03$ & \multirow[t]{3}{*}{$\mathrm{U}$} \\
\hline & ${ }^{134} \mathrm{Cs}$ & $4.4 \mathrm{E}-02 \pm 1.5 \mathrm{E}-02$ & & & ${ }^{134} \mathrm{Cs}$ & $4.9 \mathrm{E}-02 \pm 1.4 \mathrm{E}-02$ & \\
\hline & ${ }^{137} \mathrm{Cs}$ & $5.8 \mathrm{E}+00 \pm 1.0 \mathrm{E}+00$ & & & ${ }^{137} \mathrm{Cs}$ & $3.1 \mathrm{E}-01 \pm 5.7 \mathrm{E}-02$ & \\
\hline & ${ }^{152} \mathrm{Eu}$ & $4.7 \mathrm{E}-02 \pm 6.9 \mathrm{E}-02$ & U & & ${ }^{152} \mathrm{Eu}$ & $-2.7 \mathrm{E}-03 \pm 2.7 \mathrm{E}-02$ & U \\
\hline & ${ }^{154} \mathrm{Eu}$ & $-1.4 \mathrm{E}-02 \pm 3.6 \mathrm{E}-02$ & $\mathrm{U}$ & & ${ }^{154} \mathrm{Eu}$ & $-7.3 \mathrm{E}-03 \pm 2.9 \mathrm{E}-02$ & \multirow[t]{2}{*}{$\mathrm{U}$} \\
\hline & ${ }^{155} \mathrm{Eu}$ & $5.6 \mathrm{E}-02 \pm 6.8 \mathrm{E}-02$ & $\mathrm{U}$ & & ${ }^{155} \mathrm{Eu}$ & $9.8 \mathrm{E}-02 \pm 5.7 \mathrm{E}-02$ & \\
\hline & ${ }^{238} \mathrm{Pu}$ & $1.8 \mathrm{E}-03 \pm 9.5 \mathrm{E}-03$ & $\mathrm{U}$ & & ${ }^{238} \mathrm{Pu}$ & $-2.3 \mathrm{E}-03 \pm 2.3 \mathrm{E}-02$ & \multirow[t]{2}{*}{$\mathrm{U}$} \\
\hline & ${ }^{239 / 240} \mathrm{Pu}$ & $1.4 \mathrm{E}-01 \pm 4.3 \mathrm{E}-02$ & & & ${ }^{239 / 240} \mathrm{Pu}$ & $2.3 \mathrm{E}-02 \pm 1.6 \mathrm{E}-02$ & \\
\hline & ${ }^{103} \mathrm{Ru}$ & $5.1 \mathrm{E}-03 \pm 1.5 \mathrm{E}-02$ & U & & ${ }^{103} \mathrm{Ru}$ & $-5.6 \mathrm{E}-03 \pm 9.6 \mathrm{E}-03$ & $\mathrm{U}$ \\
\hline & ${ }^{106} \mathrm{Ru}$ & $2.3 \mathrm{E}-02 \pm 1.1 \mathrm{E}-01$ & $\mathrm{U}$ & & ${ }^{106} \mathrm{Ru}$ & $1.9 \mathrm{E}-02 \pm 7.9 \mathrm{E}-02$ & $\mathrm{U}$ \\
\hline & ${ }^{125} \mathrm{Sb}$ & $-2.1 \mathrm{E}-02 \pm 4.0 \mathrm{E}-02$ & $\mathrm{U}$ & & ${ }^{125} \mathrm{Sb}$ & $-1.1 \mathrm{E}-03 \pm 1.1 \mathrm{E}-02$ & $\mathrm{U}$ \\
\hline & ${ }^{113} \mathrm{Sn}$ & $-1.7 \mathrm{E}-02 \pm 2.0 \mathrm{E}-02$ & $\mathrm{U}$ & & ${ }^{113} \mathrm{Sn}$ & $-4.7 \mathrm{E}-04 \pm 4.7 \mathrm{E}-03$ & $\mathrm{U}$ \\
\hline & ${ }^{90} \mathrm{Sr}$ & $3.0 \mathrm{E}-01 \pm 4.5 \mathrm{E}-01$ & $\mathrm{U}$ & & ${ }^{90} \mathrm{Sr}$ & $-1.0 \mathrm{E}+00 \pm 1.0 \mathrm{E}+00$ & $\mathrm{U}$ \\
\hline & ${ }^{234} \mathrm{U}$ & $1.8 \mathrm{E}-01 \pm 6.3 \mathrm{E}-02$ & & & ${ }^{234} \mathrm{U}$ & $1.8 \mathrm{E}-01 \pm 6.1 \mathrm{E}-02$ & \\
\hline & ${ }^{235} \mathrm{U}$ & $8.0 \mathrm{E}-03 \pm 9.4 \mathrm{E}-03$ & & & ${ }^{235} \mathrm{U}$ & $1.1 \mathrm{E}-02 \pm 1.0 \mathrm{E}-02$ & \\
\hline & ${ }^{238} \mathrm{U}$ & $1.8 \mathrm{E}-01 \pm 6.3 \mathrm{E}-02$ & & & ${ }^{238} \mathrm{U}$ & $1.4 \mathrm{E}-01 \pm 5.0 \mathrm{E}-02$ & \\
\hline & ${ }^{65} \mathrm{Zn}$ & $3.2 \mathrm{E}-02 \pm 2.7 \mathrm{E}-02$ & $\mathrm{U}$ & & ${ }^{65} \mathrm{Zn}$ & $-1.9 \mathrm{E}-02 \pm 2.2 \mathrm{E}-02$ & $\mathrm{U}$ \\
\hline \multirow{18}{*}{$\begin{array}{c}\text { D048 } \\
(200-W)\end{array}$} & ${ }^{144} \mathrm{Ce}$ & $1.0 \mathrm{E}-01 \pm 1.3 \mathrm{E}-01$ & $\mathrm{U}$ & \multirow{18}{*}{$\begin{array}{c}\text { D050 } \\
(200-W)\end{array}$} & ${ }^{144} \mathrm{Ce}$ & $1.3 \mathrm{E}-01 \pm 2.3 \mathrm{E}-01$ & $\mathrm{U}$ \\
\hline & ${ }^{60} \mathrm{Co}$ & $1.7 \mathrm{E}-03 \pm 6.1 \mathrm{E}-03$ & $\mathrm{U}$ & & ${ }^{60} \mathrm{Co}$ & $4.8 \mathrm{E}-03 \pm 1.3 \mathrm{E}-02$ & \multirow[t]{3}{*}{$\mathrm{U}$} \\
\hline & ${ }^{134} \mathrm{Cs}$ & $2.7 \mathrm{E}-02 \pm 1.0 \mathrm{E}-02$ & & & ${ }^{134} \mathrm{Cs}$ & $6.2 \mathrm{E}-02 \pm 2.3 \mathrm{E}-02$ & \\
\hline & ${ }^{137} \mathrm{Cs}$ & $9.4 \mathrm{E}-01 \pm 1.5 \mathrm{E}-01$ & & & ${ }^{137} \mathrm{Cs}$ & $1.0 \mathrm{E}-01 \pm 3.3 \mathrm{E}-02$ & \\
\hline & ${ }^{152} \mathrm{Eu}$ & $-2.1 \mathrm{E}-03 \pm 2.1 \mathrm{E}-02$ & U & & ${ }^{152} \mathrm{Eu}$ & $2.3 \mathrm{E}-02 \pm 5.5 \mathrm{E}-02$ & U \\
\hline & ${ }^{154} \mathrm{Eu}$ & $-6.4 \mathrm{E}-04 \pm 6.4 \mathrm{E}-03$ & $\mathrm{U}$ & & ${ }^{154} \mathrm{Eu}$ & $-1.2 \mathrm{E}-02 \pm 4.3 \mathrm{E}-02$ & $\mathrm{U}$ \\
\hline & ${ }^{155} \mathrm{Eu}$ & $6.4 \mathrm{E}-02 \pm 4.3 \mathrm{E}-02$ & & & ${ }^{155} \mathrm{Eu}$ & $7.2 \mathrm{E}-02 \pm 5.9 \mathrm{E}-02$ & $\mathrm{U}$ \\
\hline & ${ }^{238} \mathrm{Pu}$ & $-4.6 \mathrm{E}-03 \pm 2.6 \mathrm{E}-02$ & $\mathrm{U}$ & & ${ }^{238} \mathrm{Pu}$ & $3.6 \mathrm{E}-03 \pm 1.1 \mathrm{E}-02$ & $\mathrm{U}$ \\
\hline & ${ }^{239 / 240} \mathrm{Pu}$ & $1.9 \mathrm{E}-02 \pm 1.4 \mathrm{E}-02$ & & & ${ }^{239 / 240} \mathrm{Pu}$ & $9.0 \mathrm{E}-03 \pm 9.7 \mathrm{E}-03$ & $\mathrm{U}$ \\
\hline & ${ }^{103} \mathrm{Ru}$ & $-7.6 \mathrm{E}-03 \pm 8.2 \mathrm{E}-03$ & $\mathrm{U}$ & & ${ }^{103} \mathrm{Ru}$ & $1.2 \mathrm{E}-02 \pm 1.6 \mathrm{E}-02$ & $\mathrm{U}$ \\
\hline & ${ }^{106} \mathrm{Ru}$ & $-1.9 \mathrm{E}-02 \pm 6.1 \mathrm{E}-02$ & $\mathrm{U}$ & & ${ }^{106} \mathrm{Ru}$ & $-3.8 \mathrm{E}-02 \pm 1.3 \mathrm{E}-01$ & $\mathrm{U}$ \\
\hline & ${ }^{125} \mathrm{Sb}$ & $1.4 \mathrm{E}-02 \pm 2.1 \mathrm{E}-02$ & $\mathrm{U}$ & & ${ }^{125} \mathrm{Sb}$ & $1.4 \mathrm{E}-02 \pm 3.9 \mathrm{E}-02$ & $\mathrm{U}$ \\
\hline & ${ }^{113} \mathrm{Sn}$ & $-5.6 \mathrm{E}-04 \pm 5.6 \mathrm{E}-03$ & $\mathrm{U}$ & & ${ }^{113} \mathrm{Sn}$ & $-7.2 \mathrm{E}-03 \pm 1.9 \mathrm{E}-02$ & $\mathrm{U}$ \\
\hline & ${ }^{90} \mathrm{Sr}$ & $-4.9 \mathrm{E}-01 \pm 4.9 \mathrm{E}-01$ & $\mathrm{U}$ & & ${ }^{90} \mathrm{Sr}$ & $-3.6 \mathrm{E}-01 \pm 4.8 \mathrm{E}-01$ & $\mathrm{U}$ \\
\hline & ${ }^{234} \mathrm{U}$ & $2.2 \mathrm{E}-01 \pm 7.0 \mathrm{E}-02$ & & & ${ }^{234} \mathrm{U}$ & $1.4 \mathrm{E}-01 \pm 5.2 \mathrm{E}-02$ & \\
\hline & ${ }^{235} \mathrm{U}$ & $1.9 \mathrm{E}-02 \pm 1.3 \mathrm{E}-02$ & & & ${ }^{235} \mathrm{U}$ & $1.6 \mathrm{E}-02 \pm 1.6 \mathrm{E}-02$ & $\mathrm{U}$ \\
\hline & ${ }^{238} \mathrm{U}$ & $1.8 \mathrm{E}-01 \pm 5.9 \mathrm{E}-02$ & & & ${ }^{238} \mathrm{U}$ & $1.2 \mathrm{E}-01 \pm 4.7 \mathrm{E}-02$ & \\
\hline & ${ }^{65} \mathrm{Zn}$ & $1.2 \mathrm{E}-03 \pm 1.2 \mathrm{E}-02$ & $\mathrm{U}$ & & ${ }^{65} \mathrm{Zn}$ & $1.3 \mathrm{E}-02 \pm 3.7 \mathrm{E}-02$ & $\mathrm{U}$ \\
\hline
\end{tabular}

$\mathrm{RQ}=$ Result Qualifier. $\mathrm{U}=$ The analyte was analyzed for but not detected. 
Table 3-4. 2008 Soil Sampling Results (pCi/g \pm total analytical uncertainty). (Sheet 11 of 24)

\begin{tabular}{|c|c|c|c|c|c|c|c|}
\hline Location & Isotope & Result \pm Error & $\mathbf{R Q}^{*}$ & Location & Isotope & Result \pm Error & $\mathbf{R Q}^{*}$ \\
\hline \multirow{18}{*}{$\begin{array}{c}\text { D052 } \\
(200-W)\end{array}$} & ${ }^{144} \mathrm{Ce}$ & $-1.3 \mathrm{E}-01 \pm 1.5 \mathrm{E}-01$ & $\mathrm{U}$ & \multirow{18}{*}{$\begin{array}{c}\text { D141 } \\
\text { (Replicate } \\
\text { of D018 } \\
\text { 200-W) }\end{array}$} & ${ }^{144} \mathrm{Ce}$ & $-5.7 \mathrm{E}-03 \pm 5.7 \mathrm{E}-02$ & $\mathrm{U}$ \\
\hline & ${ }^{60} \mathrm{Co}$ & $5.2 \mathrm{E}-03 \pm 8.0 \mathrm{E}-03$ & $\mathrm{U}$ & & ${ }^{60} \mathrm{Co}$ & $-2.4 \mathrm{E}-03 \pm 6.3 \mathrm{E}-03$ & \multirow[t]{3}{*}{$\mathrm{U}$} \\
\hline & ${ }^{134} \mathrm{Cs}$ & $5.2 \mathrm{E}-02 \pm 1.7 \mathrm{E}-02$ & & & ${ }^{134} \mathrm{Cs}$ & $3.0 \mathrm{E}-02 \pm 1.3 \mathrm{E}-02$ & \\
\hline & ${ }^{137} \mathrm{Cs}$ & $2.6 \mathrm{E}+00 \pm 4.3 \mathrm{E}-01$ & & & ${ }^{137} \mathrm{Cs}$ & $4.2 \mathrm{E}-01 \pm 6.8 \mathrm{E}-02$ & \\
\hline & ${ }^{152} \mathrm{Eu}$ & $7.2 \mathrm{E}-03 \pm 2.8 \mathrm{E}-02$ & U & & ${ }^{152} \mathrm{Eu}$ & $-1.3 \mathrm{E}-02 \pm 2.4 \mathrm{E}-02$ & U \\
\hline & ${ }^{154} \mathrm{Eu}$ & $3.9 \mathrm{E}-02 \pm 2.9 \mathrm{E}-02$ & $\mathrm{U}$ & & ${ }^{154} \mathrm{Eu}$ & $3.7 \mathrm{E}-03 \pm 2.3 \mathrm{E}-02$ & \multirow[t]{2}{*}{$\mathrm{U}$} \\
\hline & ${ }^{155} \mathrm{Eu}$ & $8.7 \mathrm{E}-02 \pm 4.8 \mathrm{E}-02$ & & & ${ }^{155} \mathrm{Eu}$ & $5.0 \mathrm{E}-02 \pm 3.5 \mathrm{E}-02$ & \\
\hline & ${ }^{238} \mathrm{Pu}$ & $2.7 \mathrm{E}-02 \pm 2.9 \mathrm{E}-02$ & $\mathrm{U}$ & & ${ }^{238} \mathrm{Pu}$ & $-1.8 \mathrm{E}-03 \pm 1.8 \mathrm{E}-02$ & $\mathrm{U}$ \\
\hline & ${ }^{239 / 240} \mathrm{Pu}$ & $1.3 \mathrm{E}-01 \pm 4.7 \mathrm{E}-02$ & & & ${ }^{239 / 240} \mathrm{Pu}$ & $1.1 \mathrm{E}-02 \pm 1.3 \mathrm{E}-02$ & $\mathrm{U}$ \\
\hline & ${ }^{103} \mathrm{Ru}$ & $-9.2 \mathrm{E}-04 \pm 9.2 \mathrm{E}-03$ & $\mathrm{U}$ & & ${ }^{103} \mathrm{Ru}$ & $3.8 \mathrm{E}-03 \pm 7.5 \mathrm{E}-03$ & $\mathrm{U}$ \\
\hline & ${ }^{106} \mathrm{Ru}$ & $-1.6 \mathrm{E}-02 \pm 7.7 \mathrm{E}-02$ & $\mathrm{U}$ & & ${ }^{106} \mathrm{Ru}$ & $-3.8 \mathrm{E}-04 \pm 3.8 \mathrm{E}-03$ & $\mathrm{U}$ \\
\hline & ${ }^{125} \mathrm{Sb}$ & $1.5 \mathrm{E}-02 \pm 2.7 \mathrm{E}-02$ & $\mathrm{U}$ & & ${ }^{125} \mathrm{Sb}$ & $2.3 \mathrm{E}-02 \pm 2.0 \mathrm{E}-02$ & $\mathrm{U}$ \\
\hline & ${ }^{113} \mathrm{Sn}$ & $4.4 \mathrm{E}-03 \pm 1.3 \mathrm{E}-02$ & $\mathrm{U}$ & & ${ }^{113} \mathrm{Sn}$ & $-7.8 \mathrm{E}-03 \pm 9.2 \mathrm{E}-03$ & $\mathrm{U}$ \\
\hline & ${ }^{90} \mathrm{Sr}$ & $-7.0 \mathrm{E}-01 \pm 7.0 \mathrm{E}-01$ & $\mathrm{U}$ & & ${ }^{90} \mathrm{Sr}$ & $-5.9 \mathrm{E}-01 \pm 5.9 \mathrm{E}-01$ & $\mathrm{U}$ \\
\hline & ${ }^{234} \mathrm{U}$ & $1.9 \mathrm{E}-01 \pm 6.3 \mathrm{E}-02$ & & & ${ }^{234} \mathrm{U}$ & $1.1 \mathrm{E}-01 \pm 4.2 \mathrm{E}-02$ & \\
\hline & ${ }^{235} \mathrm{U}$ & $2.1 \mathrm{E}-02 \pm 1.4 \mathrm{E}-02$ & & & ${ }^{235} \mathrm{U}$ & $1.1 \mathrm{E}-02 \pm 1.0 \mathrm{E}-02$ & \\
\hline & ${ }^{238} \mathrm{U}$ & $1.6 \mathrm{E}-01 \pm 5.4 \mathrm{E}-02$ & & & ${ }^{238} \mathrm{U}$ & $9.3 \mathrm{E}-02 \pm 3.7 \mathrm{E}-02$ & \\
\hline & ${ }^{65} \mathrm{Zn}$ & $1.2 \mathrm{E}-02 \pm 2.1 \mathrm{E}-02$ & $\mathrm{U}$ & & ${ }^{65} \mathrm{Zn}$ & $1.1 \mathrm{E}-04 \pm 1.1 \mathrm{E}-03$ & $\mathrm{U}$ \\
\hline D142 & ${ }^{144} \mathrm{Ce}$ & $-5.5 \mathrm{E}-02 \pm 1.1 \mathrm{E}-01$ & $\bar{U}$ & D054 & ${ }^{144} \mathrm{Ce}$ & $9.4 \mathrm{E}-02 \pm 1.6 \mathrm{E}-01$ & $\mathrm{U}$ \\
\hline (Replicate & ${ }^{60} \mathrm{Co}$ & 7.7E-03 \pm 7.6E-03 & $\mathrm{U}$ & $(200-E)$ & ${ }^{60} \mathrm{Co}$ & $-7.2 \mathrm{E}-04 \pm 6.6 \mathrm{E}-03$ & $\mathrm{U}$ \\
\hline of D028 & ${ }^{134} \mathrm{Cs}$ & $3.6 \mathrm{E}-02 \pm 1.2 \mathrm{E}-02$ & & & ${ }^{134} \mathrm{Cs}$ & $5.0 \mathrm{E}-02 \pm 1.4 \mathrm{E}-02$ & \\
\hline $200-W)$ & ${ }^{137} \mathrm{Cs}$ & $3.7 \mathrm{E}-01 \pm 6.2 \mathrm{E}-02$ & & & ${ }^{137} \mathrm{Cs}$ & $5.8 \mathrm{E}-01 \pm 1.0 \mathrm{E}-01$ & \\
\hline & ${ }^{152} \mathrm{Eu}$ & $-1.2 \mathrm{E}-02 \pm 1.9 \mathrm{E}-02$ & $\mathrm{U}$ & & ${ }^{152} \mathrm{Eu}$ & $1.2 \mathrm{E}-02 \pm 2.8 \mathrm{E}-02$ & $\mathrm{U}$ \\
\hline & ${ }^{154} \mathrm{Eu}$ & $-1.0 \mathrm{E}-02 \pm 2.3 \mathrm{E}-02$ & $\mathrm{U}$ & & ${ }^{154} \mathrm{Eu}$ & $-2.7 \mathrm{E}-02 \pm 2.7 \mathrm{E}-02$ & $\mathrm{U}$ \\
\hline & ${ }^{155} \mathrm{Eu}$ & $3.0 \mathrm{E}-02 \pm 3.2 \mathrm{E}-02$ & $\mathrm{U}$ & & ${ }^{155} \mathrm{Eu}$ & $8.2 \mathrm{E}-03 \pm 3.8 \mathrm{E}-02$ & $\mathrm{U}$ \\
\hline & ${ }^{238} \mathrm{Pu}$ & $1.5 \mathrm{E}-02 \pm 3.0 \mathrm{E}-02$ & $\mathrm{U}$ & & ${ }^{238} \mathrm{Pu}$ & $6.0 \mathrm{E}-03 \pm 1.7 \mathrm{E}-02$ & $\mathrm{U}$ \\
\hline & ${ }^{239 / 240} \mathrm{Pu}$ & $1.5 \mathrm{E}-02 \pm 1.5 \mathrm{E}-02$ & $\mathrm{U}$ & & ${ }^{239 / 240} \mathrm{Pu}$ & $4.0 \mathrm{E}-03 \pm 1.1 \mathrm{E}-02$ & $\mathrm{U}$ \\
\hline & ${ }^{103} \mathrm{Ru}$ & $2.4 \mathrm{E}-03 \pm 7.2 \mathrm{E}-03$ & $\mathrm{U}$ & & ${ }^{103} \mathrm{Ru}$ & $-3.3 \mathrm{E}-04 \pm 3.3 \mathrm{E}-03$ & $\mathrm{U}$ \\
\hline & ${ }^{106} \mathrm{Ru}$ & $6.1 \mathrm{E}-03 \pm 5.9 \mathrm{E}-02$ & $\mathrm{U}$ & & ${ }^{106} \mathrm{Ru}$ & $3.3 \mathrm{E}-02 \pm 6.7 \mathrm{E}-02$ & $\mathrm{U}$ \\
\hline & ${ }^{125} \mathrm{Sb}$ & $-1.8 \mathrm{E}-03 \pm 1.8 \mathrm{E}-02$ & $\mathrm{U}$ & & ${ }^{125} \mathrm{Sb}$ & $1.1 \mathrm{E}-02 \pm 2.1 \mathrm{E}-02$ & $\mathrm{U}$ \\
\hline & ${ }^{113} \mathrm{Sn}$ & $-1.0 \mathrm{E}-02 \pm 8.6 \mathrm{E}-03$ & $\mathrm{U}$ & & ${ }^{113} \mathrm{Sn}$ & $-1.2 \mathrm{E}-03 \pm 1.0 \mathrm{E}-02$ & $\mathrm{U}$ \\
\hline & ${ }^{90} \mathrm{Sr}$ & $-7.4 \mathrm{E}-01 \pm 7.4 \mathrm{E}-01$ & $\mathrm{U}$ & & ${ }^{90} \mathrm{Sr}$ & $-6.0 \mathrm{E}-01 \pm 6.0 \mathrm{E}-01$ & $\mathrm{U}$ \\
\hline & ${ }^{234} \mathrm{U}$ & $1.2 \mathrm{E}-01 \pm 4.3 \mathrm{E}-02$ & & & ${ }^{234} \mathrm{U}$ & $1.4 \mathrm{E}-01 \pm 4.9 \mathrm{E}-02$ & \\
\hline & ${ }^{235} \mathrm{U}$ & $1.3 \mathrm{E}-02 \pm 1.3 \mathrm{E}-02$ & $\mathrm{U}$ & & ${ }^{235} \mathrm{U}$ & $1.0 \mathrm{E}-02 \pm 9.3 \mathrm{E}-03$ & \\
\hline & ${ }^{238} \mathrm{U}$ & $9.2 \mathrm{E}-02 \pm 3.6 \mathrm{E}-02$ & & & ${ }^{238} \mathrm{U}$ & $1.4 \mathrm{E}-01 \pm 4.9 \mathrm{E}-02$ & \\
\hline & ${ }^{65} \mathrm{Zn}$ & $2.2 \mathrm{E}-02 \pm 1.9 \mathrm{E}-02$ & U & & ${ }^{65} \mathrm{Zn}$ & $-1.6 \mathrm{E}-03 \pm 1.6 \mathrm{E}-02$ & $\mathrm{U}$ \\
\hline
\end{tabular}

$\overline{\mathrm{RQ}}=$ Result Qualifier. $\mathrm{U}=$ The analyte was analyzed for but not detected. 
Table 3-4. 2008 Soil Sampling Results (pCi/g \pm total analytical uncertainty). (Sheet 12 of 24)

\begin{tabular}{|c|c|c|c|c|c|c|c|}
\hline Location & Isotope & Result \pm Error & $\mathbf{R Q}^{*}$ & Location & Isotope & Result \pm Error & $\mathbf{R Q}^{*}$ \\
\hline D056 & ${ }^{144} \mathrm{Ce}$ & $6.0 \mathrm{E}-02 \pm 1.9 \mathrm{E}-01$ & $\mathrm{U}$ & D058 & ${ }^{144} \mathrm{Ce}$ & $7.7 \mathrm{E}-02 \pm 1.8 \mathrm{E}-01$ & $\mathrm{U}$ \\
\hline \multirow[t]{17}{*}{ (200-E) } & ${ }^{60} \mathrm{Co}$ & $4.1 \mathrm{E}-03 \pm 9.8 \mathrm{E}-03$ & $\mathrm{U}$ & $(200-E)$ & ${ }^{60} \mathrm{Co}$ & $-3.5 \mathrm{E}-03 \pm 6.9 \mathrm{E}-03$ & U \\
\hline & ${ }^{134} \mathrm{Cs}$ & $3.8 \mathrm{E}-02 \pm 2.0 \mathrm{E}-02$ & & & ${ }^{134} \mathrm{Cs}$ & $3.4 \mathrm{E}-02 \pm 1.1 \mathrm{E}-02$ & \\
\hline & ${ }^{137} \mathrm{Cs}$ & $1.6 \mathrm{E}+00 \pm 2.4 \mathrm{E}-01$ & & & ${ }^{137} \mathrm{Cs}$ & $4.9 \mathrm{E}+00 \pm 8.9 \mathrm{E}-01$ & \\
\hline & ${ }^{152} \mathrm{Eu}$ & $-9.6 \mathrm{E}-03 \pm 6.0 \mathrm{E}-02$ & $\mathrm{U}$ & & ${ }^{152} \mathrm{Eu}$ & $1.3 \mathrm{E}-02 \pm 4.8 \mathrm{E}-02$ & $\mathrm{U}$ \\
\hline & ${ }^{154} \mathrm{Eu}$ & $-1.5 \mathrm{E}-03 \pm 1.5 \mathrm{E}-02$ & $\mathrm{U}$ & & ${ }^{154} \mathrm{Eu}$ & $-1.9 \mathrm{E}-02 \pm 2.3 \mathrm{E}-02$ & $\mathrm{U}$ \\
\hline & ${ }^{155} \mathrm{Eu}$ & $5.2 \mathrm{E}-02 \pm 4.2 \mathrm{E}-02$ & $\mathrm{U}$ & & ${ }^{155} \mathrm{Eu}$ & $1.9 \mathrm{E}-02 \pm 4.3 \mathrm{E}-02$ & $\mathrm{U}$ \\
\hline & ${ }^{238} \mathrm{Pu}$ & $4.2 \mathrm{E}-03 \pm 2.2 \mathrm{E}-02$ & $\mathrm{U}$ & & ${ }^{238} \mathrm{Pu}$ & $-1.2 \mathrm{E}-02 \pm 1.6 \mathrm{E}-02$ & $\mathrm{U}$ \\
\hline & ${ }^{239 / 240} \mathrm{Pu}$ & $6.3 \mathrm{E}-03 \pm 7.4 \mathrm{E}-03$ & & & ${ }^{239 / 240} \mathrm{Pu}$ & $1.8 \mathrm{E}-02 \pm 1.3 \mathrm{E}-02$ & \\
\hline & ${ }^{103} \mathrm{Ru}$ & $-1.2 \mathrm{E}-02 \pm 1.5 \mathrm{E}-02$ & $\mathrm{U}$ & & ${ }^{103} \mathrm{Ru}$ & $-4.4 \mathrm{E}-03 \pm 1.3 \mathrm{E}-02$ & $\mathrm{U}$ \\
\hline & ${ }^{106} \mathrm{Ru}$ & $-4.5 \mathrm{E}-02 \pm 1.0 \mathrm{E}-01$ & $\mathrm{U}$ & & ${ }^{106} \mathrm{Ru}$ & $-2.6 \mathrm{E}-02 \pm 8.2 \mathrm{E}-02$ & $\mathrm{U}$ \\
\hline & ${ }^{125} \mathrm{Sb}$ & $-4.0 \mathrm{E}-04 \pm 4.0 \mathrm{E}-03$ & $\mathrm{U}$ & & ${ }^{125} \mathrm{Sb}$ & $-1.3 \mathrm{E}-02 \pm 3.2 \mathrm{E}-02$ & $\mathrm{U}$ \\
\hline & ${ }^{113} \mathrm{Sn}$ & $-1.2 \mathrm{E}-02 \pm 1.7 \mathrm{E}-02$ & $\mathrm{U}$ & & ${ }^{113} \mathrm{Sn}$ & $8.7 \mathrm{E}-03 \pm 1.6 \mathrm{E}-02$ & $\mathrm{U}$ \\
\hline & ${ }^{90} \mathrm{Sr}$ & $-9.4 \mathrm{E}-01 \pm 9.4 \mathrm{E}-01$ & $\mathrm{U}$ & & ${ }^{90} \mathrm{Sr}$ & $-4.2 \mathrm{E}-01 \pm 4.2 \mathrm{E}-01$ & $\mathrm{U}$ \\
\hline & ${ }^{234} \mathrm{U}$ & $1.7 \mathrm{E}-01 \pm 5.9 \mathrm{E}-02$ & & & ${ }^{234} \mathrm{U}$ & $1.4 \mathrm{E}-01 \pm 4.9 \mathrm{E}-02$ & \\
\hline & ${ }^{235} \mathrm{U}$ & $2.1 \mathrm{E}-02 \pm 1.5 \mathrm{E}-02$ & & & ${ }^{235} \mathrm{U}$ & $1.1 \mathrm{E}-02 \pm 1.0 \mathrm{E}-02$ & \\
\hline & ${ }^{238} \mathrm{U}$ & $2.3 \mathrm{E}-01 \pm 7.4 \mathrm{E}-02$ & & & ${ }^{238} \mathrm{U}$ & $1.4 \mathrm{E}-01 \pm 4.9 \mathrm{E}-02$ & \\
\hline & ${ }^{65} \mathrm{Zn}$ & $5.4 \mathrm{E}-03 \pm 2.7 \mathrm{E}-02$ & $\mathrm{U}$ & & ${ }^{65} \mathrm{Zn}$ & $2.8 \mathrm{E}-02 \pm 2.0 \mathrm{E}-02$ & $\mathrm{U}$ \\
\hline \multirow{18}{*}{$\begin{array}{c}\text { D060 } \\
(200-E)\end{array}$} & ${ }^{144} \mathrm{Ce}$ & $4.4 \mathrm{E}-02 \pm 2.1 \mathrm{E}-01$ & $\mathrm{U}$ & D062 & ${ }^{144} \mathrm{Ce}$ & $4.5 \mathrm{E}-02 \pm 1.3 \mathrm{E}-01$ & $\mathrm{U}$ \\
\hline & ${ }^{60} \mathrm{Co}$ & $6.6 \mathrm{E}-03 \pm 1.1 \mathrm{E}-02$ & $\mathrm{U}$ & $(200-E)$ & ${ }^{60} \mathrm{Co}$ & $8.8 \mathrm{E}-04 \pm 5.9 \mathrm{E}-03$ & $\mathrm{U}$ \\
\hline & ${ }^{134} \mathrm{Cs}$ & $5.0 \mathrm{E}-02 \pm 2.0 \mathrm{E}-02$ & & & ${ }^{134} \mathrm{Cs}$ & $3.9 \mathrm{E}-02 \pm 1.2 \mathrm{E}-02$ & \\
\hline & ${ }^{137} \mathrm{Cs}$ & $4.8 \mathrm{E}-01 \pm 7.7 \mathrm{E}-02$ & & & ${ }^{137} \mathrm{Cs}$ & $1.1 \mathrm{E}+00 \pm 1.8 \mathrm{E}-01$ & \\
\hline & ${ }^{152} \mathrm{Eu}$ & $-1.5 \mathrm{E}-03 \pm 1.5 \mathrm{E}-02$ & $\mathrm{U}$ & & ${ }^{152} \mathrm{Eu}$ & $2.2 \mathrm{E}-03 \pm 2.2 \mathrm{E}-02$ & U \\
\hline & ${ }^{154} \mathrm{Eu}$ & $-1.1 \mathrm{E}-02 \pm 3.9 \mathrm{E}-02$ & $\mathrm{U}$ & & ${ }^{154} \mathrm{Eu}$ & $-1.9 \mathrm{E}-02 \pm 2.0 \mathrm{E}-02$ & $\mathrm{U}$ \\
\hline & ${ }^{155} \mathrm{Eu}$ & $1.0 \mathrm{E}-01 \pm 5.2 \mathrm{E}-02$ & & & ${ }^{155} \mathrm{Eu}$ & $5.5 \mathrm{E}-02 \pm 3.6 \mathrm{E}-02$ & \\
\hline & ${ }^{238} \mathrm{Pu}$ & $2.4 \mathrm{E}-03 \pm 2.4 \mathrm{E}-02$ & $\mathrm{U}$ & & ${ }^{238} \mathrm{Pu}$ & $2.3 \mathrm{E}-03 \pm 4.0 \mathrm{E}-03$ & $\mathrm{U}$ \\
\hline & ${ }^{239 / 240} \mathrm{Pu}$ & $2.3 \mathrm{E}-03 \pm 2.3 \mathrm{E}-02$ & $\mathrm{U}$ & & ${ }^{239 / 240} \mathrm{Pu}$ & $6.9 \mathrm{E}-03 \pm 1.2 \mathrm{E}-02$ & $\mathrm{U}$ \\
\hline & ${ }^{103} \mathrm{Ru}$ & $1.0 \mathrm{E}-02 \pm 1.7 \mathrm{E}-02$ & $\mathrm{U}$ & & ${ }^{103} \mathrm{Ru}$ & $-5.1 \mathrm{E}-03 \pm 1.1 \mathrm{E}-02$ & $\mathrm{U}$ \\
\hline & ${ }^{106} \mathrm{Ru}$ & $-2.6 \mathrm{E}-03 \pm 2.6 \mathrm{E}-02$ & $\mathrm{U}$ & & ${ }^{106} \mathrm{Ru}$ & $8.7 \mathrm{E}-03 \pm 5.9 \mathrm{E}-02$ & U \\
\hline & ${ }^{125} \mathrm{Sb}$ & $6.4 \mathrm{E}-03 \pm 3.5 \mathrm{E}-02$ & $\mathrm{U}$ & & ${ }^{125} \mathrm{Sb}$ & $-1.4 \mathrm{E}-03 \pm 1.4 \mathrm{E}-02$ & $\mathrm{U}$ \\
\hline & ${ }^{113} \mathrm{Sn}$ & $-8.3 \mathrm{E}-03 \pm 1.8 \mathrm{E}-02$ & $\mathrm{U}$ & & ${ }^{113} \mathrm{Sn}$ & $-1.5 \mathrm{E}-02 \pm 1.5 \mathrm{E}-02$ & U \\
\hline & ${ }^{90} \mathrm{Sr}$ & $-4.8 \mathrm{E}-01 \pm 4.8 \mathrm{E}-01$ & $\mathrm{U}$ & & ${ }^{90} \mathrm{Sr}$ & $-4.4 \mathrm{E}-01 \pm 4.4 \mathrm{E}-01$ & $\mathrm{U}$ \\
\hline & ${ }^{234} \mathrm{U}$ & $1.9 \mathrm{E}-01 \pm 6.5 \mathrm{E}-02$ & & & ${ }^{234} \mathrm{U}$ & $2.0 \mathrm{E}-01 \pm 6.6 \mathrm{E}-02$ & \\
\hline & ${ }^{235} \mathrm{U}$ & $1.4 \mathrm{E}-02 \pm 1.4 \mathrm{E}-02$ & $\mathrm{U}$ & & ${ }^{235} \mathrm{U}$ & $1.4 \mathrm{E}-02 \pm 1.2 \mathrm{E}-02$ & \\
\hline & ${ }^{238} \mathrm{U}$ & $1.7 \mathrm{E}-01 \pm 5.8 \mathrm{E}-02$ & & & ${ }^{238} \mathrm{U}$ & $1.8 \mathrm{E}-01 \pm 6.1 \mathrm{E}-02$ & \\
\hline & ${ }^{65} \mathrm{Zn}$ & $-1.4 \mathrm{E}-02 \pm 3.2 \mathrm{E}-02$ & $\mathrm{U}$ & & ${ }^{65} \mathrm{Zn}$ & $-6.9 \mathrm{E}-03 \pm 1.7 \mathrm{E}-02$ & $\mathrm{U}$ \\
\hline
\end{tabular}

$\overline{\mathrm{RQ}}=$ Result Qualifier. $\mathrm{U}=$ The analyte was analyzed for but not detected. 
Table 3-4. 2008 Soil Sampling Results (pCi/g \pm total analytical uncertainty). (Sheet 13 of 24)

\begin{tabular}{|c|c|c|c|c|c|c|c|}
\hline Location & Isotope & Result \pm Error & $\mathbf{R Q}^{*}$ & Location & Isotope & Result \pm Error & RQ* \\
\hline D064 & ${ }^{144} \mathrm{Ce}$ & $7.2 \mathrm{E}-03 \pm 7.2 \mathrm{E}-02$ & $\mathrm{U}$ & D066 & ${ }^{144} \mathrm{Ce}$ & $2.7 \mathrm{E}-02 \pm 1.8 \mathrm{E}-01$ & $\mathrm{U}$ \\
\hline \multirow[t]{17}{*}{$(200-E)$} & ${ }^{60} \mathrm{Co}$ & $-1.6 \mathrm{E}-03 \pm 7.9 \mathrm{E}-03$ & $\mathrm{U}$ & (200-E) & ${ }^{60} \mathrm{Co}$ & $2.4 \mathrm{E}-03 \pm 6.5 \mathrm{E}-03$ & $\mathrm{U}$ \\
\hline & ${ }^{134} \mathrm{Cs}$ & $4.7 \mathrm{E}-02 \pm 1.8 \mathrm{E}-02$ & & & ${ }^{134} \mathrm{Cs}$ & $3.1 \mathrm{E}-02 \pm 9.0 \mathrm{E}-03$ & \\
\hline & ${ }^{137} \mathrm{Cs}$ & $6.2 \mathrm{E}-01 \pm 1.0 \mathrm{E}-01$ & & & ${ }^{137} \mathrm{Cs}$ & $1.4 \mathrm{E}+01 \pm 2.2 \mathrm{E}+00$ & \\
\hline & ${ }^{152} \mathrm{Eu}$ & $-4.7 \mathrm{E}-03 \pm 2.3 \mathrm{E}-02$ & $\mathrm{U}$ & & ${ }^{152} \mathrm{Eu}$ & $1.5 \mathrm{E}-02 \pm 3.8 \mathrm{E}-02$ & $\mathrm{U}$ \\
\hline & ${ }^{154} \mathrm{Eu}$ & $-6.2 \mathrm{E}-03 \pm 3.0 \mathrm{E}-02$ & $\mathrm{U}$ & & ${ }^{154} \mathrm{Eu}$ & $-1.2 \mathrm{E}-04 \pm 1.2 \mathrm{E}-03$ & $\mathrm{U}$ \\
\hline & ${ }^{155} \mathrm{Eu}$ & $6.2 \mathrm{E}-02 \pm 4.8 \mathrm{E}-02$ & & & ${ }^{155} \mathrm{Eu}$ & $-6.2 \mathrm{E}-03 \pm 4.1 \mathrm{E}-02$ & $\mathrm{U}$ \\
\hline & ${ }^{238} \mathrm{Pu}$ & $-3.3 \mathrm{E}-02 \pm 4.7 \mathrm{E}-02$ & $\mathrm{U}$ & & ${ }^{238} \mathrm{Pu}$ & $1.6 \mathrm{E}-02 \pm 3.6 \mathrm{E}-02$ & $\mathrm{U}$ \\
\hline & ${ }^{239 / 240} \mathrm{Pu}$ & $2.6 \mathrm{E}-03 \pm 9.0 \mathrm{E}-03$ & $\mathrm{U}$ & & ${ }^{239 / 240} \mathrm{Pu}$ & $1.9 \mathrm{E}-02 \pm 1.7 \mathrm{E}-02$ & $\mathrm{U}$ \\
\hline & ${ }^{103} \mathrm{Ru}$ & $-1.7 \mathrm{E}-03 \pm 9.6 \mathrm{E}-03$ & U & & ${ }^{103} \mathrm{Ru}$ & $8.0 \mathrm{E}-03 \pm 1.8 \mathrm{E}-02$ & $\mathrm{U}$ \\
\hline & ${ }^{106} \mathrm{Ru}$ & $2.4 \mathrm{E}-02 \pm 6.8 \mathrm{E}-02$ & $\mathrm{U}$ & & ${ }^{106} \mathrm{Ru}$ & $3.3 \mathrm{E}-02 \pm 9.6 \mathrm{E}-02$ & $\mathrm{U}$ \\
\hline & ${ }^{125} \mathrm{Sb}$ & $1.0 \mathrm{E}-02 \pm 2.2 \mathrm{E}-02$ & $\mathrm{U}$ & & ${ }^{125} \mathrm{Sb}$ & $1.4 \mathrm{E}-02 \pm 4.0 \mathrm{E}-02$ & $\mathrm{U}$ \\
\hline & ${ }^{113} \mathrm{Sn}$ & $-5.7 \mathrm{E}-03 \pm 1.1 \mathrm{E}-02$ & $\mathrm{U}$ & & ${ }^{113} \mathrm{Sn}$ & $5.0 \mathrm{E}-04 \pm 5.0 \mathrm{E}-03$ & $\mathrm{U}$ \\
\hline & ${ }^{90} \mathrm{Sr}$ & $-5.5 \mathrm{E}-01 \pm 5.5 \mathrm{E}-01$ & $\mathrm{U}$ & & ${ }^{90} \mathrm{Sr}$ & $1.4 \mathrm{E}-01 \pm 4.8 \mathrm{E}-01$ & $\mathrm{U}$ \\
\hline & ${ }^{234} \mathrm{U}$ & $1.9 \mathrm{E}-01 \pm 6.5 \mathrm{E}-02$ & & & ${ }^{234} \mathrm{U}$ & $1.4 \mathrm{E}-01 \pm 5.0 \mathrm{E}-02$ & \\
\hline & ${ }^{235} \mathrm{U}$ & $1.6 \mathrm{E}-02 \pm 1.3 \mathrm{E}-02$ & & & ${ }^{235} \mathrm{U}$ & $1.9 \mathrm{E}-02 \pm 1.4 \mathrm{E}-02$ & \\
\hline & ${ }^{238} \mathrm{U}$ & $1.7 \mathrm{E}-01 \pm 5.8 \mathrm{E}-02$ & & & ${ }^{238} \mathrm{U}$ & $1.6 \mathrm{E}-01 \pm 5.6 \mathrm{E}-02$ & \\
\hline & ${ }^{65} \mathrm{Zn}$ & $2.6 \mathrm{E}-02 \pm 2.2 \mathrm{E}-02$ & $\mathrm{U}$ & & ${ }^{65} \mathrm{Zn}$ & $2.4 \mathrm{E}-02 \pm 1.8 \mathrm{E}-02$ & $\mathrm{U}$ \\
\hline \multirow{18}{*}{$\begin{array}{c}\text { D068 } \\
(200-E)\end{array}$} & ${ }^{144} \mathrm{Ce}$ & $3.7 \mathrm{E}-02 \pm 1.5 \mathrm{E}-01$ & $\mathrm{U}$ & D070 & ${ }^{144} \mathrm{Ce}$ & $-8.3 \mathrm{E}-02 \pm 1.1 \mathrm{E}-01$ & $\mathrm{U}$ \\
\hline & ${ }^{60} \mathrm{Co}$ & $6.8 \mathrm{E}-04 \pm 5.9 \mathrm{E}-03$ & $\mathrm{U}$ & (200-E) & ${ }^{60} \mathrm{Co}$ & $-1.6 \mathrm{E}-03 \pm 5.1 \mathrm{E}-03$ & $\mathrm{U}$ \\
\hline & ${ }^{134} \mathrm{Cs}$ & $3.6 \mathrm{E}-02 \pm 1.2 \mathrm{E}-02$ & & & ${ }^{134} \mathrm{Cs}$ & $3.2 \mathrm{E}-02 \pm 8.3 \mathrm{E}-03$ & \\
\hline & ${ }^{137} \mathrm{Cs}$ & $1.1 \mathrm{E}-01 \pm 2.2 \mathrm{E}-02$ & & & ${ }^{137} \mathrm{Cs}$ & $3.7 \mathrm{E}-02 \pm 1.1 \mathrm{E}-02$ & \\
\hline & ${ }^{152} \mathrm{Eu}$ & $-9.5 \mathrm{E}-04 \pm 9.5 \mathrm{E}-03$ & $\mathrm{U}$ & & ${ }^{152} \mathrm{Eu}$ & $1.2 \mathrm{E}-02 \pm 1.9 \mathrm{E}-02$ & $\mathrm{U}$ \\
\hline & ${ }^{154} \mathrm{Eu}$ & $-1.4 \mathrm{E}-04 \pm 1.4 \mathrm{E}-03$ & U & & ${ }^{154} \mathrm{Eu}$ & $3.8 \mathrm{E}-03 \pm 1.9 \mathrm{E}-02$ & $\mathrm{U}$ \\
\hline & ${ }^{155} \mathrm{Eu}$ & $4.6 \mathrm{E}-02 \pm 3.2 \mathrm{E}-02$ & $\mathrm{U}$ & & ${ }^{155} \mathrm{Eu}$ & $2.6 \mathrm{E}-02 \pm 2.7 \mathrm{E}-02$ & $\mathrm{U}$ \\
\hline & ${ }^{238} \mathrm{Pu}$ & $-8.5 \mathrm{E}-03 \pm 2.2 \mathrm{E}-02$ & $\mathrm{U}$ & & ${ }^{238} \mathrm{Pu}$ & $-2.1 \mathrm{E}-03 \pm 2.1 \mathrm{E}-03$ & $\mathrm{U}$ \\
\hline & ${ }^{239 / 240} \mathrm{Pu}$ & $2.1 \mathrm{E}-03 \pm 4.2 \mathrm{E}-03$ & $\mathrm{U}$ & & ${ }^{239 / 240} \mathrm{Pu}$ & $4.2 \mathrm{E}-03 \pm 1.2 \mathrm{E}-02$ & $\mathrm{U}$ \\
\hline & ${ }^{103} \mathrm{Ru}$ & $3.8 \mathrm{E}-03 \pm 8.4 \mathrm{E}-03$ & $\mathrm{U}$ & & ${ }^{103} \mathrm{Ru}$ & $-4.4 \mathrm{E}-03 \pm 7.0 \mathrm{E}-03$ & $\mathrm{U}$ \\
\hline & ${ }^{106} \mathrm{Ru}$ & $1.4 \mathrm{E}-02 \pm 5.7 \mathrm{E}-02$ & $\mathrm{U}$ & & ${ }^{106} \mathrm{Ru}$ & $-3.6 \mathrm{E}-02 \pm 4.7 \mathrm{E}-02$ & $\mathrm{U}$ \\
\hline & ${ }^{125} \mathrm{Sb}$ & $-9.8 \mathrm{E}-04 \pm 9.8 \mathrm{E}-03$ & $\mathrm{U}$ & & ${ }^{125} \mathrm{Sb}$ & $4.4 \mathrm{E}-03 \pm 1.5 \mathrm{E}-02$ & $\mathrm{U}$ \\
\hline & ${ }^{113} \mathrm{Sn}$ & $2.1 \mathrm{E}-03 \pm 1.0 \mathrm{E}-02$ & $\mathrm{U}$ & & ${ }^{113} \mathrm{Sn}$ & $-6.1 \mathrm{E}-03 \pm 7.8 \mathrm{E}-03$ & $\mathrm{U}$ \\
\hline & ${ }^{90} \mathrm{Sr}$ & $4.8 \mathrm{E}-01 \pm 4.7 \mathrm{E}-01$ & & & ${ }^{90} \mathrm{Sr}$ & $-1.1 \mathrm{E}-01 \pm 4.3 \mathrm{E}-01$ & $\mathrm{U}$ \\
\hline & ${ }^{234} \mathrm{U}$ & $1.8 \mathrm{E}-01 \pm 6.5 \mathrm{E}-02$ & & & ${ }^{234} \mathrm{U}$ & $1.5 \mathrm{E}-01 \pm 5.4 \mathrm{E}-02$ & \\
\hline & ${ }^{235} \mathrm{U}$ & $1.8 \mathrm{E}-02 \pm 2.0 \mathrm{E}-02$ & $\mathrm{U}$ & & ${ }^{235} \mathrm{U}$ & $1.8 \mathrm{E}-02 \pm 1.6 \mathrm{E}-02$ & $\mathrm{U}$ \\
\hline & ${ }^{238} \mathrm{U}$ & $2.0 \mathrm{E}-01 \pm 7.0 \mathrm{E}-02$ & & & ${ }^{238} \mathrm{U}$ & $1.5 \mathrm{E}-01 \pm 5.4 \mathrm{E}-02$ & \\
\hline & ${ }^{65} \mathrm{Zn}$ & $-7.6 \mathrm{E}-03 \pm 1.7 \mathrm{E}-02$ & $\mathrm{U}$ & & ${ }^{65} \mathrm{Zn}$ & $4.5 \mathrm{E}-04 \pm 4.5 \mathrm{E}-03$ & $\mathrm{U}$ \\
\hline
\end{tabular}

$\overline{\mathrm{RQ}}=$ Result Qualifier. $\mathrm{U}=$ The analyte was analyzed for but not detected. 
Table 3-4. 2008 Soil Sampling Results (pCi/g \pm total analytical uncertainty). (Sheet 14 of 24)

\begin{tabular}{|c|c|c|c|c|c|c|c|}
\hline Location & Isotope & Result \pm Error & $\mathbf{R Q}^{*}$ & Location & Isotope & Result \pm Error & RQ* \\
\hline D072 & ${ }^{144} \mathrm{Ce}$ & $7.0 \mathrm{E}-03 \pm 7.0 \mathrm{E}-02$ & $\mathrm{U}$ & D074 & ${ }^{144} \mathrm{Ce}$ & $-7.0 \mathrm{E}-03 \pm 7.0 \mathrm{E}-02$ & $\mathrm{U}$ \\
\hline \multirow[t]{17}{*}{$(200-E)$} & ${ }^{60} \mathrm{Co}$ & $5.7 \mathrm{E}-04 \pm 5.7 \mathrm{E}-03$ & $\mathrm{U}$ & $(200-E)$ & ${ }^{60} \mathrm{Co}$ & $-2.1 \mathrm{E}-03 \pm 7.2 \mathrm{E}-03$ & $\mathrm{U}$ \\
\hline & ${ }^{134} \mathrm{Cs}$ & $2.6 \mathrm{E}-02 \pm 1.3 \mathrm{E}-02$ & & & ${ }^{134} \mathrm{Cs}$ & $2.9 \mathrm{E}-02 \pm 1.0 \mathrm{E}-02$ & \\
\hline & ${ }^{137} \mathrm{Cs}$ & $9.2 \mathrm{E}-02 \pm 2.3 \mathrm{E}-02$ & & & ${ }^{137} \mathrm{Cs}$ & $1.4 \mathrm{E}-01 \pm 2.9 \mathrm{E}-02$ & \\
\hline & ${ }^{152} \mathrm{Eu}$ & $1.7 \mathrm{E}-02 \pm 4.5 \mathrm{E}-02$ & U & & ${ }^{152} \mathrm{Eu}$ & $1.3 \mathrm{E}-02 \pm 3.8 \mathrm{E}-02$ & U \\
\hline & ${ }^{154} \mathrm{Eu}$ & $-9.9 \mathrm{E}-03 \pm 2.8 \mathrm{E}-02$ & $\mathrm{U}$ & & ${ }^{154} \mathrm{Eu}$ & $1.4 \mathrm{E}-02 \pm 2.8 \mathrm{E}-02$ & $\mathrm{U}$ \\
\hline & ${ }^{155} \mathrm{Eu}$ & $3.9 \mathrm{E}-02 \pm 3.5 \mathrm{E}-02$ & $\mathrm{U}$ & & ${ }^{155} \mathrm{Eu}$ & $3.5 \mathrm{E}-02 \pm 3.6 \mathrm{E}-02$ & $\mathrm{U}$ \\
\hline & ${ }^{238} \mathrm{Pu}$ & $7.9 \mathrm{E}-03 \pm 1.7 \mathrm{E}-02$ & $\mathrm{U}$ & & ${ }^{238} \mathrm{Pu}$ & $1.2 \mathrm{E}-01 \pm 5.5 \mathrm{E}-02$ & \\
\hline & ${ }^{239 / 240} \mathrm{Pu}$ & $2.0 \mathrm{E}-03 \pm 2.0 \mathrm{E}-02$ & $\mathrm{U}$ & & ${ }^{239 / 240} \mathrm{Pu}$ & $1.3 \mathrm{E}-02 \pm 1.1 \mathrm{E}-02$ & \\
\hline & ${ }^{103} \mathrm{Ru}$ & $1.1 \mathrm{E}-02 \pm 1.2 \mathrm{E}-02$ & $\mathrm{U}$ & & ${ }^{103} \mathrm{Ru}$ & $-3.3 \mathrm{E}-04 \pm 3.3 \mathrm{E}-03$ & $\mathrm{U}$ \\
\hline & ${ }^{106} \mathrm{Ru}$ & $2.2 \mathrm{E}-03 \pm 2.2 \mathrm{E}-02$ & $\mathrm{U}$ & & ${ }^{106} \mathrm{Ru}$ & $-3.3 E-03 \pm 3.3 E-02$ & $\mathrm{U}$ \\
\hline & ${ }^{125} \mathrm{Sb}$ & $-1.5 \mathrm{E}-02 \pm 2.6 \mathrm{E}-02$ & $\mathrm{U}$ & & ${ }^{125} \mathrm{Sb}$ & $1.7 \mathrm{E}-02 \pm 1.9 \mathrm{E}-02$ & $\mathrm{U}$ \\
\hline & ${ }^{113} \mathrm{Sn}$ & $8.0 \mathrm{E}-04 \pm 8.0 \mathrm{E}-03$ & $\mathrm{U}$ & & ${ }^{113} \mathrm{Sn}$ & $-8.2 \mathrm{E}-03 \pm 1.0 \mathrm{E}-02$ & $\mathrm{U}$ \\
\hline & ${ }^{90} \mathrm{Sr}$ & $4.0 \mathrm{E}-01 \pm 4.3 \mathrm{E}-01$ & $\mathrm{U}$ & & ${ }^{90} \mathrm{Sr}$ & $-6.6 \mathrm{E}-01 \pm 6.6 \mathrm{E}-01$ & $\mathrm{U}$ \\
\hline & ${ }^{234} \mathrm{U}$ & $1.7 \mathrm{E}-01 \pm 6.1 \mathrm{E}-02$ & & & ${ }^{234} \mathrm{U}$ & $1.4 \mathrm{E}-01 \pm 5.3 \mathrm{E}-02$ & \\
\hline & ${ }^{235} \mathrm{U}$ & $1.6 \mathrm{E}-02 \pm 1.4 \mathrm{E}-02$ & & & ${ }^{235} \mathrm{U}$ & $5.9 \mathrm{E}-03 \pm 8.5 \mathrm{E}-03$ & $\mathrm{U}$ \\
\hline & ${ }^{238} \mathrm{U}$ & $1.5 \mathrm{E}-01 \pm 5.4 \mathrm{E}-02$ & & & ${ }^{238} \mathrm{U}$ & $1.8 \mathrm{E}-01 \pm 6.3 \mathrm{E}-02$ & \\
\hline & ${ }^{65} \mathrm{Zn}$ & $-2.4 \mathrm{E}-03 \pm 2.4 \mathrm{E}-02$ & $\mathrm{U}$ & & ${ }^{65} \mathrm{Zn}$ & $2.5 \mathrm{E}-02 \pm 2.0 \mathrm{E}-02$ & $\mathrm{U}$ \\
\hline \multirow{18}{*}{$\begin{array}{c}\text { D076 } \\
(200-E)\end{array}$} & ${ }^{144} \mathrm{Ce}$ & $3.5 \mathrm{E}-02 \pm 1.6 \mathrm{E}-01$ & $\mathrm{U}$ & D078 & ${ }^{144} \mathrm{Ce}$ & $-1.8 \mathrm{E}-01 \pm 1.8 \mathrm{E}-01$ & $\mathrm{U}$ \\
\hline & ${ }^{60} \mathrm{Co}$ & $-2.0 \mathrm{E}-04 \pm 2.0 \mathrm{E}-03$ & $\mathrm{U}$ & $(200-E)$ & ${ }^{60} \mathrm{Co}$ & $-2.2 \mathrm{E}-03 \pm 7.4 \mathrm{E}-03$ & $\mathrm{U}$ \\
\hline & ${ }^{134} \mathrm{Cs}$ & $4.0 \mathrm{E}-02 \pm 1.4 \mathrm{E}-02$ & & & ${ }^{134} \mathrm{Cs}$ & $4.7 \mathrm{E}-02 \pm 1.6 \mathrm{E}-02$ & \\
\hline & ${ }^{137} \mathrm{Cs}$ & $5.4 \mathrm{E}-02 \pm 1.7 \mathrm{E}-02$ & & & ${ }^{137} \mathrm{Cs}$ & $2.2 \mathrm{E}-01 \pm 3.8 \mathrm{E}-02$ & \\
\hline & ${ }^{152} \mathrm{Eu}$ & $-9.4 \mathrm{E}-03 \pm 3.1 \mathrm{E}-02$ & $\mathrm{U}$ & & ${ }^{152} \mathrm{Eu}$ & $-9.5 \mathrm{E}-03 \pm 2.5 \mathrm{E}-02$ & $\mathrm{U}$ \\
\hline & ${ }^{154} \mathrm{Eu}$ & $-1.1 \mathrm{E}-02 \pm 2.7 \mathrm{E}-02$ & $\mathrm{U}$ & & ${ }^{154} \mathrm{Eu}$ & $-2.9 \mathrm{E}-02 \pm 2.9 \mathrm{E}-02$ & $\mathrm{U}$ \\
\hline & ${ }^{155} \mathrm{Eu}$ & $1.5 \mathrm{E}-02 \pm 3.6 \mathrm{E}-02$ & $\mathrm{U}$ & & ${ }^{155} \mathrm{Eu}$ & $4.5 \mathrm{E}-02 \pm 4.5 \mathrm{E}-02$ & $\mathrm{U}$ \\
\hline & ${ }^{238} \mathrm{Pu}$ & $-1.7 \mathrm{E}-02 \pm 3.6 \mathrm{E}-02$ & $\mathrm{U}$ & & ${ }^{238} \mathrm{Pu}$ & $-4.0 \mathrm{E}-03 \pm 2.6 \mathrm{E}-02$ & $\mathrm{U}$ \\
\hline & ${ }^{239 / 240} \mathrm{Pu}$ & $2.1 \mathrm{E}-03 \pm 2.1 \mathrm{E}-03$ & $\mathrm{U}$ & & ${ }^{239 / 240} \mathrm{Pu}$ & $8.0 \mathrm{E}-03 \pm 1.3 \mathrm{E}-02$ & $\mathrm{U}$ \\
\hline & ${ }^{103} \mathrm{Ru}$ & $-2.9 \mathrm{E}-03 \pm 1.0 \mathrm{E}-02$ & $\mathrm{U}$ & & ${ }^{103} \mathrm{Ru}$ & $3.8 \mathrm{E}-03 \pm 9.0 \mathrm{E}-03$ & $\mathrm{U}$ \\
\hline & ${ }^{106} \mathrm{Ru}$ & $-4.9 \mathrm{E}-02 \pm 7.4 \mathrm{E}-02$ & $\mathrm{U}$ & & ${ }^{106} \mathrm{Ru}$ & $2.1 \mathrm{E}-03 \pm 2.1 \mathrm{E}-02$ & $\mathrm{U}$ \\
\hline & ${ }^{125} \mathrm{Sb}$ & $-6.5 \mathrm{E}-04 \pm 6.5 \mathrm{E}-03$ & $\mathrm{U}$ & & ${ }^{125} \mathrm{Sb}$ & $1.6 \mathrm{E}-02 \pm 2.0 \mathrm{E}-02$ & $\mathrm{U}$ \\
\hline & ${ }^{113} \mathrm{Sn}$ & $-6.1 \mathrm{E}-03 \pm 1.1 \mathrm{E}-02$ & $\mathrm{U}$ & & ${ }^{113} \mathrm{Sn}$ & $-1.1 \mathrm{E}-02 \pm 1.1 \mathrm{E}-02$ & $\mathrm{U}$ \\
\hline & ${ }^{90} \mathrm{Sr}$ & $-8.4 \mathrm{E}-01 \pm 8.4 \mathrm{E}-01$ & $\mathrm{U}$ & & ${ }^{90} \mathrm{Sr}$ & $-7.1 \mathrm{E}-01 \pm 7.1 \mathrm{E}-01$ & $\mathrm{U}$ \\
\hline & ${ }^{234} \mathrm{U}$ & $2.4 \mathrm{E}-01 \pm 8.2 \mathrm{E}-02$ & & & ${ }^{234} \mathrm{U}$ & $1.4 \mathrm{E}-01 \pm 5.2 \mathrm{E}-02$ & \\
\hline & ${ }^{235} \mathrm{U}$ & $5.6 \mathrm{E}-03 \pm 1.4 \mathrm{E}-02$ & $\mathrm{U}$ & & ${ }^{235} \mathrm{U}$ & $1.5 \mathrm{E}-02 \pm 1.3 \mathrm{E}-02$ & \\
\hline & ${ }^{238} \mathrm{U}$ & $2.4 \mathrm{E}-01 \pm 7.9 \mathrm{E}-02$ & & & ${ }^{238} \mathrm{U}$ & $1.5 \mathrm{E}-01 \pm 5.4 \mathrm{E}-02$ & \\
\hline & ${ }^{65} \mathrm{Zn}$ & $5.0 \mathrm{E}-02 \pm 2.4 \mathrm{E}-02$ & & & ${ }^{65} \mathrm{Zn}$ & 8.0E-03 $\pm 3.3 \mathrm{E}-02$ & $\mathrm{U}$ \\
\hline
\end{tabular}

$\overline{\mathrm{RQ}}=$ Result Qualifier. $\mathrm{U}=$ The analyte was analyzed for but not detected. 
Table 3-4. 2008 Soil Sampling Results (pCi/g \pm total analytical uncertainty). (Sheet 15 of 24)

\begin{tabular}{|c|c|c|c|c|c|c|c|}
\hline Location & Isotope & Result \pm Error & $\mathbf{R Q}^{*}$ & Location & Isotope & Result \pm Error & $\mathbf{R Q}^{*}$ \\
\hline D080 & ${ }^{144} \mathrm{Ce}$ & $2.7 \mathrm{E}-02 \pm 1.7 \mathrm{E}-01$ & $\mathrm{U}$ & D112 & ${ }^{144} \mathrm{Ce}$ & $1.5 \mathrm{E}-02 \pm 1.0 \mathrm{E}-01$ & $\mathrm{U}$ \\
\hline \multirow[t]{17}{*}{ (200-E) } & ${ }^{60} \mathrm{Co}$ & $-1.1 \mathrm{E}-03 \pm 7.1 \mathrm{E}-03$ & $\mathrm{U}$ & (Replicate & ${ }^{60} \mathrm{Co}$ & $-2.8 \mathrm{E}-03 \pm 5.1 \mathrm{E}-03$ & $\mathrm{U}$ \\
\hline & ${ }^{134} \mathrm{Cs}$ & $4.3 \mathrm{E}-02 \pm 1.8 \mathrm{E}-02$ & & of D072 & ${ }^{134} \mathrm{Cs}$ & $2.5 \mathrm{E}-02 \pm 1.0 \mathrm{E}-02$ & \\
\hline & ${ }^{137} \mathrm{Cs}$ & 3.5E-01 $\pm 6.3 \mathrm{E}-02$ & & 200-E) & ${ }^{137} \mathrm{Cs}$ & $8.2 \mathrm{E}-02 \pm 1.8 \mathrm{E}-02$ & \\
\hline & ${ }^{152} \mathrm{Eu}$ & $5.8 \mathrm{E}-03 \pm 4.0 \mathrm{E}-02$ & $\mathrm{U}$ & & ${ }^{152} \mathrm{Eu}$ & $1.6 \mathrm{E}-02 \pm 1.8 \mathrm{E}-02$ & $\mathrm{U}$ \\
\hline & ${ }^{154} \mathrm{Eu}$ & $-1.0 \mathrm{E}-02 \pm 2.4 \mathrm{E}-02$ & $\mathrm{U}$ & & ${ }^{154} \mathrm{Eu}$ & $-1.7 \mathrm{E}-03 \pm 1.7 \mathrm{E}-02$ & $\mathrm{U}$ \\
\hline & ${ }^{155} \mathrm{Eu}$ & $4.9 \mathrm{E}-02 \pm 4.3 \mathrm{E}-02$ & $\mathrm{U}$ & & ${ }^{155} \mathrm{Eu}$ & $7.1 \mathrm{E}-02 \pm 4.2 \mathrm{E}-02$ & \\
\hline & ${ }^{238} \mathrm{Pu}$ & $8.0 \mathrm{E}-03 \pm 2.6 \mathrm{E}-02$ & $\mathrm{U}$ & & ${ }^{238} \mathrm{Pu}$ & $2.2 \mathrm{E}-03 \pm 2.1 \mathrm{E}-02$ & $\mathrm{U}$ \\
\hline & ${ }^{239 / 240} \mathrm{Pu}$ & $2.6 \mathrm{E}-02 \pm 1.8 \mathrm{E}-02$ & & & ${ }^{239 / 240} \mathrm{Pu}$ & $4.4 \mathrm{E}-03 \pm 6.3 \mathrm{E}-03$ & $\mathrm{U}$ \\
\hline & ${ }^{103} \mathrm{Ru}$ & $-4.5 \mathrm{E}-03 \pm 9.8 \mathrm{E}-03$ & $\mathrm{U}$ & & ${ }^{103} \mathrm{Ru}$ & $-3.4 \mathrm{E}-03 \pm 6.5 \mathrm{E}-03$ & $\mathrm{U}$ \\
\hline & ${ }^{106} \mathrm{Ru}$ & $-3.6 \mathrm{E}-02 \pm 7.1 \mathrm{E}-02$ & $\mathrm{U}$ & & ${ }^{106} \mathrm{Ru}$ & $2.9 \mathrm{E}-02 \pm 5.1 \mathrm{E}-02$ & $\mathrm{U}$ \\
\hline & ${ }^{125} \mathrm{Sb}$ & $1.9 \mathrm{E}-02 \pm 2.2 \mathrm{E}-02$ & $\mathrm{U}$ & & ${ }^{125} \mathrm{Sb}$ & $4.5 \mathrm{E}-03 \pm 1.5 \mathrm{E}-02$ & $\mathrm{U}$ \\
\hline & ${ }^{113} \mathrm{Sn}$ & $-4.8 \mathrm{E}-03 \pm 1.1 \mathrm{E}-02$ & $\mathrm{U}$ & & ${ }^{113} \mathrm{Sn}$ & $-2.0 \mathrm{E}-03 \pm 7.6 \mathrm{E}-03$ & $\mathrm{U}$ \\
\hline & ${ }^{90} \mathrm{Sr}$ & $-9.4 \mathrm{E}-01 \pm 9.4 \mathrm{E}-01$ & $\mathrm{U}$ & & ${ }^{90} \mathrm{Sr}$ & $9.0 \mathrm{E}-02 \pm 4.4 \mathrm{E}-01$ & $\mathrm{U}$ \\
\hline & ${ }^{234} \mathrm{U}$ & $2.2 \mathrm{E}-01 \pm 7.5 \mathrm{E}-02$ & & & ${ }^{234} \mathrm{U}$ & $1.1 \mathrm{E}-01 \pm 4.2 \mathrm{E}-02$ & \\
\hline & ${ }^{235} \mathrm{U}$ & $1.4 \mathrm{E}-02 \pm 1.5 \mathrm{E}-02$ & $\mathrm{U}$ & & ${ }^{235} \mathrm{U}$ & $1.1 \mathrm{E}-02 \pm 1.0 \mathrm{E}-02$ & \\
\hline & ${ }^{238} \mathrm{U}$ & $2.0 \mathrm{E}-01 \pm 6.8 \mathrm{E}-02$ & & & ${ }^{238} \mathrm{U}$ & $1.3 \mathrm{E}-01 \pm 4.7 \mathrm{E}-02$ & \\
\hline & ${ }^{65} \mathrm{Zn}$ & $2.3 \mathrm{E}-03 \pm 1.9 \mathrm{E}-02$ & $\mathrm{U}$ & & ${ }^{65} \mathrm{Zn}$ & $-2.2 \mathrm{E}-03 \pm 1.4 \mathrm{E}-02$ & $\mathrm{U}$ \\
\hline \multirow{18}{*}{$\begin{array}{c}\text { D082 } \\
\text { (600 Area) }\end{array}$} & ${ }^{144} \mathrm{Ce}$ & $6.5 \mathrm{E}-02 \pm 1.4 \mathrm{E}-01$ & $\mathrm{U}$ & D084 & ${ }^{144} \mathrm{Ce}$ & $-6.3 \mathrm{E}-02 \pm 1.4 \mathrm{E}-01$ & $\mathrm{U}$ \\
\hline & ${ }^{60} \mathrm{Co}$ & $-1.8 \mathrm{E}-03 \pm 6.7 \mathrm{E}-03$ & $\mathrm{U}$ & (600 Area) & ${ }^{60} \mathrm{Co}$ & $5.9 \mathrm{E}-04 \pm 5.9 \mathrm{E}-03$ & $\mathrm{U}$ \\
\hline & ${ }^{134} \mathrm{Cs}$ & $2.8 \mathrm{E}-02 \pm 9.5 \mathrm{E}-03$ & & & ${ }^{134} \mathrm{Cs}$ & $3.5 \mathrm{E}-02 \pm 1.3 \mathrm{E}-02$ & \\
\hline & ${ }^{137} \mathrm{Cs}$ & $6.6 \mathrm{E}-02 \pm 1.7 \mathrm{E}-02$ & & & ${ }^{137} \mathrm{Cs}$ & $7.7 \mathrm{E}-01 \pm 1.2 \mathrm{E}-01$ & \\
\hline & ${ }^{152} \mathrm{Eu}$ & $-1.1 \mathrm{E}-02 \pm 2.9 \mathrm{E}-02$ & U & & ${ }^{152} \mathrm{Eu}$ & $-3.6 \mathrm{E}-03 \pm 2.6 \mathrm{E}-02$ & $\mathrm{U}$ \\
\hline & ${ }^{154} \mathrm{Eu}$ & $-6.0 \mathrm{E}-03 \pm 2.4 \mathrm{E}-02$ & $\mathrm{U}$ & & ${ }^{154} \mathrm{Eu}$ & $-2.2 \mathrm{E}-02 \pm 2.4 \mathrm{E}-02$ & $\mathrm{U}$ \\
\hline & ${ }^{155} \mathrm{Eu}$ & $1.9 \mathrm{E}-02 \pm 3.7 \mathrm{E}-02$ & $\mathrm{U}$ & & ${ }^{155} \mathrm{Eu}$ & $3.2 \mathrm{E}-02 \pm 3.6 \mathrm{E}-02$ & $\mathrm{U}$ \\
\hline & ${ }^{238} \mathrm{Pu}$ & $2.6 \mathrm{E}-02 \pm 2.4 \mathrm{E}-02$ & $\mathrm{U}$ & & ${ }^{238} \mathrm{Pu}$ & $9.5 \mathrm{E}-03 \pm 1.6 \mathrm{E}-02$ & $\mathrm{U}$ \\
\hline & ${ }^{239 / 240} \mathrm{Pu}$ & $6.6 \mathrm{E}-03 \pm 1.2 \mathrm{E}-02$ & $\mathrm{U}$ & & ${ }^{239 / 240} \mathrm{Pu}$ & $4.0 \mathrm{E}-02 \pm 2.0 \mathrm{E}-02$ & \\
\hline & ${ }^{103} \mathrm{Ru}$ & $1.3 \mathrm{E}-03 \pm 8.3 \mathrm{E}-03$ & $\mathrm{U}$ & & ${ }^{103} \mathrm{Ru}$ & $1.0 \mathrm{E}-02 \pm 1.0 \mathrm{E}-02$ & $\mathrm{U}$ \\
\hline & ${ }^{106} \mathrm{Ru}$ & $-6.6 \mathrm{E}-02 \pm 6.6 \mathrm{E}-02$ & $\mathrm{U}$ & & ${ }^{106} \mathrm{Ru}$ & $2.2 \mathrm{E}-02 \pm 6.1 \mathrm{E}-02$ & $\mathrm{U}$ \\
\hline & ${ }^{125} \mathrm{Sb}$ & $7.3 \mathrm{E}-03 \pm 1.9 \mathrm{E}-02$ & $\mathrm{U}$ & & ${ }^{125} \mathrm{Sb}$ & 7.7E-03 $\pm 2.1 \mathrm{E}-02$ & $\mathrm{U}$ \\
\hline & ${ }^{113} \mathrm{Sn}$ & $-7.2 \mathrm{E}-03 \pm 1.0 \mathrm{E}-02$ & $\mathrm{U}$ & & ${ }^{113} \mathrm{Sn}$ & $3.0 \mathrm{E}-03 \pm 1.1 \mathrm{E}-02$ & $\mathrm{U}$ \\
\hline & ${ }^{90} \mathrm{Sr}$ & $-6.8 \mathrm{E}-01 \pm 6.8 \mathrm{E}-01$ & $\mathrm{U}$ & & ${ }^{90} \mathrm{Sr}$ & $-3.5 \mathrm{E}-01 \pm 4.1 \mathrm{E}-01$ & $\mathrm{U}$ \\
\hline & ${ }^{234} \mathrm{U}$ & 1.9E-01 \pm 6.3E-02 & & & ${ }^{234} \mathrm{U}$ & $1.6 \mathrm{E}-01 \pm 5.4 \mathrm{E}-02$ & \\
\hline & ${ }^{235} \mathrm{U}$ & $1.6 \mathrm{E}-02 \pm 1.3 \mathrm{E}-02$ & & & ${ }^{235} \mathrm{U}$ & $2.0 \mathrm{E}-02 \pm 1.4 \mathrm{E}-02$ & \\
\hline & ${ }^{238} \mathrm{U}$ & $1.4 \mathrm{E}-01 \pm 5.0 \mathrm{E}-02$ & & & ${ }^{238} \mathrm{U}$ & $1.9 \mathrm{E}-01 \pm 6.3 \mathrm{E}-02$ & \\
\hline & ${ }^{65} \mathrm{Zn}$ & $2.1 \mathrm{E}-02 \pm 1.9 \mathrm{E}-02$ & $\mathrm{U}$ & & ${ }^{65} \mathrm{Zn}$ & $-2.9 \mathrm{E}-03 \pm 1.9 \mathrm{E}-02$ & $\mathrm{U}$ \\
\hline
\end{tabular}

$\overline{\mathrm{RQ}}=$ Result Qualifier. $\mathrm{U}=$ The analyte was analyzed for but not detected. 
Table 3-4. 2008 Soil Sampling Results (pCi/g \pm total analytical uncertainty). (Sheet 16 of 24)

\begin{tabular}{|c|c|c|c|c|c|c|c|}
\hline Location & Isotope & Result \pm Error & $\mathbf{R Q}^{*}$ & Location & Isotope & Result \pm Error & RQ* \\
\hline D086 & ${ }^{144} \mathrm{Ce}$ & $3.4 \mathrm{E}-02 \pm 1.7 \mathrm{E}-01$ & $\mathrm{U}$ & D088 & ${ }^{144} \mathrm{Ce}$ & $8.7 \mathrm{E}-02 \pm 1.4 \mathrm{E}-01$ & $\mathrm{U}$ \\
\hline \multirow[t]{17}{*}{ (600 Area) } & ${ }^{60} \mathrm{Co}$ & $-4.8 \mathrm{E}-03 \pm 8.2 \mathrm{E}-03$ & U & (600 Area) & ${ }^{60} \mathrm{Co}$ & $-1.3 \mathrm{E}-03 \pm 6.1 \mathrm{E}-03$ & U \\
\hline & ${ }^{134} \mathrm{Cs}$ & $3.9 \mathrm{E}-02 \pm 1.3 \mathrm{E}-02$ & & & ${ }^{134} \mathrm{Cs}$ & $3.7 \mathrm{E}-02 \pm 1.2 \mathrm{E}-02$ & \\
\hline & ${ }^{137} \mathrm{Cs}$ & $1.4 \mathrm{E}+00 \pm 2.3 \mathrm{E}-01$ & & & ${ }^{137} \mathrm{Cs}$ & $2.4 \mathrm{E}-01 \pm 4.4 \mathrm{E}-02$ & \\
\hline & ${ }^{152} \mathrm{Eu}$ & $-2.9 \mathrm{E}-03 \pm 2.9 \mathrm{E}-02$ & U & & ${ }^{152} \mathrm{Eu}$ & $-8.4 \mathrm{E}-03 \pm 2.5 \mathrm{E}-02$ & U \\
\hline & ${ }^{154} \mathrm{Eu}$ & $-3.5 \mathrm{E}-02 \pm 3.5 \mathrm{E}-02$ & U & & ${ }^{154} \mathrm{Eu}$ & $-9.2 \mathrm{E}-03 \pm 2.2 \mathrm{E}-02$ & $\mathrm{U}$ \\
\hline & ${ }^{155} \mathrm{Eu}$ & $3.3 \mathrm{E}-02 \pm 4.1 \mathrm{E}-02$ & $\mathrm{U}$ & & ${ }^{155} \mathrm{Eu}$ & $4.1 \mathrm{E}-02 \pm 4.3 \mathrm{E}-02$ & $\mathrm{U}$ \\
\hline & ${ }^{238} \mathrm{Pu}$ & $1.0 \mathrm{E}-02 \pm 1.8 \mathrm{E}-02$ & $\mathrm{U}$ & & ${ }^{238} \mathrm{Pu}$ & $4.2 \mathrm{E}-03 \pm 2.3 \mathrm{E}-02$ & $\mathrm{U}$ \\
\hline & ${ }^{239 / 240} \mathrm{Pu}$ & $2.3 \mathrm{E}-02 \pm 1.7 \mathrm{E}-02$ & & & ${ }^{239} / 240 \mathrm{Pu}$ & $2.1 \mathrm{E}-03 \pm 2.1 \mathrm{E}-03$ & $\mathrm{U}$ \\
\hline & ${ }^{103} \mathrm{Ru}$ & $1.3 \mathrm{E}-02 \pm 1.3 \mathrm{E}-02$ & U & & ${ }^{103} \mathrm{Ru}$ & $-3.7 \mathrm{E}-03 \pm 7.9 \mathrm{E}-03$ & $\mathrm{U}$ \\
\hline & ${ }^{106} \mathrm{Ru}$ & $1.0 \mathrm{E}-03 \pm 1.0 \mathrm{E}-02$ & $\mathrm{U}$ & & ${ }^{106} \mathrm{Ru}$ & $2.5 \mathrm{E}-02 \pm 5.7 \mathrm{E}-02$ & $\mathrm{U}$ \\
\hline & ${ }^{125} \mathrm{Sb}$ & $2.2 \mathrm{E}-02 \pm 2.6 \mathrm{E}-02$ & U & & ${ }^{125} \mathrm{Sb}$ & $-1.4 \mathrm{E}-02 \pm 1.8 \mathrm{E}-02$ & $\mathrm{U}$ \\
\hline & ${ }^{113} \mathrm{Sn}$ & $-1.7 \mathrm{E}-02 \pm 1.7 \mathrm{E}-02$ & $\mathrm{U}$ & & ${ }^{113} \mathrm{Sn}$ & $-8.6 \mathrm{E}-03 \pm 9.1 \mathrm{E}-03$ & $\mathrm{U}$ \\
\hline & ${ }^{90} \mathrm{Sr}$ & $-2.0 \mathrm{E}-01 \pm 3.9 \mathrm{E}-01$ & $\mathrm{U}$ & & ${ }^{90} \mathrm{Sr}$ & $-2.9 \mathrm{E}-01 \pm 4.4 \mathrm{E}-01$ & $\mathrm{U}$ \\
\hline & ${ }^{234} \mathrm{U}$ & $1.6 \mathrm{E}-01 \pm 5.8 \mathrm{E}-02$ & & & ${ }^{234} \mathrm{U}$ & $1.8 \mathrm{E}-01 \pm 6.1 \mathrm{E}-02$ & \\
\hline & ${ }^{235} \mathrm{U}$ & $2.6 \mathrm{E}-02 \pm 1.7 \mathrm{E}-02$ & & & ${ }^{235} \mathrm{U}$ & $1.9 \mathrm{E}-02 \pm 1.5 \mathrm{E}-02$ & \\
\hline & ${ }^{238} \mathrm{U}$ & $1.4 \mathrm{E}-01 \pm 5.2 \mathrm{E}-02$ & & & ${ }^{238} \mathrm{U}$ & $2.1 \mathrm{E}-01 \pm 6.7 \mathrm{E}-02$ & \\
\hline & ${ }^{65} \mathrm{Zn}$ & 4.7E-02 $\pm 2.5 \mathrm{E}-02$ & & & ${ }^{65} \mathrm{Zn}$ & $-7.1 \mathrm{E}-03 \pm 1.6 \mathrm{E}-02$ & $\mathrm{U}$ \\
\hline \multirow{18}{*}{$\begin{array}{c}\text { D090 } \\
\text { (600 Area) }\end{array}$} & ${ }^{144} \mathrm{Ce}$ & $3.0 \mathrm{E}-04 \pm 3.0 \mathrm{E}-03$ & $\mathrm{U}$ & D092 & ${ }^{144} \mathrm{Ce}$ & $5.2 \mathrm{E}-02 \pm 1.7 \mathrm{E}-01$ & $\mathrm{U}$ \\
\hline & ${ }^{60} \mathrm{Co}$ & $-2.6 \mathrm{E}-03 \pm 5.9 \mathrm{E}-03$ & $\mathrm{U}$ & (600 Area) & ${ }^{60} \mathrm{Co}$ & $3.4 \mathrm{E}-03 \pm 9.3 \mathrm{E}-03$ & $\mathrm{U}$ \\
\hline & ${ }^{134} \mathrm{Cs}$ & $3.8 \mathrm{E}-02 \pm 1.2 \mathrm{E}-02$ & & & ${ }^{134} \mathrm{Cs}$ & $5.8 \mathrm{E}-02 \pm 2.1 \mathrm{E}-02$ & \\
\hline & ${ }^{137} \mathrm{Cs}$ & 8.4E-01 $\pm 1.3 \mathrm{E}-01$ & & & ${ }^{137} \mathrm{Cs}$ & $9.9 \mathrm{E}-01 \pm 1.5 \mathrm{E}-01$ & \\
\hline & ${ }^{152} \mathrm{Eu}$ & $-4.8 \mathrm{E}-03 \pm 2.4 \mathrm{E}-02$ & $\mathrm{U}$ & & ${ }^{152} \mathrm{Eu}$ & $-1.3 \mathrm{E}-02 \pm 4.7 \mathrm{E}-02$ & $\mathrm{U}$ \\
\hline & ${ }^{154} \mathrm{Eu}$ & $-2.3 \mathrm{E}-02 \pm 2.3 \mathrm{E}-02$ & $\mathrm{U}$ & & ${ }^{154} \mathrm{Eu}$ & $-2.3 \mathrm{E}-02 \pm 3.1 \mathrm{E}-02$ & $\mathrm{U}$ \\
\hline & ${ }^{155} \mathrm{Eu}$ & $1.2 \mathrm{E}-03 \pm 1.2 \mathrm{E}-02$ & U & & ${ }^{155} \mathrm{Eu}$ & $5.2 \mathrm{E}-02 \pm 3.9 \mathrm{E}-02$ & $\mathrm{U}$ \\
\hline & ${ }^{238} \mathrm{Pu}$ & $1.5 \mathrm{E}-02 \pm 2.4 \mathrm{E}-02$ & $\mathrm{U}$ & & ${ }^{238} \mathrm{Pu}$ & $-1.1 \mathrm{E}-02 \pm 2.5 \mathrm{E}-02$ & $\mathrm{U}$ \\
\hline & ${ }^{239 / 240} \mathrm{Pu}$ & $2.6 \mathrm{E}-02 \pm 1.7 \mathrm{E}-02$ & & & ${ }^{239 / 240} \mathrm{Pu}$ & $1.5 \mathrm{E}-02 \pm 1.5 \mathrm{E}-02$ & $\mathrm{U}$ \\
\hline & ${ }^{103} \mathrm{Ru}$ & $-2.3 \mathrm{E}-03 \pm 8.6 \mathrm{E}-03$ & $\mathrm{U}$ & & ${ }^{103} \mathrm{Ru}$ & $-2.4 \mathrm{E}-03 \pm 1.4 \mathrm{E}-02$ & $\mathrm{U}$ \\
\hline & ${ }^{106} \mathrm{Ru}$ & $6.0 \mathrm{E}-02 \pm 5.8 \mathrm{E}-02$ & U & & ${ }^{106} \mathrm{Ru}$ & $-3.1 \mathrm{E}-02 \pm 9.3 \mathrm{E}-02$ & $\mathrm{U}$ \\
\hline & ${ }^{125} \mathrm{Sb}$ & $5.6 \mathrm{E}-04 \pm 5.6 \mathrm{E}-03$ & U & & ${ }^{125} \mathrm{Sb}$ & $1.9 \mathrm{E}-02 \pm 3.1 \mathrm{E}-02$ & $\mathrm{U}$ \\
\hline & ${ }^{113} \mathrm{Sn}$ & $-6.0 \mathrm{E}-03 \pm 9.8 \mathrm{E}-03$ & $\mathrm{U}$ & & ${ }^{113} \mathrm{Sn}$ & $-8.1 \mathrm{E}-03 \pm 1.5 \mathrm{E}-02$ & $\mathrm{U}$ \\
\hline & ${ }^{90} \mathrm{Sr}$ & $-2.9 \mathrm{E}-01 \pm 4.5 \mathrm{E}-01$ & U & & ${ }^{90} \mathrm{Sr}$ & $-2.8 \mathrm{E}-01 \pm 4.2 \mathrm{E}-01$ & $\mathrm{U}$ \\
\hline & ${ }^{234} \mathrm{U}$ & $2.2 \mathrm{E}-01 \pm 7.0 \mathrm{E}-02$ & & & ${ }^{234} \mathrm{U}$ & $1.9 \mathrm{E}-01 \pm 6.1 \mathrm{E}-02$ & \\
\hline & ${ }^{235} \mathrm{U}$ & $1.3 \mathrm{E}-02 \pm 1.1 \mathrm{E}-02$ & & & ${ }^{235} \mathrm{U}$ & $2.7 \mathrm{E}-02 \pm 1.6 \mathrm{E}-02$ & \\
\hline & ${ }^{238} \mathrm{U}$ & $1.7 \mathrm{E}-01 \pm 5.8 \mathrm{E}-02$ & & & ${ }^{238} \mathrm{U}$ & $1.8 \mathrm{E}-01 \pm 5.8 \mathrm{E}-02$ & \\
\hline & ${ }^{65} \mathrm{Zn}$ & $-1.6 \mathrm{E}-02 \pm 1.8 \mathrm{E}-02$ & $\mathrm{U}$ & & ${ }^{65} \mathrm{Zn}$ & $-4.0 \mathrm{E}-03 \pm 2.6 \mathrm{E}-02$ & $\mathrm{U}$ \\
\hline
\end{tabular}

$\overline{\mathrm{RQ}}=$ Result Qualifier. $\mathrm{U}=$ The analyte was analyzed for but not detected. 
Table 3-4. 2008 Soil Sampling Results (pCi/g \pm total analytical uncertainty). (Sheet 17 of 24)

\begin{tabular}{|c|c|c|c|c|c|c|c|}
\hline Location & Isotope & Result \pm Error & $\mathbf{R Q}^{*}$ & Location & Isotope & Result \pm Error & RQ* \\
\hline D094 & ${ }^{144} \mathrm{Ce}$ & $-1.7 \mathrm{E}-01 \pm 1.7 \mathrm{E}-01$ & $\mathrm{U}$ & D098 & ${ }^{144} \mathrm{Ce}$ & $-1.1 \mathrm{E}-02 \pm 1.1 \mathrm{E}-01$ & $\bar{U}$ \\
\hline \multirow[t]{17}{*}{ (600 Area) } & ${ }^{60} \mathrm{Co}$ & $-5.0 \mathrm{E}-03 \pm 7.3 \mathrm{E}-03$ & $\mathrm{U}$ & (600 Area) & ${ }^{60} \mathrm{Co}$ & $-5.9 \mathrm{E}-03 \pm 7.5 \mathrm{E}-03$ & $\mathrm{U}$ \\
\hline & ${ }^{134} \mathrm{Cs}$ & $2.6 \mathrm{E}-02 \pm 1.2 \mathrm{E}-02$ & & & ${ }^{134} \mathrm{Cs}$ & $4.8 \mathrm{E}-02 \pm 1.5 \mathrm{E}-02$ & \\
\hline & ${ }^{137} \mathrm{Cs}$ & $1.7 \mathrm{E}+00 \pm 2.7 \mathrm{E}-01$ & & & ${ }^{137} \mathrm{Cs}$ & 2.7E-01 \pm 4.7E-02 & \\
\hline & ${ }^{152} \mathrm{Eu}$ & $-1.3 \mathrm{E}-03 \pm 1.3 \mathrm{E}-02$ & $\mathrm{U}$ & & ${ }^{152} \mathrm{Eu}$ & $-1.7 \mathrm{E}-02 \pm 2.2 \mathrm{E}-02$ & $\mathrm{U}$ \\
\hline & ${ }^{154} \mathrm{Eu}$ & $-7.3 \mathrm{E}-03 \pm 2.0 \mathrm{E}-02$ & $\mathrm{U}$ & & ${ }^{154} \mathrm{Eu}$ & $-1.7 \mathrm{E}-03 \pm 1.7 \mathrm{E}-02$ & $\mathrm{U}$ \\
\hline & ${ }^{155} \mathrm{Eu}$ & $4.5 \mathrm{E}-02 \pm 4.0 \mathrm{E}-02$ & $\mathrm{U}$ & & ${ }^{155} \mathrm{Eu}$ & $5.4 \mathrm{E}-02 \pm 3.9 \mathrm{E}-02$ & \\
\hline & ${ }^{238} \mathrm{Pu}$ & $8.2 \mathrm{E}-03 \pm 1.1 \mathrm{E}-02$ & $\mathrm{U}$ & & ${ }^{238} \mathrm{Pu}$ & $7.8 \mathrm{E}-03 \pm 2.1 \mathrm{E}-02$ & $\mathrm{U}$ \\
\hline & ${ }^{239 / 240} \mathrm{Pu}$ & $3.1 \mathrm{E}-02 \pm 1.6 \mathrm{E}-02$ & & & ${ }^{239 / 240} \mathrm{Pu}$ & $7.8 \mathrm{E}-03 \pm 1.1 \mathrm{E}-02$ & $\mathrm{U}$ \\
\hline & ${ }^{103} \mathrm{Ru}$ & $-6.9 \mathrm{E}-03 \pm 9.6 \mathrm{E}-03$ & $\mathrm{U}$ & & ${ }^{103} \mathrm{Ru}$ & $6.9 \mathrm{E}-04 \pm 6.9 \mathrm{E}-03$ & $\mathrm{U}$ \\
\hline & ${ }^{106} \mathrm{Ru}$ & $-3.1 \mathrm{E}-02 \pm 6.1 \mathrm{E}-02$ & $\mathrm{U}$ & & ${ }^{106} \mathrm{Ru}$ & $1.1 \mathrm{E}-02 \pm 6.6 \mathrm{E}-02$ & U \\
\hline & ${ }^{125} \mathrm{Sb}$ & $-6.5 \mathrm{E}-03 \pm 2.2 \mathrm{E}-02$ & $\mathrm{U}$ & & ${ }^{125} \mathrm{Sb}$ & $-2.4 \mathrm{E}-03 \pm 1.9 \mathrm{E}-02$ & $\mathrm{U}$ \\
\hline & ${ }^{113} \mathrm{Sn}$ & $1.1 \mathrm{E}-03 \pm 1.1 \mathrm{E}-02$ & $\mathrm{U}$ & & ${ }^{113} \mathrm{Sn}$ & $-2.5 \mathrm{E}-04 \pm 2.5 \mathrm{E}-03$ & U \\
\hline & ${ }^{90} \mathrm{Sr}$ & $1.2 \mathrm{E}+00 \pm 5.0 \mathrm{E}-01$ & & & ${ }^{90} \mathrm{Sr}$ & $-4.6 \mathrm{E}-01 \pm 4.6 \mathrm{E}-01$ & $\mathrm{U}$ \\
\hline & ${ }^{234} \mathrm{U}$ & $2.0 \mathrm{E}-01 \pm 6.6 \mathrm{E}-02$ & & & ${ }^{234} \mathrm{U}$ & $1.8 \mathrm{E}-01 \pm 6.1 \mathrm{E}-02$ & \\
\hline & ${ }^{235} \mathrm{U}$ & $1.3 \mathrm{E}-02 \pm 1.4 \mathrm{E}-02$ & $\mathrm{U}$ & & ${ }^{235} \mathrm{U}$ & $2.0 \mathrm{E}-02 \pm 1.5 \mathrm{E}-02$ & \\
\hline & ${ }^{238} \mathrm{U}$ & $2.1 \mathrm{E}-01 \pm 6.7 \mathrm{E}-02$ & & & ${ }^{238} \mathrm{U}$ & $1.8 \mathrm{E}-01 \pm 6.1 \mathrm{E}-02$ & \\
\hline & ${ }^{65} \mathrm{Zn}$ & $-8.2 \mathrm{E}-03 \pm 1.8 \mathrm{E}-02$ & $\mathrm{U}$ & & ${ }^{65} \mathrm{Zn}$ & $-2.5 \mathrm{E}-03 \pm 2.0 \mathrm{E}-02$ & $\mathrm{U}$ \\
\hline \multirow{18}{*}{$\begin{array}{c}\text { D100 } \\
\text { (600 Area) }\end{array}$} & ${ }^{144} \mathrm{Ce}$ & $1.1 \mathrm{E}-01 \pm 1.4 \mathrm{E}-01$ & $\mathrm{U}$ & D102 & ${ }^{144} \mathrm{Ce}$ & $4.5 \mathrm{E}-03 \pm 4.5 \mathrm{E}-02$ & $\mathrm{U}$ \\
\hline & ${ }^{60} \mathrm{Co}$ & $-4.1 \mathrm{E}-03 \pm 7.5 \mathrm{E}-03$ & $\mathrm{U}$ & (600 Area) & ${ }^{60} \mathrm{Co}$ & $-7.7 \mathrm{E}-03 \pm 7.7 \mathrm{E}-03$ & $\mathrm{U}$ \\
\hline & ${ }^{134} \mathrm{Cs}$ & $3.9 \mathrm{E}-02 \pm 1.3 \mathrm{E}-02$ & & & ${ }^{134} \mathrm{Cs}$ & $4.6 \mathrm{E}-02 \pm 1.5 \mathrm{E}-02$ & \\
\hline & ${ }^{137} \mathrm{Cs}$ & $2.7 \mathrm{E}-03 \pm 9.1 \mathrm{E}-03$ & $\mathrm{U}$ & & ${ }^{137} \mathrm{Cs}$ & $4.0 \mathrm{E}-01 \pm 6.7 \mathrm{E}-02$ & \\
\hline & ${ }^{152} \mathrm{Eu}$ & $-2.1 \mathrm{E}-02 \pm 2.8 \mathrm{E}-02$ & $\mathrm{U}$ & & ${ }^{152} \mathrm{Eu}$ & $1.6 \mathrm{E}-03 \pm 1.6 \mathrm{E}-02$ & U \\
\hline & ${ }^{154} \mathrm{Eu}$ & $-3.4 \mathrm{E}-02 \pm 3.4 \mathrm{E}-02$ & $\mathrm{U}$ & & ${ }^{154} \mathrm{Eu}$ & $-1.3 \mathrm{E}-02 \pm 2.4 \mathrm{E}-02$ & U \\
\hline & ${ }^{155} \mathrm{Eu}$ & $5.4 \mathrm{E}-02 \pm 4.4 \mathrm{E}-02$ & $\mathrm{U}$ & & ${ }^{155} \mathrm{Eu}$ & $7.3 \mathrm{E}-02 \pm 4.4 \mathrm{E}-02$ & \\
\hline & ${ }^{238} \mathrm{Pu}$ & $1.5 \mathrm{E}-02 \pm 2.0 \mathrm{E}-02$ & $\mathrm{U}$ & & ${ }^{238} \mathrm{Pu}$ & $1.7 \mathrm{E}-03 \pm 1.7 \mathrm{E}-03$ & $\mathrm{U}$ \\
\hline & ${ }^{239 / 240} \mathrm{Pu}$ & $1.8 \mathrm{E}-03 \pm 3.6 \mathrm{E}-03$ & $\mathrm{U}$ & & ${ }^{239 / 240} \mathrm{Pu}$ & $3.3 \mathrm{E}-02 \pm 1.7 \mathrm{E}-02$ & \\
\hline & ${ }^{103} \mathrm{Ru}$ & $-1.4 \mathrm{E}-03 \pm 8.0 \mathrm{E}-03$ & $\mathrm{U}$ & & ${ }^{103} \mathrm{Ru}$ & $2.4 \mathrm{E}-03 \pm 7.8 \mathrm{E}-03$ & $\mathrm{U}$ \\
\hline & ${ }^{106} \mathrm{Ru}$ & $5.2 \mathrm{E}-02 \pm 6.8 \mathrm{E}-02$ & $\mathrm{U}$ & & ${ }^{106} \mathrm{Ru}$ & $-1.2 \mathrm{E}-02 \pm 6.3 \mathrm{E}-02$ & $\mathrm{U}$ \\
\hline & ${ }^{125} \mathrm{Sb}$ & $-7.1 \mathrm{E}-03 \pm 2.0 \mathrm{E}-02$ & $\mathrm{U}$ & & ${ }^{125} \mathrm{Sb}$ & $-1.8 \mathrm{E}-02 \pm 2.0 \mathrm{E}-02$ & $\mathrm{U}$ \\
\hline & ${ }^{113} \mathrm{Sn}$ & $-6.8 \mathrm{E}-03 \pm 9.8 \mathrm{E}-03$ & $\mathrm{U}$ & & ${ }^{113} \mathrm{Sn}$ & $-3.0 \mathrm{E}-03 \pm 9.4 \mathrm{E}-03$ & U \\
\hline & ${ }^{90} \mathrm{Sr}$ & $-1.9 \mathrm{E}-01 \pm 4.0 \mathrm{E}-01$ & $\mathrm{U}$ & & ${ }^{90} \mathrm{Sr}$ & $-3.8 \mathrm{E}-01 \pm 4.2 \mathrm{E}-01$ & $\mathrm{U}$ \\
\hline & ${ }^{234} \mathrm{U}$ & $1.9 \mathrm{E}-01 \pm 6.3 \mathrm{E}-02$ & & & ${ }^{234} \mathrm{U}$ & $1.5 \mathrm{E}-01 \pm 5.3 \mathrm{E}-02$ & \\
\hline & ${ }^{235} \mathrm{U}$ & $1.3 \mathrm{E}-02 \pm 1.4 \mathrm{E}-02$ & $\mathrm{U}$ & & ${ }^{235} \mathrm{U}$ & $1.3 \mathrm{E}-02 \pm 1.3 \mathrm{E}-02$ & $\mathrm{U}$ \\
\hline & ${ }^{238} \mathrm{U}$ & $1.9 \mathrm{E}-01 \pm 6.3 \mathrm{E}-02$ & & & ${ }^{238} \mathrm{U}$ & $1.5 \mathrm{E}-01 \pm 5.3 \mathrm{E}-02$ & \\
\hline & ${ }^{65} \mathrm{Zn}$ & $4.3 \mathrm{E}-02 \pm 2.1 \mathrm{E}-02$ & & & ${ }^{65} \mathrm{Zn}$ & $1.6 \mathrm{E}-02 \pm 2.0 \mathrm{E}-02$ & $\mathrm{U}$ \\
\hline
\end{tabular}

$\overline{\mathrm{RQ}}=$ Result Qualifier. $\mathrm{U}=$ The analyte was analyzed for but not detected. 
Table 3-4. 2008 Soil Sampling Results (pCi/g \pm total analytical uncertainty). (Sheet 18 of 24)

\begin{tabular}{|c|c|c|c|c|c|c|c|}
\hline Location & Isotope & Result \pm Error & $\mathbf{R Q}^{*}$ & Location & Isotope & Result \pm Error & RQ* \\
\hline D104 & ${ }^{144} \mathrm{Ce}$ & $5.5 \mathrm{E}-02 \pm 1.4 \mathrm{E}-01$ & $\mathrm{U}$ & D106 & ${ }^{144} \mathrm{Ce}$ & $9.0 \mathrm{E}-03 \pm 9.0 \mathrm{E}-02$ & $\mathrm{U}$ \\
\hline \multirow[t]{17}{*}{ (600 Area) } & ${ }^{60} \mathrm{Co}$ & $1.9 \mathrm{E}-03 \pm 6.3 \mathrm{E}-03$ & $\mathrm{U}$ & (600 Area) & ${ }^{60} \mathrm{Co}$ & $2.1 \mathrm{E}-03 \pm 6.2 \mathrm{E}-03$ & U \\
\hline & ${ }^{134} \mathrm{Cs}$ & $3.5 \mathrm{E}-02 \pm 1.0 \mathrm{E}-02$ & & & ${ }^{134} \mathrm{Cs}$ & $3.5 \mathrm{E}-02 \pm 9.8 \mathrm{E}-03$ & \\
\hline & ${ }^{137} \mathrm{Cs}$ & $8.2 \mathrm{E}-01 \pm 1.4 \mathrm{E}-01$ & & & ${ }^{137} \mathrm{Cs}$ & $4.0 \mathrm{E}-02 \pm 1.1 \mathrm{E}-02$ & \\
\hline & ${ }^{152} \mathrm{Eu}$ & $-3.1 \mathrm{E}-02 \pm 3.1 \mathrm{E}-02$ & $\mathrm{U}$ & & ${ }^{152} \mathrm{Eu}$ & $-1.2 \mathrm{E}-02 \pm 2.0 \mathrm{E}-02$ & $\mathrm{U}$ \\
\hline & ${ }^{154} \mathrm{Eu}$ & $-4.8 \mathrm{E}-04 \pm 4.8 \mathrm{E}-03$ & $\mathrm{U}$ & & ${ }^{154} \mathrm{Eu}$ & $-7.4 \mathrm{E}-03 \pm 2.4 \mathrm{E}-02$ & $\mathrm{U}$ \\
\hline & ${ }^{155} \mathrm{Eu}$ & $2.5 \mathrm{E}-02 \pm 4.2 \mathrm{E}-02$ & $\mathrm{U}$ & & ${ }^{155} \mathrm{Eu}$ & $6.4 \mathrm{E}-02 \pm 3.4 \mathrm{E}-02$ & \\
\hline & ${ }^{238} \mathrm{Pu}$ & $2.5 \mathrm{E}-02 \pm 1.7 \mathrm{E}-02$ & & & ${ }^{238} \mathrm{Pu}$ & $2.6 \mathrm{E}-02 \pm 2.9 \mathrm{E}-02$ & $\mathrm{U}$ \\
\hline & ${ }^{239 / 240} \mathrm{Pu}$ & $2.2 \mathrm{E}-02 \pm 1.6 \mathrm{E}-02$ & & & ${ }^{239 / 240} \mathrm{Pu}$ & $9.2 \mathrm{E}-02 \pm 3.7 \mathrm{E}-02$ & \\
\hline & ${ }^{103} \mathrm{Ru}$ & $4.4 \mathrm{E}-04 \pm 4.4 \mathrm{E}-03$ & $\mathrm{U}$ & & ${ }^{103} \mathrm{Ru}$ & $-4.3 \mathrm{E}-03 \pm 6.5 \mathrm{E}-03$ & $\mathrm{U}$ \\
\hline & ${ }^{106} \mathrm{Ru}$ & $1.0 \mathrm{E}-02 \pm 5.9 \mathrm{E}-02$ & $\mathrm{U}$ & & ${ }^{106} \mathrm{Ru}$ & $5.3 \mathrm{E}-02 \pm 5.2 \mathrm{E}-02$ & $\mathrm{U}$ \\
\hline & ${ }^{125} \mathrm{Sb}$ & $-9.2 \mathrm{E}-03 \pm 1.9 \mathrm{E}-02$ & $\mathrm{U}$ & & ${ }^{125} \mathrm{Sb}$ & $8.6 \mathrm{E}-03 \pm 1.6 \mathrm{E}-02$ & $\mathrm{U}$ \\
\hline & ${ }^{113} \mathrm{Sn}$ & $1.3 \mathrm{E}-03 \pm 9.5 \mathrm{E}-03$ & $\mathrm{U}$ & & ${ }^{113} \mathrm{Sn}$ & $-2.9 \mathrm{E}-03 \pm 7.9 \mathrm{E}-03$ & $\mathrm{U}$ \\
\hline & ${ }^{90} \mathrm{Sr}$ & $4.3 \mathrm{E}-02 \pm 4.0 \mathrm{E}-01$ & $\mathrm{U}$ & & ${ }^{90} \mathrm{Sr}$ & $8.0 \mathrm{E}-02 \pm 4.0 \mathrm{E}-01$ & $\mathrm{U}$ \\
\hline & ${ }^{234} \mathrm{U}$ & $1.5 \mathrm{E}-01 \pm 5.4 \mathrm{E}-02$ & & & ${ }^{234} \mathrm{U}$ & $2.1 \mathrm{E}-01 \pm 7.1 \mathrm{E}-02$ & \\
\hline & ${ }^{235} \mathrm{U}$ & $1.6 \mathrm{E}-02 \pm 1.3 \mathrm{E}-02$ & & & ${ }^{235} \mathrm{U}$ & $2.0 \mathrm{E}-02 \pm 1.5 \mathrm{E}-02$ & \\
\hline & ${ }^{238} \mathrm{U}$ & $1.3 \mathrm{E}-01 \pm 4.8 \mathrm{E}-02$ & & & ${ }^{238} \mathrm{U}$ & $1.9 \mathrm{E}-01 \pm 6.5 \mathrm{E}-02$ & \\
\hline & ${ }^{65} \mathrm{Zn}$ & $-3.3 \mathrm{E}-03 \pm 1.6 \mathrm{E}-02$ & $\mathrm{U}$ & & ${ }^{65} \mathrm{Zn}$ & $2.9 \mathrm{E}-02 \pm 1.7 \mathrm{E}-02$ & \\
\hline \multirow{18}{*}{$\begin{array}{c}\text { D108 } \\
\text { (600 Area) }\end{array}$} & ${ }^{144} \mathrm{Ce}$ & $4.5 \mathrm{E}-02 \pm 1.4 \mathrm{E}-01$ & $\mathrm{U}$ & D110 & ${ }^{144} \mathrm{Ce}$ & $2.5 \mathrm{E}-02 \pm 1.4 \mathrm{E}-01$ & $\mathrm{U}$ \\
\hline & ${ }^{60} \mathrm{Co}$ & $-2.2 \mathrm{E}-03 \pm 6.2 \mathrm{E}-03$ & $\mathrm{U}$ & (600 Area) & ${ }^{60} \mathrm{Co}$ & $-3.6 \mathrm{E}-03 \pm 6.7 \mathrm{E}-03$ & $\mathrm{U}$ \\
\hline & ${ }^{134} \mathrm{Cs}$ & $3.6 \mathrm{E}-02 \pm 1.6 \mathrm{E}-02$ & & & ${ }^{134} \mathrm{Cs}$ & $3.7 \mathrm{E}-02 \pm 1.5 \mathrm{E}-02$ & \\
\hline & ${ }^{137} \mathrm{Cs}$ & $2.7 \mathrm{E}-01 \pm 4.8 \mathrm{E}-02$ & & & ${ }^{137} \mathrm{Cs}$ & $1.2 \mathrm{E}-01 \pm 2.6 \mathrm{E}-02$ & \\
\hline & ${ }^{152} \mathrm{Eu}$ & $1.6 \mathrm{E}-02 \pm 1.9 \mathrm{E}-02$ & $\mathrm{U}$ & & ${ }^{152} \mathrm{Eu}$ & $1.0 \mathrm{E}-03 \pm 1.0 \mathrm{E}-02$ & $\mathrm{U}$ \\
\hline & ${ }^{154} \mathrm{Eu}$ & $1.1 \mathrm{E}-02 \pm 2.4 \mathrm{E}-02$ & $\mathrm{U}$ & & ${ }^{154} \mathrm{Eu}$ & $-2.6 \mathrm{E}-03 \pm 2.5 \mathrm{E}-02$ & $\mathrm{U}$ \\
\hline & ${ }^{155} \mathrm{Eu}$ & $5.2 \mathrm{E}-02 \pm 4.2 \mathrm{E}-02$ & $\mathrm{U}$ & & ${ }^{155} \mathrm{Eu}$ & $4.6 \mathrm{E}-02 \pm 3.7 \mathrm{E}-02$ & $\mathrm{U}$ \\
\hline & ${ }^{238} \mathrm{Pu}$ & $3.6 \mathrm{E}-03 \pm 1.1 \mathrm{E}-02$ & $\mathrm{U}$ & & ${ }^{238} \mathrm{Pu}$ & $1.9 \mathrm{E}-02 \pm 2.8 \mathrm{E}-02$ & $\mathrm{U}$ \\
\hline & ${ }^{239 / 240} \mathrm{Pu}$ & $1.4 \mathrm{E}-01 \pm 4.3 \mathrm{E}-02$ & & & ${ }^{239 / 240} \mathrm{Pu}$ & $2.1 \mathrm{E}-03 \pm 2.1 \mathrm{E}-03$ & $\mathrm{U}$ \\
\hline & ${ }^{103} \mathrm{Ru}$ & $3.8 \mathrm{E}-03 \pm 7.5 \mathrm{E}-03$ & $\mathrm{U}$ & & ${ }^{103} \mathrm{Ru}$ & $-1.4 \mathrm{E}-03 \pm 7.7 \mathrm{E}-03$ & $\mathrm{U}$ \\
\hline & ${ }^{106} \mathrm{Ru}$ & $1.8 \mathrm{E}-02 \pm 6.1 \mathrm{E}-02$ & $\mathrm{U}$ & & ${ }^{106} \mathrm{Ru}$ & $-3.8 \mathrm{E}-02 \pm 6.1 \mathrm{E}-02$ & $\mathrm{U}$ \\
\hline & ${ }^{125} \mathrm{Sb}$ & $2.7 \mathrm{E}-03 \pm 1.9 \mathrm{E}-02$ & $\mathrm{U}$ & & ${ }^{125} \mathrm{Sb}$ & $4.8 \mathrm{E}-03 \pm 1.8 \mathrm{E}-02$ & $\mathrm{U}$ \\
\hline & ${ }^{113} \mathrm{Sn}$ & $-5.1 \mathrm{E}-03 \pm 9.2 \mathrm{E}-03$ & $\mathrm{U}$ & & ${ }^{113} \mathrm{Sn}$ & $4.9 \mathrm{E}-04 \pm 4.9 \mathrm{E}-03$ & $\mathrm{U}$ \\
\hline & ${ }^{90} \mathrm{Sr}$ & $-6.8 \mathrm{E}-02 \pm 4.0 \mathrm{E}-01$ & $\mathrm{U}$ & & ${ }^{90} \mathrm{Sr}$ & $7.8 \mathrm{E}-02 \pm 4.1 \mathrm{E}-01$ & $\mathrm{U}$ \\
\hline & ${ }^{234} \mathrm{U}$ & $1.4 \mathrm{E}-01 \pm 5.0 \mathrm{E}-02$ & & & ${ }^{234} \mathrm{U}$ & $2.7 \mathrm{E}-01 \pm 8.4 \mathrm{E}-02$ & \\
\hline & ${ }^{235} \mathrm{U}$ & $1.6 \mathrm{E}-02 \pm 1.4 \mathrm{E}-02$ & $\mathrm{U}$ & & ${ }^{235} \mathrm{U}$ & $1.8 \mathrm{E}-02 \pm 1.6 \mathrm{E}-02$ & $\mathrm{U}$ \\
\hline & ${ }^{238} \mathrm{U}$ & $1.1 \mathrm{E}-01 \pm 4.2 \mathrm{E}-02$ & & & ${ }^{238} \mathrm{U}$ & $2.6 \mathrm{E}-01 \pm 8.1 \mathrm{E}-02$ & \\
\hline & ${ }^{65} \mathrm{Zn}$ & $-6.4 \mathrm{E}-06 \pm 6.4 \mathrm{E}-05$ & $\mathrm{U}$ & & ${ }^{65} \mathrm{Zn}$ & $2.1 \mathrm{E}-02 \pm 1.9 \mathrm{E}-02$ & $\mathrm{U}$ \\
\hline
\end{tabular}

$\overline{\mathrm{RQ}}=$ Result Qualifier. U = The analyte was analyzed for but not detected. 
Table 3-4. 2008 Soil Sampling Results (pCi/g \pm total analytical uncertainty). (Sheet 19 of 24)

\begin{tabular}{|c|c|c|c|}
\hline Location & Isotope & Result \pm Error & $\mathbf{R Q}^{*}$ \\
\hline D174 & ${ }^{241} \mathrm{Am}$ & $2.0 \mathrm{E}-02 \pm 2.4 \mathrm{E}-02$ & $\mathrm{U}$ \\
\hline (100-IU2/6, & ${ }^{144} \mathrm{Ce}$ & $2.4 \mathrm{E}-01 \pm 1.8 \mathrm{E}-01$ & $\mathrm{U}$ \\
\hline 600 Area) & ${ }^{60} \mathrm{Co}$ & $5.0 \mathrm{E}-05 \pm 5.0 \mathrm{E}-04$ & $\mathrm{U}$ \\
\hline \multirow[t]{16}{*}{$2 / 29 / 2008$} & ${ }^{134} \mathrm{Cs}$ & $6.1 \mathrm{E}-02 \pm 2.0 \mathrm{E}-02$ & $\mathrm{U}$ \\
\hline & ${ }^{137} \mathrm{Cs}$ & $3.6 \mathrm{E}-02 \pm 1.6 \mathrm{E}-02$ & \\
\hline & ${ }^{152} \mathrm{Eu}$ & $-8.9 \mathrm{E}-03 \pm 3.3 \mathrm{E}-02$ & $\mathrm{U}$ \\
\hline & ${ }^{154} \mathrm{Eu}$ & $-2.6 \mathrm{E}-02 \pm 3.0 \mathrm{E}-02$ & $\mathrm{U}$ \\
\hline & ${ }^{155} \mathrm{Eu}$ & $5.8 \mathrm{E}-02 \pm 5.0 \mathrm{E}-02$ & $\mathrm{U}$ \\
\hline & ${ }^{238} \mathrm{Pu}$ & $-1.5 \mathrm{E}-02 \pm 3.9 \mathrm{E}-02$ & $\mathrm{U}$ \\
\hline & ${ }^{239 / 240} \mathrm{Pu}$ & $8.5 \mathrm{E}-03 \pm 8.8 \mathrm{E}-03$ & \\
\hline & ${ }^{103} \mathrm{Ru}$ & $6.3 \mathrm{E}-04 \pm 6.3 \mathrm{E}-03$ & $\mathrm{U}$ \\
\hline & ${ }^{106} \mathrm{Ru}$ & $-8.5 \mathrm{E}-03 \pm 8.0 \mathrm{E}-02$ & $\mathrm{U}$ \\
\hline & ${ }^{125} \mathrm{Sb}$ & $4.7 \mathrm{E}-03 \pm 2.2 \mathrm{E}-02$ & $\mathrm{U}$ \\
\hline & ${ }^{113} \mathrm{Sn}$ & $-4.9 \mathrm{E}-03 \pm 1.0 \mathrm{E}-02$ & $\mathrm{U}$ \\
\hline & ${ }^{90} \mathrm{Sr}$ & $-4.0 \mathrm{E}-01 \pm 4.0 \mathrm{E}-01$ & $\mathrm{U}$ \\
\hline & ${ }^{234} \mathrm{U}$ & $2.6 \mathrm{E}-01 \pm 8.3 \mathrm{E}-02$ & \\
\hline & ${ }^{235} \mathrm{U}$ & $3.9 \mathrm{E}-02 \pm 2.3 \mathrm{E}-02$ & \\
\hline & ${ }^{238} \mathrm{U}$ & $2.0 \mathrm{E}-01 \pm 6.8 \mathrm{E}-02$ & \\
\hline & ${ }^{65} \mathrm{Zn}$ & $4.6 \mathrm{E}-03 \pm 2.4 \mathrm{E}-02$ & $\mathrm{U}$ \\
\hline \multirow{19}{*}{$\begin{array}{c}\text { D174 } \\
\text { (100-IU2/6, } \\
\text { 600 Area) } \\
\text { 4/30/2008 }\end{array}$} & ${ }^{241} \mathrm{Am}$ & $1.6 \mathrm{E}-02 \pm 1.3 \mathrm{E}-02$ & $\mathrm{U}$ \\
\hline & ${ }^{144} \mathrm{Ce}$ & $1.3 \mathrm{E}+01 \pm 8.7 \mathrm{E}+01$ & $\mathrm{U}$ \\
\hline & ${ }^{60} \mathrm{Co}$ & $-1.3 \mathrm{E}+00 \pm 4.1 \mathrm{E}+00$ & $\mathrm{U}$ \\
\hline & ${ }^{134} \mathrm{Cs}$ & $2.9 \mathrm{E}+01 \pm 9.9 \mathrm{E}+00$ & \\
\hline & ${ }^{137} \mathrm{Cs}$ & $9.4 \mathrm{E}+01 \pm 1.7 \mathrm{E}+01$ & \\
\hline & ${ }^{152} \mathrm{Eu}$ & $-7.2 \mathrm{E}+00 \pm 1.6 \mathrm{E}+01$ & $\mathrm{U}$ \\
\hline & ${ }^{154} \mathrm{Eu}$ & $-1.2 \mathrm{E}+01 \pm 1.3 \mathrm{E}+01$ & $\mathrm{U}$ \\
\hline & ${ }^{155} \mathrm{Eu}$ & $1.4 \mathrm{E}+01 \pm 2.1 \mathrm{E}+01$ & $\mathrm{U}$ \\
\hline & ${ }^{238} \mathrm{Pu}$ & $2.2 \mathrm{E}-03 \pm 2.2 \mathrm{E}-02$ & $\mathrm{U}$ \\
\hline & ${ }^{239 / 240} \mathrm{Pu}$ & $8.7 \mathrm{E}-03 \pm 1.1 \mathrm{E}-02$ & $\mathrm{U}$ \\
\hline & ${ }^{103} \mathrm{Ru}$ & $1.8 \mathrm{E}+00 \pm 4.4 \mathrm{E}+00$ & $\mathrm{U}$ \\
\hline & ${ }^{106} \mathrm{Ru}$ & $1.7 \mathrm{E}+01 \pm 3.6 \mathrm{E}+01$ & $\mathrm{U}$ \\
\hline & ${ }^{125} \mathrm{Sb}$ & $-3.9 \mathrm{E}+00 \pm 1.2 \mathrm{E}+01$ & $\mathrm{U}$ \\
\hline & ${ }^{113} \mathrm{Sn}$ & $-3.4 \mathrm{E}+00 \pm 5.8 \mathrm{E}+00$ & $\mathrm{U}$ \\
\hline & ${ }^{90} \mathrm{Sr}$ & $-9.5 \mathrm{E}-02 \pm 4.3 \mathrm{E}-01$ & $\mathrm{U}$ \\
\hline & ${ }^{234} \mathrm{U}$ & $2.7 \mathrm{E}-01 \pm 8.6 \mathrm{E}-02$ & \\
\hline & ${ }^{235} \mathrm{U}$ & $1.9 \mathrm{E}-02 \pm 1.7 \mathrm{E}-02$ & $\mathrm{U}$ \\
\hline & ${ }^{238} \mathrm{U}$ & $2.4 \mathrm{E}-01 \pm 7.9 \mathrm{E}-02$ & \\
\hline & ${ }^{65} \mathrm{Zn}$ & $7.6 \mathrm{E}+00 \pm 1.2 \mathrm{E}+01$ & $\mathrm{U}$ \\
\hline
\end{tabular}

\begin{tabular}{|c|c|c|c|}
\hline Location & Isotope & Result \pm Error & RQ* \\
\hline D174 & ${ }^{241} \mathrm{Am}$ & $3.8 \mathrm{E}-02 \pm 3.5 \mathrm{E}-02$ & $\mathrm{U}$ \\
\hline (100-IU2/6, & ${ }^{144} \mathrm{Ce}$ & $1.4 \mathrm{E}-01 \pm 1.4 \mathrm{E}-01$ & $\mathrm{U}$ \\
\hline 600 Area) & ${ }^{60} \mathrm{Co}$ & $-5.7 \mathrm{E}-03 \pm 7.9 \mathrm{E}-03$ & $\mathrm{U}$ \\
\hline \multirow[t]{16}{*}{$3 / 28 / 2008$} & ${ }^{134} \mathrm{Cs}$ & $4.4 \mathrm{E}-02 \pm 1.8 \mathrm{E}-02$ & \\
\hline & ${ }^{137} \mathrm{Cs}$ & $1.5 \mathrm{E}-02 \pm 1.2 \mathrm{E}-02$ & \\
\hline & ${ }^{152} \mathrm{Eu}$ & $-4.6 \mathrm{E}-03 \pm 2.7 \mathrm{E}-02$ & $\mathrm{U}$ \\
\hline & ${ }^{154} \mathrm{Eu}$ & $-2.7 \mathrm{E}-02 \pm 2.7 \mathrm{E}-02$ & $\mathrm{U}$ \\
\hline & ${ }^{155} \mathrm{Eu}$ & 4.3E-02 \pm 4.2E-02 & $\mathrm{U}$ \\
\hline & ${ }^{238} \mathrm{Pu}$ & $-3.3 \mathrm{E}-02 \pm 4.2 \mathrm{E}-02$ & $\mathrm{U}$ \\
\hline & ${ }^{239 / 240} \mathrm{Pu}$ & $1.0 \mathrm{E}-02 \pm 1.0 \mathrm{E}-02$ & \\
\hline & ${ }^{103} \mathrm{Ru}$ & $-1.1 \mathrm{E}-03 \pm 8.2 \mathrm{E}-03$ & $\mathrm{U}$ \\
\hline & ${ }^{106} \mathrm{Ru}$ & $1.4 \mathrm{E}-02 \pm 6.8 \mathrm{E}-02$ & $\mathrm{U}$ \\
\hline & ${ }^{125} \mathrm{Sb}$ & $2.6 \mathrm{E}-03 \pm 2.0 \mathrm{E}-02$ & $\mathrm{U}$ \\
\hline & ${ }^{113} \mathrm{Sn}$ & $-3.2 \mathrm{E}-03 \pm 9.5 \mathrm{E}-03$ & $\mathrm{U}$ \\
\hline & ${ }^{90} \mathrm{Sr}$ & $-5.0 \mathrm{E}-01 \pm 5.0 \mathrm{E}-01$ & $\mathrm{U}$ \\
\hline & ${ }^{234} \mathrm{U}$ & 2.7E-01 $\pm 8.9 \mathrm{E}-02$ & \\
\hline & ${ }^{235} \mathrm{U}$ & $3.3 \mathrm{E}-02 \pm 2.2 \mathrm{E}-02$ & \\
\hline & ${ }^{238} \mathrm{U}$ & $2.6 \mathrm{E}-01 \pm 8.8 \mathrm{E}-02$ & \\
\hline & ${ }^{65} \mathrm{Zn}$ & $3.9 \mathrm{E}-02 \pm 2.2 \mathrm{E}-02$ & \\
\hline \multirow{18}{*}{$\begin{array}{c}\text { D114 } \\
\text { (Replicate } \\
\text { of D092 } \\
600 \text { Area) }\end{array}$} & ${ }^{144} \mathrm{Ce}$ & $5.6 \mathrm{E}-03 \pm 5.6 \mathrm{E}-02$ & $\mathrm{U}$ \\
\hline & ${ }^{60} \mathrm{Co}$ & $-4.0 \mathrm{E}-03 \pm 7.7 \mathrm{E}-03$ & $\mathrm{U}$ \\
\hline & ${ }^{134} \mathrm{Cs}$ & $3.4 \mathrm{E}-02 \pm 1.1 \mathrm{E}-02$ & \\
\hline & ${ }^{137} \mathrm{Cs}$ & $1.7 \mathrm{E}+00 \pm 2.9 \mathrm{E}-01$ & \\
\hline & ${ }^{152} \mathrm{Eu}$ & $-2.2 \mathrm{E}-03 \pm 2.2 \mathrm{E}-02$ & $\mathrm{U}$ \\
\hline & ${ }^{154} \mathrm{Eu}$ & $-5.1 \mathrm{E}-03 \pm 2.5 \mathrm{E}-02$ & $\mathrm{U}$ \\
\hline & ${ }^{155} \mathrm{Eu}$ & $5.8 \mathrm{E}-02 \pm 4.4 \mathrm{E}-02$ & $\mathrm{U}$ \\
\hline & ${ }^{238} \mathrm{Pu}$ & $2.5 \mathrm{E}-02 \pm 3.0 \mathrm{E}-02$ & $\mathrm{U}$ \\
\hline & ${ }^{239 / 240} \mathrm{Pu}$ & $5.4 \mathrm{E}-02 \pm 2.6 \mathrm{E}-02$ & \\
\hline & ${ }^{103} \mathrm{Ru}$ & $-9.5 \mathrm{E}-03 \pm 9.7 \mathrm{E}-03$ & $\mathrm{U}$ \\
\hline & ${ }^{106} \mathrm{Ru}$ & $-1.3 \mathrm{E}-02 \pm 7.5 \mathrm{E}-02$ & $\mathrm{U}$ \\
\hline & ${ }^{125} \mathrm{Sb}$ & $2.9 \mathrm{E}-03 \pm 2.4 \mathrm{E}-02$ & $\mathrm{U}$ \\
\hline & ${ }^{113} \mathrm{Sn}$ & $-8.0 \mathrm{E}-03 \pm 1.1 \mathrm{E}-02$ & $\mathrm{U}$ \\
\hline & ${ }^{90} \mathrm{Sr}$ & $-5.5 \mathrm{E}-01 \pm 5.5 \mathrm{E}-01$ & $\mathrm{U}$ \\
\hline & ${ }^{234} \mathrm{U}$ & $2.3 \mathrm{E}-01 \pm 7.6 \mathrm{E}-02$ & \\
\hline & ${ }^{235} \mathrm{U}$ & $1.3 \mathrm{E}-02 \pm 1.2 \mathrm{E}-02$ & \\
\hline & ${ }^{238} \mathrm{U}$ & $2.0 \mathrm{E}-01 \pm 6.8 \mathrm{E}-02$ & \\
\hline & ${ }^{65} \mathrm{Zn}$ & $4.1 \mathrm{E}-02 \pm 2.2 \mathrm{E}-02$ & \\
\hline
\end{tabular}

$\mathrm{RQ}=$ Result Qualifier. $\mathrm{U}=$ The analyte was analyzed for but not detected. 
Table 3-4. 2008 Soil Sampling Results (pCi/g \pm total analytical uncertainty). (Sheet 20 of 24)

\begin{tabular}{|c|c|c|c|c|c|c|c|}
\hline Location & Isotope & Result \pm Error & RQ* & Location & Isotope & Result \pm Error & RQ* \\
\hline D116 & ${ }^{144} \mathrm{Ce}$ & $4.3 \mathrm{E}-02 \pm 1.1 \mathrm{E}-01$ & $\mathrm{U}$ & D117 & ${ }^{144} \mathrm{Ce}$ & $-5.8 \mathrm{E}-02 \pm 1.1 \mathrm{E}-01$ & $\mathrm{U}$ \\
\hline \multirow[t]{17}{*}{ (300 Area) } & ${ }^{60} \mathrm{Co}$ & $-2.0 \mathrm{E}-03 \pm 5.5 \mathrm{E}-03$ & $\mathrm{U}$ & (300 Area) & ${ }^{60} \mathrm{Co}$ & $-3.1 \mathrm{E}-03 \pm 5.8 \mathrm{E}-03$ & $\mathrm{U}$ \\
\hline & ${ }^{134} \mathrm{Cs}$ & $3.0 \mathrm{E}-02 \pm 8.1 \mathrm{E}-03$ & & & ${ }^{134} \mathrm{Cs}$ & $3.2 \mathrm{E}-02 \pm 1.2 \mathrm{E}-02$ & \\
\hline & ${ }^{137} \mathrm{Cs}$ & $-1.5 \mathrm{E}-03 \pm 5.8 \mathrm{E}-03$ & $\mathrm{U}$ & & ${ }^{137} \mathrm{Cs}$ & $1.4 \mathrm{E}-02 \pm 8.4 \mathrm{E}-03$ & \\
\hline & ${ }^{152} \mathrm{Eu}$ & $7.8 \mathrm{E}-03 \pm 1.9 \mathrm{E}-02$ & $\mathrm{U}$ & & ${ }^{152} \mathrm{Eu}$ & $-1.7 \mathrm{E}-03 \pm 1.7 \mathrm{E}-02$ & $\mathrm{U}$ \\
\hline & ${ }^{154} \mathrm{Eu}$ & $-1.7 \mathrm{E}-02 \pm 1.7 \mathrm{E}-02$ & $\mathrm{U}$ & & ${ }^{154} \mathrm{Eu}$ & $-6.5 \mathrm{E}-03 \pm 1.9 \mathrm{E}-02$ & $\mathrm{U}$ \\
\hline & ${ }^{155} \mathrm{Eu}$ & $2.3 \mathrm{E}-02 \pm 2.8 \mathrm{E}-02$ & $\mathrm{U}$ & & ${ }^{155} \mathrm{Eu}$ & $5.8 \mathrm{E}-03 \pm 2.8 \mathrm{E}-02$ & $\mathrm{U}$ \\
\hline & ${ }^{238} \mathrm{Pu}$ & $-2.7 \mathrm{E}-02 \pm 4.0 \mathrm{E}-02$ & $\mathrm{U}$ & & ${ }^{238} \mathrm{Pu}$ & $2.4 \mathrm{E}-03 \pm 2.4 \mathrm{E}-02$ & $\mathrm{U}$ \\
\hline & ${ }^{239 / 240} \mathrm{Pu}$ & $5.5 \mathrm{E}-03 \pm 7.9 \mathrm{E}-03$ & $\mathrm{U}$ & & ${ }^{239 / 240} \mathrm{Pu}$ & $4.9 \mathrm{E}-03 \pm 7.1 \mathrm{E}-03$ & $\mathrm{U}$ \\
\hline & ${ }^{103} \mathrm{Ru}$ & $6.4 \mathrm{E}-04 \pm 6.4 \mathrm{E}-03$ & $\mathrm{U}$ & & ${ }^{103} \mathrm{Ru}$ & $5.1 \mathrm{E}-03 \pm 7.0 \mathrm{E}-03$ & $\mathrm{U}$ \\
\hline & ${ }^{106} \mathrm{Ru}$ & $-1.1 \mathrm{E}-02 \pm 4.7 \mathrm{E}-02$ & $\mathrm{U}$ & & ${ }^{106} \mathrm{Ru}$ & $-3.5 \mathrm{E}-02 \pm 5.0 \mathrm{E}-02$ & $\mathrm{U}$ \\
\hline & ${ }^{125} \mathrm{Sb}$ & $4.7 \mathrm{E}-03 \pm 1.6 \mathrm{E}-02$ & $\mathrm{U}$ & & ${ }^{125} \mathrm{Sb}$ & $1.3 \mathrm{E}-02 \pm 1.6 \mathrm{E}-02$ & $\mathrm{U}$ \\
\hline & ${ }^{113} \mathrm{Sn}$ & $-1.7 \mathrm{E}-03 \pm 7.7 \mathrm{E}-03$ & $\mathrm{U}$ & & ${ }^{113} \mathrm{Sn}$ & $-3.0 \mathrm{E}-03 \pm 8.0 \mathrm{E}-03$ & $\mathrm{U}$ \\
\hline & ${ }^{90} \mathrm{Sr}$ & $-3.2 \mathrm{E}-01 \pm 4.4 \mathrm{E}-01$ & $\mathrm{U}$ & & ${ }^{90} \mathrm{Sr}$ & $-5.0 \mathrm{E}-01 \pm 5.0 \mathrm{E}-01$ & $\mathrm{U}$ \\
\hline & ${ }^{234} \mathrm{U}$ & $1.8 \mathrm{E}-01 \pm 6.1 \mathrm{E}-02$ & & & ${ }^{234} U$ & $6.3 \mathrm{E}-01 \pm 1.8 \mathrm{E}-01$ & \\
\hline & ${ }^{235} \mathrm{U}$ & $1.6 \mathrm{E}-02 \pm 1.3 \mathrm{E}-02$ & & & ${ }^{235} \mathrm{U}$ & $3.6 \mathrm{E}-02 \pm 2.2 \mathrm{E}-02$ & \\
\hline & ${ }^{238} \mathrm{U}$ & $2.3 \mathrm{E}-01 \pm 7.4 \mathrm{E}-02$ & & & ${ }^{238} \mathrm{U}$ & $6.6 \mathrm{E}-01 \pm 1.8 \mathrm{E}-01$ & \\
\hline & ${ }^{65} \mathrm{Zn}$ & $-1.6 \mathrm{E}-02 \pm 1.6 \mathrm{E}-02$ & $\mathrm{U}$ & & ${ }^{65} \mathrm{Zn}$ & $-1.1 \mathrm{E}-02 \pm 1.7 \mathrm{E}-02$ & $\mathrm{U}$ \\
\hline \multirow{18}{*}{$\begin{array}{c}\text { D118 } \\
\text { (300 Area) }\end{array}$} & ${ }^{144} \mathrm{Ce}$ & $6.8 \mathrm{E}-02 \pm 1.3 \mathrm{E}-01$ & $\mathrm{U}$ & D119 & ${ }^{144} \mathrm{Ce}$ & $1.6 \mathrm{E}-02 \pm 1.1 \mathrm{E}-01$ & $\mathrm{U}$ \\
\hline & ${ }^{60} \mathrm{Co}$ & $6.0 \mathrm{E}-04 \pm 6.0 \mathrm{E}-03$ & $\mathrm{U}$ & (300 Area) & ${ }^{60} \mathrm{Co}$ & $8.7 \mathrm{E}-04 \pm 6.5 \mathrm{E}-03$ & $\mathrm{U}$ \\
\hline & ${ }^{134} \mathrm{Cs}$ & $2.1 \mathrm{E}-02 \pm 1.2 \mathrm{E}-02$ & & & ${ }^{134} \mathrm{Cs}$ & $1.4 \mathrm{E}-02 \pm 8.7 \mathrm{E}-03$ & \\
\hline & ${ }^{137} \mathrm{Cs}$ & $1.1 \mathrm{E}-02 \pm 9.1 \mathrm{E}-03$ & $\mathrm{U}$ & & ${ }^{137} \mathrm{Cs}$ & 8.3E-03 $\pm 6.7 \mathrm{E}-03$ & $\mathrm{U}$ \\
\hline & ${ }^{152} \mathrm{Eu}$ & $-3.1 \mathrm{E}-02 \pm 3.5 \mathrm{E}-02$ & U & & ${ }^{152} \mathrm{Eu}$ & $8.0 \mathrm{E}-03 \pm 2.2 \mathrm{E}-02$ & $\mathrm{U}$ \\
\hline & ${ }^{154} \mathrm{Eu}$ & $-1.5 \mathrm{E}-03 \pm 1.5 \mathrm{E}-02$ & $\mathrm{U}$ & & ${ }^{154} \mathrm{Eu}$ & $3.3 \mathrm{E}-03 \pm 2.3 \mathrm{E}-02$ & $\mathrm{U}$ \\
\hline & ${ }^{155} \mathrm{Eu}$ & $3.2 \mathrm{E}-02 \pm 3.4 \mathrm{E}-02$ & U & & ${ }^{155} \mathrm{Eu}$ & 5.7E-02 $\pm 3.7 \mathrm{E}-02$ & \\
\hline & ${ }^{238} \mathrm{Pu}$ & $2.5 \mathrm{E}-03 \pm 2.5 \mathrm{E}-02$ & $\mathrm{U}$ & & ${ }^{238} \mathrm{Pu}$ & $-1.5 \mathrm{E}-02 \pm 5.3 \mathrm{E}-02$ & U \\
\hline & ${ }^{239 / 240} \mathrm{Pu}$ & $-4.9 \mathrm{E}-03 \pm 7.1 \mathrm{E}-03$ & $\mathrm{U}$ & & ${ }^{239 / 240} \mathrm{Pu}$ & $1.8 \mathrm{E}-02 \pm 1.5 \mathrm{E}-02$ & \\
\hline & ${ }^{103} \mathrm{Ru}$ & $2.5 \mathrm{E}-03 \pm 7.2 \mathrm{E}-03$ & $\mathrm{U}$ & & ${ }^{103} \mathrm{Ru}$ & $-3.8 \mathrm{E}-03 \pm 6.7 \mathrm{E}-03$ & $\mathrm{U}$ \\
\hline & ${ }^{106} \mathrm{Ru}$ & $-1.3 \mathrm{E}-02 \pm 5.8 \mathrm{E}-02$ & $\mathrm{U}$ & & ${ }^{106} \mathrm{Ru}$ & $-2.4 \mathrm{E}-02 \pm 5.7 \mathrm{E}-02$ & $\mathrm{U}$ \\
\hline & ${ }^{125} \mathrm{Sb}$ & $-8.1 \mathrm{E}-04 \pm 8.1 \mathrm{E}-03$ & $\mathrm{U}$ & & ${ }^{125} \mathrm{Sb}$ & $-5.4 \mathrm{E}-03 \pm 1.7 \mathrm{E}-02$ & $\mathrm{U}$ \\
\hline & ${ }^{113} \mathrm{Sn}$ & $1.8 \mathrm{E}-03 \pm 8.6 \mathrm{E}-03$ & $\mathrm{U}$ & & ${ }^{113} \mathrm{Sn}$ & $-2.8 \mathrm{E}-03 \pm 7.8 \mathrm{E}-03$ & $\mathrm{U}$ \\
\hline & ${ }^{90} \mathrm{Sr}$ & $-1.1 \mathrm{E}-01 \pm 4.4 \mathrm{E}-01$ & $\mathrm{U}$ & & ${ }^{90} \mathrm{Sr}$ & $1.4 \mathrm{E}-01 \pm 4.8 \mathrm{E}-01$ & $\mathrm{U}$ \\
\hline & ${ }^{234} \mathrm{U}$ & $2.2 \mathrm{E}-01 \pm 7.3 \mathrm{E}-02$ & & & ${ }^{234} \mathrm{U}$ & $1.6 \mathrm{E}+00 \pm 4.3 \mathrm{E}-01$ & \\
\hline & ${ }^{235} \mathrm{U}$ & $2.2 \mathrm{E}-02 \pm 1.6 \mathrm{E}-02$ & & & ${ }^{235} \mathrm{U}$ & $5.6 \mathrm{E}-02 \pm 2.7 \mathrm{E}-02$ & \\
\hline & ${ }^{238} \mathrm{U}$ & $2.7 \mathrm{E}-01 \pm 8.6 \mathrm{E}-02$ & & & ${ }^{238} \mathrm{U}$ & $1.6 \mathrm{E}+00 \pm 4.3 \mathrm{E}-01$ & \\
\hline & ${ }^{65} \mathrm{Zn}$ & $-6.0 \mathrm{E}-04 \pm 6.0 \mathrm{E}-03$ & $\mathrm{U}$ & & ${ }^{65} \mathrm{Zn}$ & $3.6 \mathrm{E}-02 \pm 1.8 \mathrm{E}-02$ & \\
\hline
\end{tabular}

$\overline{\mathrm{RQ}}=$ Result Qualifier. $\mathrm{U}=$ The analyte was analyzed for but not detected. 
Table 3-4. 2008 Soil Sampling Results (pCi/g \pm total analytical uncertainty). (Sheet 21 of 24)

\begin{tabular}{|c|c|c|c|c|c|c|c|}
\hline Location & Isotope & Result \pm Error & RQ* & Location & Isotope & Result \pm Error & RQ* \\
\hline D120 & ${ }^{144} \mathrm{Ce}$ & $-6.1 \mathrm{E}-02 \pm 1.0 \mathrm{E}-01$ & $\mathrm{U}$ & D121 & ${ }^{144} \mathrm{Ce}$ & $4.5 \mathrm{E}-02 \pm 1.3 \mathrm{E}-01$ & $\mathrm{U}$ \\
\hline \multirow[t]{17}{*}{ (300 Area) } & ${ }^{60} \mathrm{Co}$ & $-7.6 \mathrm{E}-03 \pm 7.6 \mathrm{E}-03$ & $\mathrm{U}$ & (300 Area) & ${ }^{60} \mathrm{Co}$ & $-1.1 \mathrm{E}-03 \pm 5.9 \mathrm{E}-03$ & $\mathrm{U}$ \\
\hline & ${ }^{134} \mathrm{Cs}$ & $3.3 \mathrm{E}-02 \pm 1.2 \mathrm{E}-02$ & & & ${ }^{134} \mathrm{Cs}$ & $3.4 \mathrm{E}-02 \pm 1.2 \mathrm{E}-02$ & \\
\hline & ${ }^{137} \mathrm{Cs}$ & $1.4 \mathrm{E}-02 \pm 9.8 \mathrm{E}-03$ & & & ${ }^{137} \mathrm{Cs}$ & $1.8 \mathrm{E}-02 \pm 1.0 \mathrm{E}-02$ & \\
\hline & ${ }^{152} \mathrm{Eu}$ & $5.6 \mathrm{E}-03 \pm 2.1 \mathrm{E}-02$ & $\mathrm{U}$ & & ${ }^{152} \mathrm{Eu}$ & $-1.3 \mathrm{E}-02 \pm 2.2 \mathrm{E}-02$ & $\mathrm{U}$ \\
\hline & ${ }^{154} \mathrm{Eu}$ & $-1.5 \mathrm{E}-02 \pm 2.4 \mathrm{E}-02$ & $\mathrm{U}$ & & ${ }^{154} \mathrm{Eu}$ & $6.6 \mathrm{E}-03 \pm 2.1 \mathrm{E}-02$ & $\mathrm{U}$ \\
\hline & ${ }^{155} \mathrm{Eu}$ & $5.8 \mathrm{E}-02 \pm 3.7 \mathrm{E}-02$ & & & ${ }^{155} \mathrm{Eu}$ & $7.6 \mathrm{E}-03 \pm 3.1 \mathrm{E}-02$ & $\mathrm{U}$ \\
\hline & ${ }^{238} \mathrm{Pu}$ & $2.4 \mathrm{E}-02 \pm 3.0 \mathrm{E}-02$ & $\mathrm{U}$ & & ${ }^{238} \mathrm{Pu}$ & $-4.5 \mathrm{E}-03 \pm 2.9 \mathrm{E}-02$ & $\mathrm{U}$ \\
\hline & ${ }^{239 / 240} \mathrm{Pu}$ & $2.3 \mathrm{E}-02 \pm 1.6 \mathrm{E}-02$ & & & ${ }^{239 / 240} \mathrm{Pu}$ & $2.2 \mathrm{E}-03 \pm 2.2 \mathrm{E}-03$ & $\mathrm{U}$ \\
\hline & ${ }^{103} \mathrm{Ru}$ & $7.5 \mathrm{E}-04 \pm 7.4 \mathrm{E}-03$ & $\mathrm{U}$ & & ${ }^{103} \mathrm{Ru}$ & $-2.7 \mathrm{E}-03 \pm 6.6 \mathrm{E}-03$ & $\mathrm{U}$ \\
\hline & ${ }^{106} \mathrm{Ru}$ & $-2.2 \mathrm{E}-02 \pm 5.8 \mathrm{E}-02$ & $\mathrm{U}$ & & ${ }^{106} \mathrm{Ru}$ & $-1.7 \mathrm{E}-02 \pm 5.2 \mathrm{E}-02$ & $\mathrm{U}$ \\
\hline & ${ }^{125} \mathrm{Sb}$ & $1.8 \mathrm{E}-02 \pm 1.8 \mathrm{E}-02$ & $\mathrm{U}$ & & ${ }^{125} \mathrm{Sb}$ & $1.5 \mathrm{E}-03 \pm 1.5 \mathrm{E}-02$ & $\mathrm{U}$ \\
\hline & ${ }^{113} \mathrm{Sn}$ & $1.1 \mathrm{E}-03 \pm 8.6 \mathrm{E}-03$ & $\mathrm{U}$ & & ${ }^{113} \mathrm{Sn}$ & $-5.8 \mathrm{E}-04 \pm 5.8 \mathrm{E}-03$ & $\mathrm{U}$ \\
\hline & ${ }^{90} \mathrm{Sr}$ & $-4.8 \mathrm{E}-01 \pm 4.8 \mathrm{E}-01$ & $\mathrm{U}$ & & ${ }^{90} \mathrm{Sr}$ & $-2.7 \mathrm{E}-01 \pm 3.9 \mathrm{E}-01$ & U \\
\hline & ${ }^{234} \mathrm{U}$ & $3.3 \mathrm{E}-01 \pm 9.9 \mathrm{E}-02$ & & & ${ }^{234} \mathrm{U}$ & $2.6 \mathrm{E}-01 \pm 8.3 \mathrm{E}-02$ & \\
\hline & ${ }^{235} \mathrm{U}$ & $3.2 \mathrm{E}-02 \pm 2.0 \mathrm{E}-02$ & & & ${ }^{235} \mathrm{U}$ & $1.7 \mathrm{E}-02 \pm 1.5 \mathrm{E}-02$ & U \\
\hline & ${ }^{238} \mathrm{U}$ & 3.3E-01 \pm 9.9E-02 & & & ${ }^{238} \mathrm{U}$ & $2.3 \mathrm{E}-01 \pm 7.6 \mathrm{E}-02$ & \\
\hline & ${ }^{65} \mathrm{Zn}$ & $1.8 \mathrm{E}-02 \pm 1.9 \mathrm{E}-02$ & $\mathrm{U}$ & & ${ }^{65} \mathrm{Zn}$ & $-6.9 \mathrm{E}-03 \pm 1.6 \mathrm{E}-02$ & $\mathrm{U}$ \\
\hline \multirow{18}{*}{$\begin{array}{c}\text { D123 } \\
\text { (300 Area) }\end{array}$} & ${ }^{144} \mathrm{Ce}$ & $1.0 \mathrm{E}-02 \pm 1.0 \mathrm{E}-01$ & $\mathrm{U}$ & D124 & ${ }^{144} \mathrm{Ce}$ & $-3.2 \mathrm{E}-01 \pm 3.2 \mathrm{E}-01$ & $\mathrm{U}$ \\
\hline & ${ }^{60} \mathrm{Co}$ & $5.7 \mathrm{E}-03 \pm 5.5 \mathrm{E}-03$ & $\mathrm{U}$ & (300 Area) & ${ }^{60} \mathrm{Co}$ & $3.5 \mathrm{E}-03 \pm 1.0 \mathrm{E}-02$ & $\mathrm{U}$ \\
\hline & ${ }^{134} \mathrm{Cs}$ & $2.7 \mathrm{E}-02 \pm 9.6 \mathrm{E}-03$ & & & ${ }^{134} \mathrm{Cs}$ & $3.9 \mathrm{E}-02 \pm 1.8 \mathrm{E}-02$ & \\
\hline & ${ }^{137} \mathrm{Cs}$ & $3.0 \mathrm{E}-02 \pm 1.2 \mathrm{E}-02$ & & & ${ }^{137} \mathrm{Cs}$ & $5.9 \mathrm{E}-02 \pm 1.9 \mathrm{E}-02$ & \\
\hline & ${ }^{152} \mathrm{Eu}$ & $-9.0 \mathrm{E}-04 \pm 9.0 \mathrm{E}-03$ & $\mathrm{U}$ & & ${ }^{152} \mathrm{Eu}$ & $-1.7 \mathrm{E}-02 \pm 4.0 \mathrm{E}-02$ & $\mathrm{U}$ \\
\hline & ${ }^{154} \mathrm{Eu}$ & $1.4 \mathrm{E}-03 \pm 1.4 \mathrm{E}-02$ & $\mathrm{U}$ & & ${ }^{154} \mathrm{Eu}$ & $1.5 \mathrm{E}-02 \pm 3.4 \mathrm{E}-02$ & $\mathrm{U}$ \\
\hline & ${ }^{155} \mathrm{Eu}$ & $4.6 \mathrm{E}-03 \pm 2.6 \mathrm{E}-02$ & U & & ${ }^{155} \mathrm{Eu}$ & $6.2 \mathrm{E}-02 \pm 4.7 \mathrm{E}-02$ & \\
\hline & ${ }^{238} \mathrm{Pu}$ & $-4.9 \mathrm{E}-03 \pm 2.4 \mathrm{E}-02$ & $\mathrm{U}$ & & ${ }^{238} \mathrm{Pu}$ & $-1.6 \mathrm{E}-02 \pm 3.1 \mathrm{E}-02$ & U \\
\hline & ${ }^{239 / 240} \mathrm{Pu}$ & $4.9 \mathrm{E}-03 \pm 7.1 \mathrm{E}-03$ & $\mathrm{U}$ & & ${ }^{239 / 240} \mathrm{Pu}$ & $1.6 \mathrm{E}-02 \pm 1.2 \mathrm{E}-02$ & \\
\hline & ${ }^{103} \mathrm{Ru}$ & $5.7 \mathrm{E}-03 \pm 6.7 \mathrm{E}-03$ & $\mathrm{U}$ & & ${ }^{103} \mathrm{Ru}$ & $-5.0 \mathrm{E}-03 \pm 1.2 \mathrm{E}-02$ & $\mathrm{U}$ \\
\hline & ${ }^{106} \mathrm{Ru}$ & $-2.8 \mathrm{E}-02 \pm 4.9 \mathrm{E}-02$ & $\mathrm{U}$ & & ${ }^{106} \mathrm{Ru}$ & $-2.2 \mathrm{E}-02 \pm 9.8 \mathrm{E}-02$ & $\mathrm{U}$ \\
\hline & ${ }^{125} \mathrm{Sb}$ & $7.2 \mathrm{E}-03 \pm 1.5 \mathrm{E}-02$ & $\mathrm{U}$ & & ${ }^{125} \mathrm{Sb}$ & $-1.3 \mathrm{E}-03 \pm 1.3 \mathrm{E}-02$ & $\mathrm{U}$ \\
\hline & ${ }^{113} \mathrm{Sn}$ & $-4.5 \mathrm{E}-03 \pm 7.5 \mathrm{E}-03$ & $\mathrm{U}$ & & ${ }^{113} \mathrm{Sn}$ & $3.5 \mathrm{E}-04 \pm 3.5 \mathrm{E}-03$ & $\mathrm{U}$ \\
\hline & ${ }^{90} \mathrm{Sr}$ & $-2.0 \mathrm{E}-01 \pm 3.9 \mathrm{E}-01$ & $\mathrm{U}$ & & ${ }^{90} \mathrm{Sr}$ & 3.1E-01 \pm 4.5E-01 & $\mathrm{U}$ \\
\hline & ${ }^{234} \mathrm{U}$ & $1.5 \mathrm{E}-01 \pm 5.4 \mathrm{E}-02$ & & & ${ }^{234} \mathrm{U}$ & $2.2 \mathrm{E}-01 \pm 7.3 \mathrm{E}-02$ & \\
\hline & ${ }^{235} \mathrm{U}$ & $1.2 \mathrm{E}-02 \pm 1.1 \mathrm{E}-02$ & & & ${ }^{235} \mathrm{U}$ & $1.6 \mathrm{E}-02 \pm 1.4 \mathrm{E}-02$ & U \\
\hline & ${ }^{238} \mathrm{U}$ & $1.5 \mathrm{E}-01 \pm 5.3 \mathrm{E}-02$ & & & ${ }^{238} \mathrm{U}$ & 2.7E-01 \pm 8.4E-02 & \\
\hline & ${ }^{65} \mathrm{Zn}$ & $-5.4 \mathrm{E}-03 \pm 1.6 \mathrm{E}-02$ & $\mathrm{U}$ & & ${ }^{65} \mathrm{Zn}$ & $1.9 \mathrm{E}-04 \pm 1.9 \mathrm{E}-03$ & $\mathrm{U}$ \\
\hline
\end{tabular}

$\overline{\mathrm{RQ}}=$ Result Qualifier. $\mathrm{U}=$ The analyte was analyzed for but not detected. 
Table 3-4. 2008 Soil Sampling Results (pCi/g \pm total analytical uncertainty). (Sheet 22 of 24)

\begin{tabular}{|c|c|c|c|c|c|c|c|}
\hline Location & Isotope & Result \pm Error & RQ* & Location & Isotope & Result \pm Error & RQ* \\
\hline D125 & ${ }^{144} \mathrm{Ce}$ & $-3.5 \mathrm{E}-02 \pm 1.3 \mathrm{E}-01$ & $\mathrm{U}$ & D126 & ${ }^{144} \mathrm{Ce}$ & $-7.8 \mathrm{E}-03 \pm 7.8 \mathrm{E}-02$ & $\mathrm{U}$ \\
\hline \multirow[t]{17}{*}{ (300 Area) } & ${ }^{60} \mathrm{Co}$ & $-2.8 \mathrm{E}-03 \pm 7.3 \mathrm{E}-03$ & U & (300 Area) & ${ }^{60} \mathrm{Co}$ & $3.3 \mathrm{E}-04 \pm 3.3 \mathrm{E}-03$ & $\mathrm{U}$ \\
\hline & ${ }^{134} \mathrm{Cs}$ & $3.8 \mathrm{E}-02 \pm 1.5 \mathrm{E}-02$ & & & ${ }^{134} \mathrm{Cs}$ & $3.3 \mathrm{E}-02 \pm 1.5 \mathrm{E}-02$ & \\
\hline & ${ }^{137} \mathrm{Cs}$ & $7.4 \mathrm{E}-02 \pm 1.8 \mathrm{E}-02$ & & & ${ }^{137} \mathrm{Cs}$ & $4.4 \mathrm{E}-02 \pm 1.5 \mathrm{E}-02$ & \\
\hline & ${ }^{152} \mathrm{Eu}$ & $-2.5 \mathrm{E}-02 \pm 2.8 \mathrm{E}-02$ & $\mathrm{U}$ & & ${ }^{152} \mathrm{Eu}$ & $-8.7 \mathrm{E}-03 \pm 2.7 \mathrm{E}-02$ & $\mathrm{U}$ \\
\hline & ${ }^{154} \mathrm{Eu}$ & $8.9 \mathrm{E}-03 \pm 2.3 \mathrm{E}-02$ & $\mathrm{U}$ & & ${ }^{154} \mathrm{Eu}$ & $-3.1 \mathrm{E}-02 \pm 3.1 \mathrm{E}-02$ & $\mathrm{U}$ \\
\hline & ${ }^{155} \mathrm{Eu}$ & $5.2 \mathrm{E}-02 \pm 3.2 \mathrm{E}-02$ & $\mathrm{U}$ & & ${ }^{155} \mathrm{Eu}$ & $7.3 \mathrm{E}-02 \pm 5.1 \mathrm{E}-02$ & \\
\hline & ${ }^{238} \mathrm{Pu}$ & $-8.3 \mathrm{E}-03 \pm 3.6 \mathrm{E}-02$ & $\mathrm{U}$ & & ${ }^{238} \mathrm{Pu}$ & $1.7 \mathrm{E}-03 \pm 1.3 \mathrm{E}-02$ & $\mathrm{U}$ \\
\hline & ${ }^{239 / 240} \mathrm{Pu}$ & $2.1 \mathrm{E}-03 \pm 2.1 \mathrm{E}-03$ & $\mathrm{U}$ & & ${ }^{239 / 240} \mathrm{Pu}$ & $2.5 \mathrm{E}-02 \pm 1.4 \mathrm{E}-02$ & \\
\hline & ${ }^{103} \mathrm{Ru}$ & $-9.8 \mathrm{E}-03 \pm 9.8 \mathrm{E}-03$ & $\mathrm{U}$ & & ${ }^{103} \mathrm{Ru}$ & $-3.9 \mathrm{E}-03 \pm 8.3 \mathrm{E}-03$ & $\mathrm{U}$ \\
\hline & ${ }^{106} \mathrm{Ru}$ & $4.3 \mathrm{E}-02 \pm 6.4 \mathrm{E}-02$ & $\mathrm{U}$ & & ${ }^{106} \mathrm{Ru}$ & $-9.2 \mathrm{E}-03 \pm 7.3 \mathrm{E}-02$ & $\mathrm{U}$ \\
\hline & ${ }^{125} \mathrm{Sb}$ & $4.5 \mathrm{E}-03 \pm 1.9 \mathrm{E}-02$ & $\mathrm{U}$ & & ${ }^{125} \mathrm{Sb}$ & $-8.6 \mathrm{E}-03 \pm 2.3 \mathrm{E}-02$ & $\mathrm{U}$ \\
\hline & ${ }^{113} \mathrm{Sn}$ & $5.4 \mathrm{E}-03 \pm 9.3 \mathrm{E}-03$ & $\mathrm{U}$ & & ${ }^{113} \mathrm{Sn}$ & $3.3 \mathrm{E}-03 \pm 1.0 \mathrm{E}-02$ & $\mathrm{U}$ \\
\hline & ${ }^{90} \mathrm{Sr}$ & $-2.2 \mathrm{E}-02 \pm 2.2 \mathrm{E}-01$ & $\mathrm{U}$ & & ${ }^{90} \mathrm{Sr}$ & $-4.6 \mathrm{E}-01 \pm 4.6 \mathrm{E}-01$ & U \\
\hline & ${ }^{234} \mathrm{U}$ & $6.1 \mathrm{E}-01 \pm 1.7 \mathrm{E}-01$ & & & ${ }^{234} \mathrm{U}$ & $2.2 \mathrm{E}+00 \pm 5.9 \mathrm{E}-01$ & \\
\hline & ${ }^{235} \mathrm{U}$ & $4.2 \mathrm{E}-02 \pm 2.3 \mathrm{E}-02$ & & & ${ }^{235} \mathrm{U}$ & $2.5 \mathrm{E}-01 \pm 8.0 \mathrm{E}-02$ & \\
\hline & ${ }^{238} \mathrm{U}$ & $5.9 \mathrm{E}-01 \pm 1.7 \mathrm{E}-01$ & & & ${ }^{238} \mathrm{U}$ & $1.6 \mathrm{E}+00 \pm 4.3 \mathrm{E}-01$ & \\
\hline & ${ }^{65} \mathrm{Zn}$ & $-1.0 \mathrm{E}-03 \pm 1.0 \mathrm{E}-02$ & $\mathrm{U}$ & & ${ }^{65} \mathrm{Zn}$ & $5.2 \mathrm{E}-02 \pm 2.3 \mathrm{E}-02$ & \\
\hline \multirow{18}{*}{$\begin{array}{c}\text { D127 } \\
\text { (300 Area) }\end{array}$} & ${ }^{144} \mathrm{Ce}$ & $-5.2 \mathrm{E}-02 \pm 1.0 \mathrm{E}-01$ & $\mathrm{U}$ & D128 & ${ }^{144} \mathrm{Ce}$ & $1.2 \mathrm{E}-01 \pm 1.4 \mathrm{E}-01$ & $\mathrm{U}$ \\
\hline & ${ }^{60} \mathrm{Co}$ & $-6.0 \mathrm{E}-04 \pm 6.0 \mathrm{E}-03$ & $\mathrm{U}$ & (300 Area) & ${ }^{60} \mathrm{Co}$ & $-5.2 \mathrm{E}-04 \pm 5.2 \mathrm{E}-03$ & $\mathrm{U}$ \\
\hline & ${ }^{134} \mathrm{Cs}$ & $3.4 \mathrm{E}-02 \pm 1.5 \mathrm{E}-02$ & & & ${ }^{134} \mathrm{Cs}$ & $4.1 \mathrm{E}-02 \pm 1.2 \mathrm{E}-02$ & \\
\hline & ${ }^{137} \mathrm{Cs}$ & $1.9 \mathrm{E}-01 \pm 3.4 \mathrm{E}-02$ & & & ${ }^{137} \mathrm{Cs}$ & $8.9 \mathrm{E}-02 \pm 1.9 \mathrm{E}-02$ & \\
\hline & ${ }^{152} \mathrm{Eu}$ & $1.1 \mathrm{E}-02 \pm 2.1 \mathrm{E}-02$ & U & & ${ }^{152} \mathrm{Eu}$ & $9.0 \mathrm{E}-03 \pm 2.6 \mathrm{E}-02$ & $\mathrm{U}$ \\
\hline & ${ }^{154} \mathrm{Eu}$ & $-2.7 \mathrm{E}-02 \pm 2.7 \mathrm{E}-02$ & $\mathrm{U}$ & & ${ }^{154} \mathrm{Eu}$ & $-2.0 \mathrm{E}-02 \pm 2.1 \mathrm{E}-02$ & $\mathrm{U}$ \\
\hline & ${ }^{155} \mathrm{Eu}$ & $5.9 \mathrm{E}-02 \pm 3.6 \mathrm{E}-02$ & & & ${ }^{155} \mathrm{Eu}$ & $4.5 \mathrm{E}-02 \pm 4.1 \mathrm{E}-02$ & U \\
\hline & ${ }^{238} \mathrm{Pu}$ & $2.7 \mathrm{E}-02 \pm 1.9 \mathrm{E}-02$ & & & ${ }^{238} \mathrm{Pu}$ & $1.6 \mathrm{E}-03 \pm 8.5 \mathrm{E}-03$ & $\mathrm{U}$ \\
\hline & ${ }^{239 / 240} \mathrm{Pu}$ & $9.0 \mathrm{E}-03 \pm 9.3 \mathrm{E}-03$ & & & ${ }^{239 / 240} \mathrm{Pu}$ & $9.8 \mathrm{E}-03 \pm 8.4 \mathrm{E}-03$ & \\
\hline & ${ }^{103} \mathrm{Ru}$ & $5.5 \mathrm{E}-04 \pm 5.5 \mathrm{E}-03$ & $\mathrm{U}$ & & ${ }^{103} \mathrm{Ru}$ & $-3.3 \mathrm{E}-03 \pm 7.2 \mathrm{E}-03$ & $\mathrm{U}$ \\
\hline & ${ }^{106} \mathrm{Ru}$ & $2.4 \mathrm{E}-02 \pm 5.6 \mathrm{E}-02$ & $\mathrm{U}$ & & ${ }^{106} \mathrm{Ru}$ & $1.9 \mathrm{E}-02 \pm 6.0 \mathrm{E}-02$ & $\mathrm{U}$ \\
\hline & ${ }^{125} \mathrm{Sb}$ & $-1.4 \mathrm{E}-02 \pm 1.8 \mathrm{E}-02$ & $\mathrm{U}$ & & ${ }^{125} \mathrm{Sb}$ & $1.1 \mathrm{E}-02 \pm 1.8 \mathrm{E}-02$ & $\mathrm{U}$ \\
\hline & ${ }^{113} \mathrm{Sn}$ & $3.6 \mathrm{E}-05 \pm 3.6 \mathrm{E}-04$ & $\mathrm{U}$ & & ${ }^{113} \mathrm{Sn}$ & $-4.8 \mathrm{E}-03 \pm 9.1 \mathrm{E}-03$ & $\mathrm{U}$ \\
\hline & ${ }^{90} \mathrm{Sr}$ & $6.0 \mathrm{E}-01 \pm 4.6 \mathrm{E}-01$ & & & ${ }^{90} \mathrm{Sr}$ & $1.0 \mathrm{E}-02 \pm 1.0 \mathrm{E}-01$ & $\mathrm{U}$ \\
\hline & ${ }^{234} \mathrm{U}$ & $2.4 \mathrm{E}-01 \pm 7.9 \mathrm{E}-02$ & & & ${ }^{234} \mathrm{U}$ & $2.6 \mathrm{E}-01 \pm 8.1 \mathrm{E}-02$ & \\
\hline & ${ }^{235} \mathrm{U}$ & $1.1 \mathrm{E}-02 \pm 1.4 \mathrm{E}-02$ & $\mathrm{U}$ & & ${ }^{235} \mathrm{U}$ & $2.5 \mathrm{E}-02 \pm 1.8 \mathrm{E}-02$ & \\
\hline & ${ }^{238} \mathrm{U}$ & $2.5 \mathrm{E}-01 \pm 8.2 \mathrm{E}-02$ & & & ${ }^{238} \mathrm{U}$ & $2.7 \mathrm{E}-01 \pm 8.4 \mathrm{E}-02$ & \\
\hline & ${ }^{65} \mathrm{Zn}$ & $1.3 \mathrm{E}-02 \pm 1.8 \mathrm{E}-02$ & $\mathrm{U}$ & & ${ }^{65} \mathrm{Zn}$ & $-1.0 \mathrm{E}-03 \pm 1.0 \mathrm{E}-02$ & $\mathrm{U}$ \\
\hline
\end{tabular}

$\overline{\mathrm{RQ}}=$ Result Qualifier. $\mathrm{U}=$ The analyte was analyzed for but not detected. 
Table 3-4. 2008 Soil Sampling Results (pCi/g \pm total analytical uncertainty). (Sheet 23 of 24)

\begin{tabular}{|c|c|c|c|c|c|c|c|}
\hline Location & Isotope & Result \pm Error & RQ* & Location & Isotope & Result \pm Error & $\mathbf{R Q}^{*}$ \\
\hline D129 & ${ }^{144} \mathrm{Ce}$ & $1.5 \mathrm{E}-01 \pm 1.3 \mathrm{E}-01$ & $\mathrm{U}$ & D131 & ${ }^{144} \mathrm{Ce}$ & $-1.5 \mathrm{E}-02 \pm 1.1 \mathrm{E}-01$ & $\mathrm{U}$ \\
\hline \multirow[t]{17}{*}{ (300 Area) } & ${ }^{60} \mathrm{Co}$ & $3.1 \mathrm{E}-03 \pm 6.7 \mathrm{E}-03$ & $\mathrm{U}$ & (Replicate & ${ }^{60} \mathrm{Co}$ & $-3.4 \mathrm{E}-03 \pm 5.4 \mathrm{E}-03$ & $\mathrm{U}$ \\
\hline & ${ }^{134} \mathrm{Cs}$ & $3.7 \mathrm{E}-02 \pm 1.4 \mathrm{E}-02$ & & of D119 & ${ }^{134} \mathrm{Cs}$ & $2.6 \mathrm{E}-02 \pm 1.0 \mathrm{E}-02$ & \\
\hline & ${ }^{137} \mathrm{Cs}$ & $2.8 \mathrm{E}-02 \pm 1.3 \mathrm{E}-02$ & & 300 Area) & ${ }^{137} \mathrm{Cs}$ & $1.8 \mathrm{E}-02 \pm 6.6 \mathrm{E}-03$ & \\
\hline & ${ }^{152} \mathrm{Eu}$ & $-1.5 \mathrm{E}-02 \pm 2.5 \mathrm{E}-02$ & $\mathrm{U}$ & & ${ }^{152} \mathrm{Eu}$ & $-2.4 \mathrm{E}-03 \pm 1.9 \mathrm{E}-02$ & $\mathrm{U}$ \\
\hline & ${ }^{154} \mathrm{Eu}$ & $-1.7 \mathrm{E}-02 \pm 3.4 \mathrm{E}-02$ & $\mathrm{U}$ & & ${ }^{154} \mathrm{Eu}$ & $9.8 \mathrm{E}-04 \pm 9.8 \mathrm{E}-03$ & $\mathrm{U}$ \\
\hline & ${ }^{155} \mathrm{Eu}$ & $3.1 \mathrm{E}-02 \pm 3.0 \mathrm{E}-02$ & $\mathrm{U}$ & & ${ }^{155} \mathrm{Eu}$ & $6.6 \mathrm{E}-02 \pm 3.7 \mathrm{E}-02$ & \\
\hline & ${ }^{238} \mathrm{Pu}$ & $-1.1 \mathrm{E}-02 \pm 3.4 \mathrm{E}-02$ & $\mathrm{U}$ & & ${ }^{238} \mathrm{Pu}$ & $1.0 \mathrm{E}-02 \pm 3.1 \mathrm{E}-02$ & $\mathrm{U}$ \\
\hline & ${ }^{239 / 240} \mathrm{Pu}$ & $2.1 \mathrm{E}-03 \pm 2.1 \mathrm{E}-02$ & $\mathrm{U}$ & & ${ }^{239 / 240} \mathrm{Pu}$ & $2.0 \mathrm{E}-03 \pm 1.1 \mathrm{E}-02$ & $\mathrm{U}$ \\
\hline & ${ }^{103} \mathrm{Ru}$ & $3.1 \mathrm{E}-04 \pm 3.1 \mathrm{E}-03$ & $\mathrm{U}$ & & ${ }^{103} \mathrm{Ru}$ & $-7.8 \mathrm{E}-03 \pm 7.8 \mathrm{E}-03$ & $\mathrm{U}$ \\
\hline & ${ }^{106} \mathrm{Ru}$ & $6.5 \mathrm{E}-03 \pm 5.6 \mathrm{E}-02$ & $\mathrm{U}$ & & ${ }^{106} \mathrm{Ru}$ & $2.1 \mathrm{E}-02 \pm 4.7 \mathrm{E}-02$ & $\mathrm{U}$ \\
\hline & ${ }^{125} \mathrm{Sb}$ & $-1.9 \mathrm{E}-03 \pm 1.7 \mathrm{E}-02$ & $\mathrm{U}$ & & ${ }^{125} \mathrm{Sb}$ & $-6.7 \mathrm{E}-03 \pm 1.5 \mathrm{E}-02$ & $\mathrm{U}$ \\
\hline & ${ }^{113} \mathrm{Sn}$ & $-6.9 \mathrm{E}-03 \pm 8.8 \mathrm{E}-03$ & $\mathrm{U}$ & & ${ }^{113} \mathrm{Sn}$ & $-6.6 \mathrm{E}-03 \pm 7.6 \mathrm{E}-03$ & $\mathrm{U}$ \\
\hline & ${ }^{90} \mathrm{Sr}$ & $-1.8 \mathrm{E}-01 \pm 4.1 \mathrm{E}-01$ & $\mathrm{U}$ & & ${ }^{90} \mathrm{Sr}$ & $4.0 \mathrm{E}-03 \pm 4.0 \mathrm{E}-02$ & $\mathrm{U}$ \\
\hline & ${ }^{234} \mathrm{U}$ & $2.7 \mathrm{E}-01 \pm 8.4 \mathrm{E}-02$ & & & ${ }^{234} U$ & $1.7 \mathrm{E}+00 \pm 4.4 \mathrm{E}-01$ & \\
\hline & ${ }^{235} \mathrm{U}$ & $8.1 \mathrm{E}-03 \pm 8.3 \mathrm{E}-03$ & & & ${ }^{235} \mathrm{U}$ & $7.5 \mathrm{E}-02 \pm 3.1 \mathrm{E}-02$ & \\
\hline & ${ }^{238} \mathrm{U}$ & $2.8 \mathrm{E}-01 \pm 8.7 \mathrm{E}-02$ & & & ${ }^{238} \mathrm{U}$ & $1.6 \mathrm{E}+00 \pm 4.2 \mathrm{E}-01$ & \\
\hline & ${ }^{65} \mathrm{Zn}$ & $-3.1 \mathrm{E}-03 \pm 1.8 \mathrm{E}-02$ & $\mathrm{U}$ & & ${ }^{65} \mathrm{Zn}$ & $-1.6 \mathrm{E}-02 \pm 1.6 \mathrm{E}-02$ & $\mathrm{U}$ \\
\hline \multirow{18}{*}{$\begin{array}{c}\text { D132 } \\
\text { (Replicate } \\
\text { of D120 } \\
\text { 300 Area) }\end{array}$} & ${ }^{144} \mathrm{Ce}$ & $4.7 \mathrm{E}-02 \pm 1.0 \mathrm{E}-01$ & $\mathrm{U}$ & D139 & ${ }^{144} \mathrm{Ce}$ & $-5.0 \mathrm{E}-02 \pm 1.2 \mathrm{E}-01$ & $\mathrm{U}$ \\
\hline & ${ }^{60} \mathrm{Co}$ & $1.9 \mathrm{E}-03 \pm 7.0 \mathrm{E}-03$ & $\mathrm{U}$ & (Replicate & ${ }^{60} \mathrm{Co}$ & $1.2 \mathrm{E}-03 \pm 5.0 \mathrm{E}-03$ & $\mathrm{U}$ \\
\hline & ${ }^{134} \mathrm{Cs}$ & $2.4 \mathrm{E}-02 \pm 9.8 \mathrm{E}-03$ & & of D118 & ${ }^{134} \mathrm{Cs}$ & $2.1 \mathrm{E}-02 \pm 8.1 \mathrm{E}-03$ & \\
\hline & ${ }^{137} \mathrm{Cs}$ & $1.8 \mathrm{E}-02 \pm 9.4 \mathrm{E}-03$ & & 300 Area) & ${ }^{137} \mathrm{Cs}$ & $1.6 \mathrm{E}-02 \pm 7.4 \mathrm{E}-03$ & \\
\hline & ${ }^{152} \mathrm{Eu}$ & $4.4 \mathrm{E}-03 \pm 2.0 \mathrm{E}-02$ & $\mathrm{U}$ & & ${ }^{152} \mathrm{Eu}$ & $-1.2 \mathrm{E}-02 \pm 2.2 \mathrm{E}-02$ & $\mathrm{U}$ \\
\hline & ${ }^{154} \mathrm{Eu}$ & $1.1 \mathrm{E}-02 \pm 2.3 \mathrm{E}-02$ & $\mathrm{U}$ & & ${ }^{154} \mathrm{Eu}$ & $-3.7 \mathrm{E}-03 \pm 1.7 \mathrm{E}-02$ & $\mathrm{U}$ \\
\hline & ${ }^{155} \mathrm{Eu}$ & $3.7 \mathrm{E}-02 \pm 2.7 \mathrm{E}-02$ & $\mathrm{U}$ & & ${ }^{155} \mathrm{Eu}$ & $3.3 \mathrm{E}-03 \pm 2.8 \mathrm{E}-02$ & $\mathrm{U}$ \\
\hline & ${ }^{238} \mathrm{Pu}$ & $-2.2 \mathrm{E}-03 \pm 2.2 \mathrm{E}-02$ & $\mathrm{U}$ & & ${ }^{238} \mathrm{Pu}$ & $3.5 \mathrm{E}-02 \pm 3.8 \mathrm{E}-02$ & $\mathrm{U}$ \\
\hline & ${ }^{239 / 240} \mathrm{Pu}$ & $1.1 \mathrm{E}-02 \pm 1.2 \mathrm{E}-02$ & $\mathrm{U}$ & & ${ }^{239 / 240} \mathrm{Pu}$ & $1.4 \mathrm{E}-02 \pm 1.5 \mathrm{E}-02$ & $\mathrm{U}$ \\
\hline & ${ }^{103} \mathrm{Ru}$ & $3.5 \mathrm{E}-03 \pm 6.1 \mathrm{E}-03$ & $\mathrm{U}$ & & ${ }^{103} \mathrm{Ru}$ & $2.3 \mathrm{E}-03 \pm 5.2 \mathrm{E}-03$ & $\mathrm{U}$ \\
\hline & ${ }^{106} \mathrm{Ru}$ & $-2.8 \mathrm{E}-02 \pm 5.6 \mathrm{E}-02$ & $\mathrm{U}$ & & ${ }^{106} \mathrm{Ru}$ & $-1.2 \mathrm{E}-02 \pm 4.8 \mathrm{E}-02$ & $\mathrm{U}$ \\
\hline & ${ }^{125} \mathrm{Sb}$ & $1.8 \mathrm{E}-02 \pm 1.7 \mathrm{E}-02$ & $\mathrm{U}$ & & ${ }^{125} \mathrm{Sb}$ & $-6.4 \mathrm{E}-03 \pm 1.5 \mathrm{E}-02$ & $\mathrm{U}$ \\
\hline & ${ }^{113} \mathrm{Sn}$ & $-2.9 \mathrm{E}-03 \pm 8.1 \mathrm{E}-03$ & $\mathrm{U}$ & & ${ }^{113} \mathrm{Sn}$ & $-5.0 \mathrm{E}-03 \pm 6.9 \mathrm{E}-03$ & $\mathrm{U}$ \\
\hline & ${ }^{90} \mathrm{Sr}$ & $-1.7 \mathrm{E}-01 \pm 4.0 \mathrm{E}-01$ & $\mathrm{U}$ & & ${ }^{90} \mathrm{Sr}$ & $-4.0 \mathrm{E}-01 \pm 4.0 \mathrm{E}-01$ & $\mathrm{U}$ \\
\hline & ${ }^{234} \mathrm{U}$ & $3.2 \mathrm{E}-01 \pm 9.9 \mathrm{E}-02$ & & & ${ }^{234} \mathrm{U}$ & $3.5 \mathrm{E}-01 \pm 1.0 \mathrm{E}-01$ & \\
\hline & ${ }^{235} \mathrm{U}$ & $2.6 \mathrm{E}-02 \pm 1.7 \mathrm{E}-02$ & & & ${ }^{235} \mathrm{U}$ & $2.1 \mathrm{E}-02 \pm 1.6 \mathrm{E}-02$ & \\
\hline & ${ }^{238} \mathrm{U}$ & $3.1 \mathrm{E}-01 \pm 9.6 \mathrm{E}-02$ & & & ${ }^{238} \mathrm{U}$ & $3.3 \mathrm{E}-01 \pm 9.9 \mathrm{E}-02$ & \\
\hline & ${ }^{65} \mathrm{Zn}$ & $-7.5 \mathrm{E}-04 \pm 7.5 \mathrm{E}-03$ & $\mathrm{U}$ & & ${ }^{65} \mathrm{Zn}$ & $-1.7 \mathrm{E}-03 \pm 1.4 \mathrm{E}-02$ & U \\
\hline
\end{tabular}

$\overline{\mathrm{RQ}}=$ Result Qualifier. $\mathrm{U}=$ The analyte was analyzed for but not detected. 
Table 3-4. 2008 Soil Sampling Results (pCi/g \pm total analytical uncertainty). (Sheet 24 of 24)

\begin{tabular}{|c|c|c|c|}
\hline Location & Isotope & Result \pm Error & RQ* \\
\hline D140 & ${ }^{144} \mathrm{Ce}$ & $1.4 \mathrm{E}-01 \pm 1.7 \mathrm{E}-01$ & $\mathrm{U}$ \\
\hline (Replicate & ${ }^{60} \mathrm{Co}$ & $-2.0 \mathrm{E}-03 \pm 1.1 \mathrm{E}-02$ & $\mathrm{U}$ \\
\hline of D123 & ${ }^{134} \mathrm{Cs}$ & $2.0 \mathrm{E}-02 \pm 1.8 \mathrm{E}-02$ & $\mathrm{U}$ \\
\hline \multirow[t]{15}{*}{300 Area) } & ${ }^{137} \mathrm{Cs}$ & $3.0 \mathrm{E}-02 \pm 1.5 \mathrm{E}-02$ & \\
\hline & ${ }^{152} \mathrm{Eu}$ & $-1.8 \mathrm{E}-02 \pm 4.2 \mathrm{E}-02$ & $\mathrm{U}$ \\
\hline & ${ }^{154} \mathrm{Eu}$ & $-1.3 \mathrm{E}-02 \pm 3.5 \mathrm{E}-02$ & $\mathrm{U}$ \\
\hline & ${ }^{155} \mathrm{Eu}$ & $-9.7 \mathrm{E}-03 \pm 4.2 \mathrm{E}-02$ & $\mathrm{U}$ \\
\hline & ${ }^{238} \mathrm{Pu}$ & $4.8 \mathrm{E}-03 \pm 3.7 \mathrm{E}-02$ & $\mathrm{U}$ \\
\hline & ${ }^{239 / 240} \mathrm{Pu}$ & $-2.4 \mathrm{E}-03 \pm 1.1 \mathrm{E}-02$ & $\mathrm{U}$ \\
\hline & ${ }^{103} \mathrm{Ru}$ & $1.7 \mathrm{E}-03 \pm 1.1 \mathrm{E}-02$ & $\mathrm{U}$ \\
\hline & ${ }^{106} \mathrm{Ru}$ & $2.1 \mathrm{E}-02 \pm 9.7 \mathrm{E}-02$ & $\mathrm{U}$ \\
\hline & ${ }^{125} \mathrm{Sb}$ & $-4.4 \mathrm{E}-03 \pm 2.9 \mathrm{E}-02$ & $\mathrm{U}$ \\
\hline & ${ }^{113} \mathrm{Sn}$ & $-3.5 \mathrm{E}-03 \pm 1.4 \mathrm{E}-02$ & $\mathrm{U}$ \\
\hline & ${ }^{90} \mathrm{Sr}$ & $-2.2 \mathrm{E}-01 \pm 4.2 \mathrm{E}-01$ & $\mathrm{U}$ \\
\hline & ${ }^{234} \mathrm{U}$ & $2.1 \mathrm{E}-01 \pm 6.9 \mathrm{E}-02$ & \\
\hline & ${ }^{235} \mathrm{U}$ & $2.7 \mathrm{E}-02 \pm 1.7 \mathrm{E}-02$ & \\
\hline & ${ }^{238} \mathrm{U}$ & $1.5 \mathrm{E}-01 \pm 5.3 \mathrm{E}-02$ & \\
\hline & ${ }^{65} \mathrm{Zn}$ & $-4.1 \mathrm{E}-03 \pm 2.8 \mathrm{E}-02$ & $\mathrm{U}$ \\
\hline \multirow{18}{*}{$\begin{array}{c}\text { D146 } \\
\text { (ERDF, } \\
\text { 200-W) }\end{array}$} & ${ }^{144} \mathrm{Ce}$ & $4.9 \mathrm{E}-02 \pm 1.5 \mathrm{E}-01$ & $\mathrm{U}$ \\
\hline & ${ }^{60} \mathrm{Co}$ & $3.8 \mathrm{E}-03 \pm 8.1 \mathrm{E}-03$ & $\mathrm{U}$ \\
\hline & ${ }^{134} \mathrm{Cs}$ & $3.5 \mathrm{E}-02 \pm 1.1 \mathrm{E}-02$ & \\
\hline & ${ }^{137} \mathrm{Cs}$ & $8.3 \mathrm{E}-03 \pm 9.8 \mathrm{E}-03$ & $\mathrm{U}$ \\
\hline & ${ }^{152} \mathrm{Eu}$ & $-1.3 \mathrm{E}-02 \pm 3.3 \mathrm{E}-02$ & $\mathrm{U}$ \\
\hline & ${ }^{154} \mathrm{Eu}$ & $1.6 \mathrm{E}-02 \pm 3.0 \mathrm{E}-02$ & $\mathrm{U}$ \\
\hline & ${ }^{155} \mathrm{Eu}$ & $6.0 \mathrm{E}-02 \pm 5.1 \mathrm{E}-02$ & $\mathrm{U}$ \\
\hline & ${ }^{238} \mathrm{Pu}$ & 2.7E-02 \pm 3.3E-02 & $\mathrm{U}$ \\
\hline & ${ }^{239 / 240} \mathrm{Pu}$ & $8.3 \mathrm{E}-03 \pm 1.0 \mathrm{E}-02$ & $\mathrm{U}$ \\
\hline & ${ }^{103} \mathrm{Ru}$ & $2.3 \mathrm{E}-03 \pm 8.4 \mathrm{E}-03$ & $\mathrm{U}$ \\
\hline & ${ }^{106} \mathrm{Ru}$ & $-3.9 \mathrm{E}-02 \pm 7.2 \mathrm{E}-02$ & $\mathrm{U}$ \\
\hline & ${ }^{125} \mathrm{Sb}$ & $-9.1 \mathrm{E}-03 \pm 2.1 \mathrm{E}-02$ & $\mathrm{U}$ \\
\hline & ${ }^{113} \mathrm{Sn}$ & $-1.6 \mathrm{E}-03 \pm 1.1 \mathrm{E}-02$ & $\mathrm{U}$ \\
\hline & ${ }^{90} \mathrm{Sr}$ & $-6.3 \mathrm{E}-01 \pm 6.3 \mathrm{E}-01$ & $\mathrm{U}$ \\
\hline & ${ }^{234} \mathrm{U}$ & $1.3 \mathrm{E}-01 \pm 4.7 \mathrm{E}-02$ & \\
\hline & ${ }^{235} \mathrm{U}$ & $4.5 \mathrm{E}-03 \pm 9.1 \mathrm{E}-03$ & $\mathrm{U}$ \\
\hline & ${ }^{238} \mathrm{U}$ & $1.7 \mathrm{E}-01 \pm 5.8 \mathrm{E}-02$ & \\
\hline & ${ }^{65} \mathrm{Zn}$ & $4.8 \mathrm{E}-04 \pm 4.8 \mathrm{E}-03$ & $\mathrm{U}$ \\
\hline
\end{tabular}

\begin{tabular}{clcc} 
Location & Isotope & Result \pm Error & RQ $^{*}$ \\
\hline D130 & ${ }^{144} \mathrm{Ce}$ & $-5.3 \mathrm{E}-02 \pm 1.1 \mathrm{E}-01$ & $\mathrm{U}$ \\
(400 Area) $)$ & ${ }^{60} \mathrm{Co}$ & $2.4 \mathrm{E}-03 \pm 5.7 \mathrm{E}-03$ & $\mathrm{U}$ \\
& ${ }^{134} \mathrm{Cs}$ & $3.3 \mathrm{E}-02 \pm 1.1 \mathrm{E}-02$ & \\
& ${ }^{137} \mathrm{Cs}$ & $2.0 \mathrm{E}-02 \pm 1.1 \mathrm{E}-02$ & \\
& ${ }^{152} \mathrm{Eu}$ & $1.6 \mathrm{E}-02 \pm 2.0 \mathrm{E}-02$ & $\mathrm{U}$ \\
& ${ }^{154} \mathrm{Eu}$ & $-2.1 \mathrm{E}-02 \pm 2.1 \mathrm{E}-02$ & $\mathrm{U}$ \\
& ${ }^{155} \mathrm{Eu}$ & $2.0 \mathrm{E}-02 \pm 2.7 \mathrm{E}-02$ & $\mathrm{U}$ \\
& ${ }^{238} \mathrm{Pu}$ & $-2.6 \mathrm{E}-02 \pm 3.7 \mathrm{E}-02$ & $\mathrm{U}$ \\
& ${ }^{239 / 240} \mathrm{Pu}$ & $4.7 \mathrm{E}-03 \pm 9.5 \mathrm{E}-03$ & $\mathrm{U}$ \\
& ${ }^{103} \mathrm{Ru}$ & $-4.3 \mathrm{E}-03 \pm 5.6 \mathrm{E}-03$ & $\mathrm{U}$ \\
& ${ }^{106} \mathrm{Ru}$ & $-3.7 \mathrm{E}-02 \pm 4.7 \mathrm{E}-02$ & $\mathrm{U}$ \\
& ${ }^{125} \mathrm{Sb}$ & $-9.0 \mathrm{E}-03 \pm 1.6 \mathrm{E}-02$ & $\mathrm{U}$ \\
& ${ }^{113} \mathrm{Sn}$ & $-7.6 \mathrm{E}-03 \pm 7.7 \mathrm{E}-03$ & $\mathrm{U}$ \\
& ${ }^{90} \mathrm{Sr}$ & $-2.7 \mathrm{E}-01 \pm 4.2 \mathrm{E}-01$ & $\mathrm{U}$ \\
& ${ }^{234} \mathrm{U}$ & $2.2 \mathrm{E}-01 \pm 7.3 \mathrm{E}-02$ & \\
& ${ }^{235} \mathrm{U}$ & $2.9 \mathrm{E}-02 \pm 1.8 \mathrm{E}-02$ & \\
& ${ }^{238} \mathrm{U}$ & $2.1 \mathrm{E}-01 \pm 6.9 \mathrm{E}-02$ & \\
& ${ }^{65} \mathrm{Zn}$ & $-1.9 \mathrm{E}-03 \pm 1.6 \mathrm{E}-02$ & $\mathrm{U}$
\end{tabular}

RQ = Result Qualifier. U = The analyte was analyzed for but not detected. 
This page intentionally left blank. 


\subsection{VEGETATION MONITORING}

The radionuclide content of vegetation was measured to evaluate long-term trends in environmental accumulation of radioactivity in the 100, 200/600, and 300/400 Areas. Vegetation samples were collected on or near facilities that store, handle, or dispose of radioactive waste. The number of vegetation samples collected in 2008 and their locations are shown in Table 4-1.

Table 4-1. Number and Locations of Vegetation Samples Collected Near Hanford Facilities and Operations in 2008.

\begin{tabular}{ccccccc} 
& \multicolumn{6}{c}{ Operational Area } \\
\cline { 2 - 7 } $\begin{array}{c}\text { Number of } \\
\text { Samples }\end{array}$ & $\mathbf{1 0 0 - N}$ & 200-East & 200-West $^{\mathrm{a}}$ & $\mathbf{3 0 0}^{\mathrm{a}}$ & $\mathbf{4 0 0}$ & $\mathbf{6 0 0}^{\mathrm{a}}$ \\
\hline 71 & 3 & 12 & 25 & 15 & 1 & 15
\end{tabular}

${ }^{\mathrm{a}}$ Number of samples includes one or more Replicate Samples.

Vegetation sampling locations are illustrated in Figures 4-1 through 4-6. Radionuclide analyses indicated that strontium-90, cesium-137, plutonium-238, plutonium-239/240, and uranium were detectable vegetation samples in 2008. Historically, the predominant radionuclides observed in vegetation samples were activation and fission products in the 100 Areas, fission products in the 200 Areas, and uranium in the 300 Area.

A summary of near-facility vegetation sampling results for selected radionuclides collected during 2008 is presented in Table 4-2. Historical vegetation sampling results for the 100-N, 200/600, and 300/400 Areas are displayed in Table 4-3. The 2008 vegetation sampling results for all areas are provided in Table 4-4.

Strontium-90 vegetation samples for this report period showed a frequent occurrence of negative (i.e., less than zero) concentrations. This was primarily due to changes in laboratory background correction calculations that were implemented during 2003. Both historical and current values are within accepted statistical ranges as evidenced by laboratory quality assurance (QA) and performance evaluation programs.

Additional discussion of the 2008 vegetation results can be found in Section 10.10.2 of PNNL-18427. 
Figure 4-1. 2008 Vegetation Sampling Locations, 100 N Area.

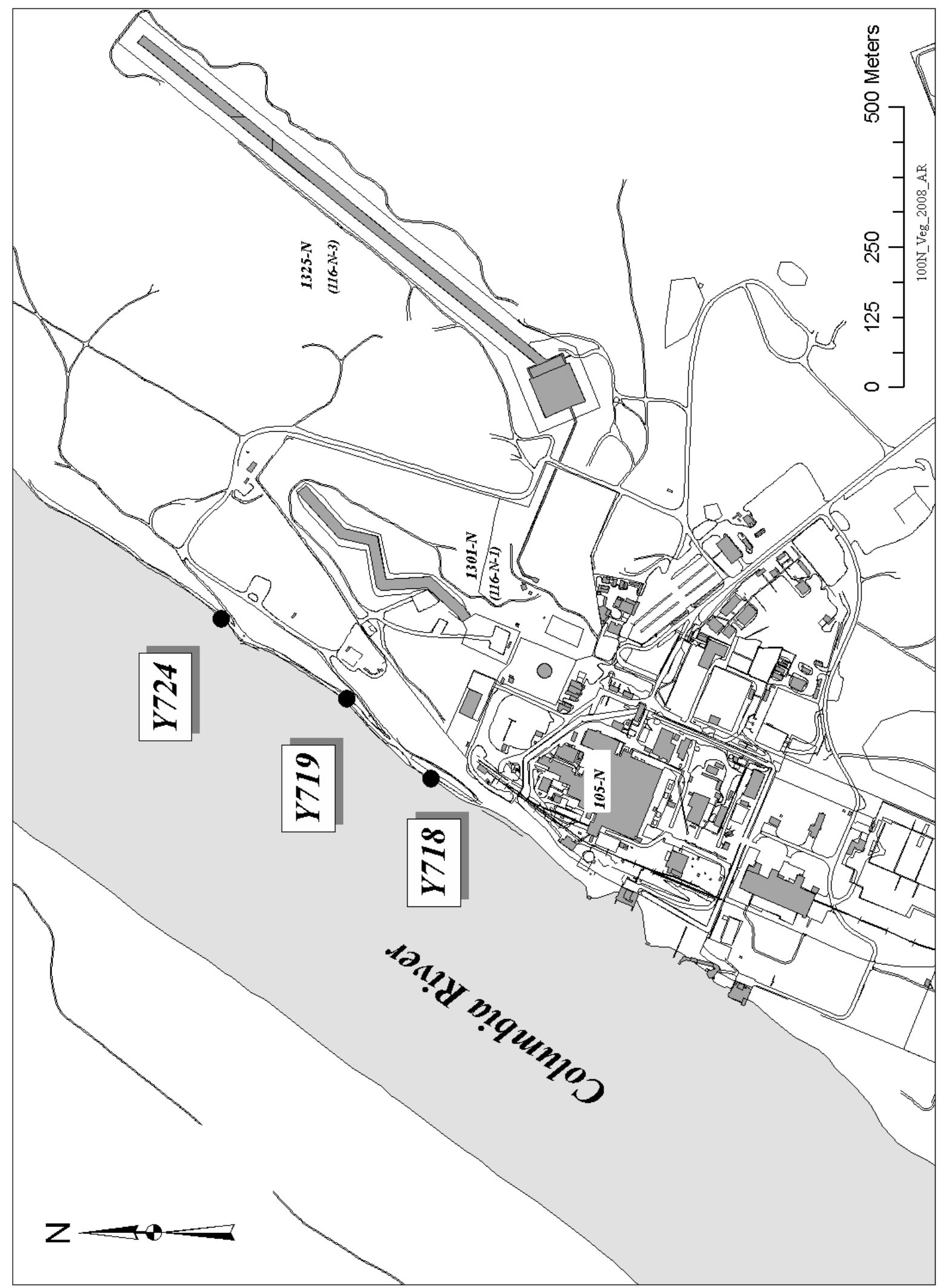


Figure 4-2. 2008 Vegetation Sampling Locations, 200 East Area.

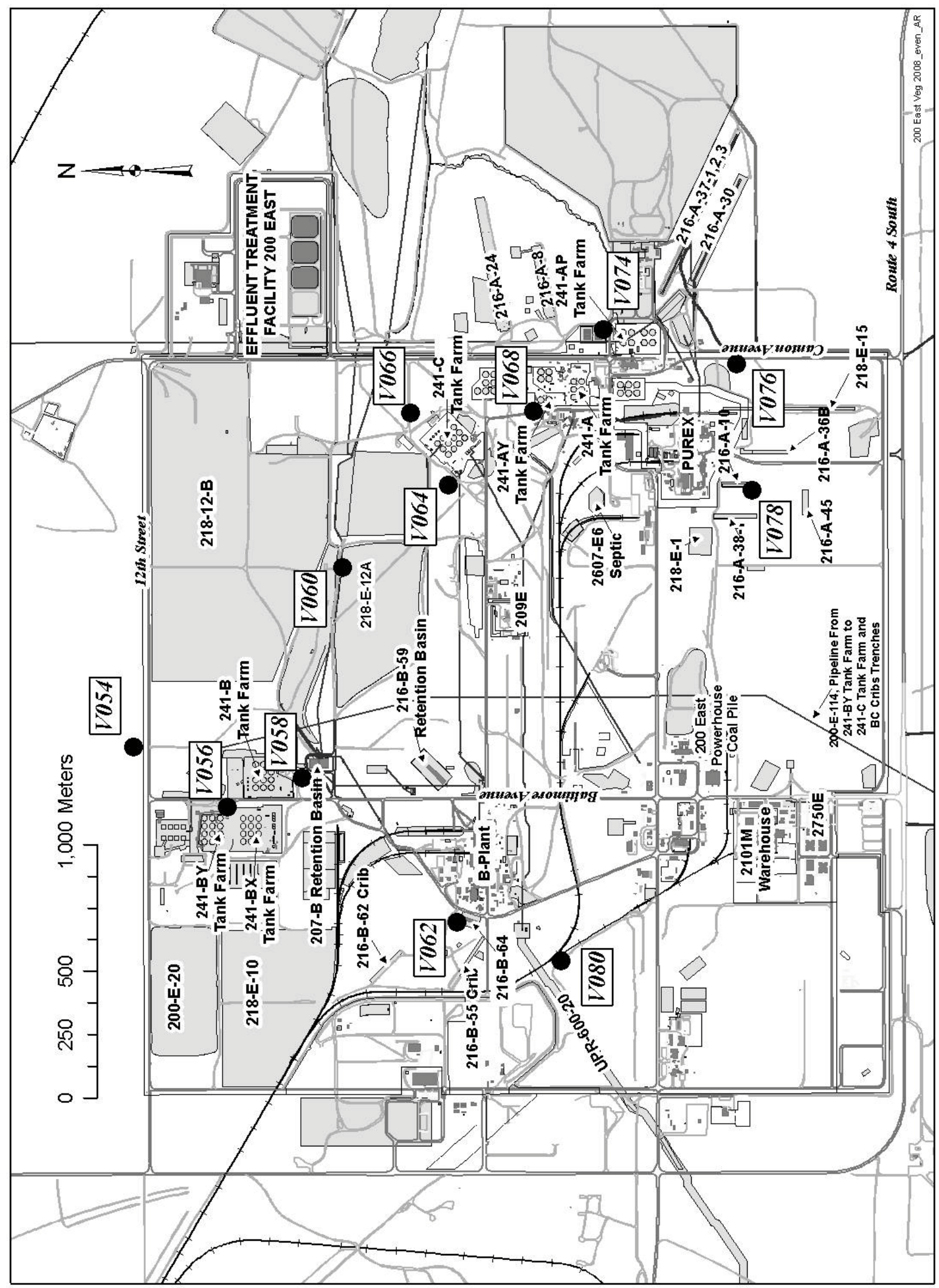


Figure 4-3. 2008 Vegetation Sampling Locations, 200 West Area.

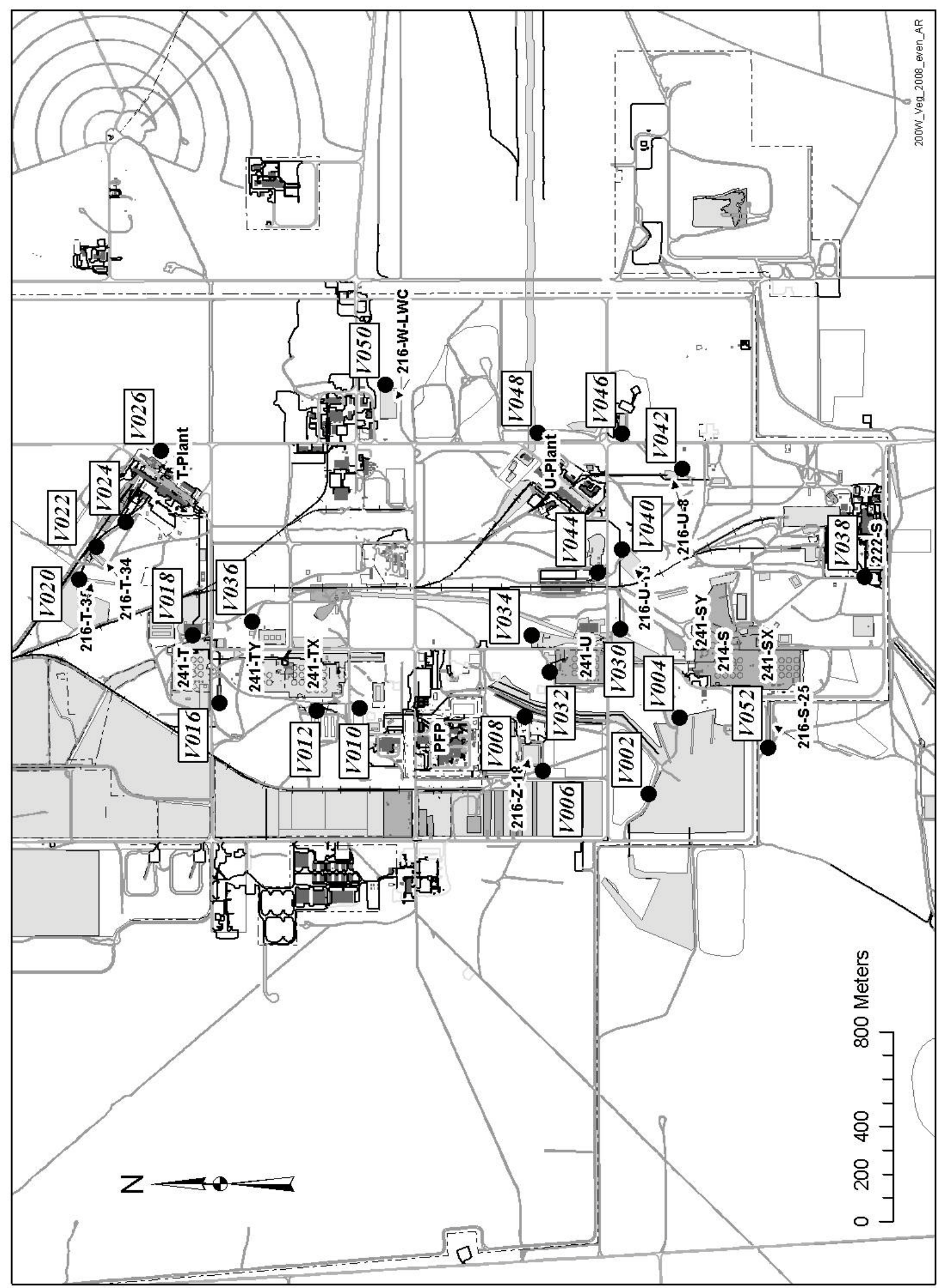


Figure 4-4. 2008 Vegetation Sampling Locations, 300 Area.

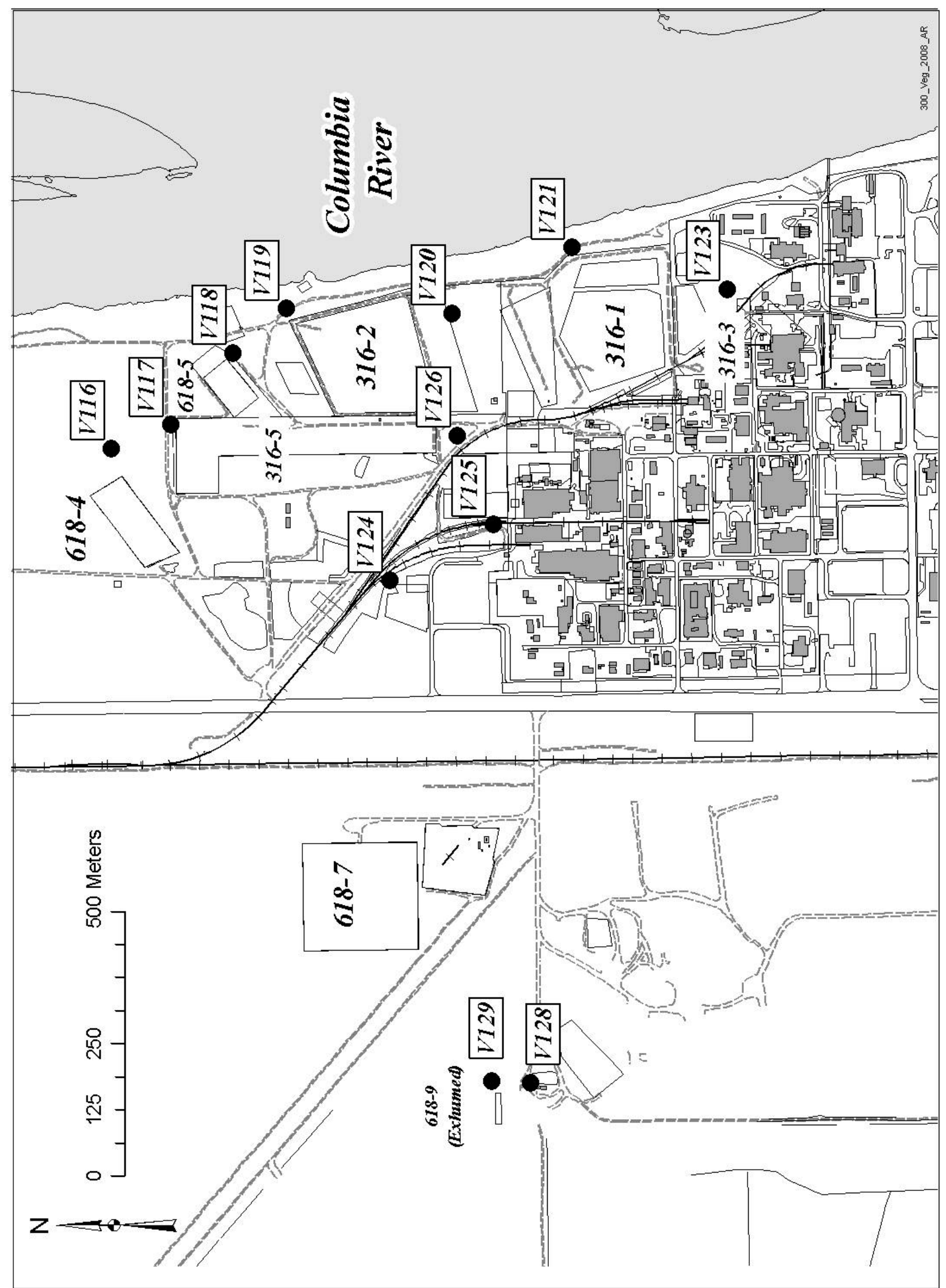


Figure 4-5. 2008 Vegetation Sampling Locations, 400 Area

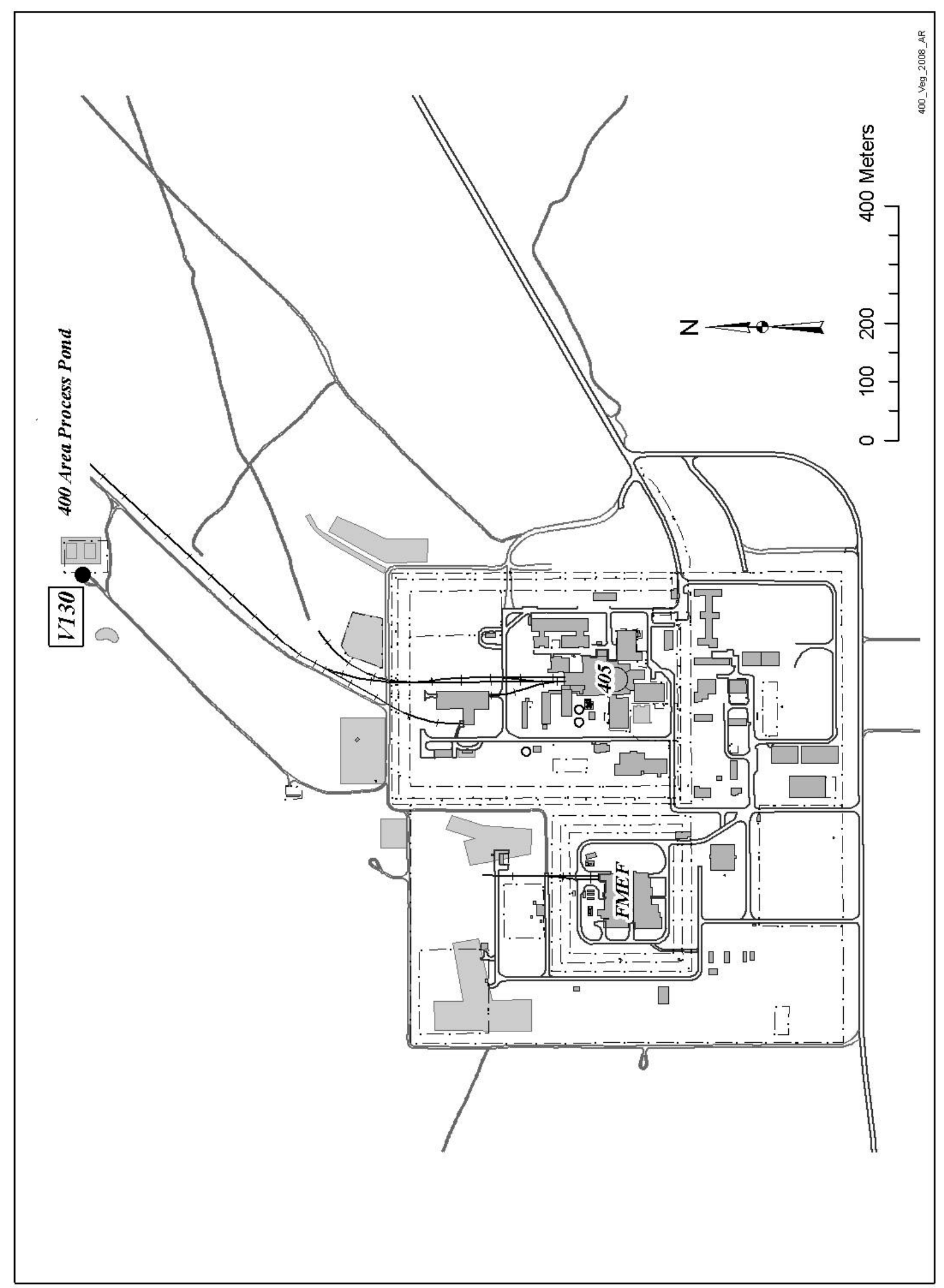


Figure 4-6. 2008 Vegetation Sampling Locations, 600 Area.

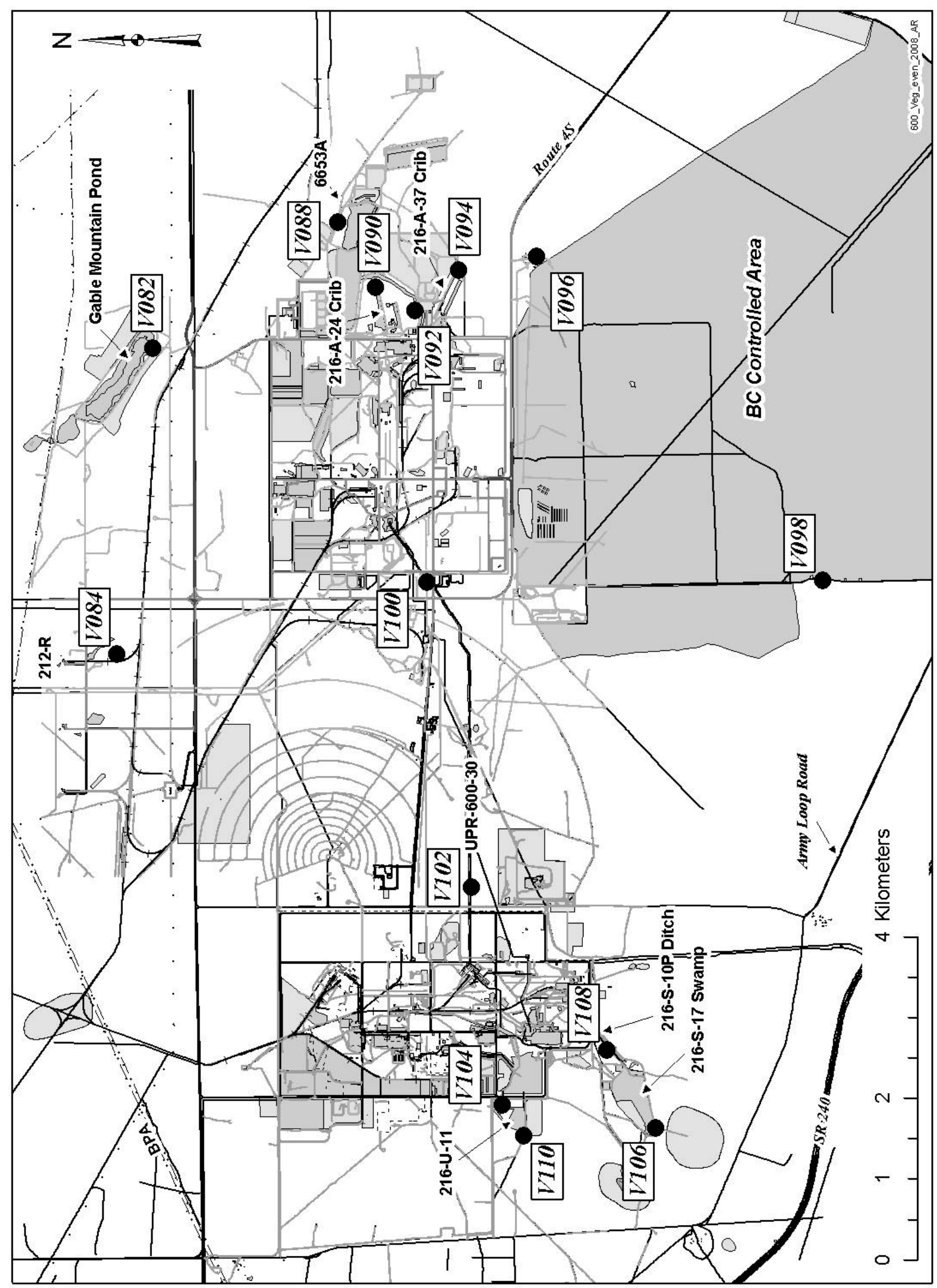


Table 4-2. Summary of Near-Facility Vegetation Sampling Results (pCi/g) ${ }^{\mathrm{a}}$ for Selected Radionuclides, 2008.

\begin{tabular}{ccccccc}
\multicolumn{2}{c}{ Number of $_{\text {Isotope }}$} & Samples $^{\mathbf{b}}$ & Detects & Average $^{\mathbf{c}}$ & Maximum $^{\mathbf{d}}$ & \multicolumn{2}{c}{ Location } \\
Area & Site \\
\hline${ }^{144} \mathrm{Ce}$ & 71 & 0 & $-8.8 \mathrm{E}-02 \pm 6.8 \mathrm{E}-01$ & $6.4 \mathrm{E}-01 \pm 5.0 \mathrm{E}-01^{\mathrm{e}}$ & 200 West & V038 \\
${ }^{60} \mathrm{Co}$ & 71 & 0 & $-8.9 \mathrm{E}-03 \pm 7.3 \mathrm{E}-02$ & $1.1 \mathrm{E}-01 \pm 9.5 \mathrm{E}-02^{\mathrm{e}}$ & 200 West & V026 \\
${ }^{134} \mathrm{Cs}$ & 71 & 0 & $8.2 \mathrm{E}-03 \pm 6.6 \mathrm{E}-02$ & $1.4 \mathrm{E}-01 \pm 2.5 \mathrm{E}-01^{\mathrm{e}}$ & 600 Area & V110 \\
${ }^{137} \mathrm{Cs}$ & 71 & 12 & $3.6 \mathrm{E}-02 \pm 1.2 \mathrm{E}-01$ & $2.2 \mathrm{E}-01 \pm 1.1 \mathrm{E}-01$ & 200 West & V042 \\
${ }^{152} \mathrm{Eu}$ & 71 & 1 & $-8.8 \mathrm{E}-04 \pm 1.8 \mathrm{E}-01$ & $3.7 \mathrm{E}-01 \pm 3.4 \mathrm{E}-01$ & 200 East & V076 \\
${ }^{154} \mathrm{Eu}$ & 71 & 0 & $-3.8 \mathrm{E}-02 \pm 3.1 \mathrm{E}-01$ & $2.1 \mathrm{E}-01 \pm 1.2 \mathrm{E}-01^{\mathrm{e}}$ & 200 East & V060 \\
${ }^{155} \mathrm{Eu}$ & 71 & 0 & $-3.9 \mathrm{E}-03 \pm 2.2 \mathrm{E}-01$ & $2.7 \mathrm{E}-01 \pm 2.2 \mathrm{E}-01^{\mathrm{e}}$ & 200 West & V042 \\
${ }^{238} \mathrm{Pu}$ & 71 & 6 & $3.7 \mathrm{E}-03 \pm 3.4 \mathrm{E}-02$ & $8.7 \mathrm{E}-02 \pm 4.7 \mathrm{E}-02$ & 300 Area & V120 \\
${ }^{239 / 240} \mathrm{Pu}$ & 71 & 17 & $1.4 \mathrm{E}-02 \pm 9.5 \mathrm{E}-02$ & $3.6 \mathrm{E}-01 \pm 9.7 \mathrm{E}-02$ & 200 West & V034 \\
${ }^{103} \mathrm{Ru}$ & 71 & 0 & $-7.7 \mathrm{E}-03 \pm 9.2 \mathrm{E}-02$ & $1.2 \mathrm{E}-01 \pm 1.2 \mathrm{E}-01^{\mathrm{e}}$ & 600 Area & V082 \\
${ }^{106} \mathrm{Ru}$ & 71 & 0 & $-4.9 \mathrm{E}-02 \pm 9.4 \mathrm{E}-01$ & $1.1 \mathrm{E}+00 \pm 8.9 \mathrm{E}-01^{\mathrm{e}}$ & 300 Area & V128 \\
${ }^{125} \mathrm{Sb}$ & 71 & 0 & $-5.5 \mathrm{E}-03 \pm 1.8 \mathrm{E}-01$ & $2.0 \mathrm{E}-01 \pm 2.0 \mathrm{E}-01^{\mathrm{e}}$ & 200 East & V054 \\
${ }^{113} \mathrm{Sn}$ & 71 & 0 & $-9.3 \mathrm{E}-03 \pm 6.5 \mathrm{E}-02$ & $7.3 \mathrm{E}-02 \pm 7.0 \mathrm{E}-02^{\mathrm{e}}$ & 300 Area & V138 \\
${ }^{90} \mathrm{Sr}$ & 71 & 3 & $-1.5 \mathrm{E}-01 \pm 6.9 \mathrm{E}-01$ & $6.5 \mathrm{E}-01 \pm 2.6 \mathrm{E}-01$ & 200 East & V058 \\
${ }^{234} \mathrm{U}$ & 71 & 66 & $1.7 \mathrm{E}-02 \pm 1.6 \mathrm{E}-02$ & $4.8 \mathrm{E}-02 \pm 1.8 \mathrm{E}-02$ & 300 Area & V119 \\
${ }^{235} \mathrm{U}$ & 71 & 20 & $3.7 \mathrm{E}-03 \pm 4.5 \mathrm{E}-03$ & $1.0 \mathrm{E}-02 \pm 7.5 \mathrm{E}-03$ & $100-\mathrm{N}$ & Y718 \\
${ }^{238} \mathrm{U}$ & 71 & 59 & $1.2 \mathrm{E}-02 \pm 1.8 \mathrm{E}-02$ & $5.5 \mathrm{E}-02 \pm 2.0 \mathrm{E}-02$ & $100-\mathrm{N}$ & V119 \\
${ }^{65} \mathrm{Zn}$ & 71 & 1 & $-3.3 \mathrm{E}-02 \pm 3.6 \mathrm{E}-01$ & $7.1 \mathrm{E}-01 \pm 2.3 \mathrm{E}-01$ & $100-\mathrm{N}$ & V118
\end{tabular}

\footnotetext{
${ }^{\mathrm{a}} 1 \mathrm{pCi}=0.037 \mathrm{~Bq}$.

${ }^{\mathrm{b}}$ Includes replicate samples

${ }^{\mathrm{c}}$ Average \pm two standard deviations

${ }^{\mathrm{d}}$ Maximum \pm analytical uncertainty

${ }^{\mathrm{e}}$ Maximum value reported is a non detect
} 
Table 4-3. Average Radionuclide Concentrations (pCi/g) ${ }^{\mathrm{a}}$ in Hanford Vegetation, 1998 through 2008.

\begin{tabular}{|c|c|c|c|c|c|c|}
\hline \multicolumn{7}{|c|}{ 100-N Area } \\
\hline Year & ${ }^{60} \mathrm{Co}$ & ${ }^{90} \mathrm{Sr}$ & ${ }^{137} \mathrm{Cs}$ & ${ }^{234} \mathbf{U}$ & ${ }^{238} \mathbf{U}$ & ${ }^{239,240} \mathrm{Pu}$ \\
\hline 1998 & $6.2 \mathrm{E}-01 \pm 6.5 \mathrm{E}-01$ & $1.2 \mathrm{E}+01 \pm 6.0 \mathrm{E}+00$ & $3.8 \mathrm{E}+01 \pm 6.5 \mathrm{E}+01$ & $1.4 \mathrm{E}-02 \pm 6.0 \mathrm{E}-03$ & $8.7 \mathrm{E}-03 \pm 4.4 \mathrm{E}-03$ & $4.2 \mathrm{E}-03 \pm 2.3 \mathrm{E}-03$ \\
\hline 1999 & $6.1 \mathrm{E}-01 \pm 5.9 \mathrm{E}-01$ & $9.1 \mathrm{E}+01 \pm 1.0 \mathrm{E}+02$ & $2.5 \mathrm{E}+02 \pm 2.5 \mathrm{E}+02$ & $2.8 \mathrm{E}-02 \pm 1.0 \mathrm{E}-03$ & $2.1 \mathrm{E}-02 \pm 7.0 \mathrm{E}-03$ & $2.2 \mathrm{E}-02 \pm 1.0 \mathrm{E}-02$ \\
\hline 2000 & $4.8 \mathrm{E}-02 \pm 3.2 \mathrm{E}-02$ & $5.7 \mathrm{E}+00 \pm 8.7 \mathrm{E}+00$ & $2.0 \mathrm{E}-01 \pm 1.2 \mathrm{E}-01$ & $3.3 \mathrm{E}-02 \pm 2.7 \mathrm{E}-02$ & $2.4 \mathrm{E}-02 \pm 1.8 \mathrm{E}-02$ & $9.1 \mathrm{E}-03 \pm 8.3 \mathrm{E}-03$ \\
\hline 2001 & $8.9 \mathrm{E}-01 \pm 1.3 \mathrm{E}+00$ & $3.5 \mathrm{E}+00 \pm 3.4 \mathrm{E}+00$ & $3.8 \mathrm{E}-01 \pm 2.2 \mathrm{E}-01$ & $9.8 \mathrm{E}-03 \pm 2.4 \mathrm{E}-03$ & $9.2 \mathrm{E}-03 \pm 2.9 \mathrm{E}-03$ & $2.4 \mathrm{E}-02 \pm 2.5 \mathrm{E}-02$ \\
\hline 2002 & 3.7E-03 \pm 3.7E-02 & $5.4 \mathrm{E}+00 \pm 1.8 \mathrm{E}+01$ & $2.4 \mathrm{E}-03 \pm 8.4 \mathrm{E}-03$ & $9.8 \mathrm{E}-03 \pm 4.5 \mathrm{E}-03$ & $5.1 \mathrm{E}-03 \pm 2.9 \mathrm{E}-03$ & $1.9 \mathrm{E}-03 \pm 5.3 \mathrm{E}-03$ \\
\hline 2003 & $6.6 \mathrm{E}-02 \pm 6.8 \mathrm{E}-02$ & $1.4 \mathrm{E}+01 \pm 4.5 \mathrm{E}+01$ & $1.5 \mathrm{E}-01 \pm 1.5 \mathrm{E}-01$ & $6.8 \mathrm{E}-03 \pm 2.1 \mathrm{E}-03$ & $4.6 \mathrm{E}-03 \pm 2.9 \mathrm{E}-03$ & $-2.8 \mathrm{E}-04 \pm 7.0 \mathrm{E}-03$ \\
\hline 2004 & $1.5 \mathrm{E}-02 \pm 1.8 \mathrm{E}-01$ & $1.1 \mathrm{E}+01 \pm 5.1 \mathrm{E}+01$ & $4.5 \mathrm{E}-02 \pm 8.7 \mathrm{E}-02$ & $9.3 \mathrm{E}-03 \pm 7.8 \mathrm{E}-03$ & $4.8 \mathrm{E}-03 \pm 2.7 \mathrm{E}-03$ & Not Detected \\
\hline 2005 & Not Detected & $5.4 \mathrm{E}+00 \pm 1.9 \mathrm{E}+01$ & Not Detected & $5.0 \mathrm{E}-03 \pm 2.3 \mathrm{E}-03$ & $5.8 \mathrm{E}-03 \pm 3.6 \mathrm{E}-03$ & Not Detected \\
\hline 2006 & Not Detected & $2.8 \mathrm{E}+00 \pm 7.4 \mathrm{E}+00$ & Not Detected & $1.2 \mathrm{E}-02 \pm 1.3 \mathrm{E}-02$ & $7.7 \mathrm{E}-03 \pm 9.9 \mathrm{E}-03$ & Not Detected \\
\hline 2007 & Not Detected & Not Detected & Not Detected & $1.6 \mathrm{E}-02 \pm 2.5 \mathrm{E}-03$ & $6.2 \mathrm{E}-03 \pm 8.3 \mathrm{E}-03$ & Not Detected \\
\hline 2008 & Not Detected & Not Detected & Not Detected & $7.2 \mathrm{E}-03 \pm 4.7 \mathrm{E}-03$ & $8.1 \mathrm{E}-03 \pm 1.9 \mathrm{E}-03$ & Not Detected \\
\hline \multicolumn{7}{|c|}{$\underline{200 / 600 ~ A r e a s ~}$} \\
\hline Year & ${ }^{60} \mathrm{Co}$ & ${ }^{90} \mathrm{Sr}$ & ${ }^{137} \mathrm{Cs}$ & ${ }^{234} \mathbf{U}$ & ${ }^{238} \mathbf{U}$ & ${ }^{239,240} \mathbf{P u}$ \\
\hline 1998 & Not Detected & $3.3 \mathrm{E}-01 \pm 1.3 \mathrm{E}-01$ & $2.1 \mathrm{E}-01 \pm 9.0 \mathrm{E}-02$ & $1.6 \mathrm{E}-02 \pm 3.0 \mathrm{E}-03$ & $9.7 \mathrm{E}-03 \pm 1.3 \mathrm{E}-03$ & $1.8 \mathrm{E}-02 \pm 8.0 \mathrm{E}-03$ \\
\hline 1999 & Not Detected & $7.9 \mathrm{E}-01 \pm 3.8 \mathrm{E}-01$ & $1.3 \mathrm{E}-01 \pm 4.0 \mathrm{E}-02$ & $3.3 \mathrm{E}-02 \pm 6.0 \mathrm{E}-03$ & $2.3 \mathrm{E}-02 \pm 4.0 \mathrm{E}-03$ & $1.4 \mathrm{E}-02 \pm 4.0 \mathrm{E}-03$ \\
\hline 2000 & Not Detected & $1.3 \mathrm{E}+00 \pm 8.0 \mathrm{E}-01$ & $1.6 \mathrm{E}-01 \pm 6.0 \mathrm{E}-02$ & $2.0 \mathrm{E}-02 \pm 3.0 \mathrm{E}-02$ & $1.4 \mathrm{E}-02 \pm 2.0 \mathrm{E}-03$ & $3.3 \mathrm{E}-02 \pm 2.8 \mathrm{E}-02$ \\
\hline 2001 & Not Detected & $1.0 \mathrm{E}+00 \pm 6.2 \mathrm{E}-01$ & $1.7 \mathrm{E}-01 \pm 6.5 \mathrm{E}-02$ & $1.9 \mathrm{E}-02 \pm 2.8 \mathrm{E}-03$ & $1.8 \mathrm{E}-02 \pm 2.6 \mathrm{E}-03$ & $2.1 \mathrm{E}-02 \pm 7.1 \mathrm{E}-03$ \\
\hline 2002 & $3.2 \mathrm{E}-04 \pm 1.8 \mathrm{E}-03$ & $3.2 \mathrm{E}-01 \pm 1.1 \mathrm{E}+00$ & $8.9 \mathrm{E}-02 \pm 4.2 \mathrm{E}-01$ & $1.6 \mathrm{E}-02 \pm 1.6 \mathrm{E}-02$ & $1.4 \mathrm{E}-02 \pm 1.5 \mathrm{E}-02$ & $8.8 \mathrm{E}-03 \pm 2.4 \mathrm{E}-02$ \\
\hline 2003 & $1.6 \mathrm{E}-02 \pm 2.1 \mathrm{E}-01$ & $1.5 \mathrm{E}+00 \pm 1.0 \mathrm{E}+01$ & $2.7 \mathrm{E}-01 \pm 2.0 \mathrm{E}+00$ & $1.0 \mathrm{E}-02 \pm 9.7 \mathrm{E}-03$ & $8.4 \mathrm{E}-03 \pm 9.0 \mathrm{E}-03$ & $2.7 \mathrm{E}-03 \pm 7.9 \mathrm{E}-03$ \\
\hline 2004 & Not Detected & $2.2 \mathrm{E}-01 \pm 8.8 \mathrm{E}+00$ & $4.2 \mathrm{E}-02 \pm 1.4 \mathrm{E}-01$ & $9.7 \mathrm{E}-03 \pm 1.0 \mathrm{E}-02$ & $8.2 \mathrm{E}-03 \pm 9.3 \mathrm{E}-03$ & $2.9 \mathrm{E}-03 \pm 1.0 \mathrm{E}-02$ \\
\hline 2005 & Not Detected & $1.4 \mathrm{E}-01 \pm 1.1 \mathrm{E}+00$ & $3.0 \mathrm{E}-02 \pm 1.3 \mathrm{E}-01$ & $\mathrm{E}-02 \pm 9.5 \mathrm{E}-03$ & $8.9 \mathrm{E}-03 \pm 9.7$ & $2.6 \mathrm{E}-03 \pm 6.6 \mathrm{E}-03$ \\
\hline 2006 & Not Detected & $3.2 \mathrm{E}-01 \pm 1.1 \mathrm{E}+00$ & $5.7 \mathrm{E}-02 \pm 5.0 \mathrm{E}-01$ & $1.6 \mathrm{E}-02 \pm 1.6 \mathrm{E}-02$ & $-02 \pm 1.5$ & $8.8 \mathrm{E}-03 \pm 2.4 \mathrm{E}-02$ \\
\hline 2007 & Not Detected & $-1.0 \mathrm{E}-01 \pm 3.4 \mathrm{E}-01$ & $3.4 \mathrm{E}-02 \pm 3.8 \mathrm{E}-01$ & $1.4 \mathrm{E}-02 \pm 1.3 \mathrm{E}-02$ & $1.2 \mathrm{E}-02 \pm 1.4 \mathrm{E}-02$ & $3.2 \mathrm{E}-03 \pm 1.1 \mathrm{E}-02$ \\
\hline 2008 & Not Detected & $-1.8 \mathrm{E}-01 \pm 7.8 \mathrm{E}-01$ & $4.6 \mathrm{E}-02 \pm 1.3 \mathrm{E}-01$ & $1.6 \mathrm{E}-02 \pm 1.0 \mathrm{E}-02$ & $1.0 \mathrm{E}-02 \pm 1.1 \mathrm{E}-02$ & $1.8 \mathrm{E}-02 \pm 1.1 \mathrm{E}-01$ \\
\hline \multicolumn{7}{|c|}{$\underline{300 / 400 \text { Areas }}$} \\
\hline Year & ${ }^{60} \mathrm{Co}$ & ${ }^{90} \mathrm{Sr}$ & ${ }^{137} \mathrm{Cs}$ & ${ }^{234} \mathbf{U}$ & ${ }^{238} \mathbf{U}$ & ${ }^{239,240} \mathbf{P u}$ \\
\hline 1998 & Not Detected & $1.0 \mathrm{E}-01 \pm 6.0 \mathrm{E}-02$ & Not Detected & $4.6 \mathrm{E}-02 \pm 3.3 \mathrm{E}-02$ & $4.4 \mathrm{E}-02 \pm 3.6 \mathrm{E}-02$ & $8.4 \mathrm{E}-03 \pm 4.5 \mathrm{E}-03$ \\
\hline 1999 & Not Detected & $4.5 \mathrm{E}-01 \pm 7.0 \mathrm{E}-02$ & Not Detected & $9.4 \mathrm{E}-02 \pm 5.3 \mathrm{E}-02$ & $8.9 \mathrm{E}-01 \pm 5.9 \mathrm{E}-02$ & $7.1 \mathrm{E}-03 \pm 3.2 \mathrm{E}-03$ \\
\hline 2000 & Not Detected & $2.1 \mathrm{E}-01 \pm 3.0 \mathrm{E}-02$ & Not Detected & $1.8 \mathrm{E}-02 \pm 1.9 \mathrm{E}-02$ & $1.7 \mathrm{E}-02 \pm 1.9 \mathrm{E}-02$ & $9.1 \mathrm{E}-03 \pm 2.4 \mathrm{E}-03$ \\
\hline 2001 & Not Detected & $2.6 \mathrm{E}-01 \pm 1.1 \mathrm{E}-01$ & Not Detected & $9.8 \mathrm{E}-02 \pm 8.0 \mathrm{E}-02$ & $1.1 \mathrm{E}-01 \pm 8.8 \mathrm{E}-02$ & $5.8 \mathrm{E}-03 \pm 1.5 \mathrm{E}-03$ \\
\hline 2002 & Not Detected & $2.1 \mathrm{E}-01 \pm 4.7 \mathrm{E}-01$ & $1.1 \mathrm{E}-02 \pm 7.9 \mathrm{E}-02$ & $3.2 \mathrm{E}-02 \pm 5.5 \mathrm{E}-02$ & $2.9 \mathrm{E}-02 \pm 5.8 \mathrm{E}-02$ & $-3.6 \mathrm{E}-04 \pm 7.2 \mathrm{E}-04$ \\
\hline 2003 & $5.0 \mathrm{E}-03 \pm 3.8 \mathrm{E}-02$ & $-8.2 \mathrm{E}-02 \pm 2.0 \mathrm{E}-01$ & $-9.4 \mathrm{E}-03 \pm 4.4 \mathrm{E}-02$ & $4.3 \mathrm{E}-02 \pm 1.1 \mathrm{E}-01$ & $3.6 \mathrm{E}-02 \pm 1.9 \mathrm{E}-01$ & $1.7 \mathrm{E}-03 \pm 1.7 \mathrm{E}-02$ \\
\hline 2004 & Not Detected & Not Detected & Not Detected & $3.3 \mathrm{E}-01 \pm 8.8 \mathrm{E}-02$ & $2.5 \mathrm{E}-02 \pm 7.3 \mathrm{E}-02$ & Not Detected \\
\hline 2005 & Not Detected & Not Detected & Not Detected & $3.0 \mathrm{E}-02 \pm 6.7 \mathrm{E}-02$ & $2.4 \mathrm{E}-02 \pm 5.9 \mathrm{E}-02$ & $3.8 \mathrm{E}-03 \pm 8.9 \mathrm{E}-03$ \\
\hline 2006 & Not Detected & Not Detected & Not Detected & $4.2 \mathrm{E}-02 \pm 1.1 \mathrm{E}-01$ & $3.6 \mathrm{E}-02 \pm 1.0 \mathrm{E}-01$ & $2.8 \mathrm{E}-03 \pm 6.6 \mathrm{E}-03$ \\
\hline 2007 & Not Detected & Not Detected & Not Detected & $2.3 \mathrm{E}-02 \pm 2.6 \mathrm{E}-02$ & $1.9 \mathrm{E}-02 \pm 2.6 \mathrm{E}-02$ & Not Detected \\
\hline 2008 & Not Detected & Not Detected & Not Detected & $2.4 \mathrm{E}-02 \pm 2.1 \mathrm{E}-02$ & $1.9 \mathrm{E}-02 \pm 2.9 \mathrm{E}-02$ & $2.3 \mathrm{E}-03 \pm 7.7 \mathrm{E}-03$ \\
\hline
\end{tabular}

$\overline{\text { (a) } \pm 2 \text { standard deviations }}$ 
Table 4-4. 2008 Vegetation Sampling Results (pCi/g \pm total analytical uncertainty). (Sheet 1 of 18)

\begin{tabular}{|c|c|c|c|c|c|c|c|}
\hline Location & Isotope & Result \pm Error & $\mathbf{R Q}^{*}$ & Location & Is otope & Result \pm Error & $\mathbf{R Q *}$ \\
\hline Y718 & ${ }^{144} \mathrm{Ce}$ & $-1.4 \mathrm{E}-01 \pm 5.3 \mathrm{E}-01$ & $\mathrm{U}$ & Y719 & ${ }^{144} \mathrm{Ce}$ & $1.0 \mathrm{E}-01 \pm 4.5 \mathrm{E}-01$ & $\mathrm{U}$ \\
\hline (N Springs & ${ }^{60} \mathrm{Co}$ & $-5.1 \mathrm{E}-02 \pm 5.1 \mathrm{E}-02$ & $\mathrm{U}$ & (N Springs & ${ }^{60} \mathrm{Co}$ & $3.0 \mathrm{E}-02 \pm 3.6 \mathrm{E}-02$ & $\mathrm{U}$ \\
\hline \multirow[t]{16}{*}{ Shoreline) } & ${ }^{134} \mathrm{Cs}$ & $2.1 \mathrm{E}-02 \pm 4.1 \mathrm{E}-02$ & $\mathrm{U}$ & Shoreline) & ${ }^{134} \mathrm{Cs}$ & $3.6 \mathrm{E}-03 \pm 3.6 \mathrm{E}-02$ & $\mathrm{U}$ \\
\hline & ${ }^{137} \mathrm{Cs}$ & $-4.5 \mathrm{E}-02 \pm 4.5 \mathrm{E}-02$ & $\mathrm{U}$ & & ${ }^{137} \mathrm{Cs}$ & $-7.7 \mathrm{E}-03 \pm 3.9 \mathrm{E}-02$ & $\mathrm{U}$ \\
\hline & ${ }^{152} \mathrm{Eu}$ & $-1.3 \mathrm{E}-02 \pm 1.2 \mathrm{E}-01$ & $\mathrm{U}$ & & ${ }^{152} \mathrm{Eu}$ & $-5.5 \mathrm{E}-02 \pm 1.0 \mathrm{E}-01$ & $\mathrm{U}$ \\
\hline & ${ }^{154} \mathrm{Eu}$ & $-3.0 \mathrm{E}-02 \pm 1.4 \mathrm{E}-01$ & $\mathrm{U}$ & & ${ }^{154} \mathrm{Eu}$ & $-1.3 \mathrm{E}-01 \pm 1.3 \mathrm{E}-01$ & $\mathrm{U}$ \\
\hline & ${ }^{155} \mathrm{Eu}$ & $2.8 \mathrm{E}-02 \pm 1.2 \mathrm{E}-01$ & $\mathrm{U}$ & & ${ }^{155} \mathrm{Eu}$ & $1.6 \mathrm{E}-02 \pm 1.0 \mathrm{E}-01$ & $\mathrm{U}$ \\
\hline & ${ }^{238} \mathrm{Pu}$ & $1.7 \mathrm{E}-03 \pm 2.4 \mathrm{E}-03$ & $\mathrm{U}$ & & ${ }^{238} \mathrm{Pu}$ & $-1.8 \mathrm{E}-03 \pm 3.6 \mathrm{E}-03$ & $\mathrm{U}$ \\
\hline & ${ }^{239 / 240} \mathrm{Pu}$ & 8.7E-04 $\pm 1.7 \mathrm{E}-03$ & $\mathrm{U}$ & & ${ }^{239 / 240} \mathrm{Pu}$ & $9.2 \mathrm{E}-04 \pm 9.2 \mathrm{E}-03$ & $\mathrm{U}$ \\
\hline & ${ }^{103} \mathrm{Ru}$ & $-1.5 \mathrm{E}-02 \pm 4.8 \mathrm{E}-02$ & $\mathrm{U}$ & & ${ }^{103} \mathrm{Ru}$ & $-2.9 \mathrm{E}-02 \pm 4.7 \mathrm{E}-02$ & $\mathrm{U}$ \\
\hline & ${ }^{106} \mathrm{Ru}$ & $3.2 \mathrm{E}-01 \pm 3.6 \mathrm{E}-01$ & $\mathrm{U}$ & & ${ }^{106} \mathrm{Ru}$ & $-8.1 \mathrm{E}-02 \pm 3.2 \mathrm{E}-01$ & $\mathrm{U}$ \\
\hline & ${ }^{125} \mathrm{Sb}$ & $-6.6 \mathrm{E}-02 \pm 1.3 \mathrm{E}-01$ & $\mathrm{U}$ & & ${ }^{125} \mathrm{Sb}$ & $-8.1 \mathrm{E}-02 \pm 1.1 \mathrm{E}-01$ & $\mathrm{U}$ \\
\hline & ${ }^{113} \mathrm{Sn}$ & $-4.5 \mathrm{E}-02 \pm 5.7 \mathrm{E}-02$ & $\mathrm{U}$ & & ${ }^{113} \mathrm{Sn}$ & $-4.3 \mathrm{E}-02 \pm 4.8 \mathrm{E}-02$ & $\mathrm{U}$ \\
\hline & ${ }^{90} \mathrm{Sr}$ & $4.0 \mathrm{E}-03 \pm 4.0 \mathrm{E}-02$ & $\mathrm{U}$ & & ${ }^{90} \mathrm{Sr}$ & $4.1 \mathrm{E}-01 \pm 2.3 \mathrm{E}-01$ & \\
\hline & ${ }^{234} \mathrm{U}$ & $4.3 \mathrm{E}-03 \pm 6.3 \mathrm{E}-03$ & $\mathrm{U}$ & & ${ }^{234} \mathrm{U}$ & $7.2 \mathrm{E}-03 \pm 7.0 \mathrm{E}-03$ & $\mathrm{U}$ \\
\hline & ${ }^{235} \mathrm{U}$ & $1.0 \mathrm{E}-02 \pm 7.5 \mathrm{E}-03$ & & & ${ }^{235} \mathrm{U}$ & $4.9 \mathrm{E}-03 \pm 4.6 \mathrm{E}-03$ & \\
\hline & ${ }^{238} \mathrm{U}$ & $8.5 \mathrm{E}-03 \pm 5.8 \mathrm{E}-03$ & & & ${ }^{238} \mathrm{U}$ & $9.0 \mathrm{E}-03 \pm 6.1 \mathrm{E}-03$ & \\
\hline & ${ }^{65} \mathrm{Zn}$ & $6.0 \mathrm{E}-02 \pm 1.0 \mathrm{E}-01$ & $\mathrm{U}$ & & ${ }^{65} \mathrm{Zn}$ & $-2.2 \mathrm{E}-01 \pm 2.2 \mathrm{E}-01$ & $\mathrm{U}$ \\
\hline Y724 & ${ }^{144} \mathrm{Ce}$ & $1.5 \mathrm{E}-01 \pm 5.2 \mathrm{E}-01$ & $\mathrm{U}$ & V002 & ${ }^{144} \mathrm{Ce}$ & $2.9 \mathrm{E}-01 \pm 9.9 \mathrm{E}-01$ & $\mathrm{U}$ \\
\hline (N Springs & ${ }^{60} \mathrm{Co}$ & $8.6 \mathrm{E}-03 \pm 4.2 \mathrm{E}-02$ & $\mathrm{U}$ & $(200-W)$ & ${ }^{60} \mathrm{Co}$ & $-5.2 \mathrm{E}-03 \pm 5.2 \mathrm{E}-02$ & $\mathrm{U}$ \\
\hline \multirow[t]{16}{*}{ Shoreline) } & ${ }^{134} \mathrm{Cs}$ & $2.6 \mathrm{E}-03 \pm 2.6 \mathrm{E}-02$ & $\mathrm{U}$ & & ${ }^{134} \mathrm{Cs}$ & 4.8E-02 $\pm 9.0 \mathrm{E}-02$ & $\mathrm{U}$ \\
\hline & ${ }^{137} \mathrm{Cs}$ & $-7.1 \mathrm{E}-03 \pm 4.2 \mathrm{E}-02$ & $\mathrm{U}$ & & ${ }^{137} \mathrm{Cs}$ & $2.8 \mathrm{E}-02 \pm 9.3 \mathrm{E}-02$ & $\mathrm{U}$ \\
\hline & ${ }^{152} \mathrm{Eu}$ & $-2.5 \mathrm{E}-02 \pm 1.2 \mathrm{E}-01$ & $\mathrm{U}$ & & ${ }^{152} \mathrm{Eu}$ & $5.4 \mathrm{E}-02 \pm 2.5 \mathrm{E}-01$ & $\mathrm{U}$ \\
\hline & ${ }^{154} \mathrm{Eu}$ & $5.2 \mathrm{E}-03 \pm 5.2 \mathrm{E}-02$ & $\mathrm{U}$ & & ${ }^{154} \mathrm{Eu}$ & $-1.8 \mathrm{E}-01 \pm 2.4 \mathrm{E}-01$ & $\mathrm{U}$ \\
\hline & ${ }^{155} \mathrm{Eu}$ & $-2.1 \mathrm{E}-02 \pm 1.2 \mathrm{E}-01$ & $\mathrm{U}$ & & ${ }^{155} \mathrm{Eu}$ & $-4.0 \mathrm{E}-02 \pm 2.5 \mathrm{E}-01$ & $\mathrm{U}$ \\
\hline & ${ }^{238} \mathrm{Pu}$ & $2.3 \mathrm{E}-03 \pm 3.5 \mathrm{E}-03$ & $\mathrm{U}$ & & ${ }^{238} \mathrm{Pu}$ & $-5.5 \mathrm{E}-03 \pm 7.3 \mathrm{E}-03$ & $\mathrm{U}$ \\
\hline & ${ }^{239 / 240} \mathrm{Pu}$ & $-7.8 \mathrm{E}-04 \pm 2.7 \mathrm{E}-03$ & $\mathrm{U}$ & & ${ }^{239 / 240} \mathrm{Pu}$ & $7.9 \mathrm{E}-04 \pm 3.5 \mathrm{E}-03$ & $\mathrm{U}$ \\
\hline & ${ }^{103} \mathrm{Ru}$ & $1.7 \mathrm{E}-02 \pm 5.2 \mathrm{E}-02$ & $\mathrm{U}$ & & ${ }^{103} \mathrm{Ru}$ & $5.6 \mathrm{E}-04 \pm 5.6 \mathrm{E}-03$ & $\mathrm{U}$ \\
\hline & ${ }^{106} \mathrm{Ru}$ & $6.6 \mathrm{E}-02 \pm 3.6 \mathrm{E}-01$ & $\mathrm{U}$ & & ${ }^{106} \mathrm{Ru}$ & $4.8 \mathrm{E}-01 \pm 8.2 \mathrm{E}-01$ & $\mathrm{U}$ \\
\hline & ${ }^{125} \mathrm{Sb}$ & $-7.8 \mathrm{E}-02 \pm 1.3 \mathrm{E}-01$ & $\mathrm{U}$ & & ${ }^{125} \mathrm{Sb}$ & $-1.3 \mathrm{E}-01 \pm 2.7 \mathrm{E}-01$ & $\mathrm{U}$ \\
\hline & ${ }^{113} \mathrm{Sn}$ & $4.0 \mathrm{E}-02 \pm 5.4 \mathrm{E}-02$ & $\mathrm{U}$ & & ${ }^{113} \mathrm{Sn}$ & $-1.0 \mathrm{E}-02 \pm 1.0 \mathrm{E}-01$ & $\mathrm{U}$ \\
\hline & ${ }^{90} \mathrm{Sr}$ & $-2.1 \mathrm{E}-01 \pm 2.2 \mathrm{E}-01$ & $\mathrm{U}$ & & ${ }^{90} \mathrm{Sr}$ & $2.0 \mathrm{E}-03 \pm 2.0 \mathrm{E}-02$ & $\mathrm{U}$ \\
\hline & ${ }^{234} \mathrm{U}$ & $1.0 \mathrm{E}-02 \pm 7.1 \mathrm{E}-03$ & & & ${ }^{234} \mathrm{U}$ & $1.1 \mathrm{E}-02 \pm 8.2 \mathrm{E}-03$ & \\
\hline & ${ }^{235} \mathrm{U}$ & $2.8 \mathrm{E}-03 \pm 3.3 \mathrm{E}-03$ & & & ${ }^{235} \mathrm{U}$ & $9.6 \mathrm{E}-04 \pm 3.3 \mathrm{E}-03$ & $\mathrm{U}$ \\
\hline & ${ }^{238} \mathrm{U}$ & $6.8 \mathrm{E}-03 \pm 5.1 \mathrm{E}-03$ & & & ${ }^{238} \mathrm{U}$ & $4.4 \mathrm{E}-03 \pm 4.8 \mathrm{E}-03$ & $\mathrm{U}$ \\
\hline & ${ }^{65} \mathrm{Zn}$ & 8.3E-04 $\pm 8.3 \mathrm{E}-03$ & $\mathrm{U}$ & & ${ }^{65} \mathrm{Zn}$ & $-1.7 \mathrm{E}-01 \pm 1.9 \mathrm{E}-01$ & $\mathrm{U}$ \\
\hline
\end{tabular}

RQ = Result Qualifier. $\mathrm{U}=$ The analyte was analyzed for but not detected. 
Table 4-4. 2008 Vegetation Sampling Results (pCi/g \pm total analytical uncertainty). (Sheet 2 of 18)

\begin{tabular}{|c|c|c|c|c|c|c|c|}
\hline Location & Isotope & Result \pm Error & $\mathbf{R Q *}$ & Location & Isotope & Result \pm Error & $\mathbf{R Q} *$ \\
\hline V004 & ${ }^{144} \mathrm{Ce}$ & 2.3E-01 \pm 3.3E-01 & $\mathrm{U}$ & V006 & ${ }^{144} \mathrm{Ce}$ & $1.2 \mathrm{E}-01 \pm 4.7 \mathrm{E}-01$ & $\mathrm{U}$ \\
\hline \multirow[t]{17}{*}{$(200-W)$} & ${ }^{60} \mathrm{Co}$ & $4.2 \mathrm{E}-03 \pm 2.4 \mathrm{E}-02$ & $\mathrm{U}$ & $(200-W)$ & ${ }^{60} \mathrm{Co}$ & $-7.5 \mathrm{E}-03 \pm 3.8 \mathrm{E}-02$ & $\mathrm{U}$ \\
\hline & ${ }^{134} \mathrm{Cs}$ & $1.4 \mathrm{E}-02 \pm 2.5 \mathrm{E}-02$ & $\mathrm{U}$ & & ${ }^{134} \mathrm{Cs}$ & $5.3 \mathrm{E}-03 \pm 4.0 \mathrm{E}-02$ & $\mathrm{U}$ \\
\hline & ${ }^{137} \mathrm{Cs}$ & 6.7E-02 \pm 3.9E-02 & & & ${ }^{137} \mathrm{Cs}$ & $-6.2 \mathrm{E}-03 \pm 4.0 \mathrm{E}-02$ & $\mathrm{U}$ \\
\hline & ${ }^{152} \mathrm{Eu}$ & $-5.8 \mathrm{E}-02 \pm 6.9 \mathrm{E}-02$ & $\mathrm{U}$ & & ${ }^{152} \mathrm{Eu}$ & $-8.2 \mathrm{E}-02 \pm 1.1 \mathrm{E}-01$ & $\mathrm{U}$ \\
\hline & ${ }^{154} \mathrm{Eu}$ & $1.2 \mathrm{E}-02 \pm 7.4 \mathrm{E}-02$ & $\mathrm{U}$ & & ${ }^{154} \mathrm{Eu}$ & $-5.9 \mathrm{E}-02 \pm 1.0 \mathrm{E}-01$ & $\mathrm{U}$ \\
\hline & ${ }^{155} \mathrm{Eu}$ & $4.1 \mathrm{E}-02 \pm 7.4 \mathrm{E}-02$ & $\mathrm{U}$ & & ${ }^{155} \mathrm{Eu}$ & $-1.7 \mathrm{E}-02 \pm 1.1 \mathrm{E}-01$ & $\mathrm{U}$ \\
\hline & ${ }^{238} \mathrm{Pu}$ & $5.5 \mathrm{E}-03 \pm 5.9 \mathrm{E}-03$ & $\mathrm{U}$ & & ${ }^{238} \mathrm{Pu}$ & $3.1 \mathrm{E}-03 \pm 4.7 \mathrm{E}-03$ & $\mathrm{U}$ \\
\hline & ${ }^{239 / 240} \mathrm{Pu}$ & $6.6 \mathrm{E}-03 \pm 6.4 \mathrm{E}-03$ & $\mathrm{U}$ & & ${ }^{239 / 240} \mathrm{Pu}$ & $2.1 \mathrm{E}-03 \pm 3.0 \mathrm{E}-03$ & $\mathrm{U}$ \\
\hline & ${ }^{103} \mathrm{Ru}$ & $2.2 \mathrm{E}-02 \pm 2.7 \mathrm{E}-02$ & $\mathrm{U}$ & & ${ }^{103} \mathrm{Ru}$ & $-4.3 \mathrm{E}-03 \pm 4.3 \mathrm{E}-02$ & $\mathrm{U}$ \\
\hline & ${ }^{106} \mathrm{Ru}$ & $-2.0 \mathrm{E}-01 \pm 2.2 \mathrm{E}-01$ & $\mathrm{U}$ & & ${ }^{106} \mathrm{Ru}$ & $-1.8 \mathrm{E}-01 \pm 3.7 \mathrm{E}-01$ & $\mathrm{U}$ \\
\hline & ${ }^{125} \mathrm{Sb}$ & $3.5 \mathrm{E}-02 \pm 7.9 \mathrm{E}-02$ & $\mathrm{U}$ & & ${ }^{125} \mathrm{Sb}$ & $5.4 \mathrm{E}-03 \pm 5.4 \mathrm{E}-02$ & $\mathrm{U}$ \\
\hline & ${ }^{113} \mathrm{Sn}$ & $-1.1 \mathrm{E}-02 \pm 3.3 \mathrm{E}-02$ & $\mathrm{U}$ & & ${ }^{113} \mathrm{Sn}$ & $3.7 \mathrm{E}-02 \pm 5.1 \mathrm{E}-02$ & $\mathrm{U}$ \\
\hline & ${ }^{90} \mathrm{Sr}$ & $-5.1 \mathrm{E}-02 \pm 2.3 \mathrm{E}-01$ & $\mathrm{U}$ & & ${ }^{90} \mathrm{Sr}$ & $-4.2 \mathrm{E}-01 \pm 4.2 \mathrm{E}-01$ & $\mathrm{U}$ \\
\hline & ${ }^{234} \mathrm{U}$ & $1.1 \mathrm{E}-02 \pm 7.4 \mathrm{E}-03$ & & & ${ }^{234} \mathrm{U}$ & $9.0 \mathrm{E}-03 \pm 7.6 \mathrm{E}-03$ & $\mathrm{U}$ \\
\hline & ${ }^{235} \mathrm{U}$ & $-9.8 \mathrm{E}-08 \pm 9.8 \mathrm{E}-07$ & $\mathrm{U}$ & & ${ }^{235} \mathrm{U}$ & 3.3E-03 $\pm 3.9 \mathrm{E}-03$ & \\
\hline & ${ }^{238} \mathrm{U}$ & $9.9 \mathrm{E}-03 \pm 6.5 \mathrm{E}-03$ & & & ${ }^{238} \mathrm{U}$ & $1.4 \mathrm{E}-02 \pm 8.3 \mathrm{E}-03$ & \\
\hline & ${ }^{65} \mathrm{Zn}$ & $-1.2 \mathrm{E}-01 \pm 1.2 \mathrm{E}-01$ & $\mathrm{U}$ & & ${ }^{65} \mathrm{Zn}$ & $-1.9 \mathrm{E}-01 \pm 1.9 \mathrm{E}-01$ & $\mathrm{U}$ \\
\hline V008 & ${ }^{144} \mathrm{Ce}$ & $-5.7 \mathrm{E}-02 \pm 5.7 \mathrm{E}-01$ & $\mathrm{U}$ & V010 & ${ }^{144} \mathrm{Ce}$ & $-4.2 \mathrm{E}-01 \pm 5.3 \mathrm{E}-01$ & $\mathrm{U}$ \\
\hline \multirow[t]{17}{*}{$(200-W)$} & ${ }^{60} \mathrm{Co}$ & $1.4 \mathrm{E}-02 \pm 5.7 \mathrm{E}-02$ & $\mathrm{U}$ & $(200-W)$ & ${ }^{60} \mathrm{Co}$ & 4.6E-03 $\pm 4.5 \mathrm{E}-02$ & $\mathrm{U}$ \\
\hline & ${ }^{134} \mathrm{Cs}$ & $-2.3 \mathrm{E}-02 \pm 5.6 \mathrm{E}-02$ & $\mathrm{U}$ & & ${ }^{134} \mathrm{Cs}$ & $2.8 \mathrm{E}-02 \pm 5.6 \mathrm{E}-02$ & $\mathrm{U}$ \\
\hline & ${ }^{137} \mathrm{Cs}$ & $2.7 \mathrm{E}-02 \pm 6.1 \mathrm{E}-02$ & $\mathrm{U}$ & & ${ }^{137} \mathrm{Cs}$ & $2.0 \mathrm{E}-02 \pm 5.5 \mathrm{E}-02$ & $\mathrm{U}$ \\
\hline & ${ }^{152} \mathrm{Eu}$ & $1.6 \mathrm{E}-01 \pm 1.6 \mathrm{E}-01$ & $\mathrm{U}$ & & ${ }^{152} \mathrm{Eu}$ & $-6.1 \mathrm{E}-03 \pm 6.1 \mathrm{E}-02$ & $\mathrm{U}$ \\
\hline & ${ }^{154} \mathrm{Eu}$ & $-1.0 \mathrm{E}-01 \pm 1.8 \mathrm{E}-01$ & $\mathrm{U}$ & & ${ }^{154} \mathrm{Eu}$ & $-1.6 \mathrm{E}-02 \pm 1.6 \mathrm{E}-01$ & $\mathrm{U}$ \\
\hline & ${ }^{155} \mathrm{Eu}$ & $5.6 \mathrm{E}-02 \pm 1.7 \mathrm{E}-01$ & $\mathrm{U}$ & & ${ }^{155} \mathrm{Eu}$ & $-8.2 \mathrm{E}-02 \pm 1.4 \mathrm{E}-01$ & $\mathrm{U}$ \\
\hline & ${ }^{238} \mathrm{Pu}$ & $8.1 \mathrm{E}-04 \pm 8.1 \mathrm{E}-04$ & $\mathrm{U}$ & & ${ }^{238} \mathrm{Pu}$ & $1.9 \mathrm{E}-02 \pm 9.3 \mathrm{E}-03$ & \\
\hline & ${ }^{239 / 240} \mathrm{Pu}$ & $1.9 \mathrm{E}-02 \pm 9.1 \mathrm{E}-03$ & & & ${ }^{239 / 240} \mathrm{Pu}$ & $1.8 \mathrm{E}-01 \pm 4.7 \mathrm{E}-02$ & \\
\hline & ${ }^{103} \mathrm{Ru}$ & $-3.1 \mathrm{E}-02 \pm 6.6 \mathrm{E}-02$ & $\mathrm{U}$ & & ${ }^{103} \mathrm{Ru}$ & $-1.3 \mathrm{E}-02 \pm 5.5 \mathrm{E}-02$ & $\mathrm{U}$ \\
\hline & ${ }^{106} \mathrm{Ru}$ & $-1.4 \mathrm{E}-01 \pm 5.1 \mathrm{E}-01$ & $\mathrm{U}$ & & ${ }^{106} \mathrm{Ru}$ & $-2.9 \mathrm{E}-01 \pm 4.9 \mathrm{E}-01$ & $\mathrm{U}$ \\
\hline & ${ }^{125} \mathrm{Sb}$ & $-9.3 \mathrm{E}-02 \pm 1.9 \mathrm{E}-01$ & $\mathrm{U}$ & & ${ }^{125} \mathrm{Sb}$ & $6.9 \mathrm{E}-02 \pm 1.4 \mathrm{E}-01$ & $\mathrm{U}$ \\
\hline & ${ }^{113} \mathrm{Sn}$ & $2.1 \mathrm{E}-02 \pm 7.5 \mathrm{E}-02$ & $\mathrm{U}$ & & ${ }^{113} \mathrm{Sn}$ & $1.9 \mathrm{E}-02 \pm 6.2 \mathrm{E}-02$ & $\mathrm{U}$ \\
\hline & ${ }^{90} \mathrm{Sr}$ & $-2.3 \mathrm{E}-01 \pm 2.3 \mathrm{E}-01$ & $\mathrm{U}$ & & ${ }^{90} \mathrm{Sr}$ & $-2.6 \mathrm{E}-01 \pm 2.6 \mathrm{E}-01$ & $\mathrm{U}$ \\
\hline & ${ }^{234} \mathrm{U}$ & $7.0 \mathrm{E}-03 \pm 5.8 \mathrm{E}-03$ & & & ${ }^{234} \mathrm{U}$ & $7.8 \mathrm{E}-03 \pm 5.5 \mathrm{E}-03$ & \\
\hline & ${ }^{235} \mathrm{U}$ & $8.6 \mathrm{E}-03 \pm 6.1 \mathrm{E}-03$ & & & ${ }^{235} \mathrm{U}$ & $9.4 \mathrm{E}-04 \pm 1.9 \mathrm{E}-03$ & $\mathrm{U}$ \\
\hline & ${ }^{238} \mathrm{U}$ & $3.5 \mathrm{E}-03 \pm 3.6 \mathrm{E}-03$ & & & ${ }^{238} \mathrm{U}$ & $2.3 \mathrm{E}-02 \pm 1.1 \mathrm{E}-02$ & \\
\hline & ${ }^{65} \mathrm{Zn}$ & $-1.5 \mathrm{E}-01 \pm 1.5 \mathrm{E}-01$ & $\mathrm{U}$ & & ${ }^{65} \mathrm{Zn}$ & $-1.8 \mathrm{E}-01 \pm 1.8 \mathrm{E}+00$ & $\mathrm{U}$ \\
\hline
\end{tabular}

RQ = Result Qualifier. $\mathrm{U}=$ The analyte was analyzed for but not detected. 
Table 4-4. 2008 Vegetation Sampling Results (pCi/g \pm total analytical uncertainty). (Sheet 3 of 18)

\begin{tabular}{|c|c|c|c|c|c|c|c|}
\hline Location & Is otope & Result \pm Error & $\mathbf{R Q}^{*}$ & Location & Is otope & Result \pm Error & $\mathbf{R Q *}$ \\
\hline V012 & ${ }^{144} \mathrm{Ce}$ & $-1.5 \mathrm{E}-02 \pm 1.5 \mathrm{E}-01$ & $\mathrm{U}$ & V016 & ${ }^{144} \mathrm{Ce}$ & $5.4 \mathrm{E}-01 \pm 9.0 \mathrm{E}-01$ & $\mathrm{U}$ \\
\hline \multirow[t]{17}{*}{$(200-W)$} & ${ }^{60} \mathrm{Co}$ & $8.4 \mathrm{E}-04 \pm 8.4 \mathrm{E}-03$ & $\mathrm{U}$ & $(200-W)$ & ${ }^{60} \mathrm{Co}$ & $-5.7 \mathrm{E}-02 \pm 7.5 \mathrm{E}-02$ & $\mathrm{U}$ \\
\hline & ${ }^{134} \mathrm{Cs}$ & $6.8 \mathrm{E}-03 \pm 4.0 \mathrm{E}-02$ & $\mathrm{U}$ & & ${ }^{134} \mathrm{Cs}$ & $-1.9 \mathrm{E}-02 \pm 8.7 \mathrm{E}-02$ & $\mathrm{U}$ \\
\hline & ${ }^{137} \mathrm{Cs}$ & $8.4 \mathrm{E}-02 \pm 4.7 \mathrm{E}-02$ & & & ${ }^{137} \mathrm{Cs}$ & $1.4 \mathrm{E}-01 \pm 1.2 \mathrm{E}-01$ & \\
\hline & ${ }^{152} \mathrm{Eu}$ & $3.7 \mathrm{E}-03 \pm 3.7 \mathrm{E}-02$ & $\mathrm{U}$ & & ${ }^{152} \mathrm{Eu}$ & $-8.9 \mathrm{E}-03 \pm 8.9 \mathrm{E}-02$ & $\mathrm{U}$ \\
\hline & ${ }^{154} \mathrm{Eu}$ & $-1.2 \mathrm{E}-02 \pm 1.1 \mathrm{E}-01$ & $\mathrm{U}$ & & ${ }^{154} \mathrm{Eu}$ & $-5.6 \mathrm{E}-02 \pm 2.5 \mathrm{E}-01$ & $\mathrm{U}$ \\
\hline & ${ }^{155} \mathrm{Eu}$ & $-3.3 \mathrm{E}-02 \pm 9.5 \mathrm{E}-02$ & $\mathrm{U}$ & & ${ }^{155} \mathrm{Eu}$ & $-7.4 \mathrm{E}-02 \pm 2.1 \mathrm{E}-01$ & $\mathrm{U}$ \\
\hline & ${ }^{238} \mathrm{Pu}$ & $2.9 \mathrm{E}-03 \pm 6.4 \mathrm{E}-03$ & $\mathrm{U}$ & & ${ }^{238} \mathrm{Pu}$ & $1.5 \mathrm{E}-02 \pm 9.3 \mathrm{E}-03$ & \\
\hline & ${ }^{239 / 240} \mathrm{Pu}$ & $2.4 \mathrm{E}-02 \pm 1.1 \mathrm{E}-02$ & & & ${ }^{239 / 240} \mathrm{Pu}$ & $9.2 \mathrm{E}-04 \pm 3.2 \mathrm{E}-03$ & $\mathrm{U}$ \\
\hline & ${ }^{103} \mathrm{Ru}$ & $1.5 \mathrm{E}-02 \pm 4.3 \mathrm{E}-02$ & $\mathrm{U}$ & & ${ }^{103} \mathrm{Ru}$ & $-3.9 \mathrm{E}-02 \pm 9.1 \mathrm{E}-02$ & $\mathrm{U}$ \\
\hline & ${ }^{106} \mathrm{Ru}$ & $-8.1 \mathrm{E}-02 \pm 3.2 \mathrm{E}-01$ & $\mathrm{U}$ & & ${ }^{106} \mathrm{Ru}$ & $-8.5 \mathrm{E}-01 \pm 8.5 \mathrm{E}-01$ & $\mathrm{U}$ \\
\hline & ${ }^{125} \mathrm{Sb}$ & $6.5 \mathrm{E}-02 \pm 8.5 \mathrm{E}-02$ & $\mathrm{U}$ & & ${ }^{125} \mathrm{Sb}$ & $1.4 \mathrm{E}-01 \pm 2.2 \mathrm{E}-01$ & $\mathrm{U}$ \\
\hline & ${ }^{113} \mathrm{Sn}$ & $2.5 \mathrm{E}-03 \pm 2.5 \mathrm{E}-02$ & $\mathrm{U}$ & & ${ }^{113} \mathrm{Sn}$ & $-6.6 \mathrm{E}-02 \pm 1.0 \mathrm{E}-01$ & $\mathrm{U}$ \\
\hline & ${ }^{90} \mathrm{Sr}$ & $-2.5 \mathrm{E}-01 \pm 2.5 \mathrm{E}-01$ & $\mathrm{U}$ & & ${ }^{90} \mathrm{Sr}$ & $-6.2 \mathrm{E}-01 \pm 6.2 \mathrm{E}-01$ & $\mathrm{U}$ \\
\hline & ${ }^{234} \mathrm{U}$ & $1.3 \mathrm{E}-02 \pm 7.7 \mathrm{E}-03$ & & & ${ }^{234} \mathrm{U}$ & $1.4 \mathrm{E}-02 \pm 9.2 \mathrm{E}-03$ & \\
\hline & ${ }^{235} \mathrm{U}$ & $8.3 \mathrm{E}-04 \pm 8.3 \mathrm{E}-04$ & $\mathrm{U}$ & & ${ }^{235} \mathrm{U}$ & $4.2 \mathrm{E}-03 \pm 6.0 \mathrm{E}-03$ & $\mathrm{U}$ \\
\hline & ${ }^{238} \mathrm{U}$ & $9.9 \mathrm{E}-03 \pm 6.6 \mathrm{E}-03$ & & & ${ }^{238} \mathrm{U}$ & $1.9 \mathrm{E}-02 \pm 1.0 \mathrm{E}-02$ & \\
\hline & ${ }^{65} \mathrm{Zn}$ & $3.0 \mathrm{E}-02 \pm 9.8 \mathrm{E}-02$ & $\mathrm{U}$ & & ${ }^{65} \mathrm{Zn}$ & $-4.7 \mathrm{E}-01 \pm 4.7 \mathrm{E}-01$ & $\mathrm{U}$ \\
\hline V018 & ${ }^{144} \mathrm{Ce}$ & $-2.4 \mathrm{E}-01 \pm 5.4 \mathrm{E}-01$ & $\mathrm{U}$ & V020 & ${ }^{144} \mathrm{Ce}$ & $-2.0 \mathrm{E}-01 \pm 9.8 \mathrm{E}-01$ & $\mathrm{U}$ \\
\hline \multirow[t]{17}{*}{$(200-W)$} & ${ }^{60} \mathrm{Co}$ & $6.7 \mathrm{E}-03 \pm 5.7 \mathrm{E}-02$ & $\mathrm{U}$ & $(200-W)$ & ${ }^{60} \mathrm{Co}$ & $1.1 \mathrm{E}-02 \pm 8.4 \mathrm{E}-02$ & $\mathrm{U}$ \\
\hline & ${ }^{134} \mathrm{Cs}$ & $-1.5 \mathrm{E}-03 \pm 1.5 \mathrm{E}-02$ & $\mathrm{U}$ & & ${ }^{134} \mathrm{Cs}$ & $-4.6 \mathrm{E}-02 \pm 1.0 \mathrm{E}-01$ & $\mathrm{U}$ \\
\hline & ${ }^{137} \mathrm{Cs}$ & $1.9 \mathrm{E}-01 \pm 8.2 \mathrm{E}-02$ & & & ${ }^{137} \mathrm{Cs}$ & 8.3E-02 \pm 8.6E-02 & $\mathrm{U}$ \\
\hline & ${ }^{152} \mathrm{Eu}$ & $8.5 \mathrm{E}-02 \pm 1.5 \mathrm{E}-01$ & $\mathrm{U}$ & & ${ }^{152} \mathrm{Eu}$ & $3.1 \mathrm{E}-02 \pm 2.4 \mathrm{E}-01$ & $\mathrm{U}$ \\
\hline & ${ }^{154} \mathrm{Eu}$ & $-4.8 \mathrm{E}-02 \pm 1.7 \mathrm{E}-01$ & $\mathrm{U}$ & & ${ }^{154} \mathrm{Eu}$ & $-1.6 \mathrm{E}-01 \pm 2.5 \mathrm{E}-01$ & $\mathrm{U}$ \\
\hline & ${ }^{155} \mathrm{Eu}$ & $-2.2 \mathrm{E}-02 \pm 1.4 \mathrm{E}-01$ & $\mathrm{U}$ & & ${ }^{155} \mathrm{Eu}$ & $-1.3 \mathrm{E}-01 \pm 2.5 \mathrm{E}-01$ & $\mathrm{U}$ \\
\hline & ${ }^{238} \mathrm{Pu}$ & $5.1 \mathrm{E}-03 \pm 6.5 \mathrm{E}-03$ & $\mathrm{U}$ & & ${ }^{238} \mathrm{Pu}$ & $2.7 \mathrm{E}-03 \pm 4.8 \mathrm{E}-03$ & $\mathrm{U}$ \\
\hline & ${ }^{239 / 240} \mathrm{Pu}$ & $8.6 \mathrm{E}-04 \pm 8.6 \mathrm{E}-03$ & $\mathrm{U}$ & & ${ }^{239 / 240} \mathrm{Pu}$ & $8.9 \mathrm{E}-04 \pm 1.8 \mathrm{E}-03$ & $\mathrm{U}$ \\
\hline & ${ }^{103} \mathrm{Ru}$ & $2.9 \mathrm{E}-02 \pm 5.8 \mathrm{E}-02$ & $\mathrm{U}$ & & ${ }^{103} \mathrm{Ru}$ & $-3.2 \mathrm{E}-02 \pm 8.5 \mathrm{E}-02$ & $\mathrm{U}$ \\
\hline & ${ }^{106} \mathrm{Ru}$ & $2.3 \mathrm{E}-02 \pm 2.3 \mathrm{E}-01$ & $\mathrm{U}$ & & ${ }^{106} \mathrm{Ru}$ & 4.3E-01 $\pm 8.2 \mathrm{E}-01$ & $\mathrm{U}$ \\
\hline & ${ }^{125} \mathrm{Sb}$ & $-1.6 \mathrm{E}-02 \pm 1.4 \mathrm{E}-01$ & $\mathrm{U}$ & & ${ }^{125} \mathrm{Sb}$ & $-1.5 \mathrm{E}-02 \pm 1.5 \mathrm{E}-01$ & $\mathrm{U}$ \\
\hline & ${ }^{113} \mathrm{Sn}$ & $-4.1 \mathrm{E}-04 \pm 4.1 \mathrm{E}-03$ & $\mathrm{U}$ & & ${ }^{113} \mathrm{Sn}$ & $-1.4 \mathrm{E}-02 \pm 9.7 \mathrm{E}-02$ & $\mathrm{U}$ \\
\hline & ${ }^{90} \mathrm{Sr}$ & $-3.0 \mathrm{E}-01 \pm 3.0 \mathrm{E}-01$ & $\mathrm{U}$ & & ${ }^{90} \mathrm{Sr}$ & $-3.9 \mathrm{E}-01 \pm 3.9 \mathrm{E}-01$ & $\mathrm{U}$ \\
\hline & ${ }^{234} \mathrm{U}$ & $2.9 \mathrm{E}-02 \pm 1.3 \mathrm{E}-02$ & & & ${ }^{234} \mathrm{U}$ & $1.2 \mathrm{E}-02 \pm 8.6 \mathrm{E}-03$ & \\
\hline & ${ }^{235} \mathrm{U}$ & $3.8 \mathrm{E}-03 \pm 3.9 \mathrm{E}-03$ & & & ${ }^{235} \mathrm{U}$ & $2.9 \mathrm{E}-03 \pm 3.4 \mathrm{E}-03$ & \\
\hline & ${ }^{238} \mathrm{U}$ & $1.5 \mathrm{E}-02 \pm 8.2 \mathrm{E}-03$ & & & ${ }^{238} \mathrm{U}$ & $8.9 \mathrm{E}-03 \pm 6.6 \mathrm{E}-03$ & \\
\hline & ${ }^{65} \mathrm{Zn}$ & $-2.8 \mathrm{E}-01 \pm 2.8 \mathrm{E}-01$ & $\mathrm{U}$ & & ${ }^{65} \mathrm{Zn}$ & $-4.3 \mathrm{E}-01 \pm 4.3 \mathrm{E}-01$ & $\mathrm{U}$ \\
\hline
\end{tabular}

RQ = Result Qualifier. $\mathrm{U}=$ The analyte was analyzed for but not detected. 
Table 4-4. 2008 Vegetation Sampling Results (pCi/g \pm total analytical uncertainty). (Sheet 4 of 18)

\begin{tabular}{|c|c|c|c|c|c|c|c|}
\hline Location & Isotope & Result \pm Error & RQ* & Location & Isotope & Result \pm Error & $\mathbf{R Q *}$ \\
\hline V022 & ${ }^{144} \mathrm{Ce}$ & $-9.8 \mathrm{E}-02 \pm 5.9 \mathrm{E}-01$ & $\mathrm{U}$ & V024 & ${ }^{144} \mathrm{Ce}$ & $-2.2 \mathrm{E}-01 \pm 5.2 \mathrm{E}-01$ & $\mathrm{U}$ \\
\hline \multirow[t]{17}{*}{$(200-W)$} & ${ }^{60} \mathrm{Co}$ & $-1.6 \mathrm{E}-02 \pm 5.7 \mathrm{E}-02$ & $\mathrm{U}$ & $(200-W)$ & ${ }^{60} \mathrm{Co}$ & $-2.7 \mathrm{E}-03 \pm 2.7 \mathrm{E}-02$ & $\mathrm{U}$ \\
\hline & ${ }^{134} \mathrm{Cs}$ & $4.9 \mathrm{E}-02 \pm 6.0 \mathrm{E}-02$ & $\mathrm{U}$ & & ${ }^{134} \mathrm{Cs}$ & $2.4 \mathrm{E}-02 \pm 5.0 \mathrm{E}-02$ & $\mathrm{U}$ \\
\hline & ${ }^{137} \mathrm{Cs}$ & 5.3E-02 \pm 5.7E-02 & $\mathrm{U}$ & & ${ }^{137} \mathrm{Cs}$ & $-7.3 \mathrm{E}-03 \pm 4.5 \mathrm{E}-02$ & $\mathrm{U}$ \\
\hline & ${ }^{152} \mathrm{Eu}$ & $9.0 \mathrm{E}-02 \pm 1.4 \mathrm{E}-01$ & $\mathrm{U}$ & & ${ }^{152} \mathrm{Eu}$ & $-7.4 \mathrm{E}-02 \pm 1.3 \mathrm{E}-01$ & $\mathrm{U}$ \\
\hline & ${ }^{154} \mathrm{Eu}$ & $5.6 \mathrm{E}-03 \pm 5.6 \mathrm{E}-02$ & $\mathrm{U}$ & & ${ }^{154} \mathrm{Eu}$ & $-7.1 \mathrm{E}-03 \pm 7.1 \mathrm{E}-02$ & $\mathrm{U}$ \\
\hline & ${ }^{155} \mathrm{Eu}$ & $1.0 \mathrm{E}-01 \pm 1.6 \mathrm{E}-01$ & $\mathrm{U}$ & & ${ }^{155} \mathrm{Eu}$ & $-1.6 \mathrm{E}-01 \pm 1.6 \mathrm{E}-01$ & $\mathrm{U}$ \\
\hline & ${ }^{238} \mathrm{Pu}$ & $1.0 \mathrm{E}-03 \pm 4.5 \mathrm{E}-03$ & $\mathrm{U}$ & & ${ }^{238} \mathrm{Pu}$ & $-9.8 \mathrm{E}-04 \pm 9.8 \mathrm{E}-03$ & $\mathrm{U}$ \\
\hline & ${ }^{239 / 240} \mathrm{Pu}$ & $3.0 \mathrm{E}-03 \pm 3.5 \mathrm{E}-03$ & & & ${ }^{239 / 240} \mathrm{Pu}$ & $5.9 \mathrm{E}-03 \pm 5.8 \mathrm{E}-03$ & $\mathrm{U}$ \\
\hline & ${ }^{103} \mathrm{Ru}$ & $-1.2 \mathrm{E}-02 \pm 5.6 \mathrm{E}-02$ & $\mathrm{U}$ & & ${ }^{103} \mathrm{Ru}$ & $1.1 \mathrm{E}-03 \pm 1.1 \mathrm{E}-02$ & $\mathrm{U}$ \\
\hline & ${ }^{106} \mathrm{Ru}$ & $-1.9 \mathrm{E}-01 \pm 5.1 \mathrm{E}-01$ & $\mathrm{U}$ & & ${ }^{106} \mathrm{Ru}$ & $6.8 \mathrm{E}-01 \pm 8.3 \mathrm{E}-01$ & $\mathrm{U}$ \\
\hline & ${ }^{125} \mathrm{Sb}$ & $1.4 \mathrm{E}-01 \pm 1.3 \mathrm{E}-01$ & $\mathrm{U}$ & & ${ }^{125} \mathrm{Sb}$ & $-7.7 \mathrm{E}-02 \pm 1.1 \mathrm{E}-01$ & $\mathrm{U}$ \\
\hline & ${ }^{113} \mathrm{Sn}$ & $1.7 \mathrm{E}-03 \pm 1.7 \mathrm{E}-02$ & $\mathrm{U}$ & & ${ }^{113} \mathrm{Sn}$ & $1.1 \mathrm{E}-02 \pm 5.8 \mathrm{E}-02$ & $\mathrm{U}$ \\
\hline & ${ }^{90} \mathrm{Sr}$ & $-4.3 \mathrm{E}-01 \pm 4.3 \mathrm{E}-01$ & $\mathrm{U}$ & & ${ }^{90} \mathrm{Sr}$ & $-2.2 \mathrm{E}-01 \pm 2.2 \mathrm{E}-01$ & $\mathrm{U}$ \\
\hline & ${ }^{234} \mathrm{U}$ & $1.4 \mathrm{E}-02 \pm 8.8 \mathrm{E}-03$ & & & ${ }^{234} \mathrm{U}$ & $1.3 \mathrm{E}-02 \pm 7.9 \mathrm{E}-03$ & \\
\hline & ${ }^{235} \mathrm{U}$ & $5.1 \mathrm{E}-03 \pm 4.7 \mathrm{E}-03$ & & & ${ }^{235} \mathrm{U}$ & $2.7 \mathrm{E}-03 \pm 3.2 \mathrm{E}-03$ & \\
\hline & ${ }^{238} \mathrm{U}$ & $1.5 \mathrm{E}-02 \pm 9.1 \mathrm{E}-03$ & & & ${ }^{238} \mathrm{U}$ & $1.2 \mathrm{E}-02 \pm 7.0 \mathrm{E}-03$ & \\
\hline & ${ }^{65} \mathrm{Zn}$ & $-3.6 \mathrm{E}-02 \pm 1.3 \mathrm{E}-01$ & $\mathrm{U}$ & & ${ }^{65} \mathrm{Zn}$ & $3.4 \mathrm{E}-02 \pm 1.0 \mathrm{E}-01$ & $\mathrm{U}$ \\
\hline V026 & ${ }^{144} \mathrm{Ce}$ & $-6.0 \mathrm{E}-01 \pm 1.0 \mathrm{E}+00$ & $\mathrm{U}$ & V030 & ${ }^{144} \mathrm{Ce}$ & $-1.4 \mathrm{E}+00 \pm 1.5 \mathrm{E}+00$ & $\mathrm{U}$ \\
\hline \multirow[t]{17}{*}{$(200-W)$} & ${ }^{60} \mathrm{Co}$ & $1.1 \mathrm{E}-01 \pm 9.5 \mathrm{E}-02$ & $\mathrm{U}$ & $(200-W)$ & ${ }^{60} \mathrm{Co}$ & $-6.5 \mathrm{E}-02 \pm 1.3 \mathrm{E}-01$ & $\mathrm{U}$ \\
\hline & ${ }^{134} \mathrm{Cs}$ & $-5.1 \mathrm{E}-02 \pm 1.0 \mathrm{E}-01$ & $\mathrm{U}$ & & ${ }^{134} \mathrm{Cs}$ & $4.4 \mathrm{E}-02 \pm 1.5 \mathrm{E}-01$ & $\mathrm{U}$ \\
\hline & ${ }^{137} \mathrm{Cs}$ & $5.1 \mathrm{E}-02 \pm 9.7 \mathrm{E}-02$ & $\mathrm{U}$ & & ${ }^{137} \mathrm{Cs}$ & $1.3 \mathrm{E}-01 \pm 1.5 \mathrm{E}-01$ & $\mathrm{U}$ \\
\hline & ${ }^{152} \mathrm{Eu}$ & $-9.6 \mathrm{E}-02 \pm 3.0 \mathrm{E}-01$ & $\mathrm{U}$ & & ${ }^{152} \mathrm{Eu}$ & $1.6 \mathrm{E}-01 \pm 4.5 \mathrm{E}-01$ & $\mathrm{U}$ \\
\hline & ${ }^{154} \mathrm{Eu}$ & $-1.0 \mathrm{E}-01 \pm 2.8 \mathrm{E}-01$ & $\mathrm{U}$ & & ${ }^{154} \mathrm{Eu}$ & $-2.4 \mathrm{E}-01 \pm 3.9 \mathrm{E}-01$ & $\mathrm{U}$ \\
\hline & ${ }^{155} \mathrm{Eu}$ & $-2.9 \mathrm{E}-02 \pm 2.4 \mathrm{E}-01$ & $\mathrm{U}$ & & ${ }^{155} \mathrm{Eu}$ & $-5.7 \mathrm{E}-01 \pm 5.7 \mathrm{E}-01$ & $\mathrm{U}$ \\
\hline & ${ }^{238} \mathrm{Pu}$ & $4.8 \mathrm{E}-03 \pm 1.5 \mathrm{E}-02$ & $\mathrm{U}$ & & ${ }^{238} \mathrm{Pu}$ & $1.9 \mathrm{E}-03 \pm 8.9 \mathrm{E}-03$ & $\mathrm{U}$ \\
\hline & ${ }^{239 / 240} \mathrm{Pu}$ & $1.6 \mathrm{E}-02 \pm 9.6 \mathrm{E}-03$ & & & ${ }^{239 / 240} \mathrm{Pu}$ & $5.7 \mathrm{E}-03 \pm 4.8 \mathrm{E}-03$ & \\
\hline & ${ }^{103} \mathrm{Ru}$ & $-1.5 \mathrm{E}-02 \pm 1.0 \mathrm{E}-01$ & $\mathrm{U}$ & & ${ }^{103} \mathrm{Ru}$ & $-1.1 \mathrm{E}-01 \pm 1.5 \mathrm{E}-01$ & $\mathrm{U}$ \\
\hline & ${ }^{106} \mathrm{Ru}$ & $-5.8 \mathrm{E}-01 \pm 8.8 \mathrm{E}-01$ & $\mathrm{U}$ & & ${ }^{106} \mathrm{Ru}$ & $2.7 \mathrm{E}-01 \pm 1.3 \mathrm{E}+00$ & $\mathrm{U}$ \\
\hline & ${ }^{125} \mathrm{Sb}$ & $6.8 \mathrm{E}-02 \pm 2.5 \mathrm{E}-01$ & $\mathrm{U}$ & & ${ }^{125} \mathrm{Sb}$ & $-1.0 \mathrm{E}-01 \pm 3.9 \mathrm{E}-01$ & $\mathrm{U}$ \\
\hline & ${ }^{113} \mathrm{Sn}$ & $1.9 \mathrm{E}-02 \pm 1.2 \mathrm{E}-01$ & $\mathrm{U}$ & & ${ }^{113} \mathrm{Sn}$ & $-5.0 \mathrm{E}-02 \pm 1.8 \mathrm{E}-01$ & $\mathrm{U}$ \\
\hline & ${ }^{90} \mathrm{Sr}$ & $-2.1 \mathrm{E}-01 \pm 2.1 \mathrm{E}-01$ & $\mathrm{U}$ & & ${ }^{90} \mathrm{Sr}$ & $-6.3 \mathrm{E}-01 \pm 6.3 \mathrm{E}-01$ & $\mathrm{U}$ \\
\hline & ${ }^{234} \mathrm{U}$ & $1.3 \mathrm{E}-02 \pm 7.8 \mathrm{E}-03$ & & & ${ }^{234} \mathrm{U}$ & $1.5 \mathrm{E}-02 \pm 8.5 \mathrm{E}-03$ & \\
\hline & ${ }^{235} \mathrm{U}$ & $2.6 \mathrm{E}-03 \pm 3.1 \mathrm{E}-03$ & & & ${ }^{235} \mathrm{U}$ & $1.9 \mathrm{E}-03 \pm 3.8 \mathrm{E}-03$ & $\mathrm{U}$ \\
\hline & ${ }^{238} \mathrm{U}$ & $1.1 \mathrm{E}-02 \pm 6.5 \mathrm{E}-03$ & & & ${ }^{238} \mathrm{U}$ & $6.1 \mathrm{E}-03 \pm 6.0 \mathrm{E}-03$ & $\mathrm{U}$ \\
\hline & ${ }^{65} \mathrm{Zn}$ & $1.3 \mathrm{E}-01 \pm 2.1 \mathrm{E}-01$ & $\mathrm{U}$ & & ${ }^{65} \mathrm{Zn}$ & $-3.2 \mathrm{E}-02 \pm 3.2 \mathrm{E}-01$ & $\mathrm{U}$ \\
\hline
\end{tabular}

RQ = Result Qualifier. $\mathrm{U}=$ The analyte was analyzed for but not detected. 
Table 4-4. 2008 Vegetation Sampling Results (pCi/g \pm total analytical uncertainty). (Sheet 5 of 18)

\begin{tabular}{|c|c|c|c|c|c|c|c|}
\hline Location & Isotope & Result \pm Error & $\mathbf{R Q *}$ & Location & Isotope & Result \pm Error & $\mathbf{R Q *}$ \\
\hline V032 & ${ }^{144} \mathrm{Ce}$ & $-8.4 \mathrm{E}-02 \pm 5.8 \mathrm{E}-01$ & $\mathrm{U}$ & V034 & ${ }^{144} \mathrm{Ce}$ & $5.0 \mathrm{E}-01 \pm 5.4 \mathrm{E}-01$ & $\mathrm{U}$ \\
\hline \multirow[t]{17}{*}{$(200-W)$} & ${ }^{60} \mathrm{Co}$ & $-8.0 \mathrm{E}-02 \pm 8.2 \mathrm{E}-02$ & $\mathrm{U}$ & $(200-W)$ & ${ }^{60} \mathrm{Co}$ & $-1.5 \mathrm{E}-02 \pm 5.4 \mathrm{E}-02$ & $\mathrm{U}$ \\
\hline & ${ }^{134} \mathrm{Cs}$ & $-2.4 \mathrm{E}-02 \pm 5.6 \mathrm{E}-02$ & $\mathrm{U}$ & & ${ }^{134} \mathrm{Cs}$ & $-1.2 \mathrm{E}-02 \pm 5.4 \mathrm{E}-02$ & $\mathrm{U}$ \\
\hline & ${ }^{137} \mathrm{Cs}$ & $2.9 \mathrm{E}-02 \pm 5.5 \mathrm{E}-02$ & $\mathrm{U}$ & & ${ }^{137} \mathrm{Cs}$ & $1.3 \mathrm{E}-02 \pm 5.0 \mathrm{E}-02$ & $\mathrm{U}$ \\
\hline & ${ }^{152} \mathrm{Eu}$ & $-5.1 \mathrm{E}-02 \pm 1.6 \mathrm{E}-01$ & $\mathrm{U}$ & & ${ }^{152} \mathrm{Eu}$ & $3.9 \mathrm{E}-02 \pm 1.4 \mathrm{E}-01$ & $\mathrm{U}$ \\
\hline & ${ }^{154} \mathrm{Eu}$ & $1.7 \mathrm{E}-01 \pm 1.7 \mathrm{E}-01$ & $\mathrm{U}$ & & ${ }^{154} \mathrm{Eu}$ & $-4.8 \mathrm{E}-02 \pm 1.5 \mathrm{E}-01$ & $\mathrm{U}$ \\
\hline & ${ }^{155} \mathrm{Eu}$ & $7.4 \mathrm{E}-02 \pm 1.5 \mathrm{E}-01$ & $\mathrm{U}$ & & ${ }^{155} \mathrm{Eu}$ & $-6.8 \mathrm{E}-02 \pm 1.4 \mathrm{E}-01$ & $\mathrm{U}$ \\
\hline & ${ }^{238} \mathrm{Pu}$ & $8.0 \mathrm{E}-03 \pm 2.1 \mathrm{E}-02$ & $\mathrm{U}$ & & ${ }^{238} \mathrm{Pu}$ & $1.8 \mathrm{E}-02 \pm 1.7 \mathrm{E}-02$ & $\mathrm{U}$ \\
\hline & ${ }^{239 / 240} \mathrm{Pu}$ & $9.2 \mathrm{E}-03 \pm 9.5 \mathrm{E}-03$ & $\mathrm{U}$ & & ${ }^{239 / 240} \mathrm{Pu}$ & $3.6 \mathrm{E}-01 \pm 9.7 \mathrm{E}-02$ & \\
\hline & ${ }^{103} \mathrm{Ru}$ & $2.0 \mathrm{E}-02 \pm 5.4 \mathrm{E}-02$ & $\mathrm{U}$ & & ${ }^{103} \mathrm{Ru}$ & $-2.9 \mathrm{E}-02 \pm 5.1 \mathrm{E}-02$ & $\mathrm{U}$ \\
\hline & ${ }^{106} \mathrm{Ru}$ & $-9.4 \mathrm{E}-04 \pm 9.4 \mathrm{E}-03$ & $\mathrm{U}$ & & ${ }^{106} \mathrm{Ru}$ & $-2.2 \mathrm{E}-01 \pm 4.8 \mathrm{E}-01$ & $\mathrm{U}$ \\
\hline & ${ }^{125} \mathrm{Sb}$ & $-8.9 \mathrm{E}-02 \pm 1.3 \mathrm{E}-01$ & $\mathrm{U}$ & & ${ }^{125} \mathrm{Sb}$ & $7.8 \mathrm{E}-02 \pm 1.2 \mathrm{E}-01$ & $\mathrm{U}$ \\
\hline & ${ }^{113} \mathrm{Sn}$ & $-6.3 \mathrm{E}-02 \pm 6.5 \mathrm{E}-02$ & $\mathrm{U}$ & & ${ }^{113} \mathrm{Sn}$ & $1.1 \mathrm{E}-02 \pm 5.8 \mathrm{E}-02$ & $\mathrm{U}$ \\
\hline & ${ }^{90} \mathrm{Sr}$ & $-7.8 \mathrm{E}-02 \pm 1.8 \mathrm{E}-01$ & $\mathrm{U}$ & & ${ }^{90} \mathrm{Sr}$ & $-2.4 \mathrm{E}+00 \pm 2.4 \mathrm{E}+00$ & $\mathrm{U}$ \\
\hline & ${ }^{234} \mathrm{U}$ & $1.7 \mathrm{E}-02 \pm 9.2 \mathrm{E}-03$ & & & ${ }^{234} \mathrm{U}$ & $1.4 \mathrm{E}-02 \pm 7.7 \mathrm{E}-03$ & \\
\hline & ${ }^{235} \mathrm{U}$ & $3.0 \mathrm{E}-03 \pm 3.5 \mathrm{E}-03$ & & & ${ }^{235} \mathrm{U}$ & $5.3 \mathrm{E}-03 \pm 4.6 \mathrm{E}-03$ & \\
\hline & ${ }^{238} \mathrm{U}$ & $9.3 \mathrm{E}-03 \pm 6.9 \mathrm{E}-03$ & & & ${ }^{238} \mathrm{U}$ & $1.0 \mathrm{E}-02 \pm 6.1 \mathrm{E}-03$ & \\
\hline & ${ }^{65} \mathrm{Zn}$ & $-5.0 \mathrm{E}-02 \pm 1.1 \mathrm{E}-01$ & $\mathrm{U}$ & & ${ }^{65} \mathrm{Zn}$ & $1.4 \mathrm{E}-01 \pm 1.3 \mathrm{E}-01$ & $\mathrm{U}$ \\
\hline V036 & ${ }^{144} \mathrm{Ce}$ & $-2.0 \mathrm{E}-01 \pm 5.1 \mathrm{E}-01$ & $\mathrm{U}$ & V038 & ${ }^{144} \mathrm{Ce}$ & $6.4 \mathrm{E}-01 \pm 5.0 \mathrm{E}-01$ & $\mathrm{U}$ \\
\hline \multirow[t]{17}{*}{$(200-W)$} & ${ }^{60} \mathrm{Co}$ & $-1.1 \mathrm{E}-03 \pm 1.1 \mathrm{E}-02$ & $\mathrm{U}$ & $(200-W)$ & ${ }^{60} \mathrm{Co}$ & $-1.3 \mathrm{E}-03 \pm 1.3 \mathrm{E}-02$ & $\mathrm{U}$ \\
\hline & ${ }^{134} \mathrm{Cs}$ & 1.7E-02 $\pm 5.4 \mathrm{E}-02$ & $\mathrm{U}$ & & ${ }^{134} \mathrm{Cs}$ & $-1.3 \mathrm{E}-04 \pm 1.3 \mathrm{E}-03$ & $\mathrm{U}$ \\
\hline & ${ }^{137} \mathrm{Cs}$ & $1.9 \mathrm{E}-01 \pm 1.1 \mathrm{E}-01$ & & & ${ }^{137} \mathrm{Cs}$ & $1.3 \mathrm{E}-01 \pm 6.9 \mathrm{E}-02$ & \\
\hline & ${ }^{152} \mathrm{Eu}$ & $-3.6 \mathrm{E}-02 \pm 1.3 \mathrm{E}-01$ & $\mathrm{U}$ & & ${ }^{152} \mathrm{Eu}$ & $6.2 \mathrm{E}-02 \pm 1.3 \mathrm{E}-01$ & $\mathrm{U}$ \\
\hline & ${ }^{154} \mathrm{Eu}$ & $-4.0 \mathrm{E}-02 \pm 1.6 \mathrm{E}-01$ & $\mathrm{U}$ & & ${ }^{154} \mathrm{Eu}$ & $-1.2 \mathrm{E}-01 \pm 1.3 \mathrm{E}-01$ & $\mathrm{U}$ \\
\hline & ${ }^{155} \mathrm{Eu}$ & $-1.0 \mathrm{E}-02 \pm 1.0 \mathrm{E}-01$ & $\mathrm{U}$ & & ${ }^{155} \mathrm{Eu}$ & $-1.1 \mathrm{E}-01 \pm 1.5 \mathrm{E}-01$ & $\mathrm{U}$ \\
\hline & ${ }^{238} \mathrm{Pu}$ & $3.8 \mathrm{E}-03 \pm 1.7 \mathrm{E}-02$ & $\mathrm{U}$ & & ${ }^{238} \mathrm{Pu}$ & $1.0 \mathrm{E}-03 \pm 1.0 \mathrm{E}-02$ & $\mathrm{U}$ \\
\hline & ${ }^{239 / 240} \mathrm{Pu}$ & $4.2 \mathrm{E}-02 \pm 1.6 \mathrm{E}-02$ & & & ${ }^{239 / 240} \mathrm{Pu}$ & $6.3 \mathrm{E}-03 \pm 6.2 \mathrm{E}-03$ & $\mathrm{U}$ \\
\hline & ${ }^{103} \mathrm{Ru}$ & $-1.5 \mathrm{E}-02 \pm 5.2 \mathrm{E}-02$ & $\mathrm{U}$ & & ${ }^{103} \mathrm{Ru}$ & $7.1 \mathrm{E}-02 \pm 5.1 \mathrm{E}-02$ & $\mathrm{U}$ \\
\hline & ${ }^{106} \mathrm{Ru}$ & $7.8 \mathrm{E}-02 \pm 4.5 \mathrm{E}-01$ & $\mathrm{U}$ & & ${ }^{106} \mathrm{Ru}$ & $6.2 \mathrm{E}-02 \pm 4.6 \mathrm{E}-01$ & $\mathrm{U}$ \\
\hline & ${ }^{125} \mathrm{Sb}$ & $-1.2 \mathrm{E}-01 \pm 1.2 \mathrm{E}-01$ & $\mathrm{U}$ & & ${ }^{125} \mathrm{Sb}$ & $8.1 \mathrm{E}-02 \pm 1.3 \mathrm{E}-01$ & $\mathrm{U}$ \\
\hline & ${ }^{113} \mathrm{Sn}$ & $1.7 \mathrm{E}-02 \pm 5.9 \mathrm{E}-02$ & $\mathrm{U}$ & & ${ }^{113} \mathrm{Sn}$ & $2.1 \mathrm{E}-02 \pm 5.7 \mathrm{E}-02$ & $\mathrm{U}$ \\
\hline & ${ }^{90} \mathrm{Sr}$ & $1.2 \mathrm{E}-01 \pm 2.5 \mathrm{E}-01$ & $\mathrm{U}$ & & ${ }^{90} \mathrm{Sr}$ & $-5.2 \mathrm{E}-02 \pm 2.0 \mathrm{E}-01$ & $\mathrm{U}$ \\
\hline & ${ }^{234} \mathrm{U}$ & $1.8 \mathrm{E}-02 \pm 9.5 \mathrm{E}-03$ & & & ${ }^{234} \mathrm{U}$ & $2.0 \mathrm{E}-02 \pm 1.0 \mathrm{E}-02$ & \\
\hline & ${ }^{235} \mathrm{U}$ & $9.6 \mathrm{E}-04 \pm 1.9 \mathrm{E}-03$ & $\mathrm{U}$ & & ${ }^{235} \mathrm{U}$ & $3.8 \mathrm{E}-03 \pm 5.5 \mathrm{E}-03$ & $\mathrm{U}$ \\
\hline & ${ }^{238} \mathrm{U}$ & $1.6 \mathrm{E}-02 \pm 9.0 \mathrm{E}-03$ & & & ${ }^{238} \mathrm{U}$ & $1.1 \mathrm{E}-02 \pm 7.1 \mathrm{E}-03$ & \\
\hline & ${ }^{65} \mathrm{Zn}$ & $3.6 \mathrm{E}-03 \pm 3.6 \mathrm{E}-02$ & $\mathrm{U}$ & & ${ }^{65} \mathrm{Zn}$ & $-7.5 \mathrm{E}-02 \pm 1.0 \mathrm{E}-01$ & $\mathrm{U}$ \\
\hline
\end{tabular}

RQ = Result Qualifier. $\mathrm{U}=$ The analyte was analyzed for but not detected. 
Table 4-4. 2008 Vegetation Sampling Results (pCi/g \pm total analytical uncertainty). (Sheet 6 of 18)

\begin{tabular}{|c|c|c|c|c|c|c|c|}
\hline Location & Isotope & Result \pm Error & RQ* & Location & Isotope & Result \pm Error & $\mathbf{R Q *}$ \\
\hline V040 & ${ }^{144} \mathrm{Ce}$ & $1.8 \mathrm{E}-01 \pm 4.7 \mathrm{E}-01$ & $\mathrm{U}$ & V042 & ${ }^{144} \mathrm{Ce}$ & $3.8 \mathrm{E}-01 \pm 1.1 \mathrm{E}+00$ & $\mathrm{U}$ \\
\hline \multirow[t]{17}{*}{$(200-W)$} & ${ }^{60} \mathrm{Co}$ & 8.6E-03 \pm 3.6E-02 & $\mathrm{U}$ & $(200-W)$ & ${ }^{60} \mathrm{Co}$ & $-2.0 \mathrm{E}-02 \pm 9.9 \mathrm{E}-02$ & $\mathrm{U}$ \\
\hline & ${ }^{134} \mathrm{Cs}$ & $-5.8 \mathrm{E}-04 \pm 5.8 \mathrm{E}-03$ & $\mathrm{U}$ & & ${ }^{134} \mathrm{Cs}$ & $-2.7 \mathrm{E}-02 \pm 1.1 \mathrm{E}-01$ & $\mathrm{U}$ \\
\hline & ${ }^{137} \mathrm{Cs}$ & $1.0 \mathrm{E}-01 \pm 4.4 \mathrm{E}-02$ & & & ${ }^{137} \mathrm{Cs}$ & $2.2 \mathrm{E}-01 \pm 1.1 \mathrm{E}-01$ & \\
\hline & ${ }^{152} \mathrm{Eu}$ & $-1.6 \mathrm{E}-02 \pm 1.1 \mathrm{E}-01$ & $\mathrm{U}$ & & ${ }^{152} \mathrm{Eu}$ & $-3.1 \mathrm{E}-02 \pm 2.8 \mathrm{E}-01$ & $\mathrm{U}$ \\
\hline & ${ }^{154} \mathrm{Eu}$ & $-2.4 \mathrm{E}-02 \pm 1.2 \mathrm{E}-01$ & $\mathrm{U}$ & & ${ }^{154} \mathrm{Eu}$ & $8.5 \mathrm{E}-02 \pm 2.8 \mathrm{E}-01$ & $\mathrm{U}$ \\
\hline & ${ }^{155} \mathrm{Eu}$ & $-2.4 \mathrm{E}-02 \pm 1.1 \mathrm{E}-01$ & $\mathrm{U}$ & & ${ }^{155} \mathrm{Eu}$ & 2.7E-01 $\pm 2.2 \mathrm{E}-01$ & $\mathrm{U}$ \\
\hline & ${ }^{238} \mathrm{Pu}$ & $2.1 \mathrm{E}-03 \pm 1.8 \mathrm{E}-02$ & $\mathrm{U}$ & & ${ }^{238} \mathrm{Pu}$ & $-3.0 \mathrm{E}-03 \pm 1.8 \mathrm{E}-02$ & $\mathrm{U}$ \\
\hline & ${ }^{239 / 240} \mathrm{Pu}$ & $3.0 \mathrm{E}-02 \pm 1.4 \mathrm{E}-02$ & & & ${ }^{239 / 240} \mathrm{Pu}$ & $1.4 \mathrm{E}-02 \pm 8.3 \mathrm{E}-03$ & \\
\hline & ${ }^{103} \mathrm{Ru}$ & $-3.0 \mathrm{E}-02 \pm 4.0 \mathrm{E}-02$ & $\mathrm{U}$ & & ${ }^{103} \mathrm{Ru}$ & $7.8 \mathrm{E}-04 \pm 7.8 \mathrm{E}-03$ & $\mathrm{U}$ \\
\hline & ${ }^{106} \mathrm{Ru}$ & $5.2 \mathrm{E}-02 \pm 3.5 \mathrm{E}-01$ & $\mathrm{U}$ & & ${ }^{106} \mathrm{Ru}$ & 4.7E-01 \pm 8.9E-01 & $\mathrm{U}$ \\
\hline & ${ }^{125} \mathrm{Sb}$ & $7.3 \mathrm{E}-02 \pm 1.0 \mathrm{E}-01$ & $\mathrm{U}$ & & ${ }^{125} \mathrm{Sb}$ & $6.0 \mathrm{E}-02 \pm 2.7 \mathrm{E}-01$ & $\mathrm{U}$ \\
\hline & ${ }^{113} \mathrm{Sn}$ & $1.3 \mathrm{E}-02 \pm 4.7 \mathrm{E}-02$ & $\mathrm{U}$ & & ${ }^{113} \mathrm{Sn}$ & $-4.6 \mathrm{E}-02 \pm 1.3 \mathrm{E}-01$ & $\mathrm{U}$ \\
\hline & ${ }^{90} \mathrm{Sr}$ & $-4.6 \mathrm{E}-02 \pm 2.1 \mathrm{E}-01$ & $\mathrm{U}$ & & ${ }^{90} \mathrm{Sr}$ & $-9.5 \mathrm{E}-02 \pm 1.9 \mathrm{E}-01$ & $\mathrm{U}$ \\
\hline & ${ }^{234} \mathrm{U}$ & $1.7 \mathrm{E}-02 \pm 8.8 \mathrm{E}-03$ & & & ${ }^{234} \mathrm{U}$ & $1.6 \mathrm{E}-02 \pm 8.8 \mathrm{E}-03$ & \\
\hline & ${ }^{235} \mathrm{U}$ & $7.4 \mathrm{E}-03 \pm 5.5 \mathrm{E}-03$ & & & ${ }^{235} \mathrm{U}$ & $2.8 \mathrm{E}-03 \pm 3.3 \mathrm{E}-03$ & \\
\hline & ${ }^{238} \mathrm{U}$ & $1.5 \mathrm{E}-02 \pm 8.1 \mathrm{E}-03$ & & & ${ }^{238} \mathrm{U}$ & $2.2 \mathrm{E}-02 \pm 1.1 \mathrm{E}-02$ & \\
\hline & ${ }^{65} \mathrm{Zn}$ & $2.5 \mathrm{E}-02 \pm 9.9 \mathrm{E}-02$ & $\mathrm{U}$ & & ${ }^{65} \mathrm{Zn}$ & $-2.5 \mathrm{E}-01 \pm 2.5 \mathrm{E}-01$ & $\mathrm{U}$ \\
\hline V044 & ${ }^{144} \mathrm{Ce}$ & $6.5 \mathrm{E}-02 \pm 4.7 \mathrm{E}-01$ & $\mathrm{U}$ & V046 & ${ }^{144} \mathrm{Ce}$ & $5.6 \mathrm{E}-02 \pm 5.6 \mathrm{E}-01$ & $\mathrm{U}$ \\
\hline \multirow[t]{17}{*}{$(200-W)$} & ${ }^{60} \mathrm{Co}$ & $1.3 \mathrm{E}-02 \pm 3.8 \mathrm{E}-02$ & $\mathrm{U}$ & $(200-W)$ & ${ }^{60} \mathrm{Co}$ & $-5.7 \mathrm{E}-02 \pm 5.7 \mathrm{E}-02$ & $\mathrm{U}$ \\
\hline & ${ }^{134} \mathrm{Cs}$ & $2.1 \mathrm{E}-02 \pm 3.9 \mathrm{E}-02$ & $\mathrm{U}$ & & ${ }^{134} \mathrm{Cs}$ & 4.1E-02 $\pm 6.7 \mathrm{E}-02$ & $\mathrm{U}$ \\
\hline & ${ }^{137} \mathrm{Cs}$ & $5.6 \mathrm{E}-02 \pm 5.8 \mathrm{E}-02$ & $\mathrm{U}$ & & ${ }^{137} \mathrm{Cs}$ & $7.0 \mathrm{E}-02 \pm 7.1 \mathrm{E}-02$ & $\mathrm{U}$ \\
\hline & ${ }^{152} \mathrm{Eu}$ & $5.9 \mathrm{E}-02 \pm 1.1 \mathrm{E}-01$ & $\mathrm{U}$ & & ${ }^{152} \mathrm{Eu}$ & $1.5 \mathrm{E}-02 \pm 1.5 \mathrm{E}-01$ & $\mathrm{U}$ \\
\hline & ${ }^{154} \mathrm{Eu}$ & $-4.4 \mathrm{E}-02 \pm 1.1 \mathrm{E}-01$ & $\mathrm{U}$ & & ${ }^{154} \mathrm{Eu}$ & $1.7 \mathrm{E}-01 \pm 1.7 \mathrm{E}-01$ & $\mathrm{U}$ \\
\hline & ${ }^{155} \mathrm{Eu}$ & $1.8 \mathrm{E}-01 \pm 1.5 \mathrm{E}-01$ & $\mathrm{U}$ & & ${ }^{155} \mathrm{Eu}$ & 4.4E-02 $\pm 1.5 \mathrm{E}-01$ & $\mathrm{U}$ \\
\hline & ${ }^{238} \mathrm{Pu}$ & $-7.2 \mathrm{E}-03 \pm 1.2 \mathrm{E}-02$ & $\mathrm{U}$ & & ${ }^{238} \mathrm{Pu}$ & $-1.1 \mathrm{E}-02 \pm 1.9 \mathrm{E}-02$ & $\mathrm{U}$ \\
\hline & ${ }^{239 / 240} \mathrm{Pu}$ & $2.5 \mathrm{E}-02 \pm 1.2 \mathrm{E}-02$ & & & ${ }^{239 / 240} \mathrm{Pu}$ & $6.0 \mathrm{E}-03 \pm 6.5 \mathrm{E}-03$ & $\mathrm{U}$ \\
\hline & ${ }^{103} \mathrm{Ru}$ & $-7.6 \mathrm{E}-03 \pm 4.0 \mathrm{E}-02$ & $\mathrm{U}$ & & ${ }^{103} \mathrm{Ru}$ & $1.5 \mathrm{E}-02 \pm 5.5 \mathrm{E}-02$ & $\mathrm{U}$ \\
\hline & ${ }^{106} \mathrm{Ru}$ & $1.6 \mathrm{E}-01 \pm 3.7 \mathrm{E}-01$ & $\mathrm{U}$ & & ${ }^{106} \mathrm{Ru}$ & $3.4 \mathrm{E}-01 \pm 5.0 \mathrm{E}-01$ & $\mathrm{U}$ \\
\hline & ${ }^{125} \mathrm{Sb}$ & $-1.0 \mathrm{E}-02 \pm 1.0 \mathrm{E}-01$ & $\mathrm{U}$ & & ${ }^{125} \mathrm{Sb}$ & $-2.0 \mathrm{E}-02 \pm 1.3 \mathrm{E}-01$ & $\mathrm{U}$ \\
\hline & ${ }^{113} \mathrm{Sn}$ & $-1.2 \mathrm{E}-02 \pm 4.9 \mathrm{E}-02$ & $\mathrm{U}$ & & ${ }^{113} \mathrm{Sn}$ & $-1.8 \mathrm{E}-03 \pm 1.8 \mathrm{E}-02$ & $\mathrm{U}$ \\
\hline & ${ }^{90} \mathrm{Sr}$ & $-1.8 \mathrm{E}-01 \pm 2.0 \mathrm{E}-01$ & $\mathrm{U}$ & & ${ }^{90} \mathrm{Sr}$ & $-1.3 \mathrm{E}-01 \pm 1.8 \mathrm{E}-01$ & $\mathrm{U}$ \\
\hline & ${ }^{234} \mathrm{U}$ & $1.4 \mathrm{E}-02 \pm 7.8 \mathrm{E}-03$ & & & ${ }^{234} \mathrm{U}$ & $2.3 \mathrm{E}-02 \pm 1.1 \mathrm{E}-02$ & \\
\hline & ${ }^{235} \mathrm{U}$ & $8.3 \mathrm{E}-03 \pm 5.6 \mathrm{E}-03$ & & & ${ }^{235} \mathrm{U}$ & $3.7 \mathrm{E}-03 \pm 4.6 \mathrm{E}-03$ & $\mathrm{U}$ \\
\hline & ${ }^{238} \mathrm{U}$ & $1.3 \mathrm{E}-02 \pm 7.8 \mathrm{E}-03$ & & & ${ }^{238} \mathrm{U}$ & $1.5 \mathrm{E}-02 \pm 8.4 \mathrm{E}-03$ & \\
\hline & ${ }^{65} \mathrm{Zn}$ & $-2.1 \mathrm{E}-01 \pm 2.1 \mathrm{E}-01$ & $\mathrm{U}$ & & ${ }^{65} \mathrm{Zn}$ & $-1.2 \mathrm{E}-01 \pm 1.3 \mathrm{E}-01$ & $\mathrm{U}$ \\
\hline
\end{tabular}

RQ = Result Qualifier. $\mathrm{U}=$ The analyte was analyzed for but not detected. 
Table 4-4. 2008 Vegetation Sampling Results (pCi/g \pm total analytical uncertainty). (Sheet 7 of 18)

\begin{tabular}{|c|c|c|c|c|c|c|c|}
\hline Location & Isotope & Result \pm Error & RQ* & Location & Isotope & Result \pm Error & $\mathbf{R Q *}$ \\
\hline V048 & ${ }^{144} \mathrm{Ce}$ & $-8.3 \mathrm{E}-02 \pm 4.4 \mathrm{E}-01$ & $\mathrm{U}$ & V050 & ${ }^{144} \mathrm{Ce}$ & $-2.9 \mathrm{E}-01 \pm 1.0 \mathrm{E}+00$ & $\mathrm{U}$ \\
\hline \multirow[t]{17}{*}{$(200-W)$} & ${ }^{60} \mathrm{Co}$ & $-8.0 \mathrm{E}-03 \pm 5.2 \mathrm{E}-02$ & $\mathrm{U}$ & $(200-W)$ & ${ }^{60} \mathrm{Co}$ & $-6.8 \mathrm{E}-03 \pm 6.8 \mathrm{E}-02$ & $\mathrm{U}$ \\
\hline & ${ }^{134} \mathrm{Cs}$ & $3.4 \mathrm{E}-02 \pm 4.7 \mathrm{E}-02$ & $\mathrm{U}$ & & ${ }^{134} \mathrm{Cs}$ & $-3.2 \mathrm{E}-04 \pm 3.2 \mathrm{E}-03$ & $\mathrm{U}$ \\
\hline & ${ }^{137} \mathrm{Cs}$ & $1.4 \mathrm{E}-02 \pm 5.0 \mathrm{E}-02$ & $\mathrm{U}$ & & ${ }^{137} \mathrm{Cs}$ & $-8.5 \mathrm{E}-02 \pm 8.6 \mathrm{E}-02$ & $\mathrm{U}$ \\
\hline & ${ }^{152} \mathrm{Eu}$ & $4.3 \mathrm{E}-03 \pm 4.3 \mathrm{E}-02$ & $\mathrm{U}$ & & ${ }^{152} \mathrm{Eu}$ & $2.8 \mathrm{E}-02 \pm 2.3 \mathrm{E}-01$ & $\mathrm{U}$ \\
\hline & ${ }^{154} \mathrm{Eu}$ & $-4.9 \mathrm{E}-02 \pm 1.5 \mathrm{E}-01$ & $\mathrm{U}$ & & ${ }^{154} \mathrm{Eu}$ & $-1.8 \mathrm{E}-01 \pm 2.2 \mathrm{E}-01$ & $\mathrm{U}$ \\
\hline & ${ }^{155} \mathrm{Eu}$ & $6.1 \mathrm{E}-02 \pm 1.2 \mathrm{E}-01$ & $\mathrm{U}$ & & ${ }^{155} \mathrm{Eu}$ & $-1.7 \mathrm{E}-02 \pm 1.7 \mathrm{E}-01$ & $\mathrm{U}$ \\
\hline & ${ }^{238} \mathrm{Pu}$ & $-3.6 \mathrm{E}-03 \pm 9.6 \mathrm{E}-03$ & $\mathrm{U}$ & & ${ }^{238} \mathrm{Pu}$ & $1.0 \mathrm{E}-03 \pm 1.0 \mathrm{E}-02$ & $\mathrm{U}$ \\
\hline & ${ }^{239 / 240} \mathrm{Pu}$ & $8.2 \mathrm{E}-03 \pm 6.9 \mathrm{E}-03$ & $\mathrm{U}$ & & ${ }^{239 / 240} \mathrm{Pu}$ & $1.0 \mathrm{E}-03 \pm 1.0 \mathrm{E}-02$ & $\mathrm{U}$ \\
\hline & ${ }^{103} \mathrm{Ru}$ & $-1.7 \mathrm{E}-02 \pm 4.6 \mathrm{E}-02$ & $\mathrm{U}$ & & ${ }^{103} \mathrm{Ru}$ & $-5.1 \mathrm{E}-02 \pm 8.2 \mathrm{E}-02$ & $\mathrm{U}$ \\
\hline & ${ }^{106} \mathrm{Ru}$ & $-3.4 \mathrm{E}-01 \pm 4.1 \mathrm{E}-01$ & $\mathrm{U}$ & & ${ }^{106} \mathrm{Ru}$ & $-1.4 \mathrm{E}-01 \pm 7.2 \mathrm{E}-01$ & $\mathrm{U}$ \\
\hline & ${ }^{125} \mathrm{Sb}$ & $-1.5 \mathrm{E}-02 \pm 1.1 \mathrm{E}-01$ & $\mathrm{U}$ & & ${ }^{125} \mathrm{Sb}$ & $3.5 \mathrm{E}-02 \pm 2.2 \mathrm{E}-01$ & $\mathrm{U}$ \\
\hline & ${ }^{113} \mathrm{Sn}$ & $-8.5 \mathrm{E}-03 \pm 5.5 \mathrm{E}-02$ & $\mathrm{U}$ & & ${ }^{113} \mathrm{Sn}$ & $-1.9 \mathrm{E}-02 \pm 1.0 \mathrm{E}-01$ & $\mathrm{U}$ \\
\hline & ${ }^{90} \mathrm{Sr}$ & $-1.2 \mathrm{E}-01 \pm 2.0 \mathrm{E}-01$ & $\mathrm{U}$ & & ${ }^{90} \mathrm{Sr}$ & $-1.6 \mathrm{E}-01 \pm 2.2 \mathrm{E}-01$ & $\mathrm{U}$ \\
\hline & ${ }^{234} \mathrm{U}$ & 2.1E-02 \pm 9.7E-03 & & & ${ }^{234} \mathrm{U}$ & $2.1 \mathrm{E}-02 \pm 1.2 \mathrm{E}-02$ & \\
\hline & ${ }^{235} \mathrm{U}$ & 8.7E-04 $\pm 3.0 \mathrm{E}-03$ & $\mathrm{U}$ & & ${ }^{235} \mathrm{U}$ & $8.4 \mathrm{E}-03 \pm 7.0 \mathrm{E}-03$ & \\
\hline & ${ }^{238} \mathrm{U}$ & $1.0 \mathrm{E}-02 \pm 7.2 \mathrm{E}-03$ & & & ${ }^{238} \mathrm{U}$ & $3.8 \mathrm{E}-03 \pm 3.9 \mathrm{E}-03$ & \\
\hline & ${ }^{65} \mathrm{Zn}$ & $-1.7 \mathrm{E}-01 \pm 1.7 \mathrm{E}-01$ & $\mathrm{U}$ & & ${ }^{65} \mathrm{Zn}$ & $-5.2 \mathrm{E}-02 \pm 2.1 \mathrm{E}-01$ & $\mathrm{U}$ \\
\hline V052 & ${ }^{144} \mathrm{Ce}$ & $-5.7 \mathrm{E}-01 \pm 1.0 \mathrm{E}+00$ & $\mathrm{U}$ & V112 & ${ }^{144} \mathrm{Ce}$ & $-9.4 \mathrm{E}-02 \pm 6.0 \mathrm{E}-01$ & $\mathrm{U}$ \\
\hline \multirow[t]{17}{*}{$(200-W)$} & ${ }^{60} \mathrm{Co}$ & $-5.4 \mathrm{E}-02 \pm 8.9 \mathrm{E}-02$ & $\mathrm{U}$ & (Replicate of & ${ }^{60} \mathrm{Co}$ & $1.7 \mathrm{E}-02 \pm$ 5.7E-02 & $\mathrm{U}$ \\
\hline & ${ }^{134} \mathrm{Cs}$ & $3.7 \mathrm{E}-02 \pm 1.0 \mathrm{E}-01$ & $\mathrm{U}$ & V032, 200-W) & ${ }^{134} \mathrm{Cs}$ & $-2.2 \mathrm{E}-02 \pm 6.7 \mathrm{E}-02$ & $\mathrm{U}$ \\
\hline & ${ }^{137} \mathrm{Cs}$ & $-3.8 \mathrm{E}-03 \pm 3.8 \mathrm{E}-02$ & $\mathrm{U}$ & & ${ }^{137} \mathrm{Cs}$ & $-2.8 \mathrm{E}-02 \pm 5.9 \mathrm{E}-02$ & $\mathrm{U}$ \\
\hline & ${ }^{152} \mathrm{Eu}$ & $-2.5 \mathrm{E}-01 \pm 2.7 \mathrm{E}-01$ & $\mathrm{U}$ & & ${ }^{152} \mathrm{Eu}$ & $4.4 \mathrm{E}-02 \pm 1.6 \mathrm{E}-01$ & $\mathrm{U}$ \\
\hline & ${ }^{154} \mathrm{Eu}$ & $-5.7 \mathrm{E}-02 \pm 2.8 \mathrm{E}-01$ & $\mathrm{U}$ & & ${ }^{154} \mathrm{Eu}$ & $-9.7 \mathrm{E}-02 \pm 1.5 \mathrm{E}-01$ & $\mathrm{U}$ \\
\hline & ${ }^{155} \mathrm{Eu}$ & $-2.0 \mathrm{E}-01 \pm 2.4 \mathrm{E}-01$ & $\mathrm{U}$ & & ${ }^{155} \mathrm{Eu}$ & 4.3E-02 $\pm 1.6 \mathrm{E}-01$ & $\mathrm{U}$ \\
\hline & ${ }^{238} \mathrm{Pu}$ & $-5.6 \mathrm{E}-03 \pm 1.1 \mathrm{E}-02$ & $\mathrm{U}$ & & ${ }^{238} \mathrm{Pu}$ & $-3.7 \mathrm{E}-03 \pm 1.2 \mathrm{E}-02$ & $\mathrm{U}$ \\
\hline & ${ }^{239 / 240} \mathrm{Pu}$ & $5.6 \mathrm{E}-03 \pm 4.8 \mathrm{E}-03$ & & & ${ }^{239 / 240} \mathrm{Pu}$ & $9.1 \mathrm{E}-03 \pm 6.7 \mathrm{E}-03$ & \\
\hline & ${ }^{103} \mathrm{Ru}$ & $6.3 \mathrm{E}-02 \pm 9.3 \mathrm{E}-02$ & $\mathrm{U}$ & & ${ }^{103} \mathrm{Ru}$ & $3.0 \mathrm{E}-02 \pm 4.9 \mathrm{E}-02$ & $\mathrm{U}$ \\
\hline & ${ }^{106} \mathrm{Ru}$ & 3.0E-01 $\pm 8.3 \mathrm{E}-01$ & $\mathrm{U}$ & & ${ }^{106} \mathrm{Ru}$ & $-2.5 \mathrm{E}-02 \pm 2.5 \mathrm{E}-01$ & $\mathrm{U}$ \\
\hline & ${ }^{125} \mathrm{Sb}$ & $-2.0 \mathrm{E}-01 \pm 2.5 \mathrm{E}-01$ & $\mathrm{U}$ & & ${ }^{125} \mathrm{Sb}$ & $-2.7 \mathrm{E}-02 \pm 1.3 \mathrm{E}-01$ & $\mathrm{U}$ \\
\hline & ${ }^{113} \mathrm{Sn}$ & $5.5 \mathrm{E}-03 \pm 5.4 \mathrm{E}-02$ & $\mathrm{U}$ & & ${ }^{113} \mathrm{Sn}$ & $-9.0 \mathrm{E}-03 \pm 6.5 \mathrm{E}-02$ & $\mathrm{U}$ \\
\hline & ${ }^{90} \mathrm{Sr}$ & $-8.2 \mathrm{E}-02 \pm 1.9 \mathrm{E}-01$ & $\mathrm{U}$ & & ${ }^{90} \mathrm{Sr}$ & $-5.5 \mathrm{E}-02 \pm 1.9 \mathrm{E}-01$ & $\mathrm{U}$ \\
\hline & ${ }^{234} \mathrm{U}$ & $2.3 \mathrm{E}-02 \pm 1.1 \mathrm{E}-02$ & & & ${ }^{234} \mathrm{U}$ & $1.5 \mathrm{E}-02 \pm 8.4 \mathrm{E}-03$ & \\
\hline & ${ }^{235} \mathrm{U}$ & $4.6 \mathrm{E}-03 \pm 4.3 \mathrm{E}-03$ & & & ${ }^{235} \mathrm{U}$ & $-1.9 \mathrm{E}-03 \pm 3.8 \mathrm{E}-03$ & $\mathrm{U}$ \\
\hline & ${ }^{238} \mathrm{U}$ & $9.3 \mathrm{E}-03 \pm 6.5 \mathrm{E}-03$ & & & ${ }^{238} \mathrm{U}$ & $6.9 \mathrm{E}-03 \pm 5.7 \mathrm{E}-03$ & \\
\hline & ${ }^{65} \mathrm{Zn}$ & $1.2 \mathrm{E}-01 \pm 2.1 \mathrm{E}-01$ & $\mathrm{U}$ & & ${ }^{65} \mathrm{Zn}$ & $-1.7 \mathrm{E}-01 \pm 1.7 \mathrm{E}-01$ & $\mathrm{U}$ \\
\hline
\end{tabular}

RQ = Result Qualifier. $\mathrm{U}=$ The analyte was analyzed for but not detected. 
Table 4-4. 2008 Vegetation Sampling Results (pCi/g \pm total analytical uncertainty). (Sheet 8 of 18)

\begin{tabular}{|c|c|c|c|c|c|c|c|}
\hline Location & Isotope & Result \pm Error & $\mathbf{R Q *}$ & Location & Isotope & Result \pm Error & $\mathbf{R Q} *$ \\
\hline V054 & ${ }^{144} \mathrm{Ce}$ & $-1.8 \mathrm{E}-01 \pm 7.9 \mathrm{E}-01$ & $\mathrm{U}$ & V056 & ${ }^{144} \mathrm{Ce}$ & $7.5 \mathrm{E}-02 \pm 5.9 \mathrm{E}-01$ & $\mathrm{U}$ \\
\hline \multirow[t]{17}{*}{ (200-E) } & ${ }^{60} \mathrm{Co}$ & 4.3E-02 \pm 8.3E-02 & $\mathrm{U}$ & $(200-E)$ & ${ }^{60} \mathrm{Co}$ & $-5.7 \mathrm{E}-03 \pm 4.9 \mathrm{E}-02$ & $\mathrm{U}$ \\
\hline & ${ }^{134} \mathrm{Cs}$ & $-6.3 \mathrm{E}-03 \pm 6.3 \mathrm{E}-02$ & $\mathrm{U}$ & & ${ }^{134} \mathrm{Cs}$ & $-2.2 \mathrm{E}-02 \pm 5.3 \mathrm{E}-02$ & $\mathrm{U}$ \\
\hline & ${ }^{137} \mathrm{Cs}$ & $-3.4 \mathrm{E}-03 \pm 3.4 \mathrm{E}-02$ & $\mathrm{U}$ & & ${ }^{137} \mathrm{Cs}$ & $1.2 \mathrm{E}-01 \pm 1.1 \mathrm{E}-01$ & \\
\hline & ${ }^{152} \mathrm{Eu}$ & $-4.2 \mathrm{E}-02 \pm 2.0 \mathrm{E}-01$ & $\mathrm{U}$ & & ${ }^{152} \mathrm{Eu}$ & $-1.1 \mathrm{E}-01 \pm 1.5 \mathrm{E}-01$ & $\mathrm{U}$ \\
\hline & ${ }^{154} \mathrm{Eu}$ & $5.3 \mathrm{E}-02 \pm 2.4 \mathrm{E}-01$ & $\mathrm{U}$ & & ${ }^{154} \mathrm{Eu}$ & $-6.2 \mathrm{E}-02 \pm 1.4 \mathrm{E}-01$ & $\mathrm{U}$ \\
\hline & ${ }^{155} \mathrm{Eu}$ & $-1.0 \mathrm{E}-01 \pm 2.0 \mathrm{E}-01$ & $\mathrm{U}$ & & ${ }^{155} \mathrm{Eu}$ & $-2.8 \mathrm{E}-02 \pm 1.6 \mathrm{E}-01$ & $\mathrm{U}$ \\
\hline & ${ }^{238} \mathrm{Pu}$ & $1.2 \mathrm{E}-02 \pm 2.2 \mathrm{E}-02$ & $\mathrm{U}$ & & ${ }^{238} \mathrm{Pu}$ & $-4.8 \mathrm{E}-03 \pm 2.2 \mathrm{E}-02$ & $\mathrm{U}$ \\
\hline & ${ }^{239 / 240} \mathrm{Pu}$ & $1.5 \mathrm{E}-03 \pm 6.7 \mathrm{E}-03$ & $\mathrm{U}$ & & ${ }^{239 / 240} \mathrm{Pu}$ & $-1.6 \mathrm{E}-03 \pm 7.2 \mathrm{E}-03$ & $\mathrm{U}$ \\
\hline & ${ }^{103} \mathrm{Ru}$ & $-4.5 \mathrm{E}-02 \pm 8.8 \mathrm{E}-02$ & $\mathrm{U}$ & & ${ }^{103} \mathrm{Ru}$ & 4.7E-04 \pm 4.7E-03 & $\mathrm{U}$ \\
\hline & ${ }^{106} \mathrm{Ru}$ & $-4.9 \mathrm{E}-01 \pm 7.3 \mathrm{E}-01$ & $\mathrm{U}$ & & ${ }^{106} \mathrm{Ru}$ & $-2.9 \mathrm{E}-01 \pm 5.4 \mathrm{E}-01$ & $\mathrm{U}$ \\
\hline & ${ }^{125} \mathrm{Sb}$ & $2.0 \mathrm{E}-01 \pm 2.0 \mathrm{E}-01$ & $\mathrm{U}$ & & ${ }^{125} \mathrm{Sb}$ & $-3.7 \mathrm{E}-02 \pm 1.3 \mathrm{E}-01$ & $\mathrm{U}$ \\
\hline & ${ }^{113} \mathrm{Sn}$ & $-1.2 \mathrm{E}-02 \pm 9.7 \mathrm{E}-02$ & $\mathrm{U}$ & & ${ }^{113} \mathrm{Sn}$ & $1.4 \mathrm{E}-02 \pm 6.4 \mathrm{E}-02$ & $\mathrm{U}$ \\
\hline & ${ }^{90} \mathrm{Sr}$ & $-2.9 \mathrm{E}-01 \pm 2.9 \mathrm{E}-01$ & $\mathrm{U}$ & & ${ }^{90} \mathrm{Sr}$ & $2.7 \mathrm{E}-01 \pm 2.3 \mathrm{E}-01$ & \\
\hline & ${ }^{234} \mathrm{U}$ & $1.7 \mathrm{E}-02 \pm 8.7 \mathrm{E}-03$ & & & ${ }^{234} \mathrm{U}$ & $1.5 \mathrm{E}-02 \pm 7.3 \mathrm{E}-03$ & \\
\hline & ${ }^{235} \mathrm{U}$ & $2.6 \mathrm{E}-03 \pm 3.9 \mathrm{E}-03$ & $\mathrm{U}$ & & ${ }^{235} \mathrm{U}$ & $2.7 \mathrm{E}-03 \pm 3.2 \mathrm{E}-03$ & \\
\hline & ${ }^{238} \mathrm{U}$ & $1.3 \mathrm{E}-02 \pm 7.3 \mathrm{E}-03$ & & & ${ }^{238} \mathrm{U}$ & $5.8 \mathrm{E}-03 \pm 5.2 \mathrm{E}-03$ & $\mathrm{U}$ \\
\hline & ${ }^{65} \mathrm{Zn}$ & $6.9 \mathrm{E}-03 \pm 6.9 \mathrm{E}-02$ & $\mathrm{U}$ & & ${ }^{65} \mathrm{Zn}$ & $-1.8 \mathrm{E}-02 \pm 1.1 \mathrm{E}-01$ & $\mathrm{U}$ \\
\hline V058 & ${ }^{144} \mathrm{Ce}$ & $-5.2 \mathrm{E}-01 \pm 5.2 \mathrm{E}-01$ & $\mathrm{U}$ & V060 & ${ }^{144} \mathrm{Ce}$ & $1.8 \mathrm{E}-01 \pm 8.1 \mathrm{E}-01$ & $\mathrm{U}$ \\
\hline \multirow[t]{17}{*}{$(200-E)$} & ${ }^{60} \mathrm{Co}$ & $-1.6 \mathrm{E}-02 \pm 3.4 \mathrm{E}-02$ & $\mathrm{U}$ & $(200-E)$ & ${ }^{60} \mathrm{Co}$ & $-3.4 \mathrm{E}-02 \pm 5.7 \mathrm{E}-02$ & $\mathrm{U}$ \\
\hline & ${ }^{134} \mathrm{Cs}$ & $3.1 \mathrm{E}-02 \pm 3.9 \mathrm{E}-02$ & $\mathrm{U}$ & & ${ }^{134} \mathrm{Cs}$ & $1.3 \mathrm{E}-02 \pm 6.8 \mathrm{E}-02$ & $\mathrm{U}$ \\
\hline & ${ }^{137} \mathrm{Cs}$ & 4.7E-02 $\pm 4.1 \mathrm{E}-02$ & $\mathrm{U}$ & & ${ }^{137} \mathrm{Cs}$ & $-3.3 \mathrm{E}-03 \pm 3.3 \mathrm{E}-02$ & $\mathrm{U}$ \\
\hline & ${ }^{152} \mathrm{Eu}$ & $-3.6 \mathrm{E}-02 \pm 1.0 \mathrm{E}-01$ & $\mathrm{U}$ & & ${ }^{152} \mathrm{Eu}$ & $-4.1 \mathrm{E}-02 \pm 1.9 \mathrm{E}-01$ & $\mathrm{U}$ \\
\hline & ${ }^{154} \mathrm{Eu}$ & $3.5 \mathrm{E}-02 \pm 1.1 \mathrm{E}-01$ & $\mathrm{U}$ & & ${ }^{154} \mathrm{Eu}$ & $2.1 \mathrm{E}-01 \pm 1.2 \mathrm{E}-01$ & $\mathrm{U}$ \\
\hline & ${ }^{155} \mathrm{Eu}$ & $9.9 \mathrm{E}-02 \pm 1.0 \mathrm{E}-01$ & $\mathrm{U}$ & & ${ }^{155} \mathrm{Eu}$ & $6.2 \mathrm{E}-02 \pm 1.8 \mathrm{E}-01$ & $\mathrm{U}$ \\
\hline & ${ }^{238} \mathrm{Pu}$ & $-2.1 \mathrm{E}-02 \pm 3.2 \mathrm{E}-02$ & $\mathrm{U}$ & & ${ }^{238} \mathrm{Pu}$ & $1.6 \mathrm{E}-02 \pm 1.8 \mathrm{E}-02$ & $\mathrm{U}$ \\
\hline & ${ }^{239 / 240} \mathrm{Pu}$ & $1.9 \mathrm{E}-03 \pm 6.6 \mathrm{E}-03$ & $\mathrm{U}$ & & ${ }^{239 / 240} \mathrm{Pu}$ & $1.0 \mathrm{E}-03 \pm 1.0 \mathrm{E}-03$ & $\mathrm{U}$ \\
\hline & ${ }^{103} \mathrm{Ru}$ & $-6.9 \mathrm{E}-04 \pm 6.9 \mathrm{E}-03$ & $\mathrm{U}$ & & ${ }^{103} \mathrm{Ru}$ & $9.3 \mathrm{E}-03 \pm 7.3 \mathrm{E}-02$ & $\mathrm{U}$ \\
\hline & ${ }^{106} \mathrm{Ru}$ & $9.0 \mathrm{E}-02 \pm 3.1 \mathrm{E}-01$ & $\mathrm{U}$ & & ${ }^{106} \mathrm{Ru}$ & $-4.2 \mathrm{E}-01 \pm 5.9 \mathrm{E}-01$ & $\mathrm{U}$ \\
\hline & ${ }^{125} \mathrm{Sb}$ & $-1.9 \mathrm{E}-02 \pm 9.4 \mathrm{E}-02$ & $\mathrm{U}$ & & ${ }^{125} \mathrm{Sb}$ & $-3.0 \mathrm{E}-02 \pm 1.9 \mathrm{E}-01$ & $\mathrm{U}$ \\
\hline & ${ }^{113} \mathrm{Sn}$ & $-5.4 \mathrm{E}-03 \pm 4.6 \mathrm{E}-02$ & $\mathrm{U}$ & & ${ }^{113} \mathrm{Sn}$ & $-1.1 \mathrm{E}-02 \pm 8.8 \mathrm{E}-02$ & $\mathrm{U}$ \\
\hline & ${ }^{90} \mathrm{Sr}$ & $6.5 \mathrm{E}-01 \pm 2.6 \mathrm{E}-01$ & & & ${ }^{90} \mathrm{Sr}$ & $-3.9 \mathrm{E}-01 \pm 3.9 \mathrm{E}-01$ & $\mathrm{U}$ \\
\hline & ${ }^{234} \mathrm{U}$ & $9.9 \mathrm{E}-03 \pm 6.9 \mathrm{E}-03$ & & & ${ }^{234} \mathrm{U}$ & $1.5 \mathrm{E}-02 \pm 7.5 \mathrm{E}-03$ & \\
\hline & ${ }^{235} \mathrm{U}$ & 8.3E-04 \pm 8.3E-04 & $\mathrm{U}$ & & ${ }^{235} \mathrm{U}$ & $3.7 \mathrm{E}-03 \pm 3.8 \mathrm{E}-03$ & \\
\hline & ${ }^{238} \mathrm{U}$ & $8.2 \mathrm{E}-03 \pm 5.5 \mathrm{E}-03$ & & & ${ }^{238} \mathrm{U}$ & $4.5 \mathrm{E}-03 \pm 3.8 \mathrm{E}-03$ & \\
\hline & ${ }^{65} \mathrm{Zn}$ & $-2.1 \mathrm{E}-01 \pm 2.1 \mathrm{E}-01$ & $\mathrm{U}$ & & ${ }^{65} \mathrm{Zn}$ & $2.0 \mathrm{E}-02 \pm 2.0 \mathrm{E}-01$ & $\mathrm{U}$ \\
\hline
\end{tabular}

RQ = Result Qualifier. $\mathrm{U}=$ The analyte was analyzed for but not detected. 
Table 4-4. 2008 Vegetation Sampling Results (pCi/g \pm total analytical uncertainty). (Sheet 9 of 18)

\begin{tabular}{|c|c|c|c|c|c|c|c|}
\hline Location & Isotope & Result \pm Error & RQ* & Location & Isotope & Result \pm Error & $\mathbf{R Q *}$ \\
\hline V062 & ${ }^{144} \mathrm{Ce}$ & $-2.0 \mathrm{E}-01 \pm 6.8 \mathrm{E}-01$ & $\mathrm{U}$ & V064 & ${ }^{144} \mathrm{Ce}$ & $-5.0 \mathrm{E}-01 \pm 7.6 \mathrm{E}-01$ & $\mathrm{U}$ \\
\hline \multirow[t]{17}{*}{$(200-E)$} & ${ }^{60} \mathrm{Co}$ & $1.8 \mathrm{E}-02 \pm 6.5 \mathrm{E}-02$ & $\mathrm{U}$ & $(200-E)$ & ${ }^{60} \mathrm{Co}$ & $-7.2 \mathrm{E}-02 \pm 7.7 \mathrm{E}-02$ & $\mathrm{U}$ \\
\hline & ${ }^{134} \mathrm{Cs}$ & $5.5 \mathrm{E}-02 \pm 7.8 \mathrm{E}-02$ & $\mathrm{U}$ & & ${ }^{134} \mathrm{Cs}$ & $5.4 \mathrm{E}-03 \pm 5.4 \mathrm{E}-02$ & $\mathrm{U}$ \\
\hline & ${ }^{137} \mathrm{Cs}$ & $-9.3 \mathrm{E}-03 \pm 5.7 \mathrm{E}-02$ & $\mathrm{U}$ & & ${ }^{137} \mathrm{Cs}$ & 4.5E-02 \pm 7.1E-02 & $\mathrm{U}$ \\
\hline & ${ }^{152} \mathrm{Eu}$ & $6.6 \mathrm{E}-02 \pm 1.8 \mathrm{E}-01$ & $\mathrm{U}$ & & ${ }^{152} \mathrm{Eu}$ & $6.6 \mathrm{E}-02 \pm 1.9 \mathrm{E}-01$ & $\mathrm{U}$ \\
\hline & ${ }^{154} \mathrm{Eu}$ & $2.0 \mathrm{E}-01 \pm 1.9 \mathrm{E}-01$ & $\mathrm{U}$ & & ${ }^{154} \mathrm{Eu}$ & $-1.5 \mathrm{E}-01 \pm 2.2 \mathrm{E}-01$ & $\mathrm{U}$ \\
\hline & ${ }^{155} \mathrm{Eu}$ & $1.2 \mathrm{E}-01 \pm 1.7 \mathrm{E}-01$ & $\mathrm{U}$ & & ${ }^{155} \mathrm{Eu}$ & $1.0 \mathrm{E}-01 \pm 2.0 \mathrm{E}-01$ & $\mathrm{U}$ \\
\hline & ${ }^{238} \mathrm{Pu}$ & $-2.2 \mathrm{E}-03 \pm 1.5 \mathrm{E}-02$ & $\mathrm{U}$ & & ${ }^{238} \mathrm{Pu}$ & $-8.7 \mathrm{E}-04 \pm 8.7 \mathrm{E}-03$ & $\mathrm{U}$ \\
\hline & ${ }^{239 / 240} \mathrm{Pu}$ & $2.3 \mathrm{E}-04 \pm 2.3 \mathrm{E}-03$ & $\mathrm{U}$ & & ${ }^{239 / 240} \mathrm{Pu}$ & 8.7E-04 \pm 8.7E-03 & $\mathrm{U}$ \\
\hline & ${ }^{103} \mathrm{Ru}$ & $-7.9 \mathrm{E}-03 \pm 6.6 \mathrm{E}-02$ & $\mathrm{U}$ & & ${ }^{103} \mathrm{Ru}$ & $2.2 \mathrm{E}-02 \pm 7.3 \mathrm{E}-02$ & $\mathrm{U}$ \\
\hline & ${ }^{106} \mathrm{Ru}$ & $2.2 \mathrm{E}-01 \pm 5.9 \mathrm{E}-01$ & $\mathrm{U}$ & & ${ }^{106} \mathrm{Ru}$ & $1.8 \mathrm{E}-01 \pm 6.6 \mathrm{E}-01$ & $\mathrm{U}$ \\
\hline & ${ }^{125} \mathrm{Sb}$ & $-1.4 \mathrm{E}-02 \pm 1.4 \mathrm{E}-01$ & $\mathrm{U}$ & & ${ }^{125} \mathrm{Sb}$ & $-3.5 \mathrm{E}-02 \pm 1.7 \mathrm{E}-01$ & $\mathrm{U}$ \\
\hline & ${ }^{113} \mathrm{Sn}$ & $3.6 \mathrm{E}-02 \pm 6.6 \mathrm{E}-02$ & $\mathrm{U}$ & & ${ }^{113} \mathrm{Sn}$ & 7.7E-03 \pm 7.7E-02 & $\mathrm{U}$ \\
\hline & ${ }^{90} \mathrm{Sr}$ & $-9.0 \mathrm{E}-02 \pm 2.1 \mathrm{E}-01$ & $\mathrm{U}$ & & ${ }^{90} \mathrm{Sr}$ & $-1.7 \mathrm{E}-01 \pm 2.4 \mathrm{E}-01$ & $\mathrm{U}$ \\
\hline & ${ }^{234} \mathrm{U}$ & $7.2 \mathrm{E}-03 \pm 5.6 \mathrm{E}-03$ & & & ${ }^{234} \mathrm{U}$ & $1.9 \mathrm{E}-02 \pm 1.2 \mathrm{E}-02$ & \\
\hline & ${ }^{235} \mathrm{U}$ & $4.4 \mathrm{E}-03 \pm 4.1 \mathrm{E}-03$ & & & ${ }^{235} \mathrm{U}$ & $4.3 \mathrm{E}-03 \pm 4.4 \mathrm{E}-03$ & \\
\hline & ${ }^{238} \mathrm{U}$ & $9.7 \mathrm{E}-03 \pm 6.5 \mathrm{E}-03$ & & & ${ }^{238} \mathrm{U}$ & $1.1 \mathrm{E}-02 \pm 8.2 \mathrm{E}-03$ & \\
\hline & ${ }^{65} \mathrm{Zn}$ & $9.4 \mathrm{E}-02 \pm 1.6 \mathrm{E}-01$ & $\mathrm{U}$ & & ${ }^{65} \mathrm{Zn}$ & $7.9 \mathrm{E}-02 \pm 1.8 \mathrm{E}-01$ & $\mathrm{U}$ \\
\hline V066 & ${ }^{144} \mathrm{Ce}$ & $-3.2 \mathrm{E}-01 \pm 5.6 \mathrm{E}-01$ & $\mathrm{U}$ & V068 & ${ }^{144} \mathrm{Ce}$ & $4.2 \mathrm{E}-01 \pm 5.5 \mathrm{E}-01$ & $\mathrm{U}$ \\
\hline \multirow[t]{17}{*}{$(200-E)$} & ${ }^{60} \mathrm{Co}$ & $-2.0 \mathrm{E}-02 \pm 6.1 \mathrm{E}-02$ & $\mathrm{U}$ & $(200-E)$ & ${ }^{60} \mathrm{Co}$ & $7.9 \mathrm{E}-03 \pm 4.6 \mathrm{E}-02$ & $\mathrm{U}$ \\
\hline & ${ }^{134} \mathrm{Cs}$ & $-3.6 \mathrm{E}-02 \pm 5.9 \mathrm{E}-02$ & $\mathrm{U}$ & & ${ }^{134} \mathrm{Cs}$ & $-3.0 \mathrm{E}-02 \pm 4.9 \mathrm{E}-02$ & $\mathrm{U}$ \\
\hline & ${ }^{137} \mathrm{Cs}$ & $9.6 \mathrm{E}-02 \pm 7.9 \mathrm{E}-02$ & $\mathrm{U}$ & & ${ }^{137} \mathrm{Cs}$ & $1.2 \mathrm{E}-02 \pm 4.9 \mathrm{E}-02$ & $\mathrm{U}$ \\
\hline & ${ }^{152} \mathrm{Eu}$ & $-6.5 \mathrm{E}-02 \pm 1.4 \mathrm{E}-01$ & $\mathrm{U}$ & & ${ }^{152} \mathrm{Eu}$ & $2.4 \mathrm{E}-02 \pm 1.4 \mathrm{E}-01$ & $\mathrm{U}$ \\
\hline & ${ }^{154} \mathrm{Eu}$ & $3.5 \mathrm{E}-03 \pm 3.5 \mathrm{E}-02$ & $\mathrm{U}$ & & ${ }^{154} \mathrm{Eu}$ & $-3.8 \mathrm{E}-02 \pm 1.3 \mathrm{E}-01$ & $\mathrm{U}$ \\
\hline & ${ }^{155} \mathrm{Eu}$ & $4.6 \mathrm{E}-02 \pm 1.4 \mathrm{E}-01$ & $\mathrm{U}$ & & ${ }^{155} \mathrm{Eu}$ & $-1.4 \mathrm{E}-02 \pm 1.4 \mathrm{E}-01$ & $\mathrm{U}$ \\
\hline & ${ }^{238} \mathrm{Pu}$ & $5.4 \mathrm{E}-03 \pm 1.5 \mathrm{E}-02$ & $\mathrm{U}$ & & ${ }^{238} \mathrm{Pu}$ & $1.2 \mathrm{E}-02 \pm 1.0 \mathrm{E}-02$ & $\mathrm{U}$ \\
\hline & ${ }^{239 / 240} \mathrm{Pu}$ & $5.9 \mathrm{E}-02 \pm 2.2 \mathrm{E}-02$ & & & ${ }^{239 / 240} \mathrm{Pu}$ & $8.4 \mathrm{E}-04 \pm 1.7 \mathrm{E}-03$ & $\mathrm{U}$ \\
\hline & ${ }^{103} \mathrm{Ru}$ & $3.5 \mathrm{E}-03 \pm 3.5 \mathrm{E}-02$ & $\mathrm{U}$ & & ${ }^{103} \mathrm{Ru}$ & $-4.8 \mathrm{E}-02 \pm 5.2 \mathrm{E}-02$ & $\mathrm{U}$ \\
\hline & ${ }^{106} \mathrm{Ru}$ & $-1.0 \mathrm{E}+00 \pm 1.0 \mathrm{E}+00$ & $\mathrm{U}$ & & ${ }^{106} \mathrm{Ru}$ & $-1.4 \mathrm{E}-01 \pm 4.6 \mathrm{E}-01$ & $\mathrm{U}$ \\
\hline & ${ }^{125} \mathrm{Sb}$ & $-1.0 \mathrm{E}-01 \pm 1.4 \mathrm{E}-01$ & $\mathrm{U}$ & & ${ }^{125} \mathrm{Sb}$ & $1.5 \mathrm{E}-02 \pm 1.2 \mathrm{E}-01$ & $\mathrm{U}$ \\
\hline & ${ }^{113} \mathrm{Sn}$ & $5.3 \mathrm{E}-02 \pm 6.6 \mathrm{E}-02$ & $\mathrm{U}$ & & ${ }^{113} \mathrm{Sn}$ & $-4.5 \mathrm{E}-02 \pm 6.0 \mathrm{E}-02$ & $\mathrm{U}$ \\
\hline & ${ }^{90} \mathrm{Sr}$ & $-2.3 \mathrm{E}-01 \pm 2.3 \mathrm{E}-01$ & $\mathrm{U}$ & & ${ }^{90} \mathrm{Sr}$ & $-1.0 \mathrm{E}-01 \pm 2.3 \mathrm{E}-01$ & $\mathrm{U}$ \\
\hline & ${ }^{234} \mathrm{U}$ & $1.4 \mathrm{E}-02 \pm 8.1 \mathrm{E}-03$ & & & ${ }^{234} \mathrm{U}$ & $1.6 \mathrm{E}-02 \pm 9.1 \mathrm{E}-03$ & \\
\hline & ${ }^{235} \mathrm{U}$ & $3.0 \mathrm{E}-03 \pm 3.5 \mathrm{E}-03$ & & & ${ }^{235} \mathrm{U}$ & $4.5 \mathrm{E}-03 \pm 4.2 \mathrm{E}-03$ & \\
\hline & ${ }^{238} \mathrm{U}$ & $1.8 \mathrm{E}-02 \pm 9.5 \mathrm{E}-03$ & & & ${ }^{238} \mathrm{U}$ & $1.2 \mathrm{E}-02 \pm 7.0 \mathrm{E}-03$ & \\
\hline & ${ }^{65} \mathrm{Zn}$ & $9.7 \mathrm{E}-02 \pm 1.4 \mathrm{E}-01$ & $\mathrm{U}$ & & ${ }^{65} \mathrm{Zn}$ & $7.9 \mathrm{E}-02 \pm 1.7 \mathrm{E}-01$ & $\mathrm{U}$ \\
\hline
\end{tabular}

RQ = Result Qualifier. $\mathrm{U}=$ The analyte was analyzed for but not detected. 
Table 4-4. 2008 Vegetation Sampling Results (pCi/g \pm total analytical uncertainty). (Sheet 10 of 18)

\begin{tabular}{|c|c|c|c|c|c|c|c|}
\hline Location & Isotope & Result \pm Error & RQ* & Location & Is otope & Result \pm Error & RQ* \\
\hline V074 & ${ }^{144} \mathrm{Ce}$ & $-2.5 \mathrm{E}-01 \pm 5.0 \mathrm{E}-01$ & $\overline{\mathrm{U}}$ & V076 & ${ }^{144} \mathrm{Ce}$ & $-1.1 \mathrm{E}-01 \pm 8.3 \mathrm{E}-01$ & $\bar{U}$ \\
\hline \multirow[t]{17}{*}{$(200-E)$} & ${ }^{60} \mathrm{Co}$ & $-5.2 \mathrm{E}-03 \pm 3.9 \mathrm{E}-02$ & $\mathrm{U}$ & $(200-E)$ & ${ }^{60} \mathrm{Co}$ & $5.2 \mathrm{E}-02 \pm 7.4 \mathrm{E}-02$ & $\mathrm{U}$ \\
\hline & ${ }^{134} \mathrm{Cs}$ & $-7.3 \mathrm{E}-03 \pm 4.0 \mathrm{E}-02$ & $\mathrm{U}$ & & ${ }^{134} \mathrm{Cs}$ & $1.1 \mathrm{E}-02 \pm 8.3 \mathrm{E}-02$ & $\mathrm{U}$ \\
\hline & ${ }^{137} \mathrm{Cs}$ & $8.0 \mathrm{E}-02 \pm 6.5 \mathrm{E}-02$ & & & ${ }^{137} \mathrm{Cs}$ & $-3.0 \mathrm{E}-02 \pm 8.1 \mathrm{E}-02$ & $\mathrm{U}$ \\
\hline & ${ }^{152} \mathrm{Eu}$ & $5.0 \mathrm{E}-03 \pm 5.0 \mathrm{E}-02$ & $\mathrm{U}$ & & ${ }^{152} \mathrm{Eu}$ & 3.7E-01 \pm 3.4E-01 & \\
\hline & ${ }^{154} \mathrm{Eu}$ & $5.0 \mathrm{E}-02 \pm 1.2 \mathrm{E}-01$ & $\mathrm{U}$ & & ${ }^{154} \mathrm{Eu}$ & $-4.9 \mathrm{E}-02 \pm 2.2 \mathrm{E}-01$ & $\mathrm{U}$ \\
\hline & ${ }^{155} \mathrm{Eu}$ & $4.4 \mathrm{E}-02 \pm 1.2 \mathrm{E}-01$ & $\mathrm{U}$ & & ${ }^{155} \mathrm{Eu}$ & $6.7 \mathrm{E}-02 \pm 1.9 \mathrm{E}-01$ & $\mathrm{U}$ \\
\hline & ${ }^{238} \mathrm{Pu}$ & $-7.7 \mathrm{E}-04 \pm 3.4 \mathrm{E}-03$ & $\mathrm{U}$ & & ${ }^{238} \mathrm{Pu}$ & $5.0 \mathrm{E}-03 \pm 4.2 \mathrm{E}-03$ & \\
\hline & ${ }^{239 / 240} \mathrm{Pu}$ & $7.7 \mathrm{E}-04 \pm 7.7 \mathrm{E}-03$ & $\mathrm{U}$ & & ${ }^{239 / 240} \mathrm{Pu}$ & 8.3E-04 $\pm 8.3 \mathrm{E}-03$ & $\mathrm{U}$ \\
\hline & ${ }^{103} \mathrm{Ru}$ & $1.0 \mathrm{E}-02 \pm 4.5 \mathrm{E}-02$ & $\mathrm{U}$ & & ${ }^{103} \mathrm{Ru}$ & 4.7E-02 \pm 8.6E-02 & $\mathrm{U}$ \\
\hline & ${ }^{106} \mathrm{Ru}$ & 4.9E-02 $\pm 3.6 \mathrm{E}-01$ & $\mathrm{U}$ & & ${ }^{106} \mathrm{Ru}$ & $1.0 \mathrm{E}-01 \pm 7.0 \mathrm{E}-01$ & $\mathrm{U}$ \\
\hline & ${ }^{125} \mathrm{Sb}$ & $6.8 \mathrm{E}-02 \pm 1.1 \mathrm{E}-01$ & $\mathrm{U}$ & & ${ }^{125} \mathrm{Sb}$ & $-1.4 \mathrm{E}-01 \pm 2.0 \mathrm{E}-01$ & $\mathrm{U}$ \\
\hline & ${ }^{113} \mathrm{Sn}$ & $3.0 \mathrm{E}-03 \pm 3.0 \mathrm{E}-02$ & $\mathrm{U}$ & & ${ }^{113} \mathrm{Sn}$ & $-4.6 \mathrm{E}-02 \pm 9.3 \mathrm{E}-02$ & $\mathrm{U}$ \\
\hline & ${ }^{90} \mathrm{Sr}$ & $1.2 \mathrm{E}-01 \pm 2.3 \mathrm{E}-01$ & $\mathrm{U}$ & & ${ }^{90} \mathrm{Sr}$ & $-2.6 \mathrm{E}-01 \pm 2.6 \mathrm{E}-01$ & $\mathrm{U}$ \\
\hline & ${ }^{234} \mathrm{U}$ & $1.6 \mathrm{E}-02 \pm 8.6 \mathrm{E}-03$ & & & ${ }^{234} \mathrm{U}$ & $1.1 \mathrm{E}-02 \pm 6.9 \mathrm{E}-03$ & \\
\hline & ${ }^{235} \mathrm{U}$ & $1.9 \mathrm{E}-03 \pm 3.8 \mathrm{E}-03$ & $\mathrm{U}$ & & ${ }^{235} \mathrm{U}$ & $9.9 \mathrm{E}-04 \pm 2.0 \mathrm{E}-03$ & $\mathrm{U}$ \\
\hline & ${ }^{238} \mathrm{U}$ & $6.1 \mathrm{E}-03 \pm 5.5 \mathrm{E}-03$ & $\mathrm{U}$ & & ${ }^{238} \mathrm{U}$ & $4.5 \mathrm{E}-03 \pm 4.9 \mathrm{E}-03$ & $\mathrm{U}$ \\
\hline & ${ }^{65} \mathrm{Zn}$ & $-2.0 \mathrm{E}-01 \pm 2.0 \mathrm{E}-01$ & $\mathrm{U}$ & & ${ }^{65} \mathrm{Zn}$ & $5.4 \mathrm{E}-02 \pm 1.8 \mathrm{E}-01$ & $\mathrm{U}$ \\
\hline V078 & ${ }^{144} \mathrm{Ce}$ & $2.2 \mathrm{E}-02 \pm 2.2 \mathrm{E}-01$ & $\bar{U}$ & V080 & ${ }^{144} \mathrm{Ce}$ & $4.4 \mathrm{E}-01 \pm 6.6 \mathrm{E}-01$ & $\overline{\mathrm{U}}$ \\
\hline \multirow[t]{17}{*}{$(200-E)$} & ${ }^{60} \mathrm{Co}$ & $2.9 \mathrm{E}-02 \pm 5.1 \mathrm{E}-02$ & $\mathrm{U}$ & (200-E) & ${ }^{60} \mathrm{Co}$ & $-3.3 \mathrm{E}-02 \pm 6.0 \mathrm{E}-02$ & $\mathrm{U}$ \\
\hline & ${ }^{134} \mathrm{Cs}$ & $2.7 \mathrm{E}-02 \pm 5.6 \mathrm{E}-02$ & $\mathrm{U}$ & & ${ }^{134} \mathrm{Cs}$ & $-2.6 \mathrm{E}-02 \pm 6.3 \mathrm{E}-02$ & $\mathrm{U}$ \\
\hline & ${ }^{137} \mathrm{Cs}$ & $-2.0 \mathrm{E}-02 \pm 5.4 \mathrm{E}-02$ & $\mathrm{U}$ & & ${ }^{137} \mathrm{Cs}$ & $-1.8 \mathrm{E}-02 \pm 6.0 \mathrm{E}-02$ & $\mathrm{U}$ \\
\hline & ${ }^{152} \mathrm{Eu}$ & 4.6E-02 $\pm 1.4 \mathrm{E}-01$ & $\mathrm{U}$ & & ${ }^{152} \mathrm{Eu}$ & $-8.4 \mathrm{E}-02 \pm 1.7 \mathrm{E}-01$ & $\mathrm{U}$ \\
\hline & ${ }^{154} \mathrm{Eu}$ & $5.5 \mathrm{E}-02 \pm 1.5 \mathrm{E}-01$ & $\mathrm{U}$ & & ${ }^{154} \mathrm{Eu}$ & $-2.4 \mathrm{E}-02 \pm 1.8 \mathrm{E}-01$ & $\mathrm{U}$ \\
\hline & ${ }^{155} \mathrm{Eu}$ & $1.6 \mathrm{E}-02 \pm 1.5 \mathrm{E}-01$ & $\mathrm{U}$ & & ${ }^{155} \mathrm{Eu}$ & $-2.0 \mathrm{E}-01 \pm 2.0 \mathrm{E}-01$ & $\mathrm{U}$ \\
\hline & ${ }^{238} \mathrm{Pu}$ & $1.6 \mathrm{E}-03 \pm 3.2 \mathrm{E}-03$ & $\mathrm{U}$ & & ${ }^{238} \mathrm{Pu}$ & $3.5 \mathrm{E}-02 \pm 1.4 \mathrm{E}-02$ & \\
\hline & ${ }^{239 / 240} \mathrm{Pu}$ & $2.3 \mathrm{E}-03 \pm 2.7 \mathrm{E}-03$ & & & ${ }^{239 / 240} \mathrm{Pu}$ & $-7.8 \mathrm{E}-04 \pm 2.7 \mathrm{E}-03$ & $\mathrm{U}$ \\
\hline & ${ }^{103} \mathrm{Ru}$ & $2.8 \mathrm{E}-02 \pm 5.6 \mathrm{E}-02$ & $\mathrm{U}$ & & ${ }^{103} \mathrm{Ru}$ & $4.5 \mathrm{E}-02 \pm 6.6 \mathrm{E}-02$ & $\mathrm{U}$ \\
\hline & ${ }^{106} \mathrm{Ru}$ & $9.8 \mathrm{E}-02 \pm 5.7 \mathrm{E}-01$ & $\mathrm{U}$ & & ${ }^{106} \mathrm{Ru}$ & $5.4 \mathrm{E}-02 \pm 5.4 \mathrm{E}-01$ & $\mathrm{U}$ \\
\hline & ${ }^{125} \mathrm{Sb}$ & $-2.1 \mathrm{E}-02 \pm 1.3 \mathrm{E}-01$ & $\mathrm{U}$ & & ${ }^{125} \mathrm{Sb}$ & 4.7E-02 $\pm 1.6 \mathrm{E}-01$ & $\mathrm{U}$ \\
\hline & ${ }^{113} \mathrm{Sn}$ & $5.5 \mathrm{E}-03 \pm 5.5 \mathrm{E}-02$ & $\mathrm{U}$ & & ${ }^{113} \mathrm{Sn}$ & $-2.4 \mathrm{E}-02 \pm 7.1 \mathrm{E}-02$ & $\mathrm{U}$ \\
\hline & ${ }^{90} \mathrm{Sr}$ & $1.2 \mathrm{E}-01 \pm 2.0 \mathrm{E}-01$ & $\mathrm{U}$ & & ${ }^{90} \mathrm{Sr}$ & $-1.1 \mathrm{E}-01 \pm 5.8 \mathrm{E}-01$ & $\mathrm{U}$ \\
\hline & ${ }^{234} \mathrm{U}$ & $1.1 \mathrm{E}-02 \pm 7.9 \mathrm{E}-03$ & & & ${ }^{234} \mathrm{U}$ & $1.4 \mathrm{E}-02 \pm 9.0 \mathrm{E}-03$ & \\
\hline & ${ }^{235} \mathrm{U}$ & $3.8 \mathrm{E}-03 \pm 3.9 \mathrm{E}-03$ & & & ${ }^{235} \mathrm{U}$ & $3.8 \mathrm{E}-03 \pm 3.9 \mathrm{E}-03$ & \\
\hline & ${ }^{238} \mathrm{U}$ & $8.8 \mathrm{E}-03 \pm 6.0 \mathrm{E}-03$ & & & ${ }^{238} \mathrm{U}$ & 1.4E-02 $\pm 9.7 \mathrm{E}-03$ & \\
\hline & ${ }^{65} \mathrm{Zn}$ & $-1.3 \mathrm{E}-02 \pm 1.2 \mathrm{E}-01$ & $\mathrm{U}$ & & ${ }^{65} \mathrm{Zn}$ & $1.4 \mathrm{E}-01 \pm 1.4 \mathrm{E}-01$ & $\mathrm{U}$ \\
\hline
\end{tabular}

RQ = Result Qualifier. $\mathrm{U}=$ The analyte was analyzed for but not detected. 
Table 4-4. 2008 Vegetation Sampling Results (pCi/g \pm total analytical uncertainty). (Sheet 11 of 18)

\begin{tabular}{|c|c|c|c|c|c|c|c|}
\hline Location & Isotope & Result \pm Error & RQ* & Location & Isotope & Result \pm Error & $\mathbf{R Q *}$ \\
\hline V082 & ${ }^{144} \mathrm{Ce}$ & $4.2 \mathrm{E}-01 \pm 1.0 \mathrm{E}+00$ & $\bar{U}$ & V084 & ${ }^{144} \mathrm{Ce}$ & $-4.4 \mathrm{E}-02 \pm 4.4 \mathrm{E}-01$ & $\bar{U}$ \\
\hline \multirow[t]{17}{*}{ (600 Area) } & ${ }^{60} \mathrm{Co}$ & $2.0 \mathrm{E}-02 \pm 1.0 \mathrm{E}-01$ & $\mathrm{U}$ & (600 Area) & ${ }^{60} \mathrm{Co}$ & $-1.3 \mathrm{E}-02 \pm 5.4 \mathrm{E}-02$ & $\mathrm{U}$ \\
\hline & ${ }^{134} \mathrm{Cs}$ & $-4.9 \mathrm{E}-02 \pm 1.0 \mathrm{E}-01$ & $\mathrm{U}$ & & ${ }^{134} \mathrm{Cs}$ & $3.8 \mathrm{E}-02 \pm 5.6 \mathrm{E}-02$ & $\mathrm{U}$ \\
\hline & ${ }^{137} \mathrm{Cs}$ & $2.3 \mathrm{E}-02 \pm 1.0 \mathrm{E}-01$ & $\mathrm{U}$ & & ${ }^{137} \mathrm{Cs}$ & $1.8 \mathrm{E}-01 \pm 6.5 \mathrm{E}-02$ & \\
\hline & ${ }^{152} \mathrm{Eu}$ & $-1.6 \mathrm{E}-02 \pm 1.6 \mathrm{E}-01$ & $\mathrm{U}$ & & ${ }^{152} \mathrm{Eu}$ & $-5.3 \mathrm{E}-02 \pm 1.5 \mathrm{E}-01$ & $\mathrm{U}$ \\
\hline & ${ }^{154} \mathrm{Eu}$ & $6.4 \mathrm{E}-02 \pm 2.9 \mathrm{E}-01$ & $\mathrm{U}$ & & ${ }^{154} \mathrm{Eu}$ & $-7.9 \mathrm{E}-02 \pm 1.5 \mathrm{E}-01$ & $\mathrm{U}$ \\
\hline & ${ }^{155} \mathrm{Eu}$ & $1.5 \mathrm{E}-01 \pm 2.6 \mathrm{E}-01$ & $\mathrm{U}$ & & ${ }^{155} \mathrm{Eu}$ & $-6.2 \mathrm{E}-03 \pm 6.2 \mathrm{E}-02$ & $\mathrm{U}$ \\
\hline & ${ }^{238} \mathrm{Pu}$ & $6.2 \mathrm{E}-03 \pm 1.1 \mathrm{E}-02$ & $\mathrm{U}$ & & ${ }^{238} \mathrm{Pu}$ & 8.9E-04 \pm 8.9E-03 & $\mathrm{U}$ \\
\hline & ${ }^{239 / 240} \mathrm{Pu}$ & $1.0 \mathrm{E}-03 \pm 1.0 \mathrm{E}-02$ & $\mathrm{U}$ & & ${ }^{239 / 240} \mathrm{Pu}$ & 8.9E-04 $\pm 4.7 \mathrm{E}-03$ & $\mathrm{U}$ \\
\hline & ${ }^{103} \mathrm{Ru}$ & $1.2 \mathrm{E}-01 \pm 1.2 \mathrm{E}-01$ & $\mathrm{U}$ & & ${ }^{103} \mathrm{Ru}$ & $-2.2 \mathrm{E}-02 \pm 6.8 \mathrm{E}-02$ & $\mathrm{U}$ \\
\hline & ${ }^{106} \mathrm{Ru}$ & $-3.0 \mathrm{E}-01 \pm 9.2 \mathrm{E}-01$ & $\mathrm{U}$ & & ${ }^{106} \mathrm{Ru}$ & $-3.6 \mathrm{E}-01 \pm 5.0 \mathrm{E}-01$ & $\mathrm{U}$ \\
\hline & ${ }^{125} \mathrm{Sb}$ & $-1.8 \mathrm{E}-02 \pm 1.8 \mathrm{E}-01$ & $\mathrm{U}$ & & ${ }^{125} \mathrm{Sb}$ & $-1.1 \mathrm{E}-01 \pm 1.4 \mathrm{E}-01$ & $\mathrm{U}$ \\
\hline & ${ }^{113} \mathrm{Sn}$ & $-1.5 \mathrm{E}-02 \pm 1.4 \mathrm{E}-01$ & $\mathrm{U}$ & & ${ }^{113} \mathrm{Sn}$ & $-5.7 \mathrm{E}-02 \pm 6.9 \mathrm{E}-02$ & $\mathrm{U}$ \\
\hline & ${ }^{90} \mathrm{Sr}$ & $-1.7 \mathrm{E}-01 \pm 1.9 \mathrm{E}-01$ & $\mathrm{U}$ & & ${ }^{90} \mathrm{Sr}$ & $1.6 \mathrm{E}-02 \pm 1.6 \mathrm{E}-01$ & $\mathrm{U}$ \\
\hline & ${ }^{234} \mathrm{U}$ & $9.9 \mathrm{E}-03 \pm 6.9 \mathrm{E}-03$ & & & ${ }^{234} \mathrm{U}$ & $1.3 \mathrm{E}-02 \pm 8.6 \mathrm{E}-03$ & \\
\hline & ${ }^{235} \mathrm{U}$ & $2.0 \mathrm{E}-03 \pm 4.0 \mathrm{E}-03$ & $\mathrm{U}$ & & ${ }^{235} \mathrm{U}$ & $3.5 \mathrm{E}-03 \pm 3.6 \mathrm{E}-03$ & \\
\hline & ${ }^{238} \mathrm{U}$ & $7.2 \mathrm{E}-03 \pm 5.4 \mathrm{E}-03$ & & & ${ }^{238} \mathrm{U}$ & $8.1 \mathrm{E}-03 \pm 5.5 \mathrm{E}-03$ & \\
\hline & ${ }^{65} \mathrm{Zn}$ & $1.8 \mathrm{E}-02 \pm 1.8 \mathrm{E}-01$ & $\mathrm{U}$ & & ${ }^{65} \mathrm{Zn}$ & $-8.4 \mathrm{E}-02 \pm 1.2 \mathrm{E}-01$ & $\mathrm{U}$ \\
\hline \multirow{18}{*}{$\begin{array}{c}\text { V088 } \\
\text { (600 Area) }\end{array}$} & ${ }^{144} \mathrm{Ce}$ & $6.2 \mathrm{E}-02 \pm 4.3 \mathrm{E}-01$ & $\mathrm{U}$ & V090 & ${ }^{144} \mathrm{Ce}$ & $-1.2 \mathrm{E}-01 \pm 6.8 \mathrm{E}-01$ & $\mathrm{U}$ \\
\hline & ${ }^{60} \mathrm{Co}$ & 8.9E-03 \pm 3.0E-02 & $\mathrm{U}$ & (600 Area) & ${ }^{60} \mathrm{Co}$ & $-3.7 \mathrm{E}-02 \pm 5.1 \mathrm{E}-02$ & $\mathrm{U}$ \\
\hline & ${ }^{134} \mathrm{Cs}$ & 8.4E-03 \pm 3.3E-02 & $\mathrm{U}$ & & ${ }^{134} \mathrm{Cs}$ & $-9.9 \mathrm{E}-03 \pm 5.4 \mathrm{E}-02$ & $\mathrm{U}$ \\
\hline & ${ }^{137} \mathrm{Cs}$ & $1.5 \mathrm{E}-02 \pm 3.2 \mathrm{E}-02$ & $\mathrm{U}$ & & ${ }^{137} \mathrm{Cs}$ & $7.4 \mathrm{E}-02 \pm 9.2 \mathrm{E}-02$ & $\mathrm{U}$ \\
\hline & ${ }^{152} \mathrm{Eu}$ & $-2.6 \mathrm{E}-02 \pm 8.9 \mathrm{E}-02$ & $\mathrm{U}$ & & ${ }^{152} \mathrm{Eu}$ & $1.2 \mathrm{E}-01 \pm 1.5 \mathrm{E}-01$ & $\mathrm{U}$ \\
\hline & ${ }^{154} \mathrm{Eu}$ & $-1.2 \mathrm{E}-02 \pm 8.3 \mathrm{E}-02$ & $\mathrm{U}$ & & ${ }^{154} \mathrm{Eu}$ & $1.5 \mathrm{E}-02 \pm 1.5 \mathrm{E}-01$ & $\mathrm{U}$ \\
\hline & ${ }^{155} \mathrm{Eu}$ & $7.1 \mathrm{E}-02 \pm 9.2 \mathrm{E}-02$ & $\mathrm{U}$ & & ${ }^{155} \mathrm{Eu}$ & $-7.0 \mathrm{E}-02 \pm 1.6 \mathrm{E}-01$ & $\mathrm{U}$ \\
\hline & ${ }^{238} \mathrm{Pu}$ & $3.6 \mathrm{E}-03 \pm 1.8 \mathrm{E}-02$ & $\mathrm{U}$ & & ${ }^{238} \mathrm{Pu}$ & $-1.1 \mathrm{E}-02 \pm 1.8 \mathrm{E}-02$ & $\mathrm{U}$ \\
\hline & ${ }^{239 / 240} \mathrm{Pu}$ & $3.6 \mathrm{E}-03 \pm 6.4 \mathrm{E}-03$ & $\mathrm{U}$ & & ${ }^{239 / 240} \mathrm{Pu}$ & $1.1 \mathrm{E}-03 \pm 1.1 \mathrm{E}-02$ & $\mathrm{U}$ \\
\hline & ${ }^{103} \mathrm{Ru}$ & 4.7E-03 \pm 4.7E-02 & $\mathrm{U}$ & & ${ }^{103} \mathrm{Ru}$ & $5.3 \mathrm{E}-03 \pm 5.3 \mathrm{E}-02$ & $\mathrm{U}$ \\
\hline & ${ }^{106} \mathrm{Ru}$ & $2.0 \mathrm{E}-01 \pm 3.3 \mathrm{E}-01$ & $\mathrm{U}$ & & ${ }^{106} \mathrm{Ru}$ & $3.6 \mathrm{E}-02 \pm 3.6 \mathrm{E}-01$ & $\mathrm{U}$ \\
\hline & ${ }^{125} \mathrm{Sb}$ & $2.7 \mathrm{E}-02 \pm 8.6 \mathrm{E}-02$ & $\mathrm{U}$ & & ${ }^{125} \mathrm{Sb}$ & $8.2 \mathrm{E}-02 \pm 1.5 \mathrm{E}-01$ & $\mathrm{U}$ \\
\hline & ${ }^{113} \mathrm{Sn}$ & $-2.3 \mathrm{E}-02 \pm 6.0 \mathrm{E}-02$ & $\mathrm{U}$ & & ${ }^{113} \mathrm{Sn}$ & $-3.9 \mathrm{E}-02 \pm 7.5 \mathrm{E}-02$ & $\mathrm{U}$ \\
\hline & ${ }^{90} \mathrm{Sr}$ & $-1.7 \mathrm{E}-01 \pm 2.0 \mathrm{E}-01$ & $\mathrm{U}$ & & ${ }^{90} \mathrm{Sr}$ & $-1.1 \mathrm{E}-01 \pm 2.0 \mathrm{E}-01$ & $\mathrm{U}$ \\
\hline & ${ }^{234} \mathrm{U}$ & $1.3 \mathrm{E}-02 \pm 8.3 \mathrm{E}-03$ & & & ${ }^{234} \mathrm{U}$ & $1.7 \mathrm{E}-02 \pm 8.7 \mathrm{E}-03$ & \\
\hline & ${ }^{235} \mathrm{U}$ & $2.8 \mathrm{E}-03 \pm 3.3 \mathrm{E}-03$ & & & ${ }^{235} \mathrm{U}$ & $2.6 \mathrm{E}-03 \pm 3.1 \mathrm{E}-03$ & \\
\hline & ${ }^{238} \mathrm{U}$ & $1.5 \mathrm{E}-02 \pm 8.1 \mathrm{E}-03$ & & & ${ }^{238} \mathrm{U}$ & $7.8 \mathrm{E}-03 \pm 5.3 \mathrm{E}-03$ & \\
\hline & ${ }^{65} \mathrm{Zn}$ & $7.5 \mathrm{E}-02 \pm 8.7 \mathrm{E}-02$ & $\mathrm{U}$ & & ${ }^{65} \mathrm{Zn}$ & $-1.1 \mathrm{E}-02 \pm 1.1 \mathrm{E}-01$ & $\mathrm{U}$ \\
\hline
\end{tabular}

RQ = Result Qualifier. $\mathrm{U}=$ The analyte was analyzed for but not detected. 
Table 4-4. 2008 Vegetation Sampling Results (pCi/g \pm total analytical uncertainty). (Sheet 12 of 18)

\begin{tabular}{|c|c|c|c|c|c|c|c|}
\hline Location & Isotope & Result \pm Error & $\mathbf{R Q}^{*}$ & Location & Is otope & Result \pm Error & RQ* \\
\hline V092 & ${ }^{144} \mathrm{Ce}$ & $2.9 \mathrm{E}-01 \pm 4.6 \mathrm{E}-01$ & $\mathrm{U}$ & V094 & ${ }^{144} \mathrm{Ce}$ & $-2.5 \mathrm{E}-01 \pm 5.6 \mathrm{E}-01$ & $\mathrm{U}$ \\
\hline \multirow[t]{17}{*}{ (600 Area) } & ${ }^{60} \mathrm{Co}$ & $-3.7 \mathrm{E}-02 \pm 4.1 \mathrm{E}-02$ & $\mathrm{U}$ & (600 Area) & ${ }^{60} \mathrm{Co}$ & $2.6 \mathrm{E}-03 \pm 2.6 \mathrm{E}-02$ & $\mathrm{U}$ \\
\hline & ${ }^{134} \mathrm{Cs}$ & 5.7E-02 \pm 5.4E-02 & $\mathrm{U}$ & & ${ }^{134} \mathrm{Cs}$ & 2.3E-02 $\pm 4.7 \mathrm{E}-02$ & $\mathrm{U}$ \\
\hline & ${ }^{137} \mathrm{Cs}$ & $1.7 \mathrm{E}-01 \pm 8.2 \mathrm{E}-02$ & & & ${ }^{137} \mathrm{Cs}$ & $-1.1 \mathrm{E}-02 \pm 4.7 \mathrm{E}-02$ & $\mathrm{U}$ \\
\hline & ${ }^{152} \mathrm{Eu}$ & $-2.2 \mathrm{E}-02 \pm 1.1 \mathrm{E}-01$ & $\mathrm{U}$ & & ${ }^{152} \mathrm{Eu}$ & $3.4 \mathrm{E}-02 \pm 1.2 \mathrm{E}-01$ & $\mathrm{U}$ \\
\hline & ${ }^{154} \mathrm{Eu}$ & $6.5 \mathrm{E}-02 \pm 1.2 \mathrm{E}-01$ & $\mathrm{U}$ & & ${ }^{154} \mathrm{Eu}$ & $6.2 \mathrm{E}-02 \pm 1.4 \mathrm{E}-01$ & $\mathrm{U}$ \\
\hline & ${ }^{155} \mathrm{Eu}$ & $-3.4 \mathrm{E}-02 \pm 1.2 \mathrm{E}-01$ & U & & ${ }^{155} \mathrm{Eu}$ & $-3.0 \mathrm{E}-02 \pm 1.5 \mathrm{E}-01$ & $\mathrm{U}$ \\
\hline & ${ }^{238} \mathrm{Pu}$ & $-1.7 \mathrm{E}-02 \pm 2.0 \mathrm{E}-02$ & $\mathrm{U}$ & & ${ }^{238} \mathrm{Pu}$ & $5.3 \mathrm{E}-03 \pm 1.2 \mathrm{E}-02$ & $\mathrm{U}$ \\
\hline & ${ }^{239 / 240} \mathrm{Pu}$ & $3.6 \mathrm{E}-02 \pm 1.6 \mathrm{E}-02$ & & & ${ }^{239 / 240} \mathrm{Pu}$ & $1.1 \mathrm{E}-03 \pm 1.1 \mathrm{E}-02$ & $\mathrm{U}$ \\
\hline & ${ }^{103} \mathrm{Ru}$ & $-4.0 \mathrm{E}-02 \pm 5.1 \mathrm{E}-02$ & $\mathrm{U}$ & & ${ }^{103} \mathrm{Ru}$ & $-1.1 \mathrm{E}-02 \pm 5.7 \mathrm{E}-02$ & $\mathrm{U}$ \\
\hline & ${ }^{106} \mathrm{Ru}$ & $1.0 \mathrm{E}-01 \pm 3.9 \mathrm{E}-01$ & $\mathrm{U}$ & & ${ }^{106} \mathrm{Ru}$ & $-2.2 \mathrm{E}-01 \pm 4.4 \mathrm{E}-01$ & $\mathrm{U}$ \\
\hline & ${ }^{125} \mathrm{Sb}$ & $-4.6 \mathrm{E}-02 \pm 1.0 \mathrm{E}-01$ & $\mathrm{U}$ & & ${ }^{125} \mathrm{Sb}$ & $-7.6 \mathrm{E}-02 \pm 1.2 \mathrm{E}-01$ & $\mathrm{U}$ \\
\hline & ${ }^{113} \mathrm{Sn}$ & $-1.2 \mathrm{E}-02 \pm 4.9 \mathrm{E}-02$ & $\mathrm{U}$ & & ${ }^{113} \mathrm{Sn}$ & $-1.7 \mathrm{E}-03 \pm 1.7 \mathrm{E}-02$ & $\mathrm{U}$ \\
\hline & ${ }^{90} \mathrm{Sr}$ & $1.5 \mathrm{E}-01 \pm 2.2 \mathrm{E}-01$ & $\mathrm{U}$ & & ${ }^{90} \mathrm{Sr}$ & $5.5 \mathrm{E}-02 \pm 2.1 \mathrm{E}-01$ & $\mathrm{U}$ \\
\hline & ${ }^{234} \mathrm{U}$ & $2.5 \mathrm{E}-02 \pm 1.2 \mathrm{E}-02$ & & & ${ }^{234} \mathrm{U}$ & $1.4 \mathrm{E}-02 \pm 8.7 \mathrm{E}-03$ & \\
\hline & ${ }^{235} \mathrm{U}$ & $6.7 \mathrm{E}-03 \pm 5.4 \mathrm{E}-03$ & & & ${ }^{235} \mathrm{U}$ & $3.5 \mathrm{E}-03 \pm 3.6 \mathrm{E}-03$ & \\
\hline & ${ }^{238} \mathrm{U}$ & $1.4 \mathrm{E}-02 \pm 8.3 \mathrm{E}-03$ & & & ${ }^{238} \mathrm{U}$ & $8.8 \mathrm{E}-03 \pm 5.8 \mathrm{E}-03$ & \\
\hline & ${ }^{65} \mathrm{Zn}$ & $1.2 \mathrm{E}-01 \pm 1.0 \mathrm{E}-01$ & $\mathrm{U}$ & & ${ }^{65} \mathrm{Zn}$ & $-7.4 \mathrm{E}-02 \pm 1.0 \mathrm{E}-01$ & $\mathrm{U}$ \\
\hline \multirow{18}{*}{$\begin{array}{c}\text { V096 } \\
\text { (600 Area) }\end{array}$} & ${ }^{144} \mathrm{Ce}$ & $-3.2 \mathrm{E}-01 \pm 7.0 \mathrm{E}-01$ & $\mathrm{U}$ & V098 & ${ }^{144} \mathrm{Ce}$ & $2.9 \mathrm{E}-02 \pm 2.9 \mathrm{E}-01$ & $\mathrm{U}$ \\
\hline & ${ }^{60} \mathrm{Co}$ & $1.1 \mathrm{E}-02 \pm 5.6 \mathrm{E}-02$ & $\mathrm{U}$ & (600 Area) & ${ }^{60} \mathrm{Co}$ & $3.5 \mathrm{E}-03 \pm 2.9 \mathrm{E}-02$ & $\mathrm{U}$ \\
\hline & ${ }^{134} \mathrm{Cs}$ & $-2.6 \mathrm{E}-02 \pm 6.0 \mathrm{E}-02$ & $\mathrm{U}$ & & ${ }^{134} \mathrm{Cs}$ & $1.9 \mathrm{E}-02 \pm 3.2 \mathrm{E}-02$ & $\mathrm{U}$ \\
\hline & ${ }^{137} \mathrm{Cs}$ & $-1.0 \mathrm{E}-02 \pm 5.6 \mathrm{E}-02$ & $\mathrm{U}$ & & ${ }^{137} \mathrm{Cs}$ & $9.2 \mathrm{E}-03 \pm 3.1 \mathrm{E}-02$ & $\mathrm{U}$ \\
\hline & ${ }^{152} \mathrm{Eu}$ & 7.7E-02 $\pm 1.5 \mathrm{E}-01$ & $\mathrm{U}$ & & ${ }^{152} \mathrm{Eu}$ & $-1.8 \mathrm{E}-02 \pm 9.3 \mathrm{E}-02$ & $\mathrm{U}$ \\
\hline & ${ }^{154} \mathrm{Eu}$ & $1.4 \mathrm{E}-02 \pm 1.4 \mathrm{E}-01$ & $\mathrm{U}$ & & ${ }^{154} \mathrm{Eu}$ & $-1.6 \mathrm{E}-02 \pm 8.9 \mathrm{E}-02$ & $\mathrm{U}$ \\
\hline & ${ }^{155} \mathrm{Eu}$ & $-9.3 \mathrm{E}-02 \pm 1.7 \mathrm{E}-01$ & $\mathrm{U}$ & & ${ }^{155} \mathrm{Eu}$ & $-5.9 \mathrm{E}-02 \pm 9.0 \mathrm{E}-02$ & $\mathrm{U}$ \\
\hline & ${ }^{238} \mathrm{Pu}$ & $1.0 \mathrm{E}-03 \pm 1.0 \mathrm{E}-02$ & $\mathrm{U}$ & & ${ }^{238} \mathrm{Pu}$ & $1.1 \mathrm{E}-02 \pm 1.6 \mathrm{E}-02$ & $\mathrm{U}$ \\
\hline & ${ }^{239 / 240} \mathrm{Pu}$ & $2.0 \mathrm{E}-03 \pm 4.0 \mathrm{E}-03$ & $\mathrm{U}$ & & ${ }^{239 / 240} \mathrm{Pu}$ & $1.5 \mathrm{E}-02 \pm 8.8 \mathrm{E}-03$ & \\
\hline & ${ }^{103} \mathrm{Ru}$ & $6.8 \mathrm{E}-02 \pm 7.2 \mathrm{E}-02$ & $\mathrm{U}$ & & ${ }^{103} \mathrm{Ru}$ & $-2.2 \mathrm{E}-03 \pm 2.2 \mathrm{E}-02$ & $\mathrm{U}$ \\
\hline & ${ }^{106} \mathrm{Ru}$ & $2.4 \mathrm{E}-01 \pm 5.4 \mathrm{E}-01$ & $\mathrm{U}$ & & ${ }^{106} \mathrm{Ru}$ & $-1.8 \mathrm{E}-01 \pm 2.9 \mathrm{E}-01$ & $\mathrm{U}$ \\
\hline & ${ }^{125} \mathrm{Sb}$ & $-5.4 \mathrm{E}-02 \pm 1.4 \mathrm{E}-01$ & $\mathrm{U}$ & & ${ }^{125} \mathrm{Sb}$ & $7.0 \mathrm{E}-02 \pm 8.1 \mathrm{E}-02$ & $\mathrm{U}$ \\
\hline & ${ }^{113} \mathrm{Sn}$ & $6.2 \mathrm{E}-02 \pm 7.2 \mathrm{E}-02$ & $\mathrm{U}$ & & ${ }^{113} \mathrm{Sn}$ & $-4.5 \mathrm{E}-03 \pm 4.5 \mathrm{E}-02$ & $\mathrm{U}$ \\
\hline & ${ }^{90} \mathrm{Sr}$ & $3.7 \mathrm{E}-02 \pm 2.1 \mathrm{E}-01$ & $\mathrm{U}$ & & ${ }^{90} \mathrm{Sr}$ & $-8.0 \mathrm{E}-02 \pm 2.1 \mathrm{E}-01$ & $\mathrm{U}$ \\
\hline & ${ }^{234} \mathrm{U}$ & $1.4 \mathrm{E}-02 \pm 8.1 \mathrm{E}-03$ & & & ${ }^{234} \mathrm{U}$ & $1.2 \mathrm{E}-02 \pm 7.9 \mathrm{E}-03$ & \\
\hline & ${ }^{235} \mathrm{U}$ & $4.9 \mathrm{E}-03 \pm 4.6 \mathrm{E}-03$ & & & ${ }^{235} \mathrm{U}$ & $7.1 \mathrm{E}-03 \pm 5.3 \mathrm{E}-03$ & \\
\hline & ${ }^{238} \mathrm{U}$ & $4.5 \mathrm{E}-03 \pm 6.1 \mathrm{E}-03$ & $\mathrm{U}$ & & ${ }^{238} \mathrm{U}$ & $4.9 \mathrm{E}-03 \pm 5.3 \mathrm{E}-03$ & $\mathrm{U}$ \\
\hline & ${ }^{65} \mathrm{Zn}$ & $-1.4 \mathrm{E}-01 \pm 1.4 \mathrm{E}-01$ & $\mathrm{U}$ & & ${ }^{65} \mathrm{Zn}$ & $4.0 \mathrm{E}-02 \pm 8.2 \mathrm{E}-02$ & $\mathrm{U}$ \\
\hline
\end{tabular}

RQ = Result Qualifier. $\mathrm{U}=$ The analyte was analyzed for but not detected. 
Table 4-4. 2008 Vegetation Sampling Results (pCi/g \pm total analytical uncertainty). (Sheet 13 of 18)

\begin{tabular}{|c|c|c|c|c|c|c|c|}
\hline Location & Isotope & Result \pm Error & $\mathbf{R Q}^{*}$ & Location & Is otope & Result \pm Error & $\mathbf{R Q}^{*}$ \\
\hline V100 & ${ }^{144} \mathrm{Ce}$ & $-2.4 \mathrm{E}-01 \pm 5.3 \mathrm{E}-01$ & $\bar{U}$ & V102 & ${ }^{144} \mathrm{Ce}$ & $-1.6 \mathrm{E}-01 \pm 4.4 \mathrm{E}-01$ & $\mathrm{U}$ \\
\hline \multirow[t]{17}{*}{ (600 Area) } & ${ }^{60} \mathrm{Co}$ & $-3.0 \mathrm{E}-02 \pm 3.9 \mathrm{E}-02$ & $\mathrm{U}$ & (600 Area) & ${ }^{60} \mathrm{Co}$ & $-3.2 \mathrm{E}-02 \pm 4.5 \mathrm{E}-02$ & $\mathrm{U}$ \\
\hline & ${ }^{134} \mathrm{Cs}$ & $1.0 \mathrm{E}-02 \pm 4.4 \mathrm{E}-02$ & $\mathrm{U}$ & & ${ }^{134} \mathrm{Cs}$ & $2.6 \mathrm{E}-02 \pm 5.1 \mathrm{E}-02$ & $\mathrm{U}$ \\
\hline & ${ }^{137} \mathrm{Cs}$ & $-3.5 \mathrm{E}-03 \pm 3.5 \mathrm{E}-02$ & $\mathrm{U}$ & & ${ }^{137} \mathrm{Cs}$ & 4.7E-02 $\pm 4.5 \mathrm{E}-02$ & $\mathrm{U}$ \\
\hline & ${ }^{152} \mathrm{Eu}$ & $-8.2 \mathrm{E}-02 \pm 1.2 \mathrm{E}-01$ & $\mathrm{U}$ & & ${ }^{152} \mathrm{Eu}$ & $-1.4 \mathrm{E}-01 \pm 1.4 \mathrm{E}-01$ & $\mathrm{U}$ \\
\hline & ${ }^{154} \mathrm{Eu}$ & $-7.8 \mathrm{E}-02 \pm 1.3 \mathrm{E}-01$ & $\mathrm{U}$ & & ${ }^{154} \mathrm{Eu}$ & $1.1 \mathrm{E}-02 \pm 1.1 \mathrm{E}-01$ & $\mathrm{U}$ \\
\hline & ${ }^{155} \mathrm{Eu}$ & $-5.4 \mathrm{E}-02 \pm 1.1 \mathrm{E}-01$ & $\mathrm{U}$ & & ${ }^{155} \mathrm{Eu}$ & $-1.9 \mathrm{E}-02 \pm 1.1 \mathrm{E}-01$ & $\mathrm{U}$ \\
\hline & ${ }^{238} \mathrm{Pu}$ & $-4.1 \mathrm{E}-03 \pm 1.9 \mathrm{E}-02$ & $\mathrm{U}$ & & ${ }^{238} \mathrm{Pu}$ & $1.1 \mathrm{E}-02 \pm 1.2 \mathrm{E}-02$ & $\mathrm{U}$ \\
\hline & ${ }^{239 / 240} \mathrm{Pu}$ & $4.1 \mathrm{E}-03 \pm 4.2 \mathrm{E}-03$ & & & ${ }^{239 / 240} \mathrm{Pu}$ & $6.8 \mathrm{E}-03 \pm 5.4 \mathrm{E}-03$ & \\
\hline & ${ }^{103} \mathrm{Ru}$ & $-5.0 \mathrm{E}-02 \pm 5.7 \mathrm{E}-02$ & $\mathrm{U}$ & & ${ }^{103} \mathrm{Ru}$ & $-4.8 \mathrm{E}-02 \pm 5.2 \mathrm{E}-02$ & $\mathrm{U}$ \\
\hline & ${ }^{106} \mathrm{Ru}$ & $1.8 \mathrm{E}-01 \pm 3.5 \mathrm{E}-01$ & $\mathrm{U}$ & & ${ }^{106} \mathrm{Ru}$ & $1.3 \mathrm{E}-01 \pm 3.7 \mathrm{E}-01$ & $\mathrm{U}$ \\
\hline & ${ }^{125} \mathrm{Sb}$ & $-5.0 \mathrm{E}-02 \pm 1.1 \mathrm{E}-01$ & $\mathrm{U}$ & & ${ }^{125} \mathrm{Sb}$ & $6.2 \mathrm{E}-02 \pm 9.9 \mathrm{E}-02$ & $\mathrm{U}$ \\
\hline & ${ }^{113} \mathrm{Sn}$ & $-2.4 \mathrm{E}-02 \pm 5.6 \mathrm{E}-02$ & $\mathrm{U}$ & & ${ }^{113} \mathrm{Sn}$ & $-6.8 \mathrm{E}-03 \pm 4.9 \mathrm{E}-02$ & $\mathrm{U}$ \\
\hline & ${ }^{90} \mathrm{Sr}$ & $-8.1 \mathrm{E}-01 \pm 8.1 \mathrm{E}-01$ & $\mathrm{U}$ & & ${ }^{90} \mathrm{Sr}$ & $-3.3 \mathrm{E}-01 \pm 4.1 \mathrm{E}-01$ & $\mathrm{U}$ \\
\hline & ${ }^{234} \mathrm{U}$ & $1.6 \mathrm{E}-02 \pm 9.6 \mathrm{E}-03$ & & & ${ }^{234} \mathrm{U}$ & $2.7 \mathrm{E}-02 \pm 1.2 \mathrm{E}-02$ & \\
\hline & ${ }^{235} \mathrm{U}$ & $5.6 \mathrm{E}-03 \pm 4.8 \mathrm{E}-03$ & & & ${ }^{235} \mathrm{U}$ & $8.5 \mathrm{E}-03 \pm 6.0 \mathrm{E}-03$ & \\
\hline & ${ }^{238} \mathrm{U}$ & $8.6 \mathrm{E}-03 \pm 6.8 \mathrm{E}-03$ & & & ${ }^{238} \mathrm{U}$ & $1.9 \mathrm{E}-02 \pm 9.7 \mathrm{E}-03$ & \\
\hline & ${ }^{65} \mathrm{Zn}$ & $6.9 \mathrm{E}-02 \pm 1.0 \mathrm{E}-01$ & $\mathrm{U}$ & & ${ }^{65} \mathrm{Zn}$ & $-3.3 \mathrm{E}-02 \pm 1.1 \mathrm{E}-01$ & $\mathrm{U}$ \\
\hline \multirow{18}{*}{$\begin{array}{c}\text { V104 } \\
\text { (600 Area) }\end{array}$} & ${ }^{144} \mathrm{Ce}$ & $3.8 \mathrm{E}-02 \pm 3.8 \mathrm{E}-01$ & $\mathrm{U}$ & V106 & ${ }^{144} \mathrm{Ce}$ & $-1.3 \mathrm{E}-01 \pm 1.1 \mathrm{E}+00$ & $\mathrm{U}$ \\
\hline & ${ }^{60} \mathrm{Co}$ & $-2.8 \mathrm{E}-02 \pm 4.1 \mathrm{E}-02$ & $\mathrm{U}$ & (600 Area) & ${ }^{60} \mathrm{Co}$ & $5.3 \mathrm{E}-02 \pm 1.0 \mathrm{E}-01$ & $\mathrm{U}$ \\
\hline & ${ }^{134} \mathrm{Cs}$ & $1.7 \mathrm{E}-02 \pm 4.2 \mathrm{E}-02$ & $\mathrm{U}$ & & ${ }^{134} \mathrm{Cs}$ & $-2.1 \mathrm{E}-02 \pm 1.4 \mathrm{E}-01$ & $\mathrm{U}$ \\
\hline & ${ }^{137} \mathrm{Cs}$ & $2.3 \mathrm{E}-02 \pm 4.2 \mathrm{E}-02$ & $\mathrm{U}$ & & ${ }^{137} \mathrm{Cs}$ & $1.9 \mathrm{E}-02 \pm 9.4 \mathrm{E}-02$ & $\mathrm{U}$ \\
\hline & ${ }^{152} \mathrm{Eu}$ & $8.4 \mathrm{E}-02 \pm 1.0 \mathrm{E}-01$ & $\mathrm{U}$ & & ${ }^{152} \mathrm{Eu}$ & $1.4 \mathrm{E}-01 \pm 2.6 \mathrm{E}-01$ & $\mathrm{U}$ \\
\hline & ${ }^{154} \mathrm{Eu}$ & $1.1 \mathrm{E}-01 \pm 1.2 \mathrm{E}-01$ & $\mathrm{U}$ & & ${ }^{154} \mathrm{Eu}$ & $-1.1 \mathrm{E}-01 \pm 3.0 \mathrm{E}-01$ & $\mathrm{U}$ \\
\hline & ${ }^{155} \mathrm{Eu}$ & $-2.7 \mathrm{E}-02 \pm 9.7 \mathrm{E}-02$ & $\mathrm{U}$ & & ${ }^{155} \mathrm{Eu}$ & $2.2 \mathrm{E}-02 \pm 2.2 \mathrm{E}-01$ & $\mathrm{U}$ \\
\hline & ${ }^{238} \mathrm{Pu}$ & $1.0 \mathrm{E}-02 \pm 1.2 \mathrm{E}-02$ & $\mathrm{U}$ & & ${ }^{238} \mathrm{Pu}$ & $-1.1 \mathrm{E}-02 \pm 1.5 \mathrm{E}-02$ & $\mathrm{U}$ \\
\hline & ${ }^{239 / 240} \mathrm{Pu}$ & $1.0 \mathrm{E}-03 \pm 3.5 \mathrm{E}-03$ & $\mathrm{U}$ & & ${ }^{239 / 240} \mathrm{Pu}$ & $9.2 \mathrm{E}-04 \pm 9.2 \mathrm{E}-03$ & $\mathrm{U}$ \\
\hline & ${ }^{103} \mathrm{Ru}$ & $-7.8 \mathrm{E}-03 \pm 5.0 \mathrm{E}-02$ & $\mathrm{U}$ & & ${ }^{103} \mathrm{Ru}$ & $-3.5 \mathrm{E}-02 \pm 1.3 \mathrm{E}-01$ & $\mathrm{U}$ \\
\hline & ${ }^{106} \mathrm{Ru}$ & $-1.7 \mathrm{E}-02 \pm 1.7 \mathrm{E}-01$ & $\mathrm{U}$ & & ${ }^{106} \mathrm{Ru}$ & $-4.1 \mathrm{E}-01 \pm 9.8 \mathrm{E}-01$ & $\mathrm{U}$ \\
\hline & ${ }^{125} \mathrm{Sb}$ & $6.1 \mathrm{E}-02 \pm 9.4 \mathrm{E}-02$ & $\mathrm{U}$ & & ${ }^{125} \mathrm{Sb}$ & 8.7E-02 $\pm 2.5 \mathrm{E}-01$ & $\mathrm{U}$ \\
\hline & ${ }^{113} \mathrm{Sn}$ & $3.6 \mathrm{E}-02 \pm 4.8 \mathrm{E}-02$ & $\mathrm{U}$ & & ${ }^{113} \mathrm{Sn}$ & $-9.2 \mathrm{E}-02 \pm 1.2 \mathrm{E}-01$ & $\mathrm{U}$ \\
\hline & ${ }^{90} \mathrm{Sr}$ & $-5.2 \mathrm{E}-02 \pm 2.0 \mathrm{E}-01$ & $\mathrm{U}$ & & ${ }^{90} \mathrm{Sr}$ & $1.4 \mathrm{E}-01 \pm 2.6 \mathrm{E}-01$ & $\mathrm{U}$ \\
\hline & ${ }^{234} \mathrm{U}$ & $3.0 \mathrm{E}-02 \pm 1.3 \mathrm{E}-02$ & & & ${ }^{234} \mathrm{U}$ & $1.6 \mathrm{E}-02 \pm 8.5 \mathrm{E}-03$ & \\
\hline & ${ }^{235} \mathrm{U}$ & $4.8 \mathrm{E}-03 \pm 4.5 \mathrm{E}-03$ & & & ${ }^{235} \mathrm{U}$ & $1.9 \mathrm{E}-03 \pm 3.8 \mathrm{E}-03$ & $\mathrm{U}$ \\
\hline & ${ }^{238} \mathrm{U}$ & $1.6 \mathrm{E}-02 \pm 8.6 \mathrm{E}-03$ & & & ${ }^{238} \mathrm{U}$ & $6.0 \mathrm{E}-03 \pm 5.4 \mathrm{E}-03$ & $\mathrm{U}$ \\
\hline & ${ }^{65} \mathrm{Zn}$ & $-5.3 \mathrm{E}-02 \pm 9.4 \mathrm{E}-02$ & $\mathrm{U}$ & & ${ }^{65} \mathrm{Zn}$ & $1.6 \mathrm{E}-01 \pm 2.3 \mathrm{E}-01$ & $\mathrm{U}$ \\
\hline
\end{tabular}

RQ = Result Qualifier. $\mathrm{U}=$ The analyte was analyzed for but not detected. 
Table 4-4. 2008 Vegetation Sampling Results (pCi/g \pm total analytical uncertainty). (Sheet 14 of 18)

\begin{tabular}{|c|c|c|c|c|c|c|c|}
\hline Location & Isotope & Result \pm Error & $\mathbf{R Q}^{*}$ & Location & Is otope & Result \pm Error & $\mathbf{R Q}^{*}$ \\
\hline V108 & ${ }^{144} \mathrm{Ce}$ & $-2.9 \mathrm{E}-01 \pm 4.8 \mathrm{E}-01$ & $\mathrm{U}$ & V110 & ${ }^{144} \mathrm{Ce}$ & $-4.0 \mathrm{E}-02 \pm 4.0 \mathrm{E}-01$ & $\mathrm{U}$ \\
\hline \multirow[t]{17}{*}{ (600 Area) } & ${ }^{60} \mathrm{Co}$ & $-5.8 \mathrm{E}-03 \pm 5.0 \mathrm{E}-02$ & $\mathrm{U}$ & (600 Area) & ${ }^{60} \mathrm{Co}$ & $5.1 \mathrm{E}-02 \pm 1.9 \mathrm{E}-01$ & $\mathrm{U}$ \\
\hline & ${ }^{134} \mathrm{Cs}$ & 5.7E-02 \pm 4.9E-02 & $\mathrm{U}$ & & ${ }^{134} \mathrm{Cs}$ & $1.4 \mathrm{E}-01 \pm 2.5 \mathrm{E}-01$ & $\mathrm{U}$ \\
\hline & ${ }^{137} \mathrm{Cs}$ & $6.6 \mathrm{E}-02 \pm 5.4 \mathrm{E}-02$ & $\mathrm{U}$ & & ${ }^{137} \mathrm{Cs}$ & $-1.1 \mathrm{E}-01 \pm 2.1 \mathrm{E}-01$ & $\mathrm{U}$ \\
\hline & ${ }^{152} \mathrm{Eu}$ & $4.0 \mathrm{E}-02 \pm 1.2 \mathrm{E}-01$ & $\mathrm{U}$ & & ${ }^{152} \mathrm{Eu}$ & $-1.8 \mathrm{E}-01 \pm 6.2 \mathrm{E}-01$ & $\mathrm{U}$ \\
\hline & ${ }^{154} \mathrm{Eu}$ & $-1.6 \mathrm{E}-02 \pm 1.3 \mathrm{E}-01$ & $\mathrm{U}$ & & ${ }^{154} \mathrm{Eu}$ & $-1.1 \mathrm{E}+00 \pm 1.1 \mathrm{E}+00$ & $\mathrm{U}$ \\
\hline & ${ }^{155} \mathrm{Eu}$ & $-7.0 \mathrm{E}-02 \pm 1.3 \mathrm{E}-01$ & $\mathrm{U}$ & & ${ }^{155} \mathrm{Eu}$ & $1.9 \mathrm{E}-02 \pm 1.9 \mathrm{E}-01$ & $\mathrm{U}$ \\
\hline & ${ }^{238} \mathrm{Pu}$ & $-1.3 \mathrm{E}-02 \pm 1.9 \mathrm{E}-02$ & $\mathrm{U}$ & & ${ }^{238} \mathrm{Pu}$ & $1.2 \mathrm{E}-02 \pm 9.8 \mathrm{E}-03$ & $\mathrm{U}$ \\
\hline & ${ }^{239 / 240} \mathrm{Pu}$ & $1.1 \mathrm{E}-03 \pm 4.9 \mathrm{E}-03$ & $\mathrm{U}$ & & ${ }^{239 / 240} \mathrm{Pu}$ & $9.8 \mathrm{E}-04 \pm 3.4 \mathrm{E}-03$ & $\mathrm{U}$ \\
\hline & ${ }^{103} \mathrm{Ru}$ & $8.8 \mathrm{E}-05 \pm 8.8 \mathrm{E}-04$ & $\mathrm{U}$ & & ${ }^{103} \mathrm{Ru}$ & $-2.2 \mathrm{E}-01 \pm 3.1 \mathrm{E}-01$ & $\mathrm{U}$ \\
\hline & ${ }^{106} \mathrm{Ru}$ & $2.5 \mathrm{E}-02 \pm 2.5 \mathrm{E}-01$ & $\mathrm{U}$ & & ${ }^{106} \mathrm{Ru}$ & $-2.8 \mathrm{E}+00 \pm 2.8 \mathrm{E}+00$ & $\mathrm{U}$ \\
\hline & ${ }^{125} \mathrm{Sb}$ & $1.4 \mathrm{E}-03 \pm 1.4 \mathrm{E}-02$ & $\mathrm{U}$ & & ${ }^{125} \mathrm{Sb}$ & $-3.2 \mathrm{E}-01 \pm 5.6 \mathrm{E}-01$ & $\mathrm{U}$ \\
\hline & ${ }^{113} \mathrm{Sn}$ & $-3.0 \mathrm{E}-02 \pm 5.9 \mathrm{E}-02$ & $\mathrm{U}$ & & ${ }^{113} \mathrm{Sn}$ & $-5.8 \mathrm{E}-03 \pm 5.8 \mathrm{E}-02$ & $\mathrm{U}$ \\
\hline & ${ }^{90} \mathrm{Sr}$ & $1.6 \mathrm{E}-01 \pm 2.9 \mathrm{E}-01$ & $\mathrm{U}$ & & ${ }^{90} \mathrm{Sr}$ & $-3.5 \mathrm{E}-02 \pm 1.8 \mathrm{E}-01$ & $\mathrm{U}$ \\
\hline & ${ }^{234} \mathrm{U}$ & $1.9 \mathrm{E}-02 \pm 9.7 \mathrm{E}-03$ & & & ${ }^{234} \mathrm{U}$ & $1.8 \mathrm{E}-02 \pm 1.0 \mathrm{E}-02$ & \\
\hline & ${ }^{235} \mathrm{U}$ & $1.9 \mathrm{E}-03 \pm 2.7 \mathrm{E}-03$ & $\mathrm{U}$ & & ${ }^{235} \mathrm{U}$ & $4.6 \mathrm{E}-03 \pm 4.3 \mathrm{E}-03$ & \\
\hline & ${ }^{238} \mathrm{U}$ & $1.2 \mathrm{E}-02 \pm 7.6 \mathrm{E}-03$ & & & ${ }^{238} \mathrm{U}$ & $5.1 \mathrm{E}-03 \pm 4.4 \mathrm{E}-03$ & \\
\hline & ${ }^{65} \mathrm{Zn}$ & $7.1 \mathrm{E}-02 \pm 1.1 \mathrm{E}-01$ & $\mathrm{U}$ & & ${ }^{65} \mathrm{Zn}$ & $-6.5 \mathrm{E}-01 \pm 6.5 \mathrm{E}-01$ & $\mathrm{U}$ \\
\hline \multirow{18}{*}{$\begin{array}{c}\text { V114 } \\
\text { (600 Area) }\end{array}$} & ${ }^{144} \mathrm{Ce}$ & $-2.8 \mathrm{E}-01 \pm 4.0 \mathrm{E}-01$ & $\mathrm{U}$ & V116 & ${ }^{144} \mathrm{Ce}$ & $-6.0 \mathrm{E}-01 \pm 6.6 \mathrm{E}-01$ & $\mathrm{U}$ \\
\hline & ${ }^{60} \mathrm{Co}$ & $-3.0 \mathrm{E}-02 \pm 4.1 \mathrm{E}-02$ & $\mathrm{U}$ & (300 Area) & ${ }^{60} \mathrm{Co}$ & $1.4 \mathrm{E}-03 \pm 1.4 \mathrm{E}-02$ & $\mathrm{U}$ \\
\hline & ${ }^{134} \mathrm{Cs}$ & $-8.2 \mathrm{E}-03 \pm 4.0 \mathrm{E}-02$ & $\mathrm{U}$ & & ${ }^{134} \mathrm{Cs}$ & $-2.0 \mathrm{E}-02 \pm 7.2 \mathrm{E}-02$ & $\mathrm{U}$ \\
\hline & ${ }^{137} \mathrm{Cs}$ & $5.2 \mathrm{E}-02 \pm 6.6 \mathrm{E}-02$ & $\mathrm{U}$ & & ${ }^{137} \mathrm{Cs}$ & $6.3 \mathrm{E}-02 \pm 6.3 \mathrm{E}-02$ & $\mathrm{U}$ \\
\hline & ${ }^{152} \mathrm{Eu}$ & $2.8 \mathrm{E}-02 \pm 9.3 \mathrm{E}-02$ & $\mathrm{U}$ & & ${ }^{152} \mathrm{Eu}$ & $-4.5 \mathrm{E}-02 \pm 1.9 \mathrm{E}-01$ & $\mathrm{U}$ \\
\hline & ${ }^{154} \mathrm{Eu}$ & $7.4 \mathrm{E}-02 \pm 1.1 \mathrm{E}-01$ & $\mathrm{U}$ & & ${ }^{154} \mathrm{Eu}$ & $-9.9 \mathrm{E}-02 \pm 1.8 \mathrm{E}-01$ & $\mathrm{U}$ \\
\hline & ${ }^{155} \mathrm{Eu}$ & $-3.7 \mathrm{E}-02 \pm 1.0 \mathrm{E}-01$ & $\mathrm{U}$ & & ${ }^{155} \mathrm{Eu}$ & $-2.3 \mathrm{E}-02 \pm 1.8 \mathrm{E}-01$ & $\mathrm{U}$ \\
\hline & ${ }^{238} \mathrm{Pu}$ & $6.0 \mathrm{E}-03 \pm 2.0 \mathrm{E}-02$ & $\mathrm{U}$ & & ${ }^{238} \mathrm{Pu}$ & $3.4 \mathrm{E}-03 \pm 3.0 \mathrm{E}-02$ & $\mathrm{U}$ \\
\hline & ${ }^{239 / 240} \mathrm{Pu}$ & $3.6 \mathrm{E}-03 \pm 5.4 \mathrm{E}-03$ & $\mathrm{U}$ & & ${ }^{239 / 240} \mathrm{Pu}$ & $-6.8 \mathrm{E}-03 \pm 1.4 \mathrm{E}-02$ & $\mathrm{U}$ \\
\hline & ${ }^{103} \mathrm{Ru}$ & $1.1 \mathrm{E}-02 \pm 5.0 \mathrm{E}-02$ & $\mathrm{U}$ & & ${ }^{103} \mathrm{Ru}$ & $1.0 \mathrm{E}-02 \pm 5.9 \mathrm{E}-02$ & $\mathrm{U}$ \\
\hline & ${ }^{106} \mathrm{Ru}$ & $-3.6 \mathrm{E}-01 \pm 3.7 \mathrm{E}-01$ & $\mathrm{U}$ & & ${ }^{106} \mathrm{Ru}$ & $2.3 \mathrm{E}-01 \pm 5.1 \mathrm{E}-01$ & $\mathrm{U}$ \\
\hline & ${ }^{125} \mathrm{Sb}$ & $9.8 \mathrm{E}-02 \pm 9.1 \mathrm{E}-02$ & $\mathrm{U}$ & & ${ }^{125} \mathrm{Sb}$ & $6.5 \mathrm{E}-02 \pm 1.5 \mathrm{E}-01$ & $\mathrm{U}$ \\
\hline & ${ }^{113} \mathrm{Sn}$ & $1.4 \mathrm{E}-02 \pm 4.5 \mathrm{E}-02$ & $\mathrm{U}$ & & ${ }^{113} \mathrm{Sn}$ & $-4.5 \mathrm{E}-03 \pm 4.5 \mathrm{E}-02$ & $\mathrm{U}$ \\
\hline & ${ }^{90} \mathrm{Sr}$ & $-1.8 \mathrm{E}-01 \pm 2.0 \mathrm{E}-01$ & $\mathrm{U}$ & & ${ }^{90} \mathrm{Sr}$ & $-2.7 \mathrm{E}-01 \pm 2.7 \mathrm{E}-01$ & $\mathrm{U}$ \\
\hline & ${ }^{234} \mathrm{U}$ & $1.9 \mathrm{E}-02 \pm 1.9 \mathrm{E}-02$ & $\mathrm{U}$ & & ${ }^{234} \mathrm{U}$ & $9.3 \mathrm{E}-03 \pm 7.8 \mathrm{E}-03$ & $\mathrm{U}$ \\
\hline & ${ }^{235} \mathrm{U}$ & $2.9 \mathrm{E}-03 \pm 5.9 \mathrm{E}-03$ & $\mathrm{U}$ & & ${ }^{235} \mathrm{U}$ & $9.3 \mathrm{E}-04 \pm 0.0 \mathrm{E}+00$ & $\mathrm{U}$ \\
\hline & ${ }^{238} \mathrm{U}$ & $-8.0 \mathrm{E}-03 \pm 1.4 \mathrm{E}-02$ & $\mathrm{U}$ & & ${ }^{238} \mathrm{U}$ & $1.1 \mathrm{E}-02 \pm 6.9 \mathrm{E}-03$ & \\
\hline & ${ }^{65} \mathrm{Zn}$ & $6.5 \mathrm{E}-02 \pm 9.8 \mathrm{E}-02$ & $\mathrm{U}$ & & ${ }^{65} \mathrm{Zn}$ & $2.0 \mathrm{E}-01 \pm 1.5 \mathrm{E}-01$ & $\mathrm{U}$ \\
\hline
\end{tabular}

RQ = Result Qualifier. $\mathrm{U}=$ The analyte was analyzed for but not detected. 
Table 4-4. 2008 Vegetation Sampling Results (pCi/g \pm total analytical uncertainty). (Sheet 15 of 18)

\begin{tabular}{|c|c|c|c|c|c|c|c|}
\hline Location & Isotope & Result \pm Error & RQ* & Location & Isotope & Result \pm Error & $\mathbf{R Q *}$ \\
\hline V117 & ${ }^{144} \mathrm{Ce}$ & $-3.2 \mathrm{E}-01 \pm 1.1 \mathrm{E}+00$ & $\bar{U}$ & V118 & ${ }^{144} \mathrm{Ce}$ & $-3.4 \mathrm{E}-01 \pm 8.9 \mathrm{E}-01$ & $\bar{U}$ \\
\hline \multirow[t]{17}{*}{ (300 Area) } & ${ }^{60} \mathrm{Co}$ & $-1.0 \mathrm{E}-01 \pm 1.1 \mathrm{E}-01$ & $\mathrm{U}$ & (300 Area) & ${ }^{60} \mathrm{Co}$ & $-5.9 \mathrm{E}-02 \pm 7.9 \mathrm{E}-02$ & $\mathrm{U}$ \\
\hline & ${ }^{134} \mathrm{Cs}$ & $6.4 \mathrm{E}-02 \pm 1.1 \mathrm{E}-01$ & $\mathrm{U}$ & & ${ }^{134} \mathrm{Cs}$ & $4.3 \mathrm{E}-03 \pm 4.3 \mathrm{E}-02$ & $\mathrm{U}$ \\
\hline & ${ }^{137} \mathrm{Cs}$ & $1.8 \mathrm{E}-02 \pm 1.2 \mathrm{E}-01$ & $\mathrm{U}$ & & ${ }^{137} \mathrm{Cs}$ & $3.1 \mathrm{E}-02 \pm 9.1 \mathrm{E}-02$ & $\mathrm{U}$ \\
\hline & ${ }^{152} \mathrm{Eu}$ & $4.2 \mathrm{E}-02 \pm 2.7 \mathrm{E}-01$ & $\mathrm{U}$ & & ${ }^{152} \mathrm{Eu}$ & $8.3 \mathrm{E}-03 \pm 8.3 \mathrm{E}-02$ & $\mathrm{U}$ \\
\hline & ${ }^{154} \mathrm{Eu}$ & $-1.9 \mathrm{E}-01 \pm 2.9 \mathrm{E}-01$ & $\mathrm{U}$ & & ${ }^{154} \mathrm{Eu}$ & $-2.5 \mathrm{E}-01 \pm 2.5 \mathrm{E}-01$ & $\mathrm{U}$ \\
\hline & ${ }^{155} \mathrm{Eu}$ & $1.7 \mathrm{E}-01 \pm 2.6 \mathrm{E}-01$ & $\mathrm{U}$ & & ${ }^{155} \mathrm{Eu}$ & $-5.8 \mathrm{E}-02 \pm 2.2 \mathrm{E}-01$ & $\mathrm{U}$ \\
\hline & ${ }^{238} \mathrm{Pu}$ & $8.1 \mathrm{E}-02 \pm 5.8 \mathrm{E}-02$ & & & ${ }^{238} \mathrm{Pu}$ & $-1.2 \mathrm{E}-02 \pm 4.8 \mathrm{E}-02$ & $\mathrm{U}$ \\
\hline & ${ }^{239 / 240} \mathrm{Pu}$ & 4.3E-03 $\pm 4.3 \mathrm{E}-02$ & $\mathrm{U}$ & & ${ }^{239 / 240} \mathrm{Pu}$ & $6.1 \mathrm{E}-03 \pm 6.1 \mathrm{E}-02$ & $\mathrm{U}$ \\
\hline & ${ }^{103} \mathrm{Ru}$ & $-1.2 \mathrm{E}-01 \pm 1.2 \mathrm{E}-01$ & $\mathrm{U}$ & & ${ }^{103} \mathrm{Ru}$ & $-1.7 \mathrm{E}-02 \pm 8.2 \mathrm{E}-02$ & $\mathrm{U}$ \\
\hline & ${ }^{106} \mathrm{Ru}$ & $-6.9 \mathrm{E}-01 \pm 9.0 \mathrm{E}-01$ & $\mathrm{U}$ & & ${ }^{106} \mathrm{Ru}$ & $1.7 \mathrm{E}-01 \pm 7.0 \mathrm{E}-01$ & $\mathrm{U}$ \\
\hline & ${ }^{125} \mathrm{Sb}$ & $-1.4 \mathrm{E}-01 \pm 2.7 \mathrm{E}-01$ & $\mathrm{U}$ & & ${ }^{125} \mathrm{Sb}$ & $-8.0 \mathrm{E}-02 \pm 2.0 \mathrm{E}-01$ & $\mathrm{U}$ \\
\hline & ${ }^{113} \mathrm{Sn}$ & $-1.2 \mathrm{E}-01 \pm 1.2 \mathrm{E}-01$ & $\mathrm{U}$ & & ${ }^{113} \mathrm{Sn}$ & $-3.7 \mathrm{E}-02 \pm 9.5 \mathrm{E}-02$ & $\mathrm{U}$ \\
\hline & ${ }^{90} \mathrm{Sr}$ & $-1.2 \mathrm{E}-01 \pm 1.8 \mathrm{E}-01$ & $\mathrm{U}$ & & ${ }^{90} \mathrm{Sr}$ & $-1.3 \mathrm{E}-01 \pm 1.8 \mathrm{E}-01$ & $\mathrm{U}$ \\
\hline & ${ }^{234} \mathrm{U}$ & $1.9 \mathrm{E}-02 \pm 1.0 \mathrm{E}-02$ & & & ${ }^{234} \mathrm{U}$ & $3.1 \mathrm{E}-02 \pm 1.4 \mathrm{E}-02$ & \\
\hline & ${ }^{235} \mathrm{U}$ & $2.8 \mathrm{E}-03 \pm 4.2 \mathrm{E}-03$ & $\mathrm{U}$ & & ${ }^{235} \mathrm{U}$ & $9.8 \mathrm{E}-04 \pm 5.2 \mathrm{E}-03$ & U \\
\hline & ${ }^{238} \mathrm{U}$ & $1.6 \mathrm{E}-02 \pm 8.5 \mathrm{E}-03$ & & & ${ }^{238} \mathrm{U}$ & $1.6 \mathrm{E}-02 \pm 8.6 \mathrm{E}-03$ & \\
\hline & ${ }^{65} \mathrm{Zn}$ & $1.7 \mathrm{E}-01 \pm 2.7 \mathrm{E}-01$ & $\mathrm{U}$ & & ${ }^{65} \mathrm{Zn}$ & $7.1 \mathrm{E}-01 \pm 2.3 \mathrm{E}-01$ & \\
\hline V119 & ${ }^{144} \mathrm{Ce}$ & $-2.9 \mathrm{E}-02 \pm 2.9 \mathrm{E}-01$ & $\mathrm{U}$ & V120 & ${ }^{144} \mathrm{Ce}$ & $4.5 \mathrm{E}-02 \pm 4.5 \mathrm{E}-01$ & $\mathrm{U}$ \\
\hline \multirow[t]{17}{*}{ (300 Area) } & ${ }^{60} \mathrm{Co}$ & $-7.2 \mathrm{E}-02 \pm 1.2 \mathrm{E}-01$ & $\mathrm{U}$ & (300 Area) & ${ }^{60} \mathrm{Co}$ & $-2.3 \mathrm{E}-02 \pm 4.1 \mathrm{E}-02$ & $\mathrm{U}$ \\
\hline & ${ }^{134} \mathrm{Cs}$ & $-4.3 \mathrm{E}-02 \pm 1.2 \mathrm{E}-01$ & $\mathrm{U}$ & & ${ }^{134} \mathrm{Cs}$ & $1.9 \mathrm{E}-02 \pm 4.8 \mathrm{E}-02$ & $\mathrm{U}$ \\
\hline & ${ }^{137} \mathrm{Cs}$ & $6.0 \mathrm{E}-02 \pm 1.2 \mathrm{E}-01$ & $\mathrm{U}$ & & ${ }^{137} \mathrm{Cs}$ & $-2.4 \mathrm{E}-02 \pm 4.7 \mathrm{E}-02$ & $\mathrm{U}$ \\
\hline & ${ }^{152} \mathrm{Eu}$ & $9.1 \mathrm{E}-02 \pm 3.3 \mathrm{E}-01$ & $\mathrm{U}$ & & ${ }^{152} \mathrm{Eu}$ & $9.4 \mathrm{E}-02 \pm 1.3 \mathrm{E}-01$ & $\mathrm{U}$ \\
\hline & ${ }^{154} \mathrm{Eu}$ & $-4.7 \mathrm{E}-02 \pm 3.4 \mathrm{E}-01$ & $\mathrm{U}$ & & ${ }^{154} \mathrm{Eu}$ & $3.4 \mathrm{E}-02 \pm 1.3 \mathrm{E}-01$ & $\mathrm{U}$ \\
\hline & ${ }^{155} \mathrm{Eu}$ & $9.7 \mathrm{E}-02 \pm 2.8 \mathrm{E}-01$ & $\mathrm{U}$ & & ${ }^{155} \mathrm{Eu}$ & $2.8 \mathrm{E}-02 \pm 1.3 \mathrm{E}-01$ & $\mathrm{U}$ \\
\hline & ${ }^{238} \mathrm{Pu}$ & 4.3E-03 $\pm 4.3 \mathrm{E}-02$ & $\mathrm{U}$ & & ${ }^{238} \mathrm{Pu}$ & 8.7E-02 $\pm 4.7 \mathrm{E}-02$ & \\
\hline & ${ }^{239 / 240} \mathrm{Pu}$ & $4.3 \mathrm{E}-03 \pm 4.3 \mathrm{E}-02$ & $\mathrm{U}$ & & ${ }^{239 / 240} \mathrm{Pu}$ & $5.5 \mathrm{E}-03 \pm 1.1 \mathrm{E}-02$ & $\mathrm{U}$ \\
\hline & ${ }^{103} \mathrm{Ru}$ & $-7.5 \mathrm{E}-02 \pm 1.3 \mathrm{E}-01$ & $\mathrm{U}$ & & ${ }^{103} \mathrm{Ru}$ & $-1.9 \mathrm{E}-02 \pm 5.1 \mathrm{E}-02$ & $\mathrm{U}$ \\
\hline & ${ }^{106} \mathrm{Ru}$ & $-4.8 \mathrm{E}-01 \pm 1.1 \mathrm{E}+00$ & $\mathrm{U}$ & & ${ }^{106} \mathrm{Ru}$ & $-1.9 \mathrm{E}-02 \pm 1.9 \mathrm{E}-01$ & $\mathrm{U}$ \\
\hline & ${ }^{125} \mathrm{Sb}$ & $1.4 \mathrm{E}-02 \pm 1.4 \mathrm{E}-01$ & $\mathrm{U}$ & & ${ }^{125} \mathrm{Sb}$ & $5.6 \mathrm{E}-02 \pm 1.2 \mathrm{E}-01$ & $\mathrm{U}$ \\
\hline & ${ }^{113} \mathrm{Sn}$ & $-1.2 \mathrm{E}-02 \pm 1.2 \mathrm{E}-01$ & $\mathrm{U}$ & & ${ }^{113} \mathrm{Sn}$ & $-5.0 \mathrm{E}-03 \pm 5.0 \mathrm{E}-02$ & $\mathrm{U}$ \\
\hline & ${ }^{90} \mathrm{Sr}$ & $-2.4 \mathrm{E}-01 \pm 2.4 \mathrm{E}-01$ & $\mathrm{U}$ & & ${ }^{90} \mathrm{Sr}$ & $-5.2 \mathrm{E}-02 \pm 1.8 \mathrm{E}-01$ & $\mathrm{U}$ \\
\hline & ${ }^{234} \mathrm{U}$ & $4.8 \mathrm{E}-02 \pm 1.8 \mathrm{E}-02$ & & & ${ }^{234} \mathrm{U}$ & $3.5 \mathrm{E}-02 \pm 1.4 \mathrm{E}-02$ & \\
\hline & ${ }^{235} \mathrm{U}$ & $5.8 \mathrm{E}-03 \pm 5.7 \mathrm{E}-03$ & $\mathrm{U}$ & & ${ }^{235} \mathrm{U}$ & $9.0 \mathrm{E}-04 \pm 3.1 \mathrm{E}-03$ & $\mathrm{U}$ \\
\hline & ${ }^{238} \mathrm{U}$ & $5.5 \mathrm{E}-02 \pm 2.0 \mathrm{E}-02$ & & & ${ }^{238} \mathrm{U}$ & 4.4E-02 $\pm 1.7 \mathrm{E}-02$ & \\
\hline & ${ }^{65} \mathrm{Zn}$ & $-4.1 \mathrm{E}-03 \pm 4.1 \mathrm{E}-02$ & $\mathrm{U}$ & & ${ }^{65} \mathrm{Zn}$ & $-3.8 \mathrm{E}-03 \pm 3.8 \mathrm{E}-02$ & $\mathrm{U}$ \\
\hline
\end{tabular}

RQ = Result Qualifier. $\mathrm{U}=$ The analyte was analyzed for but not detected. 
Table 4-4. 2008 Vegetation Sampling Results (pCi/g \pm total analytical uncertainty). (Sheet 16 of 18)

\begin{tabular}{|c|c|c|c|c|c|c|c|}
\hline Location & Isotope & Result \pm Error & $\mathbf{R Q}^{*}$ & Location & Isotope & Result \pm Error & $\mathbf{R Q *}$ \\
\hline V121 & ${ }^{144} \mathrm{Ce}$ & $3.8 \mathrm{E}-01 \pm 1.1 \mathrm{E}+00$ & $\bar{U}$ & V123 & ${ }^{144} \mathrm{Ce}$ & $8.2 \mathrm{E}-02 \pm 4.1 \mathrm{E}-01$ & $\bar{U}$ \\
\hline \multirow[t]{17}{*}{ (300 Area) } & ${ }^{60} \mathrm{Co}$ & $-8.2 \mathrm{E}-02 \pm 1.0 \mathrm{E}-01$ & $\mathrm{U}$ & (300 Area) & ${ }^{60} \mathrm{Co}$ & $-1.5 \mathrm{E}-02 \pm 4.5 \mathrm{E}-02$ & $\mathrm{U}$ \\
\hline & ${ }^{134} \mathrm{Cs}$ & $3.5 \mathrm{E}-02 \pm 1.1 \mathrm{E}-01$ & $\mathrm{U}$ & & ${ }^{134} \mathrm{Cs}$ & $7.6 \mathrm{E}-03 \pm 4.2 \mathrm{E}-02$ & $\mathrm{U}$ \\
\hline & ${ }^{137} \mathrm{Cs}$ & $4.4 \mathrm{E}-02 \pm 1.2 \mathrm{E}-01$ & $\mathrm{U}$ & & ${ }^{137} \mathrm{Cs}$ & $7.5 \mathrm{E}-04 \pm 7.5 \mathrm{E}-03$ & $\mathrm{U}$ \\
\hline & ${ }^{152} \mathrm{Eu}$ & $-1.7 \mathrm{E}-01 \pm 3.0 \mathrm{E}-01$ & $\mathrm{U}$ & & ${ }^{152} \mathrm{Eu}$ & $1.6 \mathrm{E}-02 \pm 1.1 \mathrm{E}-01$ & $\mathrm{U}$ \\
\hline & ${ }^{154} \mathrm{Eu}$ & $1.2 \mathrm{E}-01 \pm 3.0 \mathrm{E}-01$ & $\mathrm{U}$ & & ${ }^{154} \mathrm{Eu}$ & $-7.6 \mathrm{E}-03 \pm 7.6 \mathrm{E}-02$ & $\mathrm{U}$ \\
\hline & ${ }^{155} \mathrm{Eu}$ & $1.2 \mathrm{E}-01 \pm 2.6 \mathrm{E}-01$ & $\mathrm{U}$ & & ${ }^{155} \mathrm{Eu}$ & $1.5 \mathrm{E}-02 \pm 1.0 \mathrm{E}-01$ & $\mathrm{U}$ \\
\hline & ${ }^{238} \mathrm{Pu}$ & $-3.8 \mathrm{E}-02 \pm 4.8 \mathrm{E}-02$ & $\mathrm{U}$ & & ${ }^{238} \mathrm{Pu}$ & $-1.7 \mathrm{E}-03 \pm 8.7 \mathrm{E}-03$ & $\mathrm{U}$ \\
\hline & ${ }^{239 / 240} \mathrm{Pu}$ & $9.4 \mathrm{E}-03 \pm 1.6 \mathrm{E}-02$ & $\mathrm{U}$ & & ${ }^{239 / 240} \mathrm{Pu}$ & $4.2 \mathrm{E}-03 \pm 3.9 \mathrm{E}-03$ & \\
\hline & ${ }^{103} \mathrm{Ru}$ & $-7.5 \mathrm{E}-02 \pm 1.2 \mathrm{E}-01$ & $\mathrm{U}$ & & ${ }^{103} \mathrm{Ru}$ & $-1.1 \mathrm{E}-02 \pm 4.1 \mathrm{E}-02$ & $\mathrm{U}$ \\
\hline & ${ }^{106} \mathrm{Ru}$ & $3.8 \mathrm{E}-01 \pm 1.0 \mathrm{E}+00$ & $\mathrm{U}$ & & ${ }^{106} \mathrm{Ru}$ & $-4.5 \mathrm{E}-02 \pm 3.9 \mathrm{E}-01$ & $\mathrm{U}$ \\
\hline & ${ }^{125} \mathrm{Sb}$ & $-3.0 \mathrm{E}-03 \pm 3.0 \mathrm{E}-02$ & $\mathrm{U}$ & & ${ }^{125} \mathrm{Sb}$ & $1.2 \mathrm{E}-02 \pm 1.0 \mathrm{E}-01$ & $\mathrm{U}$ \\
\hline & ${ }^{113} \mathrm{Sn}$ & $-3.4 \mathrm{E}-04 \pm 3.4 \mathrm{E}-03$ & $\mathrm{U}$ & & ${ }^{113} \mathrm{Sn}$ & $-1.9 \mathrm{E}-02 \pm 5.0 \mathrm{E}-02$ & $\mathrm{U}$ \\
\hline & ${ }^{90} \mathrm{Sr}$ & $-1.3 \mathrm{E}-01 \pm 1.8 \mathrm{E}-01$ & $\mathrm{U}$ & & ${ }^{90} \mathrm{Sr}$ & $-1.5 \mathrm{E}-01 \pm 1.8 \mathrm{E}-01$ & $\mathrm{U}$ \\
\hline & ${ }^{234} \mathrm{U}$ & $1.8 \mathrm{E}-02 \pm 1.1 \mathrm{E}-02$ & & & ${ }^{234} \mathrm{U}$ & $1.9 \mathrm{E}-02 \pm 9.5 \mathrm{E}-03$ & \\
\hline & ${ }^{235} \mathrm{U}$ & $4.1 \mathrm{E}-03 \pm 5.1 \mathrm{E}-03$ & $\mathrm{U}$ & & ${ }^{235} \mathrm{U}$ & $4.4 \mathrm{E}-03 \pm 4.1 \mathrm{E}-03$ & \\
\hline & ${ }^{238} \mathrm{U}$ & $1.8 \mathrm{E}-02 \pm 9.9 \mathrm{E}-03$ & & & ${ }^{238} \mathrm{U}$ & $9.7 \mathrm{E}-03 \pm 6.1 \mathrm{E}-03$ & \\
\hline & ${ }^{65} \mathrm{Zn}$ & $1.1 \mathrm{E}-01 \pm 2.5 \mathrm{E}-01$ & $\mathrm{U}$ & & ${ }^{65} \mathrm{Zn}$ & 8.8E-02 \pm 9.9E-02 & $\mathrm{U}$ \\
\hline V124 & ${ }^{144} \mathrm{Ce}$ & $2.1 \mathrm{E}-01 \pm 4.4 \mathrm{E}-01$ & $\mathrm{U}$ & V125 & ${ }^{144} \mathrm{Ce}$ & $-5.6 \mathrm{E}-01 \pm 7.4 \mathrm{E}-01$ & $\mathrm{U}$ \\
\hline \multirow[t]{17}{*}{ (300 Area) } & ${ }^{60} \mathrm{Co}$ & $-7.7 \mathrm{E}-03 \pm 4.8 \mathrm{E}-02$ & $\mathrm{U}$ & (300 Area) & ${ }^{60} \mathrm{Co}$ & $-1.9 \mathrm{E}-02 \pm 6.2 \mathrm{E}-02$ & $\mathrm{U}$ \\
\hline & ${ }^{134} \mathrm{Cs}$ & $1.0 \mathrm{E}-02 \pm 4.4 \mathrm{E}-02$ & $\mathrm{U}$ & & ${ }^{134} \mathrm{Cs}$ & $3.8 \mathrm{E}-02 \pm 6.6 \mathrm{E}-02$ & $\mathrm{U}$ \\
\hline & ${ }^{137} \mathrm{Cs}$ & $-7.9 \mathrm{E}-03 \pm 4.2 \mathrm{E}-02$ & $\mathrm{U}$ & & ${ }^{137} \mathrm{Cs}$ & $1.3 \mathrm{E}-02 \pm 6.3 \mathrm{E}-02$ & $\mathrm{U}$ \\
\hline & ${ }^{152} \mathrm{Eu}$ & $-5.9 \mathrm{E}-02 \pm 1.1 \mathrm{E}-01$ & $\mathrm{U}$ & & ${ }^{152} \mathrm{Eu}$ & $-1.6 \mathrm{E}-02 \pm 1.6 \mathrm{E}-01$ & $\mathrm{U}$ \\
\hline & ${ }^{154} \mathrm{Eu}$ & $2.9 \mathrm{E}-03 \pm 2.9 \mathrm{E}-02$ & $\mathrm{U}$ & & ${ }^{154} \mathrm{Eu}$ & $-4.7 \mathrm{E}-02 \pm 1.8 \mathrm{E}-01$ & $\mathrm{U}$ \\
\hline & ${ }^{155} \mathrm{Eu}$ & $8.7 \mathrm{E}-02 \pm 1.1 \mathrm{E}-01$ & $\mathrm{U}$ & & ${ }^{155} \mathrm{Eu}$ & $-6.3 \mathrm{E}-02 \pm 1.9 \mathrm{E}-01$ & $\mathrm{U}$ \\
\hline & ${ }^{238} \mathrm{Pu}$ & $-7.3 \mathrm{E}-03 \pm 1.6 \mathrm{E}-02$ & $\mathrm{U}$ & & ${ }^{238} \mathrm{Pu}$ & $1.1 \mathrm{E}-02 \pm 4.2 \mathrm{E}-02$ & $\mathrm{U}$ \\
\hline & ${ }^{239 / 240} \mathrm{Pu}$ & $1.5 \mathrm{E}-03 \pm 3.0 \mathrm{E}-03$ & $\mathrm{U}$ & & ${ }^{239 / 240} \mathrm{Pu}$ & $3.5 \mathrm{E}-03 \pm 7.1 \mathrm{E}-03$ & $\mathrm{U}$ \\
\hline & ${ }^{103} \mathrm{Ru}$ & $-1.4 \mathrm{E}-03 \pm 1.4 \mathrm{E}-02$ & $\mathrm{U}$ & & ${ }^{103} \mathrm{Ru}$ & $-2.4 \mathrm{E}-03 \pm 2.4 \mathrm{E}-02$ & $\mathrm{U}$ \\
\hline & ${ }^{106} \mathrm{Ru}$ & $2.1 \mathrm{E}-01 \pm 3.8 \mathrm{E}-01$ & $\mathrm{U}$ & & ${ }^{106} \mathrm{Ru}$ & $6.2 \mathrm{E}-02 \pm 5.6 \mathrm{E}-01$ & $\mathrm{U}$ \\
\hline & ${ }^{125} \mathrm{Sb}$ & $1.4 \mathrm{E}-02 \pm 1.0 \mathrm{E}-01$ & $\mathrm{U}$ & & ${ }^{125} \mathrm{Sb}$ & $1.4 \mathrm{E}-01 \pm 1.6 \mathrm{E}-01$ & $\mathrm{U}$ \\
\hline & ${ }^{113} \mathrm{Sn}$ & $-4.0 \mathrm{E}-02 \pm 5.1 \mathrm{E}-02$ & $\mathrm{U}$ & & ${ }^{113} \mathrm{Sn}$ & $-4.2 \mathrm{E}-03 \pm 4.2 \mathrm{E}-02$ & $\mathrm{U}$ \\
\hline & ${ }^{90} \mathrm{Sr}$ & $-2.0 \mathrm{E}-01 \pm 2.0 \mathrm{E}-01$ & $\mathrm{U}$ & & ${ }^{90} \mathrm{Sr}$ & $-1.2 \mathrm{E}-01 \pm 1.8 \mathrm{E}-01$ & $\mathrm{U}$ \\
\hline & ${ }^{234} \mathrm{U}$ & $2.8 \mathrm{E}-02 \pm 1.2 \mathrm{E}-02$ & & & ${ }^{234} \mathrm{U}$ & $2.3 \mathrm{E}-02 \pm 1.1 \mathrm{E}-02$ & \\
\hline & ${ }^{235} \mathrm{U}$ & $6.7 \mathrm{E}-03 \pm 5.4 \mathrm{E}-03$ & & & ${ }^{235} \mathrm{U}$ & $5.4 \mathrm{E}-03 \pm 5.9 \mathrm{E}-03$ & $\mathrm{U}$ \\
\hline & ${ }^{238} \mathrm{U}$ & $1.1 \mathrm{E}-02 \pm 6.9 \mathrm{E}-03$ & & & ${ }^{238} \mathrm{U}$ & $1.5 \mathrm{E}-02 \pm 8.7 \mathrm{E}-03$ & \\
\hline & ${ }^{65} \mathrm{Zn}$ & $-1.7 \mathrm{E}-01 \pm 1.7 \mathrm{E}-01$ & $\mathrm{U}$ & & ${ }^{65} \mathrm{Zn}$ & $-2.9 \mathrm{E}-02 \pm 1.5 \mathrm{E}-01$ & $\mathrm{U}$ \\
\hline
\end{tabular}

RQ = Result Qualifier. $\mathrm{U}=$ The analyte was analyzed for but not detected. 
Table 4-4. 2008 Vegetation Sampling Results (pCi/g \pm total analytical uncertainty). (Sheet 17 of 18)

\begin{tabular}{|c|c|c|c|c|c|c|c|}
\hline Location & Isotope & Result \pm Error & $\mathbf{R Q}^{*}$ & Location & Isotope & Result \pm Error & RQ* \\
\hline V126 & ${ }^{144} \mathrm{Ce}$ & $-1.2 \mathrm{E}-02 \pm 1.2 \mathrm{E}-01$ & $\mathrm{U}$ & V128 & ${ }^{144} \mathrm{Ce}$ & $-9.8 \mathrm{E}-01 \pm 1.0 \mathrm{E}+00$ & $\mathrm{U}$ \\
\hline \multirow[t]{17}{*}{ (300 Area) } & ${ }^{60} \mathrm{Co}$ & $-1.0 \mathrm{E}-02 \pm 4.6 \mathrm{E}-02$ & $\mathrm{U}$ & (300 Area) & ${ }^{60} \mathrm{Co}$ & $-4.5 \mathrm{E}-02 \pm 8.7 \mathrm{E}-02$ & $\mathrm{U}$ \\
\hline & ${ }^{134} \mathrm{Cs}$ & $-3.8 \mathrm{E}-02 \pm 4.8 \mathrm{E}-02$ & $\mathrm{U}$ & & ${ }^{134} \mathrm{Cs}$ & $5.3 \mathrm{E}-02 \pm 1.0 \mathrm{E}-01$ & $\mathrm{U}$ \\
\hline & ${ }^{137} \mathrm{Cs}$ & $-1.0 \mathrm{E}-02 \pm 5.5 \mathrm{E}-02$ & $\mathrm{U}$ & & ${ }^{137} \mathrm{Cs}$ & $1.6 \mathrm{E}-02 \pm 9.8 \mathrm{E}-02$ & $\mathrm{U}$ \\
\hline & ${ }^{152} \mathrm{Eu}$ & $-7.3 \mathrm{E}-02 \pm 1.3 \mathrm{E}-01$ & $\mathrm{U}$ & & ${ }^{152} \mathrm{Eu}$ & $-9.3 \mathrm{E}-03 \pm 9.3 \mathrm{E}-02$ & $\mathrm{U}$ \\
\hline & ${ }^{154} \mathrm{Eu}$ & $4.0 \mathrm{E}-02 \pm 1.3 \mathrm{E}-01$ & $\mathrm{U}$ & & ${ }^{154} \mathrm{Eu}$ & $-7.8 \mathrm{E}-02 \pm 2.6 \mathrm{E}-01$ & $\mathrm{U}$ \\
\hline & ${ }^{155} \mathrm{Eu}$ & $6.2 \mathrm{E}-03 \pm 6.2 \mathrm{E}-02$ & U & & ${ }^{155} \mathrm{Eu}$ & $1.0 \mathrm{E}-03 \pm 1.0 \mathrm{E}-02$ & $\mathrm{U}$ \\
\hline & ${ }^{238} \mathrm{Pu}$ & $6.7 \mathrm{E}-03 \pm 1.0 \mathrm{E}-02$ & $\mathrm{U}$ & & ${ }^{238} \mathrm{Pu}$ & $-4.6 \mathrm{E}-03 \pm 1.1 \mathrm{E}-02$ & $\mathrm{U}$ \\
\hline & ${ }^{239 / 240} \mathrm{Pu}$ & $9.6 \mathrm{E}-04 \pm 1.9 \mathrm{E}-03$ & $\mathrm{U}$ & & ${ }^{239 / 240} \mathrm{Pu}$ & $-2.8 \mathrm{E}-03 \pm 5.0 \mathrm{E}-03$ & $\mathrm{U}$ \\
\hline & ${ }^{103} \mathrm{Ru}$ & $-1.4 \mathrm{E}-02 \pm 5.0 \mathrm{E}-02$ & $\mathrm{U}$ & & ${ }^{103} \mathrm{Ru}$ & $6.1 \mathrm{E}-02 \pm 1.1 \mathrm{E}-01$ & $\mathrm{U}$ \\
\hline & ${ }^{106} \mathrm{Ru}$ & 4.0E-01 $\pm 4.3 \mathrm{E}-01$ & $\mathrm{U}$ & & ${ }^{106} \mathrm{Ru}$ & $1.1 \mathrm{E}+00 \pm 8.9 \mathrm{E}-01$ & $\mathrm{U}$ \\
\hline & ${ }^{125} \mathrm{Sb}$ & $1.0 \mathrm{E}-01 \pm 1.3 \mathrm{E}-01$ & $\mathrm{U}$ & & ${ }^{125} \mathrm{Sb}$ & $-8.6 \mathrm{E}-02 \pm 2.7 \mathrm{E}-01$ & $\mathrm{U}$ \\
\hline & ${ }^{113} \mathrm{Sn}$ & $4.5 \mathrm{E}-03 \pm 4.5 \mathrm{E}-02$ & $\mathrm{U}$ & & ${ }^{113} \mathrm{Sn}$ & $-4.2 \mathrm{E}-02 \pm 1.2 \mathrm{E}-01$ & $\mathrm{U}$ \\
\hline & ${ }^{90} \mathrm{Sr}$ & $1.7 \mathrm{E}-01 \pm 2.0 \mathrm{E}-01$ & $\mathrm{U}$ & & ${ }^{90} \mathrm{Sr}$ & $-1.2 \mathrm{E}-01 \pm 1.9 \mathrm{E}-01$ & $\mathrm{U}$ \\
\hline & ${ }^{234} \mathrm{U}$ & $4.4 \mathrm{E}-02 \pm 1.8 \mathrm{E}-02$ & & & ${ }^{234} \mathrm{U}$ & $1.3 \mathrm{E}-02 \pm 7.9 \mathrm{E}-03$ & \\
\hline & ${ }^{235} \mathrm{U}$ & $4.0 \mathrm{E}-03 \pm 4.1 \mathrm{E}-03$ & & & ${ }^{235} \mathrm{U}$ & $2.9 \mathrm{E}-03 \pm 4.4 \mathrm{E}-03$ & $\mathrm{U}$ \\
\hline & ${ }^{238} \mathrm{U}$ & $4.2 \mathrm{E}-02 \pm 1.6 \mathrm{E}-02$ & & & ${ }^{238} \mathrm{U}$ & $7.9 \mathrm{E}-03 \pm 6.2 \mathrm{E}-03$ & \\
\hline & ${ }^{65} \mathrm{Zn}$ & $-1.1 \mathrm{E}-01 \pm 1.3 \mathrm{E}-01$ & $\mathrm{U}$ & & ${ }^{65} \mathrm{Zn}$ & $2.5 \mathrm{E}-01 \pm 2.2 \mathrm{E}-01$ & $\mathrm{U}$ \\
\hline V129 & ${ }^{144} \mathrm{Ce}$ & $-1.7 \mathrm{E}-01 \pm 6.5 \mathrm{E}-01$ & $\mathrm{U}$ & V131 & ${ }^{144} \mathrm{Ce}$ & $-3.1 \mathrm{E}-01 \pm 4.3 \mathrm{E}-01$ & $\mathrm{U}$ \\
\hline \multirow[t]{17}{*}{ (300 Area) } & ${ }^{60} \mathrm{Co}$ & $7.5 \mathrm{E}-02 \pm 5.8 \mathrm{E}-02$ & $\mathrm{U}$ & (Replicate of & ${ }^{60} \mathrm{Co}$ & $-1.0 \mathrm{E}-02 \pm 3.3 \mathrm{E}-02$ & $\mathrm{U}$ \\
\hline & ${ }^{134} \mathrm{Cs}$ & $2.5 \mathrm{E}-02 \pm 6.8 \mathrm{E}-02$ & $\mathrm{U}$ & V116, & ${ }^{134} \mathrm{Cs}$ & $1.0 \mathrm{E}-02 \pm 5.3 \mathrm{E}-02$ & $\mathrm{U}$ \\
\hline & ${ }^{137} \mathrm{Cs}$ & $-2.6 \mathrm{E}-03 \pm 2.6 \mathrm{E}-02$ & $\mathrm{U}$ & 300 Area) & ${ }^{137} \mathrm{Cs}$ & $-2.1 \mathrm{E}-02 \pm 3.3 \mathrm{E}-02$ & $\mathrm{U}$ \\
\hline & ${ }^{152} \mathrm{Eu}$ & $-2.1 \mathrm{E}-02 \pm 1.7 \mathrm{E}-01$ & $\mathrm{U}$ & & ${ }^{152} \mathrm{Eu}$ & $-7.0 \mathrm{E}-02 \pm 9.9 \mathrm{E}-02$ & $\mathrm{U}$ \\
\hline & ${ }^{154} \mathrm{Eu}$ & $-8.9 \mathrm{E}-02 \pm 1.7 \mathrm{E}-01$ & $\mathrm{U}$ & & ${ }^{154} \mathrm{Eu}$ & $-2.2 \mathrm{E}-02 \pm 1.0 \mathrm{E}-01$ & $\mathrm{U}$ \\
\hline & ${ }^{155} \mathrm{Eu}$ & $1.7 \mathrm{E}-01 \pm 1.6 \mathrm{E}-01$ & $\mathrm{U}$ & & ${ }^{155} \mathrm{Eu}$ & $-4.4 \mathrm{E}-02 \pm 1.1 \mathrm{E}-01$ & $\mathrm{U}$ \\
\hline & ${ }^{238} \mathrm{Pu}$ & $-4.5 \mathrm{E}-03 \pm 1.3 \mathrm{E}-02$ & $\mathrm{U}$ & & ${ }^{238} \mathrm{Pu}$ & $2.1 \mathrm{E}-03 \pm 1.7 \mathrm{E}-02$ & $\mathrm{U}$ \\
\hline & ${ }^{239 / 240} \mathrm{Pu}$ & $9.0 \mathrm{E}-04 \pm 3.1 \mathrm{E}-03$ & $\mathrm{U}$ & & ${ }^{239 / 240} \mathrm{Pu}$ & $5.2 \mathrm{E}-03 \pm 7.0 \mathrm{E}-03$ & $\mathrm{U}$ \\
\hline & ${ }^{103} \mathrm{Ru}$ & $-1.9 \mathrm{E}-03 \pm 1.9 \mathrm{E}-02$ & $\mathrm{U}$ & & ${ }^{103} \mathrm{Ru}$ & $-1.9 \mathrm{E}-02 \pm 3.7 \mathrm{E}-02$ & $\mathrm{U}$ \\
\hline & ${ }^{106} \mathrm{Ru}$ & $-1.2 \mathrm{E}-01 \pm 5.3 \mathrm{E}-01$ & $\mathrm{U}$ & & ${ }^{106} \mathrm{Ru}$ & $1.4 \mathrm{E}-01 \pm 3.2 \mathrm{E}-01$ & $\mathrm{U}$ \\
\hline & ${ }^{125} \mathrm{Sb}$ & $1.5 \mathrm{E}-01 \pm 1.4 \mathrm{E}-01$ & $\mathrm{U}$ & & ${ }^{125} \mathrm{Sb}$ & $-7.8 \mathrm{E}-02 \pm 8.8 \mathrm{E}-02$ & $\mathrm{U}$ \\
\hline & ${ }^{113} \mathrm{Sn}$ & $-3.8 \mathrm{E}-02 \pm 6.7 \mathrm{E}-02$ & $\mathrm{U}$ & & ${ }^{113} \mathrm{Sn}$ & $-2.9 \mathrm{E}-02 \pm 4.1 \mathrm{E}-02$ & $\mathrm{U}$ \\
\hline & ${ }^{90} \mathrm{Sr}$ & $-1.7 \mathrm{E}-01 \pm 1.9 \mathrm{E}-01$ & $\mathrm{U}$ & & ${ }^{90} \mathrm{Sr}$ & $-2.2 \mathrm{E}-02 \pm 1.9 \mathrm{E}-01$ & $\mathrm{U}$ \\
\hline & ${ }^{234} \mathrm{U}$ & $2.2 \mathrm{E}-02 \pm 1.0 \mathrm{E}-02$ & & & ${ }^{234} \mathrm{U}$ & $1.0 \mathrm{E}-02 \pm 7.9 \mathrm{E}-03$ & \\
\hline & ${ }^{235} \mathrm{U}$ & $6.6 \mathrm{E}-03 \pm 6.5 \mathrm{E}-03$ & $\mathrm{U}$ & & ${ }^{235} \mathrm{U}$ & $1.0 \mathrm{E}-03 \pm 2.0 \mathrm{E}-03$ & $\mathrm{U}$ \\
\hline & ${ }^{238} \mathrm{U}$ & $1.1 \mathrm{E}-02 \pm 7.1 \mathrm{E}-03$ & & & ${ }^{238} \mathrm{U}$ & $3.7 \mathrm{E}-03 \pm 5.9 \mathrm{E}-03$ & $\mathrm{U}$ \\
\hline & ${ }^{65} \mathrm{Zn}$ & $-2.9 \mathrm{E}-01 \pm 2.9 \mathrm{E}-01$ & $\mathrm{U}$ & & ${ }^{65} \mathrm{Zn}$ & $-2.6 \mathrm{E}-02 \pm 7.9 \mathrm{E}-02$ & $\mathrm{U}$ \\
\hline
\end{tabular}

RQ = Result Qualifier. $\mathrm{U}=$ The analyte was analyzed for but not detected. 
Table 4-4. 2008 Vegetation Sampling Results (pCi/g \pm total analytical uncertainty). (Sheet 18 of 18)

\begin{tabular}{|c|c|c|c|}
\hline Location & Isotope & Result \pm Error & RQ* \\
\hline \multirow{18}{*}{$\begin{array}{c}\text { V132 } \\
\text { (Replicate of } \\
\text { V123, } \\
300 \text { Area) }\end{array}$} & ${ }^{144} \mathrm{Ce}$ & $2.2 \mathrm{E}-01 \pm 5.1 \mathrm{E}-01$ & $\mathrm{U}$ \\
\hline & ${ }^{60} \mathrm{Co}$ & $2.5 \mathrm{E}-02 \pm 4.1 \mathrm{E}-02$ & $\mathrm{U}$ \\
\hline & ${ }^{134} \mathrm{Cs}$ & $1.4 \mathrm{E}-02 \pm 4.4 \mathrm{E}-02$ & $\mathrm{U}$ \\
\hline & ${ }^{137} \mathrm{Cs}$ & 2.7E-03 \pm 2.7E-02 & $\mathrm{U}$ \\
\hline & ${ }^{152} \mathrm{Eu}$ & $4.5 \mathrm{E}-02 \pm 1.2 \mathrm{E}-01$ & $\mathrm{U}$ \\
\hline & ${ }^{154} \mathrm{Eu}$ & $2.9 \mathrm{E}-02 \pm 1.2 \mathrm{E}-01$ & $\mathrm{U}$ \\
\hline & ${ }^{155} \mathrm{Eu}$ & $2.5 \mathrm{E}-02 \pm 1.4 \mathrm{E}-01$ & $\mathrm{U}$ \\
\hline & ${ }^{238} \mathrm{Pu}$ & $9.9 \mathrm{E}-04 \pm 9.9 \mathrm{E}-03$ & $\mathrm{U}$ \\
\hline & ${ }^{239 / 240} \mathrm{Pu}$ & $9.9 \mathrm{E}-04 \pm 9.9 \mathrm{E}-03$ & $\mathrm{U}$ \\
\hline & ${ }^{103} \mathrm{Ru}$ & 8.7E-03 \pm 4.4E-02 & $\mathrm{U}$ \\
\hline & ${ }^{106} \mathrm{Ru}$ & $1.2 \mathrm{E}-01 \pm 4.0 \mathrm{E}-01$ & $\mathrm{U}$ \\
\hline & ${ }^{125} \mathrm{Sb}$ & $2.6 \mathrm{E}-02 \pm 1.2 \mathrm{E}-01$ & $\mathrm{U}$ \\
\hline & ${ }^{113} \mathrm{Sn}$ & $-6.9 \mathrm{E}-03 \pm 5.5 \mathrm{E}-02$ & $\mathrm{U}$ \\
\hline & ${ }^{90} \mathrm{Sr}$ & $-1.1 \mathrm{E}-01 \pm 1.9 \mathrm{E}-01$ & $\mathrm{U}$ \\
\hline & ${ }^{234} \mathrm{U}$ & $2.0 \mathrm{E}-02 \pm 1.1 \mathrm{E}-02$ & \\
\hline & ${ }^{235} \mathrm{U}$ & $3.0 \mathrm{E}-03 \pm 5.3 \mathrm{E}-03$ & $\mathrm{U}$ \\
\hline & ${ }^{238} \mathrm{U}$ & $1.3 \mathrm{E}-02 \pm 7.7 \mathrm{E}-03$ & \\
\hline & ${ }^{65} \mathrm{Zn}$ & $-4.8 \mathrm{E}-02 \pm 1.2 \mathrm{E}-01$ & $\mathrm{U}$ \\
\hline \multirow{18}{*}{$\begin{array}{c}\text { V130 } \\
\text { (400 Area) }\end{array}$} & ${ }^{144} \mathrm{Ce}$ & $3.5 \mathrm{E}-02 \pm 3.5 \mathrm{E}-01$ & $\mathrm{U}$ \\
\hline & ${ }^{60} \mathrm{Co}$ & $4.9 \mathrm{E}-03 \pm 4.0 \mathrm{E}-02$ & $\mathrm{U}$ \\
\hline & ${ }^{134} \mathrm{Cs}$ & $1.3 \mathrm{E}-02 \pm 4.0 \mathrm{E}-02$ & $\mathrm{U}$ \\
\hline & ${ }^{137} \mathrm{Cs}$ & $5.8 \mathrm{E}-04 \pm 5.8 \mathrm{E}-03$ & $\mathrm{U}$ \\
\hline & ${ }^{152} \mathrm{Eu}$ & $1.6 \mathrm{E}-02 \pm 9.1 \mathrm{E}-02$ & $\mathrm{U}$ \\
\hline & ${ }^{154} \mathrm{Eu}$ & $8.2 \mathrm{E}-02 \pm 1.2 \mathrm{E}-01$ & $\mathrm{U}$ \\
\hline & ${ }^{155} \mathrm{Eu}$ & $-1.6 \mathrm{E}-02 \pm 9.5 \mathrm{E}-02$ & $\mathrm{U}$ \\
\hline & ${ }^{238} \mathrm{Pu}$ & $8.3 \mathrm{E}-03 \pm 1.7 \mathrm{E}-02$ & $\mathrm{U}$ \\
\hline & ${ }^{239 / 240} \mathrm{Pu}$ & $2.8 \mathrm{E}-03 \pm 4.2 \mathrm{E}-03$ & $\mathrm{U}$ \\
\hline & ${ }^{103} \mathrm{Ru}$ & $1.9 \mathrm{E}-02 \pm 3.6 \mathrm{E}-02$ & $\mathrm{U}$ \\
\hline & ${ }^{106} \mathrm{Ru}$ & $7.1 \mathrm{E}-02 \pm 3.3 \mathrm{E}-01$ & $\mathrm{U}$ \\
\hline & ${ }^{125} \mathrm{Sb}$ & $1.2 \mathrm{E}-02 \pm 8.5 \mathrm{E}-02$ & $\mathrm{U}$ \\
\hline & ${ }^{113} \mathrm{Sn}$ & $2.4 \mathrm{E}-02 \pm 4.3 \mathrm{E}-02$ & $\mathrm{U}$ \\
\hline & ${ }^{90} \mathrm{Sr}$ & $1.0 \mathrm{E}-02 \pm 1.0 \mathrm{E}-01$ & $\mathrm{U}$ \\
\hline & ${ }^{234} \mathrm{U}$ & $2.0 \mathrm{E}-02 \pm 1.0 \mathrm{E}-02$ & \\
\hline & ${ }^{235} \mathrm{U}$ & $3.9 \mathrm{E}-03 \pm 4.9 \mathrm{E}-03$ & $\mathrm{U}$ \\
\hline & ${ }^{238} \mathrm{U}$ & $8.1 \mathrm{E}-03 \pm 6.3 \mathrm{E}-03$ & \\
\hline & ${ }^{65} \mathrm{Zn}$ & $-7.9 \mathrm{E}-02 \pm 8.8 \mathrm{E}-02$ & $\mathrm{U}$ \\
\hline
\end{tabular}

\begin{tabular}{clcc} 
Location & Isotope & Result \pm Error & RQ* \\
\hline V138 & ${ }^{144} \mathrm{Ce}$ & $-4.6 \mathrm{E}-01 \pm 6.5 \mathrm{E}-01$ & $\mathrm{U}$ \\
(Replicate of & ${ }^{60} \mathrm{Co}$ & $2.3 \mathrm{E}-02 \pm 6.2 \mathrm{E}-02$ & $\mathrm{U}$ \\
V118, & ${ }^{134} \mathrm{Cs}$ & $-7.2 \mathrm{E}-02 \pm 7.2 \mathrm{E}-02$ & $\mathrm{U}$ \\
300 Area) & ${ }^{137} \mathrm{Cs}$ & $1.5 \mathrm{E}-02 \pm 5.8 \mathrm{E}-02$ & $\mathrm{U}$ \\
& ${ }^{152} \mathrm{Eu}$ & $-1.1 \mathrm{E}-01 \pm 1.6 \mathrm{E}-01$ & $\mathrm{U}$ \\
& ${ }^{154} \mathrm{Eu}$ & $-1.5 \mathrm{E}-01 \pm 1.7 \mathrm{E}-01$ & $\mathrm{U}$ \\
& ${ }^{155} \mathrm{Eu}$ & $-6.4 \mathrm{E}-02 \pm 1.6 \mathrm{E}-01$ & $\mathrm{U}$ \\
& ${ }^{238} \mathrm{Pu}$ & $-8.7 \mathrm{E}-04 \pm 8.7 \mathrm{E}-03$ & $\mathrm{U}$ \\
& ${ }^{239 / 240} \mathrm{Pu}$ & $-2.6 \mathrm{E}-03 \pm 4.6 \mathrm{E}-03$ & $\mathrm{U}$ \\
& ${ }^{103} \mathrm{Ru}$ & $3.4 \mathrm{E}-02 \pm 6.3 \mathrm{E}-02$ & $\mathrm{U}$ \\
& ${ }^{106} \mathrm{Ru}$ & $-1.2 \mathrm{E}-01 \pm 5.6 \mathrm{E}-01$ & $\mathrm{U}$ \\
& ${ }^{125} \mathrm{Sb}$ & $-7.0 \mathrm{E}-02 \pm 1.4 \mathrm{E}-01$ & $\mathrm{U}$ \\
& ${ }^{113} \mathrm{Sn}$ & $7.3 \mathrm{E}-02 \pm 7.0 \mathrm{E}-02$ & $\mathrm{U}$ \\
& ${ }^{90} \mathrm{Sr}$ & $3.0 \mathrm{E}-02 \pm 2.8 \mathrm{E}-02$ & $\mathrm{U}$ \\
& ${ }^{234} \mathrm{U}$ & $1.9 \mathrm{E}-02 \pm 9.3 \mathrm{E}-03$ & \\
& ${ }^{235} \mathrm{U}$ & $3.6 \mathrm{E}-03 \pm 4.5 \mathrm{E}-03$ & $\mathrm{U}$ \\
& ${ }^{238} \mathrm{U}$ & $1.8 \mathrm{E}-02 \pm 9.2 \mathrm{E}-03$ & \\
& ${ }^{65} \mathrm{Zn}$ & $2.6 \mathrm{E}-03 \pm 2.6 \mathrm{E}-02$ & $\mathrm{U}$ \\
& & \\
\hline & &
\end{tabular}

$\mathrm{RQ}=$ Result Qualifier. $\mathrm{U}=$ The analyte was analyzed for but not detected. 
This page intentionally left blank. 


\subsection{EXTERNAL RADIATION}

External radiation fields were monitored near facilities and waste handling, storage, and disposal sites to measure and assess the impacts of operations. TLD results were used at numerous fixed locations to gather dose rate information over extended periods of time, typically three months.

In 2008, there were 124 TLD locations collecting external radiation information. The number of TLD locations in each operational area and a summary table comparing the 2007 and 2008 TLD results are provided in Table 5-1. Additional discussion of external radiation monitoring conducted near facilities and operations during 2008 can be found in Section 10.13 of PNNL-18427 (PNNL 2009a).

Table 5-1. Thermoluminscent Dosimeter Results (mrem/yr) for 2007 and 2008.

\begin{tabular}{|c|c|c|c|c|c|c|}
\hline \multirow{2}{*}{$\begin{array}{c}\text { Operational } \\
\text { Area }\end{array}$} & \multirow{2}{*}{$\begin{array}{l}\text { Number of } \\
\text { Dosimeters }\end{array}$} & \multicolumn{2}{|c|}{2007} & \multicolumn{2}{|c|}{2008} & \multirow[b]{2}{*}{ \% Change $^{c}$} \\
\hline & & Maximum $^{a}$ & Mean ${ }^{b}$ & Maximum $^{\mathrm{a}}$ & Mean ${ }^{\mathrm{b}}$ & \\
\hline $100-B C$ & 4 & $89 \pm 12$ & $85 \pm 7$ & $83 \pm 3$ & $79 \pm 6$ & $-6 \%$ \\
\hline $100-K$ & 14 & $590 \pm 15$ & $206 \pm 273$ & $574 \pm 42$ & $192 \pm 255$ & $-6 \%$ \\
\hline $100-\mathrm{N}$ & 6 & $142 \pm 153$ & $102 \pm 48$ & $106 \pm 9$ & $88 \pm 25$ & $-13 \%$ \\
\hline 200-East & 42 & $305 \pm 148$ & $110 \pm 95$ & $280 \pm 37$ & $103 \pm 80$ & $-5 \%$ \\
\hline 200-West & 24 & $241 \pm 287$ & $110 \pm 77$ & $259 \pm 135$ & $105 \pm 77$ & $-3 \%$ \\
\hline 200 -North $(212-\mathrm{R})^{\mathrm{d}}$ & 1 & $1,700 \pm 268$ & $1,700 \pm 81$ & $1,663 \pm 250$ & $1,647 \pm 26$ & $-2 \%$ \\
\hline 300 Area & 8 & $109 \pm 6$ & $87 \pm 20$ & $103 \pm 7$ & $83 \pm 17$ & $-3 \%$ \\
\hline $300 \mathrm{TEDF}^{\mathrm{e}}$ & 6 & $87 \pm 12$ & $84 \pm 4$ & $83 \pm 7$ & $81 \pm 3$ & $-3 \%$ \\
\hline $300-F F-2$ & 4 & $88 \pm 11$ & $85 \pm 5$ & $81 \pm 8$ & $79 \pm 4$ & $-6 \%$ \\
\hline 400 Area & 7 & $98 \pm 8$ & $85 \pm 12$ & $94 \pm 9$ & $81 \pm 12$ & $-3 \%$ \\
\hline $\mathrm{CVDF}^{\mathrm{f}}$ & 4 & $306 \pm 13$ & $154 \pm 205$ & $302 \pm 18$ & $151 \pm 203$ & $-1 \%$ \\
\hline $\mathrm{ERDF}^{\mathrm{g}}$ & 3 & $93 \pm 6$ & $88 \pm 8$ & $80 \pm 10$ & $79 \pm 4$ & $-10 \%$ \\
\hline $\mathrm{IDF}^{\mathrm{d}, \mathrm{h}}$ & 1 & $99 \pm 15$ & $91 \pm 13$ & $87 \pm 13$ & $85 \pm 5$ & $-5 \%$ \\
\hline
\end{tabular}

\footnotetext{
a maximum annual average \pm 2 standard deviations

b \pm 2 standard deviations

${ }^{\mathrm{c}}$ Numbers indicate a decrease (-) or increase from the 2007 mean

${ }^{\mathrm{d}}$ Maximum value represents highest quarterly value \pm analytical uncertainty

${ }^{\mathrm{e}}$ TEDF $=300$ Area Treated Effluent Disposal Facility

${ }^{\mathrm{f}} \mathrm{CVDF}=$ Cold Vacuum Drying Facility (100 K Area)

${ }^{\mathrm{g}}$ ERDF = Environmental Restoration Disposal Facility (200 West Area)

${ }^{\mathrm{h}}$ IDF $=$ Integrated Disposal Facility (200 East Area)
} 
Observations in dose rate monitoring during 2008 included the following:

- The external radiation levels measured at all operational areas during 2008 were lower than 2007 levels.

- Major cleanup activities at the 100-K Area fuel storage basins and adjacent retired reactor buildings were conducted in 2007 and 2008. Overall average dose rates measured in both the $100-\mathrm{K}$ East and 100-K West Areas in 2008 were comparable to the 2007 values. Similarly, dose rate levels measured in 2008 at monitoring stations around the 100-K Area Cold Vacuum Drying Facility (CVDF) were comparable to 2007 levels. The Hose-in-Hose project was completed in May 2007 and dose rate levels measured at the monitoring location situated adjacent to the sludge transfer route decreased to typical Hanford Site baseline levels in 2008. Quarterly dose rate levels for each of the facilities/projects at 100-K Area are presented in graph form in Figure 5-1.

- Average dose rates measured during 2008 in the 100-N Area were approximately 13\% lower than 2007 levels. Dose rates observed at the N Springs shoreline TLD location were approximately 7\% lower in 2008 than in 2007. Figure 5-2 provides historical trend plots of quarterly dose rates at the 116-N-1, 100-N Area and N-Springs monitoring locations.

- Dose rates observed in the 200-East and 200-West Areas during 2008 were comparable to levels measured in 2007. Dose rates at the 212-R Facility, while again in 2008 the highest on site, were also comparable to those measured during 2007. Figure 5-3 provides historical trend plots of quarterly dose rate levels for each of the 200 Area operational areas.

- Dose rates measured during 2008 at the Environmental Restoration Disposal Facility were approximately 10\% lower than 2007 levels.

- Dose rates measured at the 300 Area, 300 Treated Effluent Disposal Facility (TEDF), 300-FF-2 Field Remediation project, and in the 400 Area were consistent with previous years' measurements. Figure 5-4 provides historical trend plots of quarterly dose rate levels for each of these operational areas.

- Maps showing the 2008 TLD locations are provided in Figures 5-5 through 5-12 and individual 2008 TLD results are provided in Table 5-2. 
Figure 5-1. Average Quarterly Dose Rates, 100-K Area.

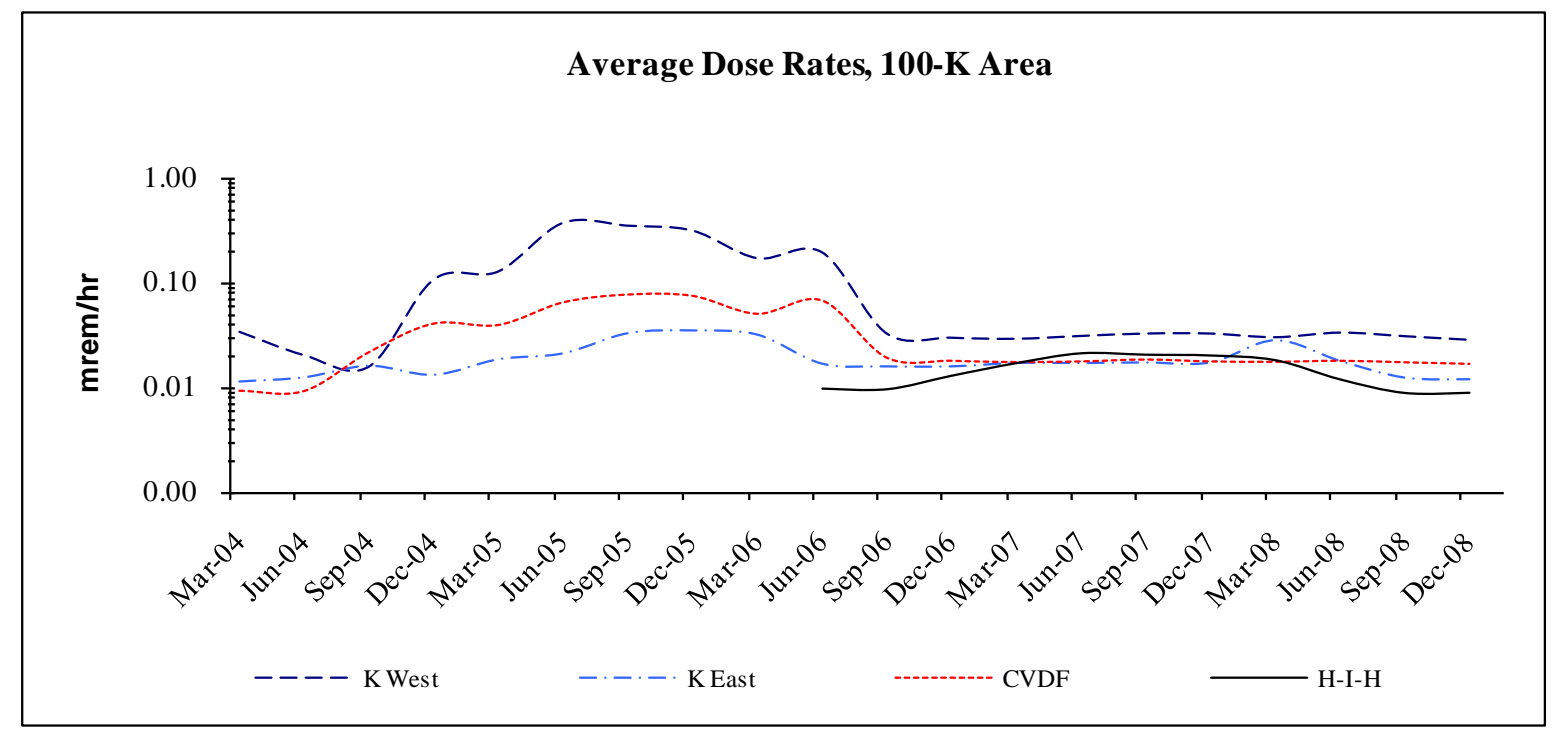

Figure 5-2. Average Quarterly Dose Rates, 100-N Area.

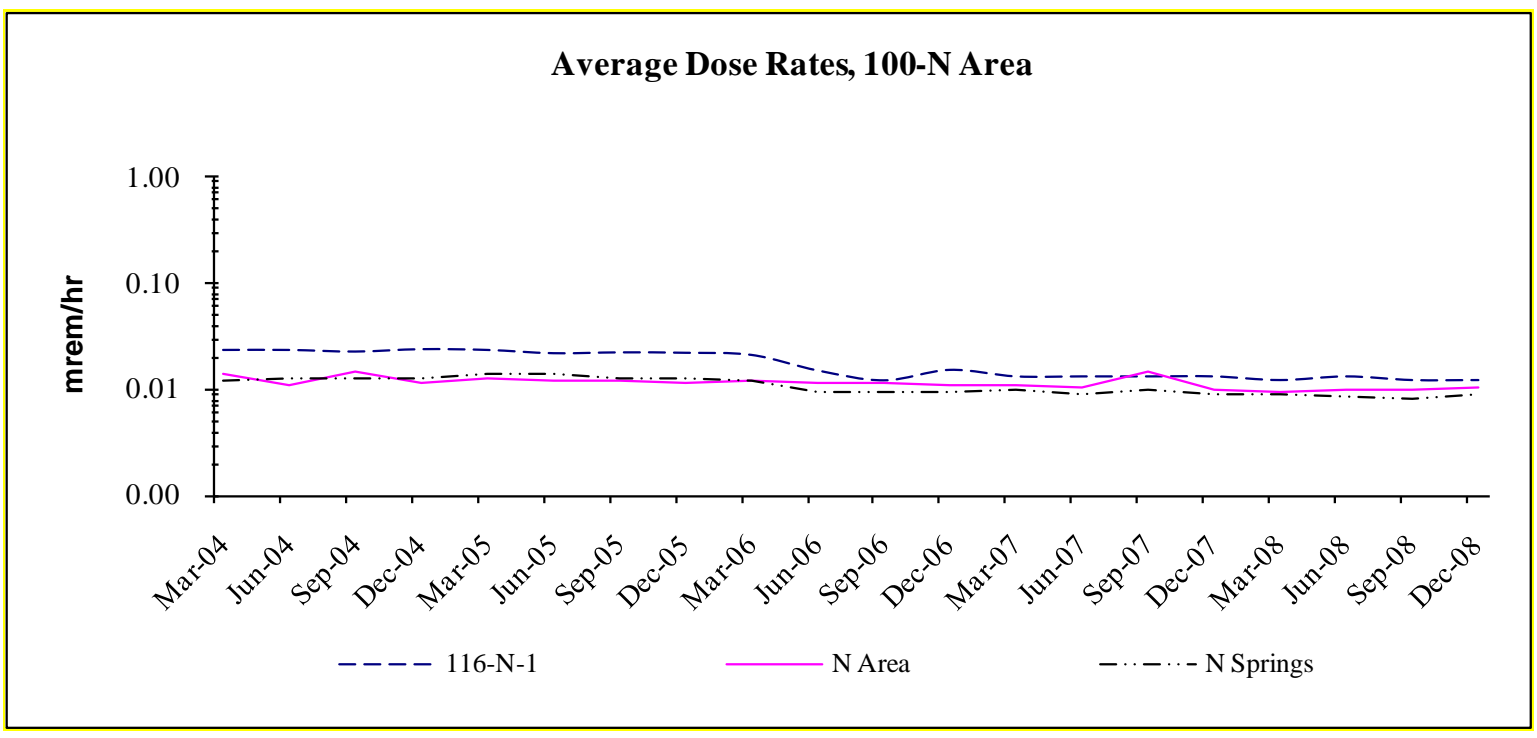


Figure 5-3. Average Quarterly Dose Rates, 200 Areas.

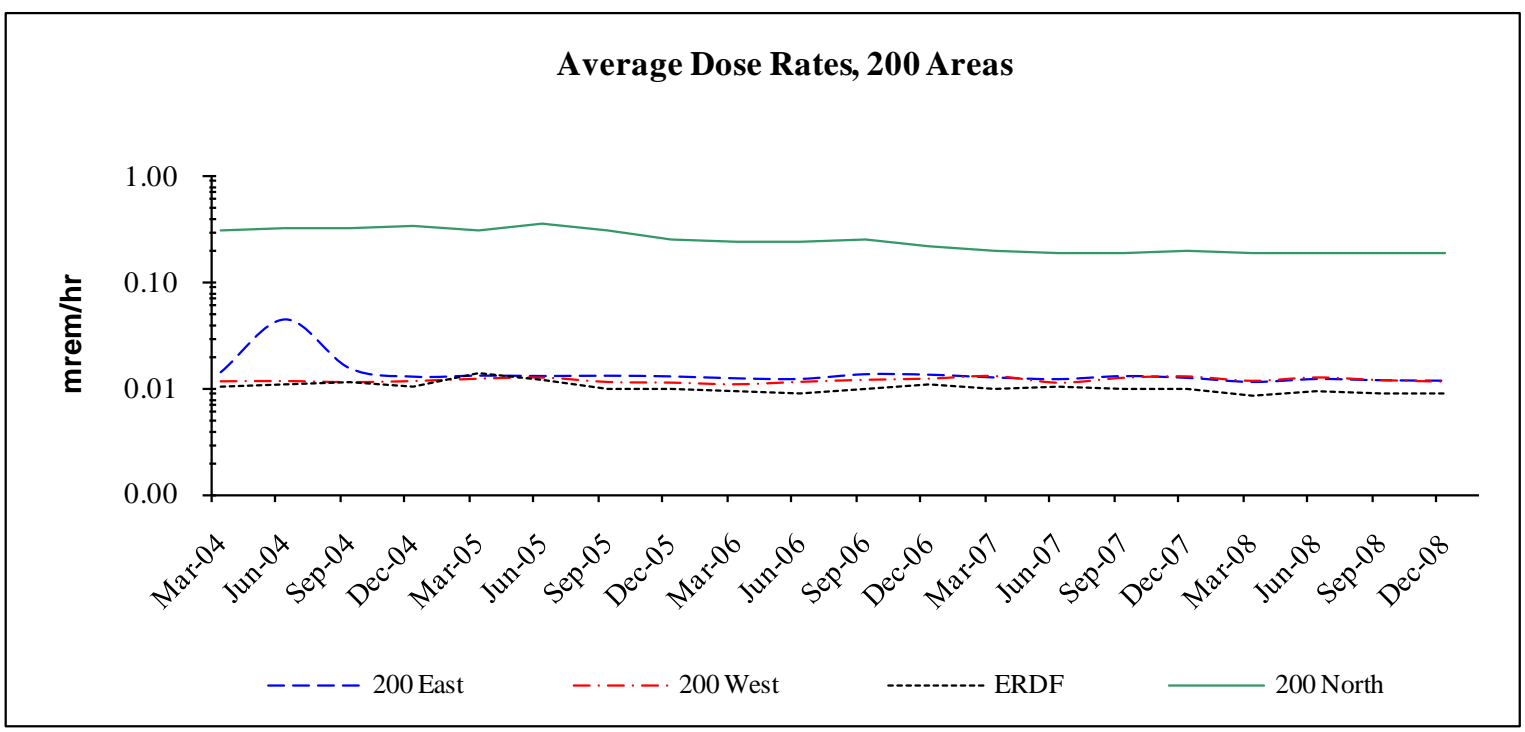

Figure 5-4. Average Quarterly Dose Rates, 300 and 400 Areas.

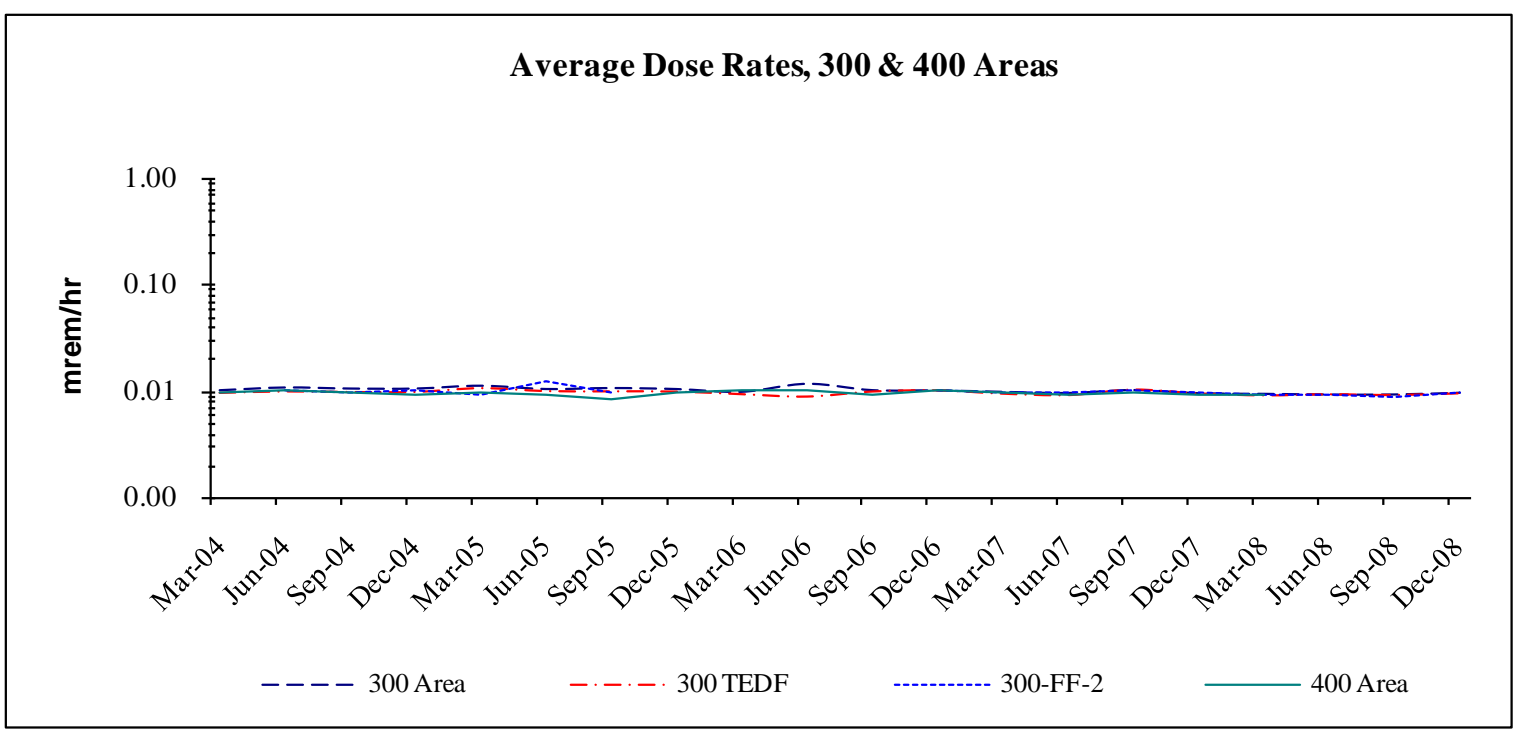


Figure 5-5. 100-B/C Area TLD Locations.

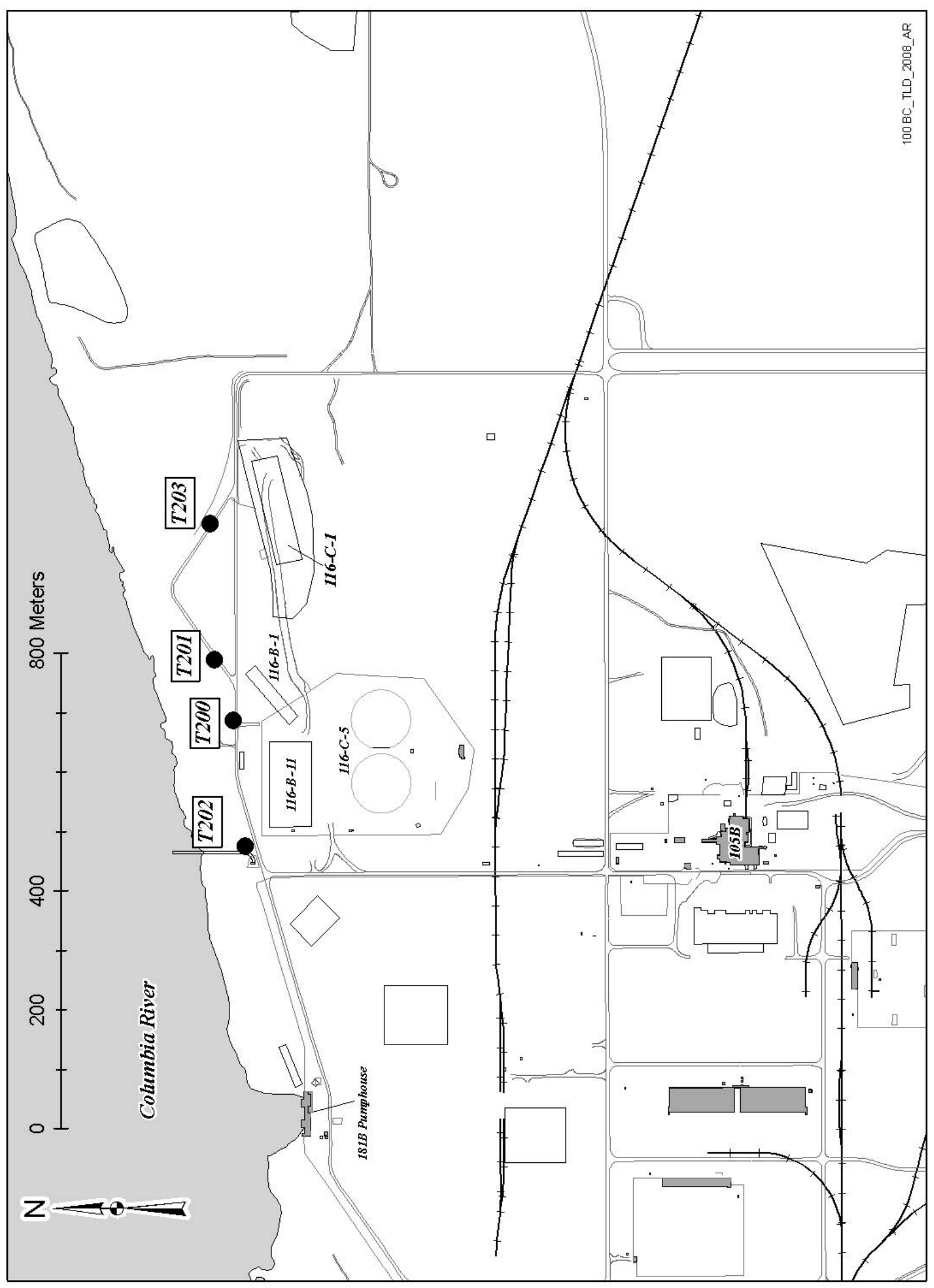


Figure 5-6. 100-K Area TLD Locations.

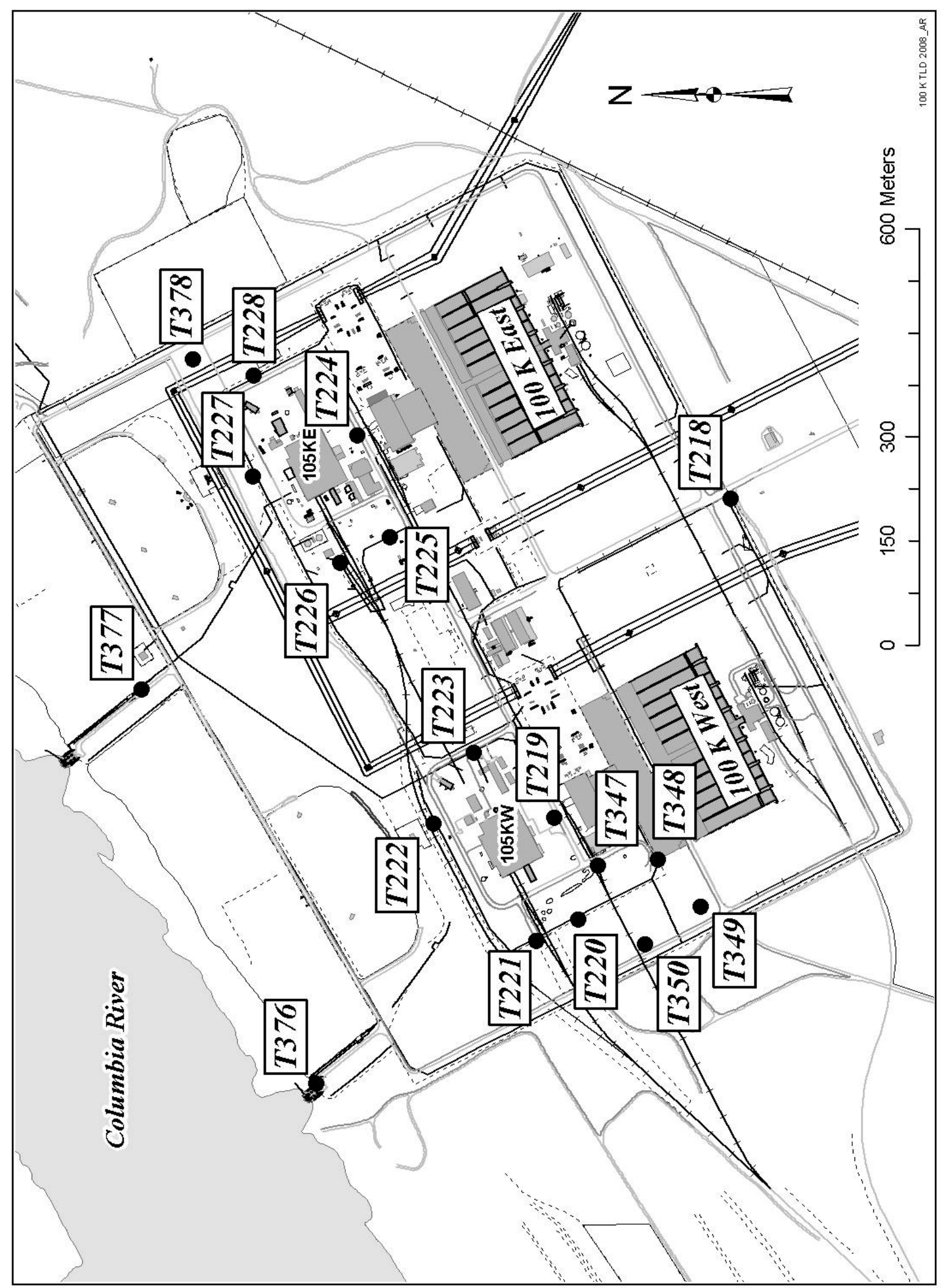


Figure 5-7. 100-N Area TLD Locations.

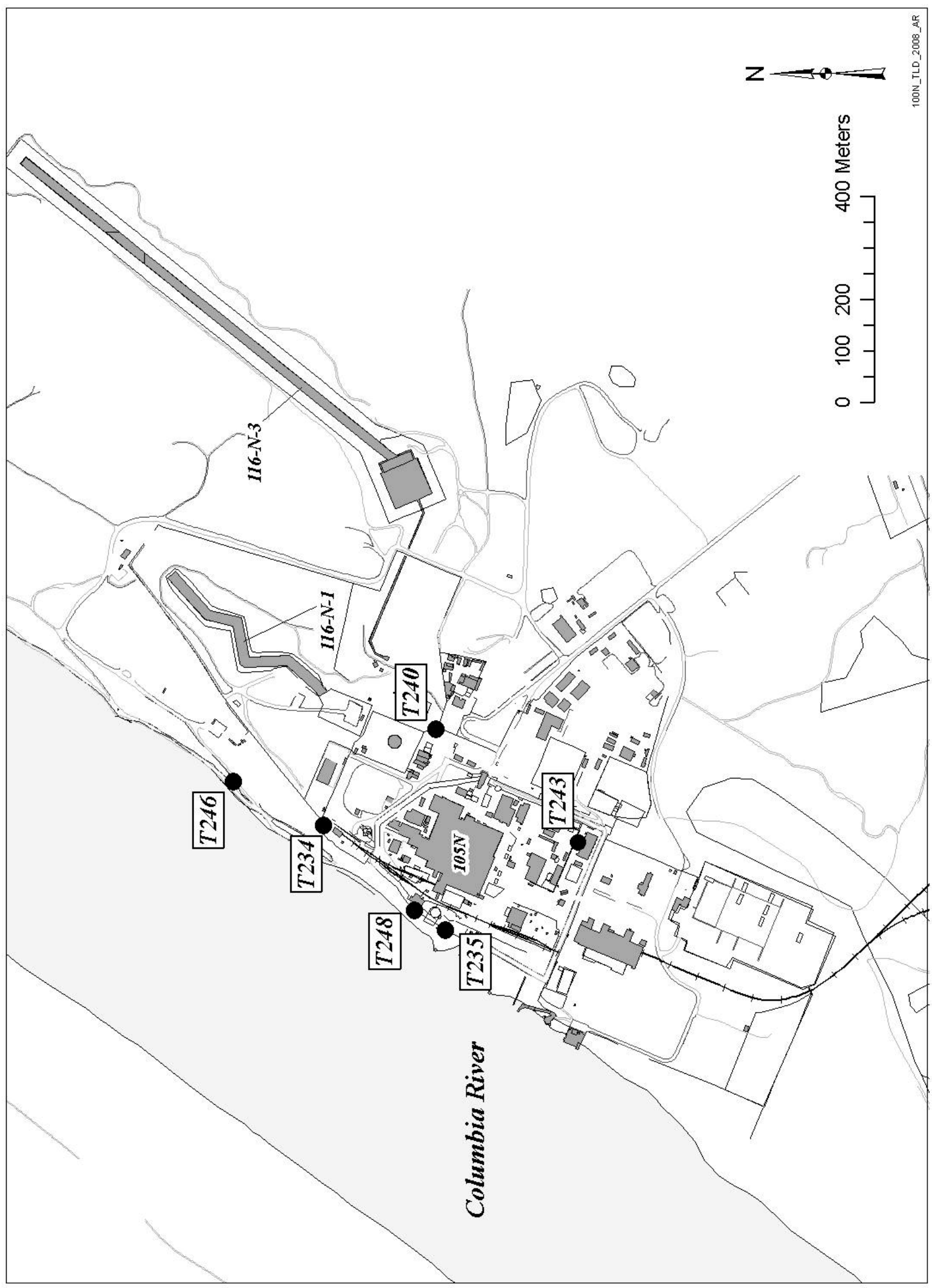


Figure 5-8. 200 East Area TLD Locations.

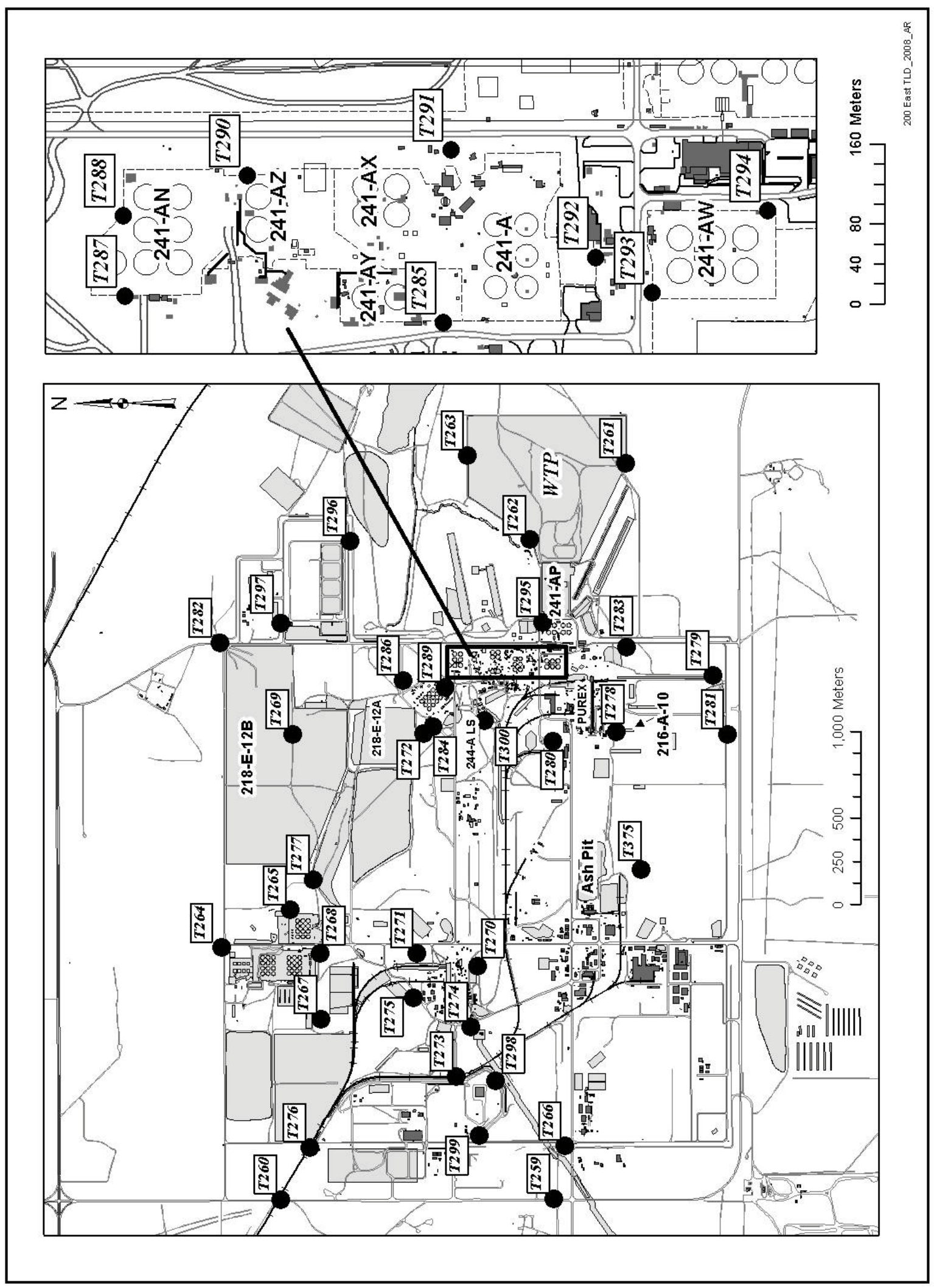


Figure 5-9. 200 West Area TLD Locations.

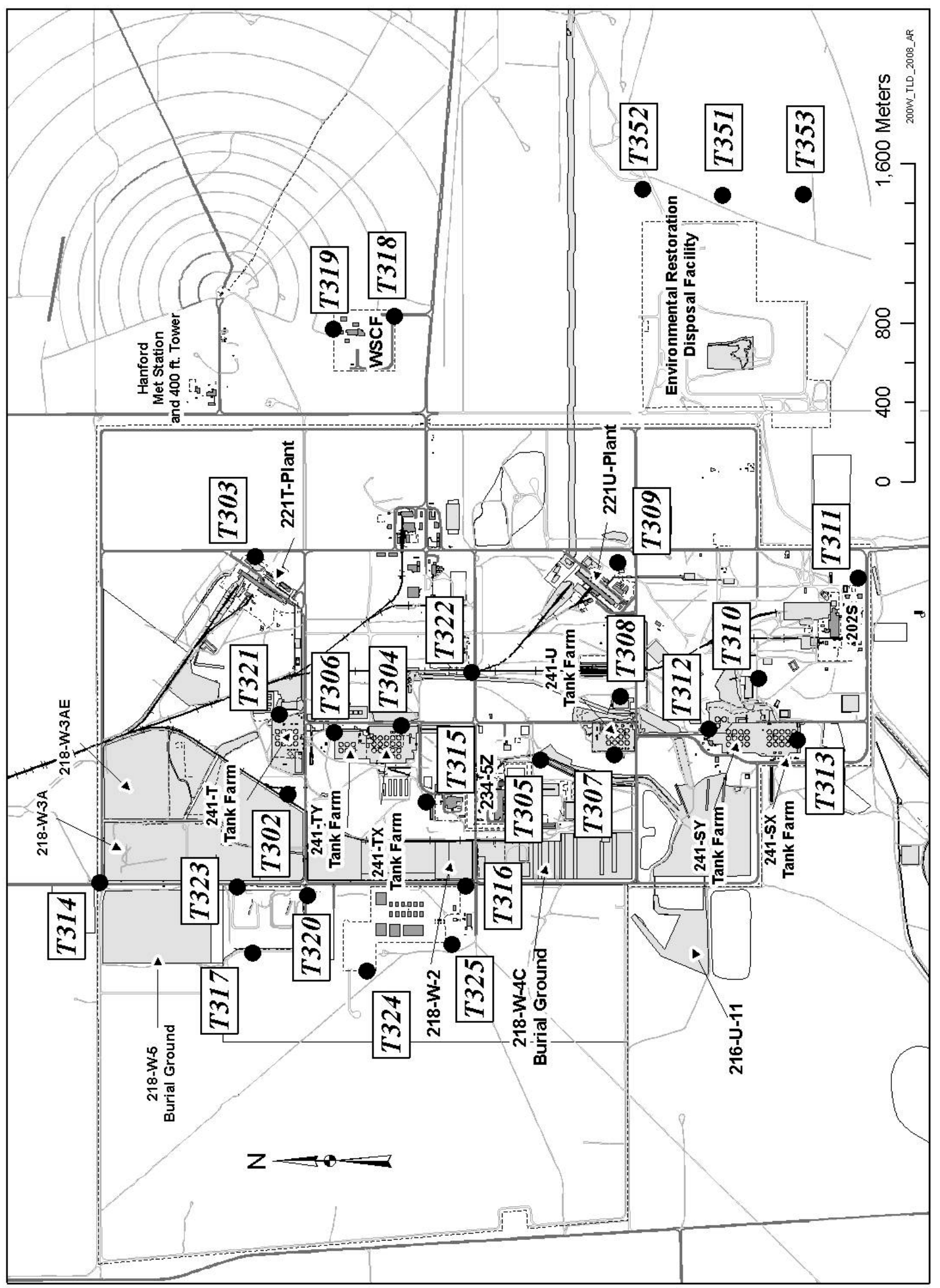


Figure 5-10. 200 North Area TLD Location.

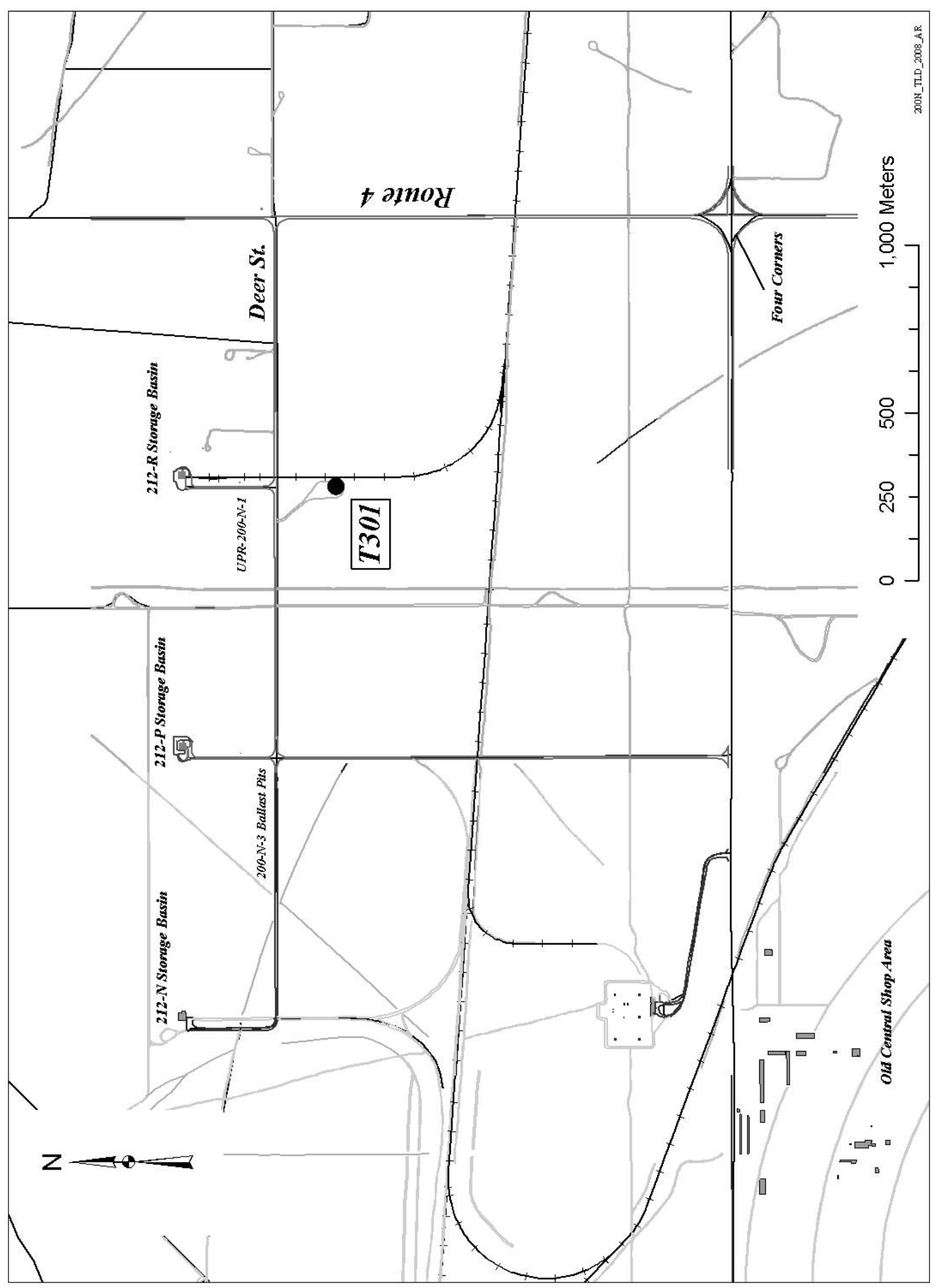


Figure 5-11. 300 Area Treated Effluent Disposal Facility and 300 Area TLD Locations.

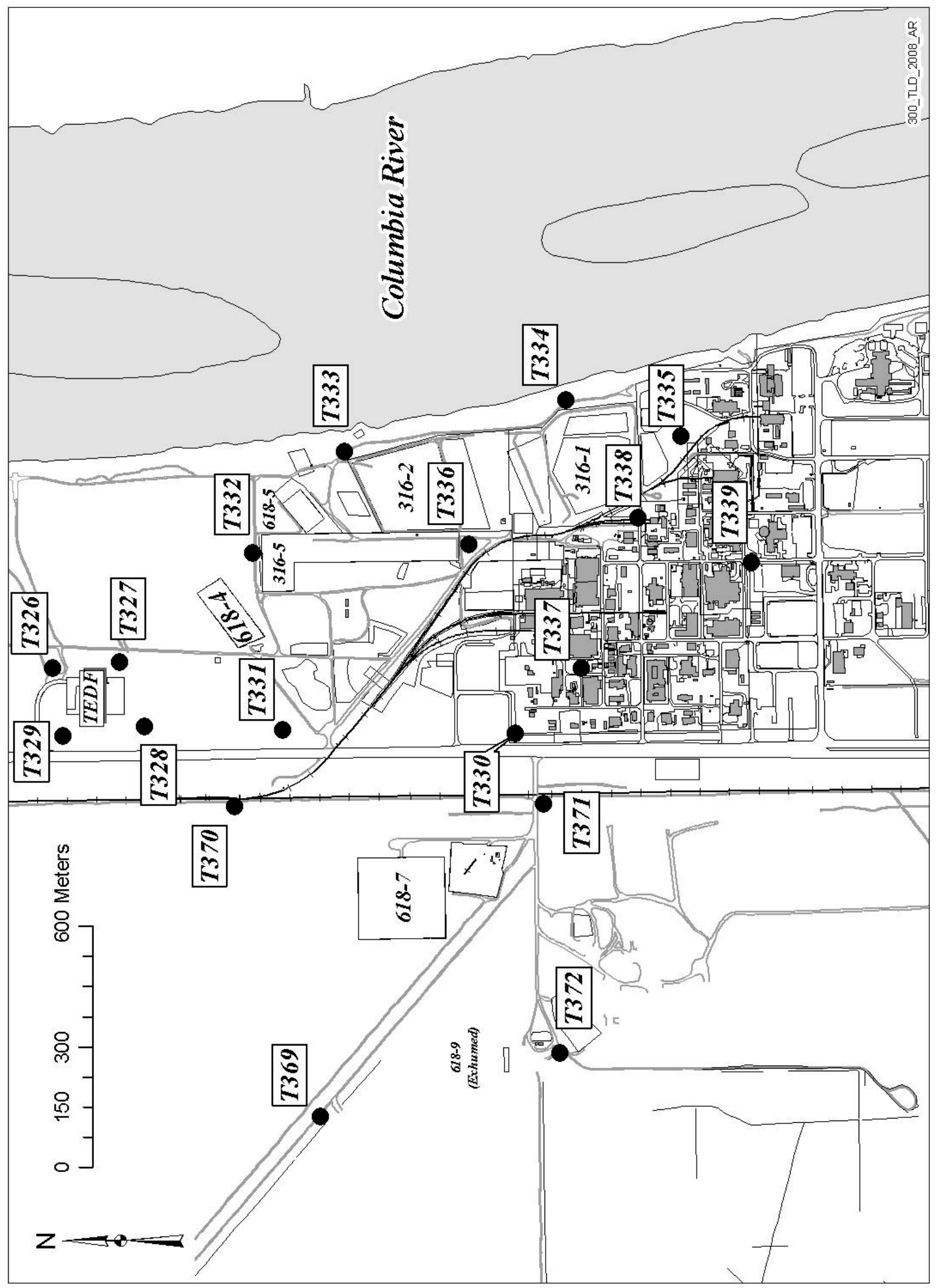


Figure 5-12. 400 Area TLD Locations.

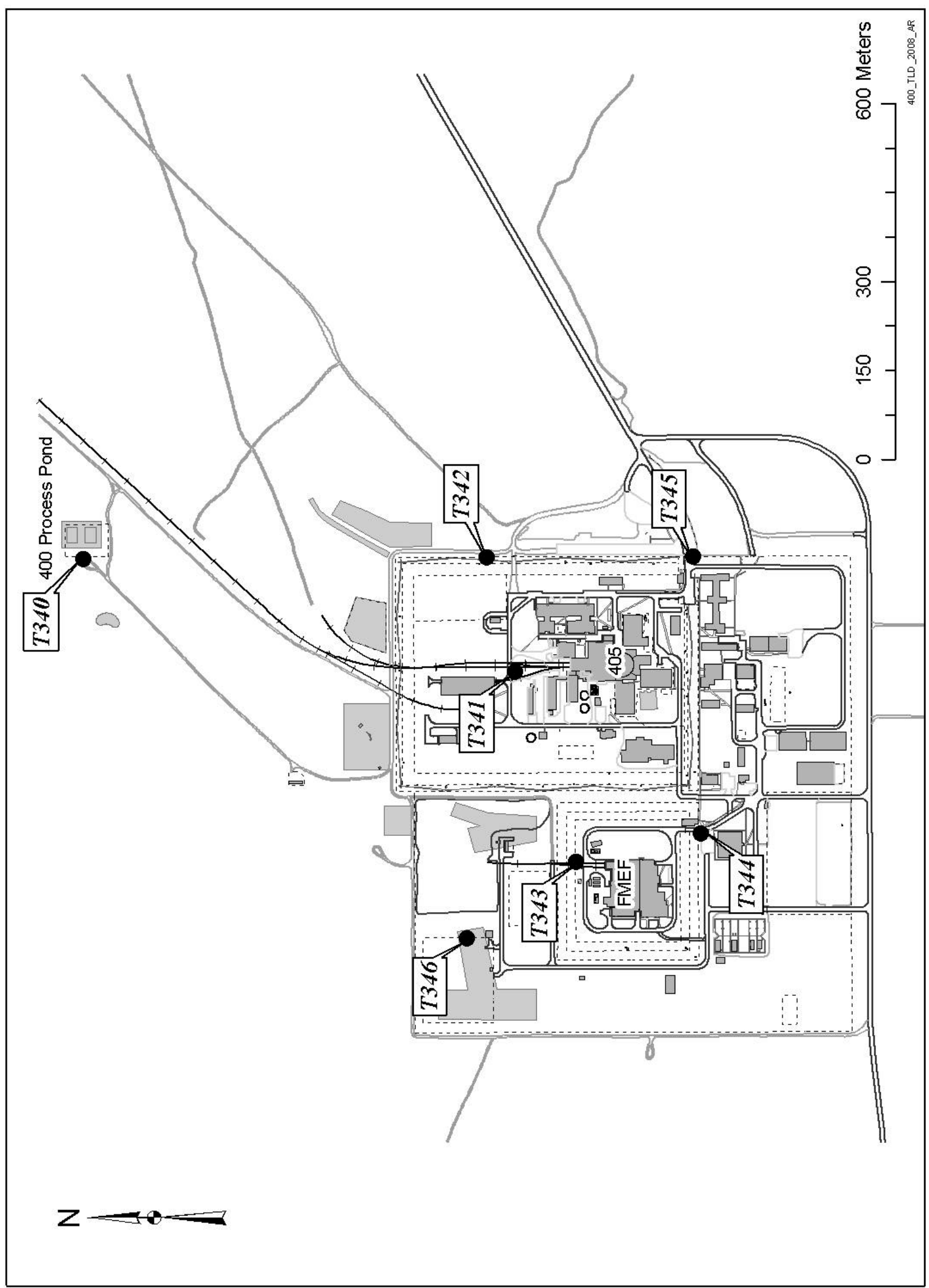


Table 5-2. 2008 TLD Results. (Sheet 1 of 14)

\begin{tabular}{|c|c|c|c|c|c|c|c|}
\hline \multicolumn{2}{|c|}{ Location } & \multirow{2}{*}{$\frac{\text { Sample Period }}{\text { 1st Quarter '08 }}$} & \multirow{2}{*}{$\frac{\text { mrem/hr }}{0.009}$} & \multirow{2}{*}{$\frac{\text { mrem/day }}{0.22}$} & \multirow{2}{*}{$\frac{\text { mrem/qtr }}{21.8}$} & \multirow{2}{*}{$\frac{\text { mrem/year }}{81}$} & \multirow{2}{*}{$\frac{\text { Days in Field }}{98}$} \\
\hline 100-B/C & $\mathrm{T} 200$ & & & & & & \\
\hline Field & $\mathrm{T} 201$ & & 0.009 & 0.22 & 21.1 & 79 & 98 \\
\hline \multirow{9}{*}{$\begin{array}{l}\text { Remediation } \\
\text { project }\end{array}$} & $\mathrm{T} 202$ & & 0.009 & 0.21 & 21.0 & 78 & 98 \\
\hline & $\mathrm{T} 203$ & & 0.009 & 0.20 & 20.0 & 75 & 98 \\
\hline & $\mathrm{T} 200$ & 2nd Quarter '08 & 0.010 & 0.23 & 19.3 & 83 & 85 \\
\hline & $\mathrm{T} 203$ & & 0.009 & 0.22 & 18.8 & 81 & 85 \\
\hline & $\mathrm{T} 200$ & 3rd Quarter '08 & 0.010 & 0.23 & 22.5 & 85 & 97 \\
\hline & $\mathrm{T} 201$ & \multicolumn{6}{|c|}{ TLD not recovered } \\
\hline & $\mathrm{T} 201$ & & 0.009 & 0.22 & 18.7 & 81 & 84 \\
\hline & $\mathrm{T} 202$ & & 0.009 & 0.22 & 18.8 & 82 & 84 \\
\hline & $\mathrm{T} 203$ & & 0.009 & 0.21 & 17.4 & 75 & 84 \\
\hline
\end{tabular}

100-B/C, Annual Averages \pm 2 Standard Deviations

\begin{tabular}{lcccc} 
& mrem/hr & mrem/day & mrem/qtr & mrem/year \\
\cline { 2 - 5 } T200 & $0.010 \pm 0.000$ & $0.23 \pm 0.01$ & $20.8 \pm 0.8$ & $83 \pm 3$ \\
T201 & $0.009 \pm 0.000$ & $0.22 \pm 0.01$ & $20.0 \pm 0.6$ & $80 \pm 3$ \\
T202 & $0.009 \pm 0.001$ & $0.21 \pm 0.01$ & $19.6 \pm 1.1$ & $78 \pm 4$ \\
T203 & $0.009 \pm 0.001$ & $0.21 \pm 0.02$ & $18.9 \pm 1.8$ & $76 \pm 7$
\end{tabular}

\begin{tabular}{|c|c|c|c|c|c|c|c|}
\hline \multicolumn{2}{|c|}{ Location } & \multirow{2}{*}{$\begin{array}{c}\text { Sample Period } \\
\text { 1st Quarter '08 }\end{array}$} & \multirow{2}{*}{$\frac{\text { mrem/hr }}{0.024}$} & \multirow{2}{*}{$\frac{\text { mrem/day }}{0.58}$} & \multirow{2}{*}{$\frac{\text { mrem/qtr }}{56.2}$} & \multirow{2}{*}{$\frac{\text { mrem/year }}{212}$} & \multirow{2}{*}{$\frac{\text { Days in Field }}{97}$} \\
\hline $100-K$ & $\mathrm{~T} 218$ & & & & & & \\
\hline \multirow[t]{27}{*}{ Area } & $\mathrm{T} 219$ & & 0.065 & 1.55 & 150.1 & 565 & 97 \\
\hline & $\mathrm{T} 220$ & & 0.025 & 0.60 & 58.5 & 220 & 97 \\
\hline & $\mathrm{T} 221$ & & 0.029 & 0.69 & 67.2 & 253 & 97 \\
\hline & $\mathrm{T} 222$ & & 0.024 & 0.56 & 54.6 & 206 & 97 \\
\hline & $\mathrm{T} 223$ & & 0.013 & 0.30 & 29.1 & 109 & 97 \\
\hline & $\mathrm{T} 224$ & & 0.016 & 0.38 & 37.1 & 140 & 97 \\
\hline & $\mathrm{T} 225$ & & 0.024 & 0.58 & 56.5 & 213 & 97 \\
\hline & $\mathrm{T} 226$ & & 0.020 & 0.47 & 45.7 & 172 & 97 \\
\hline & $\mathrm{T} 227$ & & 0.055 & 1.32 & 127.9 & 481 & 97 \\
\hline & T228 & & 0.024 & 0.56 & 54.6 & 206 & 97 \\
\hline & T376 & & 0.008 & 0.20 & 19.5 & 73 & 97 \\
\hline & T377 & & 0.009 & 0.21 & 20.1 & 76 & 97 \\
\hline & $\mathrm{T} 378$ & & 0.038 & 0.91 & 88.2 & 332 & 97 \\
\hline & $\mathrm{T} 218$ & 2nd Quarter '08 & 0.023 & 0.55 & 49.9 & 203 & 90 \\
\hline & $\mathrm{T} 219$ & & 0.067 & 1.60 & 143.6 & 583 & 90 \\
\hline & $\mathrm{T} 220$ & & 0.029 & 0.71 & 63.5 & 258 & 90 \\
\hline & $\mathrm{T} 221$ & & 0.041 & 0.98 & 88.6 & 359 & 90 \\
\hline & T222 & & 0.024 & 0.58 & 52.5 & 213 & 90 \\
\hline & $\mathrm{T} 223$ & & 0.014 & 0.33 & 29.9 & 121 & 90 \\
\hline & $\mathrm{T} 224$ & & 0.012 & 0.30 & 26.6 & 108 & 90 \\
\hline & $\mathrm{T} 225$ & & 0.021 & 0.51 & 46.1 & 187 & 90 \\
\hline & $\mathrm{T} 226$ & & 0.013 & 0.31 & 28.0 & 113 & 90 \\
\hline & $\mathrm{T} 227$ & & 0.029 & 0.69 & 62.5 & 254 & 90 \\
\hline & T228 & & 0.013 & 0.31 & 28.2 & 114 & 90 \\
\hline & T376 & & 0.009 & 0.21 & 19.2 & 78 & 90 \\
\hline & $\mathrm{T} 377$ & & 0.009 & 0.21 & 19.3 & 78 & 90 \\
\hline & $\mathrm{T} 378$ & & 0.018 & 0.43 & 38.5 & 156 & 90 \\
\hline
\end{tabular}


Table 5-2. 2008 TLD Results. (Sheet 2 of 14)

\begin{tabular}{|c|c|c|c|c|c|c|c|}
\hline \multicolumn{2}{|c|}{ Location } & \multirow{2}{*}{$\frac{\text { Sample Period }}{\text { 3rd Quarter '08 }}$} & \multirow{2}{*}{$\frac{\mathrm{mrem} / \mathrm{hr}}{0.022}$} & \multirow{2}{*}{$\frac{\mathrm{mrem} / \text { day }}{0.53}$} & \multirow{2}{*}{$\frac{\text { mrem/qtr }}{48.3}$} & \multirow{2}{*}{$\frac{\text { mrem/year }}{194}$} & \multirow{2}{*}{$\frac{\text { Days in Field }}{91}$} \\
\hline $100-K$ & $\mathrm{~T} 218$ & & & & & & \\
\hline Area & T219 & & 0.068 & 1.64 & 149.0 & 598 & 91 \\
\hline & $\mathrm{T} 220$ & & 0.025 & 0.60 & 55.5 & 220 & 92 \\
\hline & $\mathrm{T} 221$ & & 0.033 & 0.80 & 73.3 & 291 & 92 \\
\hline & T222 & & 0.021 & 0.51 & 46.6 & 187 & 91 \\
\hline & $\mathrm{T} 223$ & & 0.013 & 0.31 & 28.1 & 113 & 91 \\
\hline & $\mathrm{T} 224$ & & 0.009 & 0.22 & 20.4 & 82 & 91 \\
\hline & $\mathrm{T} 225$ & & 0.017 & 0.41 & 37.3 & 150 & 91 \\
\hline & $\mathrm{T} 226$ & & 0.011 & 0.26 & 23.5 & 94 & 91 \\
\hline & $\mathrm{T} 227$ & & 0.015 & 0.37 & 33.7 & 135 & 91 \\
\hline & $\mathrm{T} 228$ & & 0.009 & 0.21 & 19.0 & 76 & 91 \\
\hline & T376 & & 0.009 & 0.21 & 19.3 & 78 & 91 \\
\hline & T377 & & 0.008 & 0.20 & 18.4 & 74 & 91 \\
\hline & $\mathrm{T} 378$ & & 0.009 & 0.22 & 20.1 & 81 & 91 \\
\hline & $\mathrm{T} 218$ & 4th Quarter '08 & 0.022 & 0.53 & 44.8 & 192 & 85 \\
\hline & $\mathrm{T} 219$ & & 0.063 & 1.50 & 129.4 & 549 & 86 \\
\hline & $\mathrm{T} 220$ & & 0.025 & 0.60 & 50.1 & 218 & 84 \\
\hline & $\mathrm{T} 221$ & & 0.024 & 0.56 & 47.3 & 206 & 84 \\
\hline & $\mathrm{T} 222$ & & 0.023 & 0.56 & 47.3 & 203 & 85 \\
\hline & $\mathrm{T} 223$ & & 0.012 & 0.30 & 25.2 & 108 & 85 \\
\hline & $\mathrm{T} 224$ & & 0.008 & 0.19 & 16.3 & 69 & 86 \\
\hline & $\mathrm{T} 225$ & & 0.020 & 0.48 & 40.6 & 174 & 85 \\
\hline & T226 & & 0.009 & 0.23 & 19.5 & 83 & 86 \\
\hline & T227 & & 0.013 & 0.31 & 27.0 & 115 & 86 \\
\hline & T228 & & 0.009 & 0.21 & 17.7 & 75 & 86 \\
\hline & T376 & & 0.008 & 0.18 & 15.7 & 67 & 86 \\
\hline & T377 & & 0.009 & 0.21 & 18.4 & 78 & 86 \\
\hline & T378 & & 0.010 & 0.24 & 20.8 & 88 & 86 \\
\hline
\end{tabular}

100-K Area, Annual Averages \pm 2 Standard Deviations

$\mathrm{T} 218$

$\mathrm{T} 219$

$\mathrm{T} 220$

$\mathrm{T} 221$

$\mathrm{T} 222$

$\mathrm{T} 223$

$\mathrm{T} 224$

T225

T226

$\mathrm{T} 227$

$\mathrm{T} 228$

T376

T377

T378

\begin{tabular}{cccc}
$\mathbf{m r e m} / \mathbf{h r}$ & $\mathbf{m r e m} / \mathbf{d a y}$ & $\mathbf{m r e m} / \mathbf{q t r}$ & $\mathbf{m r e m} / \mathbf{y e a r}$ \\
\hline $0.023 \pm 0.002$ & $0.55 \pm 0.05$ & $50.1 \pm 4.4$ & $200 \pm 18$ \\
$0.065 \pm 0.005$ & $1.57 \pm 0.12$ & $143.4 \pm 10.5$ & $574 \pm 42$ \\
$0.026 \pm 0.004$ & $0.63 \pm 0.11$ & $57.2 \pm 9.6$ & $229 \pm 39$ \\
$0.032 \pm 0.015$ & $0.76 \pm 0.36$ & $69.5 \pm 32.5$ & $278 \pm 130$ \\
$0.023 \pm 0.002$ & $0.55 \pm 0.06$ & $50.5 \pm 5.5$ & $202 \pm 22$ \\
$0.013 \pm 0.001$ & $0.31 \pm 0.03$ & $28.2 \pm 2.9$ & $113 \pm 12$ \\
$0.011 \pm 0.007$ & $0.28 \pm 0.17$ & $25.2 \pm 15.5$ & $101 \pm 62$ \\
$0.021 \pm 0.006$ & $0.50 \pm 0.14$ & $45.4 \pm 13.2$ & $181 \pm 53$ \\
$0.013 \pm 0.009$ & $0.32 \pm 0.22$ & $29.2 \pm 19.9$ & $117 \pm 79$ \\
$0.029 \pm 0.038$ & $0.69 \pm 0.92$ & $62.9 \pm 84.2$ & $252 \pm 337$ \\
$0.014 \pm 0.014$ & $0.33 \pm 0.34$ & $30.0 \pm 30.7$ & $120 \pm 123$ \\
$0.008 \pm 0.001$ & $0.20 \pm 0.03$ & $18.5 \pm 2.7$ & $74 \pm 11$ \\
$0.009 \pm 0.000$ & $0.21 \pm 0.01$ & $19.1 \pm 1.0$ & $76 \pm 4$ \\
$0.019 \pm 0.027$ & $0.46 \pm 0.64$ & $42.0 \pm 58.4$ & $168 \pm 234$
\end{tabular}

\begin{tabular}{lccccccc}
\multicolumn{2}{c}{ Location } & Sample Period & $\mathbf{m r e m} / \mathbf{h r}$ & $\mathbf{m r e m} / \mathbf{d a y}$ & $\mathbf{m r e m} / \mathbf{q t r}$ & $\mathbf{m r e m} / \mathbf{y e a r}$ & Days in Field \\
\hline CVDF & T347 & 1st Quarter '08 & 0.035 & 0.84 & 81.6 & 307 & 97 \\
$($ 100-K & T348 & & 0.013 & 0.30 & 29.3 & 110 & 97 \\
Area) & T349 & & 0.009 & 0.23 & 21.8 & 82 & 97 \\
& T350 & & 0.012 & 0.29 & 27.7 & 104 & 97 \\
& & & & & & \\
& T347 & 2nd Quarter '08 & 0.036 & 0.85 & 76.9 & 312 & 90 \\
& T348 & & 0.013 & 0.32 & 29.0 & 118 & 90 \\
& T349 & & 0.010 & 0.25 & 22.5 & 91 & 90 \\
& T350 & & 0.012 & 0.29 & 26.4 & 107 & 90
\end{tabular}


Table 5-2. 2008 TLD Results. (Sheet 3 of 14)

\begin{tabular}{|c|c|c|c|c|c|c|c|}
\hline \multicolumn{2}{|c|}{ Location } & \multirow{2}{*}{$\begin{array}{c}\text { Sample Period } \\
\text { 3rd Quarter '08 }\end{array}$} & \multirow{2}{*}{$\frac{\text { mrem/hr }}{0.034}$} & \multirow{2}{*}{$\frac{\text { mrem/day }}{0.81}$} & \multirow{2}{*}{$\frac{\text { mrem/qtr }}{73.9}$} & \multirow{2}{*}{$\frac{\text { mrem/year }}{297}$} & \multirow{2}{*}{$\frac{\text { Days in Field }}{91}$} \\
\hline CVDF & T347 & & & & & & \\
\hline$(100-\mathrm{K}$ & T348 & & 0.013 & 0.31 & 28.6 & 115 & 91 \\
\hline \multirow[t]{5}{*}{ Area) } & Т349 & & 0.010 & 0.23 & 20.9 & 84 & 91 \\
\hline & Т350 & & 0.012 & 0.28 & 25.6 & 103 & 91 \\
\hline & T347 & 4th Quarter '08 & 0.033 & 0.80 & 68.3 & 293 & 85 \\
\hline & Т349 & & 0.009 & 0.22 & 19.1 & 82 & 85 \\
\hline & T350 & & 0.012 & 0.28 & 23.6 & 101 & 85 \\
\hline
\end{tabular}

CVDF, Annual Averages \pm 2 Standard Deviations

\begin{tabular}{lcccc} 
& mrem/hr & mrem/day & mrem/qtr & mrem/year \\
\cline { 2 - 5 } T347 & $0.035 \pm 0.002$ & $0.83 \pm 0.05$ & $75.6 \pm 4.4$ & $302 \pm 18$ \\
T348 & $0.013 \pm 0.001$ & $0.31 \pm 0.03$ & $28.0 \pm 2.9$ & $112 \pm 12$ \\
T349 & $0.010 \pm 0.001$ & $0.23 \pm 0.02$ & $21.2 \pm 2.1$ & $85 \pm 9$ \\
T350 & $0.012 \pm 0.001$ & $0.28 \pm 0.01$ & $26.0 \pm 1.3$ & $104 \pm 5$
\end{tabular}

\begin{tabular}{|c|c|c|c|c|c|c|c|}
\hline \multicolumn{2}{|c|}{ Location } & \multirow{2}{*}{$\frac{\text { Sample Period }}{\text { 1st Quarter '08 }}$} & \multirow{2}{*}{$\frac{\text { mrem/hr }}{0.009}$} & \multirow{2}{*}{$\frac{\text { mrem/day }}{0.21}$} & \multirow{2}{*}{$\frac{\text { mrem/qtr }}{20.9}$} & \multirow{2}{*}{$\frac{\text { mrem/year }}{78}$} & \multirow{2}{*}{$\frac{\text { Days in Field }}{98}$} \\
\hline $100-N$ & $\mathrm{~T} 234$ & & & & & & \\
\hline Area & $\mathrm{T} 235$ & & 0.010 & 0.25 & 24.1 & 90 & 98 \\
\hline & $\mathrm{T} 243$ & & 0.008 & 0.19 & 19.1 & 71 & 98 \\
\hline & $\mathrm{T} 246$ & & 0.009 & 0.22 & 21.1 & 79 & 98 \\
\hline & $\mathrm{T} 248$ & & 0.011 & 0.25 & 24.7 & 92 & 98 \\
\hline & $\mathrm{T} 234$ & 2nd Quarter '08 & 0.010 & 0.25 & 20.8 & 90 & 84 \\
\hline & $\mathrm{T} 235$ & & 0.011 & 0.25 & 21.2 & 92 & 84 \\
\hline & $\mathrm{T} 240$ & & 0.013 & 0.31 & 26.0 & 113 & 84 \\
\hline & $\mathrm{T} 243$ & & 0.009 & 0.21 & 17.5 & 76 & 84 \\
\hline & $\mathrm{T} 246$ & & 0.009 & 0.21 & 17.6 & 77 & 84 \\
\hline & $\mathrm{T} 235$ & & 0.010 & 0.25 & 23.9 & 90 & 97 \\
\hline & $\mathrm{T} 240$ & & 0.012 & 0.29 & 27.8 & 105 & 97 \\
\hline & $\mathrm{T} 243$ & & \multicolumn{5}{|c|}{ Monitoring Location Retired } \\
\hline & $\mathrm{T} 246$ & & 0.008 & 0.20 & 19.0 & 72 & 97 \\
\hline & $\mathrm{T} 248$ & & 0.011 & 0.27 & 25.9 & 97 & 97 \\
\hline & $\mathrm{T} 234$ & 4th Quarter '08 & 0.009 & 0.21 & 18.1 & 78 & 85 \\
\hline & $\mathrm{T} 235$ & & 0.011 & 0.25 & 21.5 & 92 & 85 \\
\hline & $\mathrm{T} 240$ & & 0.012 & 0.28 & 24.2 & 104 & 85 \\
\hline & $\mathrm{T} 246$ & & 0.009 & 0.22 & 18.8 & 81 & 85 \\
\hline & $\mathrm{T} 235$ & 1908-N Fence & $0.010 \pm 0.000$ & $0.25 \pm 0.01$ & $22.7 \pm 0.7$ & $91 \pm 3$ & \\
\hline & $\mathrm{T} 240$ & MO-536 Fenceline & $0.012 \pm 0.001$ & $0.29 \pm 0.02$ & $26.5 \pm 2.3$ & $106 \pm 9$ & \\
\hline & $\mathrm{T} 243$ & 163-N Wastepad & $0.008 \pm 0.001$ & $0.20 \pm 0.02$ & $18.3 \pm 1.7$ & $73 \pm 7$ & \\
\hline & $\mathrm{T} 246$ & N-Springs & $0.009 \pm 0.001$ & $0.21 \pm 0.02$ & $19.2 \pm 2.0$ & $77 \pm 8$ & \\
\hline & $\mathrm{T} 248$ & 107-N, South & $0.011 \pm 0.001$ & $0.27 \pm 0.02$ & $24.2 \pm 1.8$ & $97 \pm 7$ & \\
\hline
\end{tabular}


Table 5-2. 2008 TLD Results. (Sheet 4 of 14)

\begin{tabular}{|c|c|c|c|c|c|c|c|}
\hline \multicolumn{2}{|c|}{ Location } & \multirow{2}{*}{$\begin{array}{c}\text { Sample Period } \\
\text { 1st Quarter '08 }\end{array}$} & \multirow{2}{*}{$\begin{array}{c}\text { mrem } / \mathbf{h r} \\
0.009\end{array}$} & \multirow{2}{*}{$\frac{\mathrm{mrem} / \text { day }}{0.21}$} & \multirow{2}{*}{$\frac{\text { mrem/qtr }}{17.1}$} & \multirow{2}{*}{$\frac{\text { mrem/year }}{76}$} & \multirow{2}{*}{$\frac{\text { Days in Field }}{83}$} \\
\hline 200 East & $\mathrm{T} 259$ & & & & & & \\
\hline Area & $\mathrm{T} 260$ & & 0.009 & 0.21 & 16.8 & 75 & 82 \\
\hline & $\mathrm{T} 261$ & & 0.009 & 0.22 & 18.0 & 79 & 83 \\
\hline & $\mathrm{T} 262$ & & 0.008 & 0.20 & 16.8 & 74 & 83 \\
\hline & $\mathrm{T} 263$ & & 0.009 & 0.21 & 17.4 & 77 & 83 \\
\hline & $\mathrm{T} 264$ & & 0.011 & 0.26 & 21.9 & 97 & 83 \\
\hline & $\mathrm{T} 265$ & & 0.012 & 0.29 & 23.7 & 105 & 83 \\
\hline & T266 & & 0.009 & 0.21 & 17.6 & 77 & 83 \\
\hline & $\mathrm{T} 267$ & & 0.009 & 0.21 & 17.6 & 77 & 83 \\
\hline & T268 & & 0.012 & 0.30 & 24.5 & 108 & 83 \\
\hline & $\mathrm{T} 269$ & & 0.010 & 0.23 & 19.0 & 84 & 83 \\
\hline & $\mathrm{T} 270$ & & 0.014 & 0.33 & 27.0 & 119 & 83 \\
\hline & $\mathrm{T} 271$ & & 0.009 & 0.22 & 18.3 & 81 & 83 \\
\hline & $\mathrm{T} 272$ & & 0.011 & 0.27 & 22.8 & 99 & 84 \\
\hline & $\mathrm{T} 273$ & & 0.009 & 0.21 & 17.8 & 78 & 83 \\
\hline & $\mathrm{T} 274$ & & 0.009 & 0.23 & 18.7 & 82 & 83 \\
\hline & $\mathrm{T} 275$ & & 0.009 & 0.22 & 18.5 & 81 & 83 \\
\hline & $\mathrm{T} 276$ & & 0.009 & 0.21 & 17.0 & 75 & 83 \\
\hline & $\mathrm{T} 277$ & & 0.009 & 0.22 & 18.8 & 81 & 85 \\
\hline & $\mathrm{T} 278$ & & 0.009 & 0.21 & 17.5 & 76 & 84 \\
\hline & $\mathrm{T} 279$ & & 0.009 & 0.22 & 18.0 & 80 & 82 \\
\hline & $\mathrm{T} 280$ & & 0.009 & 0.21 & 17.6 & 77 & 83 \\
\hline & $\mathrm{T} 281$ & & 0.009 & 0.23 & 18.5 & 83 & 82 \\
\hline & $\mathrm{T} 282$ & & 0.009 & 0.22 & 18.2 & 79 & 84 \\
\hline & $\mathrm{T} 283$ & & 0.009 & 0.21 & 17.6 & 77 & 83 \\
\hline & $\mathrm{T} 284$ & & 0.012 & 0.28 & 23.1 & 101 & 84 \\
\hline & T285 & & 0.021 & 0.51 & 42.4 & 187 & 83 \\
\hline & $\mathrm{T} 286$ & & 0.015 & 0.35 & 29.6 & 129 & 84 \\
\hline & T287 & & 0.014 & 0.33 & 27.7 & 122 & 83 \\
\hline & T288 & & 0.022 & 0.54 & 45.0 & 196 & 84 \\
\hline & T289 & & 0.018 & 0.43 & 35.5 & 156 & 83 \\
\hline & T290 & & 0.012 & 0.29 & 24.7 & 107 & 84 \\
\hline & $\mathrm{T} 291$ & & 0.014 & 0.33 & 27.7 & 121 & 84 \\
\hline & T292 & & 0.029 & 0.70 & 57.9 & 255 & 83 \\
\hline & T293 & & 0.012 & 0.28 & 23.2 & 102 & 83 \\
\hline & T294 & & 0.017 & 0.42 & 34.5 & 152 & 83 \\
\hline & T295 & & 0.010 & 0.23 & 19.1 & 84 & 83 \\
\hline & T296 & & 0.009 & 0.22 & 18.8 & 82 & 84 \\
\hline & T297 & & 0.009 & 0.21 & 17.6 & 77 & 84 \\
\hline & T298 & & 0.008 & 0.20 & 16.6 & 73 & 83 \\
\hline & T299 & & 0.010 & 0.23 & 19.5 & 86 & 83 \\
\hline & Т300 & & 0.010 & 0.23 & 19.5 & 84 & 85 \\
\hline
\end{tabular}


Table 5-2. 2008 TLD Results. (Sheet 5 of 14)

\begin{tabular}{|c|c|c|c|c|c|c|c|}
\hline \multicolumn{2}{|c|}{ Location } & \multirow{2}{*}{$\begin{array}{c}\text { Sample Period } \\
\text { 2nd Quarter '08 }\end{array}$} & \multirow{2}{*}{$\frac{\mathbf{m r e m} / \mathbf{h r}}{0.009}$} & \multirow{2}{*}{$\frac{\text { mrem/day }}{0.22}$} & \multirow{2}{*}{$\frac{\text { mrem/qtr }}{22.6}$} & \multirow{2}{*}{$\frac{\text { mrem/year }}{80}$} & \multirow{2}{*}{$\frac{\text { Days in Field }}{104}$} \\
\hline 200 East & T259 & & & & & & \\
\hline Area & $\mathrm{T} 260$ & & 0.009 & 0.22 & 23.3 & 82 & 104 \\
\hline & $\mathrm{T} 261$ & & 0.009 & 0.22 & 23.4 & 82 & 104 \\
\hline & $\mathrm{T} 262$ & & 0.010 & 0.24 & 24.6 & 86 & 104 \\
\hline & $\mathrm{T} 263$ & & 0.009 & 0.22 & 22.9 & 80 & 104 \\
\hline & $\mathrm{T} 264$ & & 0.012 & 0.29 & 29.9 & 105 & 104 \\
\hline & $\mathrm{T} 265$ & & 0.013 & 0.31 & 32.4 & 114 & 104 \\
\hline & $\mathrm{T} 266$ & & 0.010 & 0.24 & 25.3 & 89 & 104 \\
\hline & $\mathrm{T} 267$ & & 0.011 & 0.25 & 26.2 & 92 & 104 \\
\hline & $\mathrm{T} 268$ & & 0.013 & 0.30 & 31.1 & 109 & 104 \\
\hline & $\mathrm{T} 269$ & & 0.010 & 0.24 & 25.1 & 88 & 104 \\
\hline & $\mathrm{T} 270$ & & 0.013 & 0.32 & 33.3 & 117 & 104 \\
\hline & $\mathrm{T} 271$ & & 0.010 & 0.24 & 25.2 & 88 & 105 \\
\hline & $\mathrm{T} 272$ & & 0.012 & 0.28 & 28.8 & 101 & 104 \\
\hline & $\mathrm{T} 273$ & & 0.009 & 0.22 & 23.2 & 82 & 104 \\
\hline & $\mathrm{T} 274$ & & 0.009 & 0.22 & 23.3 & 82 & 104 \\
\hline & $\mathrm{T} 275$ & & 0.010 & 0.25 & 25.7 & 90 & 104 \\
\hline & $\mathrm{T} 276$ & & 0.010 & 0.23 & 23.6 & 83 & 104 \\
\hline & $\mathrm{T} 277$ & & 0.010 & 0.25 & 25.5 & 91 & 102 \\
\hline & $\mathrm{T} 278$ & & 0.009 & 0.22 & 23.0 & 81 & 103 \\
\hline & $\mathrm{T} 279$ & & 0.009 & 0.22 & 23.5 & 82 & 105 \\
\hline & $\mathrm{T} 280$ & & 0.009 & 0.22 & 23.4 & 82 & 104 \\
\hline & $\mathrm{T} 281$ & & 0.010 & 0.24 & 24.9 & 87 & 105 \\
\hline & $\mathrm{T} 282$ & & 0.009 & 0.22 & 23.0 & 81 & 104 \\
\hline & $\mathrm{T} 283$ & & 0.010 & 0.24 & 24.6 & 86 & 104 \\
\hline & $\mathrm{T} 284$ & & 0.012 & 0.30 & 31.0 & 109 & 104 \\
\hline & $\mathrm{T} 285$ & & 0.021 & 0.51 & 52.7 & 185 & 104 \\
\hline & T286 & & 0.015 & 0.37 & 38.2 & 134 & 104 \\
\hline & $\mathrm{T} 287$ & & 0.014 & 0.33 & 34.0 & 119 & 104 \\
\hline & $\mathrm{T} 288$ & & 0.023 & 0.56 & 58.0 & 204 & 104 \\
\hline & $\mathrm{T} 289$ & & 0.018 & 0.43 & 44.8 & 157 & 104 \\
\hline & $\mathrm{T} 290$ & & 0.015 & 0.36 & 37.2 & 130 & 104 \\
\hline & $\mathrm{T} 291$ & & 0.015 & 0.35 & 36.3 & 128 & 104 \\
\hline & T292 & & 0.034 & 0.82 & 86.1 & 299 & 105 \\
\hline & T293 & & 0.012 & 0.29 & 30.4 & 107 & 104 \\
\hline & T294 & & 0.018 & 0.44 & 46.0 & 160 & 105 \\
\hline & T295 & & 0.009 & 0.22 & 22.7 & 80 & 104 \\
\hline & T296 & & 0.012 & 0.28 & 29.1 & 102 & 104 \\
\hline & T297 & & 0.009 & 0.21 & 21.9 & 77 & 104 \\
\hline & T298 & & 0.009 & 0.23 & 23.4 & 82 & 104 \\
\hline & T299 & & 0.010 & 0.23 & 24.2 & 85 & 104 \\
\hline & T300 & & 0.010 & 0.25 & 25.6 & 91 & 103 \\
\hline
\end{tabular}


Table 5-2. 2008 TLD Results. (Sheet 6 of 14)

\begin{tabular}{|c|c|c|c|c|c|c|c|}
\hline \multicolumn{2}{|c|}{ Location } & \multirow{2}{*}{$\begin{array}{c}\text { Sample Period } \\
\text { 3rd Quarter '08 }\end{array}$} & \multirow{2}{*}{$\frac{\text { mrem/hr }}{0.009}$} & \multirow{2}{*}{$\frac{\text { mrem/day }}{0.22}$} & \multirow{2}{*}{$\frac{\text { mrem/qtr }}{18.8}$} & \multirow{2}{*}{$\begin{array}{c}\text { mrem/year } \\
81\end{array}$} & \multirow{2}{*}{$\frac{\text { Days in Field }}{85}$} \\
\hline 200 East & T259 & & & & & & \\
\hline Area & $\mathrm{T} 260$ & & 0.009 & 0.22 & 18.6 & 80 & 85 \\
\hline & $\mathrm{T} 261$ & & 0.009 & 0.21 & 22.0 & 76 & 105 \\
\hline & $\mathrm{T} 262$ & & 0.008 & 0.19 & 16.5 & 71 & 85 \\
\hline & $\mathrm{T} 263$ & & 0.009 & 0.21 & 17.6 & 76 & 85 \\
\hline & $\mathrm{T} 264$ & & 0.012 & 0.29 & 24.7 & 106 & 85 \\
\hline & $\mathrm{T} 265$ & & 0.013 & 0.31 & 26.2 & 113 & 85 \\
\hline & $\mathrm{T} 266$ & & 0.009 & 0.22 & 18.8 & 81 & 85 \\
\hline & $\mathrm{T} 267$ & & 0.010 & 0.23 & 19.9 & 86 & 85 \\
\hline & $\mathrm{T} 268$ & & 0.013 & 0.30 & 25.5 & 110 & 85 \\
\hline & $\mathrm{T} 269$ & & 0.010 & 0.25 & 21.0 & 91 & 84 \\
\hline & $\mathrm{T} 270$ & & 0.014 & 0.34 & 29.1 & 125 & 85 \\
\hline & $\mathrm{T} 271$ & & 0.010 & 0.23 & 19.2 & 84 & 84 \\
\hline & $\mathrm{T} 272$ & & 0.012 & 0.29 & 24.2 & 105 & 84 \\
\hline & $\mathrm{T} 273$ & & 0.009 & 0.21 & 18.1 & 78 & 85 \\
\hline & $\mathrm{T} 274$ & & 0.009 & 0.22 & 18.7 & 80 & 85 \\
\hline & $\mathrm{T} 275$ & & 0.009 & 0.21 & 18.2 & 78 & 85 \\
\hline & $\mathrm{T} 276$ & & 0.008 & 0.20 & 17.1 & 73 & 85 \\
\hline & $\mathrm{T} 277$ & & 0.010 & 0.23 & 19.6 & 84 & 85 \\
\hline & $\mathrm{T} 278$ & & 0.009 & 0.22 & 18.8 & 81 & 85 \\
\hline & $\mathrm{T} 279$ & & 0.009 & 0.22 & 19.1 & 82 & 85 \\
\hline & $\mathrm{T} 280$ & & 0.009 & 0.22 & 18.5 & 80 & 85 \\
\hline & $\mathrm{T} 281$ & & 0.009 & 0.22 & 18.9 & 81 & 85 \\
\hline & $\mathrm{T} 282$ & & 0.009 & 0.21 & 17.2 & 75 & 84 \\
\hline & $\mathrm{T} 283$ & & 0.009 & 0.22 & 18.9 & 81 & 85 \\
\hline & $\mathrm{T} 284$ & & 0.013 & 0.30 & 25.6 & 111 & 84 \\
\hline & $\mathrm{T} 285$ & & 0.022 & 0.52 & 43.8 & 190 & 84 \\
\hline & T286 & & 0.015 & 0.35 & 29.8 & 129 & 84 \\
\hline & $\mathrm{T} 287$ & & 0.013 & 0.30 & 25.2 & 109 & 84 \\
\hline & $\mathrm{T} 288$ & & 0.024 & 0.56 & 47.4 & 206 & 84 \\
\hline & $\mathrm{T} 289$ & & 0.018 & 0.43 & 35.8 & 155 & 84 \\
\hline & $\mathrm{T} 290$ & & 0.012 & 0.30 & 25.8 & 108 & 87 \\
\hline & $\mathrm{T} 291$ & & 0.014 & 0.34 & 28.5 & 124 & 84 \\
\hline & T292 & & 0.032 & 0.76 & 63.2 & 278 & 83 \\
\hline & T293 & & 0.012 & 0.29 & 24.6 & 107 & 84 \\
\hline & T294 & & 0.019 & 0.45 & 38.2 & 166 & 84 \\
\hline & T295 & & 0.009 & 0.23 & 19.3 & 83 & 85 \\
\hline & T296 & & 0.011 & 0.26 & 22.0 & 96 & 84 \\
\hline & T297 & & 0.009 & 0.23 & 18.9 & 82 & 84 \\
\hline & T298 & & 0.009 & 0.21 & 17.5 & 75 & 85 \\
\hline & T299 & & 0.009 & 0.22 & 18.8 & 81 & 85 \\
\hline & T300 & & 0.011 & 0.26 & 22.0 & 96 & 84 \\
\hline
\end{tabular}


Table 5-2. 2008 TLD Results. (Sheet 7 of 14)

\begin{tabular}{|c|c|c|c|c|c|c|c|}
\hline \multicolumn{2}{|c|}{ Location } & \multirow{2}{*}{$\begin{array}{l}\text { Sample Period } \\
\text { 4th Quarter '08 }\end{array}$} & \multirow{2}{*}{$\frac{\mathbf{m r e m} / \mathbf{h r}}{0.009}$} & \multirow{2}{*}{$\frac{\text { mrem/day }}{0.22}$} & \multirow{2}{*}{$\frac{\text { mrem/qtr }}{20.4}$} & \multirow{2}{*}{$\frac{\text { mrem/year }}{80}$} & \multirow{2}{*}{$\frac{\text { Days in Field }}{93}$} \\
\hline 200 East & $\mathrm{T} 259$ & & & & & & \\
\hline Area & $\mathrm{T} 260$ & & 0.009 & 0.21 & 20.0 & 78 & 93 \\
\hline & T261 & & 0.009 & 0.22 & 19.9 & 80 & 91 \\
\hline & $\mathrm{T} 262$ & & 0.009 & 0.22 & 19.9 & 80 & 91 \\
\hline & $\mathrm{T} 263$ & & 0.009 & 0.22 & 19.8 & 79 & 91 \\
\hline & $\mathrm{T} 264$ & & 0.011 & 0.27 & 25.1 & 99 & 93 \\
\hline & $\mathrm{T} 265$ & & 0.013 & 0.31 & 28.8 & 113 & 93 \\
\hline & T266 & & 0.009 & 0.22 & 20.1 & 79 & 93 \\
\hline & T267 & & 0.010 & 0.23 & 21.2 & 83 & 93 \\
\hline & $\mathrm{T} 268$ & & 0.012 & 0.29 & 27.3 & 107 & 93 \\
\hline & T269 & & 0.010 & 0.24 & 22.6 & 89 & 93 \\
\hline & $\mathrm{T} 270$ & & 0.014 & 0.33 & 31.0 & 122 & 93 \\
\hline & $\mathrm{T} 271$ & & 0.010 & 0.24 & 22.0 & 86 & 93 \\
\hline & $\mathrm{T} 272$ & & 0.013 & 0.31 & 27.5 & 111 & 90 \\
\hline & $\mathrm{T} 273$ & & 0.009 & 0.20 & 19.0 & 75 & 93 \\
\hline & $\mathrm{T} 274$ & & 0.010 & 0.24 & 22.2 & 87 & 93 \\
\hline & $\mathrm{T} 275$ & & 0.010 & 0.24 & 22.2 & 87 & 93 \\
\hline & $\mathrm{T} 276$ & & 0.009 & 0.22 & 20.1 & 79 & 93 \\
\hline & $\mathrm{T} 277$ & & 0.011 & 0.25 & 23.4 & 92 & 93 \\
\hline & $\mathrm{T} 278$ & & 0.009 & 0.22 & 20.3 & 82 & 91 \\
\hline & $\mathrm{T} 279$ & & 0.009 & 0.21 & 19.3 & 77 & 91 \\
\hline & $\mathrm{T} 280$ & & 0.009 & 0.21 & 19.2 & 77 & 91 \\
\hline & $\mathrm{T} 281$ & & 0.010 & 0.24 & 21.9 & 88 & 91 \\
\hline & $\mathrm{T} 282$ & & 0.009 & 0.22 & 20.5 & 81 & 92 \\
\hline & $\mathrm{T} 283$ & & 0.010 & 0.23 & 20.8 & 83 & 91 \\
\hline & $\mathrm{T} 284$ & & 0.012 & 0.28 & 26.1 & 103 & 92 \\
\hline & $\mathrm{T} 285$ & & 0.015 & 0.35 & 32.6 & 129 & 92 \\
\hline & T286 & & 0.016 & 0.38 & 35.1 & 138 & 93 \\
\hline & $\mathrm{T} 287$ & & 0.013 & 0.32 & 29.0 & 115 & 92 \\
\hline & T288 & & 0.021 & 0.51 & 46.8 & 186 & 92 \\
\hline & T289 & & 0.017 & 0.41 & 37.4 & 148 & 92 \\
\hline & T290 & & 0.013 & 0.31 & 27.6 & 113 & 89 \\
\hline & T291 & & 0.013 & 0.31 & 28.9 & 115 & 92 \\
\hline & T292 & & 0.033 & 0.78 & 71.9 & 285 & 92 \\
\hline & T293 & & 0.012 & 0.29 & 26.8 & 106 & 92 \\
\hline & T294 & & 0.019 & 0.46 & 41.5 & 167 & 91 \\
\hline & T295 & & 0.009 & 0.22 & 20.2 & 81 & 91 \\
\hline & T296 & & 0.011 & 0.26 & 24.2 & 96 & 92 \\
\hline & T297 & & 0.010 & 0.23 & 21.2 & 84 & 92 \\
\hline & T298 & & 0.008 & 0.20 & 18.7 & 73 & 93 \\
\hline & T299 & & 0.009 & 0.22 & 20.7 & 81 & 93 \\
\hline & Т300 & & 0.011 & 0.25 & 23.2 & 92 & 92 \\
\hline
\end{tabular}


Table 5-2. 2008 TLD Results. (Sheet 8 of 14)

200 East Area, Annual Averages \pm 2 Standard Deviations

\begin{tabular}{|c|c|c|c|c|}
\hline Location & mrem/hr & mrem/day & mrem/qtr & mrem/year \\
\hline $\mathrm{T} 259$ & $0.009 \pm 0.001$ & $0.22 \pm 0.01$ & $19.7 \pm 1.2$ & $79 \pm 5$ \\
\hline $\mathrm{T} 260$ & $0.009 \pm 0.001$ & $0.22 \pm 0.02$ & $19.7 \pm 1.5$ & $79 \pm 6$ \\
\hline $\mathrm{T} 261$ & $0.009 \pm 0.001$ & $0.22 \pm 0.01$ & $19.8 \pm 1.2$ & $79 \pm 5$ \\
\hline $\mathrm{T} 262$ & $0.009 \pm 0.002$ & $0.21 \pm 0.04$ & $19.5 \pm 3.5$ & $78 \pm 14$ \\
\hline $\mathrm{T} 263$ & $0.009 \pm 0.001$ & $0.21 \pm 0.01$ & $19.5 \pm 1.1$ & $78 \pm 4$ \\
\hline $\mathrm{T} 264$ & $0.012 \pm 0.001$ & $0.28 \pm 0.03$ & $25.4 \pm 2.3$ & $102 \pm 9$ \\
\hline $\mathrm{T} 265$ & $0.013 \pm 0.001$ & $0.30 \pm 0.02$ & $27.8 \pm 2.2$ & $111 \pm 9$ \\
\hline $\mathrm{T} 266$ & $0.009 \pm 0.001$ & $0.22 \pm 0.03$ & $20.4 \pm 2.5$ & $82 \pm 10$ \\
\hline $\mathrm{T} 267$ & $0.010 \pm 0.001$ & $0.23 \pm 0.03$ & $21.2 \pm 3.0$ & $85 \pm 12$ \\
\hline $\mathrm{T} 268$ & $0.012 \pm 0.000$ & $0.30 \pm 0.01$ & $27.1 \pm 0.6$ & $108 \pm 2$ \\
\hline $\mathrm{T} 269$ & $0.010 \pm 0.001$ & $0.24 \pm 0.02$ & $22.0 \pm 1.6$ & $88 \pm 6$ \\
\hline $\mathrm{T} 270$ & $0.014 \pm 0.001$ & $0.33 \pm 0.02$ & $30.1 \pm 1.8$ & $120 \pm 7$ \\
\hline $\mathrm{T} 271$ & $0.010 \pm 0.001$ & $0.23 \pm 0.02$ & $21.2 \pm 1.6$ & $85 \pm 6$ \\
\hline $\mathrm{T} 272$ & $0.012 \pm 0.001$ & $0.29 \pm 0.03$ & $26.0 \pm 2.7$ & $104 \pm 11$ \\
\hline $\mathrm{T} 273$ & $0.009 \pm 0.001$ & $0.21 \pm 0.02$ & $19.5 \pm 1.4$ & $78 \pm 6$ \\
\hline $\mathrm{T} 274$ & $0.009 \pm 0.001$ & $0.23 \pm 0.02$ & $20.7 \pm 1.5$ & $83 \pm 6$ \\
\hline $\mathrm{T} 275$ & $0.010 \pm 0.001$ & $0.23 \pm 0.03$ & $21.1 \pm 2.6$ & $85 \pm 11$ \\
\hline $\mathrm{T} 276$ & $0.009 \pm 0.001$ & $0.21 \pm 0.02$ & $19.5 \pm 2.1$ & $78 \pm 9$ \\
\hline $\mathrm{T} 277$ & $0.010 \pm 0.001$ & $0.24 \pm 0.03$ & $21.8 \pm 2.7$ & $87 \pm 11$ \\
\hline $\mathrm{T} 278$ & $0.009 \pm 0.001$ & $0.22 \pm 0.01$ & $20.0 \pm 1.2$ & $80 \pm 5$ \\
\hline $\mathrm{T} 279$ & $0.009 \pm 0.000$ & $0.22 \pm 0.01$ & $20.1 \pm 1.1$ & $80 \pm 4$ \\
\hline $\mathrm{T} 280$ & $0.009 \pm 0.001$ & $0.22 \pm 0.01$ & $19.8 \pm 1.2$ & $79 \pm 5$ \\
\hline $\mathrm{T} 281$ & $0.010 \pm 0.001$ & $0.23 \pm 0.02$ & $21.2 \pm 1.6$ & $85 \pm 6$ \\
\hline $\mathrm{T} 282$ & $0.009 \pm 0.001$ & $0.22 \pm 0.02$ & $19.8 \pm 1.4$ & $79 \pm 6$ \\
\hline $\mathrm{T} 283$ & $0.009 \pm 0.001$ & $0.23 \pm 0.02$ & $20.6 \pm 1.8$ & $82 \pm 7$ \\
\hline $\mathrm{T} 284$ & $0.012 \pm 0.001$ & $0.29 \pm 0.03$ & $26.5 \pm 2.4$ & $106 \pm 10$ \\
\hline $\mathrm{T} 285$ & $0.020 \pm 0.007$ & $0.47 \pm 0.16$ & $43.1 \pm 14.5$ & $172 \pm 58$ \\
\hline $\mathrm{T} 286$ & $0.015 \pm 0.001$ & $0.36 \pm 0.02$ & $33.2 \pm 2.1$ & $133 \pm 8$ \\
\hline $\mathrm{T} 287$ & $0.013 \pm 0.001$ & $0.32 \pm 0.03$ & $29.1 \pm 2.7$ & $116 \pm 11$ \\
\hline $\mathrm{T} 288$ & $0.023 \pm 0.002$ & $0.54 \pm 0.05$ & $49.5 \pm 4.6$ & $198 \pm 18$ \\
\hline $\mathrm{T} 289$ & $0.018 \pm 0.001$ & $0.42 \pm 0.02$ & $38.6 \pm 2.0$ & $154 \pm 8$ \\
\hline $\mathrm{T} 290$ & $0.013 \pm 0.002$ & $0.32 \pm 0.06$ & $28.9 \pm 5.4$ & $115 \pm 21$ \\
\hline $\mathrm{T} 291$ & $0.014 \pm 0.001$ & $0.33 \pm 0.03$ & $30.5 \pm 2.8$ & $122 \pm 11$ \\
\hline T292 & $0.032 \pm 0.004$ & $0.77 \pm 0.10$ & $70.1 \pm 9.2$ & $280 \pm 37$ \\
\hline $\mathrm{T} 293$ & $0.012 \pm 0.001$ & $0.29 \pm 0.01$ & $26.4 \pm 1.1$ & $106 \pm 4$ \\
\hline $\mathrm{T} 294$ & $0.018 \pm 0.002$ & $0.44 \pm 0.04$ & $40.3 \pm 3.4$ & $161 \pm 14$ \\
\hline $\mathrm{T} 295$ & $0.009 \pm 0.000$ & $0.22 \pm 0.01$ & $20.4 \pm 1.0$ & $82 \pm 4$ \\
\hline T296 & $0.011 \pm 0.002$ & $0.26 \pm 0.05$ & $23.6 \pm 4.4$ & $94 \pm 17$ \\
\hline $\mathrm{T} 297$ & $0.009 \pm 0.001$ & $0.22 \pm 0.02$ & $20.0 \pm 1.9$ & $80 \pm 7$ \\
\hline T298 & $0.009 \pm 0.001$ & $0.21 \pm 0.02$ & $19.0 \pm 2.2$ & $76 \pm 9$ \\
\hline T299 & $0.009 \pm 0.001$ & $0.23 \pm 0.01$ & $20.8 \pm 1.2$ & $83 \pm 5$ \\
\hline Т300 & $0.010 \pm 0.001$ & $0.25 \pm 0.03$ & $22.7 \pm 2.4$ & $91 \pm 10$ \\
\hline
\end{tabular}


Table 5-2. 2008 TLD Results. (Sheet 9 of 14)

\begin{tabular}{|c|c|c|c|c|c|c|c|}
\hline \multicolumn{2}{|c|}{ Location } & \multirow{2}{*}{$\begin{array}{c}\text { Sample Period } \\
\text { 1st Quarter '08 }\end{array}$} & \multirow{2}{*}{$\frac{\mathrm{mrem} / \mathbf{h r}}{0.010}$} & \multirow{2}{*}{$\frac{\text { mrem/day }}{0.23}$} & \multirow{2}{*}{$\frac{\text { mrem/qtr }}{19.3}$} & \multirow{2}{*}{$\frac{\text { mrem/year }}{84}$} & \multirow{2}{*}{$\frac{\text { Days in Field }}{84}$} \\
\hline 200 West & T302 & & & & & & \\
\hline Area & Т303 & & 0.017 & 0.40 & 33.5 & 146 & 84 \\
\hline & Т304 & & 0.013 & 0.32 & 26.9 & 117 & 84 \\
\hline & Т305 & & 0.009 & 0.21 & 17.7 & 77 & 84 \\
\hline & T306 & & 0.012 & 0.30 & 24.8 & 108 & 84 \\
\hline & Т307 & & 0.010 & 0.25 & 20.8 & 91 & 84 \\
\hline & Т308 & & 0.010 & 0.23 & 19.6 & 85 & 84 \\
\hline & Т309 & & 0.009 & 0.22 & 18.5 & 80 & 84 \\
\hline & T310 & & 0.010 & 0.25 & 20.8 & 90 & 84 \\
\hline & T311 & & 0.008 & 0.19 & 16.0 & 70 & 84 \\
\hline & T312 & & 0.021 & 0.51 & 42.5 & 185 & 84 \\
\hline & $\mathrm{T} 313$ & & 0.017 & 0.42 & 35.0 & 152 & 84 \\
\hline & T314 & & 0.009 & 0.20 & 17.1 & 75 & 84 \\
\hline & T315 & & 0.009 & 0.22 & 18.2 & 79 & 84 \\
\hline & T316 & & 0.013 & 0.30 & 25.4 & 110 & 84 \\
\hline & T317 & & 0.009 & 0.22 & 18.5 & 80 & 84 \\
\hline & T318 & & 0.009 & 0.21 & 17.5 & 77 & 83 \\
\hline & T319 & & 0.009 & 0.22 & 18.0 & 79 & 83 \\
\hline & T320 & & 0.012 & 0.28 & 23.6 & 103 & 84 \\
\hline & $\mathrm{T} 321$ & & 0.011 & 0.25 & 21.3 & 93 & 84 \\
\hline & T322 & & 0.008 & 0.20 & 16.6 & 73 & 83 \\
\hline & T323 & & 0.009 & 0.22 & 18.1 & 79 & 84 \\
\hline & T324 & & 0.027 & 0.65 & 54.2 & 236 & 84 \\
\hline & T325 & & 0.012 & 0.30 & 24.8 & 108 & 84 \\
\hline & Т302 & 2nd Quarter '08 & 0.010 & 0.24 & 25.4 & 89 & 104 \\
\hline & Т303 & & 0.017 & 0.40 & 41.2 & 145 & 104 \\
\hline & Т304 & & 0.013 & 0.32 & 33.4 & 117 & 104 \\
\hline & Т305 & & 0.010 & 0.24 & 24.8 & 87 & 104 \\
\hline & Т306 & & 0.011 & 0.27 & 28.2 & 99 & 104 \\
\hline & Т307 & & 0.012 & 0.28 & 29.2 & 102 & 104 \\
\hline & Т308 & & 0.011 & 0.27 & 28.3 & 99 & 104 \\
\hline & Т309 & & 0.010 & 0.23 & 23.8 & 84 & 104 \\
\hline & T310 & & 0.012 & 0.28 & 29.2 & 103 & 104 \\
\hline & T311 & & 0.010 & 0.24 & 24.5 & 86 & 104 \\
\hline & T312 & & 0.014 & 0.33 & 34.3 & 121 & 104 \\
\hline & T313 & & 0.018 & 0.43 & 45.0 & 158 & 104 \\
\hline & T314 & & 0.009 & 0.21 & 22.2 & 78 & 104 \\
\hline & T315 & & 0.010 & 0.24 & 25.2 & 88 & 104 \\
\hline & T316 & & 0.013 & 0.32 & 32.8 & 115 & 104 \\
\hline & T317 & & 0.010 & 0.24 & 24.8 & 87 & 104 \\
\hline & T318 & & 0.009 & 0.22 & 23.2 & 81 & 105 \\
\hline & Т319 & & 0.010 & 0.23 & 24.3 & 84 & 105 \\
\hline & Т320 & & 0.014 & 0.33 & 34.4 & 121 & 104 \\
\hline & T321 & & 0.012 & 0.29 & 27.7 & 108 & 94 \\
\hline & T322 & & 0.009 & 0.21 & 22.3 & 78 & 104 \\
\hline & T323 & & 0.011 & 0.26 & 26.8 & 94 & 104 \\
\hline & T324 & & 0.038 & 0.91 & 94.3 & 331 & 104 \\
\hline & T325 & & 0.013 & 0.31 & 32.4 & 114 & 104 \\
\hline
\end{tabular}


Table 5-2. 2008 TLD Results. (Sheet 10 of 14)

\begin{tabular}{|c|c|c|c|c|c|c|c|}
\hline \multicolumn{2}{|c|}{ Location } & \multirow{2}{*}{$\frac{\text { Sample Period }}{\text { 3rd Quarter '08 }}$} & \multirow{2}{*}{$\begin{array}{c}\text { mrem/hr } \\
0.009\end{array}$} & \multirow{2}{*}{$\frac{\mathbf{m r e m} / \mathbf{d a y}}{0.22}$} & \multirow{2}{*}{$\frac{\text { mrem/qtr }}{18.8}$} & \multirow{2}{*}{$\frac{\text { mrem/year }}{81}$} & \multirow{2}{*}{$\frac{\text { Days in Field }}{85}$} \\
\hline 200 West & T302 & & & & & & \\
\hline Area & T303 & & 0.014 & 0.33 & 28.0 & 120 & 85 \\
\hline & T304 & & 0.013 & 0.32 & 26.8 & 115 & 85 \\
\hline & T305 & & 0.009 & 0.23 & 19.1 & 82 & 85 \\
\hline & T306 & & 0.011 & 0.26 & 22.2 & 95 & 85 \\
\hline & T307 & & 0.010 & 0.23 & 19.7 & 84 & 85 \\
\hline & $\mathrm{T} 308$ & & 0.010 & 0.25 & 20.8 & 91 & 84 \\
\hline & T309 & & 0.010 & 0.23 & 19.3 & 84 & 84 \\
\hline & $\mathrm{T} 310$ & & 0.011 & 0.26 & 22.2 & 96 & 84 \\
\hline & T311 & & 0.009 & 0.22 & 18.2 & 79 & 84 \\
\hline & T312 & & 0.015 & 0.35 & 29.4 & 128 & 84 \\
\hline & $\mathrm{T} 313$ & & 0.017 & 0.40 & 33.5 & 145 & 84 \\
\hline & T314 & & 0.009 & 0.21 & 17.3 & 75 & 84 \\
\hline & $\mathrm{T} 315$ & \multicolumn{6}{|c|}{ TLD not recovered } \\
\hline & T316 & & 0.012 & 0.29 & 24.1 & 105 & 84 \\
\hline & T317 & & 0.010 & 0.23 & 19.1 & 83 & 84 \\
\hline & T318 & & 0.009 & 0.22 & 18.6 & 81 & 84 \\
\hline & T319 & & 0.010 & 0.23 & 19.4 & 84 & 84 \\
\hline & T320 & & 0.013 & 0.31 & 25.7 & 112 & 84 \\
\hline & T321 & & 0.011 & 0.27 & 25.8 & 99 & 95 \\
\hline & $\mathrm{T} 322$ & & 0.007 & 0.16 & 13.4 & 57 & 85 \\
\hline & T323 & & 0.011 & 0.25 & 21.1 & 92 & 84 \\
\hline & T324 & & 0.033 & 0.78 & 65.9 & 286 & 84 \\
\hline & T325 & & 0.014 & 0.34 & 28.1 & 122 & 84 \\
\hline & T302 & \multirow[t]{24}{*}{ 4th Quarter '08 } & 0.010 & 0.23 & 20.8 & 84 & 90 \\
\hline & T303 & & 0.011 & 0.27 & 24.6 & 100 & 90 \\
\hline & T304 & & 0.015 & 0.35 & 31.6 & 128 & 90 \\
\hline & T305 & & 0.010 & 0.23 & 20.8 & 83 & 91 \\
\hline & T306 & & 0.011 & 0.27 & 24.3 & 99 & 90 \\
\hline & $\mathrm{T} 307$ & & 0.011 & 0.26 & 23.5 & 94 & 91 \\
\hline & T308 & & 0.011 & 0.25 & 23.2 & 92 & 92 \\
\hline & T309 & & 0.009 & 0.22 & 20.5 & 82 & 92 \\
\hline & T310 & & 0.011 & 0.26 & 24.3 & 96 & 92 \\
\hline & T311 & & 0.010 & 0.23 & 20.9 & 83 & 92 \\
\hline & $\mathrm{T} 312$ & & 0.013 & 0.32 & 29.4 & 117 & 92 \\
\hline & T313 & & 0.018 & 0.42 & 38.7 & 153 & 92 \\
\hline & T314 & & 0.009 & 0.23 & 20.6 & 83 & 91 \\
\hline & T315 & & 0.010 & 0.24 & 21.2 & 86 & 90 \\
\hline & T316 & & 0.012 & 0.30 & 27.5 & 109 & 92 \\
\hline & $\mathrm{T} 317$ & & 0.010 & 0.23 & 20.8 & 84 & 91 \\
\hline & T318 & & 0.009 & 0.20 & 18.6 & 75 & 91 \\
\hline & T319 & & 0.010 & 0.23 & 21.0 & 84 & 91 \\
\hline & T320 & & 0.013 & 0.31 & 27.9 & 112 & 91 \\
\hline & T321 & & 0.011 & 0.26 & 23.9 & 97 & 90 \\
\hline & T322 & & 0.009 & 0.22 & 19.7 & 79 & 91 \\
\hline & T323 & & 0.012 & 0.28 & 25.4 & 102 & 91 \\
\hline & T324 & & 0.020 & 0.48 & 43.3 & 174 & 91 \\
\hline & T325 & & 0.015 & 0.36 & 32.6 & 131 & 91 \\
\hline
\end{tabular}


Table 5-2. 2008 TLD Results. (Sheet 11 of 14)

\begin{tabular}{ccccc} 
Location & $\begin{array}{c}\text { 200 West Area, Annual Averages } \pm 2 \text { Standard Deviations } \\
\text { mrem/hr }\end{array}$ & mrem/day & mrem/qtr & mrem/year \\
\hline T302 & $0.010 \pm 0.001$ & $0.23 \pm 0.02$ & $21.2 \pm 1.7$ & $85 \pm 7$ \\
T303 & $0.015 \pm 0.005$ & $0.35 \pm 0.12$ & $32.0 \pm 10.9$ & $128 \pm 44$ \\
T304 & $0.014 \pm 0.001$ & $0.33 \pm 0.03$ & $29.8 \pm 2.9$ & $119 \pm 12$ \\
T305 & $0.009 \pm 0.001$ & $0.23 \pm 0.02$ & $20.7 \pm 2.1$ & $83 \pm 8$ \\
T306 & $0.011 \pm 0.001$ & $0.27 \pm 0.03$ & $25.0 \pm 2.7$ & $100 \pm 11$ \\
T307 & $0.011 \pm 0.002$ & $0.26 \pm 0.04$ & $23.4 \pm 3.8$ & $93 \pm 15$ \\
T308 & $0.011 \pm 0.001$ & $0.25 \pm 0.03$ & $23.0 \pm 2.9$ & $92 \pm 12$ \\
T309 & $0.009 \pm 0.000$ & $0.23 \pm 0.01$ & $20.6 \pm 0.9$ & $82 \pm 3$ \\
T310 & $0.011 \pm 0.001$ & $0.27 \pm 0.03$ & $24.2 \pm 2.5$ & $97 \pm 10$ \\
T311 & $0.009 \pm 0.002$ & $0.22 \pm 0.04$ & $20.0 \pm 3.6$ & $80 \pm 14$ \\
T312 & $0.016 \pm 0.007$ & $0.37 \pm 0.17$ & $34.0 \pm 16.0$ & $136 \pm 64$ \\
T313 & $0.017 \pm 0.001$ & $0.42 \pm 0.03$ & $38.1 \pm 2.6$ & $153 \pm 10$ \\
T314 & $0.009 \pm 0.001$ & $0.21 \pm 0.02$ & $19.4 \pm 1.9$ & $78 \pm 7$ \\
T315 & $0.010 \pm 0.001$ & $0.23 \pm 0.03$ & $21.2 \pm 2.4$ & $85 \pm 9$ \\
T316 & $0.013 \pm 0.001$ & $0.30 \pm 0.02$ & $27.5 \pm 2.1$ & $110 \pm 8$ \\
T317 & $0.010 \pm 0.001$ & $0.23 \pm 0.01$ & $20.9 \pm 1.3$ & $84 \pm 5$ \\
T318 & $0.009 \pm 0.001$ & $0.21 \pm 0.02$ & $19.6 \pm 1.5$ & $78 \pm 6$ \\
T319 & $0.009 \pm 0.001$ & $0.23 \pm 0.01$ & $20.8 \pm 1.3$ & $83 \pm 5$ \\
T320 & $0.013 \pm 0.002$ & $0.31 \pm 0.04$ & $28.1 \pm 3.7$ & $112 \pm 15$ \\
T321 & $0.011 \pm 0.001$ & $0.27 \pm 0.03$ & $24.8 \pm 3.2$ & $99 \pm 13$ \\
T322 & $0.008 \pm 0.002$ & $0.20 \pm 0.05$ & $18.1 \pm 5.0$ & $72 \pm 20$ \\
T323 & $0.010 \pm 0.002$ & $0.25 \pm 0.05$ & $23.0 \pm 4.8$ & $92 \pm 19$ \\
T324 & $0.030 \pm 0.015$ & $0.71 \pm 0.37$ & $64.8 \pm 33.9$ & $259 \pm 135$ \\
T325 & $0.014 \pm 0.002$ & $0.33 \pm 0.05$ & $29.7 \pm 5.0$ & $119 \pm 20$
\end{tabular}

\begin{tabular}{cccccccc}
\multicolumn{2}{c}{ Location } & Sample Period & $\mathbf{m r e m} / \mathbf{h r}$ & $\mathbf{m r e m} / \mathbf{d a y}$ & $\mathbf{m r e m} / \mathbf{q t r}$ & $\mathbf{m r e m} / \mathbf{y e a r}$ & Days in Field \\
\hline 212-R & T301 & 1st Quarter '08 & 0.186 & 4.47 & 380.0 & 1633 & 85 \\
(200 North & T301 & 2nd Quarter '08 & 0.189 & 4.53 & 466.3 & 1652 & 103 \\
Area) & T301 & 3rd Quarter '08 & 0.190 & 4.56 & 387.3 & 1663 & 85 \\
& T301 & 4th Quarter '08 & 0.187 & 4.50 & 404.8 & 1641 & 90
\end{tabular}

212-R, Annual Averages \pm 2 Standard Deviations

\begin{tabular}{ccccc} 
& $\mathbf{m r e m} / \mathbf{h r}$ & $\mathbf{m r e m} / \mathbf{d a y}$ & $\mathbf{m r e m} / \mathbf{q t r}$ & $\mathbf{m r e m} / \mathbf{y e a r}$ \\
\hline $0.188 \pm 0.003$ & $4.51 \pm 0.07$ & $411.9 \pm 6.6$ & $1647 \pm 26$
\end{tabular}

Location Sample Period

mrem/hr

mrem/day mrem/qtr mrem/year Days in Field

\begin{tabular}{ll}
\hline 300 Area & T332 \\
T333 \\
T334 \\
T335 \\
T336 \\
T337 \\
T338 \\
T339 \\
\\
T332 \\
T333 \\
T334 \\
T335 \\
T336 \\
T337 \\
T338 \\
T339
\end{tabular}

1st Quarter '08

0.009

0.009

0.009

0.010

0.009

0.009

0.012

0.010

2nd Quarter '08

0.009

0.010

0.009

0.009

0.009

0.009

0.011

0.010

$\begin{array}{ll}0.22 & 22.8 \\ 0.22 & 23.4 \\ 0.21 & 21.7 \\ 0.23 & 24.4 \\ 0.21 & 21.9 \\ 0.21 & 22.5 \\ 0.28 & 29.7 \\ 0.23 & 24.5 \\ & \\ 0.21 & 18.1 \\ 0.24 & 20.6 \\ 0.21 & 17.7 \\ 0.22 & 19.0 \\ 0.21 & 17.8 \\ 0.20 & 17.4 \\ 0.27 & 23.0 \\ 0.23 & 19.9\end{array}$

79
81
76
85
76
78
103
85
78
88
76
82
76
75
99
86

105

105

105

105

105

105

105

105

85

85

85

85

85

85

85

85 
Table 5-2. 2008 TLD Results. (Sheet 12 of 14)

\begin{tabular}{rccccccc}
\multicolumn{2}{c}{ Location } & Sample Period & mrem/hr & mrem/day & mrem/qtr & mrem/year & Days in Field \\
\hline 300 Area & T332 & 3rd Quarter '08 & 0.009 & 0.22 & 20.1 & 80 & 92 \\
& T333 & & 0.009 & 0.21 & 19.7 & 78 & 92 \\
T334 & & 0.008 & 0.20 & 18.5 & 74 & 92 \\
T335 & & 0.009 & 0.22 & 20.3 & 81 & 92 \\
T336 & & 0.009 & 0.21 & 19.6 & 78 & 92 \\
T337 & & 0.012 & 0.29 & 26.2 & 104 & 92 \\
T338 & & 0.009 & 0.22 & 20.2 & 80 & 85 \\
T339 & & & & & 81 & 85 \\
& & 0.009 & 0.22 & 18.9 & 85 & 85 \\
T332 & 4th Quarter '08 & 0.010 & 0.23 & 19.5 & 84 & 85 \\
T333 & & 0.010 & 0.23 & 19.4 & 83 & 85 \\
T334 & & 0.010 & 0.23 & 19.4 & 83 & 85
\end{tabular}

300 Area, Annual Averages \pm 2 Standard Deviations

T332

T333

T334

T335

T336

T337

T338

T339

\begin{tabular}{cccc}
$\mathbf{m r e m} / \mathbf{h r}$ & $\mathbf{m r e m} / \mathbf{d a y}$ & $\mathbf{m r e m} / \mathbf{q t r}$ & $\mathbf{m r e m} / \mathbf{y e a r}$ \\
\hline $0.009 \pm 0.000$ & $0.22 \pm 0.01$ & $19.9 \pm 0.8$ & $79 \pm 3$ \\
$0.009 \pm 0.001$ & $0.23 \pm 0.02$ & $20.7 \pm 2.1$ & $83 \pm 8$ \\
$0.009 \pm 0.001$ & $0.21 \pm 0.02$ & $19.2 \pm 2.1$ & $77 \pm 9$ \\
$0.009 \pm 0.000$ & $0.23 \pm 0.01$ & $20.7 \pm 0.9$ & $83 \pm 4$ \\
$0.009 \pm 0.001$ & $0.21 \pm 0.01$ & $19.5 \pm 1.1$ & $78 \pm 5$ \\
$0.009 \pm 0.000$ & $0.21 \pm 0.01$ & $19.1 \pm 0.9$ & $77 \pm 4$ \\
$0.012 \pm 0.001$ & $0.28 \pm 0.02$ & $25.8 \pm 1.7$ & $103 \pm 7$ \\
$0.010 \pm 0.001$ & $0.23 \pm 0.02$ & $21.1 \pm 1.4$ & $84 \pm 6$
\end{tabular}

\begin{tabular}{|c|c|c|c|c|c|c|c|}
\hline \multicolumn{2}{|c|}{ Location } & \multirow{2}{*}{$\begin{array}{c}\text { Sample Period } \\
\text { 1st Quarter '08 }\end{array}$} & \multirow{2}{*}{$\frac{\mathrm{mrem} / \mathrm{hr}}{0.009}$} & \multirow{2}{*}{$\frac{\mathrm{mrem} / \mathrm{day}}{0.22}$} & \multirow{2}{*}{$\frac{\mathbf{m r e m} / \mathbf{q t r}}{23.3}$} & \multirow{2}{*}{$\frac{\text { mrem/year }}{81}$} & \multirow{2}{*}{$\frac{\text { Days in Field }}{105}$} \\
\hline 300 TEDF & T326 & & & & & & \\
\hline & T327 & & 0.009 & 0.22 & 23.2 & 81 & 105 \\
\hline & T328 & & 0.009 & 0.22 & 22.9 & 80 & 105 \\
\hline & Т329 & & 0.009 & 0.22 & 23.5 & 82 & 105 \\
\hline & Т330 & & 0.009 & 0.22 & 22.8 & 79 & 105 \\
\hline & T331 & & 0.009 & 0.20 & 21.5 & 75 & 105 \\
\hline & T326 & 2nd Quarter '08 & 0.009 & 0.21 & 18.2 & 77 & 86 \\
\hline & T327 & & 0.010 & 0.23 & 19.6 & 83 & 86 \\
\hline & T328 & & 0.009 & 0.22 & 19.3 & 82 & 86 \\
\hline & T329 & & 0.010 & 0.23 & 19.6 & 83 & 86 \\
\hline & Т330 & & 0.009 & 0.23 & 19.3 & 83 & 85 \\
\hline & T331 & & 0.009 & 0.22 & 18.6 & 80 & 85 \\
\hline & T326 & 3rd Quarter '08 & 0.009 & 0.22 & 19.7 & 79 & 91 \\
\hline & T327 & & 0.009 & 0.22 & 20.0 & 80 & 91 \\
\hline & T328 & & 0.010 & 0.23 & 21.0 & 84 & 91 \\
\hline & Т329 & & 0.009 & 0.21 & 19.5 & 78 & 91 \\
\hline & Т330 & & 0.009 & 0.21 & 19.0 & 76 & 92 \\
\hline & T331 & & 0.010 & 0.23 & 21.2 & 84 & 92 \\
\hline & T326 & 4th Quarter '08 & 0.010 & 0.23 & 19.6 & 84 & 85 \\
\hline & T327 & & 0.009 & 0.22 & 19.0 & 81 & 85 \\
\hline & Т328 & & 0.010 & 0.24 & 20.5 & 88 & 85 \\
\hline & T329 & & 0.009 & 0.22 & 19.1 & 82 & 85 \\
\hline & Т330 & & 0.009 & 0.22 & 18.3 & 79 & 85 \\
\hline & T331 & & 0.009 & 0.23 & 19.2 & 83 & 85 \\
\hline
\end{tabular}


Table 5-2. 2008 TLD Results. (Sheet 13 of 14)

300 TEDF, Annual Averages \pm 2 Standard Deviations

\begin{tabular}{|c|c|c|c|c|}
\hline Location & mrem/hr & mrem/day & mrem/gtr & mrem/year \\
\hline Т326 & $0.009 \pm 0.001$ & $0.22 \pm 0.02$ & $20.1 \pm 1.5$ & $80 \pm 6$ \\
\hline T327 & $0.009 \pm 0.000$ & $0.22 \pm 0.01$ & $20.3 \pm 0.7$ & $81 \pm 3$ \\
\hline Т328 & $0.009 \pm 0.001$ & $0.23 \pm 0.02$ & $20.8 \pm 1.8$ & $83 \pm 7$ \\
\hline Т329 & $0.009 \pm 0.000$ & $0.22 \pm 0.01$ & $20.3 \pm 1.1$ & $81 \pm 4$ \\
\hline Т330 & $0.009 \pm 0.001$ & $0.22 \pm 0.02$ & $19.7 \pm 1.5$ & $79 \pm 6$ \\
\hline T331 & $0.009 \pm 0.001$ & $0.22 \pm 0.02$ & $20.0 \pm 2.1$ & $80 \pm 8$ \\
\hline
\end{tabular}

\begin{tabular}{|c|c|c|c|c|c|c|c|}
\hline \multicolumn{2}{|c|}{ Location } & \multirow{2}{*}{$\frac{\text { Sample Period }}{\text { 1st Quarter '08 }}$} & \multirow{2}{*}{$\frac{\text { mrem/hr }}{0.009}$} & \multirow{2}{*}{$\frac{\text { mrem/day }}{0.22}$} & \multirow{2}{*}{$\frac{\text { mrem/qtr }}{22.6}$} & \multirow{2}{*}{$\frac{\text { mrem/year }}{79}$} & \multirow{2}{*}{$\frac{\text { Days in Field }}{105}$} \\
\hline 300-FF-2 & Т369 & & & & & & \\
\hline Field & T370 & & 0.009 & 0.21 & 22.4 & 78 & 105 \\
\hline \multirow{10}{*}{$\begin{array}{c}\text { project } \\
\text { (300 Area) }\end{array}$} & Т372 & & 0.009 & 0.22 & 23.5 & 82 & 105 \\
\hline & Т369 & 2nd Quarter '08 & 0.009 & 0.21 & 18.2 & 78 & 85 \\
\hline & T371 & & 0.009 & 0.21 & 18.2 & 78 & 85 \\
\hline & T372 & & 0.009 & 0.21 & 18.2 & 78 & 85 \\
\hline & Т369 & 3rd Quarter '08 & 0.008 & 0.20 & 18.1 & 72 & 92 \\
\hline & T370 & & 0.009 & 0.21 & 18.9 & 75 & 92 \\
\hline & Т369 & 4th Quarter '08 & 0.009 & 0.22 & 18.5 & 79 & 85 \\
\hline & Т370 & & 0.009 & 0.21 & 18.1 & 78 & 85 \\
\hline & T371 & & 0.010 & 0.25 & 20.9 & 90 & 85 \\
\hline & Т372 & & 0.010 & 0.24 & 20.1 & 86 & 85 \\
\hline
\end{tabular}

300-FF-2, Annual Averages \pm 2 Standard Deviations

\begin{tabular}{lcccc} 
& mrem/hr & mrem/day & mrem/qtr & mrem/year \\
\cline { 2 - 5 } T369 & $0.009 \pm 0.001$ & $0.21 \pm 0.02$ & $19.2 \pm 1.8$ & $77 \pm 7$ \\
T370 & $0.009 \pm 0.001$ & $0.21 \pm 0.01$ & $19.5 \pm 1.3$ & $78 \pm 5$ \\
T371 & $0.009 \pm 0.002$ & $0.22 \pm 0.05$ & $19.9 \pm 4.4$ & $79 \pm 18$ \\
T372 & $0.009 \pm 0.001$ & $0.22 \pm 0.02$ & $20.3 \pm 2.0$ & $81 \pm 8$
\end{tabular}

\begin{tabular}{|c|c|c|c|c|c|c|c|}
\hline \multicolumn{2}{|c|}{ Location } & \multirow{2}{*}{$\begin{array}{c}\text { Sample Period } \\
\text { 1st Quarter '08 }\end{array}$} & \multirow{2}{*}{$\frac{\text { mrem/hr }}{0.009}$} & \multirow{2}{*}{$\frac{\text { mrem/day }}{0.21}$} & \multirow{2}{*}{$\frac{\text { mrem/qtr }}{22.5}$} & \multirow{2}{*}{$\frac{\text { mrem/year }}{78}$} & \multirow{2}{*}{$\frac{\text { Days in Field }}{105}$} \\
\hline 400 Area & T340 & & & & & & \\
\hline & T341 & & 0.010 & 0.25 & 26.3 & 92 & 105 \\
\hline & T342 & & 0.010 & 0.23 & 23.9 & 83 & 105 \\
\hline & Т343 & & 0.009 & 0.22 & 22.9 & 80 & 105 \\
\hline & T344 & & 0.009 & 0.21 & 21.9 & 76 & 105 \\
\hline & T345 & & 0.009 & 0.22 & 22.9 & 80 & 105 \\
\hline & T340 & 2nd Quarter '08 & 0.010 & 0.23 & 19.4 & 83 & 85 \\
\hline & Т341 & & 0.011 & 0.27 & 23.3 & 99 & 86 \\
\hline & T342 & & 0.010 & 0.23 & 19.8 & 84 & 86 \\
\hline & T343 & & 0.008 & 0.20 & 17.2 & 73 & 86 \\
\hline
\end{tabular}


Table 5-2. 2008 TLD Results. (Sheet 14 of 14)

\begin{tabular}{|c|c|c|c|c|c|c|c|}
\hline \multicolumn{2}{|c|}{ Location } & \multirow{2}{*}{$\frac{\text { Sample Period }}{\text { 3rd Quarter '08 }}$} & \multirow{2}{*}{$\begin{array}{c}\text { mrem/hr } \\
0.010\end{array}$} & \multirow{2}{*}{$\frac{\text { mrem/day }}{0.23}$} & \multirow{2}{*}{$\frac{\text { mrem/qtr }}{20.9}$} & \multirow{2}{*}{$\frac{\text { mrem/year }}{83}$} & \multirow{2}{*}{$\frac{\text { Days in Field }}{92}$} \\
\hline 400 Area & T340 & & & & & & \\
\hline & T341 & & 0.011 & 0.26 & 24.2 & 96 & 92 \\
\hline & T342 & & 0.010 & 0.24 & 21.5 & 86 & 91 \\
\hline & T343 & & 0.009 & 0.21 & 18.7 & 75 & 91 \\
\hline & T344 & & 0.009 & 0.21 & 18.8 & 76 & 91 \\
\hline & T345 & & 0.009 & 0.21 & 19.5 & 78 & 91 \\
\hline & T340 & 4th Quarter '08 & 0.010 & 0.24 & 20.4 & 88 & 85 \\
\hline & T341 & & 0.010 & 0.24 & 20.4 & 89 & 84 \\
\hline & T342 & & 0.009 & 0.21 & 17.6 & 75 & 85 \\
\hline & T343 & & 0.009 & 0.22 & 18.3 & 79 & 85 \\
\hline
\end{tabular}

400 Area, Annual Averages \pm 2 Standard Deviations

\begin{tabular}{lcccc} 
& $\mathbf{m r e m} / \mathbf{h r}$ & $\mathbf{m r e m} / \mathbf{d a y}$ & $\mathbf{m r e m} / \mathbf{q t r}$ & $\mathbf{m r e m} / \mathbf{y e a r}$ \\
\cline { 2 - 5 } T340 & $0.009 \pm 0.001$ & $0.23 \pm 0.02$ & $20.7 \pm 1.9$ & $83 \pm 7$ \\
T341 & $0.011 \pm 0.001$ & $0.26 \pm 0.03$ & $23.4 \pm 2.4$ & $94 \pm 9$ \\
T342 & $0.009 \pm 0.001$ & $0.23 \pm 0.03$ & $20.6 \pm 2.4$ & $82 \pm 9$ \\
T343 & $0.009 \pm 0.001$ & $0.21 \pm 0.02$ & $19.2 \pm 1.6$ & $77 \pm 6$ \\
T344 & $0.009 \pm 0.000$ & $0.21 \pm 0.01$ & $19.2 \pm 0.5$ & $77 \pm 2$ \\
T345 & $0.009 \pm 0.000$ & $0.22 \pm 0.01$ & $19.7 \pm 0.6$ & $79 \pm 2$ \\
T346 & $0.009 \pm 0.000$ & $0.22 \pm 0.01$ & $19.7 \pm 1.0$ & $79 \pm 4$
\end{tabular}

\begin{tabular}{|c|c|c|c|c|c|c|c|}
\hline \multicolumn{2}{|c|}{ Location } & \multirow{2}{*}{$\frac{\text { Sample Period }}{\text { 1st Quarter '08 }}$} & \multirow{2}{*}{$\frac{\text { mrem/hr }}{0.009}$} & \multirow{2}{*}{$\frac{\text { mrem/day }}{0.22}$} & \multirow{2}{*}{$\frac{\text { mrem/qtr }}{18.4}$} & \multirow{2}{*}{$\frac{\text { mrem/year }}{79}$} & \multirow{2}{*}{$\frac{\text { Days in Field }}{85}$} \\
\hline ERDF & T351 & & & & & & \\
\hline (200 West & T352 & & 0.009 & 0.20 & 17.3 & 75 & 85 \\
\hline & T351 & 2nd Quarter '08 & 0.010 & 0.24 & 24.5 & 86 & 104 \\
\hline & T352 & & 0.009 & 0.22 & 23.1 & 81 & 104 \\
\hline & Т351 & 3rd Quarter '08 & 0.009 & 0.20 & 17.1 & 74 & 84 \\
\hline & T352 & & 0.009 & 0.22 & 18.9 & 82 & 84 \\
\hline & Т353 & & 0.009 & 0.20 & 17.1 & 74 & 84 \\
\hline
\end{tabular}

ERDF, Annual Averages \pm 2 Standard Deviations

\begin{tabular}{lcccc} 
& mrem/hr & mrem/day & mrem/qtr & mrem/year \\
\cline { 2 - 5 } T351 & $0.009 \pm 0.001$ & $0.22 \pm 0.03$ & $20.1 \pm 2.5$ & $80 \pm 10$ \\
T352 & $0.009 \pm 0.001$ & $0.22 \pm 0.02$ & $19.6 \pm 1.9$ & $78 \pm 7$ \\
T353 & $0.009 \pm 0.001$ & $0.21 \pm 0.03$ & $19.2 \pm 2.6$ & $77 \pm 10$
\end{tabular}

\begin{tabular}{cccccccc}
\multicolumn{2}{c}{ Location } & Sample Period & mrem/hr & mrem/day & mrem/qtr & mrem/year & Days in Field \\
\hline IDF & T375 & 1st Quarter '08 & 0.009 & 0.22 & 18.5 & 81 & 83 \\
(200 East & T375 & 2nd Quarter '08 & 0.010 & 0.24 & 24.8 & 86 \\
Area) & T375 & 3rd Quarter '08 & 0.010 & 0.23 & 19.7 & 86 \\
& T375 & 4th Quarter '08 & 0.010 & 0.24 & 21.6 & 87
\end{tabular}

IDF, Annual Averages \pm 2 Standard Deviations

\begin{tabular}{cccc} 
mrem/hr & mrem/day & mrem/qtr & mrem/year \\
\hline $0.010 \pm 0.001$ & $0.23 \pm 0.01$ & $21.3 \pm 1.2$ & $85 \pm 5$
\end{tabular}




\subsection{RADIOLOGICAL SURVEYS}

In 2008, there were approximately 3,583 hectares (8,853 acres) of posted outdoor contamination areas and 584 hectares (1,443 acres) of posted underground radioactive materials areas at the Hanford Site. During 2008, several waste sites in the 100 Areas (9 hectares [22 acres]) and one waste site in the 600 Area (less than 0.4 hectare [ 1 acre]) were remediated and released from posting. A listing of these waste sites is provided in Table 6-1.

Posted contamination areas continually vary in number and size from year to year because of ongoing efforts to clean, stabilize, and remediate areas of known contamination. In conjunction, new areas of contamination are also being identified throughout the year. Survey locations, typically associated with cribs, trenches, burial grounds, tank farms, and covered ponds and ditches, are illustrated in Figures 6-1 through 6-10.

It was estimated that the external dose rate at $80 \%$ of the identified outdoor contamination areas was less than $1 \mathrm{mrem} / \mathrm{hr}$, although direct dose rate readings from isolated radioactive specks (a diameter less than $0.6 \mathrm{~cm}$ [0.25 in.]) could have been considerably higher. Contamination levels of this magnitude did not significantly add to dose rates for the public or Hanford Site workers in 2008.

Table 6-1. Waste Sites Remediated and Released From Posting During 2008.

\begin{tabular}{ccccc} 
Area & Waste Site & & Area & Waste Site \\
\hline $\mathbf{1 0 0 - B / C}$ & $118-\mathrm{B}-1$ & $\mathbf{6 0 0}$ & UPR-600-16 \\
& & & \\
$\mathbf{1 0 0 - F}$ & $118-\mathrm{F}-1$ & & & \\
& $118-\mathrm{F}-2$ & & & \\
& $118-\mathrm{F}-5 \mathrm{~F}$ & & &
\end{tabular}


Figure 6-1. 2008 Radiological Survey Locations, 100-B/C Area.

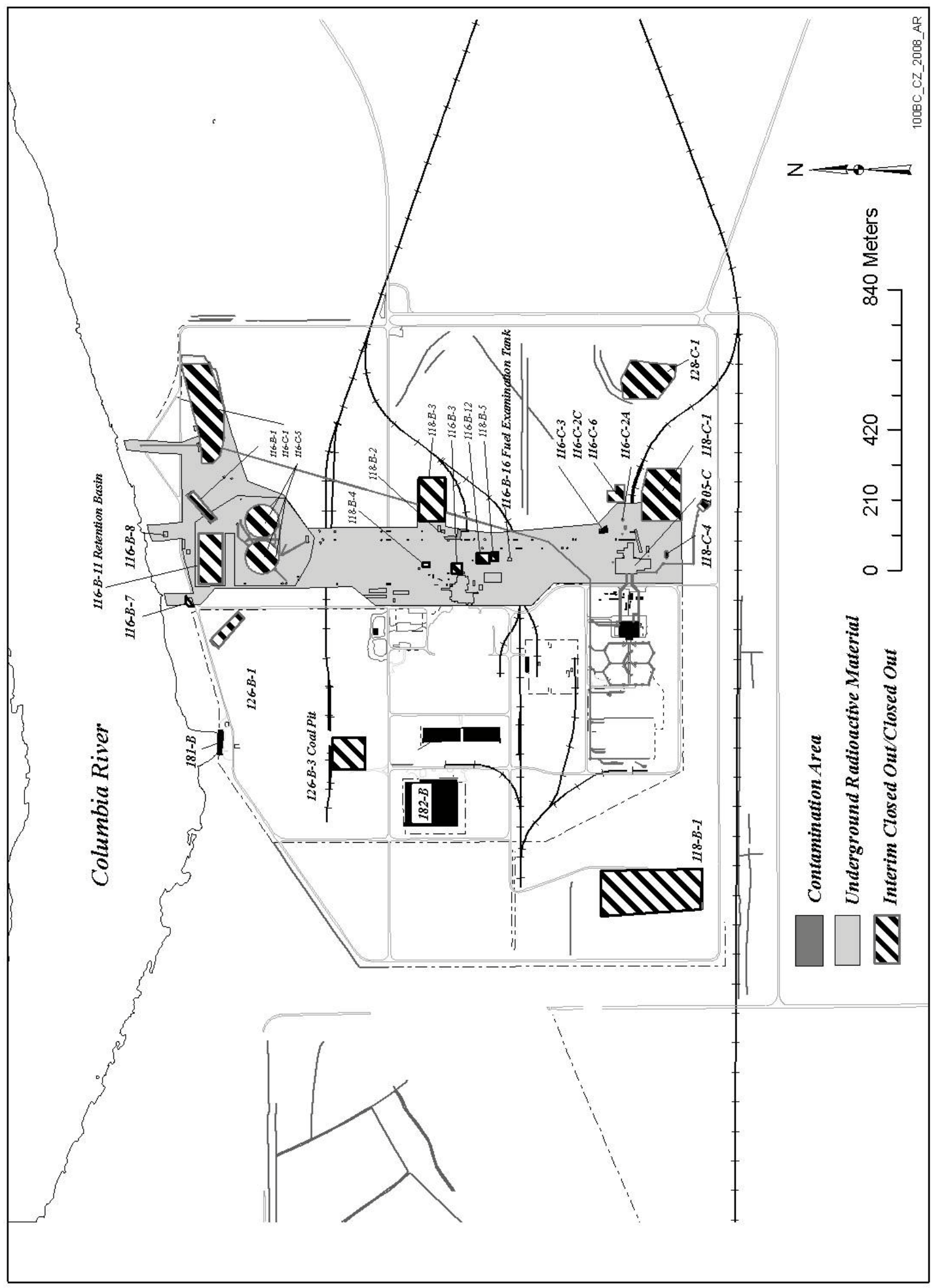


Figure 6-2. 2008 Radiological Survey Locations, 100-D/DR Area.

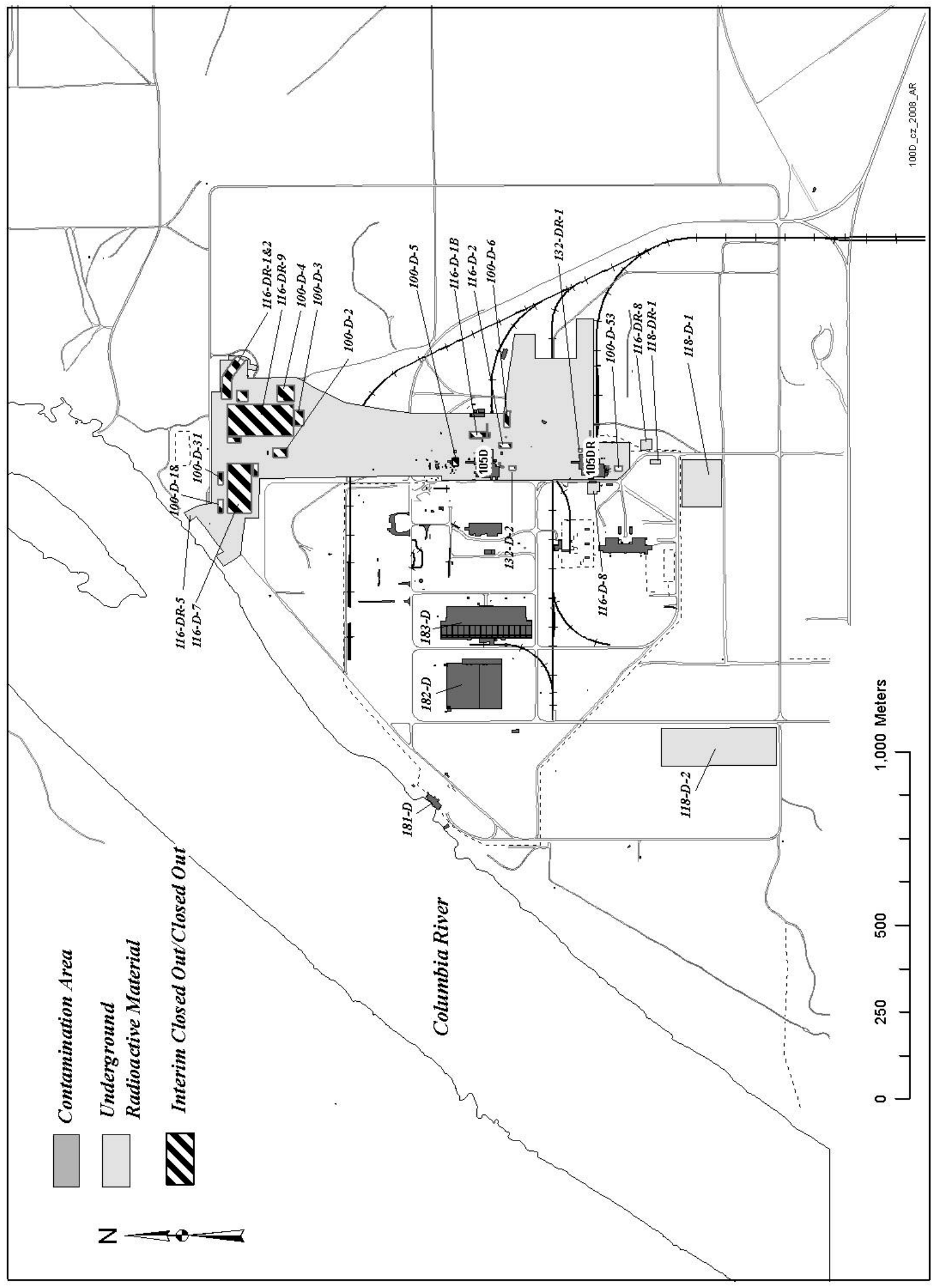


Figure 6-3. 2008 Radiological Survey Locations, 100-F Area.

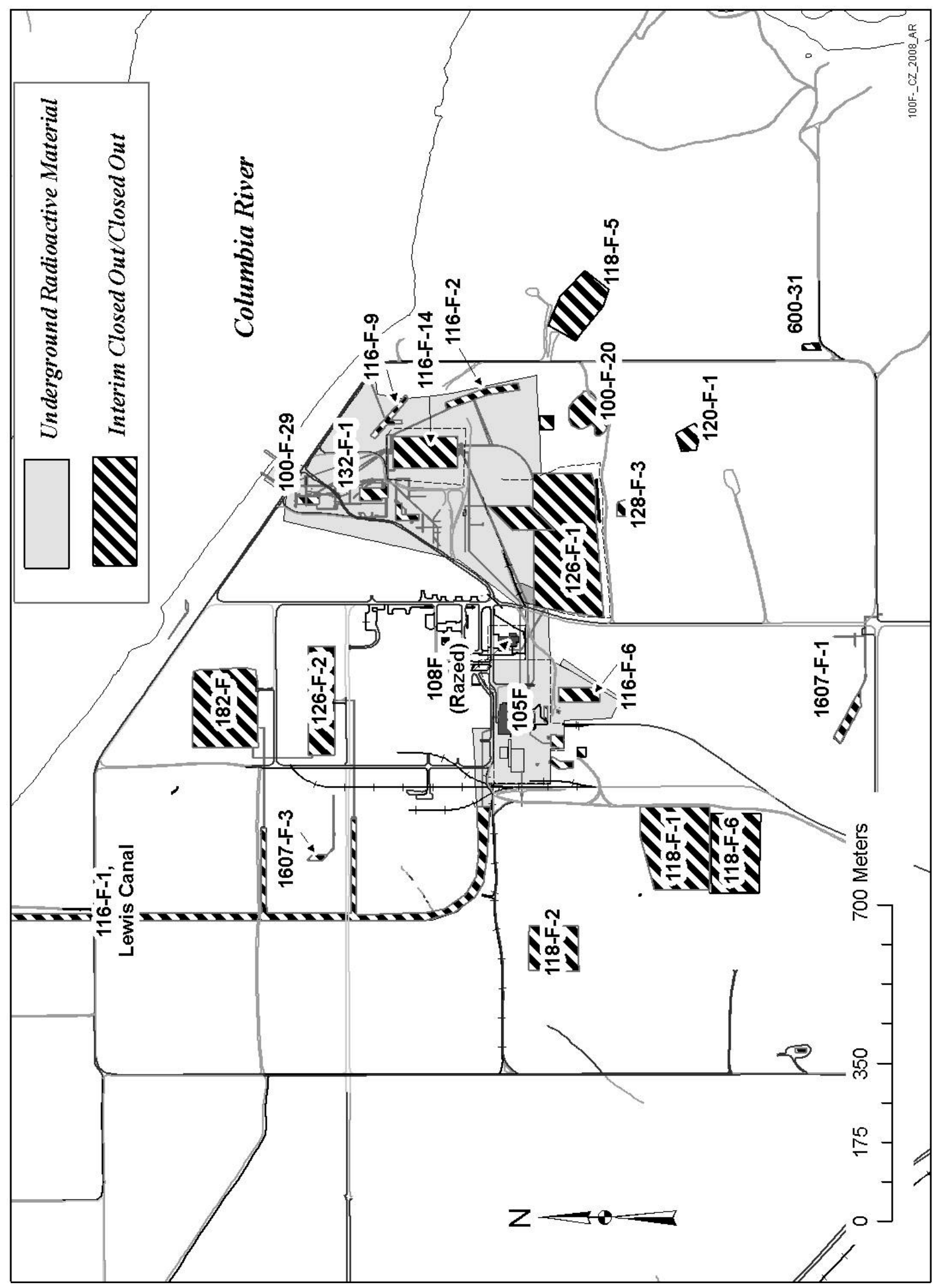


Figure 6-4. 2008 Radiological Survey Locations, 100-H Area.

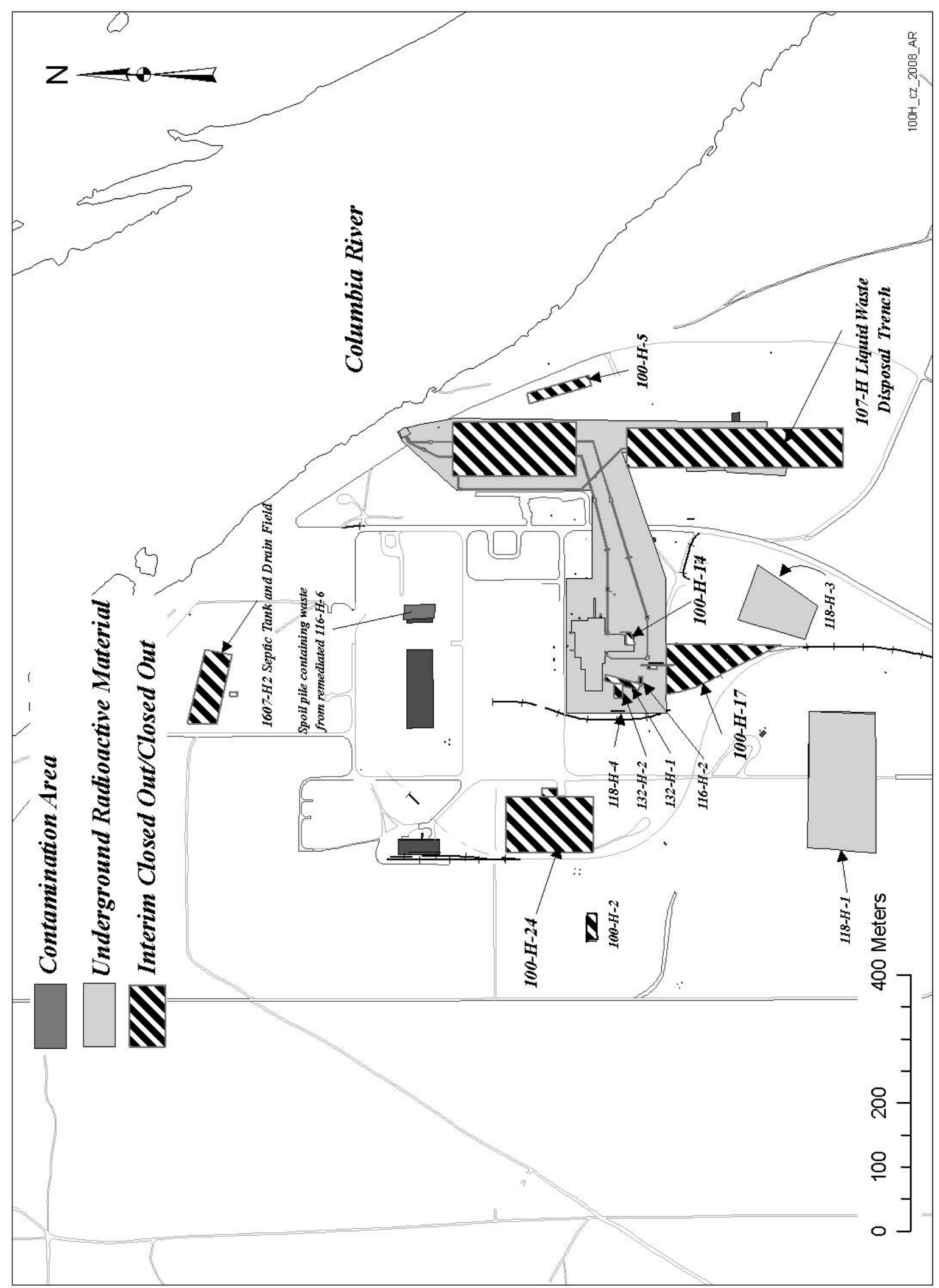


Figure 6-5. 2008 Radiological Survey Locations, 100-K Area.

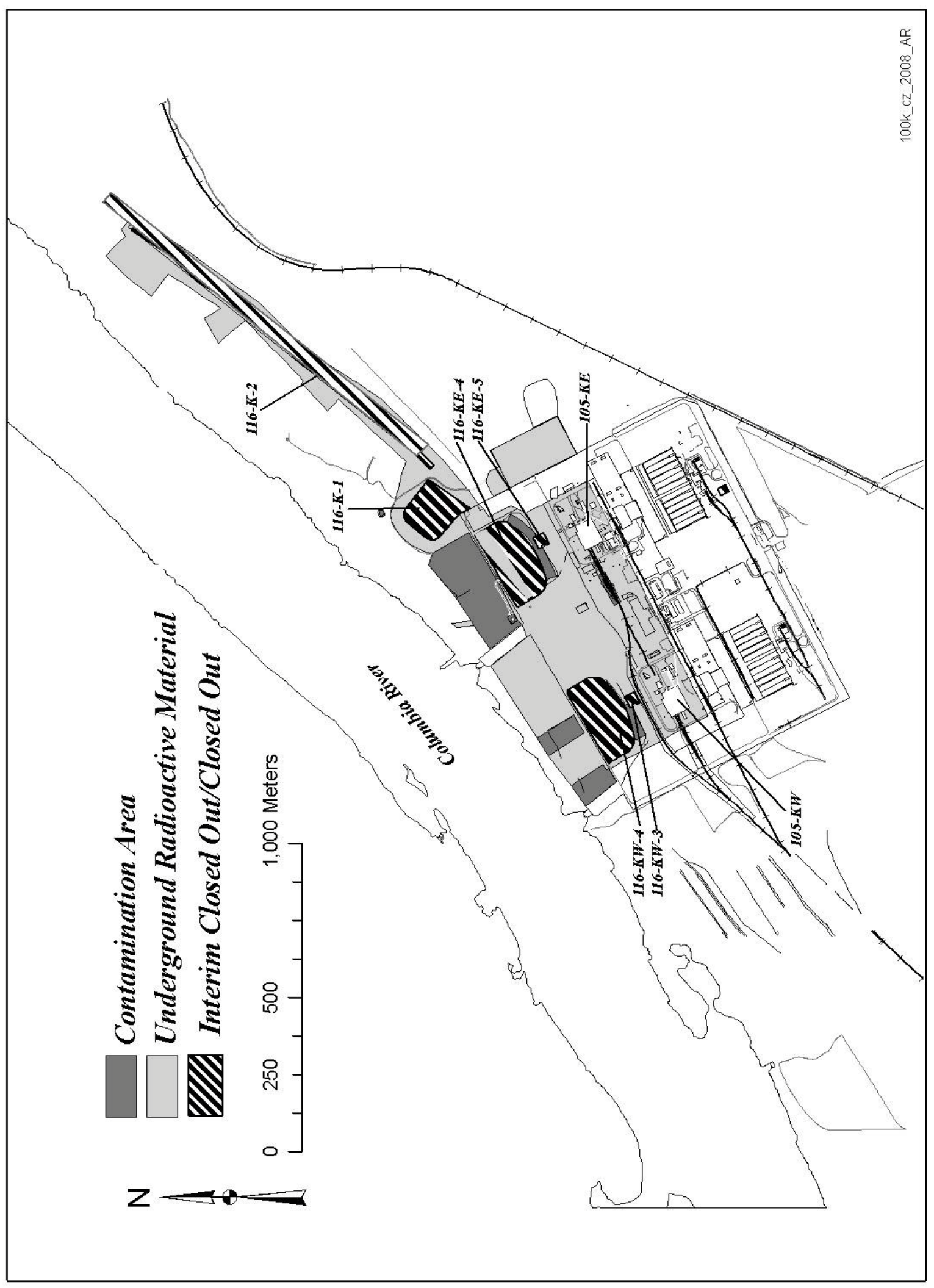


Figure 6-6. 2008 Radiological Survey Locations, 100-N Area.

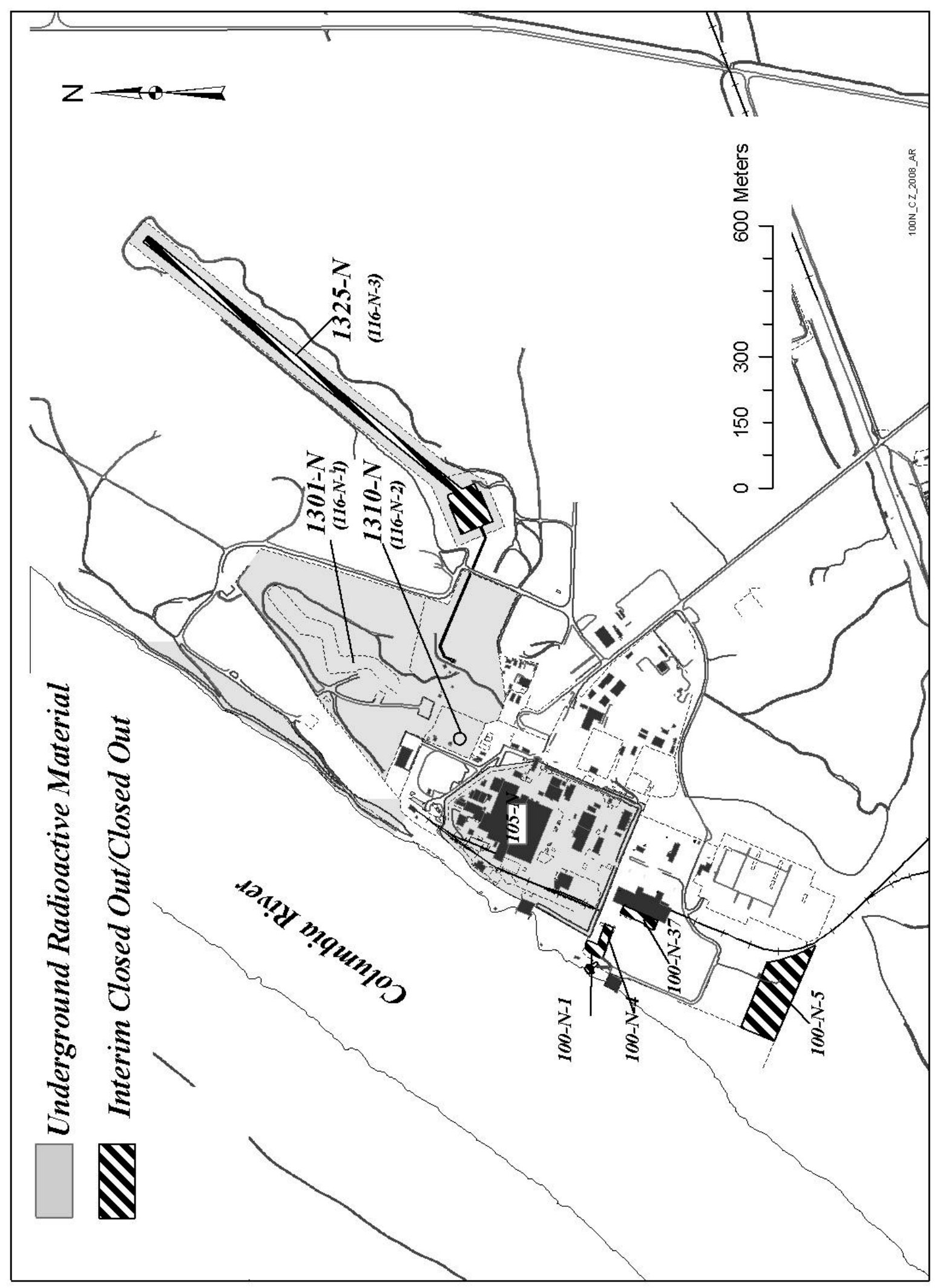


Figure 6-7. 2008 Radiological Survey Locations, 200 East Area.

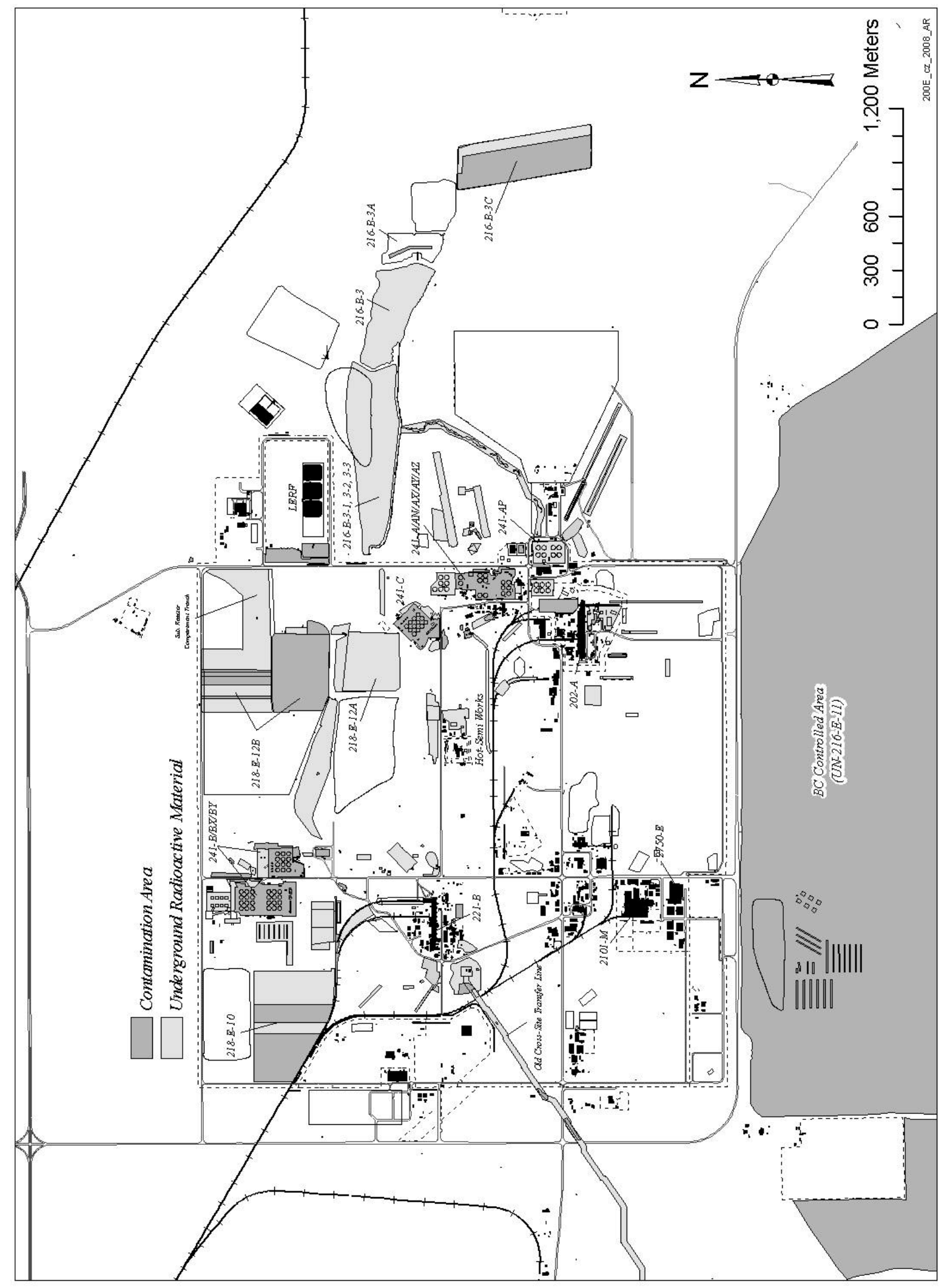


Figure 6-8. 2008 Radiological Survey Locations, 200 West Area.

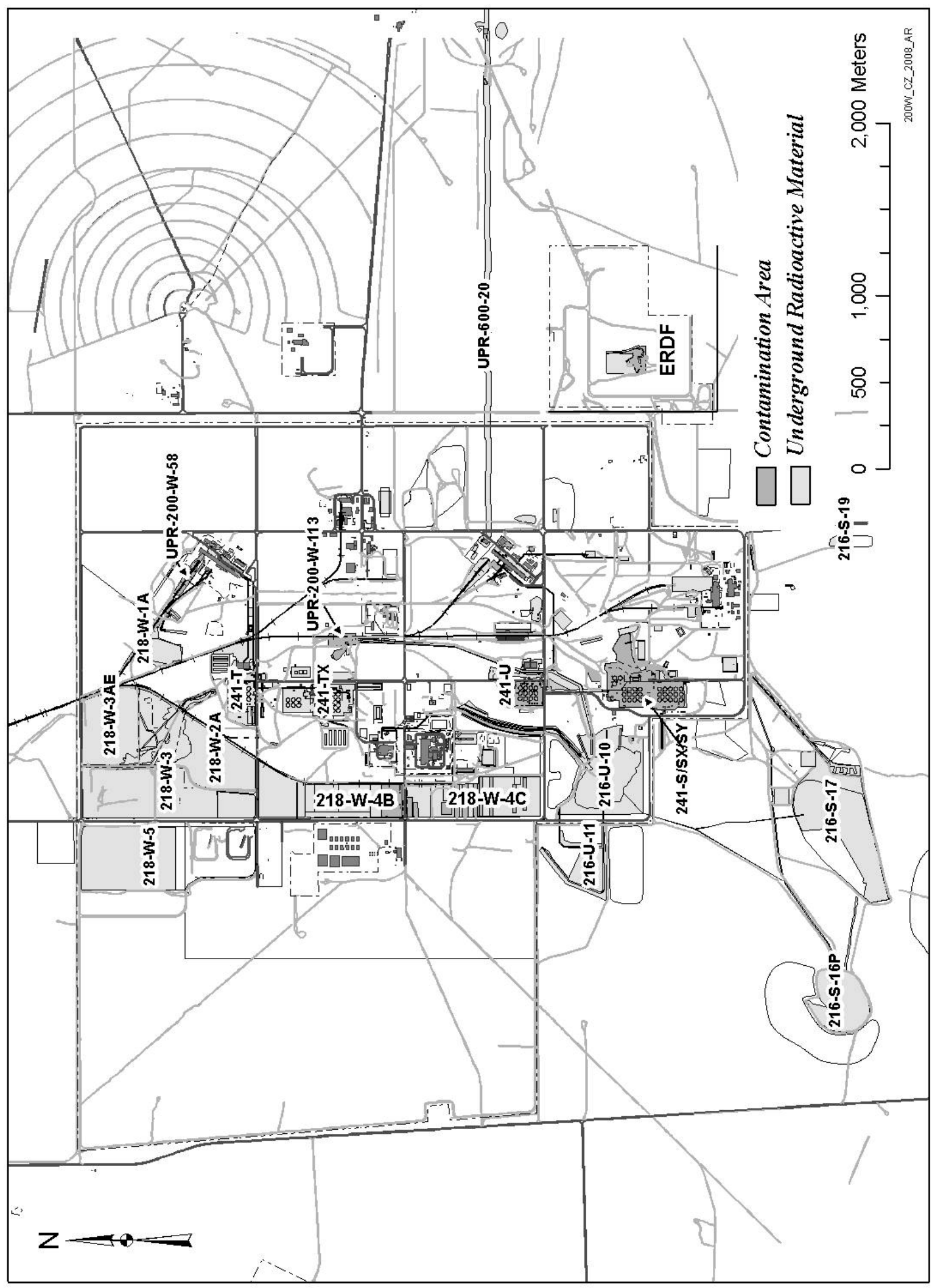


Figure 6-9. 2008 Radiological Survey Locations, 300 Area.

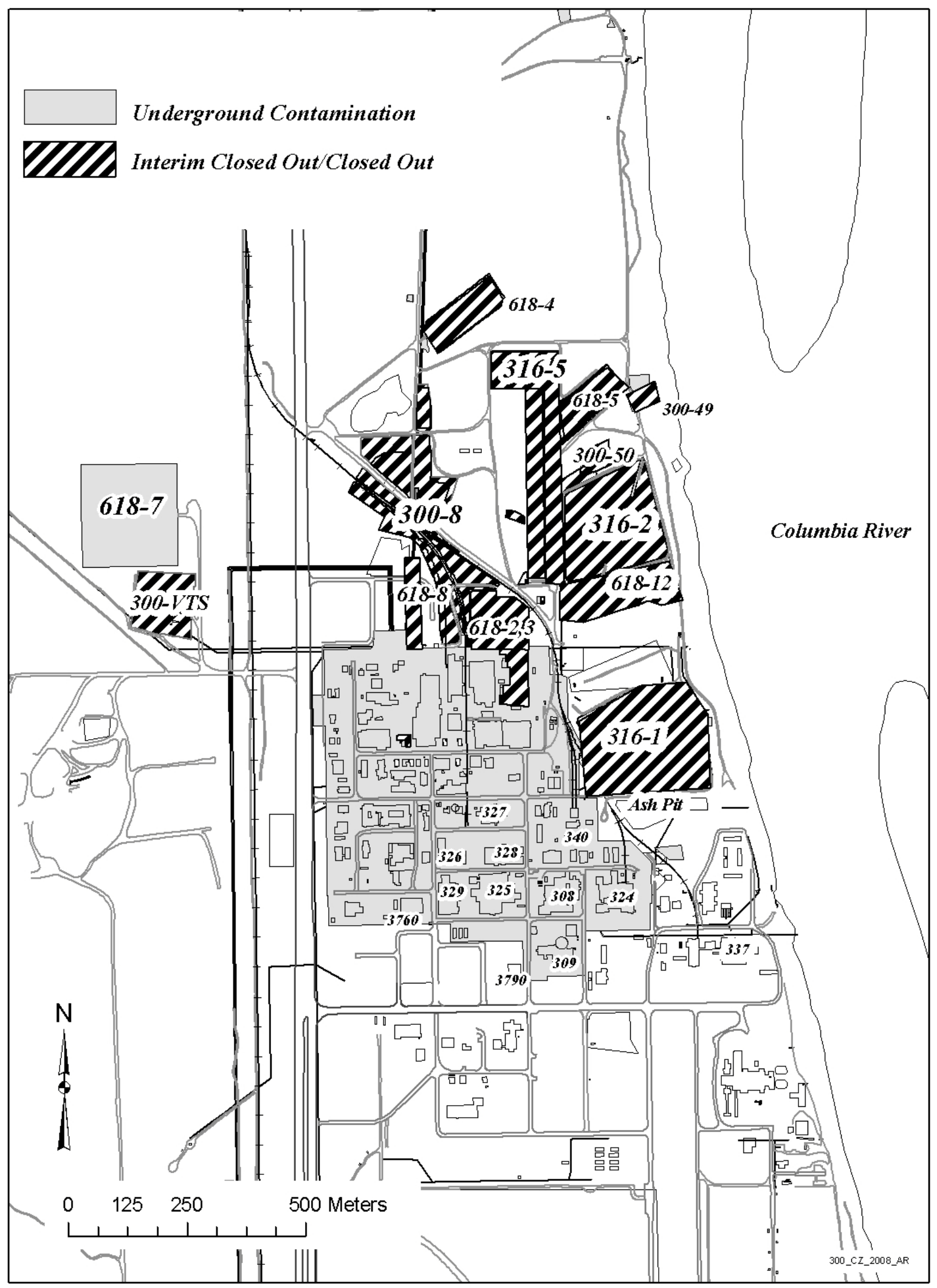


Figure 6-10. 2008 Radiological Survey Locations, 600 Area.

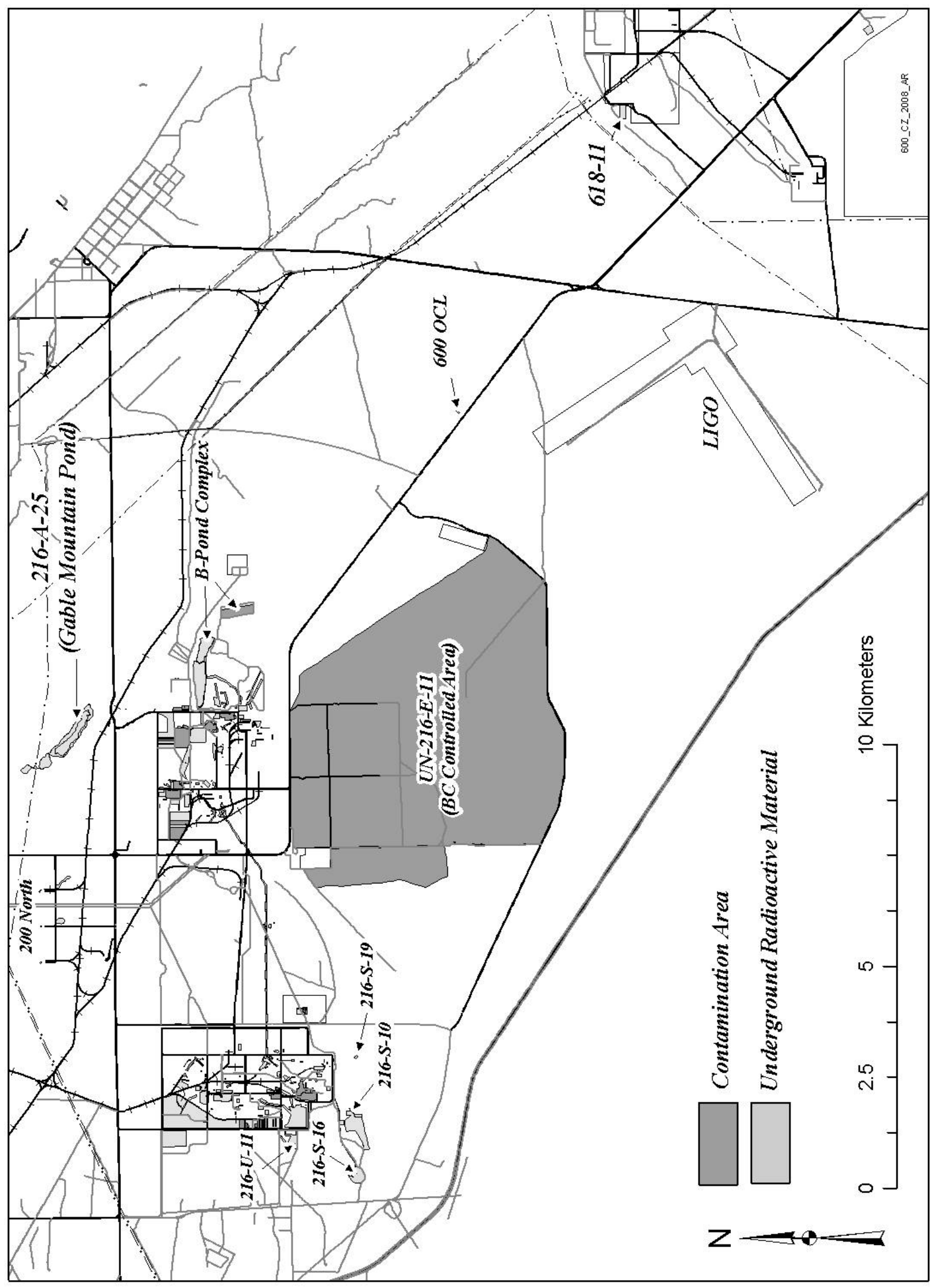


This page intentionally left blank.

6-12 


\subsection{INVESTIGATIVE SAMPLING}

Investigative samples are typically collected where known or suspected radioactive contamination was present, or to verify radiological conditions at project sites. In 2008, none of the investigative samples collected were analyzed. However, six animal samples collected in 2006 and 2007 were analyzed for radionuclides at the Waste Sampling and Characterization Facility (WSCF) laboratory and the analytical results are provided in Table 7-1. One hundred seventy-three (173) contaminated environmental samples were reported during surveillance and/or cleanup operations in 2008, and all were disposed without isotopic analyses. The locations and field readings for these contaminated samples are listed in Table 7-2.

\subsection{SOIL}

In 2008, there were 16 instances of radiological contamination in which soil was identified as the carrier of contamination. Of these, 8 were identified only as specks, or soil specks. Often, specks observed under high magnification are found to be small pieces of decomposed vegetation, most often tumbleweeds. External radioactivity levels ranged from approximately 15,000 disintegrations per minute $(\mathrm{dpm}) / 100 \mathrm{~cm}^{2}$ to more than $2,400,000 \mathrm{dpm} / 100 \mathrm{~cm}^{2}$. Contaminated areas were radiologically posted or cleaned up. The number of contamination incidents and the range of radiation dose rate levels observed in 2008 were generally within historical ranges.

\subsection{VEGETATION}

In 2008, there were 127 instances in which vegetation was identified as the carrier of radiological contamination. Thirty instances of contaminated vegetation had field readings in excess of $1,000,000 \mathrm{dpm} / 100 \mathrm{~cm}^{2}$. The radioactivity levels were all within historical ranges.

The number of contaminated vegetation incidents increased from 62 in 2007 to 127 in 2008. The increase in the number of instances in 2008 is more than twice the number observed in 2007 and the highest number reported to date. The number of contaminated tumbleweeds can be attributed to favorable growing conditions (moisture) and a resistance to the herbicide that had been used. It is expected that as contaminated vegetation continues to be identified and cleaned up, subsequent years will show a reduction in the number of these incidents. Additional discussion of Vegetation Control activities taken in 2008 can be found in Section 10.10.4 of PNNL-18427 (PNNL 2009a).

\subsection{ANIMALS}

In 2008, 30 instances of contaminated animals or animal-related contamination were identified. Of these, none were submitted to the laboratory. Three animal-related samples collected in the 200 Areas exhibited field readings in excess of 1,000,000 dpm/100 cm². 
Animals were collected either as part of an integrated pest management program or as a result of radiological surveys finding contaminated wildlife-related material (e.g., feces, nests, etc.). Animals were collected directly from or near facilities in an effort to monitor and track effectiveness of preventive measures designed to deter animal intrusion. For 2008, the number of animals found to be contaminated with radioactivity and the range of radionuclide concentrations were within historical ranges.

\subsection{SPECIAL CHARACTERIZATION SAMPLING}

No special characterization projects were conducted or completed during 2008 to ascertain the radiological, and in some cases, physical condition of specific sites or operations.

Table 7-1. Investigative Sample Results, 2008. (Sheet 1 of 2)

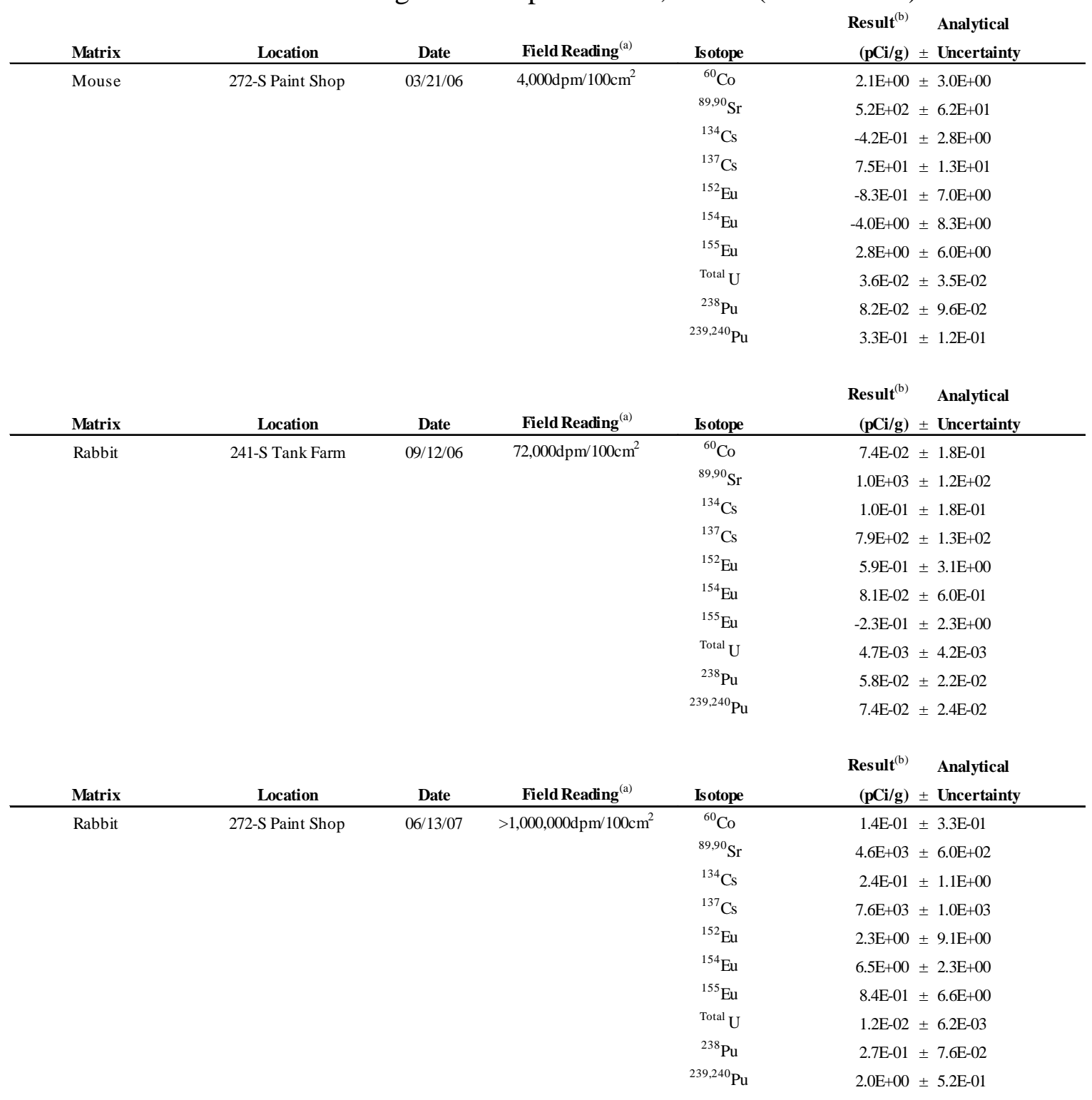


Table 7-1. Investigative Sample Results, 2008. (Sheet 2 of 2)

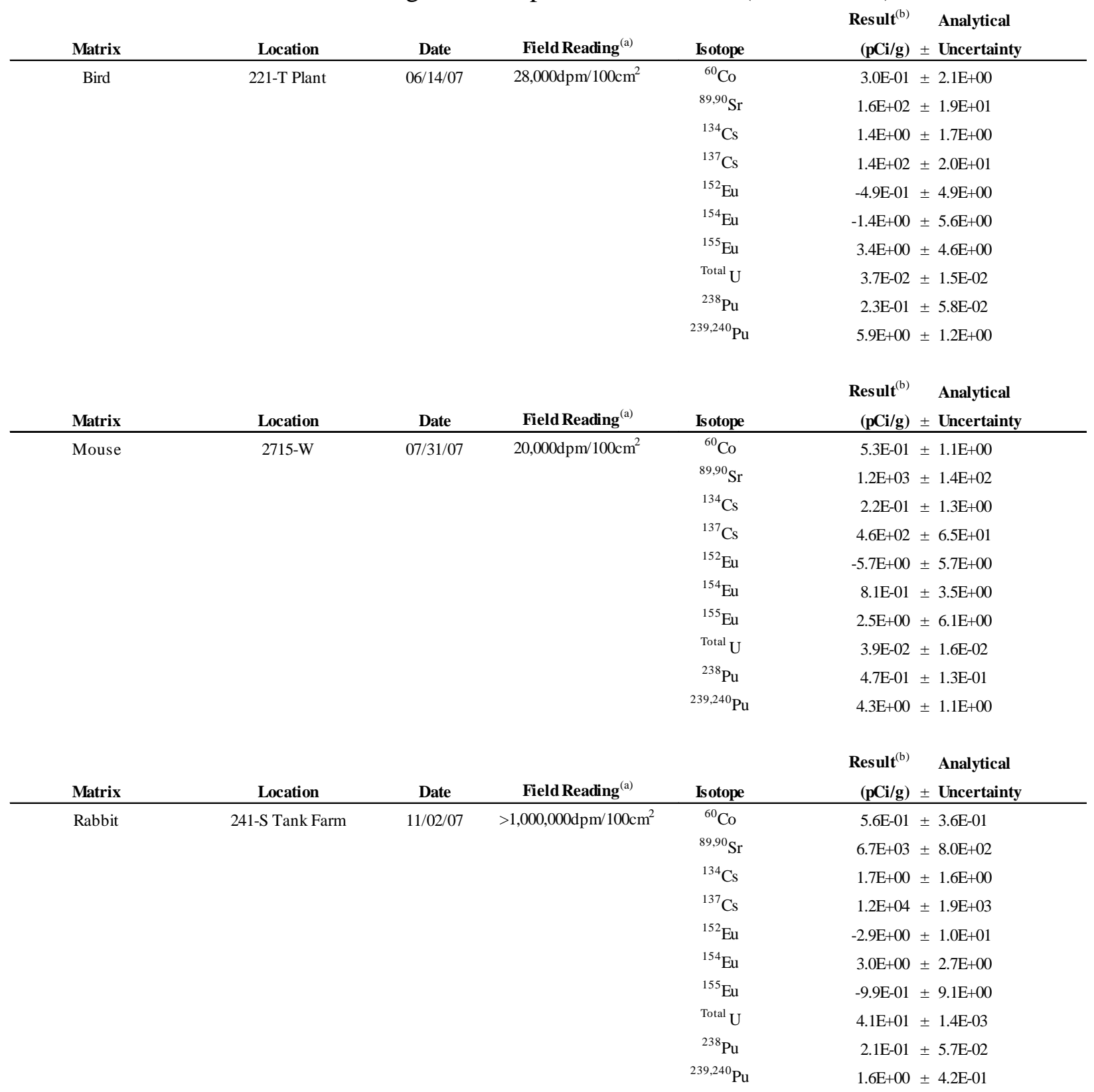

(a) $\mathrm{dpm}=$ disintegrations per minute

(b) To convert to international metric system units (SI), multiply pCi/g by 0.03704 to obtain Bq/g. 
Table 7-2. Investigative Samples Not Analyzed, 2008. (Sheet 1 of 4)

FIELD READING

SAMPLE MATRIX

LOCATION

(Beta/Gamma)

Tumbleweed

Mouse

Tumbleweeds

Tumbleweeds

Tumbleweeds (8)

Speck

Tumbleweeds

Tumbleweeds

Speck

Mouse Feces

Tumbleweed

Tumbleweeds

Tumbleweeds

Tumbleweeds (3)

Rabbit Feces

Tumbleweed

Tumbleweeds (3)

Tumbleweeds (2)

Bunch Grass

Tumbleweeds

Tumbleweeds (20)

Grass Root Balls/Sod Material

Soil

Tumbleweeds (10)

Tumbleweeds (10)

Tumbleweeds (8)

Mud Dauber Nest

Tumbleweeds (4)

Tumbleweed

Tumbleweeds $(>100)$

Tumbleweeds (30)

Tumbleweed

Tumbleweeds (>200)

Tumbleweeds (3-bags)

25'X25' area of Tumbleweeds

Soil (8 areas) and Fragment

Tumbleweeds (2)

Soi

Tumbleweed

Tumbleweed and Fragments

Tumbleweed Fragments

Tumbleweed and Fragments

Tumbleweeds (2)

Tumbleweeds and Fragments (4)

Tumbleweed

Speck

Multiple Spots (Specks)

Multiple Specks

Ant Mound

Tumbleweeds and Fragments (30)

Tumbleweeds and Fragments (2)

Rabbit Fecal Pellets (29)

Tumbleweeds (2)
Between 222-S \& REDOX

Inside 224-U

Inside Perimeter Fence @ 16th and Dayton St, Corner of 16th \& Dayton St

On top of 216-A-34 Crib

At RMA-028 @ 241-C Tank Farm

Outside the intersection of 16th \& Dayton St

Outside the Perimeter Fence at 16th \& Dayton St

Outside the Perimeter Fence 241-SX Tank Farm

Outside 209-E Gate (Hot Semiworks)

Outside 241-BX Tank Farm Perimeter Fence

221-Z Plant PFP Entrance

Outside 241-U Tank Farm

East Side of 284-W Powerhouse

Between 242-A Evaporator and 241-A Tank Farm

Along Buffalo Ave. West of 241-A/AX / AZ Tank Farms

Outside the PFP Perimeter Fence

On top of the 218-E-12A Burial Ground

On top of the 218-E-12A Burial Ground

UPR-200-W-38 on Backside of 221-T

On top of the 218-E-12A Burial Ground

UPR-600-20 between Rt 4S and 200-E Fuel Station

$16 \mathrm{sq} \mathrm{ft}$ Area inside the 216-BC Cribs

inside a $20 \mathrm{X} 10 \mathrm{ft}$ area inside the 216-BC Cribs

$10 \mathrm{X} 10 \mathrm{ft}$ area around a well inside the 216-BC Cribs

West Fenceline 241-U Tank Farm

Outside Rm 115 at 109-N

West side of PFP in Security Buffer Zone

South side of PFP in Security Buffer Zone

218-E-12B LLBG Trench 94

On top of the 216-B-9 Crib

Along Exterior Fence of 241-B Tank Farm 218-E-12B LLBG Trench 94

3-Bags (full) collected around 221-U Plant

Over old Pipeline east of 241-SX Tank Farm

On top of 218-E-12B Burial Ground

Outside200-W Area East Fenceline

inside 200-W-106 West of 241-TX

West fenceline 241-BX/BY Tank Farm

North Fenceline 241-U Tank Farm

East Perimeter Fenceline 241-SY

Northeast of 241-B Tank Farm

Along 200-West South Fenceline along Beloit Ave Outside West Fenceline 241-S Tank Farm Complex Outside West Fenceline 241-TX/TY Tank Farm

Outside the East Side of 241-A Tank Farm

Surrounding 241-ER-311 Catch Tank

Outside South Perimeter Fence 241-B Tank Farm

Inside the 218-E-12A LLBG

Inside the 218-E-12A LLBG

Adjacent to the 200-E-53 CA

On Lawn@ 242-A Evaporator

Outside East Perimeter 241-C Tank Farm
$60,000 \mathrm{dpm} / 100 \mathrm{~cm}^{2}$

$1,500 \mathrm{dpm} / 100 \mathrm{~cm}^{2}$

$36,000 \mathrm{dpm} / 100 \mathrm{~cm}^{2}$

$36,000 \mathrm{dpm} / 100 \mathrm{~cm}^{2}$

$60,000 \mathrm{dpm} / 100 \mathrm{~cm}^{2}$

$15,000 \mathrm{dpm} / 100 \mathrm{~cm}^{2}$

$60,000 \mathrm{dpm} / 100 \mathrm{~cm}^{2}$

$60,000 \mathrm{dpm} / 100 \mathrm{~cm}^{2}$

$149,000 \mathrm{dpm} / 100 \mathrm{~cm}^{2}$

$3,000 \mathrm{dpm} / 100 \mathrm{~cm}^{2}$

$54,000 \mathrm{dpm} / 100 \mathrm{~cm}^{2}$

$20,000 \mathrm{dpm} / 100 \mathrm{~cm}^{2}$

$48,000 \mathrm{dpm} / 100 \mathrm{~cm}^{2}$

$18,000 \mathrm{dpm} / 100 \mathrm{~cm}^{2}$

$20,000 \mathrm{dpm} / 100 \mathrm{~cm}^{2}$

$30,000 \mathrm{dpm} / 100 \mathrm{~cm}^{2}$

$60,000 \mathrm{dpm} / 100 \mathrm{~cm}^{2}$

$120,000 \mathrm{dpm} / 100 \mathrm{~cm}^{2}$

$5,400,000 \mathrm{dpm} / 100 \mathrm{~cm}^{2}$

$4,200,000 \mathrm{dpm} / 100 \mathrm{~cm}^{2}$

$3,900,000 \mathrm{dpm} / 100 \mathrm{~cm}^{2}$

$120,000 \mathrm{dpm} / 100 \mathrm{~cm}^{2}$

$60,000 \mathrm{dpm} / 100 \mathrm{~cm}^{2}$

$60,000 \mathrm{dpm} / 100 \mathrm{~cm}^{2}$

$30,000 \mathrm{dpm} / 100 \mathrm{~cm}^{2}$

$48,000 \mathrm{dpm} / 100 \mathrm{~cm}^{2}$

$80,000 \mathrm{dpm} / 100 \mathrm{~cm}^{2}$

$48,000 \mathrm{dpm} / 100 \mathrm{~cm}^{2}$

$30,000 \mathrm{dpm} / 100 \mathrm{~cm}^{2}$

$>1,000,000 \mathrm{dpm} / 100 \mathrm{~cm}^{2}$

$1,794,000 \mathrm{dpm} / 100 \mathrm{~cm}^{2}$

$120,000 \mathrm{dpm} / 100 \mathrm{~cm}^{2}$

$>1,000,000 \mathrm{dpm} / 100 \mathrm{~cm}^{2}$ $48,000 \mathrm{dpm} / 100 \mathrm{~cm}^{2}$

$100,000 \mathrm{dpm} / 100 \mathrm{~cm}^{2}$

2,400,000 dpm/ $100 \mathrm{~cm}^{2}$

$18,000 \mathrm{dpm} / 100 \mathrm{~cm}^{2}$

$27,000 \mathrm{dpm} / 100 \mathrm{~cm}^{2}$

$15,000 \mathrm{dpm} / 100 \mathrm{~cm}^{2}$

$6,000 \mathrm{dpm} / 100 \mathrm{~cm}^{2}$

$400,000 \mathrm{dpm} / 100 \mathrm{~cm}^{2}$

$100,000 \mathrm{dpm} / 100 \mathrm{~cm}^{2}$

$36,000 \mathrm{dpm} / 100 \mathrm{~cm}^{2}$

$240,000 \mathrm{dpm} / 100 \mathrm{~cm}^{2}$

$210,000 \mathrm{dpm} / 100 \mathrm{~cm}^{2}$

$60,000 \mathrm{dpm} / 100 \mathrm{~cm}^{2}$

$200,000 \mathrm{dpm} / 100 \mathrm{~cm}^{2}$

$300,000 \mathrm{dpm} / 100 \mathrm{~cm}^{2}$

$800,000 \mathrm{dpm} / 100 \mathrm{~cm}^{2}$

$>6,000,000 \mathrm{dpm} / 100 \mathrm{~cm}^{2}$

$400,000 \mathrm{dpm} / 100 \mathrm{~cm}^{2}$

$10,000 \mathrm{dpm} / 100 \mathrm{~cm}^{2}$

$9,000 \mathrm{dpm} / 100 \mathrm{~cm}^{2}$
DATE

01/16/08

$01 / 17 / 08$

$01 / 22 / 08$

$01 / 23 / 08$

$01 / 23 / 08$

$01 / 24 / 08$

$01 / 24 / 08$

$01 / 25 / 08$

01/31/08

02/01/08

02/04/08

02/09/08

02/09/08

02/09/08

02/11/08

02/11/08

02/12/08

02/19/08

$02 / 20 / 08$

$02 / 21 / 08$

02/21/08

02/22/08

02/25/08

$02 / 26 / 08$

02/27/08

$02 / 27 / 08$

02/28/08

02/28/08

02/29/08

03/04/08

03/04/08

03/05/08

03/06/08

03/08/08

03/12/08

03/12/08

03/12/08

03/17/08

03/18/08

03/18/08

03/19/08

03/24/08

03/24/08

03/25/08

03/26/08

03/27/08

03/28/08

03/31/08

04/01/08

04/01/08

04/07/08

04/10/08

04/10/08 
Table 7-2. Investigative Samples Not Analyzed, 2008. (Sheet 2 of 4)

FIELD READING

SAMPLEMATRIX

LOCATION

Rabbit fecal pellets (31)

North of Fenceline 241-AN Tank Farm

Northeast Fenceline 241-AN Tank Farm

Around the 242-B/BL Building

Tumbleweed Fragments (16)

Tumbleweed Fragments

Tumbleweed Fragments (10)

Tumbleweeds (4-Wind Blown) Spot

Tumbleweed Fragments \& Soil

Rabbit Feces (20)

Tumbleweeds (4)

Rabbit Feces (2)

Tumbleweed

Rabbit

Tumbleweeds (4-Attached)

Tumbleweed Fragments (10)

Tumbleweeds (5)

Rabbit Fecal Pellet

Rabbit Fecal Pellet

Tumbleweed

Tumbleweed

Tumbleweed Fragment

Tumbleweeds (Deteriorating) (3)

Tumbleweeds (Several)

Mice (2)

Mouse

Specks

Tumbleweed Fragment

Tumbleweed Fragments (2)

Tumbleweed Fragments (2)

Tumbleweeds (Wind Blown)

Tumbleweeds (Wind Blown) Specks (5)

Tumbleweed and Fragments Tumbleweed

Tumbleweed fragment \& Spots

Tumbleweed and Fragments

Tumbleweeds (50)

Tumbleweed Fragments Tumbleweeds (2)

Tumbleweeds (Wind Blown)

Tumbleweeds (Wind Blown) Tumbleweed

Tumbleweed Fragments (3)

Mouse Feces (3)

Tumbleweed

Mouse Trap

Tumbleweed

Tumbleweed Fragments (40' X100') Tumbleweeds

Decomposing Rabbit Feces (5) Tumbleweed

Tumbleweeds (4)

Tumbleweeds (Wind Blown - 15)

Northeast of 241-T Tank Farm

On Top of the 216-S-17 Pond West Fence 241-BX/BY

Along Perimeter of 241-AW Tank Farm

Along North Perimeter of 241-U Tank Farm

Near 242-S and outside 241-S Tank Farm

Near 242-S and outside 241-S Tank Farm

Near 242-S and outside 241-S Tank Farm

West Perimeter of 241-A Tank Farm along Buffalo Ave

Trapped at the 272-S Building

Inside Weather Enclosure @ 218-E-12B

Along 241-A Tank Farm Perimeter Fenceline

Along West Fenceline 241-S Tank Farm

Outside the 241-SY Tank Farm Perimeter

Outside the 241-AZ Tank Farm Perimeter

West Fenceline 241-U Tank Farm

West Fenceline 241-BX Tank Farm

South of the 241-A Tank Farm Perimeter

On top of the 218-E-4 Burial Ground

Supporting Cleanup Activities @ 241-S Tank Farm Trapped at 242-B/BL

Trapped at 224-U Building

Outside Posted CA @ 218-W-4B

Outside 241-B Tank Farm Perimeter

Outside 241-B Tank Farm Perimeter

Outside 241-C Tank Farm Perimeter

Northeast Perimeter Fence of 200-E

Northeast Perimeter Fence of 200-E 218-W-4B Trench TV7CA

Northeast Perimeter Fence of 200-E

West Perimeter Fenceline @ 241-U Tank Farm

200-E-114 PL from 241-B Tank Farm to BC Cribs

Old Transfer Line East of 241-B Tank Farm

Growing on top of the 216-U-10 Pond

On UPR-200-E-101 South of 242-B/BL

Outside 241-T Tank Farm Perimeter Fence

South of the 2727-WA Sodium Storage Area

Along 19th Street

West fence of FFS area north of 19th Ave.

Along West Perimeter Fence of 241-AZ

Inside 218-W-3AE Solid Waste Burial 221-U Plant Fenceline

241-U Tank Farm Perimeter 2727-WA Fenceline

Outside the Northeast Perimeter 218-E-12B LLBG East Fenceline 241-B Tank Farm

North Perimeter Fenceline of 241-SY Tank Farm

South Fenceline of 241-U Tank Farm West Barrier Wall of PFP

Outside PFP Security Barrier
(Beta/Gamma)

$>30,000 \mathrm{dpm} / 100 \mathrm{~cm}^{2}$

DATE

$>30,000 \mathrm{dpm} / 100 \mathrm{~cm}^{2}$

04/21/08

$04 / 21 / 08$

$04 / 23 / 08$

$04 / 29 / 08$

$04 / 29 / 08$

05/01/08

05/05/08

$05 / 05 / 08$

05/06/08

$05 / 06 / 08$

05/07/08

$05 / 08 / 08$

$05 / 09 / 08$

05/09/08

$05 / 12 / 08$

$05 / 12 / 08$

$05 / 13 / 08$

$05 / 13 / 08$

$05 / 13 / 08$

$05 / 13 / 08$

$05 / 13 / 08$

$05 / 13 / 08$

05/13/08

$05 / 14 / 08$

$05 / 14 / 08$

$05 / 15 / 08$

$05 / 19 / 08$

05/20/08

$05 / 27 / 08$

$05 / 29 / 08$

$06 / 02 / 08$

$06 / 05 / 08$

06/05/08

$06 / 12 / 08$

$06 / 12 / 08$

$06 / 17 / 08$

$06 / 23 / 08$

$06 / 30 / 08$

06/30/08

07/01/08

07/02/08

07/07/08

07/07/08

07/08/08

07/10/08

07/15/08

07/18/08

07/18/08

07/21/08

$07 / 22 / 08$

07/22/08

07/22/08

07/23/08 
Table 7-2. Investigative Samples Not Analyzed, 2008. (Sheet 3 of 4)

FIELD READING

SAMPLEMATRIX

LOCATION

(Beta/Gamma)

Tumbleweeds (Wind Blown - 4)

Tumbleweeds ( 300)

Tumbleweeds ( 300)

Rabbit Fecal Pellet (1)

Rabbit Fecal Pellets (20)

Spots in Soil Suspect Rodent Urine (3)

Tumbleweed Fragments (5)

$$
\text { Soil Speck }
$$

Tumbleweed

Soil

Tumbleweed (Wind Blown)

Tumbleweed (Wind Blown)

Tumbleweed Fragment

Tumbleweeds (5)

Tumbleweed

Tumbleweed Fragment

Tumbleweed Fragments Tumbleweeds

Tumbleweed Fragments

Speck in Ant Mound Spot

Tumbleweeds (100)

Tumbleweeds (30)

Tumbleweeds (several)

Tumbleweeds

Crickets

Tumbleweed

Tumbleweed Fragments

Tumbleweed

Rabbit Feces

Tumbleweed Fragments

Tumbleweed Fragments

Dried Mouse \& Snake

Rabbit Feces

Tumbleweed Fragments

Rabbit Feces

Cottontail Rabbit

Tumbleweed Fragments

Tumbleweeds (25)

Tumbleweeds (25)

Tumbleweeds

Tumbleweed Fragments

Tumbleweed \& Multiple Fragments

Tumbleweeds \& Fragments (50) Specks

Tumbleweed \& Fragments

Tumbleweeds (10)

Rabbit Feces

Tumbleweed

Tumbleweeds (200)

soil

Tumbleweeds Wind Blown (3)

Tumbleweeds (200)

Outside the 241-S Tank Farm Perimeter Fence

On Top of the 216-U-10 Pond

South End of the 218-E-12B LLBG

Outside 244-U Instrument Building

Around 241-SY-272 @ 241-SY Tank Farm

Outside 241-B Tank Farm Perimeter

200-E-121 along Baltimore Ave.

Perimeter of 241-SY Tank Farm

$$
\text { At 291-S REDOX Stack }
$$

Contaminated soil found under 2" @ 241-TX/TY

Outside West Perimeter Fence 241-AZ Tank Farm Near LEF/ETF

Around FFS Laydown Yard

UPR-200-W-161 Around 207-U Basin

West fenceline 241-BX/BY Tank Farm Around the $200 \mathrm{LEF} / \mathrm{ETF}$

South Perimeter Fenceline of 241-B Tank Farm Corner of 19th \& Dayton Ave

West Perimeter Fence of 218-E-16 Grout Vaults at WTP

Speck in Ant Mound on top of 200-E-111 PL

Outside the posted CA @ 216-BC-26 Crib

Inside 218-E-12B Trench 94

Outside Gate 810 200-E-110

$$
\text { 200-E-135 }
$$

Between Northeast Perimeter Fence and Canton Ave

On Glue Board from inside 242-BBL

On Top of the 216-U-10 Pond

UPR-200-E-143 Northwest of the 244-AR Lift Station 200-E-109 north of 218-E-12B

Outside North Perimeter 241-SY Tank Farm

East of 241-BX tank Farm by MO824 East of 241-C tank Farm

Under CONEX Box Inside PUREX Fence Boundary

Outside 241-SX Tank Farm Perimeter 200-E-109 North of 218-E-12B

Outside the West and North Perimeters 241-S/SX/SY

Outside North Perimeter 241-SY Tank Farm UPR-200-W-38 South of T-Plant

On Top of the 216-A-30 Crib

200-E-109 north of the Trench 94

200-E-109 north of the Trench 94

Canton Ave Side of Gate 810 N. of Trench 94

Clean Drum Storage NE Side of 221-T Plant

On top of the 218-E-12B Solid Waste Burial

$$
\text { West of 241-S Tank Farm }
$$

200-E-109, North and East of 218-E-12B

On Top of the 216-U-10 Pond

Outside 241-S \& SX Tank Farm

West of 241-BY Tank Farm

On Top of the 216-U-10 Pond

A 10 ' X 20" area on top of 200-W-78

200-E-17 LERF Perimeter Fence

On top of 216-U-10 Covered Pond
$30,000 \mathrm{dpm} / 100 \mathrm{~cm}^{2}$

$56,000 \mathrm{dpm} / 100 \mathrm{~cm}^{2}$

$900,000 \mathrm{dpm} / 100 \mathrm{~cm}^{2}$

$300,000 \mathrm{dpm} / 100 \mathrm{~cm}^{2}$

$3,600,000 \mathrm{dpm} / 100 \mathrm{~cm}^{2}$

$1,000,000 \mathrm{dpm} / 100 \mathrm{~cm}^{2}$

$40,000 \mathrm{dpm} / 100 \mathrm{~cm}^{2}$

$60,000 \mathrm{dpm} / 100 \mathrm{~cm}^{2}$

$12,000 \mathrm{dpm} / 100 \mathrm{~cm}^{2}$

$90,000 \mathrm{dpm} / 100 \mathrm{~cm}^{2}$

$60,000 \mathrm{dpm} / 100 \mathrm{~cm}^{2}$

$40,000 \mathrm{dpm} / 100 \mathrm{~cm}^{2}$

$40,000 \mathrm{dpm} / 100 \mathrm{~cm}^{2}$

$54,000 \mathrm{dpm} / 100 \mathrm{~cm}^{2}$

$12,000 \mathrm{dpm} / 100 \mathrm{~cm}^{2}$

$18,000 \mathrm{dpm} / 100 \mathrm{~cm}^{2}$

$>1,000,000 \mathrm{dpm} / 100 \mathrm{~cm}^{2}$

$42,000 \mathrm{dpm} / 100 \mathrm{~cm}^{2}$

$72,000 \mathrm{dpm} / 100 \mathrm{~cm}^{2}$

$54,000 \mathrm{dpm} / 100 \mathrm{~cm}^{2}$

$163,000 \mathrm{dpm} / 100 \mathrm{~cm}^{2}$

$>1,000,000 \mathrm{dpm} / 100 \mathrm{~cm}^{2}$

$>1,000,000 \mathrm{dpm} / 100 \mathrm{~cm}^{2}$

$18,000 \mathrm{dpm} / 100 \mathrm{~cm}^{2}$

2,940,000 dpm/100cm2

$12,000 \mathrm{dpm} / 100 \mathrm{~cm}^{2}$

$54,000 \mathrm{dpm} / 100 \mathrm{~cm}^{2}$

$100,000 \mathrm{dpm} / 100 \mathrm{~cm}^{2}$

$>1,000,000 \mathrm{dpm} / 100 \mathrm{~cm}^{2}$

$850,000 \mathrm{dpm} / 100 \mathrm{~cm}^{2}$

$>1,000,000 \mathrm{dpm} / 100 \mathrm{~cm}^{2}$

$150,000 \mathrm{dpm} / 100 \mathrm{~cm}^{2}$

$200,000 \mathrm{dpm} / 100 \mathrm{~cm}^{2}$

$500,000 \mathrm{dpm} / 100 \mathrm{~cm}^{2}$

$>1,000,000 \mathrm{dpm} / 100 \mathrm{~cm}^{2}$

$>1,000,000 \mathrm{dpm} / 100 \mathrm{~cm}^{2}$

$420,000 \mathrm{dpm} / 100 \mathrm{~cm}^{2}$

$100,000 \mathrm{dpm} / 100 \mathrm{~cm}^{2}$

$150,000 \mathrm{dpm} / 100 \mathrm{~cm}^{2}$

$>1,000,000 \mathrm{dpm} / 100 \mathrm{~cm}^{2}$

$>1,000,000 \mathrm{dpm} / 100 \mathrm{~cm}^{2}$

$>1,000,000 \mathrm{dpm} / 100 \mathrm{~cm}^{2}$ $150,000 \mathrm{dpm} / 100 \mathrm{~cm}^{2}$

$2,700,000 \mathrm{dpm} / 100 \mathrm{~cm}^{2}$

$200,000 \mathrm{dpm} / 100 \mathrm{~cm}^{2}$

$>1,000,000 \mathrm{dpm} / 100 \mathrm{~cm}^{2}$ $54,000 \mathrm{dpm} / 100 \mathrm{~cm}^{2}$

$>1,000,000 \mathrm{dpm} / 100 \mathrm{~cm}^{2}$ $60,000 \mathrm{dpm} / 100 \mathrm{~cm}^{2}$

$114,000 \mathrm{dpm} / 100 \mathrm{~cm}^{2}$

$72,000 \mathrm{dpm} / 100 \mathrm{~cm}^{2}$

$600,000 \mathrm{dpm} / 100 \mathrm{~cm}^{2}$

$900,000 \mathrm{dpm} / 100 \mathrm{~cm}^{2}$
DATE

07/23/08

$07 / 24 / 08$

07/28/08

$07 / 31 / 08$

07/31/08

08/04/08

$08 / 05 / 08$

$08 / 12 / 08$

08/13/08

$08 / 15 / 08$

$08 / 19 / 08$

$08 / 20 / 08$

$08 / 20 / 08$

$08 / 26 / 08$

$08 / 27 / 08$

$08 / 28 / 08$

$08 / 28 / 08$

$08 / 28 / 08$

$09 / 03 / 08$

09/08/08

$09 / 08 / 08$

09/13/08

09/15/08

09/15/08

09/16/08

09/17/08

09/17/08

$09 / 24 / 08$

09/26/08

09/29/08

$10 / 06 / 08$

$10 / 06 / 08$

$10 / 09 / 08$

$10 / 14 / 08$

$10 / 14 / 08$

$10 / 16 / 08$

$10 / 20 / 08$

$10 / 23 / 08$

$10 / 23 / 08$

$10 / 24 / 08$

$10 / 25 / 08$

$10 / 27 / 08$

$10 / 28 / 08$

$10 / 28 / 08$

$10 / 29 / 08$

$10 / 29 / 08$

$11 / 03 / 08$

$11 / 04 / 08$

$11 / 04 / 08$

$11 / 04 / 08$

$11 / 05 / 08$

11/06/08

11/10/08 
Table 7-2. Investigative Samples Not Analyzed, 2008. (Sheet 4 of 4)

SAMPLE MATRIX

Tumbleweeds and Fragments

Tumbleweed Fragments

Tumbleweed Fragments Tumbleweeds

Tumbleweed and Fragments

Tumbleweeds (3)

Rabbit Feces

Tumbleweed Fragments

Tumbleweed Fragments

Tumbleweed Fragments

Tumbleweeds (2)

Tumbleweed Fragment 10" Long

$$
\text { Tumbleweed }
$$

Speck (suspect rabbit urine)
FIELD READING

(Beta/Gamma)

DATE

$1,200,000 \mathrm{dpm} / 100 \mathrm{~cm}^{2} \quad 11 / 10 / 08$

$>1,000,000 \mathrm{dpm} / 100 \mathrm{~cm}^{2} \quad 11 / 17 / 08$

$120,000 \mathrm{dpm} / 100 \mathrm{~cm}^{2} \quad 11 / 17 / 08$

$18,000 \mathrm{dpm} / 100 \mathrm{~cm}^{2} \quad 11 / 17 / 08$

$>1,000,000 \mathrm{dpm} / 100 \mathrm{~cm}^{2} \quad 11 / 18 / 08$

$30,000 \mathrm{dpm} / 100 \mathrm{~cm}^{2} \quad 11 / 21 / 08$

$>1,000,000 \mathrm{dpm} / 100 \mathrm{~cm}^{2} \quad 11 / 22 / 08$

$150,000 \mathrm{dpm} / 100 \mathrm{~cm}^{2} \quad 11 / 22 / 08$

$120,000 \mathrm{dpm} / 100 \mathrm{~cm}^{2} \quad 12 / 01 / 08$

$30,000 \mathrm{dpm} / 100 \mathrm{~cm}^{2} \quad 12 / 02 / 08$

$54,000 \mathrm{dpm} / 100 \mathrm{~cm}^{2} \quad 12 / 03 / 08$

$120,000 \mathrm{dpm} / 100 \mathrm{~cm}^{2} \quad 12 / 04 / 08$

$24,000 \mathrm{dpm} / 100 \mathrm{~cm}^{2} \quad 12 / 10 / 08$

$250,000 \mathrm{dpm} / 100 \mathrm{~cm}^{2} \quad 12 / 11 / 08$ 
This page intentionally left blank. 


\subsection{QUALITY ASSURANCE}

Quality assurance (QA) may be defined as the actions necessary to provide confidence that an item, process, or program meets or exceeds the user's requirements and expectations. The near-facility environmental monitoring QA program consists of procedures and guides to demonstrate that environmental monitoring techniques and analyses are performed within established limits of acceptance. The near-facility environmental monitoring QA program and its objectives are documented in HNF-EP-0538-11, Near-Facility Environmental Monitoring Quality Assurance Project Plan (McKinney 2008).

Written operating procedures are an integral part of near-facility environmental monitoring QA. Procedures for field operations are provided in internal manual FSWO-OEM-001 (FSWO 2008) and EP-DI-611 (FH 2008). This section briefly describes the essential components of the near-facility environmental monitoring QA program.

\subsection{DOCUMENTATION}

Record keeping is a vital part of any environmental monitoring program. Maintenance of environmental data is important from a QA standpoint, from a regulatory standpoint, and for trend analyses and optimization of environmental monitoring procedures. Each phase of near-facility environmental monitoring is documented. This documentation includes environmental sample logbooks, quarterly reports, annual reports, and occurrence reports.

\subsection{SAMPLE REPLICATION}

The quality of sample collection methods and strategies is assessed by replicating the original samples and the statistical evaluation of them. Field replicates were collected during 2008 for ambient air, soil, and vegetation samples. Air sample replicate results were 95\%, soil replicate results $91 \%$, and vegetation replicate results $98 \%$, the same as the original results (see Table 8-1). 
Table 8-1. Summary of Field Replicate Results for 2008.

\begin{tabular}{|c|c|c|c|c|}
\hline \multirow[b]{2}{*}{ Medium } & \multirow[b]{2}{*}{ Radionuclide } & \multicolumn{2}{|c|}{ Number of Results } & \multirow[b]{2}{*}{ \% Agreement } \\
\hline & & Compared & In Agreement & \\
\hline \multirow[t]{17}{*}{ Air } & ${ }^{60} \mathrm{Co}$ & 2 & 2 & 100 \\
\hline & ${ }^{90} \mathrm{Sr}$ & 2 & 2 & 100 \\
\hline & ${ }^{106} \mathrm{Ru}$ & 2 & 2 & 100 \\
\hline & ${ }^{125} \mathrm{Sb}$ & 2 & 2 & 100 \\
\hline & ${ }^{134} \mathrm{Cs}$ & 2 & 2 & 100 \\
\hline & ${ }^{137} \mathrm{Cs}$ & 2 & 2 & 100 \\
\hline & ${ }^{152} \mathrm{Eu}$ & 2 & 2 & 100 \\
\hline & ${ }^{154} \mathrm{Eu}$ & 2 & 2 & 100 \\
\hline & ${ }^{155} \mathrm{Eu}$ & 2 & 2 & 100 \\
\hline & ${ }^{234} \mathrm{U}$ & 2 & 2 & 100 \\
\hline & ${ }^{235} \mathrm{U}$ & 2 & 2 & 100 \\
\hline & ${ }^{238} \mathrm{U}$ & 2 & 2 & 100 \\
\hline & ${ }^{238} \mathrm{Pu}$ & 2 & 2 & 100 \\
\hline & ${ }^{239 / 240} \mathrm{Pu}$ & 2 & 2 & 100 \\
\hline & gross $\alpha$ & 26 & 24 & 92 \\
\hline & gross $\beta$ & 26 & 24 & 92 \\
\hline & Totals: & 80 & 76 & $95 \%$ \\
\hline \multirow[t]{19}{*}{ Soil } & ${ }^{60} \mathrm{Co}$ & 9 & 9 & 100 \\
\hline & ${ }^{65} \mathrm{Zn}$ & 9 & 6 & 66 \\
\hline & ${ }^{90} \mathrm{Sr}$ & 9 & 9 & 100 \\
\hline & ${ }^{103} \mathrm{Ru}$ & 9 & 9 & 100 \\
\hline & ${ }^{106} \mathrm{Ru}$ & 9 & 9 & 100 \\
\hline & ${ }^{113} \mathrm{Sn}$ & 9 & 9 & 100 \\
\hline & ${ }^{125} \mathrm{Sb}$ & 9 & 9 & 100 \\
\hline & ${ }^{134} \mathrm{Cs}$ & 9 & 8 & 88 \\
\hline & ${ }^{137} \mathrm{Cs}$ & 9 & 7 & 77 \\
\hline & ${ }^{144} \mathrm{Ce}$ & 9 & 9 & 100 \\
\hline & ${ }^{152} \mathrm{Eu}$ & 9 & 9 & 100 \\
\hline & ${ }^{154} \mathrm{Eu}$ & 9 & 9 & 100 \\
\hline & ${ }^{155} \mathrm{Eu}$ & 9 & 6 & 66 \\
\hline & ${ }^{234} \mathrm{U}$ & 9 & 9 & 100 \\
\hline & ${ }^{235} \mathrm{U}$ & 9 & 9 & 100 \\
\hline & ${ }^{238} \mathrm{U}$ & 9 & 9 & 100 \\
\hline & ${ }^{238} \mathrm{Pu}$ & 9 & 7 & 77 \\
\hline & ${ }^{239 / 240} \mathrm{Pu}$ & 9 & 7 & 77 \\
\hline & Totals: & 162 & 149 & $91 \%$ \\
\hline \multirow[t]{19}{*}{ Vegetation } & ${ }^{60} \mathrm{Co}$ & 5 & 5 & 100 \\
\hline & ${ }^{65} \mathrm{Zn}$ & 5 & 4 & 80 \\
\hline & ${ }^{90} \mathrm{Sr}$ & 5 & 5 & 100 \\
\hline & ${ }^{103} \mathrm{Ru}$ & 5 & 5 & 100 \\
\hline & ${ }^{106} \mathrm{Ru}$ & 5 & 5 & 100 \\
\hline & ${ }^{113} \mathrm{Sn}$ & 5 & 5 & 100 \\
\hline & ${ }^{125} \mathrm{Sb}$ & 5 & 5 & 100 \\
\hline & ${ }^{134} \mathrm{Cs}$ & 5 & 5 & 100 \\
\hline & ${ }^{137} \mathrm{Cs}$ & 5 & 5 & 100 \\
\hline & ${ }^{144} \mathrm{Ce}$ & 5 & 5 & 100 \\
\hline & ${ }^{152} \mathrm{Eu}$ & 5 & 5 & 100 \\
\hline & ${ }^{154} \mathrm{Eu}$ & 5 & 5 & 100 \\
\hline & ${ }^{155} \mathrm{Eu}$ & 5 & 5 & 100 \\
\hline & ${ }^{234} \mathrm{U}$ & 5 & 5 & 100 \\
\hline & ${ }^{235} \mathrm{U}$ & 5 & 5 & 100 \\
\hline & ${ }^{238} \mathrm{U}$ & 5 & 5 & 100 \\
\hline & ${ }^{238} \mathrm{Pu}$ & 5 & 5 & 100 \\
\hline & ${ }^{239 / 240} \mathrm{Pu}$ & 5 & 5 & 100 \\
\hline & Totals: & 90 & 89 & $98 \%$ \\
\hline
\end{tabular}


Sampling methods and strategies were considered acceptable if, for a given sample medium, the overall agreement of all isotopic comparisons made between "original” and "replicate" samples were:

- Equal to or greater than $75 \%$ for air samples

- Equal to or greater than 50\% for soil and vegetation samples.

The concentrations of a sample and its replicate were considered to be "in close agreement" (meaning the concentrations are, for all practical purposes, identical) if either of the following applies:

- Each concentration falls within the error range of the other; or

- Both the concentration of the sample and its replicate are "essentially zero."

The concentrations of a sample and its replicate were considered to be "in agreement" (meaning the concentrations are close to the same value) if one of the following applies:

- On a plot, the uncertainty error bars of the sample and its replicate overlap; or

- The lower uncertainty values of both the sample and its replicate extend below the (contractual) minimum detectable concentration; or

- The relative percent difference was $<30 \%$ or the percent significant difference was $<15 \%$.

\subsection{DATA ANALYSIS}

Environmental data are reviewed to determine compliance with applicable federal and company guides. The data are analyzed both graphically and by standard statistical tests to determine trends and impacts on the environment. Newly acquired data are compared with historical data and natural background levels. Routine environmental data are stored on both magnetic media (i.e., in a computer environment) and hardcopy printouts.

\subsection{TRAINING}

To ensure quality and consistency in sample collection and handling, all personnel performing such work received formal training. All radiological control technicians are required to complete a certification program. In addition, those radiological control technicians assigned to environmental monitoring receive special classroom orientation and on-the-job training by experienced personnel. Environmental Monitoring and Investigations personnel, in addition to their formal training received while obtaining professional degrees, have received training in courses taught through Washington State University, the Harvard School of Public Health, and various other institutions. 


\subsection{SAMPLE FREQUENCY}

1. Ambient air sample filters are collected biweekly.

2. Radiological surveys of roads are performed quarterly, bimonthly, or annually.

3. The TLDs are exchanged quarterly.

4. Radiological surveys of waste sites are performed quarterly, semiannually, or annually depending on the operating status, condition, and history of the site.

5. Soil and vegetation are collected annually.

\subsection{ANALYTICAL PROCEDURES}

Three laboratories provided routine analytical support to the near-facility environmental monitoring: PNNL, WSCF, and the 222-S Analytical Laboratory. Samples are processed and/or analyzed in accordance with prescribed procedures and quality control guides that are described briefly in the following paragraphs.

\subsubsection{Pacific Northwest National Laboratory Radiation Standards and Engineering}

8.6.1.1 Thermoluminescent Dosimeters. External radiation levels are measured using TLDs. The Hanford Site uses the Harshaw 8807 dosimeter and the Harshaw 8800 reader. The TLDs are calibrated, packaged, and read by the PNNL Radiation Calibration Laboratory, Radiation Standards and Engineering Department. All TLD work is performed in accordance with formal, written procedures.

\subsubsection{Waste Sampling and Characterization Facility and 222-S Analytical Laboratory}

The WSCF and 222-S laboratories provide analytical support to near-facility environmental monitoring. Formal, written laboratory procedures are used in analyzing samples. The WSCF is used for the samples containing typical environmental levels of radioactivity. The WSCF also participates in an annual QA Task Force intercomparison program coordinated by the Radiation Protection Division of the Washington State Department of Health. The 222-S laboratory is typically used for preparation of selected samples and/or analyses of samples containing higher-than-normal levels of radioactivity. Additional discussion regarding the WSCF QA program can be found in Section 10.17 of PNNL-18427 (PNNL 2009a). 


\subsection{GLOSSARY}

Accessible Soils: Hanford soils that are not behind security fences must meet a $10 \mathrm{mrem} / \mathrm{yr}$ effective dose equivalent (EDE) limit from Hanford Site operations to the most exposed member of the public.

Average Soil Contamination: Contamination generally dispersed through the soil. Numerically, the radioactivity content averaged over a suitable mass of soil.

Background Radiation: Refers to regional levels of radioactivity produced by sources other than those of specific interest (e.g., the nuclear activities at the Hanford Site).

Becquerel (Bq): The standard international unit of radioactivity. One Becquerel is one disintegration per second or: $\mathrm{Bq}=2.7 \mathrm{E}-11 \mathrm{Ci}$.

Biological Transport: Means of biological transport may include one or more of the following processes:

- Movement of subsurface radioactivity to the surface by physiological vegetative processes.

- Dispersion of such vegetation by the wind.

- Contaminated urine and feces deposited by animals that have gained access to and ingested radioactive materials.

- Contaminated animals themselves that have ingested radioactive materials directly or ingested other contaminated animals or plants.

- Physical displacement of radioactive materials by burrowing animals.

- Nests built using contaminated materials.

Biota: The plant and animal life of a specific region.

Burial Ground: A land area specifically designated to receive contaminated solid or solidified liquid waste packages and equipment. The contaminated articles are usually placed in trenches and covered with overburden.

Calibration: Determining the deviation of an instrument from a standard traceable to the National Bureau of Standards or other recognized agency and reporting the deviations and/or eliminating them by adjustment. 
Chemical Processing: Chemical treatment of material to separate desired components selectively. At the Hanford Site, plutonium, uranium, and fission products were chemically separated from irradiated fuels.

Committed Dose Equivalent: The predicted total dose equivalent to a tissue or organ over a 50-year period after a known intake of a radionuclide into the body. It does not include contributions from external dose. Expressed in units of rem (or sievert).

Committed Effective Dose Equivalent: The sum of the committed dose equivalents to various tissues in the body, each multiplied by the appropriate weighing factor. Expressed in units of rem (or sievert).

Composite Sample: A number of samples initially collected from a sample medium and combined into a single sample; this sample is analyzed for the contaminants of concern.

\section{Comprehensive Environmental Response, Compensation, and Liability Act of 1980}

(CERCLA): Commonly known as "Superfund," CERCLA was enacted to respond to uncontrolled releases of hazardous substances to the environment, primarily at inactive sites that were not adequately addressed by the Resource Conservation and Recovery Act of 1976 (RCRA). CERCLA also applies to actively managed facilities and any onshore or offshore facility.

Controlled Area: An area where access is controlled to protect individuals from exposure to radiation and/or radioactive materials.

Contamination Area: Any area where contamination levels are greater than the values specified in Chapter 2, Table 2-2 of HNF-5173, PHMC Radiological Control Manual (FH 2007) but less than or equal to 100 times those values.

Crib: An underground structure designed to receive liquid waste that percolates into the soil directly or percolates into the soil after having traveled through a connected tile field.

Decommissioning: Actions taken to reduce the potential health and safety impacts of DOE controlled contaminated facilities. Actions could include stabilizing, reducing, or removing radioactivity or demolishing the contaminated facilities.

Decontamination: The removal of radioactive or hazardous contamination from facilities, equipment, or soils by washing, heating, chemical or electrochemical treating, mechanical cleaning, or other techniques.

Derived Concentration Guide for Public Exposure (DCG-Public): The concentration of a radionuclide in air or water that, under conditions of continuous exposure for one year by one exposure mode (e.g., ingestion of water, submersion in air, or inhalation of air), would result in an EDE equal to the annual dose limit applicable to the group exposed. For exposure of the public, the DCG is the radionuclide concentration in air or water that would result in an EDE of $100 \mathrm{mrem}(1 \mathrm{mSv})$ to a person having the characteristics of the reference man. 
Diffuse Source: A source or sources of radioactive or chemical contaminants released into the environment that do not have a defined point or origin of release (a nonpoint source).

Disposal Facility: Any facility or part of a facility where hazardous and/or radioactive waste is intentionally placed or where any land or water wastes will remain after closure.

Ditch: An open surface site for transport of liquid wastes to a pond or trench structure designed for percolation.

Ecology: State of Washington, Department of Ecology.

Effective Dose Equivalent (EDE): The summation of the products of the dose equivalent received by specified tissues of the body and a tissue-specific weighing factor. This sum is a risk-equivalent value and can be used to estimate the health-effects risk of the exposed individual. The tissue-specific weighing factor represents the fraction of the total health risk resulting from uniform whole-body irradiation that would be contributed by that particular tissue. The EDE includes the committed EDE from internal deposition of radionuclides and the EDE caused by penetrating radiation from sources outside the body. EDE is expressed in units of rem (or sievert).

Effluent: An airborne or liquid discharge from a facility after all engineered waste treatment and effluent controls have been performed. The term includes onsite discharges to the atmosphere, lagoons, ponds, cribs, injection wells, French drains, or ditches. The term does not include solid waste stored or removed for disposal or waste that is contained in retention basins or tanks before treatment and/or disposal.

Environmental Monitoring Plan: A two-part document prepared for each site, facility, or process that uses, generates, releases, or manages significant pollutants or hazardous materials.

External Radiation: Radiation originating from a source outside the body.

Facility: A processing plant, tank farm, shop, laboratory, powerhouse, or laundry. Including all contiguous land and structures, other appurtenances, and improvements on land used for recycling, reusing, reclaiming, transferring, storing, and treating of dangerous waste (including treatment, storage, and disposal sites as well as groundwater wells). (40 CFR 264, "Standards for Owners and Operators of Hazardous Waste Treatment Storage and Disposal Facilities," and WAC 173-303-040.)

Facility-Specific Environmental Monitoring: Routine environmental monitoring of all environmental media (air, biota, etc.) around facility perimeters.

Field Blank: Aliquots of analyte-free water or solvents brought to the field in sealed containers and transported to the laboratory with the sample container. Field blanks include trip blanks and equipment blanks. 
Field Duplicate: Field duplicates are collected at specified frequencies and are used to document precision. The field duplicate precision depends on the variance of waste composition, sampling techniques, and analytical technique.

Fugitive Emissions: Material that is generated incidental to an operation, process, or activity and that is released or dispersed into the open air. Fugitive emissions occur via pathways that do not allow routine measurement at the point of release.

Grab Sample: A single sample removed from a sample medium over a short time interval.

High-Level Nuclear Waste: Spent nuclear fuel or radioactive waste resulting directly from the dissolution and reprocessing of spent nuclear fuel. Secondary waste streams resulting from the dissolution and reprocessing of spent nuclear fuel are not considered high-level waste.

Inaccessible Soils: Areas from which the general public is excluded (by fences, posting, patrols, or distance), but that are still subject to meteorological effects, are subject to a $10 \mathrm{mrem} / \mathrm{yr}$ operational EDE limit.

Inactive Crib: A crib that has been designated as permanently out of service.

Inactive Radioactive Waste Site: Any waste site that is no longer needed for current operational programs and that is not currently an active waste disposal site.

Inactive Waste Sites: Inactive waste sites include units such as burial grounds, unplanned release sites, cribs, ditches, ponds, trenches, and basins, abandoned storage areas, drains, singleshell tank piping, transfer pits, and jumper boxes.

Interim Closed: Areas designated as "Interim Closed" are released from the posting requirements when the remedial actions meet the operable unit's record of decision cleanup requirements.

Less Than Detectable: An analytical term for a concentration in a sample that is lower than the minimum detection capabilities of that analytical equipment or process.

Low-Level Waste: Any gaseous, liquid, or solid radioactive waste not classified as high-level waste, transuranic waste, or spent nuclear fuel, as defined by DOE Order 435.1, Radioactive Waste Management.

Mean: Average value of a series of measurements.

Minimum Detection Limit: Smallest amount or concentration of a radionuclide or nonradioactive element that can be reliably detected in a sample. 
Near Facility Environmental Monitoring: The collection and analysis of samples of air, water, soil, biota, and other media near nuclear facilities on DOE sites and their environs and the measurement of external radiation to demonstrate compliance with applicable standards and assess radiation exposures to employees and members of the public, and the near-field environment.

Nonroutine Activities: Any actions on a large-scale ( $>2$ hectares [5 acres]), including stabilization, soil removal, fixative or sealant application, other surface treatments, or other activities that could affect future remediation activities in an inactive waste site.

Not Detected: A reporting term which describes any or all of the following: the overall analytical error was greater than the radionuclide concentration itself; or, after allowing for the subtraction of the background level of the radionuclide, the resulting concentration was less than zero; or, no radio analytical peak was detected during the analysis.

Operable Unit: A discrete area for which an incremental step can be taken toward comprehensively addressing site problems. The cleanup of a site can be divided into a number of operable units, depending on the complexity of the problems associated with the site.

Operations: In this report, this term loosely refers to Fluor Project Hanford activities including chemical processing, waste management, and decommissioning.

Point Source: A single defined point (origin) of an airborne release, such as a vent or stack.

Pond: A surface impoundment used to contain or percolate low-level liquid radioactive waste, mixed waste, or hazardous waste.

Quality Assurance: A process designed to maintain the quality of the results of a program within established limits of acceptance.

Radiation Survey: Evaluation of an area or object with portable instruments to identify radioactive materials and radiation fields present.

Radioactive Byproduct: Any radioactive material (except special nuclear material) yielded in or made radioactive by exposure to the radiation incident to the process of producing or using special nuclear material.

Radiological Control Area: An area where access is controlled to protect individuals from exposure to radiation and/or radioactive materials. Radiological control areas include, but are not limited to, areas posted as radiation areas, surface contamination, and underground radioactive materials, to describe the radiological condition of the area within.

Radiological Posting: Information in the form of signs and barriers to inform people of radiological conditions that warrant avoidance or special precautions for entry. 
Representative Sample: A sample that can be expected to exhibit the average properties of the sample source.

Retired Waste Site: A waste site that is isolated and no longer available to receive waste in any form.

Routine Activities: Any actions on a small-scale ( $<2$ hectares [5 acres]), including radioactive hot-spot removal, vegetation removal, fencing, posting, herbicide spraying, stabilization, or immediate spill response) in an inactive waste site. In general, these routine actions shall not interfere with RCRA/CERCLA response or site investigations.

Sampling System: Instrumentation and equipment that remove a part of a liquid or airborne waste stream for subsequent quantitative determination of stream parameters. The system generally employs such devices as filters, other sample collection media, or effluent traps of some kind. A continuous sampling system removes a part of the stream continuously except during sample change, maintenance, repair, or other necessary outages. A grab sampling system removes an instantaneous part of the stream or removes a part of the stream over a time period.

Sediment Column: The sediment beneath a crib. It can mean either all the sediment beneath the bottom of the crib extending to the water table or all sediment beneath a crib contaminated by radioactive materials.

Site: The location of a significant event, a prehistoric or historic occupation or activity, or a building or structure (whether standing, ruined, or vanished) where the location itself maintains historical or archeological value, regardless of the value of any existing structure.

Soil at depth: Soil below $91 \mathrm{~cm}$ (36 in.).

Soil Contamination: Contaminated soil not releasable in accordance with DOE Order 5400.5.

Solid Waste: Any discarded material that is not excluded by WAC 173-303-017(2) or that is not excluded by a variance granted under WAC 173-303-017(5). Materials are solid waste if they are: (1) abandoned by being disposed of, burned, or incinerated, or (2) accumulated, stored, or treated (but not recycled) before (or in lieu of) being abandoned by being disposed of, burned, or incinerated. In addition, a solid waste includes any material considered to be inherently wastelike.

Speck Contamination: Single grains of soil, rust particles, feces, or pieces of vegetation.

Spot Contamination: A spot or quantity of contamination less than $1 \mathrm{~cm}^{3}$ (0.06 in.) in volume, or areal contamination less than $15 \mathrm{~cm}^{2}\left(2.3 \mathrm{in}^{2}\right)$ in area.

Stabilization: The process of covering surface contaminated areas with clean backfill or topsoil. 
Standard: A specified set of rules or conditions concerned with the classification of components; delineation of procedures; definition of terms; designation of materials, performance, design, or operations; or measurements of quality in describing materials, products, systems, services, or practices. A standard is more general than a procedure or specification and more specific than a criterion.

Standard Deviation: A measure of the range of values about the mean.

Standard Error of the Mean: A measure of the uncertainty in the estimated mean of averaged values.

Surface Soil: Soil from $0 \mathrm{~cm}(0$ in.) to $5 \mathrm{~cm}$ (2 in.) deep.

Surplus Facilities: Surplus facilities include all facilities that have been accepted into a decommissioning program.

Survey: A method to detect the release, disposal, or presence of radioactive materials or hazardous substances under a specific set of conditions to determine actual or potential hazards. Such an evaluation may include, but is not limited to, tests, physical examinations, and measurements of radiation or concentrations of materials.

Suspect Waste Site: A site, believed to have been previously unknown or undocumented, that, because of characteristics present at the site or historical information about the site, is suspected of containing waste (i.e., non-dangerous, hazardous, dangerous, mixed, and radioactive).

Tank Farm: An area of large underground tanks designed to store high-level liquid waste.

Thermoluminescent Dosimeter: A chip or series of chips used for measuring external gamma radiation. It consists of a material capable of absorbing energy imparted by ionizing radiation, then emitting light as a result of thermal stimulation. A measure of that light is proportional to the radioactivity absorbed.

Total Analytical Uncertainty: All analytical measurements include some degree of uncertainty as a consequence of a series of unavoidable and unintentional inaccuracies related to the collection and analysis of samples. Examples of these inaccuracies can include errors associated with reading and recording results, sample handling and processing, instrument calibrations, numerical rounding, and randomness of radioactive decay. The total analytical uncertainty value implies that approximately $95 \%$ of the time a recount or reanalysis of the sample would give a value somewhere in the range between the initial reported value plus or minus the total analytical uncertainty.

Trip Blank: A type of field blank used to accompany sample containers to and from the field and to detect contamination or cross-contamination that occurs during sample handling and transportation. 
Uncontaminated Soil: A soil or a land area that requires no controls or restrictions in any way for radiation protection purposes and/or meets the contamination limit specifications.

Underground Radioactive Material: A radiological posting status where subsurface radioactivity is present but where surface contamination does not exceed the soil standards.

Unity Rule: If more than one radionuclide is present, the sum of the fractions represented by each radionuclide concentration divided by its respective limiting concentration (administrative control value) shall not exceed unity. This rule could also apply to parameters other than radionuclide concentration.

Unplanned Release Site: An area that was contaminated by an unplanned release of radioactive contamination, making it a radiological control area.

Unrestricted Release: Values below which unrestricted release of soils will occur will be defined in an applicable record of decision.

U.S. Environmental Protection Agency: The federal agency chartered with carrying out and monitoring the environmental regulations.

Waste Management: The activity involved with storing, disposing of, shipping, handling, and monitoring all radioactive waste.

Waste Sites: Any facility used for the planned disposal of hazardous, radioactive, toxic, or nonradioactive/nontoxic waste. 
Table 9-1. Radionuclide Nomenclature.

\begin{tabular}{lcc} 
Radionuclide & Symbol & Half-Life \\
\hline Tritium & ${ }^{3} \mathrm{H}$ & $12.3 \mathrm{yr}$ \\
Beryllium-7 & ${ }^{7} \mathrm{Be}$ & $53.28 \mathrm{~d}$ \\
Carbon-14 & ${ }^{14} \mathrm{C}$ & $5.72 \mathrm{E}+03 \mathrm{yr}$ \\
Sodium-22 & ${ }^{22} \mathrm{Na}$ & $2.6 \mathrm{yr}$ \\
Potassium-40 & ${ }^{40} \mathrm{~K}$ & $1.26 \mathrm{E}+09 \mathrm{yr}$ \\
Argon-41 & ${ }^{41} \mathrm{Ar}$ & $1.8 \mathrm{~h}$ \\
Chromium-51 & ${ }^{51} \mathrm{Cr}$ & $27.7 \mathrm{~d}$ \\
Manganese-54 & ${ }^{54} \mathrm{Mn}$ & $312 \mathrm{~d}$ \\
Cobalt-58 & ${ }^{58} \mathrm{Co}$ & $71 \mathrm{~d}$ \\
Iron-59 & ${ }^{59} \mathrm{Fe}$ & $45 \mathrm{~d}$ \\
Cobalt-60 & ${ }^{60} \mathrm{Co}$ & $5.3 \mathrm{yr}$ \\
Nickel-63 & ${ }^{63} \mathrm{Ni}$ & $100 \mathrm{yr}$ \\
Zinc-65 & ${ }^{65} \mathrm{Zn}$ & $243.8 \mathrm{~d}$ \\
Krypton-85 & ${ }^{85} \mathrm{Kr}$ & $10.7 \mathrm{yr}$ \\
Strontium-89 & ${ }^{89} \mathrm{Sr}$ & $50.5 \mathrm{~d}$ \\
Strontium-90 & ${ }^{90} \mathrm{Sr}$ & $29.1 \mathrm{yr}$ \\
Niobium-95 & ${ }^{95} \mathrm{Nb}$ & $35.0 \mathrm{~d}$ \\
Zirconium-95 & ${ }^{95} \mathrm{Zr}$ & $64.0 \mathrm{~d}$ \\
Technetium-99 & ${ }^{99} \mathrm{Tc}$ & $2.12 \mathrm{E}+05 \mathrm{yr}$ \\
Ruthenium-103 & ${ }^{103} \mathrm{Ru}$ & $39.4 \mathrm{~d}$ \\
Ruthenium-106 & ${ }^{106} \mathrm{Ru}$ & $1.0 \mathrm{yr}$ \\
Tin-113 & ${ }^{113} \mathrm{Sn}$ & $115 \mathrm{~d}$ \\
Antimony-124 & ${ }^{124} \mathrm{Sb}$ & $60 \mathrm{~d}$ \\
Antimony-125 & ${ }^{125} \mathrm{Sb}$ & $2.7 \mathrm{yr}$ \\
Iodine-129 & ${ }^{129} \mathrm{I}$ & $1.7 \mathrm{E}+07 \mathrm{yr}$ \\
Iodine-131 & ${ }^{131} \mathrm{I}$ & $8.0 \mathrm{~d}$ \\
Barium-133 & ${ }^{133} \mathrm{Ba}$ & $10.53 \mathrm{yr}$ \\
& &
\end{tabular}

\begin{tabular}{lcc}
\multicolumn{1}{c}{ Radionuclide } & Symbol & Half-Life \\
\hline Cesium-134 & ${ }^{134} \mathrm{Cs}$ & $2.1 \mathrm{yr}$ \\
Cesium-137 & ${ }^{137} \mathrm{Cs}$ & $30.3 \mathrm{yr}$ \\
Cerium-141 & ${ }^{141} \mathrm{Ce}$ & $32.5 \mathrm{~d}$ \\
Cerium-144 & ${ }^{144} \mathrm{Ce}$ & $284.6 \mathrm{~d}$ \\
Promethium-147 & ${ }^{147} \mathrm{Pm}$ & $13.4 \mathrm{~min}$ \\
Europium-152 & ${ }^{152} \mathrm{Eu}$ & $13.5 \mathrm{yr}$ \\
Europium-154 & ${ }^{154} \mathrm{Eu}$ & $8.6 \mathrm{yr}$ \\
Europium-155 & ${ }^{155} \mathrm{Eu}$ & $4.7 \mathrm{yr}$ \\
Thallium-208 & ${ }^{208} \mathrm{Tl}$ & $3.1 \mathrm{~min}$ \\
Bismuth-212 & ${ }^{212} \mathrm{Bi}$ & $60.6 \mathrm{~min}$ \\
Lead-212 & ${ }^{212} \mathrm{~Pb}$ & $10.6 \mathrm{~h}$ \\
Polonium-212 & ${ }^{212} \mathrm{Po}$ & $0.3 \mathrm{x} 10^{-6} \mathrm{~s}$ \\
Polonium-216 & ${ }^{216} \mathrm{Po}$ & $0.15 \mathrm{~s}$ \\
Radon-220 & ${ }^{220} \mathrm{Rn}$ & $55.6 \mathrm{~s}$ \\
Radium-226 & ${ }^{226} \mathrm{Ra}$ & $1.60 \mathrm{E}+03 \mathrm{yr}$ \\
Radium-228 & ${ }^{228} \mathrm{Ra}$ & $5.75 \mathrm{yr}$ \\
Thorium-232 & ${ }^{232} \mathrm{Th}$ & $1.40 \mathrm{E}+10 \mathrm{yr}$ \\
Uranium Total & $\mathrm{U}$ or & $4.50 \mathrm{E}+09 \mathrm{yr}$ \\
& $\mathrm{Uranium}$ & \\
Uranium-234 & ${ }^{234} \mathrm{U}$ & $2.40 \mathrm{E}+05 \mathrm{yr}$ \\
Uranium-235 & ${ }^{235} \mathrm{U}$ & $7.00 \mathrm{E}+08 \mathrm{yr}$ \\
Uranium-236 & ${ }^{236} \mathrm{U}$ & $2.30 \mathrm{E}+07 \mathrm{yr}$ \\
Uranium-238 & ${ }^{238} \mathrm{U}$ & $4.50 \mathrm{E}+09 \mathrm{yr}$ \\
Plutonium-238 & ${ }^{238} \mathrm{Pu}$ & $87.7 \mathrm{yr}$ \\
Plutonium-239/240 & ${ }^{239,240} \mathrm{Pu}$ & $2.40 \mathrm{E}+04 \mathrm{yr}$ \\
Plutonium-241 & ${ }^{241} \mathrm{Pu}$ & $14.4 \mathrm{yr}$ \\
Americium-241 & ${ }^{241} \mathrm{Am}$ & $433 \mathrm{yr}$ \\
& & \\
& &
\end{tabular}


This page intentionally left blank. 


\subsection{STANDARDS}

Table 10-1. U.S. Department of Energy Derived Concentration Guides. ${ }^{a}$

\begin{tabular}{ccc} 
& \multicolumn{2}{c}{ DCG } \\
\cline { 2 - 3 } Radionuclide & (pCi/m $\left.\mathbf{m}^{3}\right)$ & (pCi// $)$ \\
\hline${ }^{3} \mathrm{H}$ & $1.0 \mathrm{E}+05$ & $2.0 \mathrm{E}+06$ \\
${ }^{14} \mathrm{C}$ & $6.0 \mathrm{E}+03$ & $7.0 \mathrm{E}+04$ \\
${ }^{40} \mathrm{~K}$ & $9.0 \mathrm{E}+02$ & $7.0 \mathrm{E}+03$ \\
${ }^{41} \mathrm{Ar}$ & $1.0 \mathrm{E}+04$ & $0.0 \mathrm{E}+00$ \\
${ }^{51} \mathrm{Cr}$ & $6.0 \mathrm{E}+04$ & $1.0 \mathrm{E}+06$ \\
${ }^{54} \mathrm{Mn}$ & $2.0 \mathrm{E}+03$ & $5.0 \mathrm{E}+04$ \\
${ }^{59} \mathrm{Fe}$ & $8.0 \mathrm{E}+02$ & $2.0 \mathrm{E}+04$ \\
${ }^{58} \mathrm{Co}$ & $2.0 \mathrm{E}+03$ & $4.0 \mathrm{E}+04$ \\
${ }^{60} \mathrm{Co}$ & $8.0 \mathrm{E}+01$ & $5.0 \mathrm{E}+03$ \\
${ }^{65} \mathrm{Zn}$ & $6.0 \mathrm{E}+02$ & $9.0 \mathrm{E}+03$ \\
${ }^{85} \mathrm{Kr}$ & $3.0 \mathrm{E}+06$ & $0.0 \mathrm{E}+00$ \\
${ }^{89} \mathrm{Sr}$ & $3.0 \mathrm{E}+02$ & $2.0 \mathrm{E}+04$ \\
${ }^{90} \mathrm{Sr}$ & $9.0 \mathrm{E}+00$ & $1.0 \mathrm{E}+03$ \\
${ }^{95} \mathrm{Zr}$ & $6.0 \mathrm{E}+02$ & $4.0 \mathrm{E}+04$ \\
${ }^{95} \mathrm{Nb}$ & $3.0 \mathrm{E}+03$ & $6.0 \mathrm{E}+04$ \\
${ }^{99} \mathrm{Tc}$ & $2.0 \mathrm{E}+03$ & $1.0 \mathrm{E}+05$ \\
${ }^{103} \mathrm{Ru}$ & $2.0 \mathrm{E}+03$ & $5.0 \mathrm{E}+04$ \\
${ }^{106} \mathrm{Ru}$ & $3.0 \mathrm{E}+01$ & $6.0 \mathrm{E}+03$ \\
${ }^{113} \mathrm{Sn}$ & $1.0 \mathrm{E}+03$ & $5.0 \mathrm{E}+04$ \\
${ }^{134} \mathrm{I}$ & $4.0 \mathrm{E}+02$ & $3.0 \mathrm{E}+03$ \\
${ }^{124} \mathrm{Sb}$ & $6.0 \mathrm{E}+02$ & $1.0 \mathrm{E}+04$ \\
${ }^{125} \mathrm{Cs}$ & $2.0 \mathrm{E}+02$ & $2.0 \mathrm{E}+03$ \\
${ }^{137} \mathrm{Cs}$ & $4.0 \mathrm{E}+02$ & $3.0 \mathrm{E}+03$ \\
${ }^{141} \mathrm{Ce}$ & $1.0 \mathrm{E}+03$ & $5.0 \mathrm{E}+04$ \\
& $3.0 \mathrm{E}+01$ & $7.0 \mathrm{E}+03$ \\
& & $5.0 \mathrm{E}+04$ \\
& &
\end{tabular}

\begin{tabular}{|c|c|c|}
\hline \multirow[b]{3}{*}{ Radionuclide } & \multicolumn{2}{|c|}{ DCG } \\
\hline & Air & Liquid \\
\hline & $\left(\mathrm{pCi} / \mathrm{m}^{3}\right)$ & $(\mathrm{pCi} / \mathrm{L})$ \\
\hline${ }^{147} \mathrm{Pm}$ & $3.0 \mathrm{E}+02$ & $1.0 \mathrm{E}+05$ \\
\hline${ }^{152} \mathrm{Eu}$ & $5.0 \mathrm{E}+01$ & $2.0 \mathrm{E}+04$ \\
\hline${ }^{154} \mathrm{Eu}$ & $5.0 \mathrm{E}+01$ & $2.0 \mathrm{E}+04$ \\
\hline${ }^{155} \mathrm{Eu}$ & $3.0 \mathrm{E}+02$ & $1.0 \mathrm{E}+05$ \\
\hline${ }^{208} \mathrm{Tl}$ & $5.0 \mathrm{E}+03$ & $0.0 \mathrm{E}+00$ \\
\hline${ }^{212} \mathrm{Bi}$ & $6.0 \mathrm{E}+02$ & $1.0 \mathrm{E}+05$ \\
\hline${ }^{214} \mathrm{Bi}$ & $2.0 \mathrm{E}+03$ & $6.0 \mathrm{E}+05$ \\
\hline${ }^{212} \mathrm{~Pb}$ & $8.0 \mathrm{E}+01$ & $3.0 \mathrm{E}+03$ \\
\hline${ }^{214} \mathrm{~Pb}$ & $2.0 \mathrm{E}+03$ & $2.0 \mathrm{E}+05$ \\
\hline${ }^{212} \mathrm{Po}$ & $1.0 \mathrm{E}+00$ & $8.0 \mathrm{E}+01$ \\
\hline${ }^{216} \mathrm{Po}$ & $1.0 \mathrm{E}+00$ & $8.0 \mathrm{E}+01$ \\
\hline${ }^{220} \mathrm{Rn}$ & $3.0 \mathrm{E}+03$ & $0.0 \mathrm{E}+00$ \\
\hline${ }^{224} \mathrm{Ra}$ & $4.0 \mathrm{E}+00$ & $4.0 \mathrm{E}+02$ \\
\hline${ }^{226} \mathrm{Ra}$ & $1.0 \mathrm{E}+00$ & $1.0 \mathrm{E}+02$ \\
\hline${ }^{228} \mathrm{Ac}$ & $4.0 \mathrm{E}+01$ & $6.0 \mathrm{E}+04$ \\
\hline${ }^{232} \mathrm{Th}$ & $7.0 \mathrm{E}-03$ & $5.0 \mathrm{E}+01$ \\
\hline${ }^{\text {Total }} U$ & $1.0 \mathrm{E}-01$ & $6.0 \mathrm{E}+02$ \\
\hline${ }^{234} U$ & $9.0 \mathrm{E}-02$ & $5.0 \mathrm{E}+02$ \\
\hline${ }^{235} \mathrm{U}$ & $1.0 \mathrm{E}-01$ & $6.0 \mathrm{E}+02$ \\
\hline${ }^{236} \mathrm{U}$ & $1.0 \mathrm{E}-01$ & $5.0 \mathrm{E}+02$ \\
\hline${ }^{238} \mathrm{U}$ & $1.0 \mathrm{E}-01$ & $6.0 \mathrm{E}+02$ \\
\hline${ }^{238} \mathrm{Pu}$ & $3.0 \mathrm{E}-02$ & $4.0 \mathrm{E}+01$ \\
\hline${ }^{239,240} \mathrm{Pu}$ & $2.0 \mathrm{E}-02$ & $3.0 \mathrm{E}+01$ \\
\hline${ }^{241} \mathrm{Pu}$ & $1.0 \mathrm{E}+00$ & $2.0 \mathrm{E}+03$ \\
\hline${ }^{241} \mathrm{Am}$ & 2.0E-02 & $3.0 \mathrm{E}+01$ \\
\hline Total Alpha & 2.0E-02 & $3.0 \mathrm{E}+01$ \\
\hline Total Beta & $9.0 \mathrm{E}+00$ & $1.0 \mathrm{E}+03$ \\
\hline
\end{tabular}

${ }^{\mathrm{a}}$ From DOE Order 5400.5.

DCG $=$ derived concentration guides 
Table 10-2. EPA Concentration Levels for Environmental Compliance. ${ }^{\mathrm{a}}$ (Radionuclide Concentrations $\left[\mathrm{pCi} / \mathrm{m}^{3}\right]$ in Air)

\begin{tabular}{cc} 
Radionuclide & Concentration \\
\hline${ }^{3} \mathrm{H}$ & $1.5 \mathrm{E}+03$ \\
${ }^{14} \mathrm{C}$ & $1.0 \mathrm{E}+01$ \\
${ }^{40} \mathrm{~K}$ & $2.7 \mathrm{E}-02$ \\
${ }^{41} \mathrm{Ar}$ & $1.7 \mathrm{E}+03$ \\
${ }^{51} \mathrm{Cr}$ & $3.1 \mathrm{E}+01$ \\
${ }^{54} \mathrm{Mn}$ & $2.8 \mathrm{E}-01$ \\
${ }^{59} \mathrm{Fe}$ & $6.7 \mathrm{E}-01$ \\
${ }^{58} \mathrm{Co}$ & $6.7 \mathrm{E}-01$ \\
${ }^{60} \mathrm{Co}$ & $1.7 \mathrm{E}-02$ \\
${ }^{65} \mathrm{Zn}$ & $9.1 \mathrm{E}-02$ \\
${ }^{85} \mathrm{Kr}$ & $1.0 \mathrm{E}+06$ \\
${ }^{89} \mathrm{Sr}$ & $1.8 \mathrm{E}+00$ \\
${ }^{90} \mathrm{Sr}$ & $1.9 \mathrm{E}-02$ \\
${ }^{95} \mathrm{Zr}$ & $6.7 \mathrm{E}-01$ \\
${ }^{95} \mathrm{Nb}$ & $2.2 \mathrm{E}+00$ \\
${ }^{99} \mathrm{Tc}$ & $1.4 \mathrm{E}-01$ \\
${ }^{103} \mathrm{Ru}$ & $2.6 \mathrm{E}+00$ \\
${ }^{106} \mathrm{Ru}$ & $3.4 \mathrm{E}-01$ \\
${ }^{113} \mathrm{Sn}$ & $1.4 \mathrm{E}+00$ \\
${ }^{124} \mathrm{Sb}$ & $5.3 \mathrm{E}-01$ \\
${ }^{125} \mathrm{Sb}$ & $1.6 \mathrm{E}-01$ \\
${ }^{129} \mathrm{I}$ & $9.1 \mathrm{E}-03$ \\
${ }^{125} \mathrm{Cs}$ & $2.1 \mathrm{E}-01$ \\
\hline & $2.7 \mathrm{E}-02$ \\
&
\end{tabular}

\begin{tabular}{cc} 
Radionuclide & Concentration \\
\hline${ }^{137} \mathrm{Cs}$ & $1.9 \mathrm{E}-02$ \\
${ }^{141} \mathrm{Ce}$ & $6.3 \mathrm{E}+00$ \\
${ }^{144} \mathrm{Ce}$ & $6.2 \mathrm{E}-01$ \\
${ }^{147} \mathrm{Pm}$ & $1.1 \mathrm{E}+01$ \\
${ }^{152} \mathrm{Eu}$ & $2.0 \mathrm{E}-02$ \\
${ }^{154} \mathrm{Eu}$ & $2.3 \mathrm{E}-02$ \\
${ }^{155} \mathrm{Eu}$ & $5.9 \mathrm{E}-01$ \\
${ }^{212} \mathrm{Bi}$ & $5.6 \mathrm{E}+01$ \\
${ }^{214} \mathrm{Bi}$ & $1.4 \mathrm{E}+02$ \\
${ }^{212} \mathrm{~Pb}$ & $6.3 \mathrm{E}+00$ \\
${ }^{214} \mathrm{~Pb}$ & $1.2 \mathrm{E}+02$ \\
${ }^{224} \mathrm{Ra}$ & $1.5 \mathrm{E}-01$ \\
${ }^{226} \mathrm{Ra}$ & $3.3 \mathrm{E}-03$ \\
${ }^{228} \mathrm{Ac}$ & $3.7 \mathrm{E}+00$ \\
${ }^{232} \mathrm{Th}$ & $6.2 \mathrm{E}-04$ \\
${ }^{234} \mathrm{U}$ & $7.7 \mathrm{E}-03$ \\
${ }^{235} \mathrm{U}$ & $7.1 \mathrm{E}-03$ \\
${ }^{236} \mathrm{U}$ & $7.7 \mathrm{E}-03$ \\
${ }^{238} \mathrm{U}$ & $8.3 \mathrm{E}-03$ \\
${ }^{238} \mathrm{Pu}$ & $2.1 \mathrm{E}-03$ \\
${ }^{239 / 240} \mathrm{Pu}$ & $2.0 \mathrm{E}-03$ \\
${ }^{241} \mathrm{Pu}$ & $1.0 \mathrm{E}-01$ \\
\end{tabular}

a - from 40 CFR 61, Subpart I, Appendix E, Table 2 
Table 10-3. Inaccessible Soil Concentrations (pCi/g).

\begin{tabular}{|c|c|c|c|c|c|c|}
\hline Radionuclide & 100 B,D,K,N & $100 \mathrm{~F}, \mathrm{H}$ & 200 West Area & 200 East Area & 300 Area & 400 Area \\
\hline${ }^{3} \mathrm{H}$ & $1.4 \mathrm{E}+08$ & $7.4 \mathrm{E}+07$ & $3.7 \mathrm{E}+08$ & $2.0 \mathrm{E}+08$ & $9.5 \mathrm{E}+06$ & $1.4 \mathrm{E}+07$ \\
\hline${ }^{14} \mathrm{C}$ & $6.2 \mathrm{E}+05$ & $6.2 \mathrm{E}+05$ & $6.2 \mathrm{E}+05$ & $6.2 \mathrm{E}+05$ & $6.2 \mathrm{E}+05$ & $6.2 \mathrm{E}+05$ \\
\hline${ }^{55} \mathrm{Fe}$ & $9.7 \mathrm{E}+06$ & $9.7 \mathrm{E}+06$ & $3.6 \mathrm{E}+10$ & $1.9 \mathrm{E}+10$ & $1.0 \mathrm{E}+07$ & $1.4 \mathrm{E}+09$ \\
\hline${ }^{58} \mathrm{Co}$ & $9.8 \mathrm{E}+06$ & $9.8 \mathrm{E}+06$ & $8.1 \mathrm{E}+09$ & $4.3 \mathrm{E}+09$ & $1.2 \mathrm{E}+07$ & $3.1 \mathrm{E}+08$ \\
\hline${ }^{60} \mathrm{Co}$ & $9.9 \mathrm{E}+05$ & $9.9 \mathrm{E}+05$ & $5.7 \mathrm{E}+08$ & $3.0 \mathrm{E}+08$ & $1.0 \mathrm{E}+06$ & $9.9 \mathrm{E}+06$ \\
\hline${ }^{63} \mathrm{Ni}$ & $1.5 \mathrm{E}+08$ & $1.5 \mathrm{E}+08$ & $6.9 \mathrm{E}+09$ & $6.9 \mathrm{E}+09$ & $1.5 \mathrm{E}+08$ & $2.2 \mathrm{E}+08$ \\
\hline${ }^{90} \mathrm{Sr}^{*}$ & $8.3 \mathrm{E}+05$ & $8.3 \mathrm{E}+05$ & $2.2 \mathrm{E}+08$ & $1.2 \mathrm{E}+08$ & $8.3 \mathrm{E}+05$ & $8.4 \mathrm{E}+06$ \\
\hline${ }^{99} \mathrm{Tc}$ & $1.3 \mathrm{E}+07$ & $1.3 \mathrm{E}+07$ & $1.3 \mathrm{E}+07$ & $1.3 \mathrm{E}+07$ & $1.3 \mathrm{E}+07$ & $1.3 \mathrm{E}+07$ \\
\hline${ }^{106} \mathrm{Ru}^{*}$ & $2.0 \mathrm{E}+07$ & $2.0 \mathrm{E}+07$ & $5.7 \mathrm{E}+08$ & $3.0 \mathrm{E}+08$ & $1.5 \mathrm{E}+07$ & $2.2 \mathrm{E}+07$ \\
\hline${ }^{125} \mathrm{Sb}^{*}$ & $9.1 \mathrm{E}+06$ & $9.1 \mathrm{E}+06$ & $5.7 \mathrm{E}+09$ & $3.0 \mathrm{E}+09$ & $9.2 \mathrm{E}+06$ & $1.1 \mathrm{E}+08$ \\
\hline${ }^{129} \mathrm{I}$ & $2.8 \mathrm{E}+05$ & $2.8 \mathrm{E}+05$ & $2.8 \mathrm{E}+05$ & $2.8 \mathrm{E}+05$ & $2.2 \mathrm{E}+05$ & $2.8 \mathrm{E}+05$ \\
\hline${ }^{134} \mathrm{Cs}$ & $1.7 \mathrm{E}+04$ & $1.7 \mathrm{E}+04$ & $2.5 \mathrm{E}+08$ & $1.4 \mathrm{E}+08$ & $2.4 \mathrm{E}+04$ & $9.7 \mathrm{E}+06$ \\
\hline${ }^{137} \mathrm{Ce} *$ & $1.7 \mathrm{E}+04$ & $1.7 \mathrm{E}+04$ & $3.5 \mathrm{E}+08$ & $1.8 \mathrm{E}+08$ & $1.7 \mathrm{E}+04$ & $1.3 \mathrm{E}+07$ \\
\hline${ }^{144} \mathrm{Cs}^{*}$ & $1.4 \mathrm{E}+06$ & $1.4 \mathrm{E}+06$ & $7.4 \mathrm{E}+08$ & $4.0 \mathrm{E}+08$ & $1.9 \mathrm{E}+06$ & $2.8 \mathrm{E}+07$ \\
\hline${ }^{147} \mathrm{Pm}$ & $3.4 \mathrm{E}+07$ & $3.4 \mathrm{E}+07$ & $7.4 \mathrm{E}+09$ & $4.0 \mathrm{E}+09$ & $3.5 \mathrm{E}+07$ & $2.8 \mathrm{E}+08$ \\
\hline${ }^{152} \mathrm{Eu}$ & $4.5 \mathrm{E}+06$ & $4.5 \mathrm{E}+06$ & $1.2 \mathrm{E}+09$ & $6.2 \mathrm{E}+08$ & $4.6 \mathrm{E}+06$ & $4.5 \mathrm{E}+07$ \\
\hline${ }^{154} \mathrm{Eu}$ & $3.3 \mathrm{E}+06$ & $3.3 \mathrm{E}+06$ & $8.8 \mathrm{E}+08$ & $4.7 \mathrm{E}+08$ & $3.3 \mathrm{E}+06$ & $3.4 \mathrm{E}+07$ \\
\hline${ }^{155} \mathrm{Eu}$ & $2.3 \mathrm{E}+07$ & $2.3 \mathrm{E}+07$ & $6.9 \mathrm{E}+09$ & $3.7 \mathrm{E}+09$ & $2.4 \mathrm{E}+07$ & $2.6 \mathrm{E}+08$ \\
\hline${ }^{226} \mathrm{Ra}^{*}$ & $1.3 \mathrm{E}+05$ & $1.3 \mathrm{E}+05$ & $2.1 \mathrm{E}+05$ & $2.1 \mathrm{E}+05$ & $1.3 \mathrm{E}+05$ & $1.4 \mathrm{E}+05$ \\
\hline${ }^{227} \mathrm{Ac}^{*}$ & $2.4 \mathrm{E}+03$ & $2.4 \mathrm{E}+03$ & $5.4 \mathrm{E}+04$ & $2.9 \mathrm{E}+04$ & $1.4 \mathrm{E}+03$ & $2.1 \mathrm{E}+03$ \\
\hline${ }^{232} \mathrm{Th}^{*}$ & $2.0 \mathrm{E}+04$ & $2.0 \mathrm{E}+04$ & $2.0 \mathrm{E}+04$ & $2.0 \mathrm{E}+04$ & $4.7 \mathrm{E}+03$ & $7.1 \mathrm{E}+03$ \\
\hline${ }^{232} \mathrm{U}^{*}$ & $5.5 \mathrm{E}+04$ & $5.5 \mathrm{E}+04$ & $1.4 \mathrm{E}+05$ & $1.4 \mathrm{E}+05$ & $9.9 \mathrm{E}+03$ & $1.5 \mathrm{E}+04$ \\
\hline${ }^{233} \mathrm{U}$ & $4.5 \mathrm{E}+05$ & $4.5 \mathrm{E}+05$ & $4.5 \mathrm{E}+05$ & $4.5 \mathrm{E}+05$ & $6.7 \mathrm{E}+04$ & $1.0 \mathrm{E}+05$ \\
\hline${ }^{234} \mathrm{U}$ & $4.6 \mathrm{E}+05$ & $4.6 \mathrm{E}+05$ & $4.6 \mathrm{E}+05$ & $4.6 \mathrm{E}+05$ & $6.9 \mathrm{E}+04$ & $1.0 \mathrm{E}+05$ \\
\hline${ }^{235} \mathrm{U}^{*}$ & $4.9 \mathrm{E}+05$ & $4.9 \mathrm{E}+05$ & $4.9 \mathrm{E}+05$ & $4.9 \mathrm{E}+05$ & $7.3 \mathrm{E}+04$ & $1.1 \mathrm{E}+05$ \\
\hline${ }^{236} \mathrm{U}$ & $4.9 \mathrm{E}+05$ & $4.9 \mathrm{E}+05$ & $4.9 \mathrm{E}+05$ & $4.9 \mathrm{E}+05$ & $7.1 \mathrm{E}+04$ & $1.1 \mathrm{E}+05$ \\
\hline${ }^{238} \mathrm{U}^{*}$ & $4.7 \mathrm{E}+05$ & $4.7 \mathrm{E}+05$ & $4.7 \mathrm{E}+05$ & $4.7 \mathrm{E}+05$ & $7.7 \mathrm{E}+04$ & $1.2 \mathrm{E}+05$ \\
\hline${ }^{237} \mathrm{~Np}^{*}$ & $8.9 \mathrm{E}+02$ & $8.9 \mathrm{E}+02$ & $8.9 \mathrm{E}+02$ & $8.9 \mathrm{E}+02$ & $8.9 \mathrm{E}+02$ & $8.9 \mathrm{E}+02$ \\
\hline${ }^{238} \mathrm{Pu}$ & $1.3 \mathrm{E}+04$ & $1.3 \mathrm{E}+04$ & $8.8 \mathrm{E}+05$ & $4.7 \mathrm{E}+05$ & $1.3 \mathrm{E}+04$ & $3.4 \mathrm{E}+04$ \\
\hline${ }^{239} \mathrm{Pu}$ & $1.2 \mathrm{E}+04$ & $1.2 \mathrm{E}+04$ & $1.2 \mathrm{E}+04$ & $1.2 \mathrm{E}+04$ & $1.2 \mathrm{E}+04$ & $1.2 \mathrm{E}+04$ \\
\hline${ }^{240} \mathrm{Pu}$ & $1.2 \mathrm{E}+04$ & $1.2 \mathrm{E}+04$ & $1.4 \mathrm{E}+04$ & $1.4 \mathrm{E}+04$ & $1.2 \mathrm{E}+04$ & $1.2 \mathrm{E}+04$ \\
\hline${ }^{241} \mathrm{Pu}$ & $6.1 \mathrm{E}+05$ & $6.1 \mathrm{E}+05$ & $4.2 \mathrm{E}+07$ & $2.2 \mathrm{E}+07$ & $6.1 \mathrm{E}+05$ & $1.2 \mathrm{E}+06$ \\
\hline${ }^{241} \mathrm{Am}$ & $2.5 \mathrm{E}+04$ & $2.5 \mathrm{E}+04$ & $7.4 \mathrm{E}+05$ & $4.0 \mathrm{E}+05$ & $1.9 \mathrm{E}+04$ & $2.8 \mathrm{E}+04$ \\
\hline
\end{tabular}

Note: Asterisks mark nuclides with progeny that are assumed to be present in equilibrium amounts. However, ${ }^{234} \mathrm{U}$ was not included in the ${ }^{238} \mathrm{U}$ limits. For supporting references see WHC-SD-EN-TI-070, Soil Concentration Limits for Accessible and Inaccessible Areas. 
Table 10-4. Accessible Soil Concentrations (pCi/g).

\begin{tabular}{|c|c|c|c|c|c|c|}
\hline Radionuclide & 100 B,D,K,N & $100 \mathrm{~F}, \mathrm{H}$ & 200 West Area & 200 East Area & 300 Area & 400 Area \\
\hline${ }^{3} \mathrm{H}$ & $1.4 \mathrm{E}+08$ & $7.4 \mathrm{E}+07$ & $3.7 \mathrm{E}+08$ & $2.0 \mathrm{E}+08$ & $9.5 \mathrm{E}+06$ & $1.4 \mathrm{E}+07$ \\
\hline${ }^{14} \mathrm{C}$ & $6.2 \mathrm{E}+05$ & $6.2 \mathrm{E}+05$ & $6.2 \mathrm{E}+05$ & $6.2 \mathrm{E}+05$ & $6.2 \mathrm{E}+05$ & $6.2 \mathrm{E}+05$ \\
\hline${ }^{55} \mathrm{Fe}$ & $5.3 \mathrm{E}+05$ & $5.3 \mathrm{E}+05$ & $5.3 \mathrm{E}+05$ & $5.3 \mathrm{E}+05$ & $5.3 \mathrm{E}+05$ & $5.3 \mathrm{E}+05$ \\
\hline${ }^{58} \mathrm{Co}$ & $1.8 \mathrm{E}+01$ & $1.8 \mathrm{E}+01$ & $1.8 \mathrm{E}+01$ & $1.8 \mathrm{E}+01$ & $1.8 \mathrm{E}+01$ & $1.8 \mathrm{E}+01$ \\
\hline${ }^{60} \mathrm{Co}$ & $7.1 \mathrm{E}+00$ & $7.1 \mathrm{E}+00$ & $7.1 \mathrm{E}+00$ & $7.1 \mathrm{E}+00$ & $7.1 \mathrm{E}+00$ & $7.1 \mathrm{E}+00$ \\
\hline${ }^{63} \mathrm{Ni}$ & $2.5 \mathrm{E}+07$ & $2.5 \mathrm{E}+07$ & $2.5 \mathrm{E}+07$ & $2.5 \mathrm{E}+07$ & $2.5 \mathrm{E}+07$ & $2.5 \mathrm{E}+07$ \\
\hline${ }^{90} \mathrm{Sr}^{*}$ & $2.8 \mathrm{E}+03$ & $2.8 \mathrm{E}+03$ & $2.8 \mathrm{E}+03$ & $2.8 \mathrm{E}+03$ & $2.8 \mathrm{E}+03$ & $2.8 \mathrm{E}+03$ \\
\hline${ }^{99} \mathrm{Tc}$ & $1.0 \mathrm{E}+06$ & $1.0 \mathrm{E}+06$ & $1.0 \mathrm{E}+06$ & $1.0 \mathrm{E}+06$ & $1.0 \mathrm{E}+06$ & $1.0 \mathrm{E}+06$ \\
\hline${ }^{106} \mathrm{Ru}^{*}$ & $7.7 \mathrm{E}+01$ & $7.7 \mathrm{E}+01$ & $7.7 \mathrm{E}+01$ & $7.7 \mathrm{E}+01$ & $7.7 \mathrm{E}+01$ & $7.7 \mathrm{E}+01$ \\
\hline${ }^{125} \mathrm{Sb}^{*}$ & $3.7 \mathrm{E}+01$ & $3.7 \mathrm{E}+01$ & $3.7 \mathrm{E}+01$ & $3.7 \mathrm{E}+01$ & $3.7 \mathrm{E}+01$ & $3.7 \mathrm{E}+01$ \\
\hline${ }^{129} \mathrm{I}$ & $1.0 \mathrm{E}+04$ & $1.0 \mathrm{E}+04$ & $1.0 \mathrm{E}+04$ & $1.0 \mathrm{E}+04$ & $1.0 \mathrm{E}+04$ & $1.0 \mathrm{E}+04$ \\
\hline${ }^{134} \mathrm{Cs}$ & $1.0 \mathrm{E}+01$ & $1.0 \mathrm{E}+01$ & $1.0 \mathrm{E}+01$ & $1.0 \mathrm{E}+01$ & $1.0 \mathrm{E}+01$ & $1.0 \mathrm{E}+01$ \\
\hline${ }^{137} \mathrm{Cs}^{*}$ & $3.0 \mathrm{E}+01$ & $3.0 \mathrm{E}+01$ & $3.0 \mathrm{E}+01$ & $3.0 \mathrm{E}+01$ & $3.0 \mathrm{E}+01$ & $3.0 \mathrm{E}+01$ \\
\hline${ }^{144} \mathrm{Ce}^{*}$ & $3.3 \mathrm{E}+02$ & $3.3 \mathrm{E}+02$ & $3.3 \mathrm{E}+02$ & $3.3 \mathrm{E}+02$ & $3.3 \mathrm{E}+02$ & $3.3 \mathrm{E}+02$ \\
\hline${ }^{147} \mathrm{Pm}$ & $1.1 \mathrm{E}+06$ & $1.1 \mathrm{E}+06$ & $1.1 \mathrm{E}+06$ & $1.1 \mathrm{E}+06$ & $1.1 \mathrm{E}+06$ & $1.1 \mathrm{E}+06$ \\
\hline${ }^{152} \mathrm{Eu}$ & $1.5 \mathrm{E}+01$ & $1.5 \mathrm{E}+01$ & $1.5 \mathrm{E}+01$ & $1.5 \mathrm{E}+01$ & $1.5 \mathrm{E}+01$ & $1.5 \mathrm{E}+01$ \\
\hline${ }^{154} \mathrm{Eu}$ & $1.4 \mathrm{E}+01$ & $1.4 \mathrm{E}+01$ & $1.4 \mathrm{E}+01$ & $1.4 \mathrm{E}+01$ & $1.4 \mathrm{E}+01$ & $1.4 \mathrm{E}+01$ \\
\hline${ }^{155} \mathrm{Eu}$ & $6.3 \mathrm{E}+02$ & $6.3 \mathrm{E}+02$ & $6.3 \mathrm{E}+02$ & $6.3 \mathrm{E}+02$ & $6.3 \mathrm{E}+02$ & $6.3 \mathrm{E}+02$ \\
\hline${ }^{226} \mathrm{Ra}^{*}$ & $1.0 \mathrm{E}+01$ & $1.0 \mathrm{E}+01$ & $1.0 \mathrm{E}+01$ & $1.0 \mathrm{E}+01$ & $1.0 \mathrm{E}+01$ & $1.0 \mathrm{E}+01$ \\
\hline${ }^{227} \mathrm{Ac}^{*}$ & $1.0 \mathrm{E}+01$ & $1.0 \mathrm{E}+01$ & $1.0 \mathrm{E}+01$ & $1.0 \mathrm{E}+01$ & $1.0 \mathrm{E}+01$ & $1.0 \mathrm{E}+01$ \\
\hline${ }^{232} \mathrm{Th}^{*}$ & $5.9 \mathrm{E}+00$ & $5.9 \mathrm{E}+00$ & $5.9 \mathrm{E}+00$ & $5.9 \mathrm{E}+00$ & $5.9 \mathrm{E}+00$ & $5.9 \mathrm{E}+00$ \\
\hline${ }^{232} \mathrm{U}^{*}$ & $1.0 \mathrm{E}+01$ & $1.0 \mathrm{E}+01$ & $1.0 \mathrm{E}+01$ & $1.0 \mathrm{E}+01$ & $1.0 \mathrm{E}+01$ & $1.0 \mathrm{E}+01$ \\
\hline${ }^{233} \mathrm{U}$ & $6.3 \mathrm{E}+02$ & $6.3 \mathrm{E}+02$ & $6.3 \mathrm{E}+02$ & $6.3 \mathrm{E}+02$ & $6.3 \mathrm{E}+02$ & $6.3 \mathrm{E}+02$ \\
\hline${ }^{234} \mathrm{U}$ & $6.3 \mathrm{E}+02$ & $6.3 \mathrm{E}+02$ & $6.3 \mathrm{E}+02$ & $6.3 \mathrm{E}+02$ & $6.3 \mathrm{E}+02$ & $6.3 \mathrm{E}+02$ \\
\hline${ }^{235} \mathrm{U}^{*}$ & $1.7 \mathrm{E}+02$ & $1.7 \mathrm{E}+02$ & $1.7 \mathrm{E}+02$ & $1.7 \mathrm{E}+02$ & $1.7 \mathrm{E}+02$ & $1.7 \mathrm{E}+02$ \\
\hline${ }^{236} \mathrm{U}$ & $6.7 \mathrm{E}+02$ & $6.7 \mathrm{E}+02$ & $6.7 \mathrm{E}+02$ & $6.7 \mathrm{E}+02$ & $6.7 \mathrm{E}+02$ & $6.7 \mathrm{E}+02$ \\
\hline${ }^{238} \mathrm{U}^{*}$ & $3.7 \mathrm{E}+02$ & $3.7 \mathrm{E}+02$ & $3.7 \mathrm{E}+02$ & $3.7 \mathrm{E}+02$ & $3.7 \mathrm{E}+02$ & $3.7 \mathrm{E}+02$ \\
\hline${ }^{237} \mathrm{~Np} *$ & $4.8 \mathrm{E}+01$ & $4.8 \mathrm{E}+01$ & $4.8 \mathrm{E}+01$ & $4.8 \mathrm{E}+01$ & $4.8 \mathrm{E}+01$ & $4.8 \mathrm{E}+01$ \\
\hline${ }^{238} \mathrm{Pu}$ & $2.1 \mathrm{E}+02$ & $2.1 \mathrm{E}+02$ & $2.1 \mathrm{E}+02$ & $2.1 \mathrm{E}+02$ & $2.1 \mathrm{E}+02$ & $2.1 \mathrm{E}+02$ \\
\hline${ }^{239} \mathrm{Pu}$ & $1.9 \mathrm{E}+02$ & $1.9 \mathrm{E}+02$ & $1.9 \mathrm{E}+02$ & $1.9 \mathrm{E}+02$ & $1.9 \mathrm{E}+02$ & $1.9 \mathrm{E}+02$ \\
\hline${ }^{240} \mathrm{Pu}$ & $1.9 \mathrm{E}+02$ & $1.9 \mathrm{E}+02$ & $1.9 \mathrm{E}+02$ & $1.9 \mathrm{E}+02$ & $1.9 \mathrm{E}+02$ & $1.9 \mathrm{E}+02$ \\
\hline${ }^{241} \mathrm{Pu}$ & $1.0 \mathrm{E}+04$ & $1.0 \mathrm{E}+04$ & $1.0 \mathrm{E}+04$ & $1.0 \mathrm{E}+04$ & $1.0 \mathrm{E}+04$ & $1.0 \mathrm{E}+04$ \\
\hline${ }^{241} \mathrm{Am}$ & $1.8 \mathrm{E}+02$ & $1.8 \mathrm{E}+02$ & $1.8 \mathrm{E}+02$ & $1.8 \mathrm{E}+02$ & $1.8 \mathrm{E}+02$ & $1.8 \mathrm{E}+02$ \\
\hline
\end{tabular}

Note: Asterisks mark nuclides with progeny that are assumed to be present in equilibrium amounts. However, ${ }^{234} \mathrm{U}$ was not included in the ${ }^{238} \mathrm{U}$ limits. For supporting references see WHC-SD-EN-TI-070, Soil Concentration Limits for Accessible and Inaccessible Areas. 


\subsection{DATA SUMMARY METHODS}

Measuring any physical quantity has some degree of inherent uncertainty. This uncertainty results from the combination of all possible inaccuracies in the measurements process, including such factors as the reading of the result, the calibration of the measuring device, and numerical rounding errors.

In this report, individual radioactive measurements are accompanied by a plus or minus $( \pm)$ value, which represents the total propagated analytical uncertainty (or two-sigma counting error). The two-sigma counting error gives information on what the measurement might be if the same sample were counted again under identical conditions. The two-sigma counting error implies that approximately $95 \%$ of the time, a recount of the same sample would give a value within plus or minus the two-sigma counting error at the value reported.

Values in the tables that are less than the minimum detectable activity indicate that the reported result might have come from a sample with no radioactivity. Such values are considered below the detection limits of the measuring instrument. Also note that each radioactive measurement must have the random background radioactivity of the measuring instrument subtracted; therefore, negative results are possible, especially when the sample has very little radioactivity.

Reported averages also are accompanied by a plus or minus $( \pm)$ value, which represents two standard deviations from the mean. If the data fluctuate randomly, this is a measure of the uncertainty in the estimated average of the data because of this randomness.

Where averages of averages are reported, the plus or minus $( \pm)$ value represents two standard errors of the mean.

The mean, $\mathrm{X}$, is computed as:

$$
\mathrm{X}=\frac{1}{\mathrm{n}} \sum_{\mathrm{i}=1}^{\mathrm{n}} \mathrm{X}_{\mathrm{i}}
$$

where $\mathrm{X}_{\mathrm{i}}$ is the $\mathrm{i}^{\text {th }}$ measurement and $\mathrm{n}$ is the number of measurements.

The standard error of the mean was computed as:

$$
S E=\sqrt{\frac{S^{2}}{n}}
$$

where $S^{2}$, the variance of the $n$ measurements, was computed as: 


$$
\mathrm{S}_{\mathrm{M}}^{2}=\frac{1}{\mathrm{n}-1} \sum_{\mathrm{i}=1}^{\mathrm{n}}\left(\mathrm{X}_{\mathrm{i}}-\mathrm{X}\right)^{2}
$$

This estimator, $S^{2}$, includes the variance among the samples and the counting variance. The estimated $S^{2}$ occasionally may be less than the average counting variance. 


\subsection{REFERENCES}

10 CFR 835, “Occupational Radiation Protection,” Code of Federal Regulations, U.S. Department of Energy.

40 CFR 61, Subpart H, "National Emission Standards for Emissions of Radionuclides Other Than Radon From Department of Energy Facilities," Code of Federal Regulations, U.S. Environmental Protection Agency.

40 CFR 61, Subpart I, "National Emission Standards for Radionuclide Emissions from Federal Facilities Other Than Nuclear Regulatory Commission Licensees and not covered by Subpart H," Code of Federal Regulations, U.S. Environmental Protection Agency.

40 CFR 61, Appendix E, Table 2, "Compliance Procedures Methods for Determining Compliance with subpart I; Concentration Levels for Environmental Compliance," Code of Federal Regulations, U.S. Environmental Protection Agency.

40 CFR 264, "Standards for Owners and Operators of Hazardous Waste Treatment, Storage, and Disposal Facilities," Code of Federal Regulations, U.S. Environmental Protection Agency.

Comprehensive Environmental Response, Compensation, and Liability Act of 1980, Public Law 96-510, as amended, 42 USC 9601 et seq.

DOE, 2008, Environmental Protection Program, DOE Order 450.1A, Cancels 450.1, U.S. Department of Energy, Washington, D.C., June 4, 2008.

DOE, 2007, Environment, Safety, and Health Reporting Manual, DOE Manual 231.1-1A, Chg 2, U.S. Department of Energy, June 12, 2008.

DOE, 2001, Radioactive Waste Management, DOE Order 435.1, Chg 1, U.S. Department of Energy, Washington, D.C.

DOE, 1993, Radiation Protection of the Public and the Environment, DOE Order 5400.5, Chg 2, U.S. Department of Energy, Washington, D.C.

FH, 2007, PHMC Radiological Control Manual, HNF-5173, Rev. 5, Fluor Hanford, Inc., Richland, Washington.

FH, 2008, Environmental Protection Desk Instruction, Near-Facility Environmental Monitoring, EP-DI-611, Fluor Hanford, Inc., Richland, Washington.

FWSO. 2008. Operational Environmental Monitoring, FWSO-OEM-001, EnergySolutions Federal Services, Inc., Western Operations, Richland, Washington. 
McKinney, S. M., 2008, Near-Facility Environmental Monitoring Quality Assurance Project Plan, HNF-EP-0538-11, Fluor Hanford, Inc., Richland, Washington.

PNNL, 2009a, Hanford Site Environmental Report for Calendar Year 2008, PNNL-18427, Pacific Northwest National Laboratory, Richland, Washington.

PNNL, 2009b, Hanford Site Environmental Report for Calendar Year 2008, PNNL-18427, APP. 1, Pacific Northwest National Laboratory, Richland, Washington.

Resource Conservation and Recovery Act of 1976. 1976. Public Law 94-580, as amended, 90 Stat. 2795, 42 USC 6901 et seq.

Rittman, P. D., 1992, Soil Concentration Limits for Accessible and Inaccessible Areas, WHC-SD-EN-TI-070, Rev. 0, Westinghouse Hanford Company, Richland, Washington.

WAC 173-303-17, "Dangerous Waste Regulations; Recycling Processes Involving solid Wastes," Washington Administrative Code, Olympia, Washington.

WAC 173-303-40, “Dangerous Waste Regulations; Definitions,” Washington Administrative Code, Olympia, Washington.

WAC 173-401-710, “Operating Permit Regulations,” Washington Administrative Code, Olympia, Washington.

WAC 246-247, "Radiation Protection—Air Emissions,” Washington Administrative Code, Olympia, Washington. 


\section{DISTRIBUTION}

No. of

Copies

\section{OFFSITE}

P Kurt R. Campbell

U.S. Fish and Wildlife Service Moses Lake Field Office

Moses Lake, WA 98837

P Stuart Harris

Confederated Tribes of the Umatilla Indian Reservation

P.O. Box 638

Pendleton, OR 97801

P Greg Hughes

Hanford Reach National Monument

64 Maple Drive

Burbank, WA 99323

P R. Jim

Yakama Nation

Environmental Restoration/Waste

Management Program

P.O. Box 151

Toppenish, WA 98948

P J. E. McDonald

Energy Northwest

P.O. Box 968

MD 1020

Richland, WA 99352

P A. Palmer

U.S. Ecology, Inc.

P.O. Box 638

Richland, WA 99352
No. of

Copies

5CD Washington State Department of Health Office of Radiation Protection

Radioactive Air Emissions Program

309 Bradley Blvd., Suite 201

Richland, WA 99352

ATTN: R. S. Acselrod

J. C. Berkey

P. J. Martell, Manager

M. Priddy

J. W. Schmidt

CD Washington State Department of Health Office of Radiation Protection

Radioactive Air Emissions Program

P.O. Box 47827

Olympia, WA 98504-7827

ATTN: K. Attebery

4CD Washington State Department of Health Office of Radiation Protection

Environmental Monitoring and Assessment

P.O. Box 47825

Olympia, WA 98504-7825

ATTN: L. Albin

A. W. Conklin

J. L. Erickson

D. McBaugh

2P U.S. Environmental Protection Agency, Region 10

1200 Sixth Avenue

Seattle, WA 98101

ATTN: J. M. Leitch OAQ-107

R. W. Poeton OAQ-107 
PNNL-18427, APP. 2

No. of

Copies

2P Walla Walla University Library

Technical Services

104 S. College Avenue

College Place, WA 99324-1159

ATTN: Mark Copsey

\section{ONSITE}

E. J. Antonio (CD)

K3-54

J. G. April (CD)

J.A. Bates (CD)

T.G. Beam (CD)

L. E. Borneman (CD)

O. L. Bostic (CD)

E. M. Bowers (P, CD)

M. W. Bowman (CD)

H.C. Boynton (P)

R. C. Brunke (CD)

M. E. Burandt (CD)

S. T. Burnum (CD)

D. H. Chapin (CD)

G.M. Crummel (CD)

B. L. Curn (CD)

E. B. Dagan (CD)

A. K. Dasgupta (CD)

J. W. Demers (P, CD)

R. L. Dirkes (P)

J. N. Diven (CD)

B. J. Dixon (CD)

J. J. Dorian (P, CD)

M. C. Dorsey (P)

J.P. Duncan (CD)

J.N. Earley (CD)

R. E. Elder (CD)

R. H. Engelmann (CD)

B. G. Erlandson (CD)

J. D. Fancher (CD)

T. W. Ferns (P, CD)

J. Flack (P, CD)

J. R. Franco (CD)

B. G. Fritz (CD)
K6-66

H8-45

E6-28

T6-03

H4-02

A2-15

S5-31

S5-31

H8-15

H6-60

A3-04

A5-11

R1-51

H4-02

A3-04

S3-28

A5-17

K6-75

G3-28

H8-45

E6-28

E6-28

K6-85

E6-28

$\mathrm{X} 4-02$

H8-45

H4-02

L6-06

A5-15

A5-15

A3-04

K6-75
No. of

Copies

W. M. Glines (CD)

A5-17

L. M. Hay (P)

G2-02

W. M. Hayward (P)

N3-10

R. D. Hildebrand (CD)

A6-38

J. S. Hill (CD)

N. A. Homan (CD)

H4-02

H8-45

D. G. Horton (CD)

D. E. Jackson (CD)

R3-50

A4-52

M. F. Jarvis (CD)

A5-15

A. R. Johnson (5P)

R3-12

W.F. Johnson (CD)

R. D. Julian (P)

C. J. Kemp (CD)

R. J. Landon (CD)

H4-22

S3-25

H6-60

H4-21

S. P. Luttrell (CD)

R3-50

E6-28

R3-50

R.W. Oldham (P)

S2-42

A5-17

B. M. Pangborn (CD)

K6-75

T2-03

R1-51

E6-28

R3-32

E6-28

S2-42

K6-75

A2-15

T6-02

H4-22

K3-54

$\mathrm{X} 4-01$

T3-11

E6-28

R3-12

S7-83

H6-60

N3-30

E6-28

K3-75

A5-15

X4-01 
No of

Copies

S.R. Weil (CD)

A5-15

D. J. Wilcox (CD)

A4-52

J. W. Wilde (P)

E6-28

J. G. Woolard (P)

H4-21

D. M. Yasek (CD)

L7-10

DOE Public Reading Room (4P) ～H2-53

Hanford Site Administrative

Record (P)

H6-08

Hanford Technical Library (2P)

P8-55

LMSI Central Files (P)

B1-07 